NUREG/CR-6133

ANL-93/32

\title{
Fragmentation and Quench Behavior of Corium Melt Streams in Water
}

Manuscript Completed: January 1994

Date Published: February 1994

Prepared by

B. W. Spencer, K. Wang, C. A. Blomquist, L. M. McUmber, J. P. Schneider*

Argonne National Laboratory

9700 S. Cass Avenue

Argonne, IL 60439

\section{Prepared for}

Division of Systems Research

Office of Nuclear Regulatory Research

U.S. Nuclear Regulatory Commission

Washington, DC 20555-0001

NRC FIN A2262

*Department of Nuclear Engineering, University of Illinois, Urbana, IL 61801 


\title{
Fragmentation and Quench Behavior of Corium Melt Streams in Water
}

$$
\text { by }
$$

B. W. Spencer, S. K. Wang, C. A. Blomquist, L. M. McUmber and J. P. Schneider

\begin{abstract}
The interaction of molten core materials with water has been investigated for the pour stream mixing mode. This interaction plays a crucial role during the later stages of in-vessel core melt progression inside a light water reactor such as during the TMI-2 accident. The key issues which arise during the molten core relocation include: i) the thermal attack and possible damage to the RPV lower head from the impinging molten fuel stream and/or the debris bed, ii) the molten fuel relocation pathways including the effects of redistribution due to core support structure and the reactor lower internals, $i i i)$ the quench rate of the molten fuel through the water in the lower plenum, iv) the steam generation and hydrogen generation during the interaction, $v$ ) the transient pressurization of the primary system, and $v i$ ) the possibility of a steam explosion. In order to understand these issues, a series of six experiments (designated CCM-1 through -6) was performed in which molten corium passed through a deep pool of water in a long, slender pour stream mode. Results discussed include the transient temperatures and pressures, the rate and magnitude of steam/hydrogen generation, and the posttest debris characteristics.
\end{abstract}




\section{CONTENTS}

ABSTRACT ........................ i

EXECUTIVE SUMMARY ................... xvii

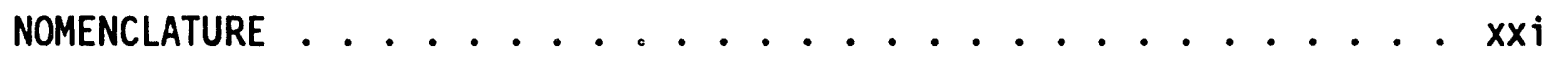

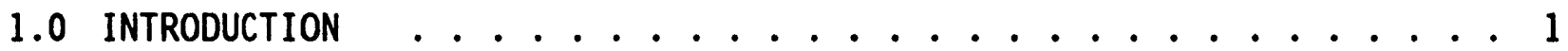

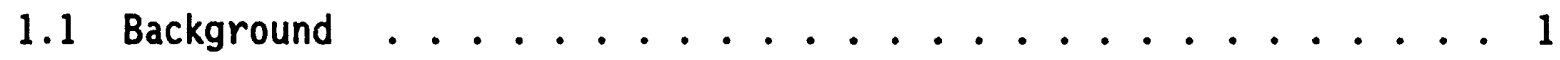

1.2 Objectives ................. 2

1.3 Scaling Considerations ............ 3

1.3.1 Water Depth/Jet Diameter ........... 3

1.3.2 Mass Length .............. 4

1.3.3 Fuel/Coolant Mass Ratio, Coolant Subcooling ....... 5

1.3.4 Vessel Ambient Pressure ............. 5

1.4 Experimental Approach . . . . . . . . . . . 6

1.5 Related Studies ................ 7

1.5.1 Simulant Material Tests ............ 7

1.5.2 Corium-Water Thermal Interaction (CWTI) Studies ....8 8 1.5.2.1 Test CWTI-9 . . . . . . . . 8

1.5.2.2 Test CWTI-10 .............. 9

1.5.3 Sandia National Laboratories Jet Program . . . . . . . 9

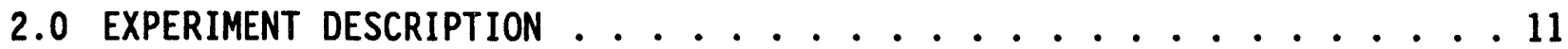

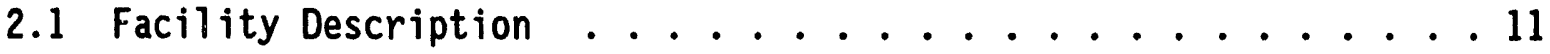

2.2 Apparatus Description .............. 11

2.2.1 Thermite Vessel/Injector ............11

2.2.2 Interaction Vesset .............. 17

2.2.3 Expansion Vesse1 .................. 17

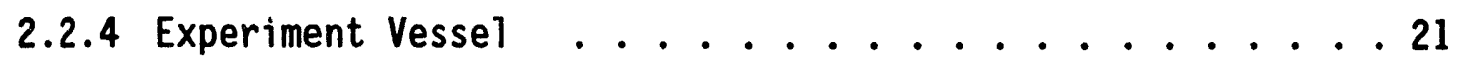

2.2.5 Ancillary Systems .............. . . 26 


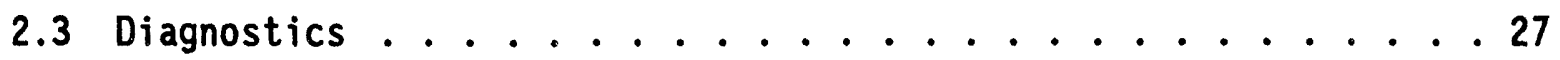

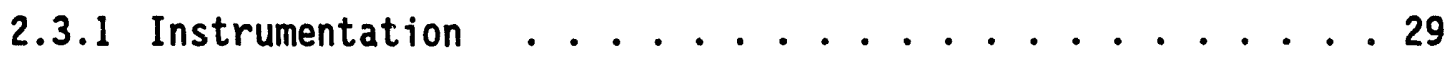

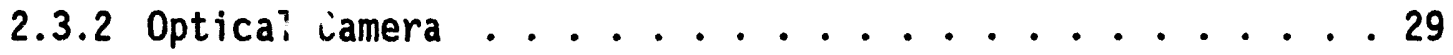

2.3.3 Flash X-ray Cine System .............. 31

2.3.4 Data Acquisition .................. 31

2.4 Test Procedures . . . . . . . . . . . . . 32

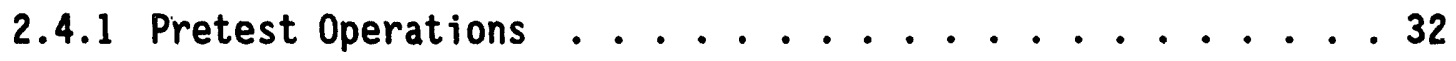

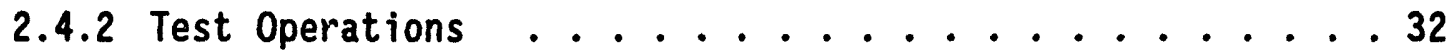

2.4.3 Posttest Operation .................. 33

2.5 Corium Characterization ........................

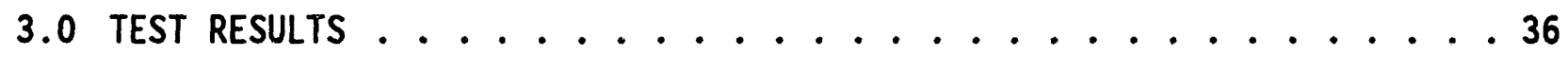

3.1 Summary of Experiment objectives ............ . 36

3.2 Test Initial Conditions . . . . . . . . . . . 36

3.3 Description of Test Results .............. 43

3.3 .1 Test $\mathrm{CCM}-1 \ldots \ldots . \ldots . \ldots . \ldots 47$

3.3 .2 Test $\mathrm{CCM}-2 \ldots \ldots \ldots \ldots . \ldots . \ldots . \ldots 52$

3.3 .3 Test $\mathrm{CCM}-3 \ldots \ldots \ldots 0$

3.3 .4 Test $\mathrm{CCM}-4 \ldots \ldots \ldots 6$

3.3 .5 Test $\mathrm{CCM}-5 \ldots \ldots \ldots \ldots \ldots$

3.3 .6 Test $\mathrm{CCM}-6 \ldots \ldots \ldots 77$

3.4 Debris Characterization .............. 88

3.4 .1 Test $\mathrm{CCM}-1 \ldots \ldots . \ldots . \ldots 88$

3.4 .2 Test $\mathrm{CCM}-2 \ldots \ldots \ldots . \ldots \ldots$

3.4 .3 Test $\mathrm{CCM}-3 \ldots \ldots . \ldots . \ldots . \ldots 94$

3.4 .4 Test $\mathrm{CCM}-4 \ldots \ldots \ldots$. . . . . . . . . . 107

3.4 .5 Test $\mathrm{CCM}-5 \ldots \ldots \ldots . \ldots . \ldots . \ldots 107$

3.4 .6 Test $\mathrm{CCM}-6 \ldots \ldots \ldots \ldots$ 
3.4.7 Particle Size Distribution ............ . 113

3.5 Gas Sample Analysis . . . . . . . . . . . . 120

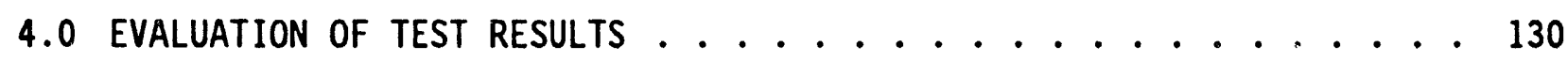

4.1 Hydrogen Generation/Metal 0xidation ........... 130

4.2 Steam Generation and Water Heating . . . . . . . . 132

4.3 Test Behavior . . . . . . . . . . . . . . . 147

4.3 .1 Test $\mathrm{CCM}-1 \ldots \ldots . . \ldots . . \ldots 147$

4.3 .2 Test CCM-2 ................... 148

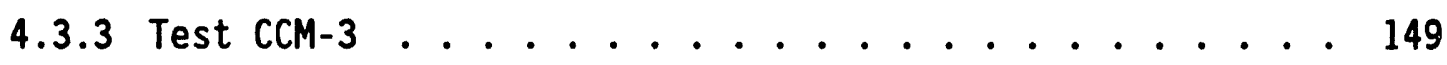

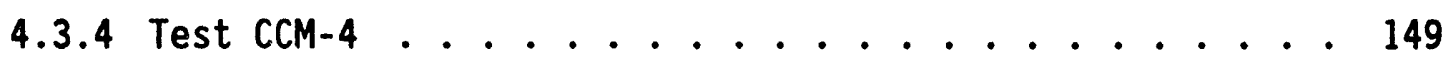

4.3 .5 Test CCM 5 . . . . . . . . . . . . . 149

4.3 .6 Test $\mathrm{CCM}-6 \ldots \ldots \ldots \ldots$

4.4 Comparison of Break-up Length and Mass Length . . . . . . . 153

5.0 MODELING OF JET BREAKUP ................... 161

5.1 Review of Previous Studies on Jet Breakup ........... 161

5.2 THIRMAL Computer code ............... . . 164

5.3 Comparisons of CCM Test Results and THIRMAL Predictions . . . 177

6.0 SUMMARY OF FINDINGS .................... 213

References ....................... 215

Acknowledgements .................. 219 Appendices

Appendix A. Instrumentation Location ............ A-1

A.ppendix B. Data for CCM-1 .............. B-1

Appendix C. Data for CCM-2 ............. C-1

Appendix D. Data for CCM-3 ............. D-1

Appendix E. Data for CCM-4 . . . . . . . . . E E-1

Appendix F. Data for CCM-5 .............. F-1

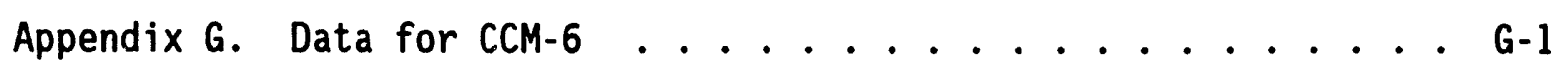




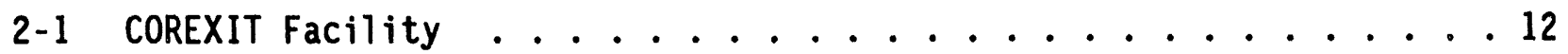

2-2 Schematic Illustration of COREXIT Facility ........... 13

2-3 4-kg Thermite Vessel with Slide Gate Assembly ......... 15

2-4 4-kg Thermite Vessel with Diaphragm Assembly .......... 15

2-5 12-kg Thermite Vessel with Diaphragm Assembly .......... 16

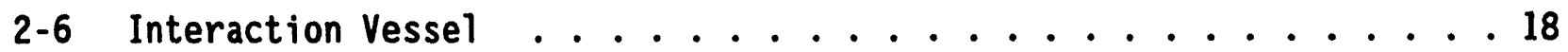

2-7 Expansion Vessel with Interaction Vessel for Tests CCM-1, 3 and 4 . 19

2-8 Expansion Vessel with Interaction Vessel for Test CCM-2 . . . . . 20

2-9 View Looking Down into Bottom Region of Expansion Vesse1 . . . . 22

2-10 Instrument and Gas Sampling Assembly for the Expansion Vessel

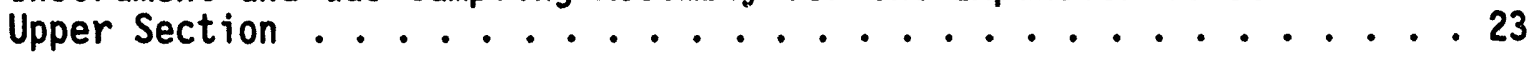

2-11 Test Apparatus for Experiment CCM-6 . . . . . . . . . . 24

2-12 Experiment Vessel Internal Structure for Test CCM-5 and $6 \ldots 25$

2-13 Impingement-Plate Holder .................. 28

2-14 Enthalpy of Thermite Generated Corium Referenced to $20^{\circ} \mathrm{C} \ldots 35$

3-1 Comparison of Thermite Vessel Pressure (PTS-1) with Interaction Vessel Pressure (PTS-2) for Experiment CCM-1 ..........440

3-2 Comparison of Thermite Vessel Pressure (PTS-1) with Interaction Vessel Pressure (PTS-2) for Experiment CCM-2 ..........40

3-3 Comparison of Thermite Vessel Pressure (PTS-1) with Interaction Vessel Pressure (PTS-2) for Experiment CCM-3 ..........41

3-4 Comparison of Thermite Vessel Pressure (PTS-1) with Interaction Vessel Pressure (PTS-2) for Experiment CCM-4 ......... 41

3-5 Comparison of Thermite Vessel Pressure (PTS-1) with Experiment Vessel Pressure (PTS-9) for Experiment CCM-5 ..........42

3-6 Comparison of Thermite Vessel Pressure (PTS-1) with Experiment Vessel Pressure (PTS-9) for Experiment CCM-6 . . . . . . . 42

3-7 X-Ray Photography of Corium Jets Emerging from the Impingement Holes for Experiment CCM-2 .............. . 54

3-8 View of Vapor Separator in the Thermite Vessel Used in Experiment

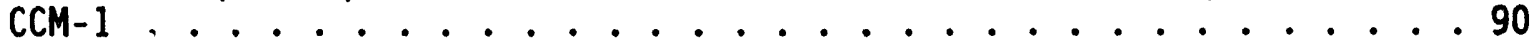


3-9 View of Expansion Region and Porous Grid Plate in the Thermite Vessel Used in Experiment CCM-1 .............. 90

3-10 View of Bottom of Thermite Vessel Used in Experiment CCM-1 . . . .91

3-11 View of Bottom of the Expansion Vessel Used in Experiment CCM-1 . . .91

3-12 View of Underside of Thermite Vesse1 Head for Experiment CCM-1 . . . 92

3-13 View of Debris in the Bottom of the Thermite Vessel Used in Experi-

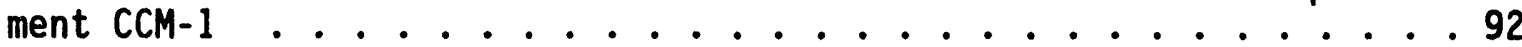

3-14 Close-Up View of Debris from the Interaction Vessel Base for Experiment $\mathrm{CCM}-1$................... . . 93

3-15 Agglomerated Particle Debris from the Interaction Vessel Base for Experiment $\mathrm{CCM}-1$. . . . . . . . . . . . . 95

3-16 Variation of Particle Size and Shape from the Interaction Vessel Debris Bed for Experiment CCM-1 . . . . . . . . . . . . 96

3-17 Debris Particle from the Interaction Vessel Base for Experiment

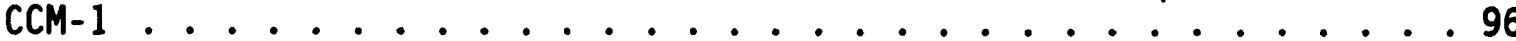

3-18 Spherically Shaped Debris Particle from the Interaction Vessel Base for Experiment CCM-1 . . . . . . . . . . . . . . . . .997

3-19 Oblong Debris Particle from the Interaction Vessel Base for Experi-

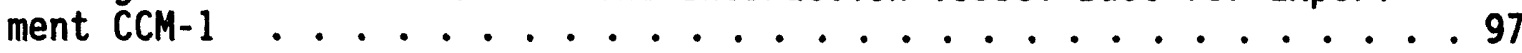

3-20 Hollow Particles from the Interaction-Vessel Debris Bed for Experiment CCM-1 ....................... 98

3-21 Bottom of Impingement Plate in the Interaction Vessel for Experiment CCM-2 ....................... 98

3-22 Underside of Interaction Vessel Head for Experiment CCM-2 . . . . .99

3-23 Debris on Side Wall of Interaction Vessel for Experiment CCM-2 . . . 99

3-24 Debris Adhering to a Thermocouple and the Side Wall of the Interaction Vessel for Experiment CCM-2 ........... 100

3-25 View of Debris Surface on the Base of the Interaction Vessel for Experiment CCM-2 ............... 100

3-26 Close-up View of Debris Surface on the Base of the Interaction Vessel for Experiment CCM-2 ............. 101

3-27 View of Debris Bed on the Interaction Vessel Base After Sectioning for Experiment CCM-2 . . . . . . . . . . . . 101

3-28 Cross-Sectional View of Debris Bed on the Interaction Vessel Base for Experiment CCM-2 .............. 102 
3-29 View of the Bottom of the Expansion Vessel for Experiment CCM-2 . . 102

3-30 Thin Crust Layer of Corium in the Thermite Vessel Used for Experiment CCM-3 ................. 103

3-31 Debris in the Bottom of the Expansion Vessel for Experiment CCM-3 - 103

3-32 Debris Attached to the Level Probes in the CCM-3 Interaction Vesse1 ................... 104

3-33 Surface of Debris Bed in the Bottom of the Interaction Vessel for Experiment $\mathrm{CCM}-3$

3-34 View of Debris-Bed Accumulation on the CCM-3 Interaction Vessel Base

3-35 Sectioned Debris Bed on the Base of the Interaction Vessel for Experiment $\mathrm{CCM}-3$

3-36 Debris Particle with Metallic Inclusion from the Base of the Interaction Vessel for Experiment $\mathrm{CCM}-3$. . . . . . . . . . . 106

3-37 Agglomerated Debris Particle from the CCM-3 Interaction Vessel Base ................... 108

3-38 Oblong Debris Particle with Holes from the CCM-3 Interaction Vessel Base

3-39 View of Debris in the Bottom of the Expansion Vessel for Experiment

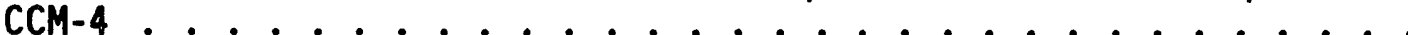

3-40 Corium Debris at the Inlet to the Pipeway in the Interaction Vessel for Experiment

3-41 Surface View of the Debris on the Interaction Vessel Base for Experiment $\mathrm{CCM}-4$

3-42 Debris Bed on the Base of the Interaction Vessel for Experiment CCM-4

3-43 Close-Up View of the Debris Bed on the Interaction-Vessel Base for Experiment $\mathrm{CCM}-1$

3-44 View of the Inside of the Thermite Vessel Used in Experiment CCM-5

3-45 Posttest View into the CCM-5 Experiment Vesse1 . . . . . . . 112

3-46 Debris Deposited on the Thermocouples and Void Detectors in the CCM-5 Experiment Vesse1

3-47 Surface of Debris Bed from Experiment CCM-5 . . . . . . . . . 114

3-48 Close-Up View of Debris Bed Surface from Experiment CCM-5 . . . . . 114 
3-49 Close-Up View of Debris Surface with Scale-like Particles for Experiment CCM-5 ................ 115

3-50 Sectioned View of Debris Bed from Experiment CCM-5 . . . . . . 115

3-51 Agglomerated Particie from the Debris Bed for Experiment CCM-5 . . 116

3-52 Hollow Particles from the Debris Bed for Experiment CCM-5 . . . . 116

3-53 View of the Experiment-Vessel Bottom for Experiment CCM-5 . . . . 117

3-54 View of the Experiment-Vessel Bottom for Experiment CCM-6 . . . . 117

3-55 Debris on A Thermocouple in Experiment CCM-6 . . . . . . . 118

3-56 Surface of the Debris Bed in Experiment CCM-6 . . . . . . . 118

3-57 Sectioned View of the Debris Bed for Experiment CCM-6 . . . . . . 119

3-58 Particle-Size Distribution of Debris . . . . . . . . . 121

4-1 Estimated Water Level in CCM-2 . . . . . . . . . . 134

4-2 Estimated Water Levet in CCM-3 . . . . . . . . . . . . . 134

4-3 Observed Water Level in CCM-5 . . . . . . . . . . . . 135

4-4 Estimated Steam Generation Rate for CCM-2 Using Water Swe 11 Data ................... . 137

4-5 Estimated Steam Generation Rate for CCM-3 Using Water Swell Data ................... 137

4-6 Estimated Steam Generation Rate for CCM-5 Using Water Swe11 Data .................... 138

4-7 Estimated Steam Generation Rate for CCM-1 . . . . . . . . . . 140

4-8 Estimated Steam Generation Rate for CCM-2 . . . . . . . . . 140

4-9 Estimated Steam Generation Rate for CCM-3 . . . . . . . . . . 141

4-10 Estimated Steam Generation Rate for CCM-4 . . . . . . . . . 141

4-11 Estimated Steam Generation Rate for CCM-5 . . . . . . . . . . 142

4-12 Estimated Steam Generation Rate for CCM-6 . . . . . . . . . . 142

4-13 Thermal Response of the Thermocouples in the Interaction Vessel Water for Experiment $\mathrm{CCM}-1$............... 145

4-14 Thermal Response of the Thermocouple in the Interaction-Vesse1 Water for Experiment CCM-2 ............. 146 
4-15 Thermal Response of the Thermocouple in the Interaction-Vesse1 Water for Experiment CCM-3

4-16 Thermal Response of the Thermocouple in the Interaction-Vessel Water for Experiment $\mathrm{CCM}-4$.............. 151

4-17 Thermal Response of the Axial Centerline Thermocouples in the Experiment-Vessel Water for Experiment CCM-5 . . . . . . . . 152

4-18 Void Detector Response for Experiment CCM-5 . . . . . . . . . . 154

4-19 Thermal Response of the Thermocouples in the Water of the Experiment Vessel for Experiment CCM-5

4-20 Thermal Response of the Thermocouples on the Axial Centerline of the Water in the Experiment Vessel for Experiment CCM- 6 . . . . . 156

4-21 Void Detector Response for Experiment CCM-6 . . . . . . . . . . 157

4-22 Thermal Response of the Thermocouples in the Water of the Experiment Vessel for Experiment CCM- 6

4-23 Comparison of Mass Length and Breakup Length . . . . . . . . . 159

5-1 Configurations of Mixing Zone . . . . . . . . . . . . . 166

5-2 Film Boiling on a Spherical Particle . . . . . . . . . . 171

5-3 Space Nodalization of THIRMAL Code . . . . . . . . . . . 176

5-4 THIRMAL Prediction of Mixing Zone . . . . . . . . . . . . . 178

5-5 Comparison of Predicted Mixing Zone and Thermocouple Data at $t=0.25 \mathrm{sec}$

5-6 Comparison of Predicted Mixing Zone and Thermocouple Data at $t=0.35 \mathrm{sec}$

5-7 Comparison of Predicted Mixing Zone and Thermocouple Data at $t=0.45 \mathrm{sec}$

5-8 Comparison of Predicted Mixing Zone and Thermocouple Data at

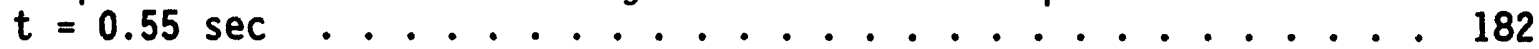

5-9 Comparison of Predicted Mixing Zone and Thermocouple Data at $t=0.65 \mathrm{sec}$

5-10 THIRMAL Prediction of Local Void Fraction in Mixing Zone . . . . 185

5-11 THIRMAL Prediction of Melt Volume Fractions in Mixing Zone at

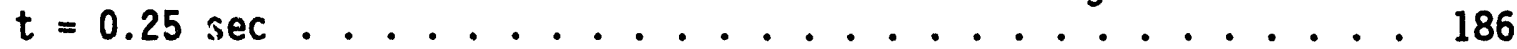

5-12 THIRMAL Prediction of Melt Volume Fractions in Mixing Zone at $t=0.35 \mathrm{sec}$ 
5-13 THIRMAL Prediction of Melt Volume Fractions in Mixing Zone at $\mathrm{t}=0.45 \mathrm{sec} \ldots \ldots \ldots . \ldots . \ldots 188$

5-14 THIRMAL Prediction of Melt Volume Fractions in Mixing Zone at $\mathrm{t}=0.55 \mathrm{sec} \ldots \ldots . \ldots . \ldots 189$

5-15 THIRMAL Prediction of Melt Volume Fractions in Mixing Zone at $\mathrm{t}=0.65 \mathrm{sec} \ldots \ldots \ldots 190$

5-16 THIRMAL Prediction of Melt Volume Fractions in Mixing Zone at $\mathrm{t}=1.15 \mathrm{sec} \ldots \ldots \ldots \ldots . . \ldots \ldots$

5-17 THIRMAL Prediction of Melt Temperatures in Mixing Zone at $\mathrm{t}=0.25 \mathrm{sec} \ldots \ldots \ldots . \ldots \ldots 3$

5-18 THIRMAL Prediction of Melt Temperatures in Mixing Zone at $\mathrm{t}=0.35 \mathrm{sec} \ldots \ldots . \ldots . \ldots 194$

5-19 THIRMAL Prediction of Melt Temperatures in Mixing Zone at $\mathrm{t}=0.45 \mathrm{sec} \ldots \ldots \ldots . \ldots \ldots$

5-20 THIRMAL Prediction of Melt Temperatures in Mixing Zone at $\mathrm{t}=0.55 \mathrm{sec} \ldots \ldots \ldots . \ldots \ldots$

5-21 THIRMAL Prediction of Melt Temperatures in Mixing Zone at $t=0.65 \mathrm{sec} \ldots \ldots \ldots 197$

5-22 THIRMAL Prediction of Melt Temperatures in Mixing Zone at $t=1.15 \mathrm{sec} \ldots \ldots \ldots 19 . \ldots \ldots$

5-23 THIRMAL Prediction of Water and Vapor Temperatures in Mixing Zone at $\mathrm{t}=0.25 \mathrm{sec} \ldots \ldots 199$

5-24 THIRMAL Prediction of Water and Vapor Temperatures in Mixing Zone at $t=0.35 \mathrm{sec} \ldots \ldots 200$

5-25 THIRMAL Prediction of Water and Vapor Temperatures in Mixing Zone at $\mathrm{t}=0.45 \mathrm{sec} \ldots \ldots 201$

5-26 THIRMAL Prediction of Water and Vapor Temperatures in Mixing Zone at $t=0.55 \mathrm{sec} \ldots \ldots 202$

5-27 THIRMAL Prediction of Water and Vapor Temperatures in Mixing Zone at $\mathrm{t}=0.65 \mathrm{sec} \ldots \ldots 203$

5-28 THIRMAL Prediction of Water and Vapor Temperatures in Mixing Zone at $\mathrm{t}=1.15 \mathrm{sec} \ldots \ldots 204$

5-29 THIRMAL Prediction of Steam and Hydrogen Generation Rates . . . . 206

5-30 THIRMAL Prediction of Total Steam and Hydrogen Generation . . . . 207

5-31 Comparison of Predicted and Measured System Pressure in Experiment Vesse1 ................. 209 
5-32 THIRMAL Prediction of Mass Accumulations of Melt Particulates on Vessel Bottom ................ 210

5-33 THIRMAL Predictions of Average Temperatures of Me1t Particulates

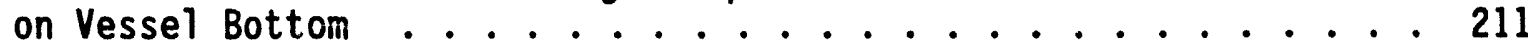

5-34 THIRMAL Prediction of Debris Particle Size Distribution . . . . . 212

\section{List of Tables}

2.1 Number of Thermocouples and Pressure Transducers Used in CCM Experiments .................. . . 30

3.1 Initial Conditions for the Interaction and Expansion Experi-

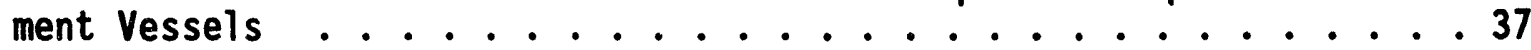

3.2 Initial Conditions for the Thermite Vessel and for Corium

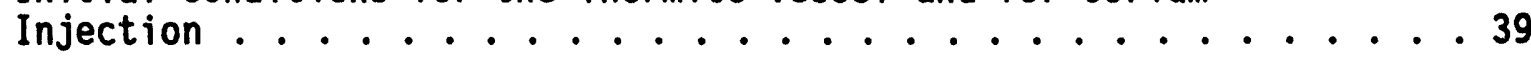

3.3 Timing Sequence for Experiment CCM-1 ............. 44

3.4 Timing Sequance for Experiments $\mathrm{CCM}-2,3$ and $4 \ldots . . \ldots 45$

3.5 Timing Sequence for Experiments CCM-5 and $6 \ldots . \ldots 46$

3.6 Experiment CCM-1 Events Observed in the Expansion Vessel from the Hycam Film ................. 48

3.7 Summary of Pressure Data for Experiment CCM-1 . . . . . . . . . . 49

3.8 Summary of Temperature Data for Experiment CCM-1 . . . . . . . 50

3.9 Experiment $\mathrm{CCM}-2$ Events Observed in the Interaction Vesse1 from the $\mathrm{X}$-ray Film .................. 53

3.10 Experiment CCM-2 Events in the Expansion Vessel as Viewed from the Hycam Film ................ 55

3.11 Summary of Pressure Data for Experiment CCM-2 . . . . . . . . . 57

3.12 Summary of Temperature Data for Experiment CCM-2 . . . . . . . 58

3.13 Events Observed from the Hycam Film for Experiment CCM-3 . . . . . 62

3.14 Summary of Pressure Data for Experiment $\mathrm{CCM}-3 \ldots \ldots 3$

3.15 Summary of Temperature Data for Experiment $\mathrm{CCM}-3 \ldots \ldots 4$

3.16 Summary of Pressure Data for Experiment CCM-4 . . . . . . . . . . 67

3.17 Summary of Temperature Data for Experiment $\mathrm{CCM}-4 \ldots 69$ 
3.18 Events Observed at the Water Surface from the Hycam Film for

Experiment CCM-5 ................ 72

3.19 Events Observed at $699 \mathrm{~mm}$ Below the Water Surface from the Hycam Film for Experiment $\mathrm{CCM}-5$.............. . 74

3.20 Summary of Pressure Data for Experiment CCM-5 . . . . . . . . 75

3.21 Summary of Temperature Data for Experiment $\mathrm{CCM}-5 \ldots 78$

3.22 Events Observed at the Water Surface from the Hycam Film for

Experiment $\mathrm{CCM}-6 \ldots \ldots . \ldots . \ldots 81$

3.23 Events Observed at $699 \mathrm{~mm}$ Below the Water Surface from the Hycam Film for Experiment $\mathrm{CCM}-6 \ldots . . . . . . . .82$

3.24 Summary of Pressure Data for Experiment $\mathrm{CCM}-6 \ldots . . . . . . .83$

3.25 Summary of Temperature Data for Experiment $\mathrm{CCM}-6 \ldots 84$

3.26 Debris Distribution ................. 89

3.27 Gas Analyses . . . . . . . . . . . . . . 123

3.28 Gas/Vapor Conditions Before, During, and After the Experiment . . 126

3.29 Adjusted Pre- and Posttest Gas/Vapor Composition . . . . . . . 128

4.1 Summary of Hydrogen Generation from the Oxidation of Corium Metals by Steam ................. 131

4.2 Summary of Steam Generation Based on PVT Data and Gas Analyses . 133

4.3 Summary of Steam Generation and Water Heatup During Jet Fall Stage.................... 144 


\section{Executive Summary}

A series of six tests was performed using the COREXIT facility at Argonne National Laboratory to investigate the phenomena associated with the quenching of molten core debris in a preexisting pool of water. The motivation for this work was to better understand the corium relocation stage of the TMI accident when $-20,000 \mathrm{~kg}$ of melt is thought to have relocated from the crucible region in the core to the vessel bottom head in about $90 \mathrm{sec}$. This relocation took place largely if not entirely beneath water and was significantly influenced by massive structures interconnected by various openings in the relocation pathway. The key questions during late, in-vessel relocation which arose in light of the TMI accident and which are of general importance pertain to:

1.) the mode of melt relocation from the original core region to the vessel bottom head (i.e., release rate, corium mass and composition, corium temperature, primary pathways);

2.) the corium/water mixing mode, steam and hydrogen generation rates, and whether or not it is possible for an energetic steam explosion to occur in the vessel bottom head region;

3.) the state of the corium as it arrives on the bottom head (i.e., melt impingement vs particle settling vs combination);

4.) the transient heatup and degradation of the head and/or penetrations which might influence early failure; and

5.) the coolability of the debris on the bottom head if a continuous source of water could be provided.

The experimental work described in this report primarily addresses points (2) - (4). The overall objective of the work was to establish a database relevant to the behavior of molten corium draining through water in a film boiling regime, and to use the information obtained together with separate effects and simulant materials tests to develop the THIRMAL model of corium thermal interaction with water. The specific objectives included:

1.) Characterize the mixing, heat transfer, and chemical reaction behavior for a corium stream flowing through water.

2.) Generate a corium debris bed representative of the quench-related solidification of the corium as it drains through water in a pourstream mode. Characterize the debris bed in terms of particle sizes, particle shapes, overall bed voidage, and density.

3.) Examine the effect of water subcooling on the mixing, steam generation, and hydrogen generation.

4.) Examine the effect of multi-stream jet geometry.

5.) Assess relevancy to actual reactor systems, including effects of scale and multiple streams. 
The tests were performed using a melt of reactor-material corium $\left(60 \% \mathrm{UO}_{2}\right.$, $16 \% \mathrm{ZrO}_{2}, 24 \%$ SSt) at a temperature of -2800 C using an exothermitic chemical reaction. The corium melt was released from a meit generator vessel via an orifice to drain into varying mass and depth of water. The controlled variables included the corium mass $(-4-12 \mathrm{~kg})$, the released stream diameter $(25.4,50.8 \mathrm{~mm})$, number of streams $(1,4)$, and water subcooling $(0-45 \mathrm{C})$. The primary scaling parameter was the (extended) length-to-diameter ratio of the corium stream in relation to the available depth of water; numerous other parameters were also scaled to be applicable to in-vessel relocation scenarios.

The system pressure was roughly atmospheric, which is much smaller than the in-vessel pressure during the TMI core relocation. However, the most important effect of the elevated system pressure is to cause the specific volume of the steam to be smaller than for atmospheric conditions, reducing the vapor flux out of the fuel/coolant mixture. This lessening of the vapor flux was reasonably approximated in the tests with subcooled water.

The key phenomena addressed in this investigation relate to the melt-water interaction, i.e., melt stream breakup, melt-water mixing, quench rate, steam generation, hydrogen generation, vessel pressurization, and debris characterizations. In order to quantify those key phenomena, various pressure transduces, thermocouples, level probes, and void probes were used to measure the pressure/temperature transients and the mixture extent. Posttest gas samples were taken, and the corium debris was examined. Test data, $X$-ray motion pictures, optical motion pictures, and posttest examinations were integrated into an interpretation of the tests events; namely, rates of quench during the corium fall stage, efficiency of quench, efficiency of fall stage solidification, applicable breakup length, extent of oxidation, and the extent of breakup and intermixing with water. The tests were performed with an inert containment atmosphere in order to measure the mass of hydrogen produced.

Test results are summarized as follows:

1.) Results indicate the most important parameters affecting the relocation phenomena are the number of corium streams, their size in relation to available water depth $\left(L / d_{i j}\right)$, and water subcooling (net stream generation);

2.) A two-stage quenching processes was indicated -- the initial stage during the transient fall through the melt (and possible spreading on the bottom) when the quench rate and steam pressurization were rapid, followed by a prolonged stage of lesser heat removal from the bed or corium layer formed on the bottom.

3.) Based on the system pressurization data, water temperature, and poot swell data, a corium fall stage quench fraction ranging from 55 to 72\% occurred with subcooled water, while the quench fraction was considerably lower with saturated water owing largely to vapor blanketing effects.

4.) Incremental oxidation of the metal constituent of the corium measured 2 to $35 \%$ of the maximum possible during the relocation. The higher oxidation occurred in saturated water.

5.) For tests in which data showed a definite change in mélt penetration 
velocity, the jet breakup lengths were found to range from $\sim 10$ to 19 jet (initial) diameters. Tests with large initial diameter of $50.8 \mathrm{~mm}$ did not appear to reach full breakup condition either because of limited melt mass or limited pool depth;

6.) The multi-jet geometry used in CCM-2 led to a significantly reduced quench fraction. In the X-ray film, the volume between the jets appeared to be highly voided, reducing the melt/water interaction. This produced a lower overall heat transfer rate, and hence a lower fall stage quench fraction.

7.) The posttest debris examination indicated that a debris bed of substantially quenched arld solidified particles was formed on the vessel bottom. Of all the tests, only in multi-stream test CCM-2 did the corium melt reagglomerate, indicating very limited quench and solidification during the jet fall stage.

8.) No ablation of the vessel bottom was found in any of the tests. Test data indicated the debris bed was cooled faster if the overlying water was subcooled.

9.) The characteristic particle dimensions ranged from -1 to $5 \mathrm{~mm}$ and showed a log-normal distribution.

10.) No steam explosion occurred in any of the tests.

Overal1, the Corium - Coolant Mixing (CCM) tests have provided an excellent database of relevant information which needs to be modeled by the THIRMAL code. Details of corium jet breakup and mixing could not be directly observed, however. 


\section{Nomenclature}

$A=$ area of mixing zone (Eq. $(5-1)), \mathrm{m}^{2}$

$A_{p}=\begin{gathered}\text { area of } \\ \text { motion }, \mathrm{m}^{2}\end{gathered}$ article projected on the plane normal to the direction of

$C=$ flow coefficient for the thermite-injector opening

$C_{d} \quad=$ particle drag coefficient

$c_{0}=$ distribution parameter

$C_{p}=$ heat capacity, $\mathrm{J} / \mathrm{kg} \cdot \mathrm{K}$

$C_{\text {sf }}=a$ constant

$d_{p}=$ spherical-particle diameter, $m$

$d_{B} \quad=$ bubble diameter, $m$

$E_{c}=$ corium energy, $M J$

$E_{R} \quad=$ chemical-reaction energy, $M J$

$E_{v} \quad=$ water-vaporization energy, $M J$

$E_{w} \quad=$ water-heating energy, $M J$

$e_{p}=$ internal energy of particle, $J$

$g=$ acceleration of gravity, $\mathrm{m} / \mathrm{s}^{2}$

$h_{p t}=$ heat transfer coefficient between particle and water, $\mathrm{W} / \mathrm{m}^{2} \cdot \mathrm{K}$

$h_{p v}=$ heat transfer coefficient between particle and vapor, $W / m^{2} \cdot K$

$h_{F B}=f i l m$ boiling heat transfer coefficient, $W / m^{2} \cdot K$

$h_{\text {MB }}=$ nucleate heat transfer coefficient, $\mathrm{W} / \mathrm{m}^{2} \cdot \mathrm{K}$

$h_{v l}=$ heat transfer coefficient between vapor and 1 iquid, $W / \mathrm{m}^{2} \cdot \mathrm{K}$

$h_{c f}=$ corium specific enthalpy at final conditions, $\mathrm{MJ} / \mathrm{kg}$

$h_{c i}=$ corium specific enthalpy at initial conditions, $\mathrm{MJ} / \mathrm{kg}$

$h_{f g}=$ latent heat of water vaporization, $\mathrm{MJ} / \mathrm{kg}$

$h_{w i}=$ water enthalpy at initial temperature, $\mathrm{MJ} / \mathrm{kg}$

$h_{w m}=$ water enthalpy at maximum temperature, $M J / k g$ 
$h_{w s}=$ water enthalpy at its saturation temperature, $\mathrm{MJ} / \mathrm{kg}$

$j_{v}=$ vapor superficial velocity, $\mathrm{m} / \mathrm{s}$

$j_{v}^{+}=j_{v} /\left(\sigma g \Delta \rho / \rho_{l}^{2}\right)^{1 / 4}$; dimensionless vapor superficial velocity

$k=$ wave number, $1 / m$; thermal conductivity, $W /(m \cdot K)$

$k_{p}=$ most probable wave number, $1 / m$

$\mathrm{m}=$ particle mass, $\mathrm{kg}$

$m_{c}=$ mass of injected corium, $\mathrm{kg}$

$\dot{m}_{c}=$ condensation rate per unit length, $\mathrm{kg} / \mathrm{m} \cdot \mathrm{sec}$

$\dot{\mathrm{m}}_{\mathrm{e}}=$ evaporation rate per unit length, $\mathrm{kg} / \mathrm{m} \cdot \mathrm{sec}$

$M_{p} \quad=$ mass of particle, $\mathrm{kg}$

$m_{i} \quad=$ mass of component " $i ", k g$

$m_{s t}=$ steam mass, $\mathrm{kg}$

$\mathrm{m}_{\mathrm{w}} \quad=$ mass of water, $\mathrm{kg}$

$M_{i} \quad=$ molecular weight of component, $i ", g / g-m o l e$

$N_{\mu l}=\mu_{1} /\left(\rho_{1} \sigma(\sigma / g \Delta \rho)^{1 / 2}\right)^{1 / 2} ;$ dimensionless liquid viscosity

$\mathrm{n}=$ total number of system moles, g-mole

$n_{i} \quad=$ number of moles of component " $i ", g$-mole

$\mathrm{P} \quad=\quad$ system pressure, $\mathrm{MPa}$

$\operatorname{Pr} \quad=$ Prandt 1 number

$\mathrm{Pe} \quad=$ Peclet number

$\dot{\mathrm{q}}_{\text {int }}=$ particle internal heat generation rate, $\mathrm{W} / \mathrm{m}^{3}$

$q_{\text {sus }}^{\prime \prime}=$ heat flux conducted into subcooled liquid, $\mathrm{J} / \mathrm{m}^{2}$

$R=$ gas constant, $8.314\left(10^{-6}\right) \mathrm{MPa} \cdot \mathrm{m}^{3} /(\mathrm{g}$ mole $\cdot \mathrm{K})$

$R_{M z}=$ radius of mixing zone, $m$

$\operatorname{Re}=$ Reynolds number 


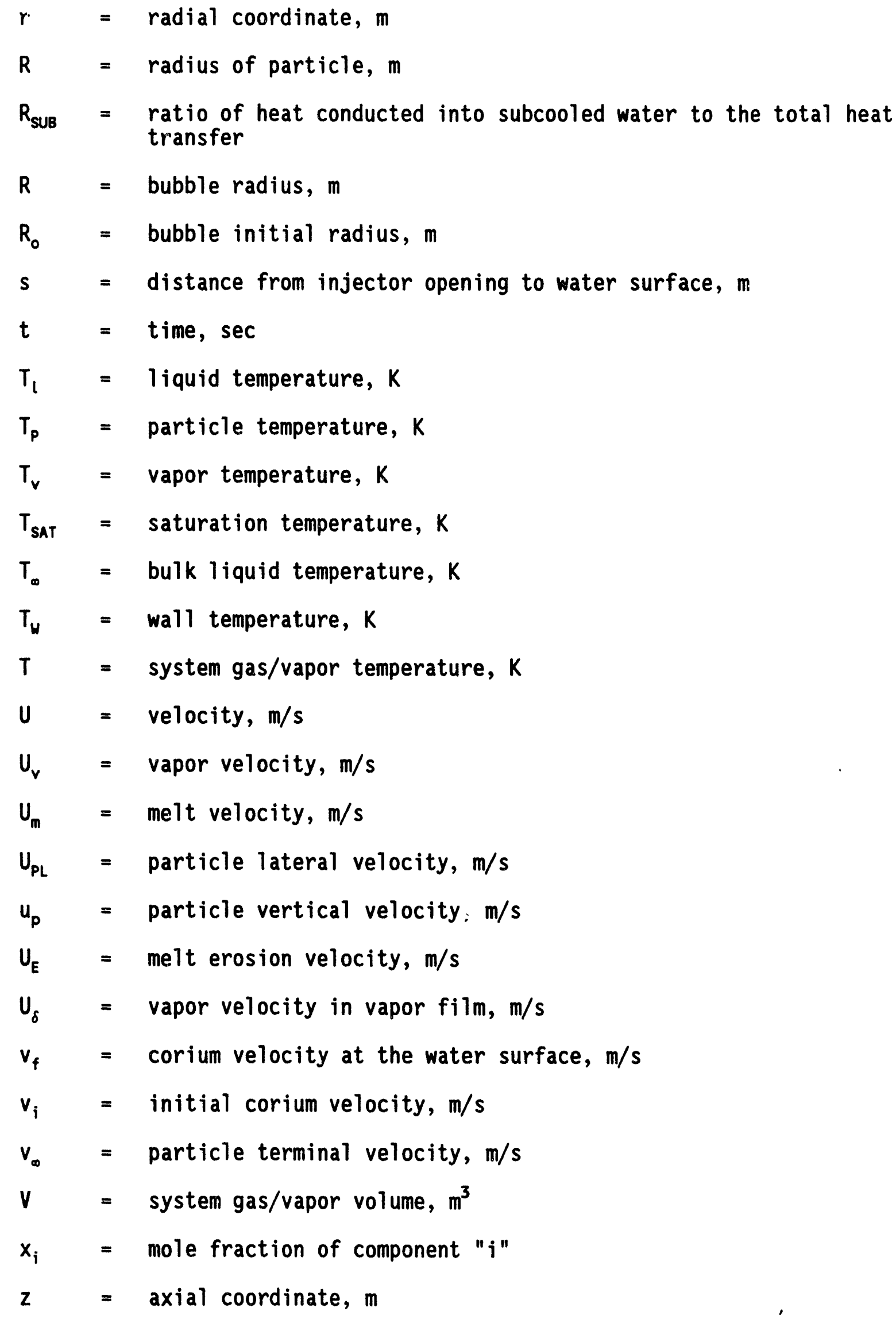


Greek Symbols

$\alpha \quad=$ void fraction

$\rho=$ density, $\mathrm{kg} / \mathrm{m}^{3}$

$\sigma=$ surface tension, $\mathrm{N} / \mathrm{m}$

$\mu \quad=\quad$ viscosity, $\mathrm{Pa} \cdot \mathrm{S}$

$\epsilon=$ Stefan-Botlzmann constant for radiation heat transfer, $\mathrm{W} / \mathrm{m}^{2} \cdot \mathrm{K}^{4}$

$\delta=$ vapor film thickness, $m$

xxiv 


\subsection{Background}

The accident at Three Mile Island led to programs in the US aimed at improving our understanding of the phenomena of severe accidents and our ability to model the processes occurring during severe accident sequences. This report addresses one of the numerous processes that came to light during investigation of that accident; i.e., the significant relocation of core materials to the reactor pressure vessel (RPV) lower head, which best current estimates place at about 20 tonnes (44,000 pounds). During the accident, molten core materials denoted as corium (a mixture of $\mathrm{UO}_{2}, \mathrm{ZrO}_{2}$, steel and other core and structural constituents) initially froze during the downward relocation process, forming a sort of crucible. Eventually the crucible was breached, and superheated melt was ejected into the surrounding (initially intact) core structure. The ejected melt ablated through structural barriers and subsequently drained downward as submerged (in water) jets by complex paths through holes of the various support members, including plates of the lower internal structure, before collecting upon the bottom of the vessel. Molten control rod materials may have flowed to the vessel lower head by a more direct downward draining path, al so through holes in the plates of the lower internal structure, but presently this remains to be confirmed. In both cases, a characteristic of the relocation process was the accumulation and spreading of melt on horizontal surfaces coupled with downward flow through available holes in the surfaces. Under such circumstances the melt may or may not actually fill the entire cross section of the flow holes through which it drains, dependent upon the rate of melt migration. At TMI, it is likely that the accumulation and spreading on the core former plates was sufficiently rapid that the melt did form streams of the same characteristic diameter as the holes in those plates $(-33 \mathrm{~mm}$ dia). However, due to spreading, this was probably not the case for the larger size holes in the lower internal structure.

The physical picture of the melt relocation involves the gravity flow of streams or rivulets of corium through water, the characteristic size of the stream being the same as or less than the size of the holes in the structural members through which they pass. The streams pass through a geometry-specific depth of water before collecting on the next horizontal surface, ultimately the vessel lower head. The key questions which arise during this process are:

1. How large is the area over which the corium mass is redistributed during the relocation?

2. To what extent does a corium stream (i.e., jet or rivulet) quench as it flows through the water?

3. What are the net steam generation and hydrogen generation during this relocation?

4. How much heat is transferred to the vessel lower head as the core materials collect on that surface? Does this heatup and/or ablation affect vessel integrity?

5. What is the morphology of the core materials collected on the vessel lower head? Is the debris likely to be coolable given a continuing presence of water? 
6. Does the relocation process create an explosive fuel/coolant mixture? If a steam explosion occurs, how does it affect the accident sequence?

The physical picture addressed above differs appreciably from concepts of melt relocation utilized in risk studies performed shortiy after the TMI accident. In those studies, the core support structure was envisioned to fail catastrophically, releasing essentially the entire core in a molten state to abruptly mix with water in the vessel lower head. Such sequences were envisioned to result in an energetic steam explosion and/or to cause abrupt failure of the reactor vessel.

\section{2 objectives}

The purpose of the present work is to investigate the physical process of melt stream relocation and quench in water. The work evolves from the picture of the relocation process as thought to have occurred during the TMI accident. However, it is additionally relevant to sequences of gradual rather than abrupt release of melt from its confining crucible, not only from the upper region of the crucible but also if localized meltout or cracking should release melt from the bottom region of the crucible.

The general objective of the study is to establish a data base relevant to the behavior of a stream of molten corium draining through water in a film boiling regime. The specific objectives include:

1. Characterize the mixing, heat transfer, and chemical reaction behavior for a corium stream flowing through water.

2. Generate a corium debris bed representative of the quench-related solidification of the corium as it drains through water in a pourstream mode. Characterize the debris bed in terms of particle sizes, particle shapes, overall bed voidage, and various density measures.

3. Examine the effect of water subcooling on the mixing, steam generation, and hydrogen generation results.

4. Examine the effect of multi-jet geometry.

5. Assess relevancy to actual reactor systems, including effects of scale and inter-stream spacing.

Applications for test results:

1. Evaluation of the progression and effects of the TMI core relocation.

2. Generic application to the best-estimate evaluations of core-melt progressions and consequences in LWR severe accidents.

3. Input for the development of models describing the mixing and heat transfer between corium and water as the melt migrates through localized breaches of the degraded boundary (crust) and flows downward into water in the lower head region. 
4. Data for evaluation of the potential coolability of the heatgenerating debris bed formed in this manner.

5. Data for evaluation of the thermal attack on the vessel lower head due to impingement and accumulation of corium.

\subsection{Scaling Considerations}

The CCM experiments were designed to model the breakup and quench of corium streams in a severe core melt accident, such as that at TMI-2. The essential scaling parameters for such an interaction, i.e., molten jet penetration of a volatile liquid, were not known a priori. Therefore, for the purpose of designing these experiments, scaling parameters had to be chosen based upon a combination of analyses of isothermal jet breakup available in the literature and assumptions regarding the effects of coolant boiling.

\subsubsection{Nater Depth/Jet Diameter}

A liquid mass, moving in a different ambient fluid, will break into irops. The time required for complete breakup of an inviscid liquid mass cari be estimated from dimensional analysis of the Navier-Stokes equation (e.g., Levich) ${ }^{1}$ :

$$
\tau-\frac{a}{U} \sqrt{\frac{\rho}{\rho^{\prime}}}
$$

where:

$$
\begin{aligned}
& \rho=\text { liquid density, } \\
& \rho^{\prime}=\text { ambient fluid density, } \\
& \tau=\text { breakup time, } \\
& a=\text { liquid mass characteristic dimension, and } \\
& U=\text { liquid mass/ambient fluid relative velocity. }
\end{aligned}
$$

The distance traveled by the liquid during breakup, i.e., the breakup length, $L_{B}$, is roughly the product of breakup time and initial liquid velocity:

$$
L_{B} \sim \sqrt{\frac{\rho}{\rho^{\prime}}}
$$

The dettailed analyses of jet breakup given by Taylor, ${ }^{2}$ Levich, ${ }^{1}$ and Epstein and Fauske $^{3}$ have all obtained essentially this relation. 
This result indicates that jet breakup length for a steady flow may be scaled by jet diameter (since the only characteristic dimension of a steady jet is its diameter). Similarly, if the jet penetrates an ambient fluid whose depth is less than the jet breakup length, the degree of breakup will be proportional to the fluid depth, scaled by jet diameter. Therefore, for a model system having the same jet/ambient fluid pair as the prototype (e.g., CCM and TMI), a degree of jet breakup comparable to that in the prototype should be obtained if the ambient fluid (e.g., water) depth in the model, scaled by jet diameter, is comparable to that of the prototype.

\subsubsection{Mass Length}

In earlier simulant material tests, ${ }^{4}$ it was observed that during initial penetration, jet material is ejected primarily from the blunted leading edge (see Fig. 5-1). At some depth, the jet trailing column I reaks, and the blunted leading edge disperses. Thereafter, the length of the continuous jet remains roughly constant, as long as the jet flow continues. That is, a quasi-steady breakup length is reached. A certain volume of jet material must be expended (i.e., ejected from the leading edge) during initial penetration; if this exceeds the volume of jet material available, the quasi-steady breakup length cannot be reached. In this case, the jet must be characterized by both its diameter and initial mass.

During initial penetration, the penetration velocity of the jet leading edge is less than that of the jet stream, because some jet fluid flows laterally out of the leading edge. The volumetric rate of jet fluid ejection from the leading edge is given approximately by

$$
\dot{v} \approx\left(U_{j}-U_{p}\right) \frac{\pi D^{2}}{4}
$$

where

$$
\begin{aligned}
\dot{V} & =\begin{array}{l}
\text { volumetric rate of jet fluid ejection from the jet leading } \\
\text { edge, }
\end{array} \\
& =\text { jet stream velocity, } \\
U_{J} & =\text { leading edge penetration velocity, and } \\
U_{P} & =\text { jet diameter. }
\end{aligned}
$$

Assuming these quantities to be constant, the length of time that penetration can be sustained is limited by the volume of jet fluid available, i.e.,

$$
T \approx \frac{V}{\dot{v}}
$$


where

$$
\begin{aligned}
& T=\text { penetration flow duration, and } \\
& V=\text { available jet fluid volume. }
\end{aligned}
$$

Let $L_{B}$ be the depth at which the entire molten volume has been expended. It may be expressed by:

$$
L_{B} \approx U_{p} T \approx \frac{U_{p}}{\left(U_{j}-U_{p}\right)} \frac{4 V}{\pi D^{2}}
$$

For the inviscid, isothermal liquid jet penetration studied by Hopkins and Robertson, 5 the ratio of penetration velocity to jet stream velocity depends only on the densities of the jet and penetrated liquids. In this case, for a given liquid pair,

$$
L_{8} \approx \frac{4 V}{\pi D^{2}}
$$

when $V$ is sufficiently small. The parameter,

$$
L_{m}=\frac{4 V}{\pi D^{2}}
$$

will be referred to here as the mass length.

This analysis suggests that for modeling jet penetration that is limited by the available jet fluid, the mass length scaled by jet diameter in the model and prototype should be comparable.

\subsubsection{Fuel/Coolant Mass Ratio, Coolant Subcooling}

The rate at which fuel can be quenched may be limited by the available heat sink, i.e., the coolant heat capacity. To ensure comparable quench rates in model and prototype, they should have the same ratio of fuel heat content to coolant heat capacity. That is, when the model and prototype utilize the same fuel and coolant materials, the fuel/coolant mass ratio of the model should be comparable to that of the prototype, with similar coolant subcooling.

\subsubsection{Vessel Ambient Pressure}

It was assumed that the vapor flux from the fuel/coolant mixture might have an important effect on the breakup of the fuel jet penetrating the coolant pool. The vapor flux is limited by the vessel ambient pressure; therefore, it was 
expected that this would be an important scaling parameter. For further discussion see section 1.4 .

\subsection{Experiment Approach}

A series of six experiments (designated as CCM-1 through 6) was performed in which molten corium $\left(60 \% \mathrm{UO}_{2}, 16 \% \mathrm{ZrO}_{2}, 24 \%\right.$ SSt at $\left.-2800 \mathrm{C}\right)$ falls through a deep pool of water in a long, slender pour stream mode. This corium/water mixing mode represents a best estimate of the downward migration of molten core material during a severe accident in which the melt penetrates through local breaches in the disruption zone boundary (crust) and flows downward under gravity head into the RPV lower internal structure. The effects of the core structure assembly in causing additional breakup and redistribution of the pour stream into rivulets were simulated by using a multi-hole plate to form multiple jets in CCM-2. The corium mixture was selected to represent a melt composition in which the zircaloy cladding is essentially fully oxidized and in which there is appreciable content of unoxidized steel from downward melt migration within the RPV.

The key phenomena addressed in this investigation relate to the melt-water interaction, i.e., melt stream breakup, melt-water mixing, quench rate, steam generation, hydrogen generation, vessel pressurization, and debris characterizations. In order to quantify those key phenomena, various pressure transducers, thermocouples, level probes, and void probes were used to measure the pressure/temperature transients and the mixture extent. Posttest gas samples were taken, and the corium debris was examined. Test data, X-ray motion pictures, optical motion pictures, and posttest examinations were integrated into an interpretation of the test events; namely, rates of quench during the jet fall stage, efficiency of quench, efficiency of fall stage solidification, applicable breakup mechanisms, extent of oxidation, and the extent of breakup and intermixing with water.

The available data of the TMI accident were used to determine the experiment scaling. Recent in-core inspection of the damaged TMI-2 reactor has revealed the presence of an estimated $20,000 \mathrm{~kg}$ of debris accumulated on the vessel lower head. According to TMI data, water always filled the lower plenum as well as the bottom region of the core up to an elevation of at least $-1 \mathrm{~m}$. Based on the configuration of the core former plate and the core structure assembly of the TMI reactor, the possible corium stream diameter ranges from 3-10 $\mathrm{cm}$. Therefore, the fuel-to-water mass ratio was -0.5 to 1 and the water depthto-jet diameter ratio was -20 to 60 .

Corresponding to the TMI sequences, fuel-to-water mass ratios of 0.03 to 0.55 were used in the CCM tests. However, the size of the available heat sink for corium quench depends on the water subcooling as well as its mass. Therefore, both saturated and highly subcooled water was used.

Water depth-to-jet diameter ratios of 20 to 40 were used, similar to the TMI values. And, since the duration of the corium drainage (and the mass therein) may vary widely in a real reactor accident, the effect of varying the 'mass length' of the corium was also investigated. The corium columns which entered the water during the initial injection stage of the CCM tests had 'mass lengths' of -15 to 55 jet diameters. 
The CCM system pressure was roughly atmospheric, which is much smaller than the quench-related in-vessel pressurization data recorded during the TMI core relocation. However, it should be recognized that the most important effect of the elevated system pressure is to cause the specific volume of the steam to be smaller than for atmospheric conditions, reducing the vapor flux out of the fuel/coolant mixture. This lessening of the vapor flux was reasonably approximated in the tests with subcooled water.

Finally, tests were performed with an inert containment atmosphere in order to measure the mass of hydrogen produced.

\subsection{Related Studies}

It is not the intent of this report to provide a definitive review of the literature related to corium-coolant mixing. Therefore, only a summary of recent relevant experimental studies is presented. For a review of 1 iterature relevant to liquid jet breakup, applicable to the behavior of molten corium pour streams, see Ginsberg.

\subsubsection{Simulant Material Tests}

Spencer, Gabor, and Cassulo ${ }^{4}$ reported an experimental study that was conducted to examine the breakup and mixing behavior of initially coherent streams of high-density molten metals flowing into water. Thirty-two tests were conducted, 28 of which used molten Wood's metal (50 w/o Bi, $25 \mathrm{w} / 0 \mathrm{~Pb}, 12.5 \mathrm{w} / 0$ $\mathrm{Cd}, 12.5 \mathrm{w} / \mathrm{O} \mathrm{Sn}$ ) for the pour stream (the other four utilized molten tin). Wood's metal melts at $70 \mathrm{C}$; tin melts at $131.8 \mathrm{C}$.

The test apparatus consisted of a glass column constructed of 6 -in. (152.4$\mathrm{mm}$ ) ID pyrex pipe joined together by flanges. The water inside the column was 1.46-m deep and initially was at room temperature (19-25 C) or at its saturation temperature (99-101 C). Air or steam were the cover gas above the water. A melt furnace/injector mounted on top of the glass column contained approximately $2 \mathrm{~kg}$ of metal. Injection was initiated by an air-drive cutter circumferentially shearing a $0.05-\mathrm{mm}$ thick stainless steel diaphragm. The melt temperature was 86$445 \mathrm{C}$ for the Wood's metal and 295-319 C for the tin.

The breakup length of the pour stream was characterized by the change in the melt leading edge penetration rate. After breakup, the leading edge velocity is that of gravity settling of particles. With no net steam generation (i.e., water temperature less than saturation), the ratio of breakup length to initial stream diameter was 20 to 25, which agrees reasonably well with the Taylor model. Greater breakup lengths, about 40 to 50 diameters, were obtained when there was net steam generation. These values were in better, though not good, agreement with the Epstein-Fauske model using steam as the ambient fluid.

Cover gas entrainment by the leading edge dominated the initial penetration stage but was reduced for the trailing pour stream column. The characteristics of the debris produced by the pour stream breakup were dependent on test conditions. Solid particles of Wood's metal were formed in tests where the water temperature was below the metal freezing point of $70 \mathrm{C}$. Globules were formed by surface-tension forces acting to reduce the surface area. Filaments (thread-like particles) also resulted from surface-tension forces acting on molten particulate that froze before globules could be fcrmed. Sheet- or flake-like particles were 
formed by freezing of the pour stream surface and separation from the flowing molten core. About $76 \mathrm{w} / 0$ of the debris particles had diameters in the range of 2.4 to $9.5 \mathrm{~mm}$. Melt temperature had little effect on the overall size distribution.

The size of the mixing zones taken as metal fragments, vapor, entrained air, and the pour stream core, increased as the melt and water temperatures increased. Steam generation increased as the water temperature rose except for one test in which film boiling occurred, producing a vapor envelope around the pour stream. This envelope reduced heat transfer and steam generation. When $t$ in at $580 \mathrm{~K}$ was dropped into water at $298 \mathrm{~K}$, a mild explosive interaction caused the mixing zone to rapidly expand to the vessel walls. When tin at $568 \mathrm{~K}$ was dropped into $373 \mathrm{~K}$ water, no explosive interaction occurred, but the steam bubbles created by the rapid boiling resulted in an expansion of the mixing zone at lower depths to the diameter of the glass vessel.

\subsubsection{Corium-Water Thermal Interaction (CWTI) Studies}

Spencer, Sienicki, and McUmber ${ }^{7}$ reported on experiments to address exvessel cavity interactions and containment loading for a LWR. A 4-5 kg capacity thermite injector was used to generate and contain the molten corium mass from a thermite reaction. The injector was bolted to the top of an interaction vessel ( 8 -in. Sch 10 stainless steel Pipe) which simulated the cavity region below the reactor pressure vessel. An expansion vessel (30-in. ID steel pipe) acted as the containment building and was connected to the interaction vessel by $4-$ in. Sch 10 pipe that represented the pathway between the reactor cavity and its containment building. Corium $\left(60 \% \mathrm{UO}_{2}, 16 \% \mathrm{ZrO}_{2}, 24 \%\right.$ steel) was injected into the interaction vessel through a slide gate mechanism for gravity tests and through a ruptured diaphragm for the high-pressure tests. Tests CWTI-9 and -10 were gravity melt injections into a deep pool of water and so are applicable to corium behavior in the lower head region of the reactor pressure vessel.

\subsubsection{Test CWTI-9}

In this test $2.18 \mathrm{~kg}$ of molten corium drained under gravity head plus an initial $0.04 \mathrm{MPa}$ pressure differential into $31 \mathrm{~cm}$ of $94 \mathrm{C}$ water. From flash $X$-ray photographs, it was determined that the corium stream entered the interaction vessel with a diameter of $2.2 \mathrm{~cm}$ and a velocity of $3.7 \mathrm{~m} / \mathrm{s}$.

A total mass of corium debris of $2.39 \mathrm{~kg}(0.11 \mathrm{~kg}$ more mass than injected) was recovered from the interaction and expansion vessels. Only $13 \%(318 \mathrm{~g})$ of this material had been swept into the expansion vessel by the dispersal of water and steam. The remaining material $(2068 \mathrm{~g})$ collected on the bottom of the interaction vessel. Its top surface was extremely irregular in contour and consisted of a thin, fragile upper crust which was severely undercut with large voids beneath it. There was only a small amount of loose material on top of the crust. The corium layer was uniformly spread out on the base to about $1.5 \mathrm{~cm}$ thickness. Much of the aggregate was fragile and fell apart into chunks as the base was removed. Although some individual particles (globules) were present in this material, most of the loose debris had the appearance of sintered agglomerates with characteristic dimensions of 5 to $15 \mathrm{~mm}$. This loose material could be easily crumbled into smaller fragments. 
The water in the interaction vessel was rapidly heated to its saturation temperature upon entry of the corium. Its temperature continued to rise, remaining very close to saturation temperature as the system pressurized from steam and hydrogen formation. The mass of steam and hydrogen generated was $970 \mathrm{~g}$ and $18.1 \mathrm{~g}$, respectively. The steel base of the interaction vessel reached a maximum temperature of $500 \mathrm{C}$, which indicated that the corium was not completely quenched by traversing the water pool.

\subsubsection{Test CWTI-10}

This test was to be identical to test CWTI-9 with the exception that the system was at room temperature $(25 \mathrm{C})$. $1.31 \mathrm{~kg}$ of molten corium was injected with a differential pressure of $0.08 \mathrm{MPa}$ through the $2.54 \mathrm{~cm}$ diameter opening into $36-\mathrm{cm}$ deep water. Almost no pressurization of the system occurred, because the highly subcooled water rapidly condensed any steam that was generated. A small amount of hydrogen $(1.9 \mathrm{~g})$ was produced during the corium pour time. An average peak water temperature of $56 \mathrm{C}$ was obtained, and the base of the interaction vessel was heated to a maximum temperature of $415 \mathrm{C}$.

All the debris $(1.61 \mathrm{~kg})$ was found at the bottom of the interaction vessel and was qualitatively similar to that found in test CWTI-9. It showed evidence of stratification, consisting of a solidified melt layer directly on top of the base, above which were a considerable number of spheroidal particles of both metal and oxide material cemented into an aggregate.

\subsubsection{Sandia National Laboratories Jet Program}

Marsha $11^{8}$ and Marshall and Berman 9 have reported the results of four series of experiments: 1) jets of molten iron/alumina falling through air (MDJET Series); 2) jets of water falling through air (WAT series); 3) jets of molten iron/alumina falling through water (EJET series); and 4) isothermal jets falling through water (IJET series). Only the EJET series is relevant to this discussion.

The EJET series of experiments were conducted to study the mixing characteristics and explosive nature of boiling jets. A single jet of molten iron/alumina at a temperature of about $2700 \mathrm{~K}$ was gravity poured into saturated and highly subcooled water whose depth was $1.57 \mathrm{~m}$. The water was contained in a $56-\mathrm{cm}$ square lucite vessel with an open top. The iron/alumina melt was generated by a thermite reaction inside a crucible located above the water reservoir. After melting through a $0.64 \mathrm{~cm}$-thick steel plate covering a $12.7-\mathrm{cm}$ diameter hole in the bottom of the reaction crucible, the melt fell about $30 \mathrm{~cm}$ onto a mixer plate assembly. This plate contained an orifice through which the molten material flowed into the water whose surface was at the bottom of the plate.

For test EJET-0, the melt mass was $37.6 \mathrm{~kg}$, the jet diameter was $3.8 \mathrm{~cm}$ and the water temperature was $303 \mathrm{~K}$. As the melt entered the water, it fragmented into particles having drop diameters which were less than the initial jet diameter. The mixture region almost immediately grew to twice the initial jet diameter, and then continued to grow at a much reduced rate until about $2.5 \mathrm{~s}$ into the pour. At this time, a rapid expansion of the mixture region occurred that filled the entire water chamber. 
The melt mass for tests EJET-1,2,3, and 4 was $47 \mathrm{~kg}$. For test EJET-1, the jet diameter was $3.8 \mathrm{~cm}$ and the wate temperature was $362 \mathrm{~K}$, about $4 \mathrm{~K}$ subcooled. Again, significant fragmentation of the melt occurred as it fell through the water. The mixture region immediately grew to be twice the initial jet diameter and then propagated, at that diameter, into the water chamber for about $600 \mathrm{~ms}$. No further growth occurred until about $1.3 \mathrm{~s}$ into the pour when a second rapid expansion of the mixture region occurred that filled the entire chamber.

The EJET-2 test had a $7.6 \mathrm{~cm}$-diameter jet and a water temperature of $361 \mathrm{~K}$. There was significant fragmentation of the molten jet as it entered the water. A mixing plateau occurred almost immediately, but the second mixture region expansion was somewhat earlier than observed in the EJET-1 test. The duration of the mixing plateau appeared to decrease with the increase in jet diameter.

A $16.3 \mathrm{~cm}$-diameter hole in the bottom of the reaction crucible was used as the orifice for the melt injection for tests EJET-3 and 4 . Water temperatures were $359 \mathrm{~K}$ and $303 \mathrm{~K}$, respectively. Nonuniform features at the melt-through plug in the bottom of the crucible resulted in a jet with a leading edge that was smaller in diameter than the actual jet diameter. The early mixing phase for test EJET-3 was similar to that of the previous experiments in that a plateau occurred almost immediately. No information on test EJET-4 was given due to problems with the high-speed cameras.

Spontaneously triggered steam explosions were not observed in these tests. As noted earlier, significant fragmentation of the jet occurred and appeared to be very transient and nonlinear. Water subcooling affected the transient mixing behavior; for highly subcooled water, the jet mixture region penetrated further into the water before the rapid radial expansion.

Steam generation appears to have played an important role in the observed jet breakup and mixing process. However, this role may have been enhanced by the test geometry, in which the steam flow out of the mixture is restricted and deflected by the orifice plate. 
The Corium Ex-vessel Interactions (COREXIT) facility used for the CWTI experiments was adapted for the corium-coolant mixing (CCM) tests. This facility is shown in Figs. 2-1 and 2-2. The COREXIT facility consisted of a containment cell, CCM experiment apparatus, systems for instrumentation, heater control, cover gas addition, automatic sequence control, data acquisition, optical photography, and flash $X$-ray.

For tests CCM-1 through 4, the CCM test apparatus consisted of a thermite reaction vessel, an interaction vessel, an expansion vessel and the pipeway connecting the interaction and expansion vessels. For tests CCM-5 and 6, the thermite reaction vessel was placed in an experiment vessel.

The thermite vessel was used to contain the molten corium mass from a thermite reaction and inject it into the water inside the interaction or experiment vessel. The expansion vessel was used to reduce system pressure by providing a space for the collection of steam, gas and particulates generated by the corium water interaction.

Details of the experiment apparatus, diagnostics systems, test procedures, and corium thermite reaction are presented in the following sub-sections.

\subsection{Facility Description}

The containment cell shown in Fig. 2-2 was constructed so that experiments with real reactor materials could be conducted. It is doubly contained and was designed to withstand a fuel-coolant interaction (vapor explosion) of maximum thermal efficiency, including the simultaneous effects of blast wave, missile impact, and hydrostatic pressurization, for up to $12 \mathrm{~kg}$ of molten fuel. The cubical cell measures $2.1 \mathrm{~m}$ on a side. The steel plate wall thickness is $19 \mathrm{~mm}$. A sliding door on the front and rear of the cell provides a 610-mm wide entryway. On top of the cell is a circular opening for insertion of the expansion vessel, which is bolted to the roof to form a pressure seal. The estimated free volume of the cell is $8 \mathrm{~m}^{3}$.

Located outside the cell are the inerting gas bottles and piping system, diagnostic equipment and ancillary systems for operation and control of the experiment.

\subsection{Apparatus Description}

The experiment apparatus includes the thermite vessel, interaction vessel, expansion vessel, experiment vessel, and ancillary systems. Details of this equipment are provided in the following sub-sections.

\subsubsection{Thermite Vessel/Injector}

Thermite reaction vessel, thermite vessel, and thermite injector are synonymous names for the device used to contain the thermite reaction and to inject the corium melt into the interaction vessel. Two thermite vessels were used for the CCM tests. One had a reactant capacity of 4-5 kg; the second had a capacity of about $12 \mathrm{~kg}$. Ignition of the reaction powders was triggered by passing electric current through an igniter wire (two for the large vessel) em- 


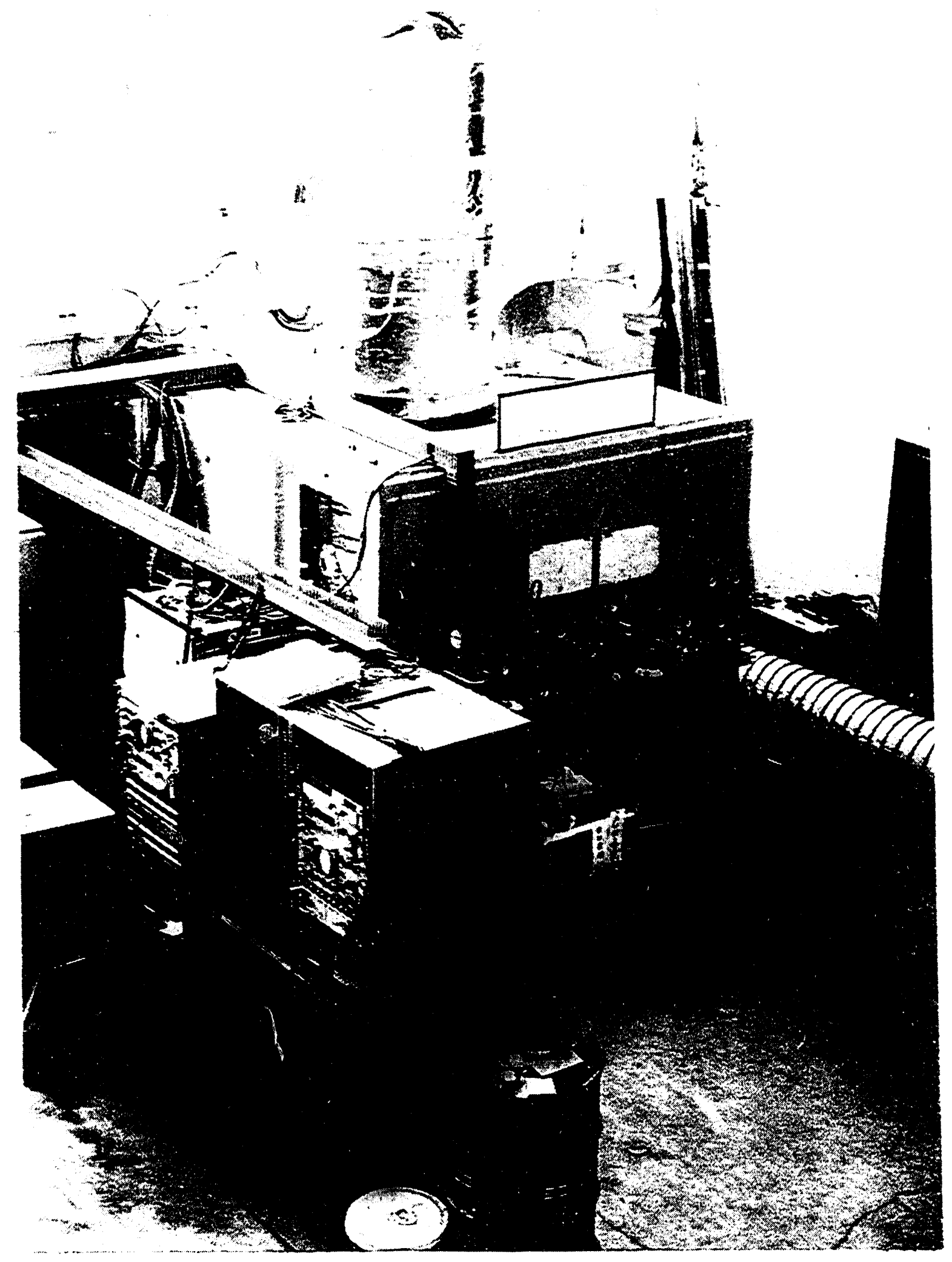

Figure 2-1. COREXIT Facility. 


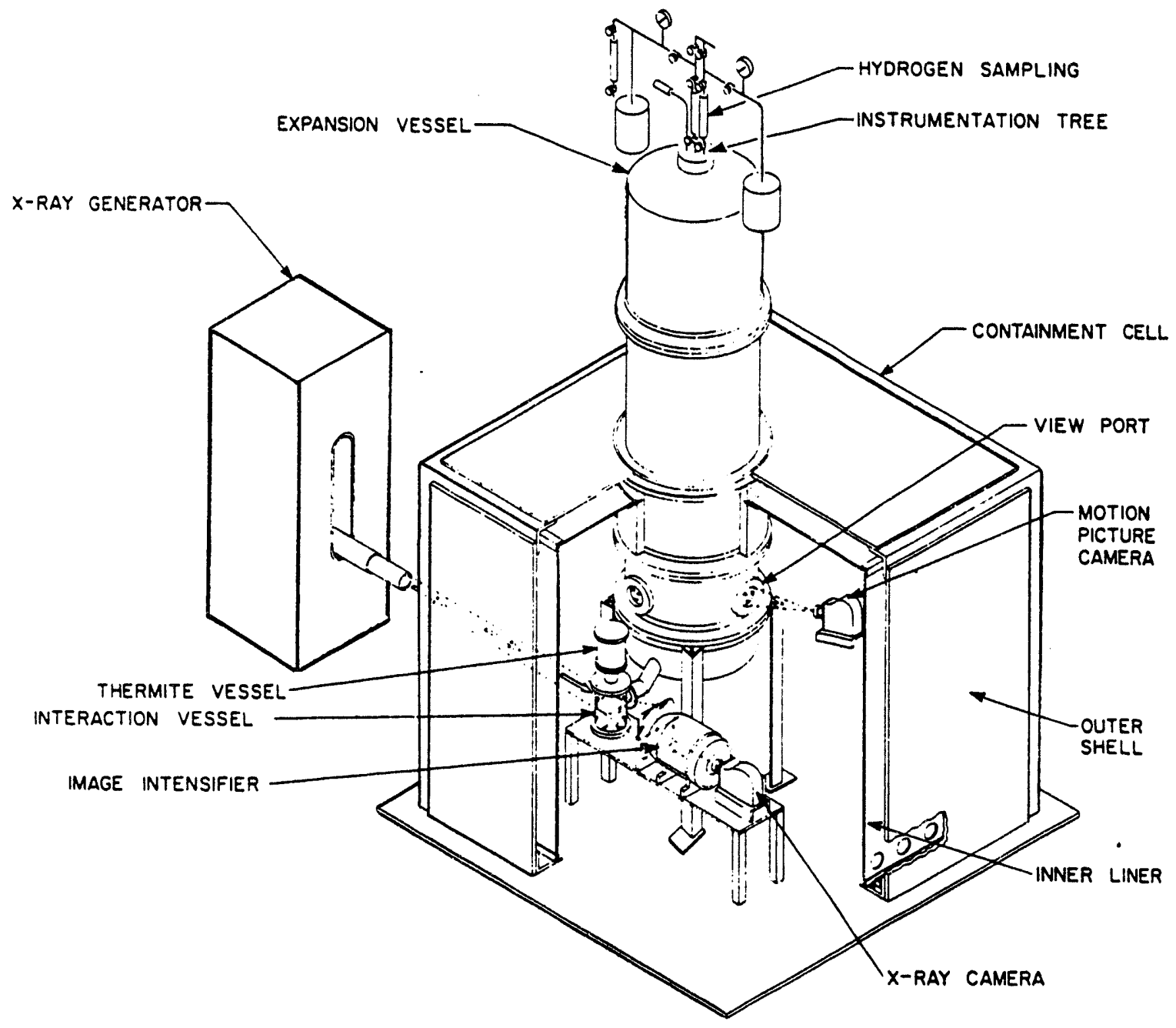

Figure 2-2. Schematic Illustration of COREXIT Facility. 
bedded in the upper region of the powder. Melt was released either by a pistonactuated slide mechanism or by failure of a $0.025-\mathrm{mm}$ thick stainless steel diaphragm.

The small thermite vessel, used for tests $\mathrm{CCM}-1$ and 3 , is illustrated in Fig. 2-3 with the piston-actuated slide assembly and in Fig. 2-4 with the diaphragm holder. The vessel body was constructed from a $232-\mathrm{mm}$ long piece of 5-in. Sch. 160 stainless steel pipe. The inside diameter of the pipe was machined to accept a $229-\mathrm{mm}$ long ceramic (Coors Mullite ${ }^{\odot}$ ) sleeve with a nominal $114-\mathrm{mm} O D$ and a $6.4-\mathrm{mm}$ wall thickness. For the top section of the vessel, a $76-\mathrm{mm}$ long piece of $8-\mathrm{in}$. Sch. 40 pipe was welded to the body adaptor plate. The top cover was an 8-in. 150 lb blind flange that bolted to the upper part of the body and had pipe taps for instrumentation, gas, and vent connections.

A tungsten grid plate (38\% open) rests atop the ceramic sleeve and is provided to inhibit the molten materials from splashing into the upper vapor region. This plate is held in place by a perforated stainless steel cylinder and a vapor separator pan. At the bottom of the vessel is a refractory cone (fired lava type A) that tapers from the sleeve ID to the 25.4-mm discharge opening.

This sleeve rests on the bottom head, which bolts to the injector body, and contains the slide or diaphragm assembly. The igniter wire was a $240-\mathrm{mm}$ length of 20-AWG (0.8-mm diameter) Tophet $A$ nickel-chrome wire that was zig-zagged across the inside of the vessel. Silver soldered to the ignitor wire were 20-Awg enameled copper wire ends that exited through openings in the Mullite sleeve and vessel body $210-\mathrm{mm}$ above the vessel bottom.

The slide assembly consisted of two halves with the 19-mm thick upper half containing an insert with a 25.4-mm diameter hole. The bottom half is machined to accept a refractory (lava) insert with a $50-\mathrm{mm}$ diameter hole that aligns with the same size hole in the slide housing when opened. Rods attached to the ends of the slide extend through seals in the slide assembly and are connected to an air cylinder. A twisted enamel coated wire was placed just above the slide with a Toop extending about $13-\mathrm{mm}$ up into the reactants.

This burn wire pair indicates when the thermite reaction front reaches the bottom of the injector. When the molten material burns away the coating and shorts the pair, an external circuit is energized and starts a timer that controls the opening of the slide.

The diaphragm assembly is a 25.4-mm thick plate with an insert that has a 25.4-mm diameter hole. A $0.025-\mathrm{mm}$ thick stainless steel diaphragm was cemented to the top of the insert with RTV. A burn wire pair as described above was placed above the diaphragm and, when energized, starts a timer that controls other test equipment.

The $12-\mathrm{kg}$ injector shown in Fig. $2-5$ is similar to the smaller thermite vessel. The body consists of a section of 8 -in. Sch. 80 pipe with a modified class 150 weld-neck flange on each end. A modified blind flange that adapts to the slide gate mechanism or accommodates a diaphragm holder, forms the bottom of the injector. The top section is a 10 -in. modified class 150 weld-neck flange with a flat head. The body of the vessel was bored for insertion of a 229-mm long Mullite liner that has a nominal $191 \mathrm{~mm} O D$ and a 7.9-mm wall thickness. At the bottom of the vessel is a refractory cone (fired lava type A) that tapers from the sleeve ID to a 50.8-mm discharge opening. A tungsten grid plate, which 


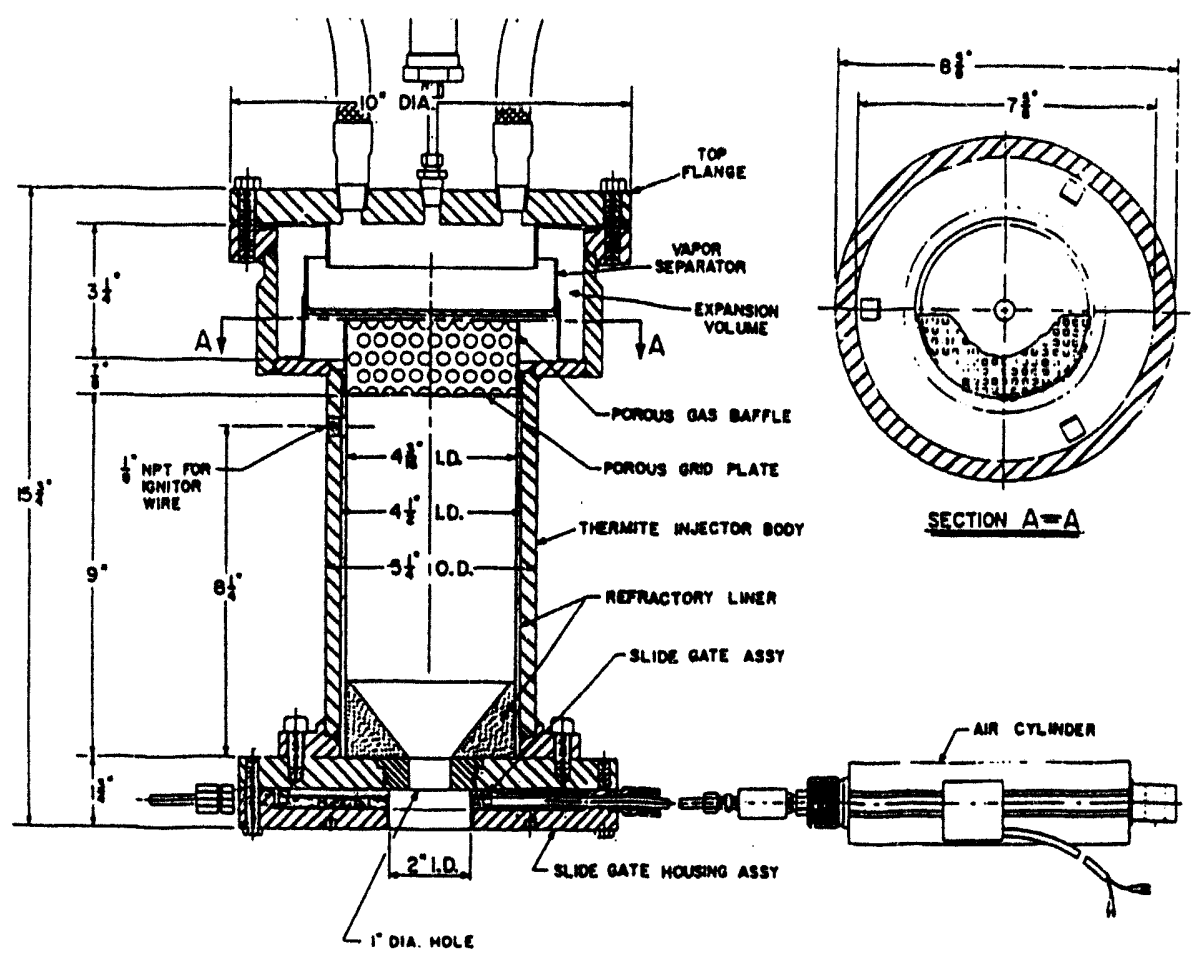

Figure 2-3. 4-kg Thermite Vessel with Slide Gate Assembly.

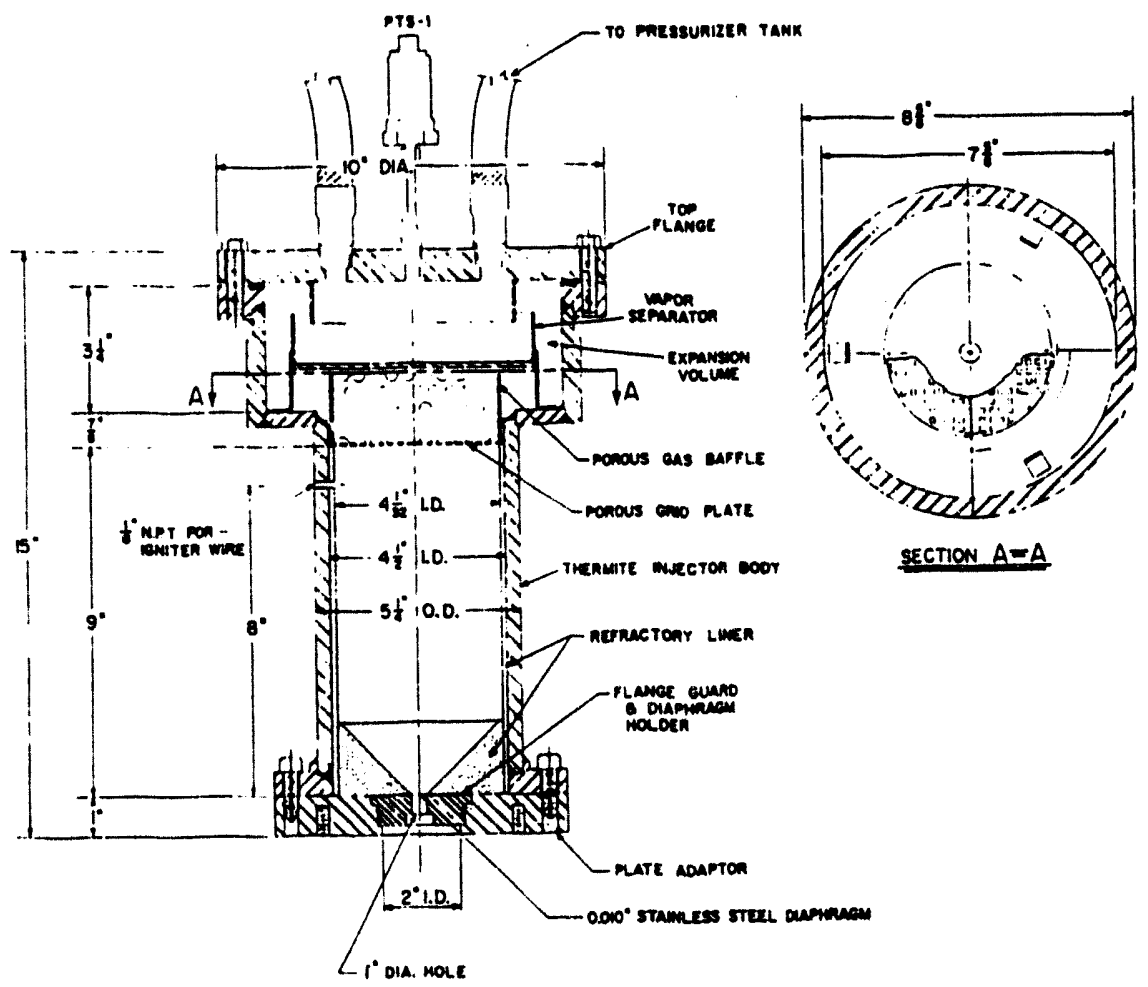

Figure 2-4. 4-kg Thermite Vessel with Diaphragm Assembly. 


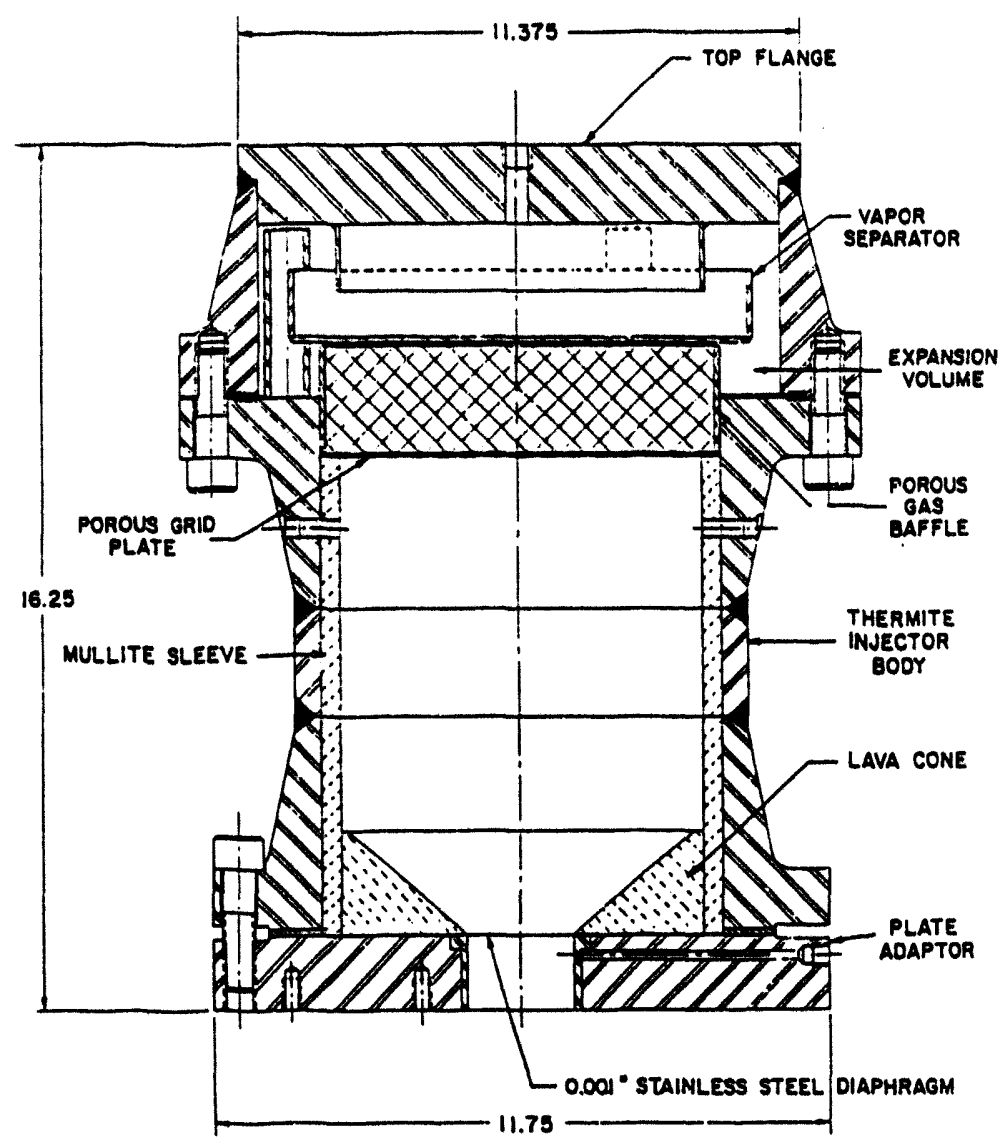

NOTE:

ALL DIMENSIONS IN INCHES.

Figure 2-5. 12-kg Thermite Vessel with Diaphragm Assembly. 
is $0.25-\mathrm{mm}$ thick and has 233 holes with a $6.35 \mathrm{~mm}$ diameter, rests atop the ceramic liner. The plate is held in place by a wire mesh cylindrical gas baffle and a vapor separatior. Access ports are provided for the burn wires, igniters, inerting gas and instrumentation. Two igniters, in parallel, are positioned in the upper portion of the reactants. Each igniter is constructed from a $177-\mathrm{mm}$ length of 20 Awg $(0.8 \mathrm{~mm}$ diameter) wire that is zig-zagged across the inside of the vessel. The material for the vessel is type 304 stainless steel and the design pressure is $5.2 \mathrm{MPa}$ at $100 \mathrm{C}$.

\subsubsection{Interaction Vessel}

The interaction vessel, shown in Fig. 2-6, was used for tests CCM-1, 3, and 4. It consists of sections of $8-\mathrm{in}$. Sch. $10(211.6-\mathrm{mm}$ ID) stainless steel pipe welded to class $150 \mathrm{slip-on}$ flanges. The upper section is $711-\mathrm{mm}$ long, has two rows of four 1/4-in. pipe couplings for instruments, and a 4-in. Sch. 10 (108-mm ID) discharge pipeway. The middle section is $457-\mathrm{mm}$ long and also has two rows of four $1 / 4-$ in. pipe couplings for instruments. The lower section is 305-mm long and is machined to accept a 12.7-mm thick base plate, through which 1.6-mm OD sheathed thermocouples are inserted. The base plate is supported by a $25.4-\mathrm{mm}$ thick plate that rests on jack screws attached to the vessel bottom.

The top flange contains penetrations for instruments and also has a centrally mounted $50.8-\mathrm{mm}$ ID, $139.7-\mathrm{mm}$ long flanged spool piece that extends 38.1 $\mathrm{mm}$ into the interaction vessel. This spool piece bolts to the thermite vessel slide or diaphragm assembly, and serves as a thermal spacer to minimize heating of the thermite vessel as the interaction vessel is heated. At the outlet of the spool piece is a $0.025-\mathrm{mm}$ thick stainless steel diaphragm that isolates the pretest interaction vessel atmosphere from the bottom of the thermite vessel. Corium rapidly passes through this diaphragm upon release from the thermite vessel. When the slide assembly is utilized, a burn wire pair is placed below this diaphragm which energizes a timer that starts the $X$-ray system.

The discharge pipeway consists of an elbow section and a U-bend section of 4-in. Sch. 10 pipe (108.2 mm ID). The downward facing elbow section is formed from two short pieces of pipe welded to a $90^{\circ}$ long-radius elbow. One pipe end is welded to the upper section of the interaction vessel $15.24-\mathrm{mm}$ below its top flange. The other end is attached to a 4 -in. class 150 slip-on flange, that is bolted to a similar flange on the $U$-bend section. This section has an upward facing long radius $180^{\circ}$ return with pipe stubs welded to each end. The shortstub end is also welded to a $4-i n$. class 150 slip-on flange that provides the connection to the expansion vessel as shown in Fig. 2-7.

Coaxial heater wires, thermocouples, and ceramic fiber insulation (WRP-XA) are installed on the exterior surfaces of the pipeway and interaction vessel.

For test CCM-2, the interaction vessel consisted of only the upper and base sections as described earlier and this arrangement is shown attached to the expansion vessel in Fig. 2-8. Tests CCM-5 and 6 did not use an interaction vessel.

\subsubsection{Expansion Vessel}

The expansion vessel configuration for experiments CCM-1 through 4 is shown in either Fig. 2-7 or 2-8 with the appropriate interaction vessel. The expansion 


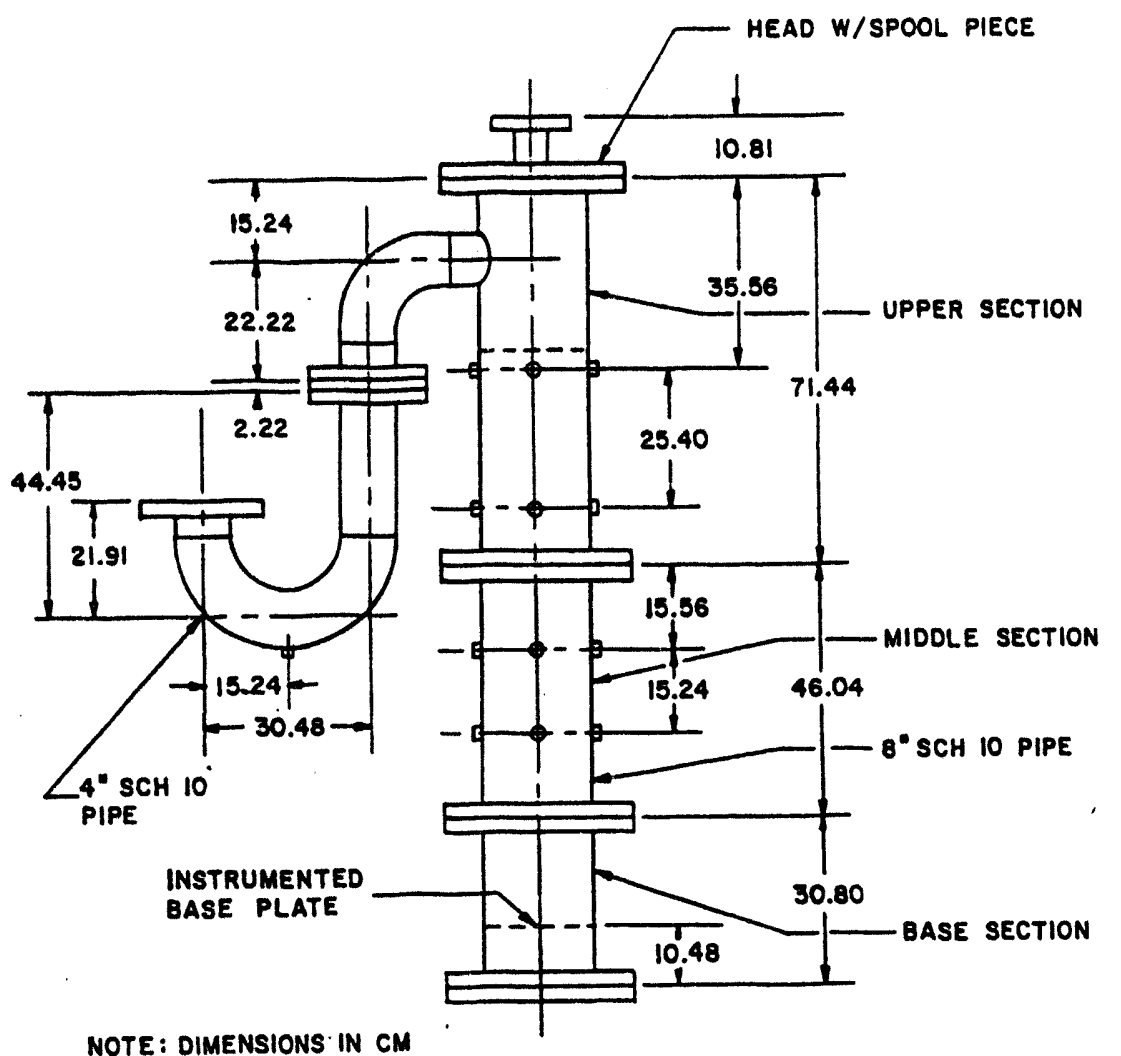

Figure 2-6. Interaction Vessel. 


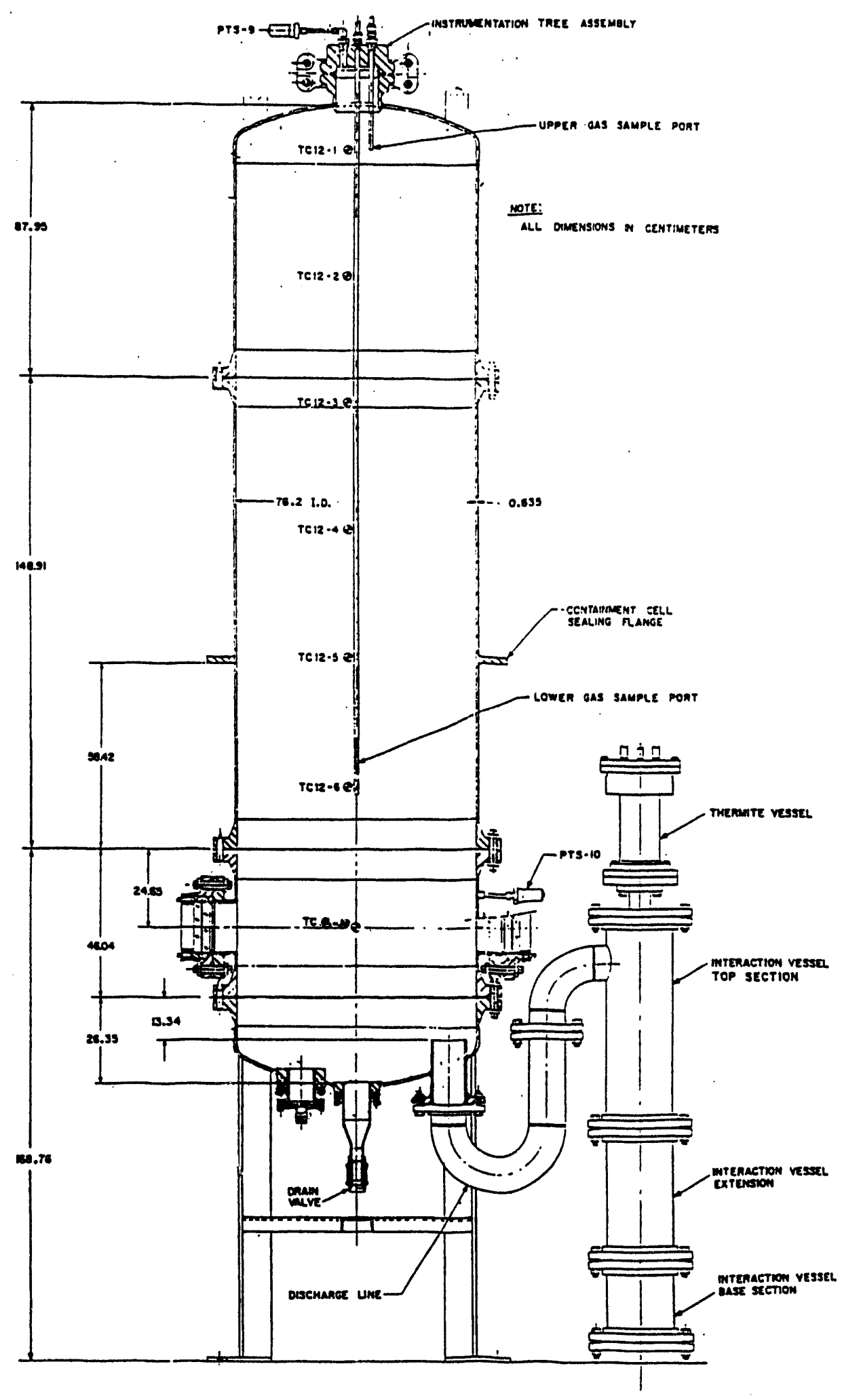

Figure 2-7. Expansion Vessel with Interaction Vessel for Tests CCM-1, 3 and 4. 


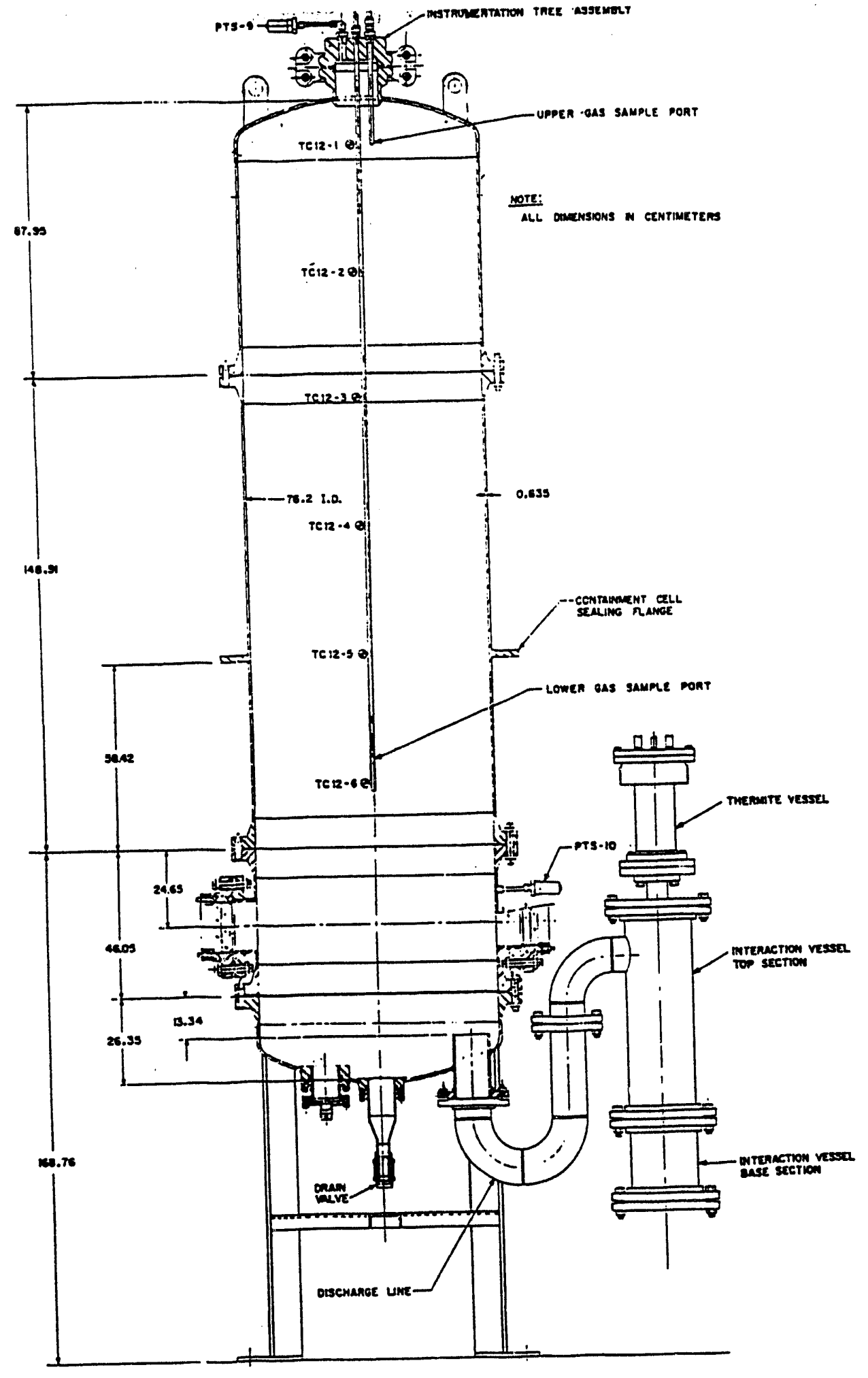

Figure 2-8. Expansion Vessel with Interaction Vessel for Test CCM-2. 
vessel is used for the accumulation of steam and hydrogen that are generated by the corium water interaction. It consists of four flanged sections: a bottom section, a multi-viewport section, a middle section, and a top section. These sections are constructed from $762-\mathrm{mm}$ ID carbon steel pipe with a wall thickness of $6.35 \mathrm{~mm}$. Its inside height is $3.09 \mathrm{~m}$ and its volume is $1.377 \mathrm{~m}^{3}$. Looking downward into the bottom section of the vessel as shown in Fig. 2-9, three penetrations are visible. The central and adjacent penetrations are packed glands for 3 -in. pipe $(88.9 \mathrm{~mm} 0 \mathrm{OD})$, and have flat disk covers. The central penetration is used as a drain and has a $19-\mathrm{mm}$ high circumferential opening below the cover. The adjacent penetration was not used. A piece of 4-in. Sch. 40 (102.2-mm ID) pipe penetrates the lower head at a radius of $295 \mathrm{~mm}$ and extends $101.6 \mathrm{~mm}$ into the vessel above its lowest penetration. This pipe stub has a lower flange that bolts to a mating flange on the discharge pipeway and provides an entry for the steam, hydrogen and particulate generated in the interaction vessel.

The multi-viewport section of the vessel has three viewports. Two of the ports are in line and are offset $50.8 \mathrm{~mm}$ from the vessel centerline. They are $152.4 \mathrm{~mm}$ in diameter and one of them is used for an optical camera, which was focused on the pipeway outlet. The third view port has a $76.2-\mathrm{mm}$ diameter, is located $90^{\circ}$ from the other ports and was not used for the first four tests. There are penetrations in the vessel wall for gas and vacuum 1 ines, instruments, and a gas sample line.

The middle section extends through the top of the containment cell. A ring welded to the outer surface of the vessel and another ring bolted to the top of the cell form a seal for the opening between the middle section and the cell. The top section contains an instrumentation assembly consisting of a Graylock fitting and blind flange that was modified for tubing and instrumentation penetrations as shown in Fig. 2-10. A central 12.7-mm OD gas sample tube extends downward from the flange to about $2.3 \mathrm{~m}$ into the vessel and provides support for six thermocouples. The second gas sample tube also has a $12.7-\mathrm{mm} 00$ and extends $269.9 \mathrm{~mm}$ into the vessel.

\subsubsection{Experiment Vesse1}

The test apparatus for experiments CCM-5 and 6 consisted of the thermite vessel mounted inside an experiment vessel (formerly expansion vessel) as depicted in Fig. 2-11. The 12-kg thermite vessel was mounted atop a unistrut framework. Suspended from the lower part of this framework was a 565-mm ID, 149-mm deep stainless steel pan with a particle trap that fitted over the pipeway outlet. The opering in the particle trap was covered and the pipe stub was blocked off with a blind flange outside the vessel at the normal discharge pipeway junction. The unistrut framework (shown in Fig. 2-12) rests on the bottom of the experiment vessel and is centered in the vessel with adjustable spacers. The framework also provides support for thermocouple leads and for lights.

The experiment vessel for test CCM-5 also consists of four flanged sections: a bottom section, a multi-viewport section, a single-viawport section, and a top section. The bottom section and multi-viewport sections are identical to those described for the expansion vessel with a few changes. The flat disk cover over the central opening was removed and a steam/gas line was joined into the drain line below the valve. All three view ports were used for high-speed photo- 


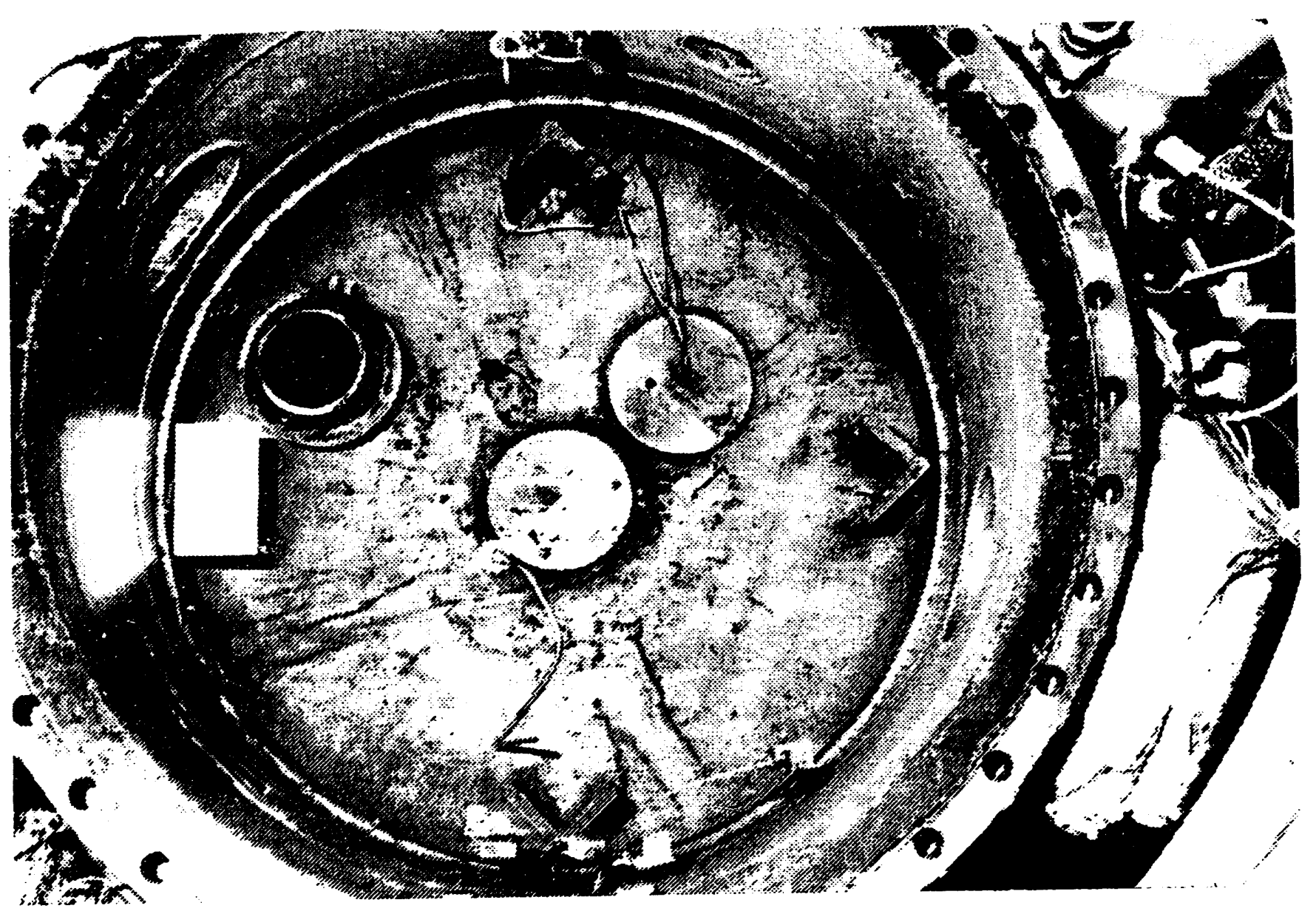

Figure 2-9. View Looking Down into Bottom Region of Expansion Vessel. 


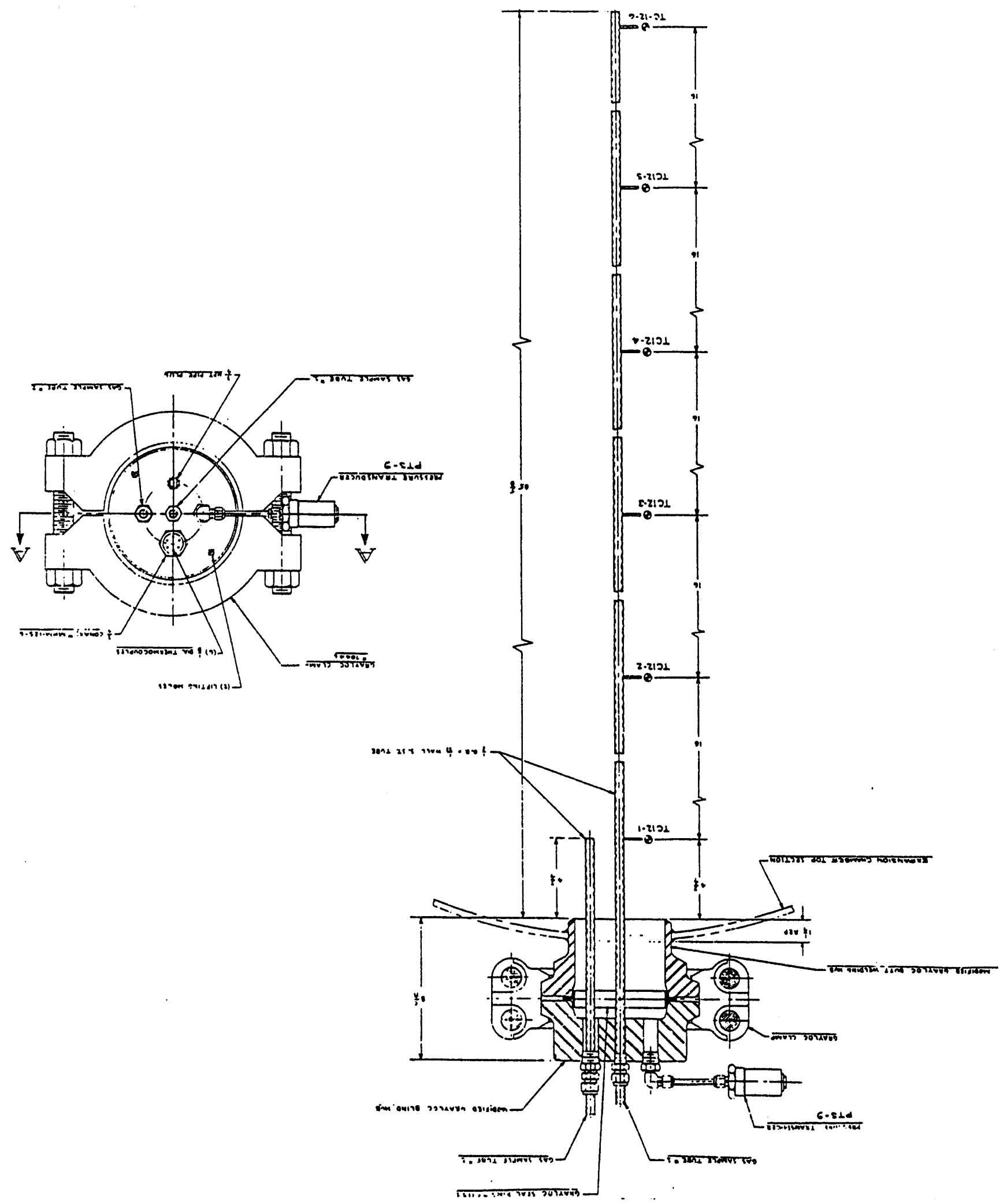

Figure 2-10. Instrumentation and Gas Sampling Assembly for the Expansion-Vessel Upper Section. 


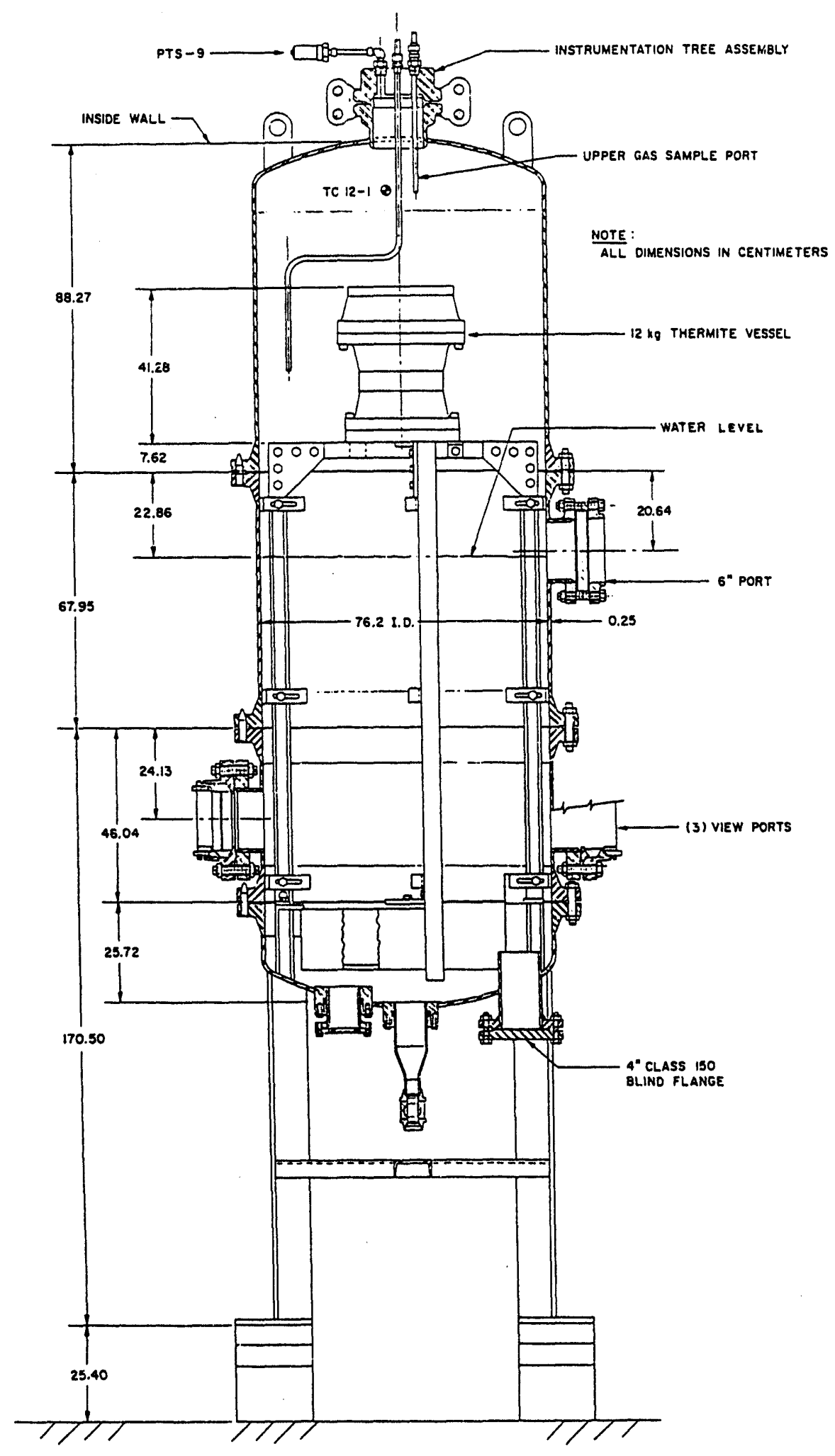

Figure 2-11. Test Apparatus for Experiment CCM-6. 


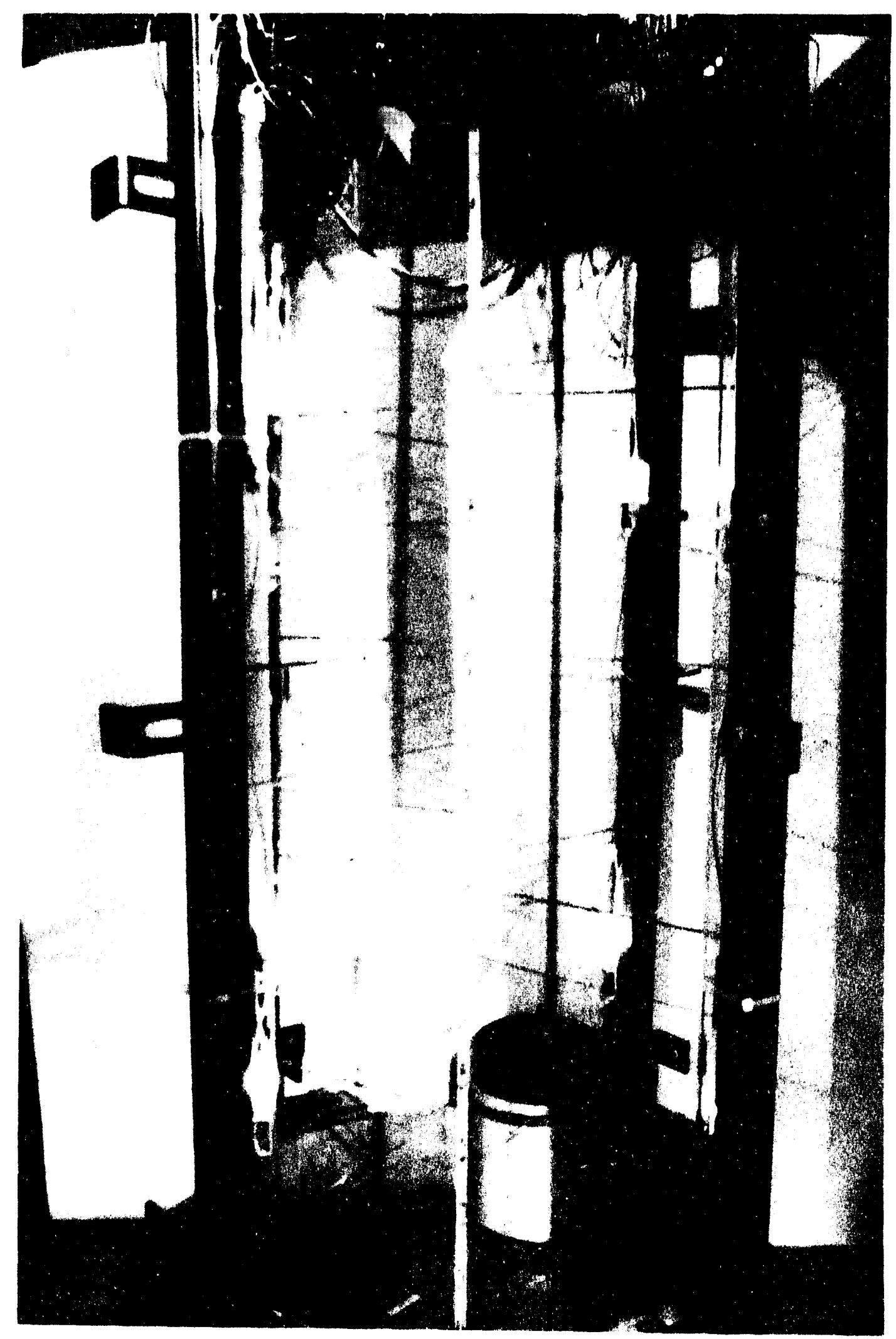

Figure 2-12. Experiment-Vessel Internal Structure for Test CCM-5 and 6. 
graphy. An overflow drain line was installed on the inside of the vessel.

The 1.49-m long middle section of the expansion vessel was removed and a $762-\mathrm{mm}$ ID, 676-mm long pipe section with a 152.4-mm diameter viewport was installed. This section contained numerous wall penetrations that were used for instrumentation, igniter leads, and gas purge lines. One penetration contained a $17.5-\mathrm{mm}$ diameter sight window. The top section of the experiment vessel was the same as used for the expansion vessel with some modification to the instrumentation assembly. The central gas sample tube was shortened and curved to fit near the vessel wall (305-mm radius) as shown in Fig. 2-11. The bottom of the tube is about $732 \mathrm{~mm}$ below the instrumentation assembly head. With the exception of thermocouple TC12-1, which was left in its original location, the thermocouples that were attached to the central gas sample tube were formed into a partial circular or helical coil with a $610-\mathrm{mm}$ diameter. Vessel disassembly occurred at the flange joint between the top and single viewport sections. The vessel inside height was $2.28 \mathrm{~m}$ and its empty volume was $1.006 \mathrm{~m}^{3}$. With the addition of the thermite vessel, the unistrut framework and catch pan, the free volume is reduced to $0.972 \mathrm{~m}^{3}$.

For test CCM-6, the experiment vessel comprised all the sections used for test CCM-5, plus the addition of the $1.49-\mathrm{m}$ long middle section of the expansion vessel. This section was placed between the top and single-viewport sections. It was added to increase the volume of the vessel so that steam generation would not be inhibited by pressurization. The inside height of the experiment vessel was $3.77 \mathrm{~m}$ and its empty volume was $1.687 \mathrm{~m}^{3}$; with test internals the vessel volume is reduced to $1.652 \mathrm{~m}^{3}$. Vessel disassembly was at the flange joint between the middle and single viewport sections.

\subsubsection{Ancillary Systems}

Ancillary systems include the temperature controllers, timers, slide actuation controls, thermite igniter, gas sample system, temperature reference junctions, a vent tank and an impingement plate holder for test CCM-2.

The temperature controllers were plug-in types (Weather Measure model $T P C-1)$ and were mounted in a control chassis. They operated load relays that routed power to the cell through multiconductor cables with 21-pin connectors (Amphenol type MS3102A). A mating connector was wired to terminal strips inside the cell, to which the heater wires were attached.

Because of safety considerations, all tests were conducted remotely and controlled by timer actuated signals. Nine plug-in timers (ATC model \#335B) were mounted in the heater control chassis.

Opening of the slide gate at the bottom of the thermite vessel occurs by automatically actuating an air cylinder attached to the slide. This air cylinder was connected to a reservoir through a solenoid operated ball valve. Manually operated valves were also provided for blocking slide operation prior to the test.

Ignition of the thermite reactants was accomplished by passing a direct current through the igniter wire. The DC power was supplied by a Sorensen SRL 60-30 power supply (60V at 35A) that can be operated in either a voltage or current limited mode. For the CCM tests, the current limited mode was used to 
insure a sufficient duration of heating for the reactants to ignite. Typically the current was set at $20 \mathrm{~A}$ and then adjusted with a dummy igniter wire in air. The current was varied so that several seconds of wire heating occurred before it melted.

A gas sample system was used to obtain one sample of the expansion or experiment vessel atmosphere prior to a test and three samples approximately 5 min after test initiation. The two gas sample lines shown in Figs. 2-7, 2-8, 2-10, and 2-11 along with a line from a penetration in the multi-viewport section of the vessel were connected to a manifold assembly mounted atop the instrumentation assembly. The manifold assembly consisted of $150-\mathrm{ml}$ sample bottles and vacuum chambers whose volumes were sufficiently large so as to entirely purge the connecting lines during sample extraction. This arrangement assured that a representative sample of the vessel atmosphere was obtained. The sample bottles, lines, and vacuum chambers were trace heated to about $150 \mathrm{C}$ to minimize temperature differences and prevent vapor condensation. Manual valves were closed at each end of the sample bottles immediately after sample extraction.

A heated temperature reference junction was used for all tests and a room temperature reference junction was added for tests CCM-5 and 6 . The heated junction was a well insulated block of aluminum that was electrically heated to an automatically controlled temperature of about $66 \mathrm{C}$. A thermocouple supplied the junction temperature to the data acquisition system. The room temperature junction was a well insulated terminal block. The output of an electrical circuit containing a two terminal monolithic integrated circuit temperature transducer (Analog Devices No. AD592) was adjusted to the millivolt equivalence of room temperature and supplied to the data acquisition system.

A stainless steel vent tank (248 $\mathrm{mm}$ ID, $940 \mathrm{~mm}$ high) was located inside the containment cell and was connected with tubing the top of the thermite vessel. The connecting line contained two solenoid actuated valves in series, whose opening and closing were timer actuated. This arrangement was used for tests CCM-1 through 4 and was provided to alleviate any pressure buildup in the thermite vessel during the reaction so that a near gravity pour would occur. In addition, an equalizer line between the thermite vessel and the expansion vessel was used. This line contained a solenoid actuated valve that was opened by a timer signal after the valves on the line to the vent tank were closed. For test CCM- 6 the vent tank arrangement but not the equalizer line was used.

In test CCM-2, an impingement plate holder (see Fig. 2-13) was bolted to the underside of the head of the interaction vessel. This device was provided to convert a single pour stream from the thermite vessel into four pour streams. It is constructed from a 308-mm long piece of $69.9-\mathrm{mm}$ ID stainless steel.tubing with a $0.3-\mathrm{mm}$ thick wall. One end is a flange for bolting to the vessel head and welded to the other end is a $6.35-\mathrm{mm}$ thick impingement plate. This plate has four 20.2-mm diameter holes on a 26.4-mm square pitch.

\subsection{Diagnostics}

Diagnostic equipment includes instrumentation, optical cameras, a flash $X$-ray cine system, and a data acquisition system. 

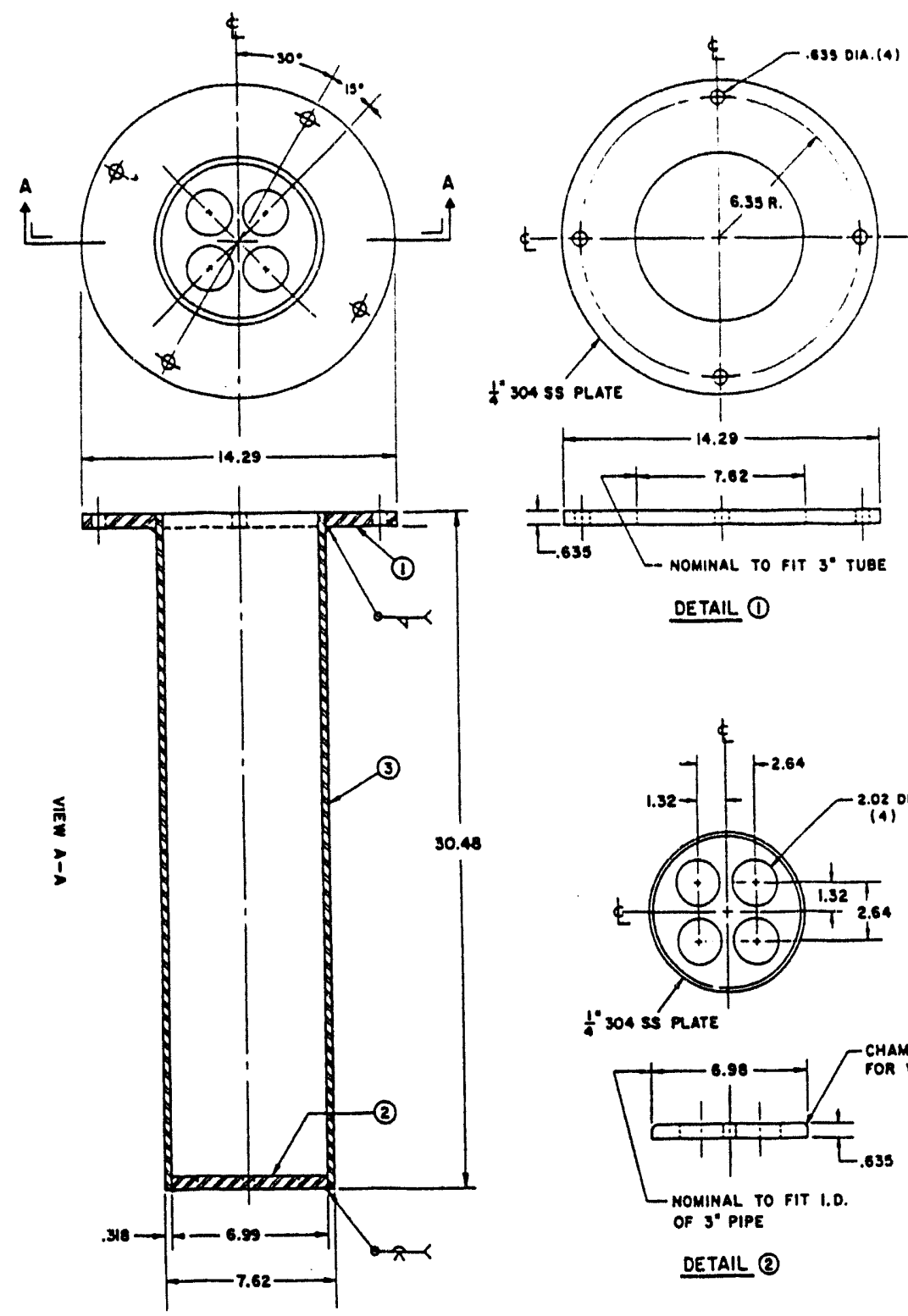

IMPINGEMENT - PLATE HOLDER

MOTE:

ALL OIMENSIONS IN CENTIMETERS.
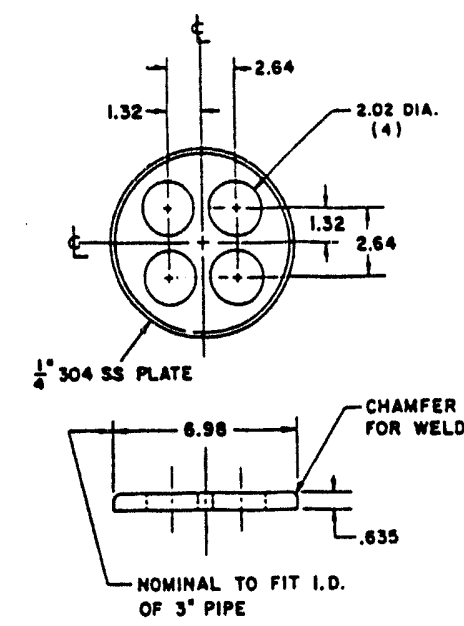

DETAIL (2)

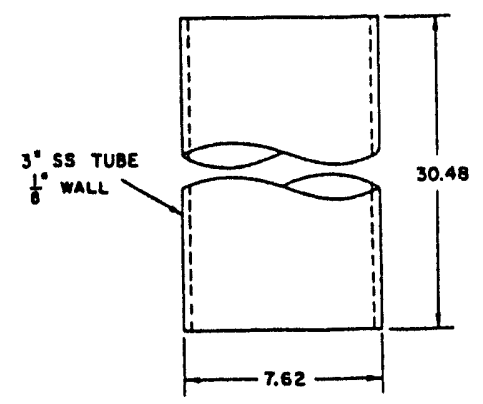

DETAIL (3)

Figure 2-13. Impingement-Plate Holder. 


\subsubsection{Instrumentation}

Instrumentation varied with each of the tests; details are presented in Appendix A. Thermocouples and pressure transducers were used in all the tests. The thermocouples were 1.59-mm OD stainless steel sheathed chromel-alumel with a grounded junction or a bare tip. Pressure transducers were primarily a strain gage type but a few piezoelectric transducers were used in some of the tests to detect a potential steam explosion. Table 2.1 gives the general location and number of thermocouples and pressure transducers used in each test.

For tests CCM-2,3, and 4, five conductivity sensing level detectors were installed through the head of the interaction vessel via a Conax connector and positioned above the water surface. These detectors were fabricated from 3.18-mm OD stainless steel sheathed coaxial heater cable by removing a short section of the outer sheath from the cable end to expose the central wire. Silicon rubber was then applied to the wire to insulate it from the sheath with a small tip of the wire exposed. The upper end of the wire was connected to a $15 \mathrm{~V} \mathrm{DC}$ voltage source through a current limiting resistor and an output sensing resistor. The water in the interaction vessel and the vessel wall complete the electrical circuit. When the rising water surface contacts the exposed tip of the detector, the circuit is completed. This causes a voltage drop across the sensing resistor that results in an output voltage signal being sent to a tape recorder. For tests CCM-2 and 3, the probe tip was located at $3.18,9.52,22.22,34.92$, and $60.32 \mathrm{~mm}$ above the water surface. In test CCM-4, the probe tip height above the water surface was $6.35,12.7,19.05,25.4$, and $31.75 \mathrm{~mm}$.

A photo cell was installed in the wall of the spool piece on the interaction vessel head in order to evaluate this instrument's ability to detect the duration of the corium pour.

For tests CCM-5 and 6,12 conductivity sensing void detectors were positioned at various locations in the water inside the experiment vessel. These were similar to the level detectors. The central wire was attached to a barrier strip, located above the water surface. An extension lead was connected to the same terminal on the barrier strip and these junctions were insulated with an epoxy sealant. The extension lead was run through two resistors in series to the positive side of a $\pm 15 V D C$ power supply. The negative side of the power supply was attached to the vessel wall to provide a return path for the electrical current. A twisted pair of wires, attached to the ends of the first series resistor, provides the signal path to a tape deck. With the probe tip immersed in water, the resistance of the water completes the circuit and current flows through the resistor and generates a voltage which is recorded. With a void around the probe, current flow will cease and no voltage will be generated. Vapor bubbles should decrease the generated voltage depending on the steam/water ratio surrounding the tip.

\subsubsection{Optical Camera}

For tests CCM-1 through 6, a 16-mm high-speed motion picture camera (Hycam model 40-0004) with a 12.2-m film capacity was positioned at the 152-mm view port in the multi-viewport section of the expansion vessel. This viewport was opposite the discharge pipeway exit in the vessel and the camera was focused on this region. Four lights (BHB-250W Halogen) were positioned around the lens $(25 \mathrm{~mm})$ to light the vessel interior. The camera and lights were controlled by 
Table 2.1. Number of Thermocouples and Pressure Transducers Used in CCM Experiments

\begin{tabular}{|c|c|c|c|c|c|c|c|c|c|c|c|c|}
\hline Test No. & $\mathrm{TC}^{\mathrm{CCM}-}$ & $\begin{array}{l}-1 \\
P T * *\end{array}$ & $T^{\mathrm{CCM}-2}$ & & $\begin{array}{r}\mathrm{CCM}^{\mathrm{C}}-3 \\
\mathrm{P}\end{array}$ & & $\mathrm{TC}^{\mathrm{CCM}-}$ & $\begin{array}{l}-4 \\
\text { PT } \\
\end{array}$ & ${ }_{T C}^{C C M}$ & $\begin{array}{l}-5 \\
\text { PT } \\
\end{array}$ & TC & $\begin{array}{l}-6 \\
\text { PT } \\
\end{array}$ \\
\hline Thermite-Vessel Gas Space & - & 1 & 1 & 1 & 1 & 1 & 1 & 1 & 1 & 1 & 1 & 1 \\
\hline Interaction-Vessel Gas Space & 2 & 1 & 2 & 1 & 2 & 1 & 2 & 1 & - & - & - & - \\
\hline Interaction-Vessel Water & 8 & 8 & 4 & 4 & 8 & 4 & 8 & 1 & - & & - & - \\
\hline Interaction-Vessel Base (Water) & 8 & - & 8 & - & 8 & - & 8 & - & 5 & - & - & - \\
\hline Pipeway U-bend & 1 & - & 1 & - & 1 & - & 1 & - & - & - & - & - \\
\hline Expansion-Vessel Gas Space & 7 & 2 & 7 & 2 & 7 & 2 & 7 & 2 & - & - & - & - \\
\hline Experiment-Vessel Gas Space & - & - & - & - & - & - & - & - & 6 & 3 & 6 & 3 \\
\hline Experiment-Vessel Water & - & - & - & - & - & - & - & - & 37 & 3 & 37 & 3 \\
\hline Experiment-Vessel Pan (Water) & - & - & - & - & - & - & 1 & - & 9 & - & 9 & - \\
\hline
\end{tabular}

$\star T C=$ Thermocouple

$\star \star \mathrm{PT}=$ Pressure Transducer 
automatic sequence timers. An internal timing generator marks one edge of the film to establish true film speed. An external timing generator marker marks the other edge of the film with a train of pulses coincident with a burn wire closure. The camera was operated at nominally 1,000 pictures per second for a total filming time of $16 \mathrm{sec}$.

For tests CCM-5 and 6, three additional Hycam cameras and one video camera were used. A Hycam camera was positioned at each of the few remaining ports on the multi-port section of the experiment vessel. The camera at the 152-mm diameter port was angled upward while the camera at the 76.2-mm diameter port was normal to the window surface. The remaining Hycam camera was located at the 152 $\mathrm{mm}$ diameter port on the single port section of the experiment vessel. At this position the camera viewed the water surface. The framing speed for all Hycam cameras was 500 frames/s. A video camera was positioned at the $17.5-\mathrm{mm}$ diameter sight window, which was $180^{\circ}$ from the $152-\mathrm{mm}$ diameter port and on the same plane.

\subsubsection{Flash X-ray Cine System}

The events inside the interaction vessel were recorded with a flash $X$-ray cine system (Hewlett Packard model 760/273). The X-ray source was located outside the containment cell. Its voltage was $350 \mathrm{kV}$ and it was capable of emitting 250 pulses at up to 250 pictures per second framing rate. The $X$-ray pulses with approximately $50 \mathrm{~ns}$ in duration, were collimated and directed to the target through a plexiglass window on the door of the containment cell. An image intensifier (Varian VXI-145) was located inside the containment cell. It received the $X$-rays transmitted through the test section, intensified the image, and displayed it on a 1-in. green phosphorous screen, where it was photographed by a 16-mm motion picture camera (Photosonics model P). A shutter pulse from the camera triggered the $X$-ray pulses and for these tests a pulse rate of 200 pulses/s was used. The image diameter was $229 \mathrm{~mm}$ diameter.

\subsubsection{Data Acquisition}

All test data were recorded in analog format on 28-channel tape recorders operated at 60 ips (DC-60 kHz band). Two Honeywell tape recorders (Model 96 and a Model 101) were used for all the tests. A third tape recorder (Honeywell Model 101) was used for experiments CCM-5 and 6. For redundancy, data was also recorded on fibre optics oscillograph recorders (Honeywell 1558). Signals from the pressure transducers were connected to the oscillograph with tape compatible modules (Honeywell 1887). Outputs from these modules were connected to the tape recorder through a plug and jack panel so that signals could be monitored on a digital voltmeter (Hewlett-Packard Model 3455A) for set-up and calibration of the oscillograph and tape deck channels. Some of the test and heated wall thermocouples were connected to a 100-channel data logger (Doric Digitrend 210) and to a plug and jack panel. The data logger was used to monitor the system temperatures during heat-up and to record system temperatures immediately preceding and following a test. One channel of each tape recorder was dedicated to an IRIG B time code signal. The time of thermite ignition and the reaction duration were determined by recording the current through the igniter wire and the burn wire contact. The voltage output from the $X$-ray system was recorded to show the exact times of individual pulses.

Data from the FM tapes were digitized with a 32-channel digitizer (CAMAC Model 1500) via a DEC PDP $11 / 34$ computer. The digitized data was displayed on 
a screen (Tektronic Model 4014-1) for viewing. Data plots were scaled, labeled, and plotted on a Versatec Model 1200A printer. A DEC PDP 11/70 was also used for data processing and transfer to the central IBM system.

\subsection{Test Procedures}

A description of pretest, test, and posttest operations follows.

\subsubsection{Pretest Operations}

Because of equipment differences, the pretest operations for experiments CCM-1 through 4 are different from those for tests CCM-5 and 6 . For experiments CCM-1, 3, and 4, the top section of the interaction vessel was bolted to the middle section, which was then bolted to the base section. The pipeway was attached to the top section and the assembled vessel was positioned inside the containment cell. The free end of the pipeway was then bolted to the flanged stub on the bottom of the expansion vesse1. Thermocouples and pressure transducers were then installed. After water was added to the interaction vessel, the top head with its instrumentation was installed and the system was leak checked.

Assembly of the thermite vessel began by bolting the body of the vessel to the slide or diaphragm assembly. When the diaphragm assembly was used the $0.025-\mathrm{mm}$ thick diaphragm was cemented to the diaphragm holder with RTV. The lava cone was wrapped with asbestos paper to ensure a snug fit as it was placed in the bottom of the vessel. The ceramic liner was installed and a burn wire pair positioned above and below the slide or diaphragm assembly.

After weighing the empty thermite vessel, it was transferred into the glove box and loaded with the thermite reactants. The mixture was compacted with a plastic rod during loading and the igniter wire was imbedded in the top portion of the mixture.

For tests CCM-5 and 6, the catch pan and unistrut framework with thermocouples and lights were installed in the lower sections of the experiment vessel. The thermite vessel was bolted to the unistrut framework. The igniter wire, burn wires, thermocouples, and electrical power lines for the lights wire attached to their respective leads that penetrated the side wall of the vessel. The water was heated to the desired temperature (approximately $50 \mathrm{C}$ ) by using an electrical hot water heater and heat from the experiment vessel walls. Argon sparging was used to prevent thermal layering. After the desired temperatures were achieved, the vessel gas space was purged with argon until its oxygen content was less than $1 \%$. The remainder of the pretest operations were the same as for the previous tests except that the $X$-ray system was not used and additional cameras were installed.

\subsubsection{Test Operations}

Because of safety considerations resulting from the use of the cine $X$-ray system or from a potential leak of radioactive materials, the tests were conducted from a remote location and controlled by timers. After the start switch was energized, the timers started the tape recorders, visicorders, igniter current and opened the vent tank valves. When the thermite reaction front caused closure of the burn wire in the bottom of the thermite vesse1, another timer was 
started that turned on the camera lights, started the Hycam camera, placed timing marks on the film, closed the vent tank valves, opened and closed the equalizer line valve, and opened the slide gate when used. The burn wire pair below the slide gate or diaphragm assembly controlled the start of the $X$-ray sequence. For tests CCM-5 and 6, only the initial timer was used for test control. Timing details for each test are presented in Section 3. The duration of each test was approximately 5 min.

\subsubsection{Posttest Operation}

After the test, the experiment area was surveyed for release of radioactive materials; three gas samples and a data logger scan were taken. After allowing the test apparatus to cool, another data logger scan was taken.

For the first four tests, after the system was depressurized the thermite vessel was removed and weighed to determine the amount of corium that had been injected. The thermite vessel was disassembled, during which photographs were taken, and the corium debris removed, weighed and stored. The head was unbolted from the interaction vessel and cleaned. Water was syphoned from the vessel and filtered. The top and middle sections of the vassel were removed and cleaned. After the debris bed on the vessel bottom was dried, the base and debris were separated from the interaction vessel bottom section.

The upper portion of the expansion vessel was unbolted from its lower part and cleaned. Water was drained and filtered from the bottom of the vessel and, after drying, the solid debris were recovered. At all stages of disassembly, appropriate photographs were taken and the debris were weighed and stored for further analyses.

The analog data tapes were digitized and appropriate plots were made.

For tests CCM-5 and 6, the experiment vesse1 top section was removed after system depressurization. The thermite vessel was lifted off the unistrut framework, disassembled, and cleaned. Water was drained and filtered from the bottom of the vessel. All electrical and instrumentation leads were disconnected. After all water had been evaporated from the experiment vessel, the unistrut framework and bottom pan were removed for cleaning and debris removal. Any remaining debris in the bottom of the vessel were then retrieved.

\subsection{Corium Characterization}

The corium selected for these tests had the same composition as the corium used in the CWTI tests (see Ref. 7). action:

The corium melt was generated by the following exothermic chemical re-

$$
\begin{aligned}
& 4.67 \mathrm{U}+2.83 \mathrm{Zr}+3 \mathrm{Fe}_{2} \mathrm{O}_{3}+2 \mathrm{CrO}_{3}+\mathrm{Ni}+ \\
& 4.67 \mathrm{UO}_{2}+2.83 \mathrm{ZrO}_{2}+6 \mathrm{Fe}+2 \mathrm{Cr}+\mathrm{Ni}+2.2 \mathrm{MJ} / \mathrm{Kg}
\end{aligned}
$$

The reaction products have a mass composition of about $60 \% \mathrm{UO}_{2}, 16 \% \mathrm{ZrO}_{2}$, and $24 \%$ steel $(67 \% \mathrm{Fe}, 21 \% \mathrm{Cr}, 12 \% \mathrm{Ni})$ by weight. 
Using the heats of formation for the oxides in Eq. 2.1 together with enthalpy data, a final "adiabatic reaction temperature" of $3372 \mathrm{C}$ is predicted. No additional thermodynamic processes are considered beyond the formation of the materials on the right hand side of Eq. 2.1, but in reality, additional processes are expected to occur. The oxides $\mathrm{UO}_{2}$ and $\mathrm{ZrO}_{2}$ form a solution in which one oxide is dissolved within the other. The heat of solution accompanying this reaction will alter the energy balance slightly. More significant, however, is the fact that at the high temperature predicted for the reaction products, the more volatile constituents may boil, thereby preventing the attainment of the adiabatic reaction temperature. Over the range of interest here, steel exerts the greatest vapor pressure and would be expected to boil off first. The thermite reactions took place at atmospheric pressure. Using the steel heat of vaporization at its atmospheric boiling temperature, up to $25 \%$ of the steel is predicted to have been boiled off at atmospheric pressure. The final reaction products have a temperature of $2807 \mathrm{C}$ (the nominal steel boiling temperature) and an enthalpy of $1.82 \mathrm{MJ} / \mathrm{kg}$, referenced to $20 \mathrm{C}$ (see Fig. 2-14).

The corium reaction products consist of two immiscible melts. The first melt is a low melting temperature metallic phase (the steel). The second phase is an oxide, which is a solid solution of $\mathrm{ZrO}_{2}$ in $\mathrm{UO}_{2}$. The temperature at which the corium begins to freeze is the liquidus temperature of the $\mathrm{UO}_{2}-\mathrm{ZrO}_{2}$ system. The $\mathrm{ZrO}_{2}$ presence amounts to $21 \%$ by mass (38\% mole fraction). From the data of Lambertson and Mueller, the liquidus temperature for this oxide solution is $2650 \mathrm{C}$ and the solidus temperature is $2570 \mathrm{C}$. Hence the melt has a "superheat" of about $160 \mathrm{C}$ referenced to the onset of oxide phase freezing; the melt superheat is about 1350 C compared to the steel phase liquidus temperature of 1460 C.

The molten reaction products form a pool at the bottom of the reaction vessel with a thin crust layer on the vessel walls. Typically, very small argon gas bubbles appear in the melt, a residue of the initial gas fraction in the packed powders. Due to the similarity of densities between the metallic and oxidic reaction, the stainless steel remains dispersed in the $\mathrm{UO}_{2} / \mathrm{ZrO}_{2}$ oxide phase.

Sample reactions were run in a small reaction vessel for all batches of depleted uranium (DU) powders used in the CWTI ${ }^{\top}$ tests. Samples of the gases from the reaction chamber were analyzed for impurities. The principal impurity was typically hydrogen. The maximum amount of hydrogen present was measured to be $0.25 \mathrm{mg} / \mathrm{g}$ reaction powders. Hence, the maximum hydrogen liberated during the reaction of a $4-\mathrm{kg}$ thermite charge was $1 \mathrm{~g}$ maximum, or about $0.5 \mathrm{~g}$-mole. 


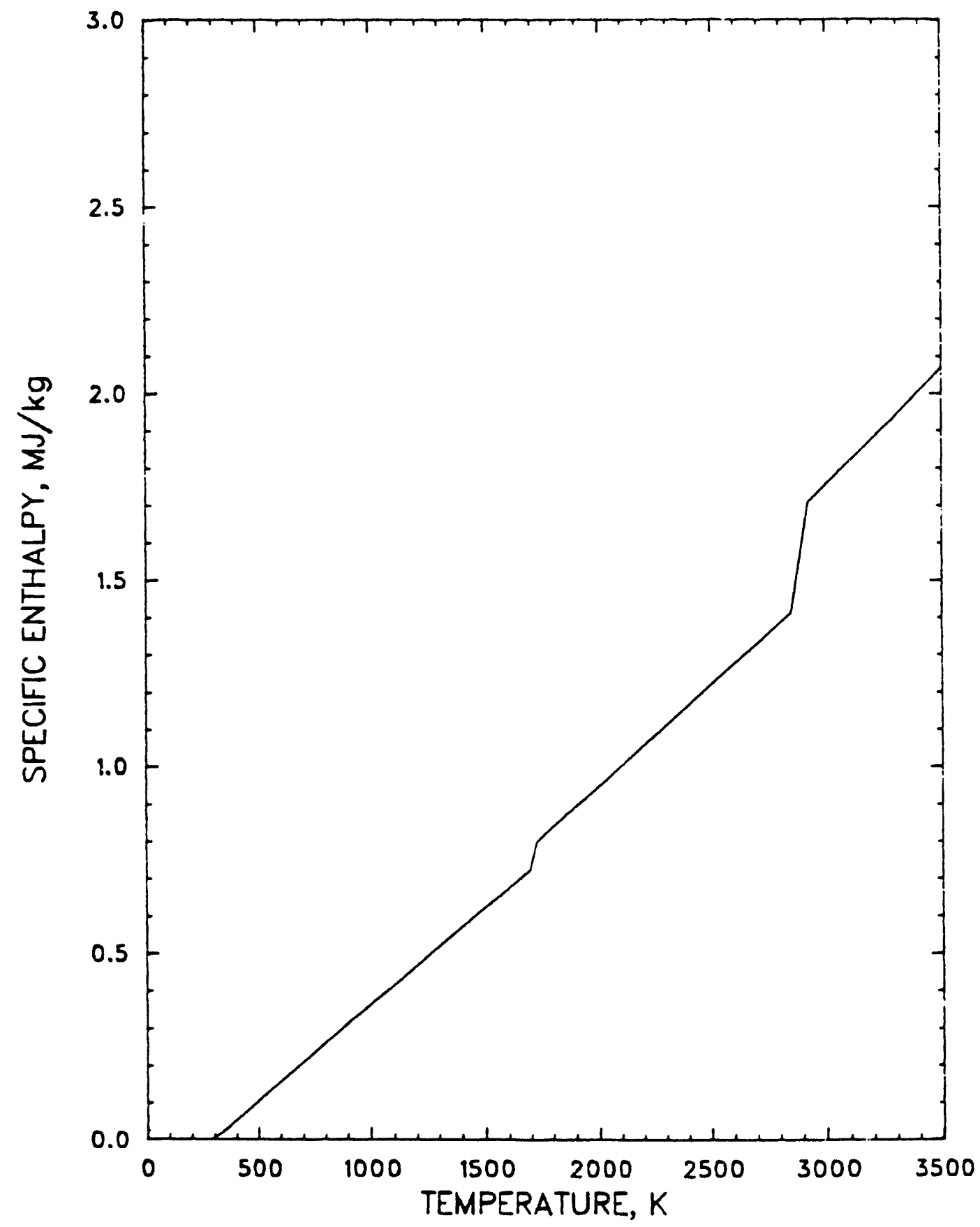

Figure 2-14. Enthalpy of Thermite Generated Corium Referenced to $20^{\circ} \mathrm{C}$. 
Following a brief summary of the test objectives, a description of the initial conditions and results is given for each test. Characterization of the debris recovered from the test apparatus, and gas sample analysis data, are also presented.

\subsection{Summary of Experiment Objectives}

The test program objectives were given in Section 1.2. Here, specific objectives for each test are given.

Experiment CCM-1 was the reference test with approximately $3-\mathrm{kg}$ of corium injected through a $25.4-\mathrm{mm}$ diameter hole into subcooled (approximately $43 \mathrm{C}$ ) water (depth $1.06 \mathrm{~m}$ ). The breakup behavior of the single pour stream, its debris characterization, and steam generation were to be examined.

In test CCM-2, a single larger diameter $(50.8 \mathrm{~mm})$ pour stream was broken into four pour streams by impingement on a submerged plate containing four holes (20.2-mm diameter) on a 26.4- $\mathrm{mm}$ square pitch. This test addressed the case of an array of pour streams which could be formed as the corium flows through core support and flow diffuser plates in the lower region of the reactor pressure vesse1. The objective was to examine the mixing behavior of perimeter jets where an adjacent body of water is continually present for mixing and entrainment. The water temperature was near saturation.

Test CCM-3 was similar to test CCM-1 with two exceptions. First, a slightly larger mass $(4.26 \mathrm{~kg})$ of corium was injected. Secondly, the water temperature was near saturation, so that by comparison with test CCM-l the effect of water subcooling on pour stream breakup, debris formation, and steam generation could be assessed.

Test CCM-4 was identical to test CCM-1 except that a larger corium mass $(10 \mathrm{~kg})$ and injection diameter $(50.8 \mathrm{~mm})$ were used. Hence, the effect of pour stream diameter could be evaluated.

For tests CCM-5 and 6 , the thermite vessel was placed inside the expansion vesse1. This provided a thirteen-fold increase in the water cross-sectional area, minimizing or eliminating the effect of confinement walls on the jet/water interaction. In test CCM-5, about $12 \mathrm{~kg}$ of corium was injected through a $50.8-\mathrm{mm}$ diameter opening into subcooled water. This test was a larger scale version of experiment CCM-1 and had the same objectives. Experiment CCM- 6 was identical to test CCM-5 except that the water temperature was near saturation. It was essentially a larger scale version of test CCM-3.

\subsection{Test Initial Conditions}

Initial conditions for the interaction vessel and the expansion/experiment vessel are shown in Table 3.1. The structure temperatures are mean values for the vesse 1 regions that were recorded just before the start of the test. The interaction vessel wall temperature was controlled to heat the water to its test temperature. For the pipeway and lower section of the expansion vessel, the 


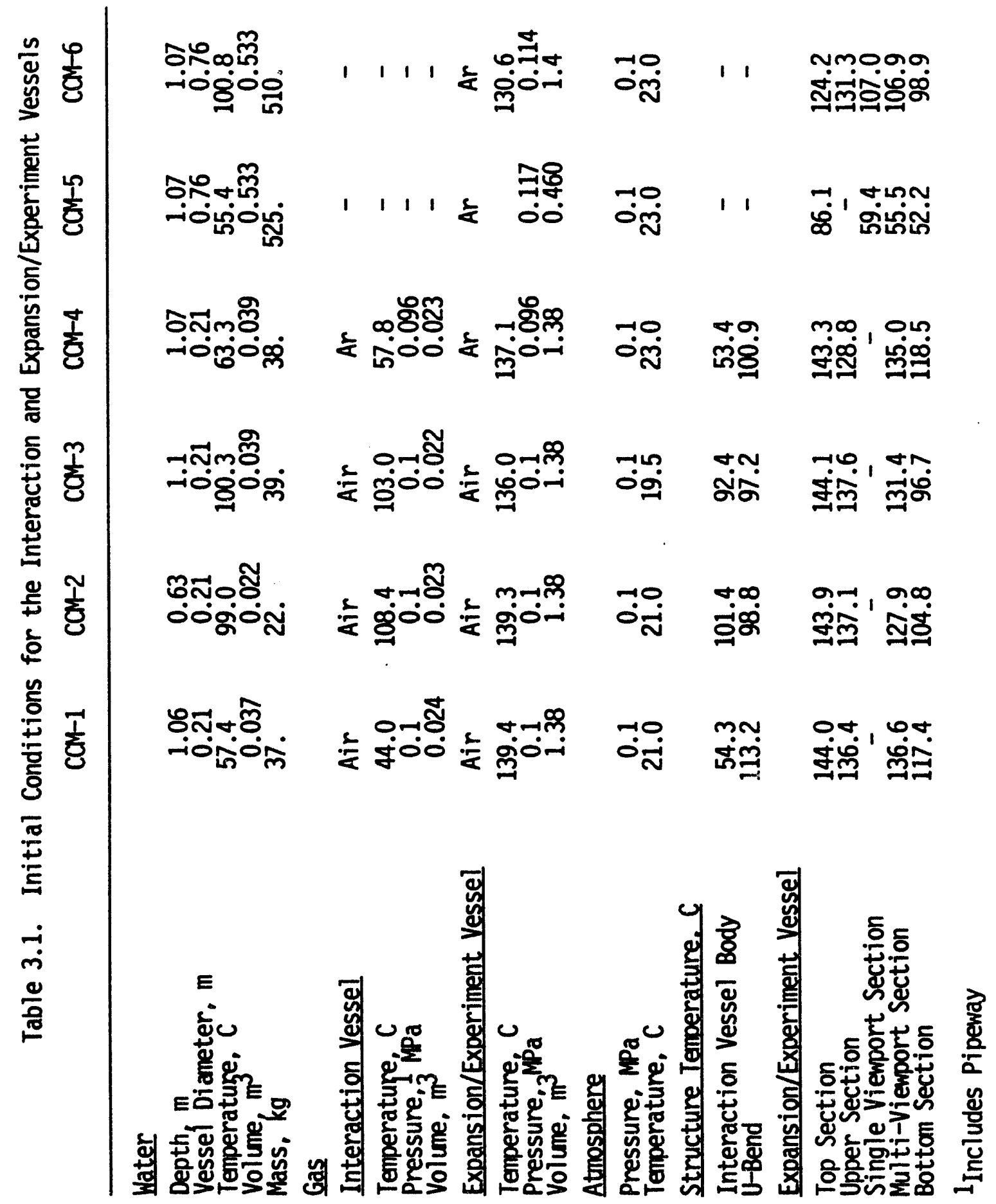


desired wall temperature was the water saturation temperature, so that steam generated from the corium-water interaction would not condense during the test, and water swept out of the interaction vessel would not boil in the U-bend or bottom of the expansion vessel. The upper sections of the expansion vessel were heated to approximately $140 \mathrm{C}$ to ensure that the steam would not condense. For the experiment vessel used in tests $\mathrm{CCM}-5$ and 6 , the temperature of the sections in contact with the water were controlied to heat the water to the desired temperature. The upper sections of the experiment vessel were heated to approximately $135 \mathrm{C}$ to prevent steam condensation. Interconnection between components, the location of the water-gas interface, and a limited number of heater zones caused some temperature variations. The initial values reported for the gas and water temperatures are mean values. Axial variations in the temperature occurred (see Section 3.3 and Appendices B-G).

The gas atmosphere inside the vessels was air for the first three tests and argon for the last three. The vessels were vented to the atmosphere during heatup to prevent pressurization. Shortly before the start of the test, the vent valve was closed. However, for tests CCM-5 and 6 , the vent line at the top of the experiment vessel was too small for complete depressurization of the system, so these tests were conducted with slightly elevated initial pressure.

Corium injection conditions are listed in Table 3.2. The size of the injection opening at the bottom of the thermite vessel determines the diameter of the corium pour stream. This diameter was $25.4 \mathrm{~mm}$ for tests CCM-1 and 3 and $50.8 \mathrm{~mm}$ for the remaining tests. The thermite reactants $(3.8$ to $12 \mathrm{~kg})$ were loaded into the thermite vessel inside a glove box with a high-purity argon environment. This 1-atm pressure cover gas was sealed into the thermite vessel.

The pressure and temperature inside the thermite vessel at the onset of injection were determined from test data. For tests CCM-1 through 4, solenoid valves on a vent line were opened during the reaction to prevent pressurization of the vessel. These valves were closed prior to injection and a valve on an equalizer line to the expansion vessel was opened. However, the pressure data for the thermite vessel show that a restriction to venting was formed. Disassembly of the solenoid valves after the early initiation of experiment CCM-4 revealed a stuck valve plunger, caused by buildup of fine products from the thermite reaction. (These materials were mildly radioactive, which is indicative of the presence of $\mathrm{UO}_{2}$.) The use of check valves on top of the thermite vessel for tests CCM-5 and 6 prevented pressurization of the vessel in these tests.

The mass of corium injected was determined by subtracting the posttest mass from the pretest mass of the thermite vessel, since some solidified corium remained on the walls and underside of the head of the thermite vessel. The mass of corium injected typically differed from the mass of corium debris retrieved from the test apparatus, as described in Section 3.4.

The thermite burn time is defined as the interval between ignition at the top of the reactants in the thermite vessel and the closure of the burn wire just above the opening in the bottom of the vessel. The duration of corium injection was inferred from the differential pressure between the thermite vessel and interaction or experiment vessel (see Figs. 3-1 through 3-6) and from motion pictures for tests CCM-5 and 6.

Corium stream velocities based on gravity flow alone, and flow with a 
Table 3.2. Initial Conditions for the Thermite Vessel and for Corium Injection

\begin{tabular}{|c|c|c|c|c|c|c|}
\hline & $\mathrm{CCH}-1$ & can-2 & $\mathrm{CCH}-3$ & $\mathrm{CCM}-4$ & CaM-5 & COH-6 \\
\hline Reactant Mass, kg & 3.784 & 12.012 & 4.8 & 10.01 & 12.012 & 12.012 \\
\hline Injected Corium Mass, kg & 2.15 & 11.15 & 3.34 & 9.24 & 11.34 & 12.79 \\
\hline Injection Diameter, mm & 25.4 & 20.0 & 25.4 & 50.8 & 50.8 & 50.8 \\
\hline Distance to Water Surface, $\mathrm{mm}$ & 454.0 & 435.0 & 412.8 & 447.7 & 339.7 & 339.7 \\
\hline Pressure at Onset of Injection, $\mathrm{MPa}$ & 0.11 & 0.175 & 0.315 & 0.355 & 0.134 & 0.202 \\
\hline Initial Gas Temperature, C & 2.63 & 29.0 & 24.0 & 20.0 & 116.9 & 100.4 \\
\hline $\begin{array}{l}\text { Gas Temperature at Onset of } \\
\text { Injection, C }\end{array}$ & 64.9 & 40.0 & 40.6 & 39.9 & 151.8 & 126.3 \\
\hline Burn Time, $s$ & 22.85 & 20.8 & 16.27 & 4.0 & 8.63 & 7.65 \\
\hline \multicolumn{7}{|l|}{ Corium Free Fall } \\
\hline Maximum Velocity at Water Surface, $\mathrm{m} / \mathrm{s}$ & 2.98 & 2.92 & 2.85 & 2.96 & 2.58 & 2.58 \\
\hline Time to Reach water Surface, s & 0.30 & 0.30 & 0.29 & 0.30 & 0.26 & 0.26 \\
\hline \multicolumn{7}{|l|}{ Corium Free Fall + Initial Velocity } \\
\hline Maximum Velocity at Water Surface, $\mathrm{m} / \mathrm{s}$ & 3.39 & 5.30 & 8.15 & 8.73 & 2.58 & 5.57 \\
\hline Time to Reach Water Surface, s & 0.18 & 0.09 & 0.05 & 0.05 & 0.26 & 0.06 \\
\hline \multicolumn{7}{|l|}{ Calculated Drain Time,s } \\
\hline Liquid Head Only & 0.99 & 0.78 & 1.19 & 0.69 & 0.78 & 0.78 \\
\hline Liquid Head + Initial $\Delta P$ & 0.54 & 0.17 & 0.16 & 0.08 & 0.78 & 0.16 \\
\hline
\end{tabular}




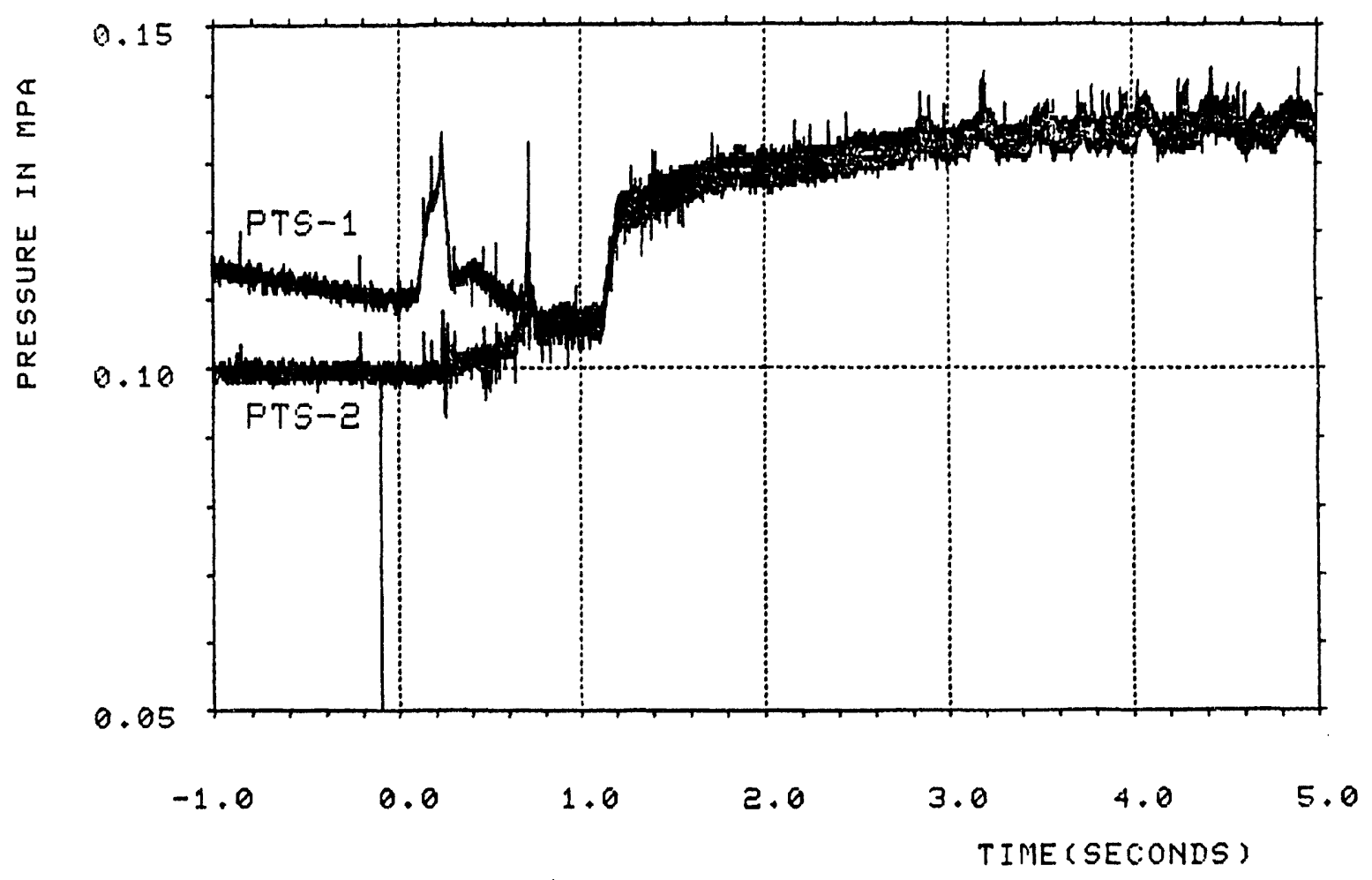

Fig. 3-1 Comparison of Thermite Vessel Pressure (PTS-1) with , Interaction Vessel Pressure (PTS-2) for Experiment CCM-1

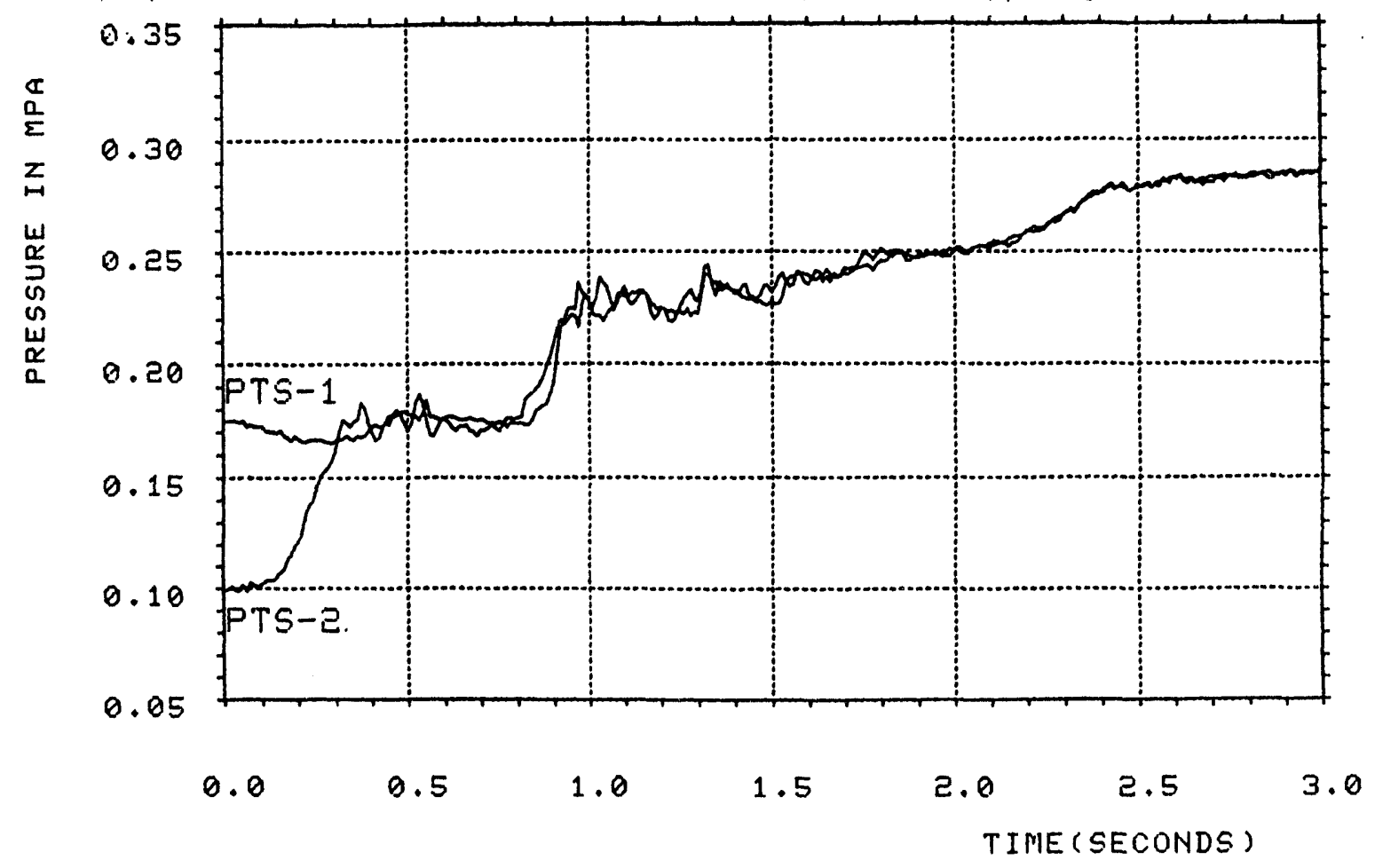

Fig. 3-2 Comparison of Thermite Vessel Pressure (PTS-1) with Interaction Vesse1 Pressure (PTS-2) for Experiment CCM-2 


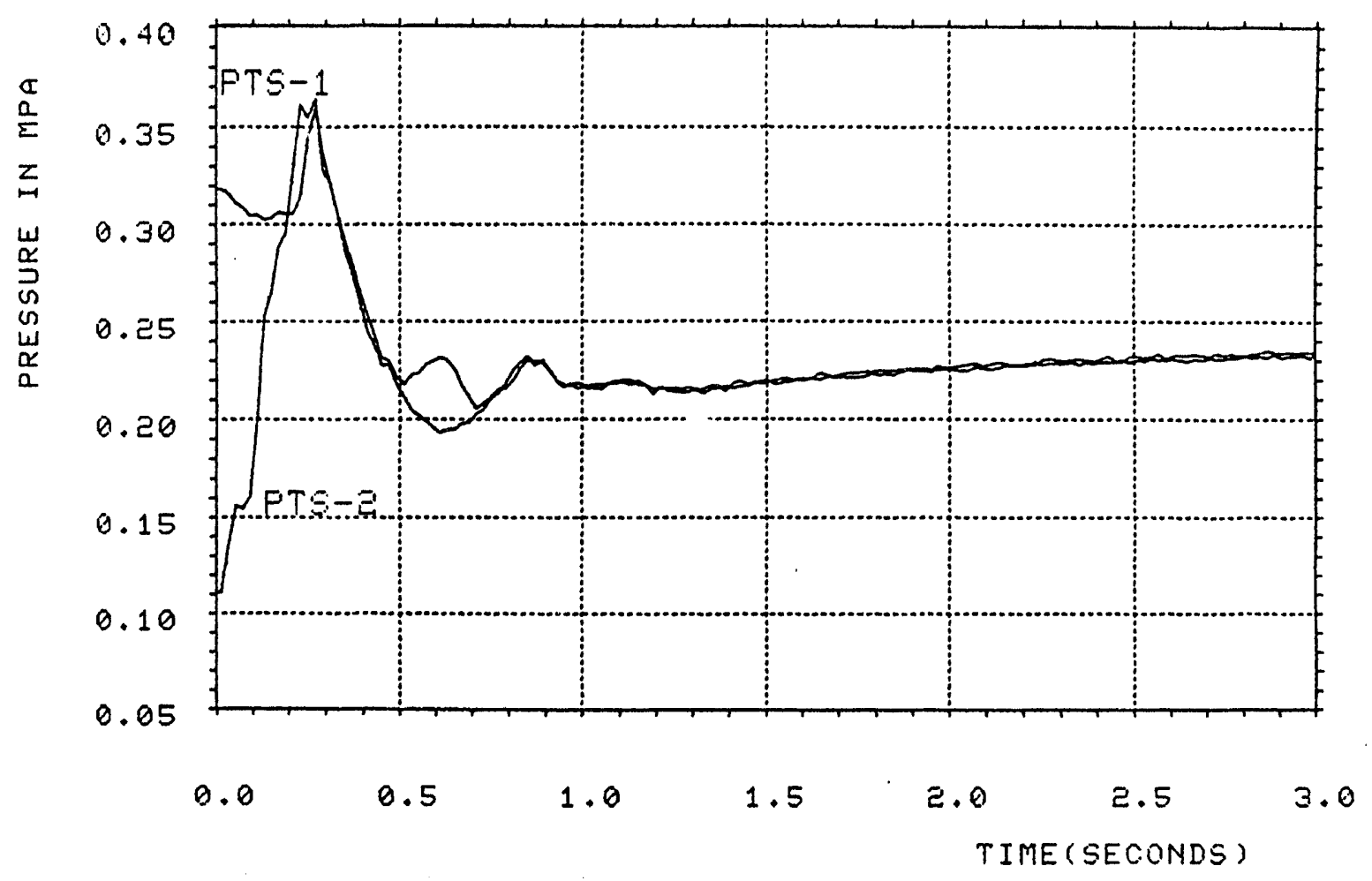

Fig. 3-3 Comparison of Thermite Vessel Pressure (PTS-1) with Interaction Vesse1 Pressure (PTS-2) for Experiment CCM-3

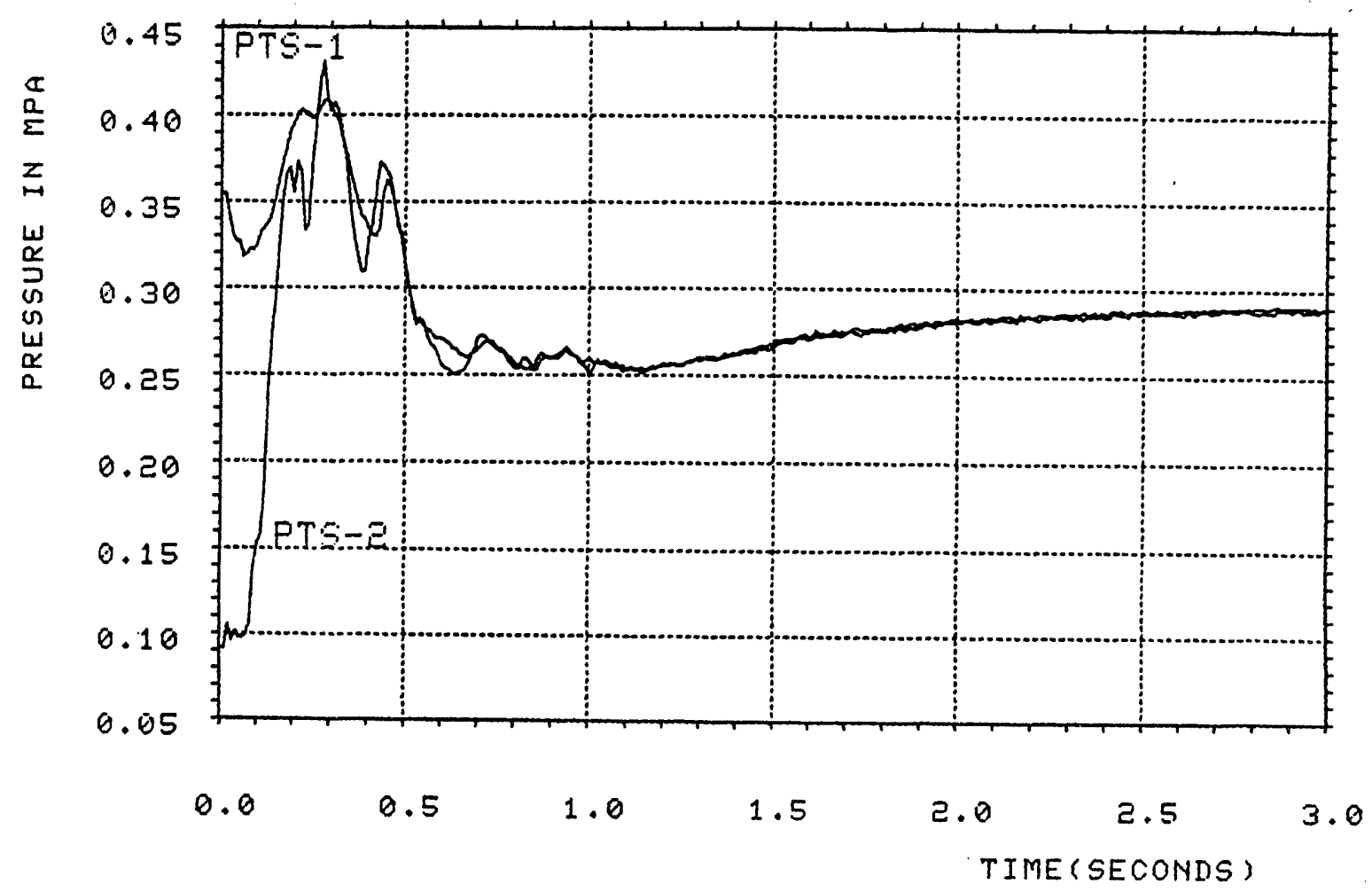

Fig. 3-4 Comparison of Thermite Vesse1 Pressure (PTS-1) with Interaction Vessel Pressure (PTS-2) for Experiment CCM-4 


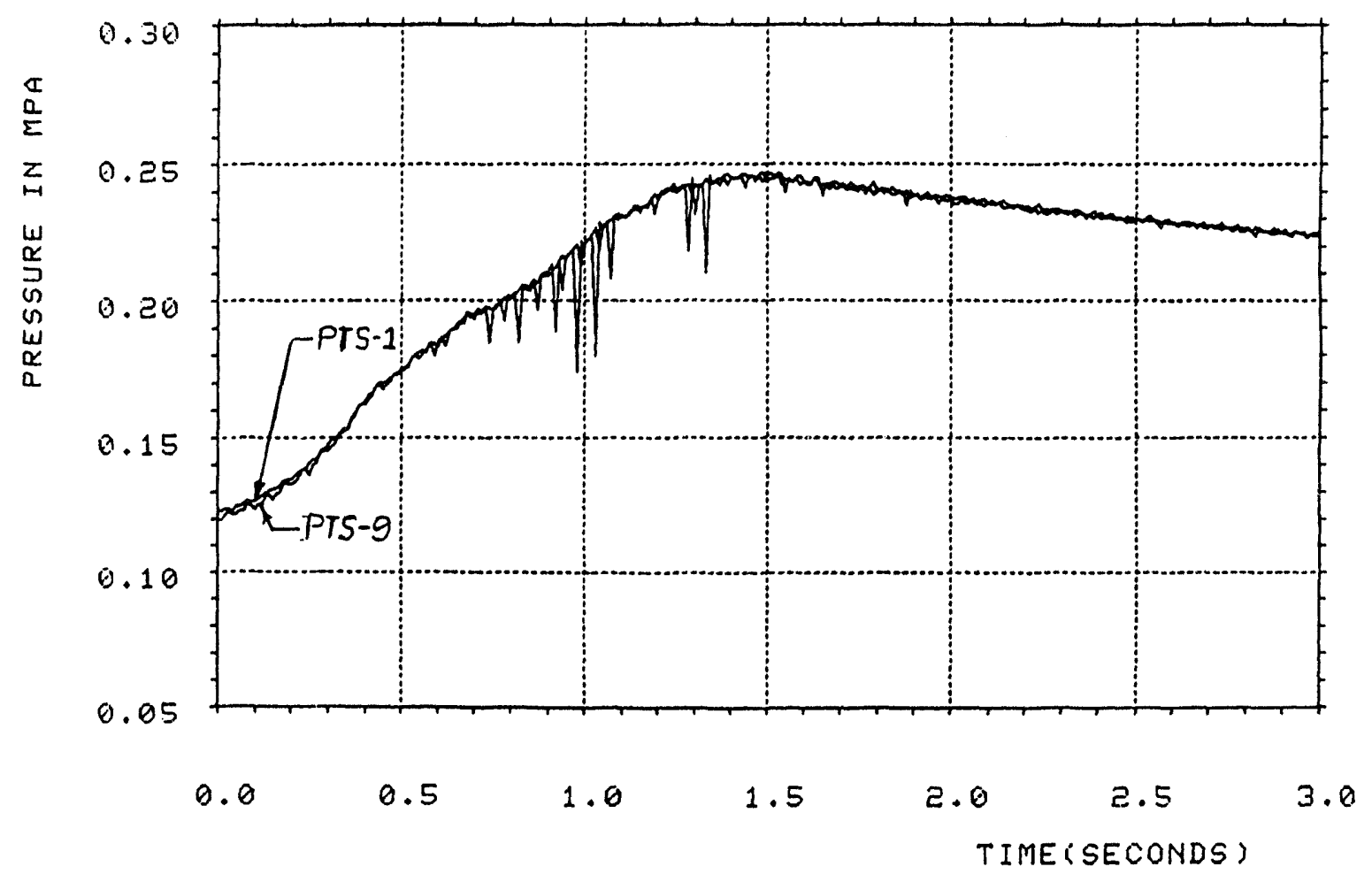

Fig. 3-5 Comparison of Thermite Vessel Pressure (PTS-1) with Experiment Vesse1 Pressure (PTS-9) for Experiment CCM-5

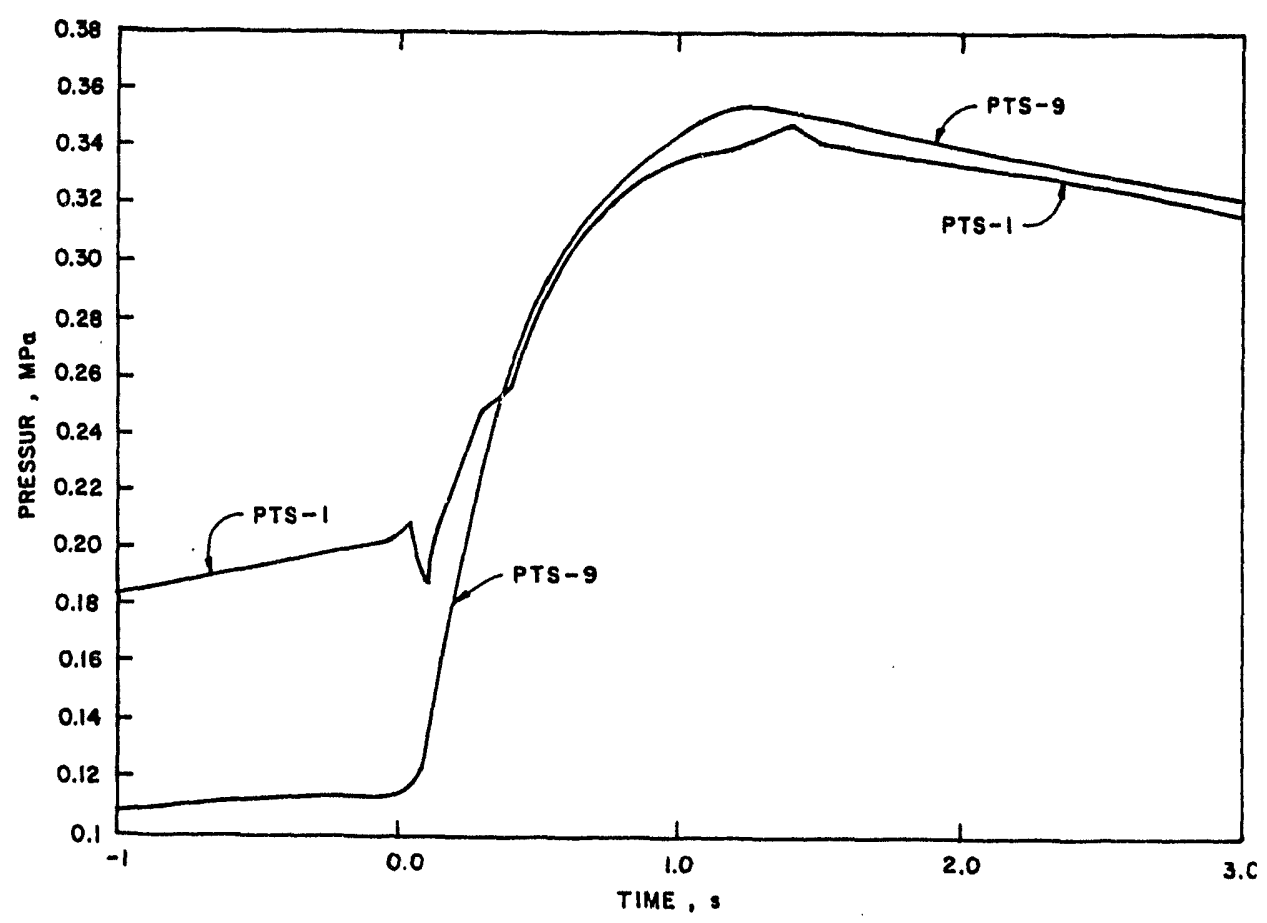

Fig. 3-6 Comparison of Thermite Vessel Pressure (PTS-1) with Experiment Vessel Pressure (PTS-9) for Experiment CCM-6 
constant pressure differential, have been calculated. The actual velocity should lie between these values. For the latter case, the velocity of the corium as it contacted the water surface was calculated as follows:

$$
v_{f}^{2}=v_{i}^{2}+2 g s
$$

where:

$$
\begin{aligned}
& \mathbf{g}=\text { acceleration of gravity, } \mathrm{m} / \mathrm{s}^{2}, \\
& \mathbf{s}=\text { distance from injector opening to water surface, } \mathrm{m}, \\
& v_{\mathbf{i}}=\text { initial corium velocity, } \mathrm{m} / \mathrm{s}, \text { and } \\
& v_{\mathbf{f}}=\text { corium velocity at water surface, } \mathrm{m} / \mathrm{s} .
\end{aligned}
$$

The initial corium velocity was estimated from the following equation:

$$
v_{i}=1000 C\left(\frac{2 \Delta P}{\rho}\right)^{1 / 2}
$$

where:

$$
\begin{aligned}
& C=\text { flow coefficient for the injector opening, } \\
& \Delta P=\text { pressure differential, } \mathrm{MPa}, \text { and } \\
& p=\text { corium density, } \mathrm{kg} / \mathrm{m}^{3} .
\end{aligned}
$$

From Ref. 12, the flow coefficient for a nozzle with the proportions of the lava cone in the thermite vessel is 0.99 . Table 3.2 lists the velocities calculated with no pressure differential, and with injector exit velocities calculated from equation (3.2) for $C=0.99$.

\subsection{Description of Test Results}

The experiments were remotely started and test events were controlled by timers. To initiate a test, an AC relay was closed; a time delay of up to 0.017 s could occur before the event started. In some instrumentation, e.g., Hycam camera starting, an additional AC relay in the circuitry could cause another similar time delay. The timer settings and corresponding test times for the experimental events are presented in Tables 3.3-3.5. Time zero for experiment CCM-1 was chosen as the time at which slide switch \#2 reached its maximum voltage. For experiments $\mathrm{CCM}_{-2}, 4,5$, and 6, time zero was designated as the closure time for the burn wire below the thermite vessel. This closure time was difficult to determine for experiment $\mathrm{CCM}-3$, so time zero was selected as the response time of PTS-2 and LP-1. 
Table 3.3. Timing Sequence for Experiment CCM-1

Event
Timer Setting, s
Test

Time, $s$

Timer start

\begin{tabular}{ll}
- & -30.978 \\
0.0 & -30.978 \\
0.0 & -30.978 \\
0.0 & -30.978 \\
4.0 & -26.978 \\
4.0 & -26.978 \\
- & -26.968 \\
- & -4.122 \\
& -4.110 \\
$0.0^{1}$ & -4.103 \\
$0.0^{1}$ & -4.103 \\
$0.0^{1}$ & -4.103 \\
$0.0^{1}$ & -4.103 \\
$0.0^{1}$ & -4.103 \\
$2.0^{1}$ & -2.103 \\
$2.0^{1}$ & -2.103 \\
$4.0^{1}$ & -0.103 \\
$4.0^{1}$ & -0.103 \\
$4.0^{1}$ & -0.103 \\
- & -0.021 \\
- & 0.00 \\
- & 0.386 \\
\hline &
\end{tabular}

$\mathrm{X}$-ray charge lock

Start two tape recorders (60 ips)

Start three visicorders (10 ips)

Open valve to accumulator

Turn on igniter power

Ignition of thermite reactants

Closure of burn wire in thermite vessel

Timer start

Ground piezoelectric transducers

Close vent valve on expansion vesse1

Start Hycam camera (1000 pps)

Start $\mathrm{X}$-ray camera (200 pps)

Turn on high-intensity lights for Hycam camera

Close valve to accumulator

Open valve on equalizer line

Close valve on equalizer line

Signal to open slide

Unground piezoelectric transducers

Coincident mark on Hycam film

Slide switch \#2 at maximum voltage (test time zero)

Start of X-ray pulse train (200 pps for $1.25 \mathrm{~s}$ )

$1_{\text {Based on closure of burn wire in thermite vessel }}$ 
Table 3.4. Timing Sequence for Experiments cak-2, 3, and 4

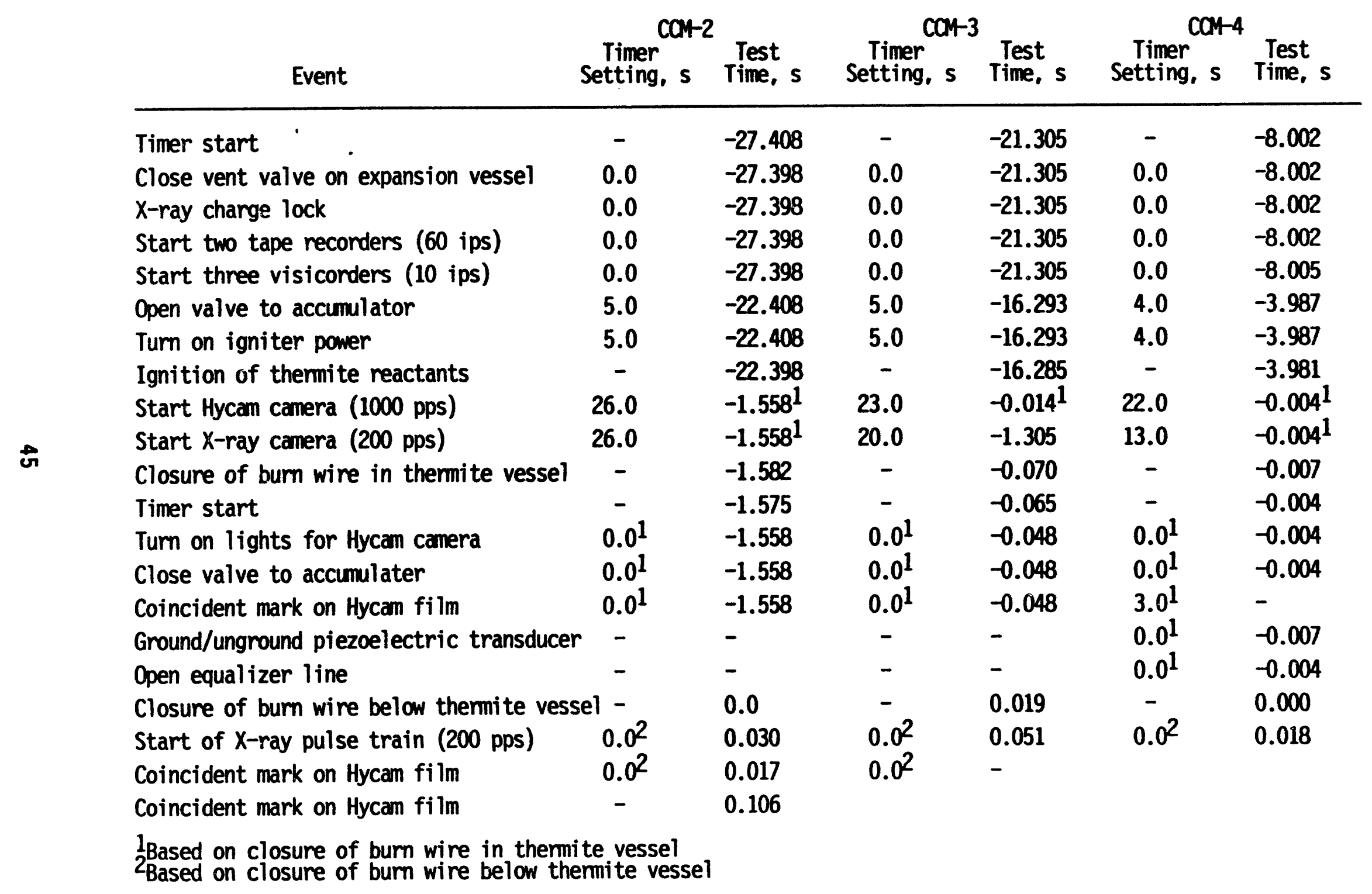


Table 3.5. Timing Sequence for Experiments CCM-5 and 6

\begin{tabular}{|c|c|c|c|c|}
\hline \multirow[b]{2}{*}{ Event } & \multicolumn{2}{|c|}{ CCM- 5} & \multicolumn{2}{|c|}{ CCM- 6} \\
\hline & $\begin{array}{l}\text { Timer } \\
\text { Setting, } s\end{array}$ & $\begin{array}{l}\text { Test } \\
\text { Time, } s\end{array}$ & $\begin{array}{c}\text { Timer } \\
\text { Setting, s }\end{array}$ & $\begin{array}{c}\text { Test } \\
\text { Time, s }\end{array}$ \\
\hline
\end{tabular}

\begin{tabular}{|c|c|c|c|c|}
\hline Timer start & - & -13.370 & - & -11.650 \\
\hline Start three tape decks (60 ips) & 0.0 & -13.362 & 0.0 & -11.660 \\
\hline Start seven visicorders (10 ips) & 0.0 & -13.362 & 0.0 & -11.660 \\
\hline Start video recorder & 0.0 & -13.362 & 0.0 & -11.660 \\
\hline Open vent line to accumulator & - & - & 4.0 & -7.650 \\
\hline Switch on ignitor power & 4.0 & -9.353 & 4.0 & -7.650 \\
\hline Ignition of thermite reactants & - & -9.342 & - & -7.640 \\
\hline $\begin{array}{l}\text { Turn on lights inside experiment } \\
\text { vessel }\end{array}$ & 10.0 & -3.355 & 8.0 & -3.643 \\
\hline Start four Hycam cameras (500 pps) & 12.0 & -1.320 & 10.0 & -1.648 \\
\hline Coincident mark on Hycam film & 15.0 & 1.637 & 13.0 & 1.350 \\
\hline $\begin{array}{l}\text { Closure of burn wire inside } \\
\text { thermite vessel }\end{array}$ & - & -0.716 & - & -0.038 \\
\hline Timer start & - & -0.713 & - & $\sim 0.01$ \\
\hline $\begin{array}{l}\text { Closure of burn wire below } \\
\text { thermite vessel }\end{array}$ & - & 0.0 & - & 0.0 \\
\hline Close vent line to accumulator & - & - & $0.5^{1}$ & 0.51 \\
\hline Coincident mark on Hycam film & $1.0^{1}$ & 0.296 & $1.0^{1}$ & 1.018 \\
\hline Coincident mark on Hycam film & $3.0^{1}$ & 2.304 & $3.0^{1}$ & 3.018 \\
\hline
\end{tabular}

$1_{\text {Based on closure of burn wire in thermite vessel }}$ 
In the following subsections, the events observed from $X$-ray films and Hycam pictures, where available, are presented along with a description of the pressure and temperature data. Debris characterization and results of gas analyses are discussed in later sections.

\subsubsection{Test CCM-1}

The thermite burn time for this test was $22.85 \mathrm{~s}$. The slide was opened $4.02 \mathrm{~s}$ after burn wire closure. A $2.15 \mathrm{~kg}$ mass of corium flowed into the interaction vessel in $0.63 \mathrm{~s}$, with an initial pressure differential of $0.012 \mathrm{MPa}$. The corium fell $454 \mathrm{~mm}$ through air before contacting the $57 \mathrm{C}$ water surface. In this test, the $X$-ray pictures started $0.39 \mathrm{~s}$ after time zero, which was too 1 ate to observe the initial corium injection.

Because of the poor quality and late timing of the X-ray pictures, only the events observed in the expansion vessel from the Hycam film are presented in Table 3.6.

Plots of the data that were digitized from the magnetic tapes are presented in Appendix B. A summary of the pressure data is presented in Table 3.7 .

Upon ignition, the thermite vessel pressurized mildly as the thermite reaction proceeded to completion. The pressure in the gas space of the thermite vessel was monitored by PTS-1. The pressure in the gas/vapor space of the interaction vessel was measured by PTS-2; it remained constant from its initial value of $0.1 \mathrm{MPa}$ until about $0.23 \mathrm{~s}$, when some small-magnitude pressure spikes occurred (see Fig. 3-1). From this point there was a slow increase in pressure until a pressure spike of $0.03 \mathrm{MPa}$ magnitude occurred at about $0.71 \mathrm{~s}$, which was caused by rapid steam generation. Although the steam generation process which produced this pressure spike was abrupt and vigorous, the relatively long rise time of $0.2 \mathrm{~s}$ indicates that it was not due to a steam explosion. The spike subsided as steam and levitated water droplets underwent blowdown into the expansion vessel via the discharge pipeway.

At the top of the expansion vessel, the pressure was measured by PTS-9, and in its lower region by PTS-10. The responses of these sensors were nearly identical, and except for the pressure spikes are similar to the responses of PTS-1 and PTS-2. The pressures measured in the interaction vessel and expansion vessel tracked one another except during the pressure spike immediately after corium injection. The pressure data showed a constant slope between $1.1 \mathrm{~s}$ and $1.3 \mathrm{~s}$, indicating vigorous but steady steam generation during the corium fall stage. A much milder pressurization in the expansion vessel and the interaction vessel after $1.3 \mathrm{~s}$ indicates that a debris bed generating steam at a much lower rate has formed on the vessel bottom.

The pressure in the water region of the interaction vessel was measured by four strain gage pressure transducers (PTS-12, PTS-13, PTS-14, and PTS-15). Their responses were nearly identical. The first indication of a pressure change was at about $0.23 \mathrm{~s}$ with a series of small pressure spikes. Next, there was a slight increase in pressure, followed by pressure spikes at $0.58,0.66,0.71$, and $0.91 \mathrm{~s}$. The pressure ramp was similar as described for PTS-1, 2, 9, and 10, and started at $1.12 \mathrm{~s}$. The behavior from this point onward was also similar.

Table 3.8 contains a summary of the temperature data. Plots of the transient temperatures are given in Appendix B. 
Table 3.6. Experiment CCM-1 Events Observed in the Expansion Vessel from the Hycam Film

Test

Time, s

Event

-0.021 First timing mark on film

$0.587 \quad$ First droplets

0.592 Red glow in pipeway

$0.604 \quad$ Glow fades

0.995 More droplets

1.087 Glowing particle

1.091 Fireball starting to form above pipeway exit

1.098 Fireball has joined flame emerging from pipeway

1.194 Water droplets appear in center of fireball and flame is spreading outward from pipeway exit

1.238 Flame is starting to diminish and more water croplets are emerging from the pipeway

1.291 Hardly any flame

1.334 Glowing particles along with water droplets are emerging from the pipeway

1.444 Flame starting to exit laterally from the pipeway and then dies

1.499 Another laterally exiting flame

1.613 Lots of glowing particles along with a brownish colored flame or smoke

1.641 Glowing particles tend to fall shortly after exiting pipeway

1.873 No visible glowing particles; brownish-black colored flame or smoke

2.201 Occasional glowing particle

2.953 Wisps of smoke

3.705 Water droplets appear

4.178 Atmosphere is smoky, so it is hard to tell if anything is exiting pipeway

$7.000 \quad$ Vapor possibly exiting from pipeway

8.829 Lights are starting to go out 
Table 3.7. Summary of Pressure Data for Experiment CaM-1

\begin{tabular}{lcccccc}
$\begin{array}{c}\text { Sensor } \\
\text { Number }\end{array}$ & $\begin{array}{c}\text { Pressure at } \\
\text { Time Zero, } \\
\text { MPa }\end{array}$ & $\begin{array}{c}\text { Time of } \\
\text { First Pressure } \\
\text { Change, } \\
\text { S }\end{array}$ & $\begin{array}{c}\text { Maximum } \\
\text { Pressure, } \\
\text { MPa }\end{array}$ & $\begin{array}{c}\text { Time to } \\
\text { Reach } \\
\text { Maximum } \\
\text { Pressure, } \\
\text { s }\end{array}$ & $\begin{array}{c}\text { Duration } \\
\text { of Maximum } \\
\text { Pressure, } \\
\text { S }\end{array}$ & $\begin{array}{c}\text { Pressure } \\
\text { at 250 S, } \\
\text { MPa }\end{array}$ \\
\hline PTS-1 & 0.110 & 0.092 & 0.135 & 3.2 & 3.8 & 0.104 \\
PTS-2 & 0.099 & 0.227 & 0.136 & 3.2 & 3.8 & 0.118 \\
PTS-9 & 0.100 & 0.223 & 0.136 & 3.6 & 3.2 & 0.119 \\
PTS-10 & 0.101 & 0.226 & 0.132 & 3.6 & 3.1 & 0.116 \\
PTS-12 & 0.100 & 0.230 & 0.130 & 3.2 & 2.6 & 0.113 \\
PTS-13 & 0.100 & 0.230 & 0.137 & 3.2 & 2.9 & 0.116 \\
PTS-14 & 0.100 & 0.232 & 0.136 & 3.2 & 3.6 & 0.116 \\
PTS-15 & 0.100 & 0.237 & 0.139 & 3.2 & 1.4 & 0.119
\end{tabular}


Table 3.8. Sumary of Temperature Data for Experiment COH-1

$\begin{array}{ccccccc}\begin{array}{c}\text { Thenmocouple } \\ \text { Number }\end{array} & \begin{array}{c}\text { Temperature } \\ \text { at Time Zero, } \\ \text { C }\end{array} & \begin{array}{c}\text { Time of } \\ \text { First Rise in } \\ \text { Temperature, } \\ \text { S }\end{array} & \begin{array}{c}\text { Max } \\ \text { Temperature, }\end{array} & \begin{array}{c}\text { Time of } \\ \text { Maximum } \\ \text { Temperature, } \\ \text { S }\end{array} & \begin{array}{c}\text { Duration of } \\ \text { Maximum } \\ \text { Temperature, } \\ \text { S }\end{array} & \begin{array}{c}\text { Temperature } \\ \text { at 250 }\end{array} \\ \text { C, }\end{array}$


Table 3.8. Surmary of Temperature Data for Experiment CCM-1 (cont.)

\begin{tabular}{|c|c|c|c|c|c|c|}
\hline $\begin{array}{c}\text { Thermocouple } \\
\text { Number }\end{array}$ & $\begin{array}{l}\text { Temperature } \\
\text { at Time Zero, } \\
C\end{array}$ & $\begin{array}{c}\text { Time of } \\
\text { First Rise in } \\
\text { Temperature, } \\
s\end{array}$ & $\begin{array}{c}\text { Max } \\
\text { Temperature, }\end{array}$ & $\begin{array}{c}\text { Time of } \\
\text { Maximum } \\
\text { Temperature, } \\
\text { s }\end{array}$ & $\begin{array}{c}\text { Duration of } \\
\text { Maximum } \\
\text { Temperature, } \\
\text { s }\end{array}$ & $\begin{array}{c}\text { Temperature } \\
\text { at } 250 \mathrm{~s} \text {. } \\
\mathrm{C}\end{array}$ \\
\hline $8-22$ & 57.9 & 0.72 & $>410$ & - & - & 80.9 \\
\hline $8-23$ & 58.3 & 0.69 & $>440^{1}$ & - & - & - \\
\hline $8-24$ & 58.3 & 0.63 & 190 & 1.1 & - & 79.8 \\
\hline $8-25$ & 58.1 & 0.66 & 80.2 & 20.2 & 4.2 & 79.9 \\
\hline $8-26$ & 55.5 & 0.92 & 76.0 & 24.0 & 2.0 & 73.8 \\
\hline $8-27$ & 57.7 & 0.68 & 215 & 1.6 & - & 79.3 \\
\hline $8-28$ & 114.6 & 0.31 & 162 & 0.76 & 0.04 & 90.8 \\
\hline $9-2$ & 67.9 & -22.53 & 183 & 47.5 & 8.6 & 137.7 \\
\hline $12-1$ & 141.4 & 1.22 & 184 & 16.3 & 4.2 & 144.3 \\
\hline $12-2$ & 145.4 & 1.45 & 168 & 3.4 & 1.0 & 151.3 \\
\hline $12-3$ & 144.6 & 1.14 & 203 & 12.7 & 3.6 & 147.7 \\
\hline $12-4$ & 143.6 & 1.22 & 200 & 6.5 & 5.2 & 147.0 \\
\hline $12-5$ & 136.3 & 1.20 & 188 & 13.0 & 3.3 & 139.3 \\
\hline $12-6$ & 137.0 & 1.13 & 200 & 9.8 & 2.1 & 138.5 \\
\hline
\end{tabular}


The temperature in the gas/vapor region of the interaction vessel was measured by TC8-12 and TC8-13. The responses of these thermocouples were somewhat different; though they are at the same radial position and elevation, the angular positions are different. The increases in temperature are caused by steam generation or a hot slug of water/vapor. The temperature rise detected by TC8-12 at 1.12 s coincides with the pressure rise in the interaction vessel, and is attributable to steam generation. The later gradual temperature decrease results from convective cooling.

The water temperature inside the interaction vessel was measured by four thermocouples in the top section (TC8-20,21, 22, and 23), four in the middle section (TC8-24, 25, 26, and 27) and eight just above the base (TC8-6, 7, 8, 9, $16,17,18$, and 19). Their radial locations in the pool varied. In the top section of the interaction vessel, thermocouple data indicated that the water temperature increased by $20 \mathrm{C}$ in $2 \mathrm{~s}$, then slowly dropped to $76 \mathrm{C}$ in $250 \mathrm{~s}$. The sharp temperature peaks of the thermocouple data indicated that some of the thermocouples were hit by corium. Thermocouple data in the middle section and the bottom section of the interaction vessel showed that the water temperature increased only slightly during the corium jet fall stage. The steel base was heated above $500 \mathrm{C}$ as the corium collected on the base, indicating that the corium was not completely quenched by traversing the deep water pool.

The temperature in the $U$-bend of the discharge pipeway was measured by TC8-28. The temperature increased rapidly due to the expulsion of steam from the interaction vessel during the corium jet fall stage. The temperature peak was then followed by a sharp temperature drop, possibly due to the impingement of the water droplets entrained by the gas influx.

The gas/vapor temperature inside the expansion vessel was measured by six thermocouples (TC12-1 through -6). The first rise in temperature varied from $1.1 \mathrm{~s}$ for TC8-10 near the pipeway exit to $1.45 \mathrm{~s}$ for TC12-2 near the top of the expansion vesse1. However, the maximum rise in temperature for TC8-10 was about $8 \mathrm{C}$ (see Table 3.8) compared with $63 \mathrm{C}$ for TC12-6 at the next higher elevation. The mean maximum temperature for the six thermocouples on the instrument tree was $144.7 \mathrm{C}$ or a $3.3 \mathrm{C}$ rise from the initial $141.4 \mathrm{C}$ temperature.

\subsubsection{Test CCM-2}

In this experiment the burn time was $20.8 \mathrm{~s}$. Corium injection started about $1.6 \mathrm{~s}$ after the burn. A mass of $11.15 \mathrm{~kg}$ flowed through a $50.8-\mathrm{mm}$ diameter hole with an initial pressure differential of $0.076 \mathrm{MPa}$. The corium fel1 $435 \mathrm{~mm}$ through an air atmosphere before contacting the $100 \mathrm{C}$ water surface $6.4 \mathrm{~mm}$ above an impingement plate containing four $20-\mathrm{mm}$ diameter holes.

The test events observed from the $X$-ray pictures are presented in Table 3.9. The initial contact of the corium with the water surface was not recorded due to a timing problem. The $X$-ray beam was positioned $50.8 \mathrm{~mm}$ below the water surface and was aligned to view three of the four corium streams. The pictures clearly show the individual streams (see Fig. 3-7). A strong steam cross-flow is indicated by the considerable deflection of the streams.

Table 3.10 contains a 1 ist of events observed in the expansion vessel from the Hycam film. The first indication of material exiting the pipeway occurs at $0.29 \mathrm{~s}$. Subsequently, glowing particles mixed with steam emerged in a pulsating 
Table 3.9. Experiment CCM-2 Events Observed in the Interaction Vesse1 from the X-Ray Film

Test

Time, s

Event

0.248 First good view of impingement plate and corium. No center jets. Side jets exit obliquely and break up. Some material in water above bottom of impingement plate.

0.272 Center jet, left jet, partial right jet. Side jets break up about $50 \mathrm{~mm}$ below impingement plate.

0.343 Center jet is deflected to left

$0.510 \quad$ All jets visible

0.574 Left jet penetrating deeper into water

0.630 Material that was driven upward starting to descend

0.642 Center and left jets break up just below impingement plate

0.677 Four jets visible and breakup about $49 \mathrm{~mm}$ below impingement plate

0.718 Center and right jets penetrate water below viewing range

$(-160 \mathrm{~mm})$. Left jet veers off to side.

0.741 Center and right jets but only a small left jet

0.754 Center jet penetrates below viewing range while left and right jets veer to side

0.813 Center and right jets break up just below impingement plate. Left jet breaks up at about $44 \mathrm{~mm}$ below impingement plate.

0.936 Four jets visible

0.952 No left jet

1.30 Last frame. Corium still exiting impingement-plate holes 


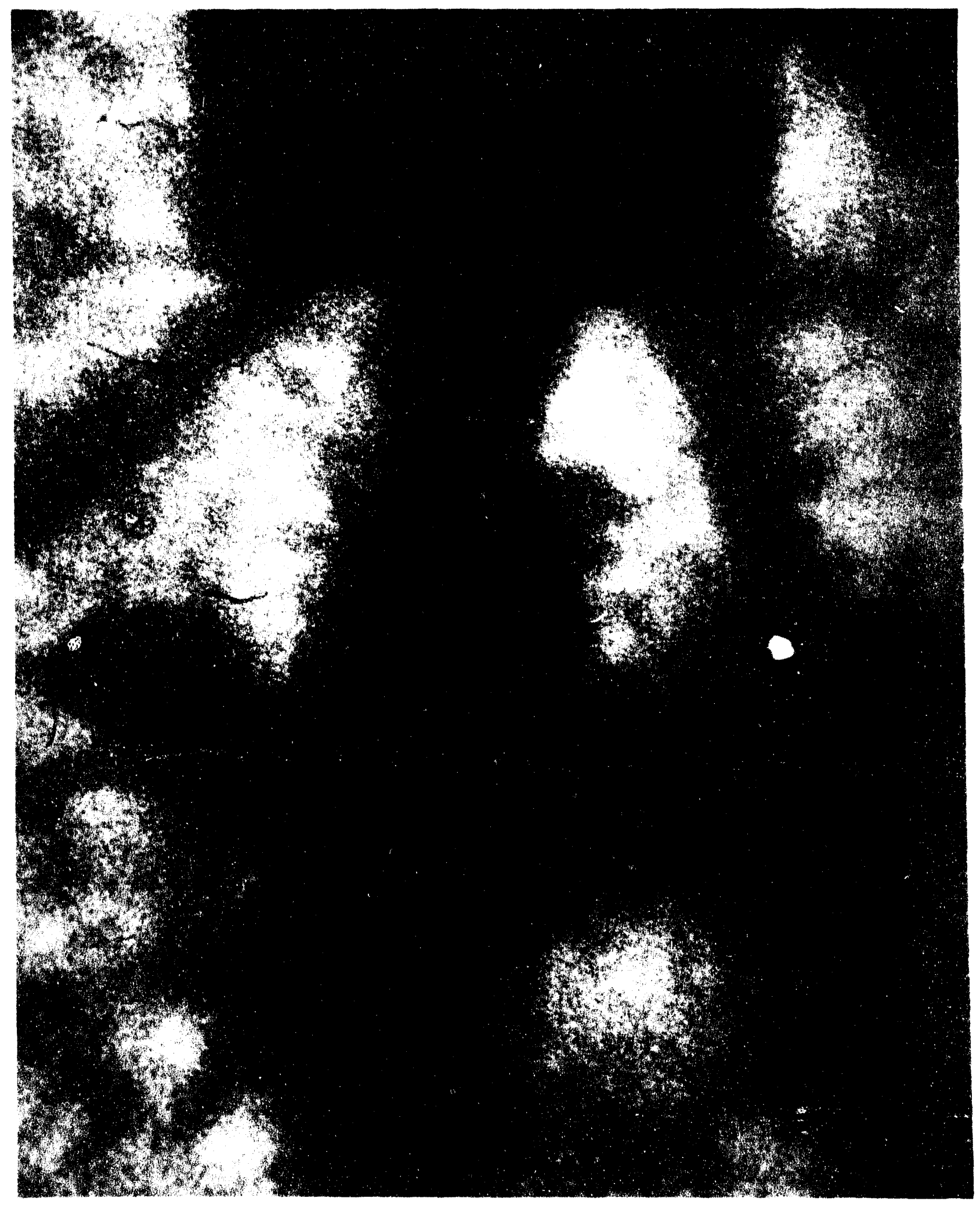

Fig. 3-7 X-Ray Photography of Corium Jets Emerging from the Impingement Holes for Experiment CCM-2 
Table 3.10. Experiment CCM-2 Events in the Expansion Vessel as Viewed from the Hycam Film

Test

Time, s

Event

0.291 First indication of material exiting pipeway

$0.399 \quad$ First glowing particle in steam flow

0.437 An increasing number of glowing particles are exiting pipeway

0.472 No glowing particles exiting

0.563 Another batch of glowing particles exiting

0.585 No glowing particles exiting

0.599 Another batch of glowing particles exiting (the particles exit in a pulsating pattern

1.009 First descending droplet or particle

1.062 Many descending particles. Some are large.

1.102 Small amount of glowing particles are exiting the pipeway

1.249 Larger amount of glowing particles are exiting the pipeway

1.603 First large descending glowing particle

1.767 Particles still exiting the pipeway

3.296 Last view of a glowing particle exiting the pipeway. View starting to cloud up from steam.

3.614 Last glowing descending particle

3.749 White, steamy atmosphere 
manner for about the next $3 \mathrm{~s}$.

Plots of the digitized data are located in Appendix C. A summary of the pressure data is given in Table 3.11.

The pressure in the gas space of the thermite vessel was measured by PTS-1. Similar to CCM-1, the pressure increased steadily until the injection of the corium. A series of pressure decreases and increases occurred during the next $0.7 \mathrm{~s}$. The pressure in the thermite vessel upon injection onset was $0.175 \mathrm{MPa}\left(\Delta \mathrm{P}_{\mathrm{mj}}=0.075 \mathrm{MPa}\right)$. The gas vapor pressure in the interaction vessel was measured by PTS-2 (see Fig. 3-2). It began to rise from its time zero value of $0.1 \mathrm{MPa}$ at about $0.06-0.09 \mathrm{~s}$. The pressure in the interaction vessel peaked several times and exceeded the pressure in the thermite vessel between 0.2 to $0.6 \mathrm{~s}$ (i.e., during the corium injection). It is conceivable that the corium pour may have been retarded or even interrupted by this differential pressure reversal. No pressure spike was observed.

The gas/vapor pressure inside the expansion vessel was measured by PTS-9 at the top of the vessel and by PTS-10 at its lower side. Their behavior wassimilar. Unlike that of the interaction vessel, the expansion vessel pressure showed a steady increase from 0 to $3 \mathrm{~s}$. The maximum pressure differential of $0.07 \mathrm{MPa}$ between the interaction vessel and the expansion vessel caused a maximum steam flow of $-90 \mathrm{~m} / \mathrm{s}$ through the pipeway. A much milder pressurization in the expansion vessel and the interaction vessel after $3 \mathrm{~s}$ indicated that a debris bed with a much lower steaming rate has formed. It is interesting to note that the pressure increase during the jet fall stage was not as rapid as CCM-1, indicating the extent of the jet breakup was not as extensive as in CCM-1.

In the water region of the interaction yessel, the pressure was measured by four transducers. PTS -12 and PTS-14 were located $63.5 \mathrm{~mm}$ below the initial water surface, and PTS-13 and PTS-15 were located $317.5 \mathrm{~mm}$ below the water surface. The behavior of these sensors was similar to that of PTS-2.

Temperatures for experiment CCM-2 were measured by 23 thermocouples. A summary of the data is presented in Table 3.12. The temperature in the gas space of the thermite vessel was measured by TC9-2.

Thermocouples TC8-12 and TC8-13 were located about $25.4 \mathrm{~mm}$ below the head of the interaction vessel to measure gas/vapor temperature. A rapid temperature increase measured by TC8-12 indicated that a high-temperature gas flux (i.e., steam, water droplets, and possibly the corium droplets) occurred during the time period between 1.3 and $2.6 \mathrm{~s}$. However, TC8-13, located in the same radial (but different angular) position showed a rather steady temperature increase. This may imply that steam generation was not uniform across the water surface. It is interesting to note that both $\mathrm{TC} 8-12$ and $\mathrm{TC} 8-13$ registered a significant temperature rise between $20 \mathrm{~s}$ to $50 \mathrm{~s}$. This is a strong indication that steam generation due to the debris bed was significant, i.e., the debris bed was not quenched.

Four thermocouples were located in the main body of the interaction vessel water. The temperature data showed that all the thermocouples were hit by the corium. Some appeared to have melted. 
Table 3.11. Sumary of Pressure Data for Experiment COH-2

\begin{tabular}{lcccc}
$\begin{array}{c}\text { Time of } \\
\begin{array}{c}\text { First Pressure } \\
\text { Change. } \\
\text { s }\end{array}\end{array}$ & $\begin{array}{c}\text { Maximum } \\
\text { Pressure, } \\
\text { MPa }\end{array}$ & $\begin{array}{c}\text { Time to } \\
\text { Reach } \\
\text { Maximum } \\
\text { Pressure, } \\
\text { s }\end{array}$ & $\begin{array}{c}\text { Duration } \\
\text { of Maximm } \\
\text { Pressure, } \\
\text { s }\end{array}$ & $\begin{array}{c}\text { Pressure } \\
\text { at 250 s, } \\
\text { MPa }\end{array}$ \\
\hline $0.03-0.05$ & 0.362 & 250 & - & 0.362 \\
$0.06-0.09$ & 0.362 & 250 & - & 0.362 \\
0.02 & 0.361 & 250 & - & 0.361 \\
$0.02-0.07$ & 0.351 & 250 & - & 0.351 \\
$0.02-0.06$ & 0.314 & 250 & - & 0.314 \\
0.04 & 0.354 & 250 & - & 0.354 \\
$0.04-0.09$ & 0.357 & 250 & - & 0.357 \\
$0.03-0.11$ & 0.386 & 250 & - & 0.386
\end{tabular}


Table 3.12. Summary of Temperature Data for Experiment Car-2

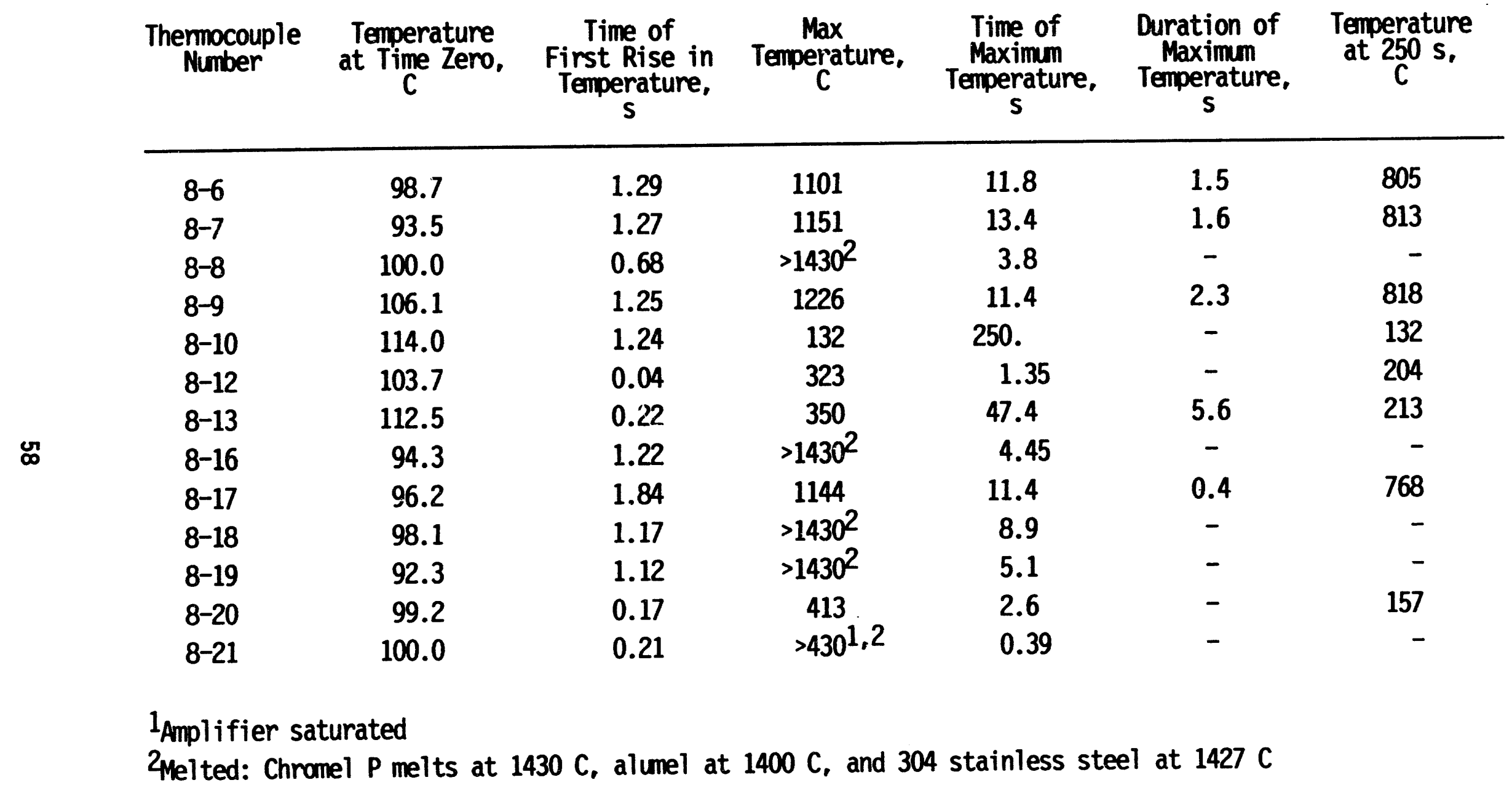


Table 3.12. Summary of Temperature Data for Experiment CaM-2 (cont.)

\begin{tabular}{ccccccc}
$\begin{array}{c}\text { Thermocouple } \\
\text { Number }\end{array}$ & $\begin{array}{c}\text { Temperature } \\
\text { at Time Zero, } \\
\text { C }\end{array}$ & $\begin{array}{c}\text { Time of } \\
\text { First Rise in } \\
\text { Temperature, } \\
\text { S }\end{array}$ & $\begin{array}{c}\text { Max } \\
\text { Temperature, } \\
\text { C }\end{array}$ & $\begin{array}{c}\text { Time of } \\
\text { Maximum } \\
\text { Temperature, } \\
\text { S }\end{array}$ & $\begin{array}{c}\text { Duration of } \\
\text { Maximum } \\
\text { Temperature, } \\
\text { S }\end{array}$ & $\begin{array}{c}\text { Temperature } \\
\text { at 250 } \\
\text { S, }\end{array}$ \\
\hline $8-22$ & 100. & 0.37 & $>4301$ & 4.4 & - & - \\
$8-23$ & 75.8 & 0.67 & $>4301$ & 0.98 & - & 131.7 \\
$8-28$ & 97.5 & 0.0 & 131.7 & 4.3 & 246. & 163.1 \\
$9-2$ & 40.3 & 0.7 & 245 & 83. & 6.8 & 136.2 \\
$12-1$ & 140.0 & 0.55 & 136.2 & 163. & 87. & 138.8 \\
$12-2$ & 146.2 & 0.53 & 138.8 & 88. & 5. & 139.4 \\
$12-3$ & 142.3 & 0.39 & 145.8 & 38. & 5. & 140.0 \\
$12-4$ & 142.0 & 0.39 & 141.7 & 58. & 14. & 140.0 \\
$12-5$ & 132.8 & 0.39 & 144.2 & 56. & 6. & 137.4 \\
$12-6$ & 132.4 & 0.47 & 137.4 & 174. & 76. &
\end{tabular}


Near the base of the interaction vessel, there were eight thermocouples. Four (TC8-6, 7, 9, and 17) were located $3.2 \mathrm{~mm}$ above the base ( $629 \mathrm{~mm}$ below the water surface). The remaining four (TC8-8, 16, 18 and 19) were positioned $6.4 \mathrm{~mm}$ above the base (625 $\mathrm{mm}$ below the water surface).

The group of thermocouples located closer to the vessel bottom all showed a steady temperature rise from $-1 \mathrm{~s}$ to $-10 \mathrm{~s}$, with a much slower decrease thereafter. The temperature increase indicates the arrival of corium debris at the vessel bottom. The high debris cooling rate between $20 \mathrm{~s}$ to $100 \mathrm{~s}$ accounts for the heatup of the interaction vessel atmosphere during this time period.

The second group of four thermocouples (TC8-8, 16, 18 and 19) were $3.2 \mathrm{~mm}$ above the first group. Whereas the first group reached a maximum temperature of 1100-1200 C, all the thermocouples in this group melted. It is conceivable that during the bed-forming period, the debris collected on the upper layer of the bed transferred less energy to the water than the debris collected earlier (i.e., on the lower layer) did, since the void fraction of the water would have been very high during the later stage of the bed forming period.

Thermocouple TC8-28 was located about $25 \mathrm{~mm}$ upstream of the center of the pipeway $U$-bend. It showed that the temperature in the pipeway increased to 130 $C$ during the steam blowdown period and stayed unchanged thereafter/.

Inside the experiment vesse1, the gas/vapor temperature was measured by 7 thermocouples. Thermocouple TC8-10 was at the vessel center at the level of the pipeway discharge. It showed a temperature response similar to TC8-28, located in the pipeway, except that the initial temperature was higher due to a preheated expansion vessel atmosphere. Thermocouples TC12-1 through 6 were positioned radially about $32 \mathrm{~mm}$ from the vessel center and were axially $406 \mathrm{~mm}$ apart. All thermocouple data show a decrease in expansion yessel temperature during the corium injection as water is displaced from the interaction vessel into the expansion vessel. Heatup of the vapor in the expansion vessel by steam and/or corium particles is indicated after $-1 \mathrm{~s}$.

Five level detectors were adjacently positioned above the water surfaces at heights of 3.2 to $60.3 \mathrm{~mm}$ (see Table A.3). Level probe LP-1 was positioned $3.2 \mathrm{~mm}$ above the water surface. It responded at time zero. For level probe LP2 , located $9.5 \mathrm{~mm}$ above the water surface, the response time was from 0.03 to $3.66 \mathrm{~s}$. Level probe LP-3 was $22.2 \mathrm{~mm}$ above the water, and its response time was from 0.07 to $3.24 \mathrm{~s}$. A response from 0.09 to $3.2 \mathrm{~s}$ was obtained for level probe LP-4, which was located $34.9 \mathrm{~mm}$ above the water surface. Level probe LP-5 was $60.3 \mathrm{~mm}$ above the water; its response times were 0.14 to $1.1 \mathrm{~s}$ and 2.48 to $2.71 \mathrm{~s}$.

From the level detector response data, the estimated pool swell velocity was $0.3-1.8 \mathrm{~m} / \mathrm{s}$. The mean swell velocity to the uppermost level probe was $0.75 \mathrm{~m} / \mathrm{s}$.

\subsubsection{Test $\mathrm{CCM}-3$}

Experiment CCM-3 had a burn time of $16.27 \mathrm{~s}$ which was $6.67 \mathrm{~s}$ shorter than that in experiment CCM-1. Corium injection started about $0.07 \mathrm{~s}$ after burn completion. A mass of $3.34 \mathrm{~kg}$ was injected through a $25.4-\mathrm{mm}$ diameter opening under an initial pressure differential of $0.215 \mathrm{MPa}$. The corium fell $413 \mathrm{~mm}$ 
through air before contacting the surface of the water, whose temperature was 100.3 C and whose depth was about $1.1 \mathrm{~m}$.

For this experiment time zero was based on the response of pressure transducer PTS-2 in the interaction vessel, rather than the normal basis for time zero, i.e., the closure of the burn wire below the thermite vessel. Consequently, the test time corresponding to the timing marks on the Hycam film cannot be precisely inferred; the events presented in Table 3.13 are referenced to film time with time zero being the first visible indication of water droplets.

Plots of the digitized data are presented in Appendix D. A summary of the pressure data is given in Table 3.14.

The pressure in the thermite injector, measured by PTS-1, began to increase at $-13 \mathrm{~s}$, i.e., $3.3 \mathrm{~s}$ after ignition of the reactants. The pressure rose from $0.1 \mathrm{MPa}$ to a peak of $0.362 \mathrm{MPa}$ at $0.27 \mathrm{~s}$. Following the sudden drop at $0.51 \mathrm{~s}$ due to the injection, the pressure increased and decreased several times and tracked the pressure in the interaction vessel.

The gas pressure inside the interaction vessel was measured by PTS-2 (see Fig. 3-3). It rose abruptly from time zero to a peak of $0.362 \mathrm{MPa}$ at $0.23 \mathrm{~s}$. Due to a large pressure difference between the thermite vessel and the interaction vessel upon the onset of injection, the corium jet velocity at water surface was calculated to be as $8.15 \mathrm{~m} / \mathrm{s}$ as listed in Table 3.2 .

The gas/vapor pressure inside the expansion vessel was measured by PTS-9 at the top of the vessel and by PTS-10 at the bottom of the vessel. From its time zero value of $0.1 \mathrm{MPa}$, the pressure measured by PTS-9 began to rise at $0.06 \mathrm{~s}$. The rate of rise was $0.14 \mathrm{MPa} / \mathrm{s}$ until up to $0.5 \mathrm{~s}$, then $0.09 \mathrm{MPa} / \mathrm{s}$ until $1.13 \mathrm{~s}$. Beyond this time the pressure rose slowly from $0.219 \mathrm{MPa}$ to $0.244 \mathrm{MPa}$ at $15 \mathrm{~s}$. The pressure remained constant for about $20 \mathrm{~s}$ and then slowly decreased to $0.218 \mathrm{MPa}$ at $300 \mathrm{~s}$. The behavior of PTS-10 was similar. Its initial response was also at $0.06 \mathrm{~s}$ but its pressure peak was $0.235 \mathrm{MPa}$. The pressure at $300 \mathrm{~s}$ was $0.21 \mathrm{MPa}$ which is $4 \%$ lower than the value obtained by PTS-9.

In the water region of the interaction vessel, four pressure transducers (PTS-12, 13, 14 and 15) were used and they were located at 76, 330, 591 and $830 \mathrm{~mm}$ below the water surface, respectively. Their behavior was similar to PTS-2 with the initial response occurring at time zero.

Temperature data from the 27 thermocouples used in the test are summarized in Table 3.15. The gas temperature inside the thermite vessel was measured by TC9-2. It increased steadily during the thermite reaction, as in the previous tests.

In the gas/vapor region of the interaction vessel, the temperature was measured by thermocouples TC8-12 and TC8-13. Data from thermocouple TC8-13 were not recorded because of a faulty data channel. The gas temperature as measured by TC8-12 had a time zero value of $105.4 \mathrm{C}$ and increased slightly from this point to $109.8 \mathrm{C}$ at $0.11 \mathrm{~s}$. A sudden temperature drop to $97.5 \mathrm{C}$ occurring at $0.3 \mathrm{~s}$ may have been caused by a slug of cooler water hitting the thermocouple.

Eight thermocouples were located in the water region of the interaction vesse1. Most were hit by corium. The water temperature rise during the jet fall 
Table 3.13. Events Observed from the Hycam Film for Experiment CCM-3

Film

Time, $s$

Event

0.0 Water droplets appear to be descending. View is dim and pipeway outlet is not visible.

0.089

First glowing particles emerging from pipeway

오

0.137

More glowing particles starting to exit pipeway

0.205

Downward moving water vapor

1.066

Larger mass of glowing particles are emerging

1.168

Dark debris particles being expelled along with glowing particles

2.023

Glowing particles visible in a smoky background

2.081

Glowing particles starting to descend. Possibly a few particles are exiting the pipeway.

4.071 Last view of glowing descending particle. Atmosphere is growing too smoky for further visualization. 
Table 3.14. Summary of Pressure Uata for Experiment Car-3

\begin{tabular}{lllcccc}
$\begin{array}{c}\text { Sensor } \\
\text { Number }\end{array}$ & $\begin{array}{c}\text { Pressure at } \\
\text { Time Zero, } \\
\text { MPa }\end{array}$ & $\begin{array}{c}\text { Time of } \\
\text { First Pressure } \\
\text { Change, } \\
\text { s }\end{array}$ & $\begin{array}{c}\text { Maximum } \\
\text { Pressure, } \\
\text { MPa }\end{array}$ & $\begin{array}{c}\text { Time to } \\
\text { Reach } \\
\text { Maximm } \\
\text { Pressure, } \\
\text { s }\end{array}$ & $\begin{array}{c}\text { Duration } \\
\text { of Maximum } \\
\text { Pressure, } \\
\text { s }\end{array}$ & $\begin{array}{c}\text { Pressure } \\
\text { at }\end{array}$ \\
$\begin{array}{lllllll}\text { MPa } \\
\text { s, }\end{array}$ \\
\hline PTS-1 & 0.318 & 0.0 & 0.361 & 0.27 & - & 0.218 \\
PTS-2 & 0.1 & 0.0 & 0.362 & 0.23 & - & 0.219 \\
PTS-9 & 0.1 & 0.06 & 0.245 & 10.0 & 20 & 0.218 \\
PTS-10 & 0.1 & 0.06 & 0.235 & 10.0 & 20 & 0.210 \\
PTS-12 & 0.1 & 0.0 & 0.322 & 0.25 & - & 0.194 \\
PTS-13 & 0.1 & 0.0 & 0.384 & 0.26 & - & 0.209 \\
PTS-14 & 0.1 & 0.0 & 0.352 & 0.27 & - & 0.212 \\
PTS-15 & 0.1 & 0.0 & 0.389 & 0.27 & - & 0.230
\end{tabular}


Table 3.15. Summary of Temperature Data for Experiment Car-3

\begin{tabular}{|c|c|c|c|c|c|c|}
\hline $\begin{array}{l}\text { Thermocouple } \\
\text { Number }\end{array}$ & $\begin{array}{l}\text { Temperature } \\
\text { at Time Zero, } \\
\text { C }\end{array}$ & $\begin{array}{l}\text { Time of } \\
\text { First Change in } \\
\text { Temperature, } \\
\text { s }\end{array}$ & $\underset{C}{\text { Max }}$ & $\begin{array}{c}\text { Time of } \\
\text { Maximum } \\
\text { Temperature, } \\
\text { s }\end{array}$ & $\begin{array}{c}\text { Duration of } \\
\text { Maximum } \\
\text { Temperature, } \\
\text { s }\end{array}$ & $\begin{array}{c}\text { Temperature } \\
\text { at } 300 \mathrm{~s} \text {. } \\
\mathrm{C}\end{array}$ \\
\hline $8-6$ & 87.5 & 1.04 & 580. & 11.87 & 1.09 & 110.0 \\
\hline $8-7$ & 90.4 & 1.00 & 116.4 & 10.79 & 19.2 & 112.5 \\
\hline $8-8$ & 97.5 & 0.90 & 134.7 & 17.9 & - & 118.3 \\
\hline $8-9$ & 98.1 & 1.33 & 277. & 1.47 & - & 120.0 \\
\hline $8-10$ & 113.8 & 0.24 & 123.3 & 8.0 & 11.4 & 118.8 \\
\hline $8-12$ & 105.4 & 0.0 & 110. & 22.8 & 1.5 & 109.2 \\
\hline $8-13$ & Bad channel & & & & & \\
\hline $8-16$ & 93.0 & 1.00 & 118.8 & 15.89 & 84. & 118.3 \\
\hline $8-17$ & 93.9 & 0.88 & 119.6 & 8.33 & 52. & 115.4 \\
\hline $8-18$ & 95.0 & 0.93 & 122.5 & 4.58 & 45. & 115.4 \\
\hline $8-19$ & 93.0 & 0.81 & 118.7 & 12.20 & 63. & 115.0 \\
\hline $8-20$ & 100.0 & 0.08 & $>466.0^{1}$ & 0.12 & - & 121.7 \\
\hline $8-21$ & 100.0 & 0.14 & $>466.0^{1}$ & 0.56 & - & 123.2 \\
\hline
\end{tabular}


Table 3.15. Summary of Temperature Data for Experiment CaM-3 (cont.)

\begin{tabular}{ccccccc}
$\begin{array}{c}\text { Themocouple } \\
\text { Number }\end{array}$ & $\begin{array}{c}\text { Temperature } \\
\text { at Time Zero, } \\
\text { C }\end{array}$ & $\begin{array}{c}\text { Time of } \\
\begin{array}{c}\text { First Change in } \\
\text { Temperature, } \\
\text { s }\end{array}\end{array}$ & $\begin{array}{c}\text { Max } \\
\text { Temperature, } \\
\text { C }\end{array}$ & $\begin{array}{c}\text { Time of } \\
\text { Maximum } \\
\text { Temperature, } \\
\text { s }\end{array}$ & $\begin{array}{c}\text { Duration of } \\
\text { Maximum } \\
\text { Temperature, } \\
\text { s }\end{array}$ & $\begin{array}{c}\text { Temperature } \\
\text { at 300 } \\
\text { S, }\end{array}$ \\
\hline $8-22$ & 100.0 & 0.13 & $>466.01$ & 0.34 & - & 122.1 \\
$8-23$ & 100.0 & 0.15 & 179.5 & 0.66 & - & 121.0 \\
$8-24$ & 101.2 & 0.28 & 136.7 & 0.60 & - & 124.6 \\
$8-25$ & 100.0 & 0.15 & $>466.01$ & 0.42 & - & 123.8 \\
$8-26$ & 90.3 & 1.11 & $>466.0$ & 1.40 & - & - \\
$8-27$ & 101.2 & 0.26 & 127.5 & 19.4 & 20.6 & 124.2 \\
$8-28$ & 98.1 & 0.05 & 123.1 & 0.33 & - & 116.7 \\
$9-2$ & 40.6 & 0.0 & 239.0 & 72.1 & - & 240.0 \\
$12-1$ & 136.0 & 0.22 & 125.0 & 6. & 34. & 121.6 \\
$12-2$ & 138.6 & 0.20 & 125.0 & 5. & 20. & 126.3 \\
$12-3$ & 139.6 & 0.08 & 130.8 & 166. & 22. & 130.0 \\
$12-4$ & 137.8 & 0.13 & 130.8 & 41. & 50. & 127.1 \\
$12-5$ & 132.6 & 0.05 & 127.9 & 22. & 6.6 & 125.8 \\
$12-6$ & 131.3 & 0.12 & 125.0 & 15. & 25. & 121.2
\end{tabular}


stage ranged from -15 to $25 \mathrm{C}$ depending on the location of the thermocouples.

Eight thermocouples were located 6.4 to $10.2 \mathrm{~mm}$ above the base of the interaction vessel. Only the thermocouple with the lowest elevation (i.e., TC8-6) registered a significant temperature increase, indicating the formation of a debris bed, while all the other thermocouples registered a much milder temperature increase indicative of water heatup. The debris bed was not thick enough to embed three of the eight thermocouples. The four others showing little temperature increase were either not embedded by the debris or were exposed only to well-quenched particles that settled on the base after being fluidized during the corium injection.

The temperature in the pipeway U-bend was measured by TC8-28 1ocated $45.7 \mathrm{~cm}$ below the pipeway outlet in the expansion vessel. The temperature in the pipeway rose to a maximum during the jet injection period, then tracked the temperature in the expansion vessel.

Inside the expansion vesse1, seven thermocouples were used to measure the gas/vapor temperature. Thermocouple TC8-10 was at the same elevation as the pipeway outlet. Thermocouples TC12-1 through -6 were positioned radially about $32 \mathrm{~mm}$ from the vessel center and $406 \mathrm{~mm}$ apart axially. All the thermocouples registered a temperature drop, which indicated blowdown of steam/water droplets from the interaction vessel. The temperature then rose gradually to a maximum of $-125 \mathrm{C}$ throughout the expansion vesse1.

The five level probes were positioned as in experiment CCM-2. Level probe LP-1 at $3.2 \mathrm{~mm}$ above the surface had a response time of -0.01 to $1.76 \mathrm{~s}$. For level probe LP-2, located $9.5 \mathrm{~mm}$ above the water surface, the initial response occurred at $0.05 \mathrm{~s}$. Level probe LP-3 was $22.2 \mathrm{~mm}$ above the water surface; response occurred at $0.07 \mathrm{~s}$ and lasted until $1.8 \mathrm{~s}$. Located $34.9 \mathrm{~mm}$ above the water surface, level probe LP-4 showed a response from 0.11 to $1.75 \mathrm{~s}$. Level probe LP-5 was $60.3 \mathrm{~mm}$ above the water surface and had a response time of $0.12 \mathrm{~s}$ to $1.65 \mathrm{~s}$.

From these data it appears that a splash wave occurred, followed by a level rise from steam generation. The mean swell velocity to the uppermost level probe is estimated to be $1.1 \mathrm{~m} / \mathrm{s}$.

\subsubsection{Test CCM-4}

In experiment CCM-4, the vent line from the chermite vessel was plugged, and the buildup of pressure in the vessel from the thermite reaction caused an early failure of the diaphragm. This limited the burn time for the reactants to $4 \mathrm{~s}$. A mass of $8.53 \mathrm{~kg}$ of corium flowed through a 50.8-mm diameter hole under an initial pressure differential of $0.259 \mathrm{MPa}$. The corium fell $448 \mathrm{~mm}$ through an argon atmosphere to the surface of the water, whose temperature was $63.3 \mathrm{C}$ and whose depth was $1.073 \mathrm{~m}$. Because of the short burn time, X-ray and Hycam pictures were not obtained. The extent of the thermite reaction is unknown.

The digitized data are presented in Appendix E, and a summary of the pressure data is given in Table 3.16, except for transducer PTK-1, whose responses are suspect. The main function of this Piezoelectric transducer was to detect a steam explosion, which did not occur. 
Table 3.16. Sumary of Pressure Data for Experiment COM-4

\begin{tabular}{lcccccc}
$\begin{array}{c}\text { Sensor } \\
\text { Number }\end{array}$ & $\begin{array}{c}\text { Pressure at } \\
\text { Time Zero, } \\
\text { MPa }\end{array}$ & $\begin{array}{c}\text { Time of } \\
\text { First Pressure } \\
\text { Change, } \\
\text { s }\end{array}$ & $\begin{array}{c}\text { Maximum } \\
\text { Pressure, } \\
\text { MPa }\end{array}$ & $\begin{array}{c}\text { Time to } \\
\text { Reach } \\
\text { Maximum } \\
\text { Pressure, } \\
\text { S }\end{array}$ & $\begin{array}{c}\text { Duration } \\
\text { of Maximum } \\
\text { Pressure, } \\
\text { S }\end{array}$ & $\begin{array}{c}\text { Pressure } \\
\text { at 300 S, } \\
\text { MPa }\end{array}$ \\
\hline PTS-1 & 0.355 & 0.0 & 0.409 & 0.29 & 0. & 0.274 \\
PTS-2 & 0.092 & 0.02 & 0.430 & 0.28 & 0. & 0.269 \\
PTS-9 & 0.094 & 0.0 & 0.307 & 15.9 & 14.1 & 0.274 \\
PTS-10 & 0.095 & 0.0 & 0.30 & 10.6 & 19.4 & 0.265 \\
PTS-12 & 0.097 & 0.0 & 0.365 & 0.27 & 0. & 0.24
\end{tabular}


The gas/vapor pressure inside the interaction vessel was measured by PTS-2 (see Fig. 3-4). At time zero the pressure was $0.092 \mathrm{MPa}$; it rose suddenly to $0.369 \mathrm{MPa}$ at $0.19 \mathrm{~s}$. The pressure underwent some fluctuations before reaching its peak of $0.430 \mathrm{MPa}$ at $0.28 \mathrm{~s}$, then declined to $0.253 \mathrm{MPa}$ at $1.13 \mathrm{~s}$. Its later behavior was similar to that of PTS 1. Due to a large pressure difference between the thermite vessel and the interaction vessel upon the onset of injection, the corium jet velocity at the water surface was calculated to be $8.73 \mathrm{~m} / \mathrm{s}$. In the expansion vessel, transducer PTS -9 measured the gas/vapor pressure at the top of the vesse1; PTS-10 was located at the lower sidewall. The behavior of these sensors was similar. At time zero, the pressure measured by PTS-9 was $0.094 \mathrm{MPa}$. Significant pressure increase occurred between 0.2 to 2.0 $s$, indicating high steam generation rate during the jet fall stage. Transducer PTS-12 measured the water pressure inside the interaction vessel at $50.8 \mathrm{~mm}$ below the water surface. Its behavior is similar to that of PTS-2 but with a lower peak value of $0.356 \mathrm{MPa}$.

Twenty seven thermocouples were used for temperature measurements. A summary of these data is given in Table 3.17. The temperature in the thermite vessel gas space was measured by TC9-2; it rose sharply from its initial value $(20 \mathrm{C})$ to $-160 \mathrm{C}$ at $2 \mathrm{~s}$.

The gas/vapor temperature in the interaction vessel was measured by thermocouples TC8-12 and TC8-13. The temperature measured by TC8-12 rose from its initial value of $58.5 \mathrm{C}$ to $115.3 \mathrm{C}$ at $2 \mathrm{~s}$. Thermocouple TC8-13 was located nearer the discharge pipeway entrance than TC8-12. Except for a spurious signal at $0.04 \mathrm{~s}$, thermocouple TC8-13 had a response similar to that of TC8-12 up to about $20 \mathrm{~s}$. Temperatures measured by these thermocouples after $20 \mathrm{~s}$ differed significantly. This indicates that steam generation was not uniform across the water surface.

In the water region of the interaction vessel, fourteen thermocouples were used, with three or four at each of four different levels (see Table A.5). At $50.8 \mathrm{~mm}$ below the water surface, thermocouples TC8-23, TC8-32, and TC8-33 were located $4.44 \mathrm{~cm}(T C 8-23)$ and $6.35 \mathrm{~cm}(T C 8-32,33)$ from the centeriline. All of these thermocouples were hit by corium, and T:8-33 was melted. The rate of the radial expansion of the corium/water mixture, based on the response time of these thermocouples, was bounded by 0.02 and $0.2 \mathrm{~m} / \mathrm{s}$. It must be noted that a short interval between corium contacts at two different radial locations does not necessarily indicate rapid radial expansion of the mixture. Rather, it may indicate that the descending mixture front is wide enough to emcompass both thermocouples.

Thermocouples TC8-20, TC8-21, and TC8-22 were located $304.8 \mathrm{~mm}$ below the water surface at radii of $88.9,57.2$, and $82.6 \mathrm{~mm}$, respectively. All of these thermocouples were hit by the corium. The rate of expansion of the corium/water mixture was estimated to be -0.02 to $0.09 \mathrm{~m} / \mathrm{s}$. Compared to that seen by the thermocouples located at the higher elevation, the rate of expansion of the corium/water mixture appears to have been relatively low.

Three thermocouples (TC8-24, TC8-25, and TC8-29) were located $562 \mathrm{~mm}$ below the water surface at radij of $76.2,34.9$, and $63.5 \mathrm{~mm}$, respectively. The temperature measured by TC8-24 shows only water heating, while the other two thermocouples were hit by corium. TC8-29 registered an earlier temperature jump than TC8-25, yet TC8-25 was located nearer the centerline. This indicates that 
Table 3.17. Sumary of Temperature Data for Experiment COM-4

$\begin{array}{ccccccc}\begin{array}{c}\text { Thermocouple } \\ \text { Number }\end{array} & \begin{array}{c}\text { Temperature } \\ \text { at Time Zero, } \\ \text { C }\end{array} & \begin{array}{c}\text { Time of } \\ \text { First Change in } \\ \text { Temperature, } \\ \text { s }\end{array} & \begin{array}{c}\text { Max } \\ \text { Temperature, } \\ \text { C }\end{array} & \begin{array}{c}\text { Time of } \\ \text { Maximm } \\ \text { Temperature, } \\ \text { s }\end{array} & \begin{array}{c}\text { Duration of } \\ \text { Maximum } \\ \text { Temperature, } \\ \text { s }\end{array} & \begin{array}{c}\text { Temperature } \\ \text { at }\end{array} \\ \text { 300 } \text { S, } \\ 8-6 & 37.1 & 0.83 & 97.9 & 2.89 & 0.0 & 78.1 \\ 8-7 & 46.2 & 0.84 & 246 . & 0.95 & 0.0 & 121.9 \\ 8-8 & 48.9 & 0.84 & 839 . & 1.15 & 0.06 & 125.5 \\ 8-9 & 50.7 & 0.88 & >1400.2 & 2.6 & - & - \\ 8-10 & 125.0 & 0.03 & 113.0 & 15.0 & 27.0 & 108.9 \\ 8-12 & 58.5 & 0.05 & 155.0 & 107 . & 10.0 & 130.4 \\ 8-13 & 57.1 & 0 . & 183.3 & 0.04 & - & 110.0 \\ 8-16 & 44.3 & 0.83 & >1400.2 & 3.67 & - & - \\ 8-17 & 46.2 & 1.67 & 1000 . & 10.28 & 1.40 & 120.0 \\ 8-18 & 48.3 & 1.59 & 1227 . & 4.40 & 1.12 & 120.3 \\ 8-19 & 41.5 & 1.82 & 417 . & 2.57 & 0.0 & 113.4 \\ 8-20 & 69.4 & 0.17 & >1400.2 & 1.17 & - & - \\ 8-21 & 60.4 & 0.13 & >1400.2 & 0.53 & - & - \\ 8-22 & 61.8 & 0.26 & 588 . & 1.15 & 0 . & 111.8 \\ 8-23 & 64.2 & 0.04 & 628 . & 0.27 & 0.06 & 123.9 \\ 8-24 & 65.0 & 0.17 & 129.2 & 65 . & 21 . & 126.7 \\ 8-25 & 61.7 & 1.06 & 843 . & 1.68 & 0 . & 128.4 \\ 8-26 & 53.8 & 0.62 & >1400.2 & 1.85 & - & - \\ 8-27 & 53.7 & 0.57 & 125.3 & 100.0 & 73 . & 121.1 \\ 8-28 & 98.4 & 0.02 & 122.5 & 1.22 & 0.17 & 113.0\end{array}$


Table 3.17. Summary of Temperature Data for Experiment CCM-4 (cont.)

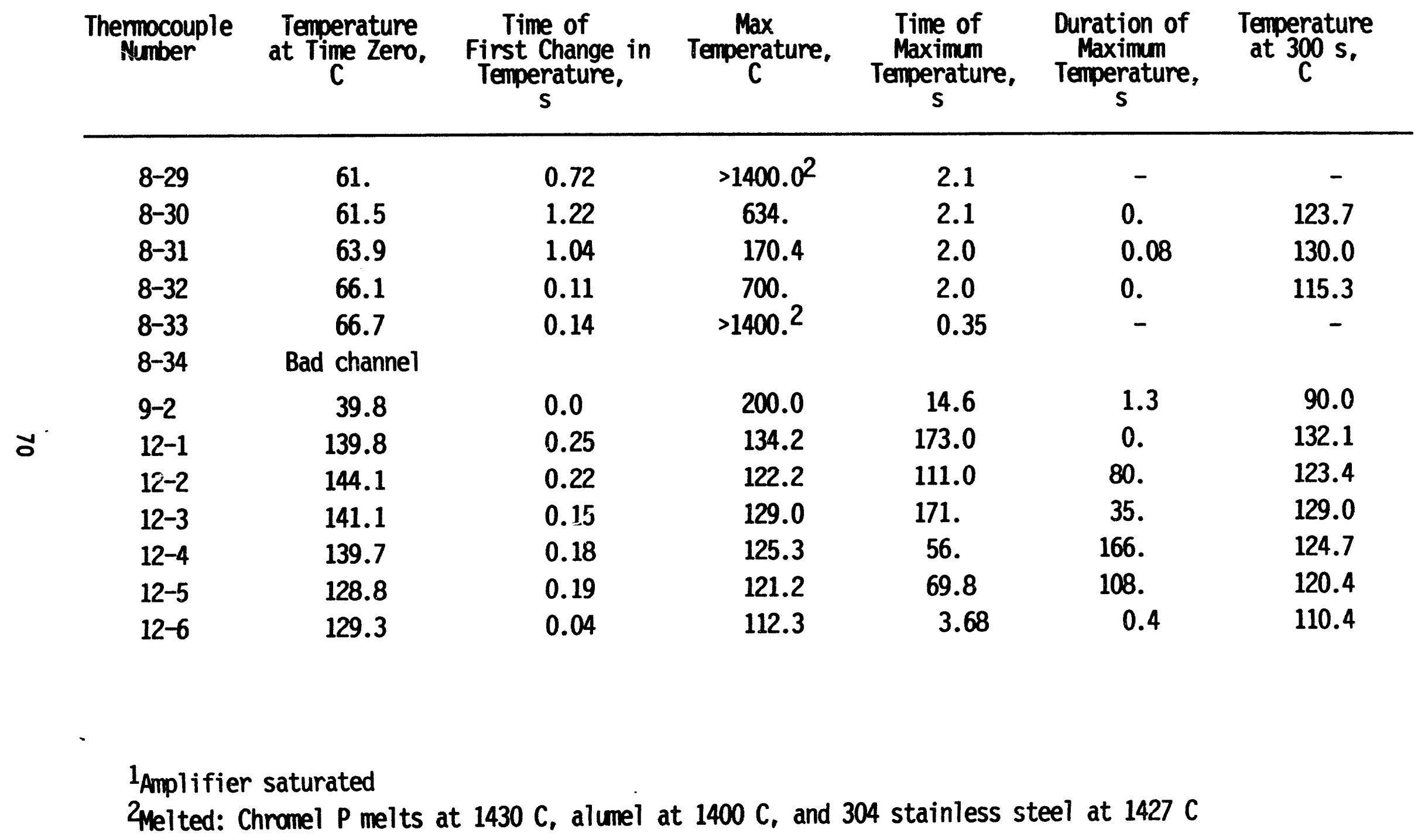


the corium jet may have moved away from the centerline during its fall.

Four thermocouples (TC8-26, TC8-27, TC8-30, and TC8-31) were located $718 \mathrm{~mm}$ below the water surface at radii of $88.9,38.1,63.5$, and $63.5 \mathrm{~mm}$, respectively. Only TC8-26 and TC8-30 were hit by corium. The maximum water temperature in the interaction vessel ranged from $-120 \mathrm{C}$ to $130 \mathrm{C}$, depending on the elevation.

Eight thermocouples were positioned 6.4 to $101.6 \mathrm{~mm}$ above the base of the interaction vessel. Based on the temperature data from these thermocouples, a debris bed of thickness -2.5 to $3.2 \mathrm{~cm}$ was formed on the vessel bottom. Debris distribution was not uniform. The TC8-17 and 18 responses indicate that a debris bed with temperature ranging from -1000 to $1300 \mathrm{C}$ quenched rapidly between $\sim 10$ to $25 \mathrm{~s}$. Similar to CCM-1, the debris bed cooling rate of this test is believed to be much higher than that of $\mathrm{CCM}-2$ due to the higher water subcooling. Furthermore, unlike CCM-2, the interaction vessel atmosphere was not heated up significantly during the debris bed cooling period; heat was mainly transferred to the subcooled water.

Thermocouple TC8-28 was located near the bottom of the pipeway U-bend. The temperature in the pipeway increased by $-25 \mathrm{C}$ during the jet fall stage and tracked the temperature in the expansion vessel thereafter.

As in the previous tests, seven thermocouples were used to measure the temperature inside the expansion vessel. Thermocouple TC8-10 was positioned at the center of the vessel, $349 \mathrm{~mm}$ above the pipeway exit. The remaining six (TC12-1 through 6) were located on a 38.1-mm radius, $406 \mathrm{~mm}$ apart axially, with TC12-1 $2.83 \mathrm{~m}$ above the pipeway exit. Their temperatures at time zero varied from a low of $125 \mathrm{C}$ for TC8-10 to a high of $144.1 \mathrm{C}$ for TC12-2 and TC12-3, with a mean of $135.4 \mathrm{C}$. All seven thermocouples showed an initial drop in temperature at 0.15 to $0.3 \mathrm{~s}$ (see Table 3.17) due to the blowdown of water droplets from the interaction vessel.

\subsubsection{Test CCM-5}

This experiment was the first to be conducted with the modified experimental apparatus, in which the thermite vessel was placed inside the expansion vessel.

About $0.72 \mathrm{~s}$ after the $8.63 \mathrm{~s}$ burn, a corium mass of $11.34 \mathrm{~kg}$ flowed through a $50.8-\mathrm{mm}$ hole with an initial pressure differential of about $0.006 \mathrm{MPa}$. The corium feli $340 \mathrm{~mm}$ through an argon/water vapor atmosphere to the surface of the water, whose mean temperature was $55.4 \mathrm{C}$ and whose depth was $1.067 \mathrm{~m}$ to the base of the collection pan.

The events observed on the Hycam film, from the camera whose field of view included the water surface, are presented in Table 3.18. The region above the water surface was completely black on the film because reactant aerosols were emitted from the thermite vessel through the check valves.

Three Hycam cameras were positiuned at viewports whose centerlines were $699 \mathrm{~mm}$ below the water surface. Two of the cameras were angled to obtain upward and downward views. The motion pictures from these cameras are similar to these obtained by the third camera, whose view was normal to the axial centerline of the water. Only the events observed on the film from this third camera are 
Table 3.18. Events Observed at the Water Surface from the Hycam Film for Experiment CCM-5

Test

Time, $s$

Event

-0.040 First corium particle at water surface. Space above water surface is black

-0.024 A number of particles are visible below the water surface

$0.044 \quad$ Brightness starts

0.061 Very bright particles at water surface

0.096 View is very bright. No visible jet but some particles are visible.

0.123 Water level starting to rise

0.463 Most of corium drop is over

0.604 Water level at maximum rise of about $55 \mathrm{~mm}$ above initial surface

0.628 Water level starting to drop

0.816 Water level stabilized at about $31 \mathrm{~mm}$ above initial surface.

White-hot particles are visible near lower edge of view port but elsewhere particles are reddish brown

0.994 Most of brightness is gone

1.260 Only a few visible glowing particles

1.941 Descending particle of about $5.6 \mathrm{~mm}$ diameter

2.102 Last visible descending particle

3.514 Last glow from particle on the view-port ledge 
presented in Table 3.19 .

Digitized data from the pressure transducers, thermocouples, and void detectors are given in Appendix F. A summary of the pressure data is given in Table 3.20.

The gas pressure inside the thermite vessel was measured by PTS-1 (see Fig. 3-5). It rose from its initial value of $0.096 \mathrm{MPa}$ at $-7.48 \mathrm{~s}$ to a maximum of $0.246 \mathrm{MPa}$ at $1.37 \mathrm{~s}$.

The gas/vapor pressure at the top of the experiment vessel was measured by pressure transducer PTS-9. At $-7.48 \mathrm{~s}$, the pressure began to increase from its initial value of $0.094 \mathrm{MPa}$, possibly due to the release of reactant aerosols from the thermite vessel into the experiment vessel. The pressure increased steadily to $0.117 \mathrm{MPa}$ at time zero, then tracked the pressure in the thermite vessel. The pressure began to increase significantly during the corium injection. Similar to the previous four tests except for CCM-2, the duration of the vessel pressurization due to corium/water interaction was around $1.5 \mathrm{~s}$. However, unlike the previous four tests, the pressure data of the thermite vessel and the experiment vessel were almost identical after the onset of corium injection. Also unlike the previous four tests, a pressure decrease occurred at $\sim 1.4 \mathrm{~s}$. This was probabiy caused by steam condensation on the "cold" experiment vessel wall. Although the vessel was preheated, a large temperature gradient existed along the vessel wall, caused by the presence of the subcooled water.

Three pressure transducers (PTS-10, PTS-12, and PTS-13) were located below the water surface. Transducers PTS-10 and PTS -12 were located $10.5 \mathrm{~mm}$ below the water surface. PTS-13 was $79.1 \mathrm{~mm}$ below the water surface. The response of these sensors were identical to that of PTS-9 except for the small differences noted in Table 3.20 .

Fifty two thermocouples were installed. Their data are summarized in Table 3.21. The maximum temperature measurable for the water thermocouples was normally 269-312 C, depending on the reference junction used. However, the amplifiers could overrange about 1.5 times before saturation, which increased the maximum temperature to 388-430 C. A temperature spike that causes amplifier saturation may not be completely shown on the digitized data plots in Appendix $E$.

The gas temperature inside the thermite vessel was measured by thermocouple TC9-2. At $-7.16 \mathrm{~s}$, i.e., $0.32 \mathrm{~s}$ after ignition of the thermite reactants, the gas temperature began to rise from its initial value of $116.9 \mathrm{C}$. The temperature rose to $151.8 \mathrm{C}$ at time zero, and reached a maximum of $222 \mathrm{C}$ at $32 \mathrm{~s}$.

Six thermocouples (TC12-1 through -6) were located in the gas/vapor region of the experiment vessel. The analog tape channel for TC12-3 was faulty. The five other thermocouples showed a temperature rise of -12 to $35 \mathrm{C}$. This axial gas/vapor temperature gradient is due to the presence of cold water in the vessel. Similar to the pressure data, significant temperature decrease occurred after the vessel reached its maximum temperature at $\sim 3$ to $4 \mathrm{~s}$. This indicates that a new thermal equilibrium was reached in the vessel atmosphere.

Thirteen thermocouples (TC8-50 through 63) were spaced $76.2 \mathrm{~mm}$ apart (TC8-63 was $152.4 \mathrm{~mm}$ below TC8-61) on the axial centerline of the experiment vessel to measure the water centerline temperature. Thermocouple TC8-59 was 
Table 3.19. Events Observed at $699 \mathrm{~mm}$ Below the Water Surface from the Hycam Film for Experiment CCM-5

Test

Time, $s$

Event

$\begin{array}{ll}-0.040 & \text { Light intensity is increasing } \\ 0.143 & \text { View is starting to get very bright } \\ 0.236 & \text { First particle is barely visible at top of frame } \\ 0.570 & \text { Glowing particles are becoming more visible } \\ 0.665 & \text { Bright particles are visible } \\ 0.752 & \text { Dim background with bright particles } \\ 0.806 & \text { View is brightening again } \\ 0.923 & \text { View dimming } \\ 1.119 & \text { 5-mm diameter particle with a } 48 \mathrm{~cm} / \mathrm{s} \text { velocity } \\ 1.305 & \text { Less bright particles are becoming visible } \\ 1.404 & \text { Light is dimming } \\ 1.540 & \text { Starting to see a vapor film around particles } \\ 1.623 & \text { Particles are mostly orangish brown } \\ 2.151 & \text { 6.5-mm diameter particle with a } 61 \mathrm{~cm} / \mathrm{s} \text { velocity } \\ 2.849 & \text { Only a few visible glowing particles descending } \\ 3.074 & \text { Light reflects off steam bubble } \\ 3.092 & \text { Hex-shaped steam bubbles rising } \\ 3.627 & \text { Black colored descending particles } \\ 4.300 & \text { Last view of glowing particle } \\ 4.440 & \text { Dark particles still descening } \\ 5.575 & \text { View dimming } \\ 5.808 & \text { Cannot see anything }\end{array}$


Table 3.20. Sumary of Pressure Data for Experiment Can-5

\begin{tabular}{|c|c|c|c|c|c|c|}
\hline $\begin{array}{l}\text { Sensor } \\
\text { Number }\end{array}$ & $\begin{array}{c}\text { Pressure at } \\
\text { Time Zero, } \\
\text { MPa }\end{array}$ & $\begin{array}{c}\text { Time of } \\
\text { First Pressure } \\
\text { Change. } \\
\mathbf{s}\end{array}$ & $\begin{array}{l}\text { Maximum } \\
\text { Pressure, } \\
\text { MPa }\end{array}$ & $\begin{array}{l}\text { Time to } \\
\text { Reach } \\
\text { Maximum } \\
\text { Pressure, } \\
\text { s }\end{array}$ & $\begin{array}{c}\text { Duration } \\
\text { of Maximum } \\
\text { Pressure, } \\
\text { s }\end{array}$ & $\begin{array}{c}\text { Pressure } \\
\text { at } 300 \mathrm{~s} \text {, } \\
\mathrm{MPa}\end{array}$ \\
\hline PTS-1 & 0.123 & 0. & 0.246 & 1.37 & 0.19 & 0.183 \\
\hline PTS-9 & 0.117 & 0. & 0.246 & 1.37 & 0.19 & 0.182 \\
\hline PTS-10 & 0.115 & 0. & 0.236 & 1.34 & 0.22 & 0.177 \\
\hline PTS-12 & 0.123 & 0. & 0.246 & 1.34 & 0.13 & 0.185 \\
\hline PTS-13 & Bad data chan & & & & & \\
\hline
\end{tabular}


faulty. The mean water temperature from the remaining 11 thermocouples was 55.2 $C$ with the highest temperature of $57.8 \mathrm{C}$ seen by TC8-60 (762 $\mathrm{mm}$ below the water surface) and the lowest temperature of $50.8 \mathrm{C}$ seen by TC8-63 (991 $\mathrm{mm}$ below the water surface). Eight of the thermocouples (TC8-50 through 56 and 58), melted as noted in Table 3.21. The primary purpose of these thermocouples was to measure the rate of corium descent. This information is presented in Section 4.

Twenty three thermocouples (TC8-20 through 43) were used to measure the water temperature in the experiment vessel at various axial and radial locations. Five thermocouples (TC8-20 through 24) were located $50.8 \mathrm{~mm}$ below the water surface at radii from 50.8 to $254 \mathrm{~mm}$. The time-zero temperature for these sensors ranged from 58.7 to $60.3 \mathrm{C}$ with a mean value of $59.6 \mathrm{C}$. All of the thermocouples except TC8-24 (178 $\mathrm{mm}$ radius) were contacted by corium; the resulting temperature rise saturated their amplifiers. After recovering from this temperature excursion in about 3 to $5 \mathrm{~s}$, the four thermocouples had temperatures of 64.9 to $66 \mathrm{C}$ at $300 \mathrm{~s}$. Based on the temperature data, the radial expansion rate of the corium/water mixture at this elevation was bounded by 0.1 and $0.5 \mathrm{~m} / \mathrm{s}$.

Six thermocouples (TC8-26 through 31) were located on radi i from 50.8 to $279 \mathrm{~mm}$ at $305 \mathrm{~mm}$ below the water surface. At time zero, the water temperature measured by these sensors ranged from 57.4 to $58.8 \mathrm{C}$ with a mean value of 58.2 C. Three of these thermocouples (TC8-26, 27, and 29 at radii of 50.8, 152, and $76.2 \mathrm{~mm}$, respectively) were contacted with a sufficient mass of corium to cause amplifier saturation. Two of the thermocouples (TC8-26 and 29) melted. The expansion rate of the corium/water mixture was estimated to be $-0.13 \mathrm{~m} / \mathrm{s}$ and was confined within a region of radius $-18 \mathrm{~cm}$.

Three thermocouples (TC8-35, 36, and 37) were located $533 \mathrm{~mm}$ below the water surface on radii of $76.2,178$, and $279 \mathrm{~mm}$. Their time-zero temperatures were 53.2, 54.0, and $54.7 \mathrm{C}$, respectively. Three thermocouples (TC8-32, 33, and 34) were located $559 \mathrm{~mm}$ below the water surface on radii of $50.8,152$, and $254 \mathrm{~mm}$. The three latter thermocouples were located $90^{\circ}$ clockwise from TC8-35 through 37. The data channel for TC8-34 was faulty, so no data were recorded. Except for TC8-32, all of the other thermocouples indicated little or no corium contact. Also, the behavior of TC8-32 and 33 indicates that the corium dispersal was not azimuthally symmetrical.

Six thermocouples (TC8-38 through 43) were located $813 \mathrm{~mm}$ below the water surface at radii from 50.8 to $279 \mathrm{~mm}$. The ir time-zero temperatures ranged from 51.3 to $53.5 \mathrm{C}$ with a mean of $51.7 \mathrm{C}$. The masses of corium that contacted TC8-41 and 42 caused only a small temperature rise of about $35 \mathrm{C}$.

Nine thermocouples (TC8-6 through 10 and 16 through 19) were positioned $6.4 \mathrm{~mm}$ above the bottom of the debris pan, i.e., $1.06 \mathrm{~m}$ below the water surface. Their time zero temperature range was 50 to $56.7 \mathrm{C}$ with a mean $53.8 \mathrm{C}$. Thermocouples TC8-6, 9, 16, and 18 with radii of $0,50.8,76.2$, and $76.2 \mathrm{~mm}$, respectively, all melted. The temperature measured by TC8-7 (50.8 mm radius) indicated that a debris bed was formed and was quenched at $-30 \mathrm{~s}$.

Twelve void detectors (VD-1 through 12) were installed in the water region of the experiment vessel in an attempt to detect the presence of steam. From the data presented in Appendix F, the pretest voltage is near zero. Non-zero voltage signals indicate voids or corium contact. 


\subsubsection{Test CCN-6}

In this test the reactant burn time was $7.65 \mathrm{~s}$. About $0.04 \mathrm{~s}$ later corium injection started. A mass of $12.0 \mathrm{~kg}$ was injected through a 50.8-mm diameter hole with an initiai pressure differential of $0.087 \mathrm{MPa}$. After falling $340 \mathrm{~mm}$ through a steam/argon atmosphere, the corium contacted the water, whose mean temperature was $100.8 \mathrm{C}$. The depth of water to the bottom of the debris collection pan was $1.067 \mathrm{~m}$, as in experiment CCM-5.

The events observed on the Hycam film from the camera whose field of view included the water surface are presented in Table 3.22. Three Hycam cameras were positioned at viewports $699 \mathrm{~mm}$ below the water surface.

Because of the failure of one of the analog tape decks at the start of the test, twenty three channels of data were not recorded. Some of this data were replotted from the backup visicorder traces and are presented in Appendix $G$ along with the digitized data from the remaining two tape decks. A summary of the pressure data is given in Table 3.24. As in past experiments, the piezoelectric pressure transducers (PTK-1, PTK-2, and PTK-3) were in place to detect possible steam explosions; none occurred.

The gas pressure inside the thermite vessel was measured by PTS-1, as shown in Fig. 3-6. It began to rise from an initial value of $0.103 \mathrm{MPa}$ at $-6 \mathrm{~s}$, i.e., about $1.6 \mathrm{~s}$ after the ignition of the thermite reactants. Even though the vent line to an accumulator tank was open, the pressure inside the vessel reached $0.195 \mathrm{MPa}$ at $-5.3 \mathrm{~s}$ before decreasing to $0.173 \mathrm{MPa}$ at $-4.0 \mathrm{~s}$. From this point on, there was a slight pressure fluctuation to $-2 \mathrm{~s}$, followed by a pressure rise to $0.202 \mathrm{MPa}$ at time zero.

The pressure in the experiment vessel rose steeply from 0 to $1.3 \mathrm{~s}$ due to steam generation, and it tracked the pressure in the thermite vessel after the onset of the corium injection. Beyond $1.3 \mathrm{~s}$, the pressure started to decrease, since the peak pressure was limited by saturation. When thermal equilibrium was established, condensation began, causing further pressure decreases.

Three strain gage pressure transducers (PTS-10, 12, and 13) were located in the water region of the experiment vessel. Transducers PTS-10 and 12 were $105 \mathrm{~mm}$ below the water surface. Their responses were similar to that of PTS -9 (see Table 3.24). Pressure transducer PTS-13 was $791 \mathrm{~mm}$ below the water surface. Its response is also similar to that of PTS-9. Piezoelectric transducer PTK-2 was located $22.2 \mathrm{~mm}$ below the water surface, and transducer PTK-3 was in the upper head of the experiment vessel. The early pressure response of PTK-2 was similar to that of PTS-12; the signal from PTK-3 was faulty.

Fifty-two thermocouples were used to measure the temperature in the CCM- 6 test apparatus. A summary of these data are presented in Table 3.25 and temperature plots are given in Appendix G. Because of the analog tape deck failure, data plots for TC8-6, 7, 8, 9, 10,34,35,36, 38, 40, 41, 42, and 50 are not included in Appendix G.

The gas temperature inside the thermite vessel was measured by TC9-2. It started to rise from its pretest value of $100.4 \mathrm{C}$ at $-5.8 \mathrm{~s}$, which is about $1.8 \mathrm{~s}$ after ignition of the thermite reactants. At time zero, the temperature was $126.3 \mathrm{C}$. Shortly afterwards at $0.08 \mathrm{~s}$, the temperature dipped slightly and then 
Table 3.21. Summary of Temperature Data for Experiment Car-5

\begin{tabular}{|c|c|c|c|c|c|c|}
\hline $\begin{array}{l}\text { Thenmocouple } \\
\text { Number }\end{array}$ & $\begin{array}{l}\text { Temperature } \\
\text { at Time Zero, } \\
C\end{array}$ & $\begin{array}{l}\text { Time of } \\
\text { First Change in } \\
\text { Temperature, } \\
\text { s }\end{array}$ & $\underset{\text { Temperature, }}{\text { Max }}$ & $\begin{array}{c}\text { Time of } \\
\text { Maximum } \\
\text { Temperature, } \\
s\end{array}$ & $\begin{array}{c}\text { Duration of } \\
\text { Maximum } \\
\text { Temperature, } \\
\text { s }\end{array}$ & $\begin{array}{c}\text { Temperature } \\
\text { at } 300 \mathrm{~s} \text {. } \\
\mathrm{C}\end{array}$ \\
\hline $8-6$ & 56.7 & 1.26 & $>1400^{2}$ & 2.0 & - & - \\
\hline $8-7$ & 55.0 & 1.19 & $>1400^{2}$ & 2.55 & - & 66.6 \\
\hline $8-8$ & 51.9 & 1.75 & 119.1 & 3.47 & 0.0 & 61.0 \\
\hline $8-9$ & 56.3 & 1.08 & $>1400^{2}$ & 2.0 & - & - \\
\hline $8-10$ & 53.6 & 1.24 & 108.1 & 1.72 & 0.0 & 56.0 \\
\hline $8-16$ & 55.0 & 1.11 & $>1400^{2}$ & 1.32 & - & - \\
\hline $8-17$ & 50.0 & 1.00 & $>1400$ & 2.17 & - & 61.2 \\
\hline $8-18$ & 51.7 & 1.22 & $>1400^{2}$ & 1.48 & - & - \\
\hline $8-19$ & Bad data channel & - & - & - & - & - \\
\hline $8-20$ & 59.8 & 0.12 & $>300^{1}$ & 0.12 & - & 66.0 \\
\hline $8-21$ & 60.3 & 0.33 & $>300^{1}$ & 1.28 & - & 66.0 \\
\hline $8-22$ & 58.7 & 0.33 & 220 & 2.90 & 0.0 & 64.9 \\
\hline $8-23$ & 59.3 & 0.33 & $>300^{1}$ & 0.68 & - & 66.0 \\
\hline $8-24$ & 60.1 & 0.33 & 69.2 & 2.78 & 0.0 & 66.9 \\
\hline $8-26$ & 58.8 & 0.24 & $>300^{2}$ & 0.67 & - & - \\
\hline $8-27$ & 57.9 & 0.81 & $>300^{1}$ & 1.09 & - & 66.0 \\
\hline $8-28$ & 58.8 & 0.34 & 75.4 & 2.04 & 0.0 & 66.6 \\
\hline $8-29$ & 57.4 & 0.34 & $>300^{2}$ & 0.46 & - & - \\
\hline $8-30$ & 58.7 & 0.33 & 75.9 & 1.46 & 0.0 & 66.7 \\
\hline $8-31$ & 57.7 & 0.36 & 81.5 & 2.52 & 0.0 & 65.5 \\
\hline $8-32$ & 57.3 & 0.53 & $152.5^{1}$ & 0.70 & - & 67.2 \\
\hline
\end{tabular}


Table 3.21. Sumary of Temperature Data for Experiment Car-5 (cont.)

\begin{tabular}{|c|c|c|c|c|c|c|}
\hline $\begin{array}{l}\text { Thermocouple } \\
\text { Number }\end{array}$ & $\begin{array}{l}\text { Temperature } \\
\text { at Time Zero, } \\
\text { C }\end{array}$ & $\begin{array}{l}\text { Time of } \\
\text { First Change in } \\
\text { Temperature, } \\
\text { s }\end{array}$ & $\underset{\text { Temperature, }}{\text { Max }}$ & $\begin{array}{c}\text { Time of } \\
\text { Maximum } \\
\text { Temperature, } \\
\text { s }\end{array}$ & $\begin{array}{c}\text { Duration of } \\
\text { Maximum } \\
\text { Temperature, } \\
\text { s }\end{array}$ & $\begin{array}{c}\text { Temperature } \\
\text { at } 300 \mathrm{~s} \text {. } \\
\mathrm{C}\end{array}$ \\
\hline $8-33$ & 58.1 & 1.06 & $100^{1}$ & 3.53 & 0.0 & 68.1 \\
\hline $8-34$ & Bad data channel & - & - & - & - & - \\
\hline $8-35$ & 53.2 & 0.20 & $85^{1}$ & 1.0 & 0.0 & 61.7 \\
\hline $8-36$ & 54.0 & 0.86 & 65.0 & 7.09 & 0.0 & 62.4 \\
\hline $8-37$ & 54.7 & 0.42 & 65.6 & 5.83 & 0.0 & 62.2 \\
\hline $8-38$ & 53.5 & 0.17 & 284 & 0.86 & - & 63.5 \\
\hline $8-39$ & Bad data channel & - & - & - & - & - \\
\hline $8-40$ & 51.3 & 1.03 & 62.5 & 110 & 190 & 62.5 \\
\hline $8-41$ & 51.8 & 0.20 & 84.3 & 3.39 & 0.0 & 62.1 \\
\hline $8-42$ & 51.5 & 0.84 & 87.5 & 2.83 & 0.0 & 61.5 \\
\hline $8-43$ & 50.6 & 1.08 & 235.6 & 9.0 & 0.0 & 63.1 \\
\hline $8-50$ & 66.7 & 0.10 & $>1400^{\prime}$ & 0.18 & - & - \\
\hline $8-51$ & 56.3 & 0.15 & $>1400^{2}$ & 0.21 & - & - \\
\hline $8-52$ & 56.0 & 0.19 & $>1400^{2}$ & 0.29 & - & - \\
\hline $8-53$ & 55.8 & 0.23 & $>1400^{2}$ & 0.32 & - & - \\
\hline $8-54$ & 56.9 & 0.26 & $>1400^{2}$ & 0.35 & - & - \\
\hline $8-55$ & 54.9 & 0.27 & $>1400^{2}$ & 0.4 & - & - \\
\hline
\end{tabular}


Table 3.21 Summary of Temperature Data for Experiment Car-5 (cont.)

$\begin{array}{ccccccc}\begin{array}{c}\text { Thenmocouple } \\ \text { Number }\end{array} & \begin{array}{c}\text { Temperature } \\ \text { at Time Zero, } \\ \text { C }\end{array} & \begin{array}{c}\text { Time of } \\ \text { First Change in } \\ \text { Temperature, } \\ \text { S }\end{array} & \begin{array}{c}\text { Max } \\ \text { Temperature, } \\ \text { C }\end{array} & \begin{array}{c}\text { Time of } \\ \text { Maximum } \\ \text { Temperature, } \\ \text { S }\end{array} & \begin{array}{c}\text { Duration of } \\ \text { Maximum } \\ \text { Temperature, } \\ \text { s }\end{array} & \begin{array}{c}\text { Tenperature } \\ \text { at }\end{array} \\ \mathbf{3 0 0} \text { s, } \\ 8-56 & 54.9 & 0.32 & >1400^{2} & 0.93 & - & - \\ 8-57 & 56.9 & 0.36 & >1400 & 0.9 & - & 64.5 \\ 8-58 & 54.9 & 0.43 & >1400^{2} & 0.94 & - & - \\ 8-59 & \text { Bad data channel } & - & - & - & - & - \\ 8-60 & 57.8 & 0.76 & 253.0 & 1.10 & 0.0 & 66.6 \\ 8-61 & 51.5 & 1.90 & 1200 . & 2.14 & 0.0 & 59.7 \\ 8-63 & 50.8 & 1.42 & 1293 . & 1.44 & 0.0 & 64.7 \\ 9-2 & 151.8 & 0.0 & 222.5 & 32.0 & 6.4 & 173.1 \\ 12-1 & 115.8 & 0.47 & 141.2 & 4.4 & 2.5 & 126.8 \\ 12-2 & 110.7 & 0.39 & 142.4 & 3.31 & 0.16 & 123.8 \\ 12-3 & 108.3 & 0.44 & - & - & - & - \\ 12-4 & 121.9 & 0.42 & 144.0 & 3.70 & 0.89 & 130.7 \\ 12-5 & 91.9 & 0.27 & 122.9 & 3.15 & 0.15 & 98.9 \\ 12-6 & 83.2 & 0.25 & 95.5 & 3.46 & 0.22 & 75.3\end{array}$

$1_{\text {Amplifier saturated }}$

4melted: Chromel P melts at $1430 \mathrm{C}$, alumel at $1400 \mathrm{C}$, and 304 stainless steel at $1427 \mathrm{C}$ 
Table 3.22 Events Observed at the Water Surface from the Hycam Film for Experiment CCM- 6

Test

Time, s

Event

-1.149 First good view of vessel interior. Steam bubbles are rising through the water.

-0.446 Appearance of first glowing particle at the top of the frame (about $2.5 \mathrm{~mm}$ diameter)

-0.409 Above particle hits the water

-0.244 First flow at top of frame of a larger mass of corium. Only particles have been present

-0.211 Larger mass of particles are visible

- 0.180 Particles from above mass penetrate the water surface

-0.104 Bright particles are starting to be visible in the water

0.089 View is very bright

0.098 Water level is starting to rise

0.363 Water level is at top of viewing port (99 $\mathrm{mm}$ increase in level)

0.413 Water level is starting to drop

0.648 Brightness is starting to diminish

0.662 Blackness reaches bottom of viewing port

0.810 A few glowing particles are descending in a black background

0.856 More glowing particles are visible

0.886 A glowing particle alights on the ledge of the viewport

0.944 A mass of glowing particles is descending and more are forming on the view port ledge

1.047 Reddish-brown picture with glowing particles

1.082 Picture starting to blacken

1.408 Last visible glowing particle at the top of the frame

4.607 Last glow from particles on view-port ledge 
Table 3.23 Events Observed at $699 \mathrm{~mm}$ Below the Water Surface from the Hycam Film for Experiment CCM- 6

Test

Time, s

Event

-1.131 First view of interior of experiment vessel. Relatively dark.

0.093 Picture is starting to brighten

0.364 First visible particle at top right of frame

0.412 Above particle reaches horizontal centerline of view port

0.685 Bright picture but many particles are visible

0.800 Picture is still bright but more particles are visible. The particles are varied in size and shape. Some are spherical. One particle has about a 4.5-mm diameter.

0.991 Tan-colored background with bright particles. One particle is about $3 \mathrm{~mm}$ in diameter. Some upward moving steam bubbles are visible.

1.067 Particle descending with a velocity of about $0.4 \mathrm{~m} / \mathrm{s}$

1.258 Rising steam bubbles are more visible

1.333 Some dark descending particles are visible

1.637 A $3-\mathrm{mm}$ diameter particle is descending with a velocity of about $0.4 \mathrm{~m} / \mathrm{s}$

1.743 More particles are visible

1.768 A particle has a diameter of about $2.4 \mathrm{~mm}$ and is descending with a velocity of about $1 \mathrm{~m} / \mathrm{s}$

1.789 A bunch of large particles (6.5 to $8.5 \mathrm{~mm}$ diameter) are descending

1.952 There is a mixture of large particles (not spherical). Some are bright and some are dark.

2.217 Rising corium particles are visible.

3.016 Most of particles have descended. There are some rising black particles

3.033 Picture is brownish-black colored with visible steam bubbles and some glowing particles

3.480 Dim particle glow at bottom of an all black frame 
Table 3.24 Sumary of Pressure Data for Experiment COH-6

\begin{tabular}{|c|c|c|c|c|c|c|c|}
\hline & $\begin{array}{l}\text { Sensor } \\
\text { Number }\end{array}$ & $\begin{array}{c}\text { Pressure at } \\
\text { Time Zero, } \\
\text { MPa }\end{array}$ & $\begin{array}{c}\text { Time of } \\
\text { First Pressure } \\
\text { Change, } \\
\mathbf{s}\end{array}$ & $\begin{array}{l}\text { Maximum } \\
\text { Pressure, } \\
\text { MPa }\end{array}$ & $\begin{array}{c}\text { Time to } \\
\text { Reach } \\
\text { Maximm } \\
\text { Pressure, } \\
\text { s }\end{array}$ & $\begin{array}{l}\text { Duration } \\
\text { of Maximum } \\
\text { Pressure. } \\
\text { s }\end{array}$ & $\begin{array}{c}\text { Pressure } \\
\text { at } 90 \mathrm{~s}, \\
\mathrm{MPa}\end{array}$ \\
\hline & PTS-1 & 0.202 & 0.0 & 0.348 & 1.40 & 0.0 & 0.217 \\
\hline & PTS-9 & 0.114 & 0.0 & 0.354 & 1.30 & 0.0 & 0.222 \\
\hline & PTS-10 & 0.117 & 0.0 & 0.345 & 1.35 & 0.0 & 0.219 \\
\hline & PTS-12 & 0.124 & 0.0 & 0.358 & 1.30 & 0.0 & 0.226 \\
\hline \multirow[t]{2}{*}{$\underset{\omega}{\infty}$} & PTS-13 & 0.126 & 0.0 & 0.417 & 1.40 & 0.0 & 0.269 \\
\hline & PTK-2 & 0.1 & 0.0 & 0.360 & 1.12 & 0.4 & 0.135 \\
\hline
\end{tabular}


Table 3.25 Summary of Temperature Data for Experiment Car-6

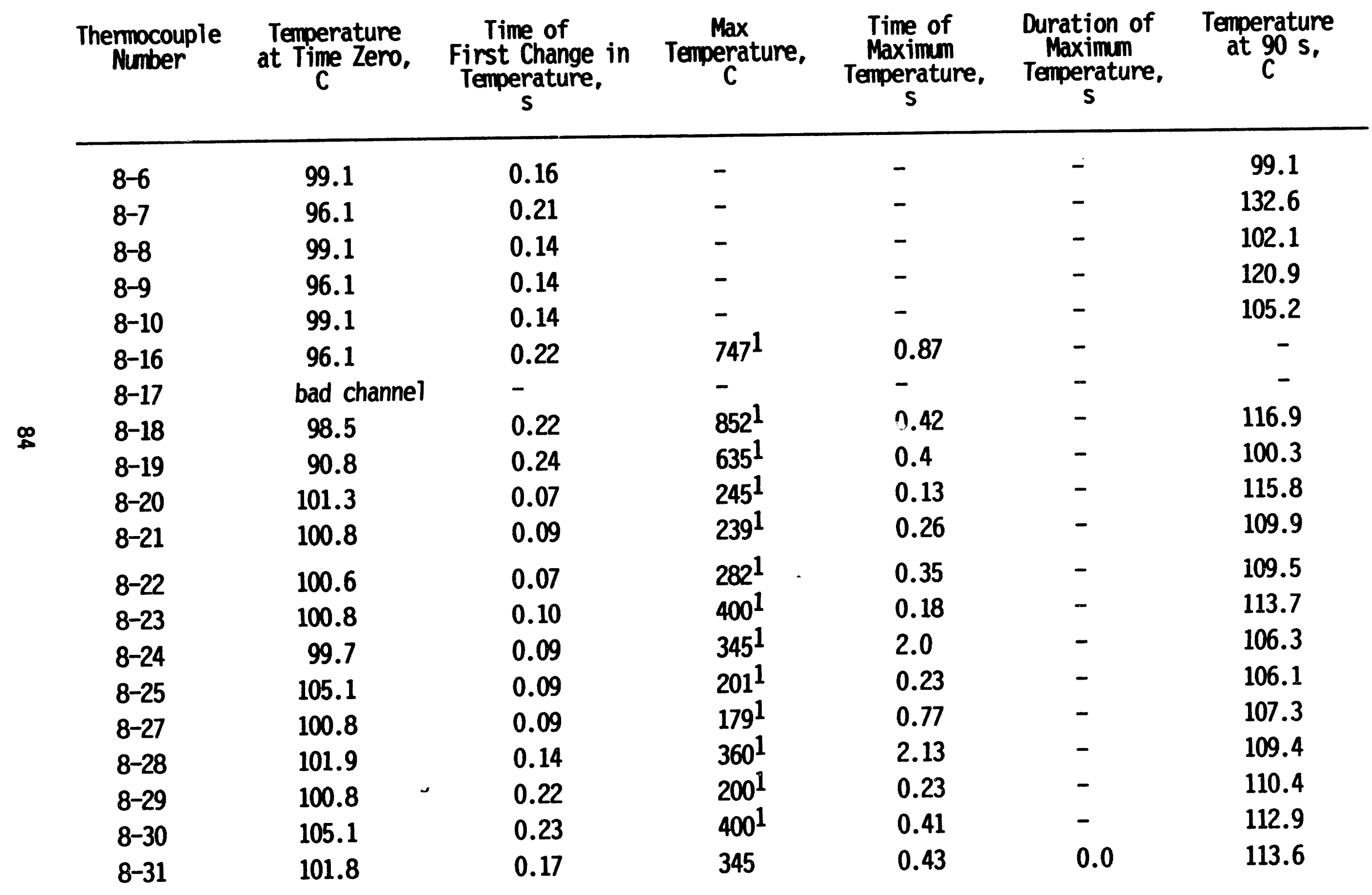


Table 3.25 Sumary of Temperature Data for Experiment Can-6 (cont.)

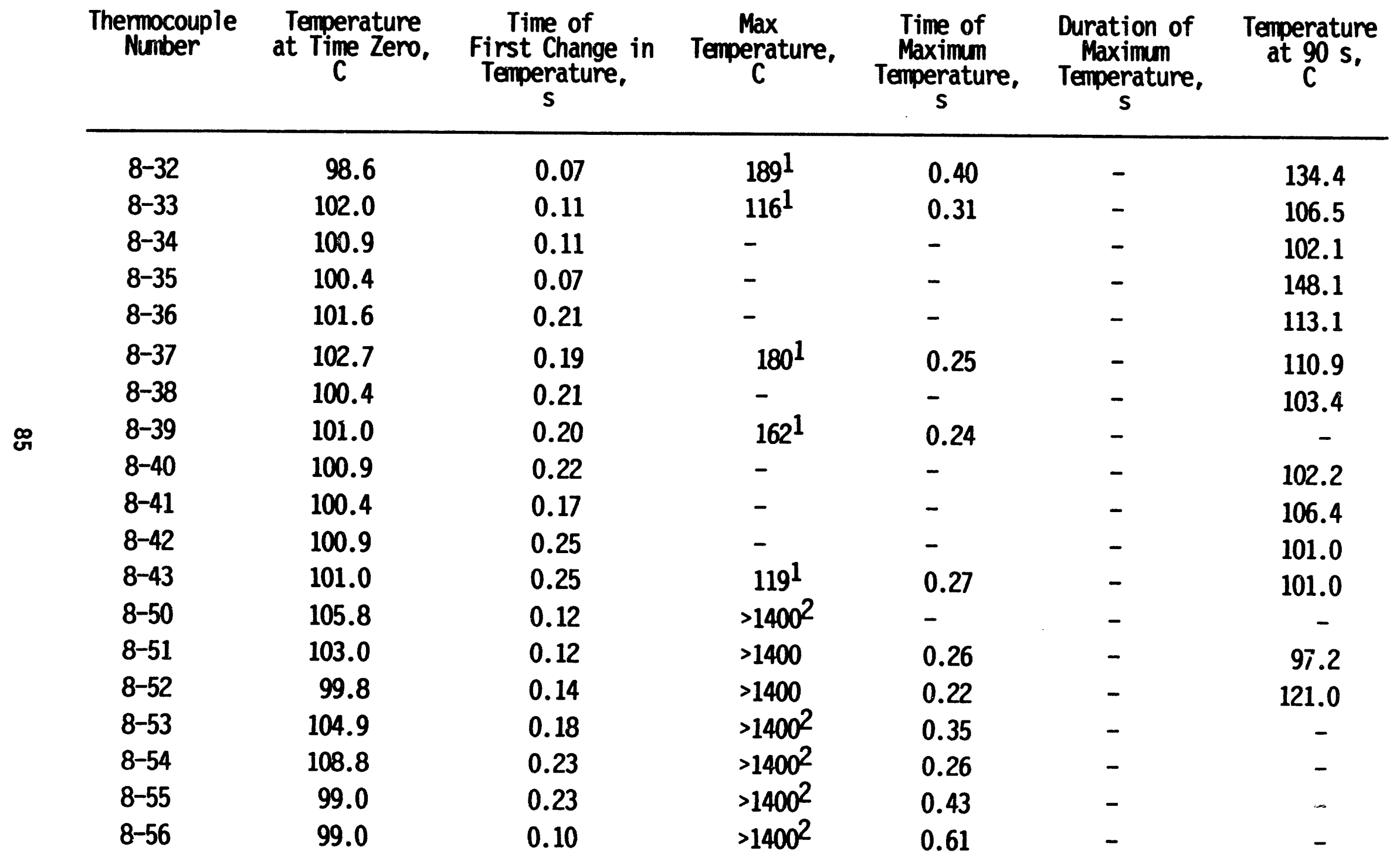


Table 3.25 Sumary of Temperature Data for Experiment COH-6 (cont.)

\begin{tabular}{|c|c|c|c|c|c|c|}
\hline $\begin{array}{c}\text { Thermocouple } \\
\text { Number }\end{array}$ & $\begin{array}{l}\text { Temperature } \\
\text { at Time Zero, } \\
\text { C }\end{array}$ & $\begin{array}{l}\text { Time of } \\
\text { First Change in } \\
\text { Temperature, } \\
\text { s }\end{array}$ & $\underset{\text { Temperature, }}{\operatorname{Max}}$ & $\begin{array}{c}\text { Time of } \\
\text { Maximum } \\
\text { Temperature, } \\
s\end{array}$ & $\begin{array}{c}\text { Duration of } \\
\text { Maximum } \\
\text { Temperature, } \\
\text { s }\end{array}$ & $\begin{array}{c}\text { Temperature } \\
\text { at } 300 \mathrm{~s} \text {, } \\
\mathrm{C}\end{array}$ \\
\hline $8-57$ & 94.6 & 0.14 & 813. & 0.52 & 0.0 & 110.7 \\
\hline $8-58$ & 99.0 & 0.19 & 307 & 0.47 & 0.0 & 107.6 \\
\hline $8-59$ & 103.9 & 0.19 & $>1400^{2}$ & 0.35 & - & - \\
\hline $8-60$ & 108.4 & 0.12 & $>1400^{2}$ & 0.42 & - & - \\
\hline $8-61$ & 99.3 & 0.13 & 728 & 0.65 & 0.0 & 111.1 \\
\hline $8-63$ & 102.7 & 0.22 & 325 & 0.97 & 0.0 & 109.3 \\
\hline $9-2$ & 126.3 & 0.08 & 191.3 & 26.3 & 13.7 & 170.9 \\
\hline $12-1$ & 130.2 & 0.27 & 168.5 & 9.5 & 1.0 & 132.0 \\
\hline $12-2$ & 133.8 & 0.25 & 166.0 & 12.9 & 5.0 & 140.5 \\
\hline $12-3$ & 130.2 & 0.20 & 165.0 & 14.0 & 1.0 & 136.3 \\
\hline $12-4$ & 132.9 & 0.25 & 174.5 & 7.9 & 6.1 & 139.2 \\
\hline $12-5$ & 128.6 & 0.28 & 162.3 & 13.6 & 3.2 & 131.2 \\
\hline $12-6$ & 127.1 & 0.06 & 189.3 & 5.8 & 1.0 & 132.2 \\
\hline
\end{tabular}


went through a series of fluctuations as it rose to $182.5 \mathrm{C}$ at $0.77 \mathrm{~s}$. At this point, the indicated temperature dropped abruptly and oscillated between 0 and $100 \mathrm{C}$. This behavior is indicative of amplifier saturation.

Six thermocouples (TC12-1 through 6) were located in the gas/vapor region. Thermocouple TC12-1 was the farthest $(2.46 \mathrm{~m})$ from the water surface; TC12-6 was the closest $(1.77 \mathrm{~m})$. The region bounded by these sensors was fairly uniform in temperature, with the time zero temperature range being 127.1 to $132.9 \mathrm{C}$ with a mean of 130.5 C. The responses of these sensors were similar except that TC12-2 and TC12-5 show a small temperature drop in the 3-5 s range, whereas the other thermocouples show an increase in temperature.

The maximum temperatures ranged from $162.3 \mathrm{C}$ for TC12-5 to $189.3 \mathrm{C}$ for TC12-6, with a mean of $170.9 \mathrm{C}$, and occurred between 6 and $18 \mathrm{~s}$. Unlike CCM-5, the temperature increased significantly during -5 to $15 \mathrm{~s}$. This indicates that steam generation was substantial during the debris bed cooling period.

Thirteen thermocouples (TC8-50 through 63) were located along the axial centerline of the experiment vessel with TC8-50 at the water surface and the remainder spaced $76.2 \mathrm{~mm}$ apart, except that TC8-55 was $102 \mathrm{~mm}$ below TC8-54, TC8-56 was $50.8 \mathrm{~mm}$ below TC8-55, and TC8-63 was $152 \mathrm{~mm}$ below TC8-61. At time zero these thermocouples showed a temperature range of 94.6 to $108.4 \mathrm{C}$ with a mean of $102.2 \mathrm{C}$. Nine of these thermocouples showed temperatures above $1400 \mathrm{C}$, with seven definitely melting. The other two (TC8-51 and 52) had temperatures of 97.2 and $121.0 \mathrm{C}$ at $90 \mathrm{~s}$, which agrees with the temperatures measured by other thermocouples at this time. The responses of these thermocouples also gave a measure of the rate of corium descent; this information is presented in Section 4.

Twenty three thermocouples (TC8-20 through 43) were located in the water region of the expansion vessel. Six thermocouples (TC8-20 through 25) were located $50.8 \mathrm{~mm}$ below the water surface at radii of 50.8 to $279 \mathrm{~mm}$. At time zero their temperatures ranged from 99.7 to $105.1 \mathrm{C}$ with a mean of $101.4 \mathrm{C}$. All of these thermocouples were hit by corium. The rate of radial expansion of the corium/water mixture was bounded by 0.2 and $1.2 \mathrm{~m} / \mathrm{s}$.

Five thermocouples (TC8-27 through 31) were located $305 \mathrm{~mm}$ below the water surface at radii ranging from $76.2 \mathrm{~mm}$ for TC8-29 to $279 \mathrm{~mm}$ for TC8-31. At time zero the temperature measured by these sensors ranged from 100.8 to $105.1 \mathrm{C}$ with a mean at 102.1 C. A11 of these thermocouples except TC8-31 experienced amplifier saturation when contacted by the corium. The rate of radial expansion of the corium/water mixture at this elevation was $-0.1 \mathrm{~m} / \mathrm{s}$.

Three thermocouples, TC8-35, 36, and 37, were located $533 \mathrm{~mm}$ below the water surface at radii of $76.2,178$, and $279 \mathrm{~mm}$ respectively. At time zero their temperatures were $100.4,101.6$, and $102.7 \mathrm{C}$ with a mean of $101.6 \mathrm{C}$. No tape data are available for TC8-35 and 36. The amplifier for TC8-37 was saturated from about 0.25 to $3.4 \mathrm{~s}$. At $90 \mathrm{~s}$ the temperature measured by TC8-35 was $148.1 \mathrm{C}$, compared with $113.1 \mathrm{C}$ for TC8-36 and $110.9 \mathrm{C}$ for TC8-37. Corium adhering to the thermocouple could have caused this higher temperature.

Three thermocouples, TC8-32, 33, and 34, were located $559 \mathrm{~mm}$ below the water surface at radii of $50.8,152$, and $252 \mathrm{~mm}$, respectively. At time zero, their temperatures were $98.6,102.0$, and $100.9 \mathrm{C}$ with a mean of $100.5 \mathrm{C}$. No tape data are available for TC8-34. For thermocouple TC8-32, amplifier saturation 
occurred from about $0.4 \mathrm{~s}$ to $7 \mathrm{~s}$. The data indicate that these thermor:ouples were hit by small masses of corium.

Six thermocouples (TC8-38 through 43) were located $813 \mathrm{~mm}$ below the water surface, 50.8 to $279 \mathrm{~mm}$ from the vessel axial centerline. At time zero their temperatures ranged from 100.4 to $101.0 \mathrm{C}$ with a mean of $100.8 \mathrm{C}$. Data plots for TC8-38, 40, 41, and 42 are not available.

Nine thermocouples (TC8-6 through 10 and 16 through 19) were positioned $6.2 \mathrm{~mm}$ above the bottom of the debris pan (1.06 $\mathrm{m}$ below the water surface). At time zero the temperature measured by the eight thermocouples ranged from 90.8 to 99.1 C with a mean of 97.2 C. Data from thermocouples TC8-16 and $19 /$ show that significant debris cooling occurred from 5 to $15 \mathrm{~s}$. This corresponds to the duration of the vessel temperature increase.

The same twelve void detectors used in experiment CCM-5 were utilized in experiment CCM- 6 . Their data has been used to estimate the corium dispersal as discussed in Section 4.

\subsection{Debris Characterization}

After each experiment the test apparatus was disassembled and the corium debris from the thermite reaction was removed and weighed. The distribution of the debris is noted in Table 3.26. Typically, there was a difference between the mass of corium injected as given in Table 3.2 and the mass of the recovered debris. Complete debris recovery from the large apparatus used in the CCM experiments was difficult. Offsetting the possible debris mass loss is a possible mass gain due to oxidation of the metallic constituents of the thermite by air or steam. The principal oxides formed are assumed to be $\mathrm{Fe}_{3} \mathrm{O}_{4}, \mathrm{Cr}_{2} \mathrm{O}_{3}$, and NiO, whose masses are 38,46 , and $27 \%$ greater than the metal. Hence, if ali the metals in a $3.78-\mathrm{kg}$ thermite mixture (CCM-1 reactant mass) were completely oxidized, the mass gain would be $352 \mathrm{~g}$, an increase of $9.3 \%$. For a $12-\mathrm{kg}$ mixture, the mass gain could be as much as $1118 \mathrm{~g}$, a $9.3 \%$ increase.

\subsubsection{Test CCM-1}

An inspection of the thermite vessel after its disassembly revealed a fine olive colored powder in its upper section and a small amount of debris in the vapor separator (Fig. 3-8). Some globules of metal and oxide were on the ledge of the expansion region as can be seen in Fig. 3-9. In this figure a portion of the tungsten porous grid plate was missing. This hole resulted from dissolution of the tungsten by $\mathrm{UO}_{2}$. Some solidified corium was also present on the grid plate, on the side of the thermite vessel, and on the mullite sleeve and lavite cone (see Fig. 3-10).

Inside the expansion vessel, debris was found only in two small patches on the vessel wall. There was no visible debris on the bottom of the vessel (see Fig. 3-11). The $11 \mathrm{~g}$ of debris recovered from the expansion vessel is about $0.5 \%$ of the injected corium.

In the interaction vesse1, there was some debris on the underside of the vessel head (Fig. 3-12), but the walls of the vessel were clean. The debris bed on the vessel bottom is shown in Fig. 3-13. A closeup view of some of this material is shown in Fig. 3-14. The debris bed consisted of loosely bound and 
Table 3.26 Debris Distribution

\begin{tabular}{|c|c|c|c|c|c|c|}
\hline Test & $\mathrm{CCM}-1$ & $\mathrm{CCM}-2$ & $\mathrm{CCM}-3$ & $\mathrm{COM}-4$ & $\mathrm{COM}-5$ & $\mathrm{CCM}-6$ \\
\hline Mixed Reactants Mass, g & 3784 & 12012 & 4800 & 10010 & 12012 & 12012 \\
\hline Load Cell Reactants Mass, g & 3870 & 12372 & 4750 & 10370 & 12301 & 12228 \\
\hline Reactant Mass Difference, g & 96 & 360 & -50 & 360 & 289 & 216 \\
\hline Load Cell Injected Mass, g & 2150 & 11147 & 3340 & 9240 & 11340 & 12792 \\
\hline Load Cell Retained Mass, g & 1720 & 1225 & 1410 & 1130 & 961 & -564 \\
\hline \multicolumn{7}{|l|}{ Interaction Vesel Debris, $\mathrm{g}$} \\
\hline Underside of Head & 101 & 227 & 218 & 196 & & \\
\hline Side Walls & 0 & 393 & 0 & 0 & & \\
\hline Base & 2371 & 8258 & 2569 & 6090 & NA & NA \\
\hline Impingement Plate Holder & $N A^{\star}$ & 496 & NA & NA & & \\
\hline Subtotal & 2472 & 9374 & 2787 & 6286 & & \\
\hline Pipeway Debris, g & 0 & 36 & 82 & 102 & MA & NA \\
\hline Expansion Vessel Debris, g & 11 & 1139 & 925 & 2282 & MA & NA \\
\hline \multicolumn{7}{|l|}{ Experiment Vessel Debris, g } \\
\hline Bottom & NA & NA & NA & NA & 1001 & 2443 \\
\hline & & & & & 9662 & 9082 \\
\hline Subtotal & & & & & 10623 & 11525 \\
\hline Recovered Debris, g & 2483 & 10549 & 3794 & 8670 & 10623 & 11525 \\
\hline Thermite Vessel Debris, $\mathrm{g}$ & 1014 & 1600 & 1145 & 583 & 1432 & 313 \\
\hline Total Debris, $\mathrm{g}$ & 3497 & 12149 & 4939 & 9253 & 12055 & 11838 \\
\hline $\begin{array}{l}\text { Mixed Reactant Mass Minus } \\
\text { Debris Mass, g }\end{array}$ & 287 & -137 & -139 & 757 & -43 & 174 \\
\hline
\end{tabular}

*NA: Not Applicable 


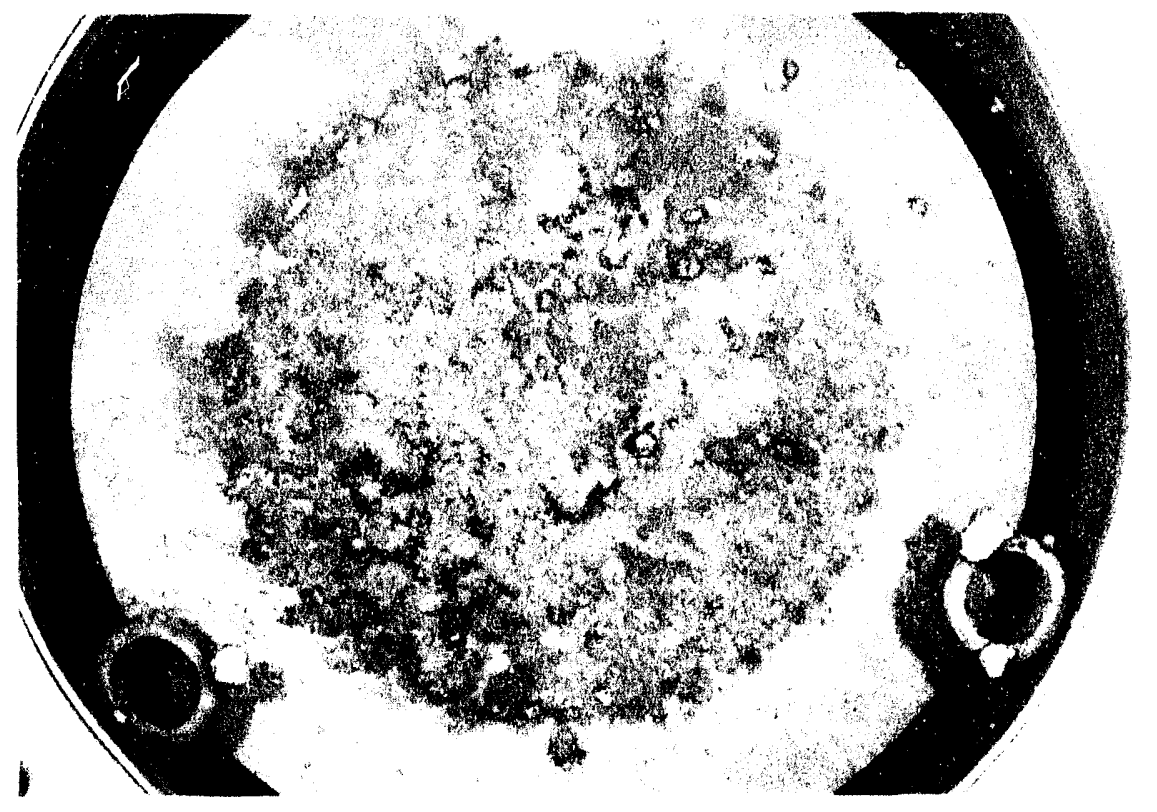

Fig. 3-8 View of Vapor Separator in the Thermite Vessel Used in Experiment CCM-1

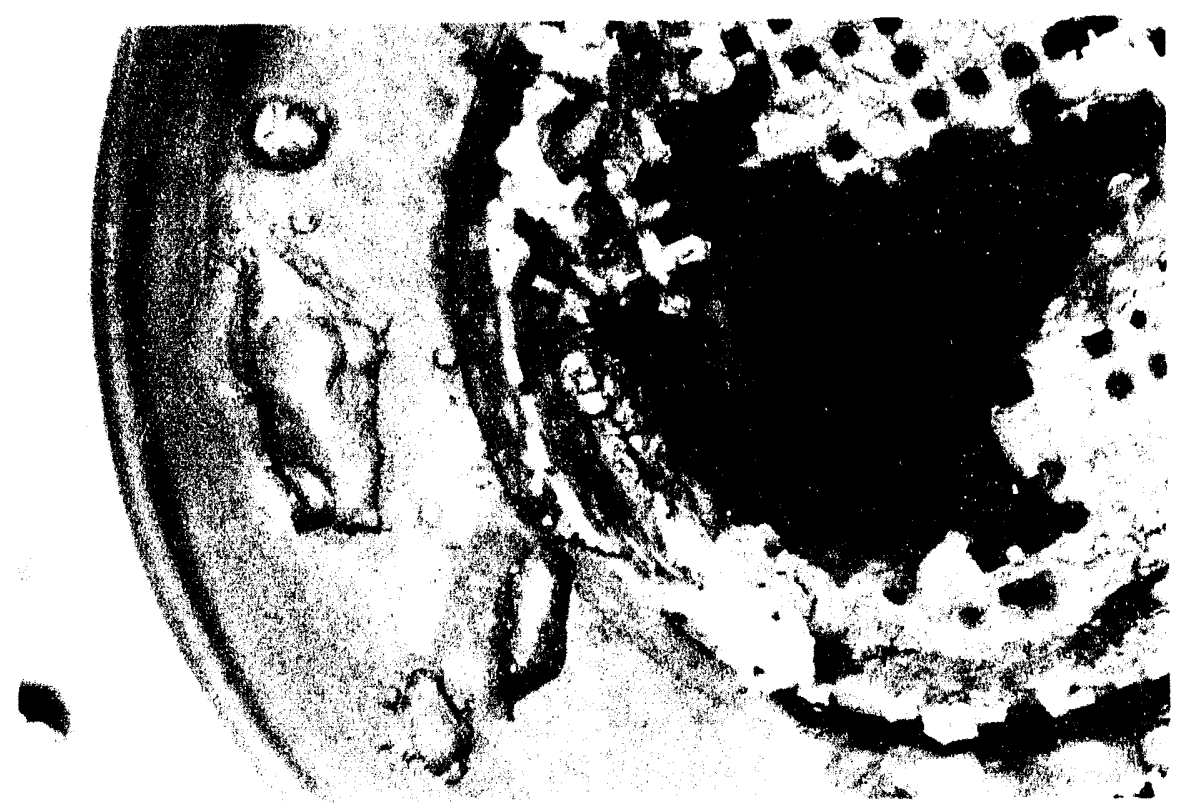

Fig. 3-9 View of Expansion Region and Porous Grid Plate in the Thermite Vessel Used in Experiment CCM-1 


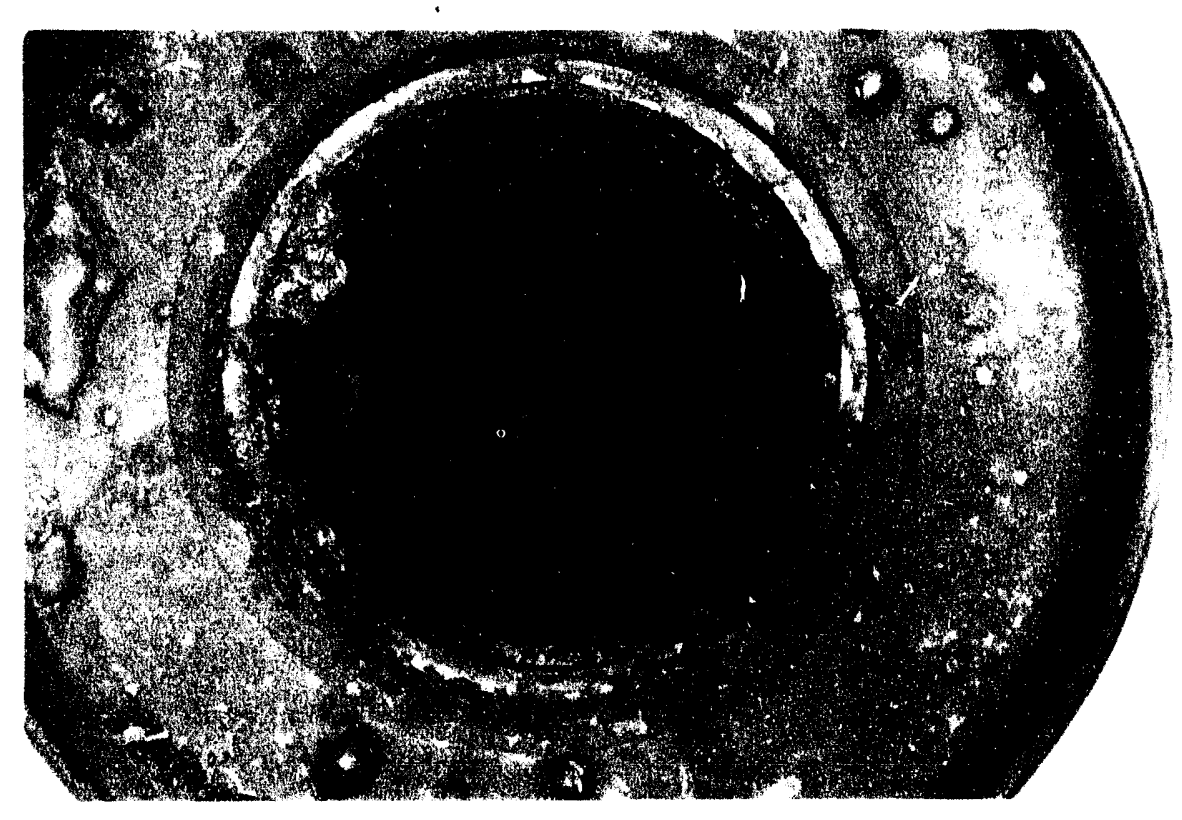

Fig. 3-10 View of Bottom of Thermite Vessel Used in Experiment CCM-1

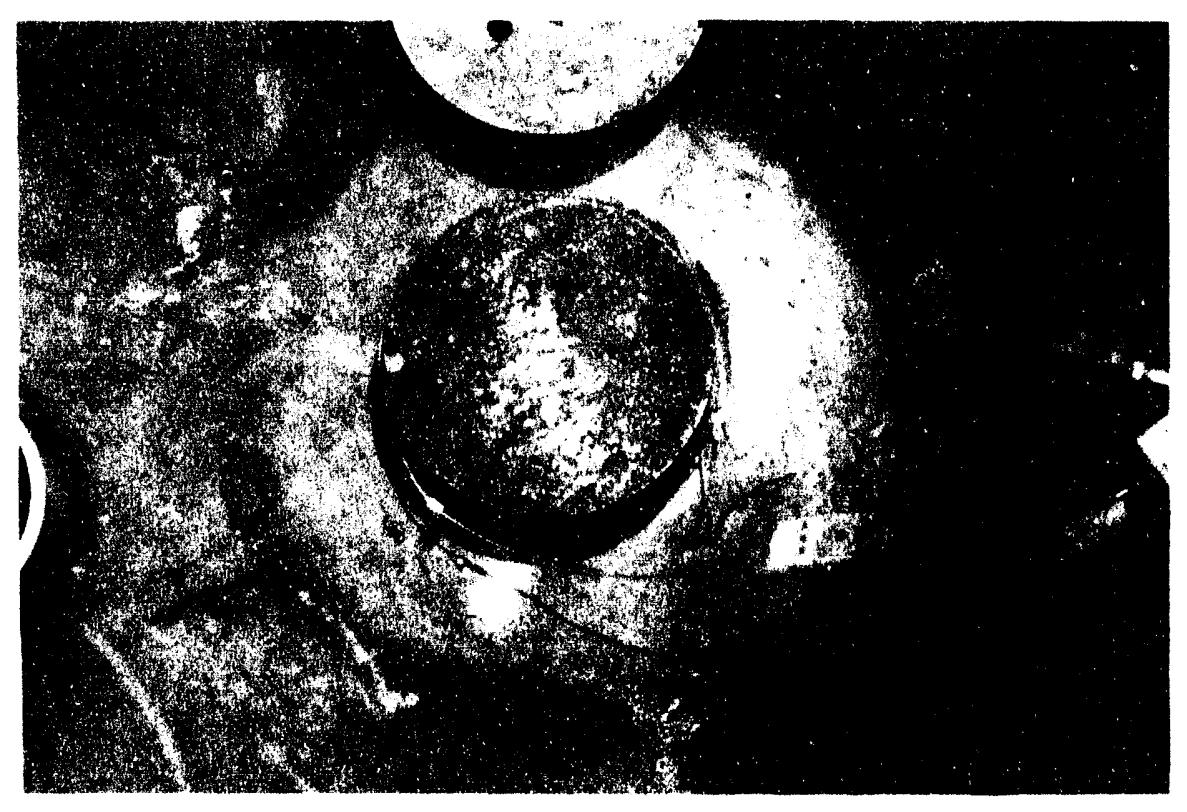

Fi8. 3.11 View of Botton of the Expansion Vessel Used in Experiment CCM-1 


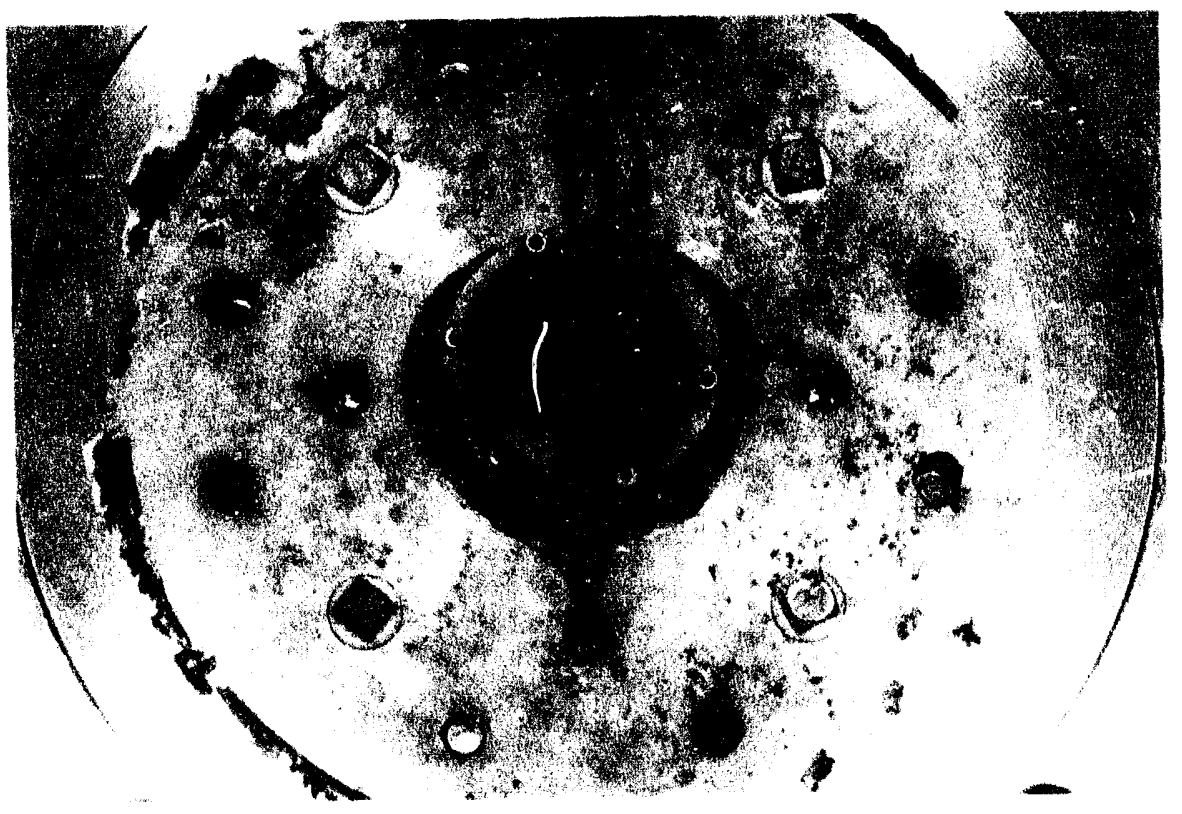

Fig. 3-12 View of Underside of Thermite Vessel Head for Experiment CCM-1

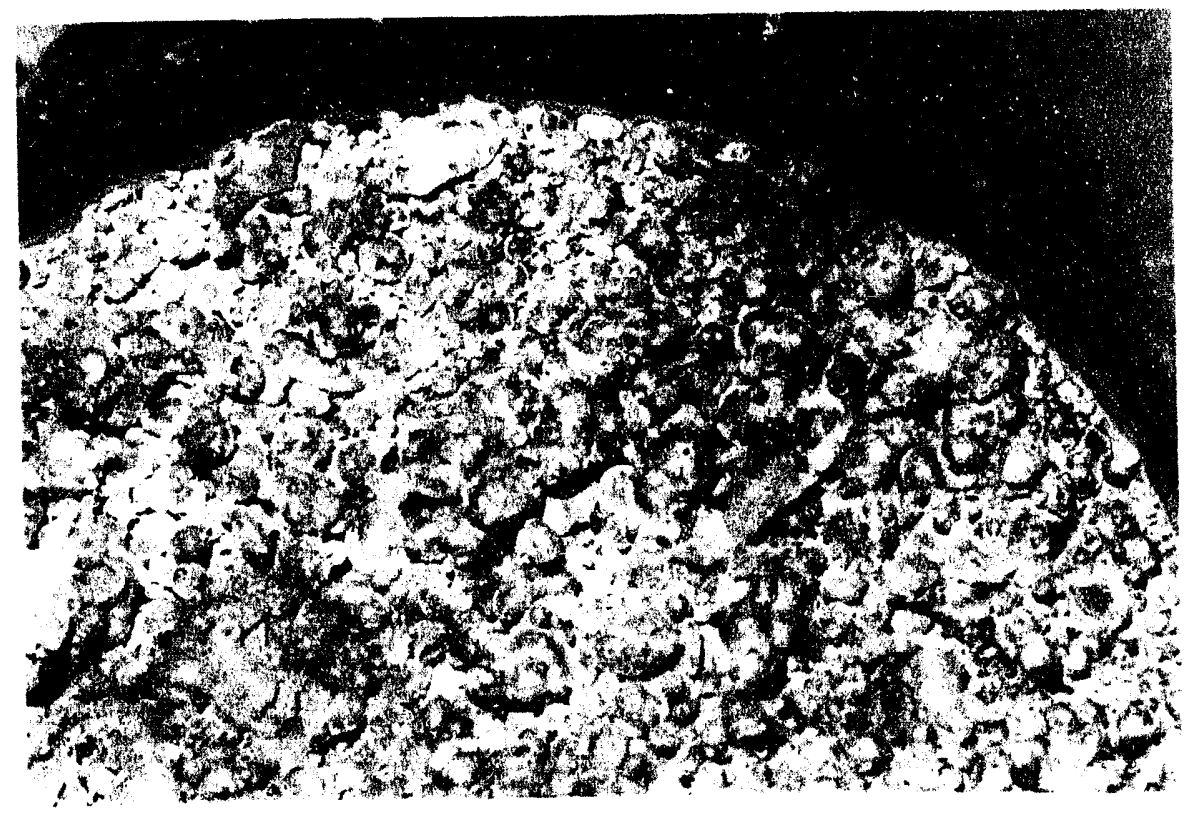

Fig. 3-13 View of Debris in the Bottom of the Thermite Vessel Used in Experiment CCM-1 

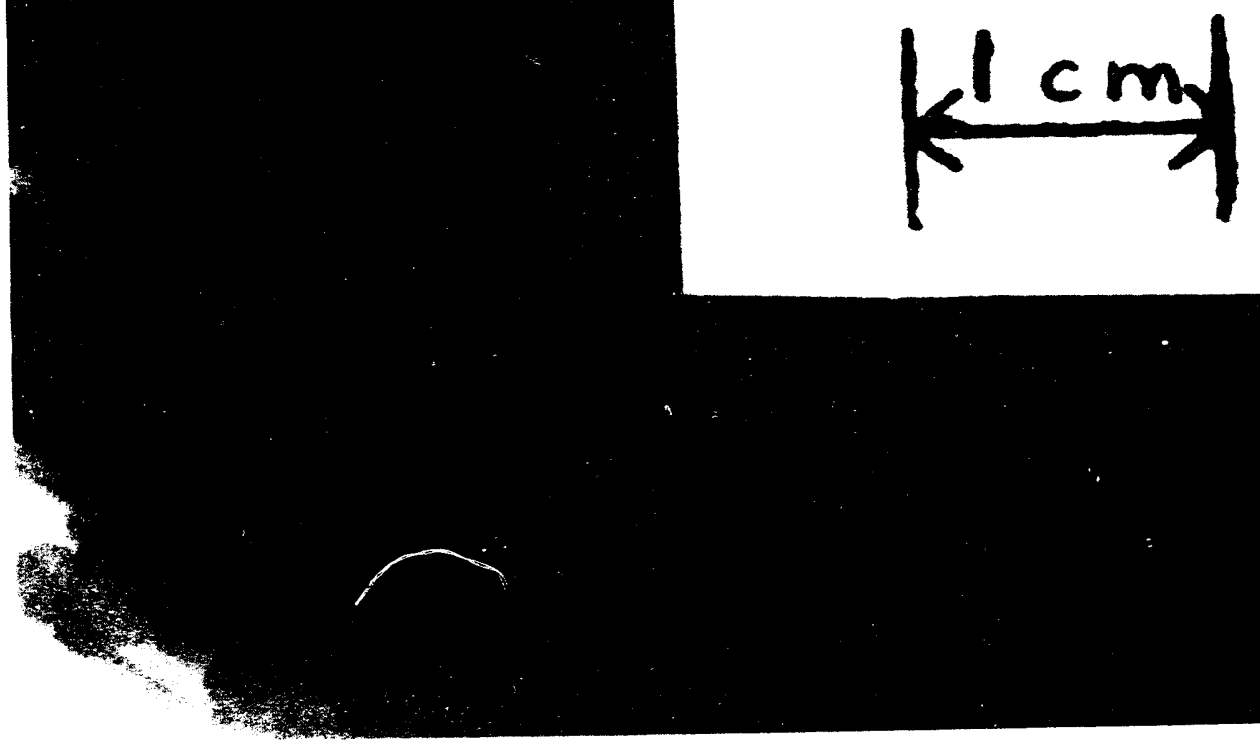

Fig. 3-14 Close-Up View of Debris from the Interaction Vessel Base for Experiment CCM-1 
sintered particles with a depth of 15.9 to $27 \mathrm{~mm}$ (Fig. 3-15). The vessel wall and base showed no attack from the corium.

From the bed dimensions and a corium solid density of $8710 \mathrm{~kg} / \mathrm{m}^{3}$, a bed porosity of $53 \%$ is estimated. A volumetric displacement measurement of the density of a representative debris sample confirmed this estimated value.

The oxide and metallic particles were similar in appearance. Many oxide particles had metallic inclusions. All sizes and shapes of particles were present (Figs. 3-16 and 3-17). The vessel wall and base showed no attack from the corium. There were fragments of broken crust and many spheroidal (Fig. 3-18) and ellipsoidal fragments (Fig. 3-19). Spheroidal fragments having diameters of over $10 \mathrm{~mm}$ were found; however, a diameter of $3 \mathrm{~mm}$ was more typical. Many of the particles were hollow shells (Figs. 3-18, 3-19, and 3-20).

\subsubsection{Test CCM-2}

In experiment CCM-2, $84 \%$ of the injected corium was recovered from che interaction vessel and about $10 \%$ from the expansion vessel.

In the interaction vessel, the impingement plate holder had a small amount of corium debris on the inside wall of the tubing and some on the bottom of the impingement plate (Fig. 3-21). The holes in the impingement plate were open and suffered no erosion. Patches of corium adhered to the underside of the vessel head (Fig. 3-22) and to the vessel sidewalls (Figs. 3-23 and 3-24).

Eighty-eight percent of the debris in the interaction vessel was found on its base. The surface of this debris bed (Figs. 3-25 and 3-26) had a different appearance from that in experiment CCM-1. It was rough and had a reddish brown color with some black colored material interspersed. There was a large globule of metal visible, along with an oblong piece of greyish colored debris. Some small spherodal particles were present. The material was sintered together in large chunks with noticeable voids (Figs, 3-27 and 3-28). The base showed no erosion. The bed thickness was relatively uniform, about $45 \mathrm{~mm}$. The estimated porosity was 39\%. A sample of this material had a measured density of 5930 $\mathrm{kg} / \mathrm{m}^{3}, 68 \%$ of solid corium density.

of the debris swept into the expansion vessel, $36 \mathrm{~g}$ was deposited in the pipeway, while the remaining material was found on the bottom of the expansion vessel (Fig. 3-29).

\subsubsection{Test CCM-3}

Inside the thermite vessel there was a thin layer of corium where the tungsten grid plate was located (Fig. 3-30). The debris accumulation in the bottom of the expansion vessel is shown in Fig. 3-31. This material consisted of fine powder, particles of scale, and filament-shaped particles. Some debris was attached to the level probes (Fig. 3-32).

Ninety-two percent of the corium debris in the interaction vessel was found on the base. This material was similar to the CCM-1 debris. Its surface is shown in Fig. 3-33. The thickness of the debris bed varied from 19 to $38 \mathrm{~mm}$ as shown in Fig. 3-34. A cross-sectional view is shown in Fig. 3-35. The debris was loosely bound and sintered. Some of the particles are shown in Figs. 3-36, 


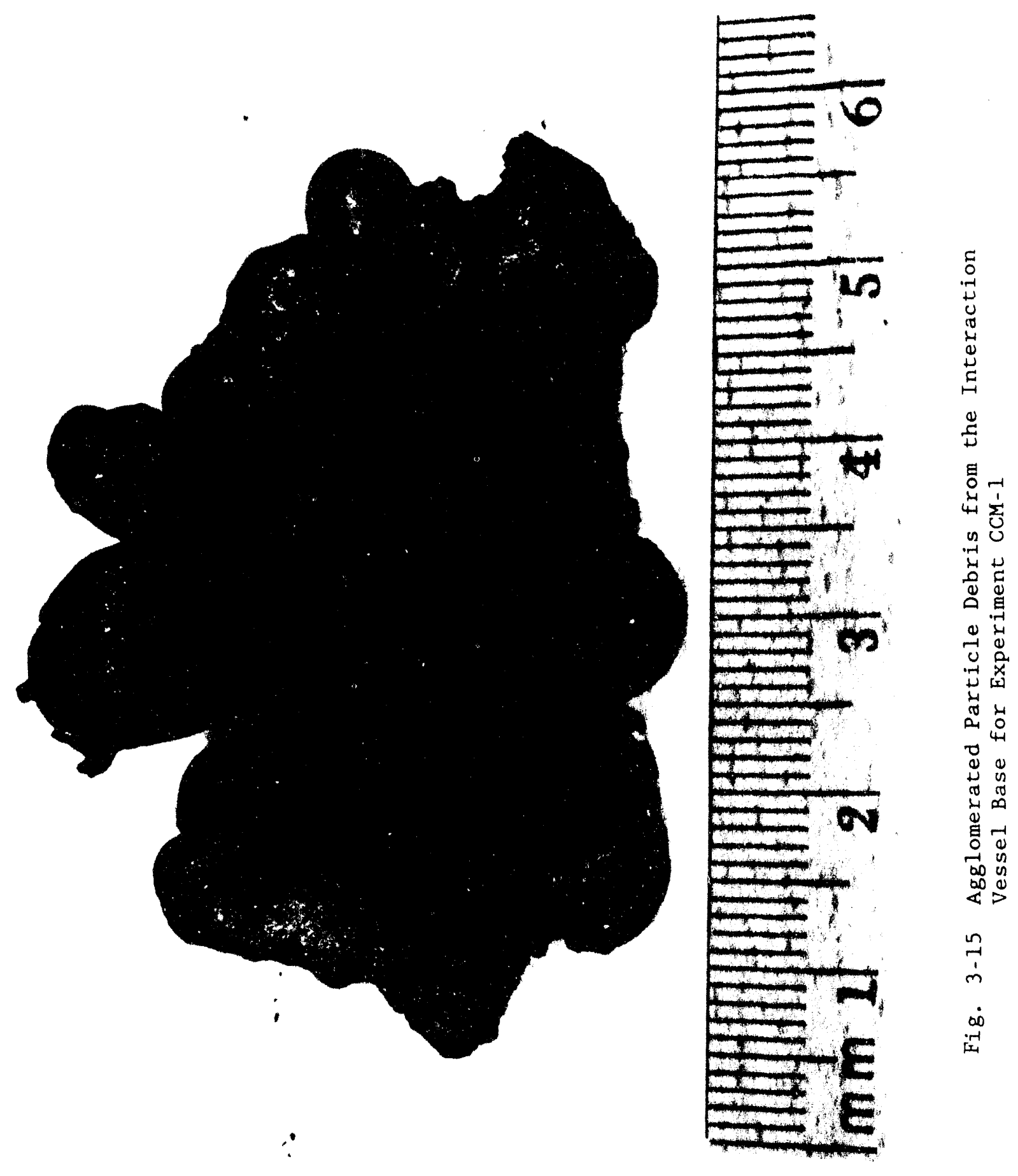




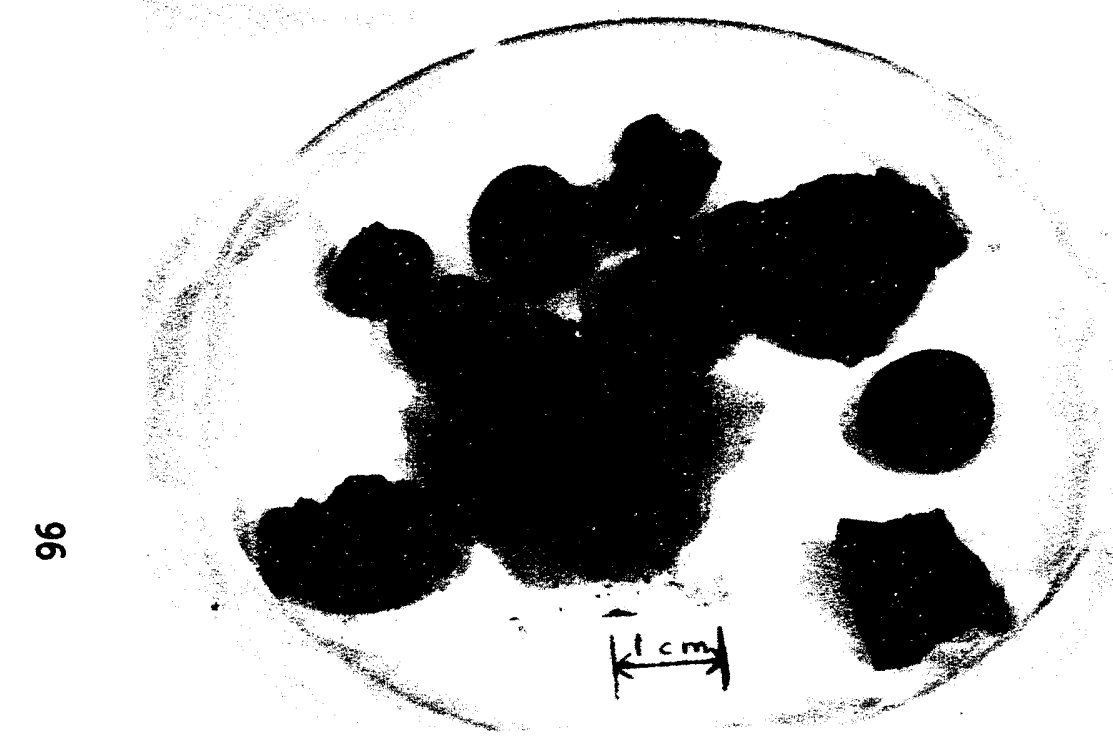

Fig. 3-16

Variation of Particle Size and Shape from the Interaction Vessel Debris Bed for Experiment CCM-1
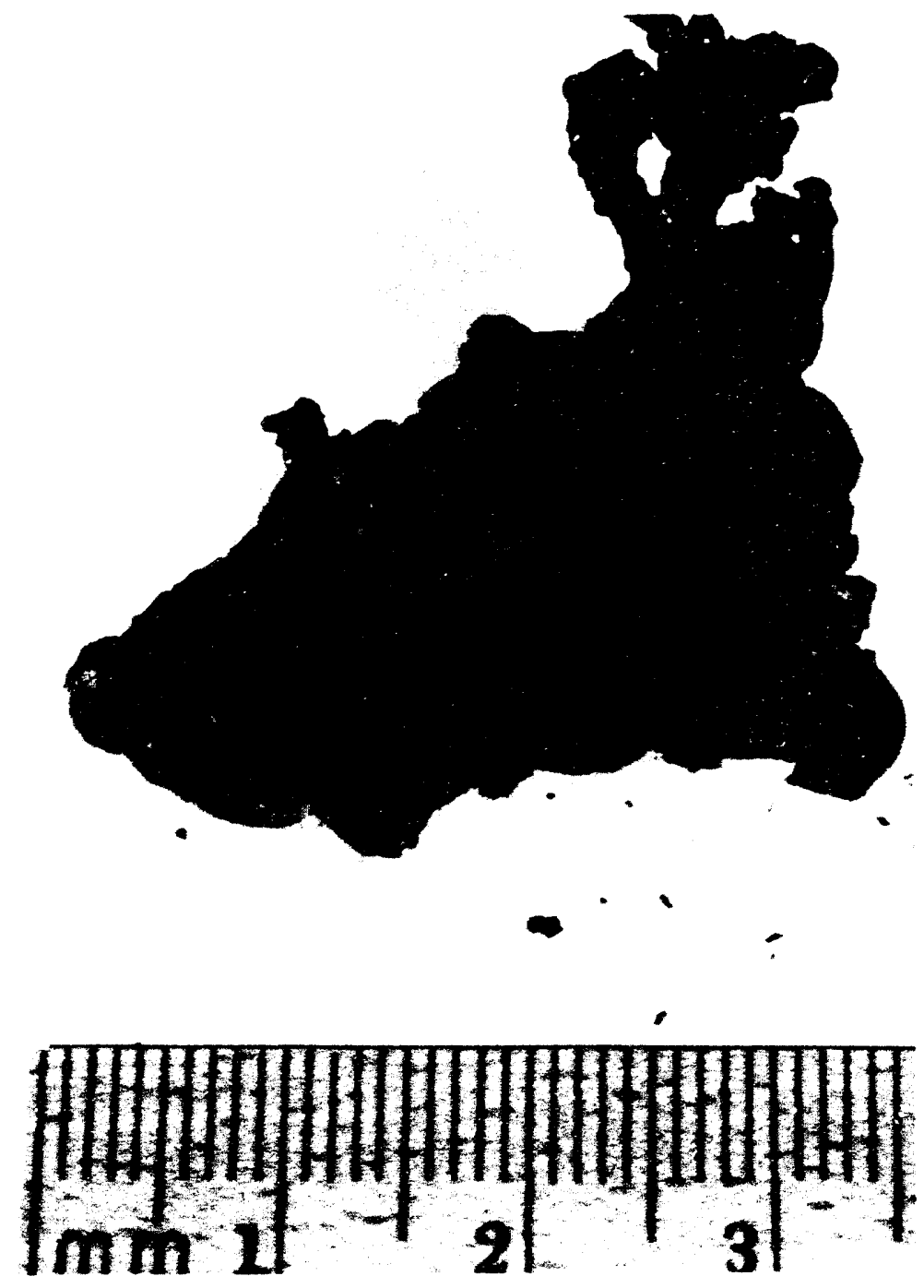

Fig. 3-17

Debris Particle from the Interaction Jessel Base for Experiment CCM-1 


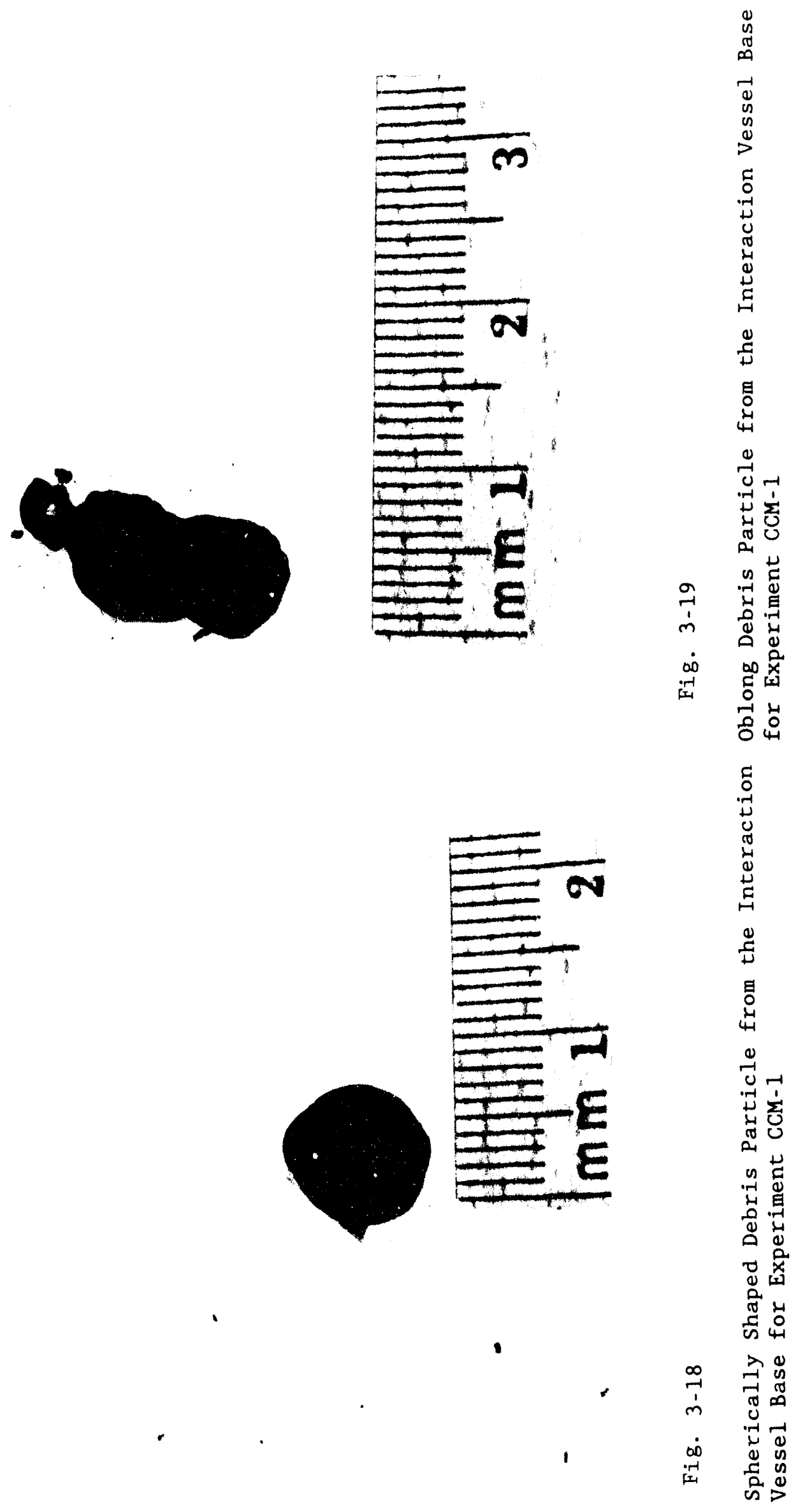




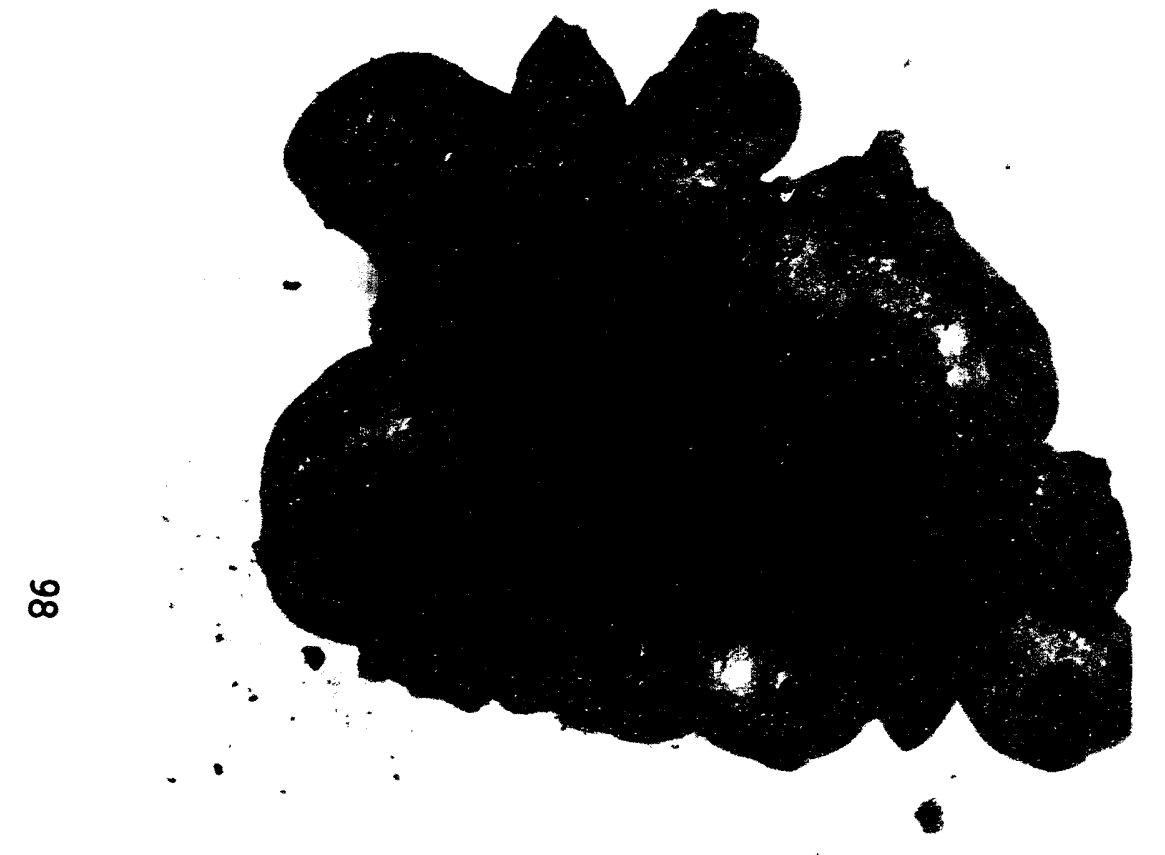

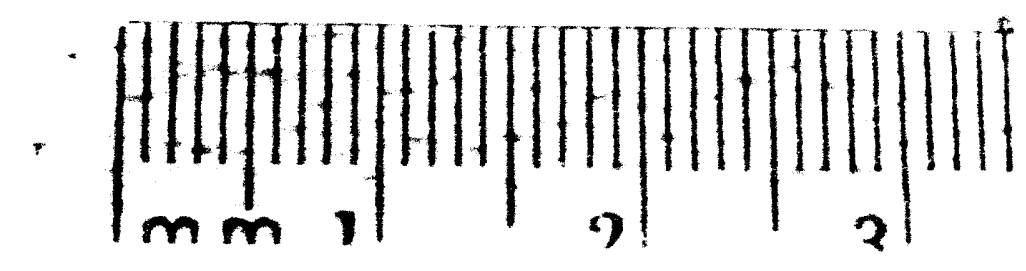

Fig. $\quad 3-20$

Hollow Particles from the Interaction-Vessel Debris Bed for Experiment CCM-1

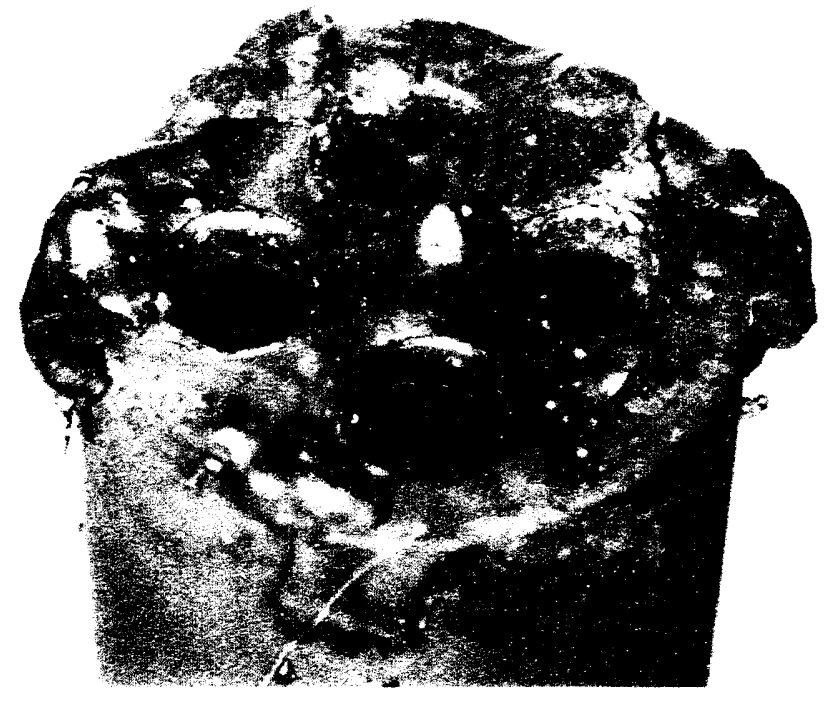

Fig. $\quad 3-21$

Bottom of Impingement Plate in the Interaction Vessel for Experiment CCM-2 


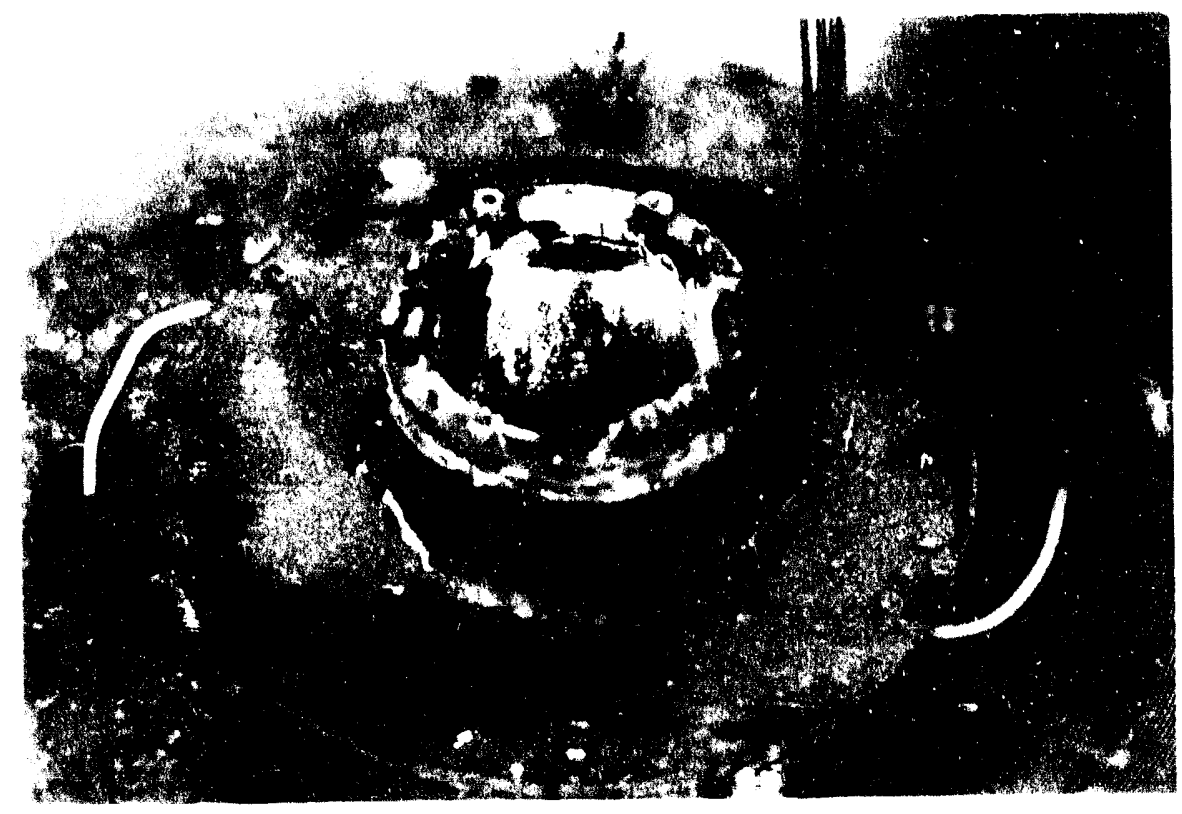

Fig. 3-22 Underside of Interaction Vessel Head for Experiment CCM-2

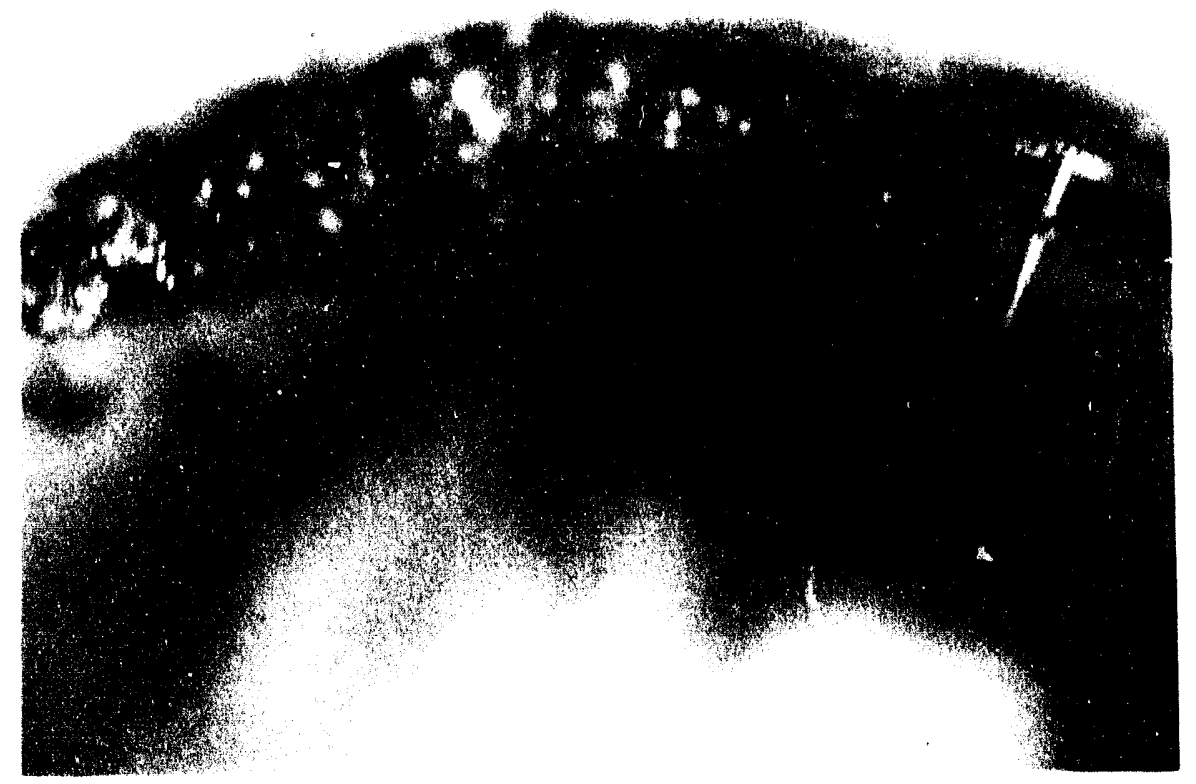

Fig. 3-23 Debris on Side Wall of Interaction Vessel for Experiment CCM-2 


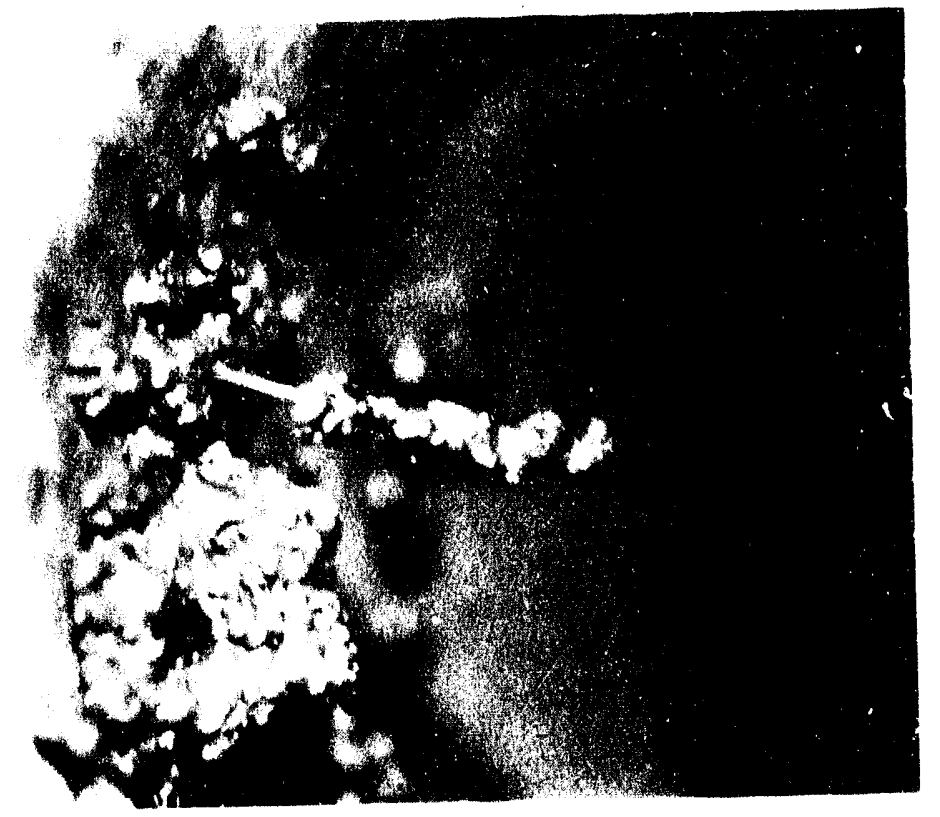

Fig. 3-24 Debris Adhering to a Thermocouple and the Side Wall of the Interaction Vessel for Experiment CCM-2

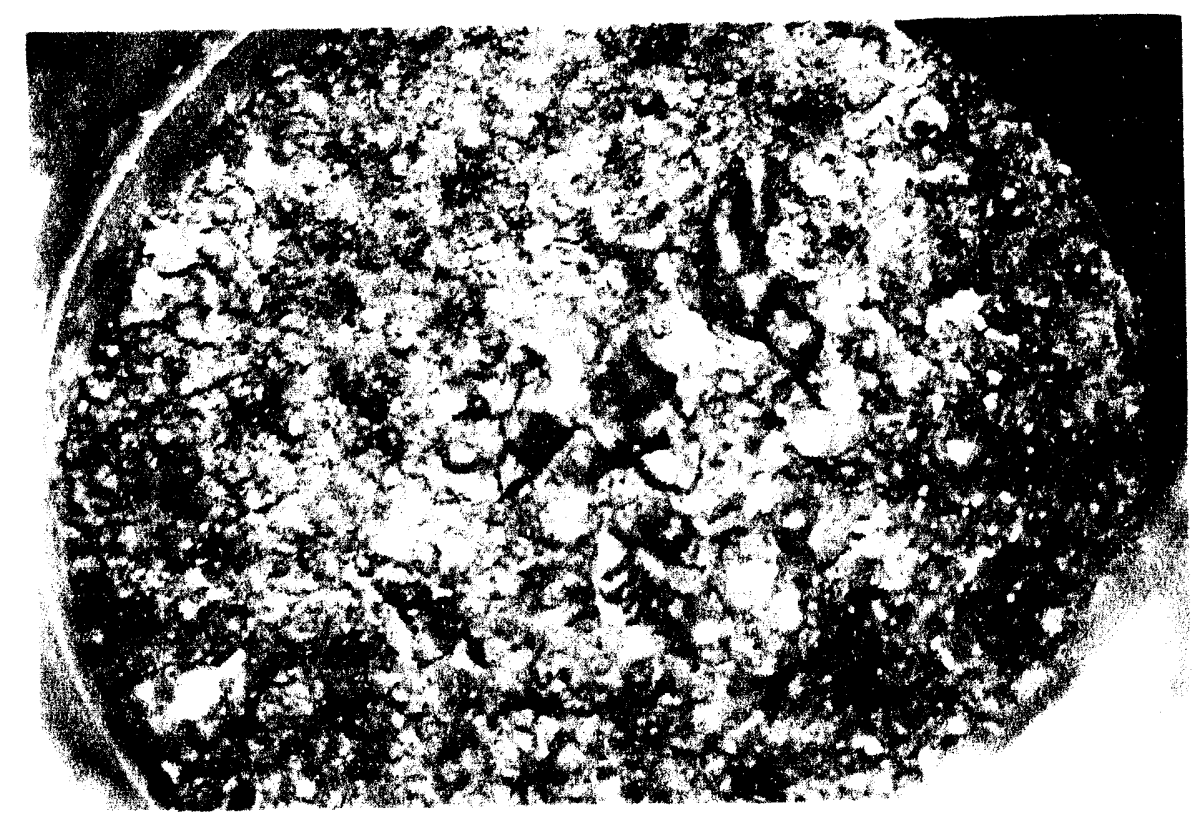

Fig. 3-25 View of Debris Surface on the Base of the Interaction Vessel for Experiment CCM-2 


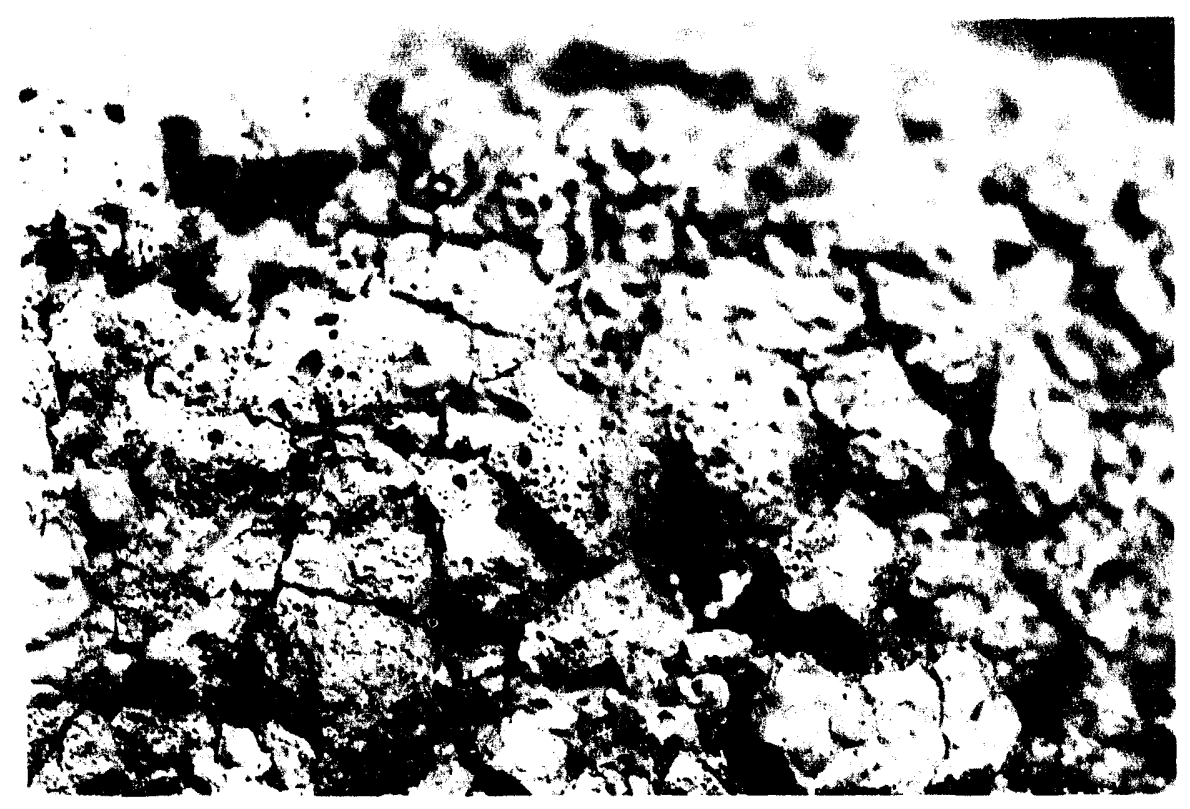

Fig. 3-26 Close-up View of Debris Surface on the Base of the Interaction Vessel for Experiment CCM-2

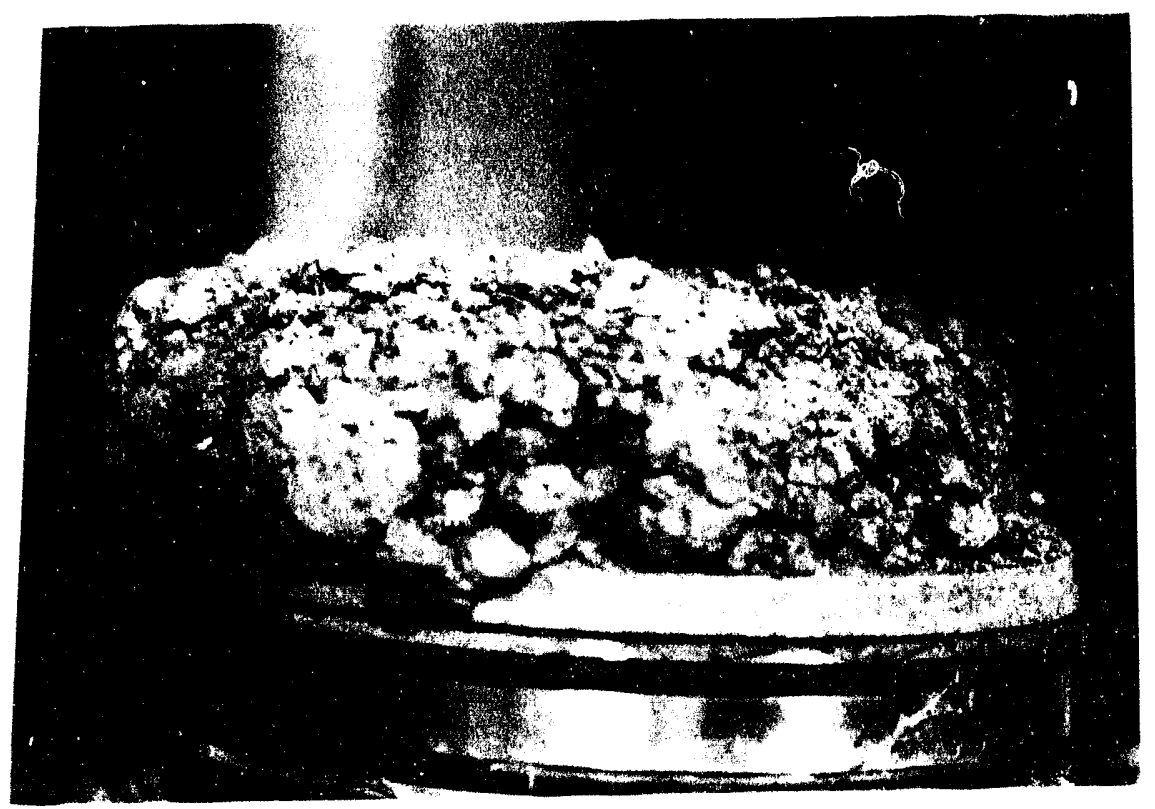

Fig. 3-27 View of Debris Bed on the Interaction Vessel Base After Sectioning for Experiment CCM-2 


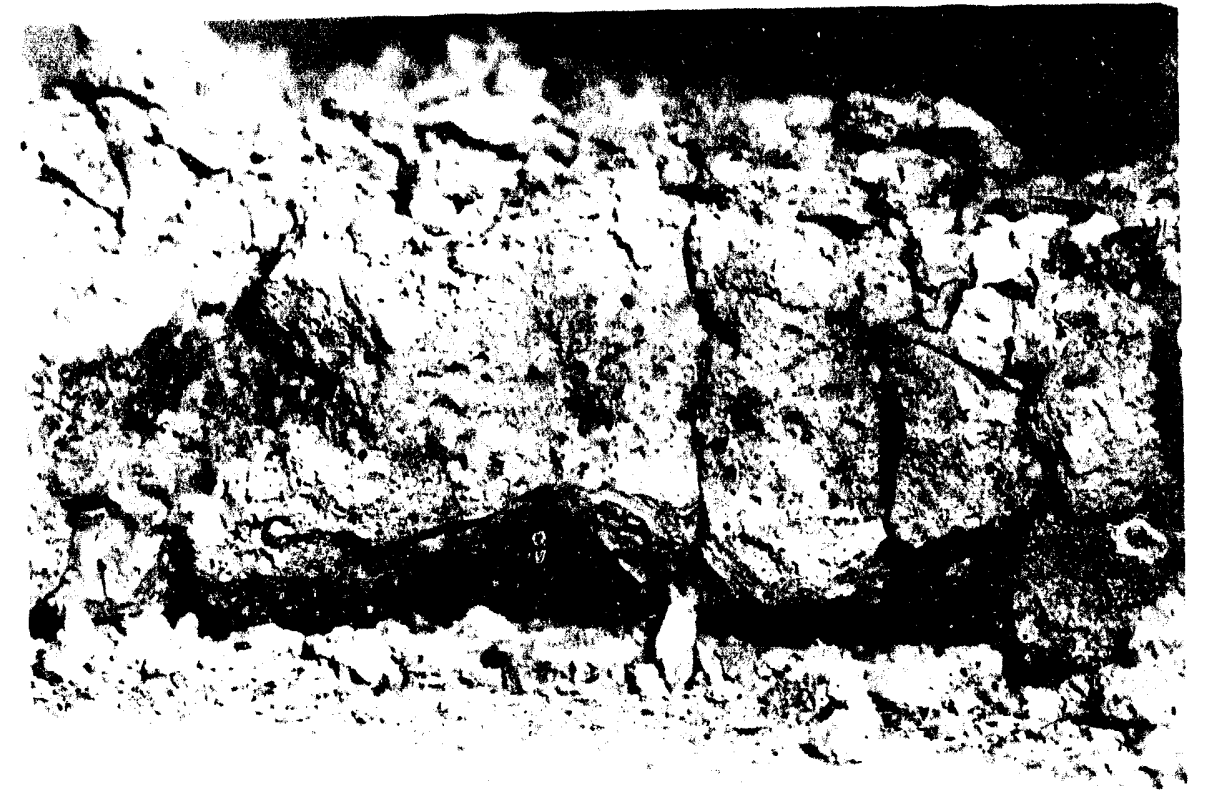

Fig. 3-28 Cross-Sectional View of Debris Bed on the Interaction Vessel Base for Experiment CCM-2

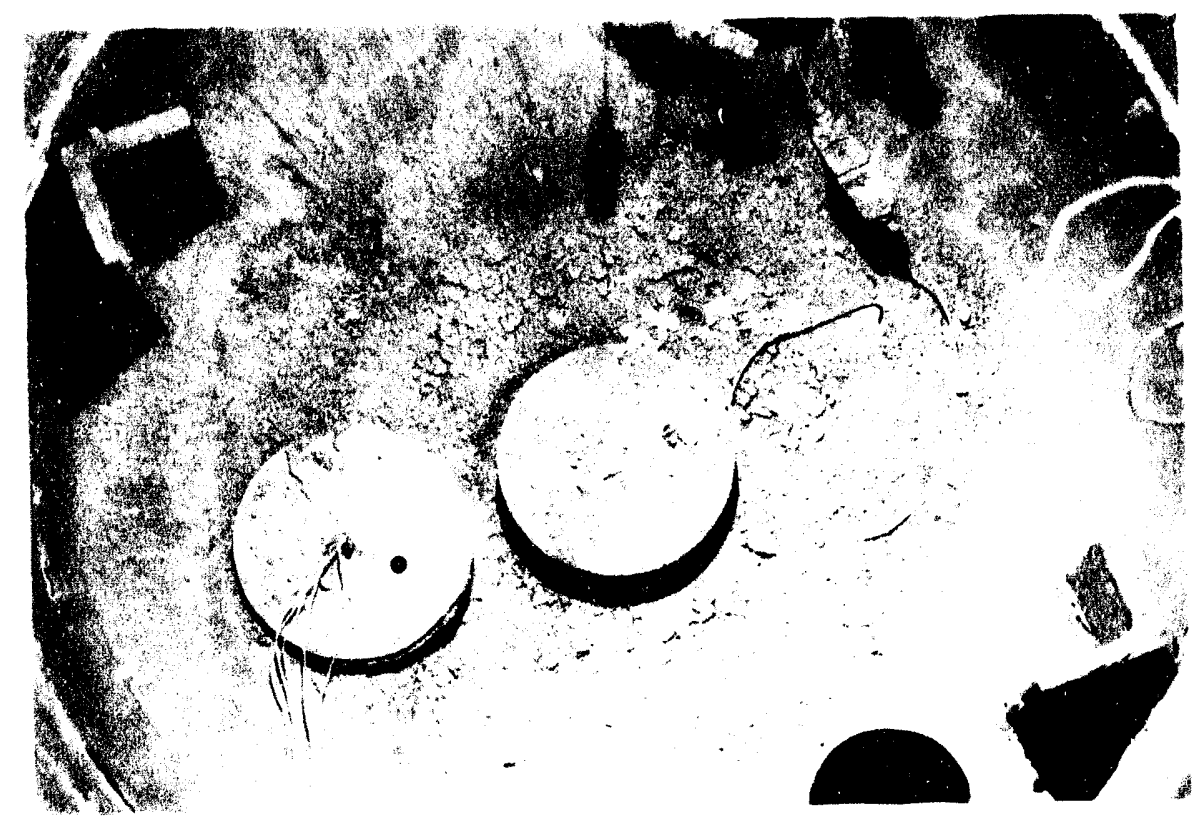

Fig. 3-29 View of the Bottom of the Expansion Vessel for Experiment CCM-2 


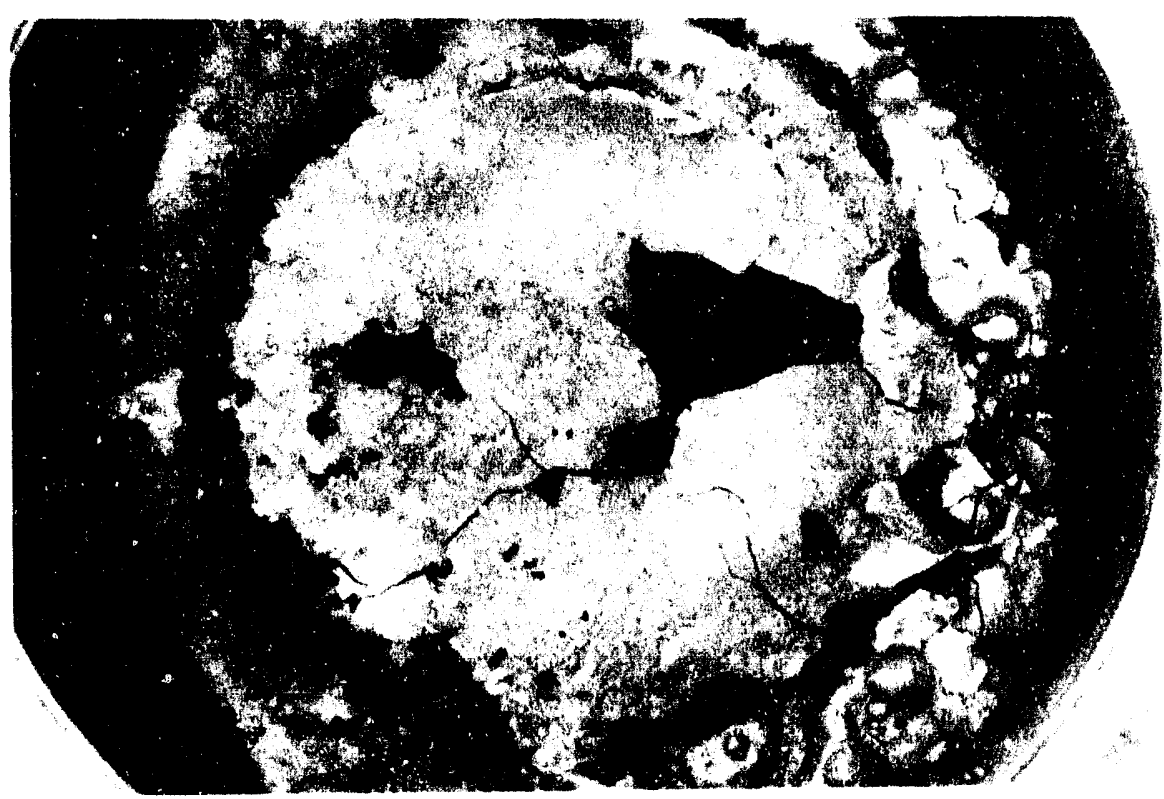

Fig. 3-30 Thin Crust Layer of Corium in the Thermite Vessel Used for Experiment CCM-3

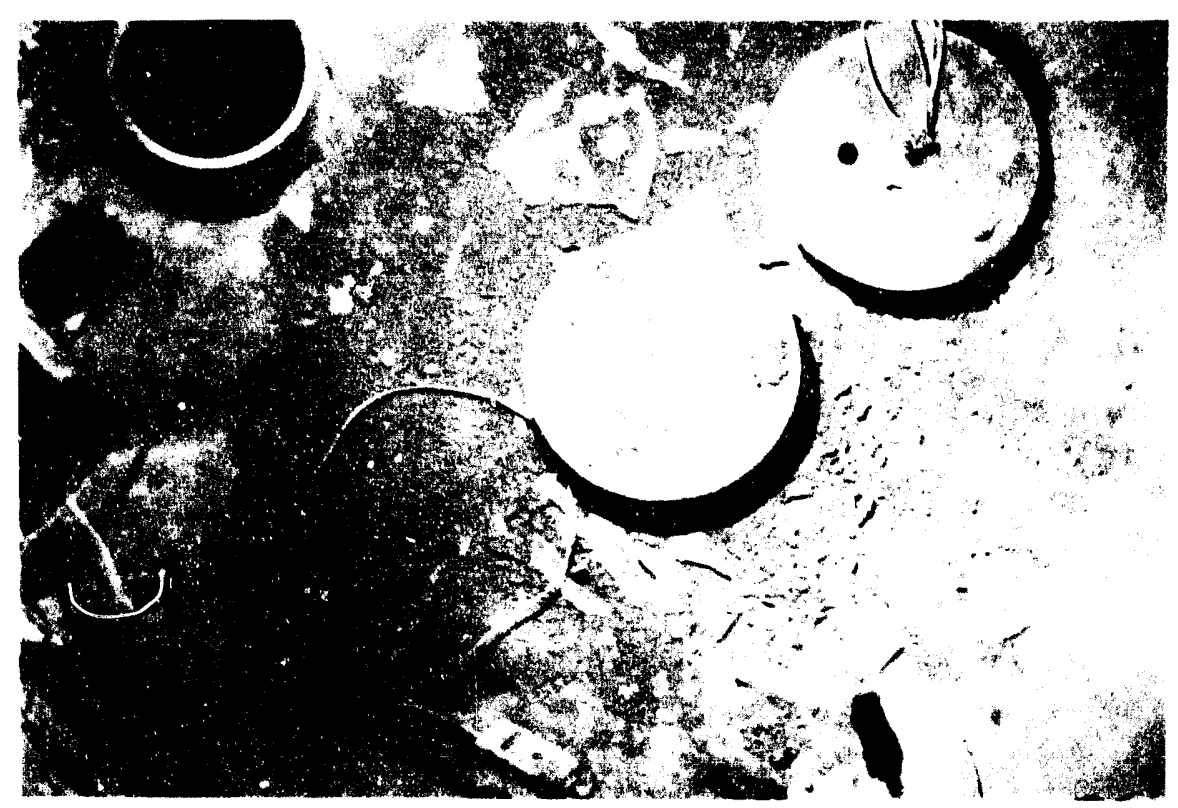

Fig. 3-31 Debris in the Bottom of the Expansion Vessel for Experiment CCM-3 


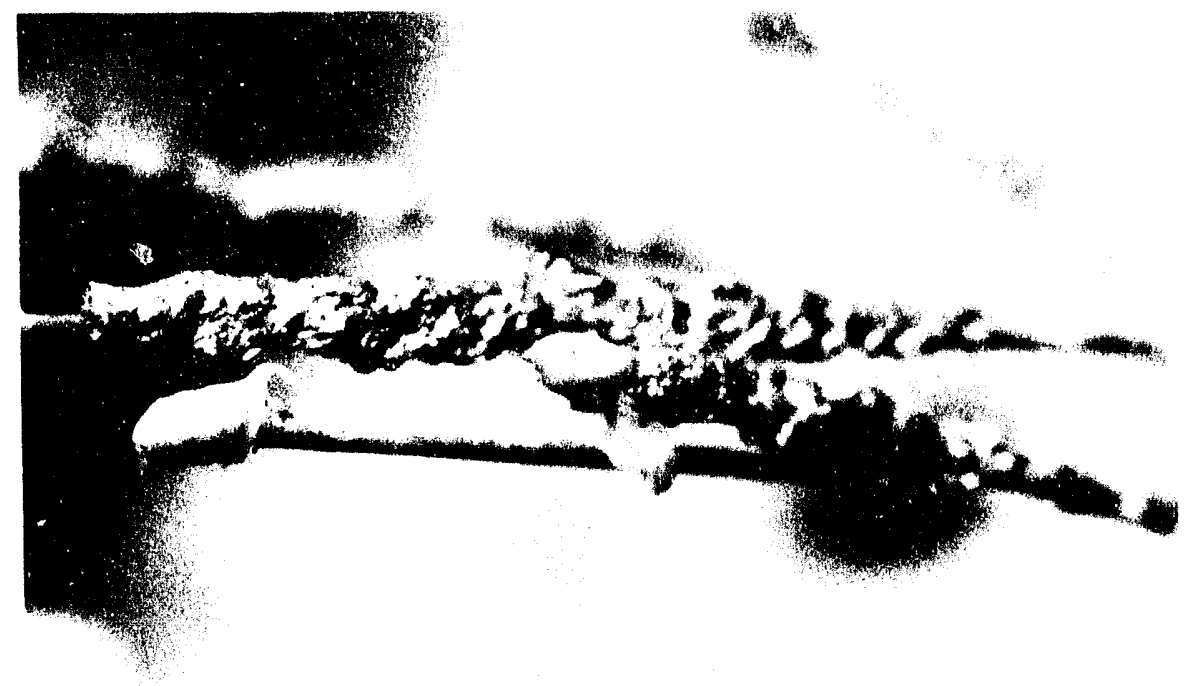

Fig. 3-32 Debris Attached to the Level Probes in the CCM-3 Interaction Vessel

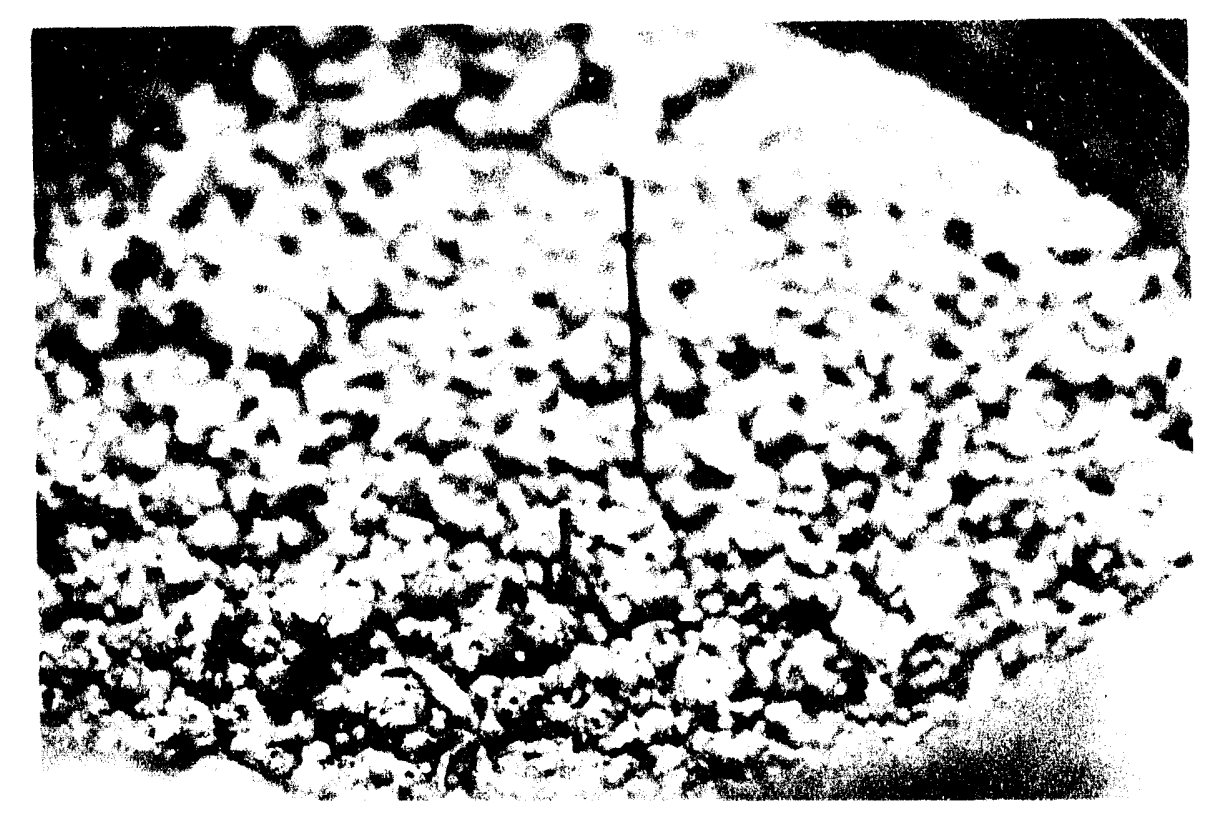

Fig. 3-33 Surface of Debris Bed in the Bottom of the Interaction Vessel for Experiment CCM-3 


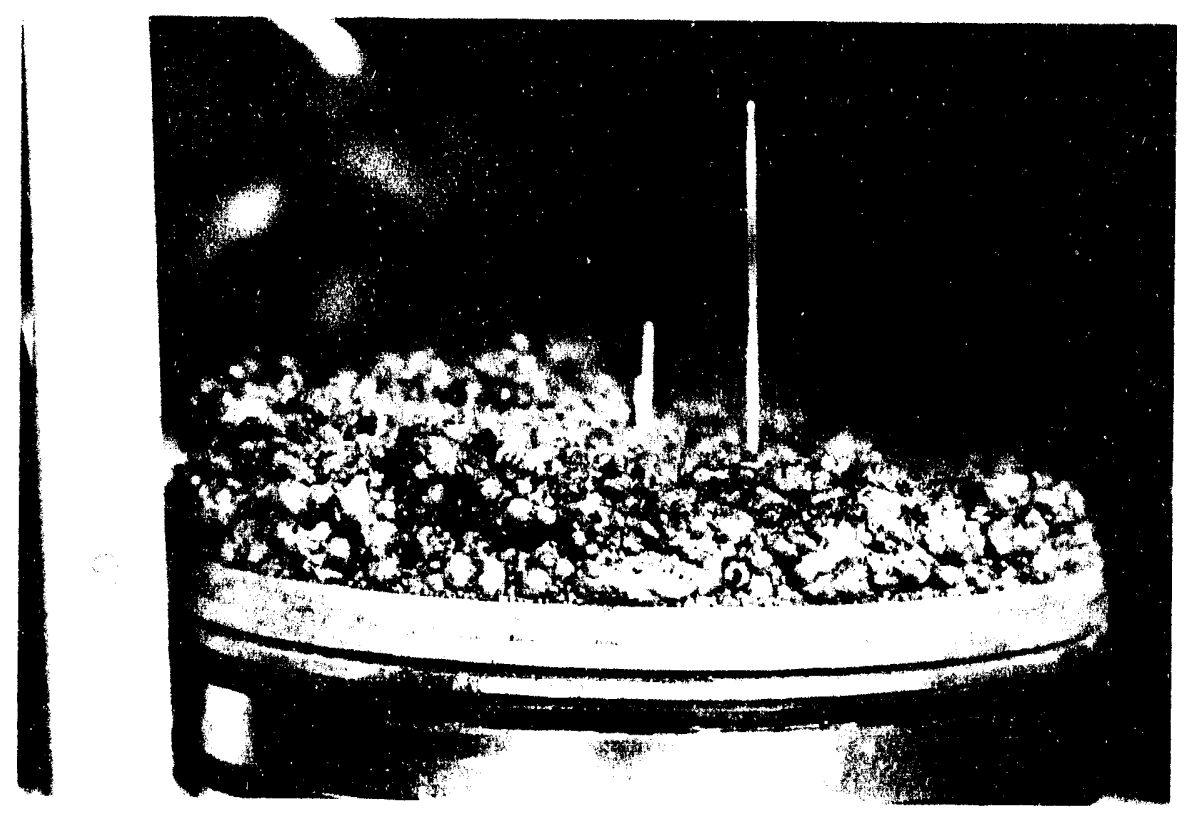

Fig. 3-34 View of Debris-Bed Accumulation on the CCM-3 Interaction Vessel Base

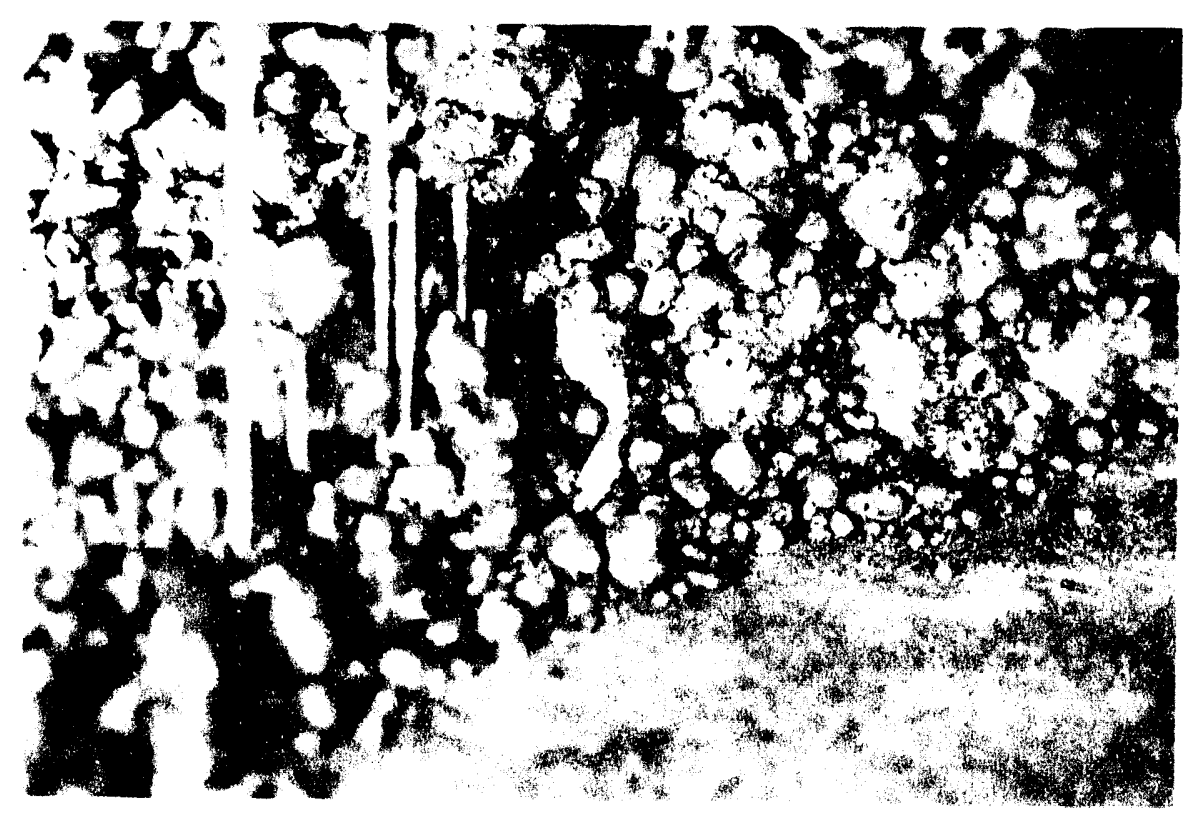

Fig. 3-35 Sectioned Debris Bed on the Base of the Interaction Vessel for Experiment CCM-3 


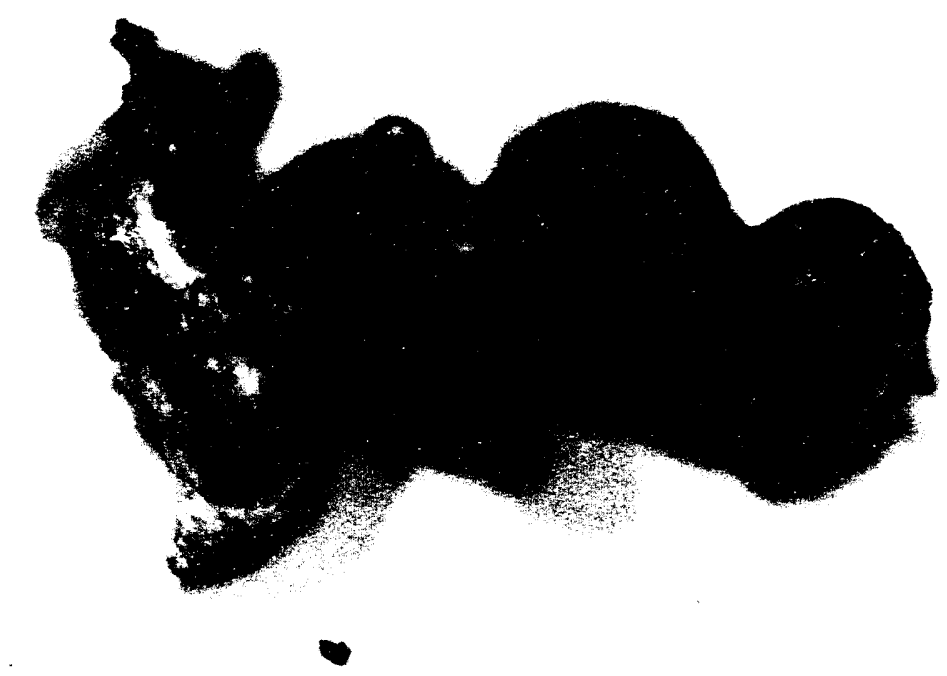

Fig. 3-36 Debris Particle with Metallic Inclusion from the Base of the Interaction Vessel for Experiment CCM-3 
3-37, and 3-38. The particle shown in Fig. 3-36 has an obvious metallic inclusion but generally it was difficult to distinguish between the metallic and non-metallic debris. Many of the particles were spheroidal. Agglomerated particles (Fig. 3-37) were plentiful but fragile. A large number of the particles were hollow, such as that shown in Fig. 3-37.

The interaction vessel base suffered no erosion. The debris bed was estimated to be $65 \%$ voidęd. The measured density of a representative sample of the debris was $5220 \mathrm{~kg} / \mathrm{m}^{3}, 60 \%$ of solid corium density.

\subsubsection{Test $\mathrm{CCM}-4$}

The thermite vessel was relatively clean with a small amount of blackish scale on the mullite sleeve and lava cone. The tungsten grid plate was missing a 15-mm diameter section from its center. Some corium debris was on the ledge of the expansion volume adjacent to the porous gas baffle, which was intact. A small amount of olive-colored powder was inside the vapor separator. The debris in the bottom of the expansion vessel is pictured in Fig. 3-39. This material was $26 \%$ of the recovered debris from the expansion and interaction vessels.

When the head was removed from the interaction vessel, its underside was found to be in a condition similar to that of the previous tests. Inside the vessel the water and vessel walls had a reddish color, due to the presence of unreacted corium trioxide $\left(\mathrm{CrO}_{3}\right)$ and iron oxide $\left(\mathrm{Fe}_{2} \mathrm{O}_{3}\right)$ and/or vessel oxidation by condensing vapor. Patches of corium scale adhered to the walls of the vessel and to some of the thermocouples. There was an accumulation of debris at the entrance of the pipeway (Fig. 3-40).

The surface of the debris bed had a coarse appearance, as shown in Fig. 3-41, and a central tapered mass of material around the thermocouples (Fig. 3-42). A close-up view of this central mass is shown in Fig. 3-43. From a sample of the base material, a measured density of $5560 \mathrm{~kg} / \mathrm{m}^{3}, 63.8 \%$ of solid corium density, was obtained.

\subsubsection{Test CCN-5}

In this experiment the mass of debris recovered from the experiment vessel was $94 \%$ of the injected corium mass. The total mass of debris was $0.4 \%$ greater than the initial mass of the reactants.

When the experiment vessel was opened, a fine olive-colored powder, which was mildly radioactive, was found on the outside of the thermite vessel and its support structure. An $X$-ray diffraction analysis of this powder showed that it consisted of $\alpha-\mathrm{Fe}$, a spinal material $\left(\mathrm{Fe}_{3} \mathrm{O}_{4}, \mathrm{FeCrO}_{4}\right.$, and $\left.\mathrm{Fe}_{2} \mathrm{O}_{3}\right), \mathrm{UO}_{2}, \mathrm{ZrO}_{2}$, and a very small amount of $\mathrm{Zr}$. These are products of the thermite reaction. Inside the thermite vessel, the tungsten grid plate and the stainless steel gas baffle were missing. The underside of the vessel head was coated with the same olive colored powder. This material was also found inside the relief valves. On the ledge of expansion region of the vessel, Fig. 3-44, there were debris scale and metallic globules. A layer of olive-colored corium was on the mullite sleeve. The lava cone was relatively clean with some debris near the discharge hole.

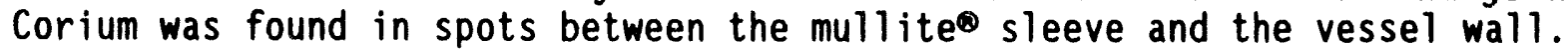




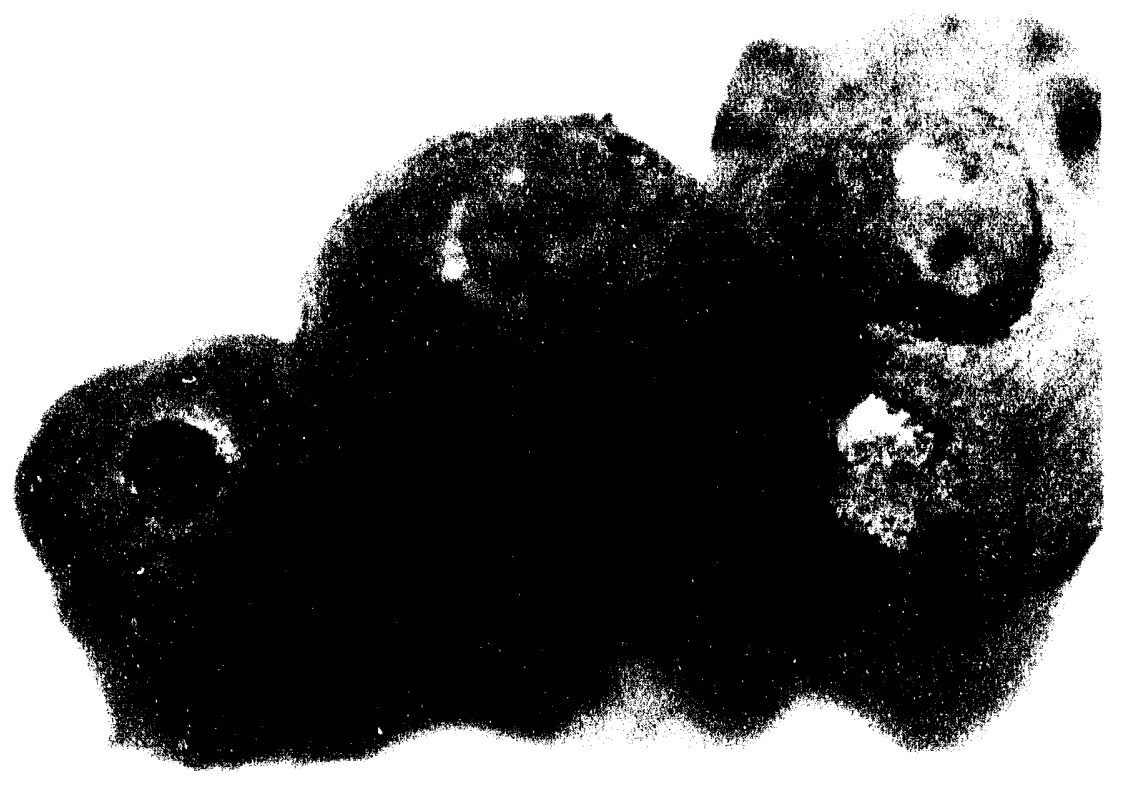

Fig. 3-37 Agglomerated Debris Particle from the CCM-3 Interaction Vessel Base

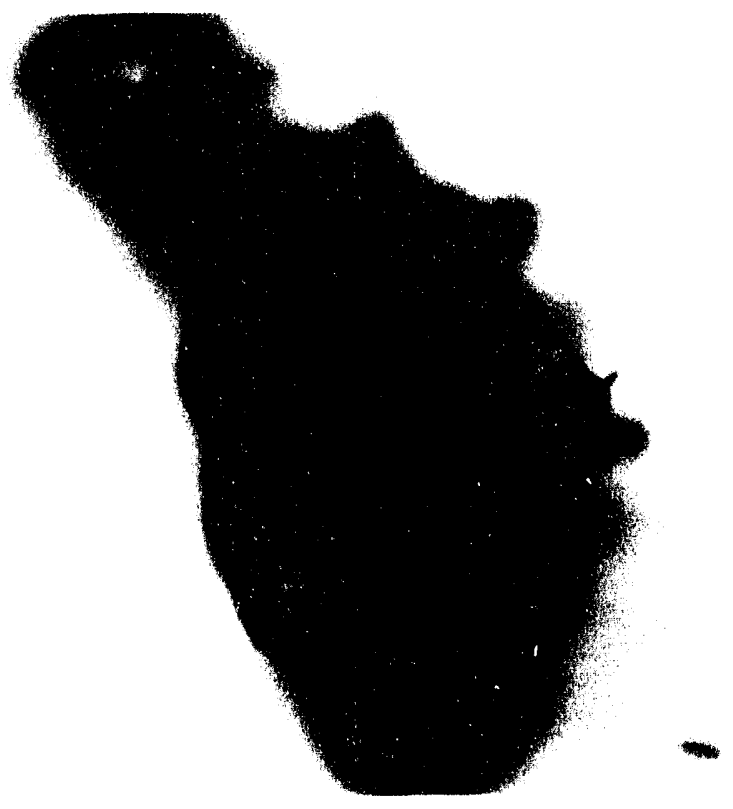

F1g. 3-38 Oblong Debris Particle with Holes from the CCM-3 Interaction Vessel Base 


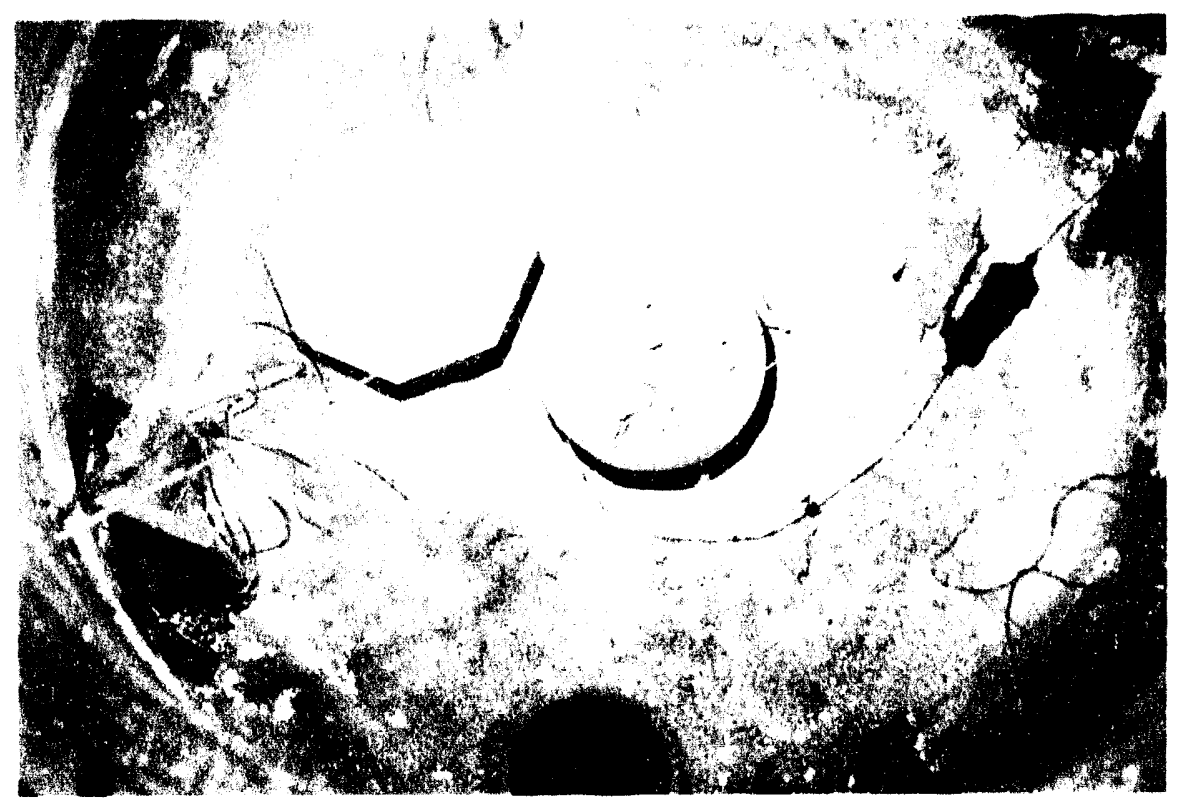

F1g. 3-39 View of Debris in the Bottom of the Expansion Vessel for Experiment CCM-4

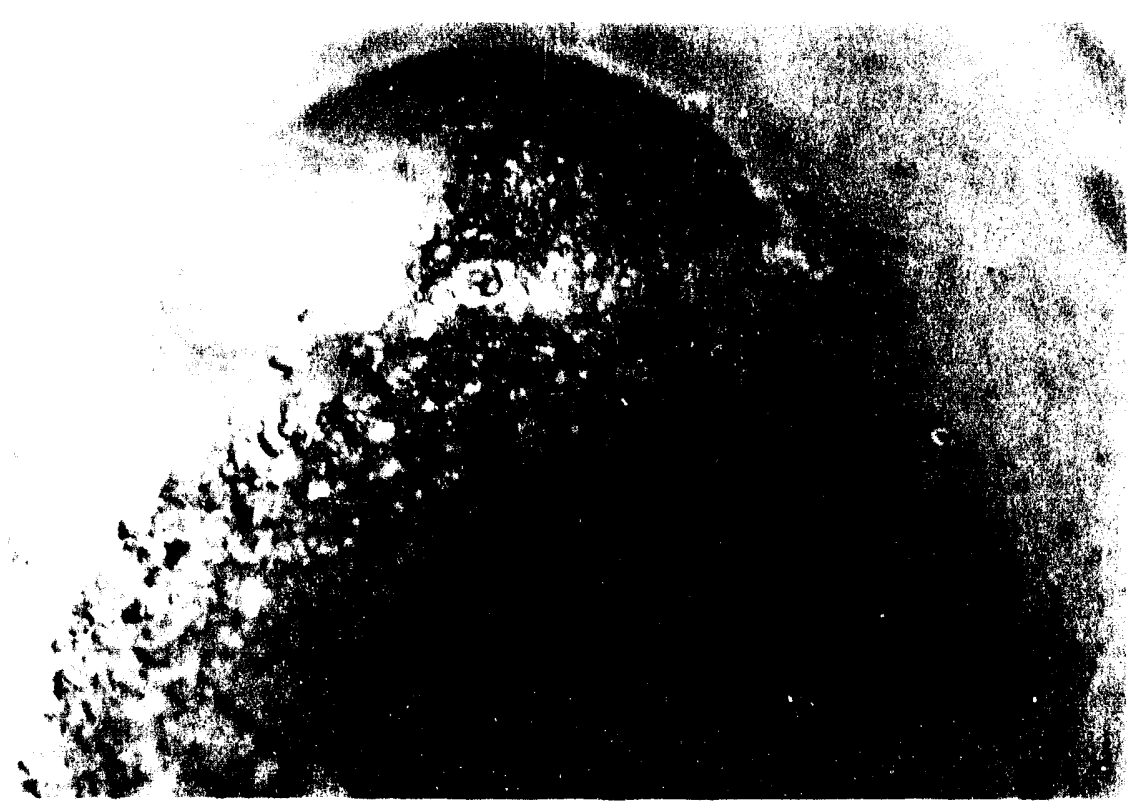

F1g. 3-40 Corium Debris at the Inlet to the Pipeway in the Interaction Vessel for Experiment CCM-4 


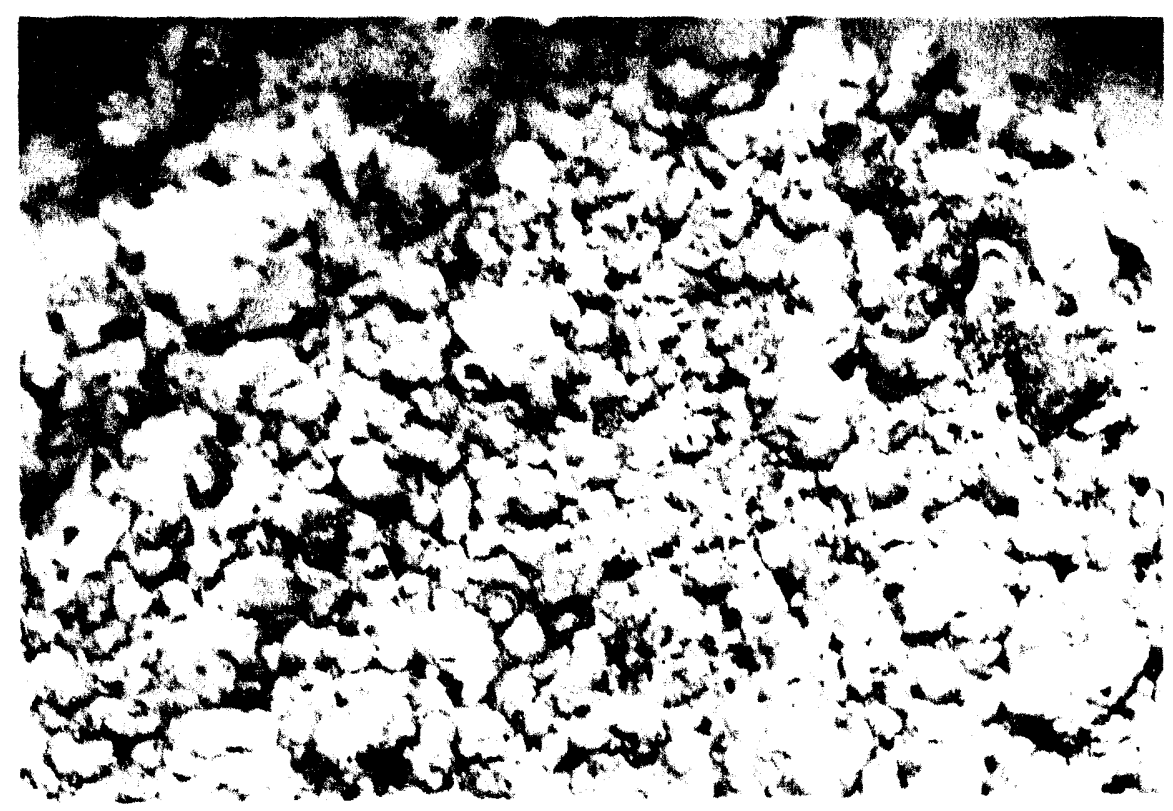

Fig. 3-41 Surface View of the Debris on the Interaction Vessel Base for Experiment CCM-4

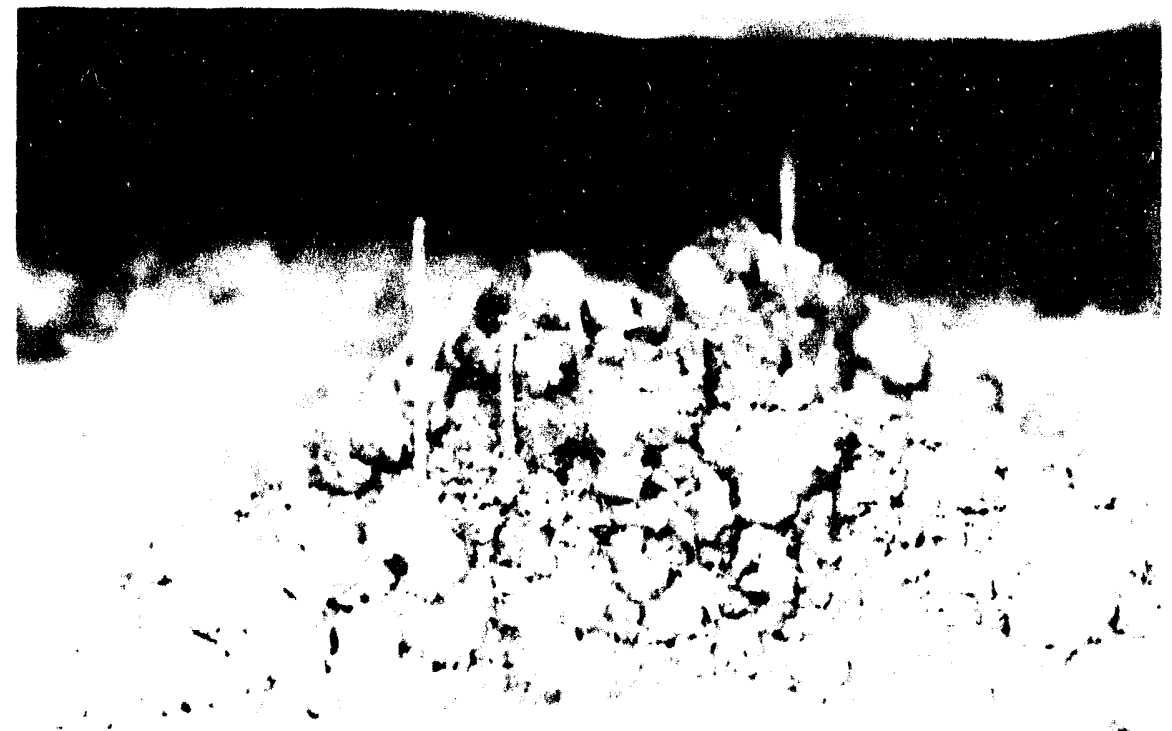

Fig. 3-42 Debris Bed on the Base of the Interaction Vessel for Experiment CCM- 4 


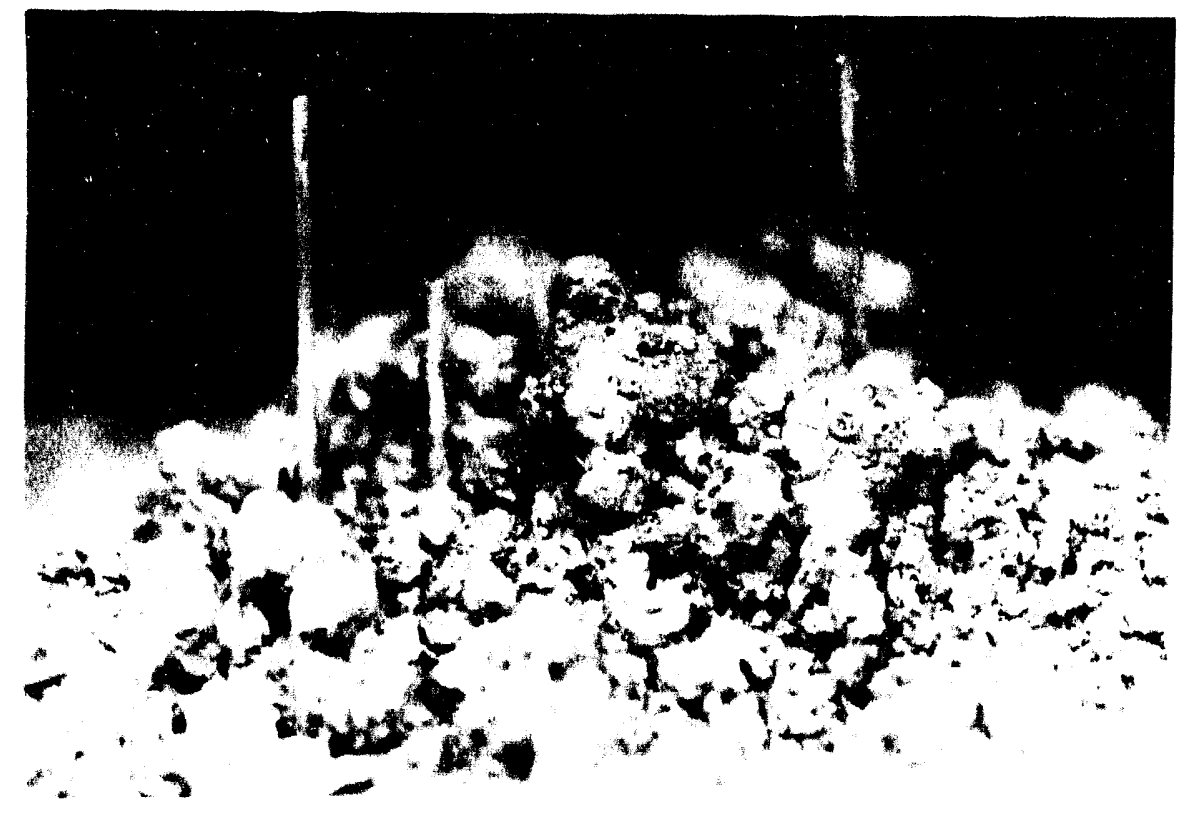

Fig. 3-43 Close-Up View of the Debris Bed on the Interaction-Vessel Base for Experiment CCM-1

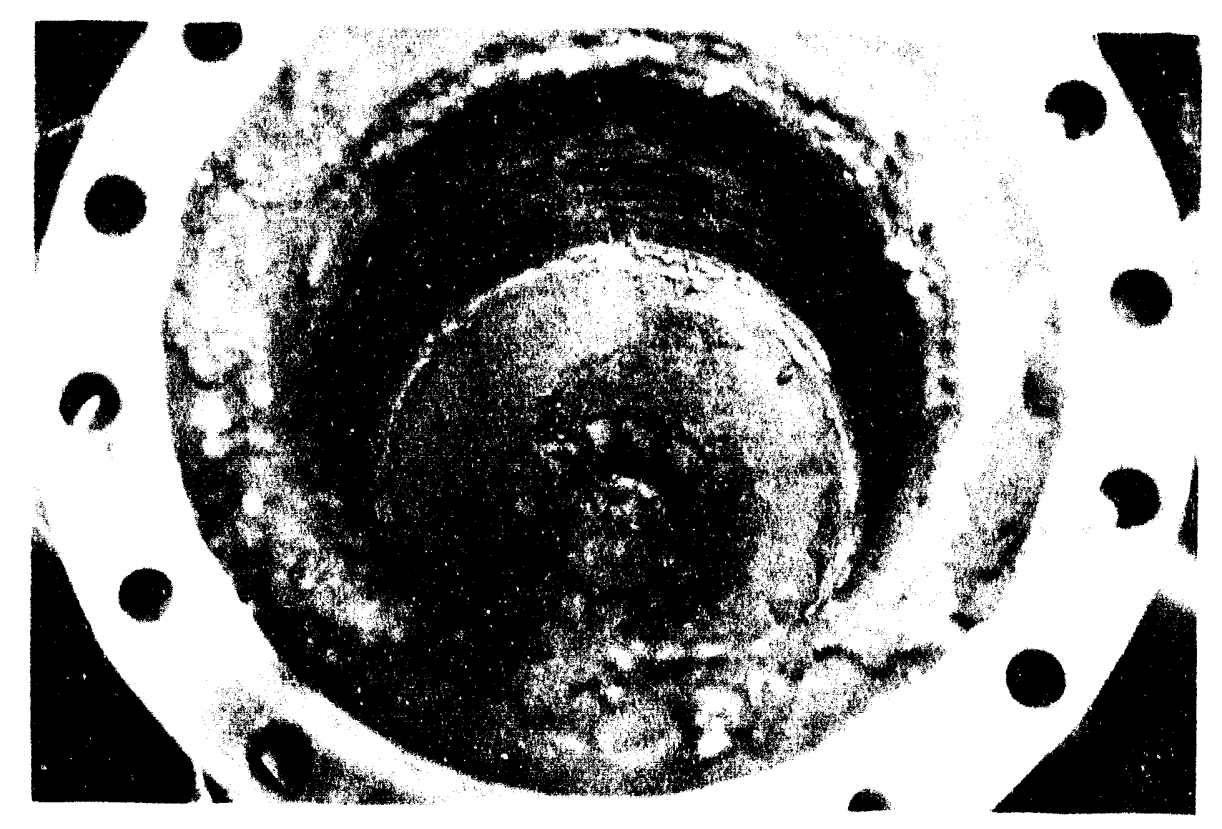

Fig. 3-44 View of the Inside of the Thermite Vessel Used in Experiment CCM-5 


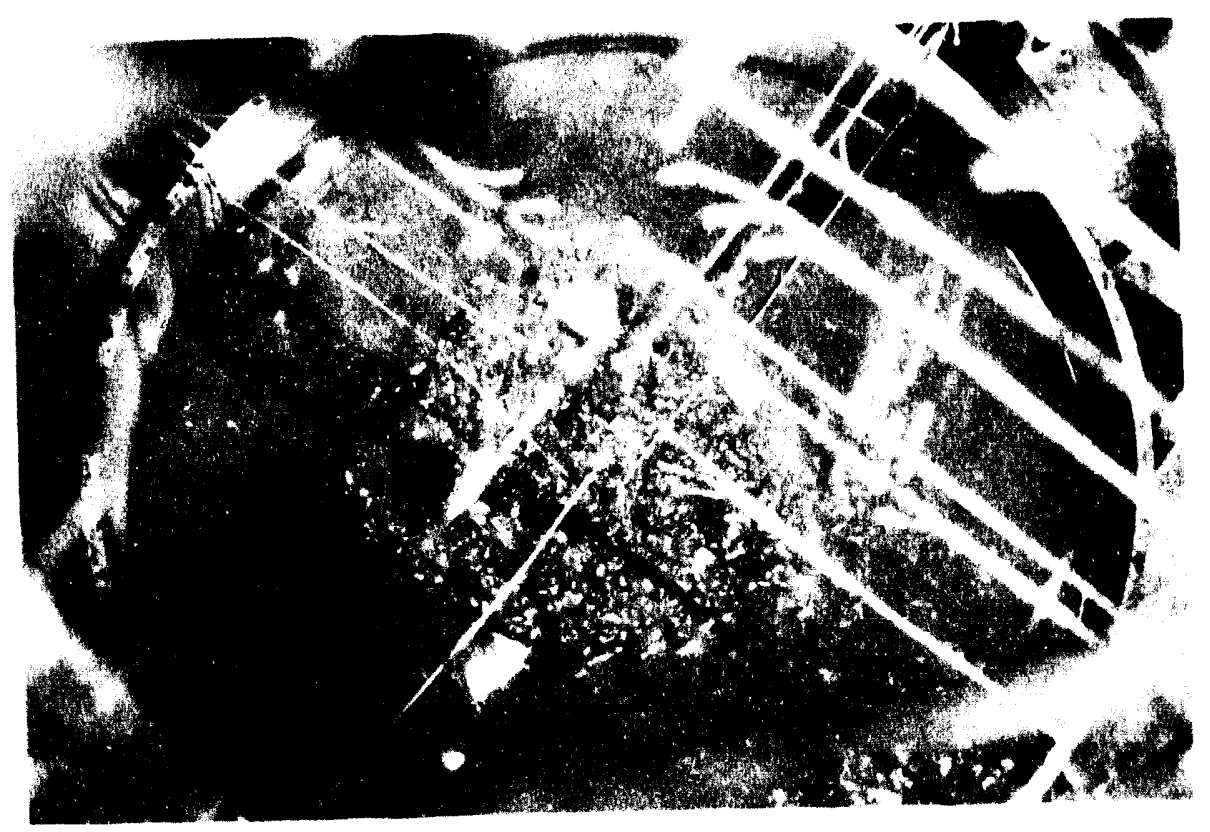

Fig. 3-45 Posttest View into the CCM-5 Experiment Vesse1
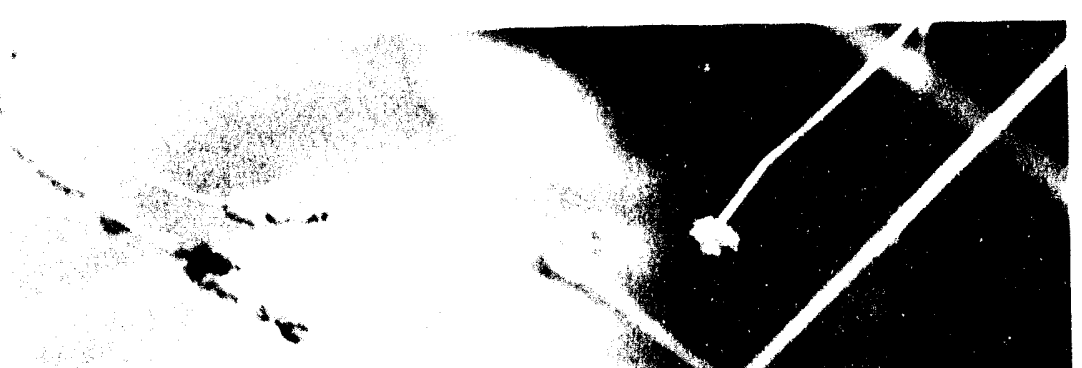

w

.

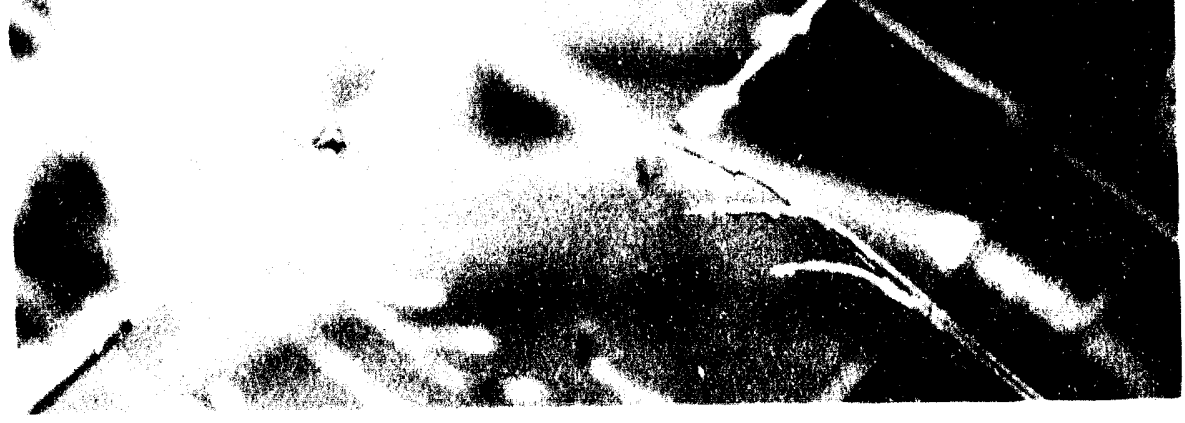

Fig. 3-46 Debris Deposited on the Thermocouples and Void Detectors in the CCM-5 Experiment Vesse1 
A view of the experiment vessel is shown in Fig. 3-45. Some debris adhered to the thermocouples and void detectors, Fig. 3-46, and some was found on the view ports and outside the catch pan on its support brackets. The debris was concentrated in the center of the pan (the central mass was approximately $250 \mathrm{~mm}$ in diameter and $42 \mathrm{~mm}$ thick), but was skewed toward one side. This material had a rough surface with particles of various shapes and sizes as shown in Figs. 3-47, 3-48, and 3-49. The particles were brittle, many were hollow, and some were sintered together. A cross-sectional view of the debris bed is shown in Fig. 3-50. Some small globules of stainless steel were actached to the pan bottom. A large agglomerated mass is shown in Fig. 3-51. Hollow particles are shown in Fig. 3-52. The measured density of a sample of the debris was 5800 $\mathrm{kg} / \mathrm{m}^{3}, 66.6 \%$ of solid corium density. Another debris sample with attached metal globules had a measured density of $6820 \mathrm{~kg} / \mathrm{m}^{3}$.

Ninety-four percent of the debris recovered from the experiment vessel was found outside the catch pan. A view of the bottom of the experiment vessel is given in Fig. 3-53. The debris in this region consisted of fine powder, coarse particles, and pieces of scale.

\subsubsection{Test CCM-6}

When the thermite vessel was disassembled, its interior condition was found to be similar to that observed in experiment CCM-5. There was an olive-colored powder on the underside of the vessel head and on the vapor separator. A small amount of particulate was found inside the vapor separator. Most of the tungsten grid plate had been dissolved and about $21 \%$ of the stainless steel gas baffle had been melted. A thin layer of black-colored corium adhered to the sides of the mullite sleeve and part of the ceramic cone near the discharge hole. These surfaces were covered with an olive-colored powder. An X-ray diffraction analysis of material taken from the accumulator vent lines showed a spinel type pattern, i.e., $\mathrm{FeCr}_{2} \mathrm{O}_{4}$, with a very small amount of $\mathrm{UO}_{2}$. A SEM-EDS analysis indicated that $C r>F e>U$.

On the inside of the experiment vessel some corium debris was found on the ledges of the view ports, on the catch pan supports, and on the bottom of the vessel (Fig. 3-54). The mass of debris outside the pan was $21 \%$ of the total debris mass recovered from the vessel. This fraction is 2.4 times that found in this region in experiment CCM-5. As in the previous tests, corium adhered to some of the void detectors and thermocouples (Fig. 3-55).

The debris bed surface appearance, (Fig. 3-56), was similar to that of previous tests, except in CCM- 6 , most of the debris are orange-colored particles indicating a incich nigher extent of oxidation. This is due to the saturated water wiich serves as a stem-enriched environment for the oxication to occur. The bed depth is greatest, about $40 \mathrm{~mm}$, near the center. The bed liass distribution was skewed to one side. The bed cross section is shown in fig. 3-57. The measured density of a sample of the bed material was $5530 \mathrm{~kg} / \mathrm{m}^{3}, 63.5 \%$ of solid corium density.

\subsubsection{Particle Size Distribution}

The debris were sieved through 6350-, 3360-, and 1700-micron screens. A representative sample of the material whose particle size was less than 1700 microns was further sized with a sonic sifter. The sifter contained a stack of 

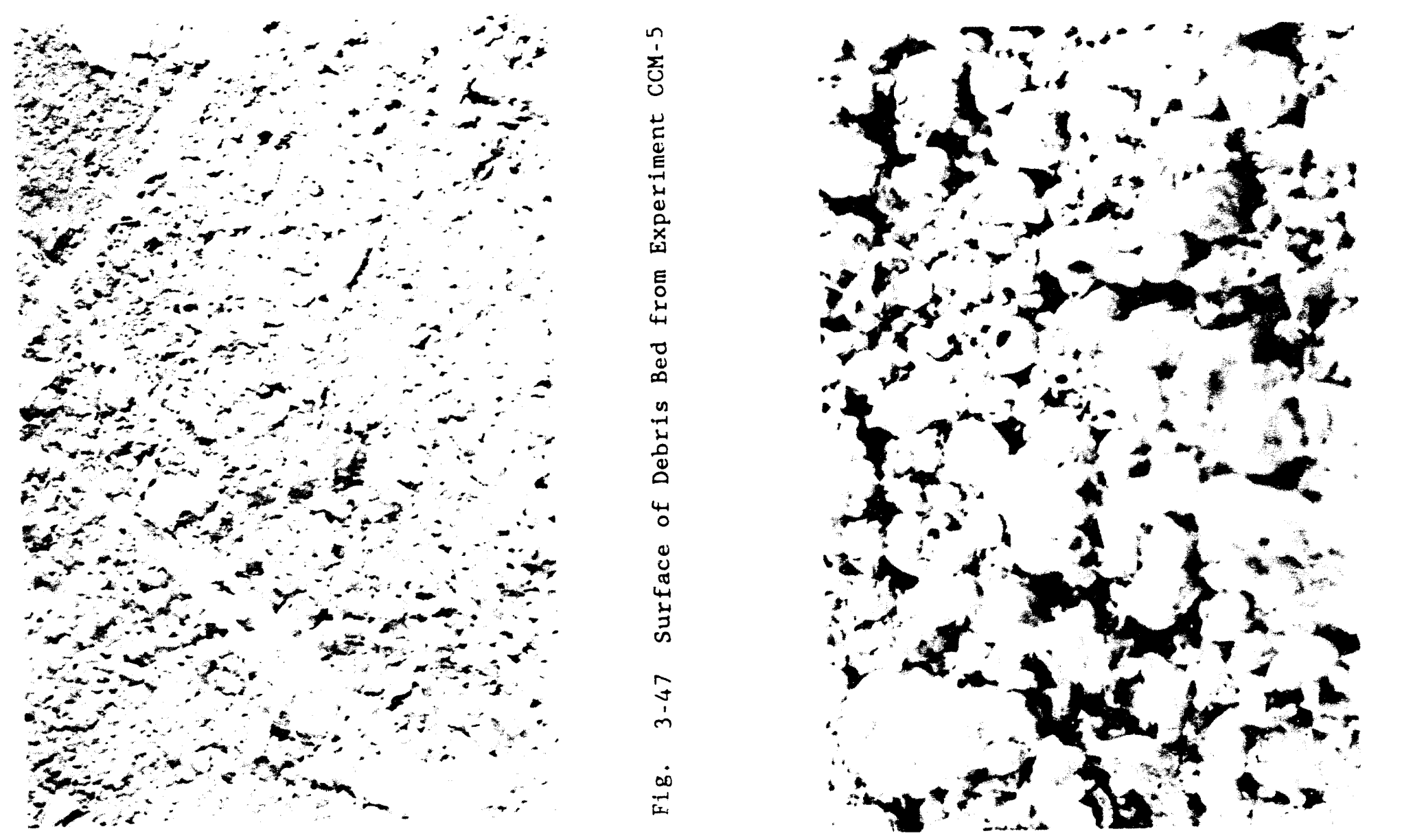


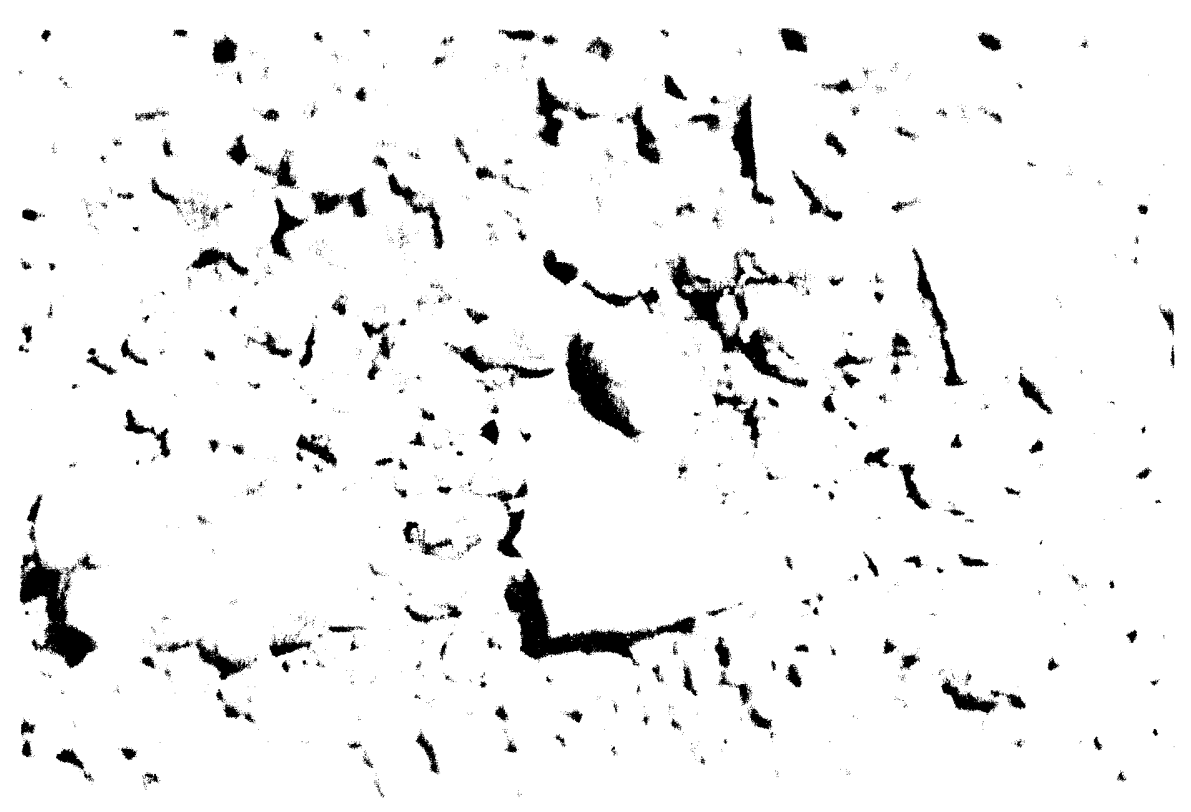

Fig. 3-49 Close-Up View of Debris Surface with Scale-1ike Particles for Experiment CCM-5

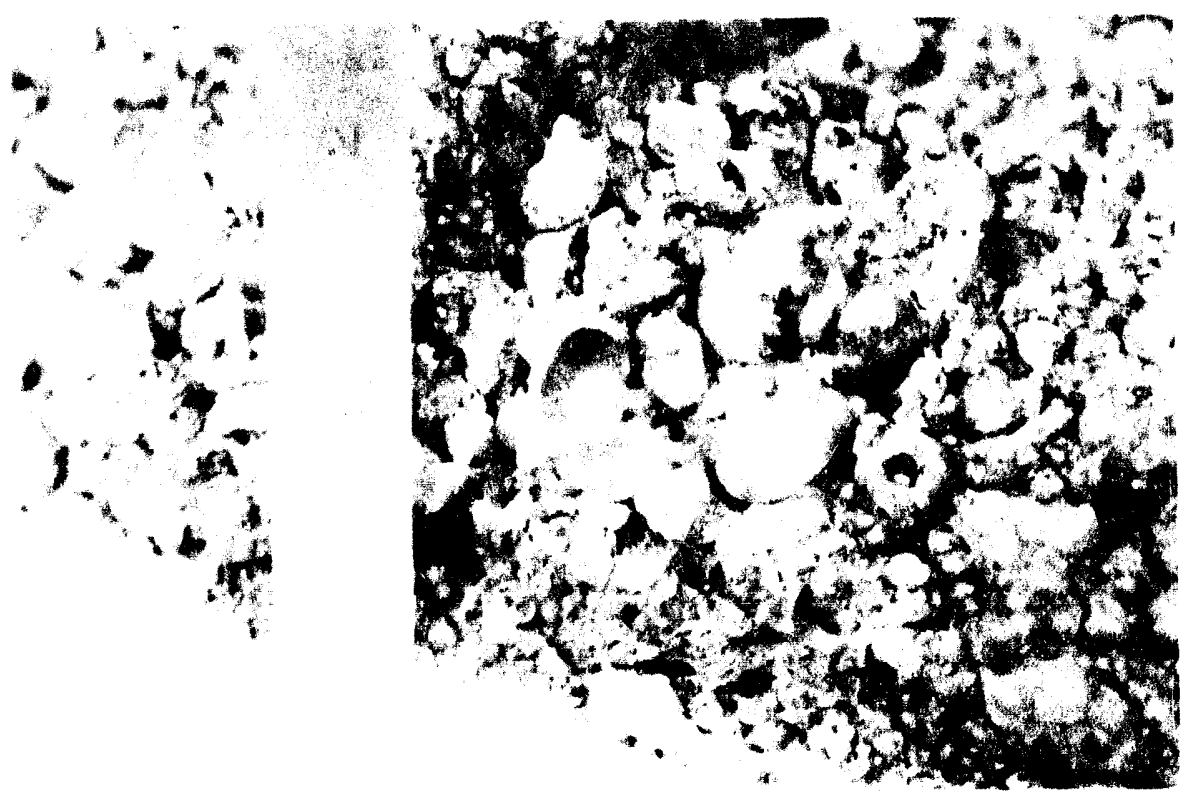

Fig. 3-50 Sectioned View of Debris Bed from Experiment CCM-5 


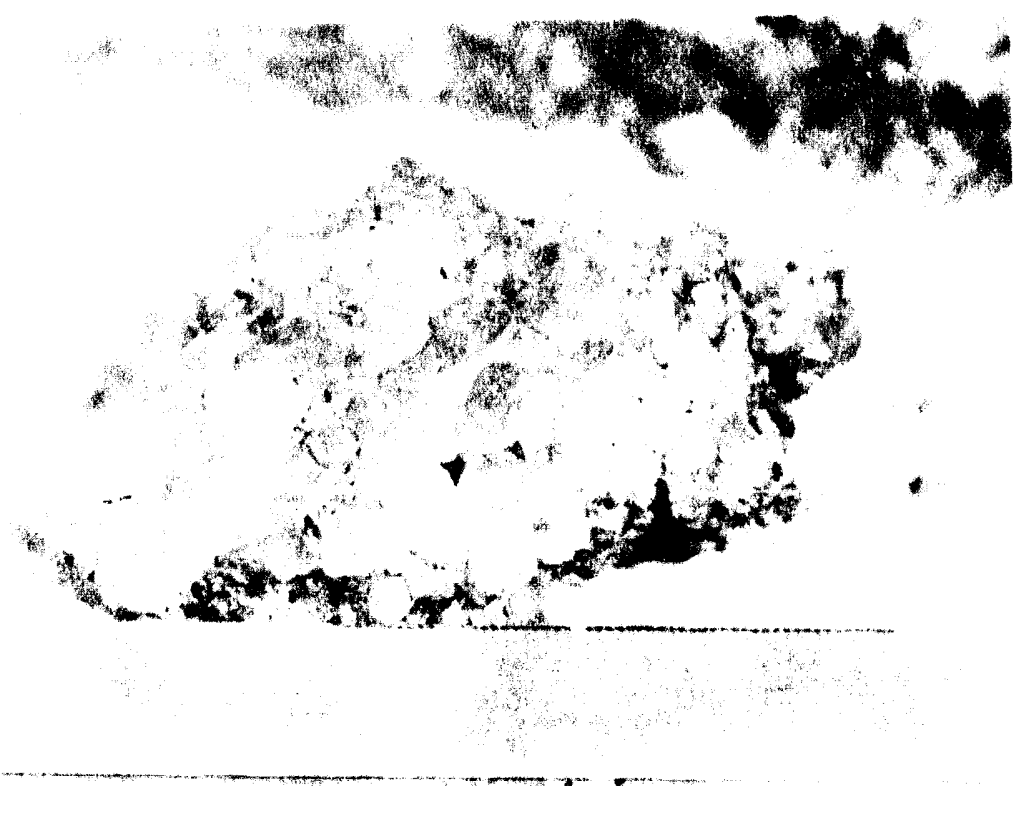

Fig. 3-51 Agglomerated Particle from the Debris Bed for Experiment CCM-5

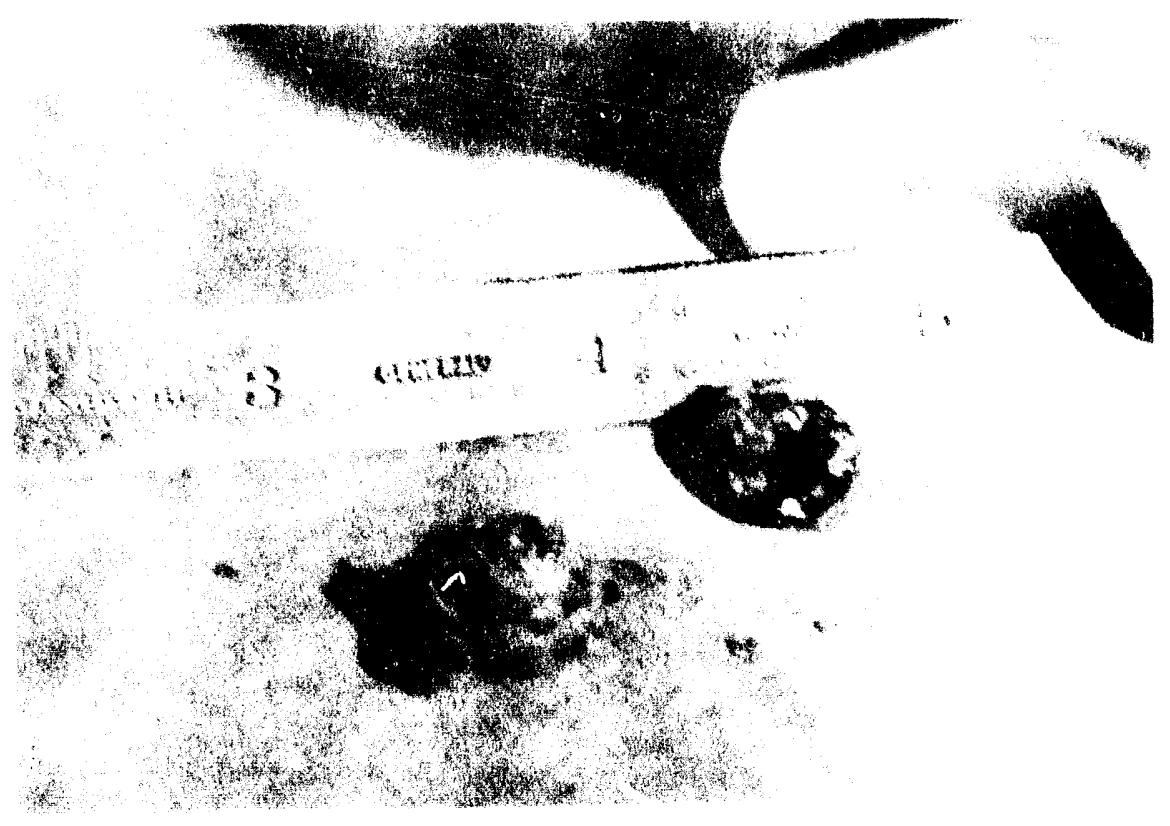

Fig. 3-52 Hollow Particles from the Debris Bed for Experiment CCM-5 


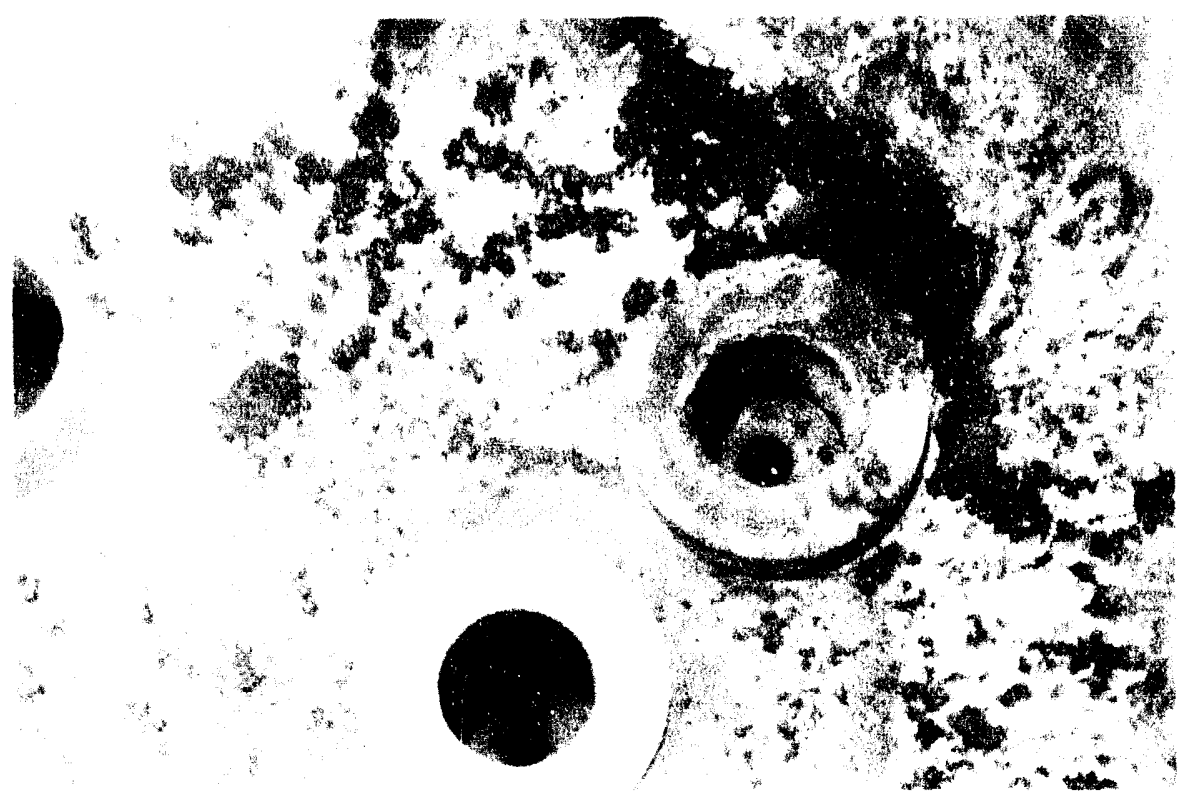

Fig. 3-53 View of the Experiment-Vessel Bottom for Experiment CCM-5

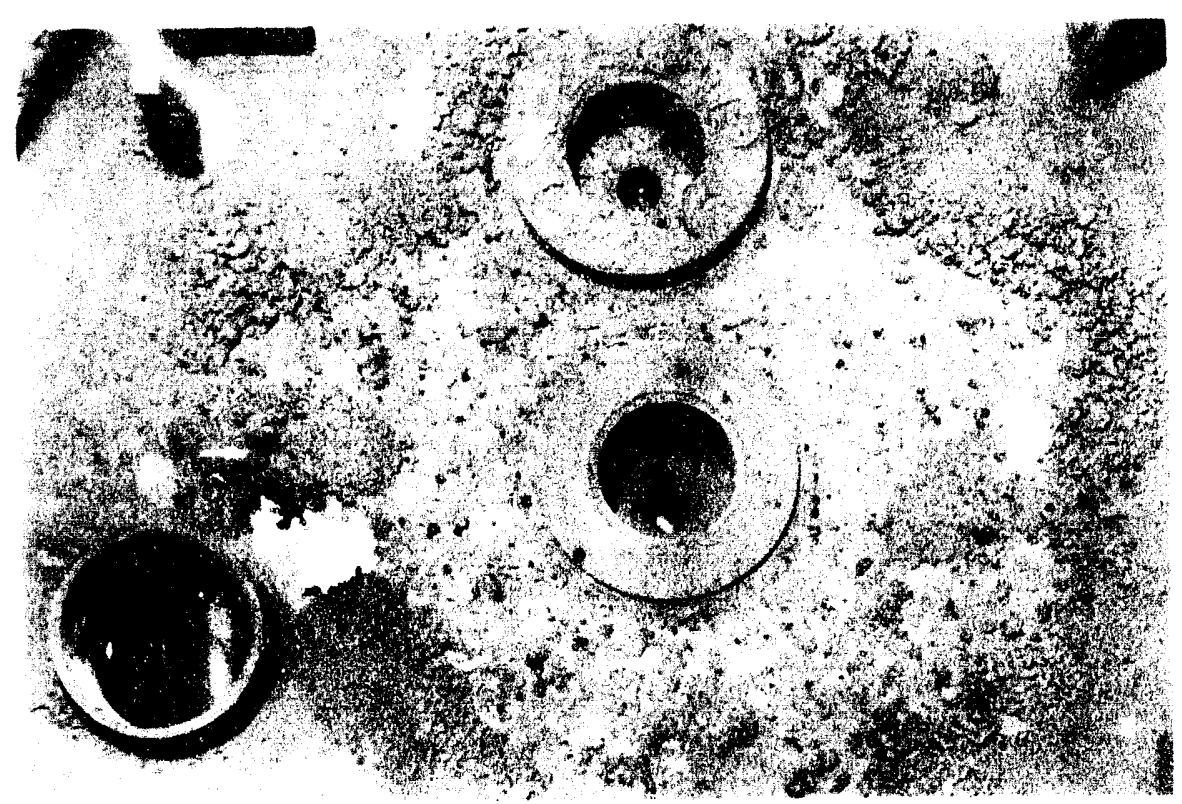

Fig. 3-54 View of the Experiment-Vessel Bottom for Experiment CCM- 6 


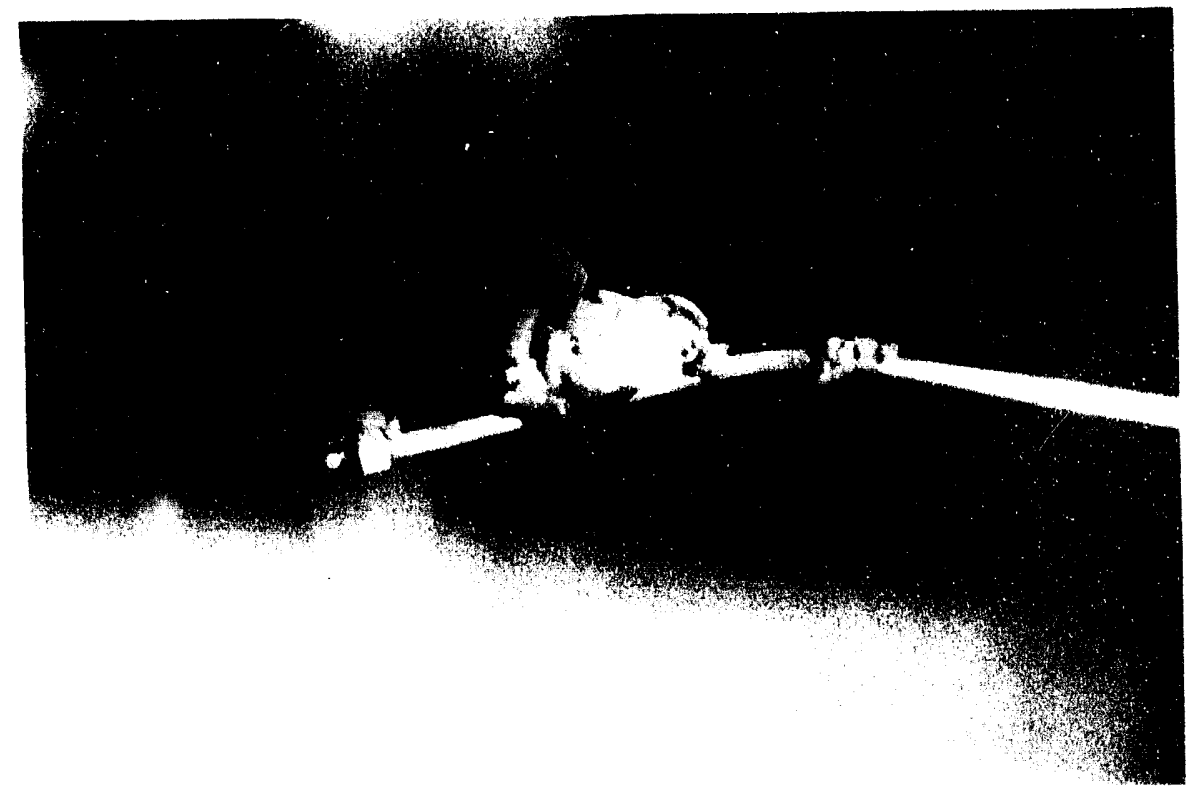

Fig. 3-55 Debris on A Thermocouple in Experiment CCM-6

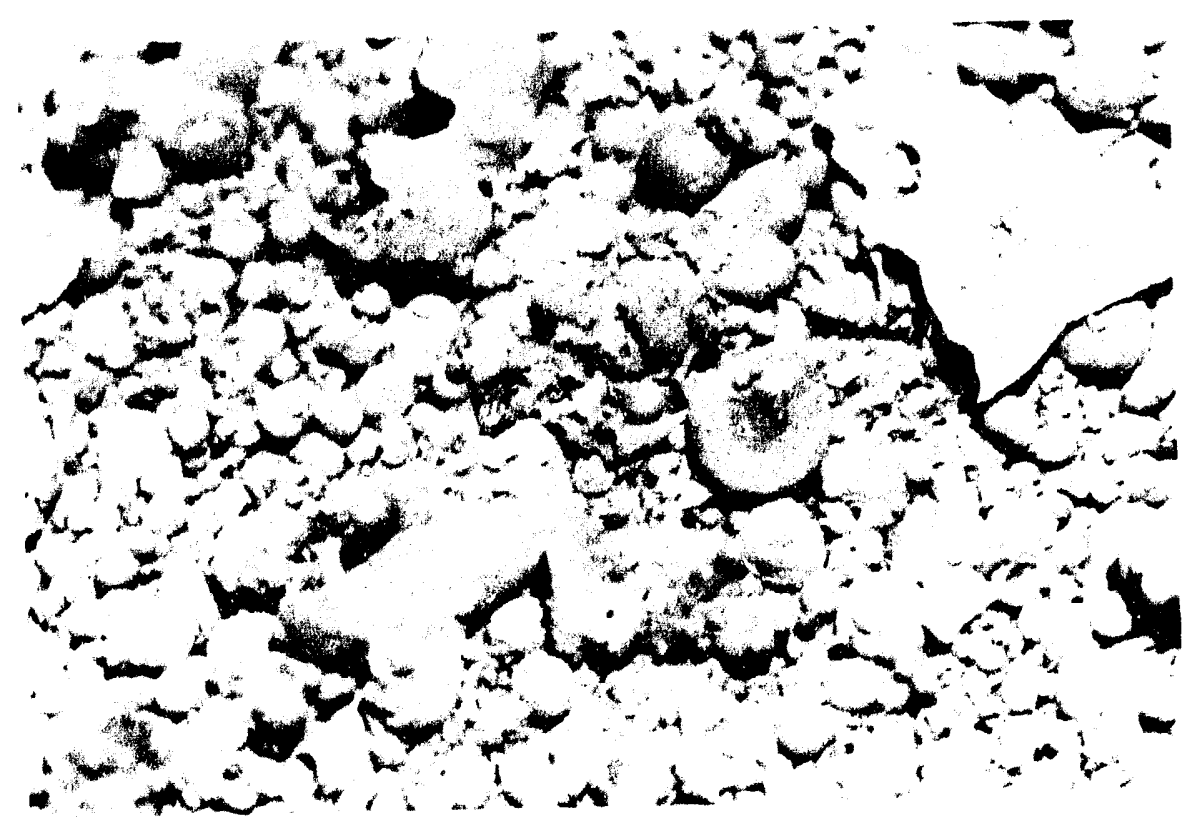

Fig. 3-56 Surface of the Debris Bed in Experiment CCM-6 


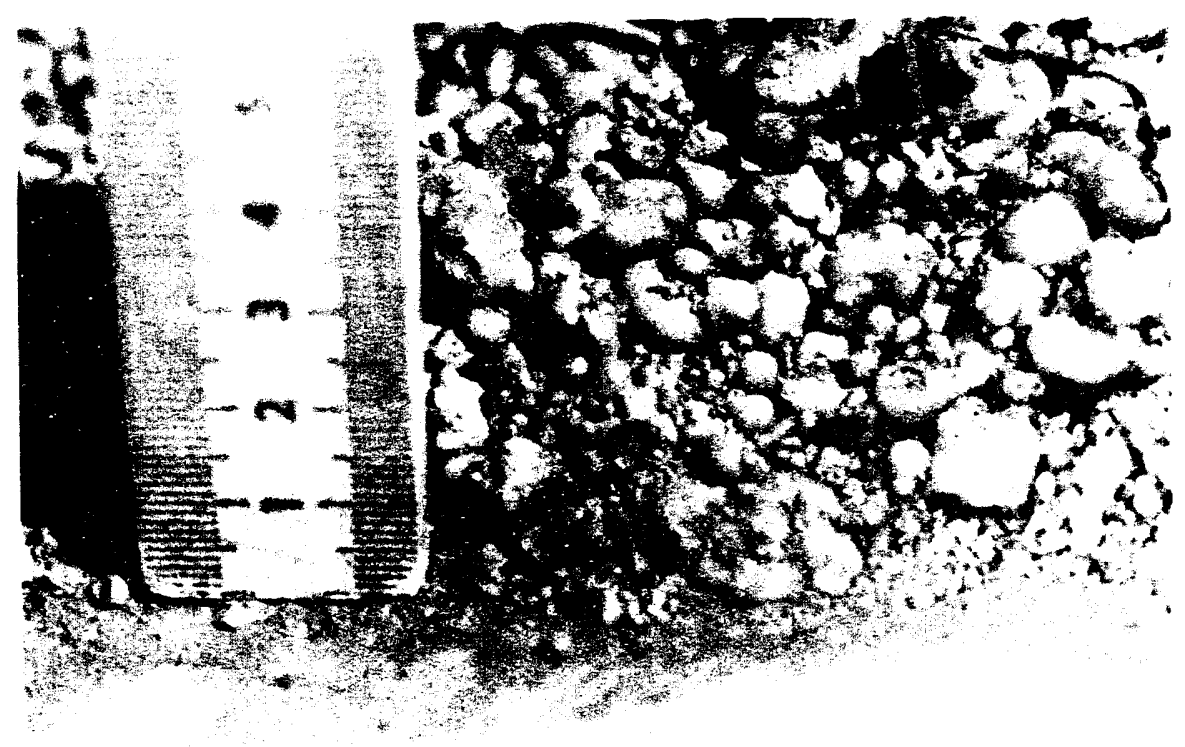

Fig. 3-57 Sectioned View of the Debris Bed for Experiment CCM-6 
six screens whose openings ranged from 53 to 841 microns. For CCM-2, the debris from the interaction vessel base was largely agglomerated, and sieving was not feasible. The particle size distribution of the debris from each test is plotted on a logarithmic probability graph in Fig. 3-58.

The log-normal distribution has the following functional form:

$$
f(d)=\frac{N}{\sqrt{2} \pi} \frac{1}{\beta} \frac{1}{d} \exp \left\{-\frac{1}{2 \beta^{2}}[\ln (d)-\alpha]^{2}\right\}
$$

where:

$$
\begin{aligned}
& N \quad=\text { the mass of particles, } \\
& d \quad=\text { the particle diameter, } \\
& f(d)=\text { the mass distribution function (i.e., mass per size range), } \\
& \alpha, \beta=\text { constants. }
\end{aligned}
$$

The log normal distribution plots as a straight line on a log probability graph. The constants $\alpha$ and $\beta$ can be obtained graphically from such a plot. The parameter $\alpha$ is related to median diameter, i.e.,

$$
e^{\alpha}=50 \% \text { size }
$$

The parameter $\beta$ characterizes the slope of the log-normal distribution, i.e.,

$$
e^{\beta}=\frac{84.1 \% \text { size }}{50 \% \text { size }}=\frac{50 \% \text { size }}{15.8 \% \text { size }}
$$

The parameter e $\beta$ can be interpreted as the standard deviation, $\alpha$, of the particle diameter. In other words, $68 \%$ of the total debris mass is composed of particles of sizes ranging from $\bar{d} / \sigma$ to $\sigma \bar{d}$, where $\bar{d}$ is the median diameter.

As shown in Fig. 3-58, the debris from each test showed a log-normal size distribution. The mass median particle size ranges from $\sim 1$ to $5 \mathrm{~mm}$.

\subsection{Gas Sample Aanlys is}

For each test, a sample of the pretest atmosphere and three samples of the posttest atmosphere were taken from the expansion/experiment vessel. The expansion/experiment vessel was vented through a small opening at the top of the vesse 1; the pretest gas sample was taken from a port near the middle of the vesse1, and so should have been representative of the system atmosphere.

Gas samples were analyzed by mass spectrometry in the Analytical Chemistry Lab at ANL. The results of the gas analyses are given in Table 3.27. The samples were analyzed several days after the tests, and so had cooled to room 


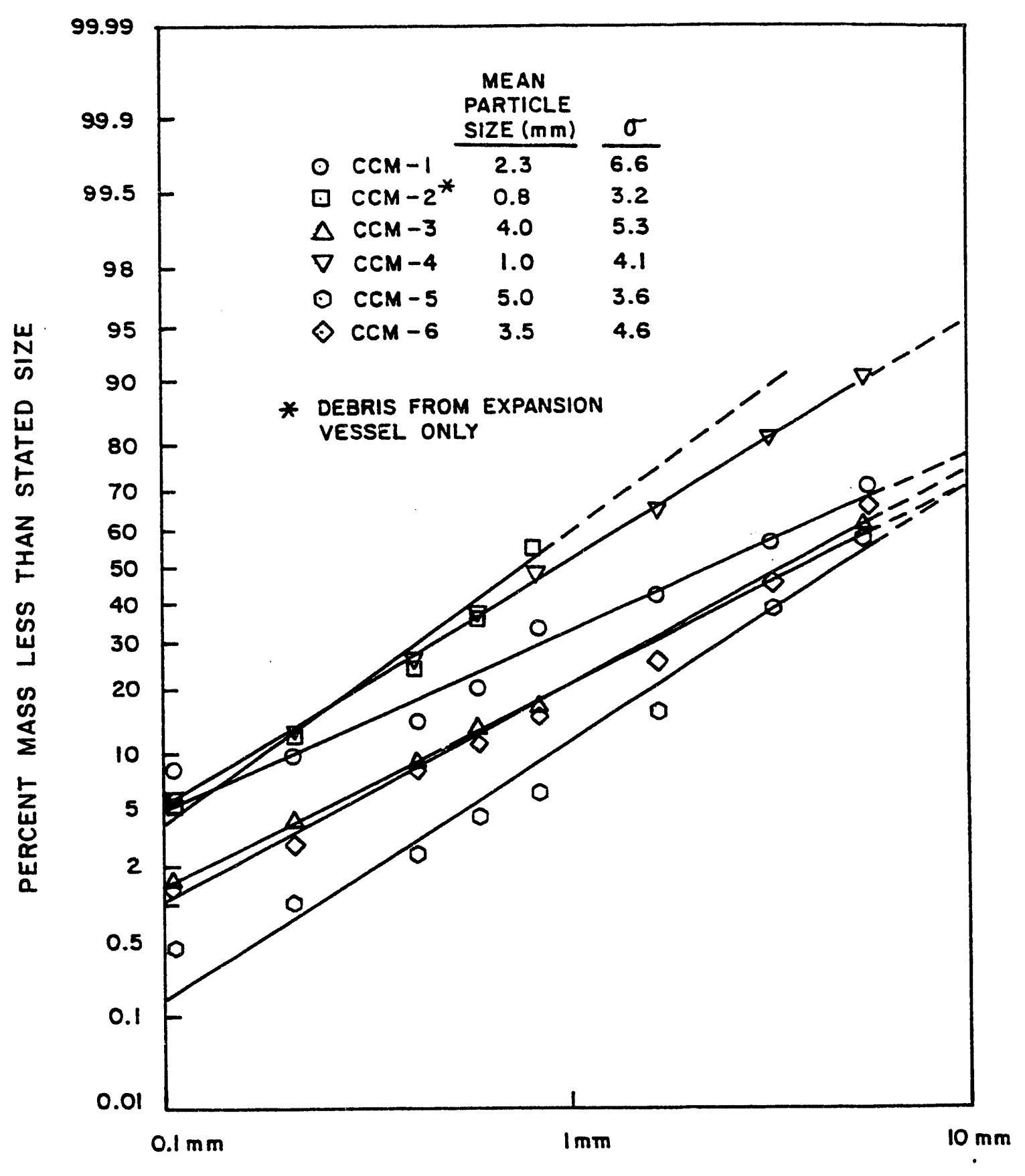

SIEVE OPENING SIZE, $\mathrm{mm}$

Fig. 3-58 Particle-Size Distribution of Debris 
BLANK PAGE 
Table 3.27 Gas Analyses

Volume or Mole Fraction

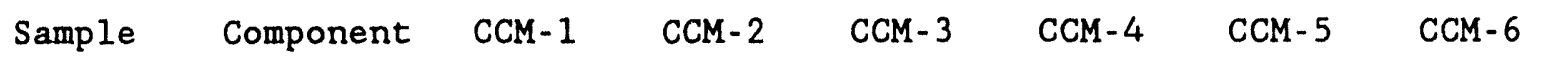

\begin{tabular}{|c|c|c|c|c|c|c|c|}
\hline \multirow[t]{7}{*}{ Pretest } & $\mathrm{H}_{2}$ & 0.0002 & 0.0020 & 0.0000 & -- & 0.0000 & 0.0000 \\
\hline & $\mathrm{H}_{2} \mathrm{O}$ & 0.0004 & 0.0050 & 0.0008 & -- & 0.0005 & 0.0289 \\
\hline & $\mathrm{N}_{2}$ & 0.7818 & 0.7830 & 0.7904 & 0.0370 & 0.0695 & 0.1918 \\
\hline & $\mathrm{O}_{2}$ & 0.2080 & 0.1990 & 0.1987 & 0.0130 & 0.0121 & 0.0000 \\
\hline & $\mathrm{Ar}$ & 0.0090 & 0.0108 & 0.0099 & 0.9500 & 0.9169 & 0.7578 \\
\hline & $\mathrm{CO}_{2}$ & 0.0006 & $\underline{0.0002}$ & 0.0002 & $\cdots$ & $\underline{0.0010}$ & $\underline{0.0216}$ \\
\hline & & 1.0000 & 1.0000 & 1.0000 & 1.0000 & 1.0000 & 1.0001 \\
\hline Posttest & $\mathrm{H}_{2}$ & 0.0024 & 0.6220 & 0.7060 & 0.0100 & 0.5050 & 0.9050 \\
\hline \multirow[t]{8}{*}{ Upper } & $\mathrm{H}_{2} \mathrm{O}$ & 0.0006 & 0.0390 & 0.0007 & 0.2000 & 0.0030 & 0.0160 \\
\hline & $\mathrm{N}_{2}$ & 0.7900 & 0.2660 & 0.2290 & 0.0300 & 0.0390 & 0.0110 \\
\hline & $\mathrm{O}_{2}$ & 0.1970 & 0.0680 & 0.0510 & 0.0100 & 0.0002 & 0.0000 \\
\hline & Ar & 0.0100 & 0.0043 & 0.0064 & 0.750 & 0.4410 & 0.0490 \\
\hline & $\mathrm{CO}_{2}$ & 0.0008 & 0.0005 & 0.0009 & -- & 0.0024 & 0.0163 \\
\hline & $\mathrm{CH}_{4}$ & -- & 0.0009 & - & $\cdots$ & 0.0003 & 0.0003 \\
\hline & CO & $\cdots$ & $\cdots$ & $\underline{0.0060}$ & $\ldots$ & $\underline{0.0090}$ & 0.0030 \\
\hline & & 1.0008 & 1.0007 & 1.0000 & 2.0000 & 0.9999 & 1.0006 \\
\hline Posttest & $\mathrm{H}_{2}$ & 0.0047 & 0.6000 & 0.6950 & & 0.5000 & 0.9040 \\
\hline \multirow[t]{8}{*}{ Middle } & $\mathrm{H}_{2} \mathrm{O}$ & 0.0017 & 0.0760 & 0.0009 & & 0.0050 & 0.0220 \\
\hline & $\mathrm{N}_{2}$ & 0.8050 & 0.2780 & 0.2300 & & 0.0370 & 0.0110 \\
\hline & $\mathrm{O}_{2}$ & 0.1780 & 0.0400 & 0.0510 & NA & 0.0082 & 0.0000 \\
\hline & Ar & 0.0100 & 0.0046 & 0.0062 & & 0.4380 & 0.0470 \\
\hline & $\mathrm{CO}_{2}$ & 0.0005 & 0.0005 & 0.0014 & & 0.0024 & 0.0135 \\
\hline & $\mathrm{CH}_{4}$ & $\cdots$ & 0.0011 & -- & & 0.0002 & 0.0002 \\
\hline & CO & $\ldots$ & - & $\underline{0.0150}$ & $\longrightarrow$ & $\underline{0.0090}$ & 0.0030 \\
\hline & & 0.9999 & 1.0002 & 0.9995 & & 0.9998 & 1.0007 \\
\hline
\end{tabular}


Table 3.27 Gas Analyses (cont.)

Volume or Mole Fraction

Sample Component CCM-1 CCM-2 CCM-3 CCM-4 CCM-5 $\quad$ CCM-6

\begin{tabular}{|c|c|c|c|c|c|c|c|}
\hline Posttest & $\mathrm{H}_{2}$ & 0.0044 & 0.6240 & 0.7080 & & 0.5060 & 0.8860 \\
\hline \multirow[t]{8}{*}{ Lower } & $\mathrm{H}_{2} \mathrm{O}$ & 0.0002 & 0.0580 & 0.0003 & & 0.0030 & 0.0250 \\
\hline & $\mathrm{N}_{2}$ & 0.7940 & 0.2500 & 0.2150 & & 0.0380 & 0.0110 \\
\hline & $\mathrm{O}_{2}$ & 0.1910 & 0.0620 & 0.0530 & NA & 0.0094 & 0.0000 \\
\hline & Ar & 0.0100 & 0.0039 & 0.0063 & & 0.4310 & 0.0470 \\
\hline & $\mathrm{CO}_{2}$ & 0.0008 & 0.0005 & 0.0019 & & 0.0025 & 0.0280 \\
\hline & $\mathrm{CH}_{4}$ & $\cdots$ & 0.0014 & $\cdots$ & & 0.0002 & 0.0002 \\
\hline & $\mathrm{CO}$ & $\cdots$ & $\ldots$ & $\underline{0.0160}$ & & $\underline{0.0100}$ & $\underline{0.0030}$ \\
\hline & & 1.0004 & 0.9998 & 1.0005 & & 1.0001 & 1.0002 \\
\hline Posttest & $\mathrm{H}_{2}$ & 0.0038 & 0.6151 & 0.7030 & 0.0100 & 0.5037 & 0.8979 \\
\hline \multirow[t]{8}{*}{ Mean } & $\mathrm{H}_{2} \mathrm{O}$ & 0.0008 & 0.0577 & 0.0006 & 0.2000 & 0.0037 & 0.0210 \\
\hline & $\mathrm{N}_{2}$ & 0.7961 & 0.2646 & 0.2247 & 0.0300 & 0.0380 & 0.0110 \\
\hline & $\mathrm{O}_{2}$ & 0.1886 & 0.0567 & 0.0517 & 0.0100 & 0.0059 & 0.0000 \\
\hline & Ar & 0.0100 & 0.0043 & 0.0063 & 0.7500 & 0.4367 & 0.0476 \\
\hline & $\mathrm{CO}_{2}$ & 0.0007 & 0.0005 & 0.0014 & $\cdots$ & 0.0024 & 0.0193 \\
\hline & $\mathrm{CH}_{4}$ & $\cdots$ & 0.0011 & - & $\cdots$ & 0.0002 & 0.0002 \\
\hline & $\mathrm{CO}$ & - & 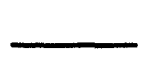 & 0.0123 & $\cdots$ & $\underline{0.0093}$ & $\underline{0.0030}$ \\
\hline & & 1.0000 & 1.0000 & 1.0000 & 1.0000 & 0.9999 & 1.0000 \\
\hline
\end{tabular}


temperature. If water vapor in a sample has condensed, the mole fraction measured will be that of a saturated atmosphere. Therefore, the water data from mass spectrometry may not accurately represent lest conditions. However, the mole fraction of steam present at the time of posttest sampling, and by inferring the mass of steam generated during the test, may be found from PVT data.

Assuming that the gases and vapor are in thermal equilibrium, and that their mixture behaves as an ideal gas when the temperature is above the dew point, the number of g-moles present can be estimated from PVT data using the following equation:

$n=\frac{P V}{R T}$

where

$$
\begin{aligned}
& n=\text { number of gram moles, } \\
& P=\text { system pressure, MPa, } \\
& R=\text { gas constant } 8.314\left(10^{-6}\right) \frac{m^{3} \mathrm{MPa}}{g-\text { mole } \mathrm{K}} \\
& T=\text { system temperature, } \mathrm{K}, \\
& V=\text { system volume, } \mathrm{m}^{3} .
\end{aligned}
$$

In experiments CCM-1 through 4, the pretest system volume was $1.377 \mathrm{~m}^{3}$ for the expansion vessel and $0.012 \mathrm{~m}^{3}$ for the pipeway. The volume in the interaction vessel gas region was about $0.012 \mathrm{~m}^{3}$; it varied slightly for each of these tests because of the different elevation of the water surface. The temperatures and volumes of each of these regions were used to determine the number of moles in the system. For experiment CCM-5, the free volume in the gas/vapor space of the experiment vessel was $0.462 \mathrm{~m}^{3}$. In experiment $\mathrm{CCM}-6$, it was $1.15 \mathrm{~m}^{3}$. Table 3.28 contains the pretest atmosphere data; the pressure and temperature values are means.

Since the mass of inert gases in the system remains constant throughout the test, the inert gas fraction obtained from the pretest and posttest gas analyses may be compared to determine the number of moles in the system at the time of posttest sampling.

When the total number of moles present is known for a given time, the number of moles of steam present at that time can be found by taking pressure and temperature measurements after the system has cooled, i.e., after the steam has condensed. Using these data in Eq. 3.6, the number of moles of noncondensible gases present after a test can be determined. The number of moles of steam present at posttest sampling is simply the total number of moles (from inert gas fraction data) less the number of moles of noncondensibles.

However, two complicating factors should be mentioned. If the mass of steam generated is 1arge enough to saturate the gas, condensation could occur. In addition, condensation could occur along the walls of the vessel while the central region of the vessel contained gas and vapor at a temperature above its 
Table 3.28. Gas/Vapor Conditions Before, During, and After the Experiment CCM-1 CCM-2 CCM-3 CCM-4 CCM-5 CCM-6

Pretest

\begin{tabular}{|c|c|c|c|c|c|c|}
\hline $\begin{array}{l}\mathrm{P}, \mathrm{MPa} \\
\mathrm{T}, \mathrm{K} \\
\mathrm{n}, \mathrm{g} \text {-mole }\end{array}$ & $\begin{array}{c}0.1 \\
411.6 \\
40.843\end{array}$ & $\begin{array}{c}0.1 \\
408.9 \\
41.222\end{array}$ & $\begin{array}{c}0.1 \\
406.0 \\
41.529\end{array}$ & $\begin{array}{l}0.096 \\
408.6 \\
39.312\end{array}$ & $\begin{array}{c}0.094 \\
368.6 \\
14.588\end{array}$ & $\begin{array}{c}0.114 \\
403.8 \\
39.052\end{array}$ \\
\hline
\end{tabular}

Maximum Pressure

$\begin{array}{lcccccc}t, s & 5.0 & 250.0 & 15.0 & 15.0 & 1.4 & 1.3 \\ \mathrm{P}, \mathrm{MPa} & 0.135 & 0.364 & 0.245 & 0.303 & 0.246 & 0.354 \\ \mathrm{~T}, \mathrm{~K} & 440.2 & 410.9 & 398.6 & 389.3 & 389.6 & 417.4 \\ \mathrm{n}, \mathrm{g} \text {-moles } & 51.680 & 149.937 & 103.749 & 131.835 & 35.058 & 117.315\end{array}$

End of Test $(t-250 \mathrm{~s})$
$P, \mathrm{MPa}$
$T, K$
n, g-mole

\begin{tabular}{cccccc}
0.118 & \multicolumn{1}{c}{0.364} & 0.216 & 0.270 & 0.182 & 0.221 \\
414.0 & 410.9 & 397.6 & 394.5 & 384.3 & 403.9 \\
48.134 & 149.937 & 91.699 & 115.279 & 26.295 & 75.687
\end{tabular}

End of Day

P, MPa

$T, K$

n, g-mole

Next Day
$\mathbf{P}, \mathbf{M P a}$
$T, K$
n, g-mole

$\begin{array}{cccccc}0.124 & 0.221 & 0.130 & 0.219 & 0.162 & \cdots \\ 410.1 & 393.6 & 386.1 & 388.9 & 385.1 & \cdots \\ 51.075 & 95.034 & 56.833 & 95.384 & 23.351 & \cdots\end{array}$

$\begin{array}{cccccc}0.086 & 0.088 & 0.101 & 0.087 & 0.103 & 0.064 \\ 306.9 & 312.4 & 308.3 & 310.4 & 343.0 & 311.3 \\ 47.873 & 47.678 & 55.297 & 47.476 & 16.673 & 28.438\end{array}$


dew point. Therefore, the mass of steam present at posttest sampling and the total mass of steam generated during a test may not be strictly equivalent. However, for this analysis, equivalence is assumed.

Finally, for the posttest and post-condensation data to be used directly for determining the mass of steam generated, the system has to be leak tight. Although a leak check for positive pressure was performed before each test, the system may leak under vacuum. Calculations have shown that the post-condensation pressure should have been lower than that observed, indicating air in-leakage. This has been taken into account in the estimates of the pre- and posttest gas composition presented in Table 3.29. 
Table 3.29 Adjusted Pre- and Posttest Gas/Vapor Composition

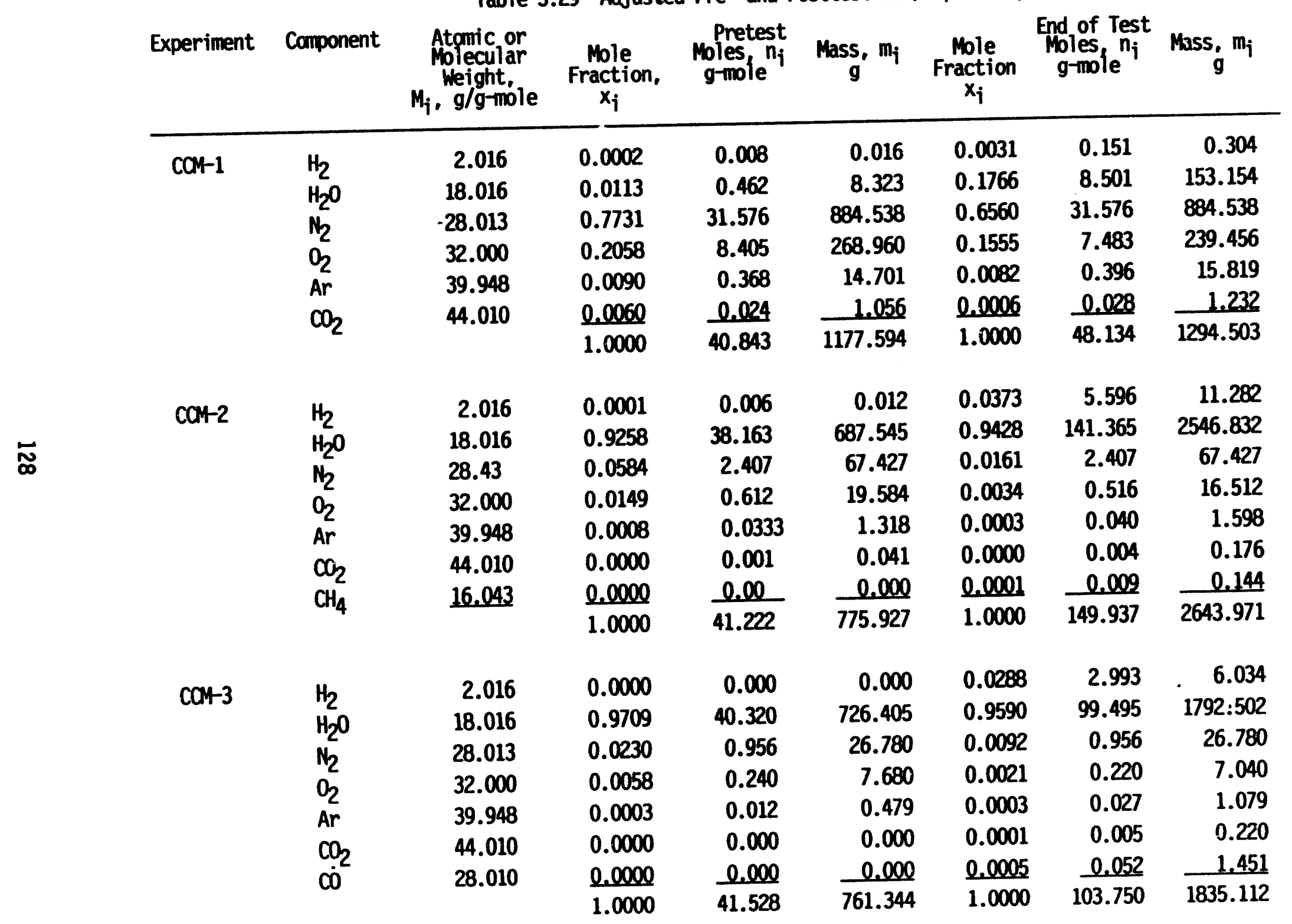


Table 3.29 Adjusted Pre- and Posttest Gas,'Vapor Composition (cont.)

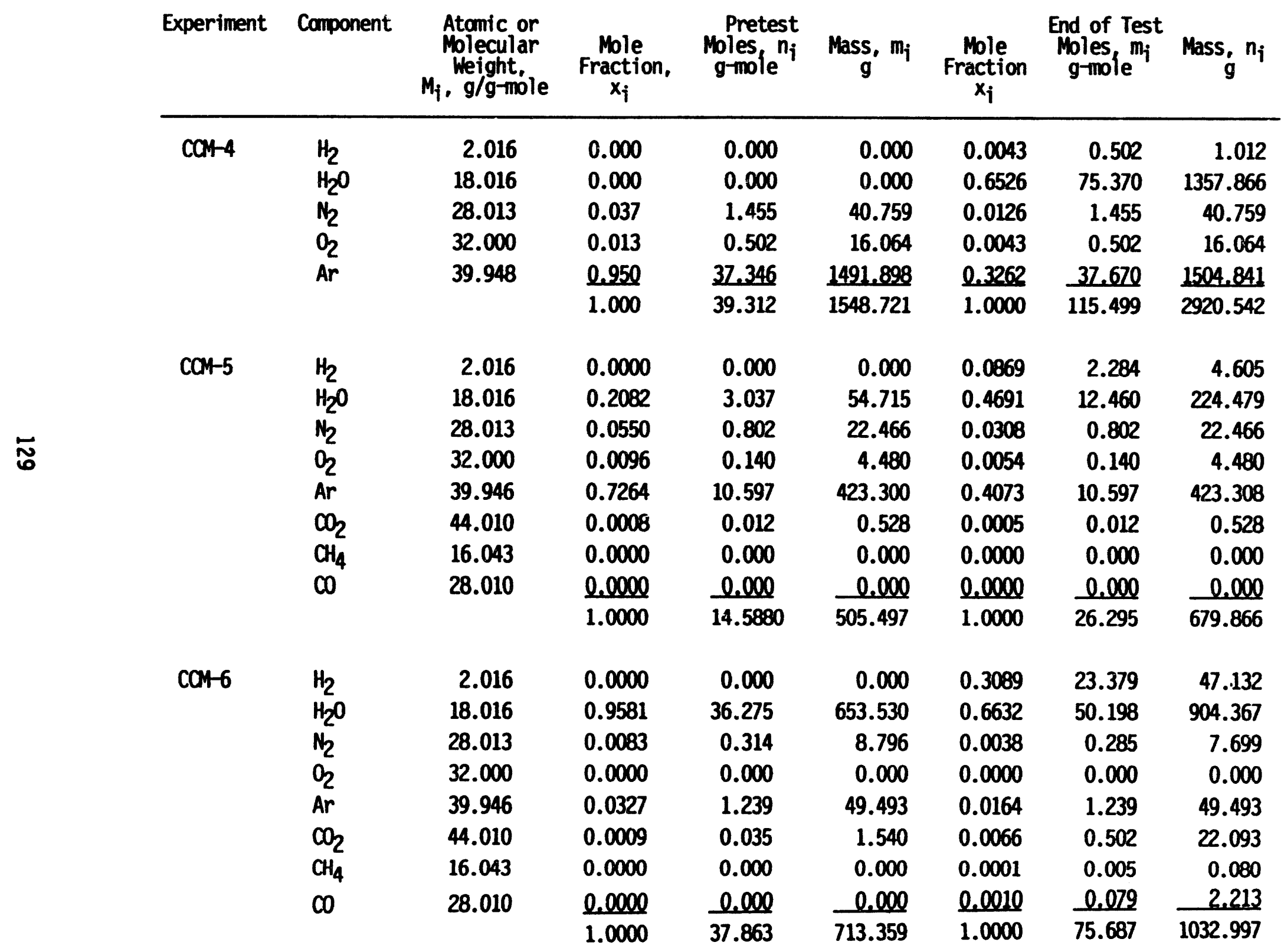




\subsection{EYALUATION OF TEST RESULTS}

\subsection{Hydrogen Generation/Metal Oxidation}

When the metallic constituents of the stainless steel in the corium contact water or sterm, oxidation occurs and hydrogen is liberated. Based on thermodynamic limitations of the stainless steel-steam reaction, Baker ${ }^{3}$ states that the local ratio of the partial pressures of steam to hydrogen determines the reaction products. Chromium tends to react even in the presence of a large excess of hydrogen to form $\mathrm{Cr}_{2} \mathrm{O}_{3}$. However, the exceptional stability of the spinel compound, $\mathrm{FeO} \cdot \mathrm{Cr}_{2} \mathrm{O}_{3}$, suggests that it would be the likely product in a hydrogen rich atmosphere because of the excess of iron over chromium in the metal. The remainder of the iron should form $\mathrm{FeO}$ (or $\mathrm{Fe}_{0.95} \mathrm{O}$, Wustite) only when the local steam to hydrogen ratio is greater than about 8.5 . Nickel oxide, Ni0, and the spinel compound $\mathrm{Fe}_{3} \mathrm{O}_{4}$ would form only in the presence of a large excess of steam, and $\mathrm{Fe}_{2} \mathrm{O}_{3}$ formation is negligible. Reaction products observed under conditions of excess steam include $\mathrm{FeO}, \mathrm{NiO}, \mathrm{Cr}_{2} \mathrm{O}_{3}$ and the spinel compounds $\mathrm{FeO} \cdot \mathrm{Cr}_{2} \mathrm{O}_{3}, \mathrm{Fe}_{3} \mathrm{O}_{4}$ and $\mathrm{NiO} \cdot \mathrm{Cr}_{2} \mathrm{O}_{3}$.

From the above discussion, it is obvious that a number of metallic oxides are possible, but for these analyses, the following exothermic reactions are assumed.

$$
\begin{array}{ll}
2 \mathrm{Cr}+3 \mathrm{H}_{2} \mathrm{O} \rightarrow \mathrm{Cr}_{2} \mathrm{O}_{3}+3 \mathrm{H}_{2} & 3877.4 \mathrm{~J} / \mathrm{g} \mathrm{Cr} \\
\mathrm{Ni}+\mathrm{H}_{2} \mathrm{O} \rightarrow \mathrm{NiO}+\mathrm{H}_{2} & 42.9 \mathrm{~J} / \mathrm{g} \mathrm{Ni} \\
3 \mathrm{Fe}+4 \mathrm{H}_{2} \mathrm{O} \rightarrow \mathrm{Fe}_{3} \mathrm{O}_{4}+4 \mathrm{H}_{2} & 900.6 \mathrm{~J} / \mathrm{g} \mathrm{Fe}
\end{array}
$$

From the results of the gas analyses, Section 3.5, and noting the vigorous corium water interaction, it was concluded that $\mathrm{Fe}_{3} \mathrm{O}_{4}$ would have been formed.

This conclusion is supported by XRD examination of the corium debris from the CWTI tests, which found $\mathrm{Fe}_{3} \mathrm{O}_{4}$ but no $\mathrm{FeO}$.

Equations $4.1,2$ and 3 were used to estimate the completeness of oxidation of the metallic constituents of corium in these tests. It was assumed that the hydrogen was generated from these reactions only. Other sources of hydrogen, namely oxidation of $\mathrm{UO}_{2}$, oxidation of structural materials in the apparatus, and hydrogen impurities in the reactants (shown to be small in Section 2.5), were neglected. It was further assumed that only the corium that exited the thermite vessel was available for reaction with steam, i.e., oxidation of the crust residue in the injector was neglected because of the initial inert atmosphere. Both nickel and chromium are resistant to oxidation and form stable oxide films. Iron oxide tends to flake off at high temperatures and thus expose the base metal to additional oxidation. However, it was assumed that the mass of hydrogen generated was proportional to the mass of metal reactants, i.e., to form $1 \mathrm{~g}$ of $\mathrm{H}_{2}$ requires $4.299 \mathrm{~g}$ of $\mathrm{Cr}, 2.426 \mathrm{~g}$ of $\mathrm{Ni}$ and $13.851 \mathrm{~g}$ of $\mathrm{Fe}$ be oxidized by steam.

The maximum amount of hydrogen that can be formed if all of the metals in the injected corium were oxidized is given in Table 4.1 along with the amount determined from the gas analyses in Section 3.5. The efficiency of hydrogen generation as defined as 
Table 4.1

Summary of Hydrogen Generation from the Oxidation of Corium Metals by Steam

\begin{tabular}{|c|c|c|c|c|c|c|}
\hline & CCM-1 & $\mathrm{CCM}-2$ & $\mathrm{CCM}-3$ & $\mathrm{CCM}-4$ & $\mathrm{CCM}-5$ & $\mathrm{CCM}-6$ \\
\hline \multicolumn{7}{|l|}{ Injected mass, g-mole } \\
\hline $\mathrm{Fe}$ & 6.1 & 31.7 & 9.5 & 26.3 & 32.3 & 34.2 \\
\hline $\mathrm{Cr}$ & 2.0 & 10.5 & 3.2 & 8.7 & 10.7 & 11.3 \\
\hline $\mathrm{Ni}$ & 1.0 & 5.3 & 1.6 & 4.4 & 5.4 & 5.7 \\
\hline \multicolumn{7}{|l|}{ Injected mass, $\mathrm{g}$} \\
\hline $\mathrm{Fe}$ & 342 & 1772 & 531 & 1469 & 1803 & 1910 \\
\hline $\mathrm{Cr}$ & 105 & 546 & 164 & 453 & 556 & 589 \\
\hline $\mathrm{Ni}$ & 60 & 312 & 94 & 259 & 318 & 336 \\
\hline $\mathrm{H}_{2}$ moles formed, $\mathrm{g} / \mathrm{mole}$ & 0.15 & 5.59 & 2.99 & 0.50 & 2.28 & 23.38 \\
\hline $\mathrm{H}_{2}$ mass formed. $\mathrm{g}$ & 0.30 & 11.3 & 6.0 & 1.0 & 4.6 & 47.1 \\
\hline Theoretical maximum $\mathrm{H}_{2}$ moles, g-mo is & 12.2 & 63.4 & 19.0 & 52.5 & 64.5 & 67.3 \\
\hline Theoretica 1 maximum $\mathrm{H}_{2}$ mass, $\mathrm{g}$ & 24.7 & 127.8 & 38.3 & 105.9 & 130.0 & 135.7 \\
\hline $\mathrm{H}_{2}$ generation efficiency. $\%$ & 1 & 9 & 16 & 1 & 2 & 1.35 \\
\hline Heat liberated, $\mathrm{MJ}$ & 0.01 & 0.33 & 0.18 & 0.03 & 0.13 & 1.38 \\
\hline Mole $\mathrm{H}_{2} /$ mole steam & 0.02 & 0.05 & 0.05 & 0.01 & 0.17 & 0.30 \\
\hline
\end{tabular}




$$
\mathrm{H}_{2} \text { generation efficiency }=100 \frac{\text { Measured } \mathrm{H}_{2} \text { Generation }}{\text { Maximum Theoretical } \mathrm{H}_{2} \text { Generation }}
$$

These data are also presented in Table 4.1 and can be interpreted as a measure of the completeness of the metal phase oxidation process in the water/steam environment. Table 4.1 also gives the heat calculated to have been liberated during the reaction process.

From the data in Table 4.1, the experiments with subcooled water (CCM-1, 4, and 5) have hydrogen generation efficiencies of about $1 \%$. For the remaining tests with saturated water, the efficiency of steam generation varied from 7.5 to $34.7 \%$.

\subsection{Steam Generation and Mater Heating}

Most steam generation occurs during the descent of the corium through the water. Some generation can also occur after the debris bed is formed at the bottom of the interaction or experiment vessel. The mass of steam that was generated in each test, estimated from PVT data and the gas analyses presented in Section 3.5, is given in Table 4.2 .

The investigation of the steam generation was focused on the short-term quenching of the corium, i.e., during the jet fall stage, rather than the longterm debris bed cooling. However, the stean generation during the jet fall stage cannot be measured directly. In order to estimate the sieam generation during the jet fall stage, two methods were used: analysis of pool swell data, and analysis of the expansion vessel (or experiment vessel) pressurization data.

In CCM-2 and 3 , five level detectors were adjacently positioned above the water surface at heights of 3.2 to $60.3 \mathrm{~mm}$. From level probe responses, the global void fraction of the pool can be obtained. Unfortunately, the highest elevation that water level could have reached is not known because the level probes were not positioned high enough. However, the thermal response of thermocouple TC8-12, which was located $27 \mathrm{~cm}$ and $25 \mathrm{~cm}$ above the water surface in CCM-2 and 3, respectively, indicated that a high-temperature water/corium slug may have hit this thermocouple during the interval 1.3 to 2.6 s during test CCM-2. There was no such indication in CCM-3. Based on this information and extrapolation of the level probe data, the water levels can be roughly estimated as shown in Figs. 4-1 and 4-2. The water level of CCM-5, as shown in Fig. 4-3, was observed from a view port located at the same elevation as the initial water level. Pool swell data for tests CCM-1, 4, and 6 are not available.

The water level, $\mathrm{H}$, in a bubbling or pool boiling system can be given in terms of an average (global) pool void fraction, $\alpha$, as 
Table 4.2

Summary of Steam Generation Based on PVT Data and Gas Analyses

\begin{tabular}{|c|c|c|c|c|c|c|}
\hline & CCM-1 & CCM-2 & CCM-3 & CCM-4 & CCM-5 & CCM-6 \\
\hline Maximum Pressure, MPa & 0.135 & 0.364 & 0.245 & 0.303 & 0.246 & 0.354 \\
\hline Time of occurrence, s & 5.0 & 250.0 & 15.0 & 15.0 & 1.4 & 1.3 \\
\hline Temperature, K & 440.2 & 411 & 399 & 389 & 390 & 417 \\
\hline System moles, g-mole & 51.7 & 150 & 103.8 & 131.8 & 35.6 & 117.3 \\
\hline Steam Basis & & & & & & \\
\hline Pressure, MPa & 0.118 & 0.364 & 0.245 & 0.283 & 0.193 & 0.350 \\
\hline Time of occurrence, s & 250.0 & 250.0 & 15.0 & 15.0 & 30.0 & 1.3 \\
\hline Temperature, K & 414.0 & 410.9 & 398.6 & 389.3 & 384.1 & 417.4 \\
\hline System moles, g-mole & 48.1 & 149.9 & 103.8 & 120.5 & 27.9 & 116 \\
\hline Water moles, g-mole & 8.5 & 141.4 & 99.5 & 80.3 & 14.1 & 90.5 \\
\hline Water mole fraction & 0.177 & 0.943 & 0.959 & 0.609 & 0.504 & 0.780 \\
\hline Net steam moles, g-mole & 8.0 & 103.2 & 59.2 & 80.3 & 11.0 & 54.2 \\
\hline Steam generated, g-mole & 8.5 & 108.8 & 62.2 & 80.8 & 13.3 & 77.6 \\
\hline Steam generated, g & 152 & 1960 & 1119 & 1456 & 240 & 1398 \\
\hline
\end{tabular}




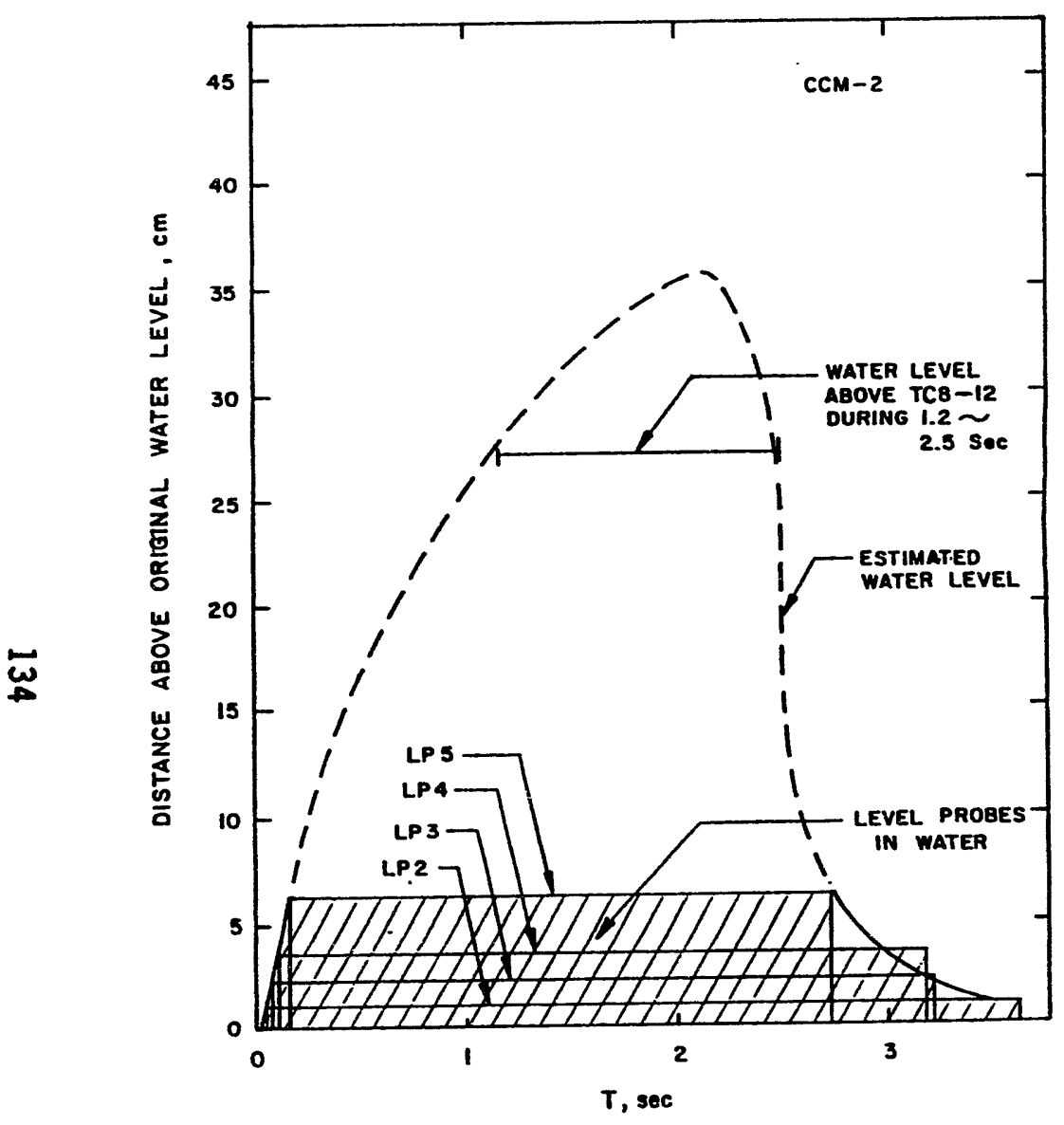

Fig. 4-1 Estimated Water Level in CCM-2

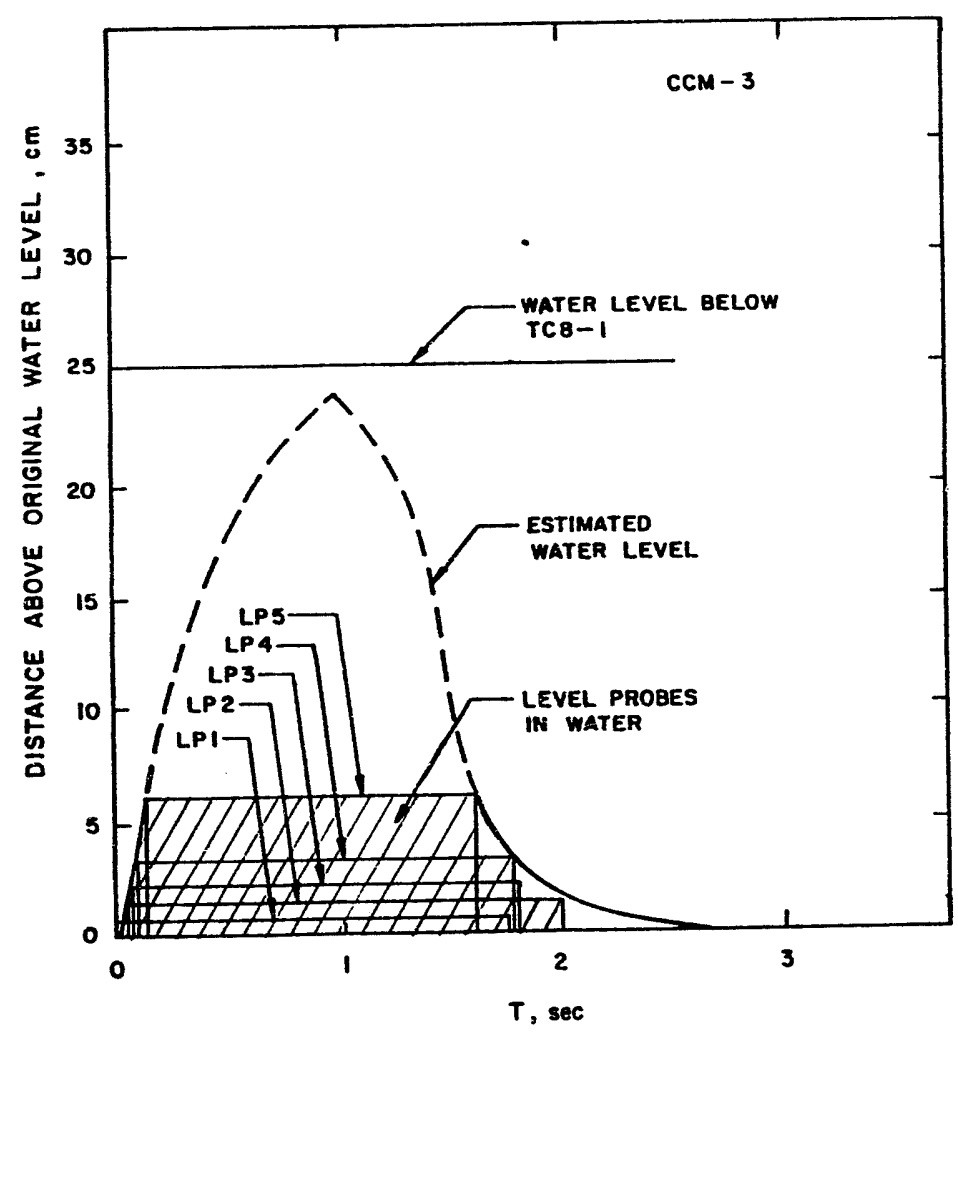

Fig. 4-2 Estimated Water Level in CCM-3 


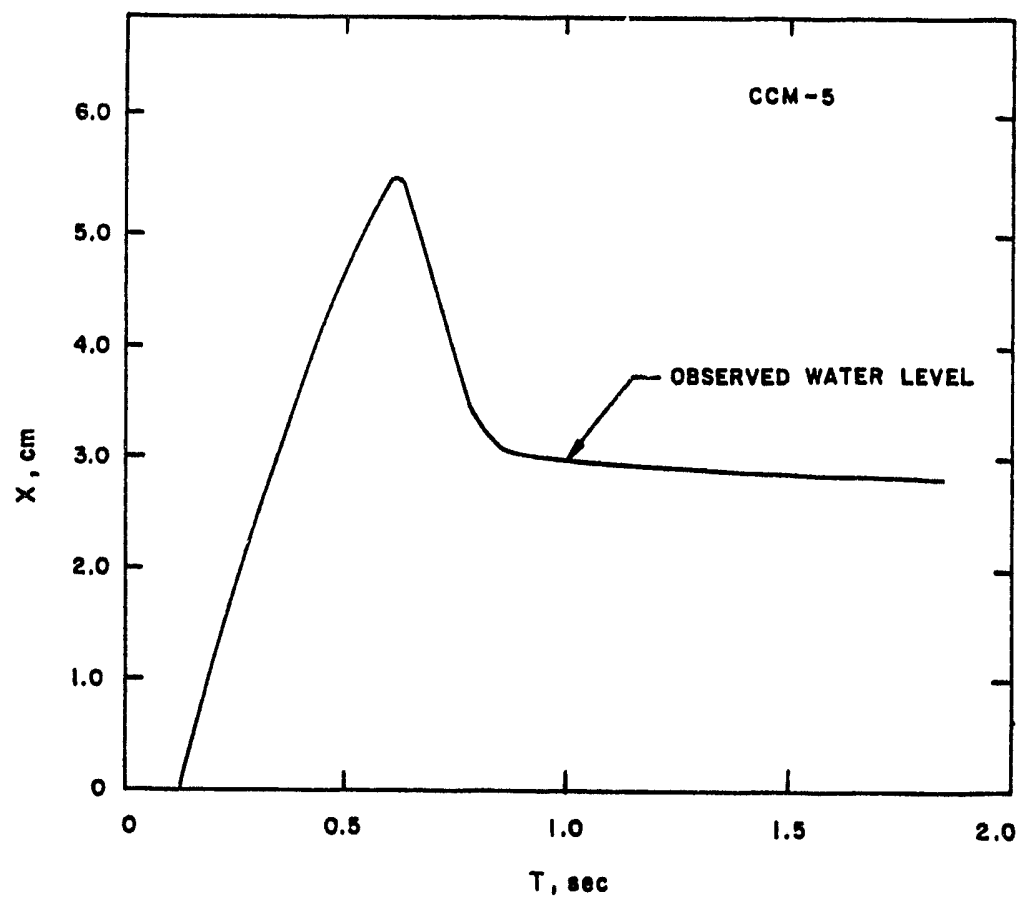

Fig. 4-3 Observed Water Level in CCM-5 


$$
H=\frac{H_{0}}{1-\alpha}
$$

where $H_{0}$ is the water level without void, i.e., the collapsed level of the pool. Kataoka and Ishiji ${ }^{14}$ correlated the pool void fraction using a modified drift flux formulation. They concluded that for large gas fluxes, the pool void fraction is given by the following expression:

$$
\alpha=\left\{C_{0}+0.03\left(\frac{\rho_{g}}{\rho_{f}}\right)^{-0.157} N_{\mu f}^{-0.562} / j_{g}+\right\}^{-1}
$$

where:

$$
\begin{aligned}
& c_{o}=1.2-0.2\left(\rho_{g} / \rho_{f}\right)^{1 / 2}, \\
& N_{\mu f}=\mu_{f}\left(\rho_{f} \sigma \sqrt{\sigma / g \Delta \rho}\right)^{0.5} \\
& j_{g}^{+}=j_{g} /\left(\sigma g \Delta \rho / \rho_{f}^{2}\right)^{1 / 4}, \\
& j_{g}=\text { gas superficial velocity }
\end{aligned}
$$

From the pool global void fraction, assuming hydrogen generation is negligible, the steam generation rate can be estimated using Eq. 4.6. Steam generation rates estimated using this relation and pool swell data are shown in Figs. 4-4, 4-5 and 4-6.

The pool swell velocity registered by the level probes can also be used to estimate the global void fraction. Assuming that the pool swell velocity is a constant, Eq. 4.7 can be integrated to yield

$$
\alpha(t)=\left[\frac{v_{L s} t}{H_{0}}+1\right]^{-1}
$$

The steam generation rate estimated from the pool swell velocity is also shown in Figs. 4-4 and 5, using the average pool swell velocity to the uppermost level 

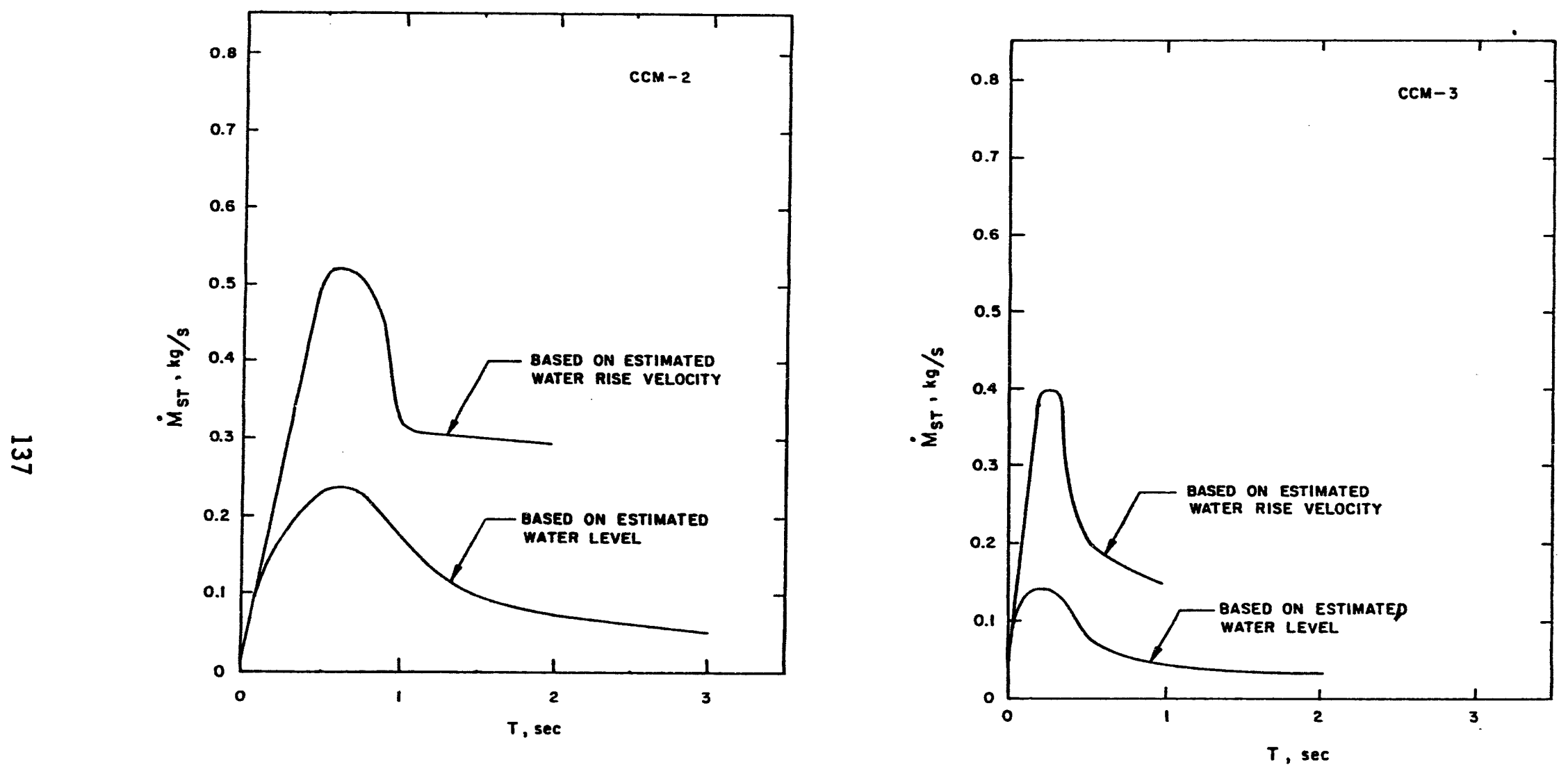

Fig. 4-4 Estimated Steam Generation Rate for CCM-2 Using Water Swell Data

Fig. 4-5 Estimated Steam Generation Rate for CCM-3 Using Water Swell Data. 


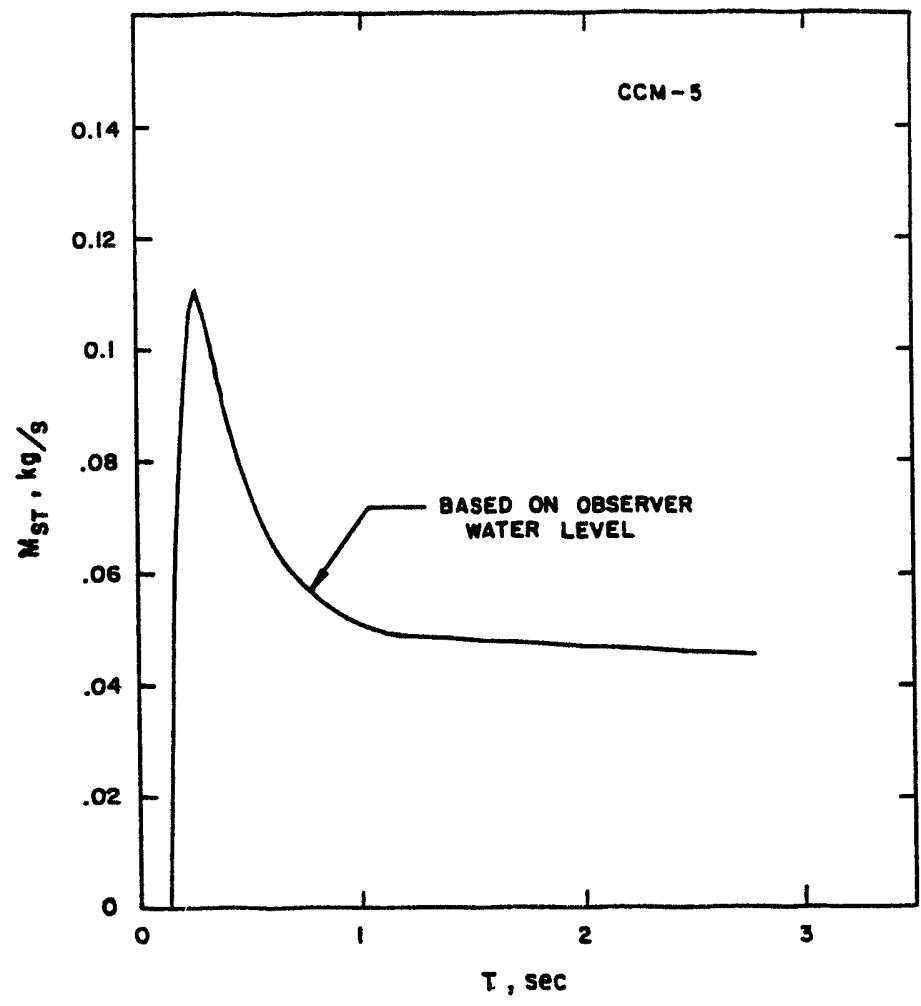

Fig. 4-6 Estimated Steam Generation Rate for CCM-5 Using Water Swell Data 
probe, i.e., $0.75 \mathrm{~m} / \mathrm{s}$ for $\mathrm{CCM}-5$ and $1.1 \mathrm{~m} / \mathrm{s}$ for $\mathrm{CCM}-3$. In general, a higher steam generation rate was predicted from the pool swell velocity than from the pool level data.

Another, perhaps more credible method to estimate the steam generation rate during the jet fall stage is the use of expansion vessel (or experiment vessel) pressurization data. The total flow rate of gas and steam is reflected by the rate of pressure rise in the expansion vessel for CCM-1 through 4 and the experiment vessel for $\mathrm{CCM}-5$ and 6 . Assuming that the gas/steam mixture behaves as a perfect gas during the system pressurization, the steam generation rate can be estimated using the relation:

$$
\dot{m}_{s t}=\frac{X_{s t} M_{s t} V_{e v}}{R_{t} T_{m}} \frac{d P_{e v}}{d t}-\frac{m_{m}}{T_{m}} \frac{d T_{m}}{d t}
$$

where

$$
\begin{aligned}
& X=\text { mole fraction, } \\
& M=\text { molecular weight, } \\
& m_{m}=\text { the mean mass of the mixture, } \\
& T_{m}=\text { the mean temperature of the mixture, and } \\
& R_{u}=\text { the universal gas constant; }
\end{aligned}
$$

and the subscripts st, ev and $m$ refer to steam, expansion vessel (or experiment vesse1), and mixture, respectively.

The second term on the RHS of Eg. (4.8) is typically two orders of magnitude smaller than the first term, and may be neglected. Steam generation rates calculated from pressurization data are given in Figs. 4-7 through 12 .

In general, the steam generation rate predicted using the pressurization data is higher than predicted using the Kataoka and Ishi ${ }^{\text {la }}$ correlation (which predicts the gas flux based on the global void fraction). Since the local void fraction in the corium/water mixture must have been much higher than the global void fraction estimated from the pool swell data, the steam generation rates are probably underestimated by this method. In addition, the region in the immediate vicinity of the corium jet may be so highly voided that a vapor channel with ap annular type flow is created. The drift flux correlation of Kataoka and Ishii is applicable only for bubbly or slug flow; therefore, its use here may not be appropriate.

The energy required to vaporize the water is given by

$$
E_{s}=m_{s t}\left(h_{f g}\right)
$$

where 


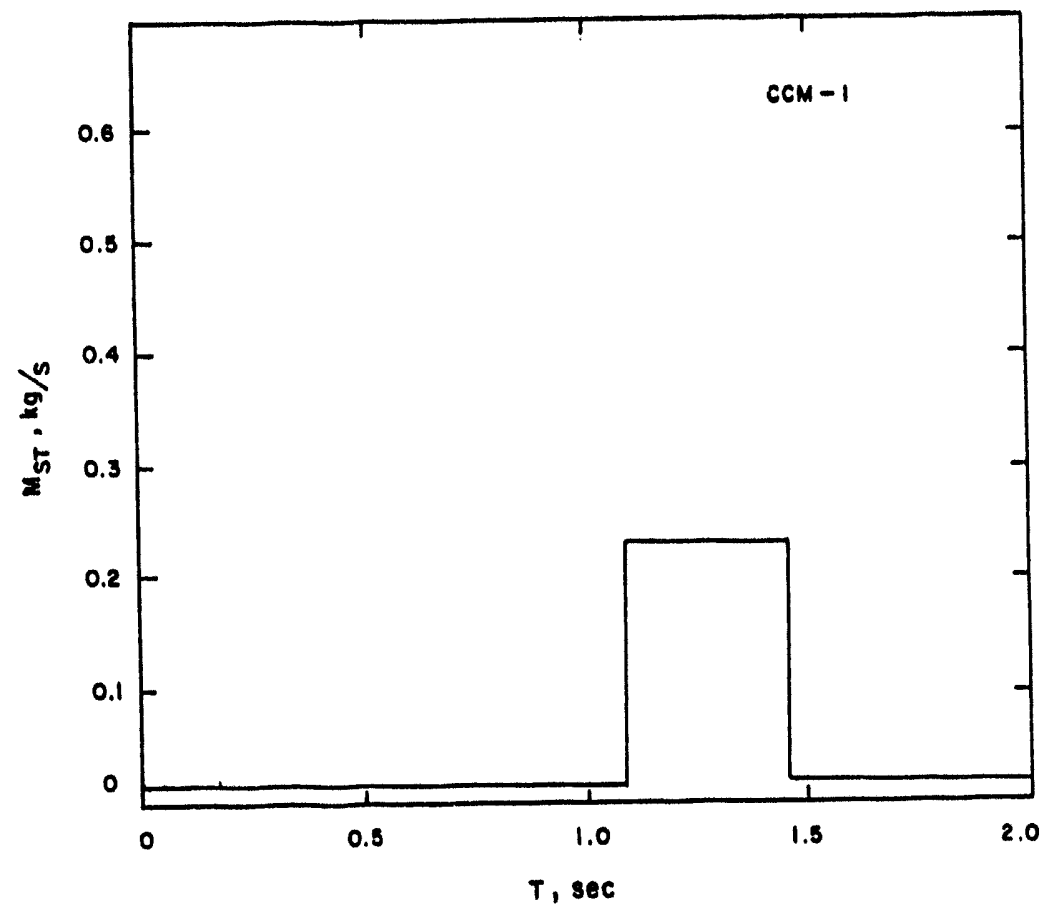

Fig. 4-7 Estimated Steam Generation Rate for CCM-1

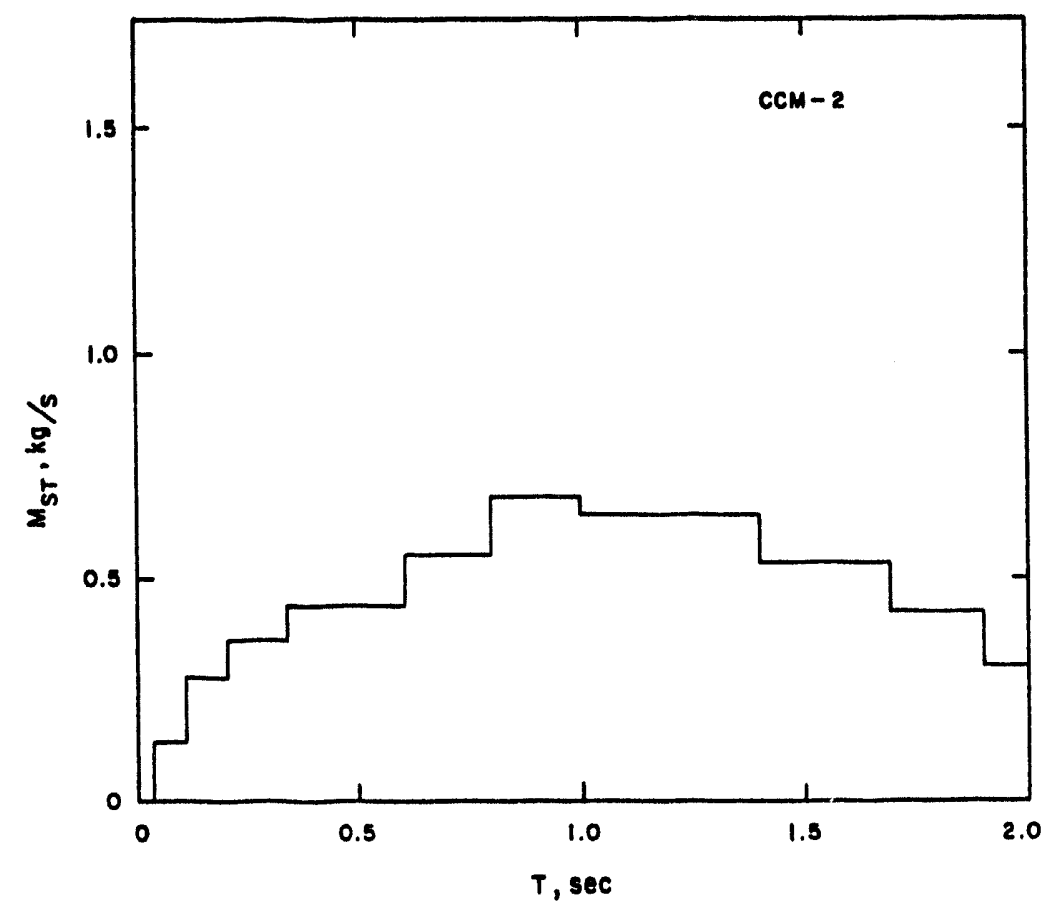

Fig. 4-8 Estimated Steam Generation Rate for CCM-2 


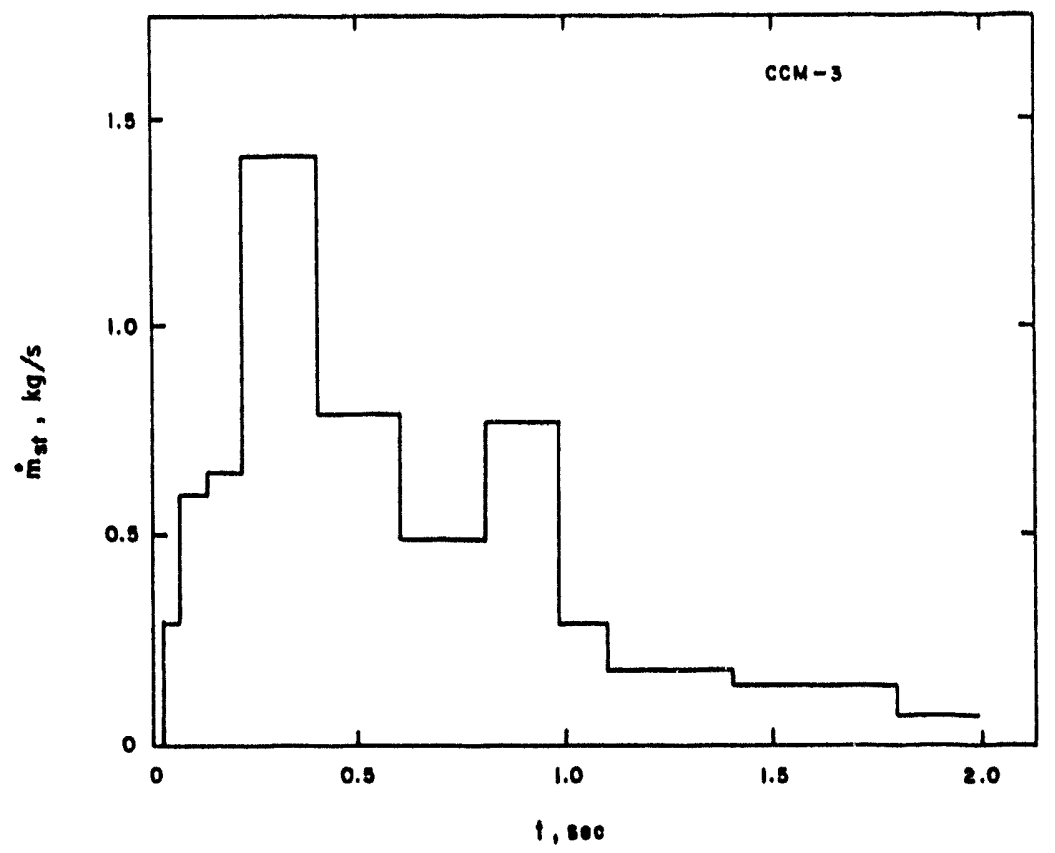

Fi8. 4-9 Estimated Steam Generation Rate for CCM-3

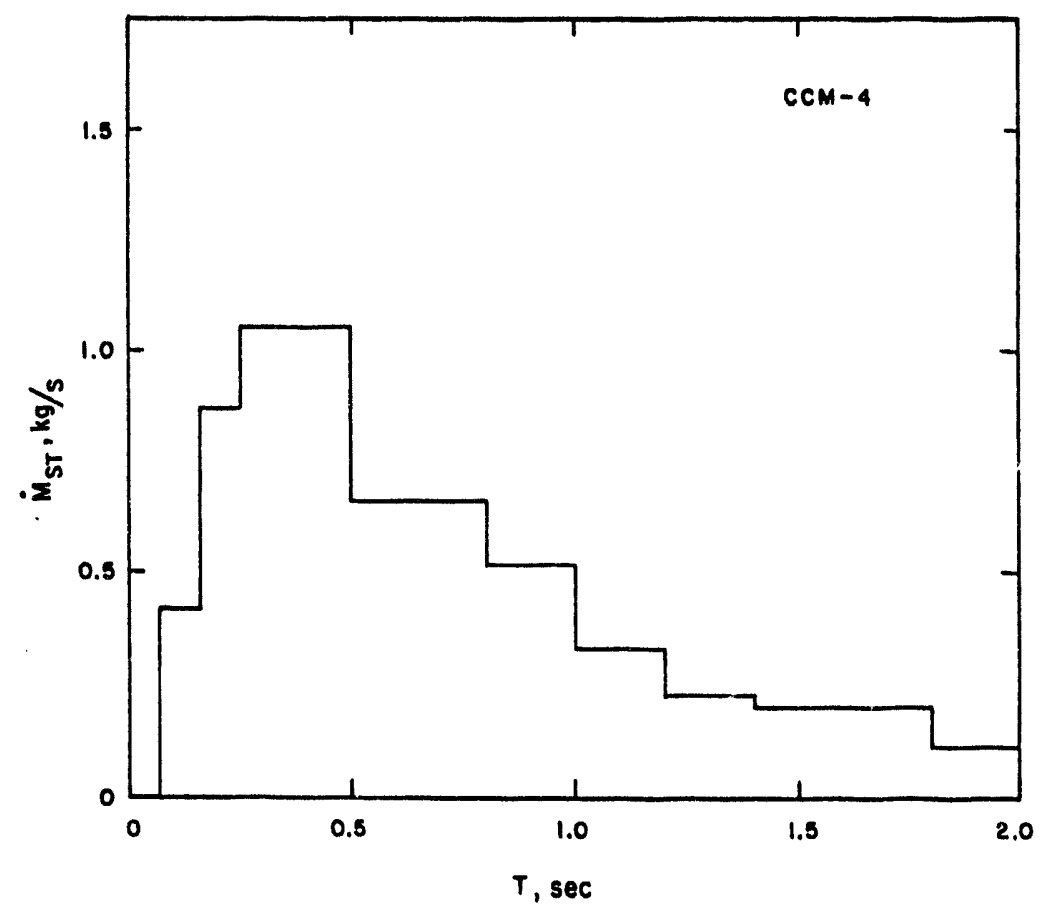

Fig. 4-10 Estimated Steam Generation Rate for CCM-4 


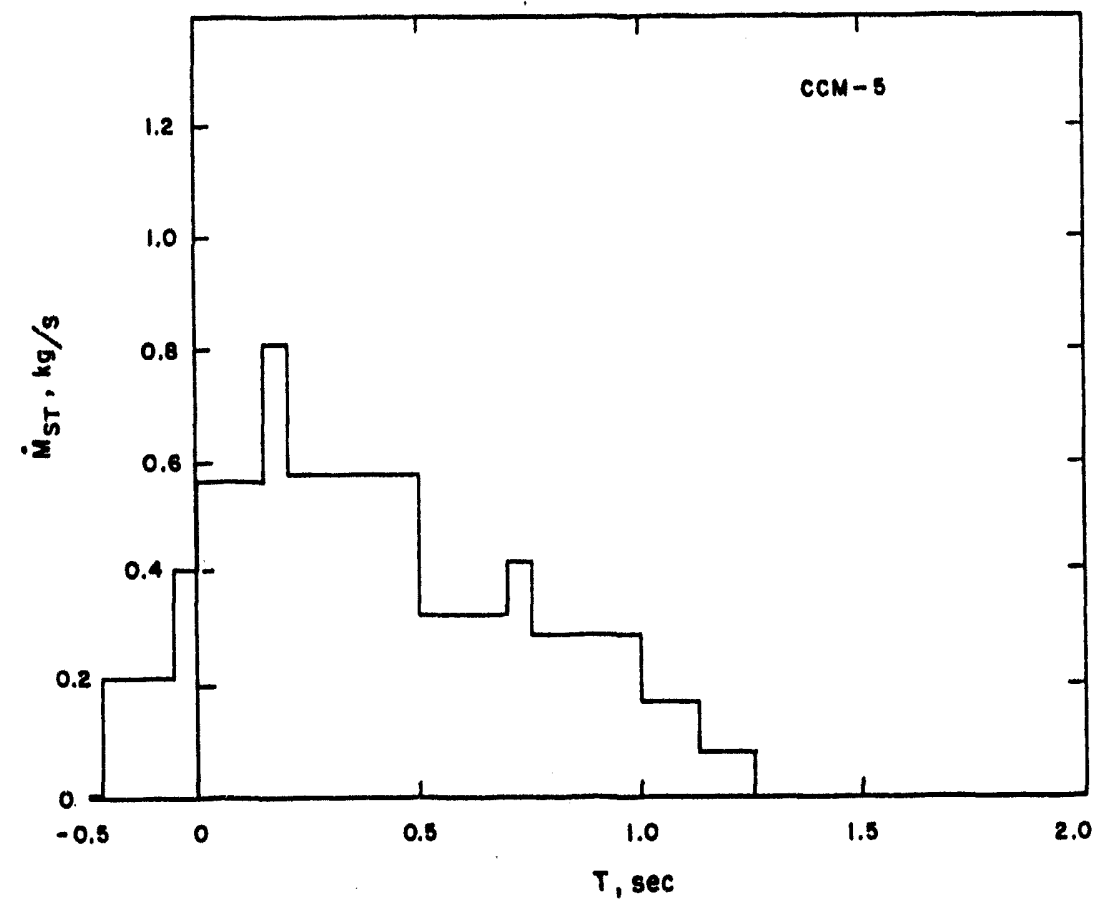

Fig. 4-11 Estimated Steam Generation Rate for CCM-5

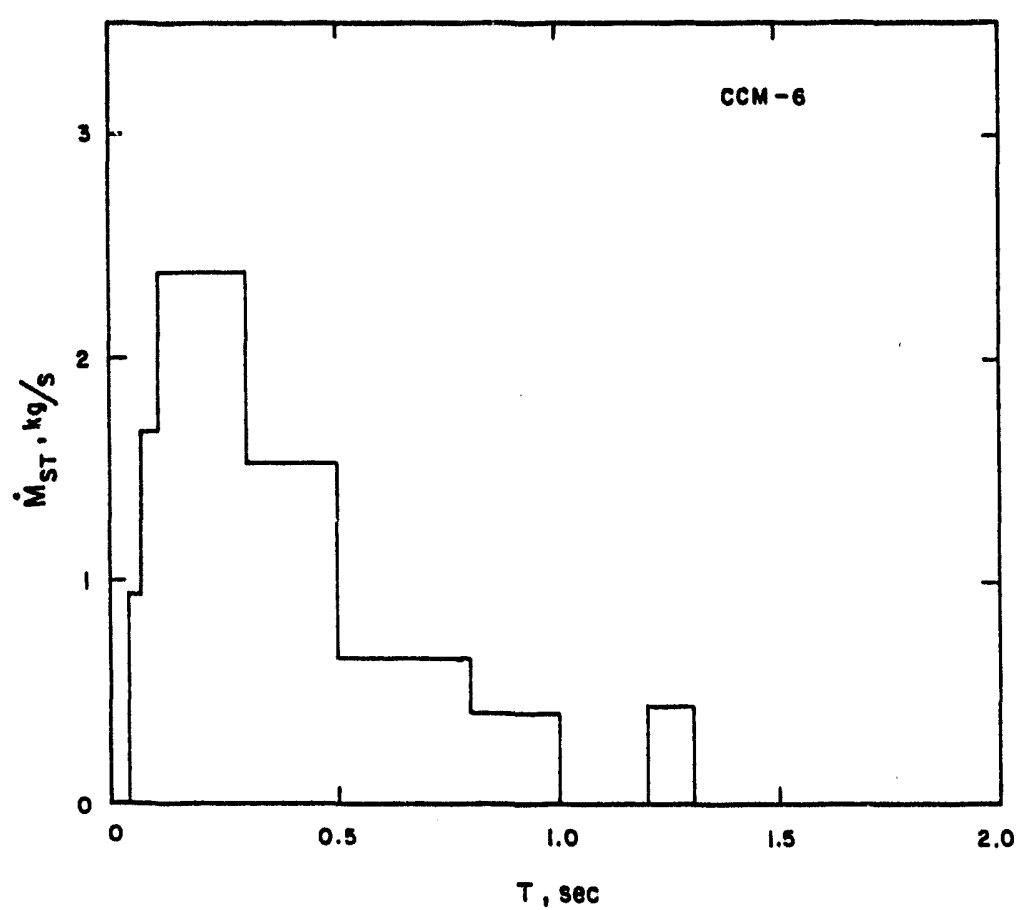

Fig. 4-12 Estimated Steam Generation Rate for CCM-6 
$E_{s}=$ water vaporization energy, $M J$,

$h_{f g}=$ latent heat of vaporization of water, $M J / k g$, and

$\mathrm{m}_{\mathrm{st}}=$ steam mass, $\mathrm{kg}$.

The latent heat of vaporization, obtained from steam tables at the mean system pressure, is about $2.2 \mathrm{MJ} / \mathrm{kg}$.

The corium energy content listed in Table 4.3 was calculated by

$$
E_{c}=m_{c}\left(h_{c i}-h_{c f}\right)
$$

where

$E_{c}=$ corium energy, MJ,

$h_{c i}=$ corium specific enthalpy at initial conditions, $\mathrm{MJ} / \mathrm{kg}$,

$h_{c f}=$ corium specific enthalpy at final conditions, $M J / k g$, and

$m_{c}=$ corium mass injected, $\mathrm{kg}$.

Corium specific enthalpy data referenced to $20 \mathrm{C}$ are given in Fig. 2-14. At injection conditions the enthalpy is assumed to be $1.8 \mathrm{MJ}$; for the final condition the enthalpy is based on the maximum water temperature. The portion of this energy that is converted into steam during the jet fall stage can be expressed as a percentage generation fraction where

$$
\text { Steam Generation Fraction }=\left[\frac{E_{s}}{E_{c}}\right] 100 .
$$

These fractions are given in Table 4.3 and, as one would expect, higher values were obtained for the tests with saturated water, since condensation occurs when the water is subcooled.

The energy transferred to the water was calculated using the equations below (Eq. 4.13 for water that was initially subcooled and Eq. 4.14 for saturated water). The calculated water heating is given in Table 4.3.

$$
\begin{aligned}
& E_{w}=m_{s t}\left(h_{w s}-h_{w i}\right)+\left(m_{w}-m_{s t}\right)\left(h_{w-m}-h_{w i}\right) \\
& E_{w}=\left[h_{w}-\frac{m_{s t}}{2}\right]\left(h_{w m}-h_{w i}\right)
\end{aligned}
$$

where

$$
E_{w} \text { = water heating energy, } M J \text {, }
$$


Table 4.3

Summary of Steam Generation and Water Heatup During Jet Fall Stage

\begin{tabular}{|c|c|c|c|c|c|c|}
\hline & $\mathrm{CCM}-1$ & CCM-2 & $\mathrm{CCM}-3$ & $\mathrm{CCH}-4$ & $\mathrm{CCM}-5$ & CCM-6 \\
\hline Injected Corium Energy, $E_{c}, M J$ & 3.8 & 19.4 & 5.9 & 16.2 & 20.3 & 21.1 \\
\hline Duration of Jet Fall Stage, ${ }^{*} \mathrm{~s}$ & 1.3 & 2.6 & 1.8 & 2.0 & 1.4 & 1.3 \\
\hline Mass of Steam Generated During Jet Fall Stage, ${ }^{\star \star} \mathrm{kg}$ & 0.11 & 0.93 & 0.60 & 1.05 & 0.5 & 1.8 \\
\hline Steam Generation Energy During Jet Fall Stage, $E_{\mathbf{s}}, \mathrm{MJ}$ & 0.25 & 2.04 & 1.25 & 2.32 & 1.11 & 4.0 \\
\hline Steam Generation Fraction, $\frac{E_{\mathbf{g}}}{E_{c}}, \chi$ & 7 & 11 & 21 & 14 & 5 & 19 \\
\hline Water Heating Energy During Jet Fall Stage, $E_{w}$. MJ & 2.0 & 1.8 & 1.5 & 6.7 & 10.1 & 3.5 \\
\hline Water Heating Fraction, $\frac{E_{w}}{E_{c}}, x$ & 66 & 9 & 25 & 41 & 50 & 17 \\
\hline Chemical Reaction Energy, $E_{R}, M J$ & 0.009 & 0.32 & 0.18 & 0.03 & 0.13 & 1.38 \\
\hline $\begin{array}{l}\text { Corium Quenching Fraction During Jet Fall Stage, } \\
\left(E_{s}+E_{w}\right) /\left(E_{c}+E_{r}\right), x\end{array}$ & 72 & 20 & 45 & 55 & 55 & 33 \\
\hline
\end{tabular}

*Based on pressurization data.

**Based on Eq. 4.9. 


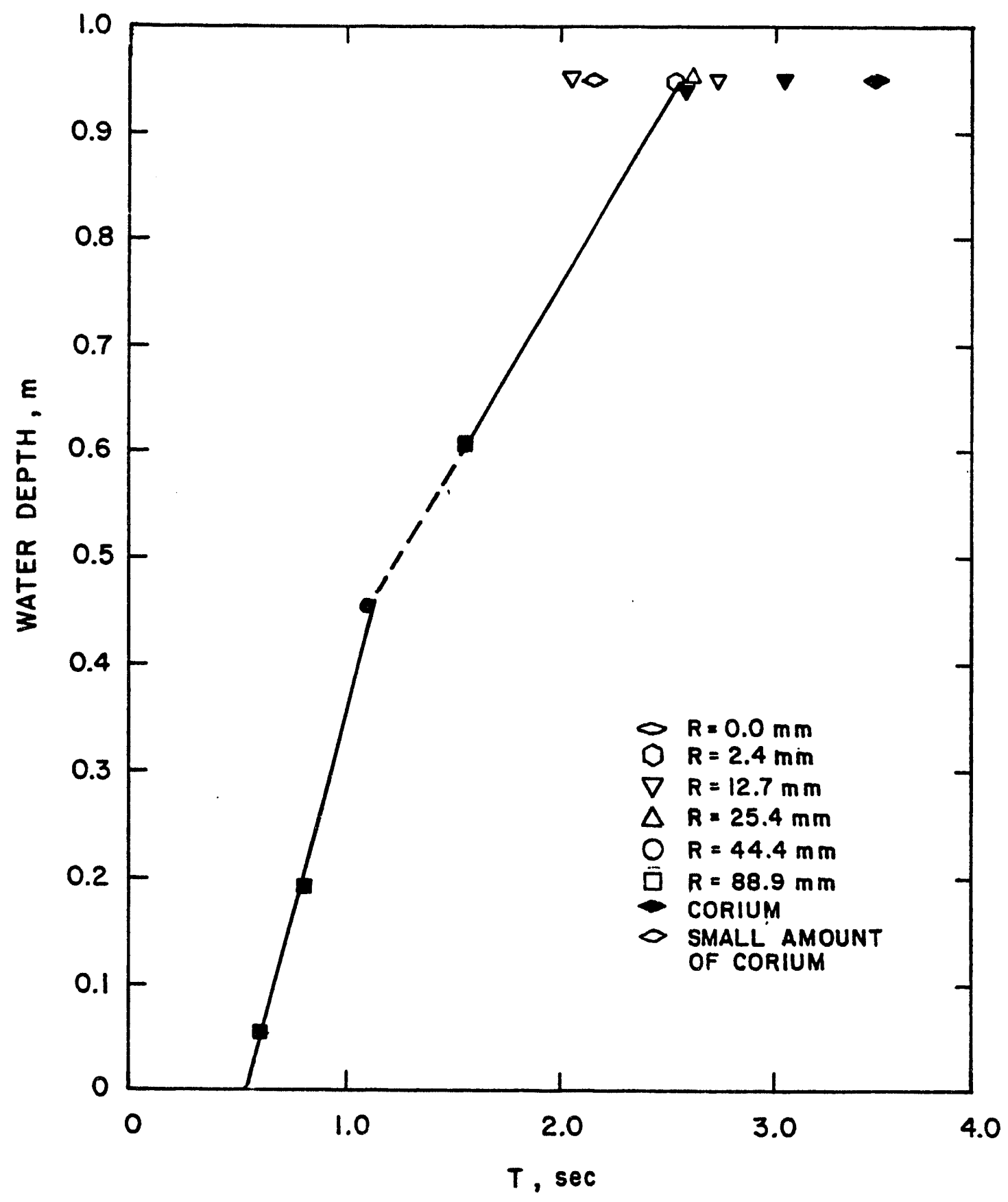

Fig. 4-13 Thermal Response of the Thermocouples in the Interaction Vessel Water for Experiment CCM-1 


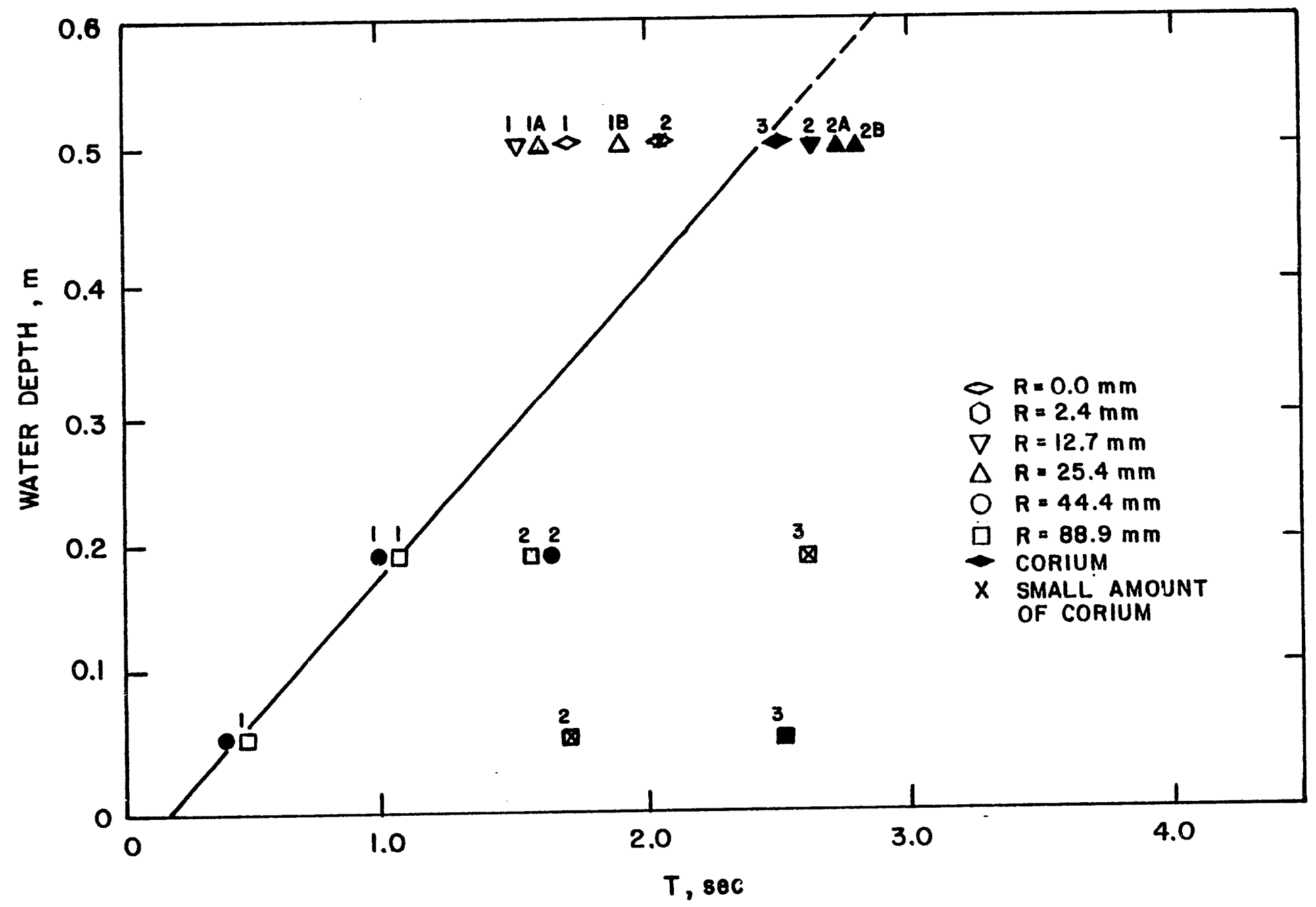

Fig. 4-14 Thermal Response of the Thermocouple in the Interaction-Vessel Water for Experiment CCM-2 
$h_{w i}=$ enthalpy of water at initial temperature, $\mathrm{MJ} / \mathrm{kg}$,

$h=$ enthalpy of water at maximum temperature, MJ/kg,

$h_{\mathrm{wS}}=$ enthalpy of saturated water, $\mathrm{MJ} / \mathrm{kg}$,

$m_{\text {st }}=$ mass of steam generated, $\mathrm{kg}$, and

$m_{w}=$ mass of water, $\mathrm{kg}$.

The fraction of corium energy that is converted into water heating is expressed as a percentage by

water heating fraction $=\left[\begin{array}{l}E_{w} \\ E_{c}\end{array}\right] 100$.

These fractions are presented in Table 4.3. It is clear that most corium energy is converted into water heating if the water is subcooled.

The total fraction of available energy (corium energy plus chemical energy from hydrogen generation) that is converted into water heating and steam generation is expressed as a percentage by

$$
\text { Total energy fraction }=\left[\frac{E_{w}+E_{s t}}{E_{c}+E_{R}}\right] 100 .
$$

As shown in Table 4.3, except for CCM-2, significant corium quench occurred during the jet fall stage for all the tests. In general, a corium quench fraction ranging from 55 to $72 \%$ occurred in subcooled water, while a lower quench fraction ranging from 20 to $45 \%$ occurred in saturated water. These large quench fractions indicate that a debris bed of solidified and substantially quenched particles was formed, allowing minimal thermal attack upon the vessel bottom. Furthermore, it takes -21\% of the total energy for a corium stream (with an initial temperature of $3080 \mathrm{~K}$ ) to be solidified. Therefore, except for (CM-2, all the corium streams would have been solidified during the fall stage. For CCM-2, the quench fraction may have been reduced by the multiple-jet geometry; the volume between the jets appeared to be highly voided. As described in Section 3.4 , the posttest examination of $\mathrm{CCM}-2$ showed that the debris is sintered together in large, reagglomerated chunks, which confirms that the extent of corium quench was very limited during the jet fall stage. Another possible explanation for the low quench fraction is the shallow pool (i.e., $40 \%$ shallower than other tests), which allowed less time for corium quench during the jet fall stage.

\subsection{Test Behavior}

Basic analysis of test results is presented below.

\subsubsection{Test CCM-1}

There was about a $0.22 \mathrm{~s}$ delay between the time the slide started to open 
and the ejection of corium from the thermite vessel, caused by a thin layer of solidified material across the exit hole of the thermite vessel. From Table 3.2 , the estimated time for the corium to reach the surface of the water was $0.3 \mathrm{~s}$ for free fall and $0.18 \mathrm{~s}$ for an initial pressure differential of $0.01 \mathrm{MPa}$ and a discharge coefficient of 1 for the injector hole. Using a delay time of 0.22 , the calculated time for corium to reach the burn wire is $0.362 \mathrm{~s}$ which falls between the 0.352 and 0.386 s estimated time based on the start of the X-ray camera. Correspondingly, the estimated time for the corium to reach the water surface is $0.48 \mathrm{~s}$ at a velocity of $3.39 \mathrm{~m} / \mathrm{s}$. This contact time agrees well with the extrapolated time of $0.5 \mathrm{~s}$ obtained from the water thermocouple response to corium contact shown in Fig. 4-13.

The data in Fig. 4-13 have been used to estimate the breakup length of the corium stream. Here the same criteria as used by Spencer ${ }^{4}$ (in related experiments with simulant materials) has been applied. That is, the depth at which the penetration velocity of the melt slows significantly is taken to be the end of the more or less continuous stream, the slower velocity at greater depths being indicative of a swarm of small particles. From Fig. 4-13, the particle terminal velocity is about $0.4 \mathrm{~m} / \mathrm{s}$. The terminal velocity of a smooth corium sphere with a diameter of $2.3 \mathrm{~mm}$ (the median debris particle size for this test) is about $0.6 \mathrm{~m} / \mathrm{s}$. However, most of the debris particles cannot be characterized as spherical, and many of the particles were hollow. Therefore, a terminal velocity well below $0.6 \mathrm{~m} / \mathrm{s}$ is to be expected.

The penetration velocity (from Fig. 4-13) slowed noticeably between contacting the thermocouple at the $45.74 \mathrm{~cm}$ depth and that at $60.98 \mathrm{~cm}$. This indicates a breakup length between 18 and 24 jet diameters.

From the gas analyses, $8.501 \mathrm{~g}$-moles of steam were generated, exclusive of the amount needed to produce the $0.151 \mathrm{~g}$-mole of hydrogen. The oxygen depletion was $0.922 \mathrm{~g}$-mole. Based on the quantity of hydrogen generated and the oxygen depleted, the mass of metal that was oxidized was $32 \mathrm{~g}$.

\subsubsection{Test CCM-2}

With an initial pressure differential of $0.075 \mathrm{MPa}$ between the thermite and interaction vessels, a discharge velocity of $1.35 \mathrm{~m} / \mathrm{s}$ is calculated. Therefore, a corium velocity at the water surface of $5.30 \mathrm{~m} / \mathrm{s}$ with a fall time of $0.19 \mathrm{~s}$ is predicted. From the limited thermal response data shown in Figure 4-14, an extrapolated contact time of the corium with the water surface is $0.3 \mathrm{~s}$, in fair agreement with the calculated entry time.

A continuous flow of corium from the grid plate holes was observed from the $X$-ray data presented in Table 3.9. Corium was still flowing from the grid plate holes at $1.3 \mathrm{~s}$ when the last $X$-ray picture was taken.

When the corium stream from the thermite vessel impacts the grid plate, it is broken into four streams, as described in Section 3.3.2. It is possible to determine only a lower bound for the breakup length of these streams from the thermocouple data (Fig. 4-14), because the corium penetration velocity appears to have been relatively constant over the instrumented depth of 25 jet diameters.

The pressure behavior of the system is one of dynamic equilibrium. As early as $1 \mathrm{~s}$, gas-vapor equilibrium in the system, as the atmosphere becomes 
saturated, is indicated by the slow pressure and temperature increase. The generation of hydrogen and the increase in temperature from the steam and hot walls of the vessel allow the system pressure to rise while condensation occurs at an increasing dew point. Finally, the vessel wall temperature controls the condensation process.

\subsubsection{Test CCM-3}

A corium mass of $3.34 \mathrm{~kg}$ was injected into the interaction vessel through a 25.4-mm diameter hole under an initial differential pressure of $0.215 \mathrm{MPa}$. With this pressure differential the estimated corium velocity at the water surface is $8.15 \mathrm{~m} / \mathrm{s}$. However, the temperature response of the thermo coupler in the interaction vessel indicates that the initial penetration velocity of the corium was around $-1.2 \mathrm{~m} / \mathrm{sec}$ as shown in Fig. 4-15. This indicates that the effects of the initial pressure differential between the thermite vessel and the interaction vessel on the jet initial velocity were not significant.

The $59 \mathrm{~g}$-moles of steam were generated during the test, exclusive of the amount needed for the formation of $3.0 \mathrm{~g}$-moles of hydrogen. This amount of hydrogen is $15.7 \%$ of the theoretical maximum that could be formed. Oxygen depletion amounted to $0.02 \mathrm{~g}$-mole.

From Fig. 4-15, the corium stream breakup length is between 18 and 36 jet diameters. The relatively late response times of several of the thermocouples can be attributed to contact by fluidized corium drops.

\subsubsection{Test CCM-4}

Time zero for the test was chosen as the closure time for the burn wire below the thermite-vessel diaphragm. At $0.062 \mathrm{~s}$, the pressure inside the interaction vessel starts to rise rapidly; this time agrees with the closure of the burn wire below the injection tube. Because of the premature failure of the diaphragm, the reaction time for the corium was short, and the injected corium may have contained unreacted powder or aerosols.

After the corium entered the water, it descended at a velocity of $1.34 \mathrm{~m} / \mathrm{s}$ (see Fig. 4-16). This is also much lower than that calculated using the pressure differential between the thermite vessel and the interaction vessel. The thermocouple data suggest that the jet may have remained largely intact to a depth of at least 12 diameters; however, no firm conclusion is possible.

During the test, there were $8 \mathrm{~g}$-moles of steam formed including the $0.5 \mathrm{~g}$ mole needed to produce an equivalent number of moles of hydrogen.

\subsubsection{Test CCM-5}

Experiment CCM-5 was the first test with the modified experimental apparatus in which the thermite vessel was mounted inside the experiment vessel.

A plot of the responses of the thermocouples on the axial centerline of the experiment vessel are shown in ig. 4-17. Corium contact is indicated by the shaded circles. A water-corium contact time of $0.15 \mathrm{~s}$ can be inferred from these data. This time is in reasonable agreement with the contact time obtained from the Hycam film. The corium velocity at the water surface corresponding to this 


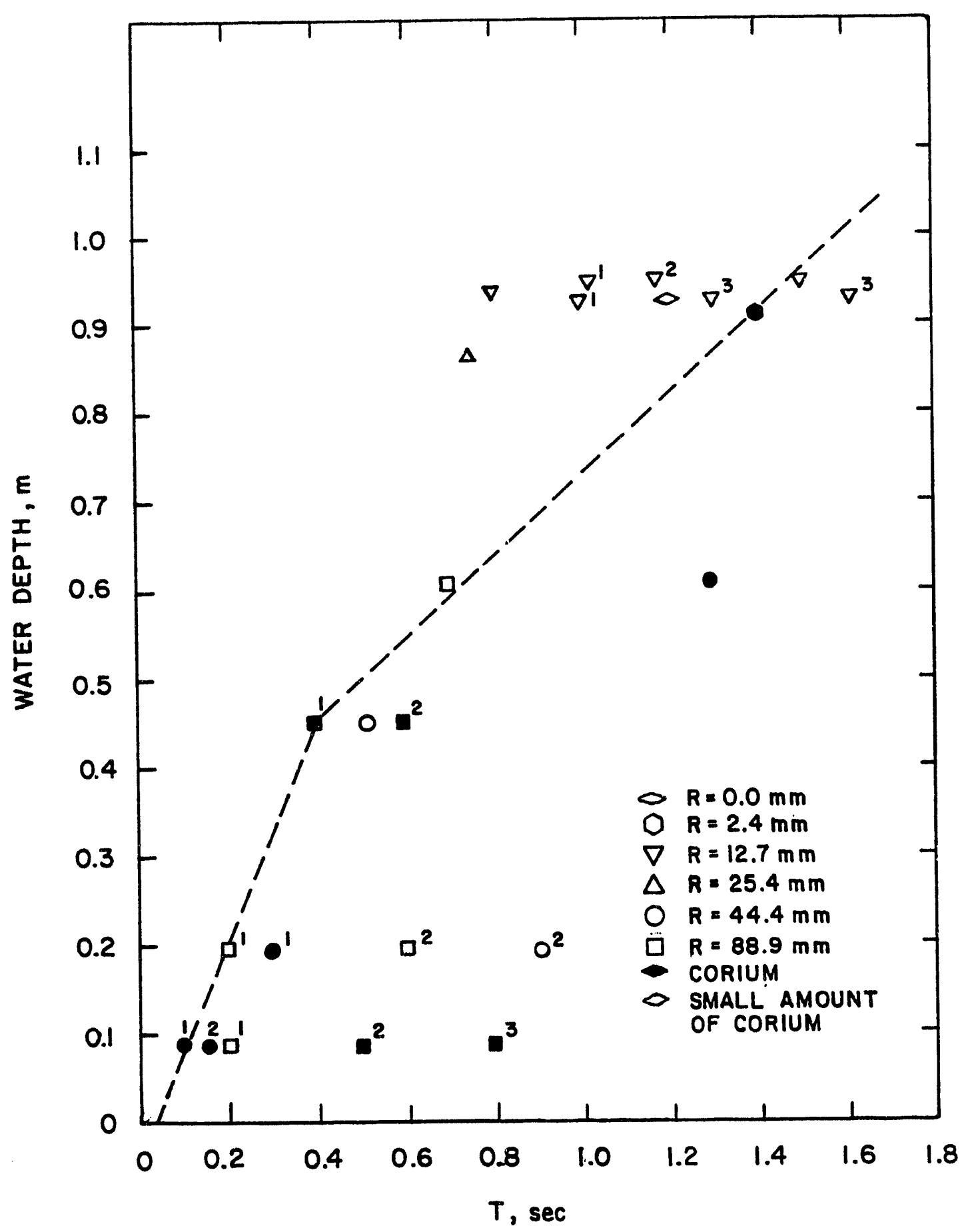

Fig. 4-15 Thermal Response of the Thermocouple in the Interaction-Vessel Water for Experiment CCM-3 


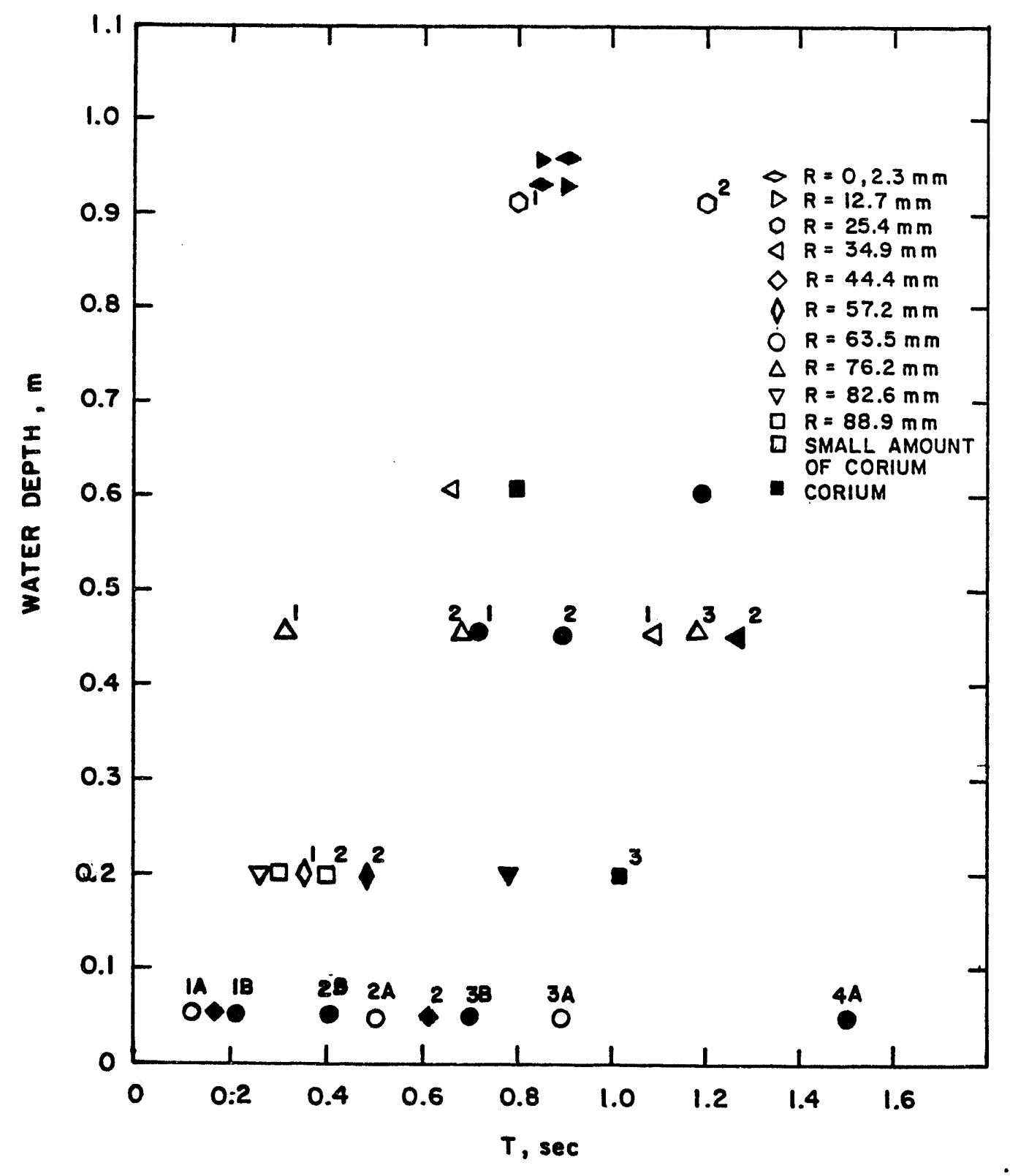

Fig. 4-16 Thermal Response of the Thermocouple in the Interaction-Vessel Water for Experiment CCM-4 


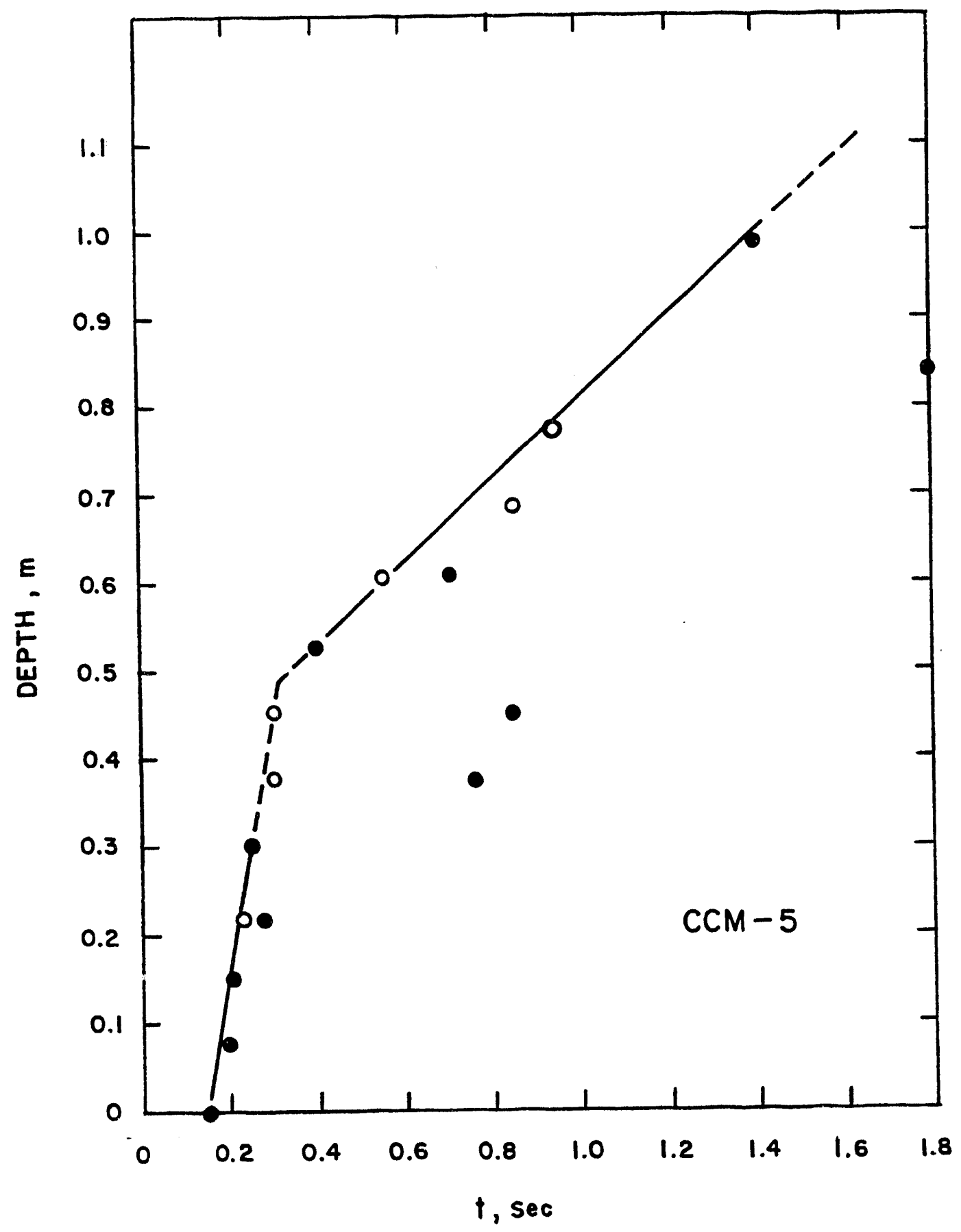

Fig. 4-17 Thermal Response of the Axial Centerline Thermocouples in the Experiment-Vessel Water for Experiment CCM-5 
contact time is $3.4 \mathrm{~m} / \mathrm{s}$.

As the corium descended through the water, it contacted or was near enough to the thermocouples and void detectors to produce responses. The responses of the void detectors are shown in Fig. 4-18 and those of all thermocouples in Fig. 4-19. It is not possible to determine if the void detector responses were caused by steam or adhering corium. However, because steam can be present at a given depth only after the arrival of corium, a void detector response gives an upper bound on the arrival time of the corium stream or particle swarm. In Fig. 4-19, only the strongest responses have been assumed to indicate corium contact. Multiple responses are indicated, e.g., 1, 2, 3, above the symbols; corium contact is indicated by a shaded symbol. From the Hycam pictures (Tables 3.18 and 3.19 ), the corium injection duration was about $0.5 \mathrm{~s}$ although particulates were observed in the water as 1 ate as $4.5 \mathrm{~s}$. Therefore, the 1ate thermal responses were probably caused by fluidized corium due to water circulation in the vessel.

Both the axial centerline thermocouples show a significant decrease in the corium penetration velocity, indicating break-up, at a depth of about 10 corium stream diameters.

During the test there were $13 \mathrm{~g}$-moles of steam generated, of which 2 g-moles were used to form an equivalent number of moles of hydrogen.

\subsubsection{Test CCM-6}

This experiment was similar to test CCM-5, except that the gas/vapor space was increased by a factor of 2.48 , and the water was at its saturation temperature of $101.5 \mathrm{C}$. A plot of the responses of the thermocouples located on the axial centerline of the water in the experiment vessel is shown in Fig. 4-20. The responses of the void detectors are shown in Fig. 4-21. The open data symbols represent a small signal increase, possibly an indication of an expulsion of gas and particulate from the thermite vessel preceding the main body of the melt.

The responses of the thermocouples located below the water free surface are shown in Fig. 4-23. Shaded symbols again represent corium contact. Numbers above the symbols indicate multiple responses. At a water depth of $1.06 \mathrm{~m}$, there are two thermocouples at radii of 50.8,76.2, 152.4 and $177.8 \mathrm{~mm}, 180^{\circ}$ apart at four angular orientations (see Appendix G). The two thermocouples are designated $A$ and $B$.

The thermocouple data indicate jet breakup within 14 jet diameters. This is confirmed by Hycam pictures which show that breakup was complete at that depth. During the test, a total of $77.04 \mathrm{~g}$-moles of steam were generated. $23.379 \mathrm{~g}$-moles of hydrogen were formed from steam-metal reactions.

\subsection{Comparison of Break-up Length and Mass Length}

As mentioned in Section 1.3.2, maximum jet penetration distance is limited by initial available jet fluid if the "mass length" is not too much greater than the jet breakup length. The initial available jet fluid expressed in terms of mass length compared with the measured jet break-up length as shown in Figure 4-23. 


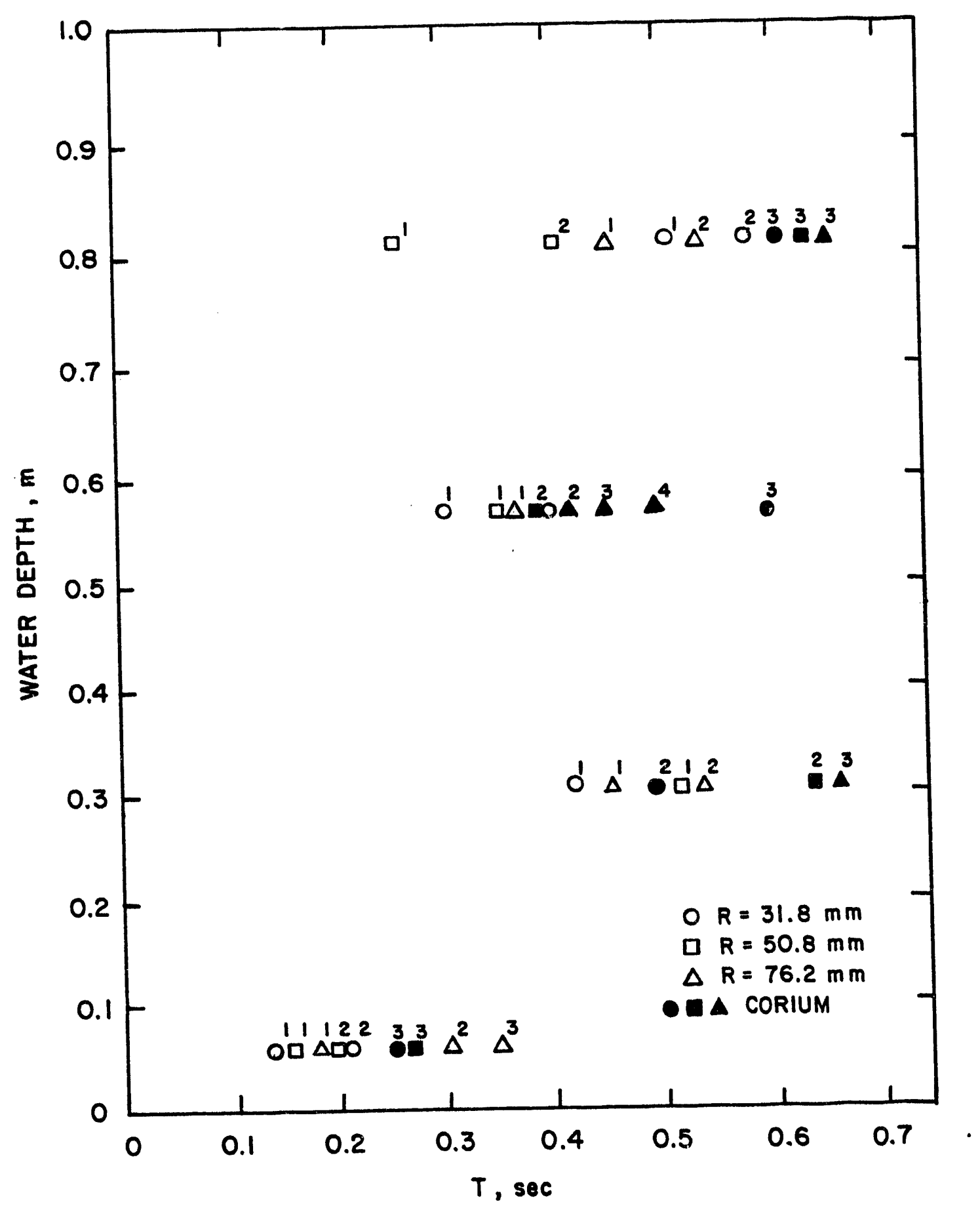

Fig. 4-18 Void Detector Response for Experiment CCM-5 


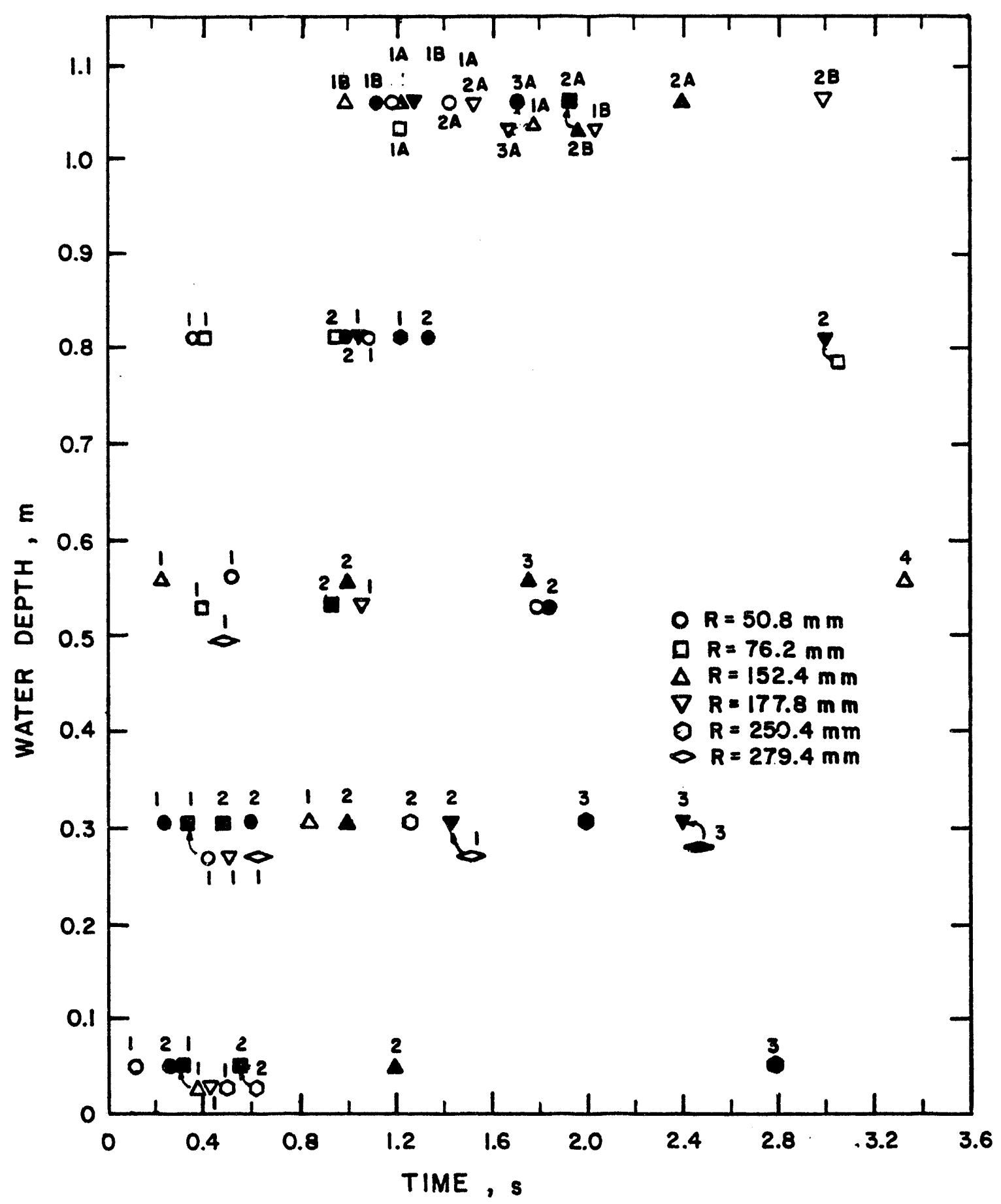

Fig. 4-19 Thermal Response of the Thermocouples in the Water of the Experiment Vessel for Experiment CCM-5 


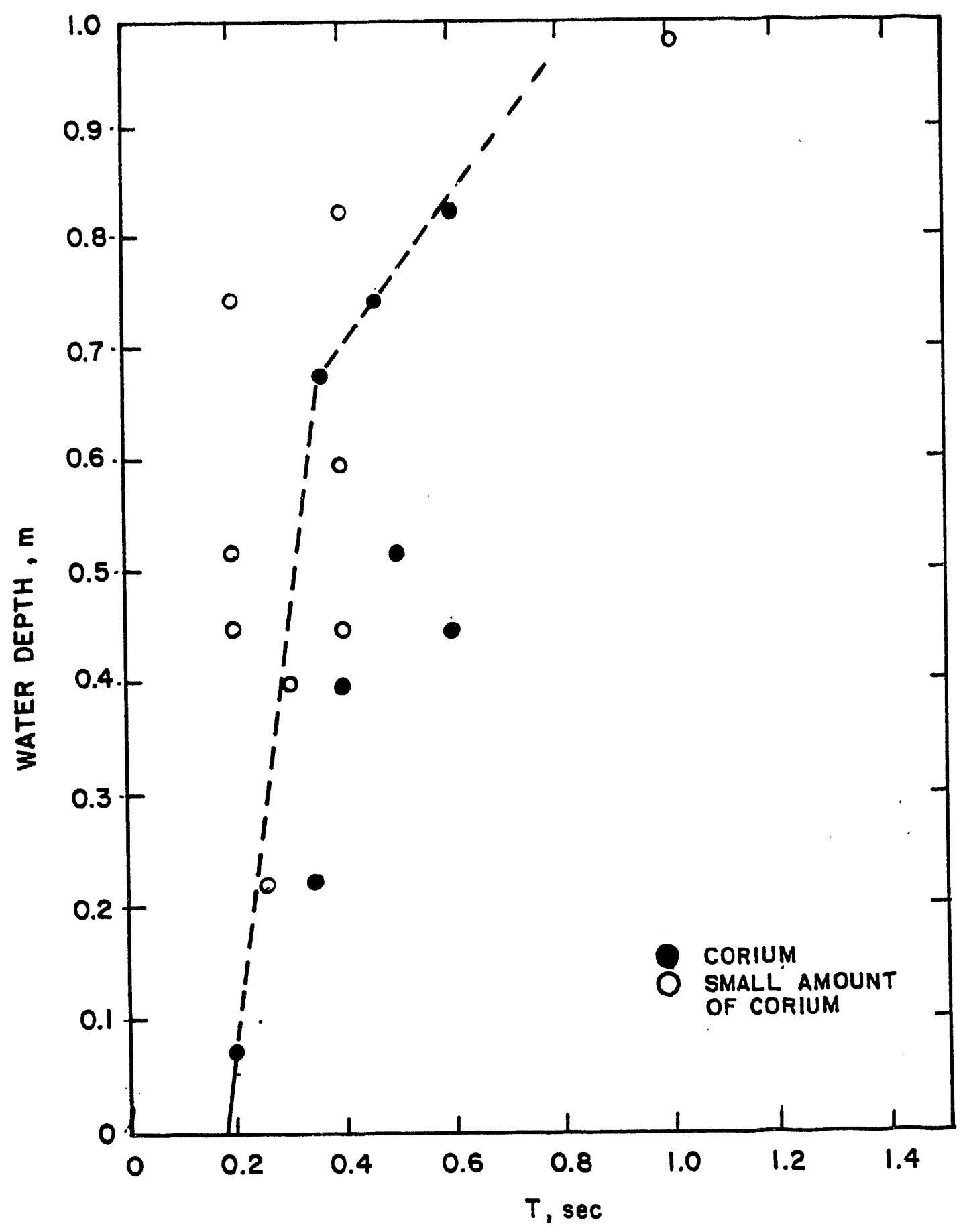

Fig. 4-20 Thermal Response of the Thermocouples on the Axial Centerline of the Water in the Experiment Vessel for Experiment CCM- 6 


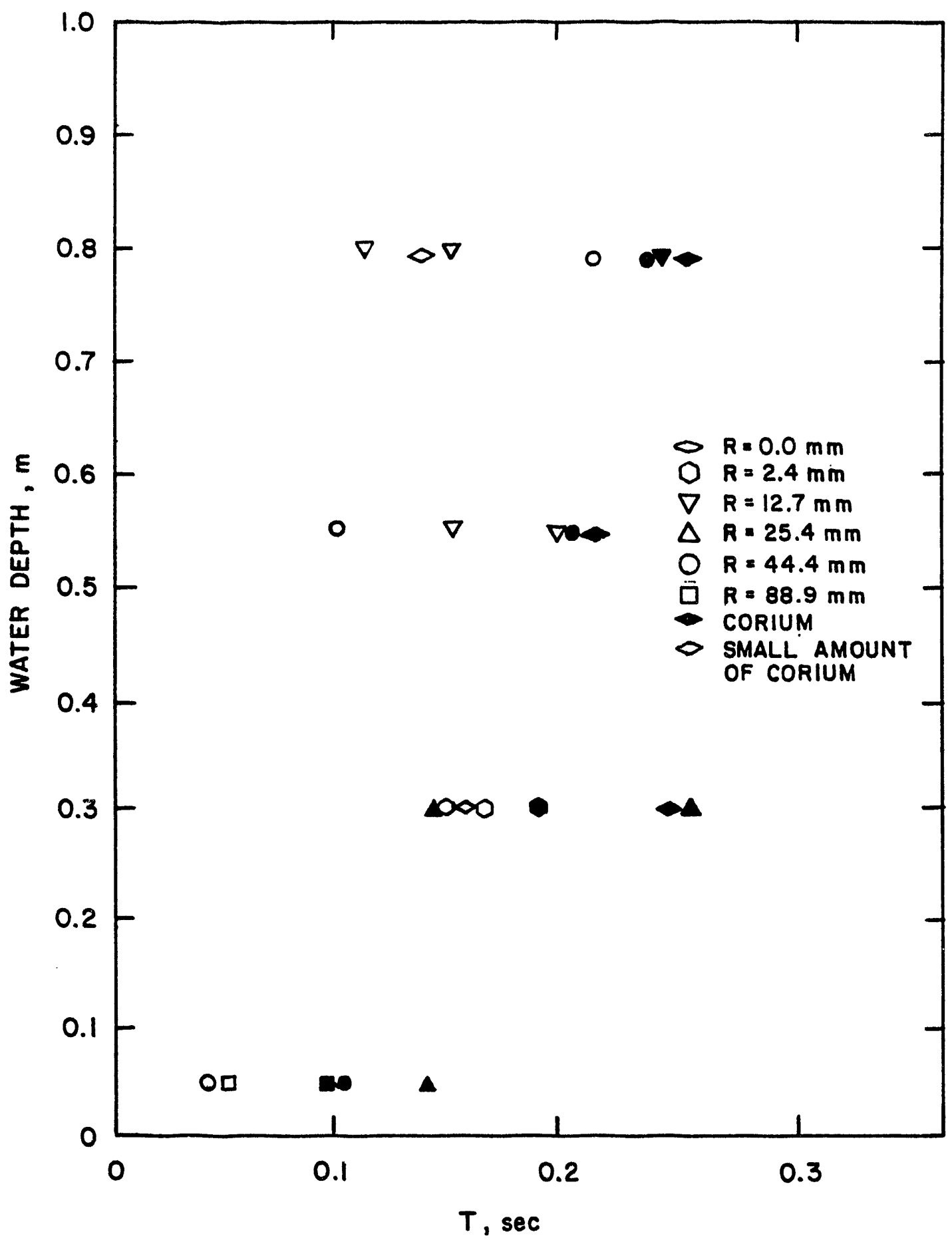

Fig. 4-21 Void Detector Response for Experiment CCM-6 


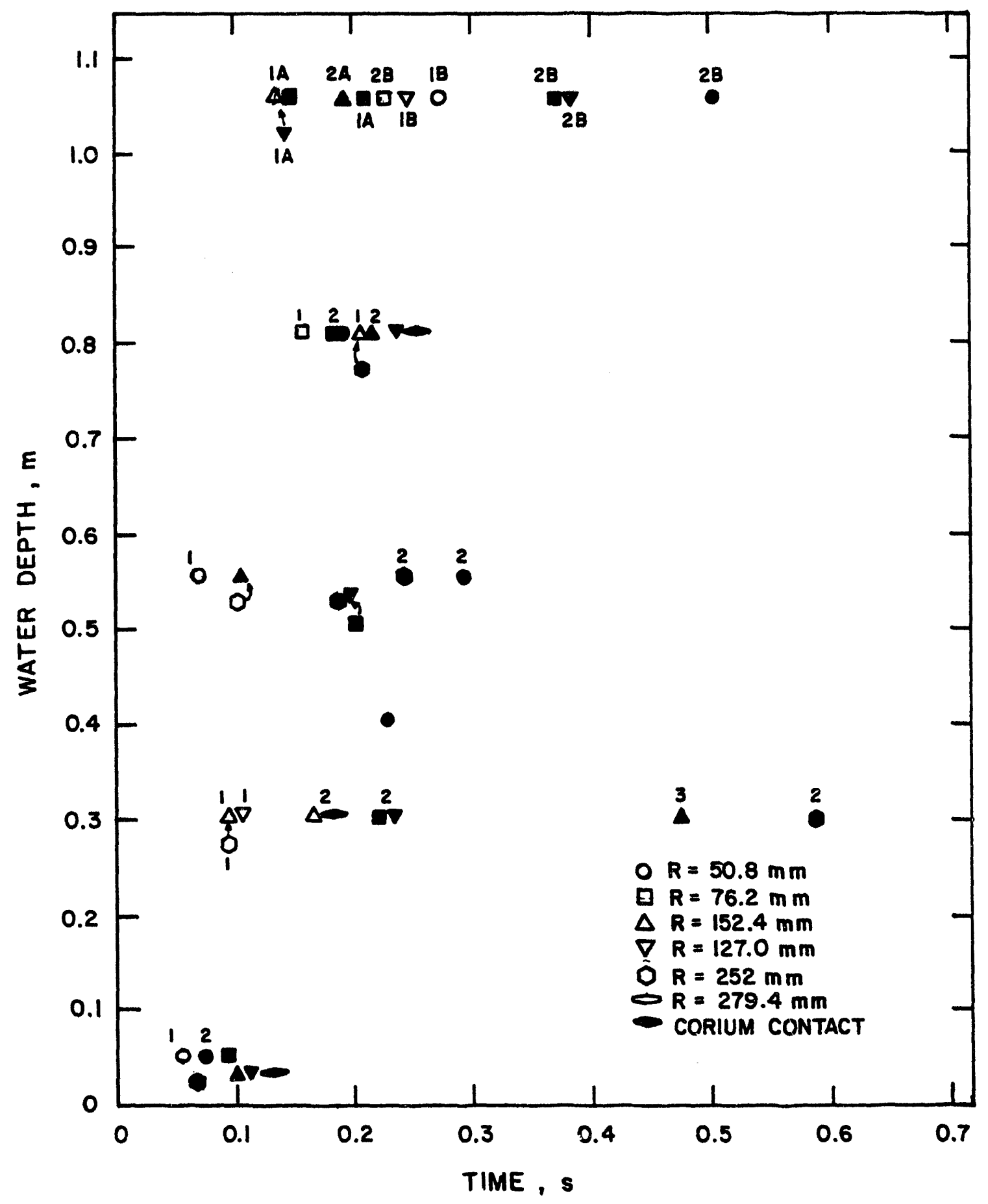

Fig. 4-22 Thermal Response of the Thermocouples in the Water of the Experiment Vessel for Experiment CCM- 6 


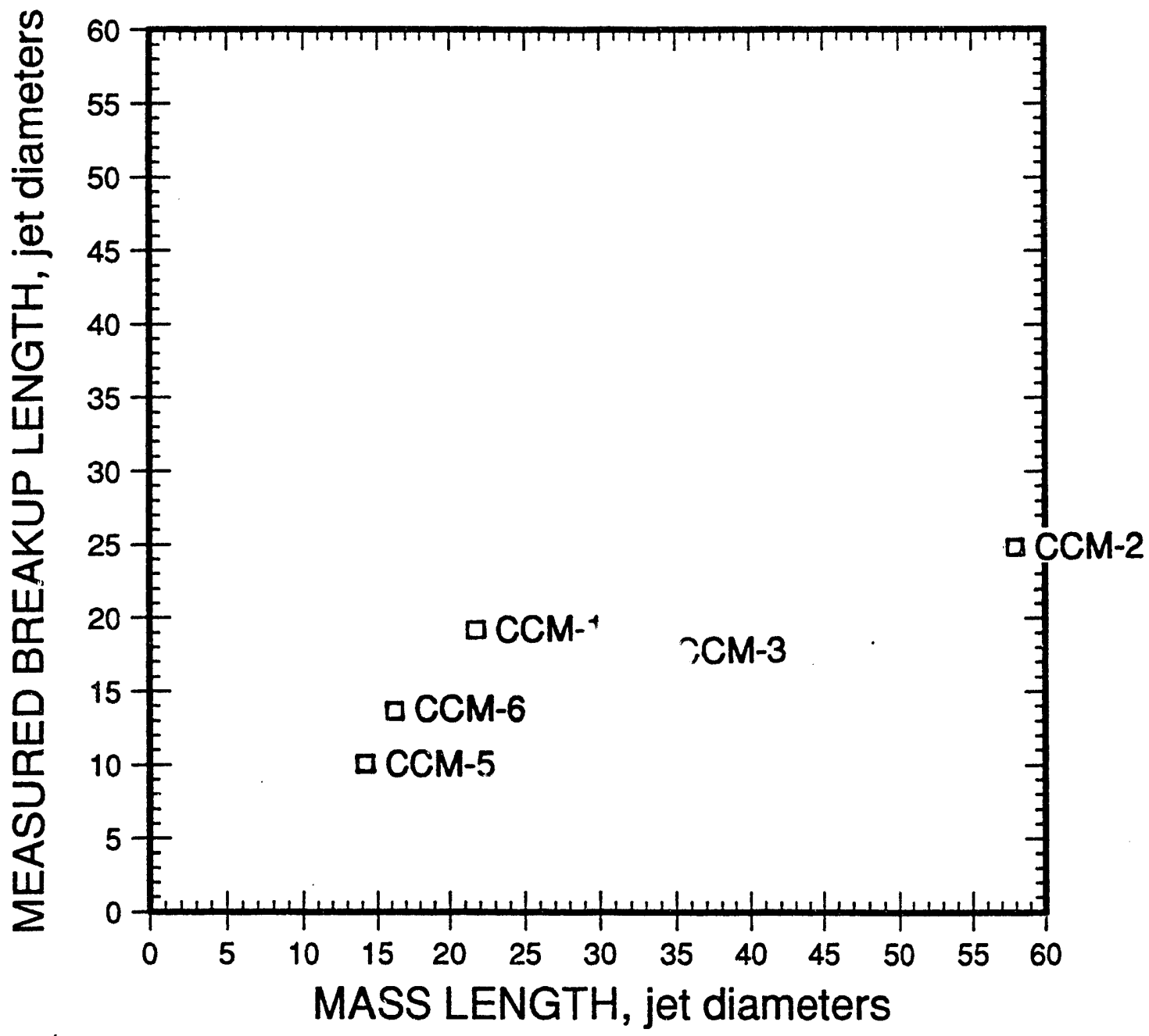

Figure 4-23. Comparison of Mass Length and Breakup Length. 
It is clear that for CCM-1,5 and 6 , the jet break-up length is proportional to the initial mass length, i.e. melt drainage stopped before the jet reached a a quasi-steady breakup length. On the other hand, quasi-steady breakup may have been achieved for CCM-2 and 3 , since the mass lengths were much longer than the breakup length for these two tests. 


\subsection{Review of Previous Studies on Jet Breakup}

On the basis of theoretical considerations, the interactions that melt jets undergo with water are expected to encompass a number of different regimes ${ }^{1,6}$. The different regimes reflect the various instability processes that can cause a jet to break into fragments or droplets. Specifically, the existence of individual regimes corresponds to the predominance of a particular instability/breakup mechanism. Jets having large diameters exceeding the Taylor wavelength are expected to breakup up at the leading edge due to the Taylor instability ${ }^{15}$. In contrast, jets having sufficiently small diameters will fragment along their length as the result of capillary wave growths ${ }^{16,17}$. For intermediate diameters, the breakup behavior is expected to include a regime where droplets are formed by erosion of the jet column arising from the growth of Kelvin-Helmholtz instabilities ${ }^{2}$. Although three regimes are discussed here for the purposes of illustration, it is possible that other regimes and breakup processes also exist, depending additionally upon other jet and coolant variables including jet velocity and coolant temperature.

Typically, analytical studies of jet instability examine the growth of infinitesimally small surface waves on an initially undisturbed liquid cylinder ${ }^{16,18}$. This allows linearization of the governing equations, making the problem tractable. Although linear instability analysis has often been used to predict jet breakup length, it is strictly correct for small perturbations only; when a surface wave reaches a finite amplitude, the linearized equations are no longer applicable. Although these (and more advanced) analyses are valuable for making qualitative predictions about jet breakup, the numerical val"es obtained should generally be considered order-of-magnitude estimates only.

The observed breakup processes of liquid jets in gases fall into three general regimes. These may be termed laminar (Rayleigh), turbulent, and Weber number controlled ${ }^{19}$. In Rayleigh and turbulent breakup, capillary forces drive the breakup. In the Weber number controlled regime, breakup is driven by dynamic pressure forces created by the motion of the jet relative to the ambient fluid. Atomization is an extreme example of Weber number controlled breakup. It occurs when the ambient Weber number is very high.

Windquist mapped jet breakup regime described in the literature on liquid jets in air as a function of jet Reynolds and Ohnesorge numbers, as first proposed by ohnesorge ${ }^{20}$. These dimensionless groups include all the relevant variables for horizontal liquid jets issuing into the same ambient fluid (i.e., atmospheric air). The breakup regime passes from Rayleigh breakup to turbulent breakup to atomization as the Ohnesorge number is decreased and the Reynolds number is increased.

A comprehensive review of liquid jet breakup literature relevant to water reactor severe accidents has been made by Ginsberg. ${ }^{6}$. He constructed a qualitative plot of breakup length as a function of the ambient Weber number. According to Ginsberg, when We anb $<1$ approximately, Rayleigh breakup occurs. As We increases, the breakup becomes turbulent. At very high We $(>100)$, atomization may occur. However, Ginsberg notes that atomization is typically seen only when the jet nozzle is very small. 
Liquid threads or laminar jets in a vacuum are subject only to capillary instability. The capillary instability of inviscid liquid threads was first analyzed by Rayleigh ${ }^{21}$. He showed that the liquid cylinder breaks into uniform drops whose diameter is about twice that of the jet. Weber derived an expression for jet breakup length, taking into account the effect of jet viscosity

$$
\frac{L}{D}=C\left(\sqrt{W e_{j}}+\frac{3 W e_{j}}{R e_{j}}\right) .
$$

Grant and Middleman correlated their experiment data with a very similar expression, supporting Weber's analysis ${ }^{23}$. Tomotika analyzed the breakup of viscous liquid threads in viscous liquids ${ }^{17}$. He found that the size of the drops produced by the breakup increases with the ambient fluid/jet viscosity ratio. For a value near unity, the drop diameter is about six times that of the jet. Experiment data are in good agreement with Tomotika's findings ${ }^{24}$.

Turbulent jet breakup length is also typically correlated by jet Weber number, but is strongly affected by the jet turbulence intensity $y^{25}$. The transition from laminar (Rayleigh) to turbulent breakup is affected by nozzle. geometry ${ }^{26}$. However, this effect disappears when the jet turbulence is very well developed ${ }^{27}$.

G. I. Taylor made the first analysis of the breakup of atomizing jets. Applying the results of his study of wind-generated ripples on the surface of a viscous fluid ${ }^{28}$, he predicted that the breakup length of a low melting point metal jet in water should depend only on the jet-ambient fluid density ratio ${ }^{2}$.

$$
\frac{L}{D} \sim \sqrt{\frac{W e_{j}}{W e_{a}}}=\sqrt{\frac{\rho_{j}}{\rho_{a}}} .
$$

Taylor suggested a constant of proportionality of 5 . This relation was later rederived by Levich'.

Jets may break up due to the effects of the relative motion of the jet and ambient fluid without significant atomization. Biakup of water jets in counterflowing air was observed by Hoty and Taylor ${ }^{29}$. In their experiments, atomization occurred only very near the nozzle, and did not cause the loss of a significant part of the jet fluid. Further from the nozzle, the jet developed helical waves. The eventual breakup of the jets was shown to be due to form drag on these waves. Some explanation of this behavior is suggested by the analysis of Lin and Kang ${ }^{30}$ and Green and Laws ${ }^{31}$. Lin and Kang showed that atomization will be damped out if the perturbations on the jet surface at the nozzle are not sufficiently large. Green and Laws showed that a 'twisting' flow satisfies the equations of motion for ideal fluid jets.

Helical or sinuous waves have also been seen in water/gas inverted annular flows. However, when the relative velocity is sufficiently high, a transition to drop ejection from roll waves occurs ${ }^{32}$ with the jet breakup length being correlated by 


$$
\frac{L}{D}=695\left(R e_{j}\right)^{-0.53} \sqrt{W e_{j}}\left(\frac{W e_{G, \text { rel }}}{\alpha^{2}}\right)^{-0.545}
$$

The last term in brackets is roughly the ambient Weber number and $\alpha$ is the void fraction of the water/gas inverted annular flow. Thus, the last two terms make up an expression very similar to that of Taylor. The first term might be supposed to be a correction for jet turbulence.

Viscous jets have been shown analytically to be subject to long wavelength instability caused by dynamic pressure forces $33,34,35$. Small diameter water jets in water ${ }^{36}$ and molten uranium alloy jets in liquid sodium ${ }^{37}$ have been observed to undergo 'pedal breakdown' in which the jet is repeatedly deflected laterally.

Spencer et. al., photographed the breakup of Wood's metal streams poured into water ${ }^{4}$. They varied the metal temperature and water subcooling to achieve film boiling, nucleate boiling, and negligible boiling on the metal surface. Breakup lengths increased with increasing melt and water temperature about 20 to $>50$ initial jet diameters.

A series of experiments with water jets penetrating liquid nitrogen and Freon-11 was conducted by Saito et. al. ${ }^{38}$. Jet diameter and velocity were varied for each of the continuous phase liquids. Because the jet and continuous phase liquid densities were similar, the dispersed jet fluid did not fall to the bottom of the pool, but was swept out by the vapor flow. Saito et. al. termed the length of the jet/vapor column beneath the pool surface 'penetration length.' They obtained an extremely good correlation for penetration length as a function of jet/continuous phase density ratio and Froude number,

$$
\frac{L}{D}=2.1 \int \overline{\frac{\rho_{j}}{\rho_{c}}} \mathrm{Fr}^{1 / 2} .
$$

An analytical study which predicts the behavior of jet breakup using a linear Kelvin-Helmholtz instability analysis to examine the stability at the jet/steam and steam/water interfaces of a steam sheet was first proposed by

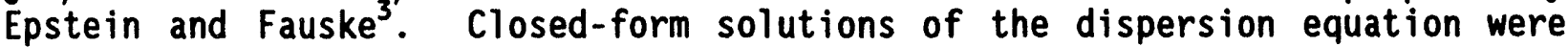
established in the limits of very thick and very thin steam films. It is concluded that the presence of a thick steam film may reduce by more than one order of magnitude the degree of instability of a core-melt jet predicted in the absence of the steam film. According to Epstein and Fauske's analysis, jet breakup length is also a function of the density ratio, similar to Taylor's prediction, i.e., Equation (5-2). However, the ambient fluid is taken to be either steam or water, depending on whether the steam film is thick or thin.

Another approach to the prediction of jet breakup is the use of a drop fragmentation model. Pilch correlated data in the literature for liquid drop breakup in gases ${ }^{39}$. He found that the largest droplets remaining after breakup were much larger than would be expected from the stability criteria We $=W_{\text {crit }}$, due to the deceleration of the drop during breakup. He also found that the time for complete breakup fell within the range of $T+=3-6$, even though the actual breakup times varied over two orders of magnitude. The dimensionless time, $\mathrm{T}^{+}$, is defined as $T D / \epsilon V$ where $\epsilon$ is the square root of jet to ambient fluid density 
ratio.

Chu and Corradini developed a drop breakup model following Pilch's idea that breakup is caused by Rayleigh-Taylor instabilities on the windward side of the drop ${ }^{40}$. They later used this model to approximate the injection of jet fluid from the jet leading edge during its initial penetration of the ambient liquid. Their drop breakup model assumes that a drop is formed as a result of RayleighTaylor instability and then undergoes a number of subdivisions such that

$$
\frac{d}{D}=\operatorname{Exp}\left(-C_{1}(T+)^{c_{2}} W^{c_{3}}\right)
$$

where $C_{1}$ depends on $\epsilon$, and $C_{2}$ as well as $C_{3}$ are constants. Good agreement was obtained with the observed rate of breakup of a mass or iron-alumina generated by a thermite reaction in one of the FITS tests at Sandia.

It is important to note that an upper limit log-normal drop diameter distribution is produced by many breakup processes including both hydrodynamic breakup and vapor explosion ${ }^{41}$. Thus, it is not possible to make more than gross generalizations about the breakup process from the drop diameter data.

Analysis of drop diameter data is further complicated by the fact that the diameters of dispersed melt drops in volatile liquids may not be determined solely by hydrodynamic breakup. Drops may also expand before solidifying, leaving voided interiors. Brauer et, al., proposed that this phenomenon is due to vaporization of entrapped liquid ${ }^{42}$.

\subsection{THIRMAL Computer Code}

The Thermal-Hydrodynamic Interaction and Reaction of Melt and Liquid (THIRMAL) computer code has been developed to provide capability to calculate the breakup and quenching behavior of melt entering water as a jet. In this model the jet material is assumed to be dispersed from the coherent jet column as well as from the vortex ball formed at the jet leading edge. Kelvin-Helmholtz instability is assumed to be the surface erosion mechanism on the coherent column following the method of Fauske and Epstein. ${ }^{3}$ Shearing by the ambient fluid accounts for the material eroded from the leading edge. The most probable sizes of particles dispersed from the column and the leading edge are thus predicted from different dispersion mechanisms. A detailed description on the interfacial instability as well as the jet breakup mechanisms is documented in Refs. [43] and [44]. Given the diameter, velocity, and temperature of melt entering the water, THIRMAL mechanistically calculates the time dependent jet penetration distance, breakup of the jet into droplets, quenching of the jet column and individual droplets, void formation and boilup of the surrounding water, steam/hydrogen formation due to melt-water interaction, steam condensation within subcooled water, and the resulting system pressurization. THIRMAL calculates the erosion of the jet into droplets by shearing of the leading edge and interfacial instability along the vapor covered jet column. Heat transfer from the jet to surrounding water by film boiling is considered. Local conditions of temperature and velocity within the jet are calculated using a multicell, Lagrangian numerical formulation. Temperatures and velocities of droplets formed from the breakup of the jet column are also obtained from Lagrangian calculations along 
the droplet/particle trajectories.

The assumption that a continuous vapor film exists between the melt jet and surrounding water during the interaction, as described in Refs. [43] and [44], becomes inadequate when a continuous liquid phase no longer exists in the vicinity of the jet. Specifically, when the steam generation rate is high enough to globaliy levitate the liquid phase, water may be dispersed in the form of droplets while steam becomes the continuous phase. When this flow dispersion occurs, the jet surface erosion is no longer driven by the steam flow in the thin vapor film but rather by the steam flow surrounding the jet. In general, the steam velocity is much higher in a thin vapor film than for a steam flow distributed over the vapor continuous region. As a result, erosion of the jet is typically reduced when dispersed flow occurs. In order to identify the flow regime of the multiphase (i.e., melt particles/water/steam mixture) region, the detailed local void fraction, phase temperature, and velocity in the mixing region become important. Furthermore, the characteristics of the jet debris depends strongly on the history of melt particulates in the multiphase mixing region. In simulating the flow conditions of the multiphase mixing region, previous investigators usually solved governing equations of mass, momentum, and energy for all the phases (i.e., water, steam, and melt particulates). The resulting numerical process generally involves solving nine conservation equations (for one dimension), one matching equation, and one equation of state (see e.g., References [45] and [46]). In the THIRMAL code, a simple onedimensional multiphase flow model using drift-flux correlations is solved to calculate the local flow conditions as described below.

In the THIRMAL code, the two-dimensional effects on the jet breakup as described above are estimated by using a "mixing zone" approximation. The mixing zone is the region containing the jet column, dispersed particulate, steam and water. The geometry of the mixing zone can be considered as a cylinder with diameter and length varying in space and time, as sketched in Figure 5-1. The existence of a finite mixing zone is demonstrated by recent Wood's metal/Freon experments carried out at ANL. ${ }^{47}$ The single-phase water region (i.e., region outside of the mixing zone) is not considered in the model equations. The onedimensional mass equation for the steam is

$$
\frac{\partial}{\partial t}\left(\alpha \rho_{v} A\right)+\frac{\partial}{\partial z}\left(\alpha \rho_{v} A U_{v}\right)=\dot{m}_{e}-\dot{m}_{c}
$$

where

$$
\begin{aligned}
& \dot{m}_{e}=\text { evaporation rate per unit length, and } \\
& \dot{m}_{c}=\text { condensation rate per unit length. }
\end{aligned}
$$

The cross-sectional area of the mixing zone, $A$, is defined as the area where the dispersed particles have migrated laterally. As analyzed in Reference [44], the perpendicular components of the velocity of the eroded material with respect to the jet surface is the erosion velocity, $U_{E}$. For a high temperature melt surface in the film boiling regime, the erosion velocity has been shown to be $[44]$ 


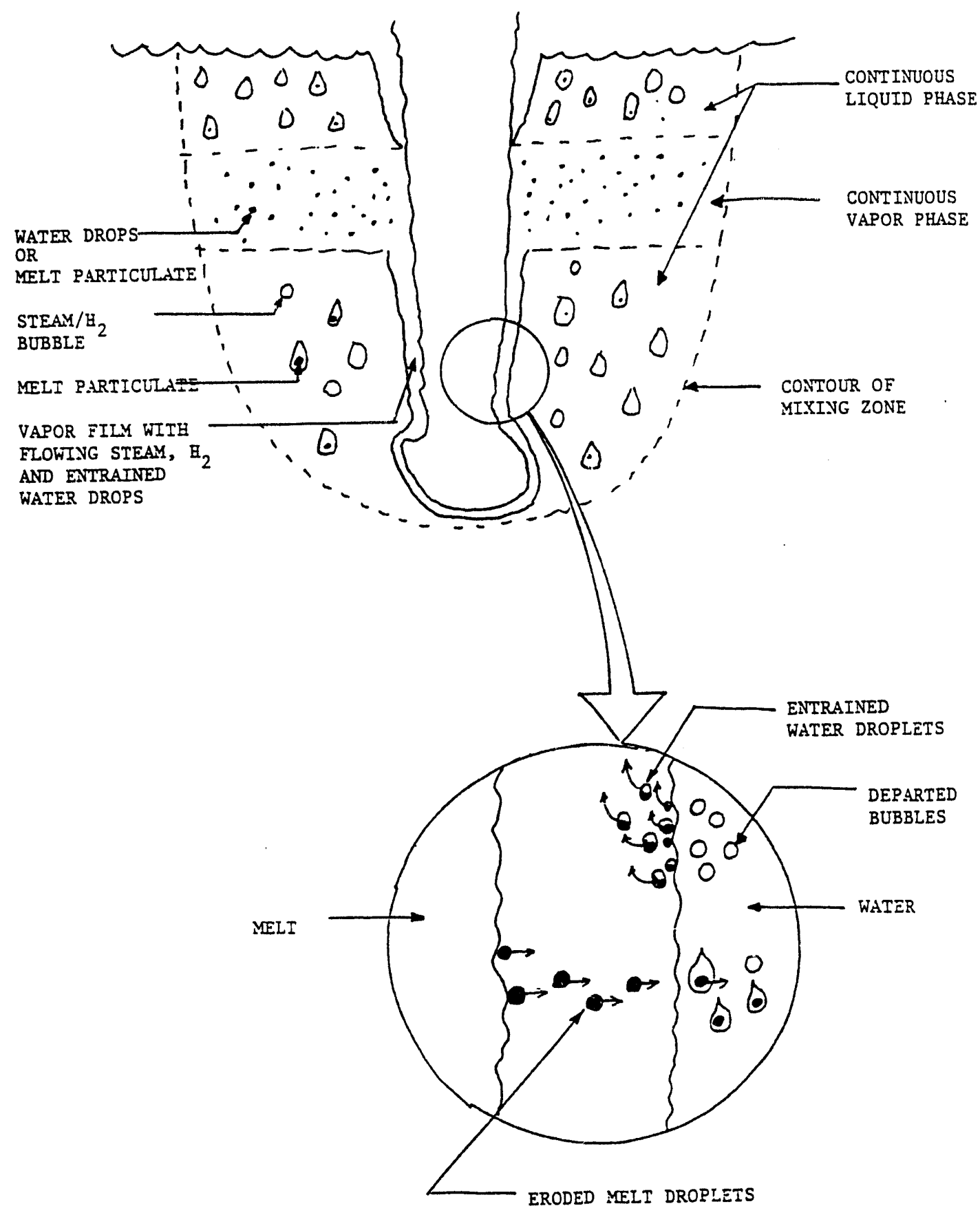

Figure 5-1. Configurations of Mixing Zone 


$$
U_{E}=\frac{\left[K \rho_{v} \rho_{m}\left(U_{v}-U_{m}\right)^{2}-\sigma_{m} K_{p}\left(K \rho_{v}+\rho_{m}\right)\right]^{1 / 2}}{K \rho_{v}+\rho_{m}}
$$

where $K=\left(1+\cosh k_{p} \delta\right) / \sinh k_{p} \delta$, and the most probable wave number, $k_{p}$, is

where

$$
k_{p}=\frac{2 \rho_{v} u_{v}^{2} K}{3 \sigma_{m}}
$$

$$
\delta=\text { vapor film thickness, }
$$

and $v$ and $m$ denote vapor and melt, respectively. After a melt droplet is eroded from the jet surface with erosion velocity $U_{E}$, the lateral motion of the particulate is governed by the equation,

$$
\frac{1}{6} \pi d_{p}^{3} \rho_{P} \frac{d U_{P L}}{d t}=-C_{D}\left(\frac{1}{4} \pi d_{P}^{2}\right)\left(\frac{1}{2} \bar{\rho} U_{P L}^{2}\right) ; U_{P L}(0)=U_{E}
$$

where

$$
\begin{aligned}
& d_{p} \quad=\text { particle diameter, } \\
& U_{P L}=\text { particle lateral velocity, } \\
& \bar{\rho} \quad=\rho_{l}(1-\alpha)+\rho_{v}(\alpha) \text { the average density, and } \\
& C_{D} \quad=\text { drag coefficient. }
\end{aligned}
$$

The lateral distance that a particle can migrate is obtained by integrating Equation (5-9) twice to yield

$$
R_{M Z}=\int_{0}^{t} U_{P L} d t=\frac{1}{c}\left[\ln \left(c t+\frac{1}{U_{E}}\right]-\ln \frac{1}{U_{E}}\right]
$$

where $R_{M z}$ is the radius of the mixing zone and

$$
C=\frac{3}{4} \frac{C_{D} \bar{\rho}}{d_{p} \rho_{m}}
$$

In solving Equation (5-6), the area of mixing zone is determined by numerically integrating Equation (5-10). Additionally, the code also allows the mixing zone of the cell at $\left(z_{i}, t_{j}\right)$ to be replaced by the cell at $\left(z_{i-1}, t_{j}\right)$ at the time $t_{j+k}$ traced., if the mixing zone of the cell at $\left(z_{i-1}, t_{j}\right)$ is greater than that of the cell at $\left(z_{i}, t_{j}\right)$. This means a cell can replace a smaller cell right above it after $k$ time steps when all the vapor has risen into the new cell, with the time lag determined by $k=\delta z /\left(\delta t U_{v}\right)$. Note that the courant criterion for 
stability has been applied in selecting the time step, i.e., $U_{v} \delta t / \delta z<1$.

The basic assumption of the model in solving for the flow conditions of the mixing zone is that the void fraction and vapor superficial velocity can be related by a single correlation, thus elmininating of the vapor momentum equation. A further simplification is made by assuming that the liquid velocity is negligible compared to the vapor velocity. This means that the mixing zone is treated as a quasi-static bubbling pool with little net liquid flow. Kataoka and Ishij ${ }^{14}$ correlated a large number of experimental data for pool void fraction over wide ranges of gas flux, vessel diameter, system pressure, and liquid physical properties. They concluded that in bubbling or pool boiling systems, the void fraction can be represented by a drift flux correlations as

$$
\alpha=\frac{1}{c_{0}+\sqrt{2} / j_{v}{ }^{+}} ; \text {for } j_{v}{ }^{+} \leq 0.5
$$

and

$$
\alpha=\frac{1}{c_{0}+0.03\left[\frac{\rho_{v}}{\rho_{l}}\right]^{-0.157} N_{\mu l}^{-0.562} / j_{v}{ }^{+}} ; \text {for } j_{v}{ }^{+} \geq 0.5
$$

where

$$
\begin{aligned}
& j_{v}^{+}=j_{v} /\left(\sigma g \Delta \rho / \rho_{l}^{2}\right)^{1 / 4} ; \text { dimensionless gas superficial velocity, } \\
& c_{0} \approx 1.2-0.2\left[\frac{\overline{\rho_{v}}}{\rho_{l}} ;\right. \text { the distribution parameter, } \\
& N_{\mu l}=\mu_{l} /\left(\rho_{l} \sigma(\sigma / g \Delta \rho)^{1 / 2}\right)^{1 / 2} \text {, dimensionless liquid viscosity, }
\end{aligned}
$$

and $v$ and 1 denote vapor and 1 iquid phase, respectively.

The vapor velocity, $U_{y}$, in Equation $(5-6)$ is then replaced by either Equation (5-11) or Equation (5-12) depending on the gas superficial velocity and the void fraction can thus be determined, if the liquid evaporation rate and the vapor condensation rate are known.

The source term of Equation (5-6), the liquid evaporation rate depends on the number and temperature of melt particles which are either eroded from the jet or transported from elsewhere in the mixing zone. In order to obtain a temperature history of each group of particulates, their motion is tracked in Lagrangian coordinates rather than in Eulerian coordinates. Based upon the force balance on a single spherical particle in the axial direction, the velocity of particle can obtained by solving the equation, 


$$
\frac{1}{6} \pi \phi_{p}^{3} \rho_{p} \frac{d U_{p}}{d t}=-\left(\rho_{p}-\bar{\rho}\right)\left(\frac{1}{6} \pi \phi_{p}^{3}\right) g+F
$$

where

$$
F=\left\{\begin{array}{l}
0.5 A_{p} C_{D} \rho_{v}\left(U_{v}-U_{p}\right)\left|U_{v}-U_{p}\right| \text { for dispersed flow } \\
0.5 A_{p} C_{D} \rho_{l} U_{p}^{2} \text { for non-dispersed flow }
\end{array}\right.
$$

At each numerical grid cell during one time step, steam generated from the particulates (either just eroded from the jet or convected from the adjacent cells) is summed to yield the source term of Equation (5-6).

The energy (temperature) of each particulate is obtained from

$$
m_{p} \frac{d e_{p}}{d t}=m_{p} \dot{q}_{i n t}+h_{p l} \pi d_{p}^{2}\left(T_{l}-T_{p}\right)+h_{p v}\left(T_{v}-T_{p}\right)
$$

where

$$
\begin{aligned}
& e_{p}=\text { internal energy of particle, } \\
& \dot{q}_{i n t}=\text { particle internal heat generation rate, } \\
& h_{p l}=\text { heat transfer coefficient between particle and liquid, and } \\
& h_{p v}=\text { heat transfer coefficient between particle and vapor. }
\end{aligned}
$$

For a liquid-continuous regime, the third term on the right hand side of Equation (5-14) is set to zero, since the energy transferred from the particles is predominantly absorbed by the liquid. If flow dispersion occurs, particles are suspended in a vapor-continuous phase and the forced convective heat transfer coefficient is given by the spherical particle correlation,

$$
h_{p v}=\frac{k_{v}}{d_{p}}\left(2.0+0.6 \operatorname{Re}_{p}^{1 / 2} \operatorname{Pr}_{v}^{1 / 3}\right)
$$

where

$k=$ thermal conductivity,

Re $=$ Reynolds number,

$\operatorname{Pr} \quad=$ Prandtl number, and subscript $v$ denotes the vapor phase.

Under these conditions, heat transfer between suspenced particulates and water droplets is assumed to be zero (i.e., the second term on the right hand side of Equation (5-14) is zero). 
For a particle in a film boiling heat transfer regime, previous experimental and analytical studies on the heat transfer coefficients do not generally give satisfactory agreements, especially at high particle temperatures or high liquid subcooling. In the THIRMAL code, an analytical model for film boiling heat transfer from a spherical particle is incorporated that has been developed on the basis of observation of high temperature melt/water interactions. Usually, a descending particle is covered by a thin vapor blanket on its lower section and a stretched, roughly elliptical shaped vapor dome behind it. As the vapor dome grows due to steam generation, buoyancy overcomes surface tension to cause a steam bubble to depart from the vapor dome and rise to the water surface, as sketched in Figure 5-2. By assuming a linear temperature profile in the yapor film and a parabolic profile for both the liquid velocity and temperature in the liquid boundary layer, and by applying the integral momentum and energy equations, the vapor film thickness, vapor velocity, and liquid boundary layer thickness can be determined as describer. in Reference [43]. The heat transfer coefficient is

$$
h_{F B}=\frac{k}{\delta}+\varepsilon\left(T_{p}^{2}+T_{S A T}^{2}\right)\left(T_{p}+T_{S A T}\right)
$$

where

$\delta \quad=$ vapor film thickness, and

$\epsilon=$ Stefan-Boitzmann constant for radiation heat transfer.

If the liquid is subcooled, the temperature distribution in the liquid boundary layer can be described approximately by

$$
\frac{U_{\delta}}{R+\delta} \frac{\partial T_{l}}{\partial \theta}=\alpha_{l} \frac{\partial^{2} T_{1}}{\partial r^{2}}
$$

where

$$
\begin{aligned}
& U_{\delta} \quad=\text { the vapor velocity in the vapor film, } \\
& R \quad=\text { radius of the particle and } \\
& \alpha_{l} \quad=\text { liquid thermal conductivity. }
\end{aligned}
$$

The solution to Eq. $(5-17)$ is

$$
\frac{T_{l}-T_{\infty}}{T_{S A T} T_{\infty}}=\operatorname{erfc}\left(\frac{r}{2 \sqrt{\alpha_{1}(R+\delta) \phi}}\right)
$$

where

$$
\phi=\int_{0}^{\theta} \frac{\sin ^{4} \theta}{U_{\delta}} d \theta
$$




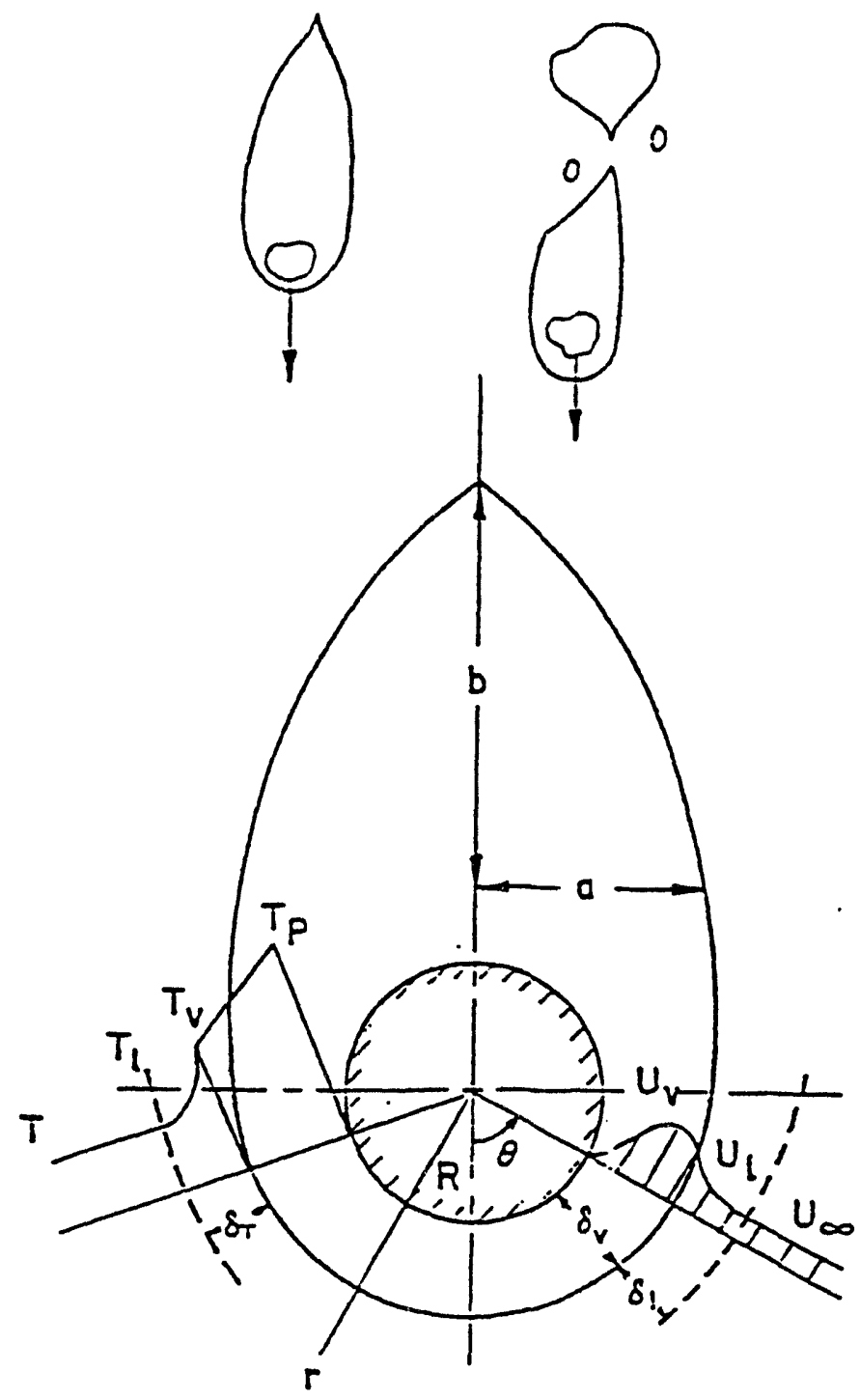

Figure 5-2. Film Boiling on a Spherical Particle 
The heat flux conducted into the subcooled liquid is

$$
q_{S U B}^{\prime \prime}=K_{1} \frac{\left(T_{S A T}-T_{\infty}\right) \sin ^{2} \theta}{\sqrt{\pi \alpha_{1}(R+\delta) \theta}}
$$

Therefore, the ratio of the heat conducted into the subcooled liquid to the total heat transferred from the particle is

$$
R_{S U B} \Delta=\frac{\int_{0}^{\pi} q_{S U B}^{\prime \prime}(R+\delta)^{2} d \theta}{\left(T_{P}-T_{S A T}\right) \int_{0}^{\pi} h_{F B} R^{2} d \theta}
$$

The ratio of the heat which is converted into the evaporation of liquid is, therefore, $\left(1-R_{\text {SUB }}\right)$.

Once the temperature of the particle has dropped below the minimum film boiling temperature, heat transfer will occur by nucleate boiling. A correlation suggested by Rohsenow [48] is used in THIRMAL code:

$$
\frac{C_{p l}^{3}\left(T_{w}-T_{S A T}\right)^{2}}{h_{f g}}=C_{s f}^{3}\left[\frac{h_{M B}}{\mu_{l} h_{f g}}\left(\frac{\sigma}{g \Delta \rho}\right)^{1 / 2}\right] P r_{l}^{3}
$$

where

$h_{\text {MB }}=$ nucleate heat transfer coefficient,

$T_{w} \quad=$ wall temperature, and

$C_{s f}=0.006$ to 0.014 , depending on the material of the surface.

When nucleate boiling occurs, it is assumed that all of the heat transferred from the particle generates the steam, since the liquid surrounding the particle is, at least locally, well saturated.

The energy equations of the vapor and liquid phases can then be formulated once the source terms generated by the melt particles are determined. Similar to Equation (5-6), the energy equations are 


$$
\begin{aligned}
& \frac{\partial}{\partial t}\left(\alpha \rho_{v} e A\right)+\frac{\partial}{\partial z}\left(\alpha \rho_{v} e_{v} A U_{v}\right)=-p\left[\frac{\partial}{\partial t}(\alpha A)+\frac{\partial}{\partial z}\left(\alpha A U_{v}\right)\right] \\
& +\sum_{i}\left(1-R_{s U B}\right)_{i} h_{p i} d_{p i}^{2} \pi\left(T_{p i}-T_{l}\right) \\
& +\sum_{i} h_{p v i} d_{p i}^{2} \pi\left(T_{p i}-T_{v}\right) \\
& +\frac{\sigma \alpha}{d_{B}} h_{v i}\left(T_{l}-T_{v}\right) A \\
& -\dot{m}_{c} h_{f g}
\end{aligned}
$$

for the vapor, and

$$
\begin{aligned}
& \frac{\partial}{\partial t}\left((1-\alpha) \rho_{l} e_{l} A\right)=-p\left[\frac{\partial((1-\alpha) A)}{\partial t}\right] \\
& +\sum_{i} R_{\text {SUBi }} h_{p l i} d_{p i}^{2} \pi\left(T_{\pi}-T_{l}\right) \\
& -\frac{6 \alpha}{d_{B}} h_{v l}\left(T_{l}-T_{v}\right) A+\dot{m}_{c} h_{f g}
\end{aligned}
$$

for the liquid phase. Note that the evaporation rate, $\dot{m}_{e}$, in Eq. (5-6) is defined as

$$
\dot{m}_{e}=\frac{\sum_{i}\left(1-R_{\text {SUB }}\right)_{i} h_{P l i} d_{p}^{2} \pi\left(T_{p i}-T_{l}\right)}{h_{f g}}
$$

The heat transfer coefficient between liquid and vapor, $h_{v l}$, can be correlated similarly to Eq. (5-15). For convective heat transfer between a liquid droplet and a vapor-continuous phase, i.e., as in dispersed flow, it yields:

$$
h_{v t}=\frac{k}{d_{d}}\left(2.0+0.6 \operatorname{Re}_{d}^{1 / 2} \operatorname{Pr}_{v}^{1 / 3}\right)
$$

where subscript $d$ denotes water droplets. For bubbles suspended in a liquidcontinuous phase, heat transfer from the bubble to the ambient liquid is given by [44]

where

$$
h_{v e}=\frac{k_{v}}{d_{B}}\left[2.0+1.13(\text { Pe K })^{1 / 2}\right]
$$


Pe $\quad=d_{B} U_{w} / \alpha$, Peclet number and

$K_{s} \quad=a$ constant depending on bubble velocity.

At this point, the only unknown is the steam condensation rate, $\dot{m}_{c}$. In the THIRMAL model, a semiemperical correlation for bubble collapse due to condensation in a subcooled liquid developed by Hewitt and Parker ${ }^{50}$ is applied to determine the condensation rate. According to Reference [50], the total collapsed volume of a bubble in subcooled water can be correlated as

$$
\frac{\Delta V}{V}=1-\left(1+\frac{2}{\sqrt{\pi}} J_{a} \frac{\sqrt{\alpha \Delta t}}{R_{0}}\left(\frac{1.44}{\Delta T_{\text {sus }}}\right)^{0.75}\right)^{-3}
$$

where

$$
\begin{array}{ll}
V & =\text { initial bubble volume, } \\
\Delta V & =\text { bubble collapsed volume due to condensation, } \\
J_{a} & =\rho_{l} C p_{l} \Delta T_{\text {sub }} / \rho_{v} h_{f g}, \text { Jacob number, } \\
R_{0} & =\text { initial bubble radius, and } \\
\Delta T_{\text {SUB }} & =\text { water subcooling. }
\end{array}
$$

Therefore, the total steam condensation rate at each cell can be approximated by $A \delta z \alpha \rho_{v} \frac{\Delta V}{V} / \delta t$, and the mass sink term in Eq. (5-6) becomes

$$
\dot{m}_{c}=\operatorname{A\alpha \rho } \frac{\Delta V}{V} / \delta t
$$

Note that in applying Equation (5-27), a constant bubble initial radius, $R_{0}$, is assumed for each cell. The bubble initial radius is approximated by the maximum stable bubble radius i.e.,

$$
R_{0}=(3 \sigma / \Delta \rho g)^{1 / 2}
$$

In the THIRMAL model, pressure gradients other than the hydrostatic head of the water pool are neglected. This assumption is valid as long as an abrupt pressure discontinuity such as occurs during a steam explosion is absent.

The numerical scheme for solving the conservation equations uses a finite difference method with upwind/donor cell differencing of the convective terms. The mass and energy equations are partial differential equations of the form,

$$
\frac{\partial}{\partial t}\left(\alpha_{i} \phi_{i}\right)+\frac{\partial}{\partial z}\left(\alpha_{i} U_{i} \phi_{i}\right)=S_{i}
$$


where

$\phi_{i}=\rho A$. for the mass equation, and

$\phi_{i}=e \rho A$ for the energy equations.

The finite difference form of Equation (5-30) is

$$
\frac{(\alpha \phi)_{I}^{J+1}-(\alpha \phi)_{I}^{J}}{\delta t}+\frac{(\alpha U \phi)_{I+1 / 2}^{J}-(\alpha U \phi)_{I-1 / 2}^{J}}{\delta z}=S_{I}^{J}
$$

where $I$ and $J$ are the indices of discretization in space and time, respectively. The spatial nodalization is sketched in Figure 5-3 and the convective terms are defined as

$$
\begin{aligned}
& (\alpha \cup \phi)_{I+1 / 2}=(\alpha F)_{e}, \text { and } \\
& (\alpha \cup \phi)_{I-1 / 2}=(\alpha F)_{W}
\end{aligned}
$$

In order to avoid numerical instabitity in the discretized flow field equation, Equation (5-31), the donor-cell method is applied and Equation (5-31) is formulated as

$$
(\alpha \phi)_{I}^{J+1}=(\alpha \phi)_{I}^{J}+S_{I}^{J} \delta t-\frac{\delta t}{\delta z}\left[(\alpha F)_{e}^{J}-(\alpha F)_{W}^{J}\right]
$$

where

$$
\begin{aligned}
& (\alpha \mathrm{F})_{e}=\alpha_{1}\left(\max \left[F_{e}, 0\right]\right)-\alpha_{1+1}\left(\max \left[-F_{e}, 0\right]\right) \\
& (\alpha F)_{w}=\alpha_{1-1}\left(\max \left[F_{w}, 0\right]\right)-\alpha_{1}\left(\max \left[-F_{w}, 0\right]\right)
\end{aligned}
$$

Solutions of $(\alpha \Phi)_{1}^{J+1}$ are then obtained by solving the tridiagonal linear system of Equation (5-33).

The general procedure for obtaining the flow field solution is as follows:

i) Determine the erosion rate by assuming a zero void fraction mixing zone;

ii) Solve the momentum and energy equations of the particles in Lagrangian coordinates to yield the source and sink terms for the conservation equations;

iii) Apply the drift flux correlation to yield a single unknown in the vapor mass equation, Equation (5-6), and solve for the void fraction. Check for flow dispersion; 


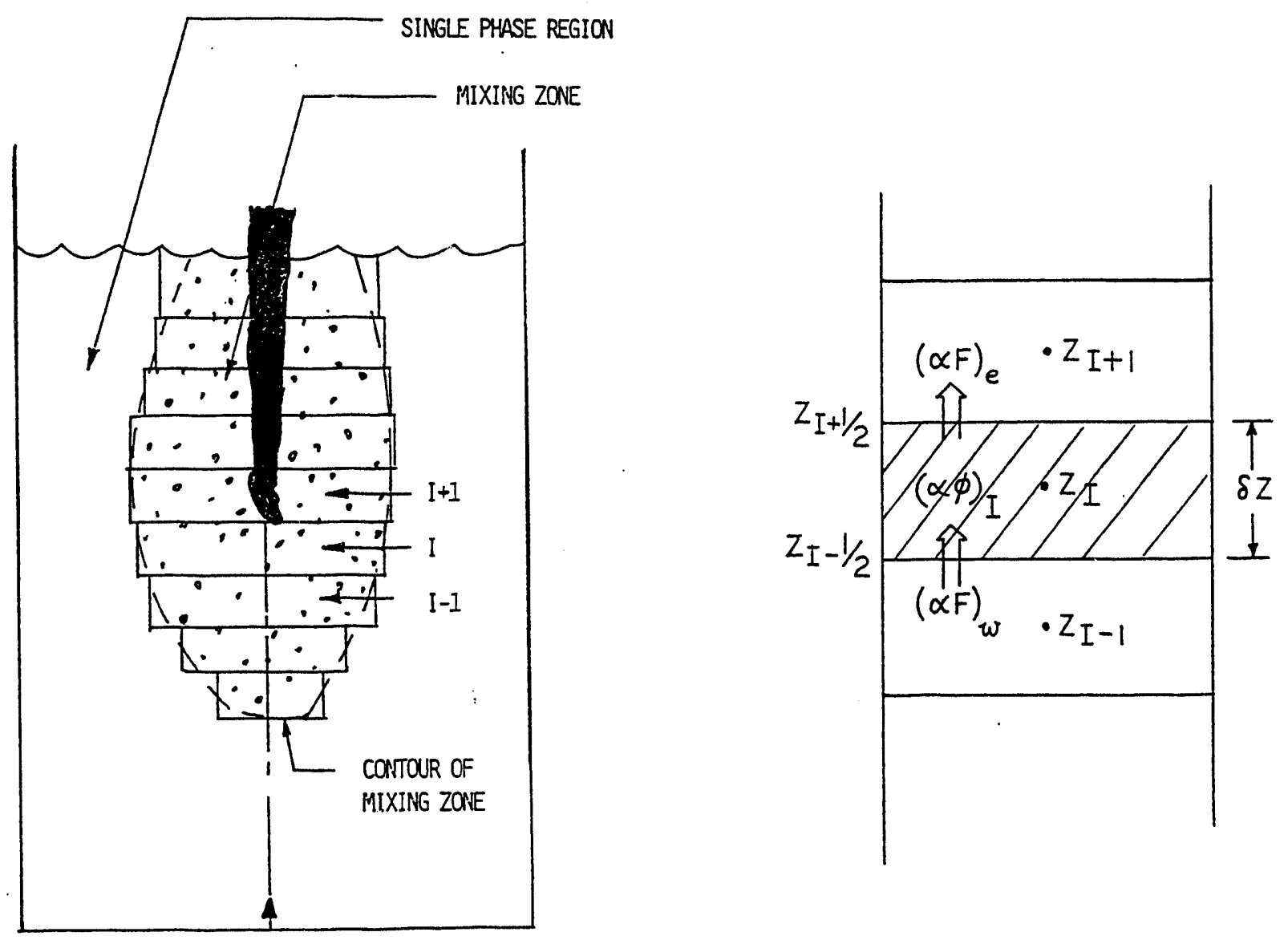

Figure 5-3. Space Nodalization of THIRMAL Code 
iv) Determine the vapor and liquid temperatures using the energy conservation equations, Equations (5-22) and (5-23);

v) Determine the system pressure based on the vapor exit temperature and system geometry;

vi) Iterate on Steps i) through v) using the updated flow and system conditions;

The occurrence of dispersed flow is constantly checked when the vapor superficial veiocity is determined. In THIRMAL, a dispersion criterion developed by Leung et. al. ${ }^{51}$ is used. The threshold steam superficial velocity is correlated by

$$
j_{v c} \geq 1.4 \frac{(g \sigma \Delta \rho)^{1 / 4}}{\rho_{v}^{1 / 2}}
$$

where $j_{v c}$ is the minimum steam superficial velocity for the onset of dispersed flow.

When this criterion is met, the code switches the local mixing zone geometry from a vapor film to a disnersed flow channel. The erosion velocity, as expressed in Equation (5-7), ul Jer this condition is obtained simply by setting the vapor film thickness to a large number. Thus, Equation (5-7) becomes

$$
U_{E}=\left(\frac{\rho_{v} U^{2}-\sigma_{m} k_{p}}{\rho_{m}}\right)^{1 / 2}
$$

and

$$
k_{\mathrm{p}} \approx \frac{2 \rho_{\mathrm{v}} \mathrm{v}^{2}}{3 \sigma_{\mathrm{m}}}
$$

It is interesting to note that Equations (5-35) and (5-36) are the same as obtained by Levich ${ }^{1}$ for the erosion at the boundary between two media with nonzero relative velocity.

\subsection{Comparisons of CCM Test Results and THIRMAL Predictions}

In this subsection, a few representative THIRMAL calculations are compared with test data in order to evalute the THIRMAL code. Here, a complete set of THIRMAL predictions is compared with the CCM-5 test data. The comparisons include the jet breakup length, steam and hydrogen generation, water and vapor temperatures, local flow conditions, and debris characterization.

The size of the mixing zone, i.e., the melt/water/steam mixture, as predicted by THIRMAL is shown in Figure 5-4 at various times during the interaction. Also shown are the responses of the thermocouples located in the vessel (Figures 5-5 to 5-9). In these figures, the curves represent the code 


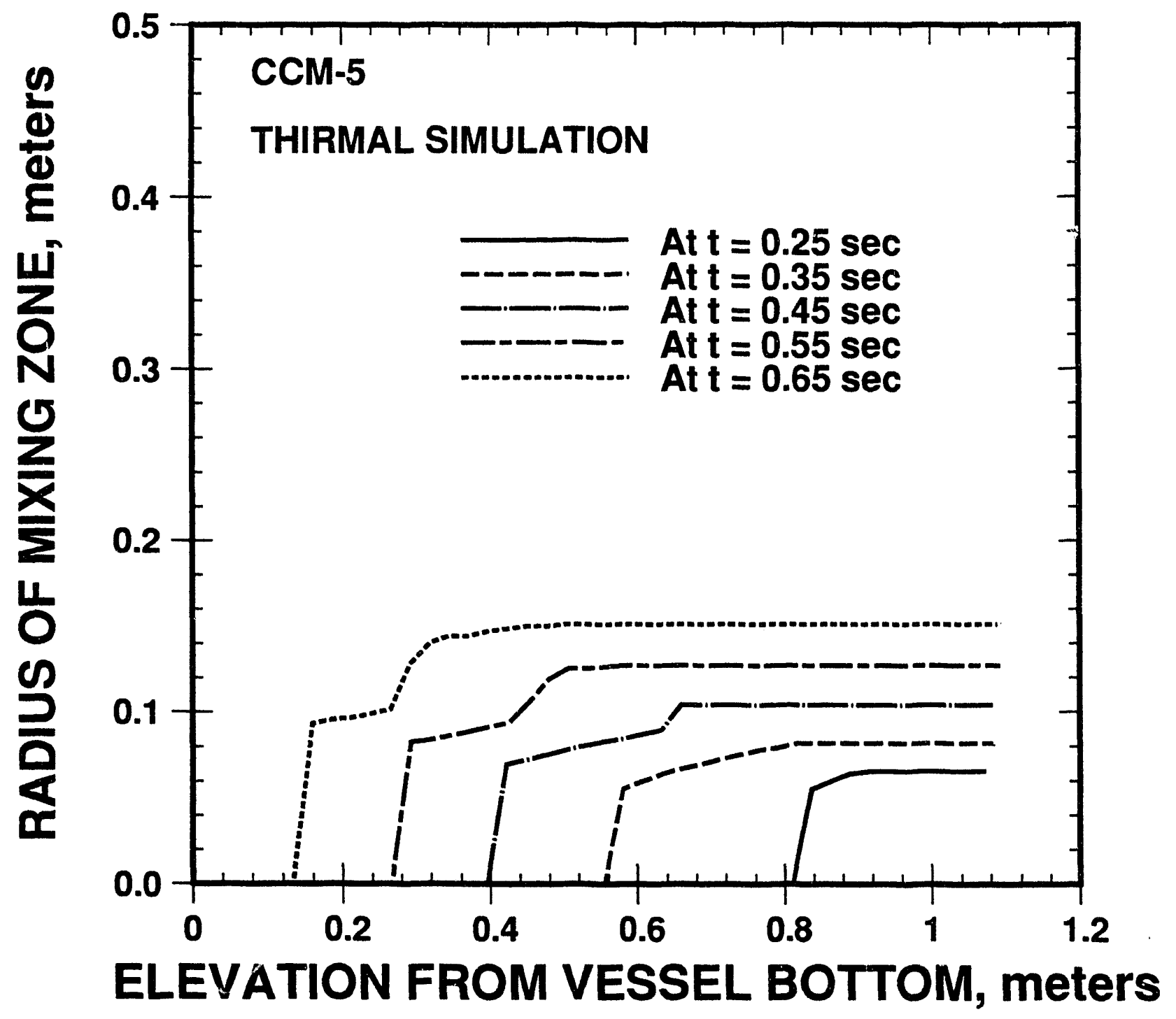

Figure 5-4. THIRMAL Prediction of Mixing Zone 


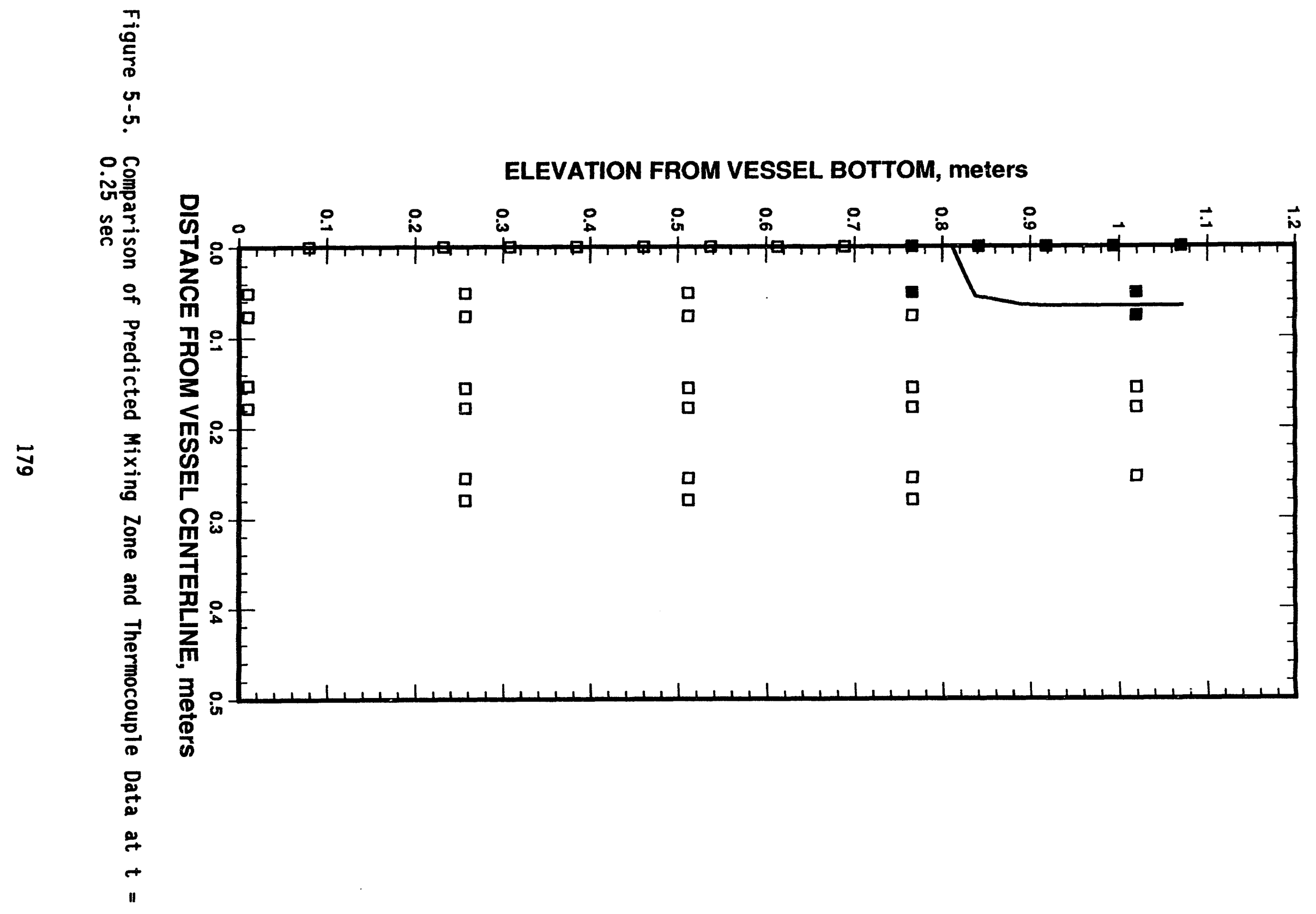




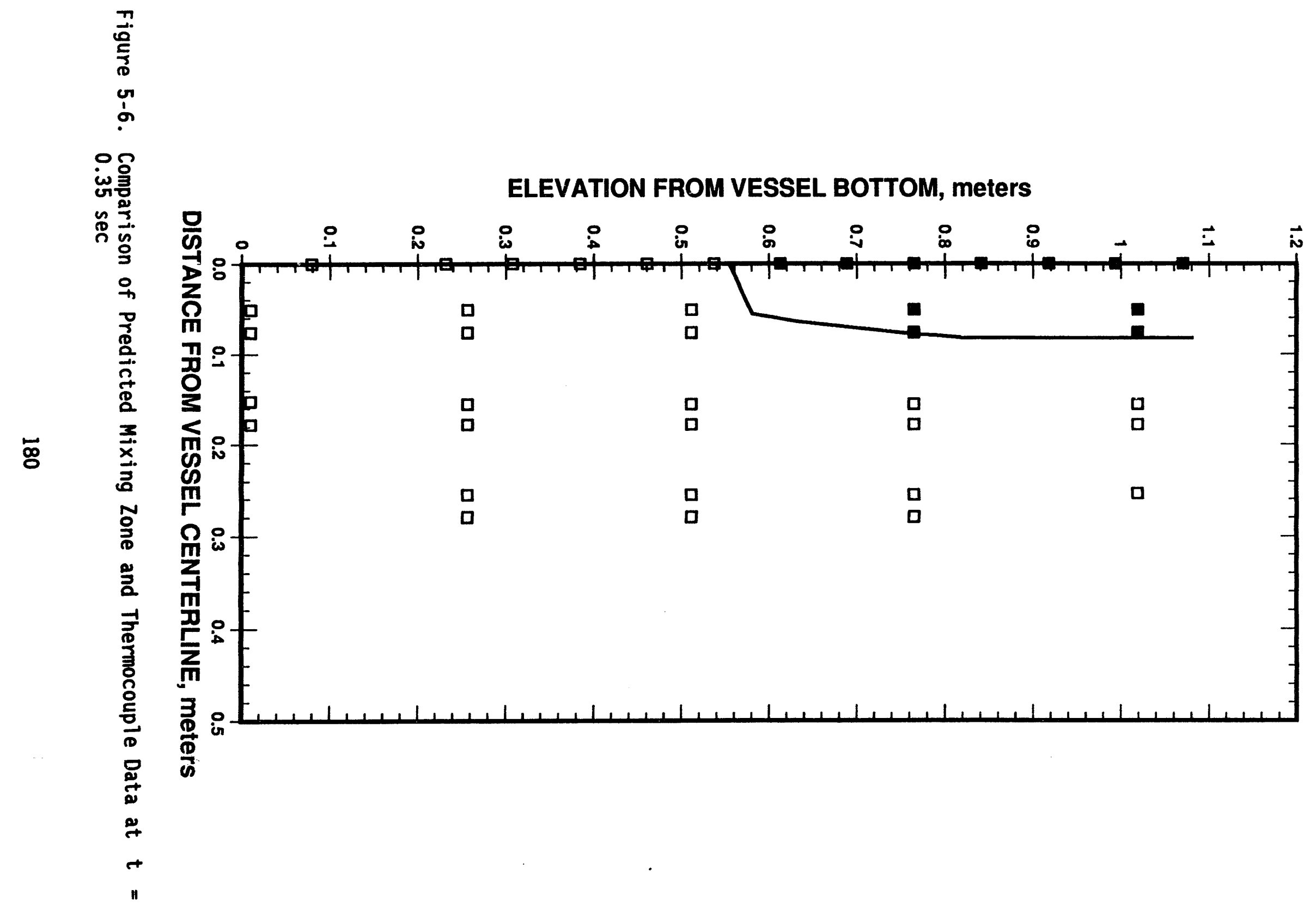




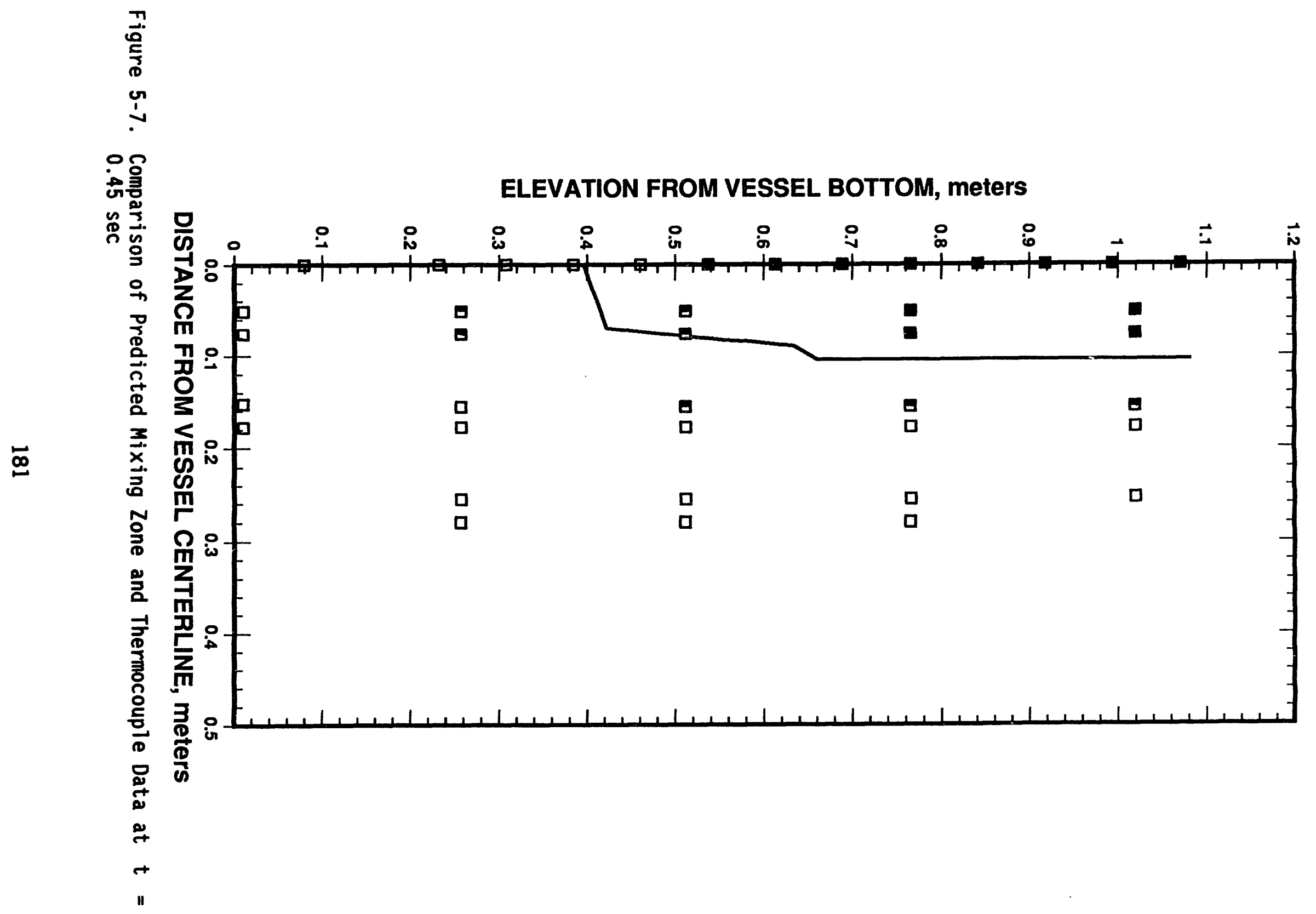




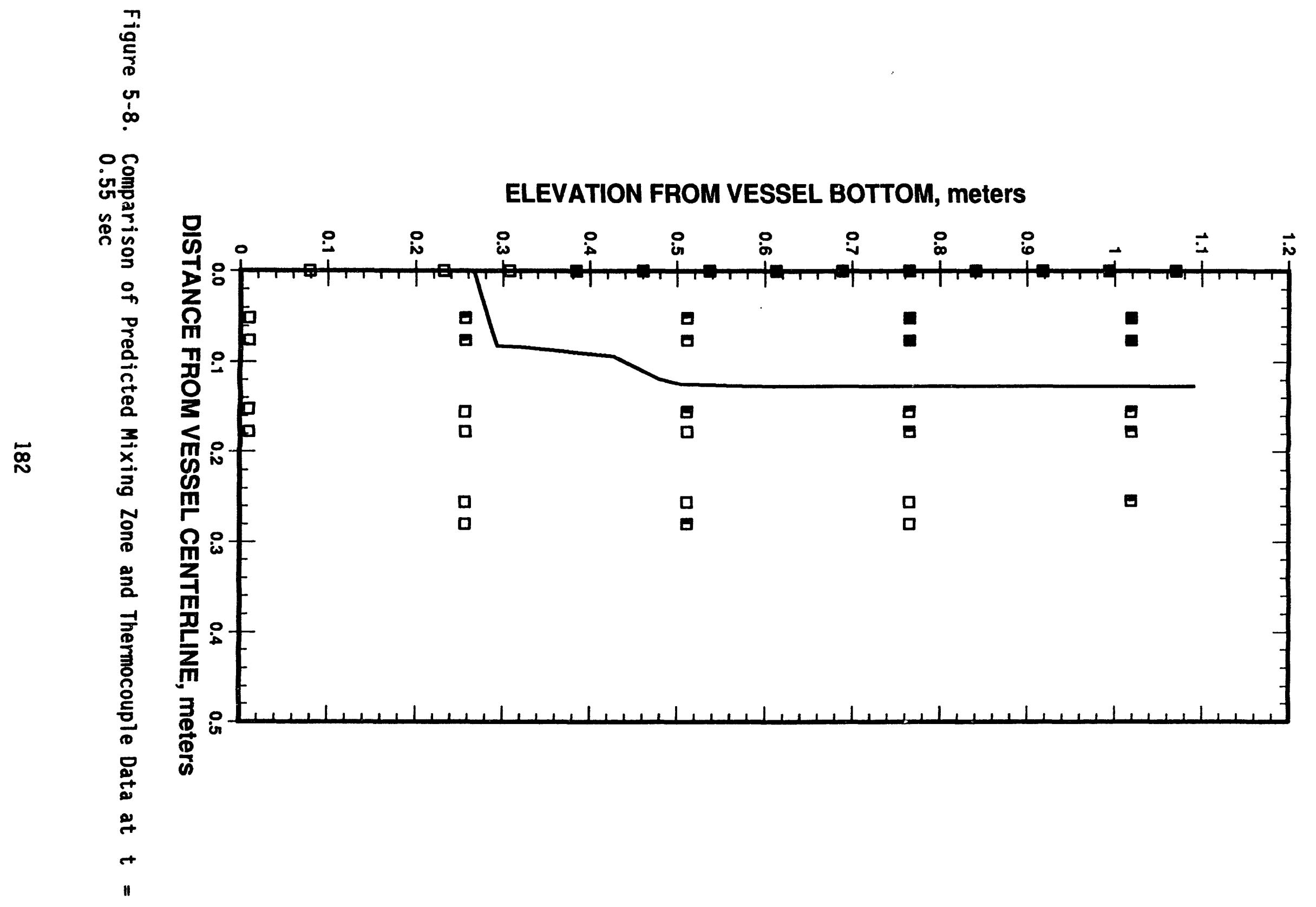




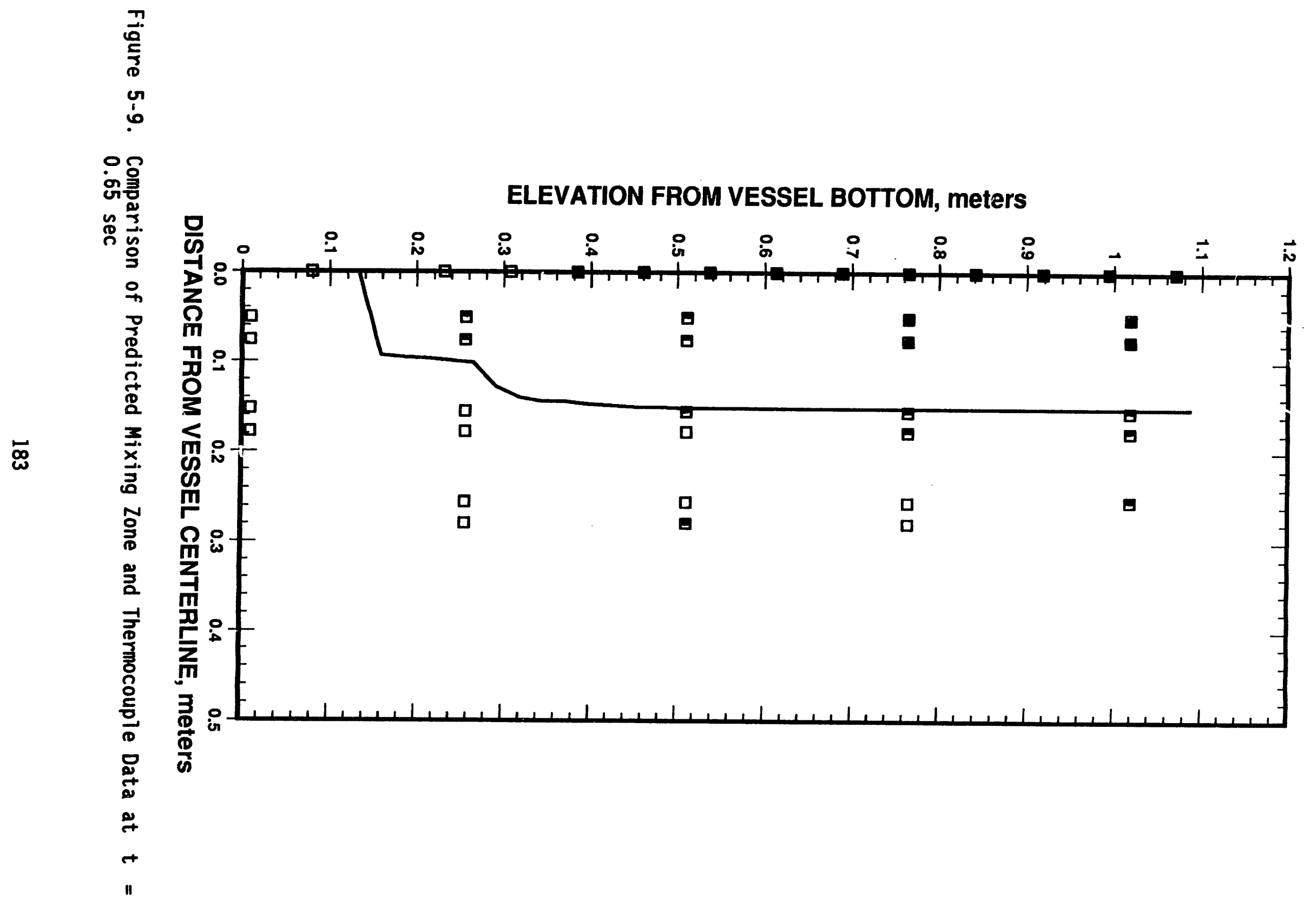


prediction; the symbols represent the locations of thermocouples. The closed symbols are the thermocouples which have been hit by corium melt (indicated by a temperature rise of $>500 \mathrm{~K})$; the semi-closed symbols denote a minor ( 200 to $500 \mathrm{~K})$ temperature rise due to contact by the steam/water mixture. The open symbols denote thermocouples with no significant temperature rise before the time indicated. In general, the code prediction of the mixing zone agrees very well with the thermocouple responses. Before the jet breakup occurs, the penetration velocity of the lower front of the mixing zone (i.e., the downward propagation velocity) can be considered as the jet leading edge velocity. THIRMAL predicts that jet breaks up around $0.37 \mathrm{sec}$. As shown in Figures 5-5 and 5-6 for $t=0.25$ sec and $t=0.35 \mathrm{sec}$ (i.e., prior to the occurrence of the jet breakup), the leading edge velocity of the jet is predicted as -2.5 to $2.7 \mathrm{~m} / \mathrm{sec}$, while a roughly constant velocity of $\sim 3 \mathrm{~m} / \mathrm{sec}$ is estimated using the temperature response of the axial centerline thermocouples as indicated in Figure 4-17. After the jet breakup occurs (i.e., $t>0.37 \mathrm{sec}$ ), THIRMAL predicts a constant penetration velocity of $-1.4 \mathrm{~m} / \mathrm{sec}$ for the mixing zone representing a settling velocity of the eroded melt particulates. Also shown in Figure 4-17, for $t \geq 0.3$ the temperature response of the centerline thermocouples shows a much lower penetration velocity of $\sim 0.33 \mathrm{~m} / \mathrm{sec}$ which presumably represents the melt particulates settling velocity. The discrepancy between the measured and predicted penetration velocity of the mixing zone after jet breaks up is possibly due to water circulation which may have retarded the melt particulates from settling toward the vessel bottom. This effect is believed to be more pronounced for very fine particulates. The THIRMAL code, using the pool bubbling assumption and the drift-flux approximation, however, does not model any water circulation in the vessel. As a result, the predicted settling velocity of the melt particulates may be overestimated.

The extent of the melt/water interaction and the jet erosion, as predicted by the THIRMAL code, can be illustrated by the local volume fraction of each phase in the mixing zone at various instants during the interaction. Corresponding to Figure 5-4, the local void fraction averaged over the mixing zone is shown in Figure 5-10. In general, the void fraction in the mixing zone is very high, especially when the jet breakup occurs. From $t=0.25 \mathrm{sec}$ to $t=$ $0.35 \mathrm{sec}$, the void fraction increases rapidly as jet breakup is approached. From $t=0.35 \mathrm{sec}$ to $t=0.45 \mathrm{sec}$, only a slight increase in the void fraction is predicted since a quasi-steady jet breakup is achieved (i.e., the mass injection rate of the jet is approximately the same as the jet erosion rate; the coherent jet length stays a constant). From $t=0.45 \mathrm{sec}$ to $t=0.55 \mathrm{sec}$, the void fraction starts to drop, since the melt injection has stopped. At $t=0.65 \mathrm{sec}$, the void fraction drops considerably due to spreading of melt drops in the mixing zone. According to the THIRMAL predictions, flow dispersion always occurs from the water surface to $-40 \mathrm{~cm}$ below during the first $0.5 \mathrm{sec}$. Unfortunately, due to adhering corium, the void detector responses did not give a good indication of the extent of the voided region during the experiment (see Figure 4-18).

The volume fractions of the jet column and the melt particulates in the mixing zone during the interaction are shown in Figures 5-11 to 5-16. The melt particles are further specified by THIRMAL as molten droplets or solid particles. Thus, the physical state of the accumulated melt on the vessel bottom can be predicted. As shown in Figure 5-11, the jet column is predicted to penetrate to $-30 \mathrm{~cm}$ below the water surface, its volume fraction dropping from $\sim 20 \%$ to $3 \%$ due to jet erosion. At this instant, only molten droplets are predicted to reside in the mixing zone, having a volume fraction varying from $-13 \%$ near the water 


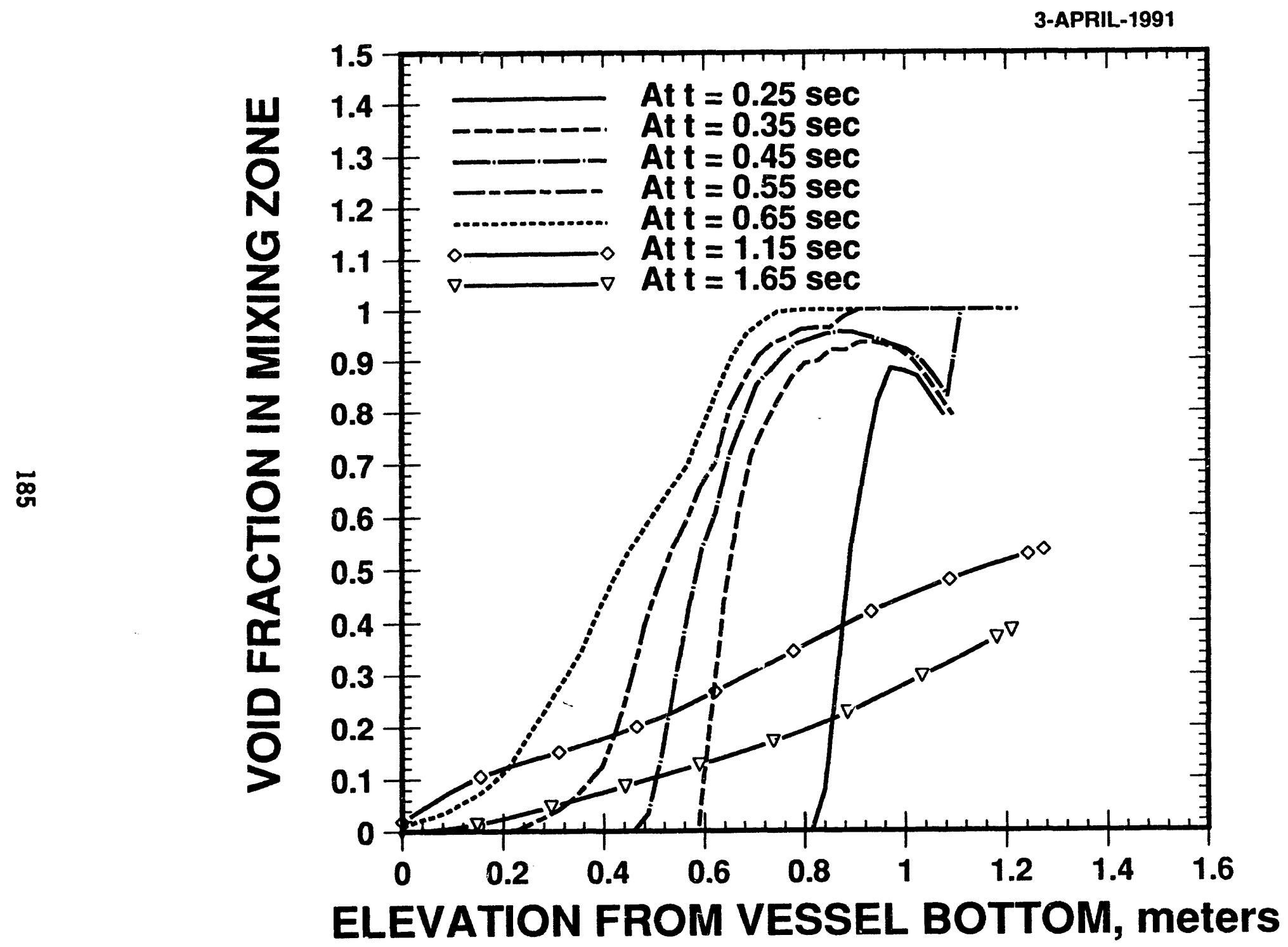

Figure 5-10. THIRMAL Prediction of Local Void Fraction in Mixing Zone 


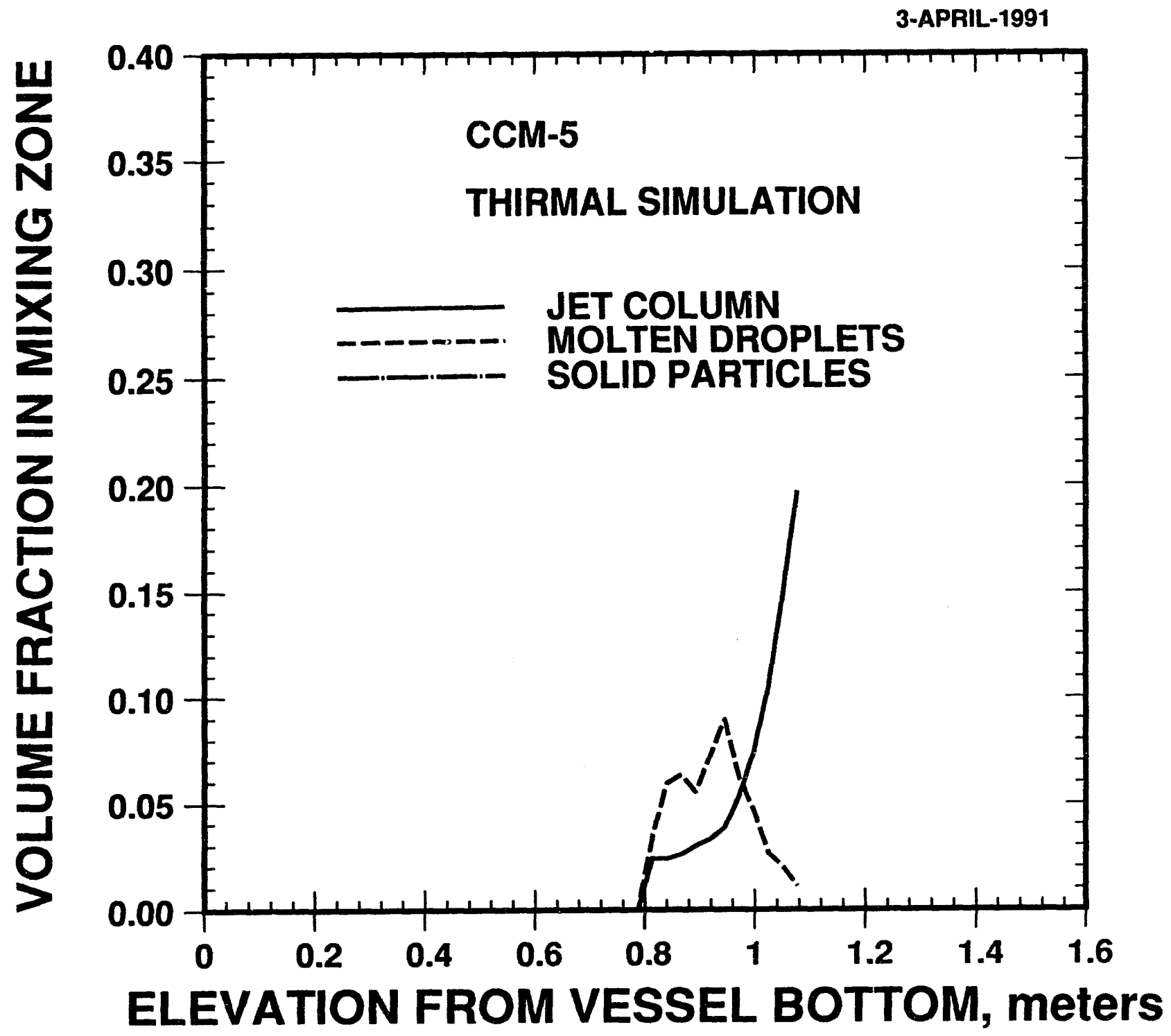

Figure 5-11. THIRMAL Prediction of Melt Volume Fractions in Mixing Zone at $t=0.25 \mathrm{sec}$ 
3-APRIL-1991

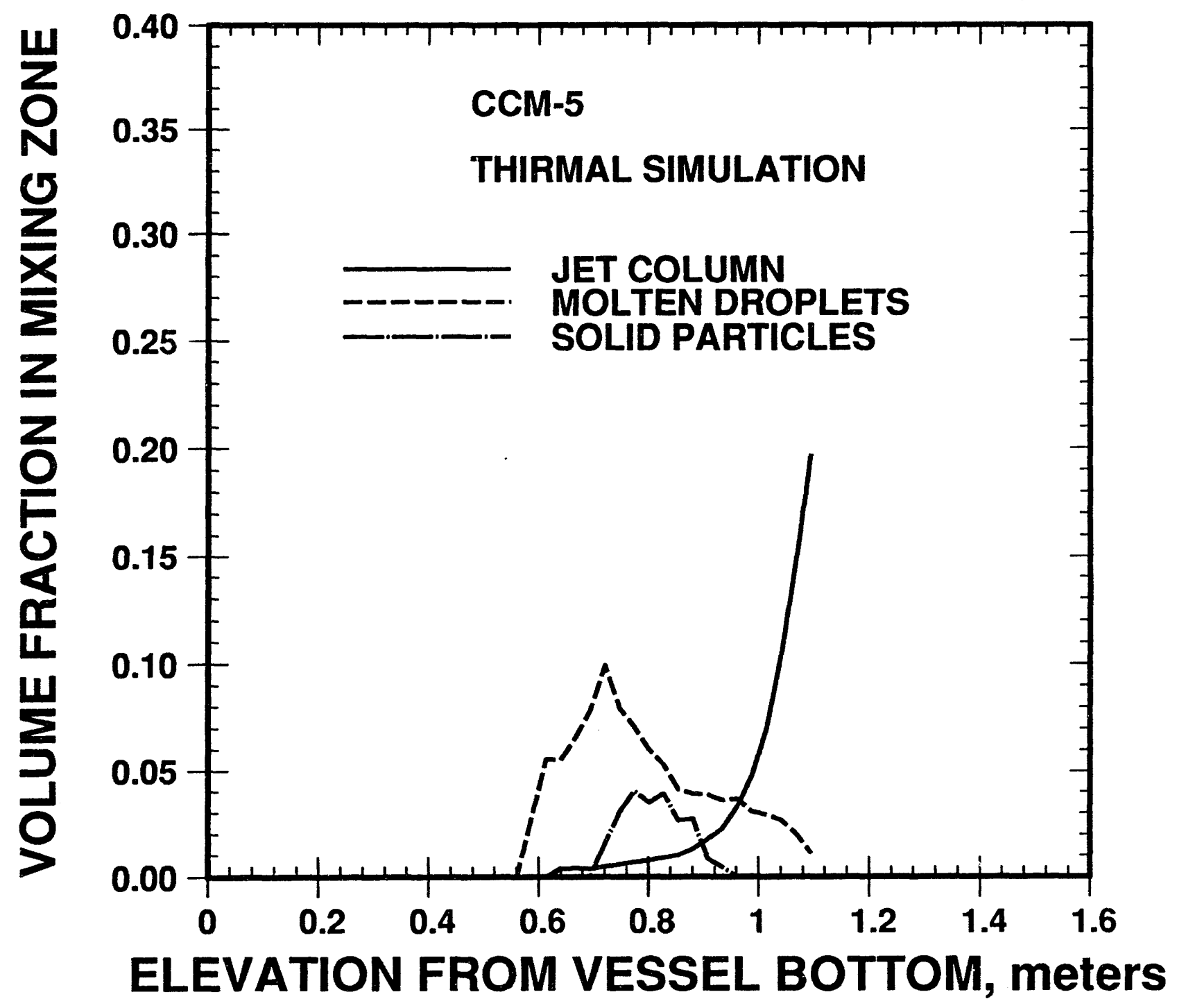

Figure 5-12. THIRMAL Prediction of Melt Volume Fractions in Mixing Zone at $t=0.35 \mathrm{sec}$ 
3-APRIL-1991

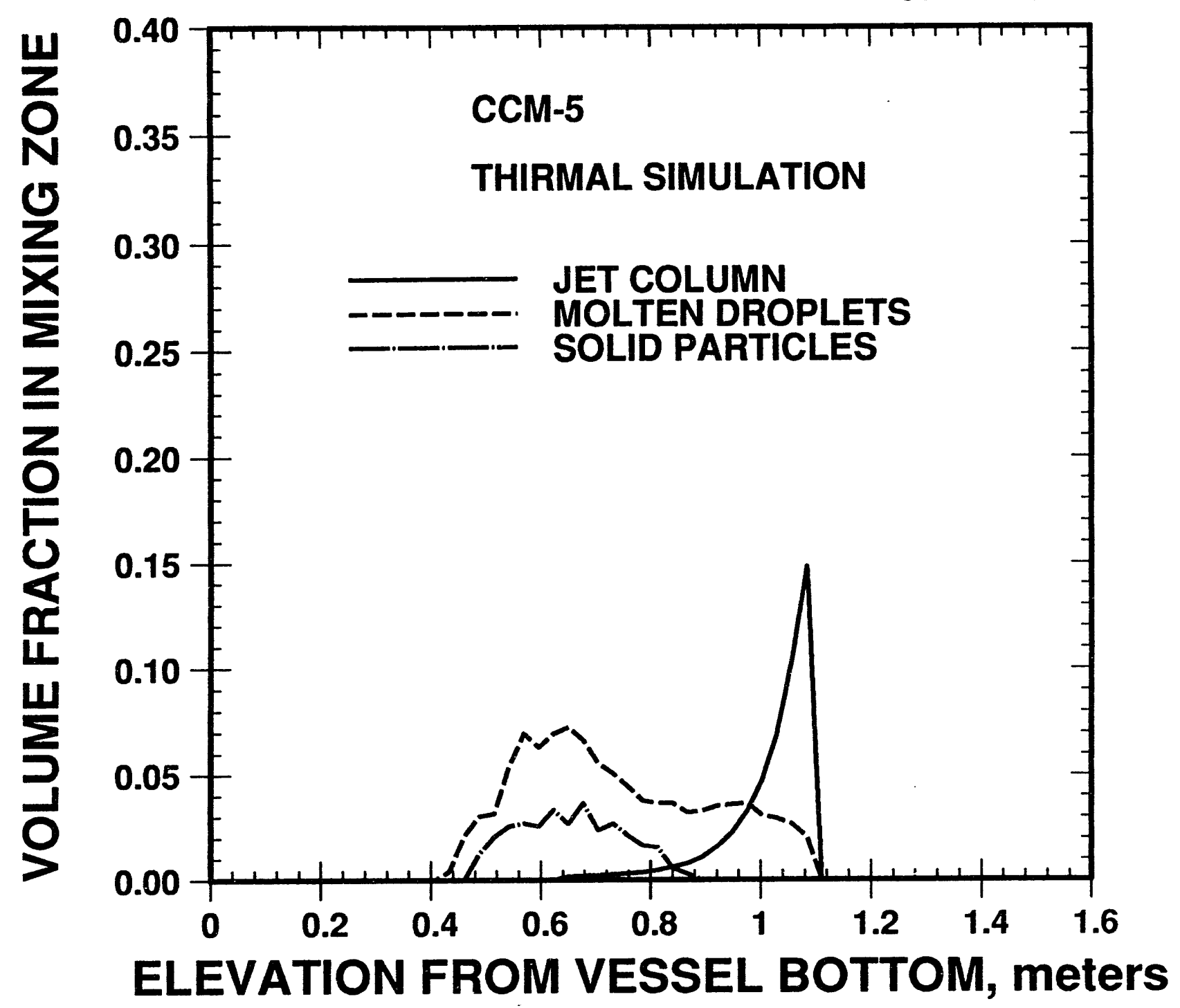

Figure 5-13. THIRMAL Prediction of Melt Volume Fractions in Mixing Zone at $t=0.45 \mathrm{sec}$ 


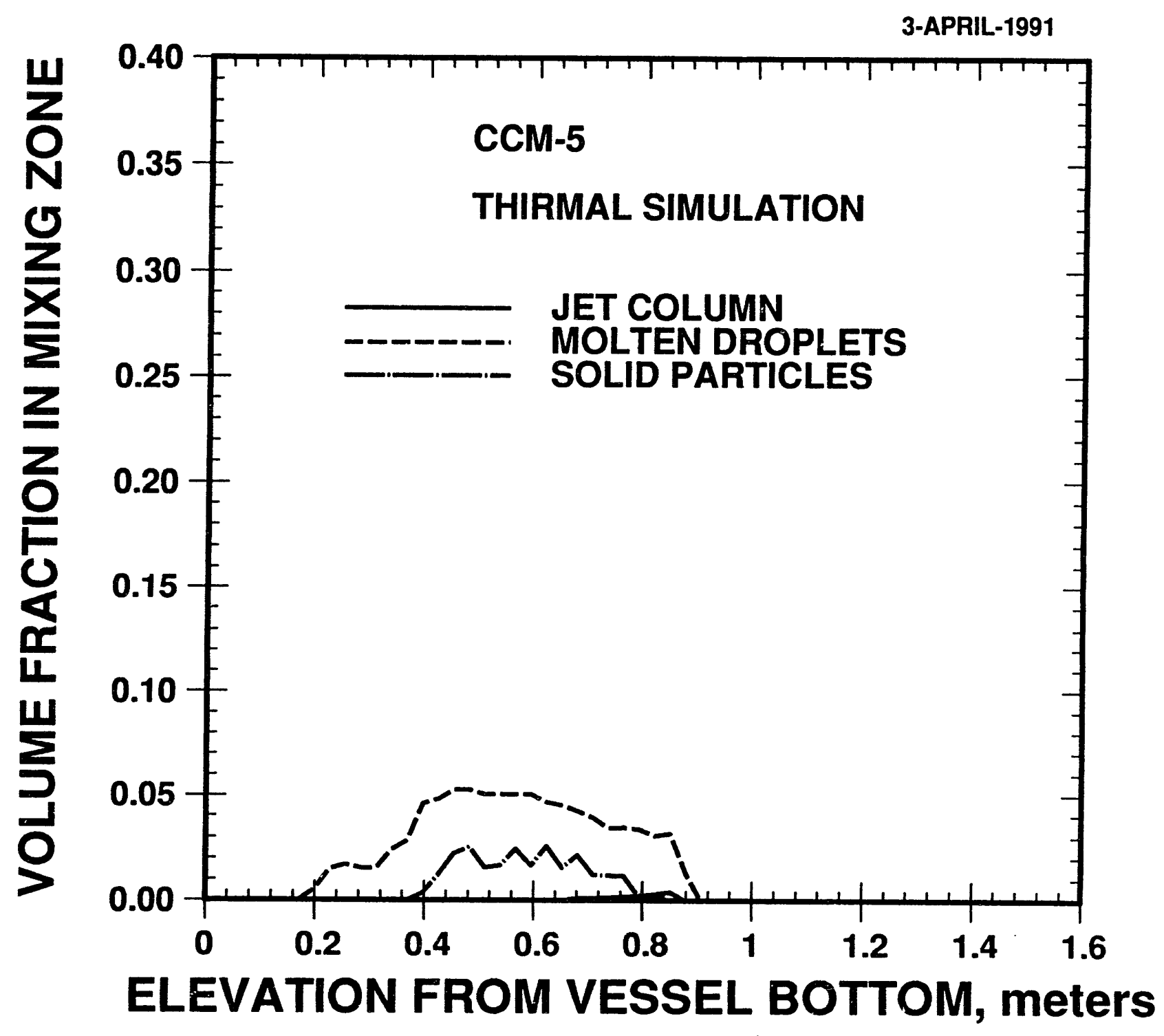

Figure 5-14. THIRMAL Prediction of Melt Volume Fractions in Mixing Zone at $t=0.55 \mathrm{sec}$ 


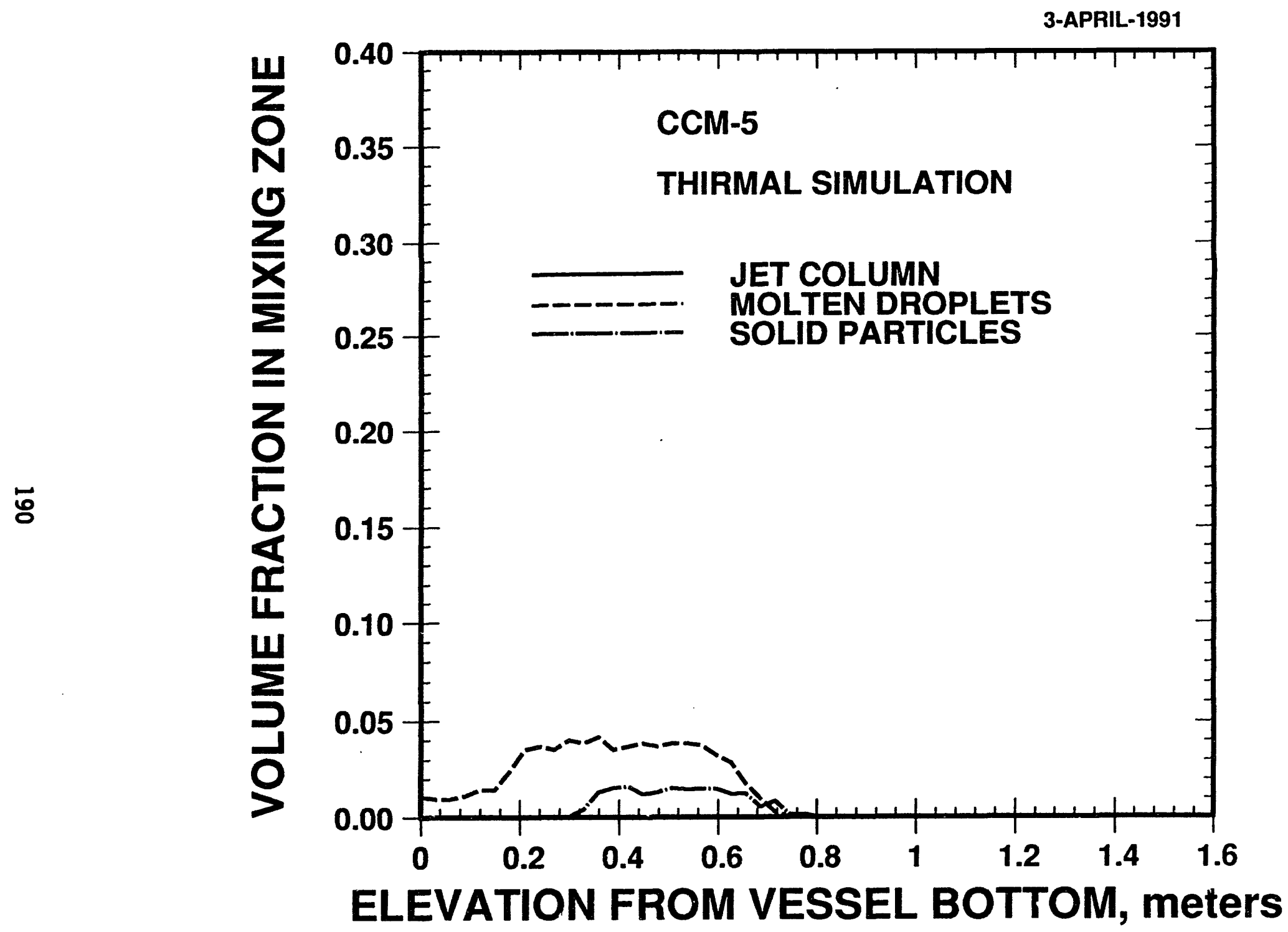

Figure 5-15. THIRMAL Prediction of Melt Volume Fractions in Mixing Zone at $t=0.65 \mathrm{sec}$ 
3-APRIL-1991

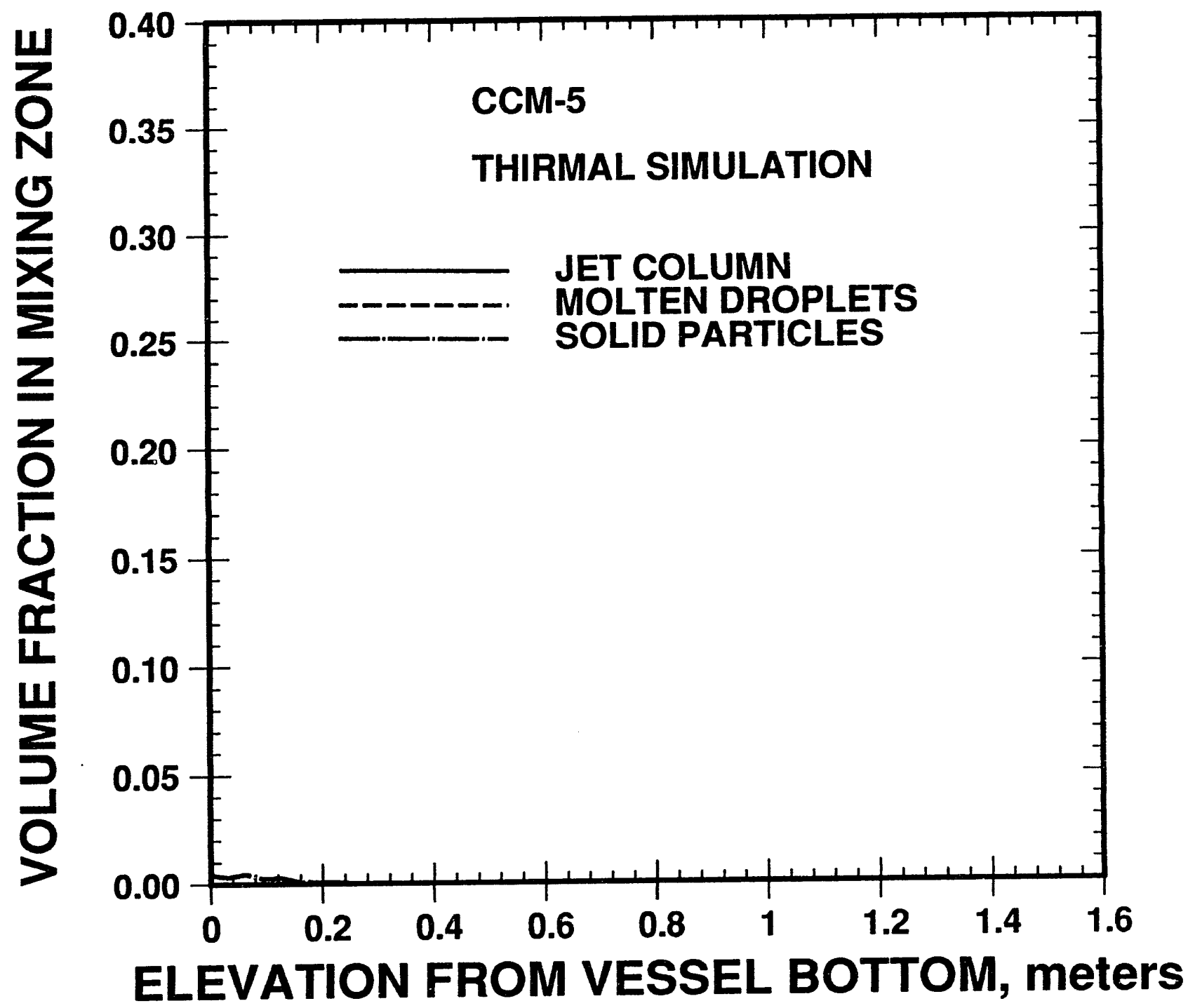

Figure 5-16. THIRMAL Prediction of Melt volume Fractions in Mixing Zone at $t=1.15 \mathrm{sec}$ 
surface to $-2 \%$ at the jet leading edge. At $t=0.35 \mathrm{sec}$, some particles are predicted to have solidified, having a maximum volume fraction of $-5 \%$, as shown in Figure 5-12. The jet leading edge is predicted to penetrate to $\sim 48 \mathrm{~cm}$ below the water surface (i.e., the breakup length). At $t=0.45 \mathrm{sec}$, quasi-steady jet breakup is achieved. The jet breakup length is predicted to be -9.4 diameters, agreeing very well with the centerline thermocouple responses, which indicate a breakup length of 10 diameters (see Figure 4-17). At $t=0.55 \mathrm{sec}$ (i.e., Figure 5-14), the mixing zone is basically dominated by the melt particulates with only a small amount of melt jet $(-1 \%)$ remaining in the mixing zone. At $t=0.65 \mathrm{sec}$, there is no melt jet remaining in the mixing zone and the amount of the molten droplets and the solid particles are roughly the same as shown in Figure 5-15. The first response from thermocouples located on the vessel bottom in CCM-5, indicating corium accumulation, was at $t-1.2 \mathrm{sec}$ (see Appendix $F$ ). However, THIRMAL predicts that the accumulation starts at $-0.8 \mathrm{sec}$. As described before, the discrepancy is probably due to the assumption of no water circulation in the THIRMAL code. After $t=1.15 \mathrm{sec}$, THIRMAL predicts that only solid particles are settling on the bottom of the vessel.

Corresponding to Figures 5-11 to 5-16, the temperatures of each phase in the mixing zone at varions instants predicted by the THIRMAL code are shown in Figures 5-17 to 5-22. In general, the molten droplets are predicted to loose superheat at the location below the jet leading edge.

The vapor and water temperature distributions predicted by the THRIMAL code are shown in Figures 5-23 to 5-28. Water heatup in general is significant at elevations above $-0.5 \mathrm{~m}$ from the vessel bottom. The total sensible heating of water during the jet fall stage (i.e., to $t-0.6 \mathrm{sec}$, the time before a jet column disappears) can be estimated from the axial variations of the water temperature, the mixing zone and the void fraction. At $t=0.65 \mathrm{sec}$, the mixing zone can be estimated to be a cylindrical water/vapor mixture with a diameter of $0.3 \mathrm{~m}$ and a height of $1.0 \mathrm{~m}$ (see Figure 5-4). Water temperature in the mixing zone can be approximated by $T_{S A T}$ at elevation $0.6 \mathrm{~m}$ to the water surface and 0.5 $\left(T_{\text {SAT }}+T_{1}\right.$ ) at elevation $0.1 \mathrm{~m}$ to $0.6 \mathrm{~m}$ (see Figure 5-27). The average void fraction can be approximated by $40 \%$ and $5 \%$ (see Figure 5-10) for the upper and lower zones defined above. Therefore, the total heat transfer to heat up water in the mixing zone can be estimated as the following equation:

$$
E_{W}=A_{M 2} \sum_{i=1,2} L_{i} \rho_{L_{i}}\left(1-\alpha_{i}\right)\left(T_{L_{i}}-T_{I}\right) C_{p_{i}}
$$

where

$$
\begin{aligned}
& E_{W}=\text { water heating energy } \\
& E_{M Z}=\text { cross-sectional area of mixing zone } \\
& L_{i}=\text { length of mixing zone of } i \text { th zone } \\
& \rho_{L_{i}}=\text { density of water of } i \text { th zone } \\
& T_{L_{i}}=\text { temperature of water of } i \text { th zone } \\
& T_{1}=\text { initial water temperature } \\
& C_{p i}=\text { heat capacity of water at } T_{L_{i}}
\end{aligned}
$$




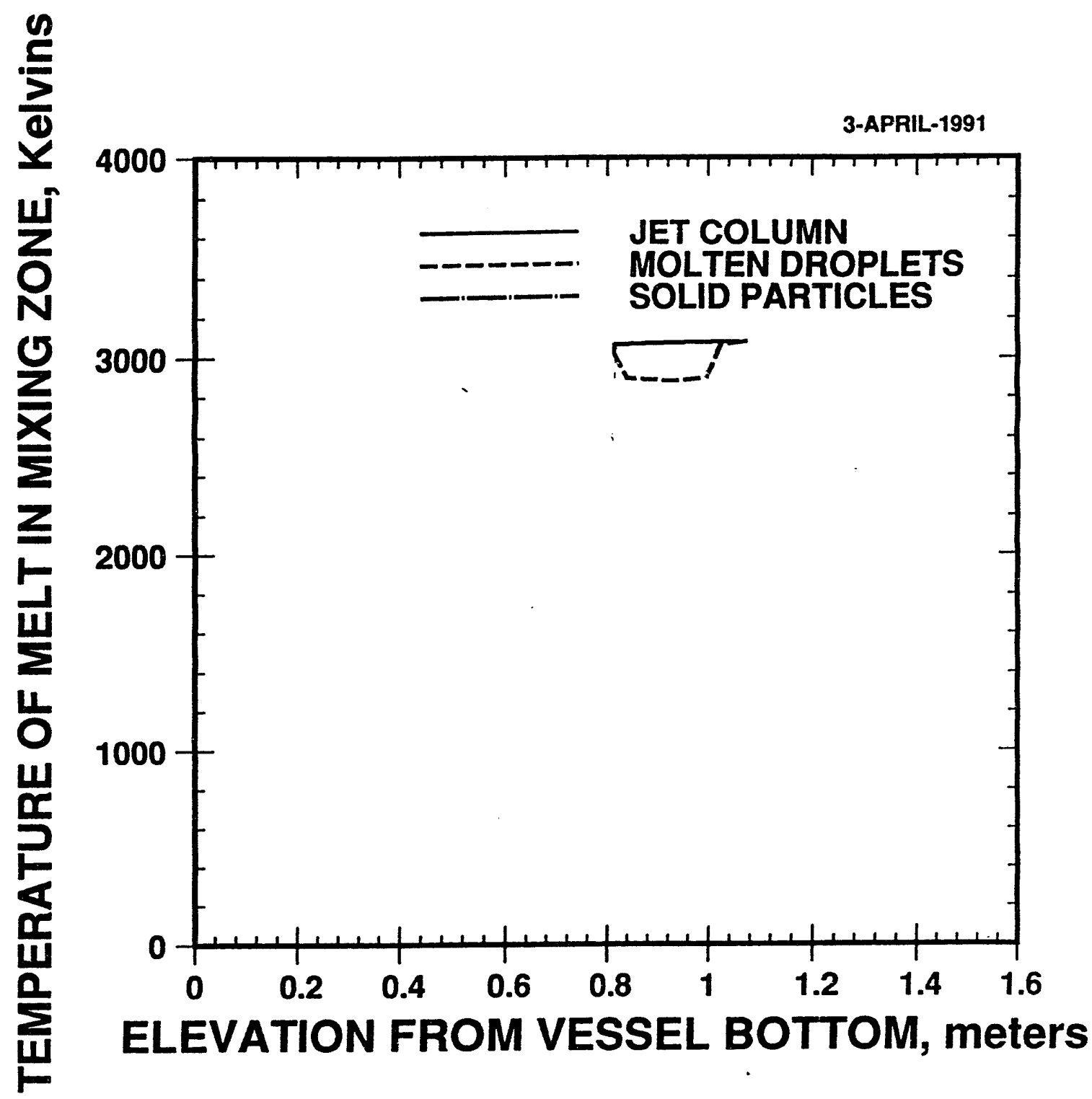

Figure 5-17. THIRMAL Prediction of Melt Temperatures in Mixing Zone at $t=0.25 \mathrm{sec}$ 


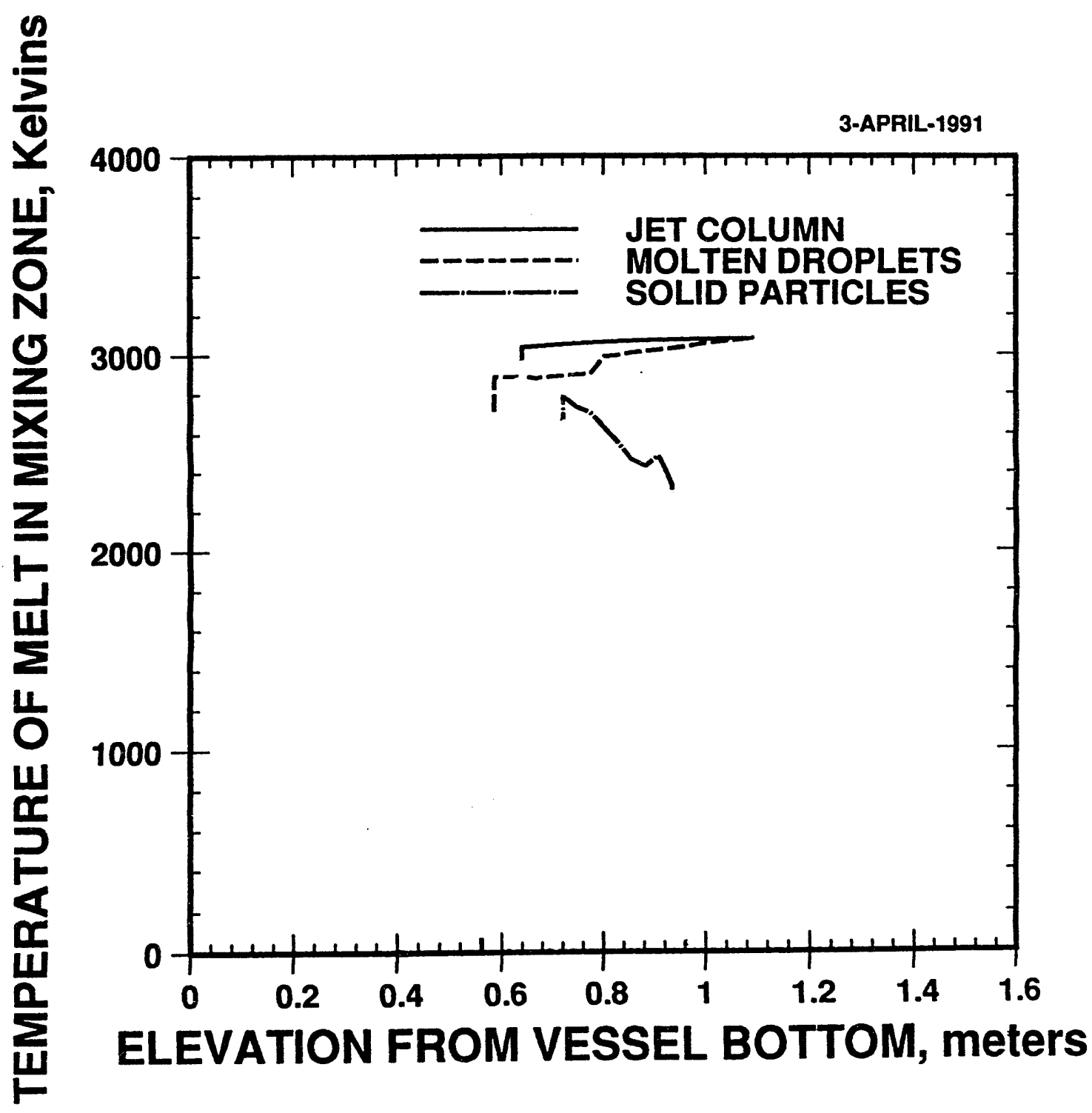

Figure 5-18. THIRMAL Prediction of Melt Temperatures in Mixing Zone at $t=0.35 \mathrm{sec}$ 


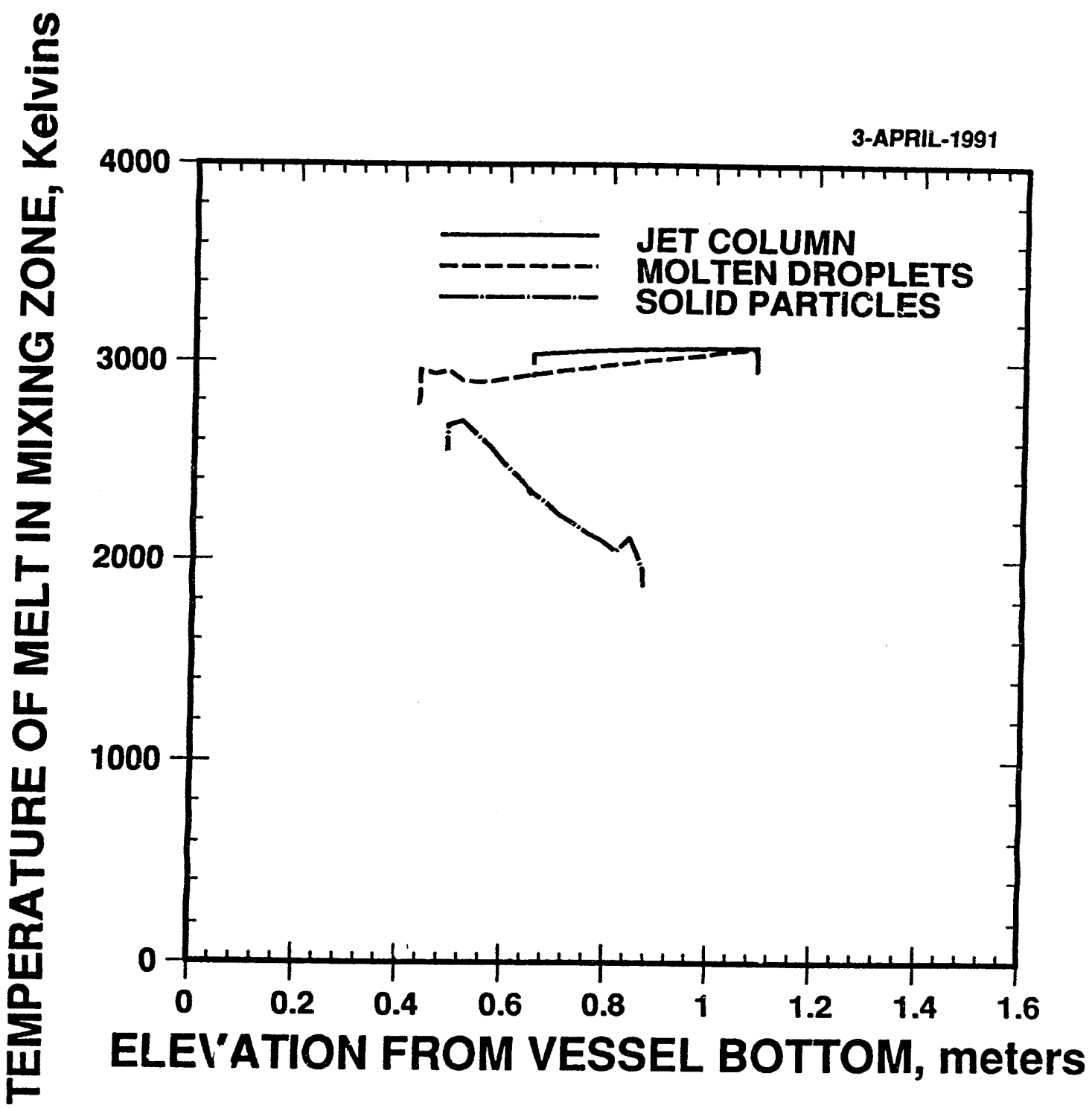

Figure 5-19. THIRMAL Prediction of Melt Temperatures in Mixing Zone at $t=0.45 \mathrm{sec}$ 


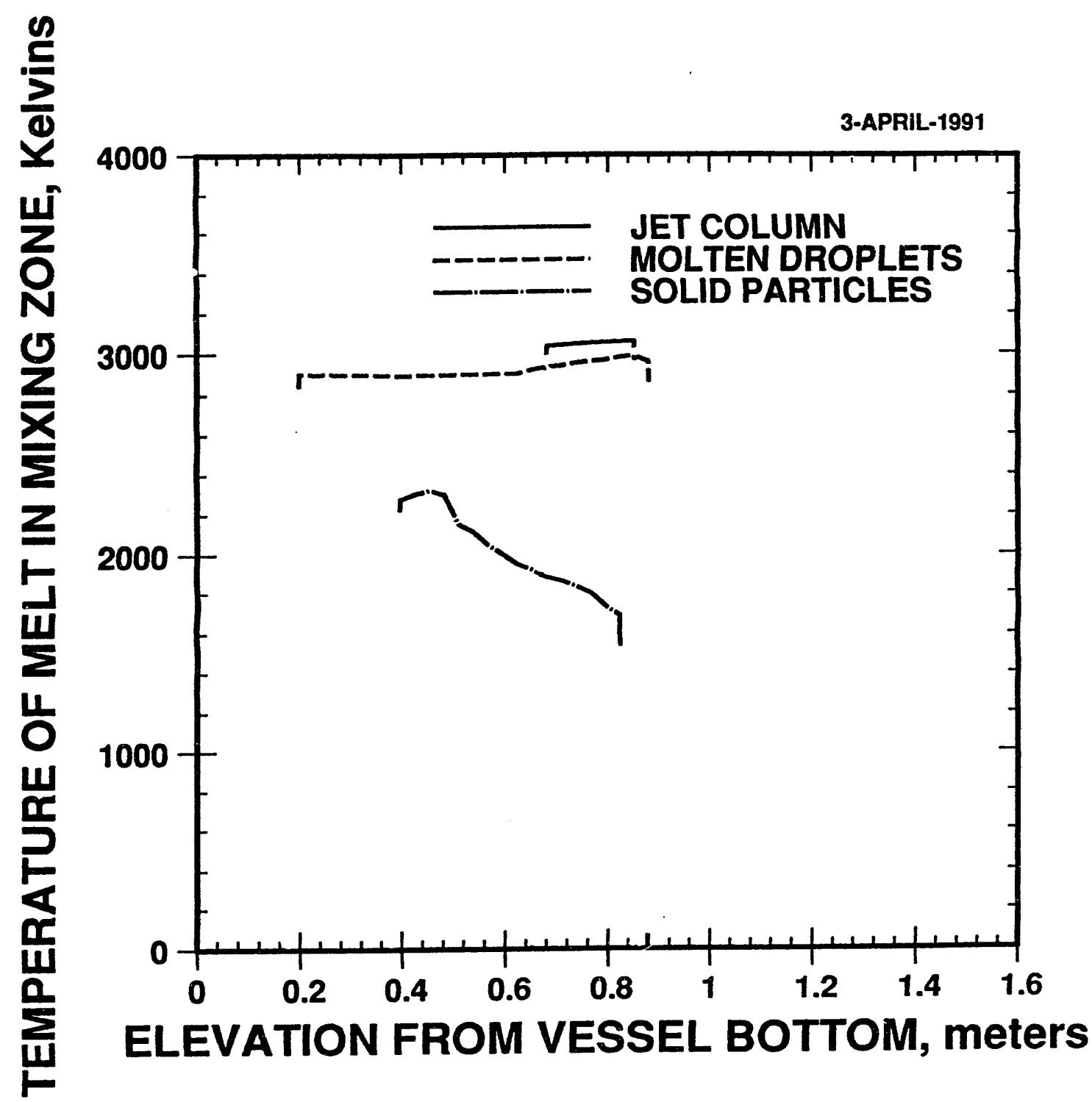

Figure 5-20. THIRMAL Prediction of Melt Temperatures in Mixing Zone at $t=0.55 \mathrm{sec}$ 


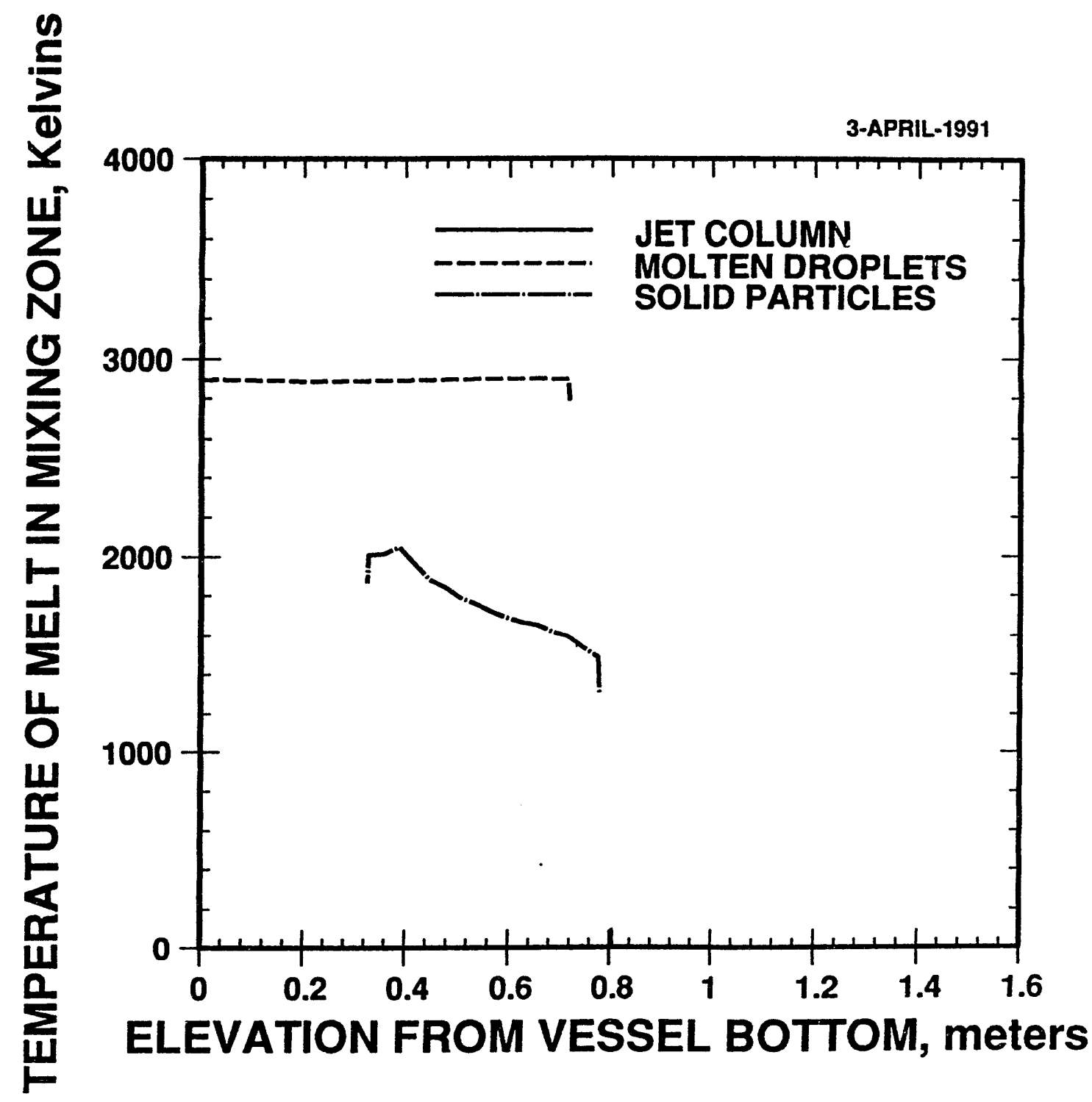

Figure 5-21. THIRMAL Prediction of Melt Temperatures in Mixing Zone at $t=0.65 \mathrm{sec}$ 


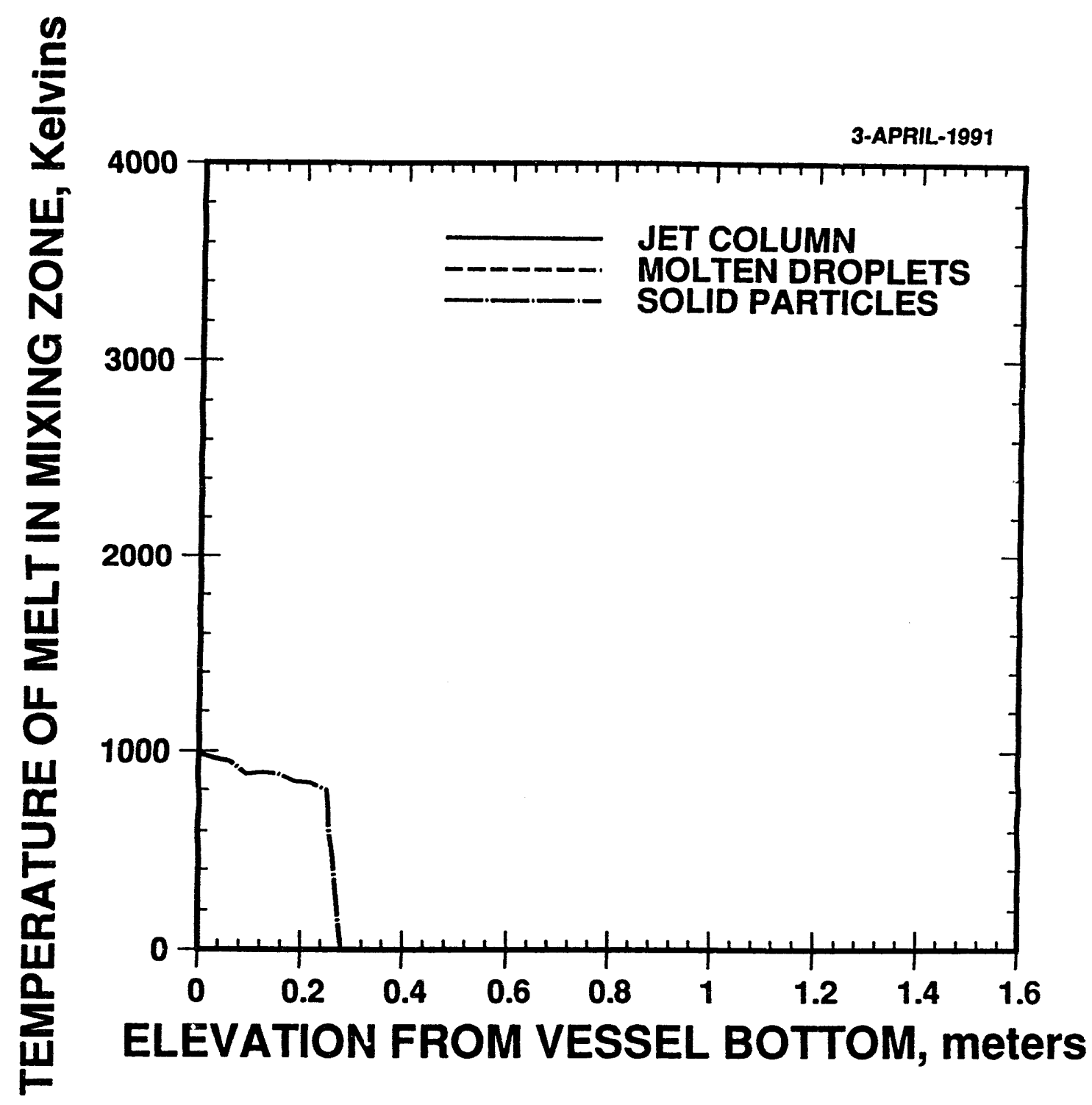

Figure 5-22. THIRMAL Prediction of Melt Temperatures in Mixing Zone at $t=1.15 \mathrm{sec}$ 


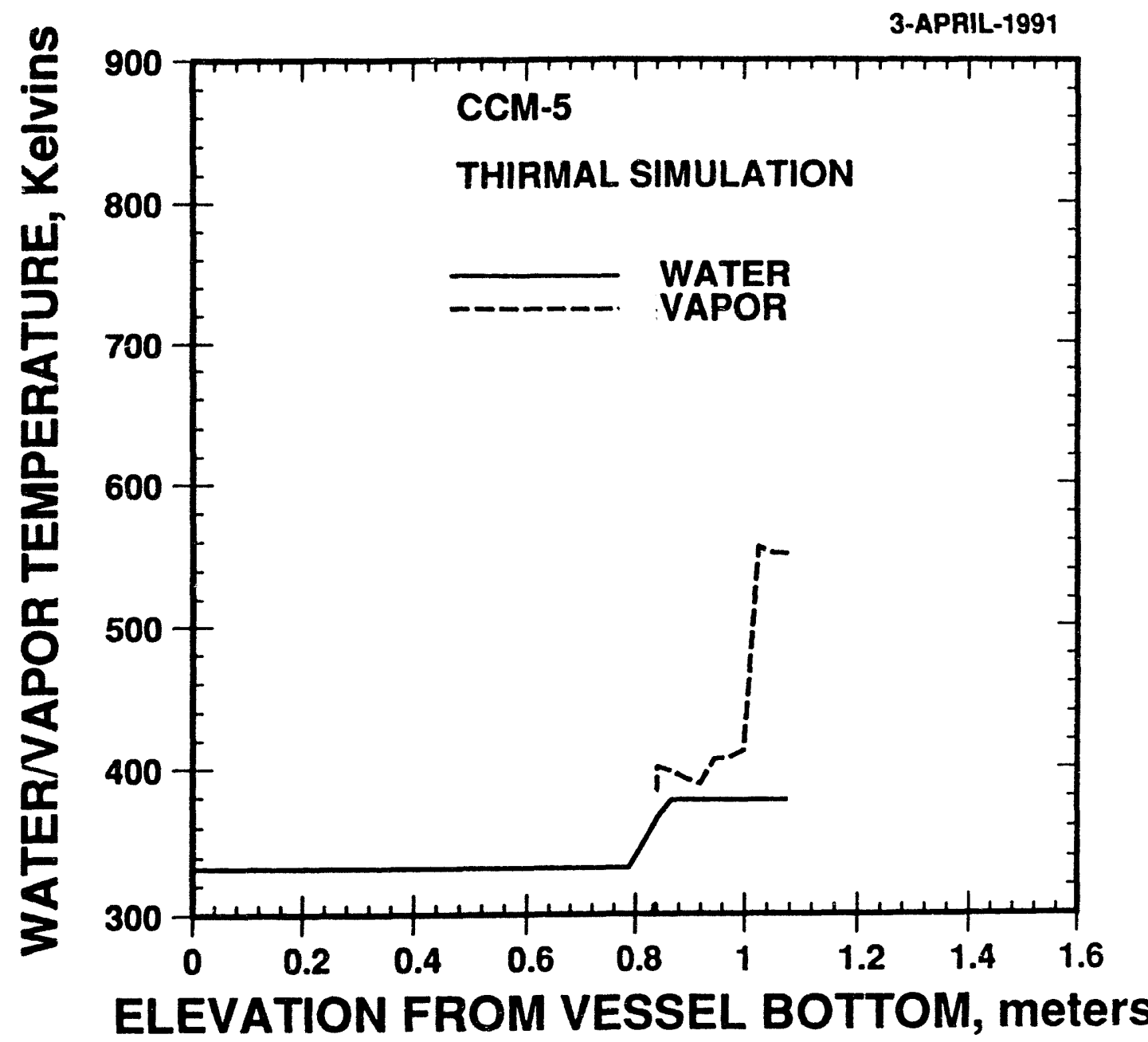

Figure 5-23. THIRMAL Prediction of Water and Vapor Temperatures in Mixing Zone at $t=0.25 \mathrm{sec}$ 


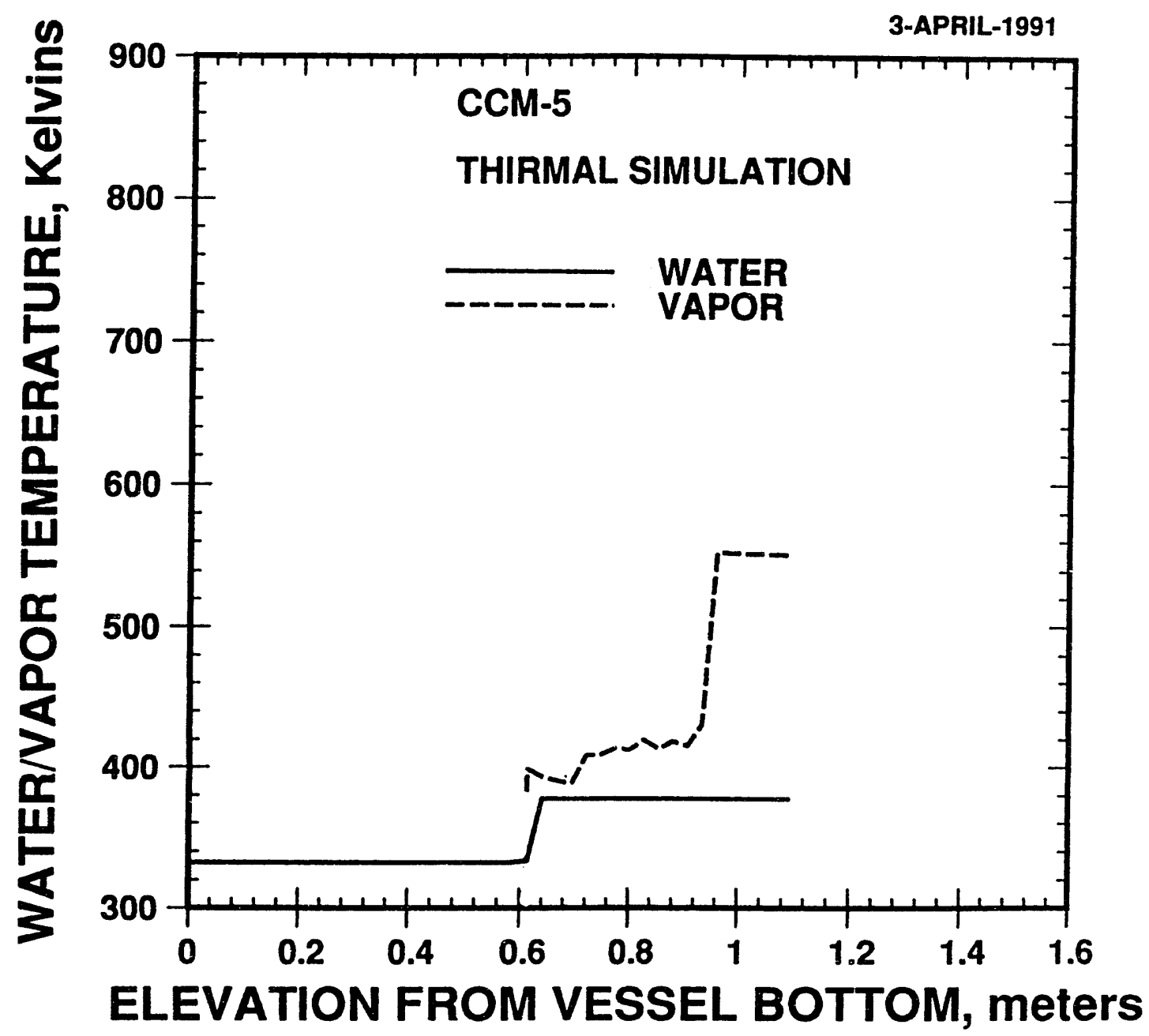

Figure 5-24. THIRMAL Prediction of Water and Vapor Temperatures in Mixing Zone at $t=0.35 \mathrm{sec}$ 


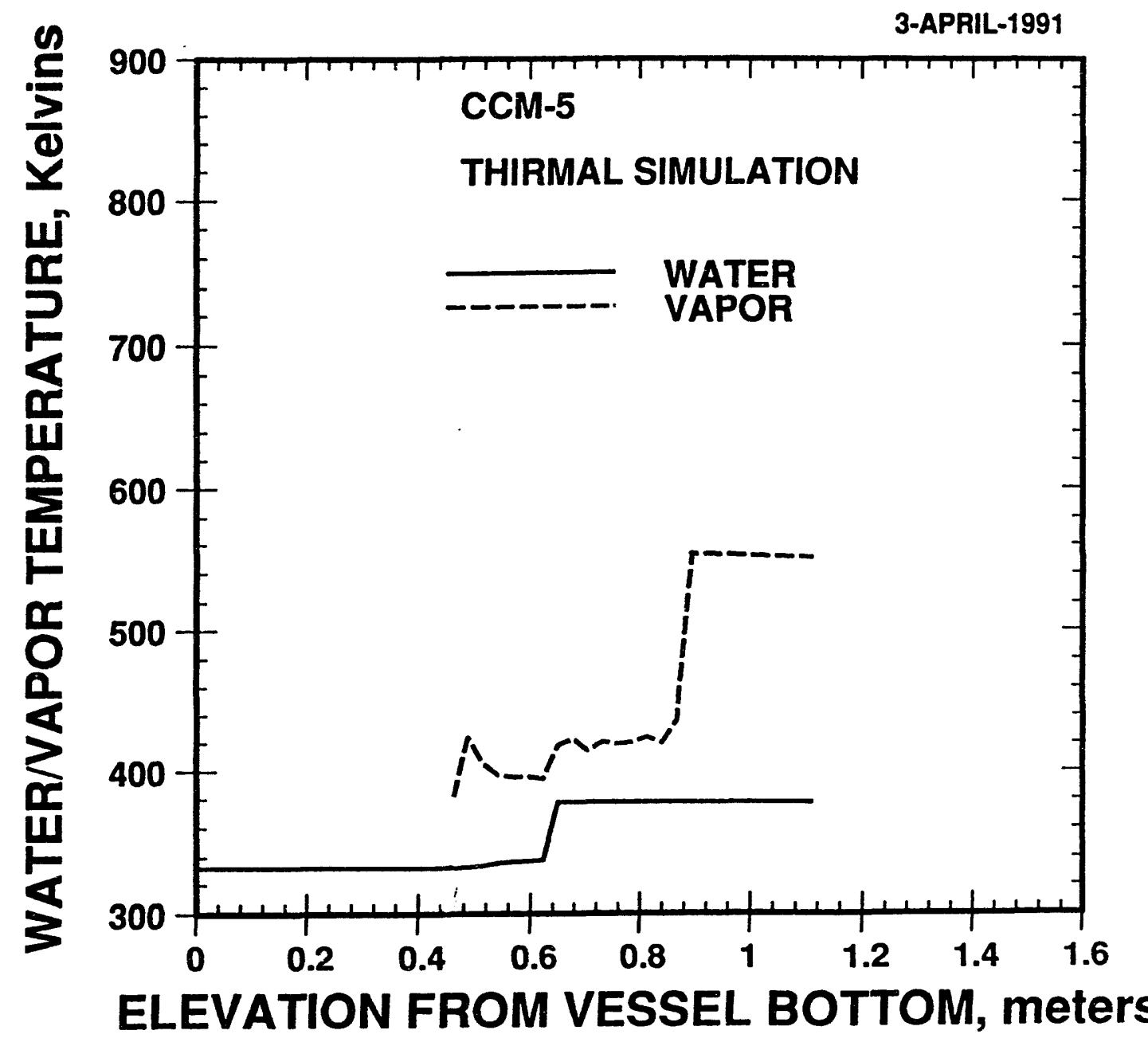

Figure 5-25. THIRMAL Prediction of Water and Vapor Temperatures in Mixing Zone at $t=0.45 \mathrm{sec}$ 


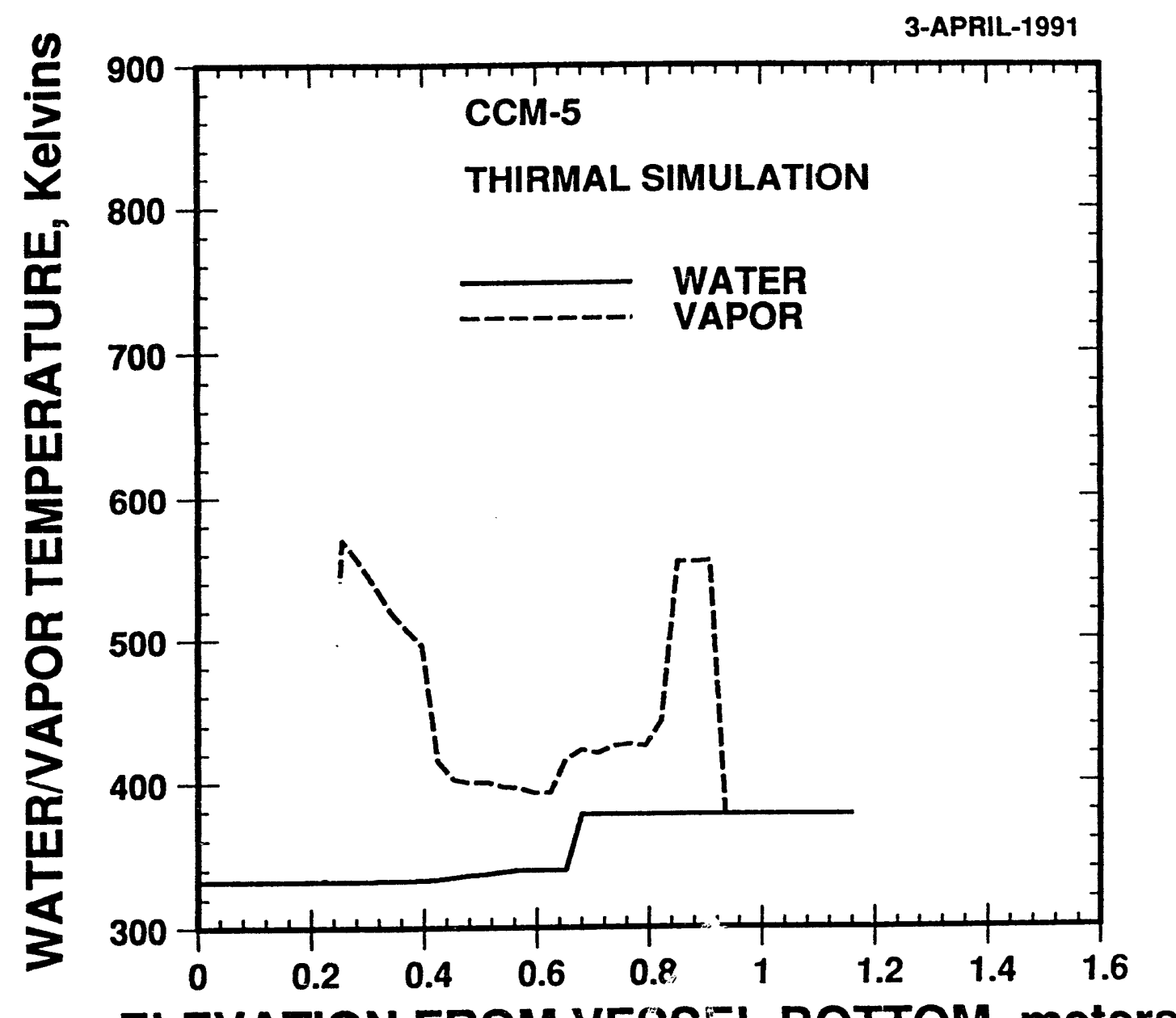

ELEVATION FROM VESSEL BOTTOM, meters

Figure 5-26. THIRMAL Prediction of Water and Vapor Temperatures in Mixing Zone at $t=0.55 \mathrm{sec}$ 


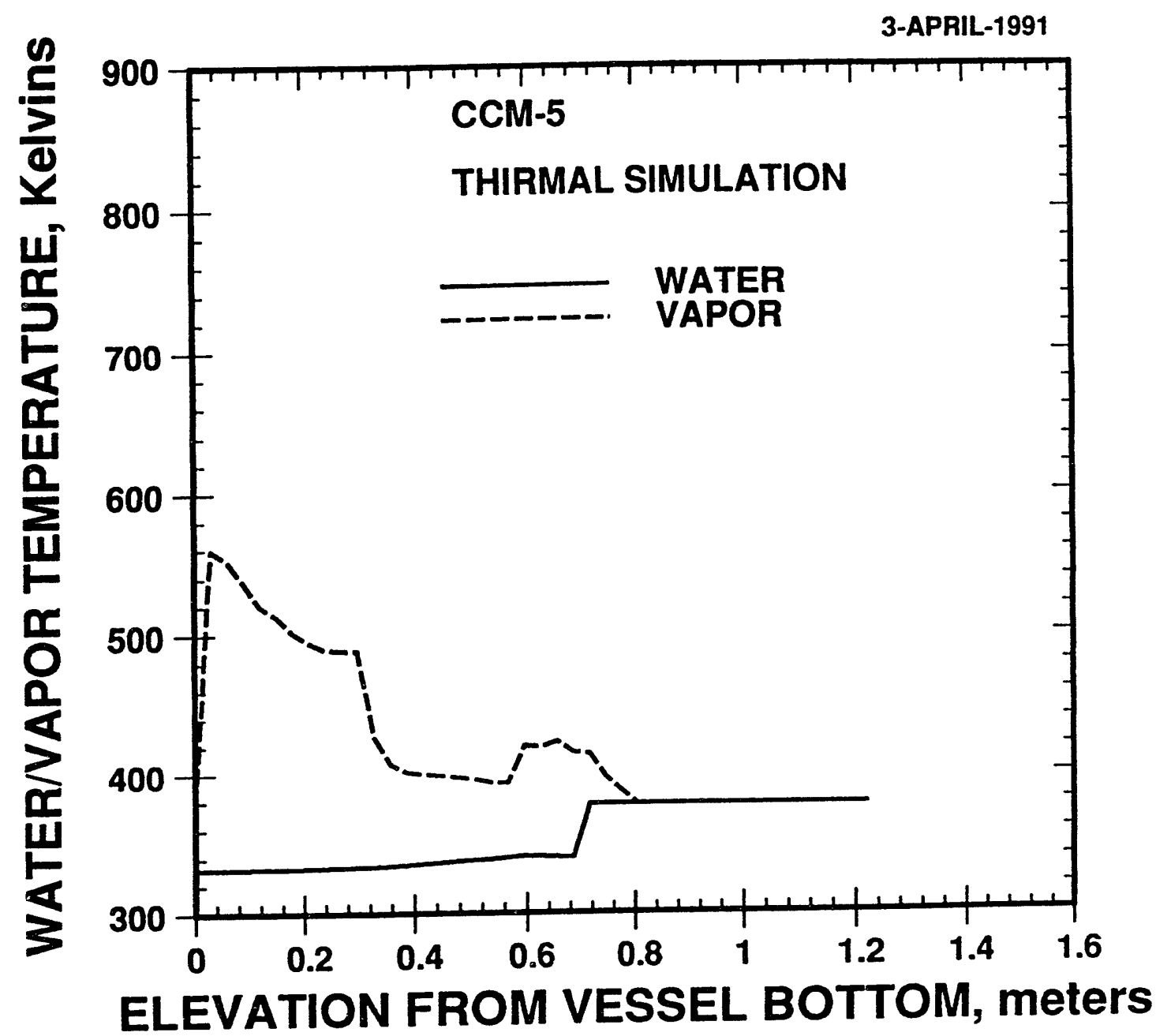

Figure 5-27. THIRMAL Prediction of Water and Vapor Temperatures in Mixing Zone at $t=0.65 \mathrm{sec}$ 


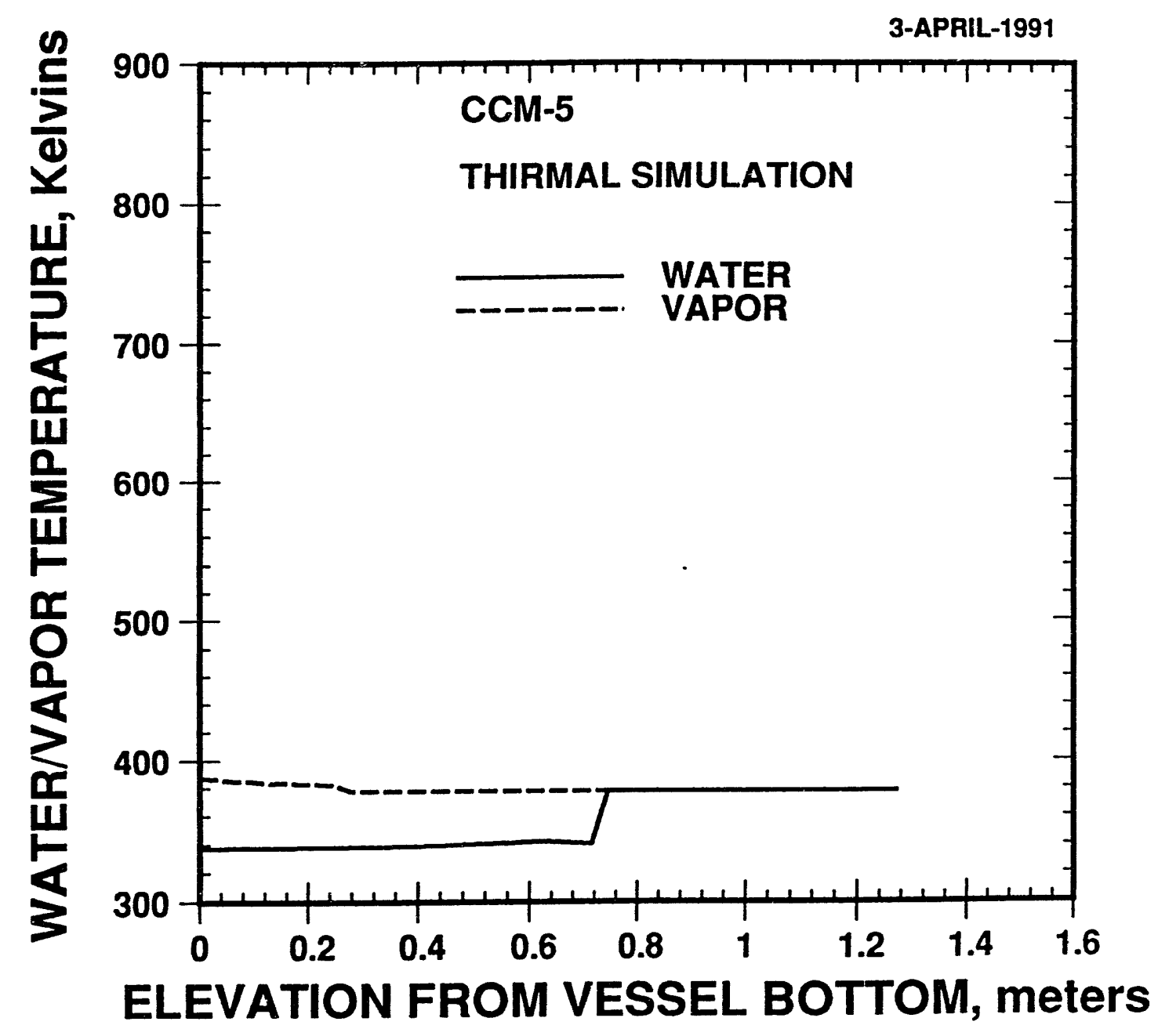

Figure 5-28. THIRMAL Prediction of Water and Vapor Temperatures in Mixing Zone at $t=1.15 \mathrm{sec}$ 
Based on the approximated temperature, void fraction and the mixing zone, the total water heating energy is estimated to be $7.1 \mathrm{MJ}$, which is consistent with the calculation based on thermocouple data (see Table 4.3).

The axial variation of the vapor temperature in the mixing zone shows a decrease toward the middle region of the mixing zone and then increases toward the water surface before a complete disruption of the jet occurs (see Figures 5-23 to 5-26). The turning point of the vapor temperature distribution occurs at the location where the flow dispersion occurs in the mixing zone. In the THIRMAL code the vapor phase in a form of bubbly flow is allowed to loose energy to the liquid continuous phase if the flow dispersion does not occur. Therefore, the average vapor temperature tends to decrease at higher elevation in the non-dispersed region. However, as the flow becomes dispersed, i.e., the vapor becomes the continuous phase, convective heat transfer from the meit particulates to the vapor phase becomes significant and THIRMAL predicts a continuous temperature increase toward the water surface. Also, the vapor temperature distributions in the dispersed region show a similar trend as the melt volume fraction (see Figures 5-11 to 5-13), since the heatup of vapor is directly proportional to the surface area of the melt material.

The steam and hydrogen generation rates predicted by the THIRMAL code are shown in Figure 5-29; the total accumulations are shown in Figure 5-30. The predicted steam generation rate qualitatively agrees with the estimate based on the system pressurization data (see Figure 4-11). The total steam generation is predicted to be around $0.5 \mathrm{~kg}$ for the first second (i.e., the jet fall stage) and agrees with the estimate based on Figure 4-11 (see Table 4-3). However, the net steam generation collected after the experiment was only $0.24 \mathrm{~kg}$, possibly due to condensation on the vessel wall. The total hydrogen generation is predicted to be $0.04 \mathrm{~kg}$ which agrees very well with the experiment data (see Table 4-1).

In the THIRMAL code, two rate limiting processes are considered in the prediction of the hydrogen generation. The first is the diffusion of water molecules through the vapor/gas film to the melt surface. Following Ref. [52], the rate of hydrogen production represented by this limitation is

$$
\frac{\mathrm{dW}}{\mathrm{dt}}=2.02 \frac{\mathrm{\Omega \Delta p}}{\mathrm{RT \delta}}
$$

The second rate limiting process is the diffusion of ferro and oxygen ions through the thickness of the oxidized layer. The rate of hydrogen generation is given by the parabolic kinetic expression [53]

$$
\frac{d W^{2}}{d t}=3.81 \times 10^{2} \exp \left(-\frac{84300}{R T}\right)
$$

where

$$
\begin{aligned}
& W \quad=\text { weight of zirconium reacted per unit surface area in } \mathrm{kg} / \mathrm{m}^{2}, \\
& t \quad=\text { time, }
\end{aligned}
$$




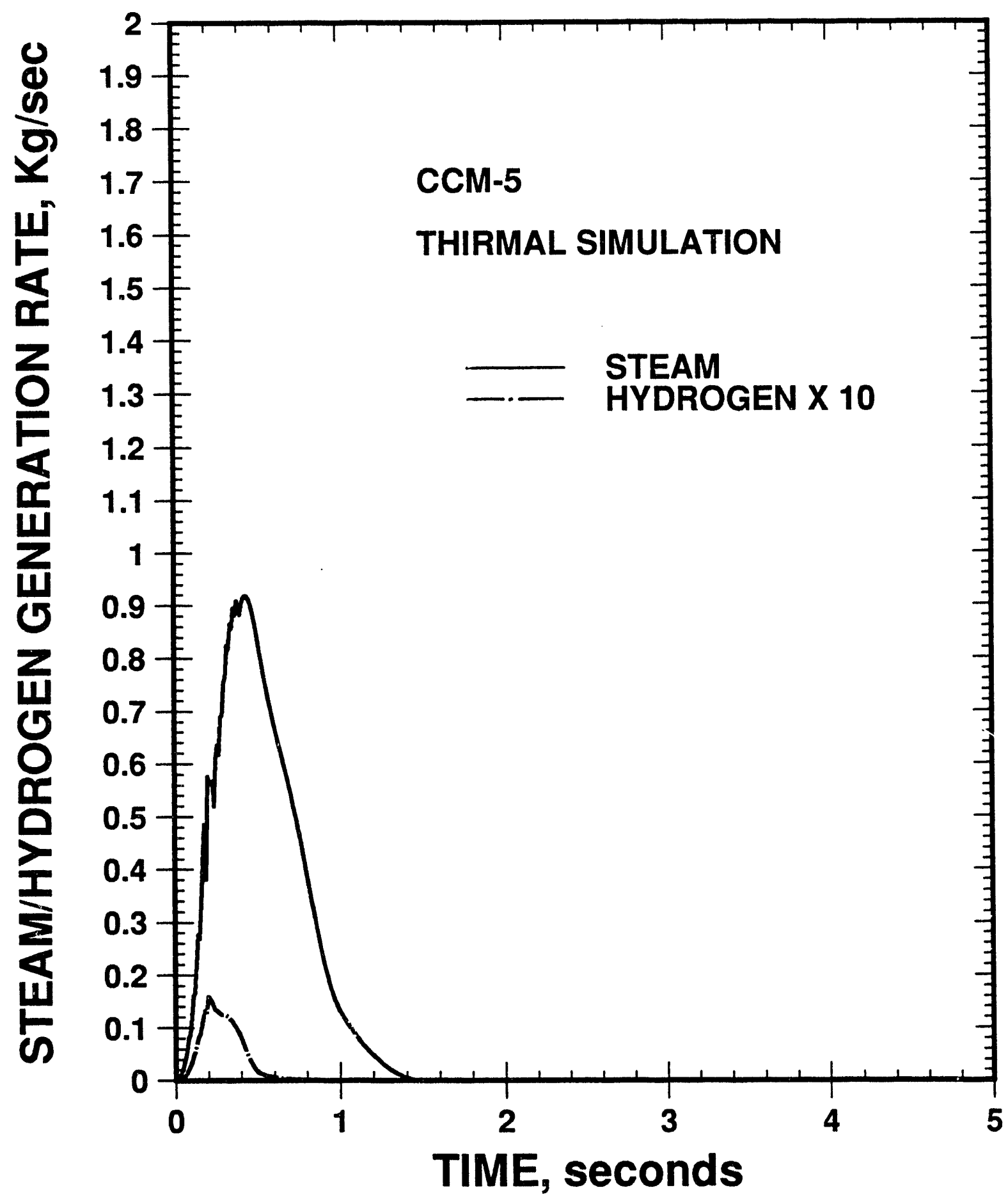

Figure 5-29. THIRMAL Prediction of Steam and Hydrogen Generation Rates 


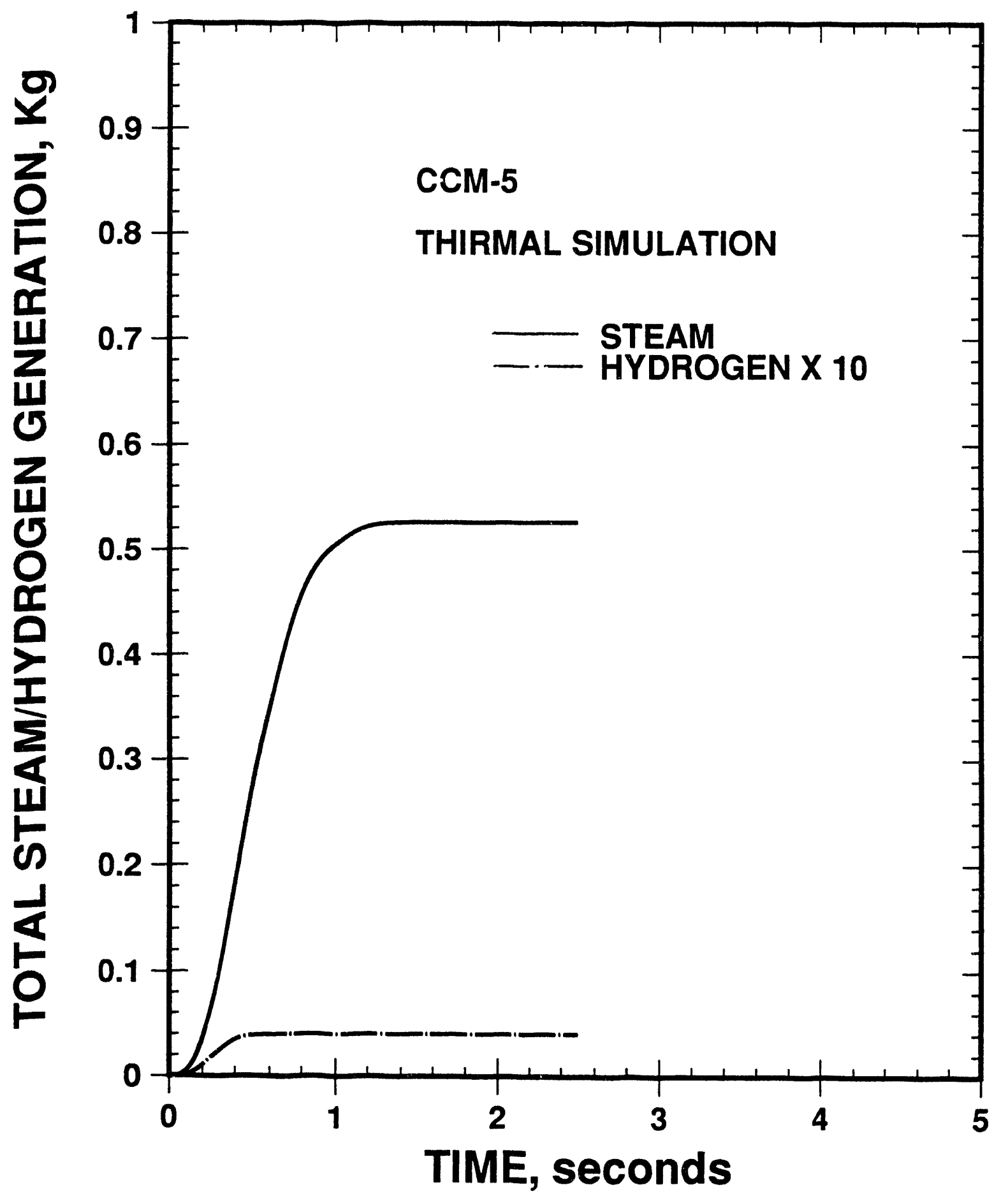

Figure 5-30. THIRMAL Prediction of Total Steam and Hydrogen Generation 
$\Omega=$ diffusion coefficient for steam and hydrogen,

$R \quad=$ gas constant,

$\delta \quad=$ vapor/gas film thickness.

In applying Eq. (5-37), the temperature, $T$, is set equal to the vapor temperature in the vapor film. In Eq. (5-38), the temperature is set equal to the melt surface temperature. In the THIRMAL code, both Eqs. $(5-37)$ and $(5-38)$ are provisionally evaluated and the lower value thus calculated is used to predict the hydrogen generation.

The system pressure, predicted by including the variations in temperature and volume of the steam/gas mixture in the experiment vessel, is shown in Figure 5-31 in comparison to the test measurements. The comparison shows a significant discrepancy for the pressure after $\sim 1 \mathrm{sec}$. This is probably due to the steam condensation on the vessel wall. However, the predicted system pressure agrees reasonably well with the measurements during the jet fall stage.

The debris characterization predicted by the THIRMAL code is shown in Figures 5-32 and 5-33. Figure 5-32 shows the mass of the accumulated melt particulates in terms of molten droplets and solid particles. It is predicted that the melt particulates start to accumulate on the vessel bottom at $\sim 0.8 \mathrm{sec}$ and $-96 \%$ of the accumulation is composed of solid particles. The prediction of a mostly solid debris bed agrees with the experiment in which a debris bed that consisted of loosely bound and sintered particles was found. Corresponding to Figure 5-32, Figure 5-33 shows the average temperatures of the accumulated particulates. The molten droplets are predicted to accumulate on the vessel bottom with the corium melting temperature. However, due to the limited amount of mass, the temperature of the accumulated molten droplets does not represent the average temperature of the debris which is better represented by the solid particles with temperatures varying from $2400 \mathrm{~K}$ to $1800 \mathrm{~K}$ in the first 2 seconds as predicted by the THIRMAL code. The temperature responses of the thermocouples in CCM-5 located on the vessel bottom varied from $\sim 1200 \mathrm{~K}$ to $1800 \mathrm{~K}$. The lower debris temperatures measured by the thermocouples compared to the code prediction may attribute to the heat loss to the vessel structure.

The prediction of the dispersed particle size distribution for CCM-5 is shown in Figure 5-34. The code predicts a median diameter of $-4.5 \mathrm{~mm}$ which agrees very well with the measurement of $5 \mathrm{~mm}$ (see Figure 3-58). The code also predicts a log-normal distribution for the dispersed particles. 


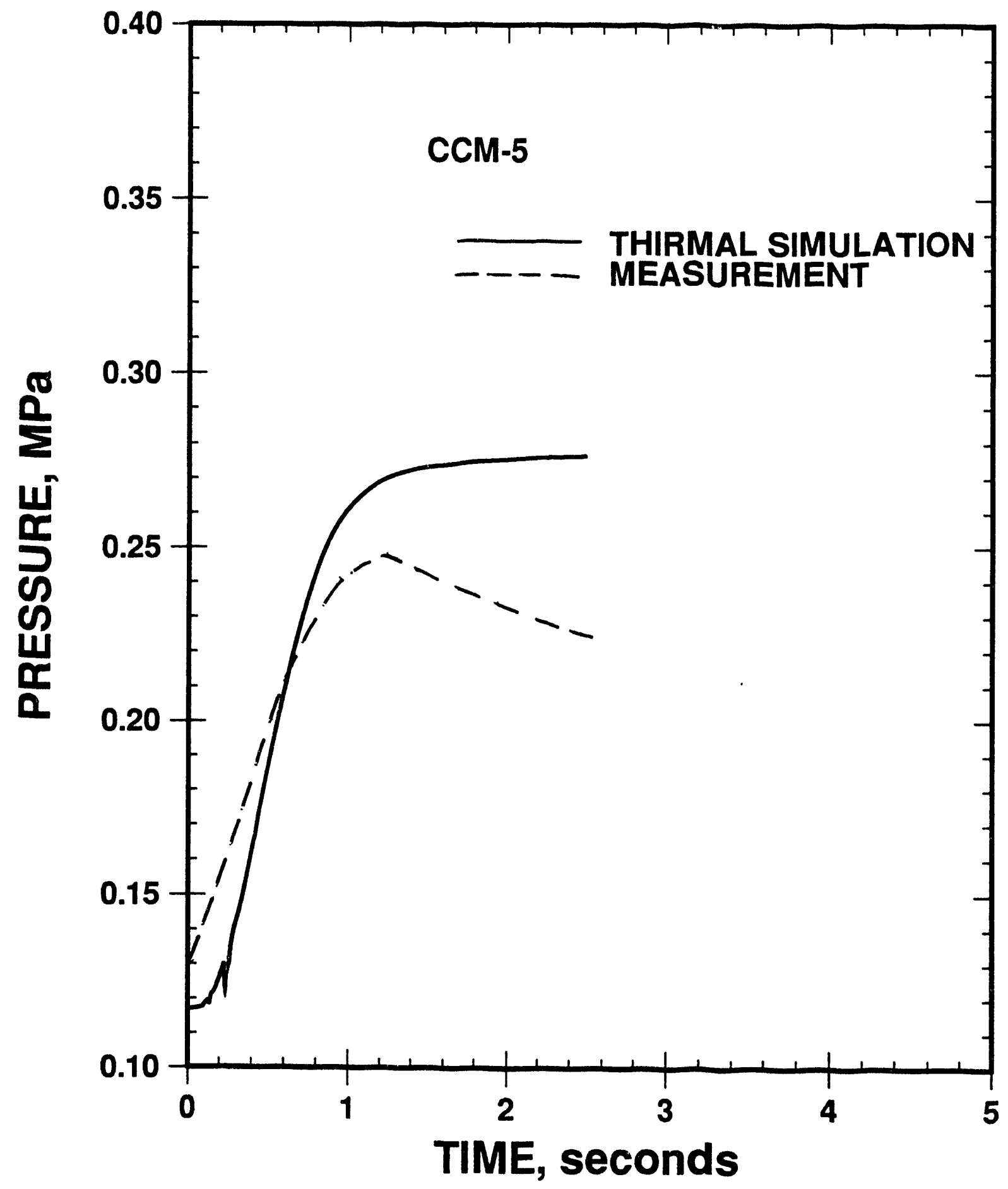

Figure 5-31. Comparison of Predicted and Measured System Pressure in Experiment Vessel 


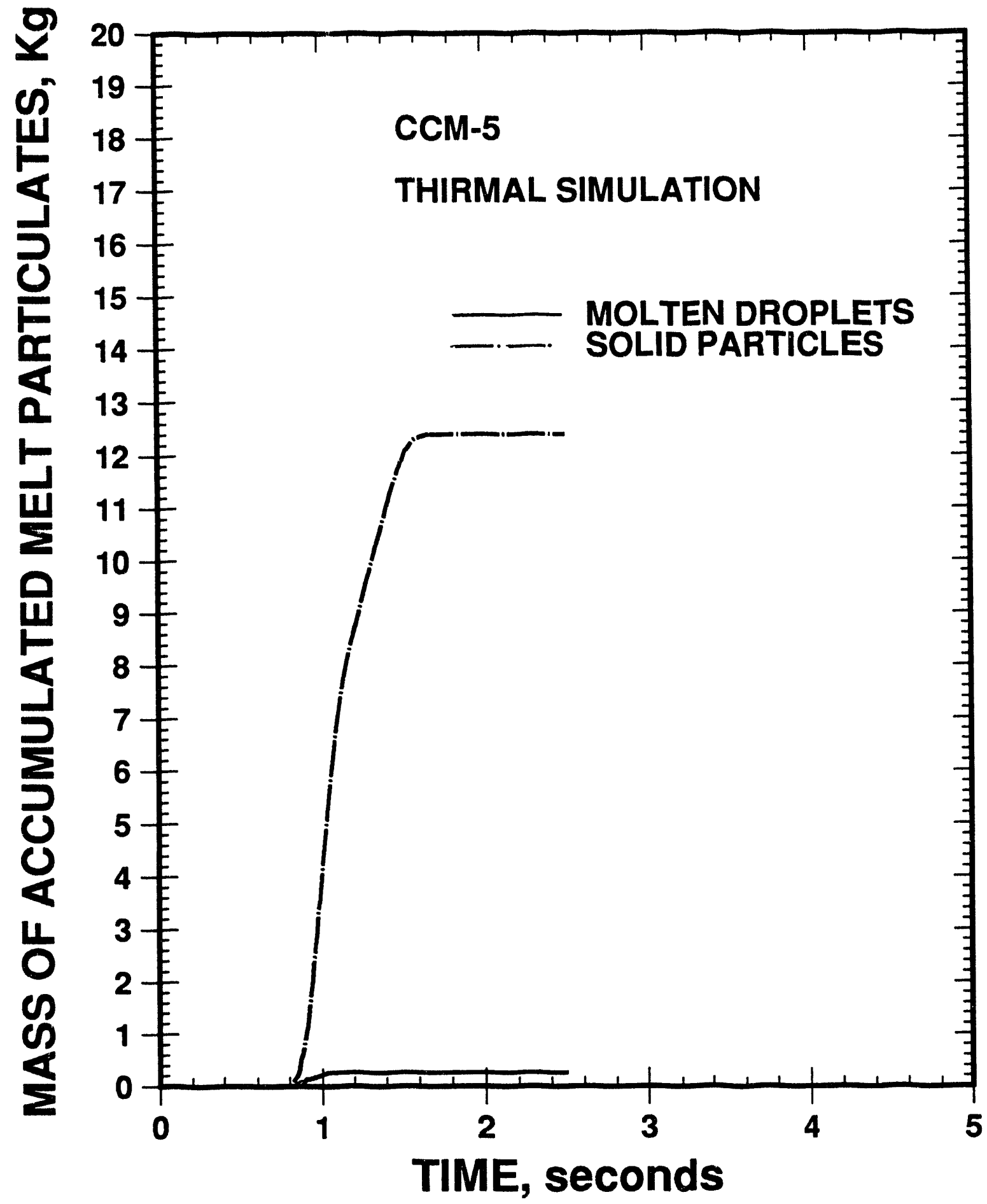

Figure 5-32. THIRMAL Prediction of Mass Accumulations of Melt Particulates on Vessel Bottom 


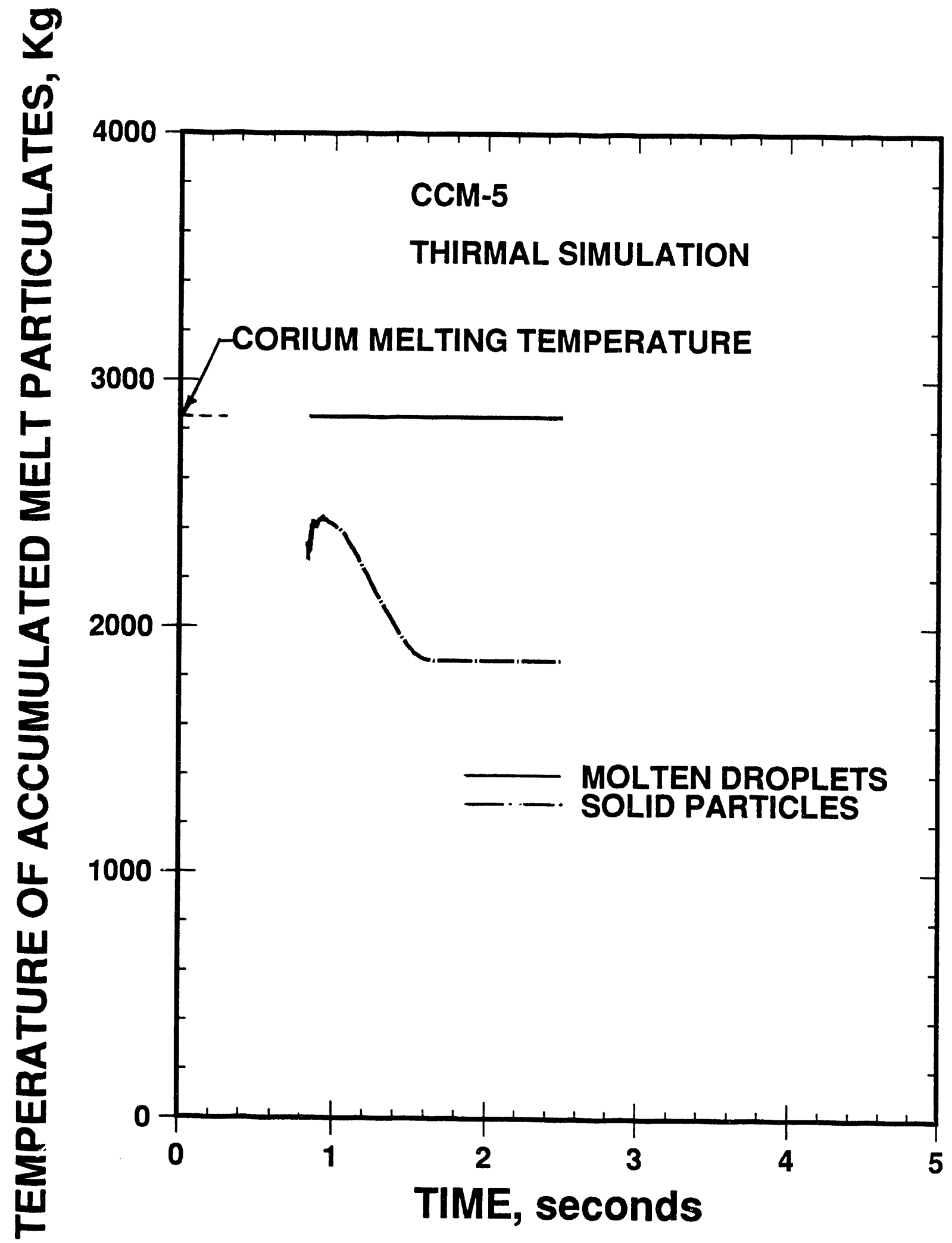

Figure 5-33. THIRMAL Predictions of Average Temperatures of Melt Particulates on Vessel Bottom 


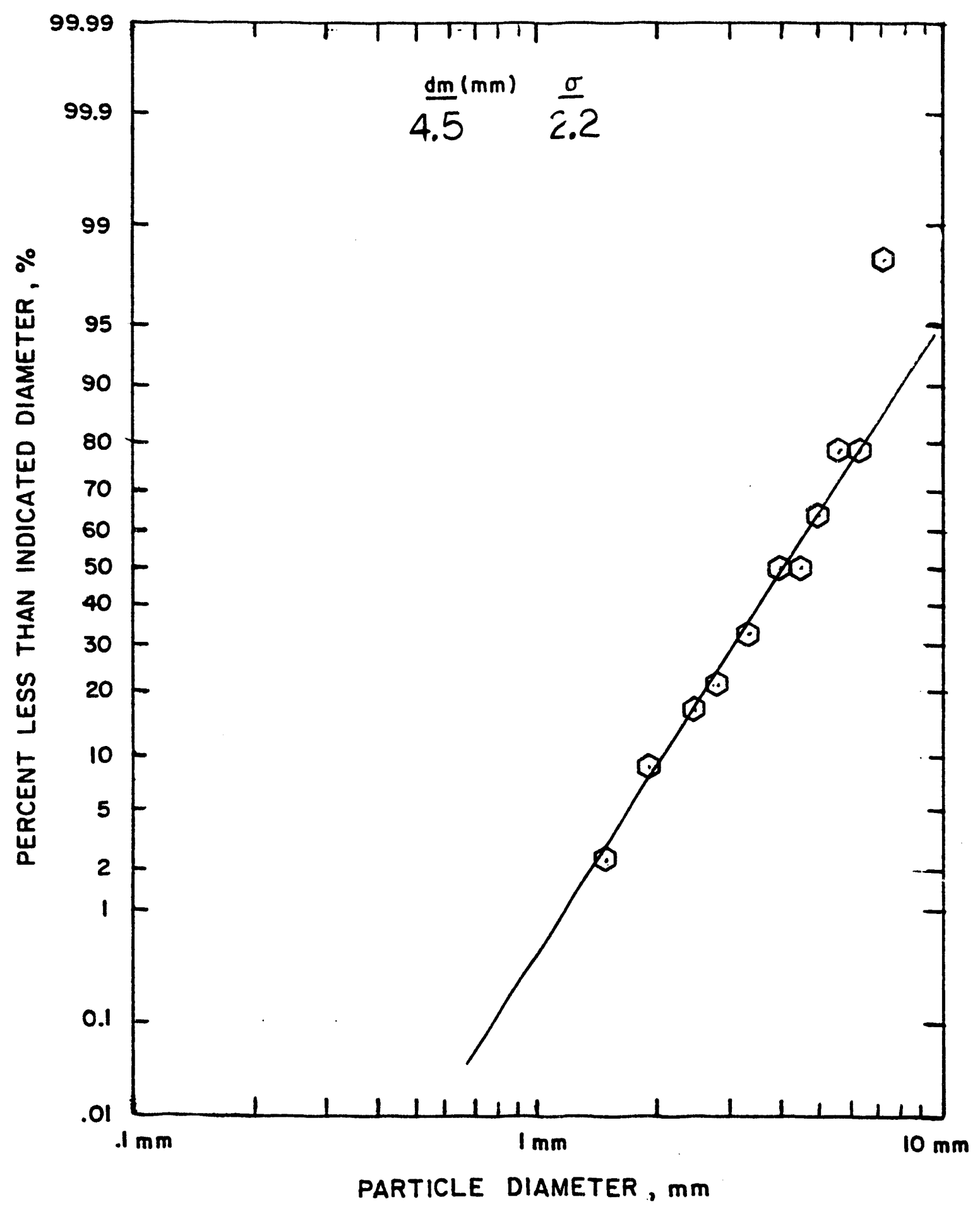

Figure 5-34. THIRMAL Prediction of Debris Particle Size Distribution 
were drawn:

From the test results, the following observations were made and conclusions

1. There were no steam explosions in any of the tests.

2. For the tests in which the thermocouple data showed a distinct change in the melt penetration velocity, jet breakup lengths were estimated to range from -10 to 19 jet diameters. For tests with low 'mass length' -todiameter ratios, i.e., CCM-5 and 6 , jet breakup length was probably limited by 'mass length'; it appears that a quasi-steady pour was not achieved. For tests with higher 'mass length' -to-diameter ratios, i.e., $\mathrm{CCM}-1,2$, and 3 , the measured breakup lengths were considerably higher. For small melt volume, corium mass, and jets diameter affect the breakup length primarily through the 'mass length'.

It is important to note that the durations of the melt injection in the CCM tests were relatively short. Thus, the observed interactions may be representative only of the initial phase of melt relocation, when corium streams first penetrate the coolant.

3. Based on the system pressurization data, water temperature, and pool swell data, a corium fall stage quench fraction ranging from 55 to $72 \%$ occurred with subcooled water, while the quench fraction was considerably lower with saturated water. As is to be expected, the larger heat sink provided by the subcooled water produced larger quench fractions.

4. The multi-jet geometry used in CCM-2 led to a significantly reduced quench fraction. In $X$-ray film, the volume between the jets appeared to be highly voided, reducing the jet surface in film boiling contact with water. This produced a lower overall heat transfer rate, and hence a lower fall stage quench fraction. However, the quench fraction may also have been reduced somewhat, in comparison with the other tests, by a relatively shallow water pool. The effect of multi-jet geometry is believed to have been less important in the TMI-2 core relocation, since the pitch ratio (i.e., the ratio of hole separation to diameter) of the TMI-2 core former plate, through which the molten core material is believed to have drained, is much higher than that used in CCM-2.

5. The metal exidation ranged from -2 to $35 \%$ of the maximum possible values and was dependent upon the water subcooling. The high oxidation rate in saturated water is due to the relatively higher steam volume fraction environment.

6. From pressurization data and calculated steam generation, a two-stage process for quenching the corium stream in a deep pool of water was indicated. Rapid heat transfer during the jet fall stage was reflected by the rapid steam pressurization rate. Heat removal from the surface of the debris layer formed at the bottom of the vessel was much slower.

7. The posttest debris examination indicated that a debris bed of solidified and substantially quenched particles was formed on the vessel bottom. of 
all the tests, only in $\mathrm{CCM}-2$ did the debris fragments reagglomerate, indicating very limited quench during the jet fall stage. As mentioned above, fall stage quench in this test was limited by voiding among the jets and/or the relatively shallow pool used in this test.

8. Based on the temperature history of the debris-water mixture at the vessel bottom, the debris bed was cooled relatively faster if the overlying water was subcooled. However, no ablation of the vessel bottom was observed in any of the tests.

9. The characteristic dimensions of the debris particles ranged between -1 to $5 \mathrm{~mm}$ and showed a log-normal distribution in all the CCM tests.

10. The initial jet velocity and water-to-corium mass ratio had no detectable effect on the jet breakup length and quench fraction.

11. The CCM test results suggest that the most important parameters affeiting molten core relocation are jet geometry (i.e., single jet vs. multiple jet) and water subcooling. The depth of the water pool also has a strong effect on the jet breakup and the configuration of the debris bed.

12. THIRMAL code has been developed to model the breakup and quench behavior of molten jet/water interactions. The code predictions are in good agreement with the test results of $\mathrm{CCM}-5$.

The CCM tests have provided an overall picture of corium-water interaction. However, the details of corium jet breakup and mixing have not yet been observed successfully. In order to develop and test models of this process, such observations of corium-water or appropriate simulant material systems are necessary. 


\section{REFERENCES}

1. Levich, V. C., Physiochemical Hydrodynamics, Prentice-Hall, Inc., Englewood Cliffs, NJ (1962).

2. Taylor, G. I., "The Dispersion of Jets of Metals at Low Melting Point in Water," The Scientific Papers of G. I. Taylor, Vol. III, ed. G. M. Batchelor (1940).

3. Epstein, M. and H. K. Fauske, "Steam Film Instability and the Mixing of Core-Melts of Jets and Water, "ANS Proc., 1985 National Heat Transfer Conference, ANS \#700101, Denver, CO (Aug. 1985).

4. Spencer, B. W., J. D. Gabor and J. C. Cassulo, "Effect of Boiling Regime on Melt Stream Breakup in Water, " Particulate Phenomena and Multiphase Transport, Vol. 3, Ed. T. N. Veziroglu, Hemisphere Publishing Co., 1987.

5. Hopkins, D. F. and J. M. Robertson, "Two-Dimensional Incompressible Fluid Jet Penetration," J. Fluid Mech., 29, Part 2, pp. 273-297 (1967).

6. Ginsberg, T., "Liquid Jet Breakup Characterization with Applications to Melt-Water Mixing," Proc. Intl. ANS/ENS Topical Mtg. on Thermal Reactor Safety, ANS \#70016, Vo1. 5, San Diego, CA (Feb. 2-6, 1986).

7. Spencer, B. W., J. J. Sienicki, and L. M. Mcumber, "Hydrodynamics and Heat Transfer Aspects of Corium-Water Interactions," EPRI NP-5127 (March 1987).

8. Marshall, B. W., Jr., "Coarse Mixing of Isothermal and Boiling Jets," Report Presented at Severe Fuel Damage Meeting, Silver Spring,. MD (May 4-8, 1987).

9. Marshal1, B.W., Jr., and Berman, M., "An Experimental Study of Isothermal and Boiling Liquid Jets, "14th Water Reactor Safety Research Information Meeting, Gaithersburg, MD (October 27-31, 1986).

10. Marshal1, B. W., Jr., D. F. Beck, and M. Berman, "Mixing of Isothermal and Boiling Molten-Core Jets with Water: The Initial Conditions for Energetic FCIs, " Proc. of the International ENS/ANS Conference on Thermal Reactor Safety, Avignon, France, October 2-7, 1988.

11. Lamberton, W. A., and M. H. Mueller, "Uranium Oxide Phase Equilibrium Systems: III, $\mathrm{UO}_{2}-\mathrm{ZrO}_{2}$, " Journal of American Ceramic Society, $\underline{\mathbf{3 5}}$, pp. $365-368$ (1953).

12. "Flow of Fluids Through Valves, Fittings, and Pipe, "Crane Technical Paper No. 410, Crane Co., Chicago, IL (1965).

13. Baker, L., Jr., "Hydrogen-Generating Reactions in LWR Severe Accidents," Proceedings International Meeting on Light Water Reactor Severe Accident Evaluation, Cambridge, MD (August 28-September 1, 1983). 
14. Kataoka, I., and M. Ishii, "Prediction of Pool Void Fraction by New Drift Flux Correlation," NUREG/CR-4657, ANL-86-29 Report, Argonne National Laboratory, Argonne, IL (June 1986).

15. Taylor, G. I., "The Instability of Liquid Surfaces When Accelerated in a Direction Perpendicular to Their Planes, Park I, "Proc. Roy. Soc. London, A-201, p. 192 (1950).

16. Lamb, H., "Hydrodynamics," Sixth edition, Cambridge University Press, reprinted by Dover Publications, New York (1945).

17. Tomotika, S., "On the Instability of a Cylindrical Thread of a Viscous Liquid Surrounded by Another Viscous Fluid," Proc. royal Society London, Series A., Vol. 150, p. 322 (1935).

18. Chandrasekhar, Hydrodynamic and Hydromagnetic Stability, Dover Publications, Inc., New York (1981).

19. Bin, A. K., "Minimum Air Entrainment Velocity of Vertical Plunging Liquid Jets," Chem. Engineering Sci., Vol. 43, No. 2, p. 379 (1988).

20. Windquist, S. C., and Corradini, M. L., "Modeling the Atomization of a Liquid Jet and Application of the Model to Large Diameter Molten Corium Jets," Masters Thesis, University of Wisconsin, 48, August 1986.

21. Rayleigh, W. S., "On the Destability of Jets," Proc. Lond. Math. Soc., Vol. 1, p. 10 (1978).

22. Weber, C., "On the Breakdown of a Fluid Jet," Zeitschrift für angewandte Mathematik and Mechanik, Vol. 11, p. 136 (1931).

23. Kitamura, Y. and Takahaski, T., "Stability of Jets in Liquid-Liquid Systems," Encyclopedia of Fluid Mechanics, Vol. 2, Chapter 19, Gulf Publishing To., Houston (1986).

24. Rumscheidt, F. D. and Mason, S. G., "Break-up of Stationary Liquid Threads," J. Cooloid Sc., 17, p. 260 (1962).

25. Ervine, D. A., McKeogh, E. and Elsawy, E. M., "Effect of Turbulence Intensity on the Rate of Air Entrainment by Plunging Water Jets, "Proc. Inst. Civ., Engrs., Park 2, $\underline{69}$ p. 425 (1980).

26. McCarthy, J. J. and Molloy, N. A., "Review of Stability of Liquid Jets and the Influence of nozzle Design," Chem. Eng. Journal, Vol. 7, p. 1 (1974).

27. Iciek, J., The Hydrodynamics of a Free Liquid Jet and Their Influence on Direct Contact Heat Transfer - I. Hydrodynamics of a Free, Cylindrical Liquid Jet," Int. J. Multiphase Flow, $\underline{8}$, p. 239 (1982). 
28. Taylor, G. I., "Generation of Ripples by Wind Blowing Over a Viscous Fluid," (1940) in G. K. Batchelor, The Scientific Papers of G. I. Taylor, Vol. 3, Cambridge University Press, Cambridge, MA (1963).

29. Hoyt, H. J. and Taylor, J. J., "Waves on Water Jets," J. Fluid Mechanics, Vol. 83, Part 1, p. 119 (1977).

30. Lin, S. P. and Kang, D. J., "Atomization of a Liquid Jet," Physics of Fluids, 30, No. 7, p. 2000 (1987).

31. Green, A. E. and Laws, N., "Ideal Fluid Jets," Int. J. Sci., Vol. 6, p. 317 (1968).

32. De Jarlais, G., Ishii, M., Linehan, J., "Hydrodynamic Stability of Inverted Annular Flow in an Adiabatic Simulation, "ASME Trans., Vol. 108, p. 84 (1986).

33. Entov, V. M. and Yuarin, A. L., "Dynamical Equations for a Liquid Jet," fluid Dynamics, Vol. 15, No. 5, September-October 1980, p. 644.

34. Yarin, A. L., "On the Dymnamical Equations for Liquid Jets," Fluid Dynamics, Vol. 18, No. 1, January-February 1983, p. 134.

35. Entov, V. M. and Yarin, A. L., "Transverse Stability of a Liquid Jet in a Counterflowing Air Stream," J. Engineering Physics, Vol. 38, No. 5, May 1980, p. 495.

36. Reynolds, A. J., "Observations of a Liquid-into-Liquid Jet," J. Fluid Mech., 14, p. 552 (1962).

37. Gabor, J. D., Purviance, R. T., Aeschlimann, R. W. and Spencer, B. W., "Characterization of Metal Fuel Fragmentation," ANL-IFR-52, Argonne National Laboratory (1986).

38. Saito, M., et. al., "Experimental Study on Penetration Behaviors of Water Jet into Freon-11 and Liquid Nitrogen," ANS Proc. 1988 National Heat Transfer Conf., Houston, TX, July 24-27, 1988.

39. Pilch, M. and Erdman, C. A., "Consideration of Liquid Drop Fragmentation in Safety Analysis," ANS Trans., 38 p. 403 (1981).

40. Chu, C. C. and Corradini, M. L., "Hydrodynamic Fragmentation of Liquid Droplets," ANS Transactions, 47 p. 306 (1984).

41. Fletcher, D. F., "The Particle Size Distribution of Solidified Metal Debris from Molten Fuel-Coolant Interaction Experiments," UKAEA Culham Laboratory Report CLM-P816 (March 1987).

42. Brauer, F. E., Green, N. W. and Messler, R. B., "Metal/Water Explosions," Nuclear Science and Engineering, 31 p. 551 (1968).

43. Wang, S. K., Blomquist, C. A., and Spencer, B. W., "Modeling of Thermal and Hydrodynamic Aspects of Molten Jet/Water Interaction," ANS Proceedings, 1989 National Heat Transfer Conference. 
44. Wang, S. K., C. A. Blomquist, and B. W. Spencer, "Interfacial Instabilities Leading to Bubble Departure during Film Boiling on Vertical Surface," ANS Proc., 1989 National Heat Transfer Conference.

45. Fletcher, D. F., and Thyagaraja, A., "A Method of Quantitatively Describing a Multi-Component Mixture," CLM-P812, Culham Laboratory, 1987.

46. Amarasooriya, W. H., and Theofanous, T. G., "Premixing of Steam Explosions: A Three-fluid Model," ANS Proc. of 1988 National Heat Transfer Conference, Vol. 3, p. 191-200, 1988.

47. Schneider, J. P., Characterization of Jet Breakup Mechanisms Obtained from Simulant Experiments of Molten Fuel Penetrating Coolant, " presented at the DOE-OER Annual Review of University Research, Savannah River site, SC, Aprit 22-24, 1991.

48. Rohsenow, W. H., "A Method of Correlating Heat Transfer Data for Surface Boiling of Liquid," Trans. ASME, 74 (37), p. 969-976, 1952.

49. Sideman, S., and Shahtai, H., "Direct Contact Heat Transfer between a Single Drop and Immisible Liquid Medium," Can. J. of Chem. Eng., June 1964 , P. 107-117, 1963.

50. Hewitt, H. C. and Parker, J. D., "Bubble Growth and Collapse in Liquid Nitrogen," Trans. ASME, J. Heat Transfer, P. 22-26 (1968).

51. Leung, J. C. M., Lambert, G. A., and Slachyra, L. J., "Transition to Dispersed Flows in a Stagnant Pool with Gas Injection," ANS Trans., Vol. 38, 1981 .

52. Baker, L., Jr., and Just, J. C., "Studies of Metal-Water Reactions at High Temperatures III: Experimental and Theoretical Studies of the Zirconium-Water Reaction," ANL-6548, Argonne National Laboratory, 1962.

53. J. T. Bittel, L. H. Sjodahl and J. F. White. "Oxidation of 304L Stainless Steel by Steam and by Air," Corrosion, 25, 1969, pp. 7-14. 


\section{ACKNOWLEDGEMENTS}

This work was funded by the U.S. Nuclear Regulatory Commission. We gratefully acknowledge the support and guidance of the NRC program managers, J. L. Telford and R. W. Wright. The efforts of L. J. Ondracek and J. Kawka in preparing the manuscript and figures are deeply appreciated. 
As mentioned in Section 2.3.1, instrumentation consisted of thermocouples and pressure transducers for all tests and level and void detectors for some of the tests. Thermocouples were $1.59-\mathrm{mm}$ OD stainless-steel sheathed chrome 1 alumel with a grounded junction except for TC 12-6 and all the water thermocouples in experiments CCM-5 and 6, which were bare junction. Pressure transducers were either strain gage or piezoelectric and their description are presented in Table A1. Sensor locations are designated by their radius from the centerline of the vessel where installed, by the angular orientation shown in Fig. A1 or $A 2$ and by the vertical elevation from the pipeway outlet for tests $C C M-1$ through 4 and from the water surface for tests CCM-5 and 6 . The thermocouple insertion pattern for the base of the interaction vesse 1 is shown in Fig. A3. Tables A2 through A7 contain the locations of the instrumentation used in experiments CCM-1 through 6 , respectively. 

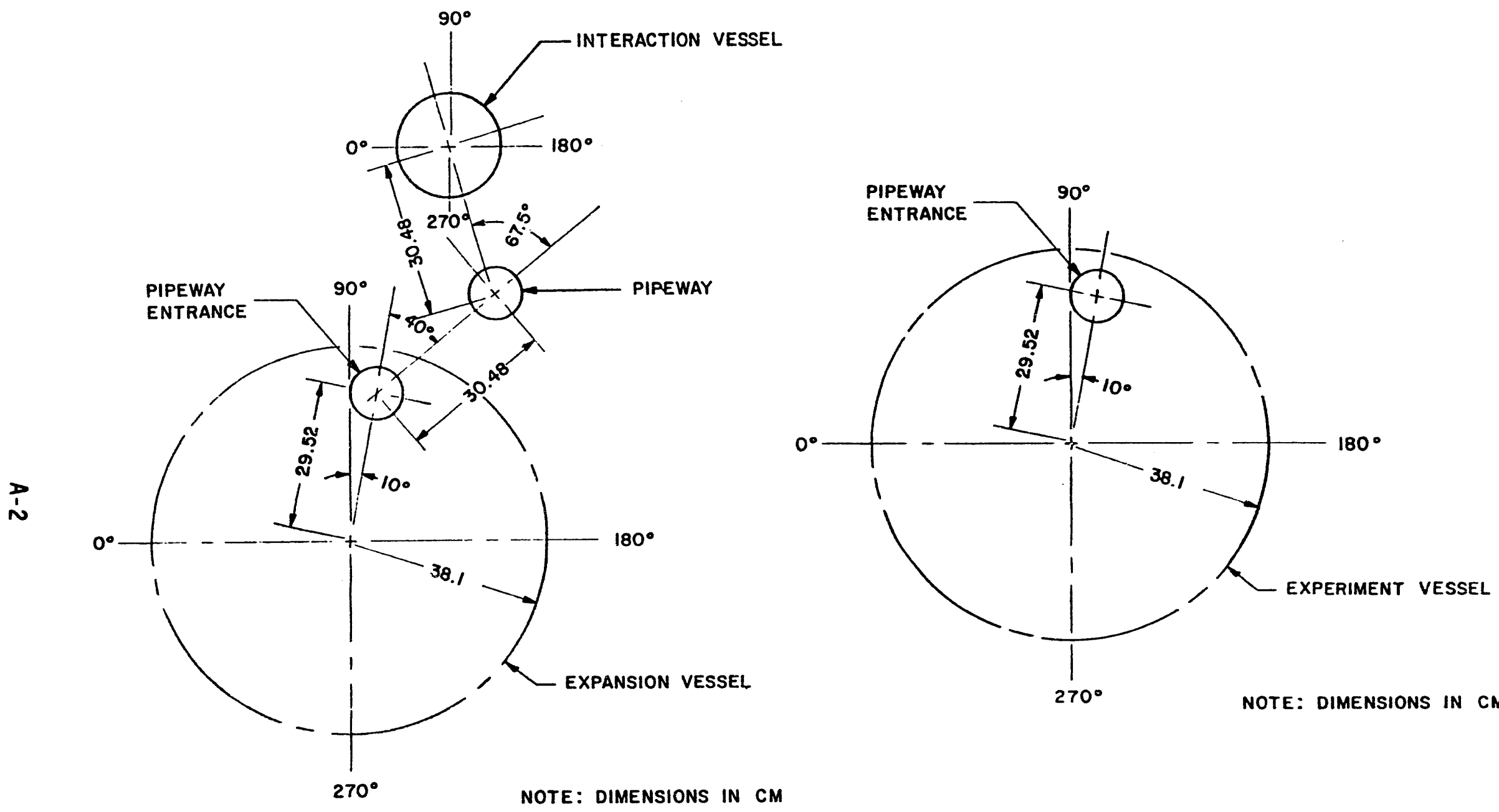

Fig. A.1.

Angular Orientation for Sensor Location for Experiments $\mathrm{CCM}-1,2,3$, and 4 .
Fig. A.2.

Angular Orientation for Sensor Location for Experiments CCM-5 and 6. 

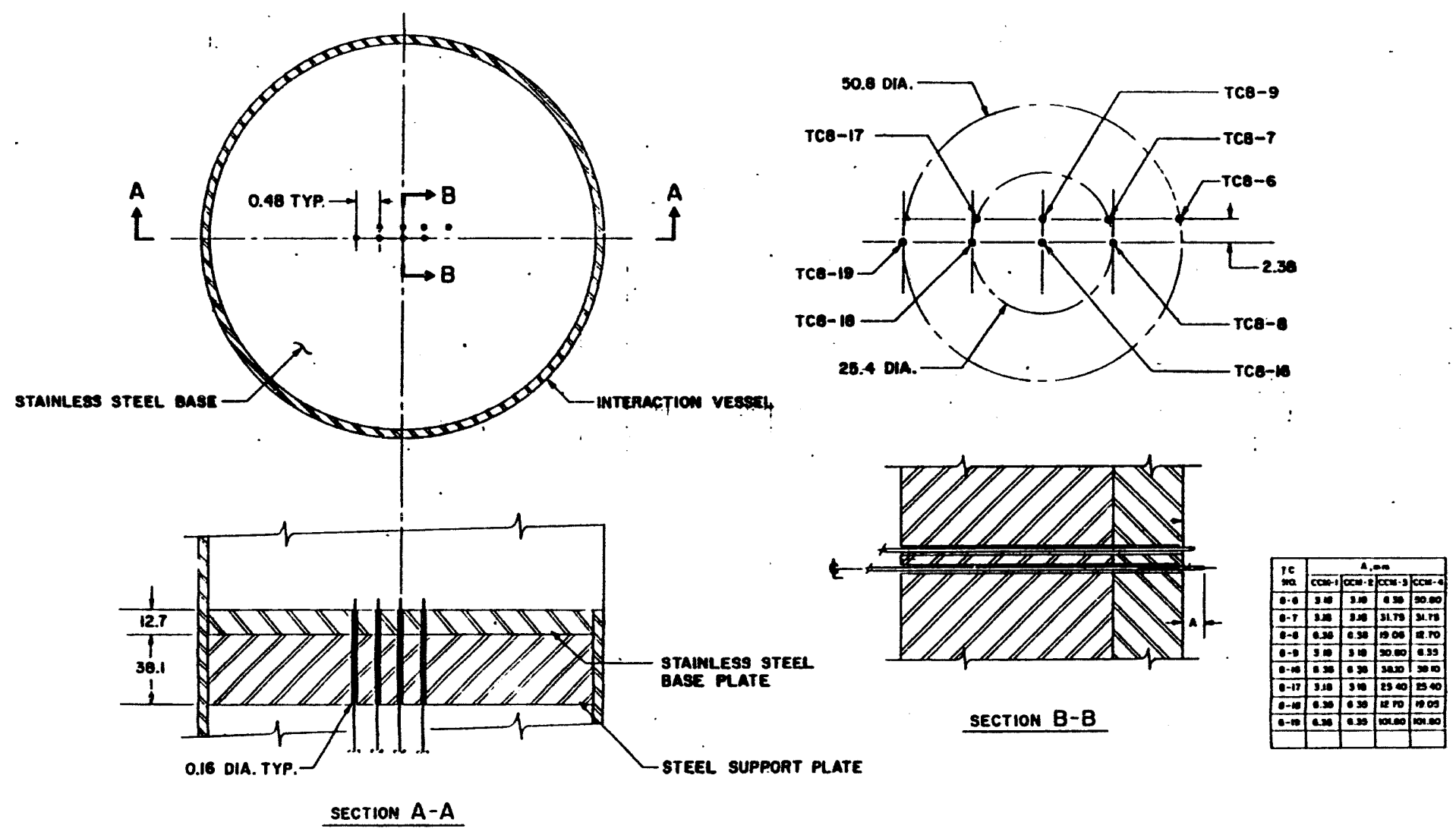

SECTION A-A

NOTE : DIMENSIONS IN MILLIMETERS

Fig. A.3. Instrumented Base for Interaction Vesse1. 
Table A1. Pressure Sensor Description and Designation

Sensor Designation

Sensor Description

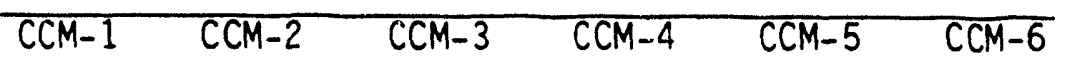

Data Sensors Mode1 PB513-G4

strain gauge pressure transducer, ser. no. 94, 0-100 psig range

PTS-1 PTS-1 PTS-1 PTS - 1

Statham Model PA732TC-100-350

strain gauge pressure transducer, ser. no. 2667, 0-100 psia range

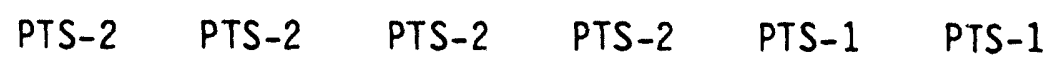

Statham Mode 1 PA732TC-150-350

strain gauge pressure transducer, ser. no. $2439,0-100$ psia range

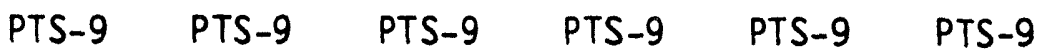

Sensotec Series T strain gauge

pressure transducer, ser. no.

33292, 0-100 psia range

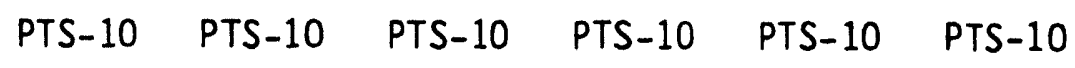

Data Sensors Model PB413B-9

strain gauge pressure transducer, ser. no. 148, 0-100 psig

PTS-12 PTS-12 PTS-12 PTS-12

Alinco Model 152-BAU62

strain gauge pressure transducer, ser. no. $34454,0-100$ psig

PTS-13 PTS-13 PTS-13

Alinco Model 151 strain gauge pressure transducer, ser. no. 26266, 0-100 psig

$\begin{array}{llll}\text { PTS-14 } & \text { PTS-14 PTS-14 -- } & \text { PTS-12 PTS-12 }\end{array}$

Statham Model P10-100G-350

strain gauge pressure transducer, ser. no. 934, 0-100 psig range

$\begin{array}{llll}\text { PTS-15 PTS-15 PTS-15 -- PTS-13 PTS-13 } & \text { PT }\end{array}$

PCB Model 112A05 piezoelectric pressure transducer, ser. no. 1987, 0-100 psig range

PTK-1 -- $\quad--\quad$ PTK-1 PTK-1 PTK-1

PCB Model 112A05 piezoelectric pressure transducer, ser. no. 2114, 0-100 psig range

PTK-2

PCB Model 112A05 piezoelectric pressure transducer, ser. no. 2339, 0-100 psig range

PTK-3 
Table A1. Pressure Sensor Description and Designation (cont'd)

\begin{tabular}{|c|c|c|c|c|c|c|}
\hline \multirow[b]{2}{*}{ Sensor Description } & \multicolumn{6}{|c|}{ Sensor Designation } \\
\hline & CCM-1 & CCM-2 & CCM-3 & CCM-4 & CCM-5 & CCM-6 \\
\hline $\begin{array}{l}\text { lodel } 112 A 05 \text { piezoelectric } \\
\text { ure transducer, ser. no. } \\
0-100 \text { psig range }\end{array}$ & PTK-4 & -- & -- & -- & -- & -- \\
\hline $\begin{array}{l}\text { ure transducer, ser. no. } \\
0-300 \text { psig range }\end{array}$ & -- & -- & -- & -- & PTK-3 & PTK-3 \\
\hline
\end{tabular}

PCB Model 112A05 piezoelectric pressure transducer, ser. no. $2340,0-100$ psig range

PCB Mode 1112 A05 piezoelectric pressure transducer, ser. no. $2307,0-300$ psig range 
Table A2. Sensor Locations for Test CCM-1

\begin{tabular}{|c|c|c|c|c|c|}
\hline $\begin{array}{l}\text { Sensor } \\
\text { No. }\end{array}$ & Location & $\begin{array}{l}\text { Elevation, } \\
\mathrm{cm}\end{array}$ & $\underset{\mathrm{cm}}{\operatorname{Radius}}{ }^{2}$ & $\begin{array}{c}\text { Angle, } \\
\text { deg. }\end{array}$ & Function \\
\hline PTS-1 & $\begin{array}{l}\text { Thermite vessel } \\
\text { top flange }\end{array}$ & 89.85 & 0.0 & 324.5 & $\begin{array}{l}\text { Pressure in gas space } \\
\text { of thermite vessel }\end{array}$ \\
\hline PTS-2 & $\begin{array}{l}\text { Interaction vesse } \\
\text { head }\end{array}$ & 41.58 & 8.57 & 147.5 & $\begin{array}{l}\text { Pressure in interaction } \\
\text { vessel vapor space }\end{array}$ \\
\hline PTS-9 & Expansion vesse 1 & $\begin{array}{l}309.57 \\
\text { top section }\end{array}$ & 4.45 & 165.0 & $\begin{array}{l}\text { Pressure in upper region } \\
\text { of expansion vesse } 1\end{array}$ \\
\hline PTS-10 & Expansion vesse 1 & 38.10 & 38.10 & 350.0 & $\begin{array}{l}\text { Pressure in lower } \\
\text { region of expansion } \\
\text { vessel }\end{array}$ \\
\hline PTS-12 & $\begin{array}{l}\text { Interaction vessel } \\
\text { upper top section }\end{array}$ & 5.70 & 11.85 & 297.5 & $\begin{array}{l}\text { Pressure in interaction } \\
\text { vessel }\end{array}$ \\
\hline PTS-13 & $\begin{array}{l}\text { Interaction vessel } \\
\text { lower top section }\end{array}$ & -19.70 & 11.85 & 342.5 & $\begin{array}{l}\text { Pressure in interaction } \\
\text { vessel }\end{array}$ \\
\hline PTS-14 & $\begin{array}{l}\text { Interaction vesse } 1 \\
\text { upper middle } \\
\text { section }\end{array}$ & -45.74 & 11.85 & 252.5 & $\begin{array}{l}\text { Pressure in interaction } \\
\text { vessel }\end{array}$ \\
\hline PTS-15 & $\begin{array}{l}\text { Interaction vesse } 1 \\
\text { lower middle } \\
\text { section }\end{array}$ & -60.98 & 11.85 & 252.5 & $\begin{array}{l}\text { Pressure in interaction } \\
\text { vessel }\end{array}$ \\
\hline PTK-1 & $\begin{array}{l}\text { Interaction vesse } 1 \\
\text { upper top section }\end{array}$ & 5.70 & 11.85 & 207.5 & $\begin{array}{l}\text { Pressure in interaction } \\
\text { vessel }\end{array}$ \\
\hline PTK-2 & $\begin{array}{l}\text { Interaction vesse } 1 \\
\text { lower top section }\end{array}$ & -19.70 & 11.85 & 252.5 & $\begin{array}{l}\text { Pressure in interaction } \\
\text { vessel }\end{array}$ \\
\hline PTK-3 & $\begin{array}{l}\text { Interaction vesse } 1 \\
\text { upper middle } \\
\text { section }\end{array}$ & -45.74 & 11.85 & 342.5 & $\begin{array}{l}\text { Pressure in interaction } \\
\text { vessel }\end{array}$ \\
\hline PTK-4 & $\begin{array}{l}\text { Interaction vesse } 1 \\
\text { lower middle } \\
\text { section }\end{array}$ & -60.98 & 11.85 & 357.5 & $\begin{array}{l}\text { Pressure in interaction } \\
\text { vessel }\end{array}$ \\
\hline
\end{tabular}

1 Pipeway outlet in expansion vessel is elevation 0.0. Water surface is elevation 9.19. Applicable for each vessel - see Fig. Al. 
Table A2. Sensor Locations for Test CCM-1 (contd.)

\begin{tabular}{|c|c|c|c|c|c|}
\hline $\begin{array}{l}\text { Sensor } \\
\text { No. }\end{array}$ & Location & $\underset{\mathrm{cm}}{\text { Elevation, }}$ & $\underset{\mathrm{cm}}{\operatorname{Radius}}, 2$ & $\begin{array}{c}\text { Angle, }{ }^{2} \\
\text { deg. }\end{array}$ & Function \\
\hline TC8-6 & $\begin{array}{l}\text { Interaction vesse } 1 \\
\text { base region }\end{array}$ & -96.86 & 2.54 & 69.6 & Water/debris temperature \\
\hline TC8-7 & $\begin{array}{l}\text { Interaction vesse } 1 \\
\text { base region }\end{array}$ & -96.86 & 1.27 & 61.4 & Water/debris temperature \\
\hline TC8-8 & $\begin{array}{l}\text { Interaction vesse } 1 \\
\text { base region }\end{array}$ & -96.54 & 1.27 & 72.5 & Water/debris temperature \\
\hline TC8-9 & $\begin{array}{l}\text { Interaction vesse } \\
\text { base region }\end{array}$ & -96.86 & 0.24 & 342.5 & Water/debris temperature \\
\hline TC8-10 & Expansion vessel & 0.0 & 0.0 & 0.0 & Gas/vapor temperature \\
\hline TC8-12 & $\begin{array}{l}\text { Interaction Vessel } \\
\text { Head Region }\end{array}$ & 39.04 & 8.57 & 327.5 & Gas/vapor temperature \\
\hline TC8-13 & $\begin{array}{l}\text { Interaction vesse } 1 \\
\text { head region }\end{array}$ & 39.04 & 8.57 & 357.5 & Gas/vapor temperature \\
\hline TC8-16 & $\begin{array}{l}\text { Interaction vesse } 1 \\
\text { base region }\end{array}$ & -96.54 & 0.0 & 0.0 & Water/debris temperature \\
\hline TC8-17 & $\begin{array}{l}\text { Interaction vesse } 1 \\
\text { base region }\end{array}$ & -96.86 & 1.27 & 263.3 & Water/debris temperature \\
\hline TC8-18 & $\begin{array}{l}\text { Interaction vessel } \\
\text { base region }\end{array}$ & -96.54 & 1.27 & 252.5 & Water/debris temperature \\
\hline TC8-19 & $\begin{array}{l}\text { Interaction vesse } 1 \\
\text { base region }\end{array}$ & -96.86 & 2.54 & 252.5 & Water/debris temperature \\
\hline TC8-20 & $\begin{array}{l}\text { Interaction vessel } \\
\text { upper top section }\end{array}$ & 5.7 & 8.89 & 27.5 & Water/vapor temperature \\
\hline TC8-21 & $\begin{array}{l}\text { Interaction vesse } 1 \\
\text { upper top section }\end{array}$ & 5.7 & 4.44 & 117.5 & Water/vapor temperature \\
\hline TC8-22 & $\begin{array}{l}\text { Interaction vesse } 1 \\
\text { lower top section }\end{array}$ & -19.7 & 8.89 & 72.5 & Water/vapor temperature \\
\hline TC8-23 & $\begin{array}{l}\text { Interaction vesse } 1 \\
\text { lower top section }\end{array}$ & -19.7 & 4.44 & 162.5 & Water/vapor temperature \\
\hline TC8-24 & $\begin{array}{l}\text { Interaction vessel } \\
\text { upper middle } \\
\text { section }\end{array}$ & -45.74 & 8.89 & 72.5 & Water/vapor temperature \\
\hline
\end{tabular}


Table A2. Sensor Locations for Test CCM-1 (contd.)

\begin{tabular}{|c|c|c|c|c|c|}
\hline $\begin{array}{l}\text { Sensor } \\
\text { No. }\end{array}$ & Location & $\underset{\mathrm{cm}}{\text { Elevation, }}$ & $\underset{\mathrm{cm}}{\text { Radius, }}, 2$ & $\begin{array}{l}\text { Angle, } 2 \\
\text { deg. }\end{array}$ & Function \\
\hline TC8-25 & $\begin{array}{l}\text { Interaction vessel } \\
\text { upper middle } \\
\text { section }\end{array}$ & -45.74 & 4.44 & 162.5 & Water/vapor temperature \\
\hline TC8-26 & $\begin{array}{l}\text { Interaction vessel } \\
\text { lower middle } \\
\text { section }\end{array}$ & -60.98 & 8.89 & 27.5 & Water/vapor temperature \\
\hline TC8-27 & $\begin{array}{l}\text { Interaction vessel } \\
\text { lower middle } \\
\text { section }\end{array}$ & -60.98 & 4.44 & 147.5 & Water/vapor temperature \\
\hline TC8-28 & Pipeway U-bend & -45.74 & - & - & Gas/vapor temperature \\
\hline TC9-2 & $\begin{array}{l}\text { Thermite vessel } \\
\text { gas space }\end{array}$ & 87.31 & 5.08 & 312.5 & Gas temperature \\
\hline TC12-1 & $\begin{array}{l}\text { Expansion vessel } \\
\text { instrument tree }\end{array}$ & 282.58 & 3.18 & 180.0 & Gas/vapor temperature \\
\hline TC12-2 & $\begin{array}{l}\text { Expansion vesse } 1 \\
\text { instrument tree }\end{array}$ & 241.94 & 3.18 & 0.0 & Gas/vapor temperature \\
\hline TC12-3 & $\begin{array}{l}\text { Expansion vessel } \\
\text { instrument tree }\end{array}$ & 201.30 & 3.18 & 315.0 & Gas/vapor temperature \\
\hline TC12-4 & $\begin{array}{l}\text { Expansicn vesse } 1 \\
\text { instrument tree }\end{array}$ & 160.66 & 3.18 & 157.5 & Gas/vapor temperature \\
\hline TC12-5 & $\begin{array}{l}\text { Expansion vessel } \\
\text { instrument tree }\end{array}$ & 120.02 & 3.18 & 0.0 & Gas/vapor temperature \\
\hline TC12-6 & $\begin{array}{l}\text { Expansion vessel } \\
\text { instrument tree }\end{array}$ & 79.38 & 3.18 & 202.5 & Gas/vapor temperature \\
\hline
\end{tabular}


Table A3. Sensor Locations for Test CCM-2

\begin{tabular}{|c|c|c|c|c|c|}
\hline $\begin{array}{l}\text { Sensor } \\
\text { No. }\end{array}$ & Location & $\begin{array}{c}\text { Elevation, } \\
\mathrm{cm}\end{array}$ & $\underset{\mathrm{cm}}{\operatorname{Radius}}, 2$ & $\begin{array}{l}\text { Angle, } \\
\text { deg }\end{array}$ & Function \\
\hline PTS-1 & $\begin{array}{l}\text { Thermite vesse } 1 \\
\text { top flange }\end{array}$ & 89.53 & 0.0 & 342.5 & $\begin{array}{l}\text { Pressure in gas space } \\
\text { of thermite vessel }\end{array}$ \\
\hline PTS-2 & $\begin{array}{l}\text { Interaction vesse } 1 \\
\text { head }\end{array}$ & 41.58 & 8.57 & 327.5 & $\begin{array}{l}\text { Pressure in interaction } \\
\text { vessel vapor space }\end{array}$ \\
\hline PTS-9 & $\begin{array}{l}\text { Expansion vesse } 1 \\
\text { top section }\end{array}$ & 309.57 & 4.45 & 165.0 & $\begin{array}{l}\text { Pressure in upper region } \\
\text { of expansion vessel }\end{array}$ \\
\hline PTS-10 & Expansion vesse 1 & 38.10 & 38.10 & 350.0 & $\begin{array}{l}\text { Pressure in lower region } \\
\text { of expansion vessel }\end{array}$ \\
\hline PTS-12 & $\begin{array}{l}\text { Interaction vessel } \\
\text { upper top section }\end{array}$ & 5.70 & 11.85 & 297.5 & $\begin{array}{l}\text { Pressure in interaction } \\
\text { vesse } 1\end{array}$ \\
\hline PTS-13 & $\begin{array}{l}\text { Interaction vesse } \\
\text { lower top section }\end{array}$ & -19.70 & 11.85 & 342.5 & $\begin{array}{l}\text { Pressure in interaction } \\
\text { vessel }\end{array}$ \\
\hline PTS-14 & $\begin{array}{l}\text { Interaction vesse } 1 \\
\text { upper top section }\end{array}$ & 5.70 & 11.85 & 207.5 & $\begin{array}{l}\text { Pressure in interaction } \\
\text { vessel }\end{array}$ \\
\hline PTS-15 & $\begin{array}{l}\text { Interaction vesse } 1 \\
\text { lower top section }\end{array}$ & -19.70 & 11.85 & 252.5 & $\begin{array}{l}\text { Pressure in interaction } \\
\text { vesse } 1\end{array}$ \\
\hline TC8-6 & $\begin{array}{l}\text { Interaction vesse } 1 \\
\text { base region }\end{array}$ & -50.82 & 2.54 & 144.6 & $\begin{array}{l}\text { Water/debris } \\
\text { temperature }\end{array}$ \\
\hline TC8-7 & $\begin{array}{l}\text { Interaction vesse } 1 \\
\text { base region }\end{array}$ & -50.82 & 1.27 & 139.2 & $\begin{array}{l}\text { Water/debris } \\
\text { temperature }\end{array}$ \\
\hline TC8-8 & $\begin{array}{l}\text { Interaction vesse } 1 \\
\text { base region }\end{array}$ & -50.51 & 1.27 & 150.0 & $\begin{array}{l}\text { Water/debris } \\
\text { temperature }\end{array}$ \\
\hline TC8-9 & $\begin{array}{l}\text { Interaction vesse } 1 \\
\text { base region }\end{array}$ & -50.82 & 0.24 & 60.0 & $\begin{array}{l}\text { Water/debris } \\
\text { temperature }\end{array}$ \\
\hline TC8-10 & Expansion vesse 1 & 0.0 & 0.0 & 0.0 & $\begin{array}{l}\text { Gas/vapor } \\
\text { temperature }\end{array}$ \\
\hline TC8-12 & $\begin{array}{l}\text { Interaction vesse } 1 \\
\text { head region }\end{array}$ & 39.04 & 8.57 & 177.5 & $\begin{array}{l}\text { Gas/vapor } \\
\text { temperature }\end{array}$ \\
\hline
\end{tabular}

${ }_{2}$ Pipeway out let in expansion vesse 1 is elevation 0.0 . Water surface is elevation 12.05 . 2 Applicable for each vessel - see Fig. Al. 
Table A3. Sensor Locations for Test CCM-2 (cont'd)

\begin{tabular}{|c|c|c|c|c|c|}
\hline $\begin{array}{l}\text { Sensor } \\
\text { No. }\end{array}$ & Location & $\begin{array}{l}\text { evation, } \\
\mathrm{cm}\end{array}$ & $\underset{\mathrm{cm}}{\text { Radius, }}$ & $\begin{array}{l}\text { Angle, } \\
\text { deg }\end{array}$ & Function \\
\hline TC8-13 & $\begin{array}{l}\text { Interaction vesse } 1 \\
\text { head region }\end{array}$ & 39.04 & 8.57 & 357.5 & $\begin{array}{l}\text { Gas/vapor } \\
\text { temperature }\end{array}$ \\
\hline TC8-16 & $\begin{array}{l}\text { Interaction vesse? } \\
\text { base region }\end{array}$ & -50.51 & 0.0 & 0.0 & $\begin{array}{l}\text { Water/debris } \\
\text { temperature }\end{array}$ \\
\hline TC8-17 & $\begin{array}{l}\text { Interaction vessel } \\
\text { base region }\end{array}$ & -50.82 & 1.27 & 340.8 & $\begin{array}{l}\text { Water/debris } \\
\text { temperature }\end{array}$ \\
\hline TC8-18 & $\begin{array}{l}\text { Interaction vessel } \\
\text { base region }\end{array}$ & -50.51 & 1.27 & 330.0 & $\begin{array}{l}\text { Water/debris } \\
\text { temperature }\end{array}$ \\
\hline TC8-19 & $\begin{array}{l}\text { Interaction vessel } \\
\text { base region }\end{array}$ & -50.82 & 2.54 & 330.0 & $\begin{array}{l}\text { Water/debris } \\
\text { temperature }\end{array}$ \\
\hline TC8-20 & $\begin{array}{l}\text { Interaction vessel } \\
\text { upper top section }\end{array}$ & 5.70 & 8.89 & 27.5 & $\begin{array}{l}\text { Water/vapor } \\
\text { temperature }\end{array}$ \\
\hline TC8-21 & $\begin{array}{l}\text { Interaction vessel } \\
\text { upper top section }\end{array}$ & 5.70 & 4.44 & 117.5 & $\begin{array}{l}\text { Water/vapor } \\
\text { temperature }\end{array}$ \\
\hline TC8-22 & $\begin{array}{l}\text { Interaction vessel } \\
\text { lower top section }\end{array}$ & -19.70 & 8.89 & 72.5 & $\begin{array}{l}\text { Water/vapor } \\
\text { temperature }\end{array}$ \\
\hline TC8-23 & $\begin{array}{l}\text { Interaction vessel } \\
\text { lower top section }\end{array}$ & -19.70 & 4.44 & 162.5 & $\begin{array}{l}\text { Water/vapor } \\
\text { temperature }\end{array}$ \\
\hline 「C8-28 & Pipeway U-bend & -45.74 & - & - & $\begin{array}{l}\text { Gas/vapor } \\
\text { temperature }\end{array}$ \\
\hline TC9-2 & $\begin{array}{l}\text { Thermite vessel } \\
\text { gas space }\end{array}$ & 86.99 & 5.08 & 312.5 & Gas temperature \\
\hline TC12-1 & $\begin{array}{l}\text { Expansion vessel } \\
\text { instrument tree }\end{array}$ & 282.58 & 3.18 & 180.0 & $\begin{array}{l}\text { Gas/vapor } \\
\text { temperature }\end{array}$ \\
\hline TC12-2 & $\begin{array}{l}\text { Expansion vessel } \\
\text { instrument tree }\end{array}$ & 241.94 & 3.18 & 0.0 & $\begin{array}{l}\text { Gas/vapor } \\
\text { temperature }\end{array}$ \\
\hline TC12-3 & $\begin{array}{l}\text { Expansion vesse } \\
\text { instrument tree }\end{array}$ & 201.30 & 3.18 & 315.0 & $\begin{array}{l}\text { Gas/vapor } \\
\text { temperature }\end{array}$ \\
\hline TC12-4 & $\begin{array}{l}\text { Expansion vessel } \\
\text { instrument tree }\end{array}$ & 160.66 & 3.18 & 157.5 & $\begin{array}{l}\text { Gas/vapor } \\
\text { temperature }\end{array}$ \\
\hline TC12-5 & $\begin{array}{l}\text { Expansion vessel } \\
\text { instrument tree }\end{array}$ & 120.02 & 3.18 & 0.0 & $\begin{array}{l}\text { Gas/vapor } \\
\text { temperature }\end{array}$ \\
\hline
\end{tabular}


Table A3. Sensor Locations for Test CCM-2 (cont'd)

\begin{tabular}{|c|c|c|c|c|c|c|c|}
\hline $\begin{array}{l}\text { Sensor } \\
\text { No. }\end{array}$ & Location & $\begin{array}{l}\text { vation, } \\
\mathrm{cm}\end{array}$ & $\underset{\mathrm{cm}}{\text { Radius, }}{ }^{2}$ & $\begin{array}{c}\text { Angle, } \\
\text { deg }\end{array}$ & \multicolumn{3}{|c|}{ Function } \\
\hline TC12-6 & $\begin{array}{l}\text { Expansion vessel } \\
\text { instrument tree }\end{array}$ & 79.38 & 3.18 & 202.5 & $\begin{array}{l}\text { Gas/va } \\
\text { temper }\end{array}$ & $\begin{array}{l}\text { por } \\
\text { ature }\end{array}$ & \\
\hline LP-1 & $\begin{array}{l}\text { Interaction vessel } \\
\text { gas space }\end{array}$ & 12.37 & 8.57 & 147.5 & Water & leve? & position \\
\hline$L P-2$ & $"$ & 13.00 & 8.57 & 147.5 & $"$ & $"$ & $"$ \\
\hline LP-3 & $"$ & 14.27 & 8.57 & 147.5 & $"$ & $"$ & $"$ \\
\hline$L P-4$ & $"$ & 15.54 & 8.57 & 147.5 & $"$ & $"$ & $"$ \\
\hline$L P-5$ & $"$ & 18.08 & 8.57 & 147.5 & $"$ & $"$ & $"$ \\
\hline
\end{tabular}


Table A4. Sensor Locations for Test CCM-3

\begin{tabular}{|c|c|c|c|c|c|c|}
\hline $\begin{array}{l}\text { Sensor } \\
\text { No. }\end{array}$ & Location & $\begin{array}{l}\text { evation, } \\
\mathrm{cm}\end{array}$ & $\underset{\mathrm{cm}}{\text { Radius, }}$ & $\begin{array}{l}\text { Angle, } \\
\text { deg }\end{array}$ & \multicolumn{2}{|c|}{ Function } \\
\hline PTS-1 & $\begin{array}{l}\text { Thermite vessel } \\
\text { top flange }\end{array}$ & 89.53 & 0.0 & 342.5 & $\begin{array}{l}\text { Pressure in } \\
\text { of thermite }\end{array}$ & $\begin{array}{l}\text { n gas space } \\
\text { e vesse } 1\end{array}$ \\
\hline PTS-2 & $\begin{array}{l}\text { Interaction vessel } \\
\text { head }\end{array}$ & 41.58 & 8.57 & 347.5 & $\begin{array}{l}\text { Pressure ir } \\
\text { vessel vapo }\end{array}$ & $\begin{array}{l}n \text { interaction } \\
\text { or space }\end{array}$ \\
\hline PTS-9 & $\begin{array}{l}\text { Expansion vessel } \\
\text { top section }\end{array}$ & 309.57 & 4.45 & 165.0 & $\begin{array}{l}\text { Pressure in } \\
\text { of expansic }\end{array}$ & $\begin{array}{l}\text { n upper region } \\
\text { on vessel }\end{array}$ \\
\hline PTS-10 & Expansion vesse? & 38.10 & 38.10 & 350.0 & $\begin{array}{l}\text { Pressure ir } \\
\text { of expans ic }\end{array}$ & $\begin{array}{l}\text { n lower region } \\
\text { on vesse } 1\end{array}$ \\
\hline PTS-12 & $\begin{array}{l}\text { Interaction vessel } \\
\text { upper top section }\end{array}$ & 5.70 & 11.85 & 297.5 & $\begin{array}{l}\text { Pressure ir } \\
\text { vesse? }\end{array}$ & in interaction \\
\hline PTS-13 & $\begin{array}{l}\text { Interaction vessel } \\
\text { lower top section }\end{array}$ & -19.70 & 11.85 & 342.5 & $"$ & $"$ \\
\hline PTS-14 & $\begin{array}{l}\text { Interaction vessel } \\
\text { upper middle section }\end{array}$ & -45.74 & 11.85 & 252.5 & $"$ & $"$ \\
\hline PTS-15 & $\begin{array}{l}\text { Interaction vessel } \\
\text { lower middle section }\end{array}$ & -19.70 & 11.85 & 252.5 & $"$ & $"$ \\
\hline TC8-6 & $\begin{array}{l}\text { Interaction vesse } 1 \\
\text { base region }\end{array}$ & -95.90 & 2.54 & 84.6 & $\begin{array}{l}\text { Water/debri } \\
\text { temperature }\end{array}$ & is \\
\hline TC8-7 & $\begin{array}{l}\text { Interaction vessel } \\
\text { base region }\end{array}$ & -93.36 & 1.27 & 79.2 & $"$ & $"$ \\
\hline TC8-8 & $\begin{array}{l}\text { Interaction vesse } 1 \\
\text { base region }\end{array}$ & -94.63 & 1.27 & 90.0 & $"$ & $"$ \\
\hline TC8-9 & $\begin{array}{l}\text { Interaction vesse } 1 \\
\text { base region }\end{array}$ & -91.46 & 0.24 & 0.0 & $"$ & $"$ \\
\hline TC8-10 & Expansion vessel & 0.0 & 0.0 & 0.0 & Gas/vapor $t$ & temperature \\
\hline TC8-12 & $\begin{array}{l}\text { Interaction vesse } 1 \\
\text { head region }\end{array}$ & 39.04 & 8.57 & 227.5 & $"$ & $"$ \\
\hline TC8-13 & $\begin{array}{l}\text { Interaction vesse } 1 \\
\text { head region }\end{array}$ & 39.04 & 8.57 & 312.5 & $"$ & $"$ \\
\hline
\end{tabular}

${ }_{2}^{1}$ Pipeway outlet in expansion vesse 1 is elevation 0.0 . Water surface is elevation 13.32 . 2 Applicable for each vessel - see Fig. Al. 
Table A4. Sensor Locations for Test CCM-3 (cont'd)

\begin{tabular}{|c|c|c|c|c|c|c|}
\hline $\begin{array}{l}\text { Sensor } \\
\text { No. }\end{array}$ & Location & $\underset{\mathrm{cm}}{\operatorname{vation}}$ & $\underset{\mathrm{Cm}}{\operatorname{Radius}, 2}$ & $\begin{array}{c}\text { Angle, } \\
\text { deg }\end{array}$ & Funct & ion \\
\hline TC8-16 & $\begin{array}{l}\text { Interaction vessel } \\
\text { base region }\end{array}$ & -92.73 & 0.0 & 0.0 & \multicolumn{2}{|c|}{$\begin{array}{l}\text { Water/debris } \\
\text { temperature }\end{array}$} \\
\hline TC8-17 & $\begin{array}{l}\text { Interaction vessel } \\
\text { base region }\end{array}$ & -94.00 & 1.27 & 280.8 & $"$ & $"$ \\
\hline TC8- 18 & $\begin{array}{l}\text { Interaction vessel } \\
\text { base region }\end{array}$ & -95.27 & 1.27 & 270.0 & $"$ & $"$ \\
\hline TC8-19 & $\begin{array}{l}\text { Interaction vessel } \\
\text { base region }\end{array}$ & -86.38 & 2.54 & 270.0 & $"$ & $"$ \\
\hline TC8-20 & $\begin{array}{l}\text { Interaction vessel } \\
\text { upper top section }\end{array}$ & 5.70 & 8.89 & 27.5 & \multicolumn{2}{|c|}{$\begin{array}{l}\text { Water/vapor } \\
\text { temperature }\end{array}$} \\
\hline TC8-21 & $\begin{array}{l}\text { Interaction vessel } \\
\text { upper top section }\end{array}$ & 5.70 & 4.44 & 117.5 & $"$ & $"$ \\
\hline TC8-22 & $\begin{array}{l}\text { Interaction vessel } \\
\text { lower top section }\end{array}$ & -19.70 & 8.89 & 72.5 & $"$ & $"$ \\
\hline TC8-23 & $\begin{array}{l}\text { Interaction vessel } \\
\text { lower top section }\end{array}$ & -19.70 & 4.44 & 162.5 & $"$ & $"$ \\
\hline TC8-24 & $\begin{array}{l}\text { Interaction vessel } \\
\text { upper middle section }\end{array}$ & -45.74 & 8.89 & 72.5 & $"$ & $"$ \\
\hline TC8-25 & $\begin{array}{l}\text { Interaction vessel } \\
\text { upper middle section }\end{array}$ & -45.74 & 4.44 & 162.5 & $"$ & $"$ \\
\hline TC8-26 & $\begin{array}{l}\text { Interaction vessel } \\
\text { lower middle section }\end{array}$ & -60.98 & 8.89 & 27.5 & $"$ & $"$ \\
\hline TC8-27 & $\begin{array}{l}\text { Interaction vessel } \\
\text { lower middle section }\end{array}$ & -60.98 & 4.44 & 147.5 & $"$ & $"$ \\
\hline TC8-28 & Pipeway U-bend & -45.74 & - & - & Gas/vapor & temperature \\
\hline TC9-2 & $\begin{array}{l}\text { Thermite vesse } 1 \\
\text { gas space }\end{array}$ & 86.99 & 5.08 & 312.5 & Gas temper & rature \\
\hline TC12-1 & $\begin{array}{l}\text { Expansion vesse } 1 \\
\text { instrument tree }\end{array}$ & 282.58 & 3.18 & 180.0 & Gas/vapor & temperature \\
\hline TC12-2 & $\begin{array}{l}\text { Expansion vesse } 1 \\
\text { instrument tree }\end{array}$ & 241.94 & 3.18 & 0.0 & $"$ & $"$ \\
\hline
\end{tabular}


Table A4. Sensor Locations for Test CCM-3 (cont'd)

\begin{tabular}{|c|c|c|c|c|c|c|c|}
\hline $\begin{array}{l}\text { Sensor } \\
\text { No. }\end{array}$ & Location & $\underset{\mathrm{cm}}{\text { Elevation, }}$ & $\underset{\mathrm{Cm}}{\operatorname{Radius}^{2}}$ & $\begin{array}{l}\text { Angle, } \\
\text { deg }\end{array}$ & \multicolumn{3}{|c|}{ Function } \\
\hline TC12-3 & $\begin{array}{l}\text { Expansion vesse } 1 \\
\text { instrument tree }\end{array}$ & 201.30 & 3.18 & 315.0 & $"$ & & $"$ \\
\hline TC12-4 & $\begin{array}{l}\text { Expansion vesse } \\
\text { instrument tree }\end{array}$ & 160.66 & 3.18 & 157.5 & Gas/vap & por $t \epsilon$ & emperature \\
\hline TC12-5 & $\begin{array}{l}\text { Expansion vesse } 7 \\
\text { instrument tree }\end{array}$ & 120.02 & 3.18 & 0.0 & $"$ & & $"$ \\
\hline TC12-6 & $\begin{array}{l}\text { Expansion vesse } 1 \\
\text { instrument tree }\end{array}$ & 79.38 & 3.18 & 202.5 & $"$ & & $"$ \\
\hline LP-1 & $\begin{array}{l}\text { Interaction vessel } \\
\text { gas space }\end{array}$ & 13.64 & $\begin{array}{l}8.57 \\
-\ldots\end{array}$ & 132.5 & Water 1 & level & position \\
\hline LP-2 & $"$ & 14.27 & 8.57 & 132.5 & $"$ & $"$ & $"$ \\
\hline LP-3 & $"$ & 15.54 & 8.57 & 132.5 & $"$ & $"$ & $"$ \\
\hline LP-4 & $"$ & 16.81 & 8.57 & 132.5 & $"$ & $"$ & $"$ \\
\hline LP-5 & $"$ & 19.35 & 8.57 & 132.5 & $"$ & $"$ & $"$ \\
\hline
\end{tabular}


Table A5. Sensor Locations for Test CCM-4

\begin{tabular}{|c|c|c|c|c|c|c|}
\hline $\begin{array}{l}\text { Sensor } \\
\text { No. }\end{array}$ & Location & cmation, 1 & $\underset{\mathrm{cm}}{\operatorname{Radius}}{ }^{2}$ & $\begin{array}{l}\text { Angle, } \\
\text { deg }\end{array}$ & Funct & ion \\
\hline PTS-1 & $\begin{array}{l}\text { Thermite vessel } \\
\text { top flange }\end{array}$ & 89.53 & 0.0 & 342.5 & \multicolumn{2}{|c|}{$\begin{array}{l}\text { Pressure in gas space of } \\
\text { thermite vessel }\end{array}$} \\
\hline PTS-2 & $\begin{array}{l}\text { Interaction vessel } \\
\text { head }\end{array}$ & 41.58 & 8.57 & 227.5 & \multicolumn{2}{|c|}{$\begin{array}{l}\text { Pressure in interaction } \\
\text { vessel vapor space }\end{array}$} \\
\hline PTS-9 & $\begin{array}{l}\text { Expansion vesse } 1 \\
\text { top section }\end{array}$ & 309.57 & 4.45 & 165.0 & \multicolumn{2}{|c|}{$\begin{array}{l}\text { Pressure in upper region } \\
\text { of expansion vessel }\end{array}$} \\
\hline PTS-10 & Expansion vesse? & 38.10 & 38.10 & 350.0 & \multicolumn{2}{|c|}{$\begin{array}{l}\text { Pressure in lower region } \\
\text { of expansion vessel }\end{array}$} \\
\hline PTS-12 & $\begin{array}{l}\text { Interaction vessel } \\
\text { upper top section }\end{array}$ & 5.70 & 11.85 & 207.5 & \multicolumn{2}{|c|}{$\begin{array}{l}\text { Pressure in interaction } \\
\text { vessel }\end{array}$} \\
\hline PTK-1 & $\begin{array}{l}\text { Interaction vesse } \\
\text { upper middle section }\end{array}$ & -45.74 & 11.85 & 257.5 & $"$ & $"$ \\
\hline TC8-6 & $\begin{array}{l}\text { Interaction vesse } 1 \\
\text { base region }\end{array}$ & -91.46 & 2.54 & 204.6 & \multicolumn{2}{|c|}{$\begin{array}{l}\text { Water/debris } \\
\text { temperature }\end{array}$} \\
\hline TC8-7 & $\begin{array}{l}\text { Interaction vesse } 1 \\
\text { base region }\end{array}$ & -93.36 & 1.27 & 199.2 & $"$ & ' \\
\hline TC8-8 & $\begin{array}{l}\text { Interaction vesse } 1 \\
\text { base region }\end{array}$ & -95.27 & 1.27 & 210 & $"$ & " \\
\hline TC8-9 & $\begin{array}{l}\text { Interaction vessel } \\
\text { base region }\end{array}$ & -95.91 & 0.24 & 120 & $"$ & 1 \\
\hline TC8-10 & Expansion vesse 1 & 0.0 & 0.0 & 0.0 & \multicolumn{2}{|c|}{ Gas/vapor temperature } \\
\hline TC8-12 & $\begin{array}{l}\text { Interaction vessel } \\
\text { head region }\end{array}$ & 39.04 & 8.57 & 282.5 & $"$ & $"$ \\
\hline TC8-13 & $\begin{array}{l}\text { Interaction vessel } \\
\text { head region }\end{array}$ & 39.04 & 8.57 & 47.5 & $"$ & $"$ \\
\hline TC8-16 & $\begin{array}{l}\text { Interaction vesse } 1 \\
\text { base region }\end{array}$ & -93.36 & 0.0 & 0.0 & \multicolumn{2}{|c|}{$\begin{array}{l}\text { Water/debris } \\
\text { temperature }\end{array}$} \\
\hline TC8-17 & $\begin{array}{l}\text { Interaction vesse } 1 \\
\text { base region }\end{array}$ & -94.00 & 1.27 & 40.8 & $"$ & $"$ \\
\hline
\end{tabular}

${ }_{2}^{1}$ Pipeway out let in expansion vesse 1 is e levation 0.0 . Water surface is elevation 10.78 . ${ }^{2}$ Applicable for each vesse 1 - see Fig. Al. 
Table A5. Sensor Locations for Test CCM-4

\begin{tabular}{|c|c|c|c|c|c|}
\hline $\begin{array}{l}\text { Sensor } \\
\text { No. }\end{array}$ & Location & $\begin{array}{l}\text { evation, } \\
\mathrm{cm}\end{array}$ & $\underset{\mathrm{Cm}}{\operatorname{Radius}}{ }^{2}$ & $\begin{array}{l}\text { Angle, } \\
\text { deg }\end{array}$ & Function \\
\hline TC8-18 & $\begin{array}{l}\text { Interaction vessel } \\
\text { base region }\end{array}$ & -94.63 & 1.27 & 30.0 & $\begin{array}{l}\text { Water/debris } \\
\text { temperature }\end{array}$ \\
\hline TC8-19 & $\begin{array}{l}\text { Interaction vesse } 1 \\
\text { base region }\end{array}$ & -86.38 & 2.54 & 30.0 & $"$ \\
\hline TC8-20 & $\begin{array}{l}\text { Interaction vessel } \\
\text { lower top section }\end{array}$ & -19.70 & 8.89 & 67.5 & $\begin{array}{l}\text { Water/vapor } \\
\text { temperature }\end{array}$ \\
\hline TC8-21 & $\begin{array}{l}\text { Interaction vessel } \\
\text { lower top section }\end{array}$ & -19.70 & 5.72 & 252.5 & $"$ \\
\hline TC8-22 & $\begin{array}{l}\text { Interaction vesse } 1 \\
\text { lower top section: }\end{array}$ & -19.70 & 8.26 & 342.5 & $"$ \\
\hline TC8-23 & $\begin{array}{l}\text { Interaction vesse } 1 \\
\text { upper top section }\end{array}$ & 5,70 & 4.44 & 117.5 & $"$ \\
\hline TC8-24 & $\begin{array}{l}\text { Interaction vessel } \\
\text { upper middle section }\end{array}$ & -45.74 & 7.62 & 162.5 & $"$ \\
\hline TC8-25 & $\begin{array}{l}\text { Interaction vessel } \\
\text { upper middle section }\end{array}$ & -45.74 & 3.49 & 67.5 & $"$ \\
\hline TC8-26 & $\begin{array}{l}\text { Interaction vessel } \\
\text { lower middle section }\end{array}$ & -60.98 & 8.89 & 297.5 & $"$ \\
\hline $\mathrm{TC} 8-27$ & $\begin{array}{l}\text { Interaction vessel } \\
\text { lower middle section }\end{array}$ & -60.98 & 3.81 & 207.5 & $"$ \\
\hline TC8-28 & Pipeway U-bend & -45.74 & - & - & Gas/vapor temperature \\
\hline TC8-29 & $\begin{array}{l}\text { Interaction vessel } \\
\text { upper middle section }\end{array}$ & -45.74 & 6.35 & 342.5 & $\begin{array}{l}\text { Water/vapor } \\
\text { temperature }\end{array}$ \\
\hline TC8-30 & $\begin{array}{l}\text { Interaction vesel } \\
\text { lower middle section }\end{array}$ & -60.98 & 6.35 & 117.5 & $"$ \\
\hline TC8-31 & $\begin{array}{l}\text { Interaction vessel } \\
\text { lower middle section }\end{array}$ & -60.98 & 6.35 & 27.5 & $"$ \\
\hline TC8-32 & $\begin{array}{l}\text { Interaction vessel } \\
\text { upper top section }\end{array}$ & 5.70 & 6.35 & 27.5 & $"$ \\
\hline TC8-33 & $\begin{array}{l}\text { Interaction vessel } \\
\text { upper top section }\end{array}$ & 5.70 & 6.35 & 297.5 & $"$ \\
\hline
\end{tabular}


Table A5. Sensor Locations for Test CCM-4

\begin{tabular}{|c|c|c|c|c|c|c|c|}
\hline $\begin{array}{l}\text { Sensor } \\
\text { No. }\end{array}$ & Location & $\begin{array}{l}\text { vation, } \\
\mathrm{cm}\end{array}$ & $\underset{\mathrm{cm}}{\text { Radius, }}{ }^{2}$ & $\begin{array}{c}\text { Angle, } \\
\text { deg }\end{array}$ & \multicolumn{3}{|c|}{ Function } \\
\hline TC8-34 & $\begin{array}{l}\text { Interaction vessel } \\
\text { lower top section }\end{array}$ & -19.70 & 1.91 & 162.5 & $"$ & $"$ & \\
\hline TC9-2 & $\begin{array}{l}\text { Thermite vessel } \\
\text { gas space }\end{array}$ & 86.99 & 5.08 & 312.5 & Gas te & emperat & ture \\
\hline TC12-1 & $\begin{array}{l}\text { Expansion vessel } \\
\text { instrument tree }\end{array}$ & 282.58 & 3.18 & 180.0 & Gas/vi & apor te & :emperature \\
\hline TC12-2 & $\begin{array}{l}\text { Expansion vesse } 1 \\
\text { instrument tree }\end{array}$ & 241.94 & 3.18 & 0.0 & $"$ & & $"$ \\
\hline TC12-3 & $\begin{array}{l}\text { Expansion vessel } \\
\text { instrument tree }\end{array}$ & 201.30 & 3.18 & 315.0 & $"$ & & $"$ \\
\hline TC12-4 & $\begin{array}{l}\text { Expansion vessel } \\
\text { instrument tree }\end{array}$ & 160.66 & 3.18 & 157.5 & $"$ & & $"$ \\
\hline TC12-5 & $\begin{array}{l}\text { Expansion vesse } 1 \\
\text { instrument tree }\end{array}$ & 120.02 & 3.18 & 0.0 & $"$ & & $"$ \\
\hline TC12-6 & $\begin{array}{l}\text { Expansion vessel } \\
\text { instrument tree }\end{array}$ & 79.38 & 3.18 & 202.5 & $"$ & & $"$ \\
\hline LP-1 & $\begin{array}{l}\text { Interaction vessel } \\
\text { gas space }\end{array}$ & 11.42 & 8.57 & 132.5 & Water & level & position \\
\hline LP-2 & $\begin{array}{l}\text { Interaction vessel } \\
\text { gas space }\end{array}$ & 12.05 & 8.57 & 132.5 & $"$ & $"$ & $"$ \\
\hline LP-3 & $\begin{array}{l}\text { Interaction vessel } \\
\text { gas space }\end{array}$ & 12.69 & 8.57 & 132.5 & $"$ & $"$ & $"$ \\
\hline$L P-4$ & $\begin{array}{l}\text { Interaction vessel } \\
\text { gas space }\end{array}$ & 13.32 & 8.57 & 132.5 & $"$ & $"$ & $"$ \\
\hline$L P-5$ & Interaction vessel & 13.96 & 8.57 & 132.5 & $"$ & $"$ & $"$ \\
\hline
\end{tabular}


Table A6. Sensor Locations for Test CCM-5

\begin{tabular}{|c|c|c|c|c|c|}
\hline $\begin{array}{l}\text { Sensor } \\
\text { No. }\end{array}$ & Location & $\begin{array}{l}\text { Elevation, } \\
\mathrm{cm}\end{array}$ & $\underset{\mathrm{cm}}{\operatorname{Radius}, 2}$ & $\begin{array}{c}\text { Angle, } \\
\text { deg }\end{array}$ & Function \\
\hline PTS-1 & $\begin{array}{l}\text { Thermite vessel } \\
\text { top flange }\end{array}$ & 67.95 & 0.0 & 90 & $\begin{array}{l}\text { Pressure in gas space } \\
\text { of thermite vesse } 1\end{array}$ \\
\hline PTS-9 & $\begin{array}{l}\text { Experiment vessel } \\
\text { top section }\end{array}$ & 123.8 & 4.44 & 180 & $\begin{array}{l}\text { Pressure in top section } \\
\text { of experimert vesse } 1\end{array}$ \\
\hline PTS-10 & $\begin{array}{l}\text { Experiment vessel } \\
\text { single-view port } \\
\text { section }\end{array}$ & -10.48 & 38.10 & 30 & $\begin{array}{l}\text { Pressure in single view- } \\
\text { port section of } \\
\text { experiment vessel }\end{array}$ \\
\hline PTS-12 & $\begin{array}{l}\text { Experiment vessel } \\
\text { single-view port } \\
\text { section }\end{array}$ & -10.48 & 38.10 & 210 & $\begin{array}{l}\text { Pressure in single view- } \\
\text { port section of } \\
\text { experiment vessel }\end{array}$ \\
\hline PTS-13 & $\begin{array}{l}\text { Experiment vessel } \\
\text { multi-view port } \\
\text { section }\end{array}$ & -79.06 & 38.10 & 210 & $\begin{array}{l}\text { Pressure in multi-view } \\
\text { port section of } \\
\text { experiment vessel }\end{array}$ \\
\hline PTK-1 & $\begin{array}{l}\text { Experiment vessel } \\
\text { multi-view port } \\
\text { section }\end{array}$ & -66.36 & 38.10 & 350 & $\begin{array}{l}\text { Pressure in single view- } \\
\text { port section of } \\
\text { experiment vessel }\end{array}$ \\
\hline PTK-2 & $\begin{array}{l}\text { Experiment vessel } \\
\text { single view-port } \\
\text { section }\end{array}$ & -2.22 & 38.10 & 330 & $\begin{array}{l}\text { Pressure in single view- } \\
\text { port section of } \\
\text { expansion vessel }\end{array}$ \\
\hline PTK-3 & $\begin{array}{l}\text { Experiment vessel } \\
\text { top section }\end{array}$ & 123.8 & 4.44 & 90 & $\begin{array}{l}\text { Pressure in top section } \\
\text { of experiment vessel }\end{array}$ \\
\hline TC8-6 & $\begin{array}{l}\text { Experiment vessel } \\
\text { bottom section }\end{array}$ & -106.04 & 0.0 & 0.0 & $\begin{array}{l}\text { Pan water/debris } \\
\text { temperature }\end{array}$ \\
\hline TC8-7 & $\begin{array}{l}\text { Experiment vessel } \\
\text { bottom section }\end{array}$ & -106.04 & 5.08 & 30 & $\begin{array}{l}\text { Pan water/debris } \\
\text { temperature }\end{array}$ \\
\hline TC8-8 & $\begin{array}{l}\text { Experiment vessel } \\
\text { bottom section }\end{array}$ & -106.04 & 15.24 & 30 & $\begin{array}{l}\text { Pan water/debris } \\
\text { temperature }\end{array}$ \\
\hline TC8-9 & $\begin{array}{l}\text { Experiment vessel } \\
\text { bottom section }\end{array}$ & -106.04 & 7.62 & 120 & $\begin{array}{l}\text { Pan water/debris } \\
\text { temperature }\end{array}$ \\
\hline TC8-10 & $\begin{array}{l}\text { Experiment vessel } \\
\text { bottom section }\end{array}$ & -106.04 & 17.78 & 120 & $\begin{array}{l}\text { Pan water/debris } \\
\text { temperature }\end{array}$ \\
\hline
\end{tabular}

1 Water surface is elevation 0.0 .
2 See Figure A.2 
Table A6. Sensor Locations for Test CCM-5 (cont'd)

\begin{tabular}{|c|c|c|c|c|c|c|c|}
\hline $\begin{array}{l}\text { Sensor } \\
\text { No. }\end{array}$ & & & $\underset{\mathrm{cm}}{\text { vation, }}$ & $\underset{\mathrm{cm}}{\operatorname{Radius},{ }^{2}}$ & $\begin{array}{c}\text { Angle, } \\
\text { deg }\end{array}$ & \multicolumn{2}{|c|}{ Function } \\
\hline TC8-16 & \multicolumn{2}{|c|}{$\begin{array}{l}\text { Experiment vessel } \\
\text { bottom section }\end{array}$} & -106.04 & 5.08 & 210 & \multicolumn{2}{|c|}{$\begin{array}{l}\text { Pan Water/Debris } \\
\text { Temperature }\end{array}$} \\
\hline TC8-17 & $"$ & $"$ & -106.04 & 15.24 & 210 & $"$ & $"$ \\
\hline TC8-18 & $"$ & $"$ & -106.04 & 7.62 & 300 & $"$ & $"$ \\
\hline TC8-19 & $"$ & $"$ & -106.04 & 17.78 & 300 & $"$ & $"$ \\
\hline TC8-20 & \multicolumn{2}{|c|}{$\begin{array}{l}\text { Experiment vessel } \\
\text { lower sections }\end{array}$} & -5.08 & 5.08 & 30 & \multicolumn{2}{|c|}{$\begin{array}{l}\text { Water/vapor } \\
\text { temperature }\end{array}$} \\
\hline TC8-21 & $"$ & $"$ & -5.08 & 15.54 & 30 & $"$ & $"$ \\
\hline TC8-22 & $"$ & $"$ & -5.08 & 25.40 & 30 & $"$ & $"$ \\
\hline TC8-23 & $"$ & $"$ & -5.08 & 7.62 & 120 & $"$ & $"$ \\
\hline TC8-24 & $"$ & $"$ & -5.08 & 17.78 & 120 & $"$ & $"$ \\
\hline TC8-26 & $"$ & $"$ & -30.48 & 5.08 & 210 & $"$ & $"$ \\
\hline TC8-27 & $"$ & $"$ & -30.48 & 15.54 & 210 & $"$ & $"$ \\
\hline TC8-28 & $"$ & $"$ & -30.48 & 25.40 & 210 & $"$ & $"$ \\
\hline TC8-29 & $"$ & $"$ & -30.48 & 7.62 & 300 & $"$ & $"$ \\
\hline TC8-30 & $"$ & $"$ & -30.48 & 17.78 & 300 & $"$ & $"$ \\
\hline TC8-31 & $"$ & $"$ & -30.48 & 27.94 & 300 & $"$ & $"$ \\
\hline TC8-32 & $"$ & $"$ & -55.88 & 5.08 & 30 & $"$ & $"$ \\
\hline TC8-33 & $"$ & $"$ & -55.88 & 15.24 & 30 & $"$ & $"$ \\
\hline TC8-34 & $"$ & $"$ & -55.88 & 25.40 & 30 & $"$ & $"$ \\
\hline TC8-35 & $"$ & $"$ & -53.34 & 7.62 & 120 & $"$ & $"$ \\
\hline TC8-36 & $"$ & " & -53.34 & 17.78 & 120 & $"$ & $"$ \\
\hline$T C 8-37$ & $"$ & $"$ & -53.34 & 27.94 & 120 & $"$ & $"$ \\
\hline TC8-38 & $"$ & $"$ & -81.28 & 5.08 & 210 & $"$ & $"$ \\
\hline TC8-39 & $"$ & $"$ & -81.28 & 15.24 & 210 & $"$ & $"$ \\
\hline
\end{tabular}


Table A6. Sensor Locations for Test CCM-5 (cont'd)

\begin{tabular}{|c|c|c|c|c|c|c|c|}
\hline $\begin{array}{l}\text { Sensor } \\
\text { No. }\end{array}$ & Locatio & & $\underset{\mathrm{cm}}{\text { evation, }}$ & $\begin{array}{c}\text { Radius, } \\
\mathrm{cm}\end{array}$ & $\begin{array}{l}\text { Angle, }{ }^{2} \\
\text { deg }\end{array}$ & \multicolumn{2}{|c|}{ Function } \\
\hline TC8-40 & $\begin{array}{l}\text { Exper iment } \\
\text { lower secti }\end{array}$ & $\begin{array}{l}\text { vessel } \\
\text { ions }\end{array}$ & -81.28 & 25.40 & 210 & \multicolumn{2}{|c|}{$\begin{array}{l}\text { Water/vapor } \\
\text { temperature }\end{array}$} \\
\hline TC8-41 & $"$ & $"$ & -81.28 & 7.62 & 300 & $"$ & $"$ \\
\hline TC8-42 & $"$ & $"$ & -81.28 & 17.78 & 300 & $"$ & $"$ \\
\hline TC8-43 & $"$ & $"$ & -81.28 & 27.94 & 300 & $"$ & $"$ \\
\hline TC8-50 & $"$ & $"$ & 0.0 & 0.0 & 0.0 & $"$ & $"$ \\
\hline TC8-51 & $"$ & $"$ & -7.62 & 0.0 & 0.0 & $"$ & $"$ \\
\hline TC8-52 & $"$ & $"$ & -15.24 & 0.0 & 0.0 & $"$ & $"$ \\
\hline TC8-53 & $"$ & $"$ & -22.86 & 0.0 & 0.0 & $"$ & $"$ \\
\hline TC8-54 & $"$ & $"$ & -30.48 & 0.0 & 0.0 & $"$ & $"$ \\
\hline TC8-55 & $"$ & $"$ & -38.10 & 0.0 & 0.0 & $"$ & $"$ \\
\hline TC8-56 & $"$ & $"$ & -45.72 & 0.0 & 0.0 & $"$ & $"$ \\
\hline TC8-57 & $"$ & $"$ & -53.34 & 0.0 & 0.0 & $"$ & $"$ \\
\hline TC8-58 & $"$ & $"$ & -60.96 & 0.0 & 0.0 & $"$ & $"$ \\
\hline TC8-59 & $"$ & $"$ & -68.58 & 0.0 & 0.0 & $"$ & $"$ \\
\hline TC8-60 & $"$ & $"$ & -76.20 & 0.0 & 0.0 & $"$ & $"$ \\
\hline TC8-61 & $"$ & $"$ & -83.82 & 0.0 & 0.0 & $"$ & $"$ \\
\hline TC8-63 & $"$ & $"$ & -99.06 & 0.0 & 0.0 & $"$ & $"$ \\
\hline TC9-2 & \multicolumn{2}{|c|}{$\begin{array}{l}\text { Thermite vessel gas } \\
\text { space }\end{array}$} & 65.41 & 5.08 & 312.5 & \multicolumn{2}{|c|}{ Gas temperature } \\
\hline TC12-1 & \multicolumn{2}{|c|}{$\begin{array}{l}\text { Experiment vessel } \\
\text { top section }\end{array}$} & 96.52 & 2.54 & 180 & \multicolumn{2}{|c|}{ Gas/vapor temperature } \\
\hline TC12-2 & $"$ & $"$ & 77.47 & 26.67 & 155 & $"$ & $"$ \\
\hline TC12-3 & $"$ & $"$ & 72.39 & 31.75 & 190 & $"$ & $"$ \\
\hline TC12-4 & $"$ & $"$ & 63.50 & 29.21 & 25 & $"$ & $"$ \\
\hline
\end{tabular}


Table A6. Sensor Locations for Test CCM-5 (cont.'d)

\begin{tabular}{|c|c|c|c|c|c|c|c|}
\hline $\begin{array}{l}\text { Sensor } \\
\text { No. }\end{array}$ & & ocation & $\begin{array}{l}\text { evation, } \\
\mathrm{cm}\end{array}$ & $\underset{\mathrm{cm}}{\text { Radius, }}, 2$ & $\begin{array}{c}\text { Angle, } \\
\text { deg }\end{array}$ & \multicolumn{2}{|c|}{ Function } \\
\hline TC12-5 & $\begin{array}{l}\text { Expe } \\
\text { Top }\end{array}$ & $\begin{array}{l}\text { iment vessel } \\
\text { sections }\end{array}$ & 45.72 & 29.21 & 270 & Gas/vapor $t$ & temperature \\
\hline TC12-6 & $"$ & $"$ & 27.94 & 29.21 & 320 & $"$ & $"$ \\
\hline VD-1 & $\begin{array}{l}\text { Expe } \\
\text { lowe }\end{array}$ & $\begin{array}{l}\text { - iment vesse } 1 \\
\text { sections }\end{array}$ & -5.08 & 3.18 & 210 & Void detect & tion \\
\hline VD-2 & $"$ & $"$ & -5.08 & 5.08 & 210 & $"$ & $"$ \\
\hline VD-3 & $"$ & $"$ & -5.08 & 7.62 & 210 & $"$ & $"$ \\
\hline VD-4 & $"$ & $"$ & -30.48 & 3.18 & 30 & $"$ & $"$ \\
\hline VD-5 & $"$ & $"$ & -30.48 & 5.08 & 30 & $"$ & $"$ \\
\hline VD-6 & $"$ & $"$ & -30.48 & 7.62 & 30 & $"$ & $"$ \\
\hline VD-7 & $"$ & $"$ & -55.88 & 3.18 & 210 & $"$ & $"$ \\
\hline VD-8 & $"$ & $"$ & -55.88 & 5.08 & 210 & $"$ & $"$ \\
\hline VD-9 & $"$ & $"$ & -55.88 & 7.62 & 210 & $"$ & $"$ \\
\hline VD -10 & $"$ & $"$ & -81.28 & 3.18 & 30 & $"$ & $"$ \\
\hline VD-11 & $"$ & $"$ & -81.28 & 5.08 & 30 & $"$ & $"$ \\
\hline VD-12 & $"$ & $"$ & -81.28 & 7.62 & 30 & $"$ & $"$ \\
\hline
\end{tabular}


Table A7. Sensor Locations for Test CCM-6

\begin{tabular}{|c|c|c|c|c|c|}
\hline $\begin{array}{l}\text { Sensor } \\
\text { No. }\end{array}$ & Location & $\underset{\mathrm{cm}}{\text { Elevation, }}$ & $\underset{\mathrm{cm}}{\text { Radius, }}$ & $\begin{array}{l}\text { Angle, } \\
\text { deg }\end{array}$ & Function \\
\hline PTS-1 & $\begin{array}{l}\text { Thermite vessel } \\
\text { top flange }\end{array}$ & 67.95 & 0.0 & 90 & $\begin{array}{l}\text { Pressure in gas } \\
\text { space of thermite } \\
\text { vesse } 1\end{array}$ \\
\hline PTS-9 & $\begin{array}{l}\text { Experiment vessel } \\
\text { top section }\end{array}$ & 273.0 & 4.44 & 180 & $\begin{array}{l}\text { Pressure in top } \\
\text { section of experi- } \\
\text { ment vesse } 1\end{array}$ \\
\hline PTS-10 & $\begin{array}{l}\text { Experiment vessel } \\
\text { single-view port } \\
\text { section }\end{array}$ & -10.48 & 38.10 & 30 & $\begin{array}{l}\text { Pressure in single view } \\
\text { port section of } \\
\text { experiment vessel }\end{array}$ \\
\hline PTS-12 & $\begin{array}{l}\text { Experiment vessel } \\
\text { single-view port } \\
\text { section }\end{array}$ & -10.48 & 38.10 & 210 & $\begin{array}{l}\text { Pressure in single view- } \\
\text { port section of } \\
\text { experiment vessel }\end{array}$ \\
\hline PTS-13 & $\begin{array}{l}\text { Experiment vessel } \\
\text { multi-view port } \\
\text { section }\end{array}$ & -79.06 & 38.10 & 210 & $\begin{array}{l}\text { Pressure in multi-view } \\
\text { port section of experiment } \\
\text { vesse } 1\end{array}$ \\
\hline PTK-1 & $\begin{array}{l}\text { Experiment vessel } \\
\text { multi-view port } \\
\text { section }\end{array}$ & -66.36 & 38.10 & 350 & $\begin{array}{l}\text { Pressure in single-view } \\
\text { port section of } \\
\text { experiment vessel. }\end{array}$ \\
\hline PTK-2 & $\begin{array}{l}\text { Experiment vessel } \\
\text { single view-port } \\
\text { section }\end{array}$ & -2.22 & 38.1 & 330 & $\begin{array}{l}\text { Pressure in single-view } \\
\text { port section of } \\
\text { expansion vesse } 1\end{array}$ \\
\hline PTK-3 & $\begin{array}{l}\text { Experiment vessel } \\
\text { top section }\end{array}$ & 273.0 & 4.44 & 90 & $\begin{array}{l}\text { Pressure in top section } \\
\text { of experiment vesse } 1\end{array}$ \\
\hline TC8-6 & $\begin{array}{l}\text { Experiment vessel } \\
\text { bottom section }\end{array}$ & -106.04 & 0.0 & 0.0 & $\begin{array}{l}\text { Pan water/debris } \\
\text { temperature }\end{array}$ \\
\hline TC8-7 & $\begin{array}{l}\text { Experiment vessel } \\
\text { bottom section }\end{array}$ & -106.04 & 5.08 & 30 & $\begin{array}{l}\text { Pan water/debris } \\
\text { temperature }\end{array}$ \\
\hline TC8-8 & $\begin{array}{l}\text { Experiment vessel } \\
\text { bottom section }\end{array}$ & -106.04 & 15.24 & 30 & $\begin{array}{l}\text { Pan water/debris } \\
\text { temperature }\end{array}$ \\
\hline TC8-9 & $\begin{array}{l}\text { Experiment vesse? } \\
\text { bottom section }\end{array}$ & -106.04 & 7.62 & 120 & $\begin{array}{l}\text { Pan water/debris } \\
\text { temperature }\end{array}$ \\
\hline
\end{tabular}

1 Water surface is elevation 0.

${ }^{2}$ See Fig. A2. 
Table A7. Sensor Locations for Test CCM-6 (cont'd)

\begin{tabular}{|c|c|c|c|c|c|c|c|}
\hline $\begin{array}{l}\text { Sensor } \\
\text { No. }\end{array}$ & & & $\underset{\mathrm{cm}}{\text { Elevation, }}$ & $\underset{\mathrm{cm}}{\operatorname{Radius},{ }^{2}}$ & $\begin{array}{c}\text { Angle, } \\
\text { deg }\end{array}$ & \multicolumn{2}{|c|}{ Function } \\
\hline TC8-10 & \multicolumn{2}{|c|}{$\begin{array}{l}\text { Experiment vessel } \\
\text { bottom section }\end{array}$} & -106.04 & 17.78 & 120 & \multicolumn{2}{|c|}{$\begin{array}{l}\text { Pan/water debris } \\
\text { temperature }\end{array}$} \\
\hline TC8-16 & $"$ & $"$ & -106.04 & 5.08 & 210 & $"$ & $"$ \\
\hline TC8-17 & $"$ & $"$ & -106.04 & 15.24 & 210 & $"$ & $"$ \\
\hline TC8-18 & $"$ & $"$ & -106.04 & 7.62 & 300 & $"$ & $"$ \\
\hline TC8-19 & $"$ & $"$ & -106.04 & 17.78 & 300 & $"$ & $"$ \\
\hline TC8-20 & \multicolumn{2}{|c|}{$\begin{array}{l}\text { Experiment vessel } \\
\text { lower sections }\end{array}$} & -5.08 & 5.08 & 30 & \multicolumn{2}{|c|}{ Water/vapor temperature } \\
\hline TC8-21 & $"$ & $"$ & -5.08 & 15.54 & 30 & $"$ & $"$ \\
\hline TC8-22 & $"$ & $"$ & -5.08 & 25.40 & 30 & $"$ & $"$ \\
\hline TC8-23 & $"$ & $"$ & -5.08 & 7.62 & 120 & $"$ & $"$ \\
\hline TC8-24 & $"$ & $"$ & -5.08 & 17.78 & 120 & $"$ & $"$ \\
\hline TC8-25 & $"$ & $"$ & -5.08 & 27.94 & 120 & $"$ & $"$ \\
\hline TC8-27 & $"$ & $"$ & -30.48 & 15.54 & 210 & $"$ & $"$ \\
\hline TC8-28 & $"$ & $"$ & -30.48 & 25.40 & 210 & $"$ & $"$ \\
\hline TC8-29 & $"$ & $"$ & -30.48 & 7.62 & 300 & $"$ & $"$ \\
\hline TC8-30 & $"$ & $"$ & -30.48 & 17.78 & 300 & $"$ & $"$ \\
\hline TC8-31 & $"$ & $"$ & -30.48 & 27.94 & 300 & $"$ & $"$ \\
\hline TC8-32 & $"$ & $"$ & -55.88 & 5.08 & 30 & $"$ & $"$ \\
\hline TC8-33 & $"$ & $"$ & -55.88 & 15.24 & 30 & $"$ & $"$ \\
\hline TC8-34 & $"$ & $"$ & -55.88 & 25.40 & 30 & $"$ & $"$ \\
\hline TC8-35 & $"$ & $"$ & -53.34 & 7.62 & 120 & $"$ & $"$ \\
\hline TC8-36 & $"$ & $"$ & -53.34 & 17.78 & 120 & $"$ & $"$ \\
\hline TC8-37 & $"$ & $"$ & -53.34 & 27.94 & 120 & $"$ & 'i \\
\hline TC8-38 & $"$ & $"$ & -81.28 & 5.08 & 210 & " & $"$ \\
\hline
\end{tabular}


Table A7. Sensor Locations for Test CCM-6 (cont'd)

\begin{tabular}{|c|c|c|c|c|c|c|c|}
\hline $\begin{array}{l}\text { Sensor } \\
\text { No. }\end{array}$ & Location & & $\begin{array}{l}\text { vation, } 1 \\
\mathrm{~cm}\end{array}$ & $\underset{\mathrm{cm}}{\operatorname{Radius},{ }^{2}}$ & $\begin{array}{c}\text { Angle, } \\
\text { deg }\end{array}$ & Functic & \\
\hline TC8-39 & $\begin{array}{l}\text { Experiment } v \\
\text { lower sectio }\end{array}$ & $\begin{array}{l}\text { lesse } 1 \\
\text { ons }\end{array}$ & -81.28 & 15.24 & 210 & Water/vapor & temperature \\
\hline TC8-40 & $"$ & $"$ & -81.28 & 25.40 & 210 & $"$ & $"$ \\
\hline TC8-41 & $"$ & $"$ & -81.28 & 7.62 & 300 & $"$ & $"$ \\
\hline TC8-42 & $"$ & $"$ & -81.28 & 17.78 & 300 & $"$ & $"$ \\
\hline TC8-43 & $"$ & $"$ & -81.28 & 27.94 & 300 & $"$ & $"$ \\
\hline TC8-50 & $"$ & $"$ & 0.0 & 0.0 & 0.0 & $"$ & $"$ \\
\hline TC8-51 & $"$ & $"$ & -7.62 & 0.0 & 0.0 & $"$ & $"$ \\
\hline TC8-52 & $"$ & $"$ & -15.24 & 0.0 & 0.0 & $"$ & $"$ \\
\hline TC8-53 & $"$ & $"$ & -22.86 & 0.0 & 0.0 & $"$ & $"$ \\
\hline TC8-54 & $"$ & $"$ & -30.48 & 0.0 & 0.0 & $"$ & $"$ \\
\hline TC8-55 & $"$ & $"$ & -40.64 & 5.08 & 0.0 & $"$ & $"$ \\
\hline TC8-56 & $"$ & $"$ & -45.72 & 0.0 & 0.0 & $"$ & $"$ \\
\hline TC8-57 & $"$ & $"$ & -53.34 & 0.0 & 0.0 & $"$ & $"$ \\
\hline TC8-58 & $"$ & $"$ & -60.96 & 0.0 & 0.0 & $"$ & $"$ \\
\hline TC8-59 & $"$ & $"$ & -68.58 & 0.0 & 0.0 & $"$ & $"$ \\
\hline TC8-60 & $"$ & $"$ & -76.20 & 0.0 & 0.0 & $"$ & $"$ \\
\hline TC8-61 & $"$ & $"$ & -83.82 & 0.0 & 0.0 & $"$ & $"$ \\
\hline TC8-63 & $"$ & $"$ & -99.06 & 0.0 & 0.0 & $"$ & $"$ \\
\hline TC9-2 & \multicolumn{2}{|c|}{$\begin{array}{l}\text { Thermite vessel } \\
\text { gas space }\end{array}$} & 65.41 & 5.08 & 312.5 & \multicolumn{2}{|c|}{ Gas temperature } \\
\hline TC12-1 & \multicolumn{2}{|c|}{$\begin{array}{l}\text { Experiment vessel } \\
\text { top section }\end{array}$} & 245.7 & 2.54 & 180 & \multicolumn{2}{|c|}{ Gas/vapor temperature } \\
\hline TC12-2 & $"$ & $"$ & 226.7 & 26.67 & 155 & $"$ & $"$ \\
\hline TC12-3 & $"$ & $"$ & 221.6 & 31.75 & 190 & $"$ & $"$ \\
\hline
\end{tabular}


Table A7. Sensor Locations for Test CCM-6 (cont'd)

\begin{tabular}{|c|c|c|c|c|c|c|c|c|}
\hline $\begin{array}{l}\text { Sensor } \\
\text { No. }\end{array}$ & & & $\begin{array}{l}\text { /ation, } 1 \\
\mathrm{~cm}\end{array}$ & $\underset{\mathrm{cm}}{\operatorname{Radius},{ }^{2}}$ & $\begin{array}{l}\text { Angle, } \\
\text { deg }\end{array}$ & \multicolumn{3}{|c|}{ Function } \\
\hline TC12-4 & \multicolumn{2}{|c|}{$\begin{array}{l}\text { Experiment vesse } \\
\text { top sections }\end{array}$} & 212.7 & 29.21 & 25 & Gas/vapor & temper & ature \\
\hline TC12-5 & $"$ & $"$ & 194.9 & 29.21 & 270 & $"$ & & $"$ \\
\hline TC12-6 & $"$ & $"$ & 177.2 & 29.21 & 320 & $"$ & & $"$ \\
\hline VD-1 & \multicolumn{2}{|c|}{$\begin{array}{l}\text { Expansion vessel } \\
\text { lower sections }\end{array}$} & -5.08 & 5.72 & 210 & \multicolumn{3}{|c|}{ Void detection } \\
\hline VD-2 & $"$ & $"$ & -5.08 & 6.99 & 210 & $"$ & $"$ & \\
\hline VD-3 & $"$ & $"$ & -5.08 & 9.52 & 210 & $"$ & $"$ & \\
\hline VD-4 & $"$ & $"$ & -30.48 & 5.08 & 30 & $"$ & $"$ & \\
\hline VD-5 & $"$ & $"$ & -30.48 & 7.30 & 30 & $"$ & $"$ & \\
\hline VD-6 & $"$ & $"$ & -30.48 & 9.52 & 30 & $"$ & $"$ & \\
\hline VD-7 & $"$ & $"$ & -55.88 & 3.81 & 210 & $"$ & $"$ & \\
\hline VD-8 & $"$ & $"$ & -55.88 & 5.72 & 210 & $"$ & $"$ & \\
\hline VD-9 & $"$ & $"$ & -55.88 & 8.26 & 210 & $"$ & $"$ & \\
\hline VD-10 & $"$ & $"$ & -81.28 & 3.18 & 30 & $"$ & $"$ & \\
\hline VD-11 & $"$ & $"$ & -81.28 & 5.72 & 30 & $"$ & $"$ & \\
\hline VD -12 & $"$ & $"$ & -81.28 & 8.26 & 30 & $"$ & $"$ & \\
\hline
\end{tabular}


APPENDIX B. DATA FOR CCM-1

PTS-1: Thermite-Vessel Gas Pressure

PTS-2: Interaction-Vessel Gas Pressure

PTS-9: Pressure at Top of Expansion Vesse1

PTS-10: Pressure at Lower Side of Expansion Vessel

PTS-12: Pressure in Upper-top Section of the Interaction Vessel

PTS-13: Pressure in Lower-top Section of the Interaction Vessel

PTS-14: Pressure in Upper Middle Section of the Interaction Vessel

PTS-15: Pressure in Lower Middle Section of the Interaction Vessel

TC8-6: Temperature at Base of Interaction Vessel

TC8-7: Temperature at Base of Interaction Vessel

TC8-8: Temperature at Base of Interaction Vesse1

TC8-9: Temperature at Base of Interaction Vessel

TC8-10: Temperature Inside Expansion Vessel $34.9 \mathrm{~cm}$ above pipeway exit

TC8-12: Temperature of Gas Space in Interaction Vessel

TC8-13: Temperature of Gas Space in Interaction Vesse1

TC8-16: Temperature at Base of Interaction Vesse1

TC8-17: Temperature at Base of Interaction Vessel

TC8-18: Temperature at Base of Interaction Vessel

TC8-19: Temperature at Base of Interaction Vessel

TC8-20: Temperature in Upper-top Section of Interaction Vessel

TC8-21: Temperature in Upper-top Section of Interaction Vesse1

TC8-22: Temperature in Lower-top Section of Interaction Vessel

TC8-23: Temperature in Lower-top Section of Interaction Vesse1

TC8-24: Temperature in Upper-middle Section of Interaction Vessel

TC8-25: Temperature in Upper-middle Section of Interaction Vessel

TC8-26: Temperature in Lower-middle Section of Interaction Vessel

TC8-27: Temperature in Lower-middle Section of Interaction Vessel

TC8-28: Temperature in Discharge Pipeway $U$-bend

TC9-2: Temperature in Thermite-Vessel Gas Space

TC12-1: Temperature Inside Exr ansion Vessel $282.58 \mathrm{~cm}$ Above Pipeway Exit

TC12-2: Temperature Inside Expansion Vesse1 $241.94 \mathrm{~cm}$ Above Pipeway Exit

TC12-3: Temperature Inside Expansion Vesse1 $201.30 \mathrm{~cm}$ Above Pipeway Exit

TC12-4: Temperature Inside Expansion Vesse1 $160.66 \mathrm{~cm}$ Above Pipeway Exit

TC12-5: Temperature Inside Expansion Vessel $120.02 \mathrm{~cm}$ Above Pipeway Exit

TC12-6: Temperature Inside Expansion Vessel $79.38 \mathrm{~cm}$ Above Pipeway Exit 


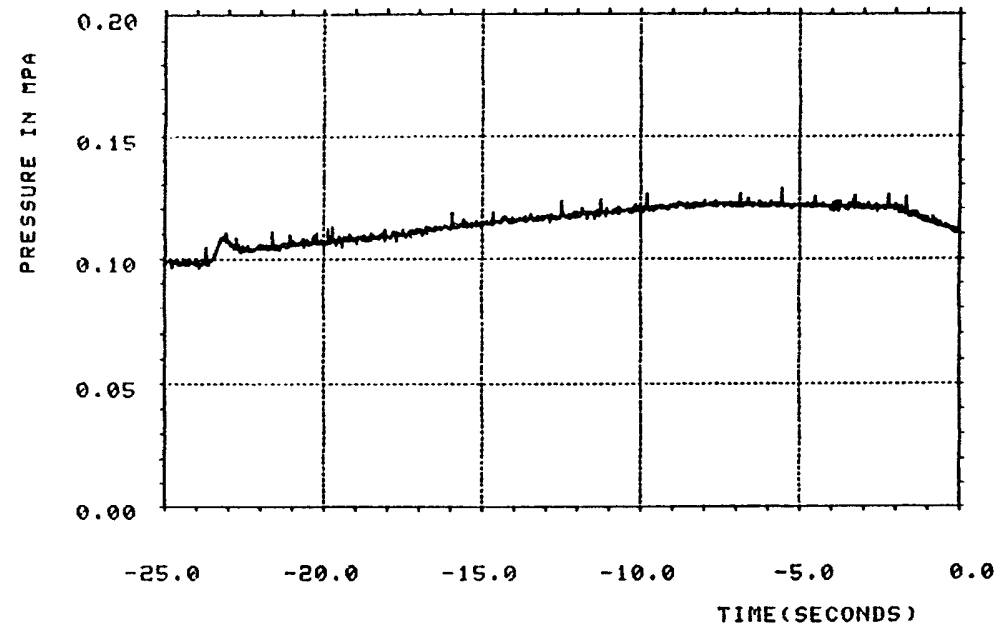

PTS-1, CCN-1, DATE 5/14/86

$\infty$

'́

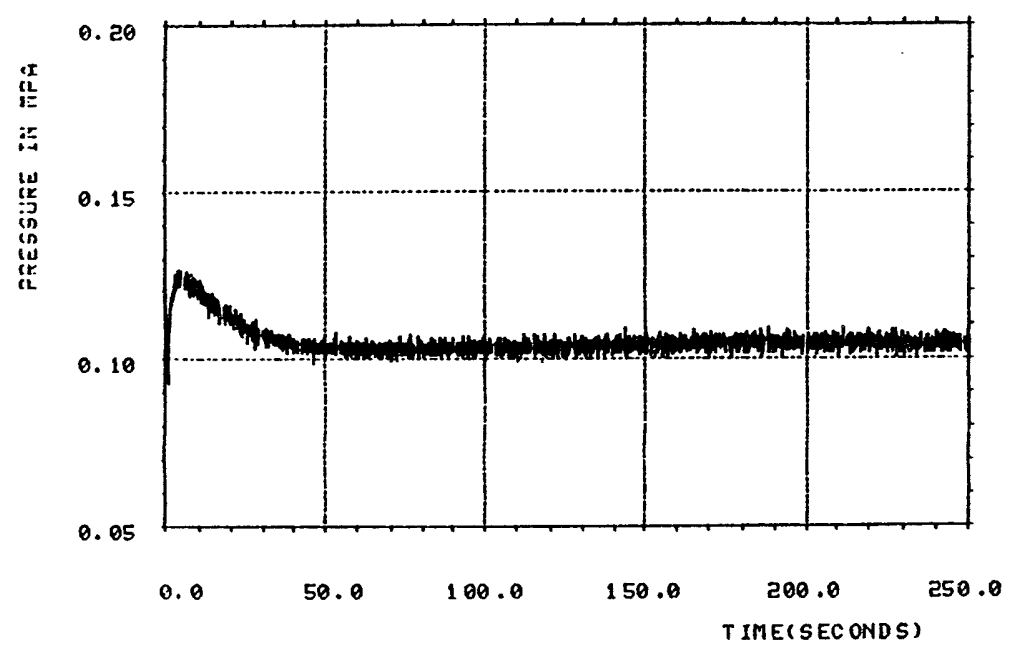

PTS-1, CCH-1, (101), DATE 5/14/86

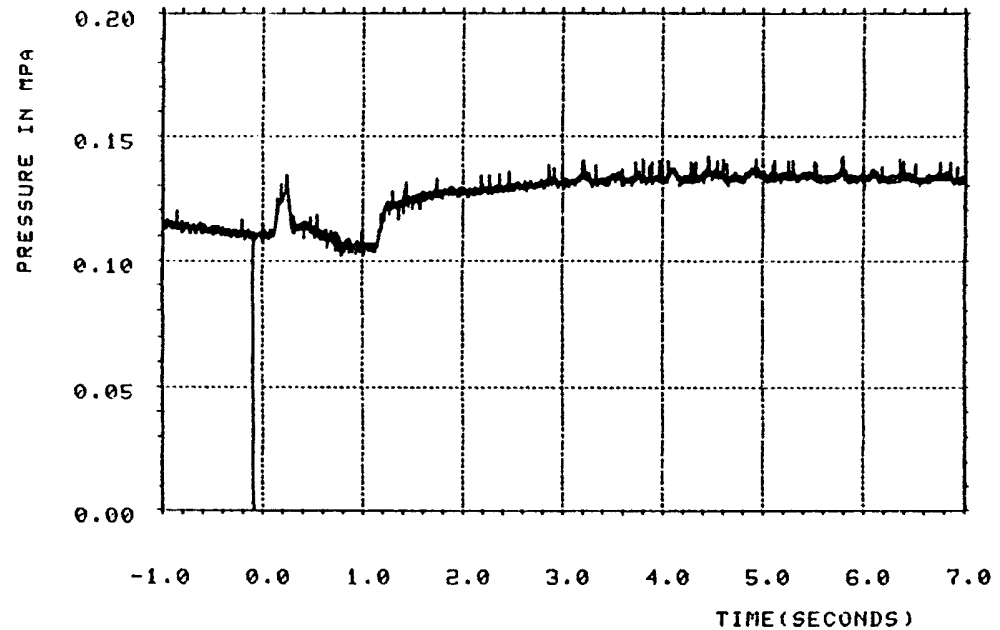

PTS-1, CCM-1, UATE 5/14/86

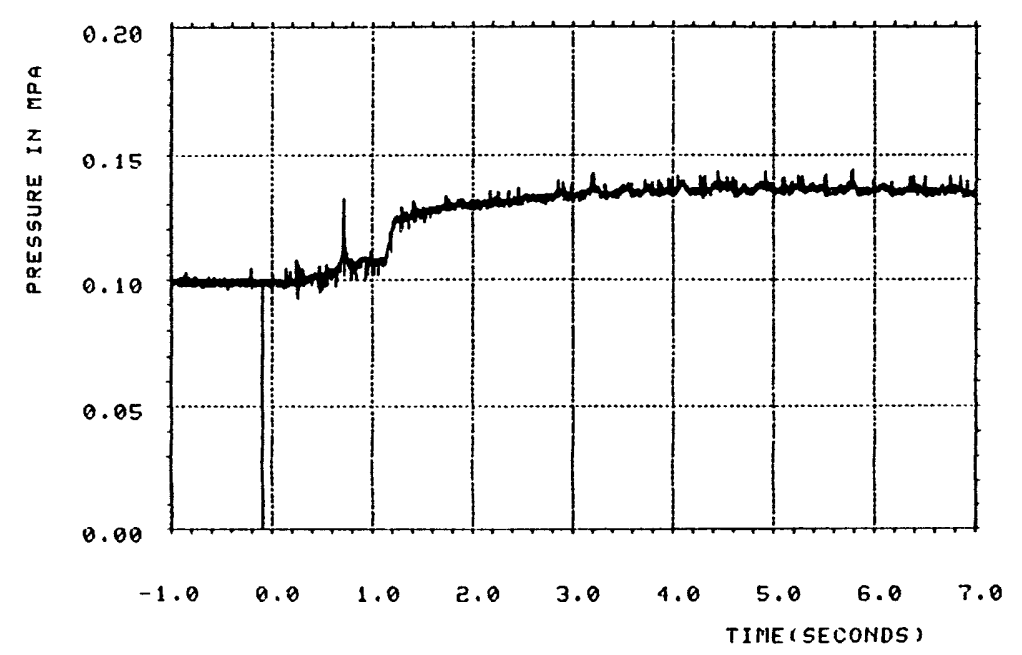

PTS-2, CCH-1, DATE 5/14/86 


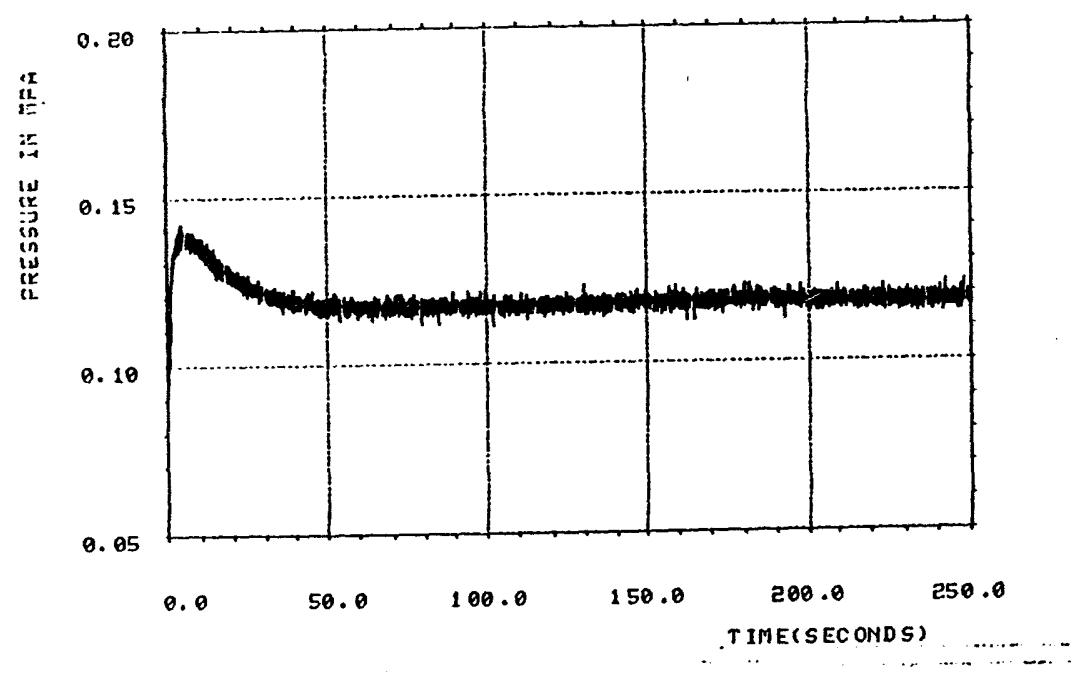

PTS-2, CCIA-1, (101), DATE 5/14/86

唄

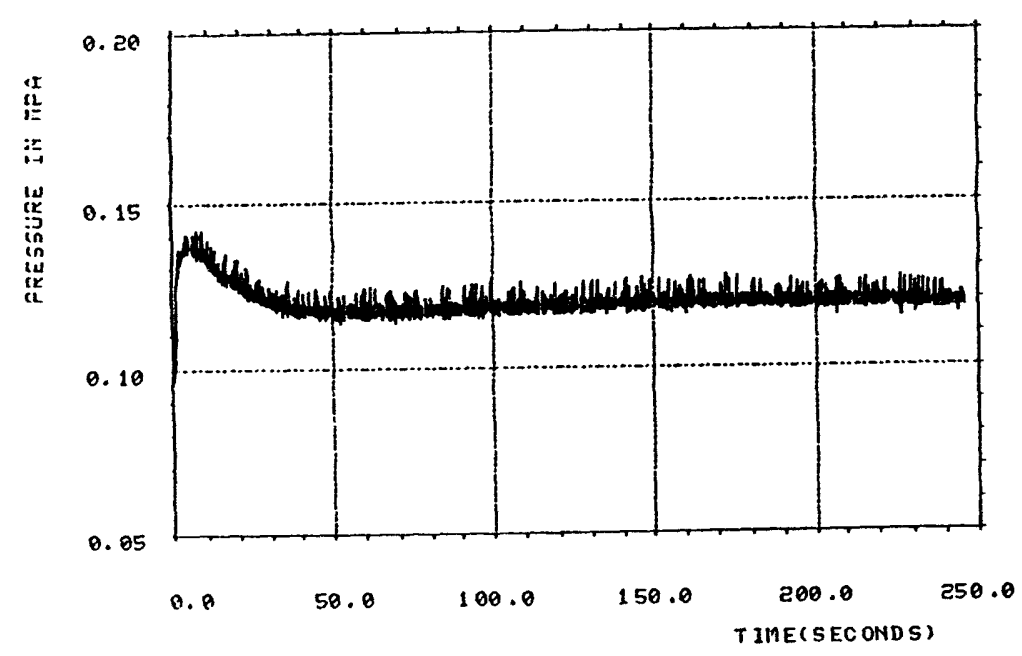

PTS-9, CCN-1, (96), DATE 5/14/86

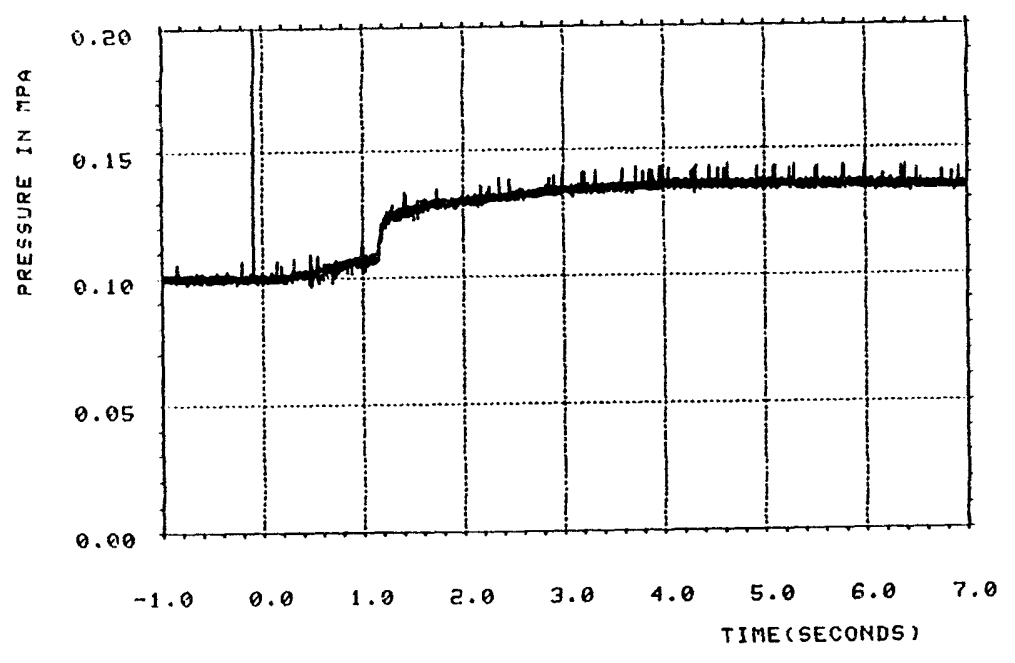

PTS-9, DUH-1, DATE 5/14/86

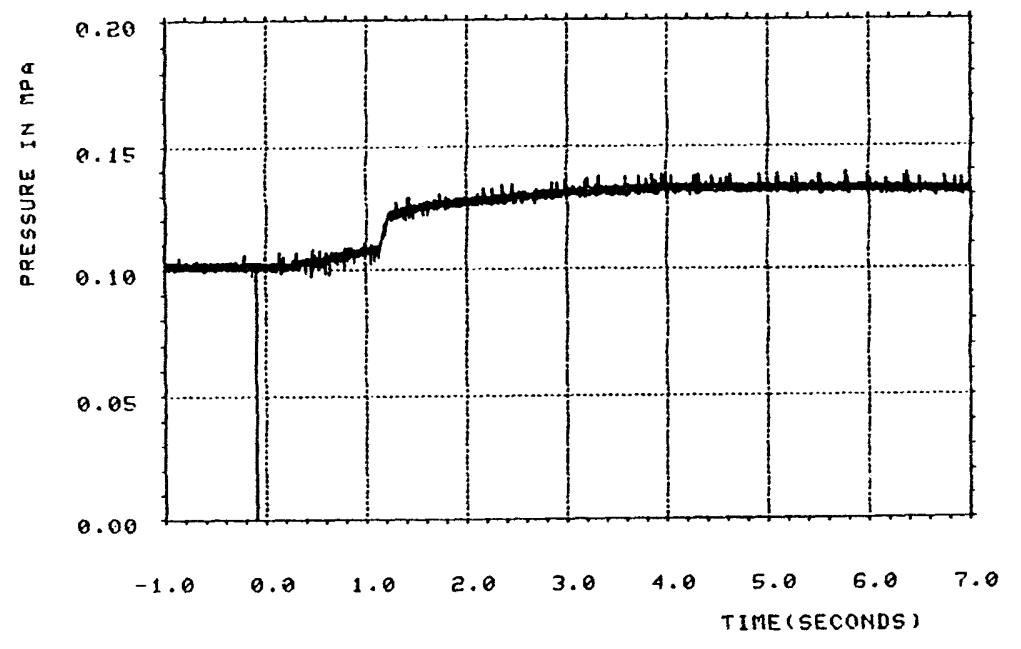

PTS-10, DOM-1, DATE 5/14/86 


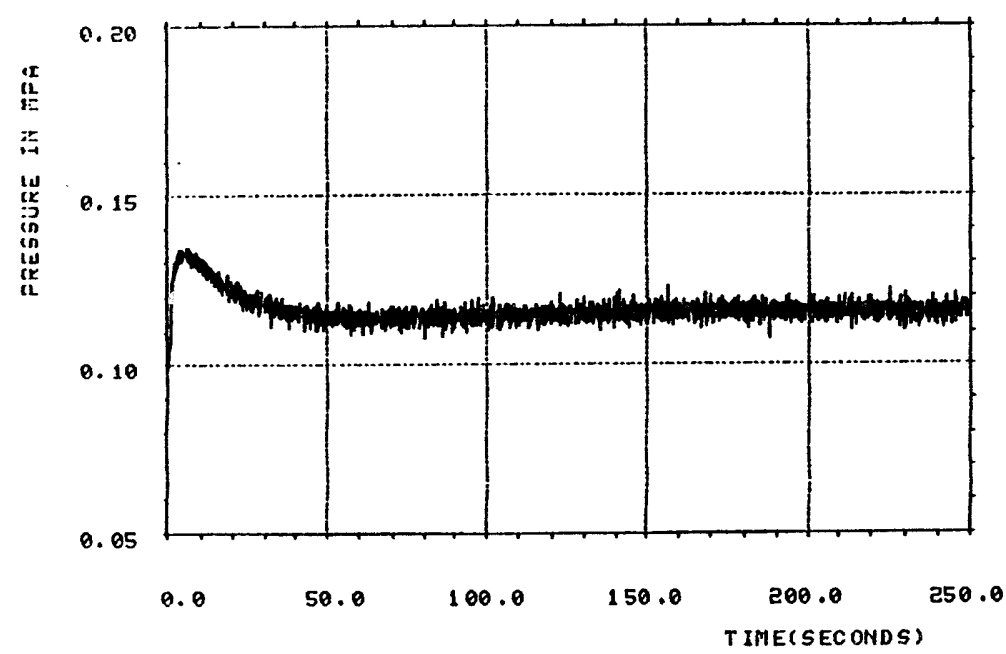

$\stackrel{\infty}{\square}$

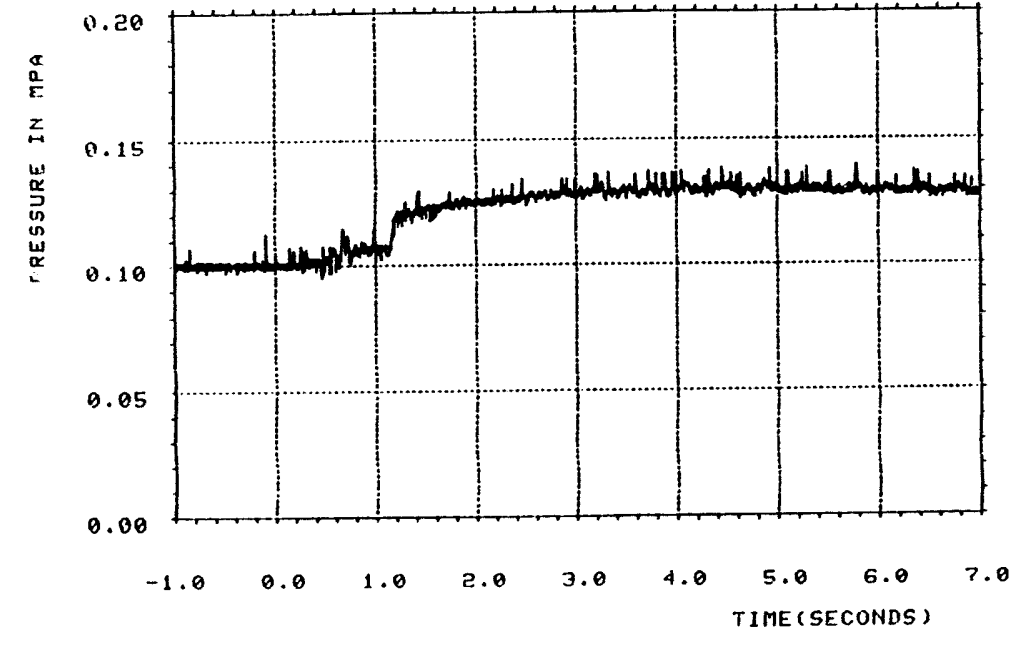

PTS-12, CON1-1, DATE 5/14/86

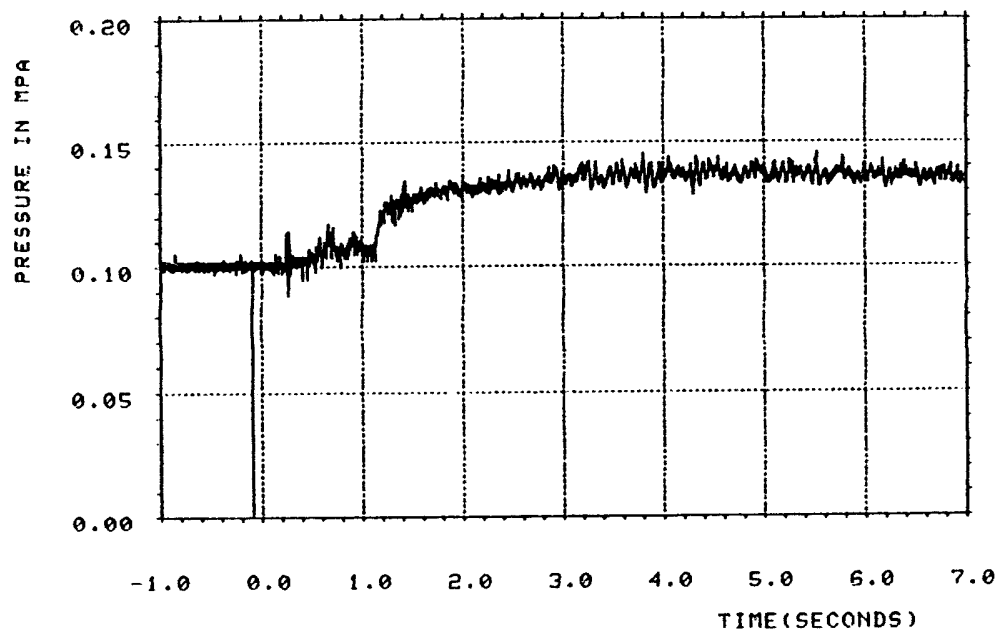

PTS-13, CCH-1, LIATE 5/14/86

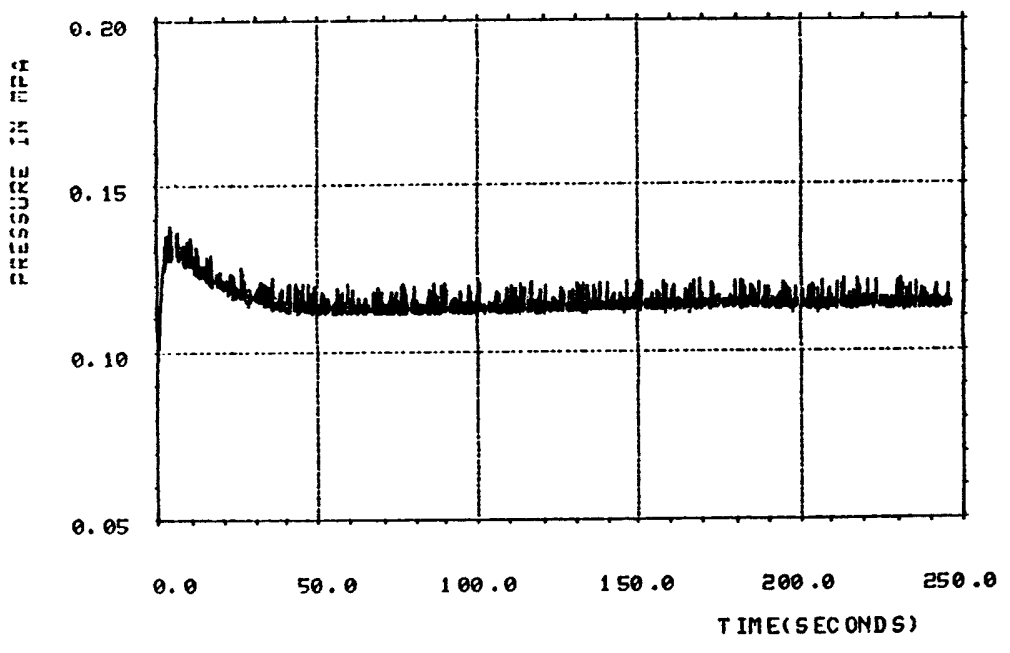

PTs-12, CCI1-1, (96), DATE 5/14/86 


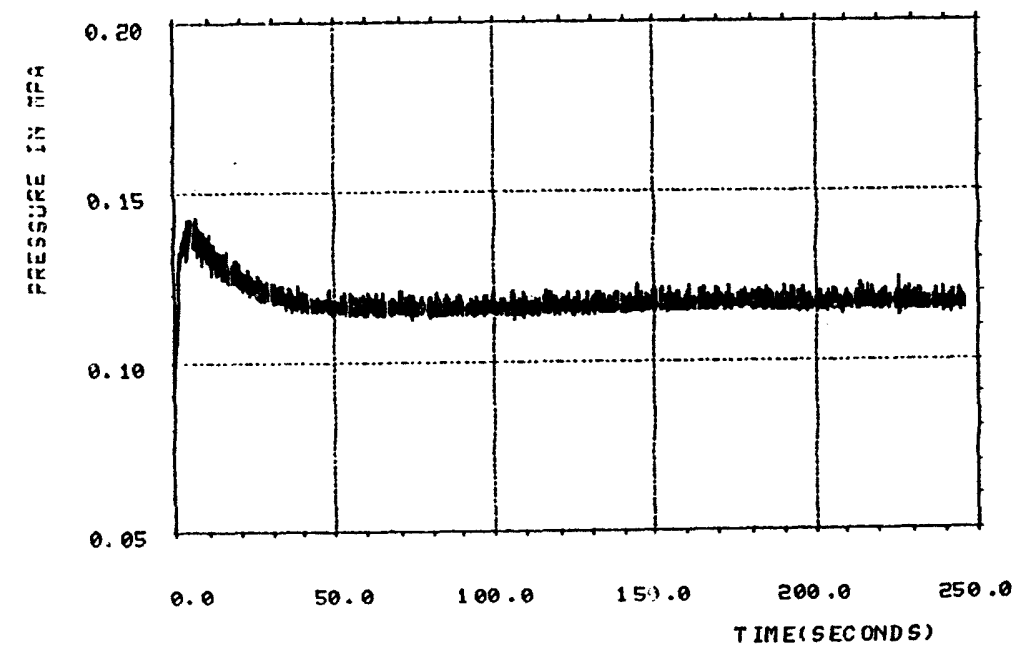

$\infty$

PTS- 13, CCII-1, (96), DATE 5/14/86.

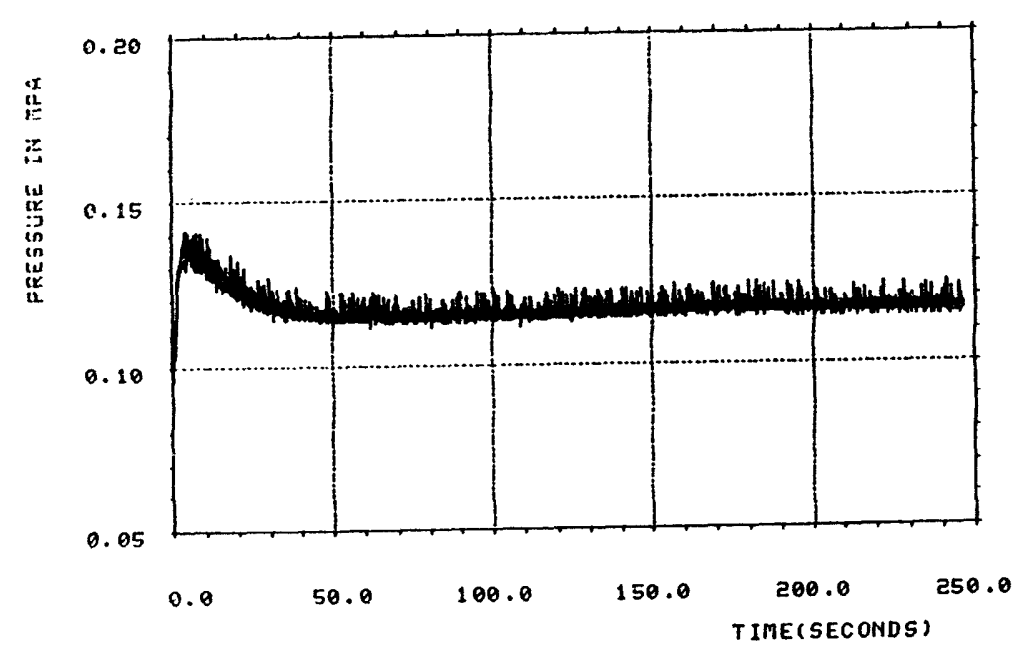

PTS-14, CCM-1, (96), DATE 5/14/86

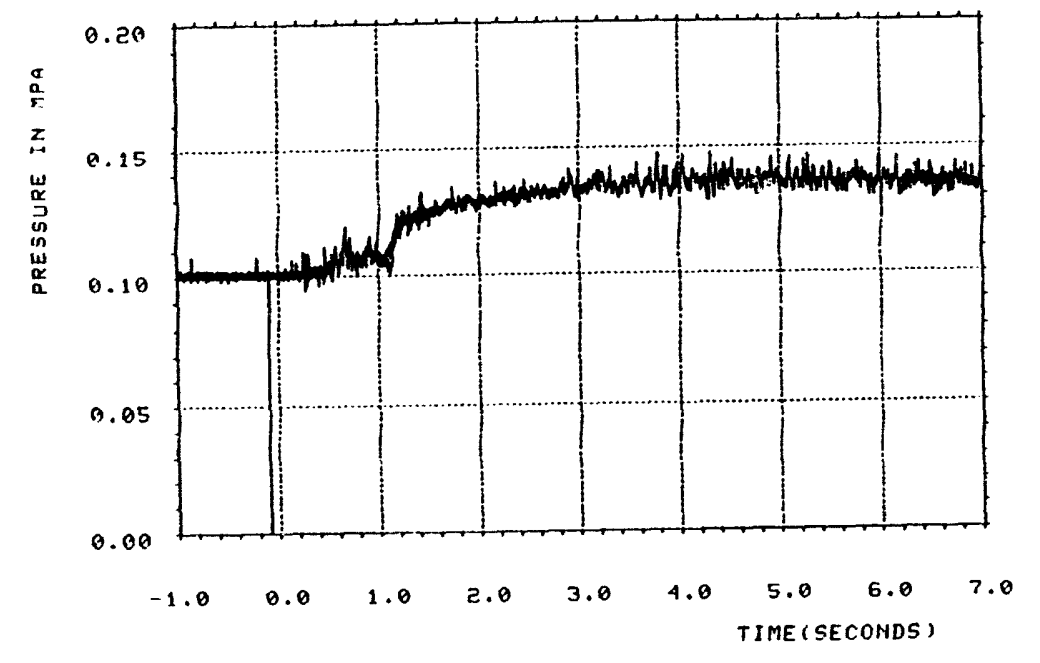

PTS-14, CH-1, [IATE 5/14/86

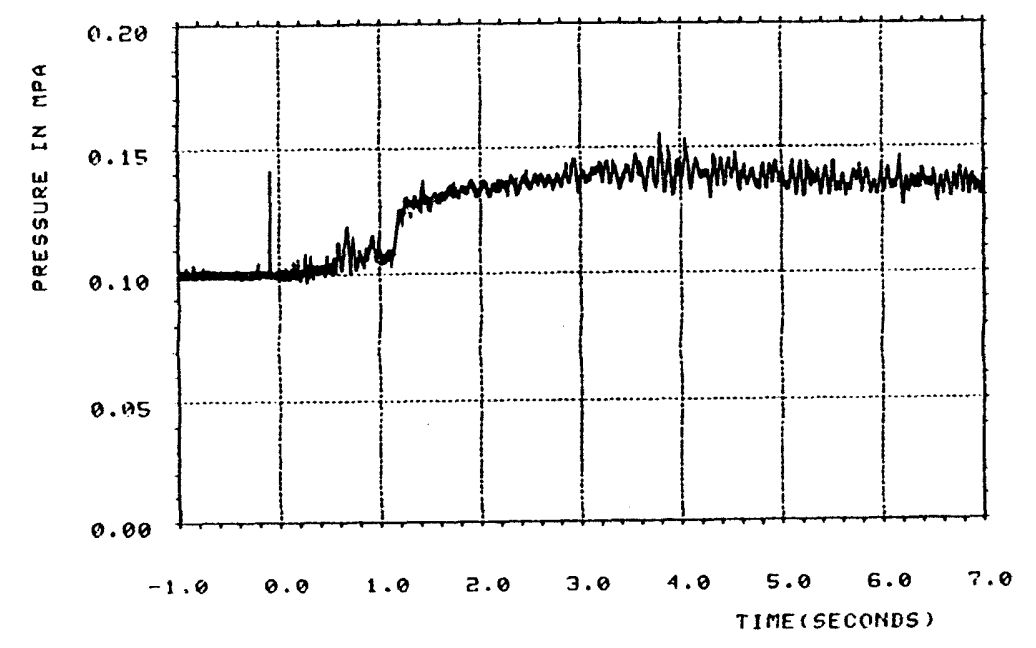

PTS-15, C.N1-1, DATE 5/14/86 


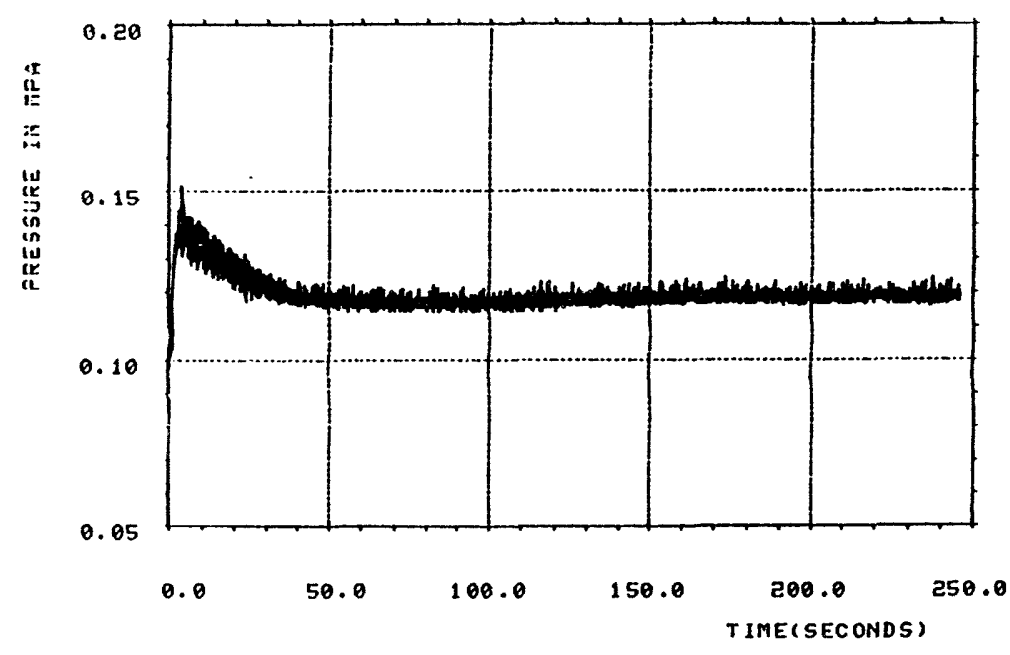

ơ

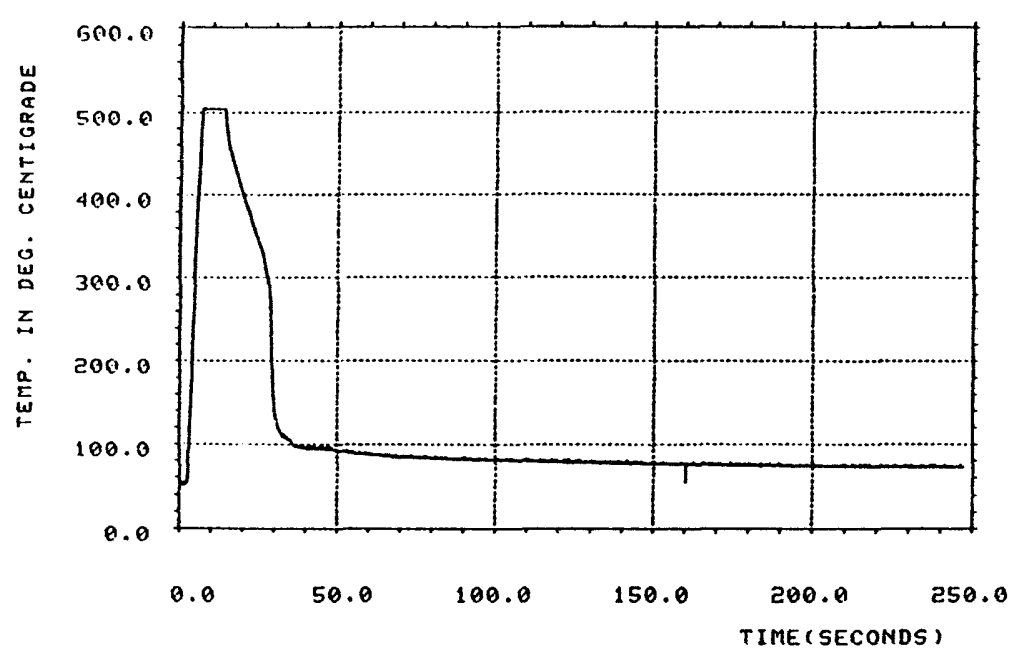

TC8-6, CCM-1, DATE 5/14/86

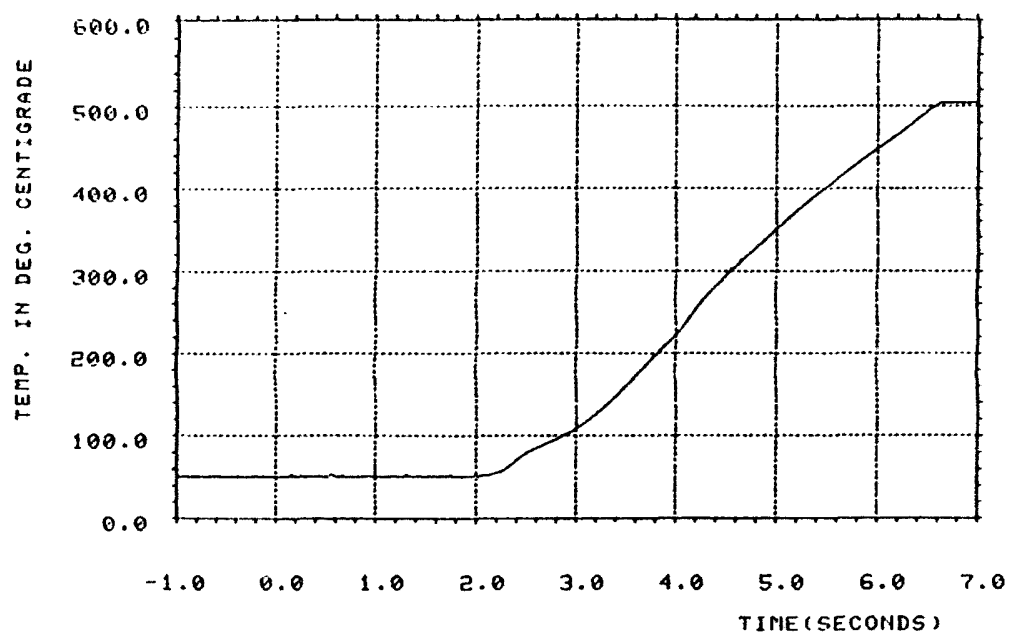

TCQ-5, CCN-1, [ATE 5/14/86

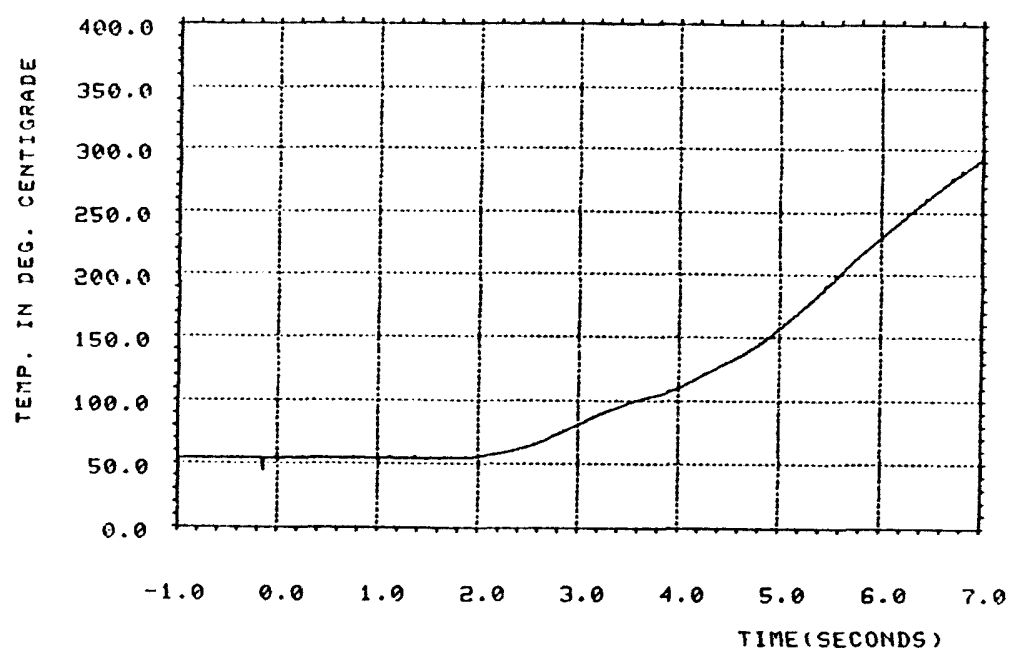

TC8-7, CCII-1, DATE $5 / 14 / 86$ 


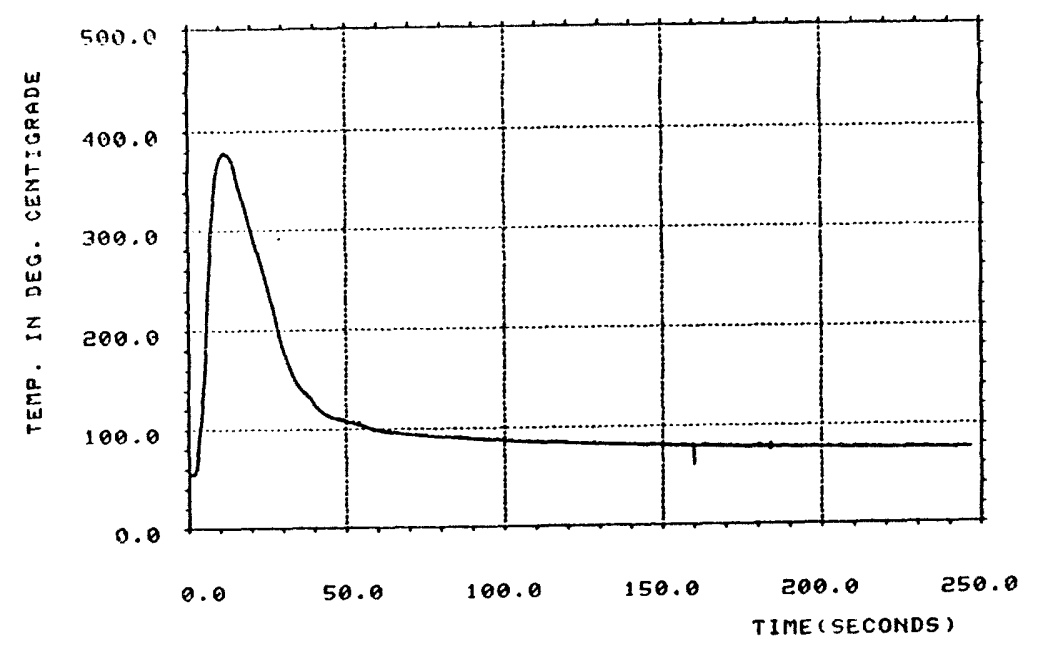

$\stackrel{\text { P }}{4}$

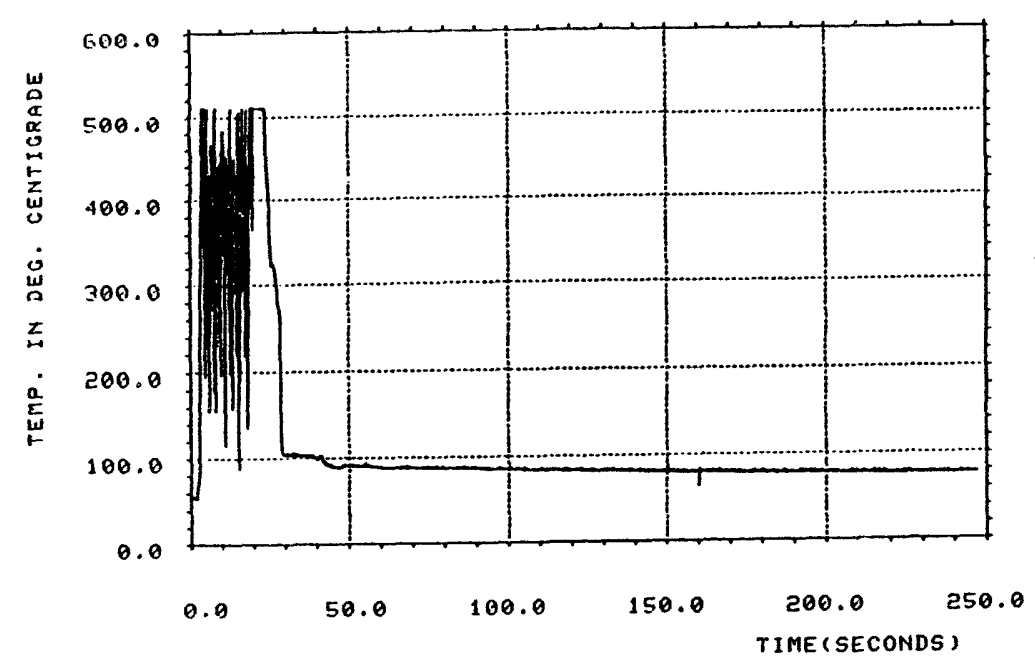

TC8-8, CCH-1, DATE 5/14/86

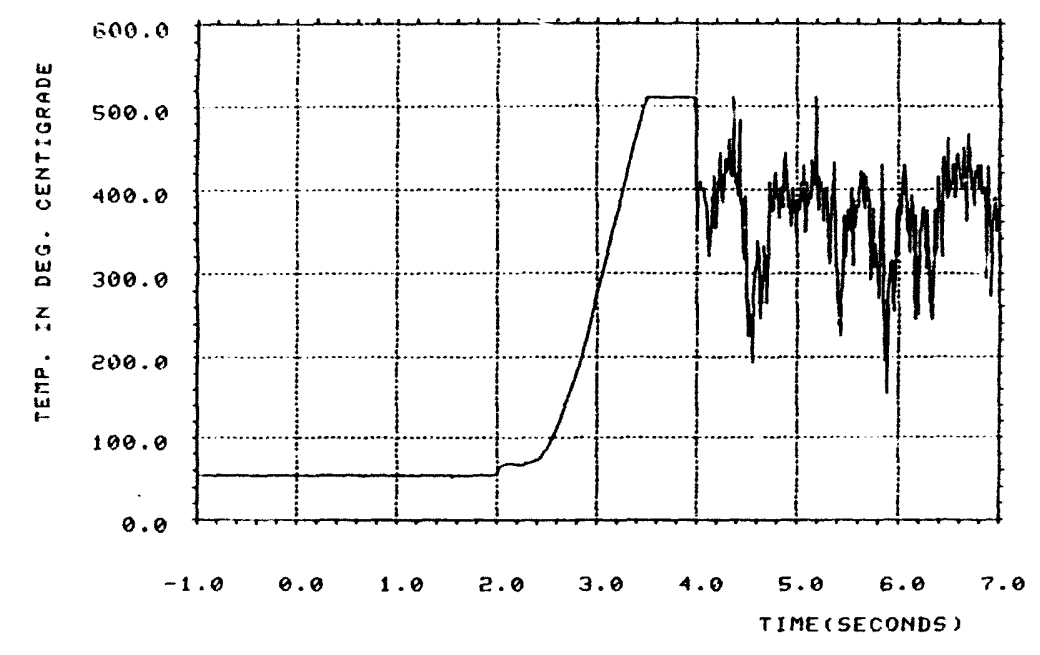

TC8-8, CCH-1, DATE 5/14/86

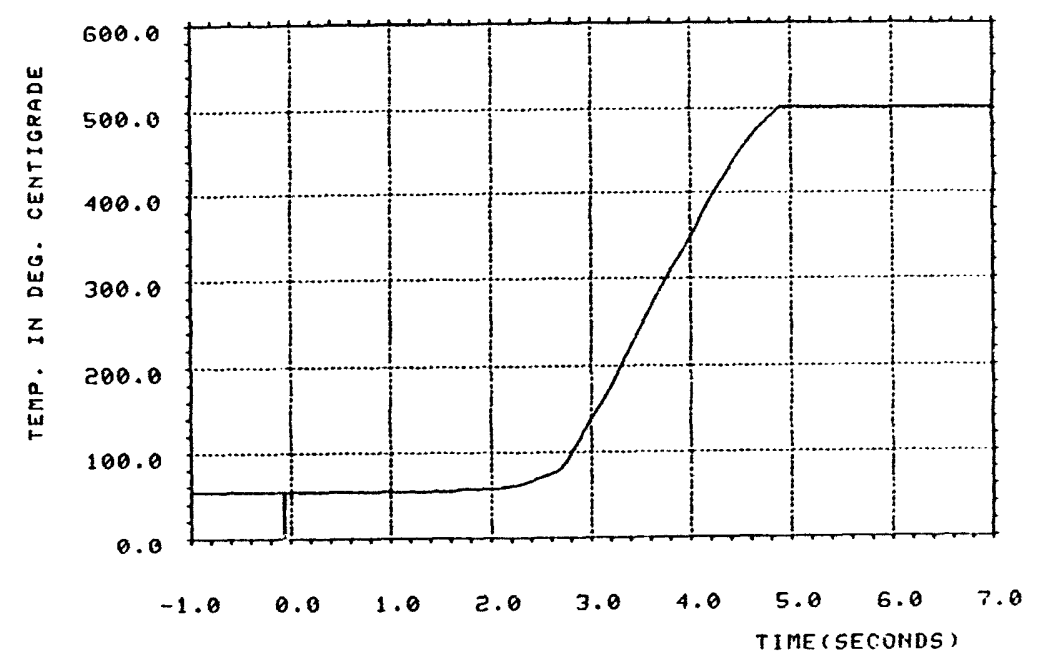

TC8-9, CCA-1, DATE 5/14/86 


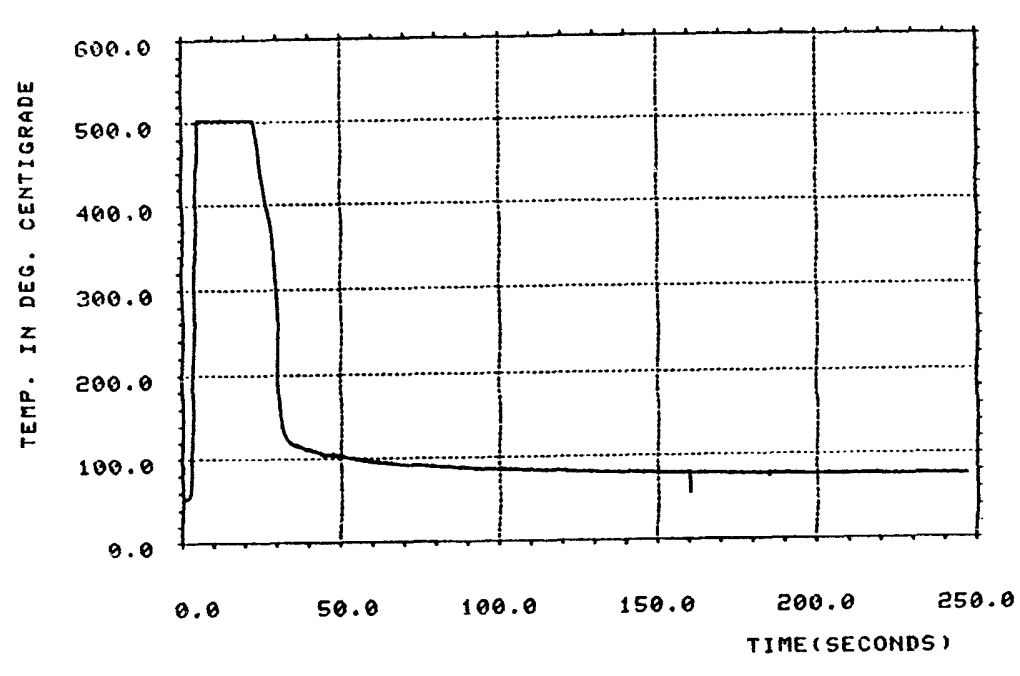

TC8-9, CCH-1, DATE 5/14/86

$\infty$
$\dot{\infty}$
$\infty$

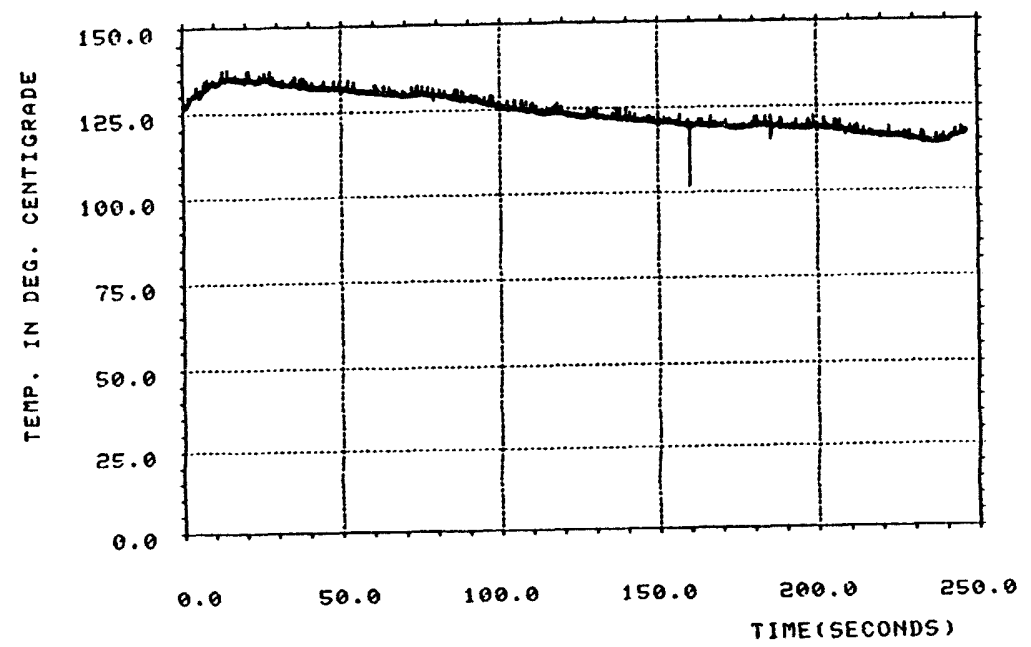

TC8-10, CCri-1, DATE 5/14/86

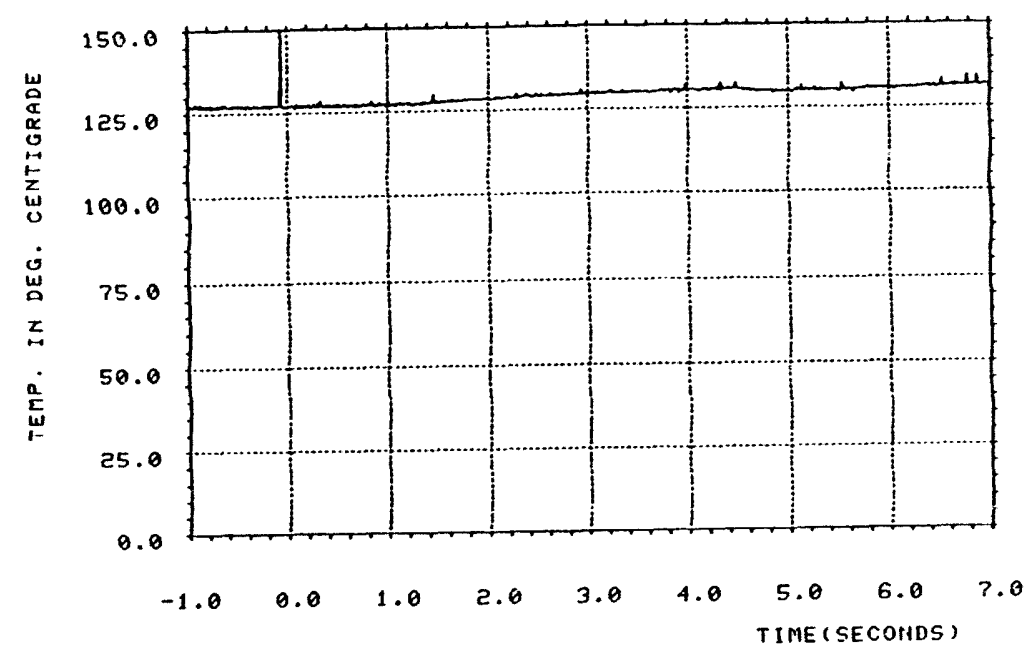

TC8-10, COH-1, DATE 5,14/86

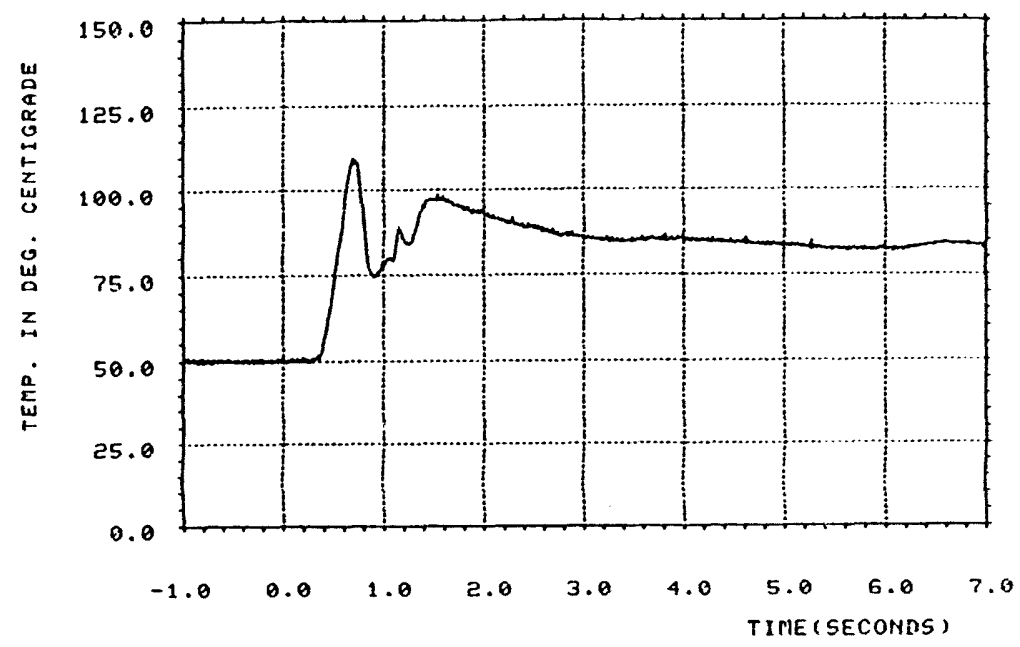

TC8-12, CCN1-1, DATE 5,14/86 


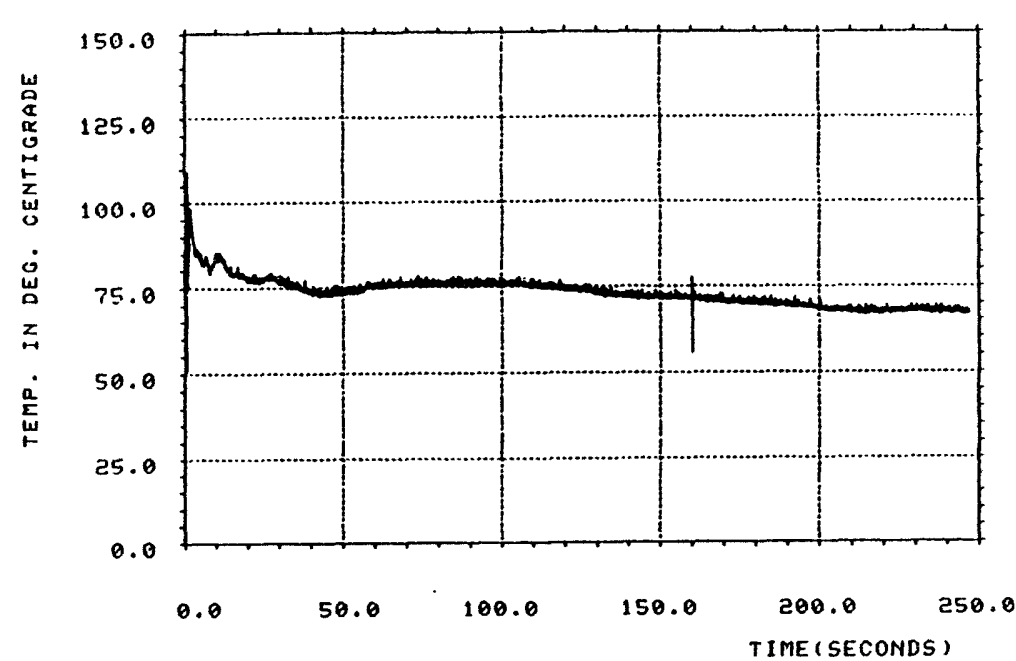

or

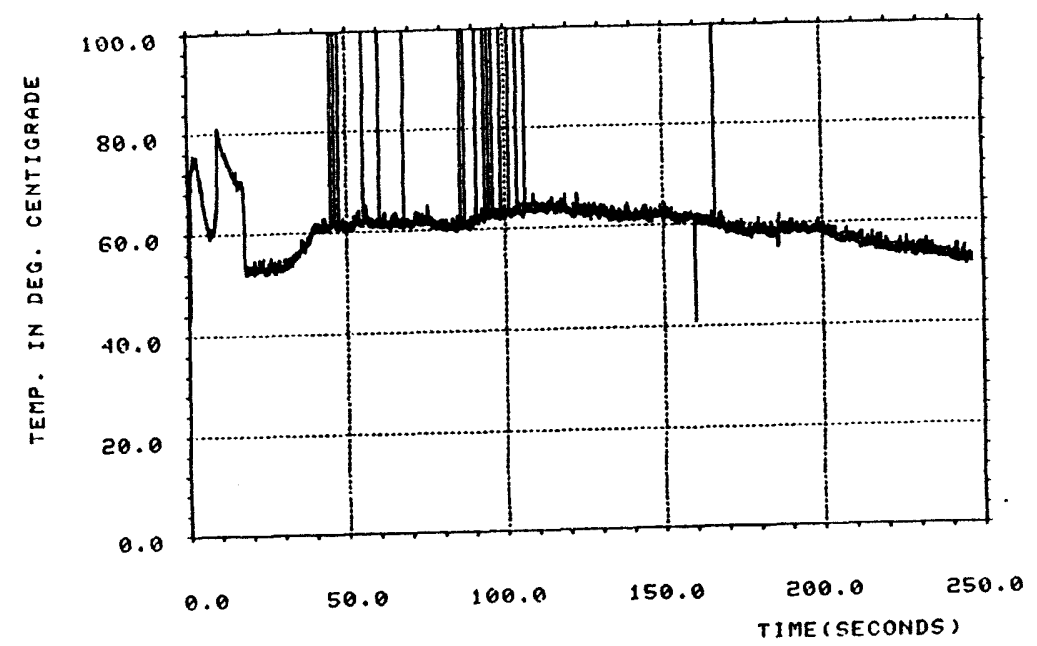

TC8-13, CCII-1, DIATE 5/14/86

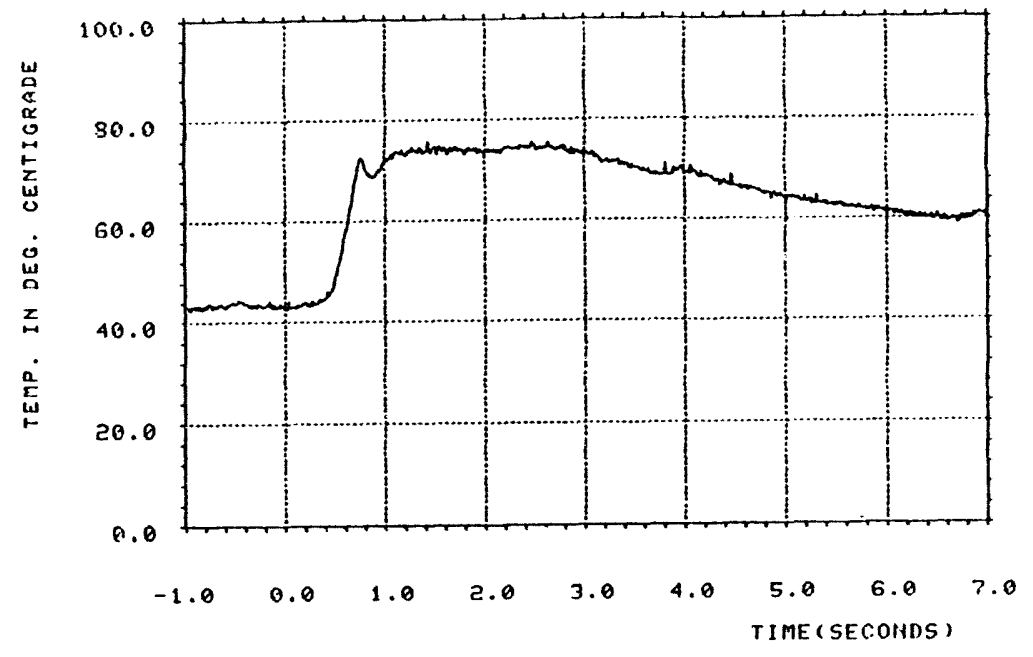

TC8-13, CCH-1, DATE 5/14/86

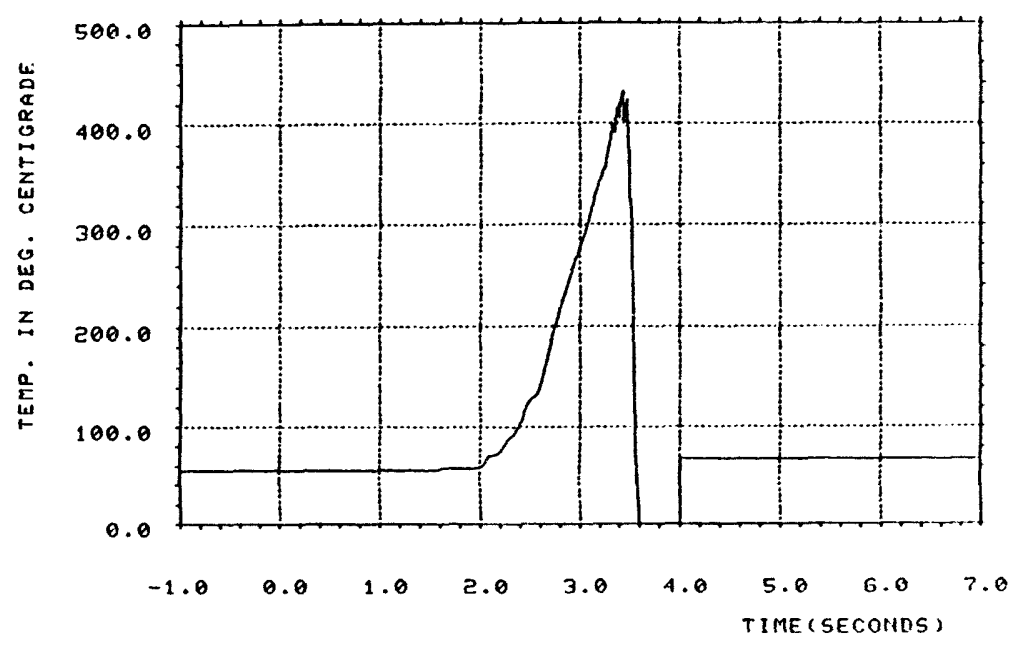

7C8-16, CCM-1, [IATE 5/14/86 


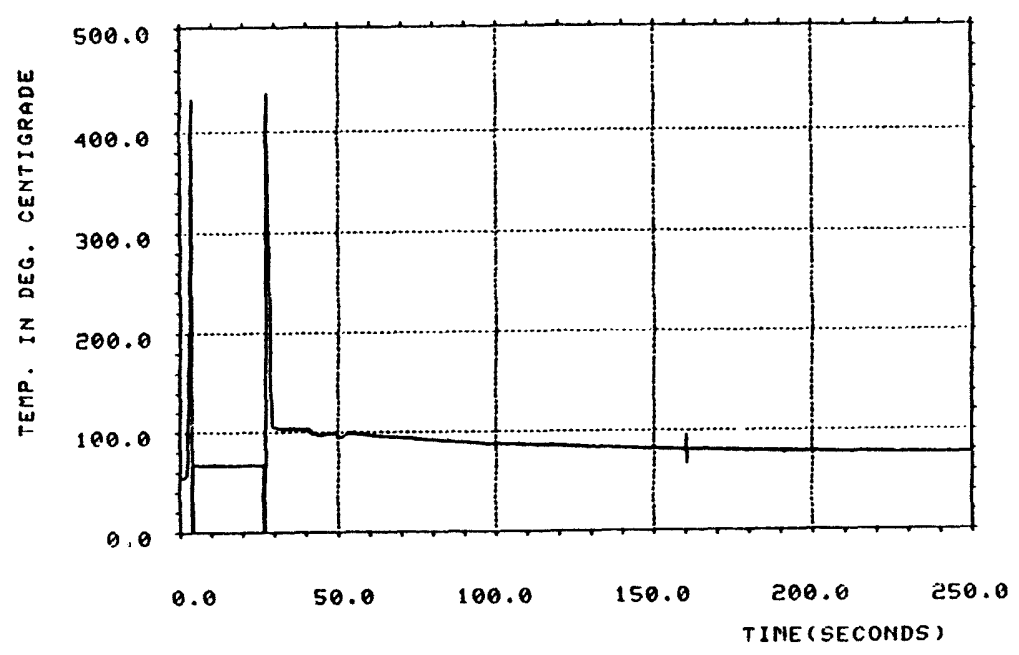

TC8-16, CCM-1, DATE 5/14/86

0
1
0

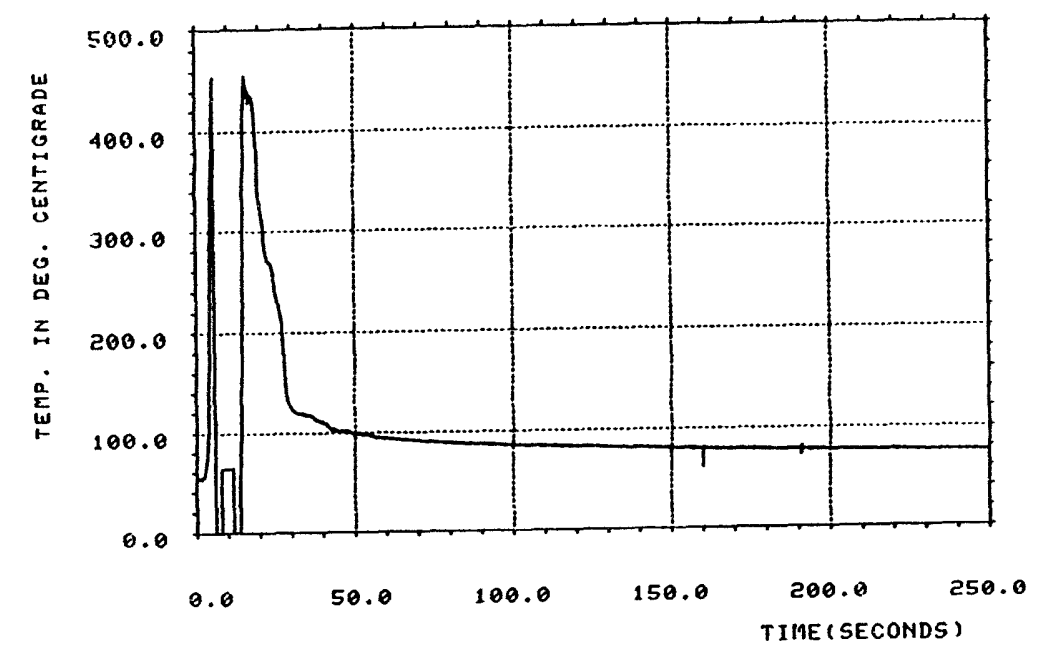

TC8-17, CCM-1, DATE 5/14/86

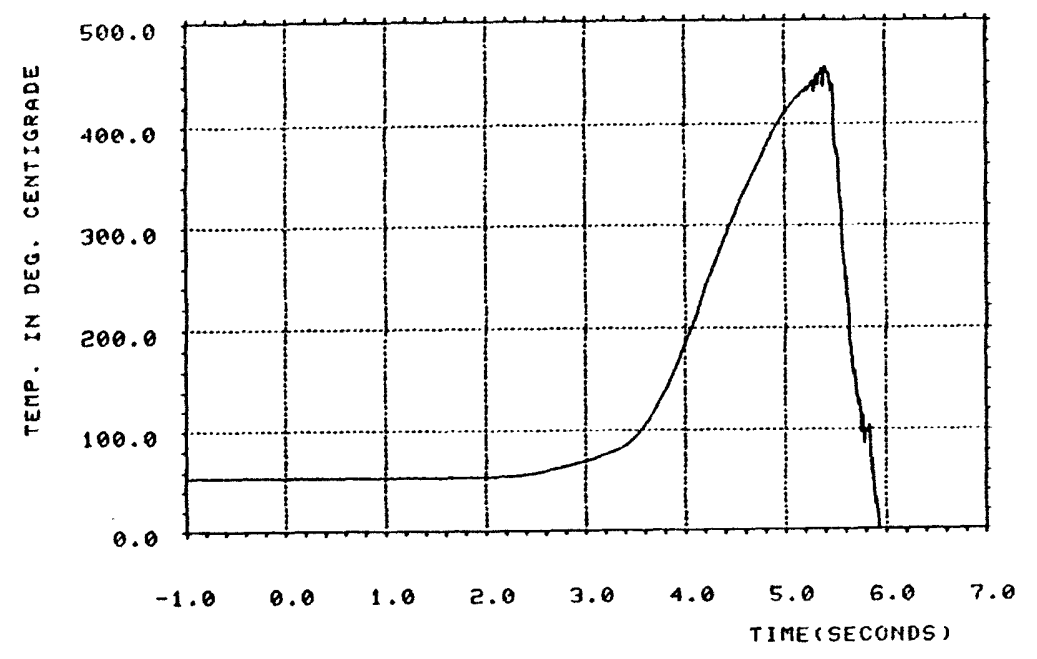

TC8-17, CCI1-1, DATE $5,14 / 86$

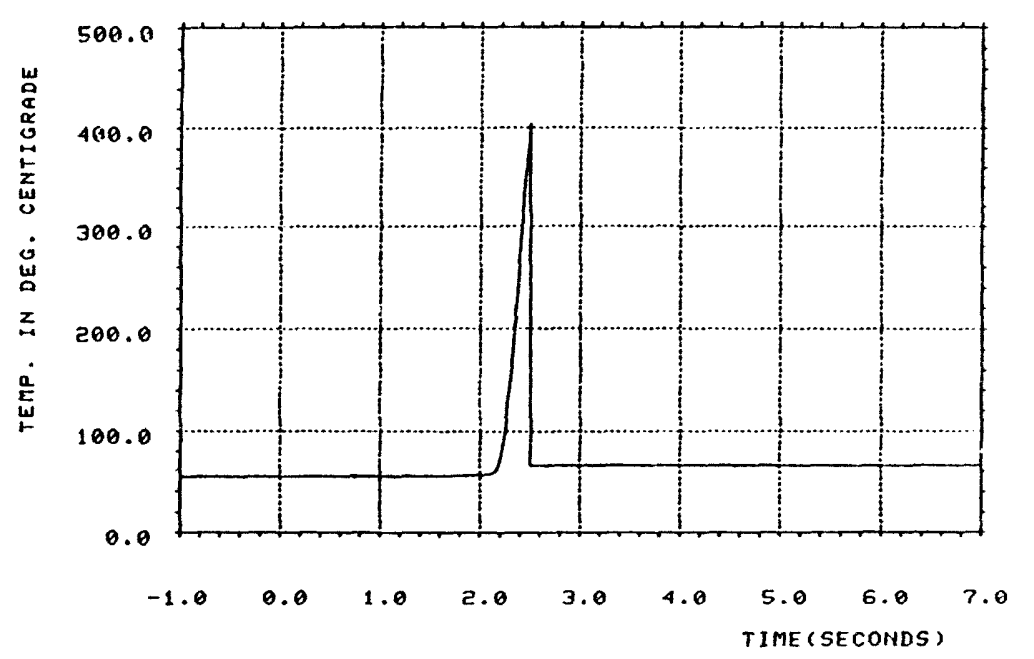

TC8-18, COM-1, DATE 5/14/86 


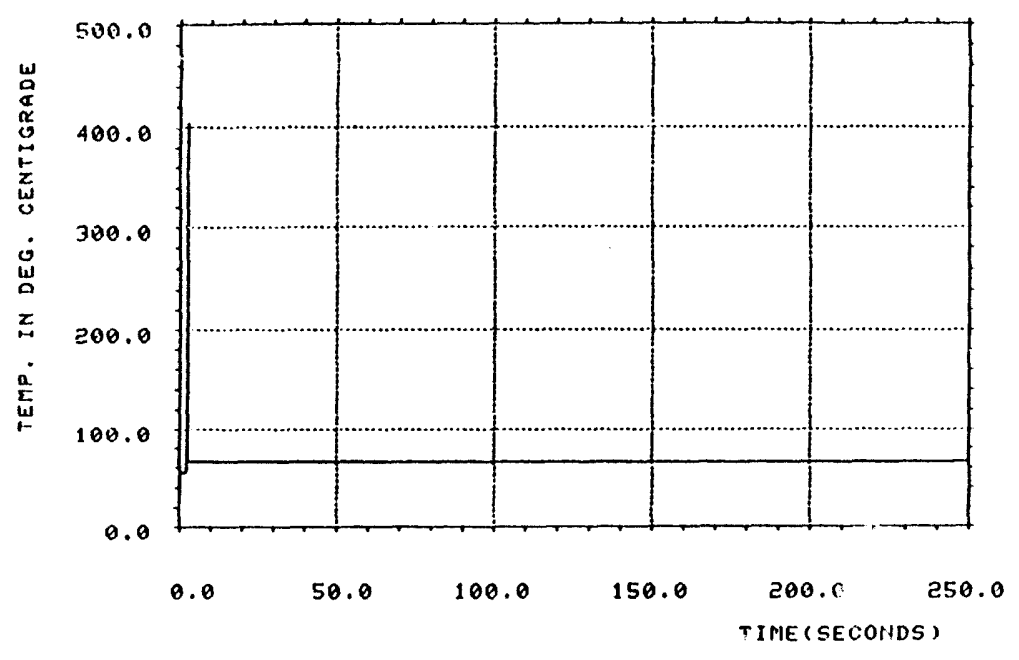

$\stackrel{\infty}{\check{1}}$

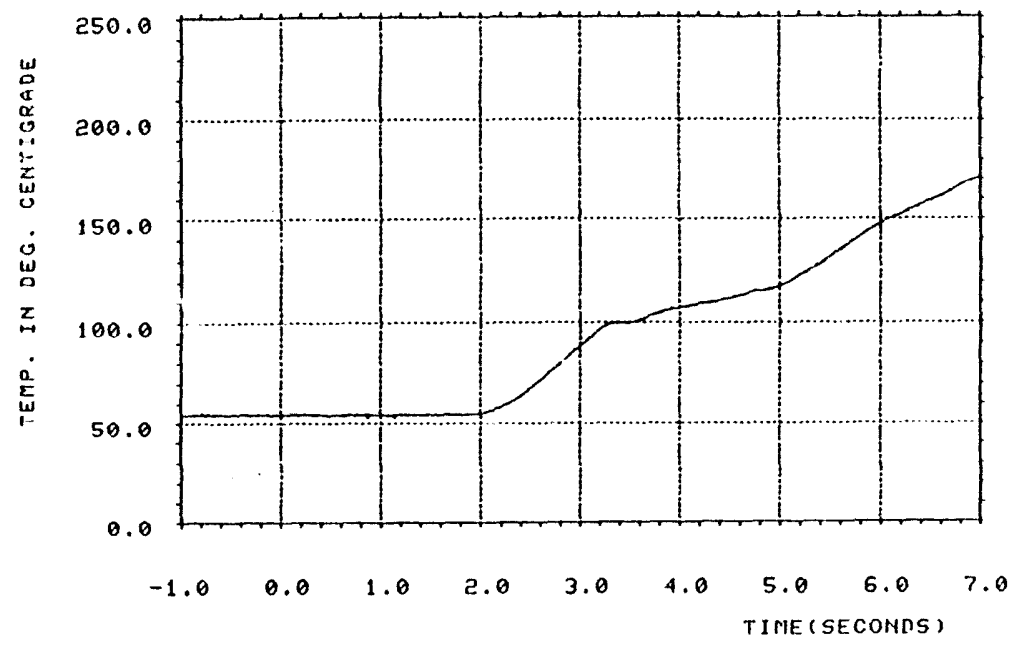

TC8-19, CCM-1, DATE 5/14/86

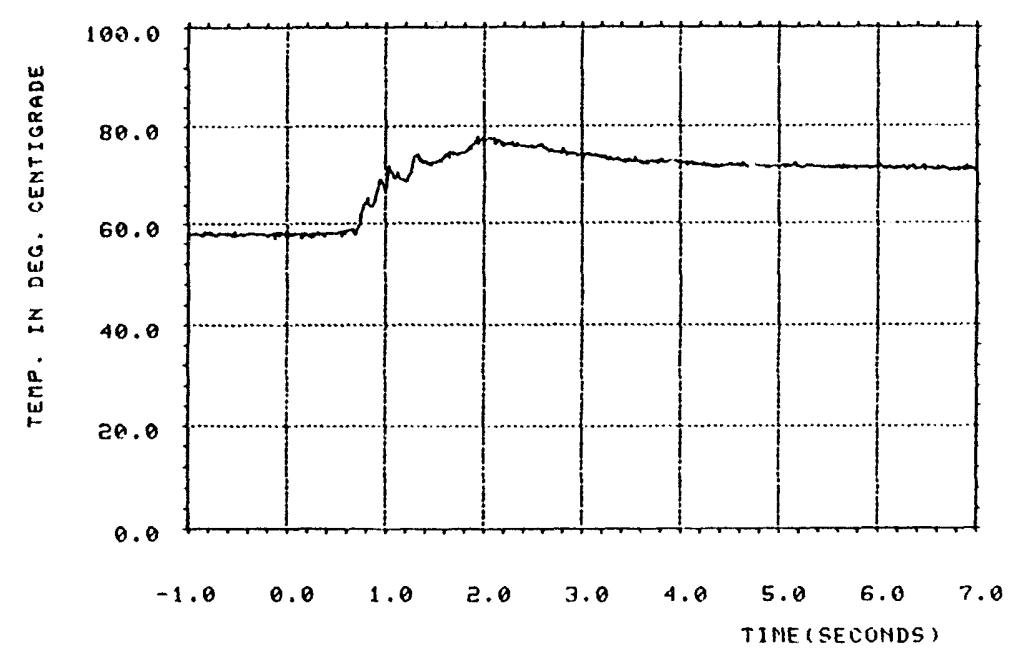

TC8-20, CCN-1, LIATE $5,14 / 86$

TC8-19, CCN-1, DATE 5/14/86

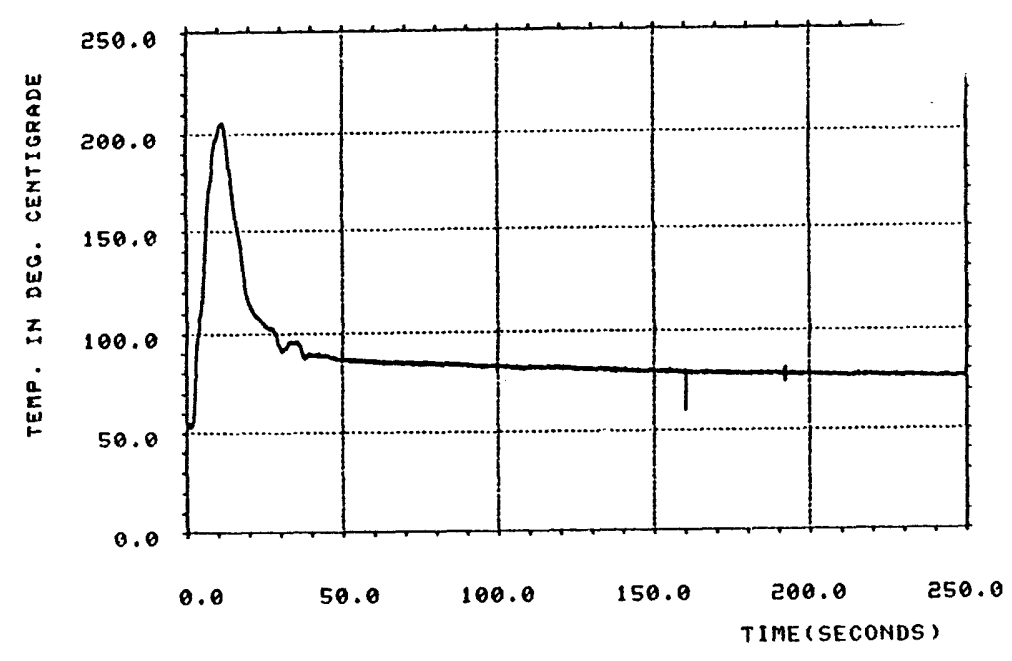




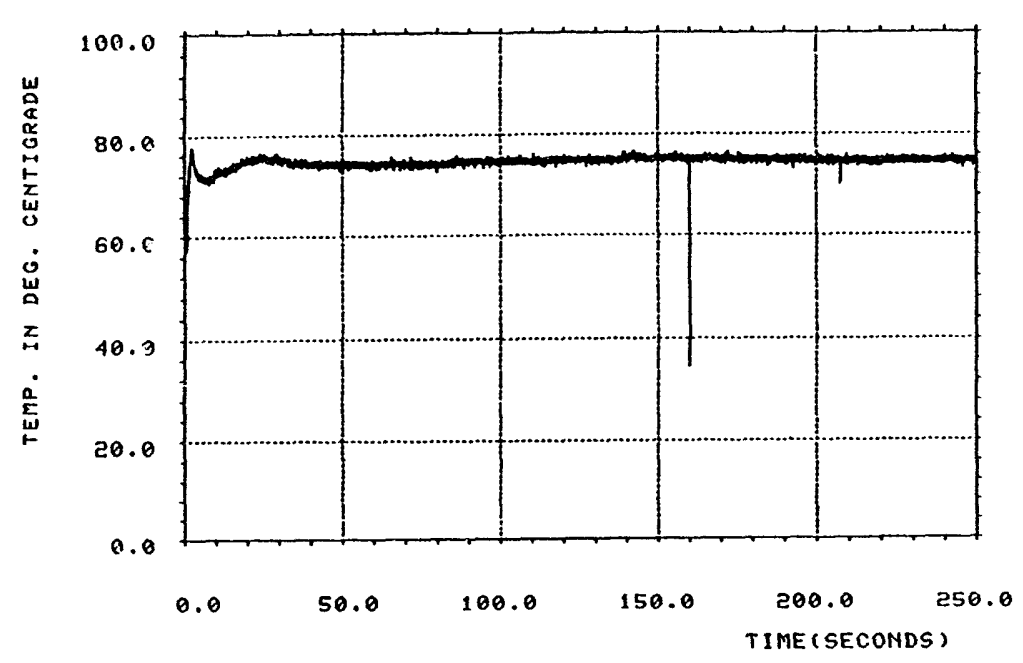

TC8-20, CCM-1, DATE 5/14/86

$\stackrel{\infty}{\stackrel{1}{N}}$

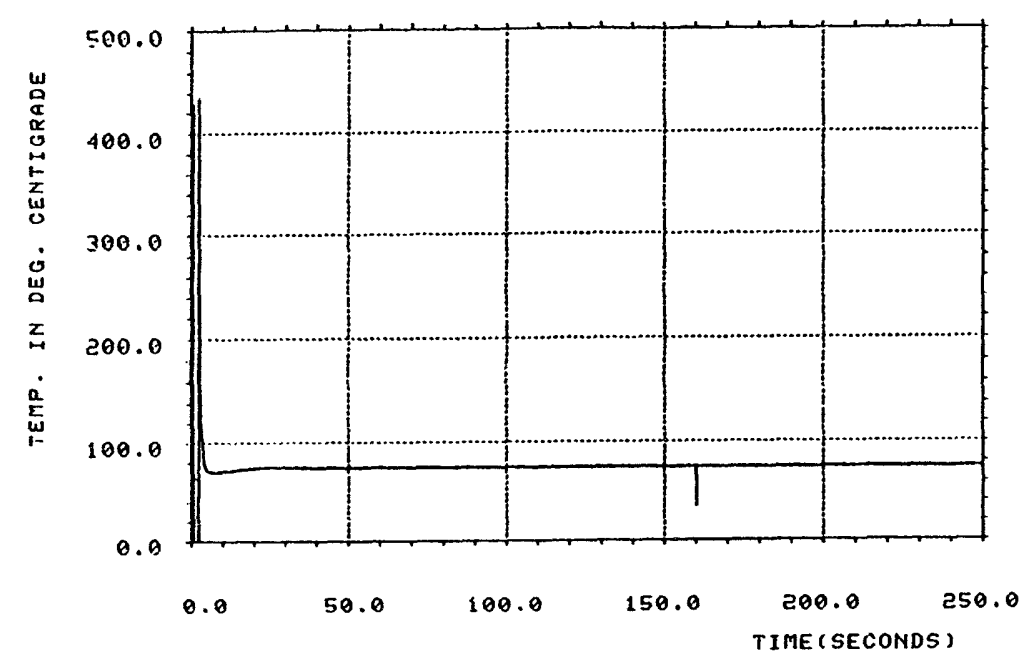

TC8-2i, CCH-1, DATE 5/14/86

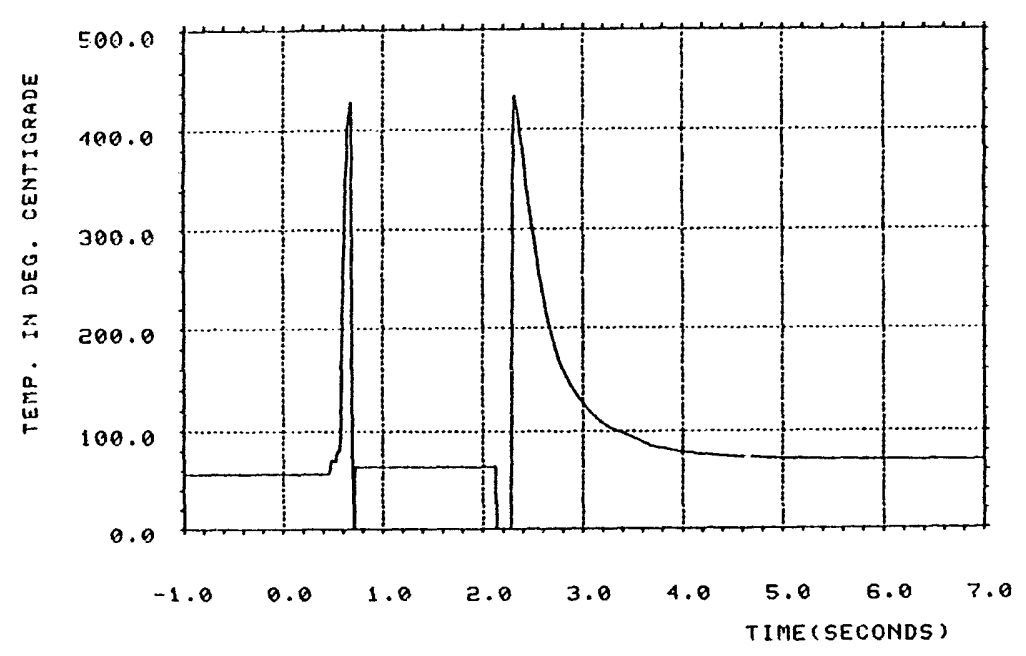

TCR-21, CCM-1, DATE 5/14/86

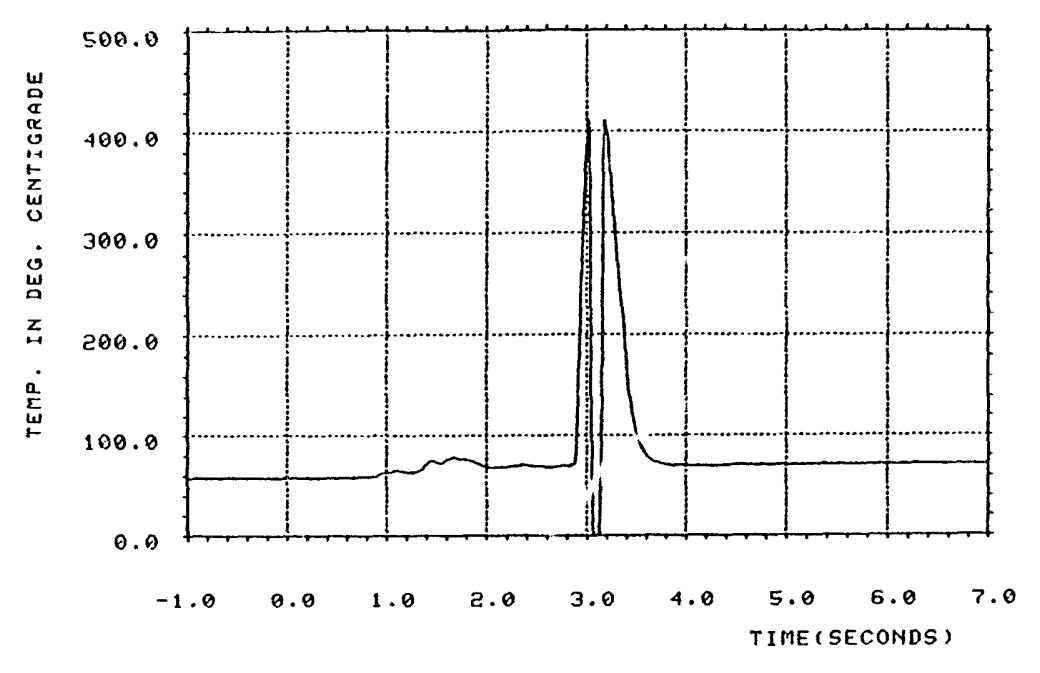

TC8-22, CCN-1, DATE 5/14/86 


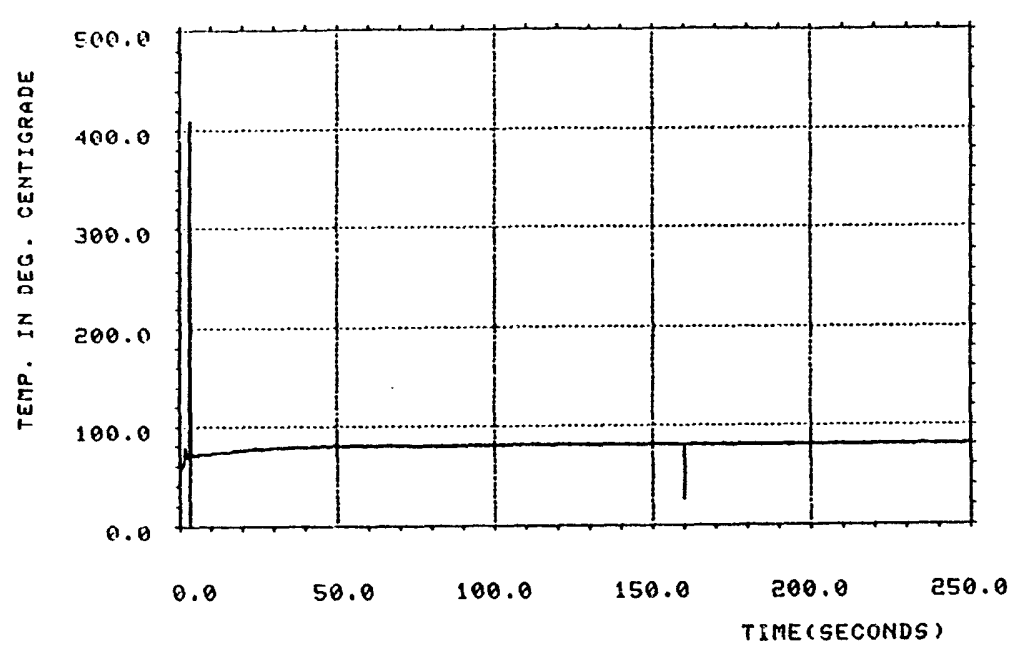

罢

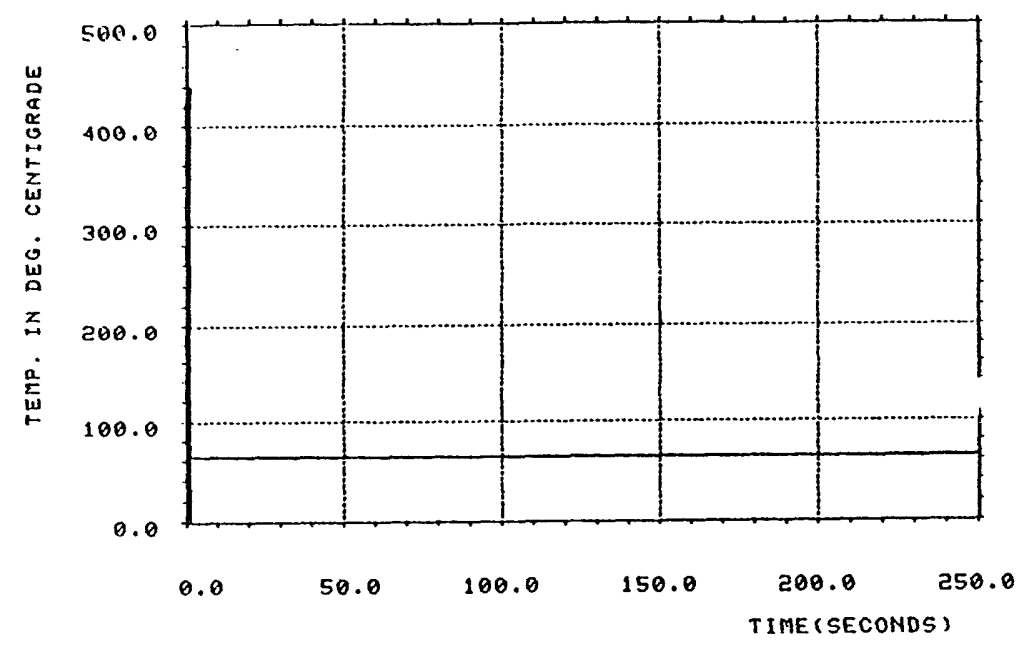

TC8-23, C.CH-1, [IATE 5/14/86

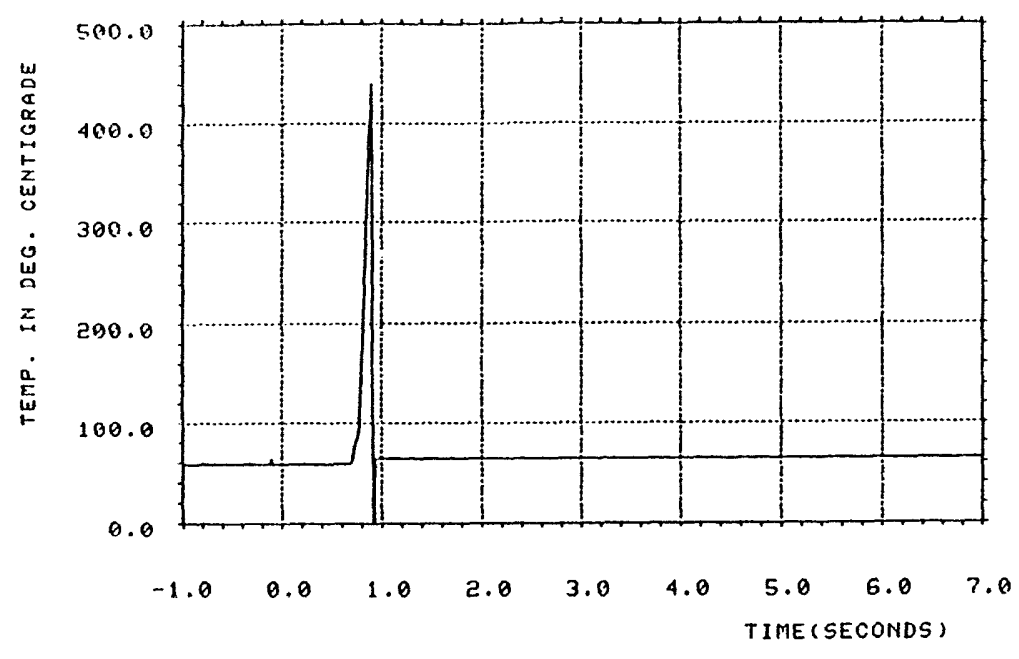

TC8-23, CCIn-1, DATE $5 / 14 / 86$

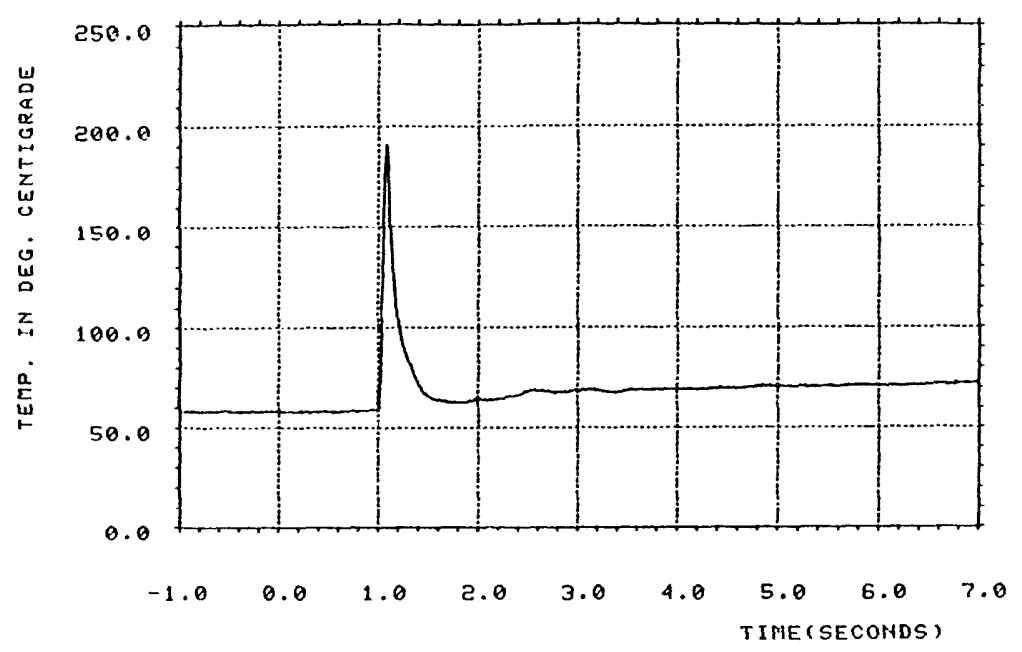

TC8-24, CCN-1, DATE 5/14/86 


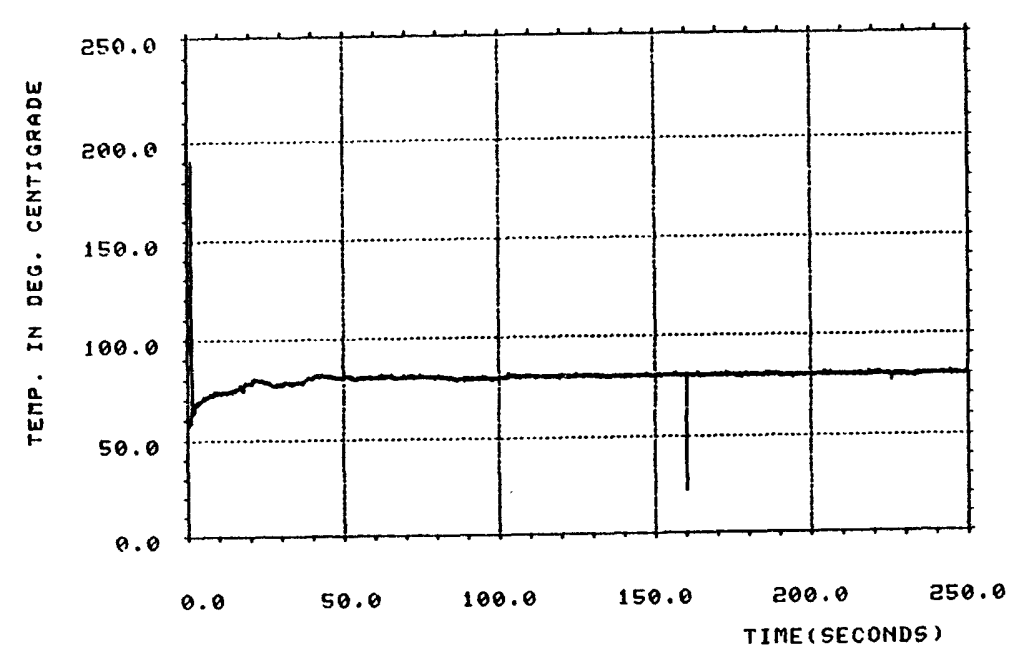

TC8-24, CCII-1, DATE 5/14/86

$\stackrel{\square}{\doteqdot}$

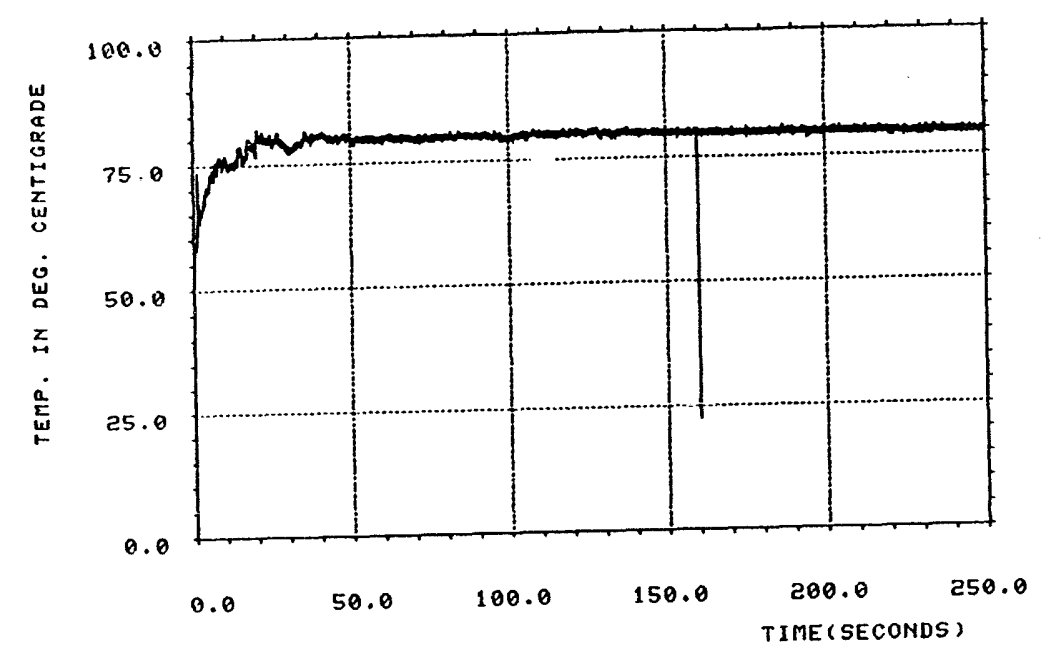

TC8-25, CCIn-1, DATE 5/14/86

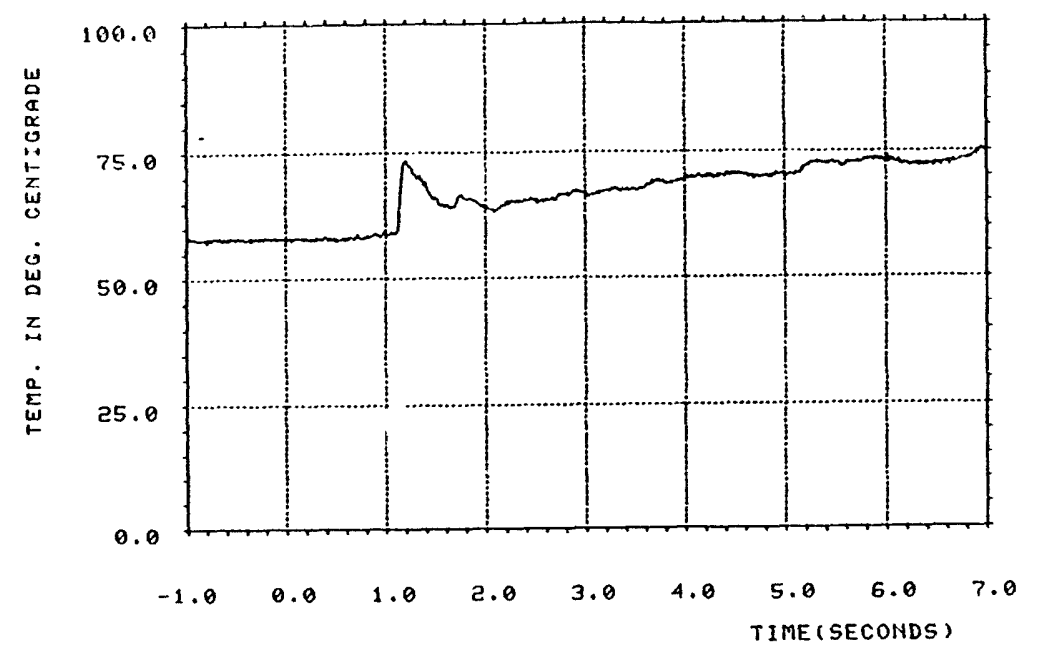

TC8-25, COH-1, DATE 5/14/86

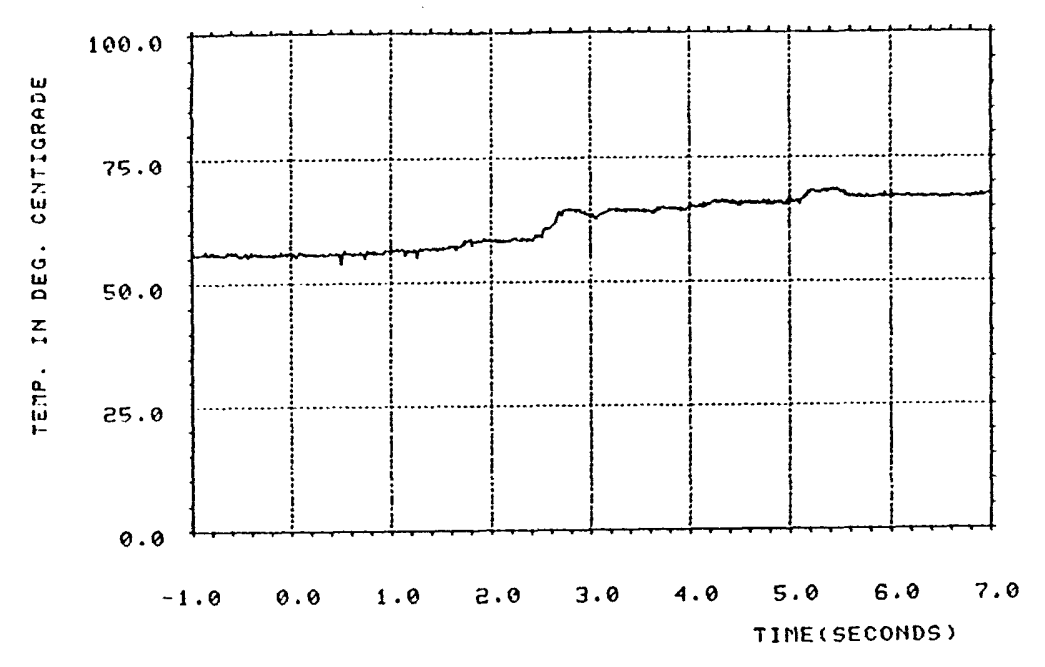

TC8-26, CCII-1, DATE 5/14/86 


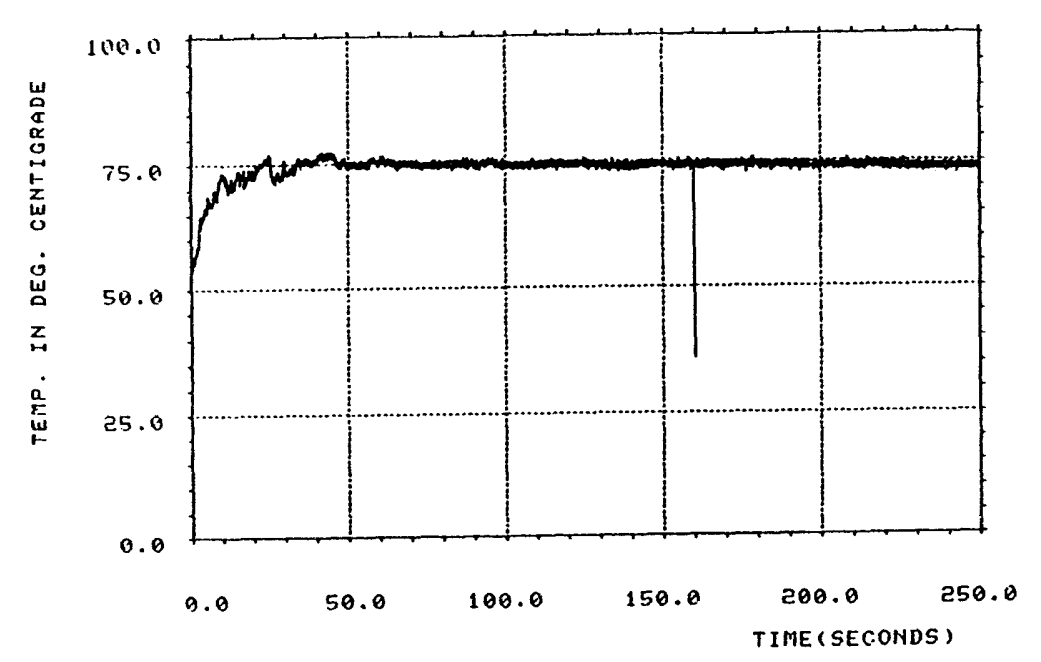

䀡

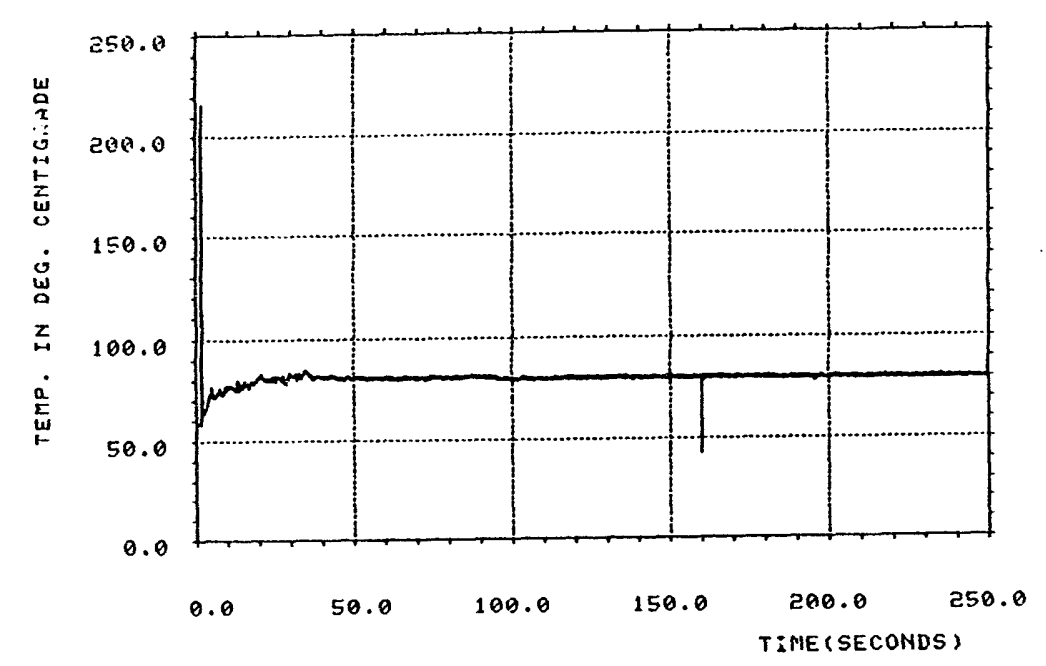

TC8-27, CCN-1, DATE 5/14/86

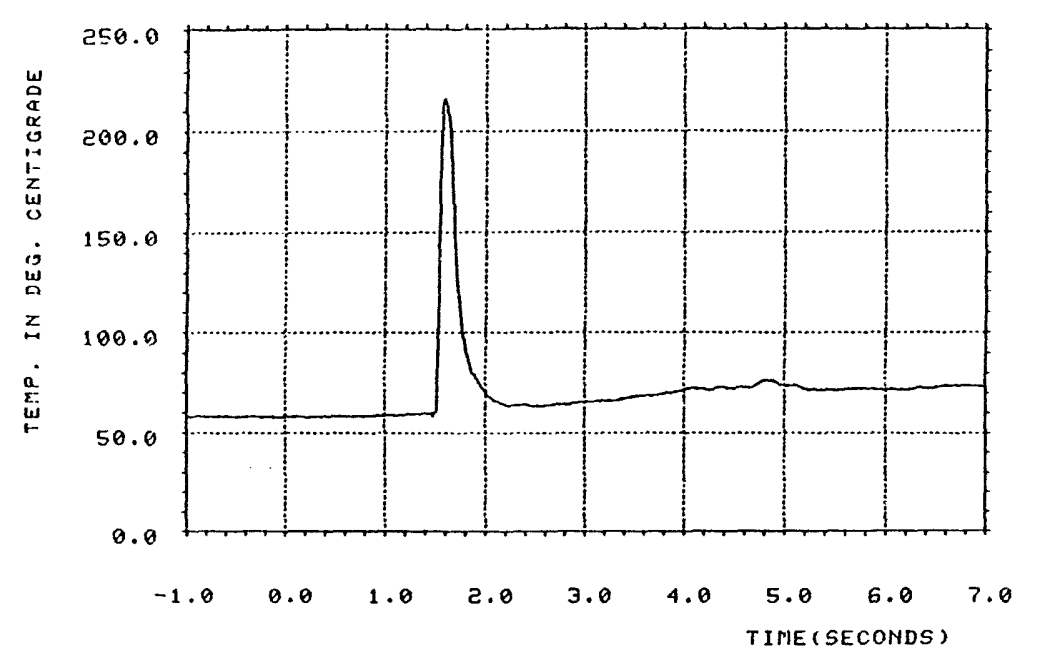

TC8-27, CCI1-1, DATE 5/14/86

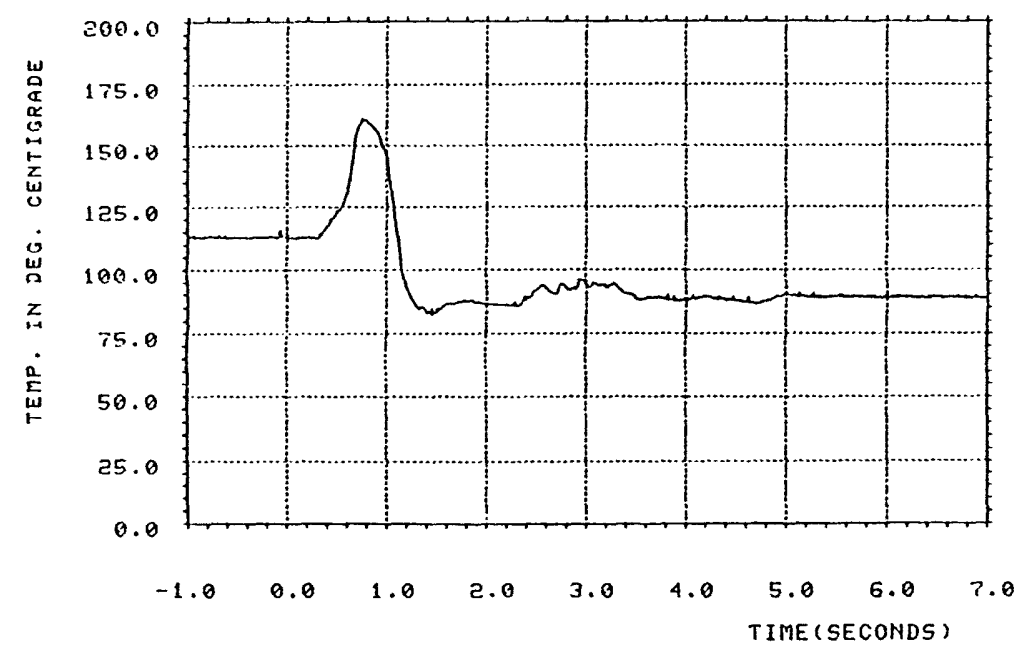

TC8-28, CCH-1, DATE 5/14/86 
嗢

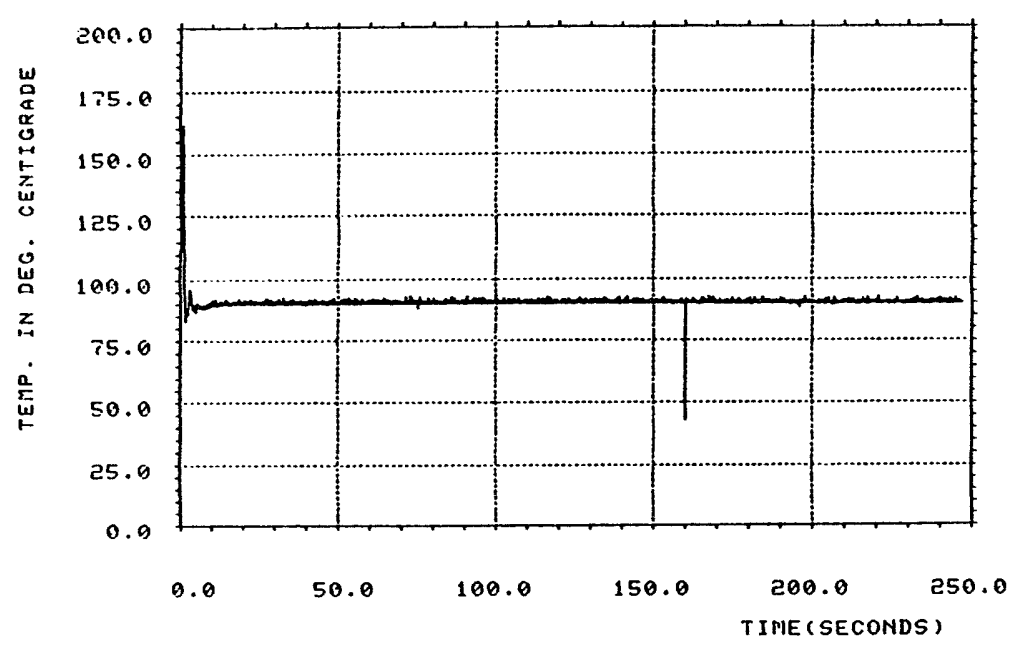

TC8-28. CCN-1, DATE 5,14/86

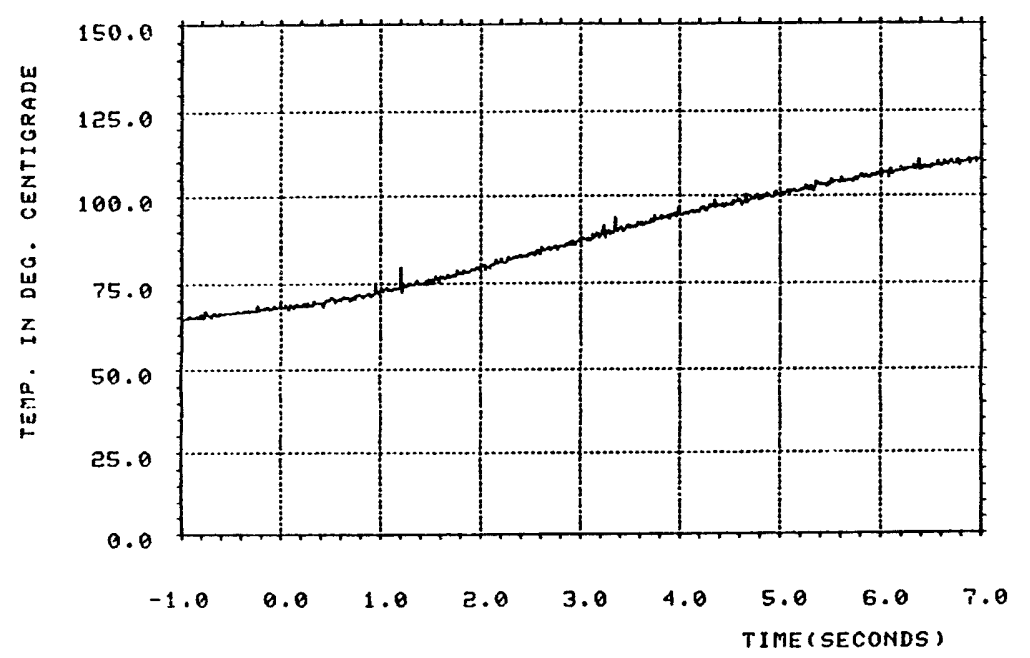

TCO-2, CON-1, DATE 5/14/86

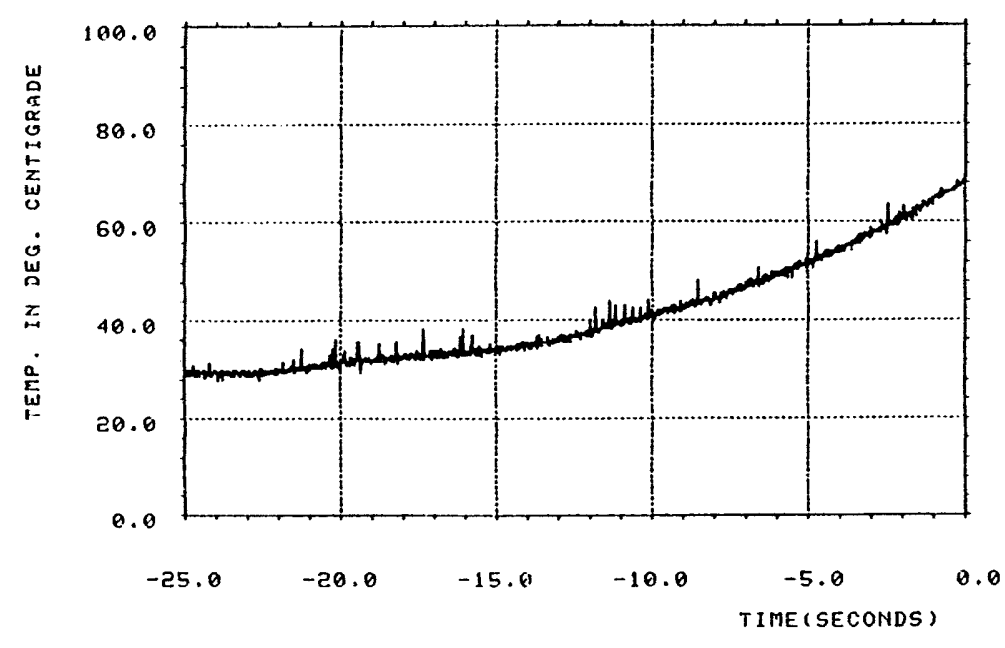

TCg-2, COH-1, DATE 5/14/86

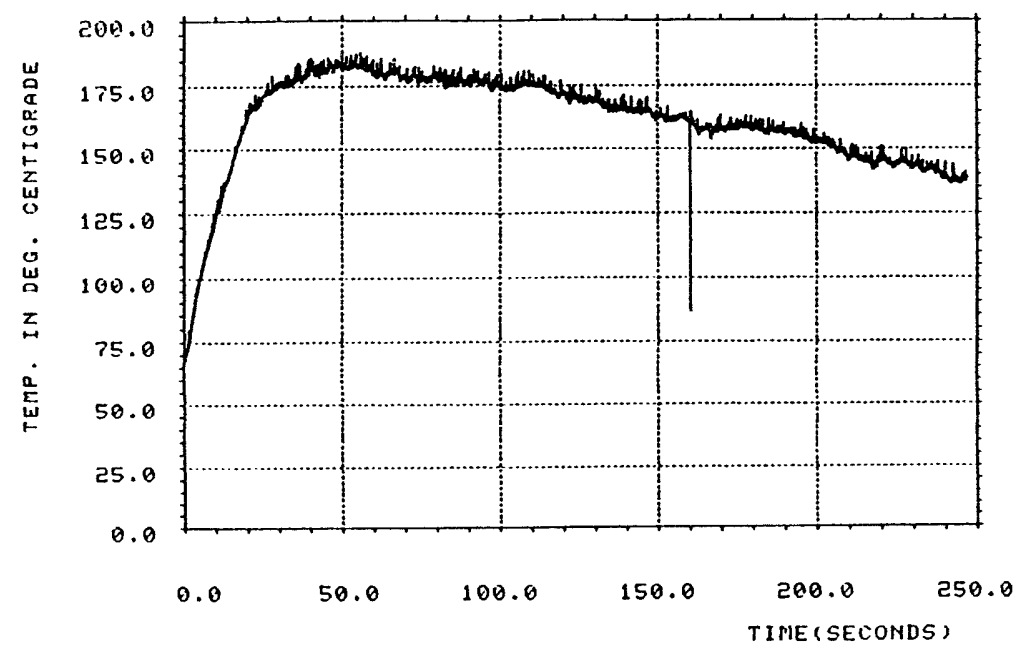

TC9-2, COH-1, DATE 5/14/86 


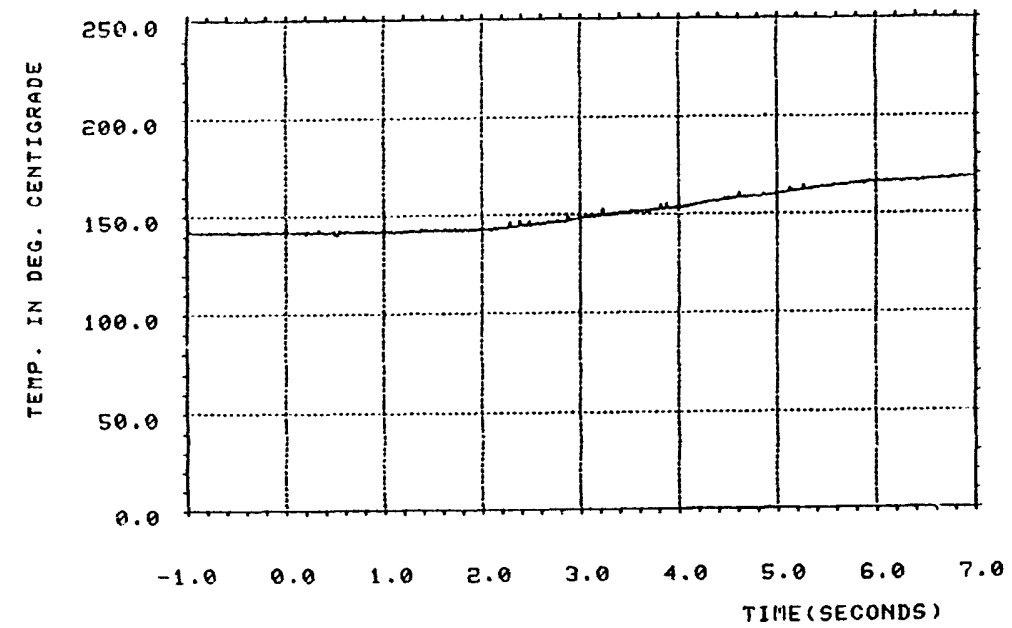

$\stackrel{1}{\sqcup}$

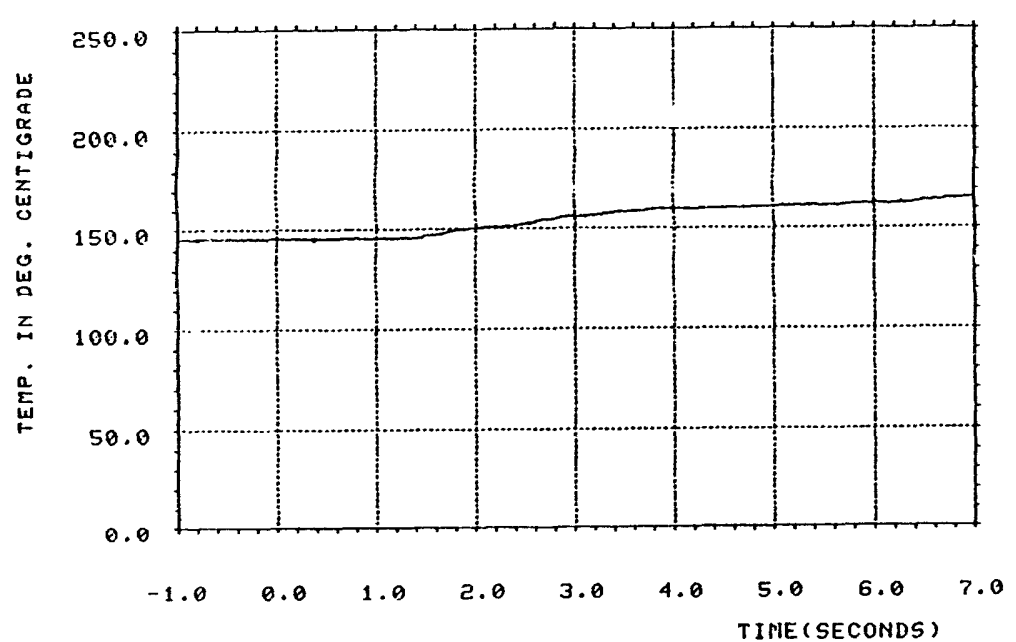

TC12-2, COM-1, DATE 5/14/86

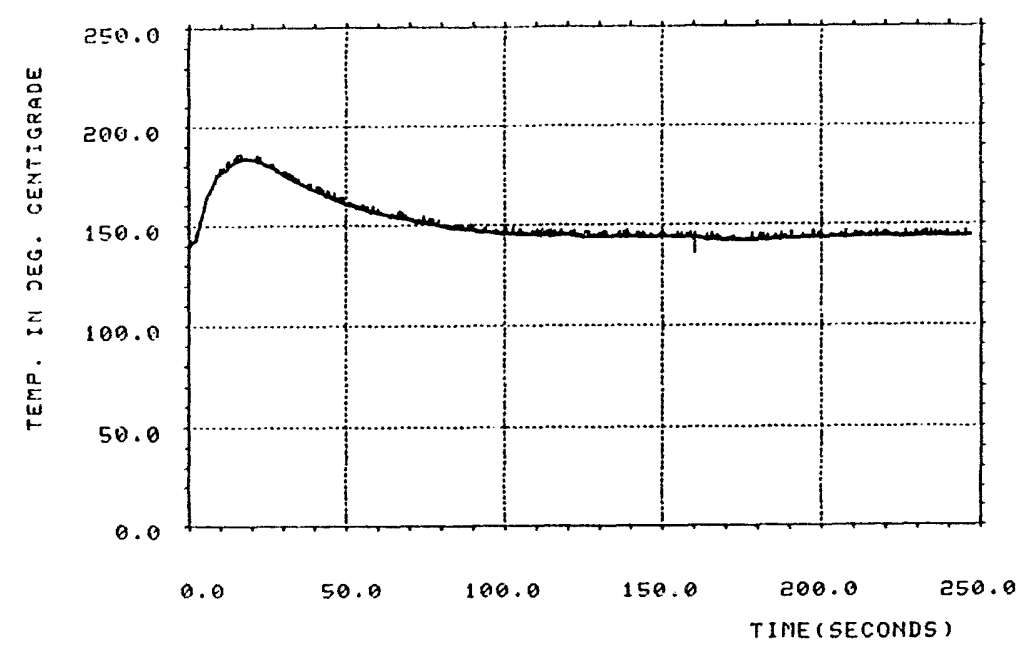

TE12-1. CON-1. DATE 5/14/86

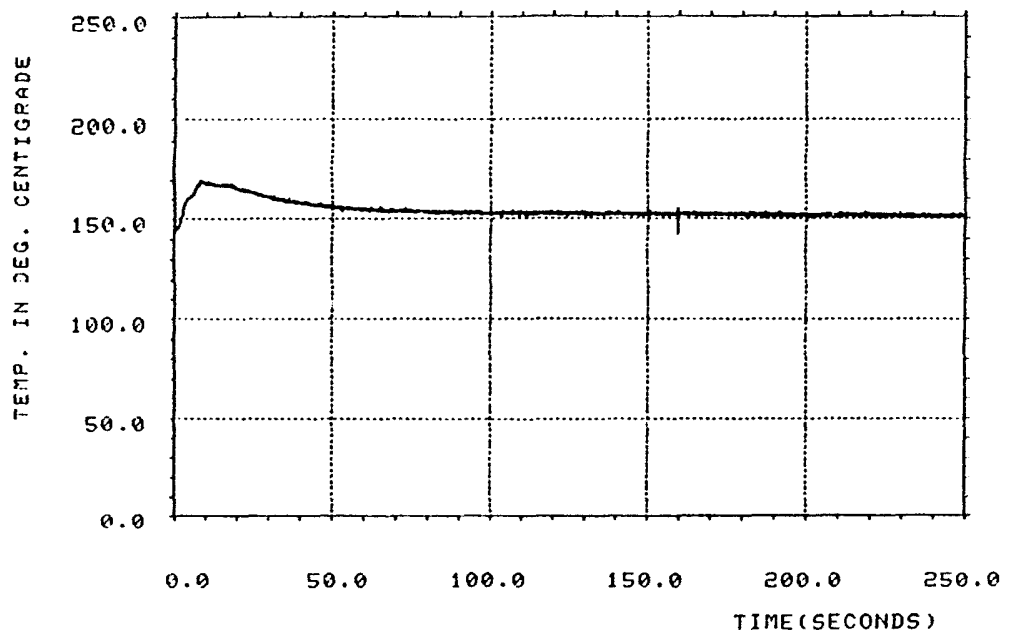

TC12-2, CCM-1, DATE 5/14/86 


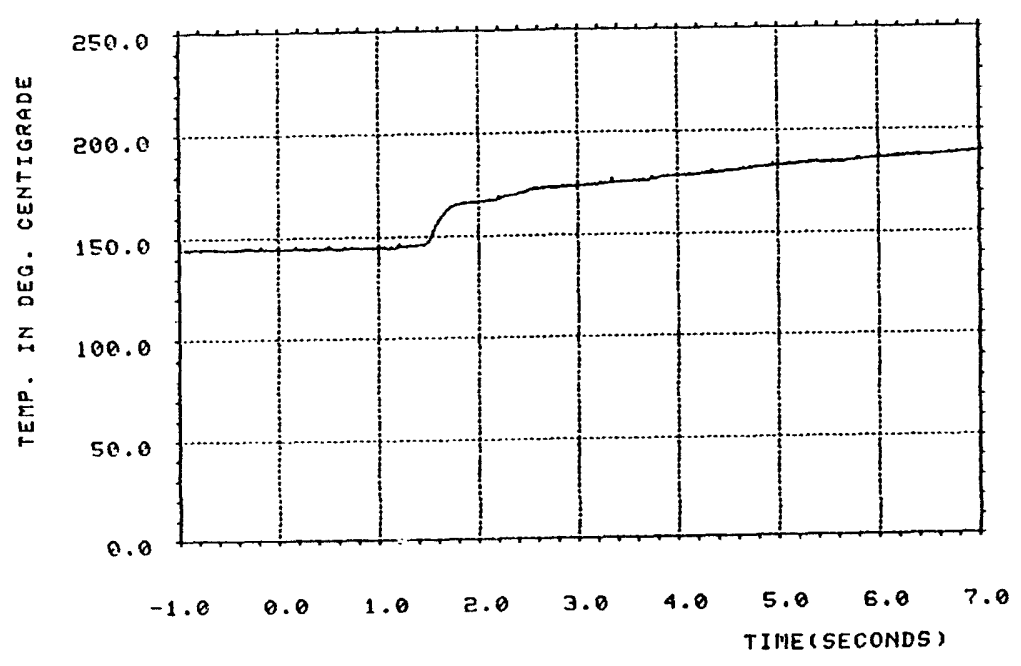

$\stackrel{\square}{\stackrel{1}{\infty}}$

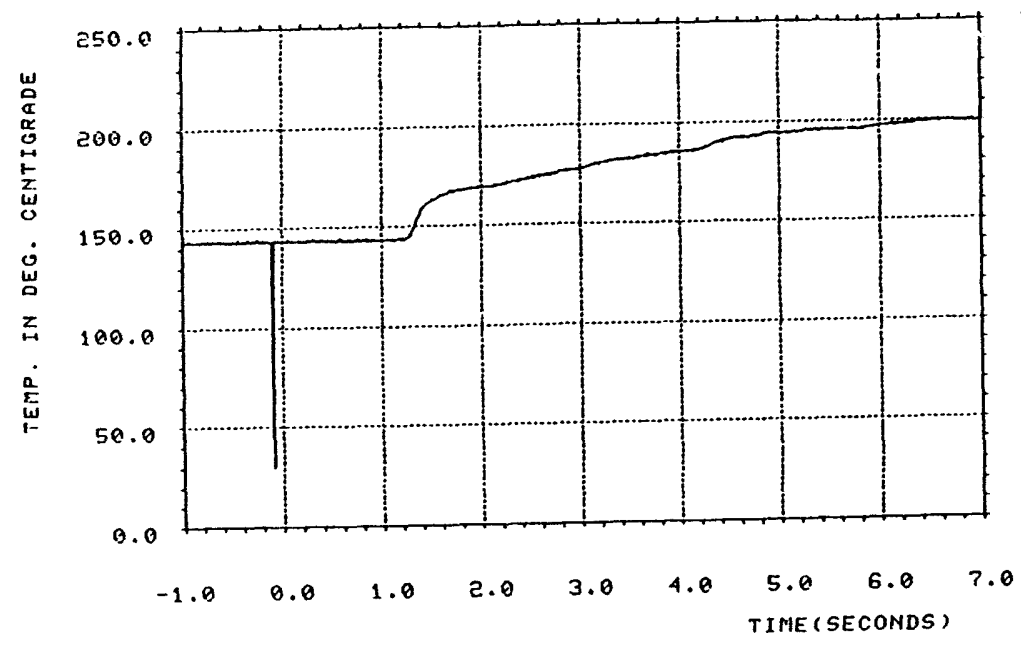

TC12-4, COM-1, DATE 5/14/86

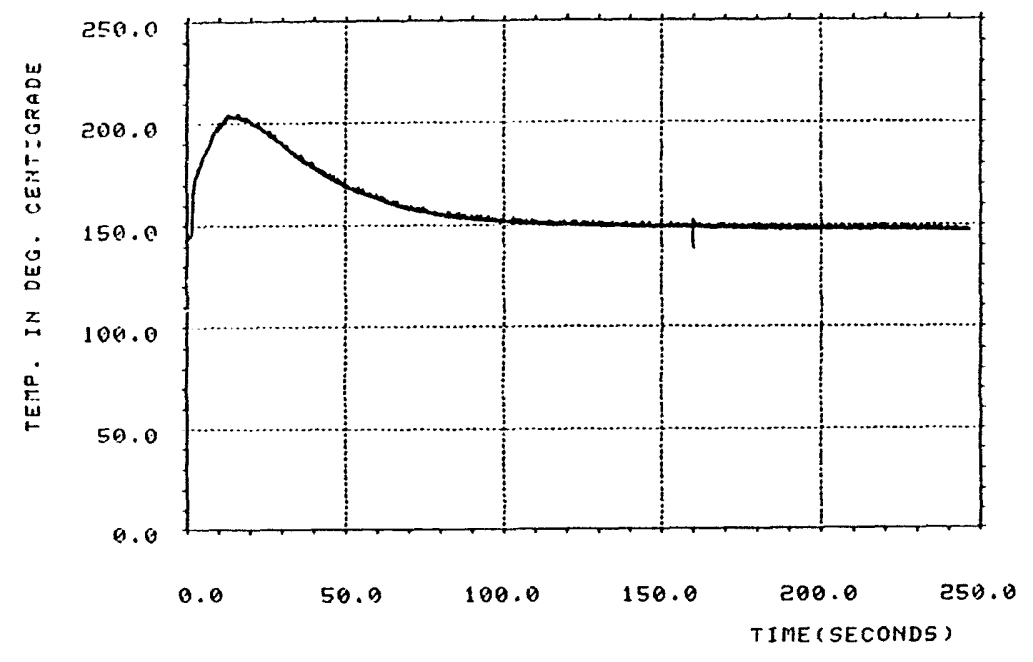

TC12-3, CCNI-1, DATE 5/14/86

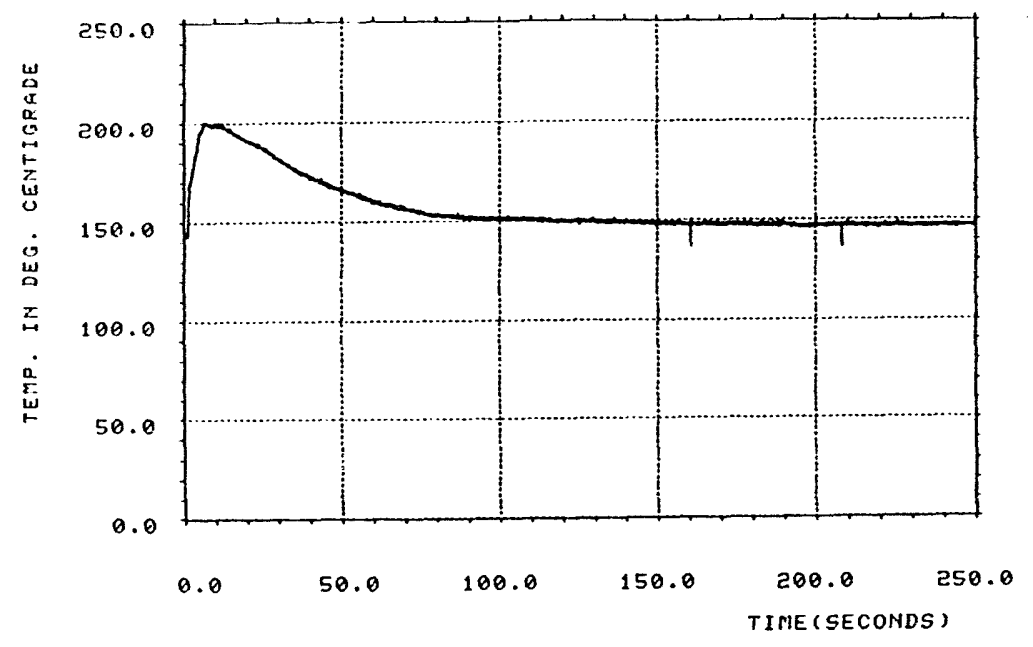

TC12-4, CDN-1, LIATE 5/14/86 


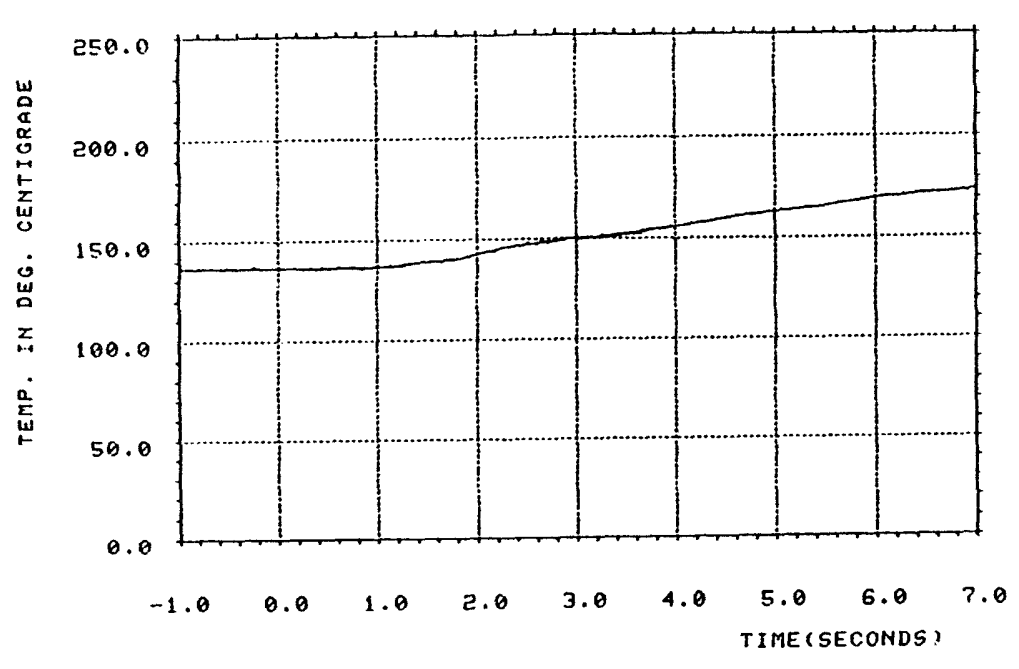

$\stackrel{p}{\dot{0}}$

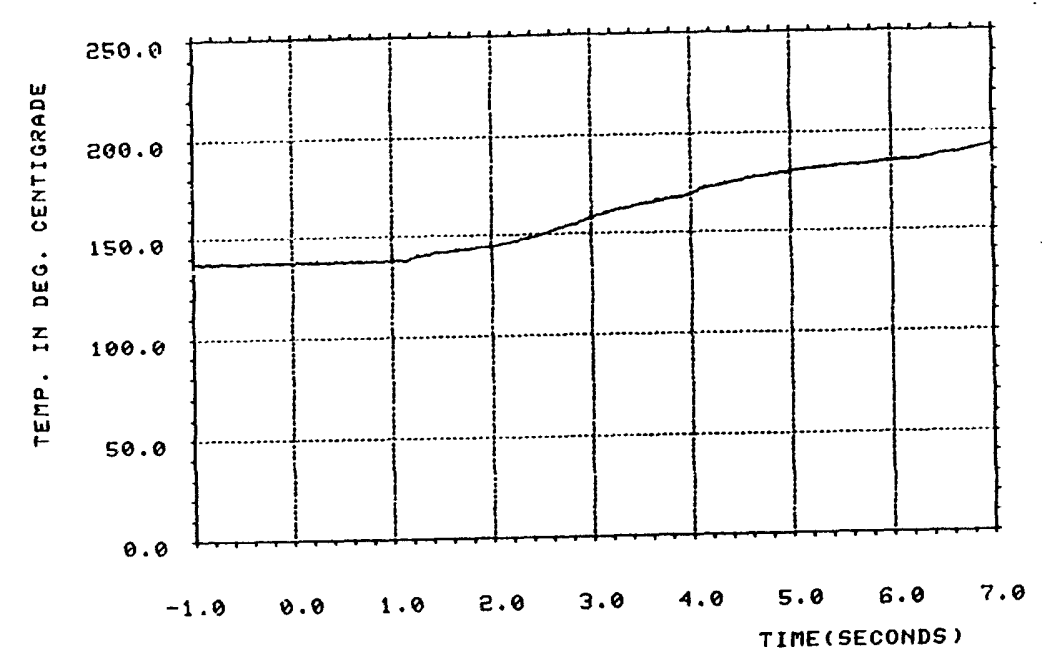

TC12-6. CCI1-1, DATE 5/14/86

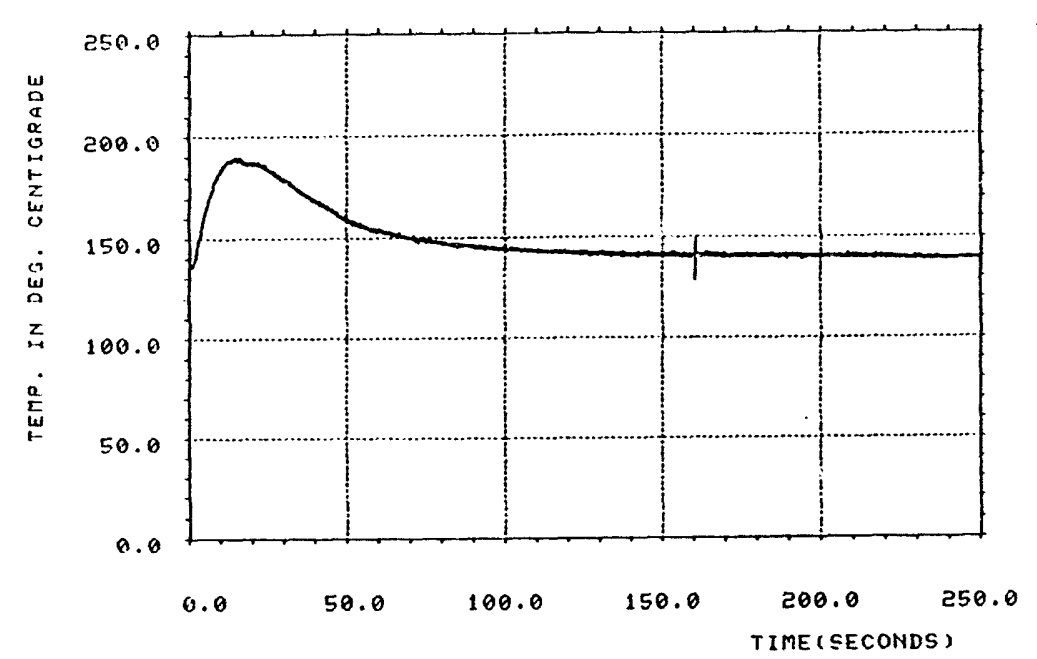

TC12-5, CCII-1, DATE 5/14/86

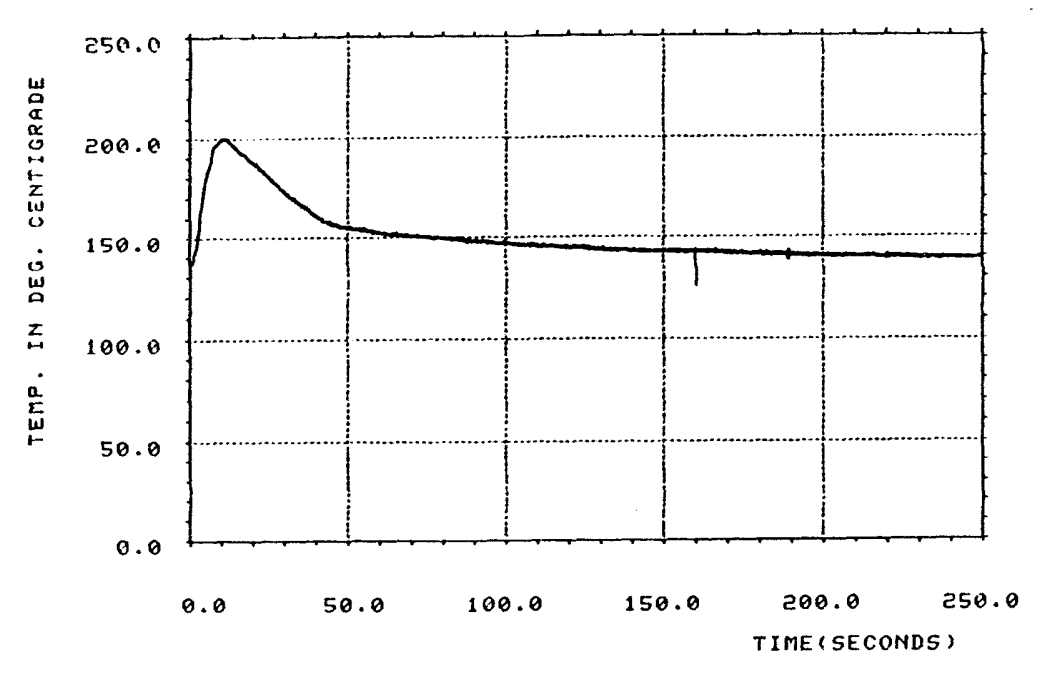

TC12-6. CCII-1, DATE 5/14/86 
APPENDIX C. DATA FOR CCM-2

\begin{abstract}
PTS-1: Thermite-Vesse1 Gas Pressure
PTS-2: Interaction-Vessel Gas Pressure

PTS-9: Pressure at Top of Expansion Vessel

PTS-10: Pressure at Lower Side of Expansion Vesse1

PTS-12: Pressure in Upper-top Section of the Interaction Vessel

PTS-13: Pressure in Lower-top Section of the Interaction Vessel

PTS-14: Pressure in Upper-top Section of the Interaction Vesse1

PTS-15: Pressure in Lower-top Section of the Interaction Vesse1

TC8-6: Temperature at Base of Interaction Vessel

TC8-7: Temperature at Base of Interaction Vessel

TC8-8: Temperature at Base of Interaction Vesse1

TC8-9: Temperature at Base of Interaction Vessel

TC8-10: Temperature Inside Expansion Vesse1 $34.9 \mathrm{~cm}$ above pipeway exit

TC8-12: Temperature of Gas Space in Interaction Vesse1

TC8-13: Temperature of Gas Space in Interaction Vessel

TC8-16: Temperature at Base of Interaction Vesse1

TC8-17: Temperature at Base of Interaction Vessel

TC8-18: Temperature at Base of Interaction Vessel

TC8-19: Temperature at Base of Interaction Vessel

TC8-20: Temperature in Upper-top Section of Interaction Vesse1

TC8-21: Temperature in Upper-top Section of Interaction Vessel

TC8-22: Temperature in Lower-top Section of Interaction Vessel

TC8-23: Temperature in Lower-top Section of Interaction Vessel

TC8-24: Temperature in Upper-middle Section of Interaction Vessel

TC8-25: Temperature in Upper-middle Section of Interaction Vessel

TC8-26: Temperature in Lower-middle Section of Interaction Vessel

TC8-27: Temperature in Upper-middle Section of Interaction Vessel

TC8-28: Temperature in Discharge Pipeway U-bend

TC9-2: Temperature in Thermite-Vessel Gas Space

TC12-1: Temperature Inside Expansion Vesse1 $282.58 \mathrm{~cm}$ Above Pipeway Exj.t

TC12-2: Temperature Inside Expansion Vessel $241.94 \mathrm{~cm}$ Above Pipeway Exit

TC12-3: Temp ature Inside Expansion Vessel $201.30 \mathrm{~cm}$ Above Pipeway Exit

TC12-4: Temperature Inside Expansion Vesse1 $160.66 \mathrm{~cm}$ Above Pipeway Exit

TC12-5: Temperature Inside Expansion Vessel $120.02 \mathrm{~cm}$ Above Pipeway Exit
\end{abstract}


APPENDIX C. DATA FOR CCM-2 (Cont'd.)

TC12-6: Temperature Inside Expansion Vesse1 $79.38 \mathrm{~cm}$ Above Pipeway Exit LP-1: Water Level in Interaction Vessel $12.37 \mathrm{~cm}$ Elevation

LP-2: Water Level in Interaction Vesse1 $13.00 \mathrm{~cm}$ Elevation

LP-3: Water Level in Interaction Vessel $14.27 \mathrm{~cm}$ Elevation

LP-4: Water Level in Interaciion Vessel $15.54 \mathrm{~cm}$ Elevation

LP-5: Water Level in Interaction Vessel $18.08 \mathrm{~cm}$ Elevation 


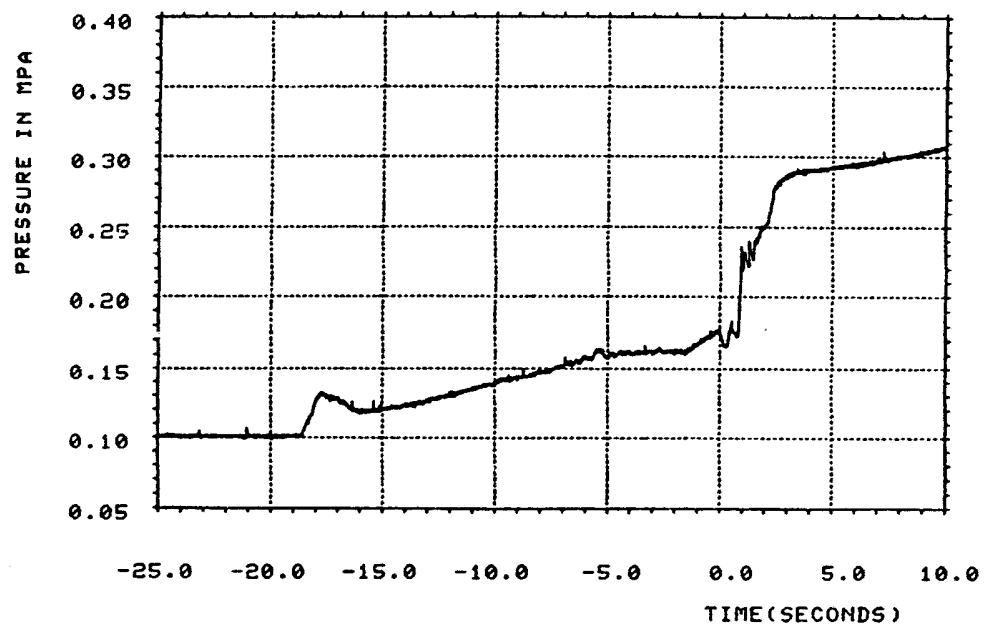

PTS-1, CCM-2, DATE $7 / 17 / 86$

$\stackrel{1}{\omega}$

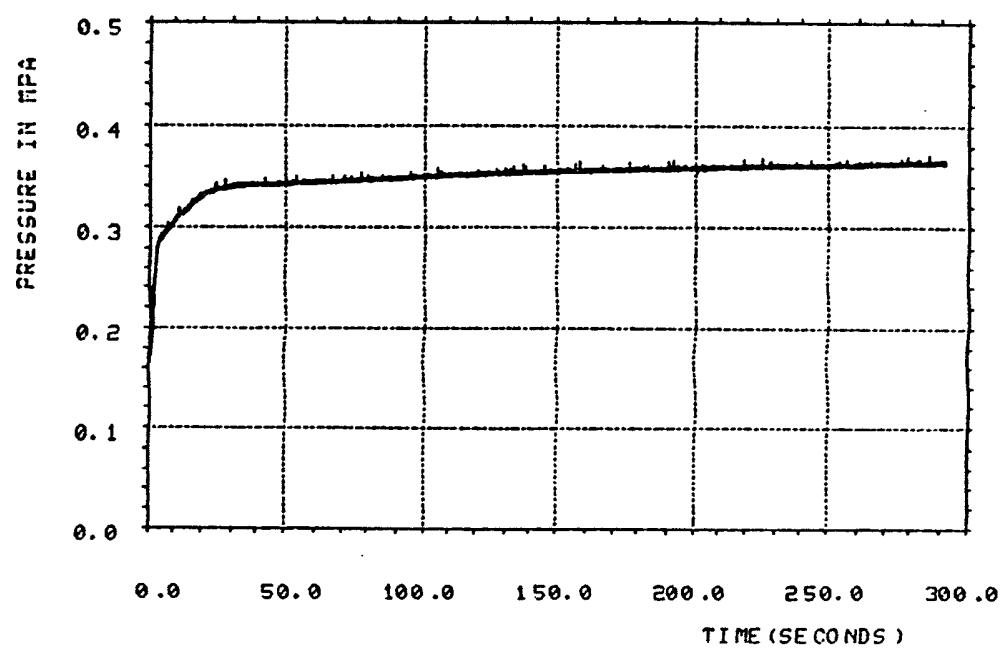

PTS-1, CCID-2, DHTE ?/17/86

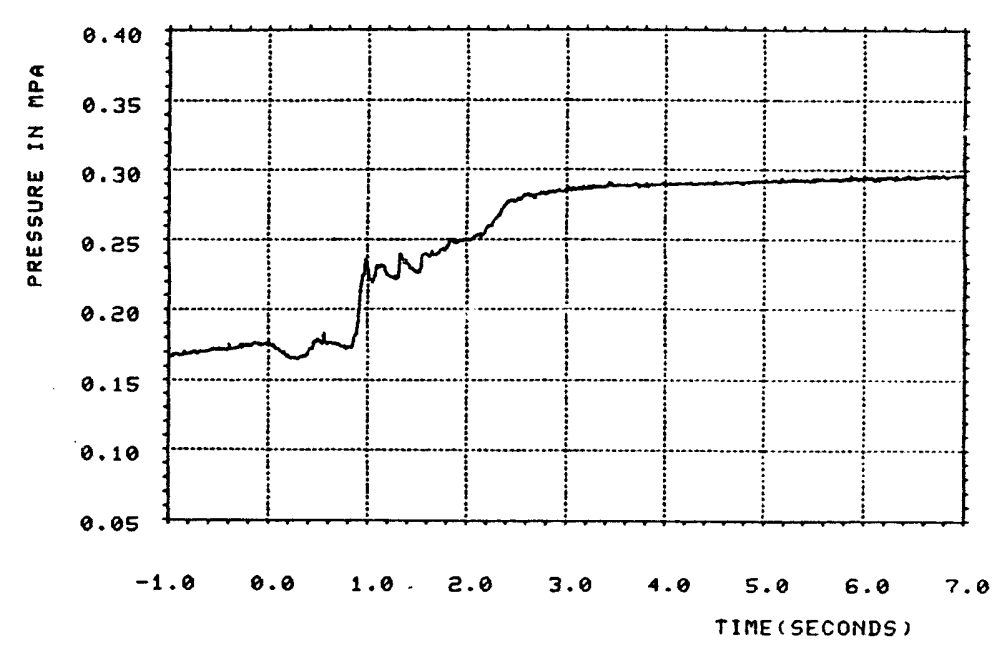

PTS-1, CCI-2, DATE $7 / 17 / 86$

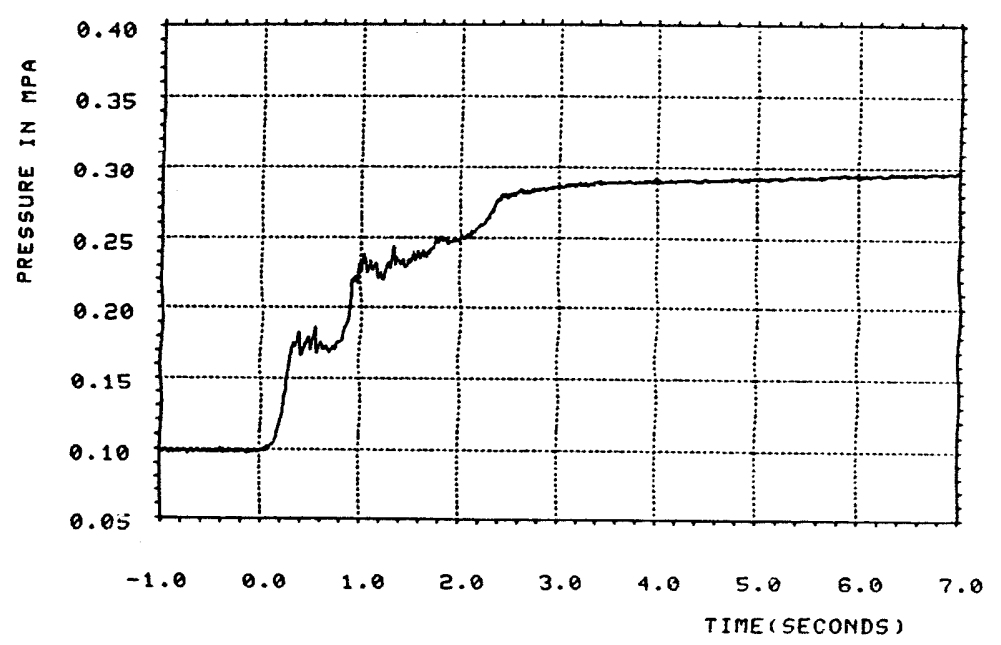

PTS-2, CCM-2, DATE $7 / 17 / 86$ 


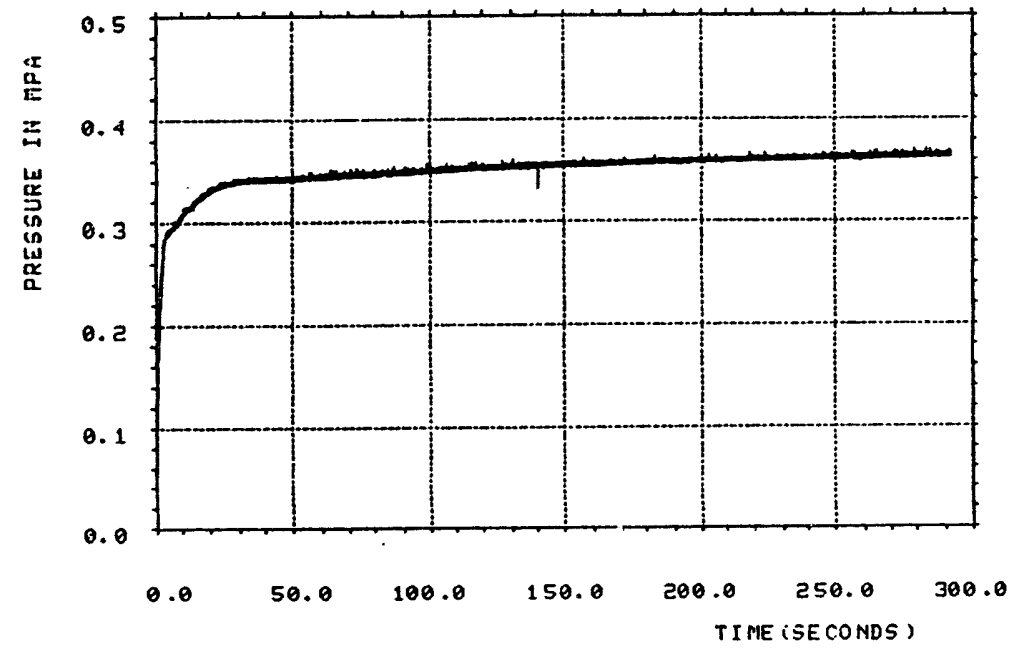

PTS-2, CCM-2, DATE $\geqslant 17 / 36$

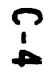

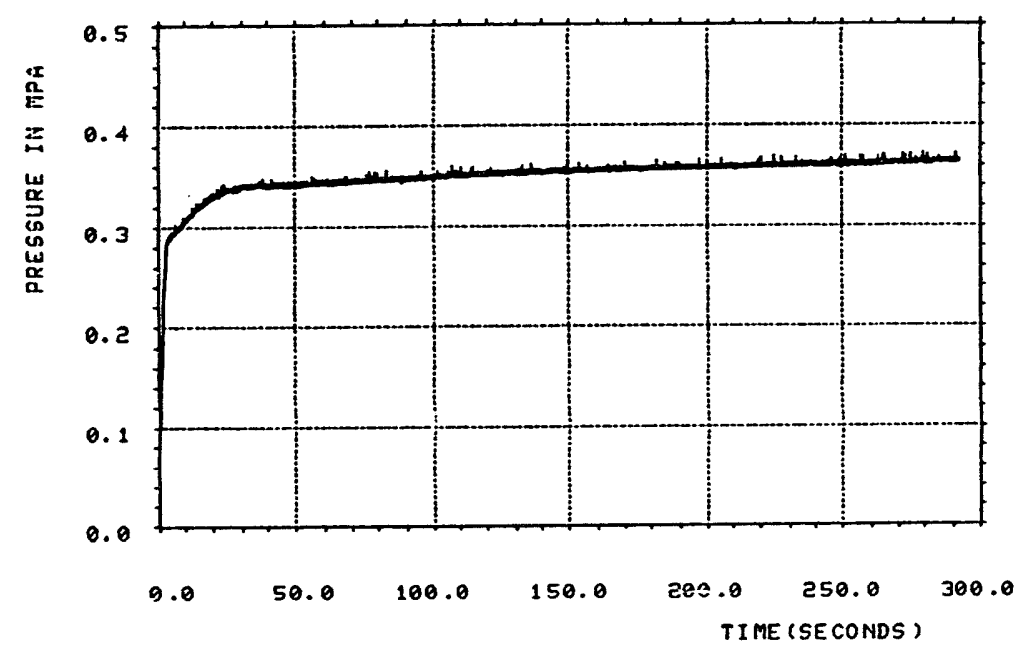

PTS-0, CCM-2, DATE $\because / 17 / 36$

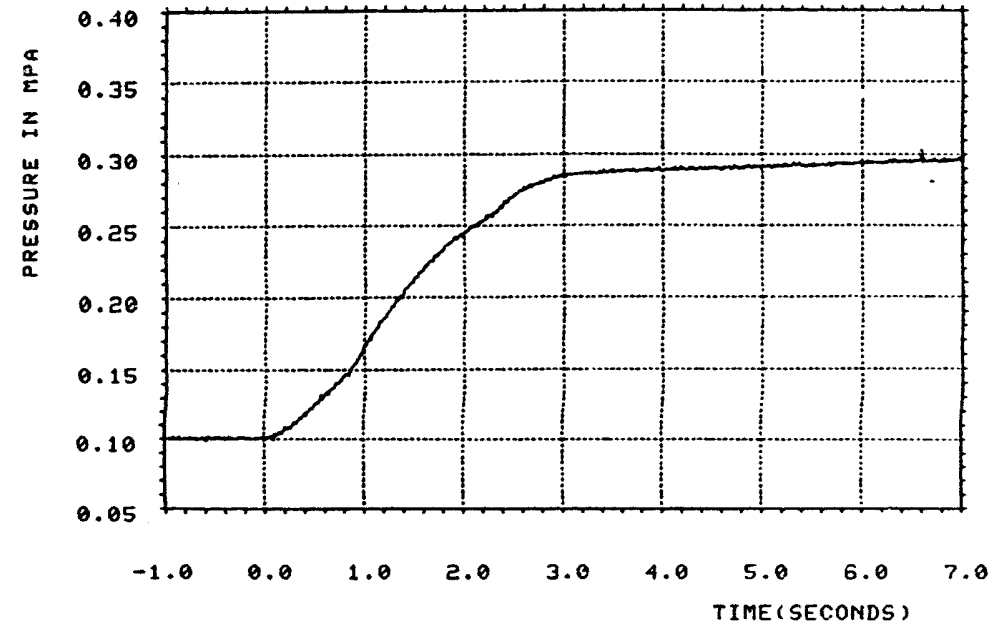

PTS-9, CCM-2, DATE 7:17:86

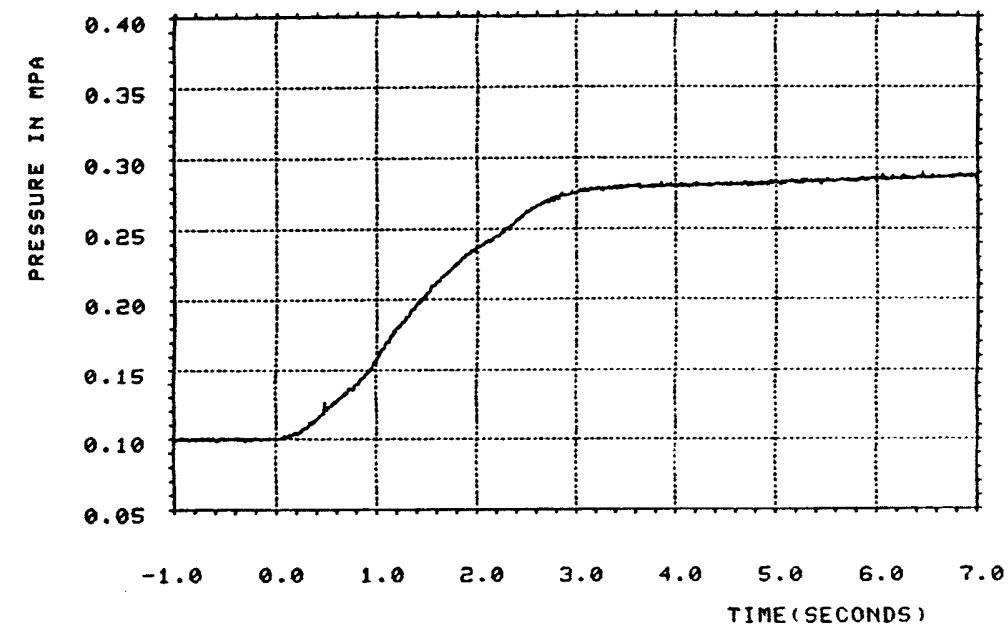

PTS-10, C.M-2, [IATE $3 / 17.86$ 


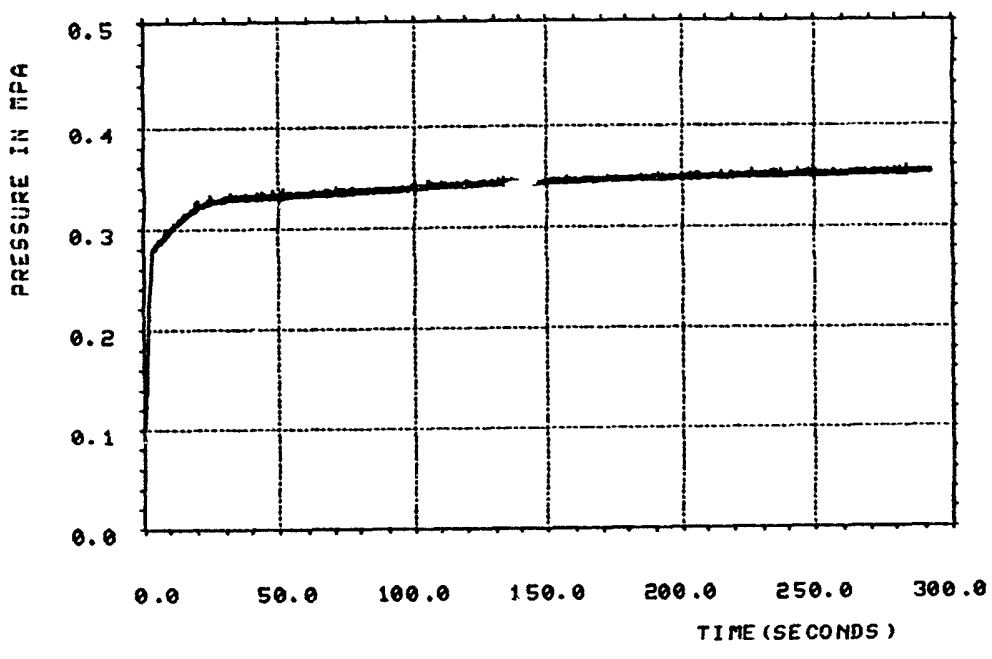

PTS-10, CCM-2, DATE $7 / 17 / 86$

in

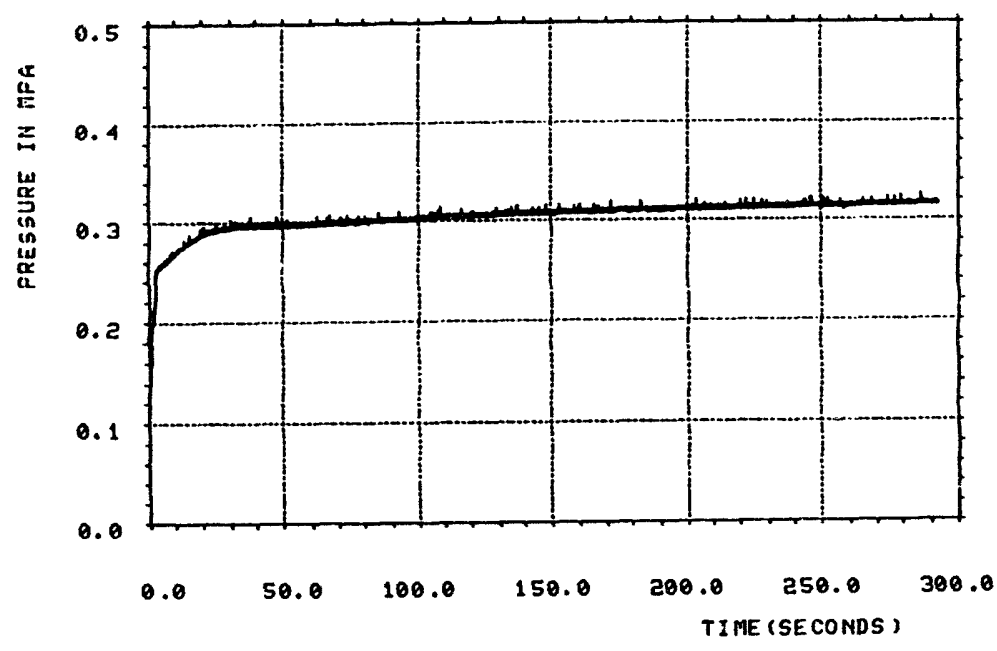

PTS-12, CCM-2, DATE $7 / 17 / 86$

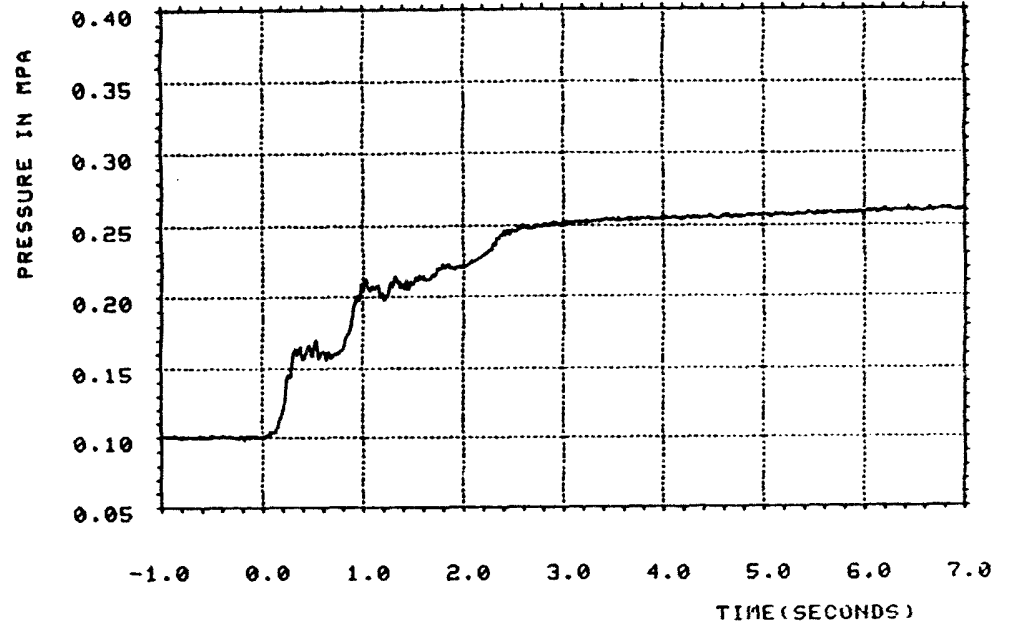

PTS-12, CCIM-2, DATE $7 / 17 / 86$

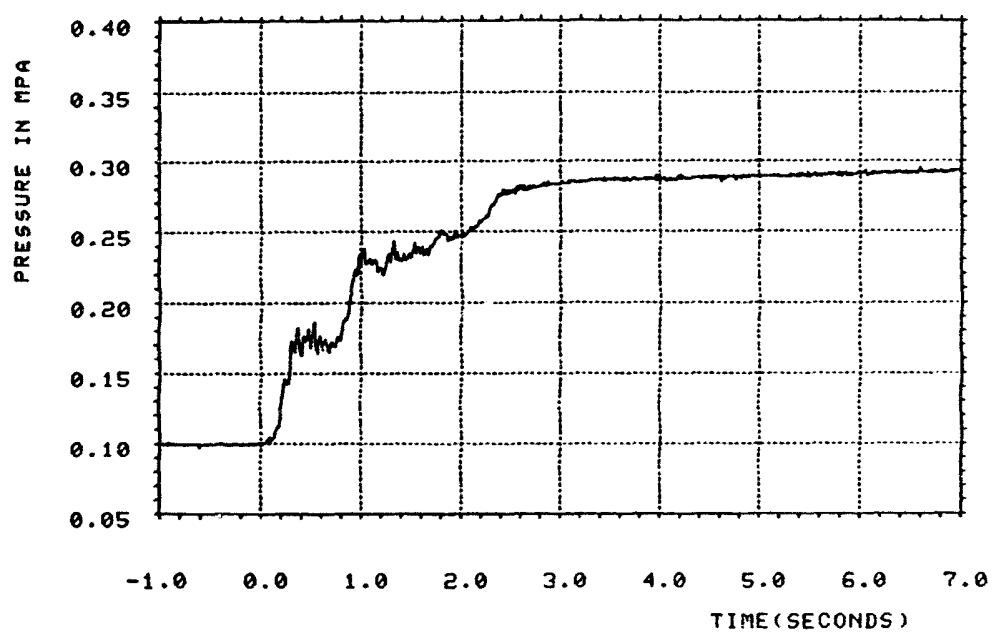

PTS-13, CCM-2, DATE $2 / 17 / 86$ 


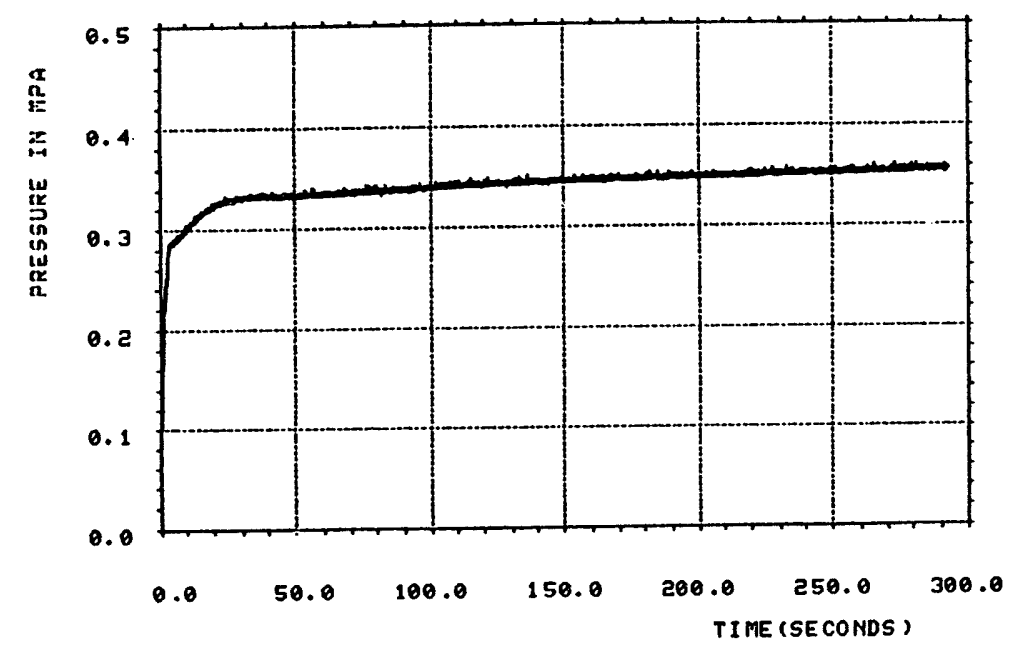

PTS -13, CCM-2, DATE $7 / 17 / 86$

ถู

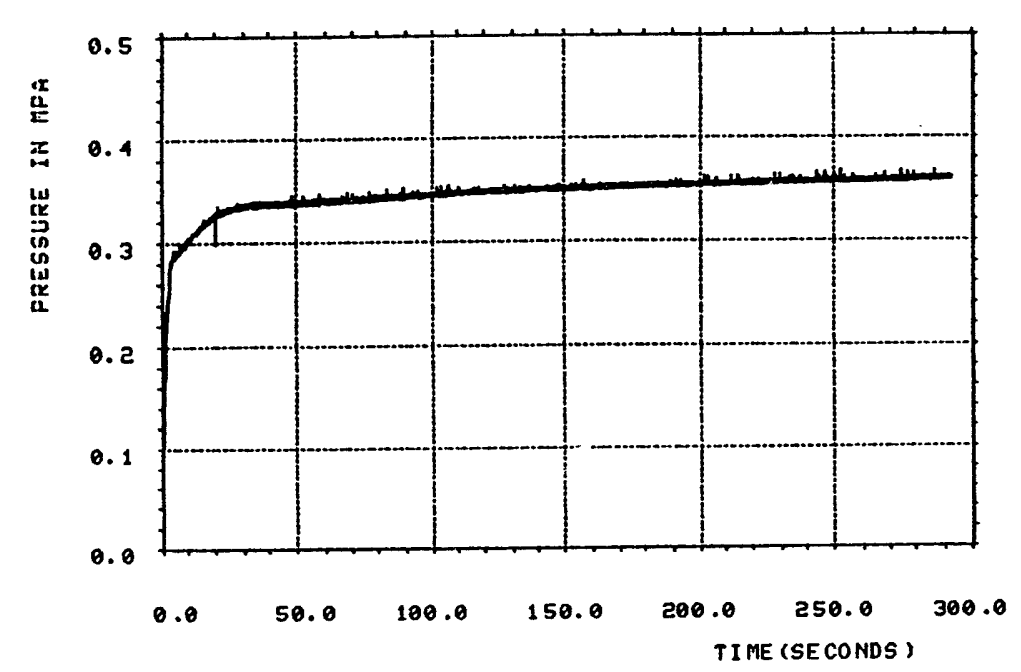

PTS-14, CCM-2, DATE $7 / 17 / 86$

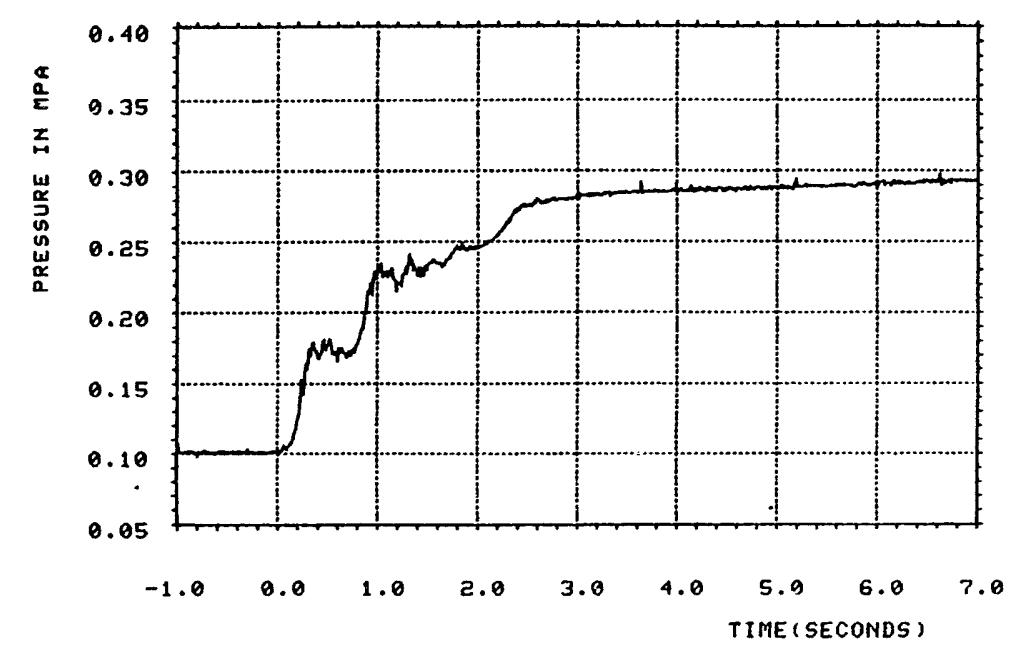

PTS-14, SCM-2, DATE $7 / 17 / 86$

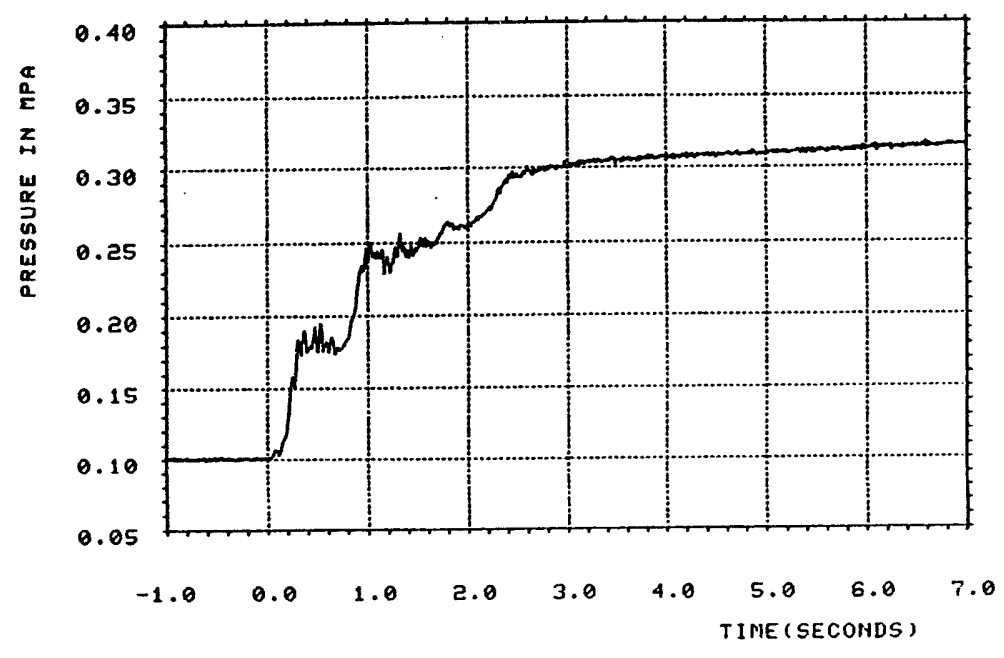

PTS-15, CCII-2, DATE $3: 17 / 86$ 
(SANOOJS) 3HI

$\begin{array}{lllllllll}0.2 & 0.9 & 0.2 & 0.0 & 0 . E & 0.5 & 0.1 & 0.0 & 0.1-\end{array}$

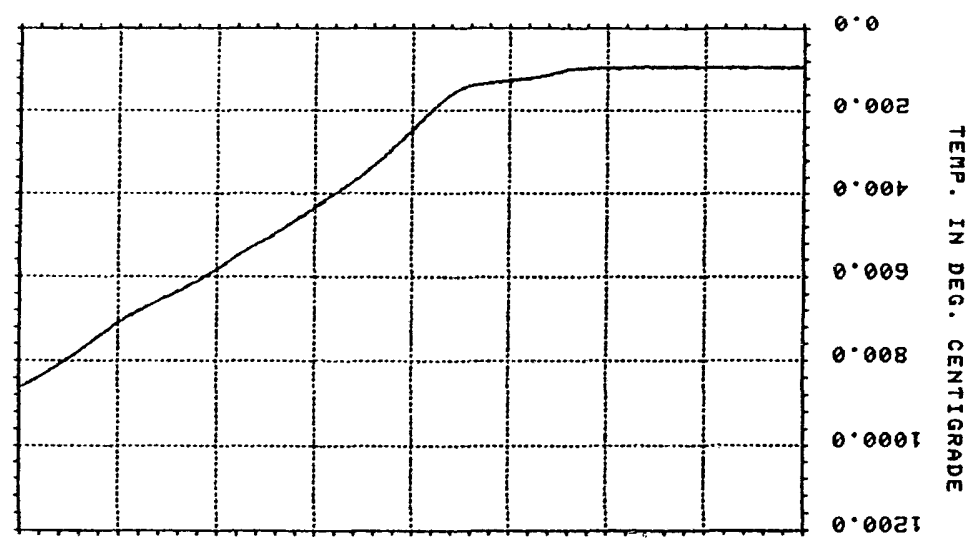

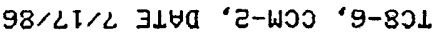

( 50 NO0 35) 3WI $\perp$
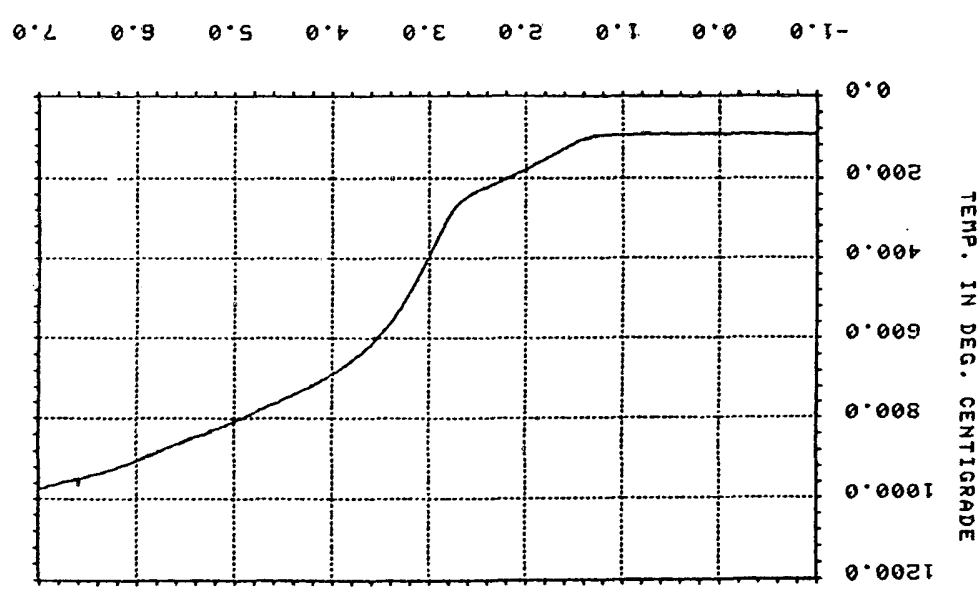

( SANOJ 3S ) 3N II

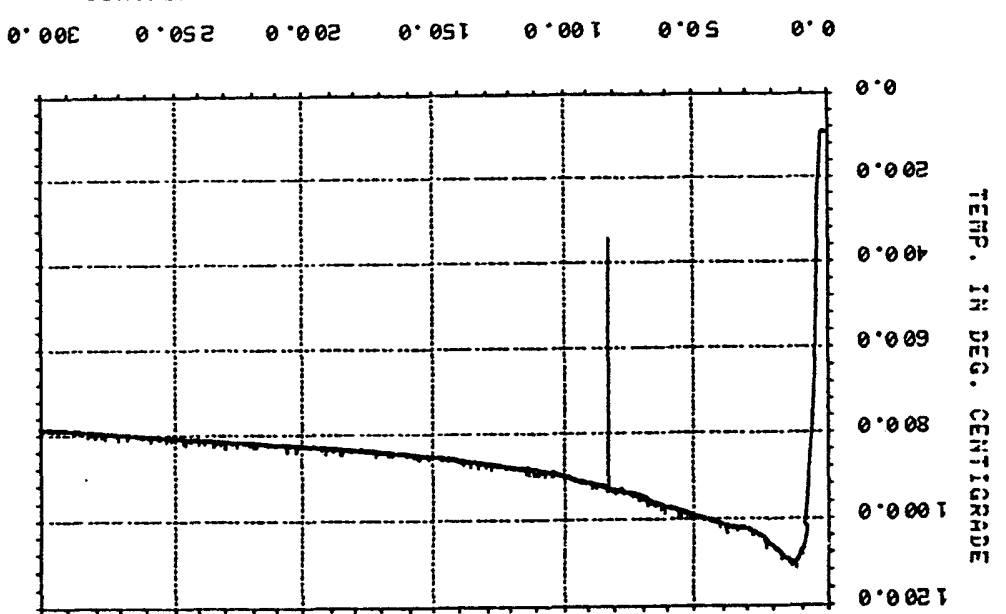

( SANOJ JS) JWIL

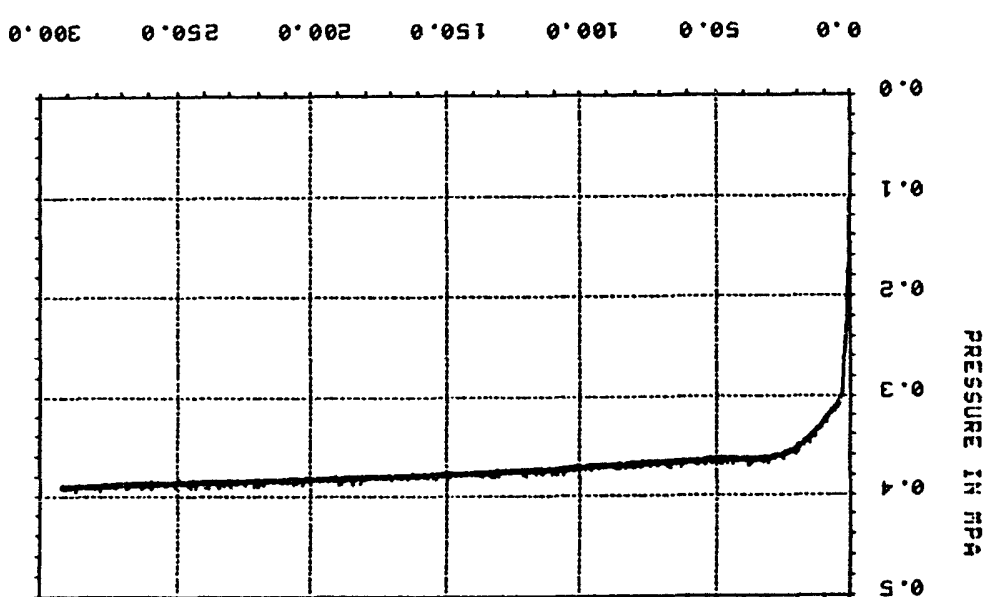


$98 / 2 I / 2 \quad 31 \forall 0 \cdot 2-w 00 \cdot 6-801$

( SONOJ 35 ) 3 II

0.00E o.0sz 0.00z 0.0St 0.001 0.0s 0.0

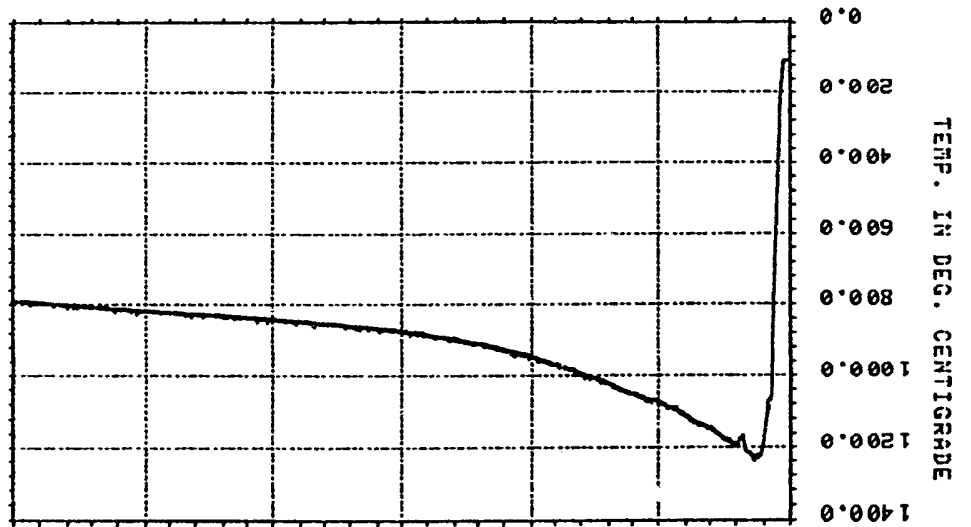

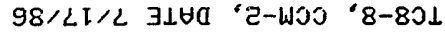

(SONOJJS) JWII

$\begin{array}{lllllllll}0.2 & 0.2 & 0.2 & 0.6 & 0 . \varepsilon & 0.5 & 0.1 & 0.0 & 0.1-\end{array}$

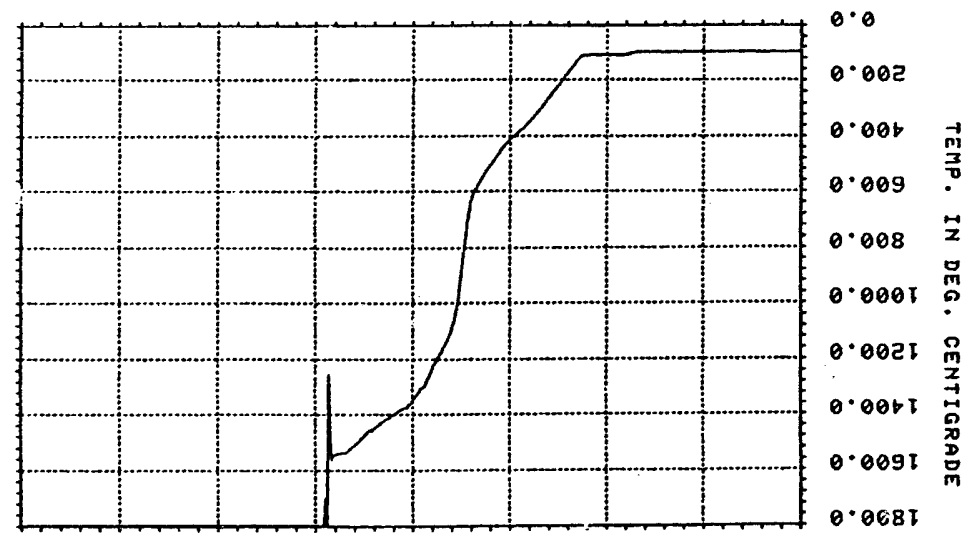

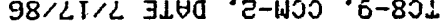

(sanOJ3S) 3WI 1

$\begin{array}{lllllllll}0.2 & 0.9 & 0.5 & 0.6 & 0 . \varepsilon & 0.2 & 0.1 & 0.0 & 0.1-\end{array}$

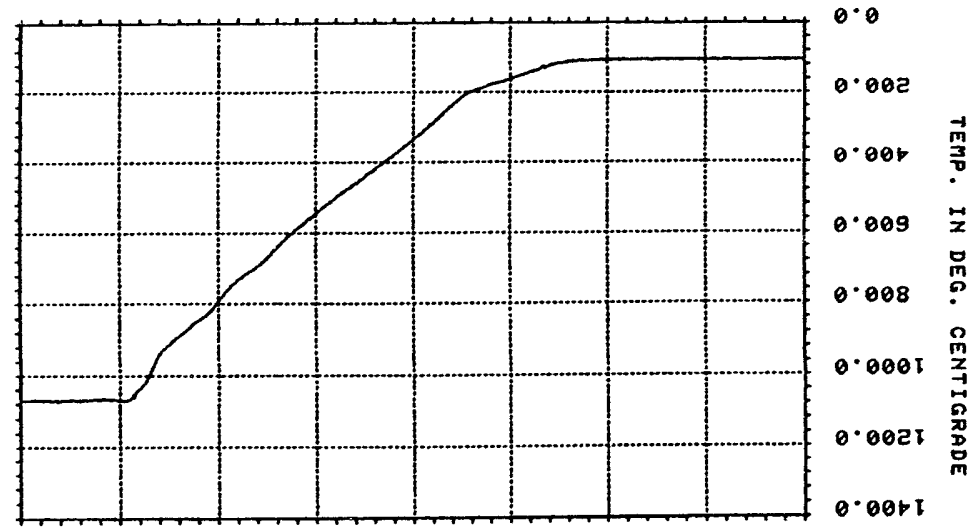

$\dot{1}$

( SANOJ 3S ) 3WIL

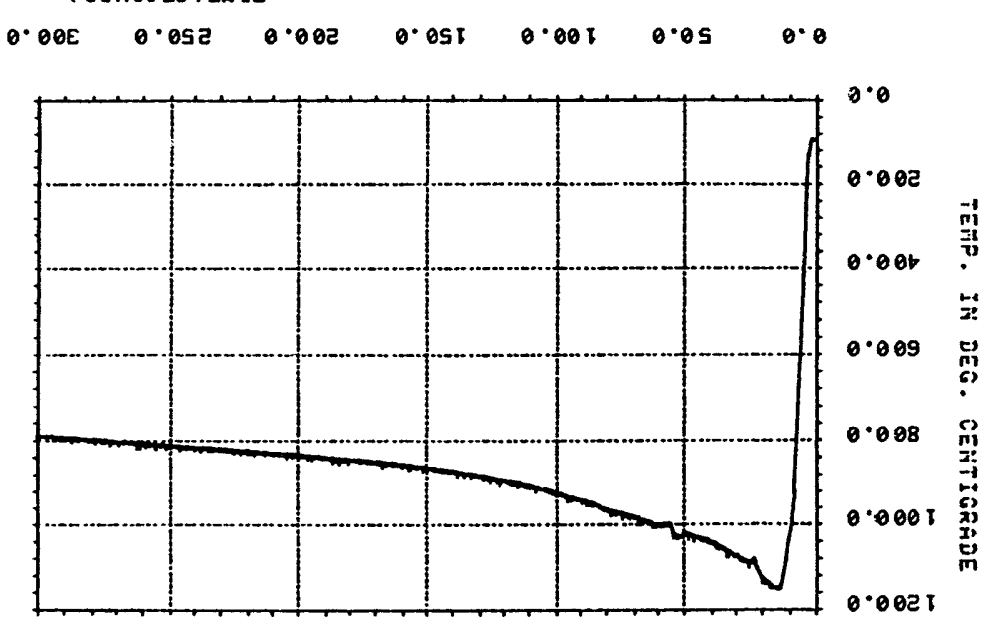




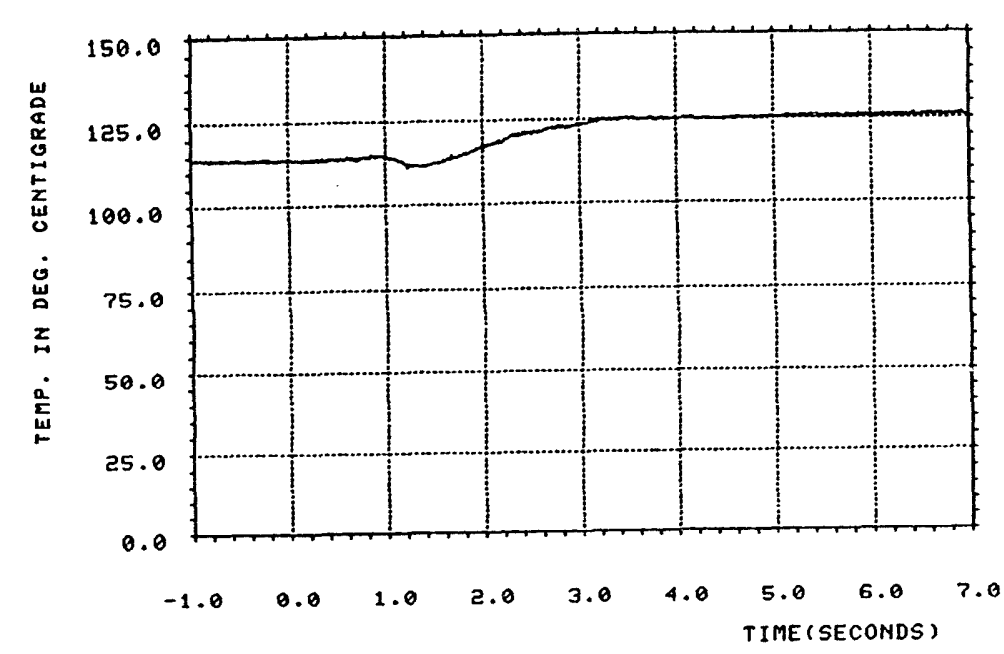

TC8-10, CCM-2, DATE $7 / 17 / 86$

\&

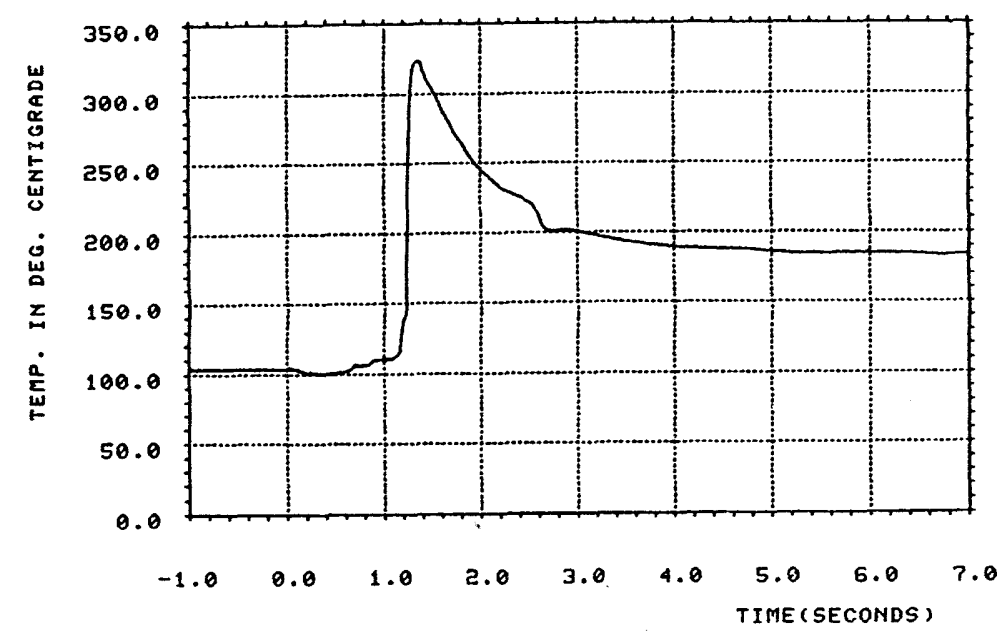

TC8-12, CCM-2, DATE $7 / 17 / 86$

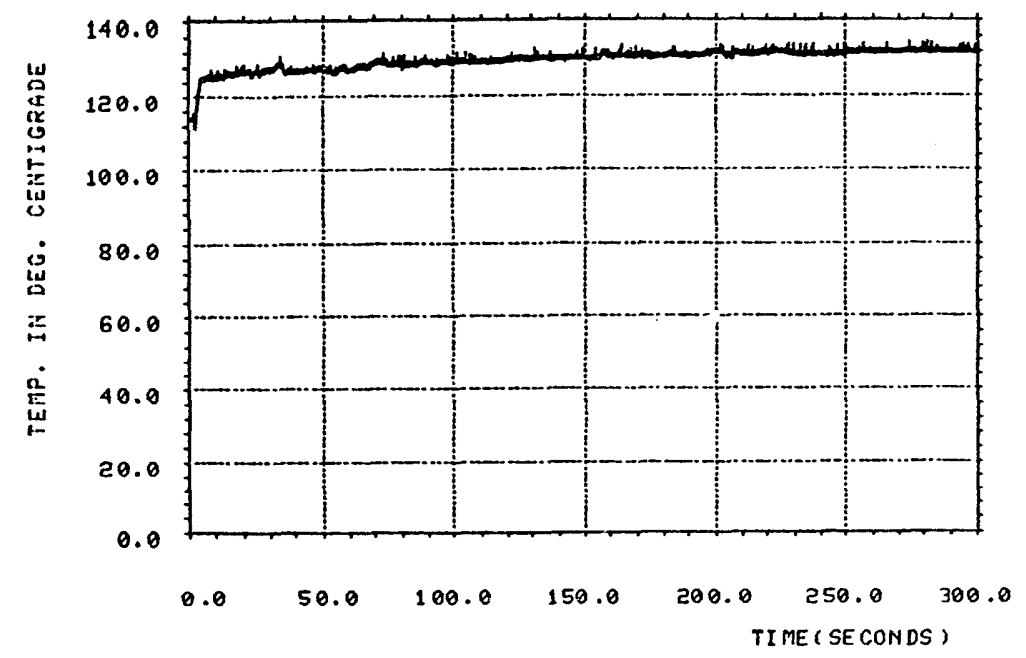

TC8-19, CCM-2, DATE $7 / 17 / 86$

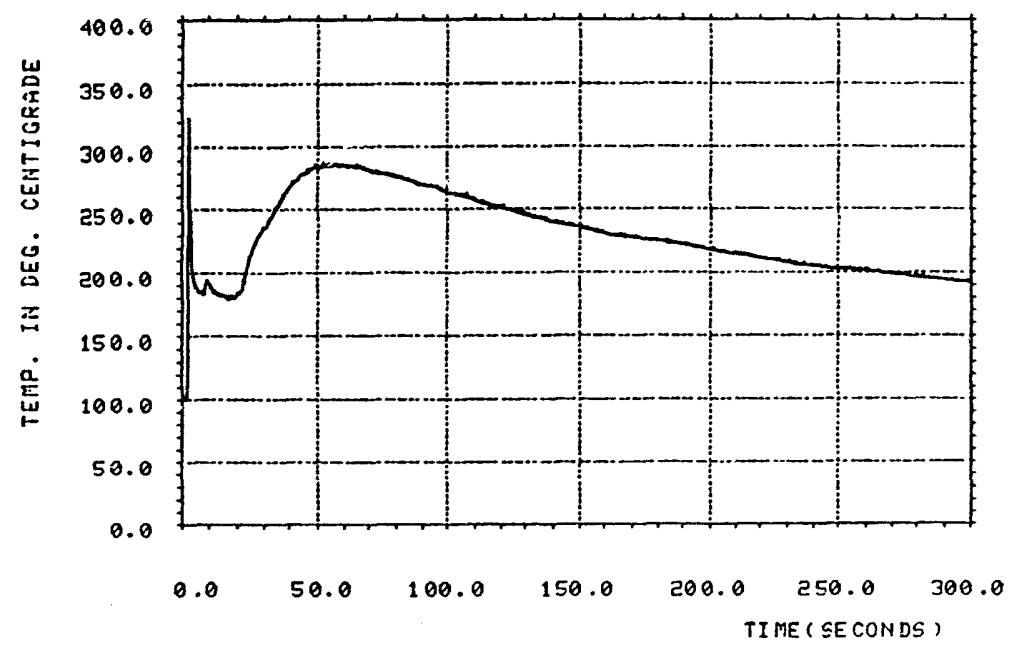

TC3-12, CCM-2, DATE $2 / 13,86$ 


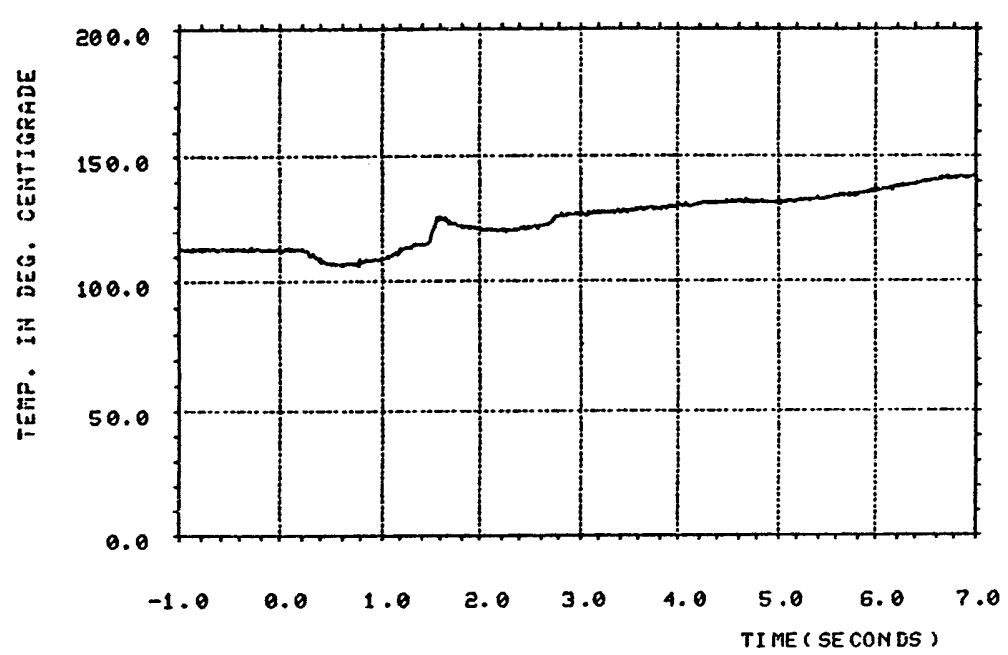

TC8-13, CCM-2, DATE $7 / 17 / 86$

$\dot{1}$

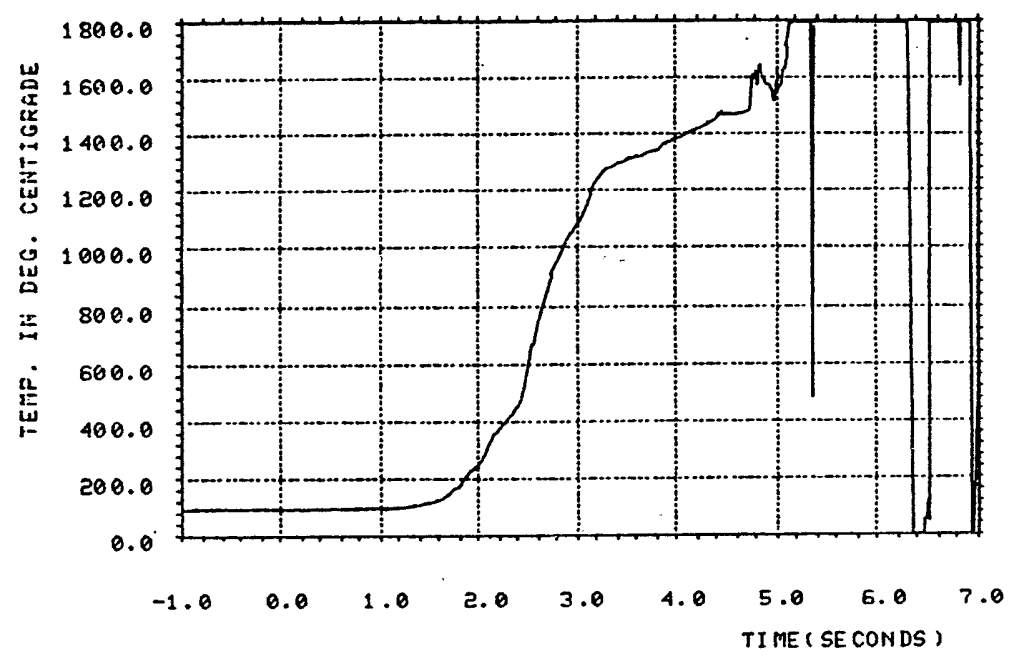

TC\&-16, CCM-2, DATE $2 / 17 / 86$

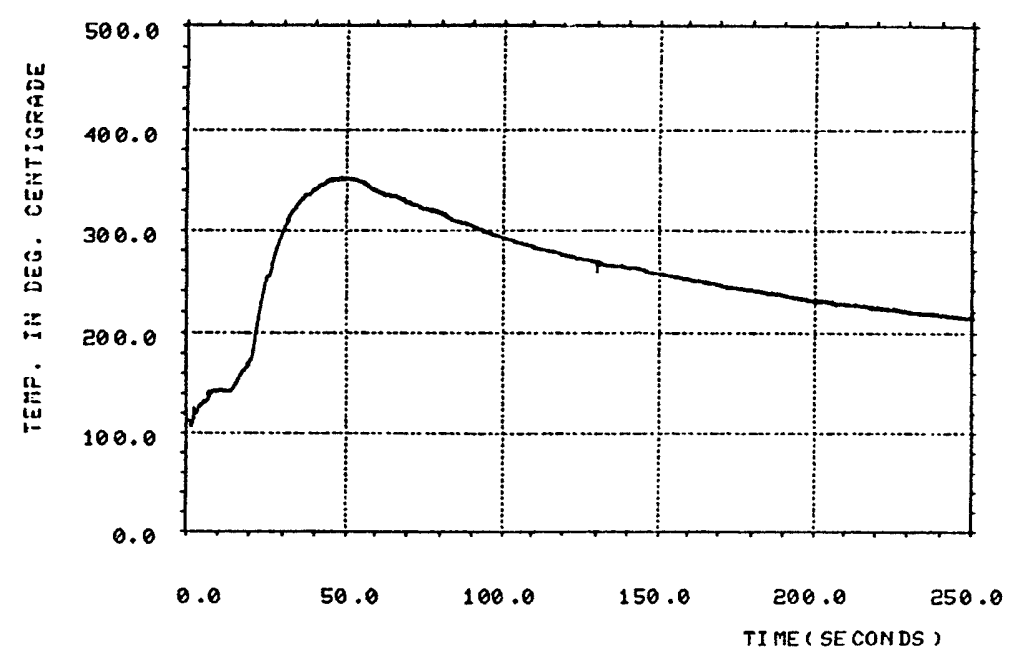

TC8-13, CCIN-2, DATE $?, 17 / 86$

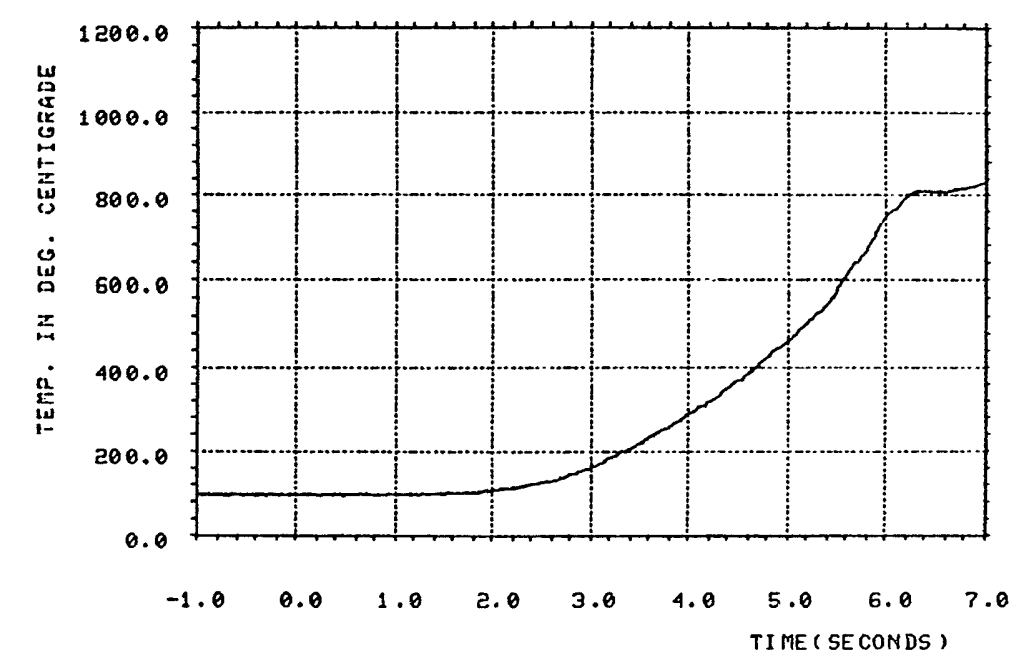

TC8-17, CCM-2, DATE $7 / 17 / 86$ 


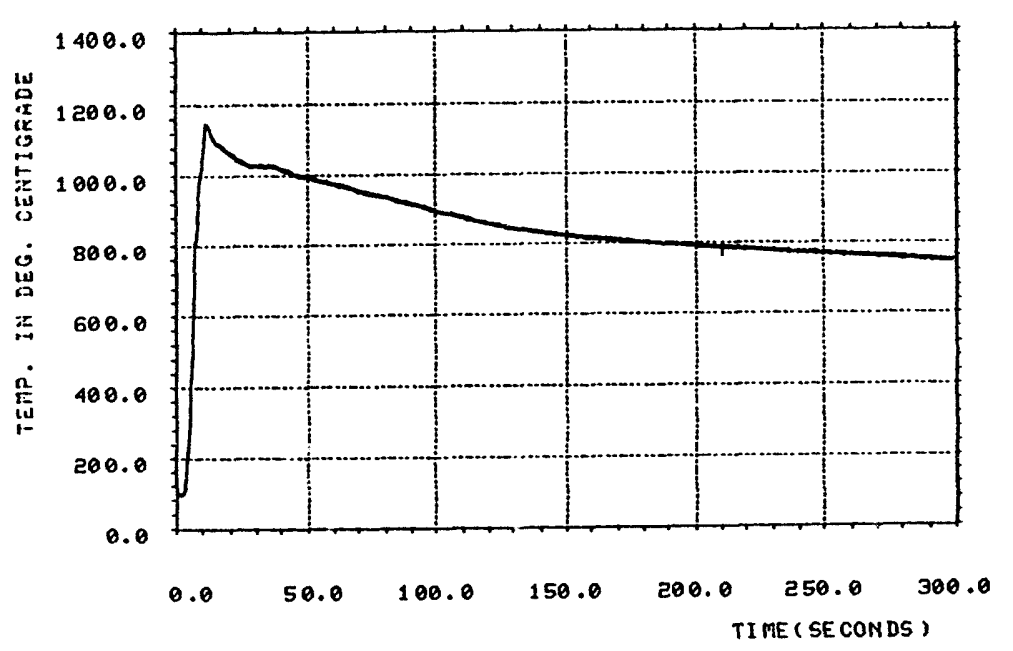

TC8-17, CCM-2, DATE $2 / 17 / 86$

$\stackrel{\Xi}{5}$

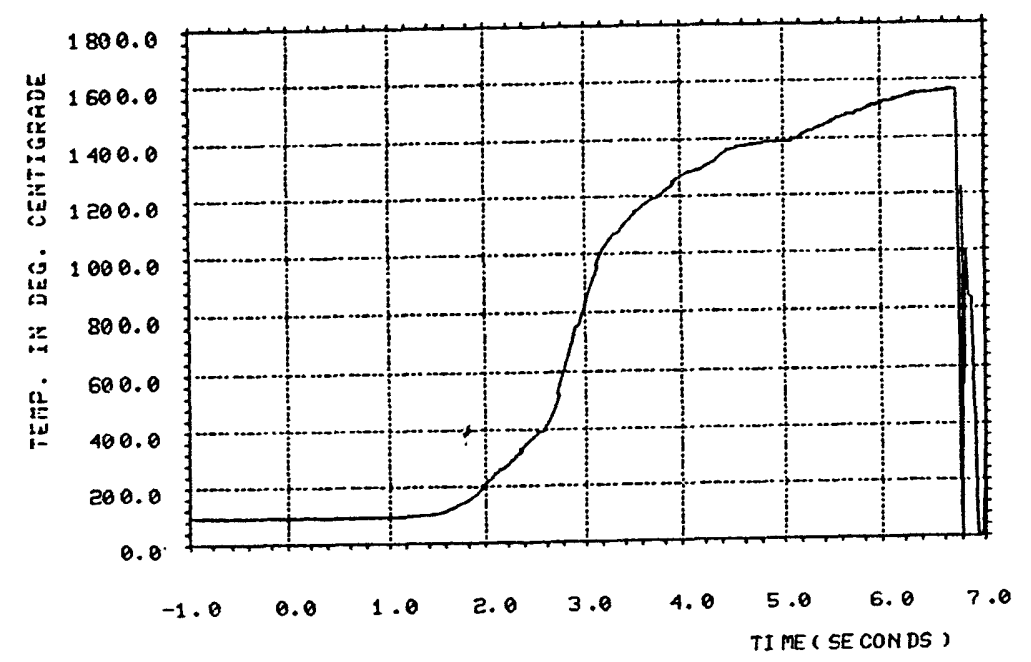

TC8-19. CCM-2, DATE 7/17/86

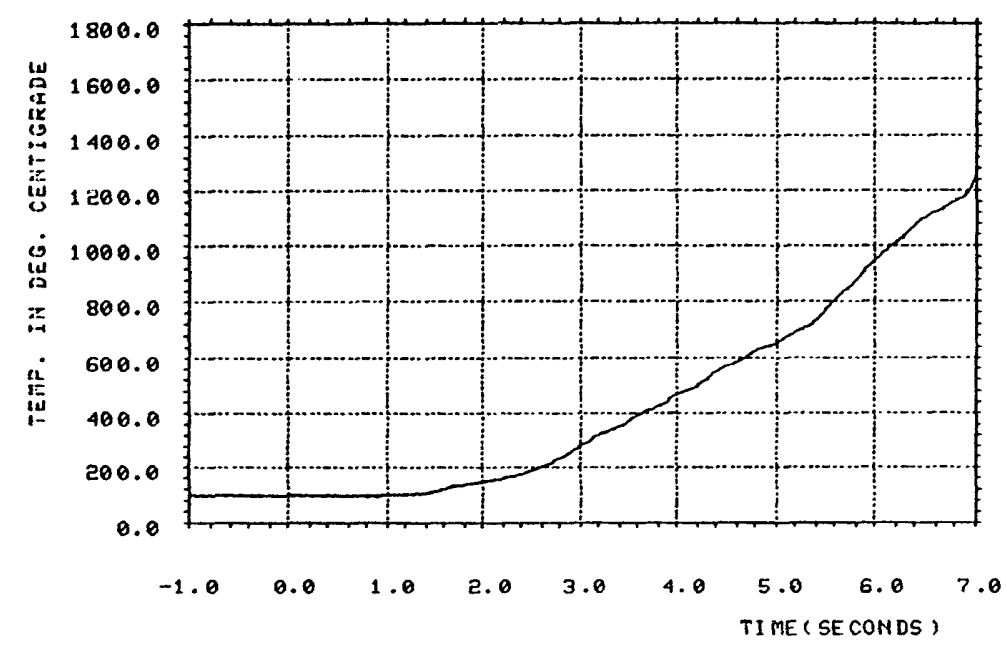

TC8-18, CCM-2, DATE $2 / 17 / 86$

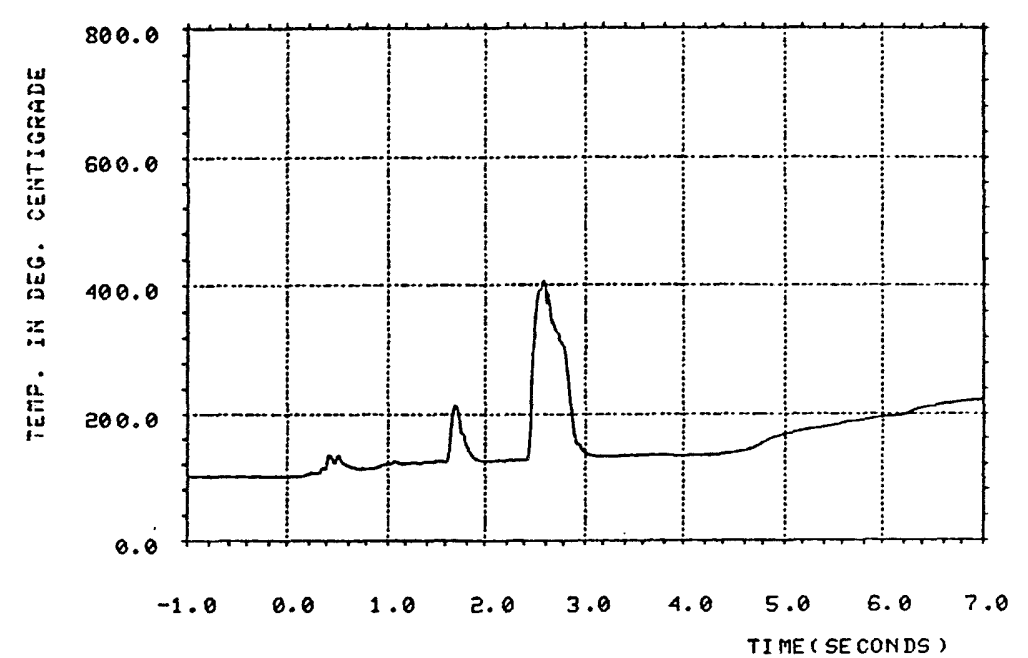

TC8-20, CCM-2, DATE $2 / 17 / 86$ 


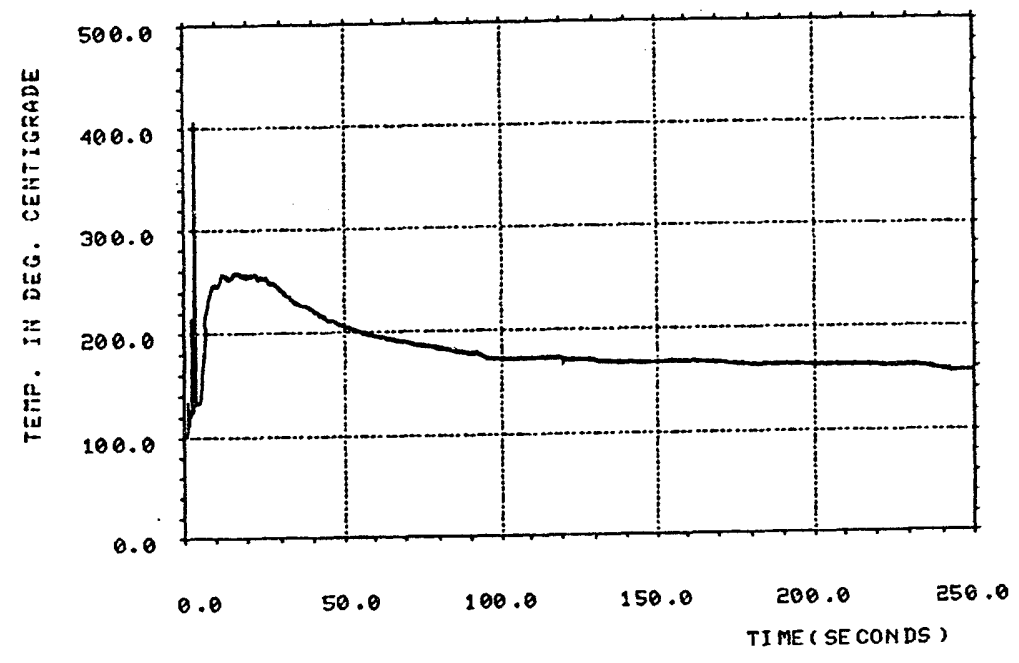

TC8-20, CCM-2, DATE $7 / 17 / 86$

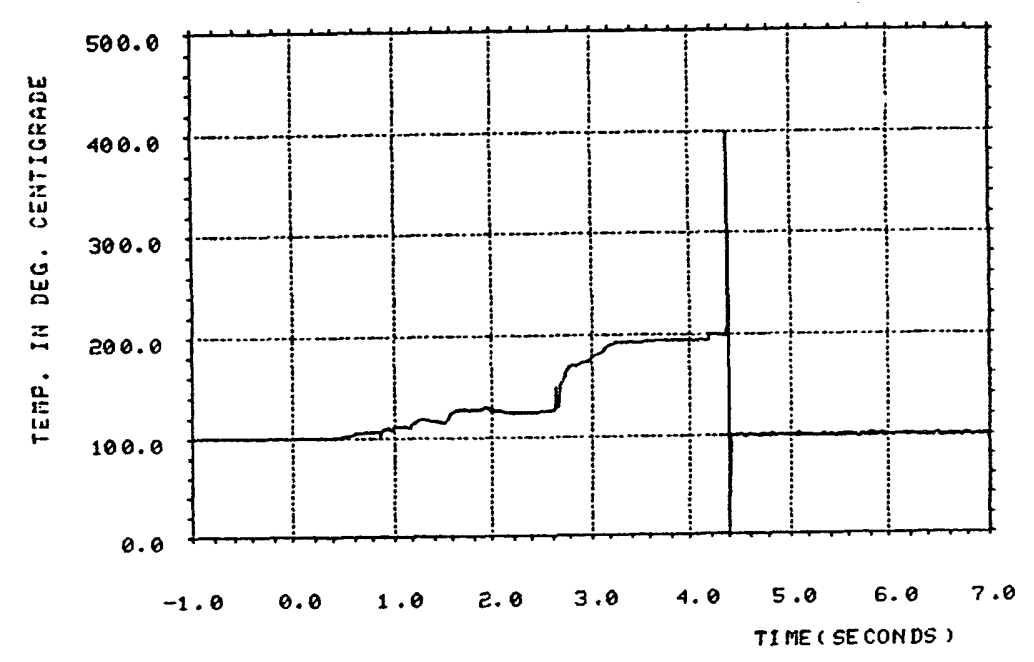

TC8-22, CCIM-2, DATE $7 / 17,86$

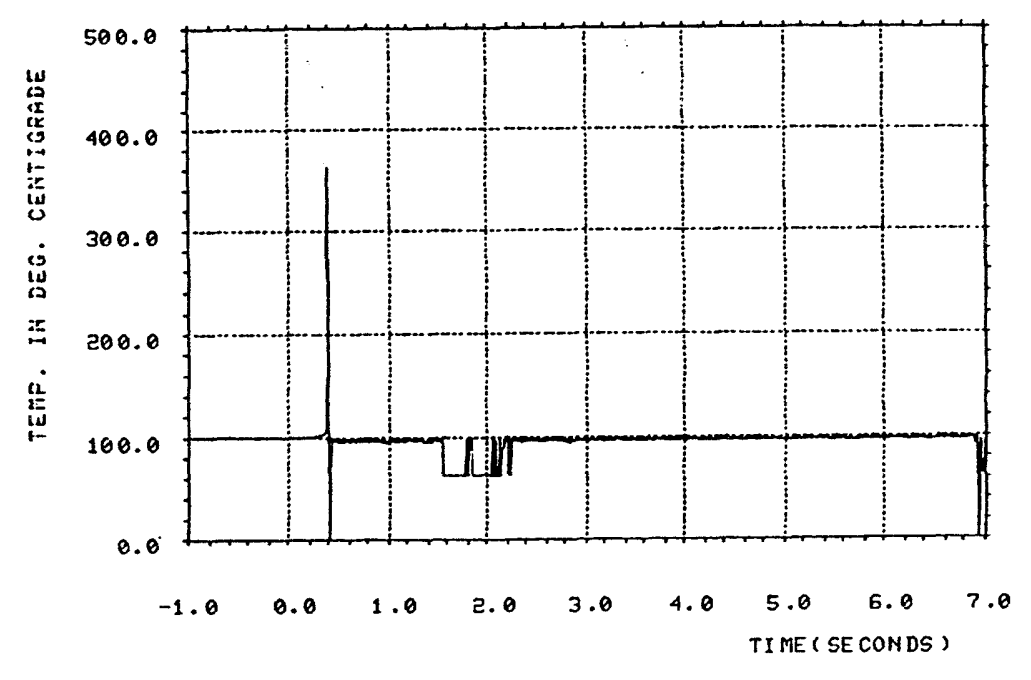

TCE-21, CCM-2, DATE $3 / 17 / 86$

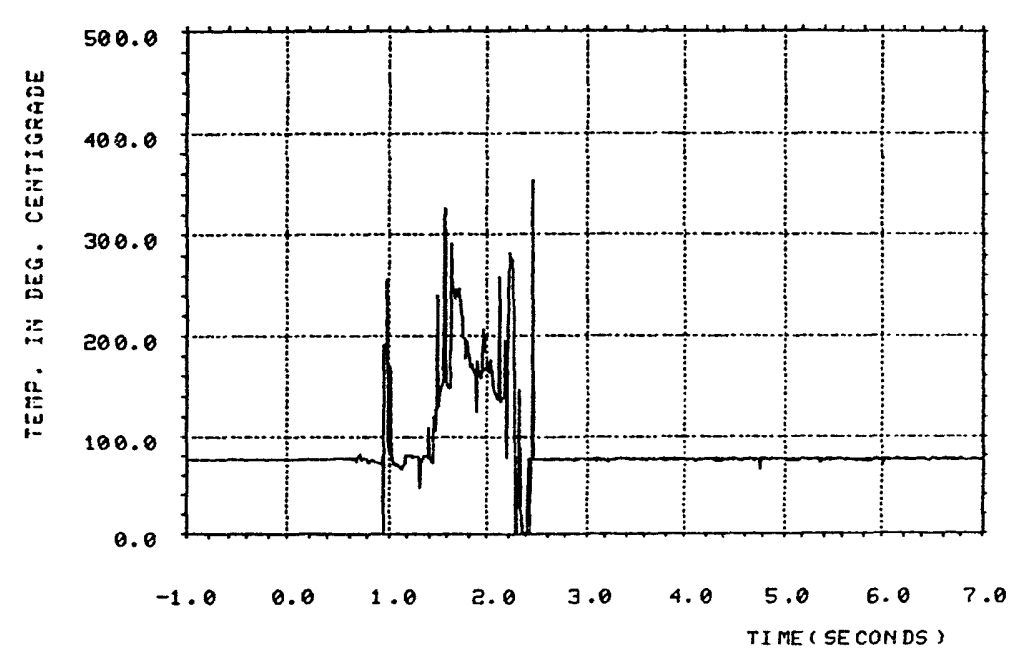

TC8-23, CCM-2, DATE 7/17/S6 


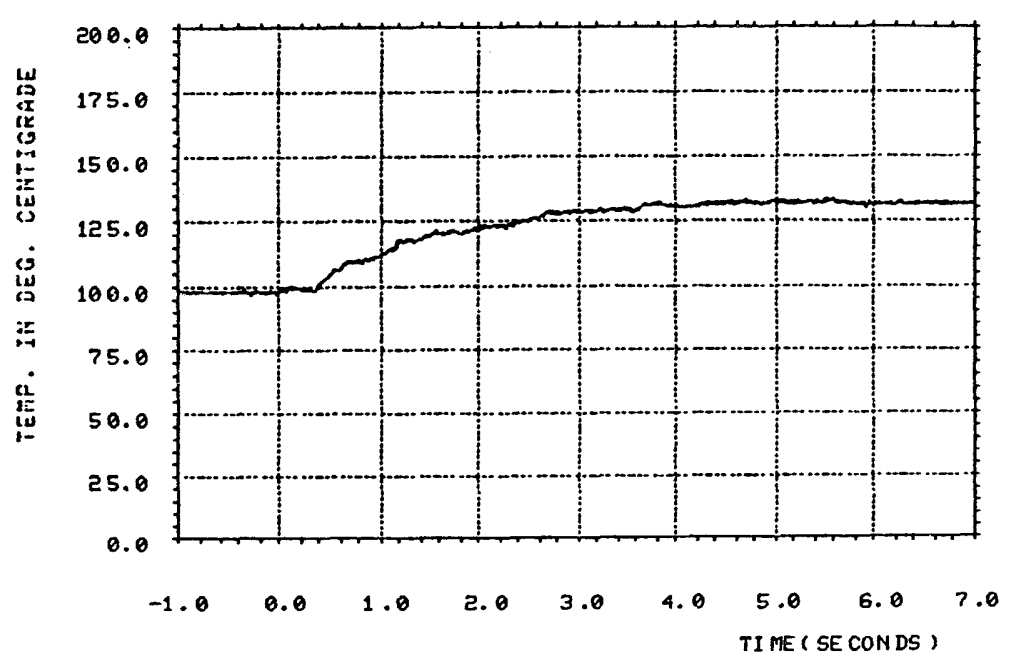

TC8-28, CCM-2, DATE $7 / 17 / 86$

$\stackrel{?}{\omega}$

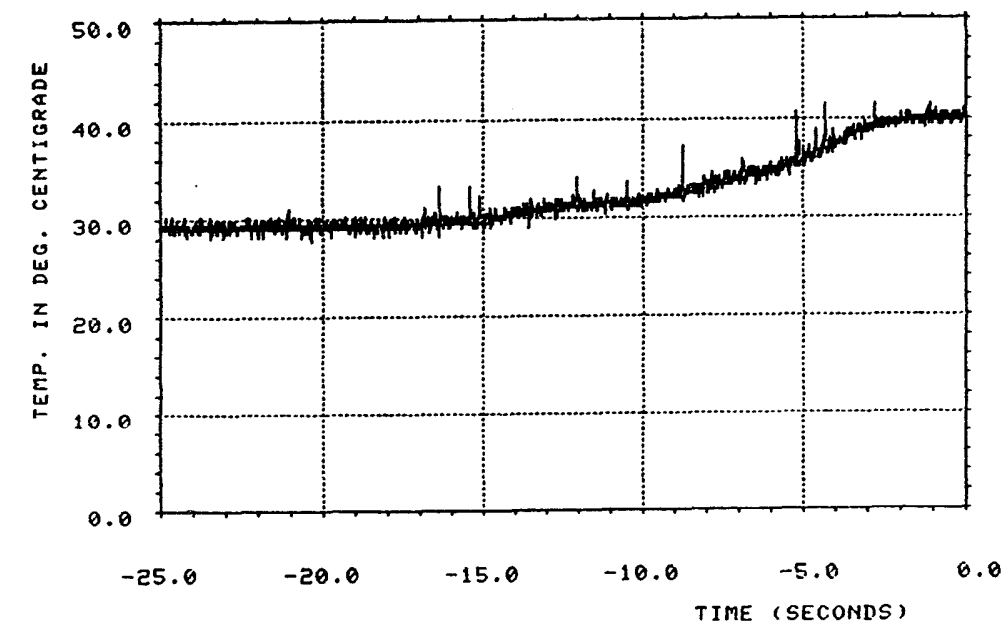

TCQ-2, CCM-2, DÁTE 7/17/86

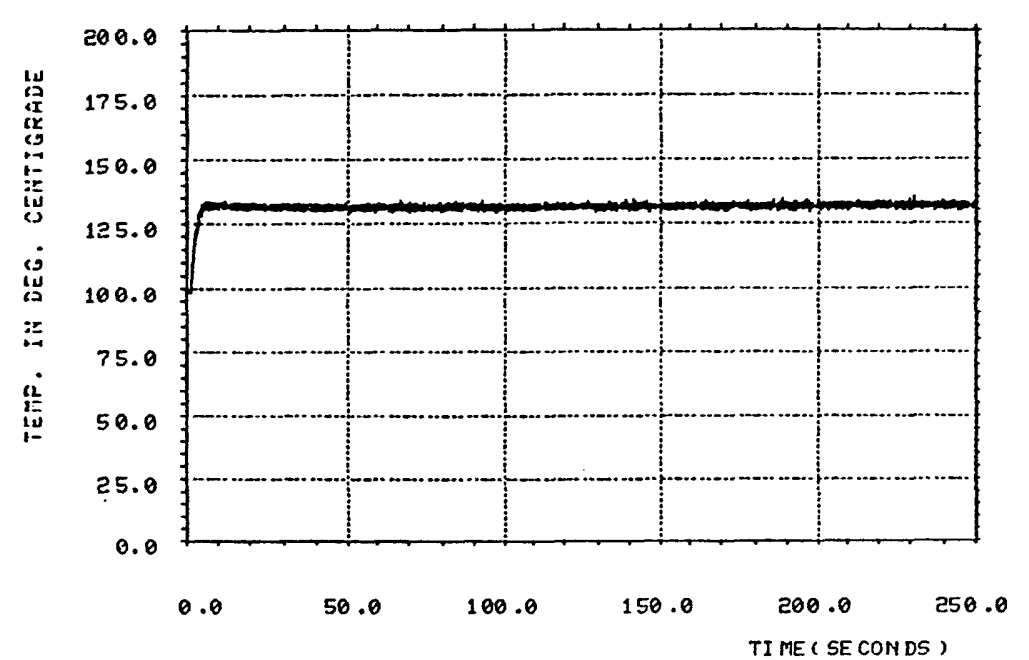

TC8-28, CCN-2, DATE $? / 17 / 86$

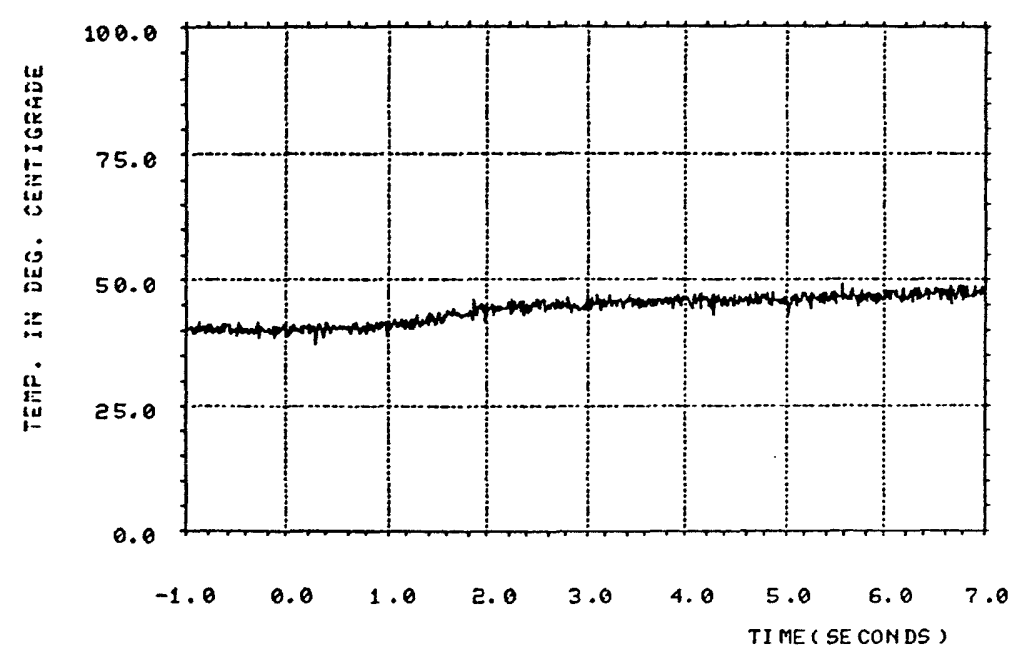

TCQ-2, CCM-2, DATE $7 / 17.86$ 


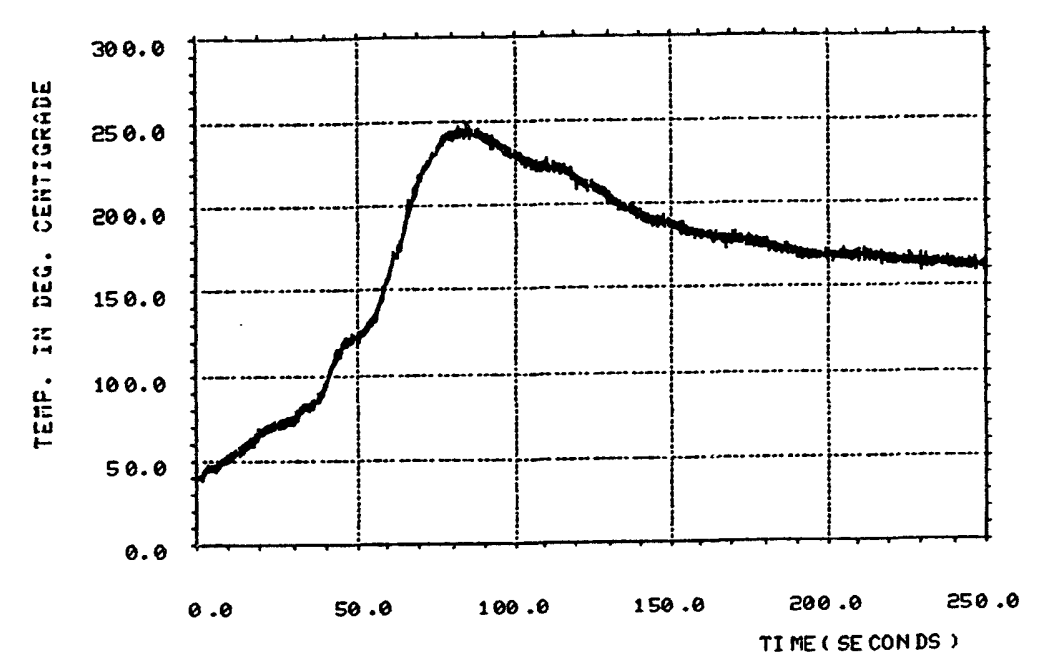

TCQ-2, CCM-2, DATE $7 / 17 / 86$

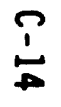

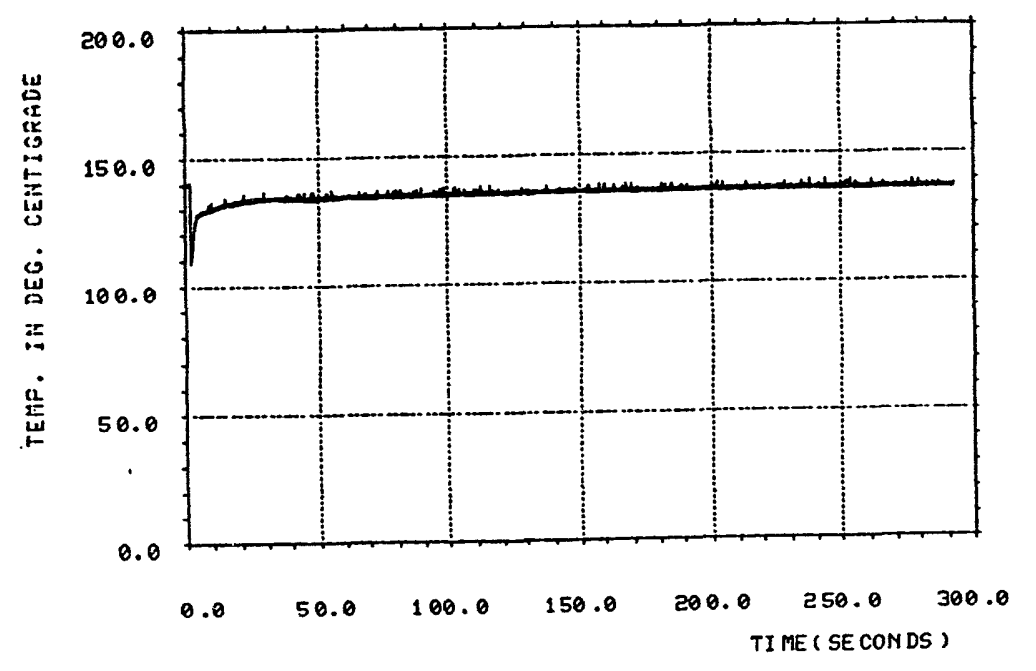

TC12-1, CCM-2, DATE ?:17:86

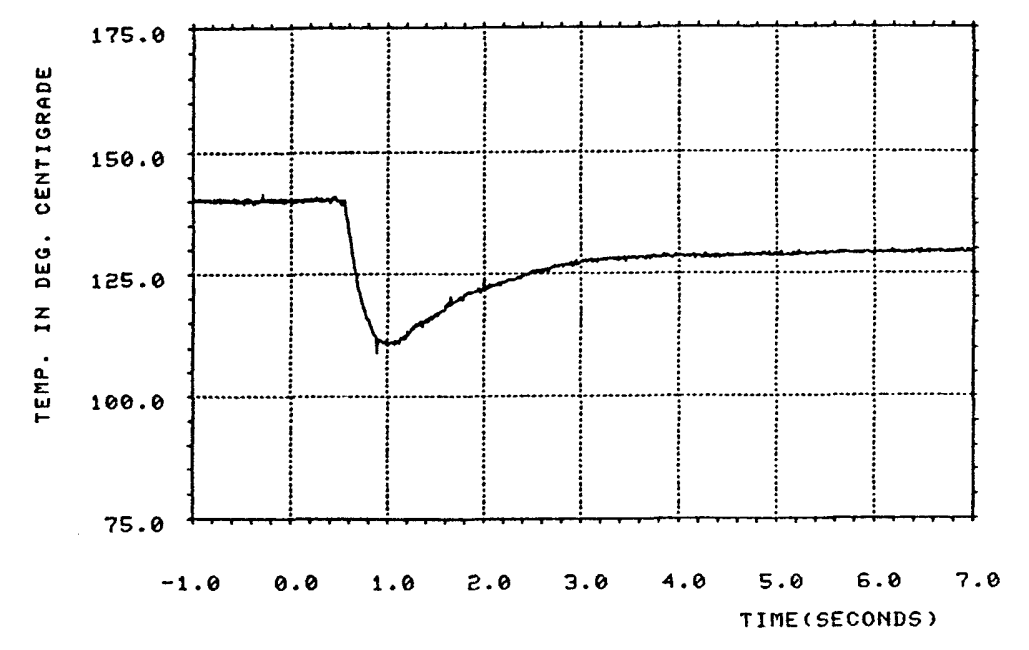

TC12-1, CCM-2, DATE $2,17 / 86$

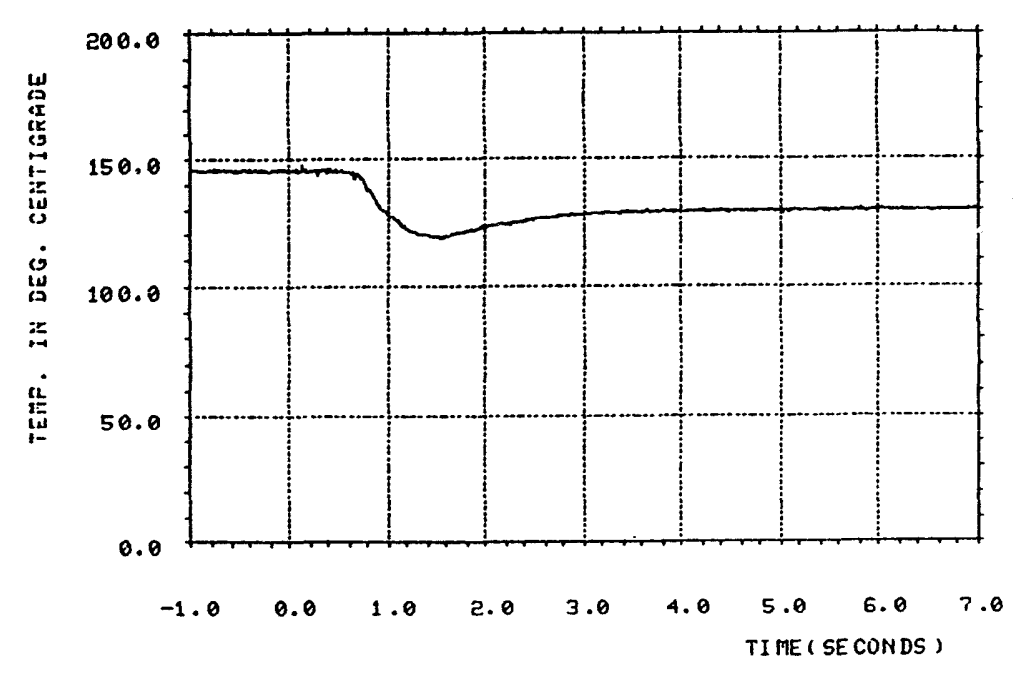

TC12-2, CCN1-2, DATE $7 / 17 / 86$ 


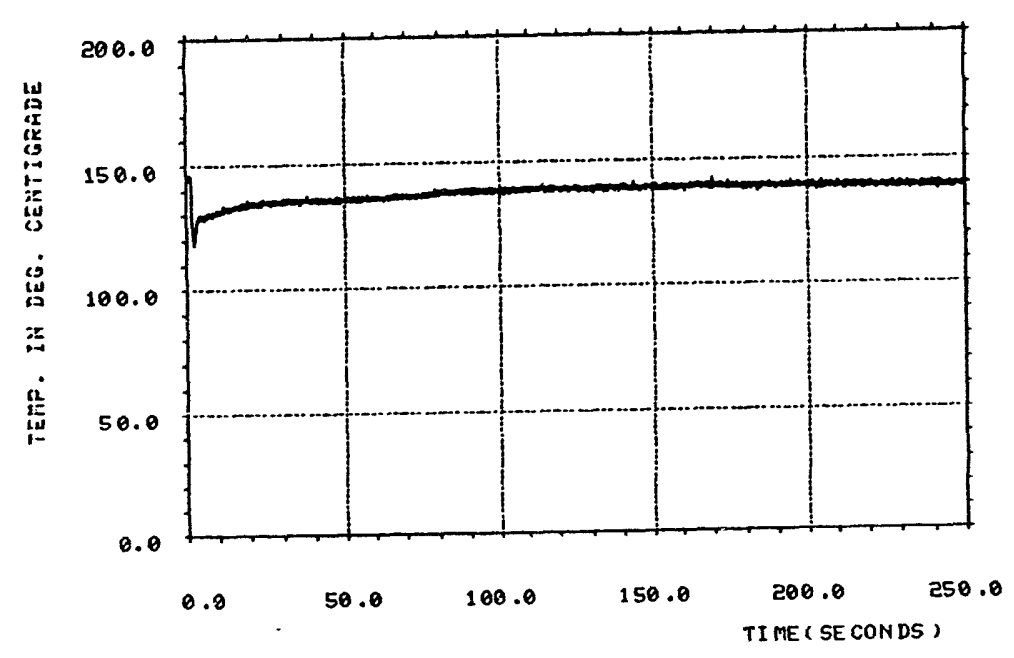

它

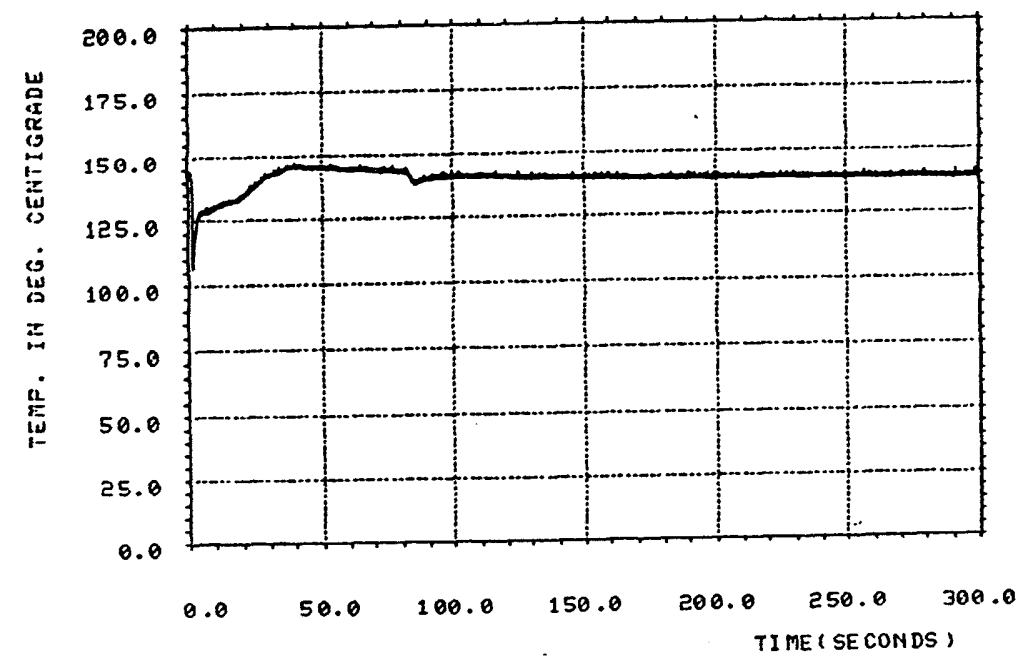

TC12-3, CCM-2, IIATE $7 / 17 / 86$

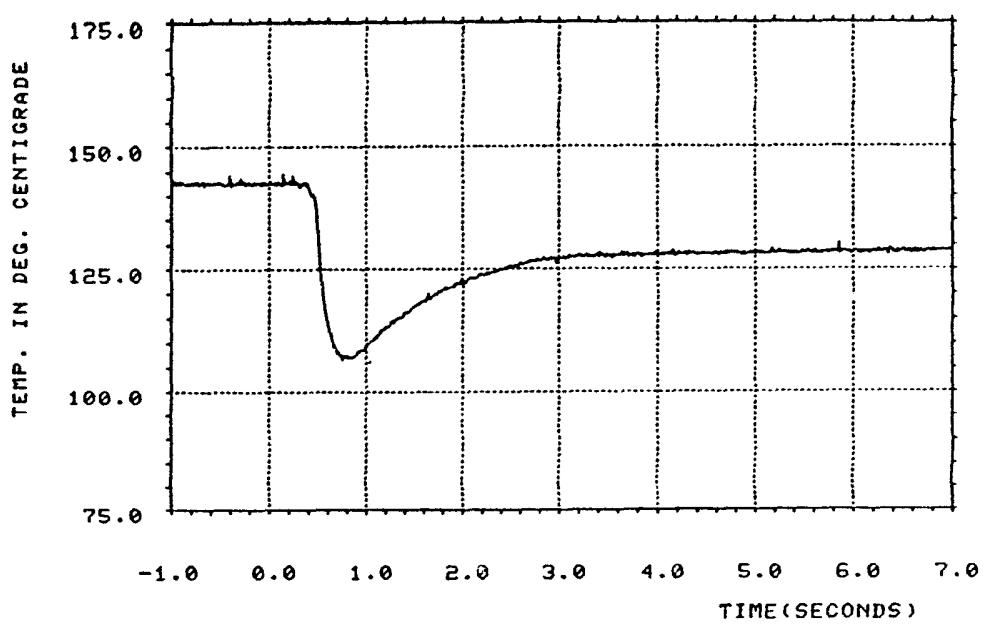

TC12-3, CCM-2, DATE $7 / 17,86$

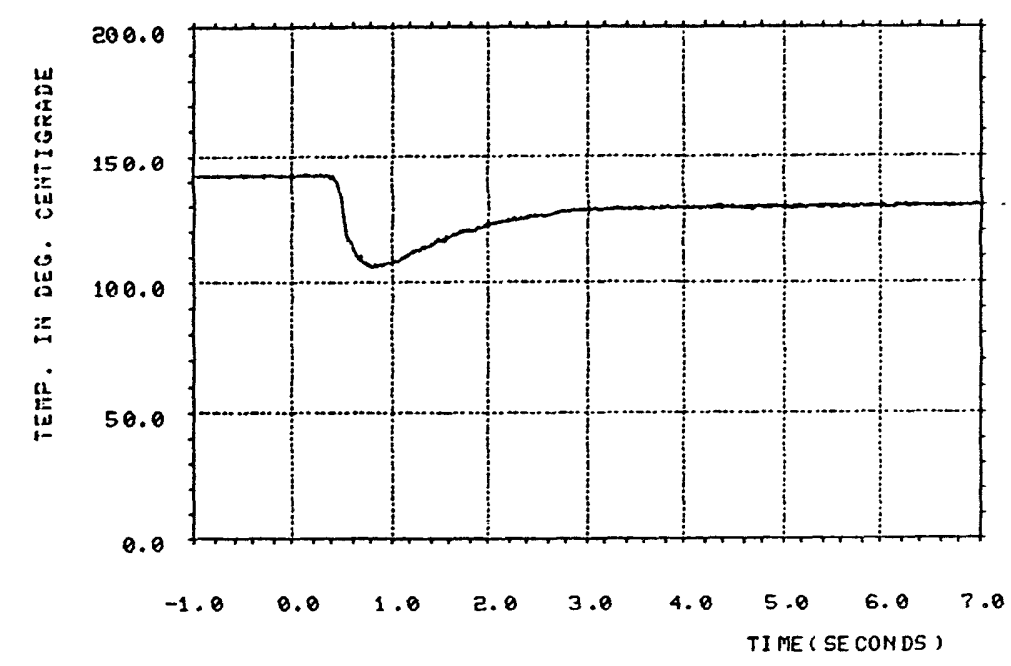

TC12-4, CCM-2, DATE $7 / 17 / 86$ 
( SONOJ 3S) 3WIL

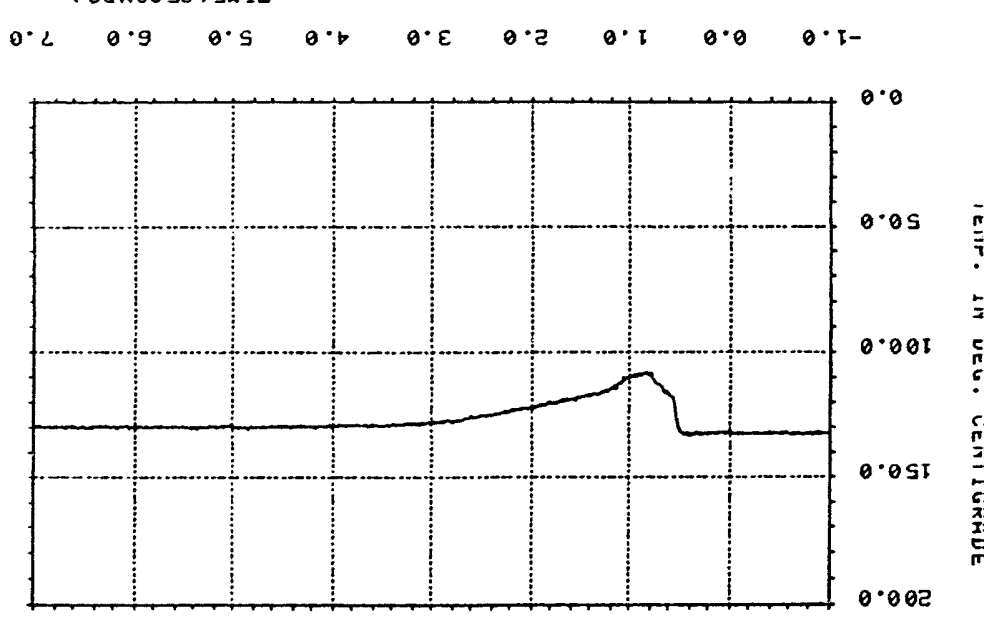

98/LL

( Sa NOJ 35 ) 3W ID
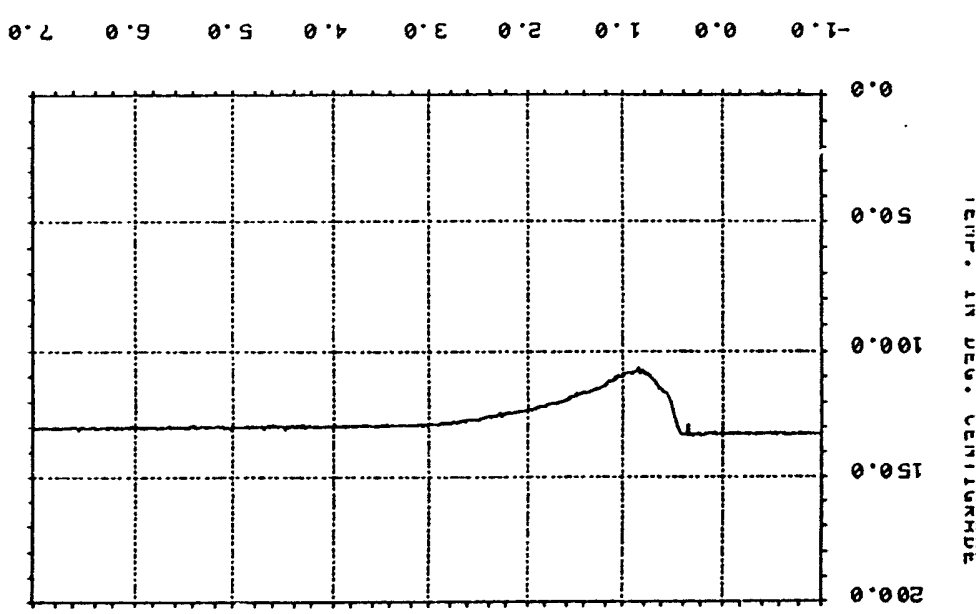

( SQ NOJ 3S ) 3W II

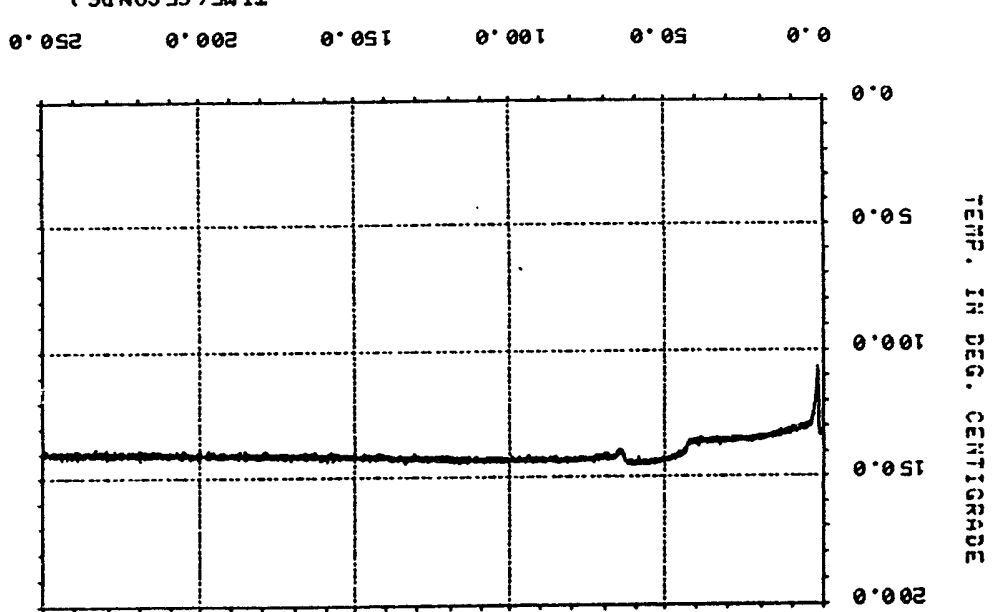

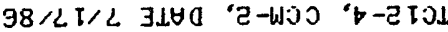

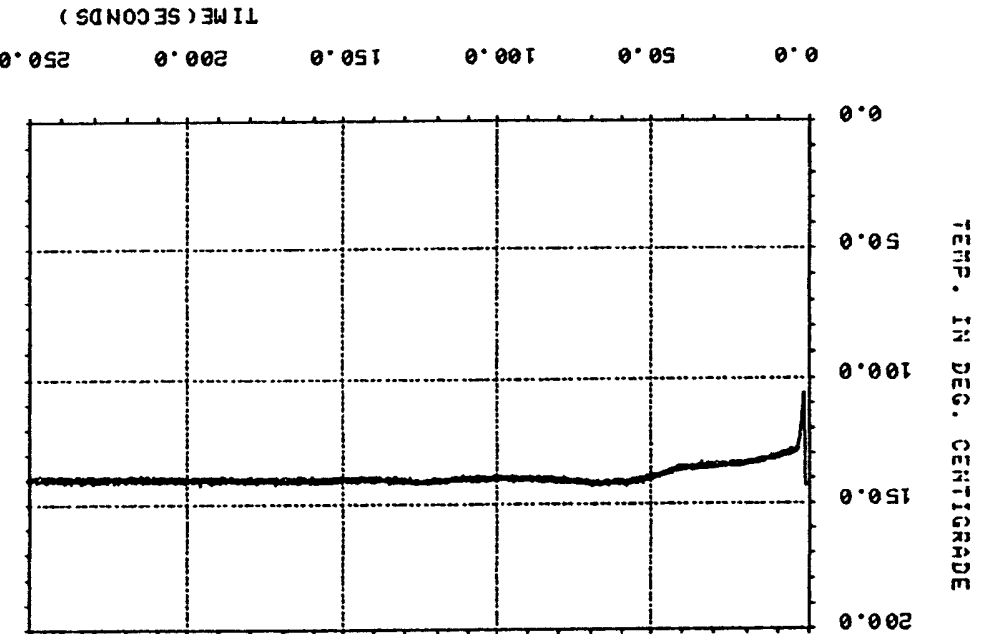




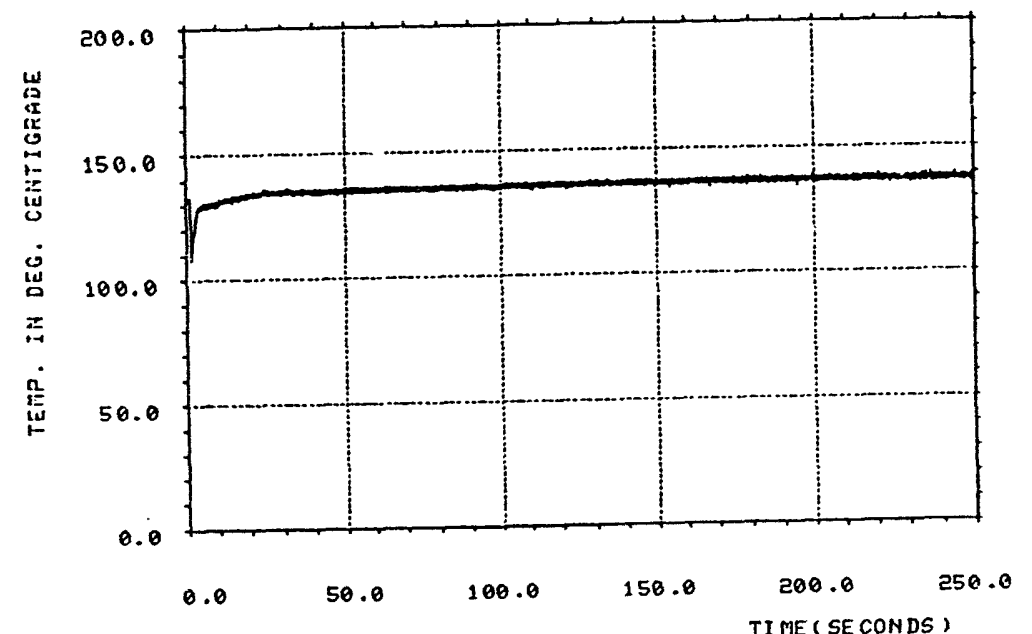

$\stackrel{\Xi}{=}$

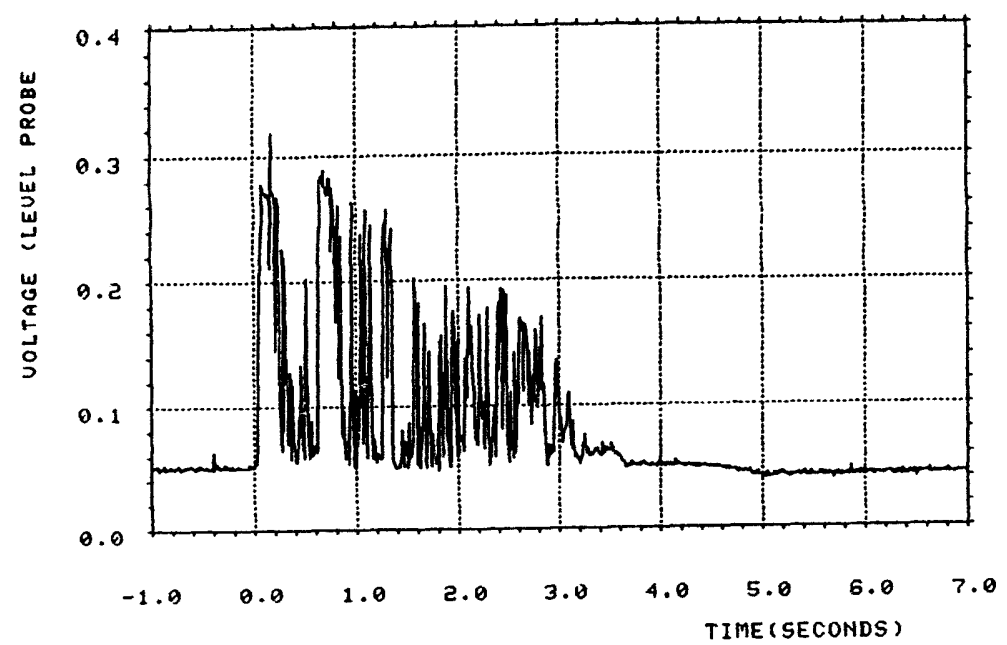

LP-2, COM-2. DATE $7 / 17 / 86$

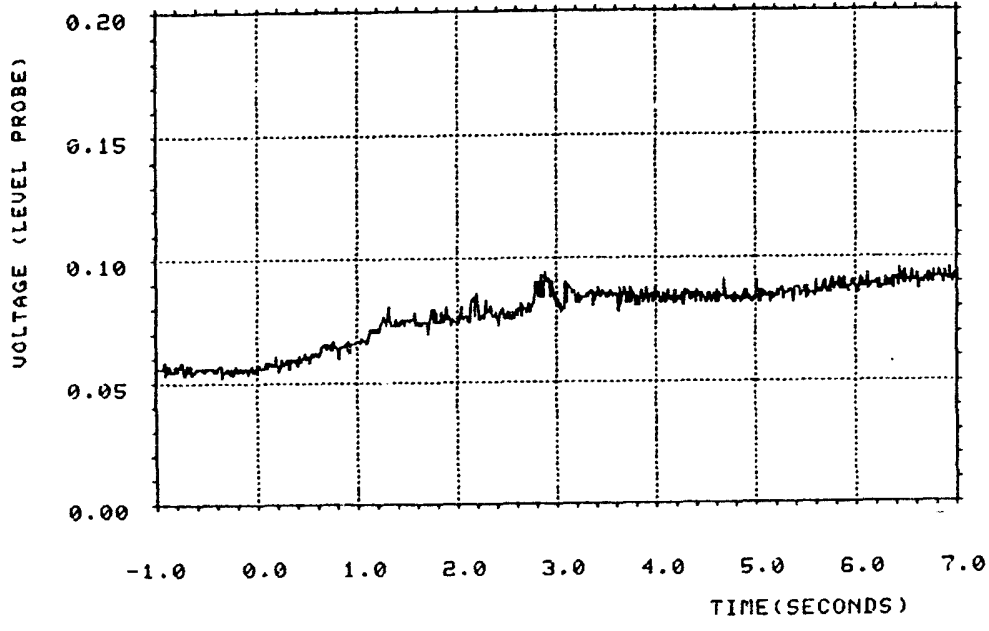

LP-1, CCII-2, DATE $? / 17 / 86$

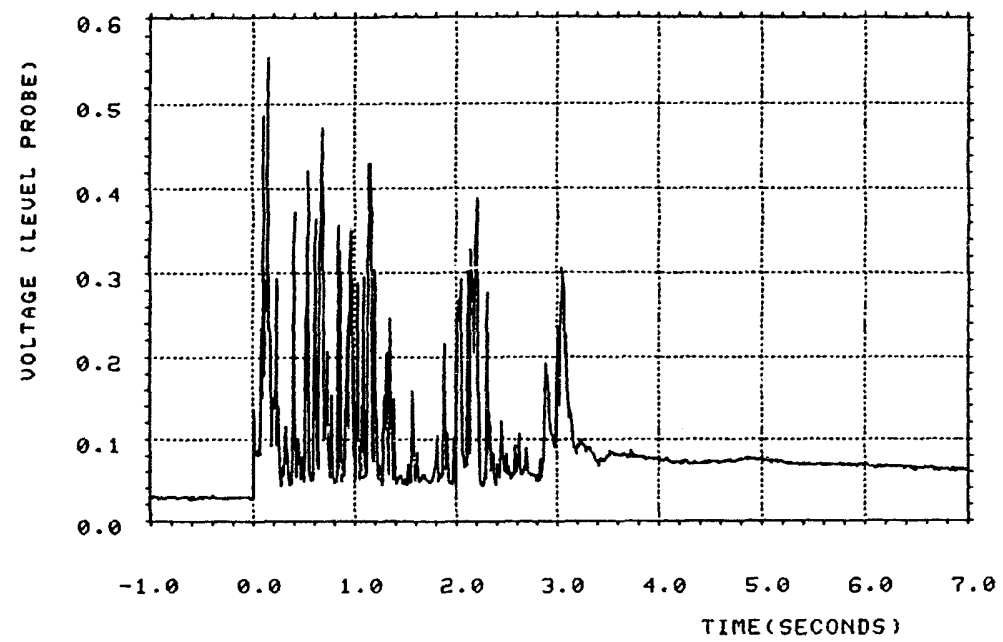

LP-3, CON-2, DATE 7/17/86 


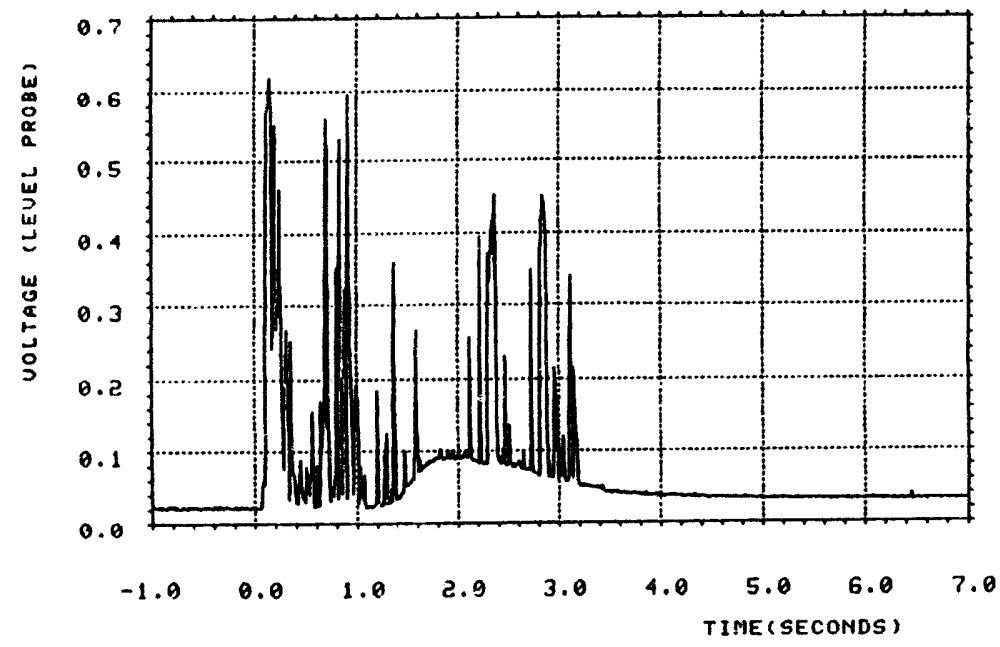

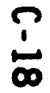

LP-4, CCM-2, DATE $7 / 17 / 86$

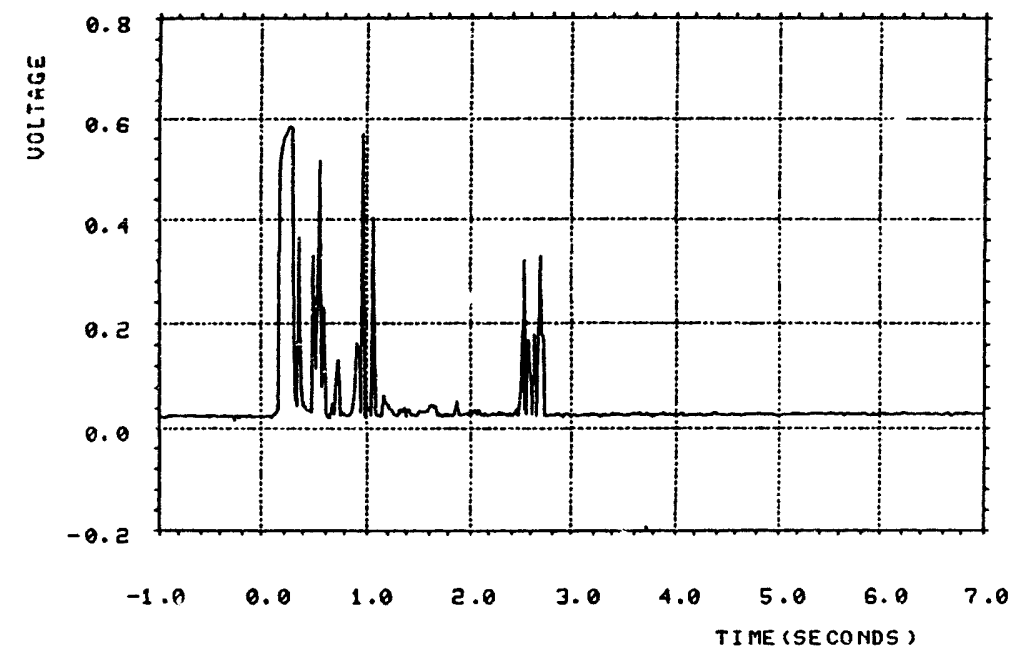

LP-5, CCM-2, DATE $7 / 17 / 86$ 
APPENDIX D. DATA FOR CCM-3

PTS-1: Thermite-Vessel Gas Pressure

PTS-2: Interaction-Vessel Gas Pressure

PTS-9: Pressure at Top of Expansion Vessel

PTS-10: Pressure of Lower Side of Expansion Vessel

PTS-12: Pressure in Upper-top Section of the Interaction Vessel

PTS-13: Pressure in Lower-top Section of the Interaction Vessel

PTS-14: Pressure in Upper Middle Section of the Interaction Vessel

PTS-15: Pressure in Lower Middle Section of the Interaction Vesse1

TC8-6: Temperature at Base of Interaction Vessel

TC8-7: Temperature at Base of Interaction Vessel

TC8-8: Temperature at Base of Interaction Vesse1

TC8-9: Temperature at Base of Interaction Vessel

TC8-10: Temperature Inside Expansion Vessel $34.9 \mathrm{~cm}$ above Pipeway Exit

TC8-12: Temperature of Gas Space in Interaction Vessel

TC8-16: Temperature at Base of Interaction Vessel

TC8-17: Temperature at Base of Interaction Vesse1

TC8-18: Temperature at Base of Interaction Vessel

TC8-19: Temperature at Base of Interaction Vessel

TC8-20: Temperature in Upper-top Section of Interaction Vessel

TC8-21: Temperature in Upper-top Section of Interaction Vesse1

TC8-22: Temperature in Lower-top Section of Interaction Vessel

TC8-23: Temperature in Lower-top Section of Interaction Vesse1

TC8-24: Temperature in Upper-middle Section of Interaction Vessel

TC8-25: Temperature in Upper-middle Section of Interaction Vessel

TC8-26: Temperature in Lower-middle Section of Interaction Vessel

TC8-27: Temperature in Lower-middle Section of Interaction Vessel

TC8-28: Temperature in Discharge Pipeway $U$-bend

TC9-2: Temperature in Thermite-vessel Gas Space

TC12-1: Temperature Inside Expansion Vessel $282.58 \mathrm{~cm}$ Above Pipeway Exit

TC12-2: Temperature Inside Expansion Vessel $241.94 \mathrm{~cm}$ Above Pipeway Exit

TC12-3: Temperature Inside Expansion Vessel $201.30 \mathrm{~cm}$ Above Pipeway Exit

TC12-4: Temperature Inside Expansion Vessel $160.66 \mathrm{~cm}$ Above Pipeway Exit

TC12-5: Temperature Inside Expansion Vessel $120.02 \mathrm{~cm}$ Above Pipeway Exit

TC12-6: Temperature Inside Expansion Vessel $79.38 \mathrm{~cm}$ Above Pipeway Exit 
APPENDIX D. DATA FOR CCM-3 (Con't.d)

LP-1: Water Level in Interaction Vessel $13.64 \mathrm{~cm}$ Elevation

LP-2: Water Level in Interaction Vessel $14.27 \mathrm{~cm}$ Elevation

LP-3: Water Level in Interaction Vessel $15.54 \mathrm{~cm}$ Elevation

LP-4: Water Level in Interaction Vessel $16.81 \mathrm{~cm}$ Elevation

LP-5: Water Level in Interaction Vessel $19.35 \mathrm{~cm}$ Elevation 


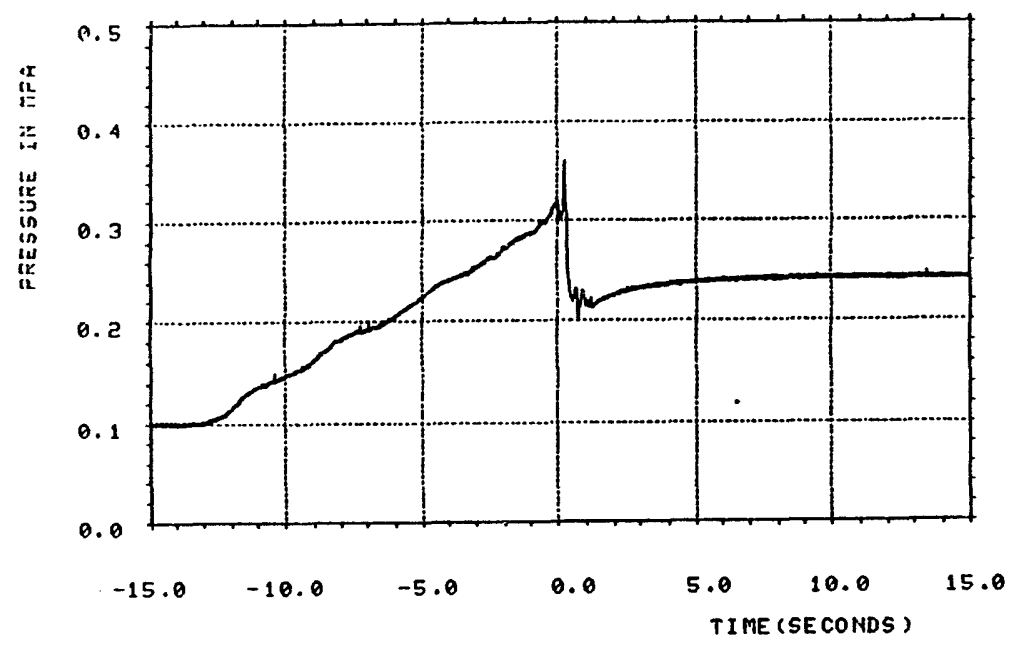

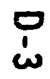

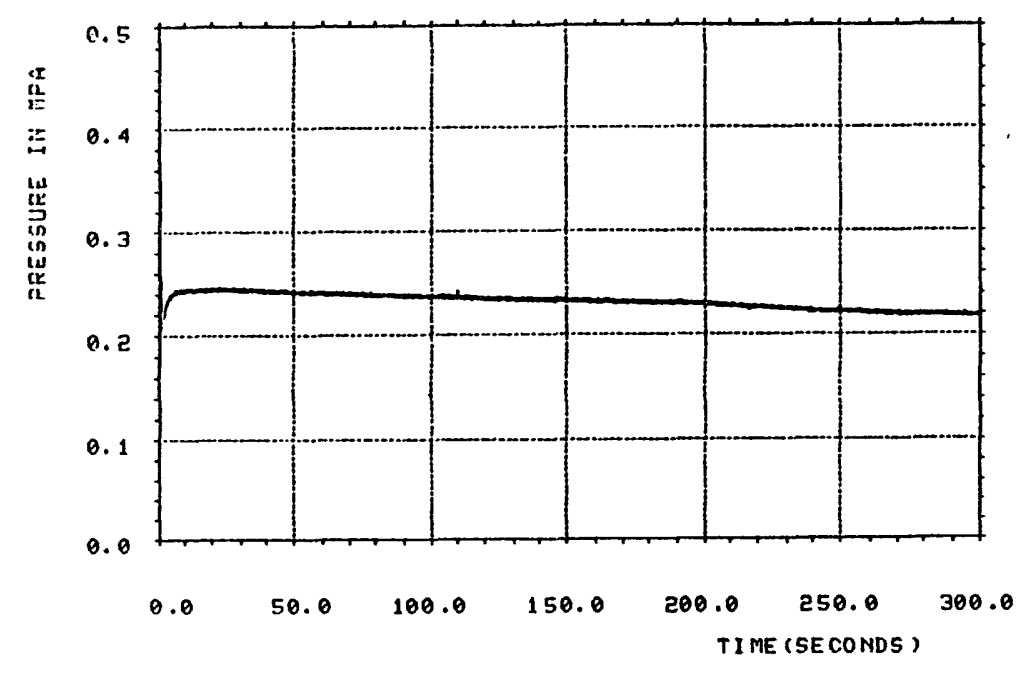

PTS-1, CCM-3, DATE 8/14/86

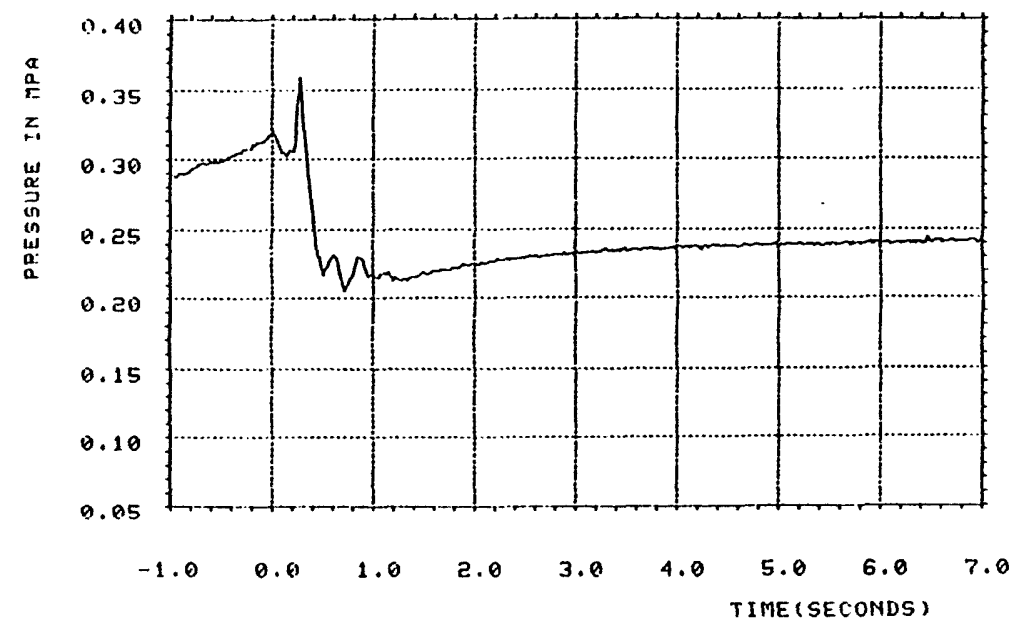

PTS-1, COCH-3, DATE 8/14/86

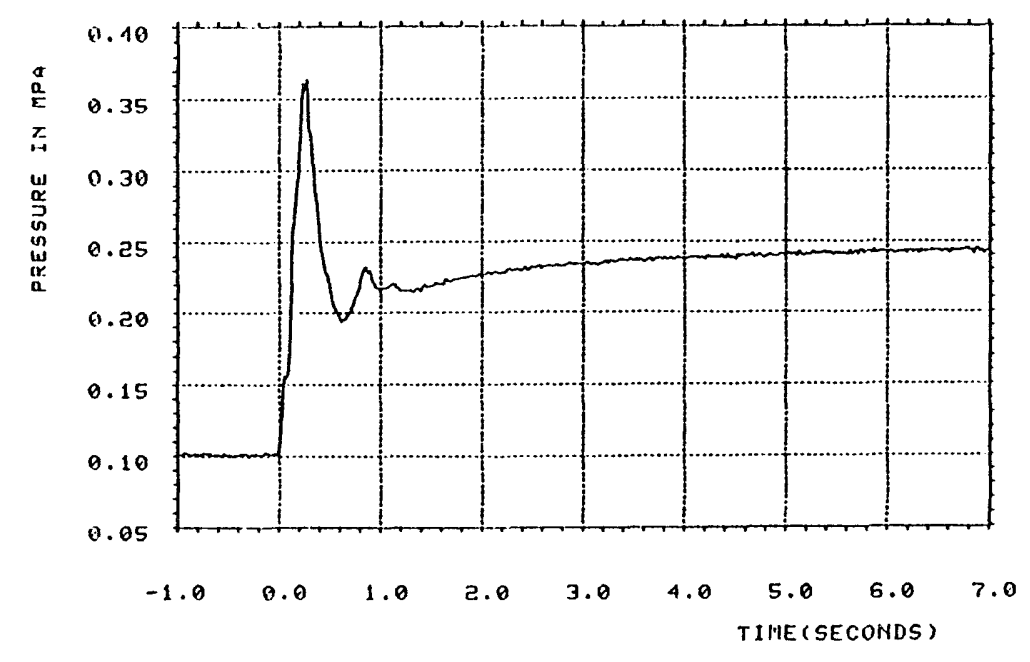

PTS-2, CCN-3, DATE 8/14/86 


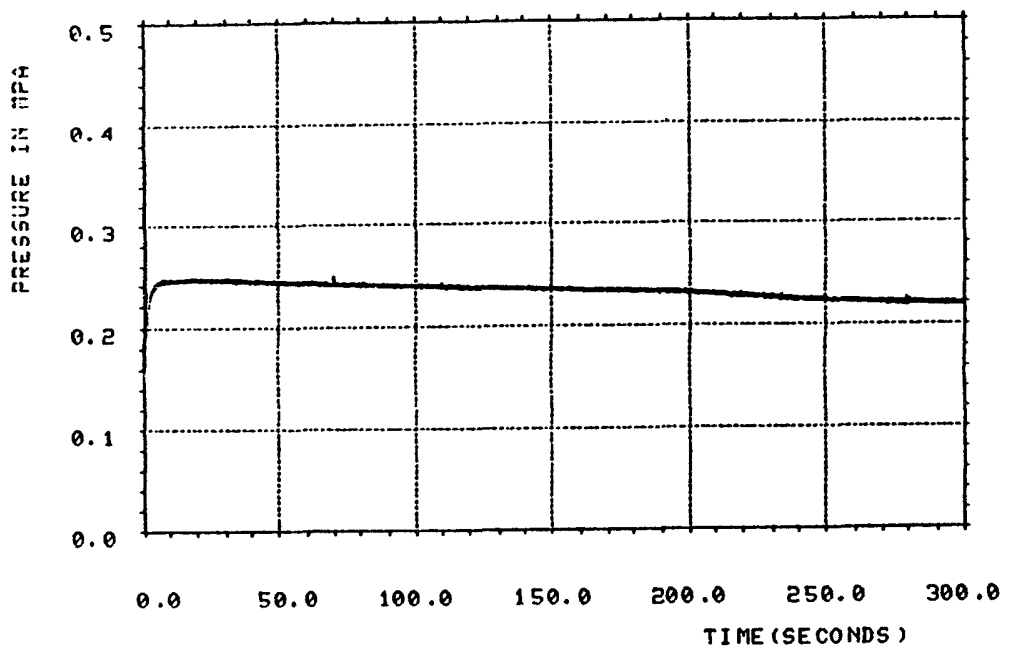

PTS-2, CCM-3, DATE 8,14/86

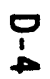

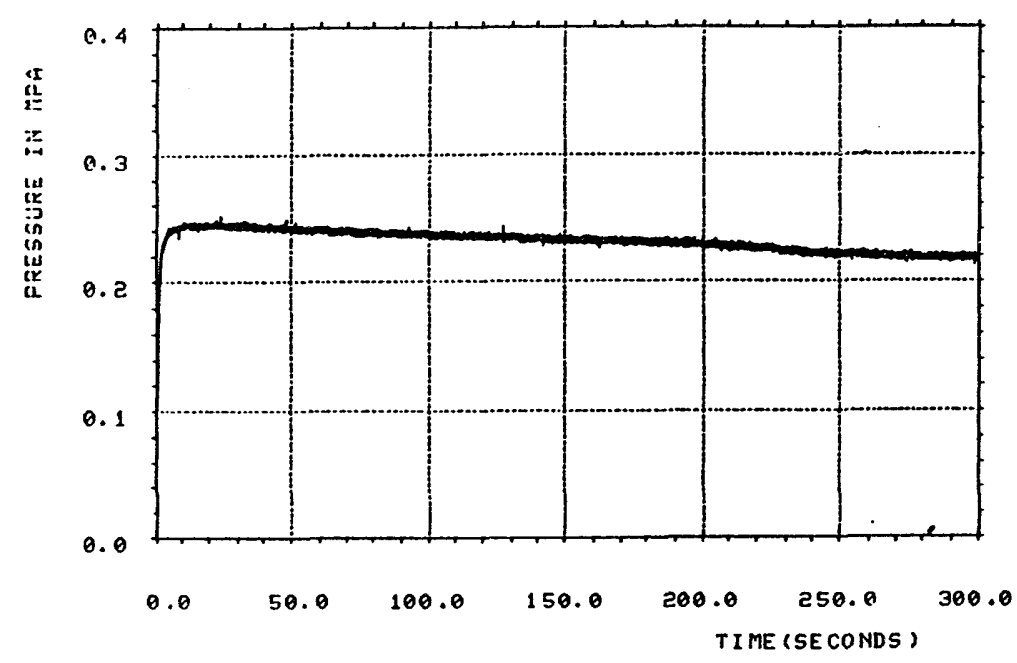

PTS-9, CCII-3, DATE 8/14/86

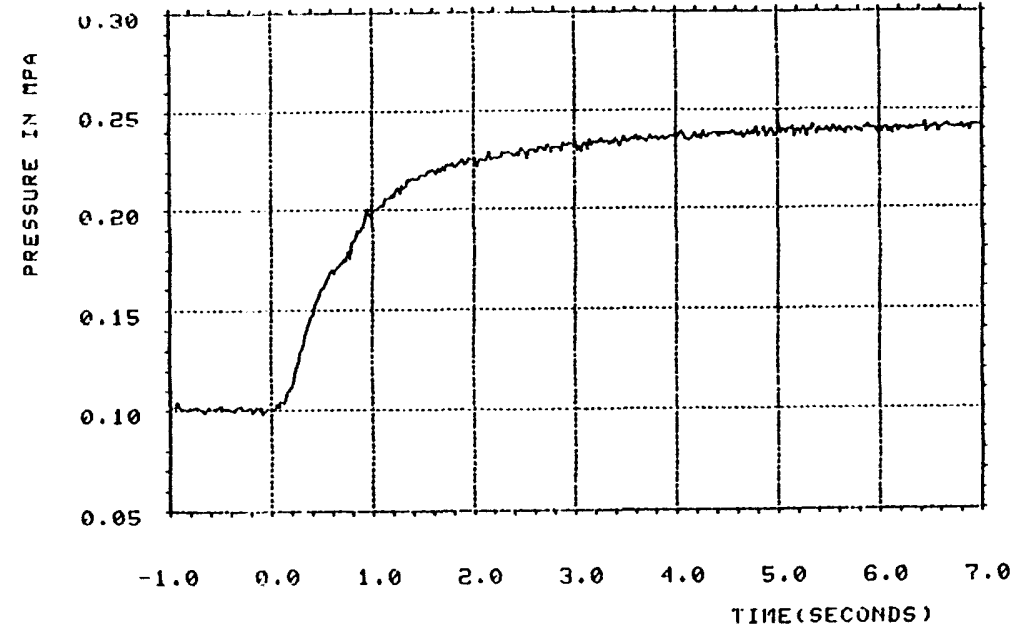

PTS-9, CCH-3, DATE 8/14/8G

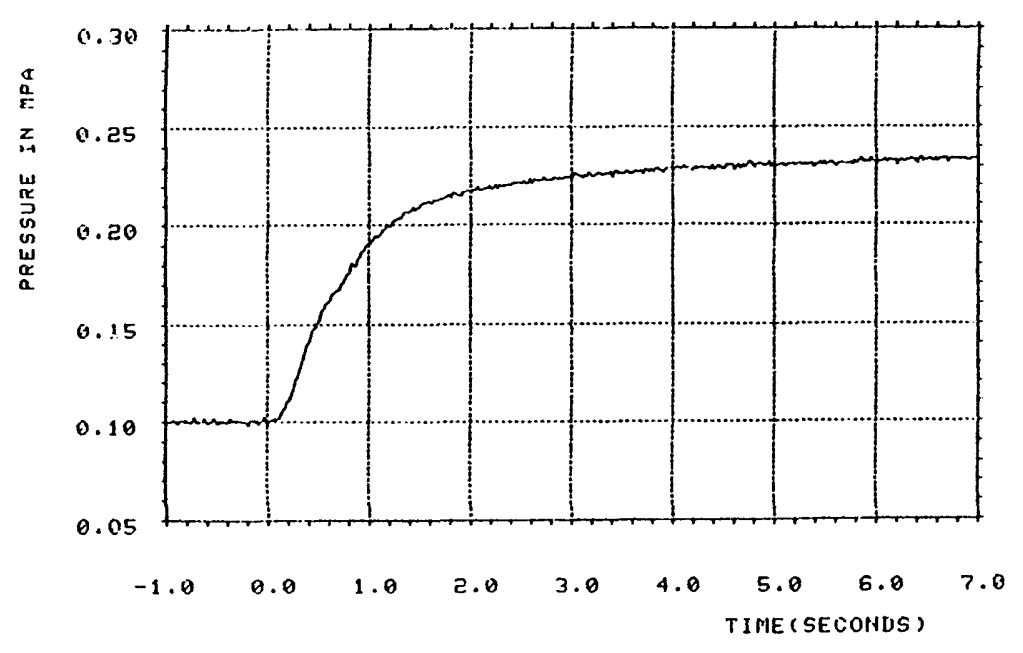

PTS-10, (CH-3, DATE 8/14/86 


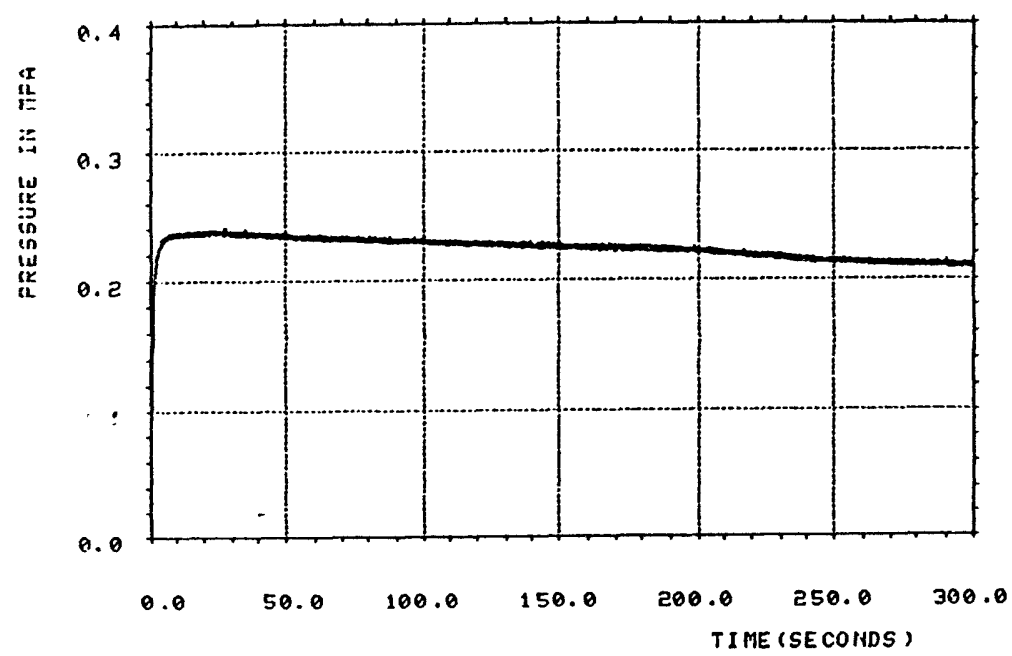

PTS-1e, CCIN-3, DATE $8 / 14 / 86$

'

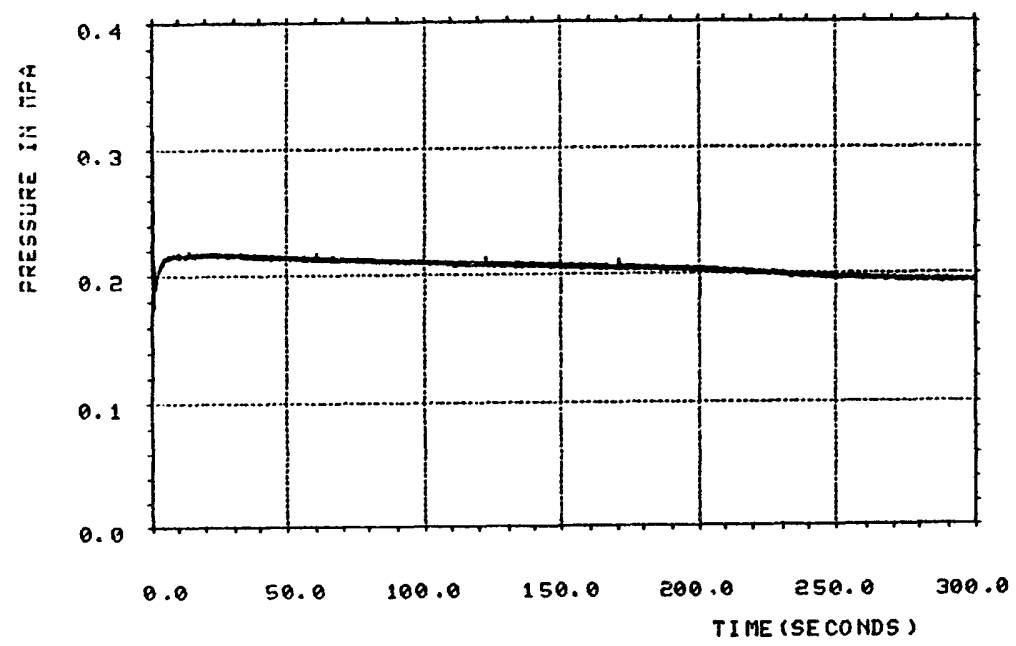

PTS-12, CCM-3, DATE $8 / 14 / 86$

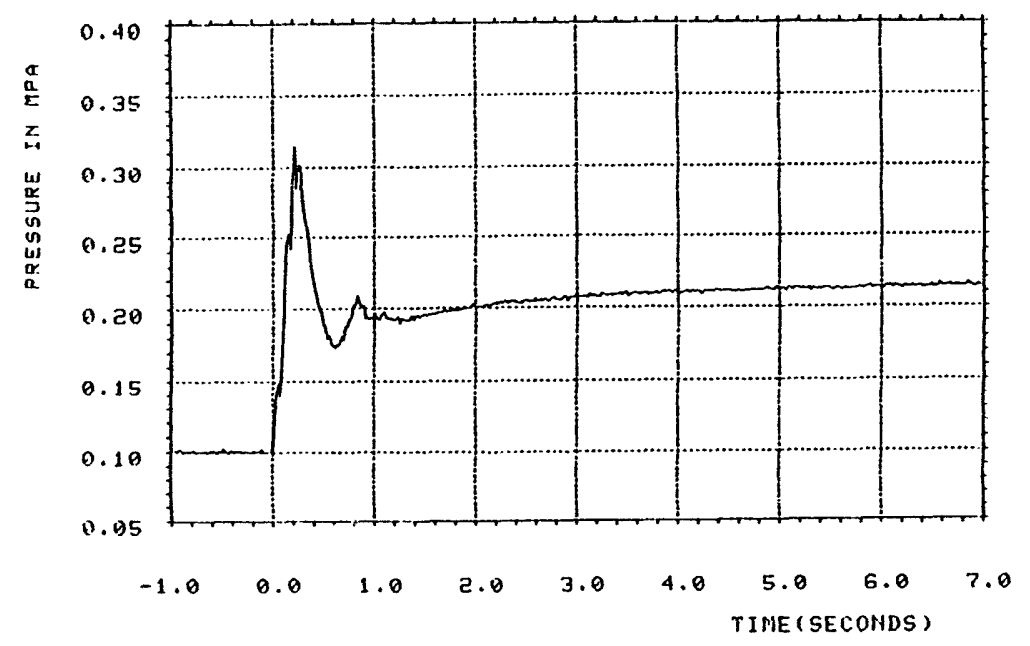

PTS-12, CON-3, DATE 8/14/86

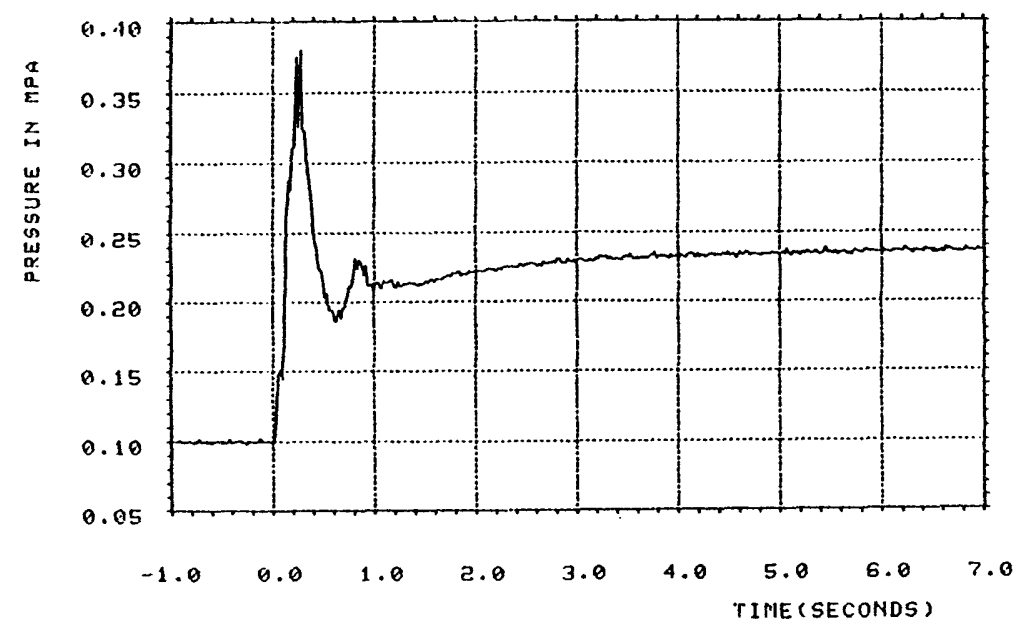

PTS-13, CCN-3, DATE $8 / 14 / 86$. 


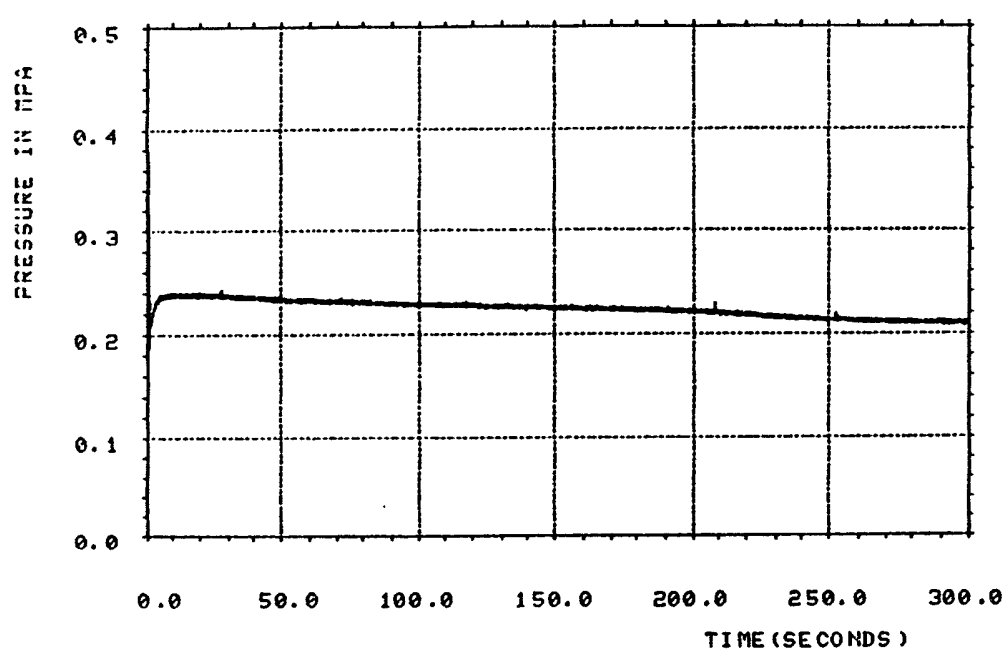

PTS-13, CCM-3, DATE $8 / 14 / 86$

ó

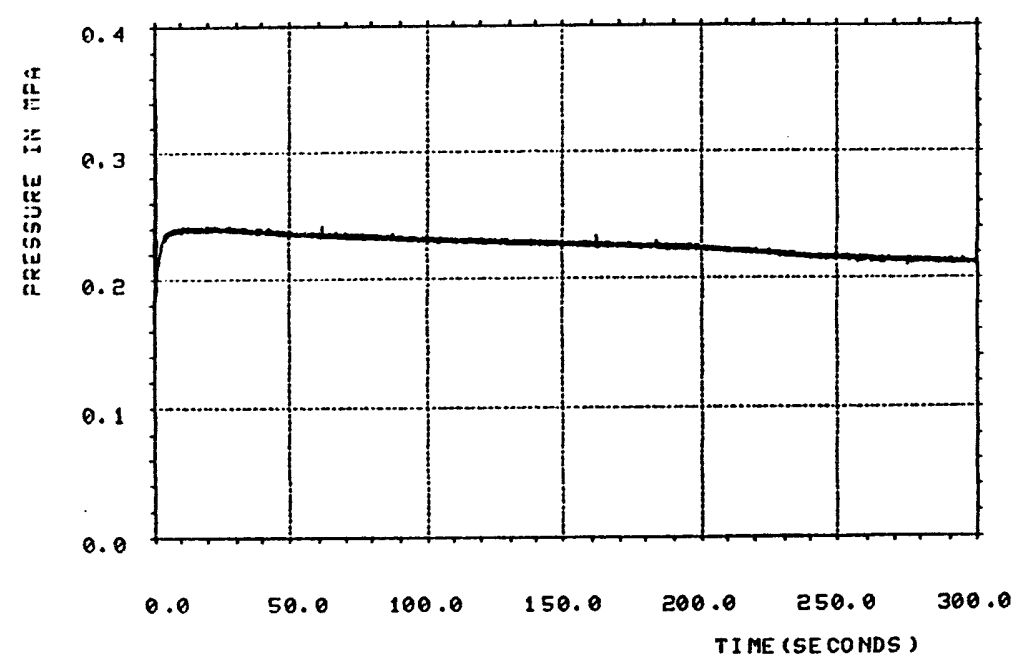

PTS-14, CCM-3, DATE 8/14/86

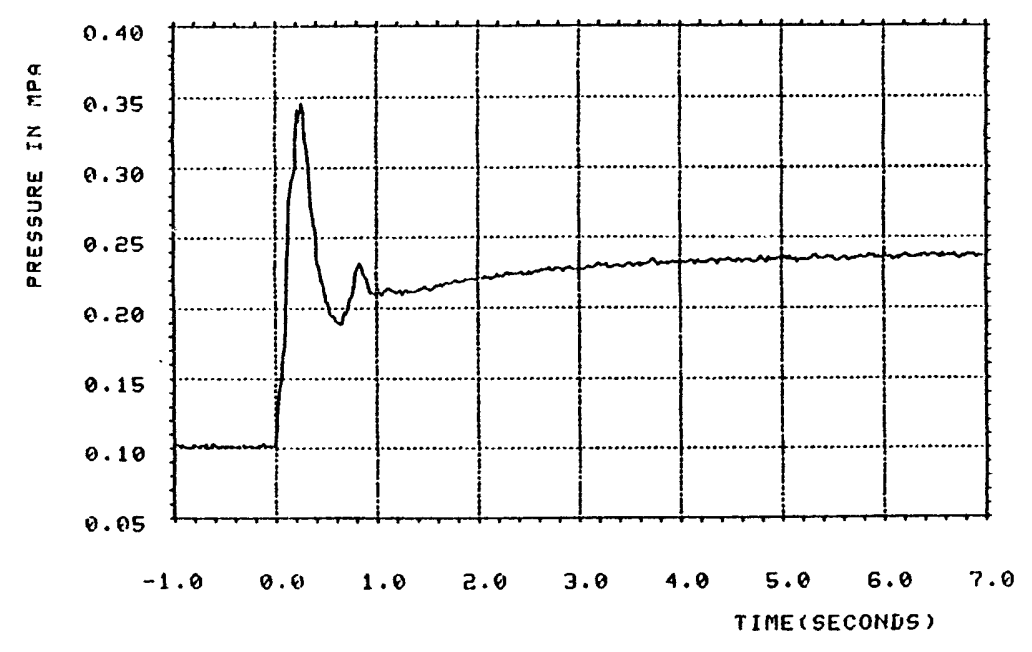

PTS-14, COH-3, LATE $8 / 14 / 86$.

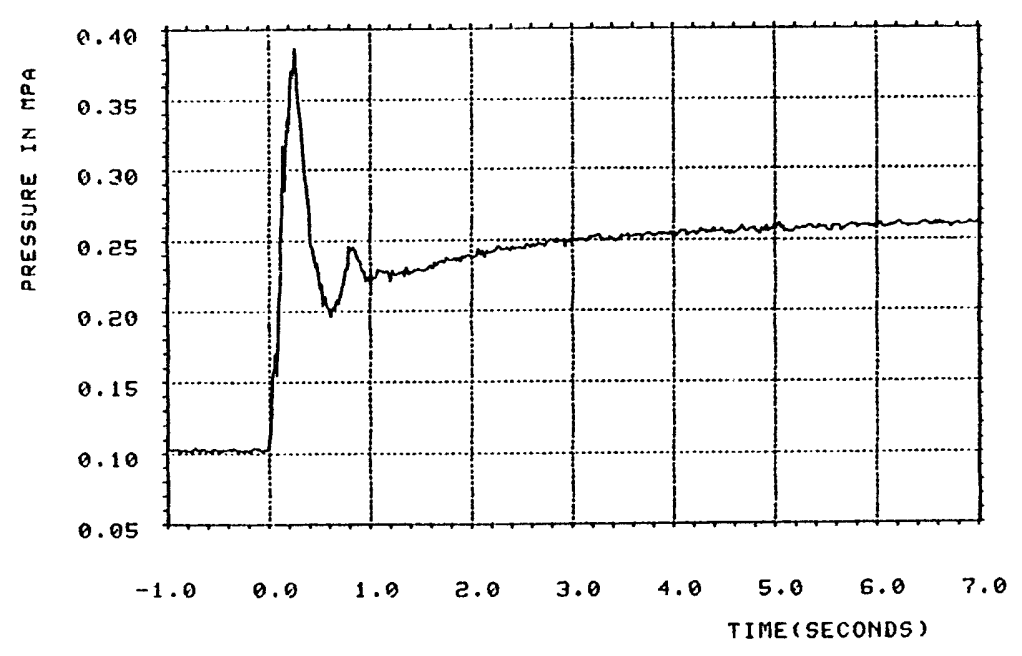

FTS-15, COH-3, WHTE 8/14/86. 


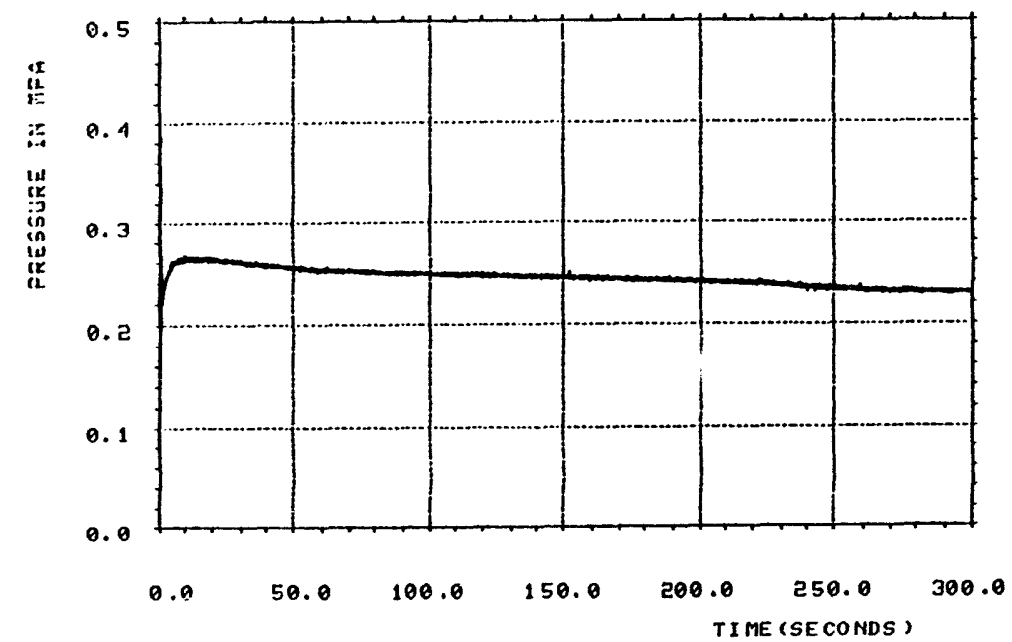

$\stackrel{1}{1}$

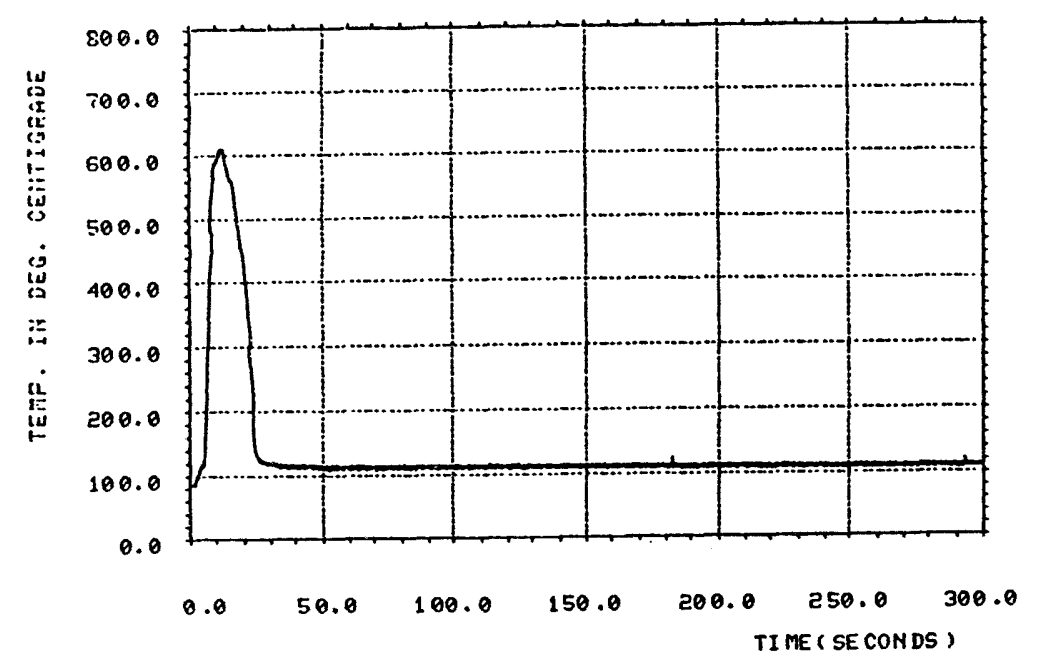

TC8-6, CCIM-3, DATE 8/14/86

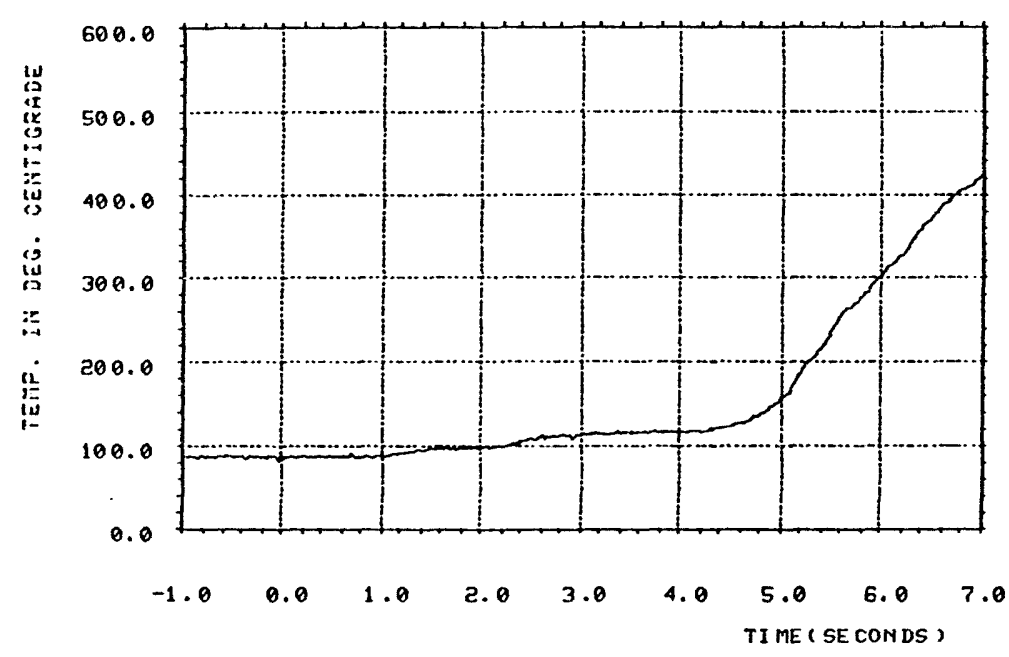

TC8-6, CCI-3, DATE 8/14/86

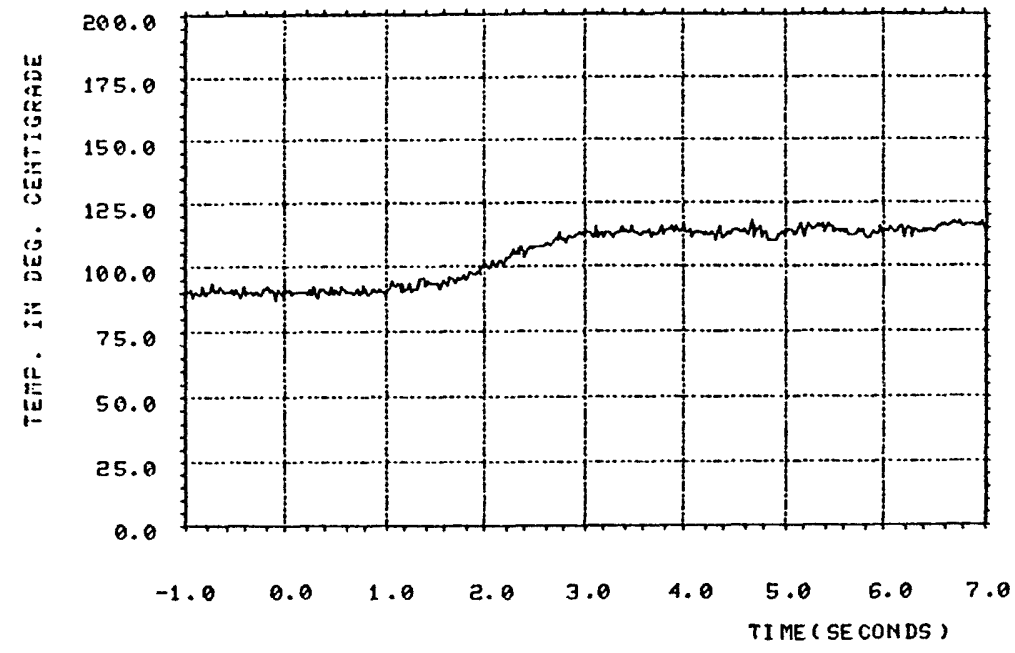

TC8-7. CCN-3, DATE 8/14/8S 


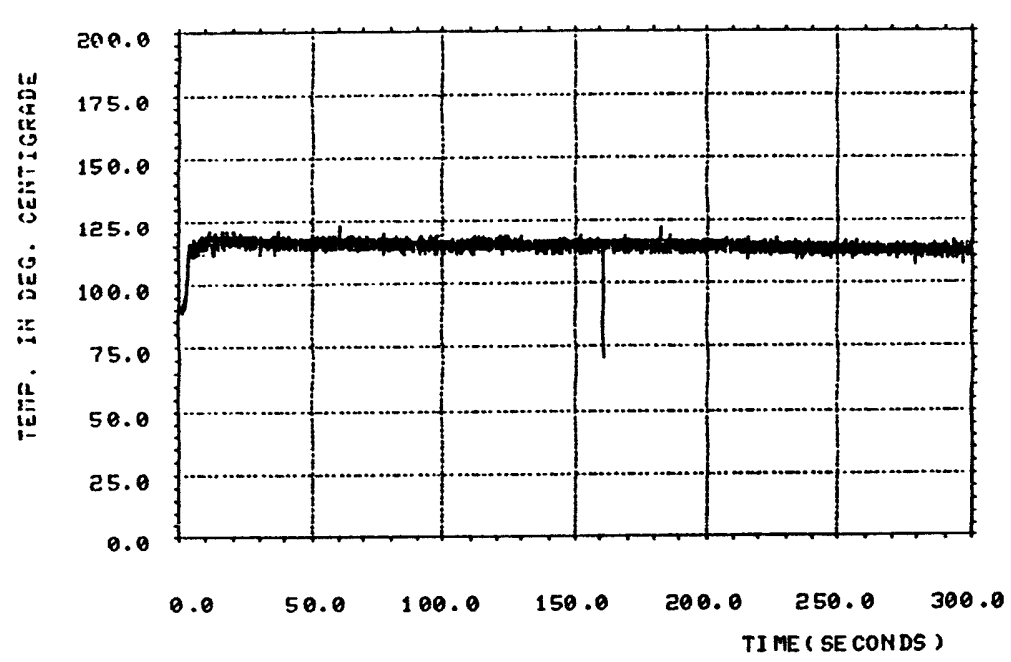

ó

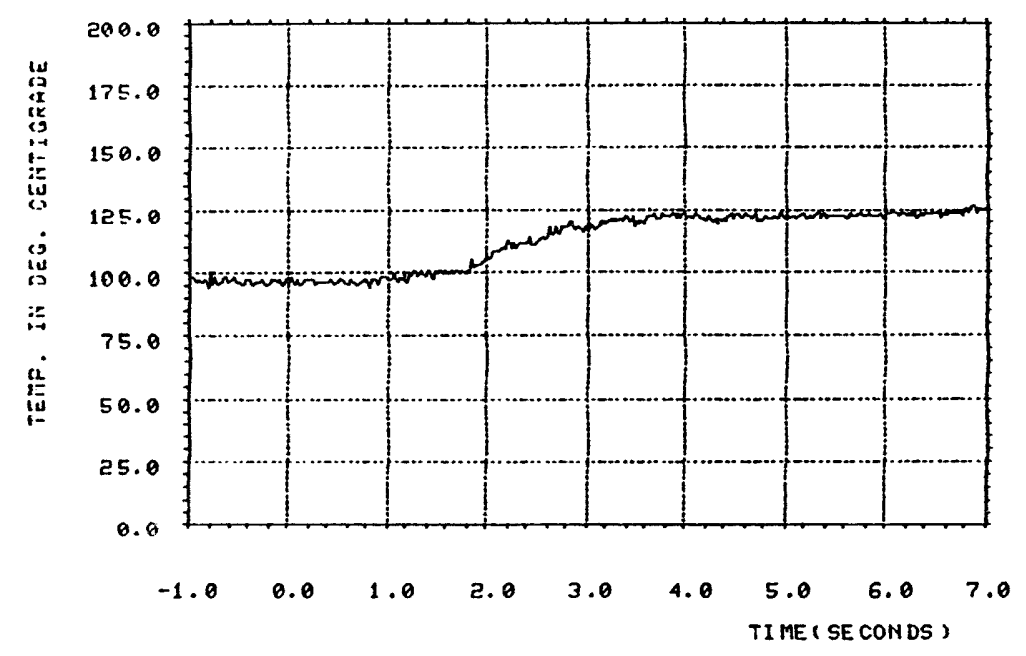

TC8-8, CCII-3, DATE $8 / 14 / 86$

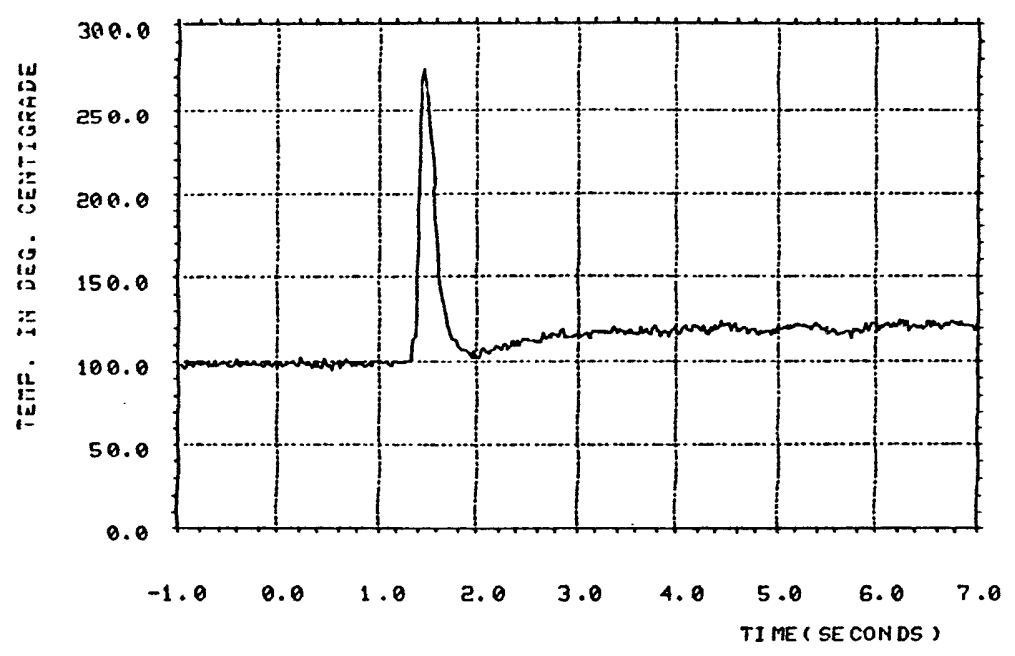

TCB-9, CCM-3, DATE 8/14/86 


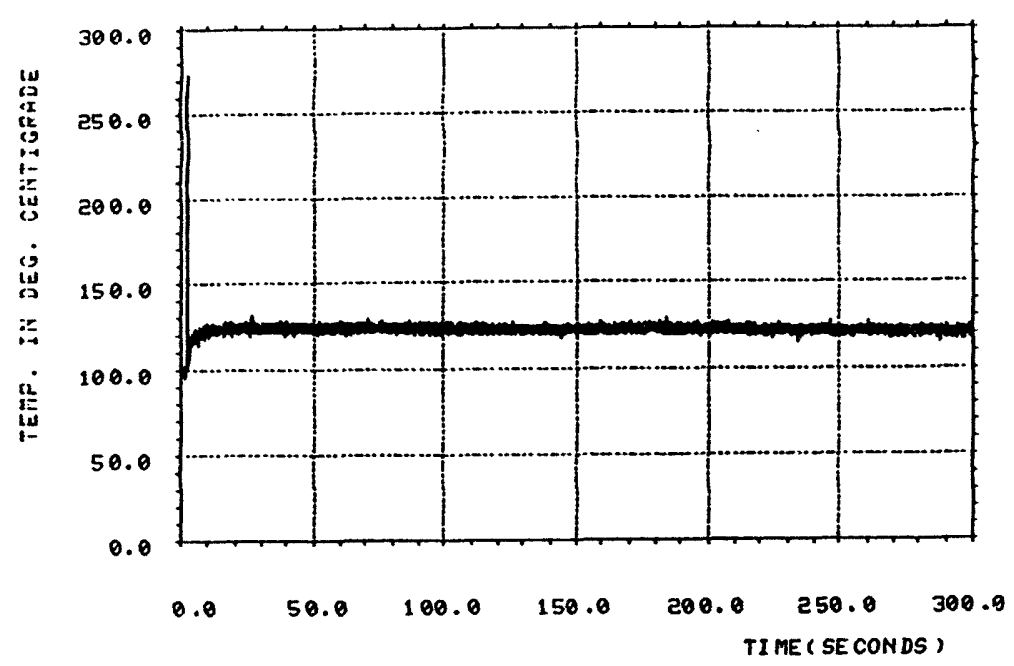

ó

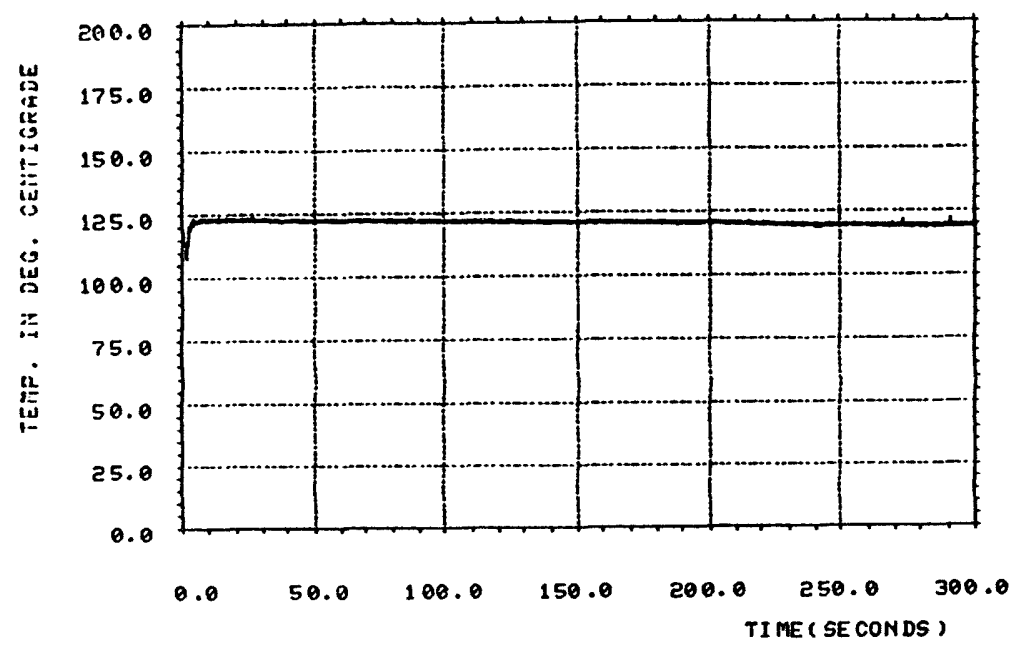

TC8-19, CCN-3, DATE 8/14/86

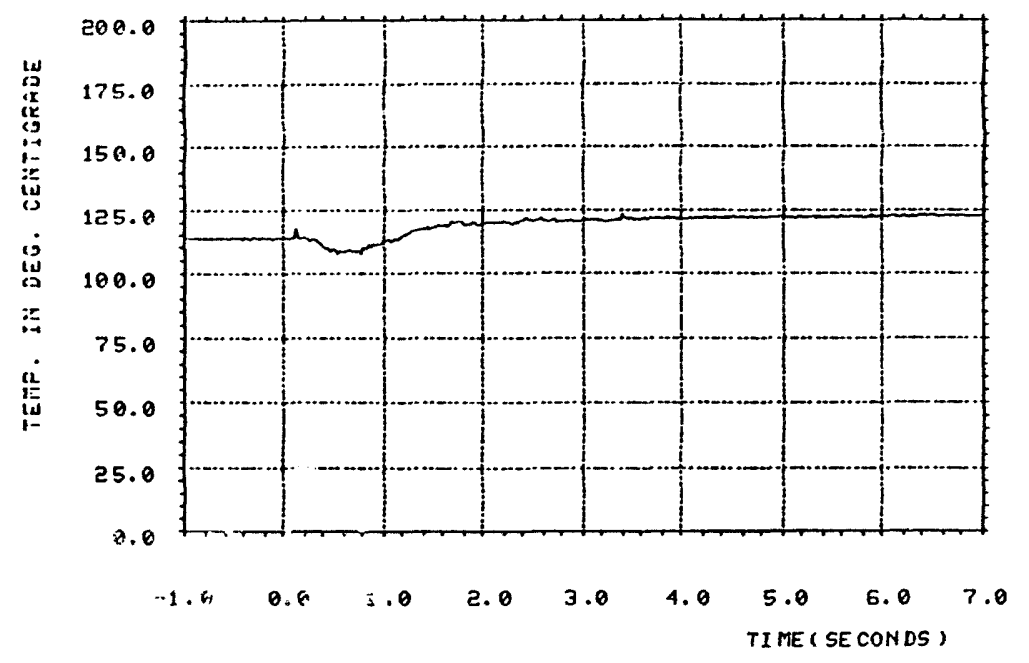

TC8-10, CCN-3, DATE 8/14/86

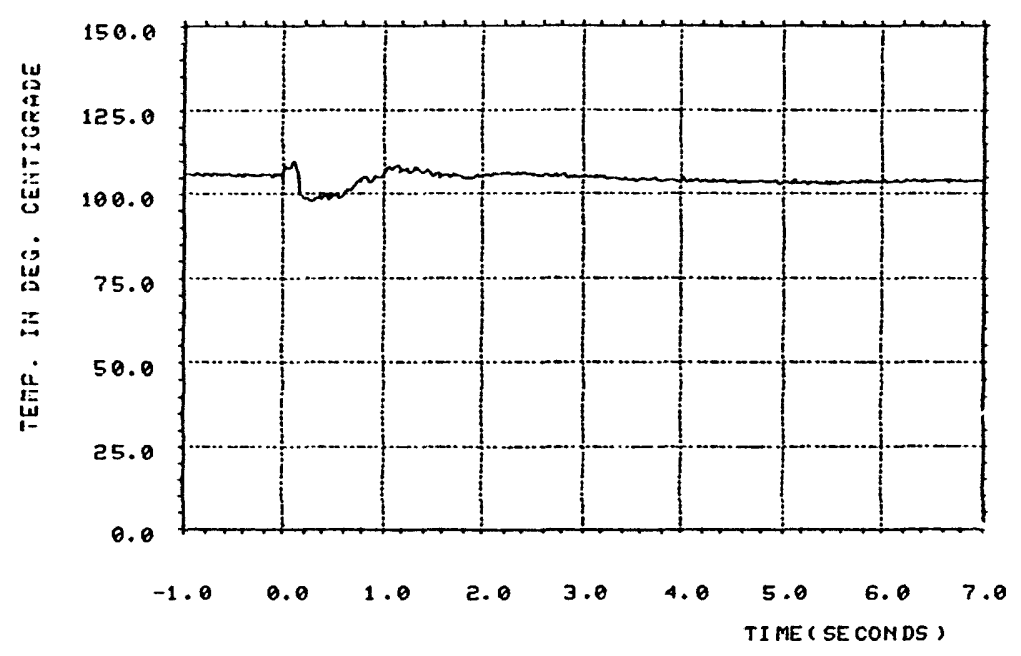

TC8-12, CCI-3, DATE 8/14/86 


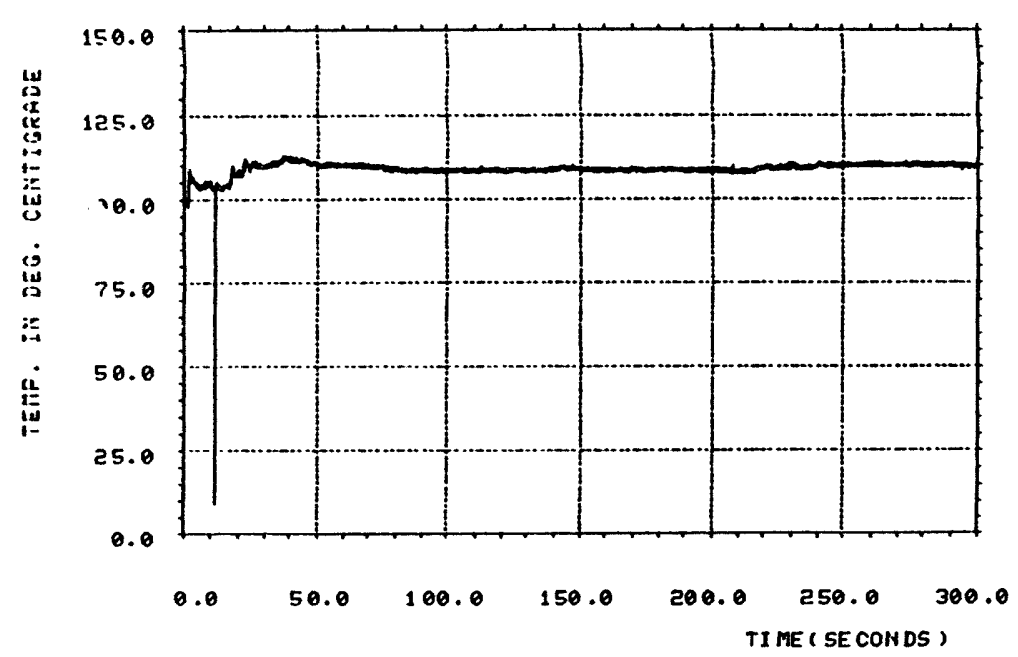

TC8-12, CCI1-3, DATE $8 / 14 / 86$

0

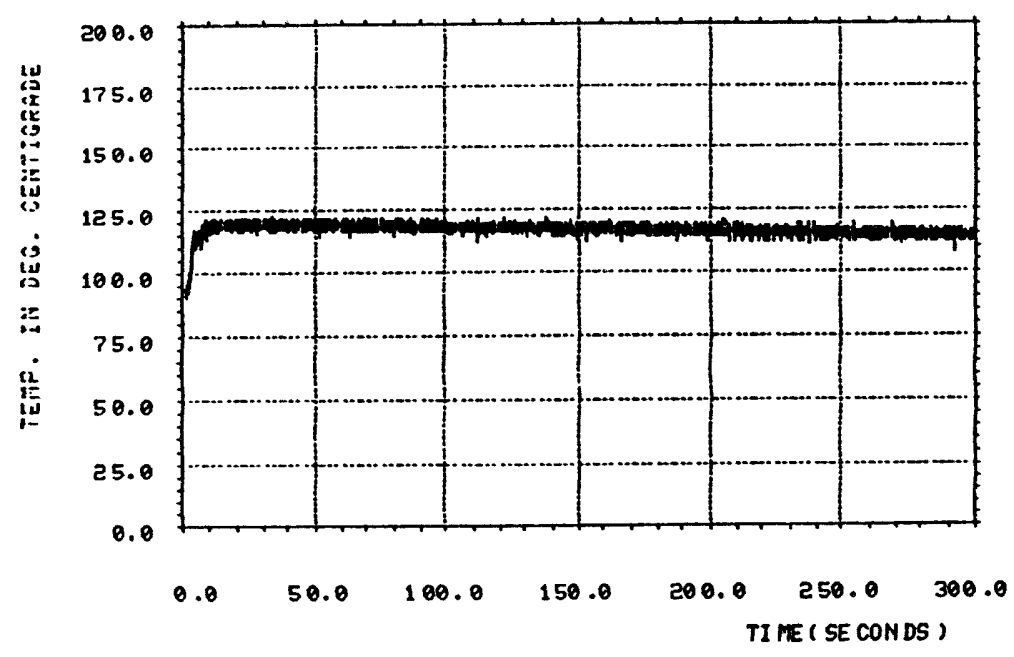

TC 8-16, CCM-3, DATE $8 / 14 / 86$

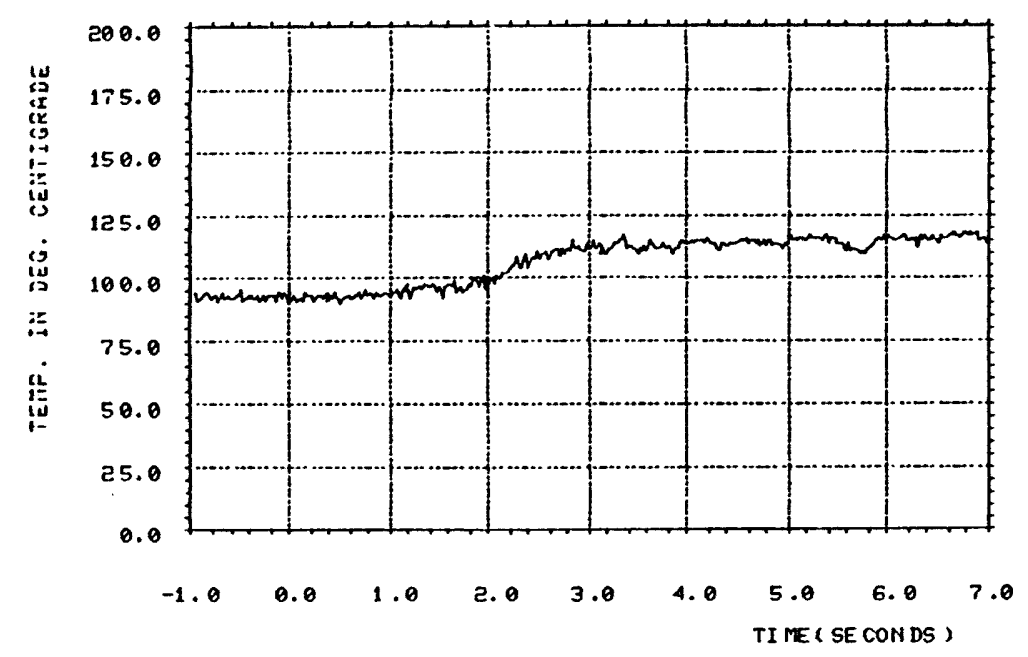

TC8-16, CCM-3, BATE 8/14/86

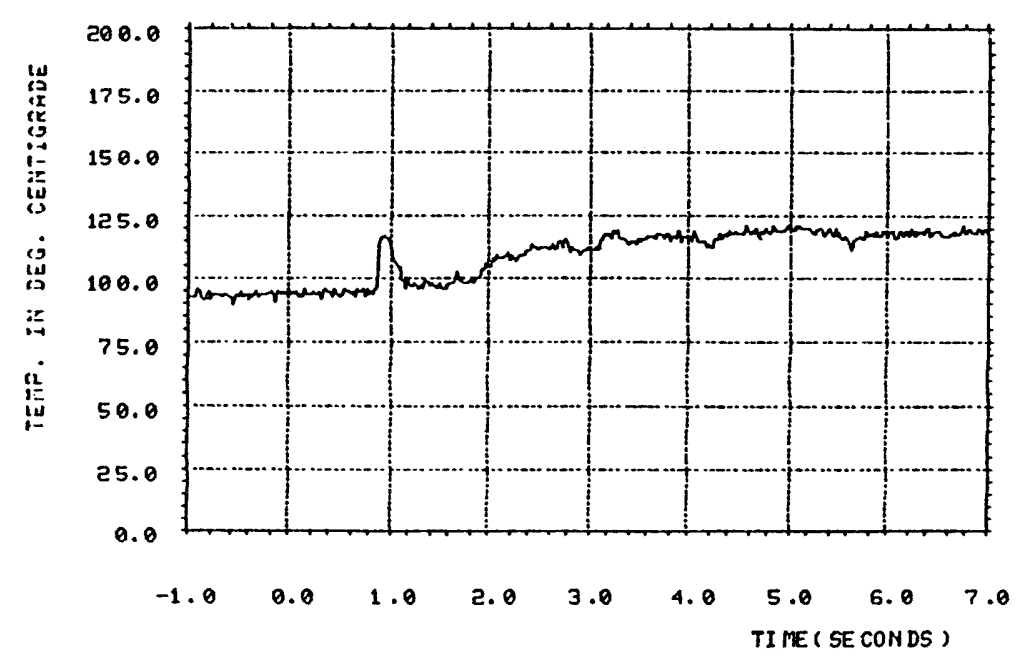

TC8-17. CCM-3, DATE 8/14/86 


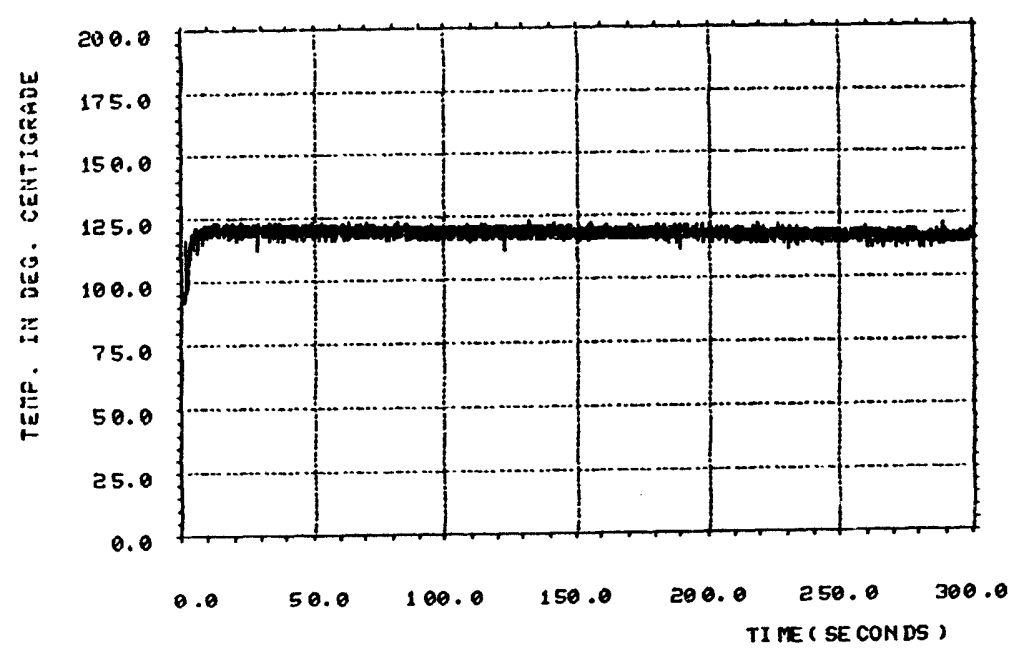

$\stackrel{\square}{\vdots}$

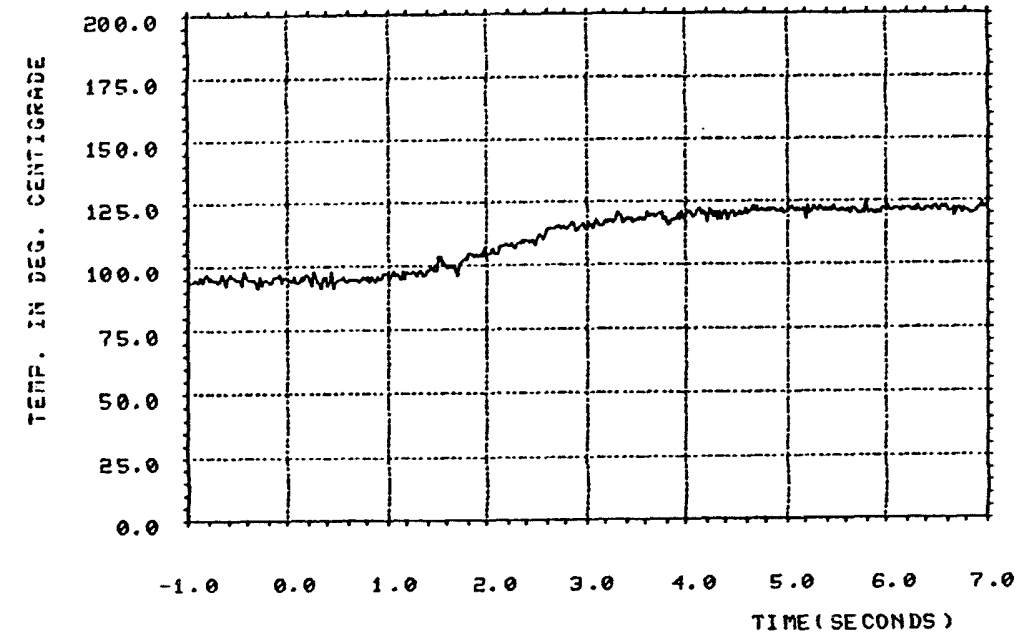

TC8-18, CCM-3, DATE 8/14/86

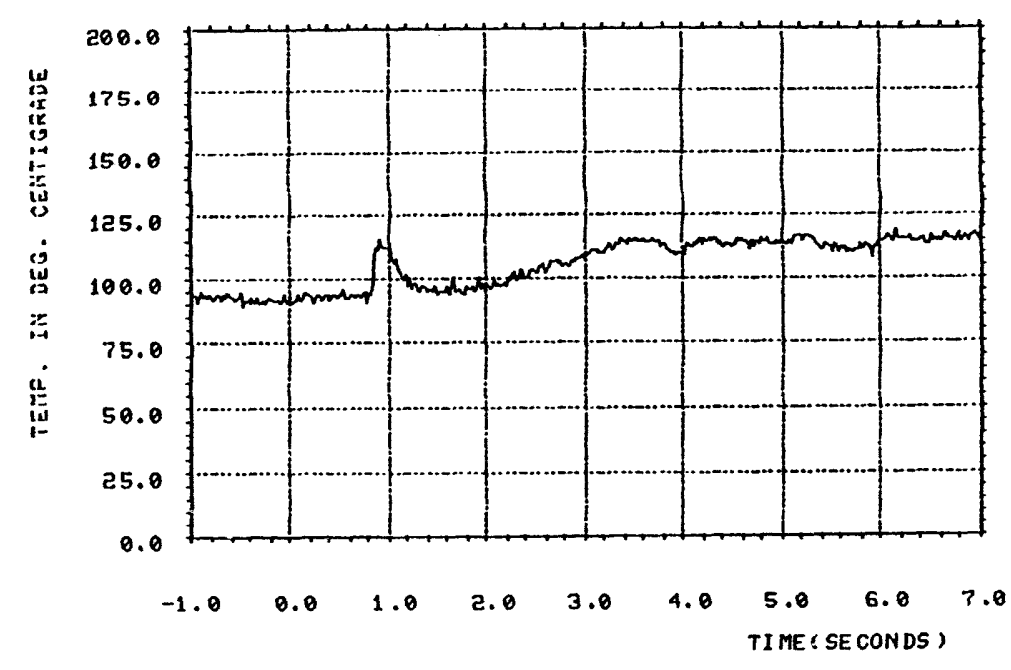

TC8-19, CCH-3, DATE 8/14/86 


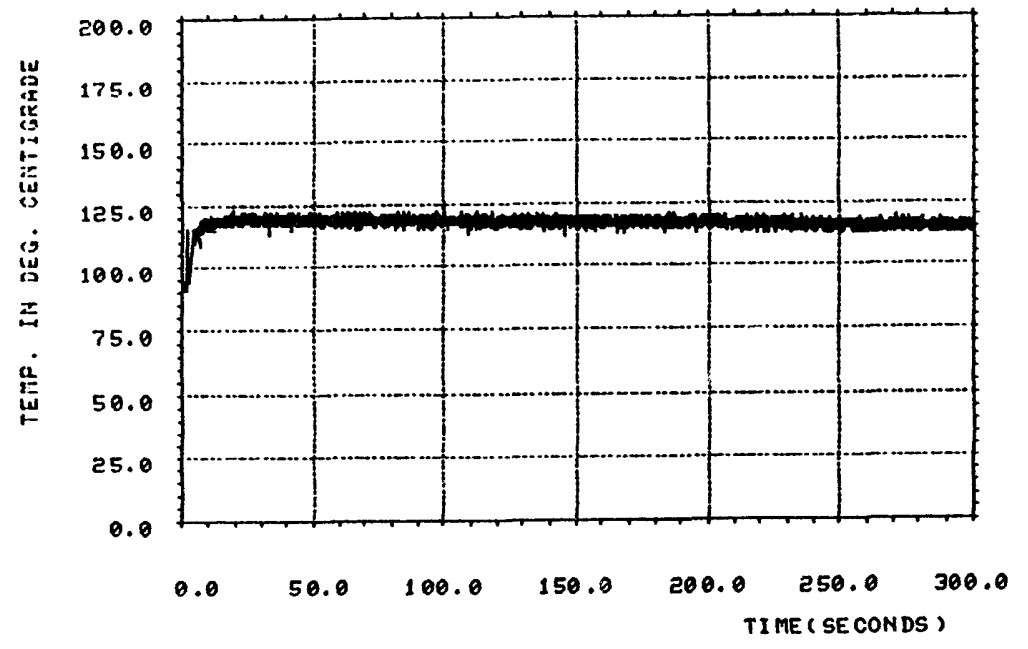

$\stackrel{1}{n}$

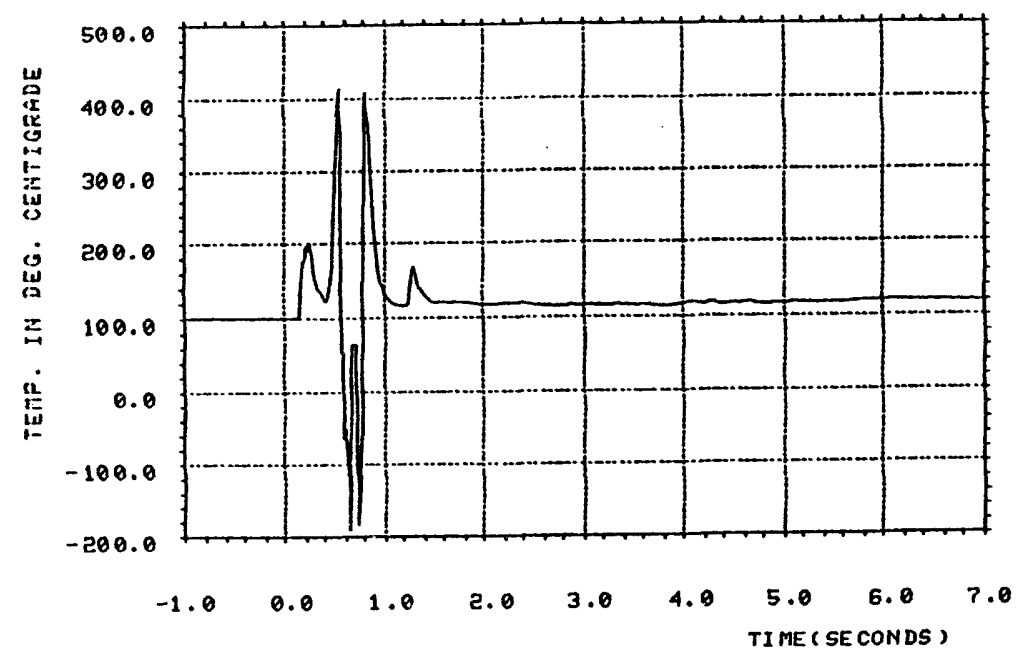

TC8-21, CCM-3, DATE 8/14/86

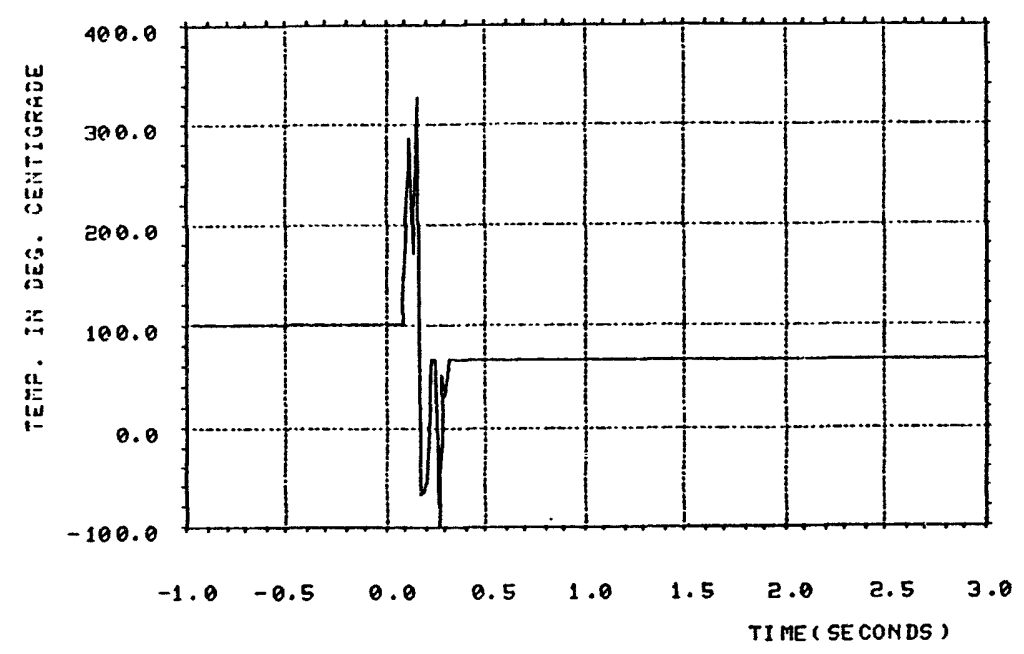

TC8-20. CCN-3, DATE $8 / 14 / 86$

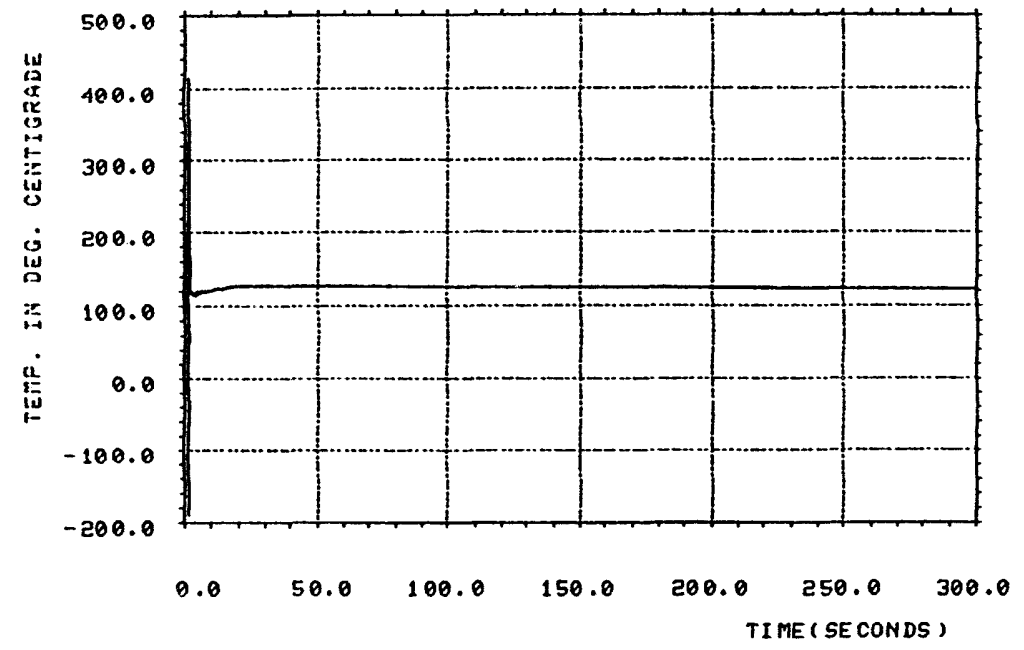

TC8-21, CCM-3, DATE $8 / 14 / 86$ 

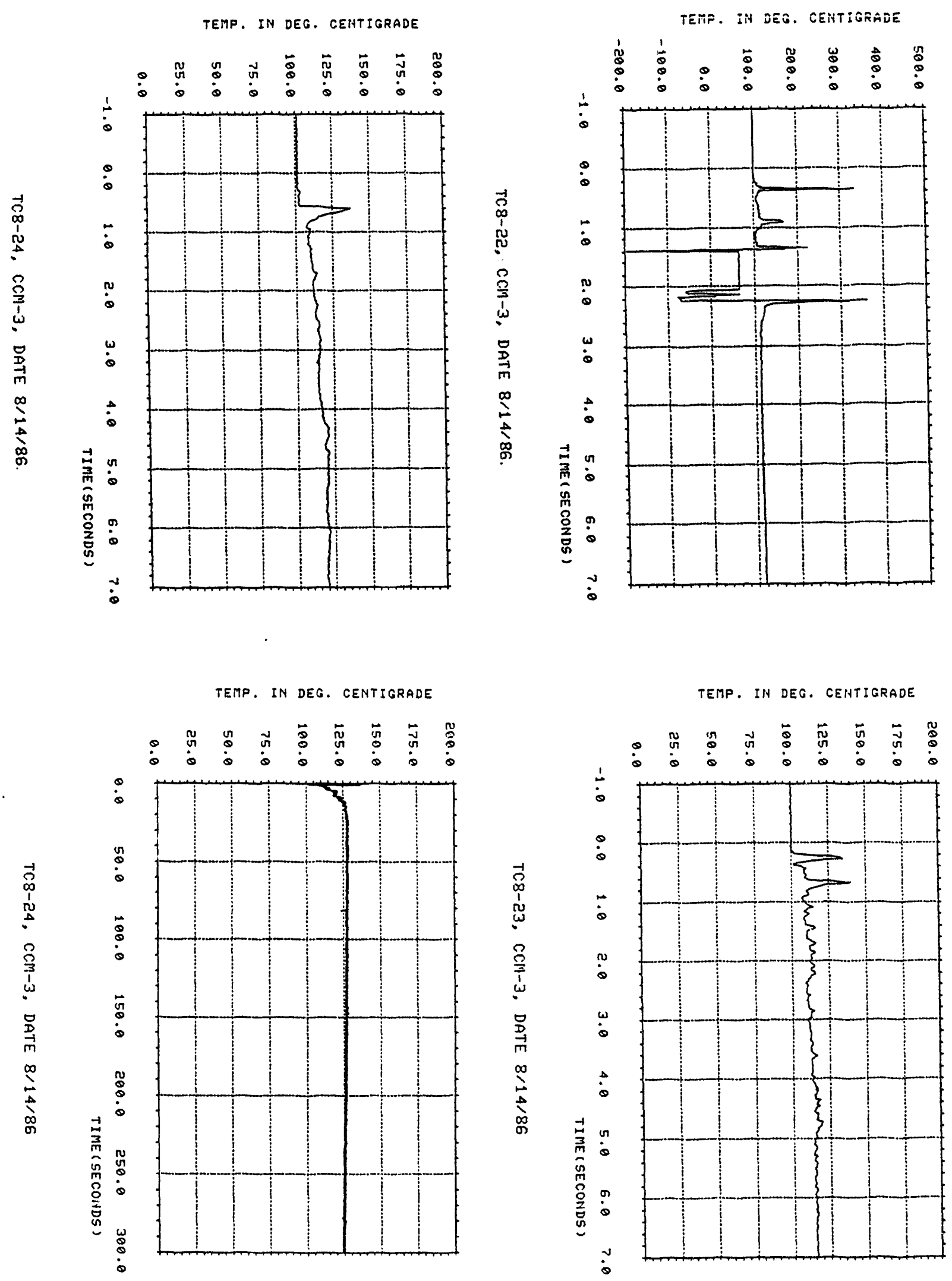


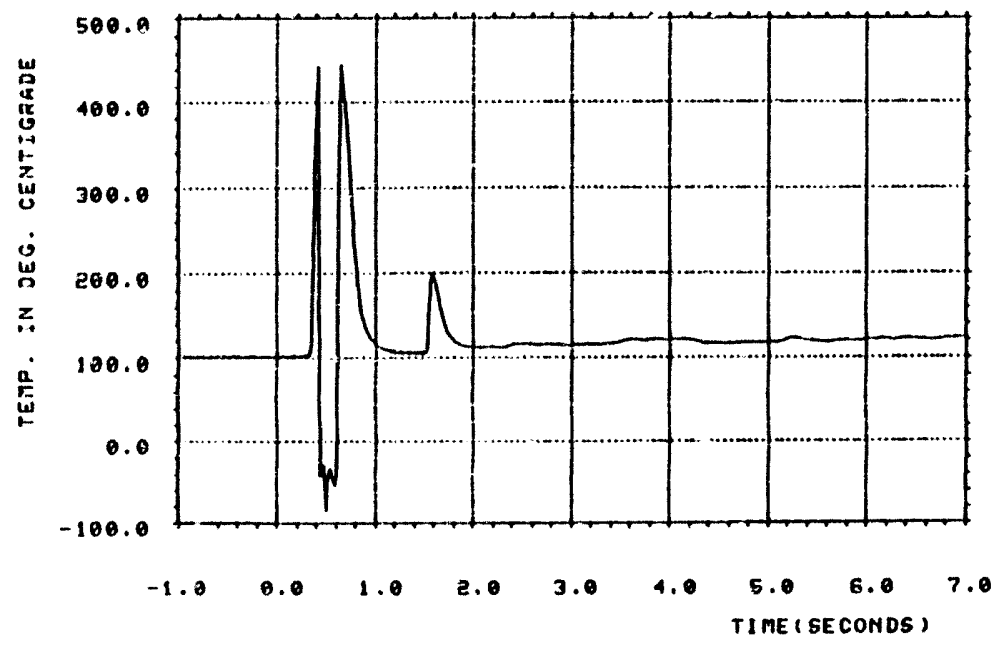

$\stackrel{0}{1}$

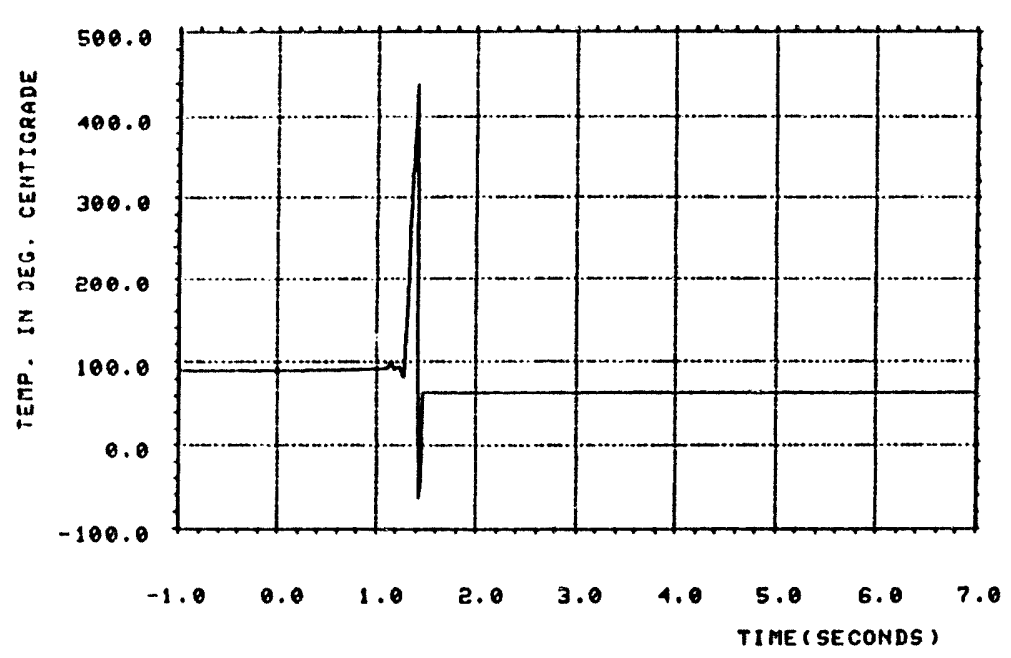

TC8-26, CCM-3, DATE 8/14/86

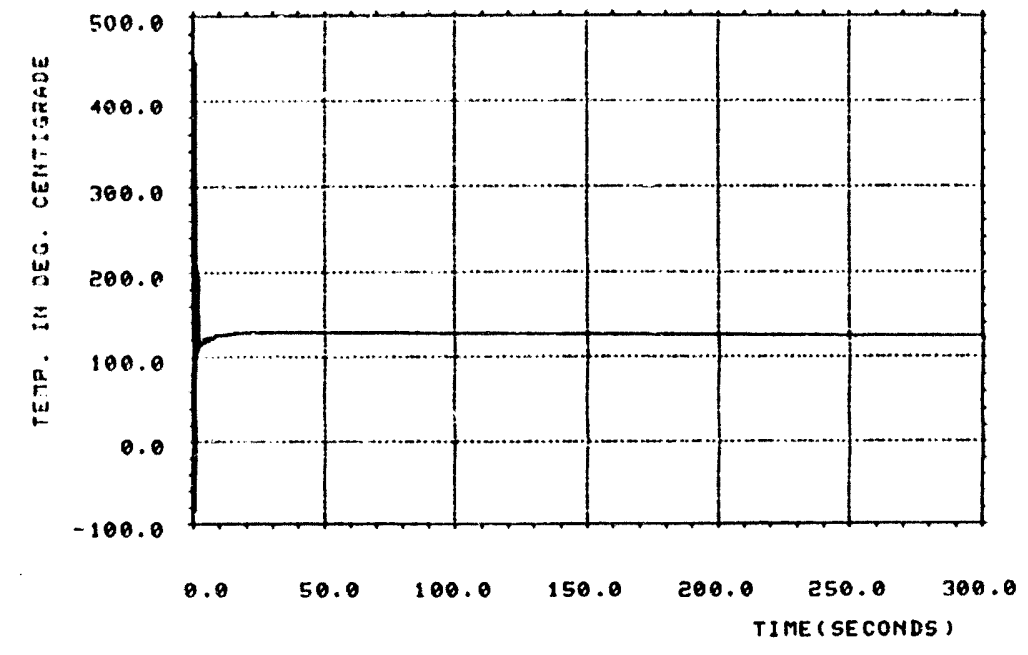

TC8-25, CCM-3, DATE $8 / 14 / 86$

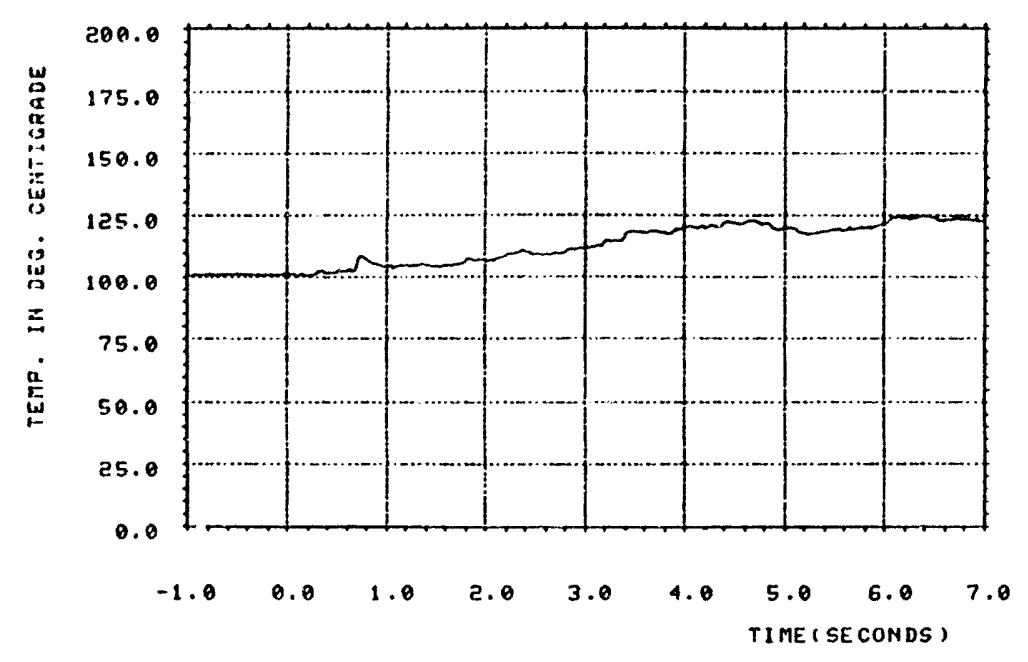

TC8-27, C.CH-3, DATE 8/14/86 


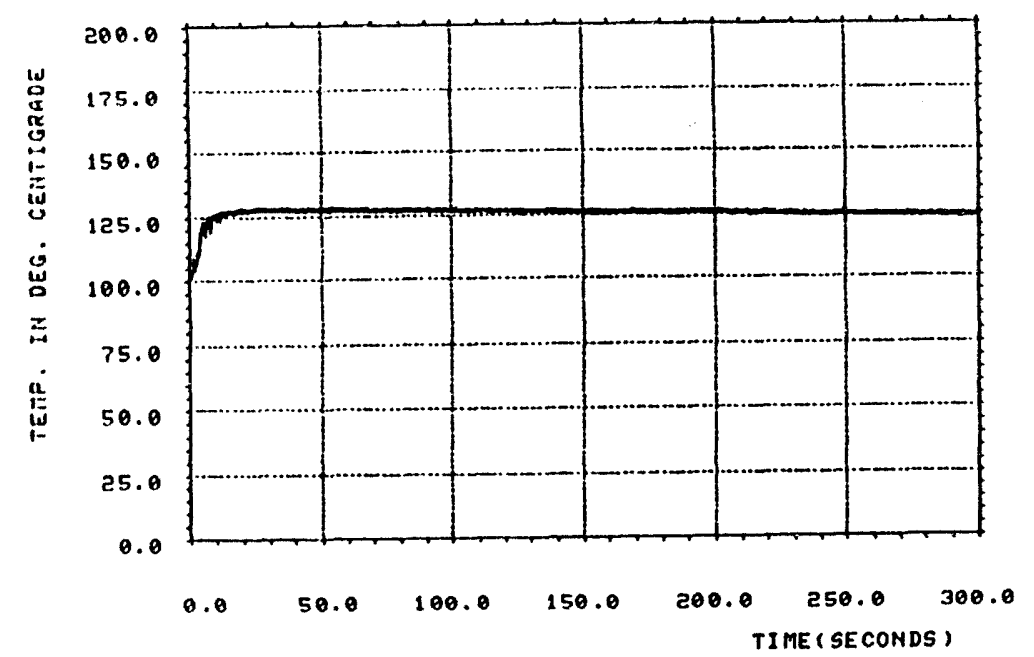

品

TC8-27, CCM-3, DATE 8/14/86

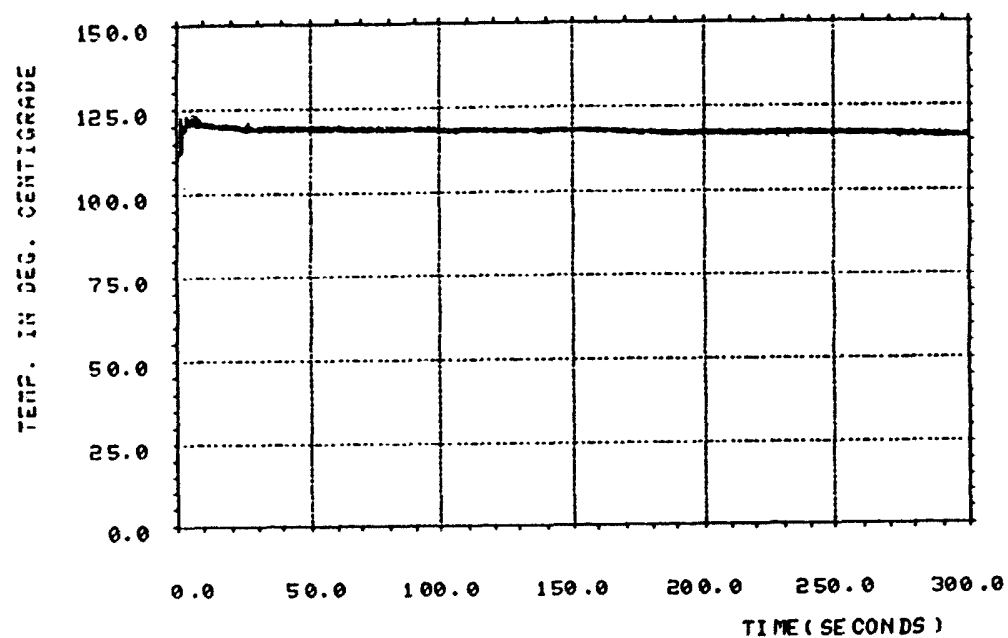

TC8-28, CCM-3, DATE $8 / 14 / 86$

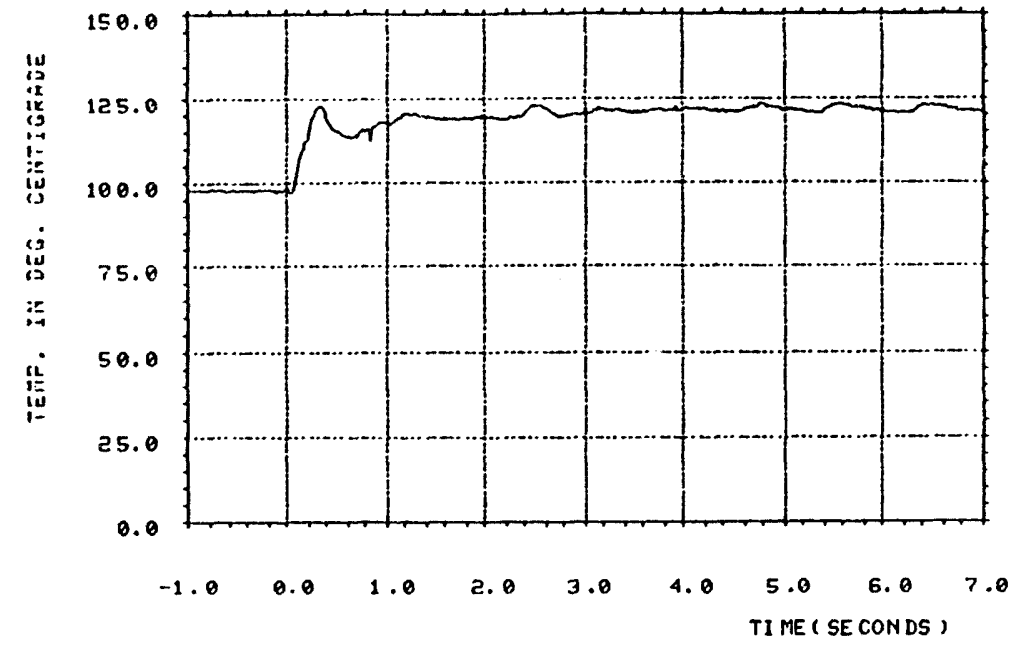

TC8-28, CCM-3, DATE 8/14/86

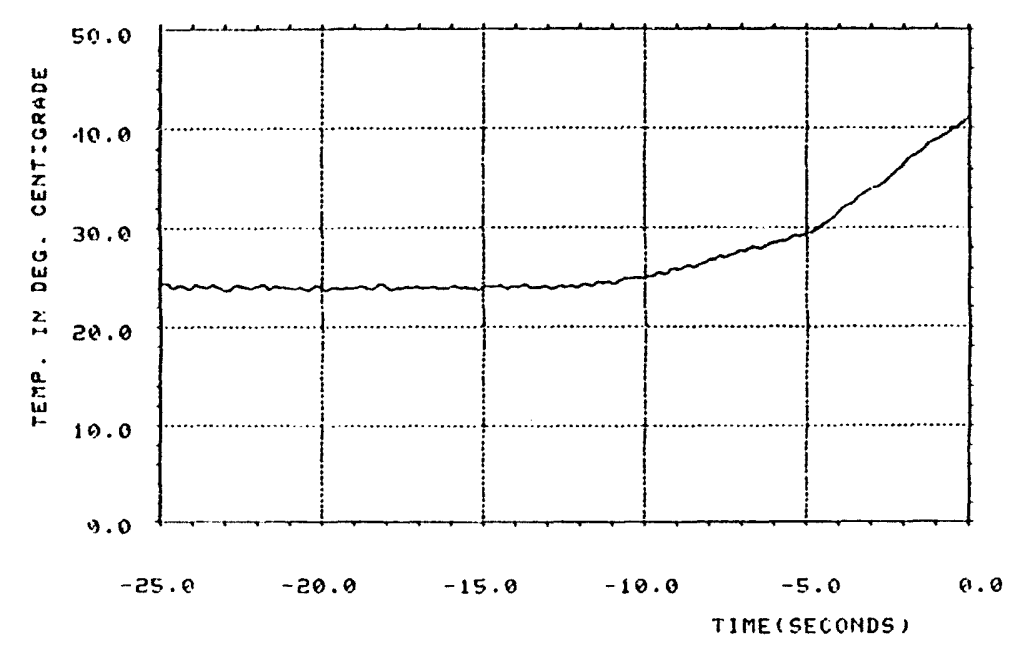

TCQ-2. VCH-3, DATE 8/14/86 


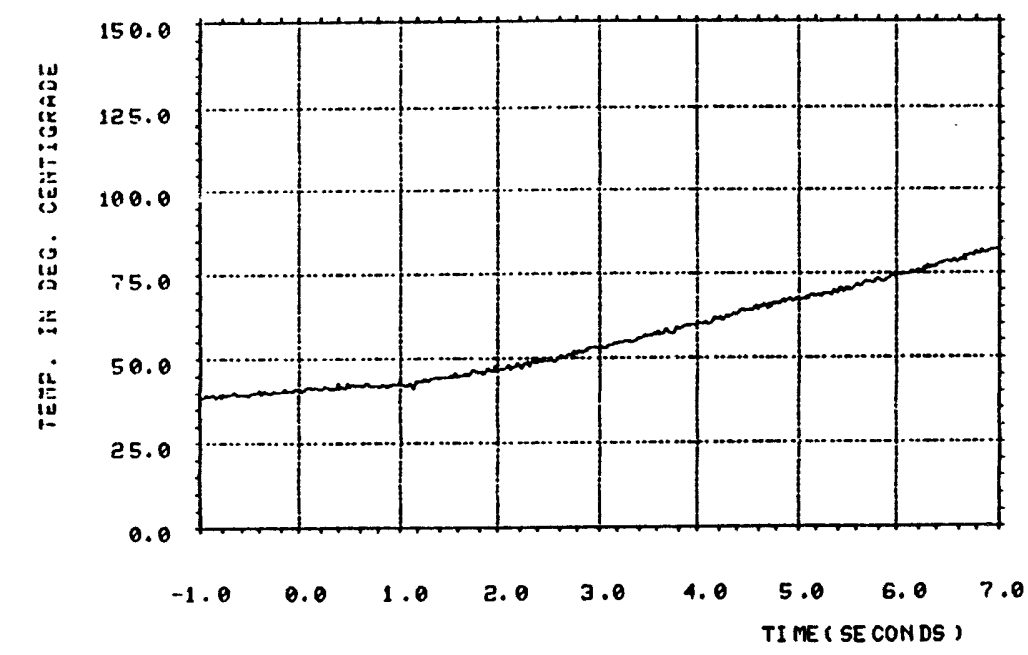

虽

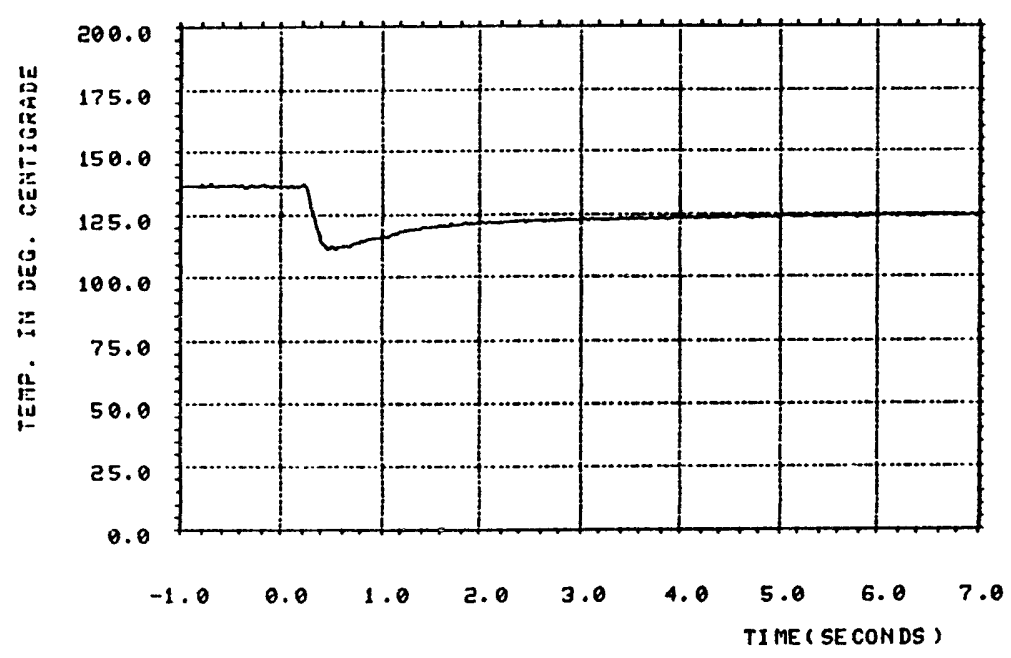

TC12-1, CCI1-3, DATE 8/14/86

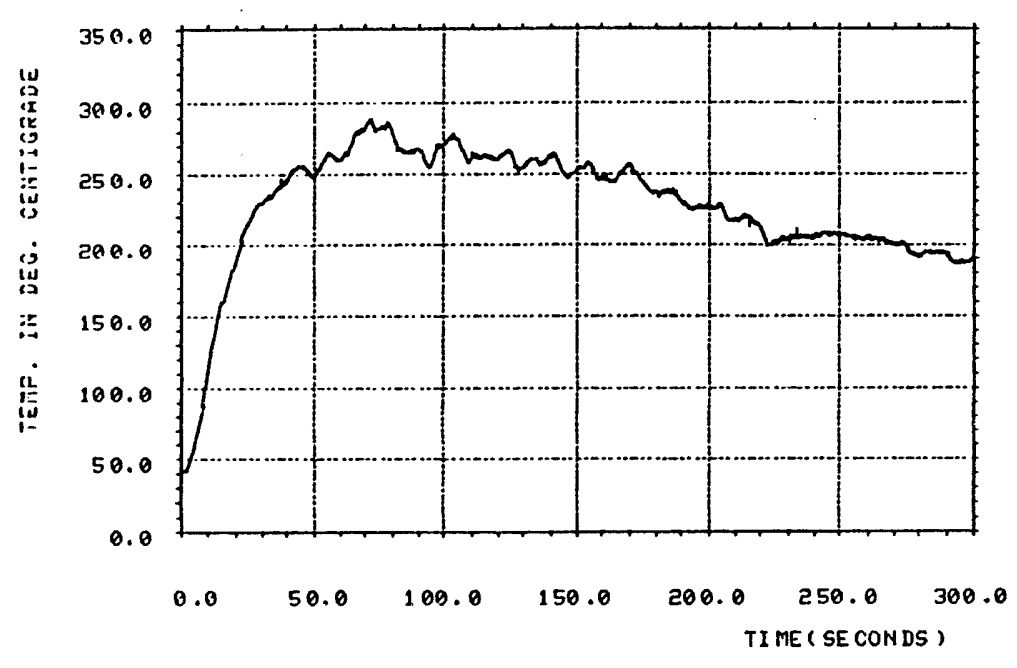

TC9-2, CCM-3, DATE $8 / 14 / 86$

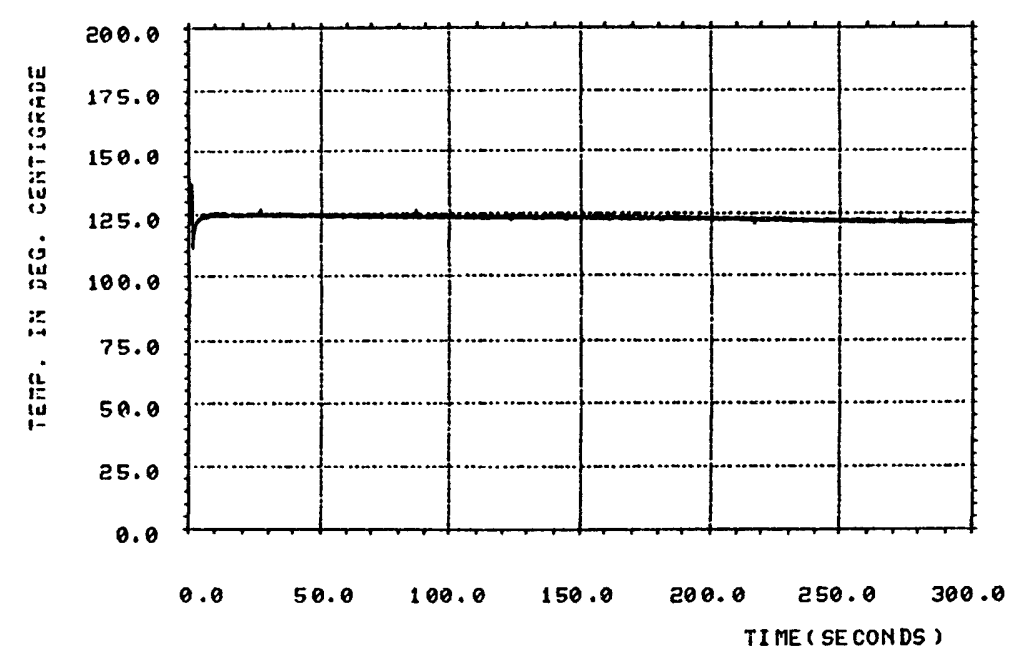

TC12-1, CCN-3, DATE 8/14/86 
( SQNOJ 3S) 3WI 1

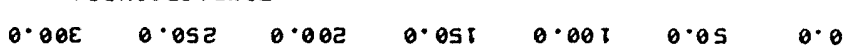

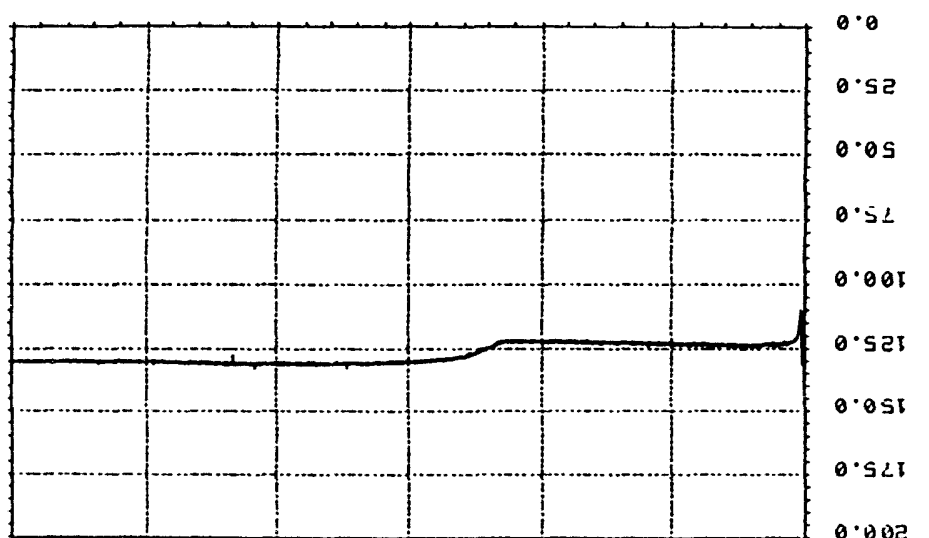

$28 /,[/ 8 \quad \exists 7 \theta d \cdot \varepsilon-N 30 \cdot 5-5107$

( SanOO 3S ) 3W IL

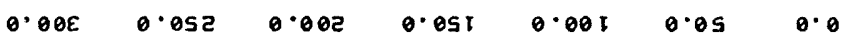

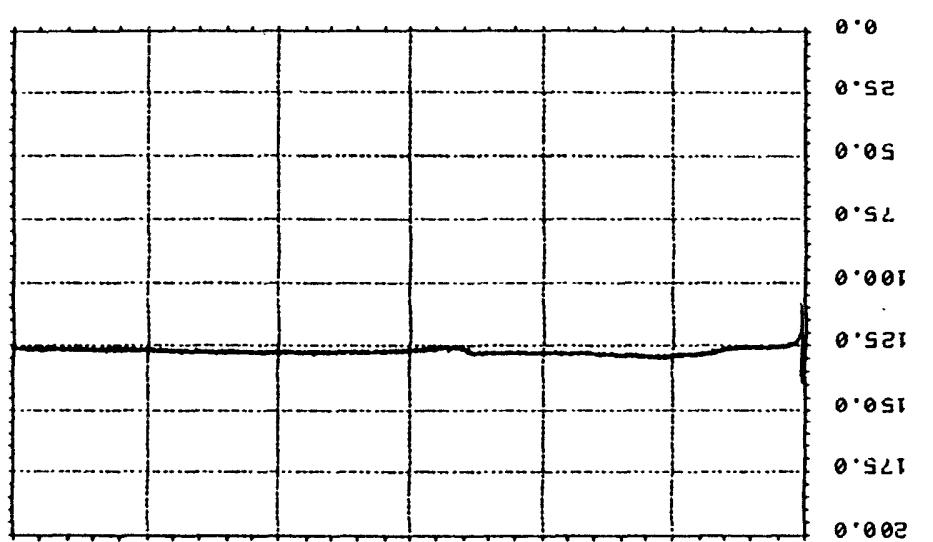

( SQNOJ32 ) 3W IN

$0.2 \quad 0.9 \quad 0.5 \quad 0.0 \quad 0 . \varepsilon \quad 0.2 \quad 0.1 \quad 0.0 \quad 0.1-$

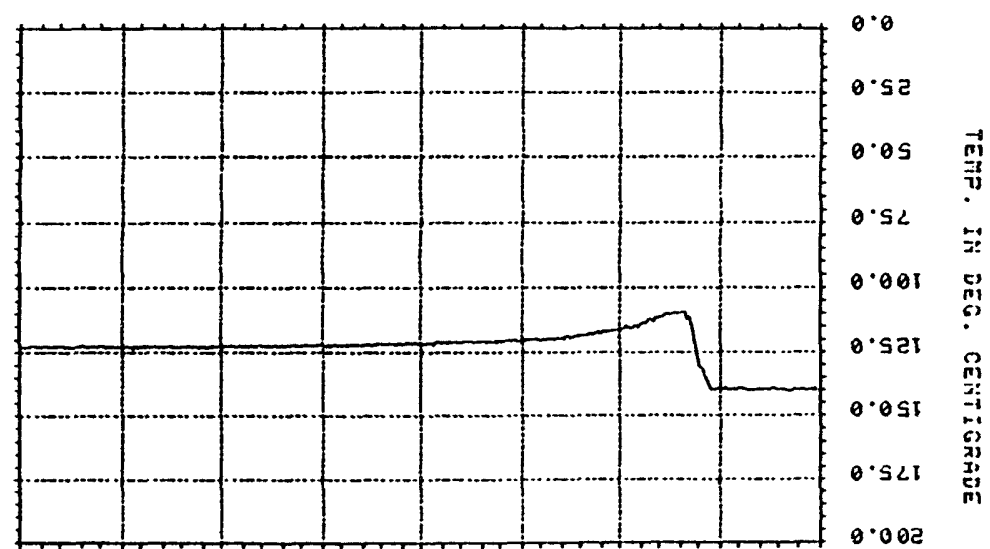

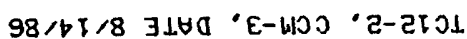

$\bar{a}$

(SANOJ 3S) JW IL

$\begin{array}{lllllllll}0.2 & 0.9 & 0.5 & 0.6 & 0 . \varepsilon & 0.5 & 0.1 & 0.0 & 0.1-\end{array}$

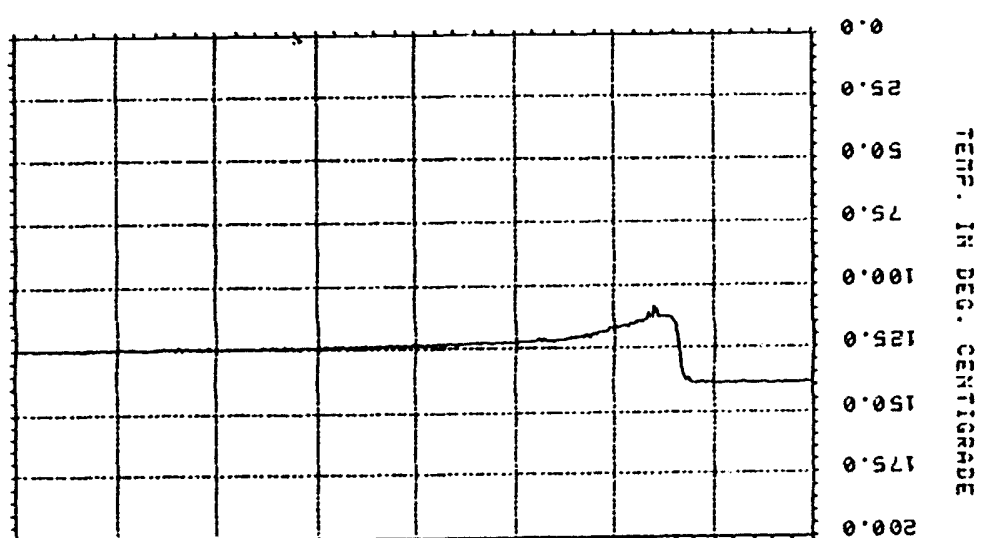




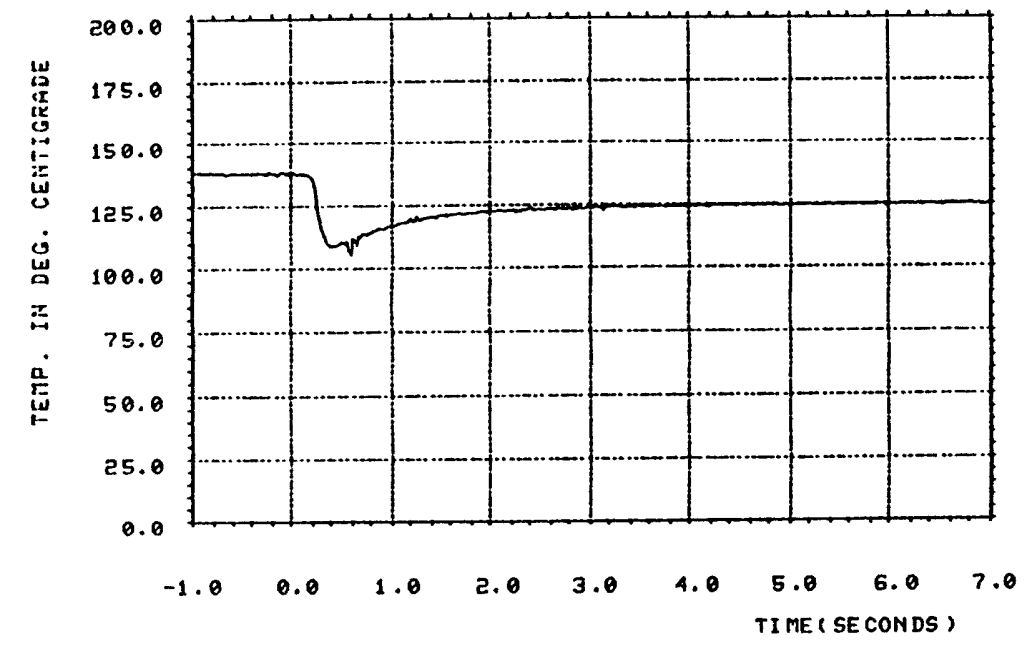

$\stackrel{?}{\infty}$

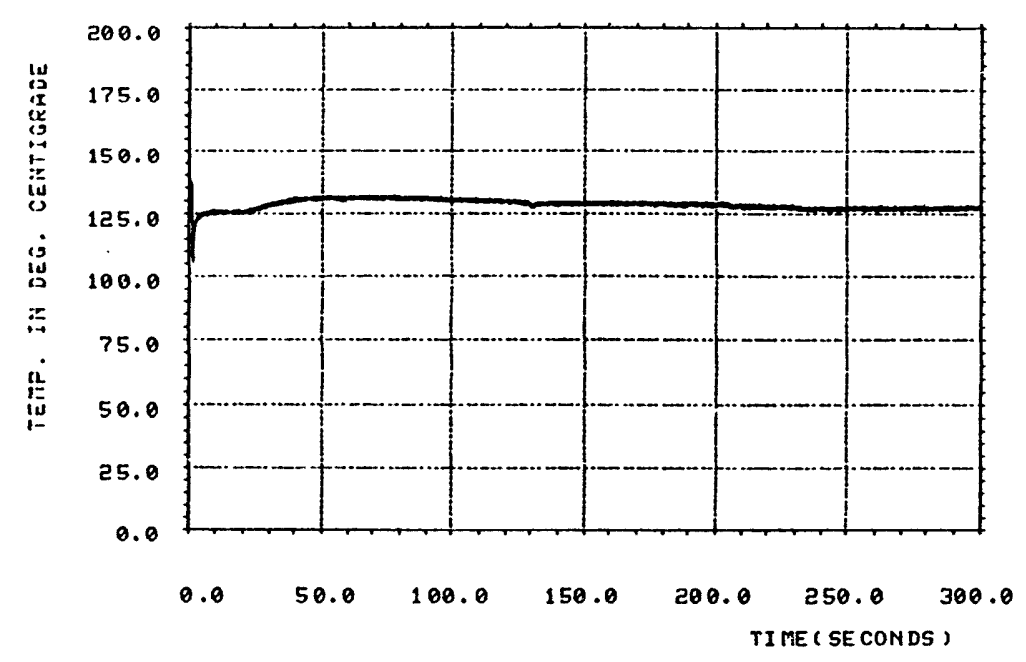

TC12-4, CCN-3, DATE $8 / 14 / 86$

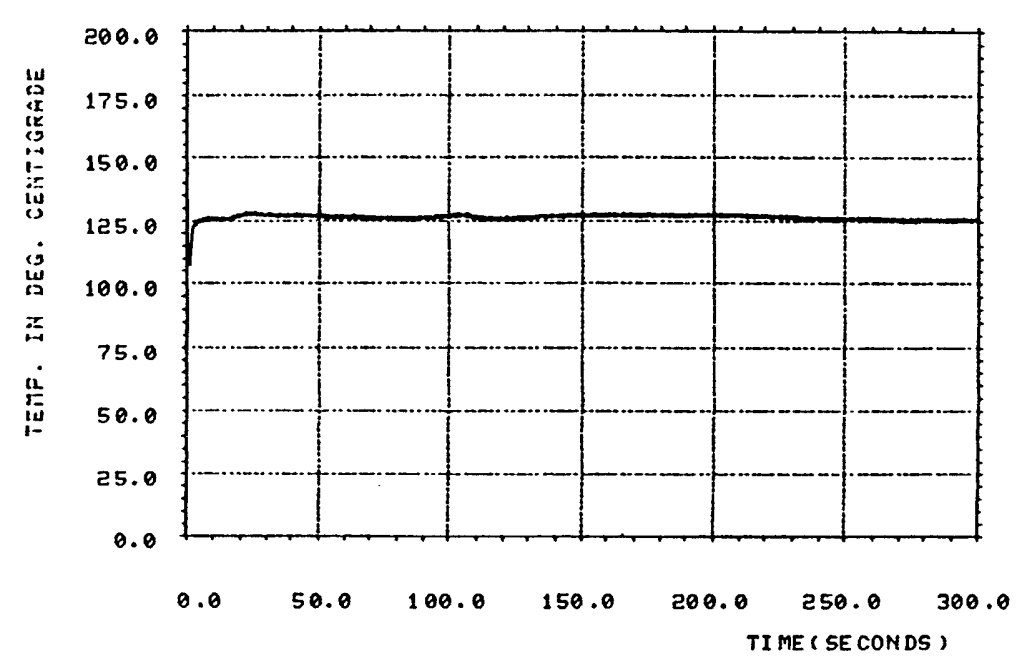

TC12-5, CCM-3, DATE $8 / 14 / 86$

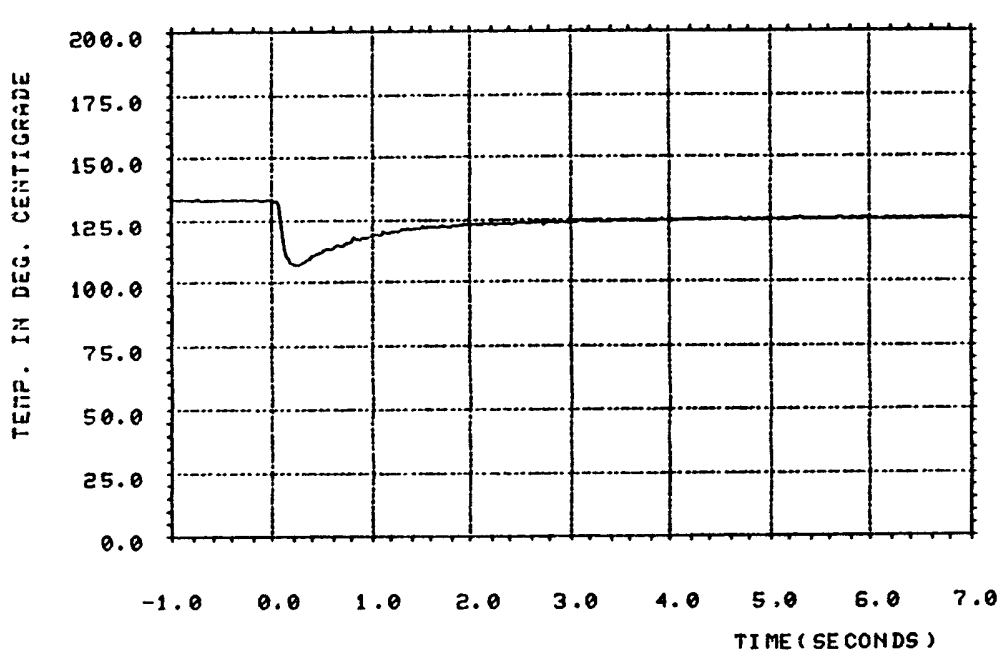

TC 12-5, CCM-3, DATE 8/14/86 


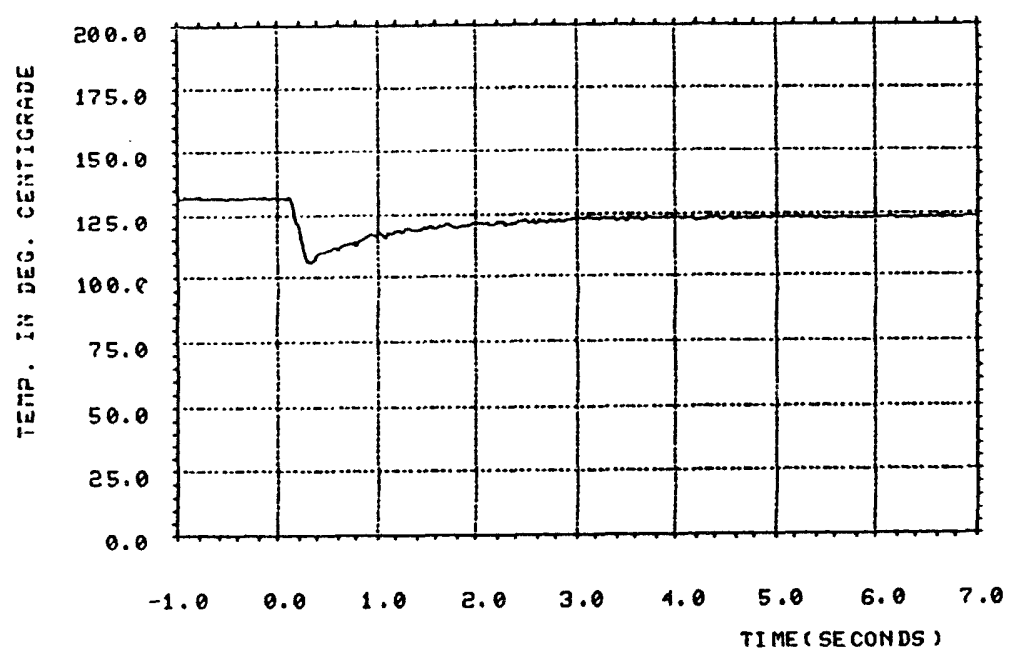

$\frac{1}{6}$

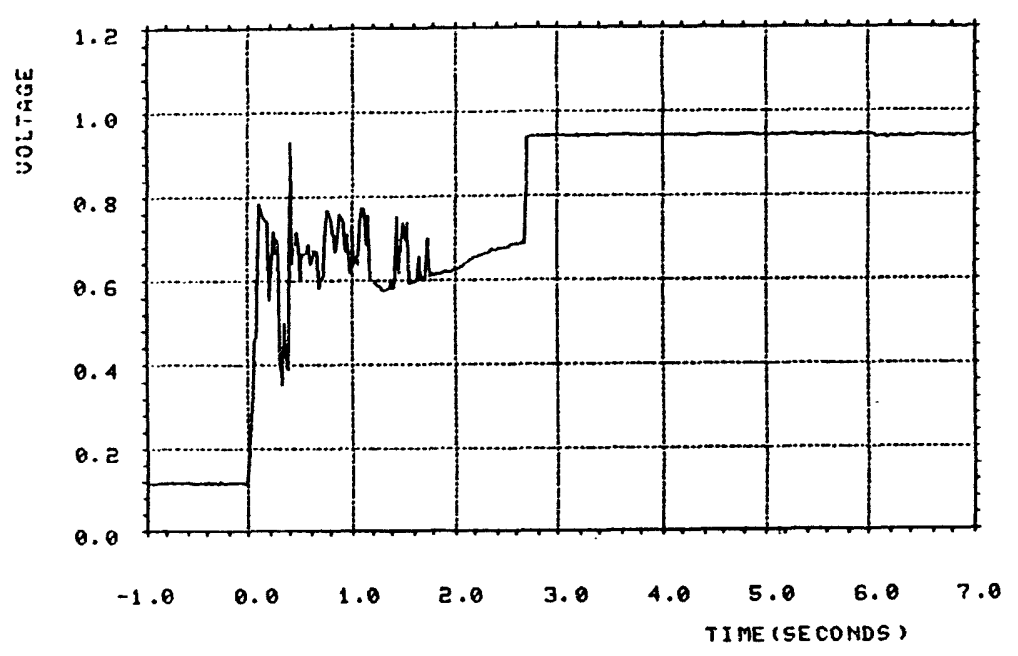

LP-1, CCM-3, DATE 8/14/86

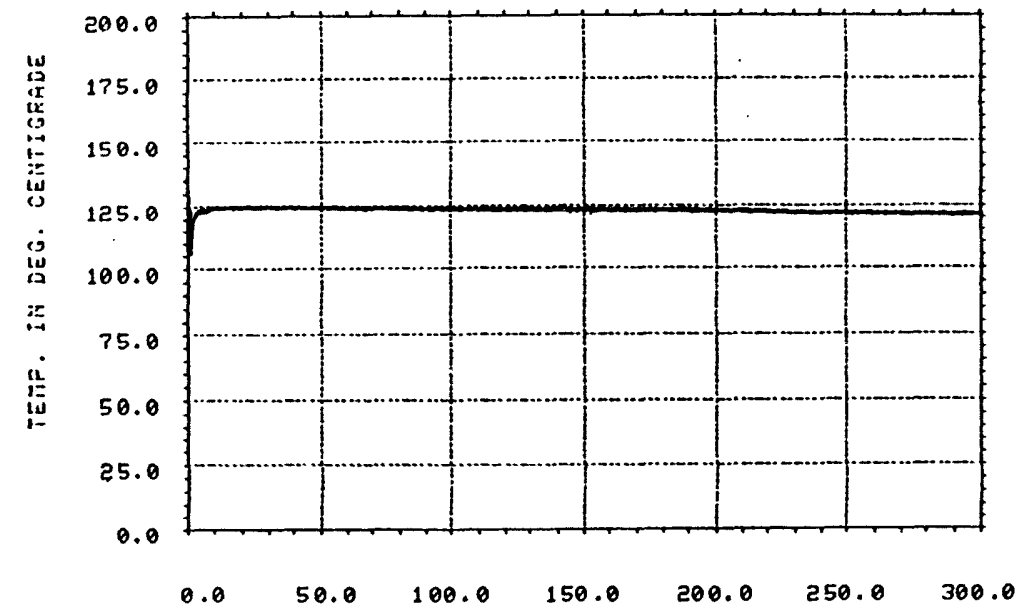

TIME ( SE CONDS )

TC12-6, CCM-3, DATE 8/14/86

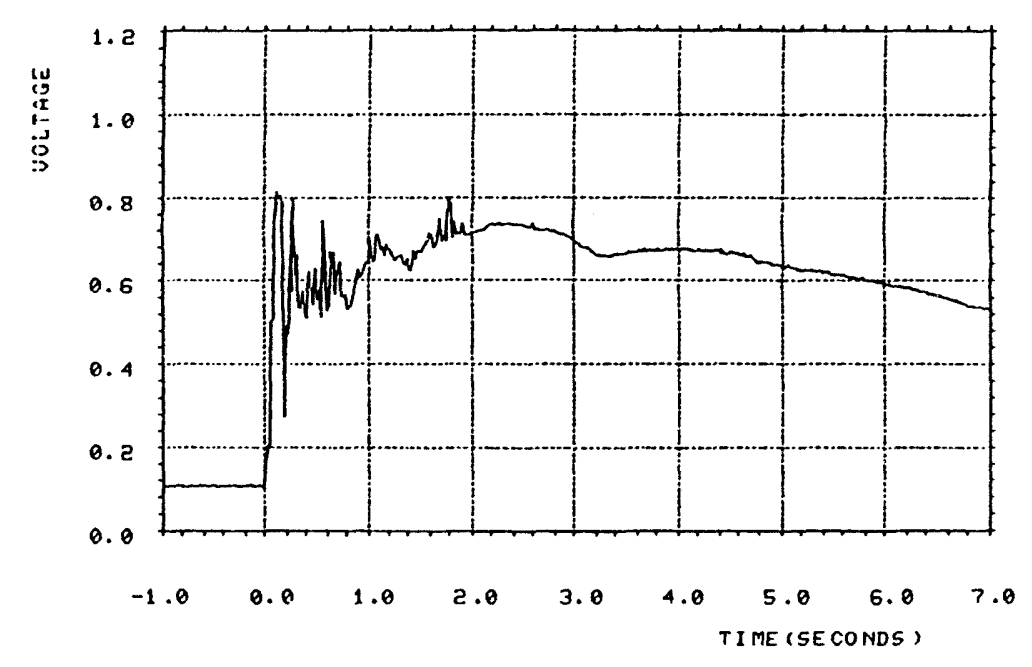

LP-2, CCN-3, DATE $3 / 14 / 86$ 


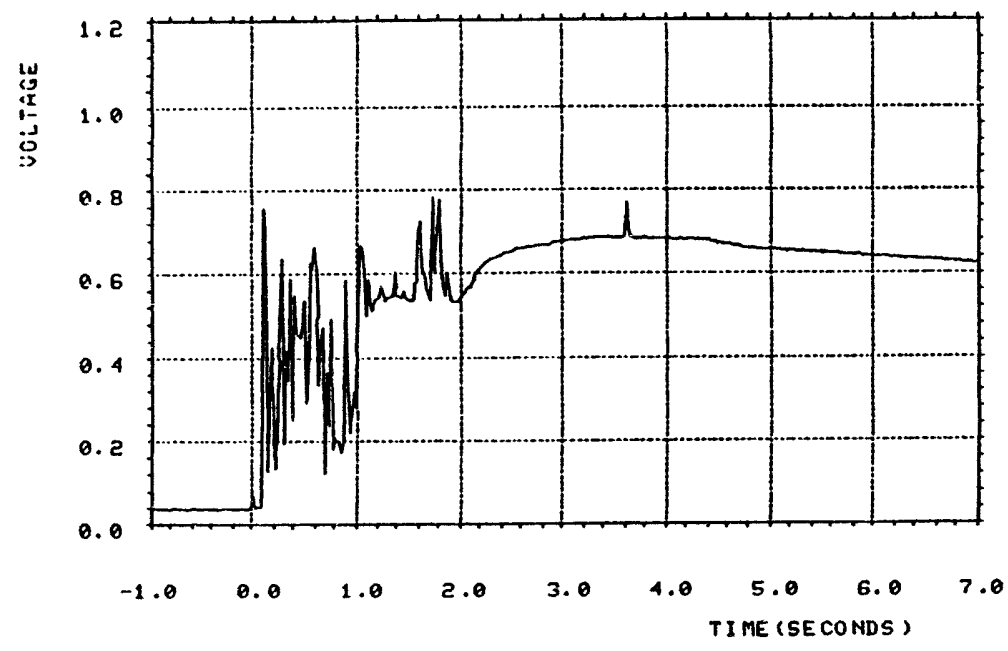

임

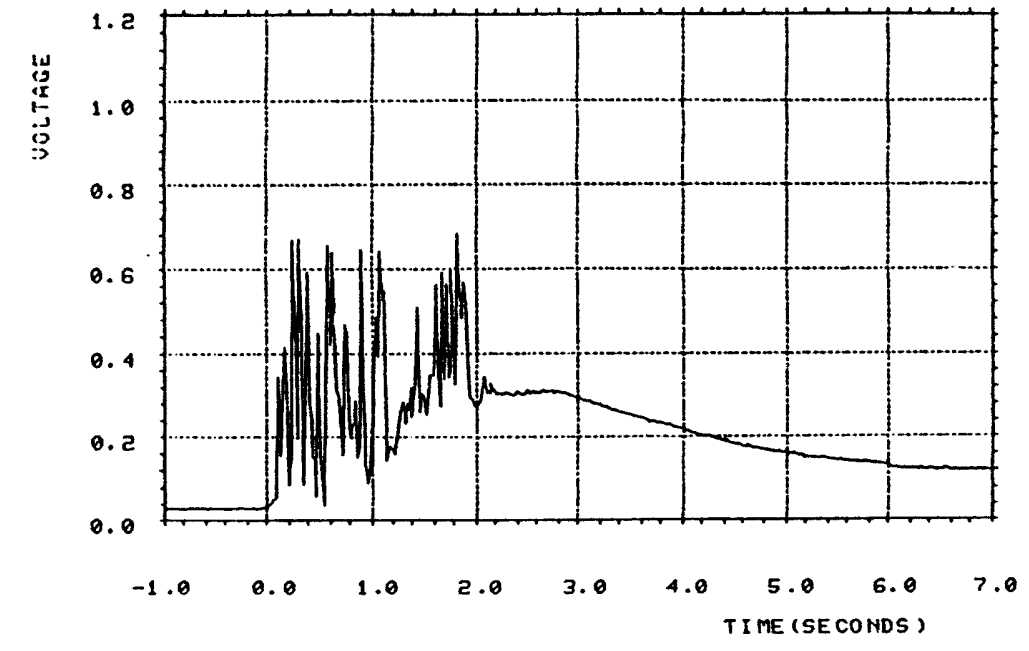

LP-4, CCM-3, DATE $8 / 14 / 86$

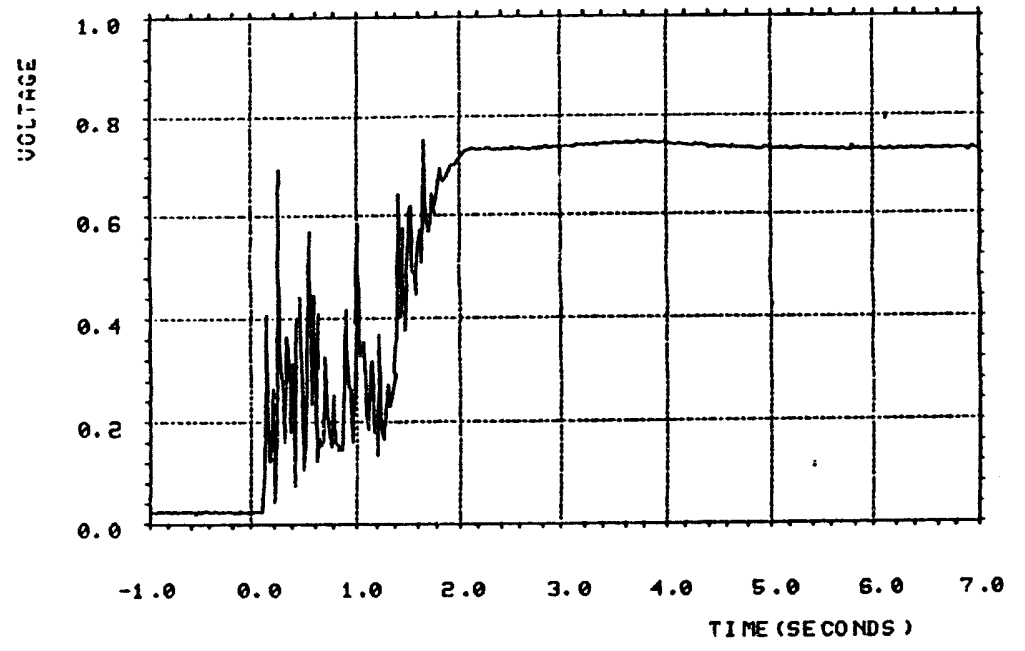

LP $-5, C C M-3,(101)$, DATE $8 / 14 / 86$ 
APPENDIX E. DATA FOR CCM-4

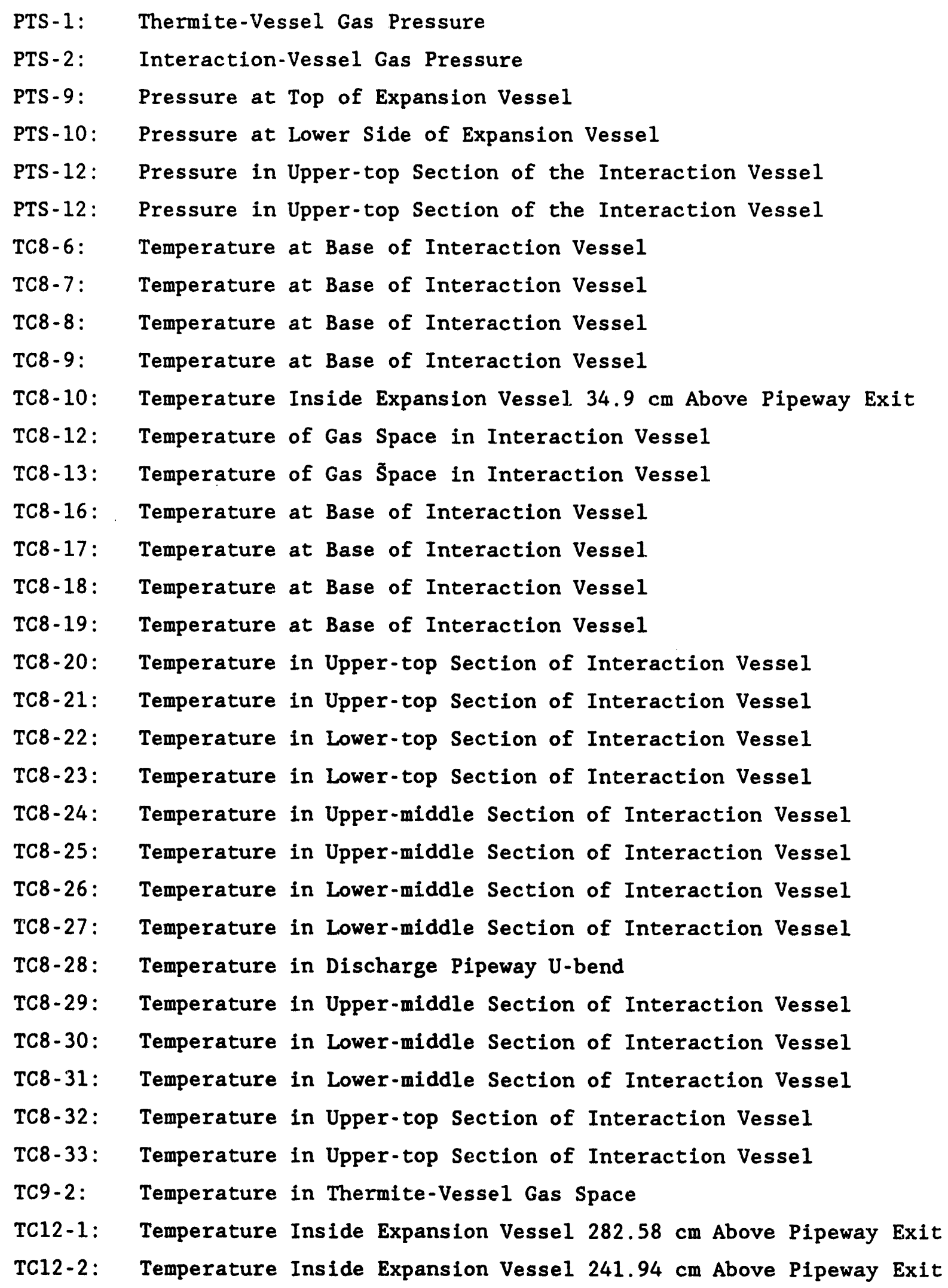


APENDIX E. DATA FOR CCM-4 (Cont'd.)

TC12-3: Temperature Inside Expansion Vesse1 $201.30 \mathrm{~cm}$ Above Pipeway Exit TC12-4: Temperature Inside Expansion Vesse1 $160.66 \mathrm{~cm}$ Above Pipeway Exit TC12-5: Temperature Inside Expansion Vessel $120.02 \mathrm{~cm}$ Above Pipeway Exit TC12-6: Temperature Inside Expansion Vesse1 $79.38 \mathrm{~cm}$ Above Pipeway Exit 


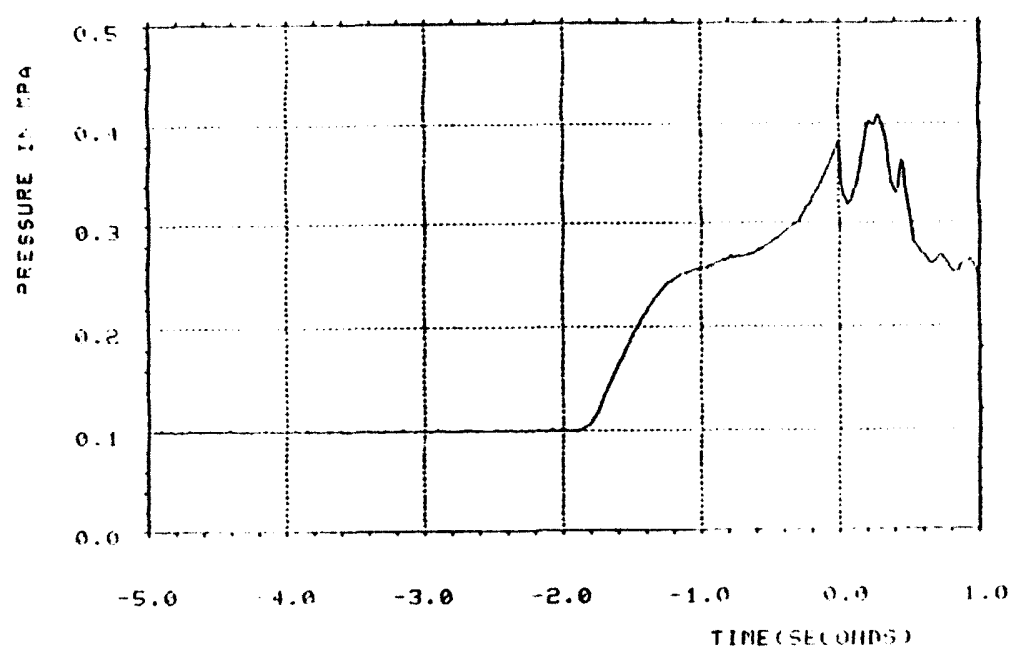

FT:-1, CCH-4, IIATE 6/4:ST

$\stackrel{m}{\omega}$

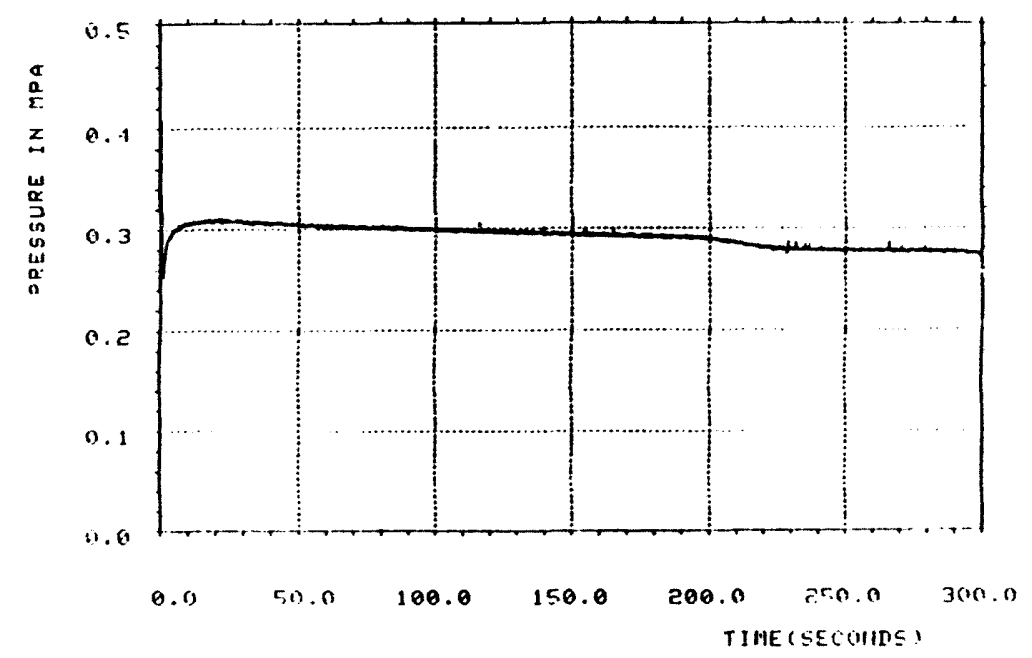

F.T $=-1$, COM-4, DHTE 6/4/87

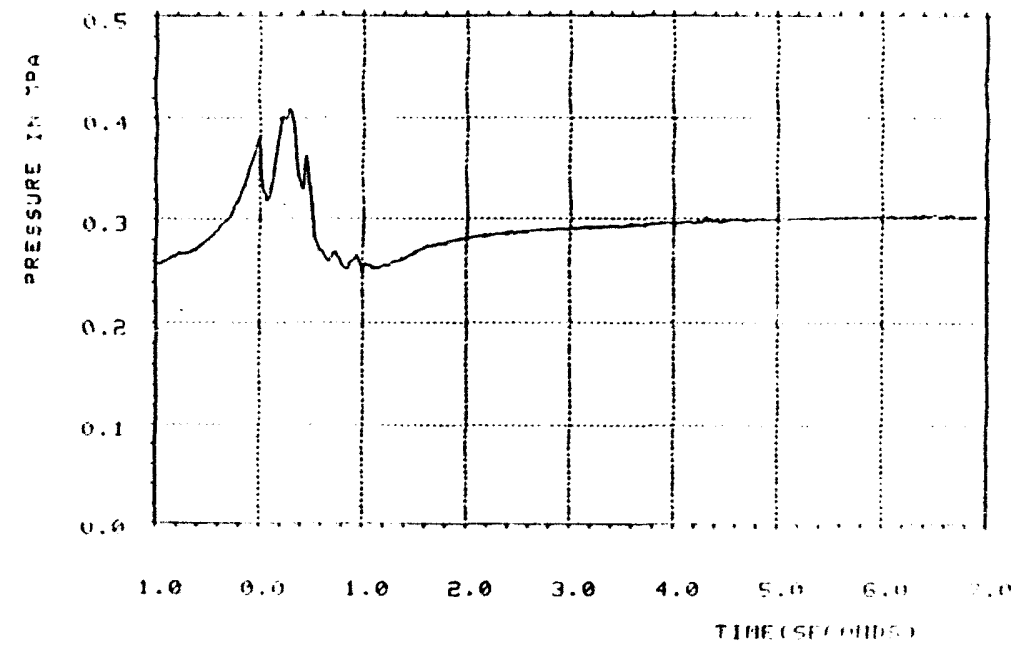

PTS-1, CON-4, [IATE 6:4,87

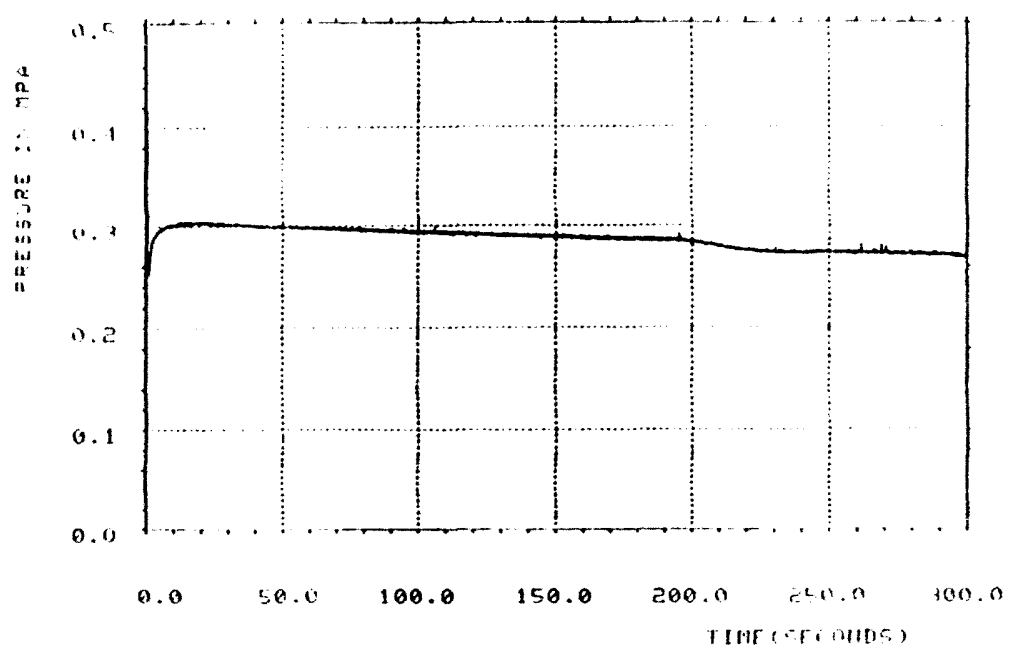

HTS-2, COM-4, DATE G/4, ST 


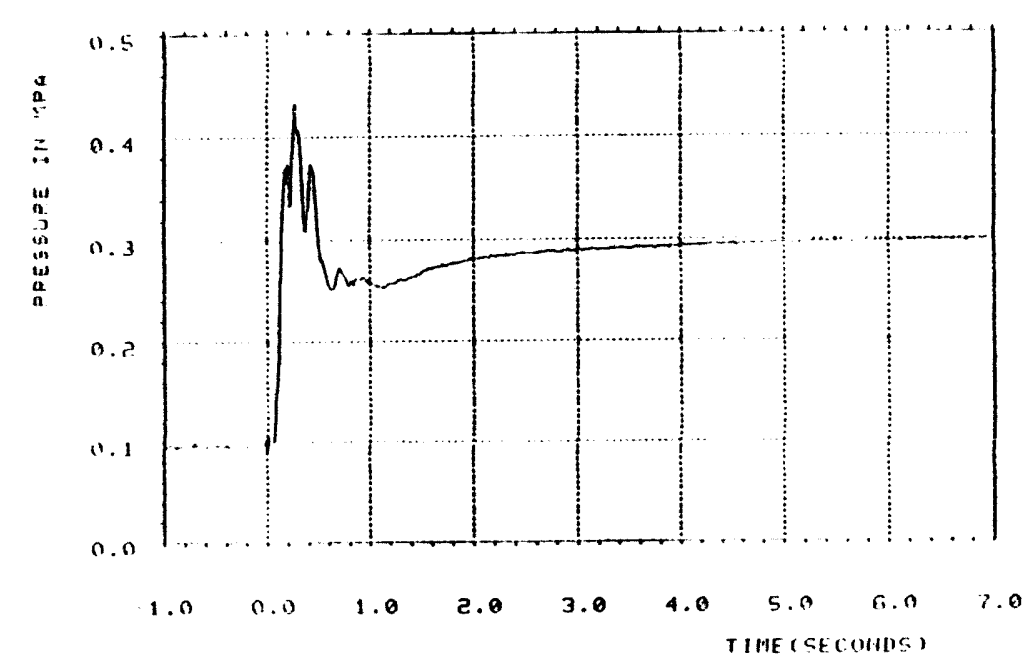

PTS-2, CON-4, DATE 6,4:S

$m$

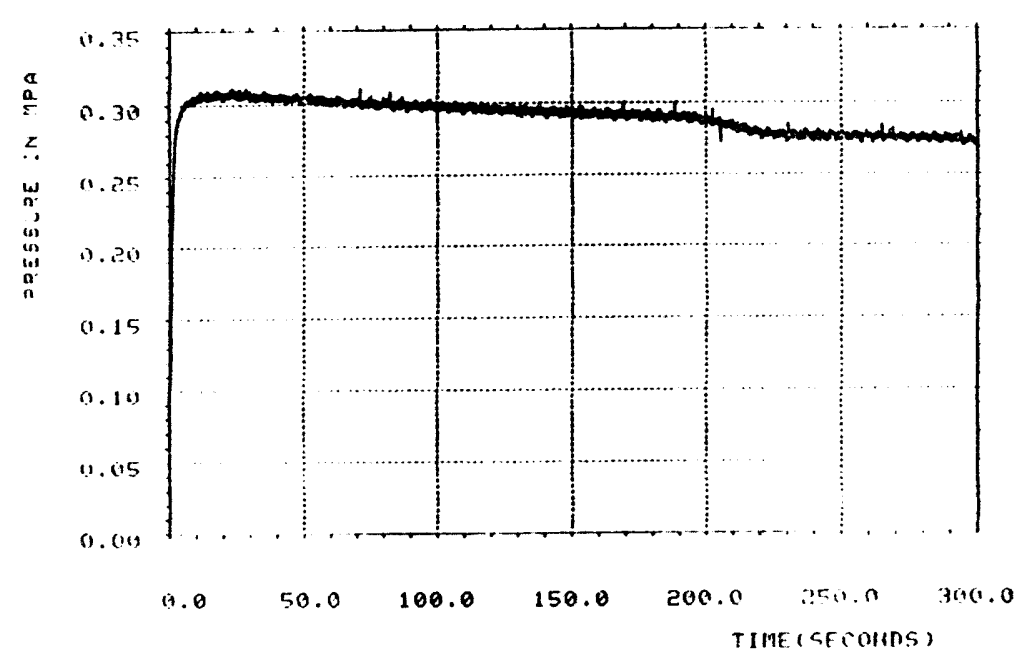

FTS-9. COM-4, DATE 6/4/8?

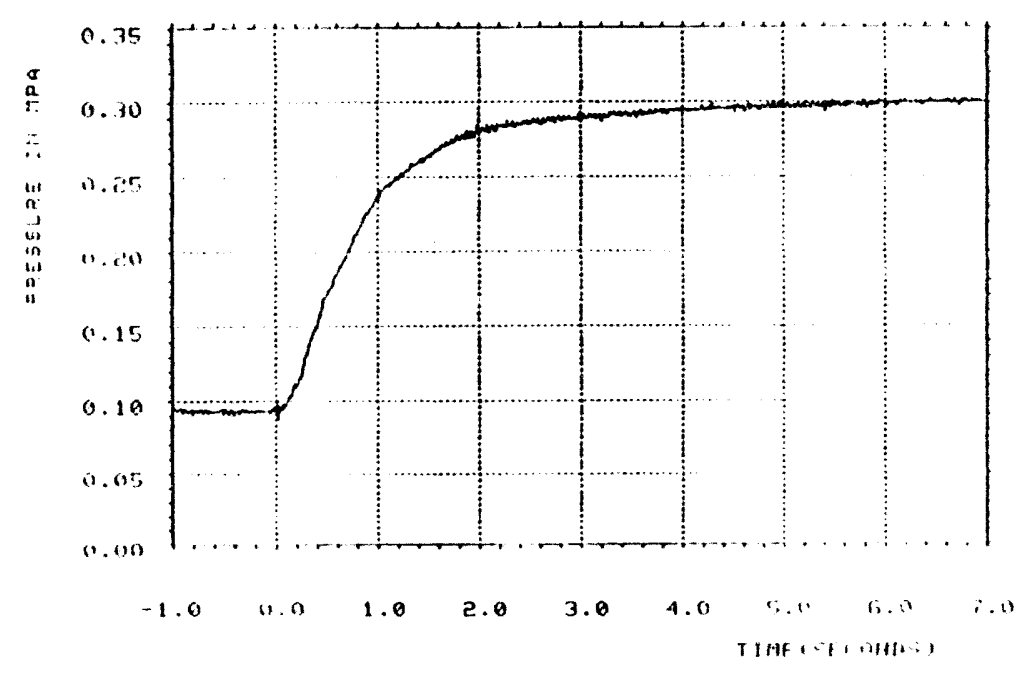

11: 9. COH-4. HATE 6\%4

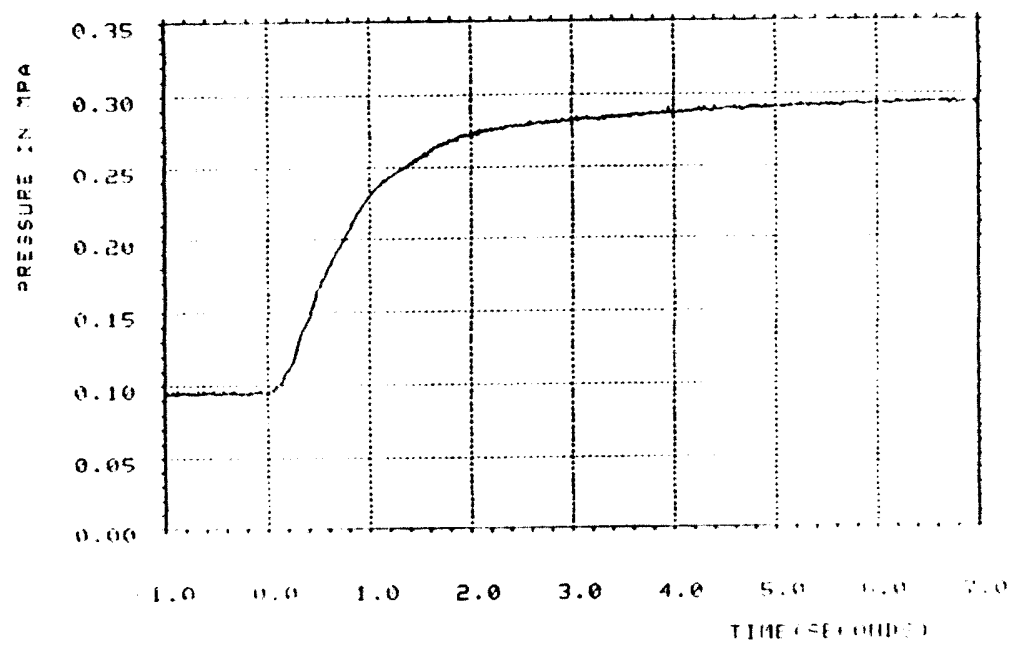

1. I11, ENA I, DATE $6 .+\ldots$ 


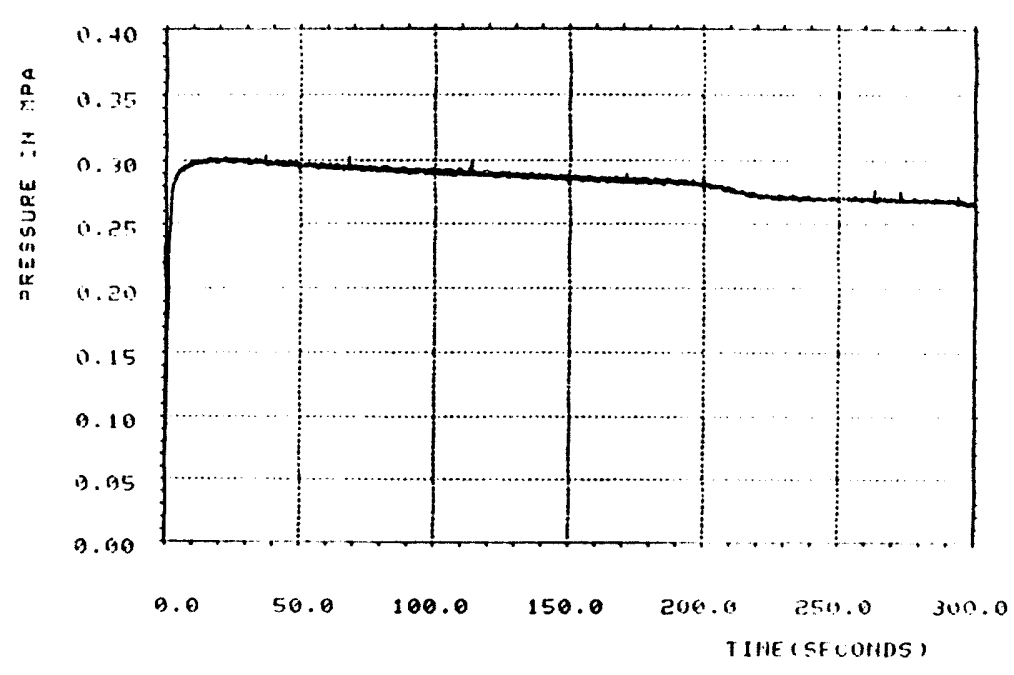

FTS-10, CCH-4, DATE 6 - $3 ?$

in

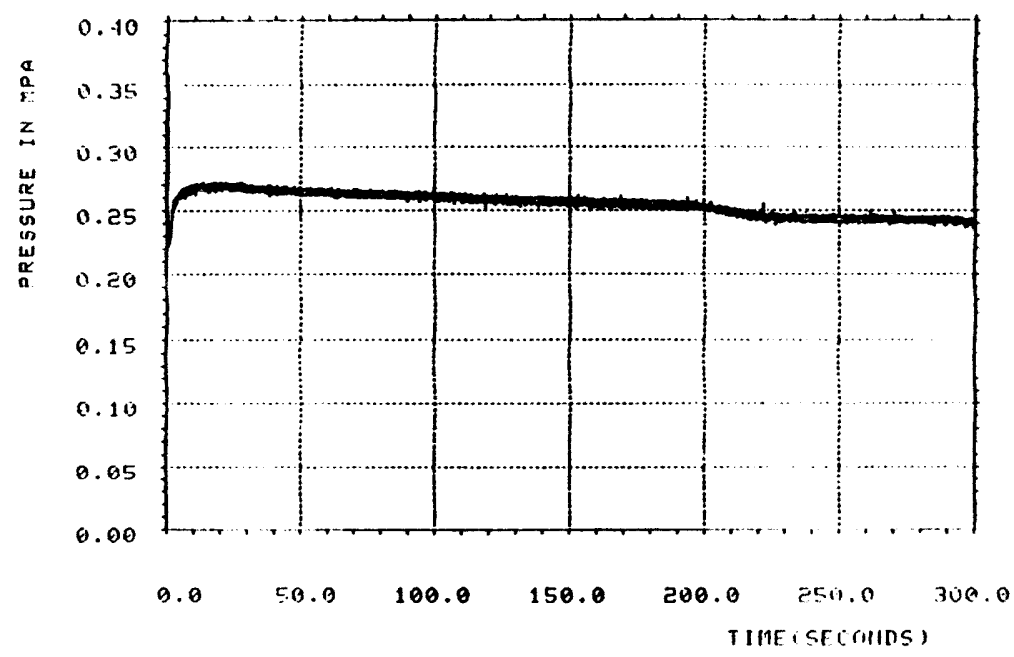

FTS-12, CON-4, DATE 6, 4 ? ?

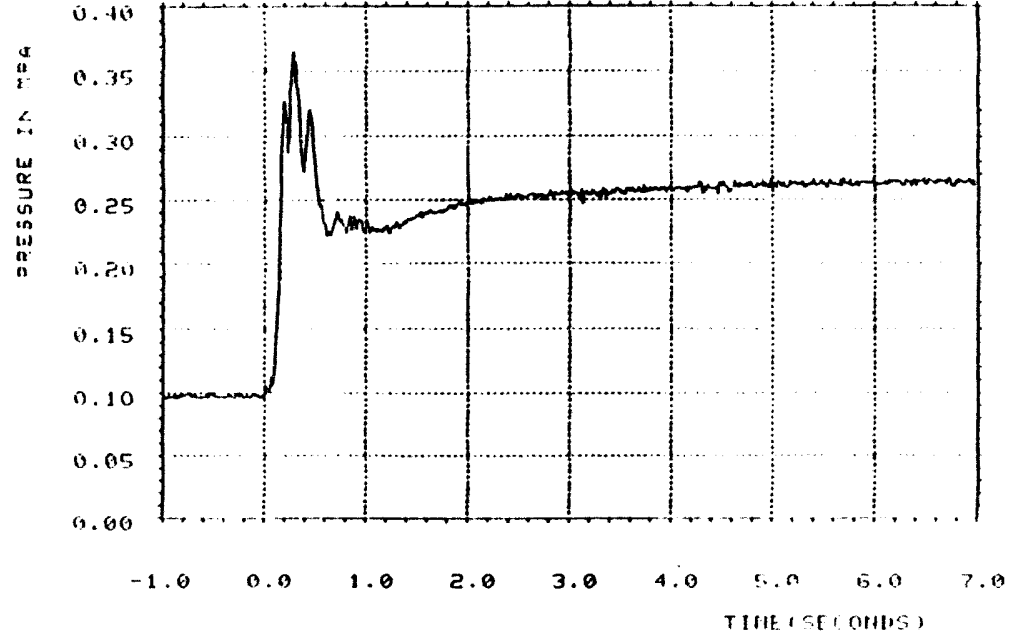

FT-12, CCH-4, DATE 6-4.

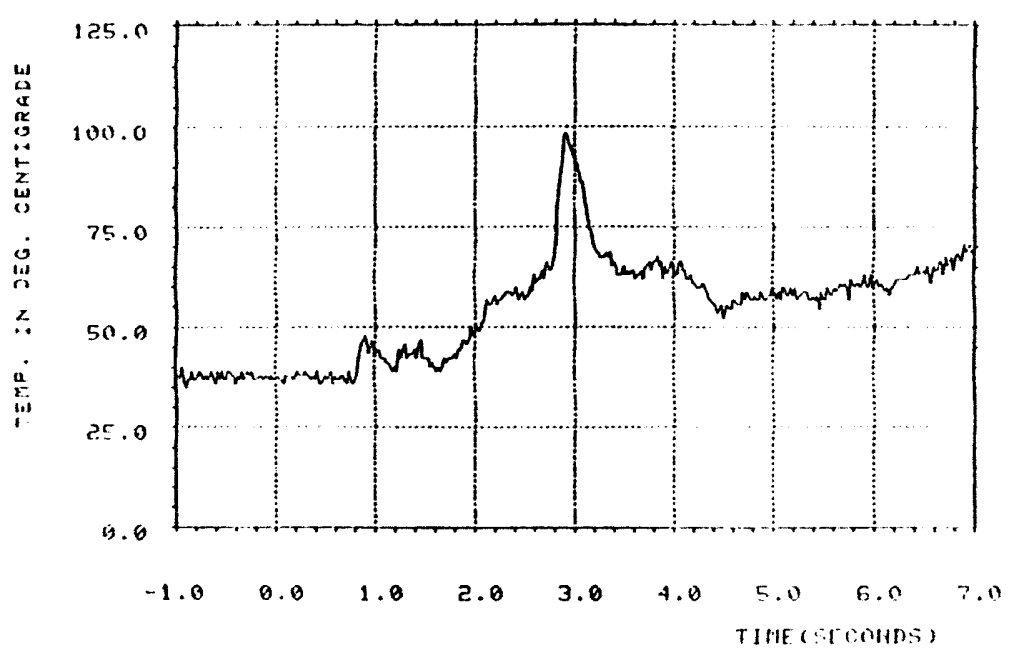

TI8-6. CCN-4, DATE 6, 4,87 


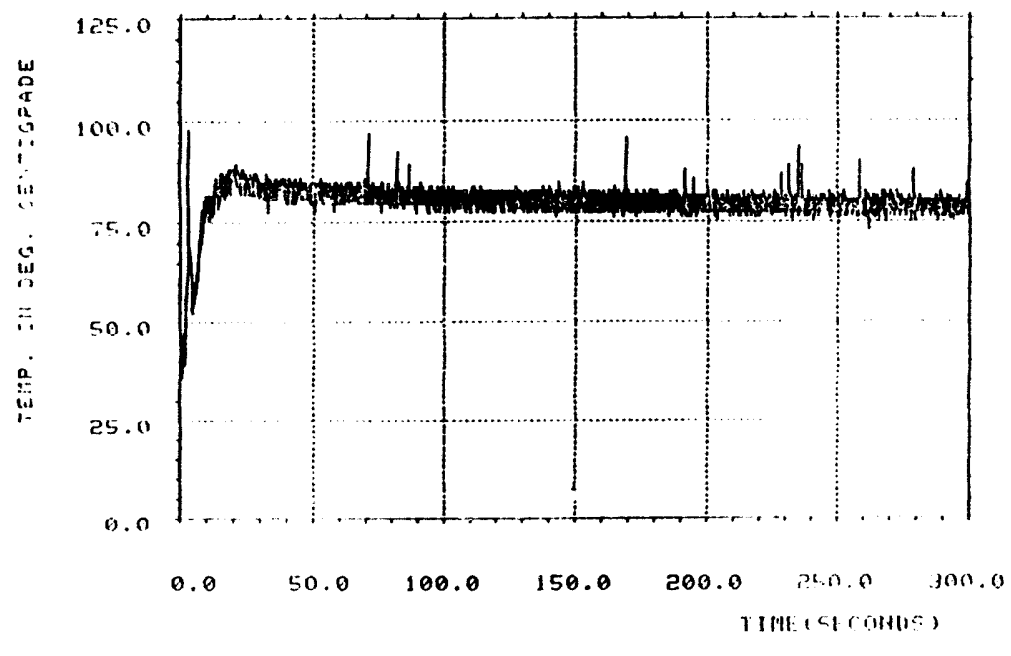

$\dot{m}$

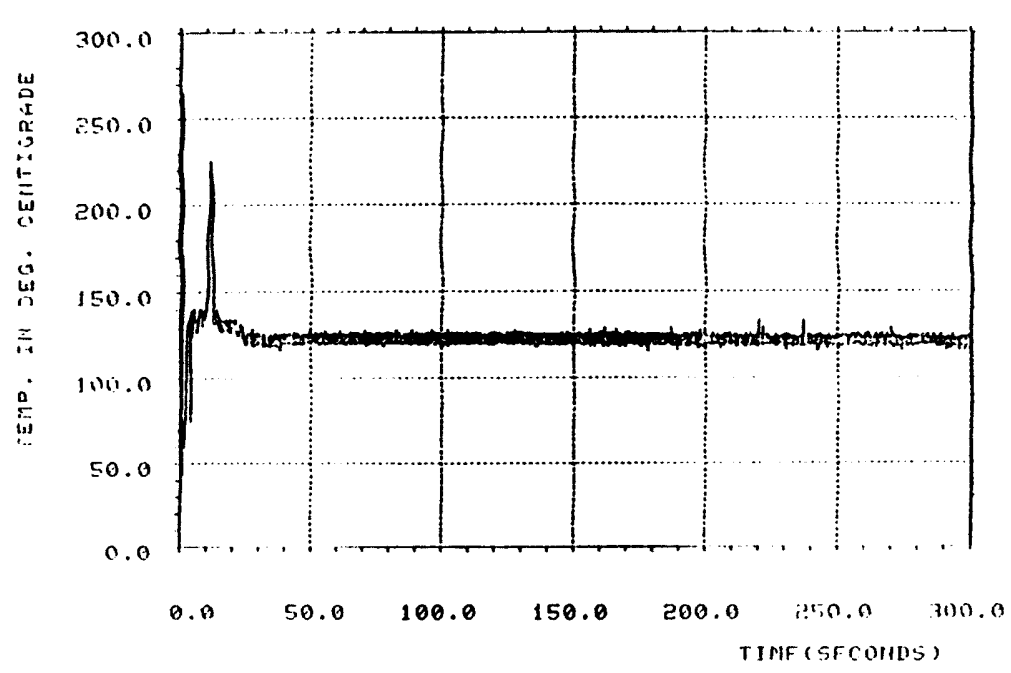

Tr:3-7, CCM-4, DATE 6/4. 87

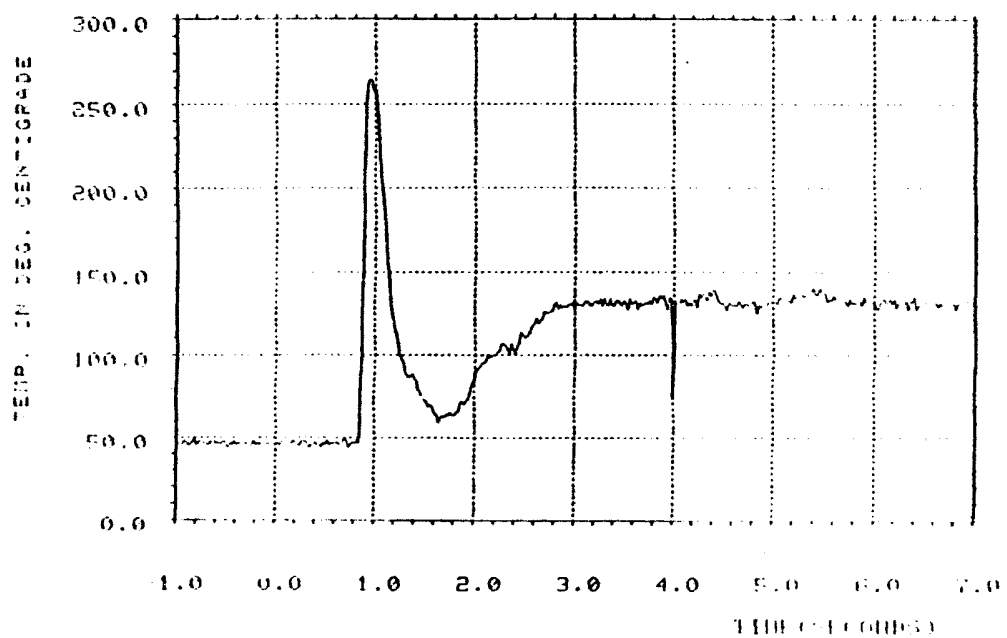

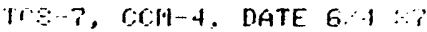

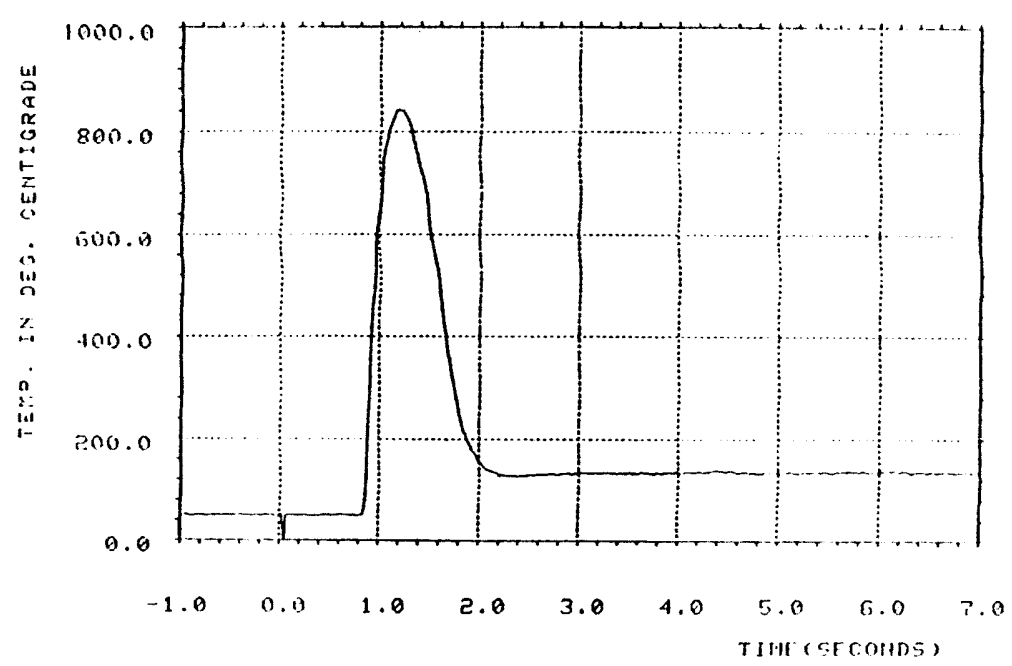

Tis-8, DCN-4, INATE $6,4,3$ 


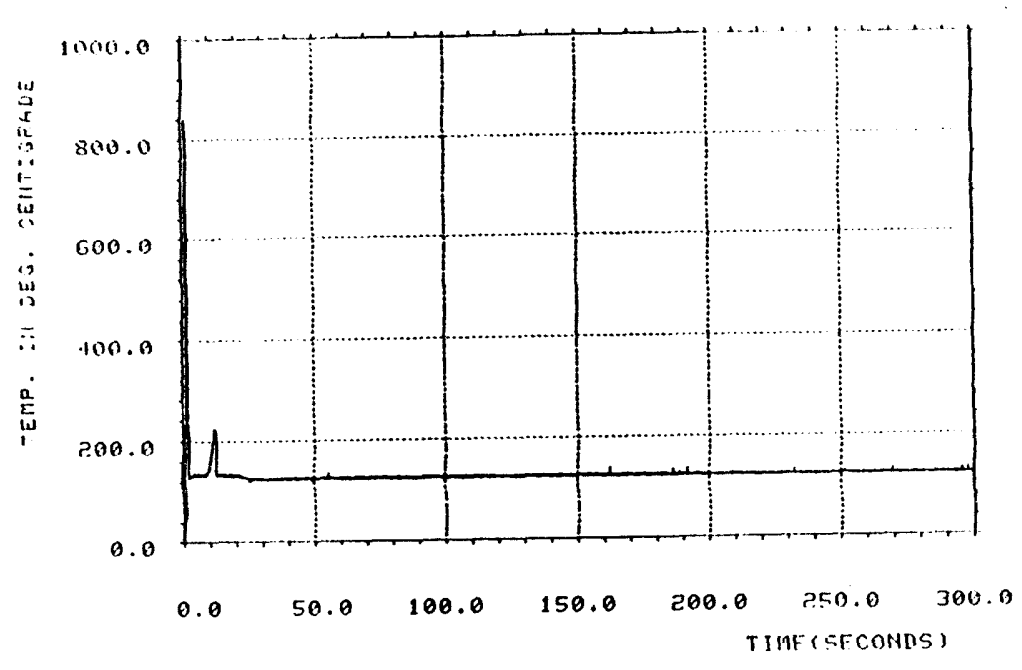

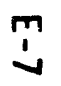

TIS-8, CCH-1, [DATE 6:4 87

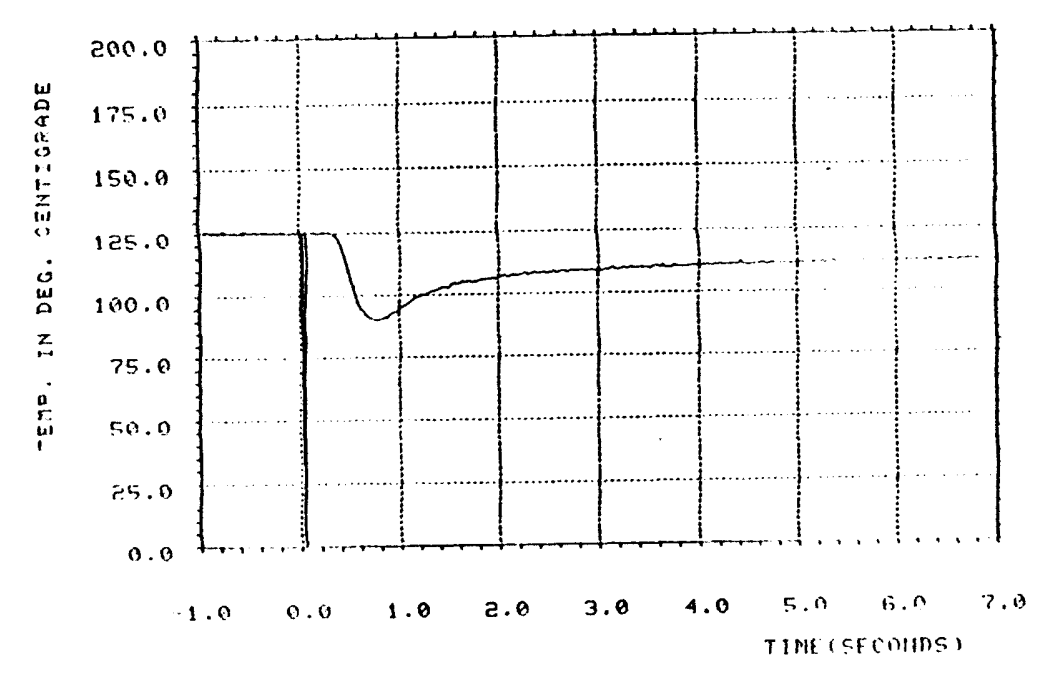

Tr8-10, CCH-4, DATE 6,4 87

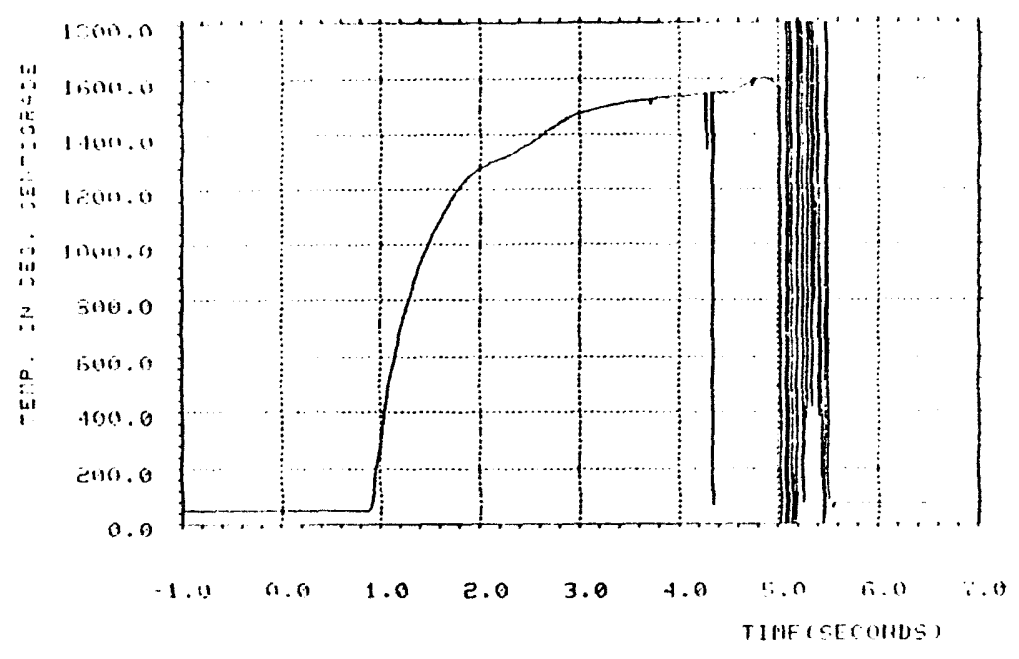

TC8-9, CCH-4, DATE E 4.3

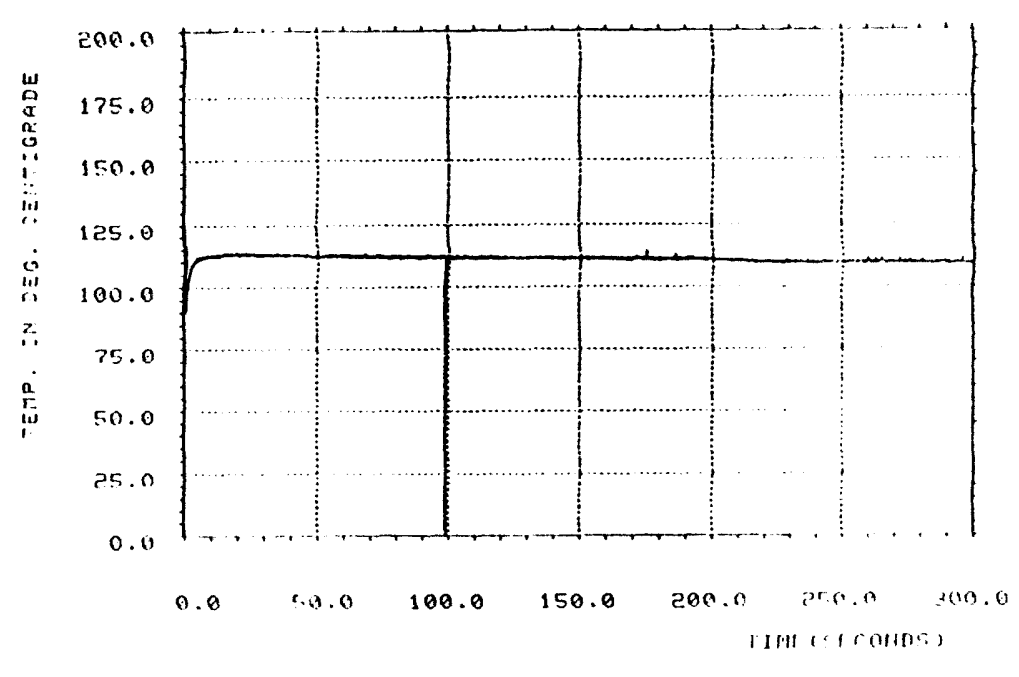

TOS 10, CCN-4, NATE 6\% 

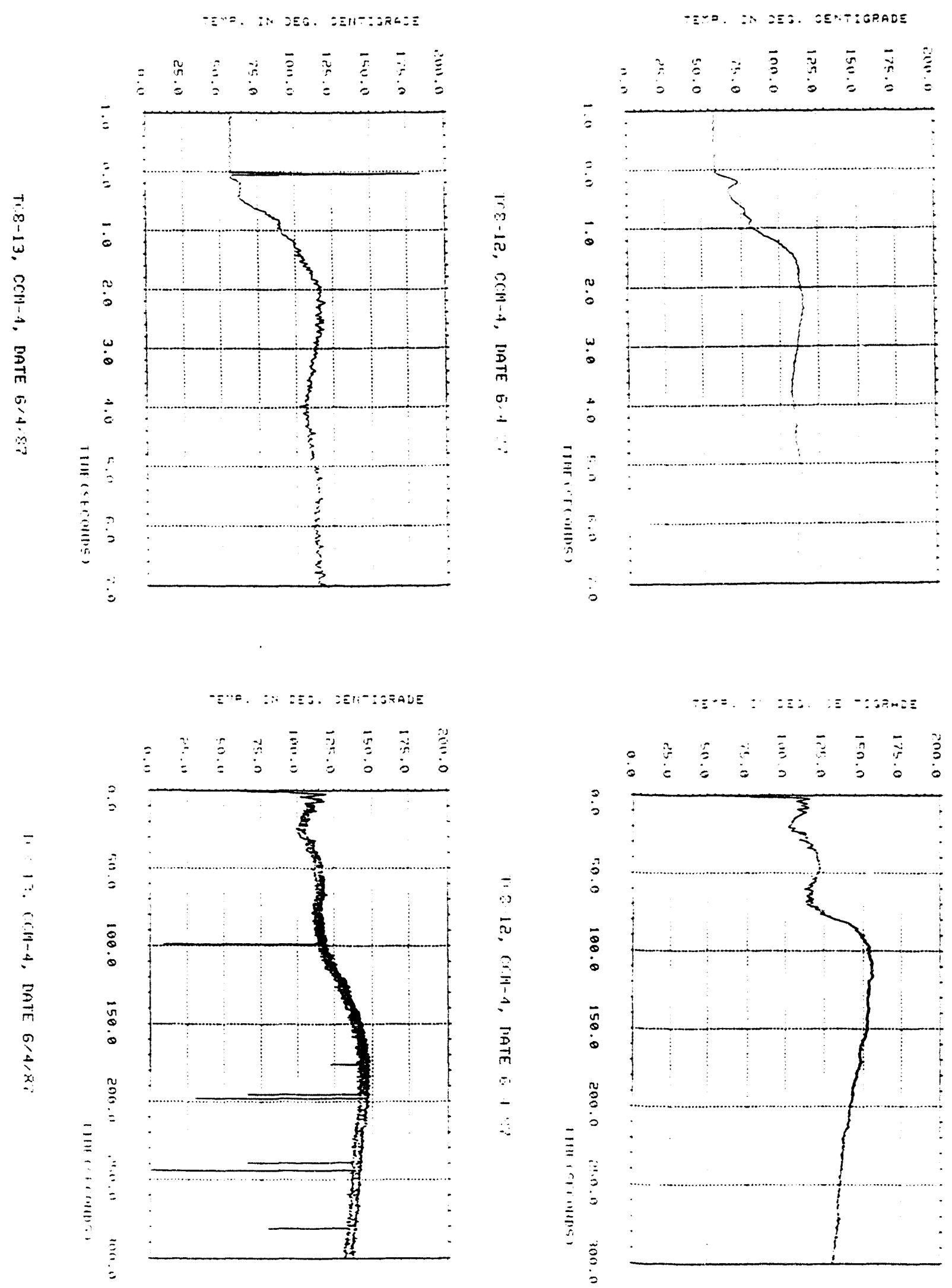


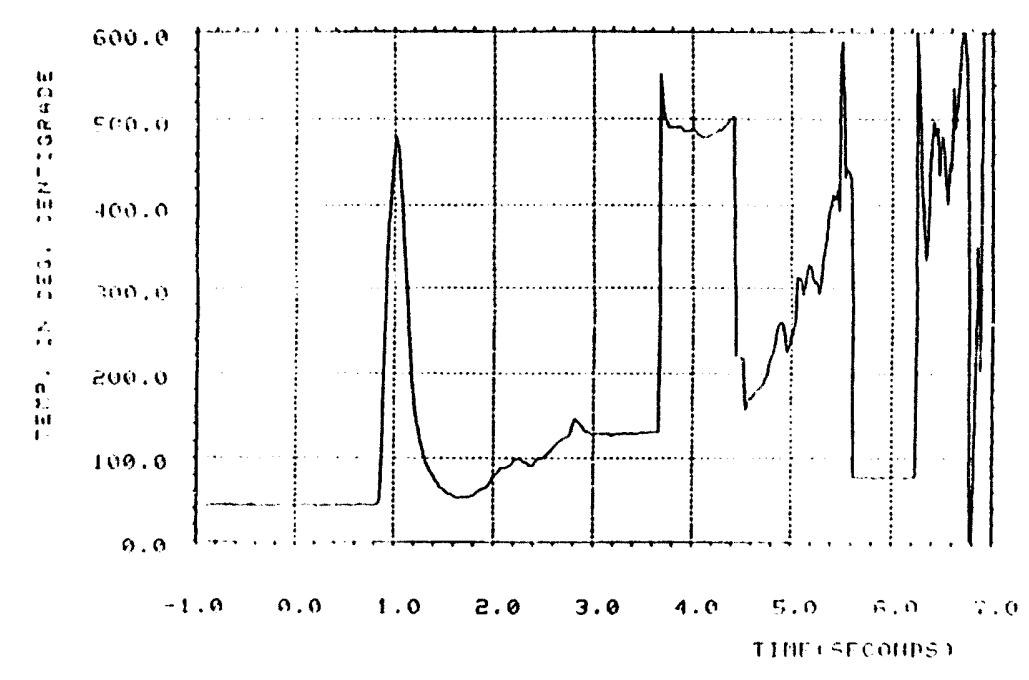

$\stackrel{m}{6}$

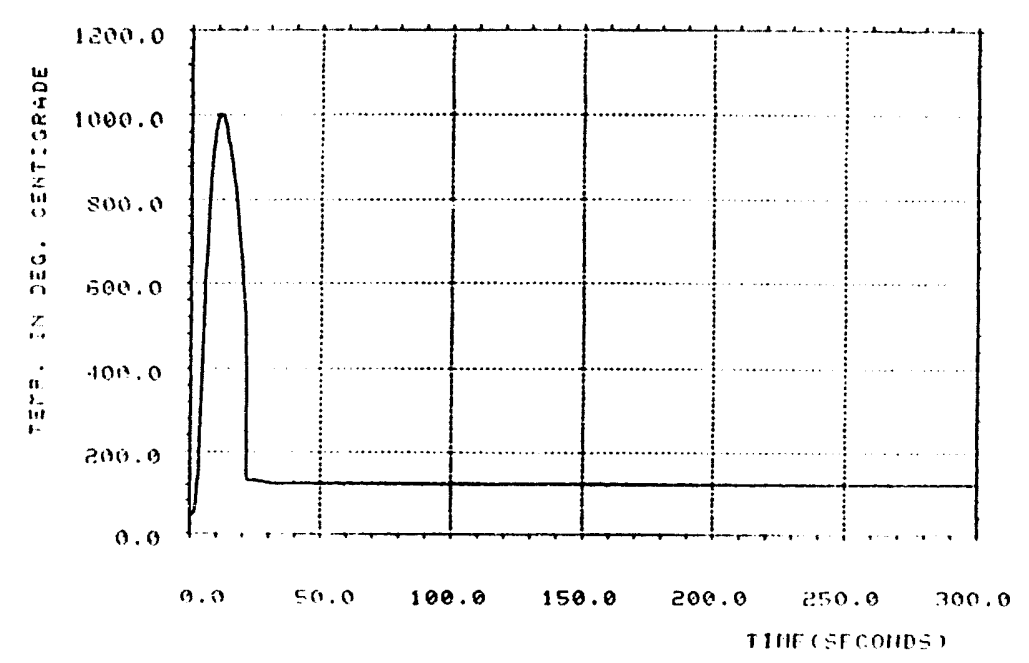

TI3-17, CCI1-4, DATE 6/4-87

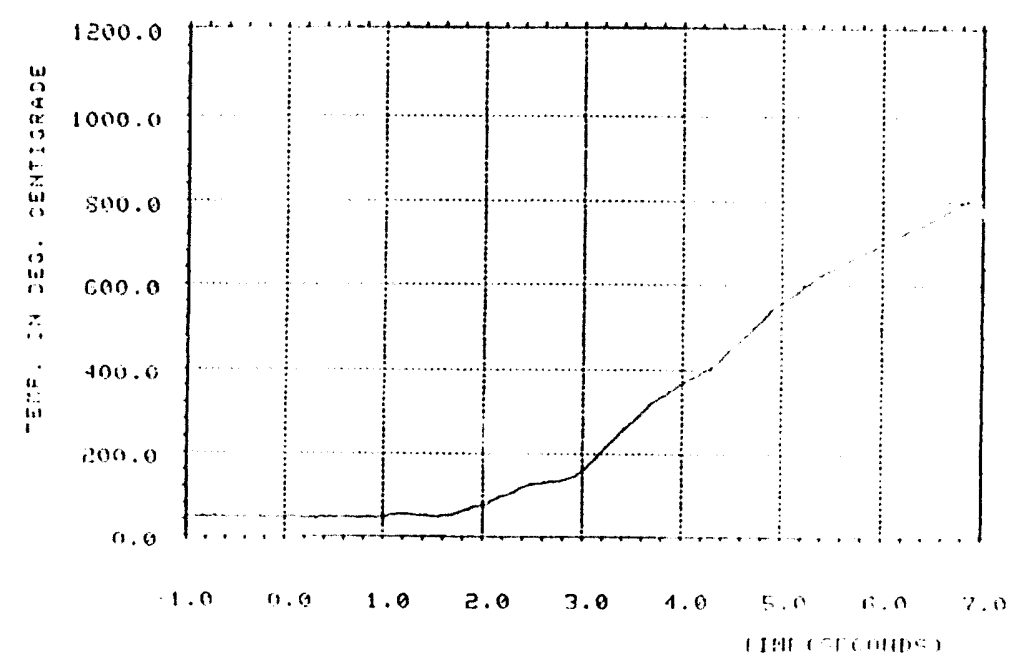

TIR-17, CCN-4. DATE $64 \therefore$

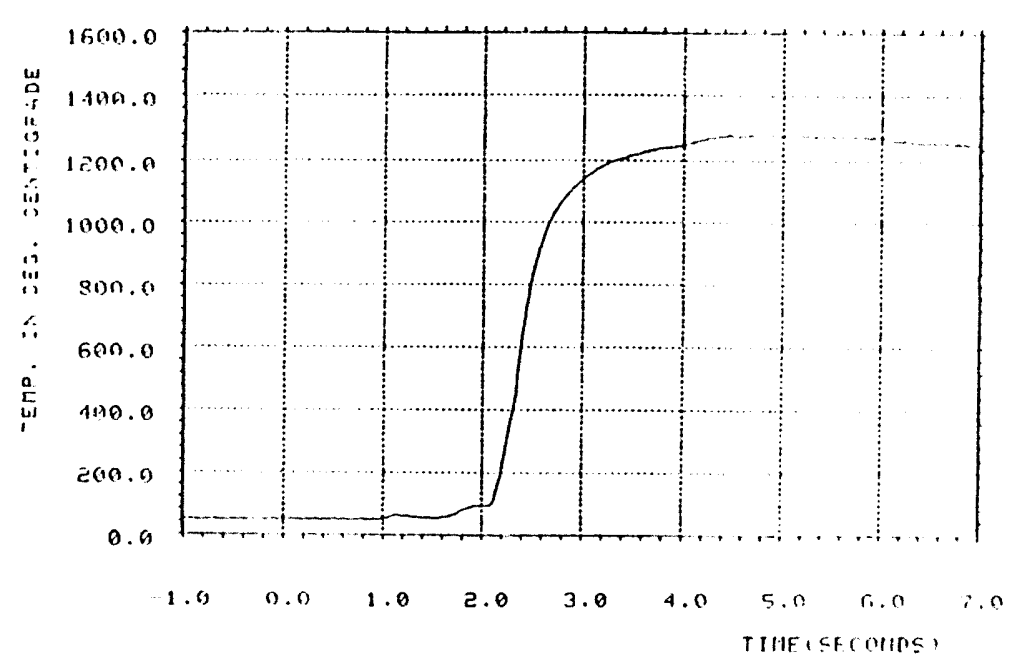

TCO-13, CCN-4, DATE 6 4.7 


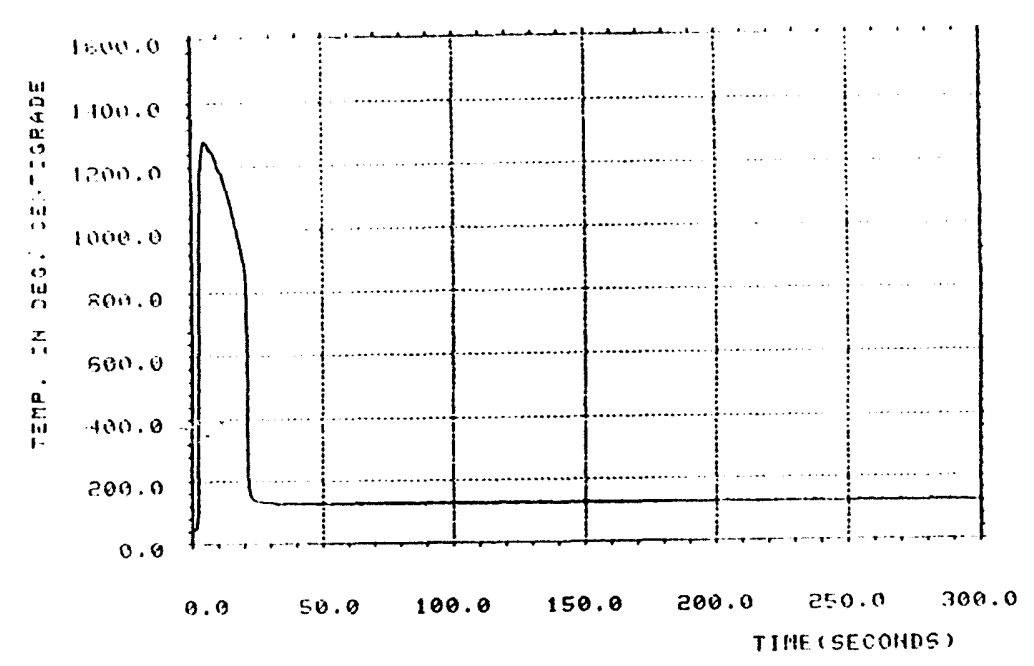

$\stackrel{\dddot{⿱}}{\circ}$

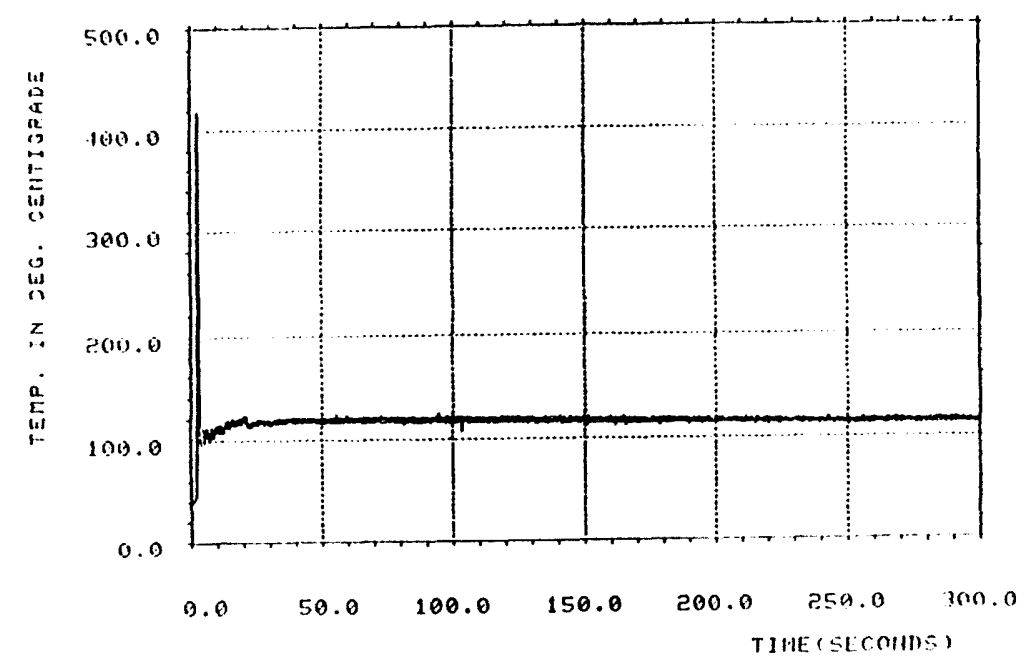

Tr8-19, cCH-4, DATE 6.4 2.

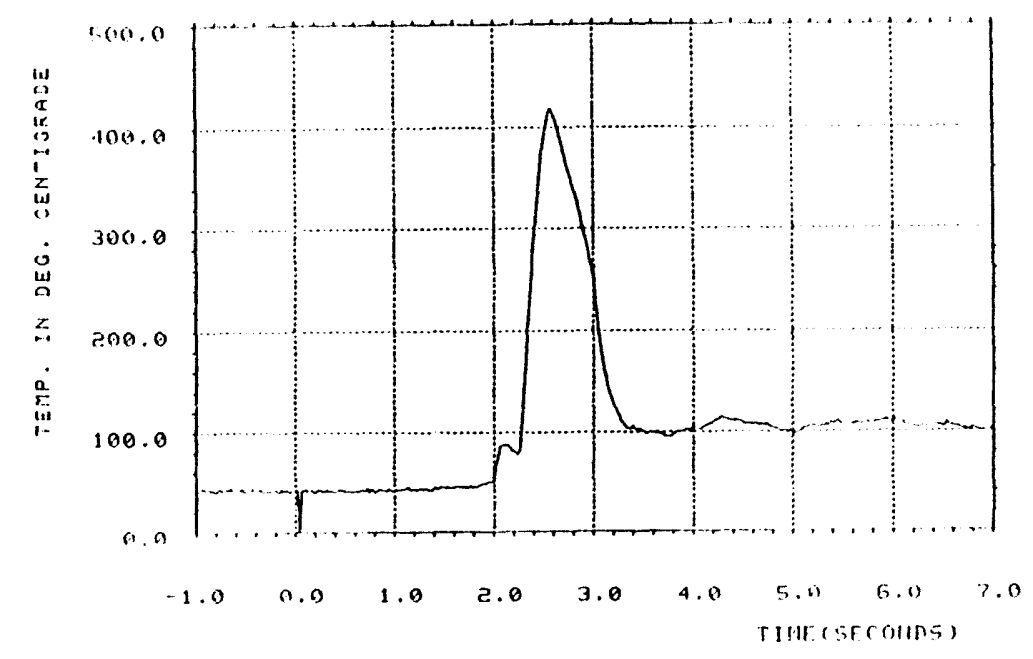

Tr:8-19, CCH-4, DATE E.4,

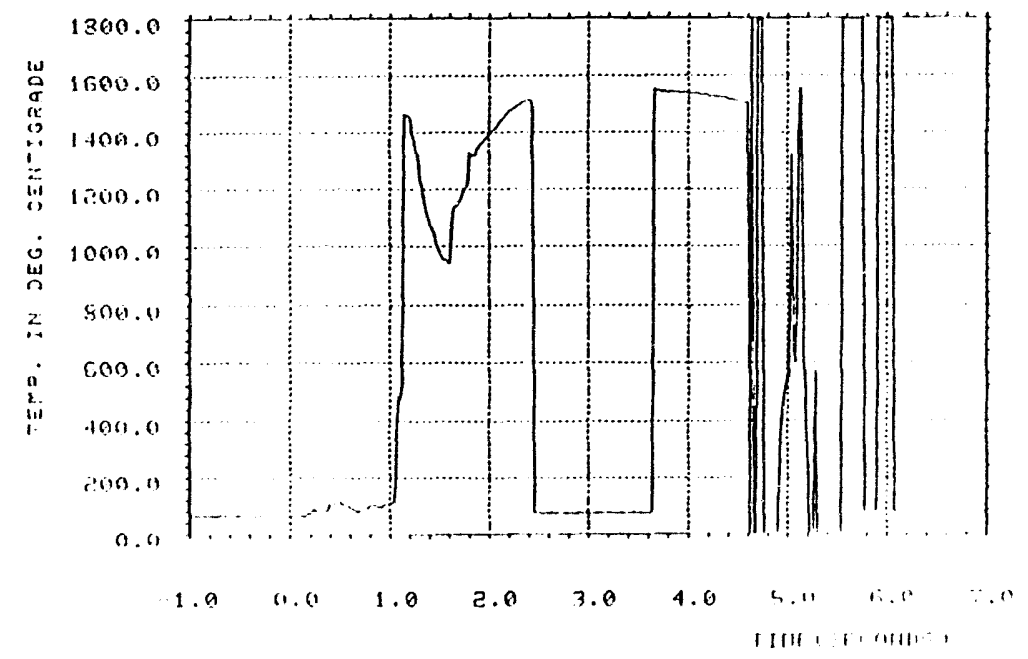

Tr - 20 , CON-4, DATE 6. 


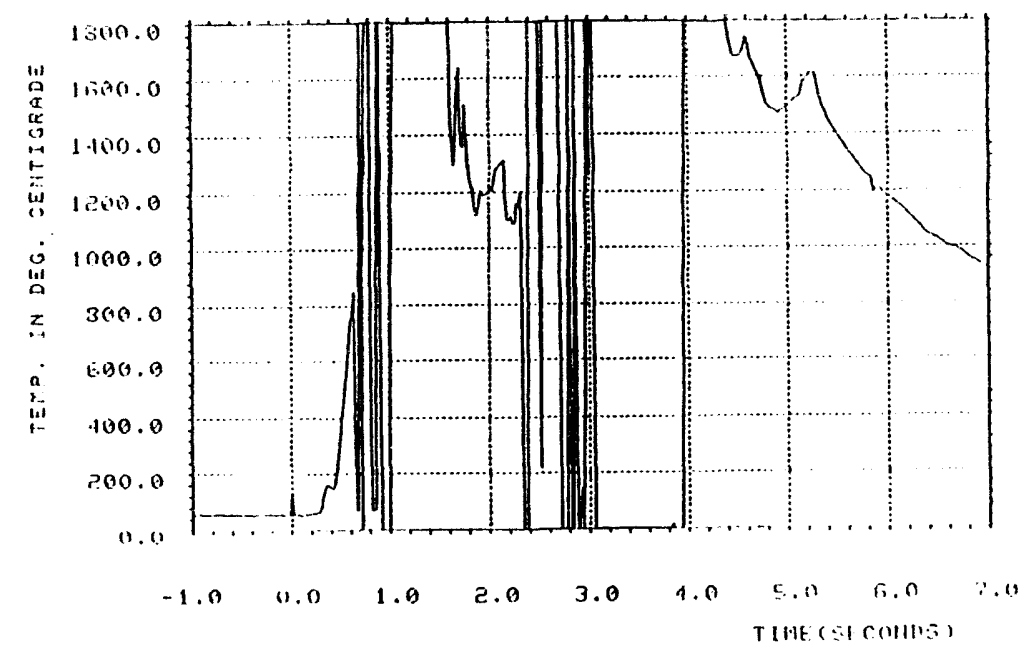

$\stackrel{9}{\dot{I}}$

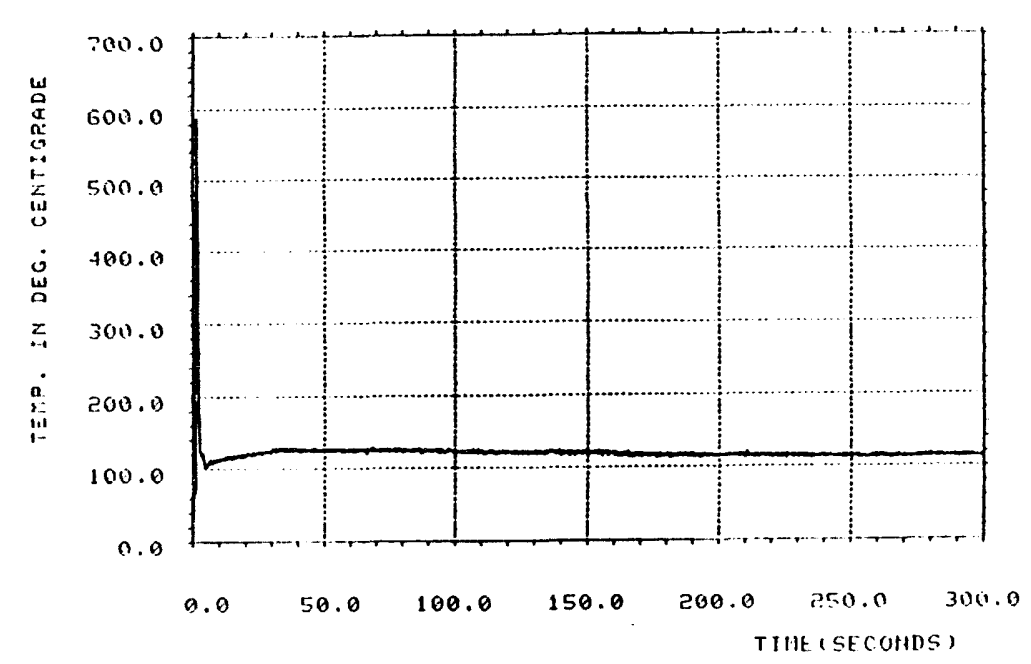

TC:8-22, CCI1-4, DATE $6 / 4: 87$

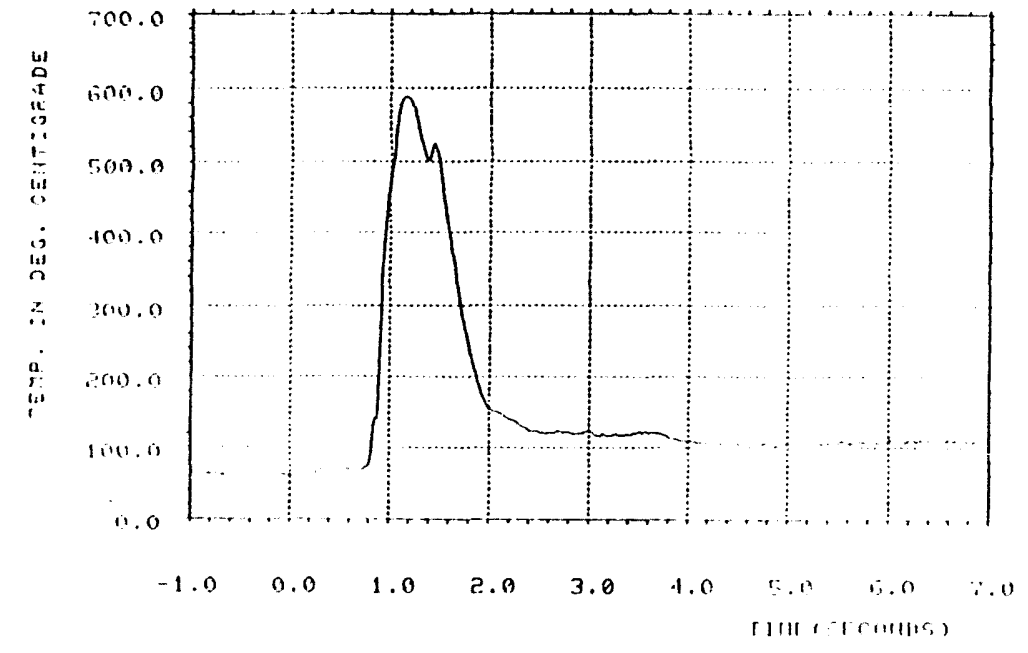

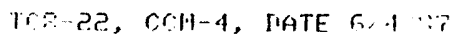

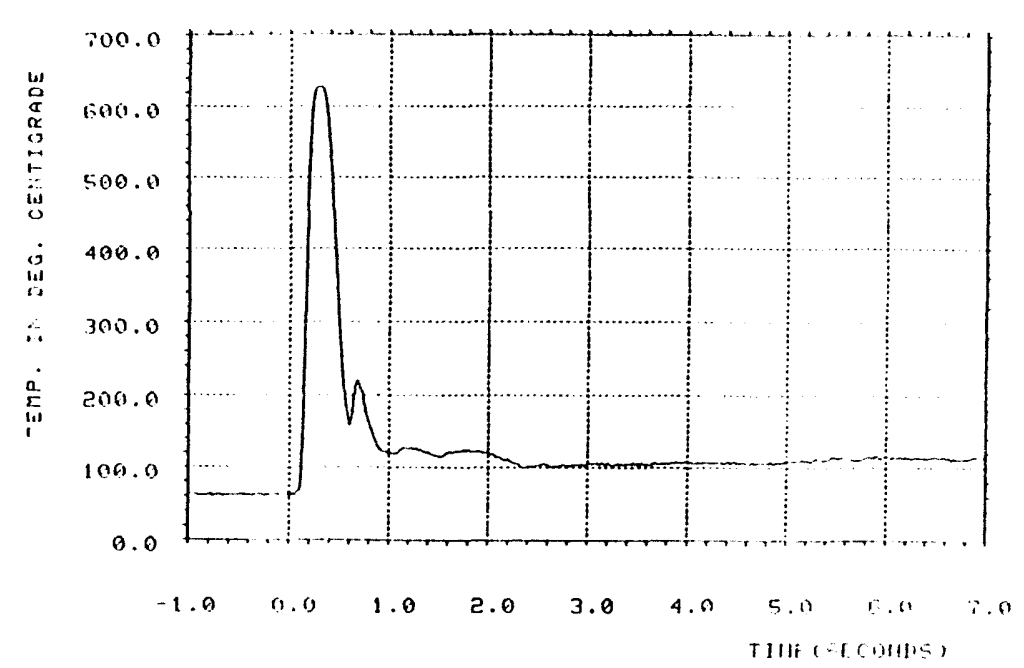

TI: 23, COM-4, DIATE $6 \div$ 


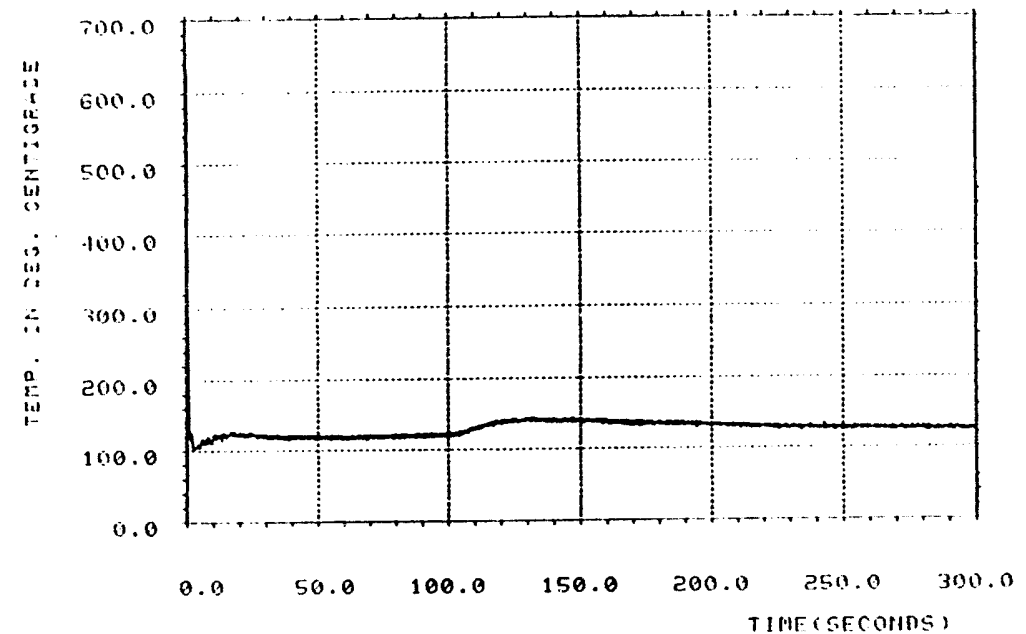

$\stackrel{m}{n}$

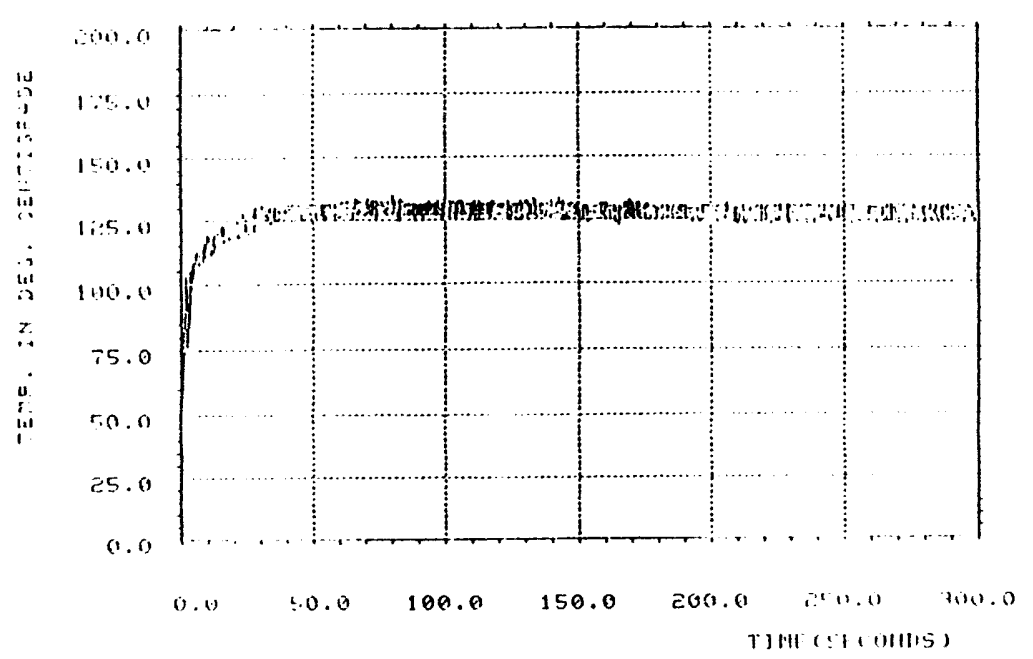

TOB-24, CCH-4, DIATE E.

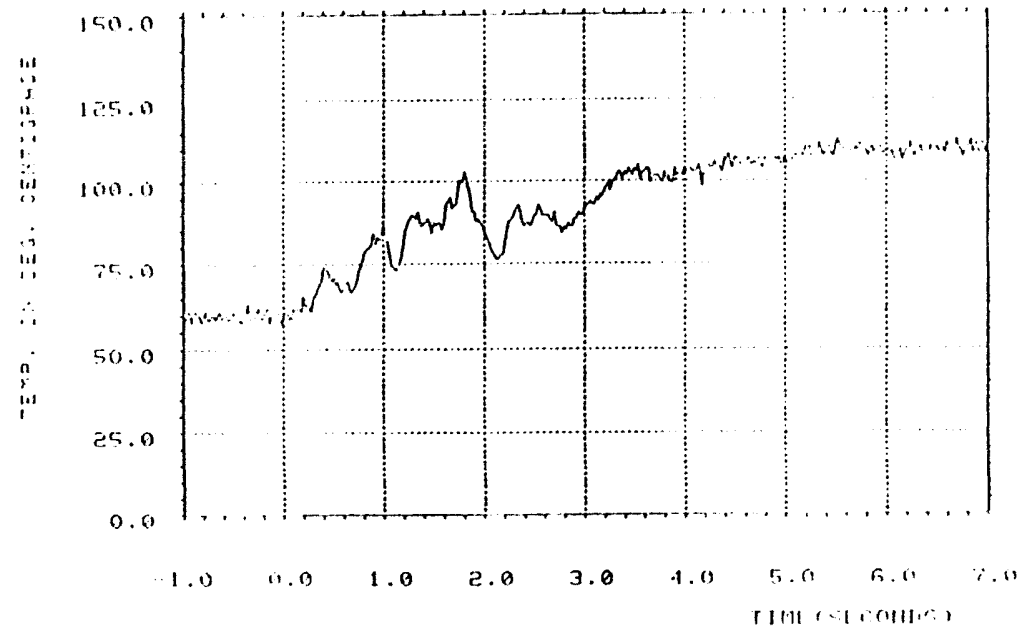

10E-24, COM-t, NATE H1

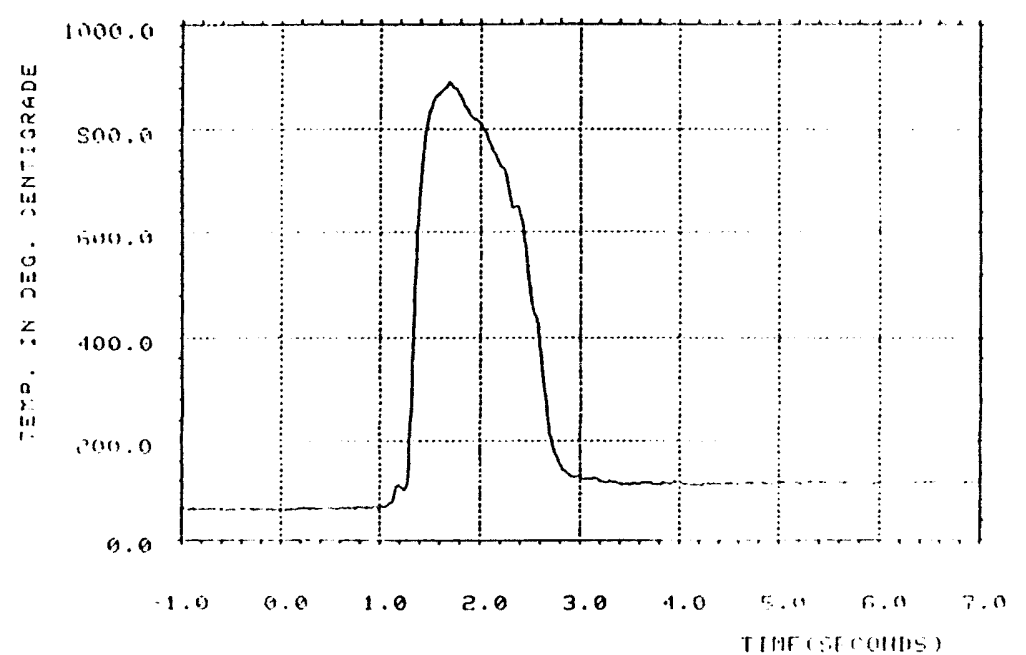

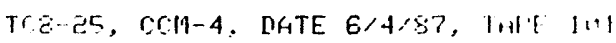




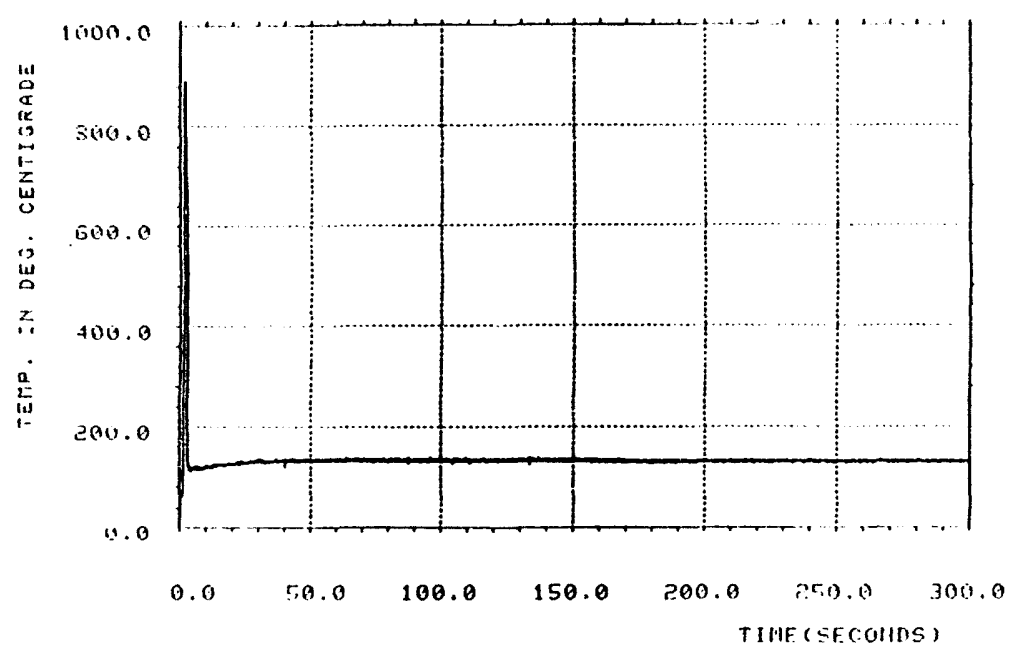

$\stackrel{m}{\dot{\omega}}$

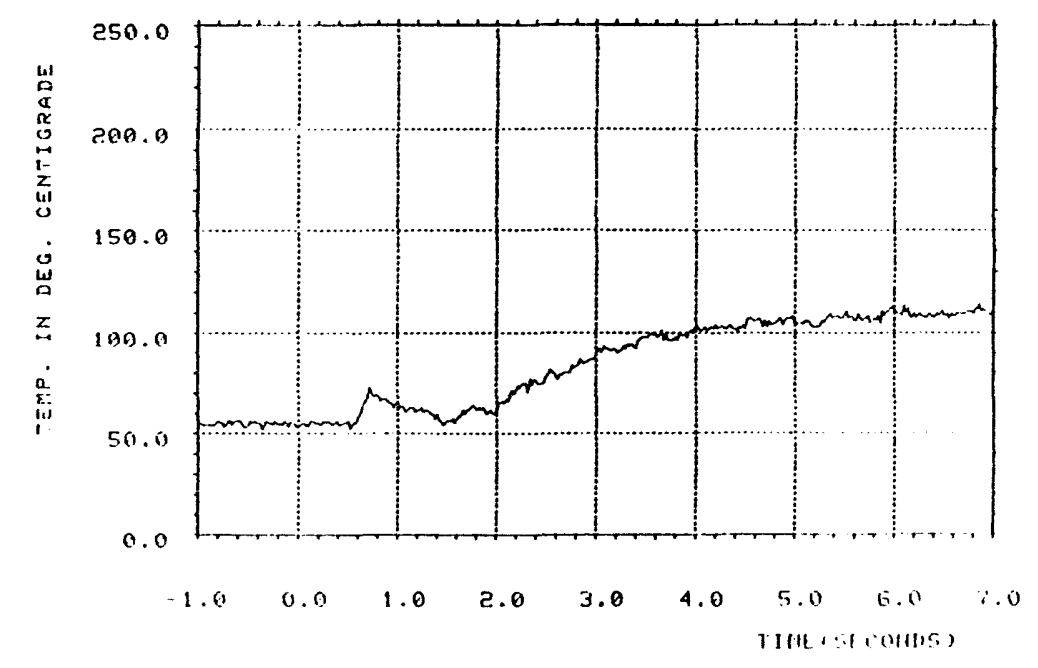

TIE-27, CCIM-4, DATE 6,

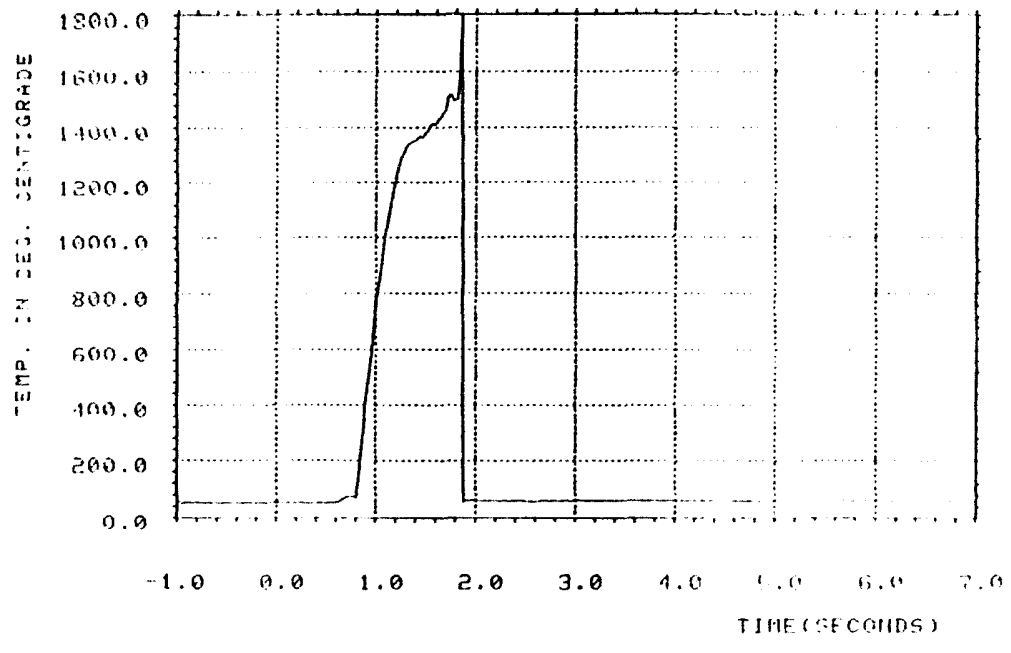

TSE-26, CNH-4, DATE 5,4,87

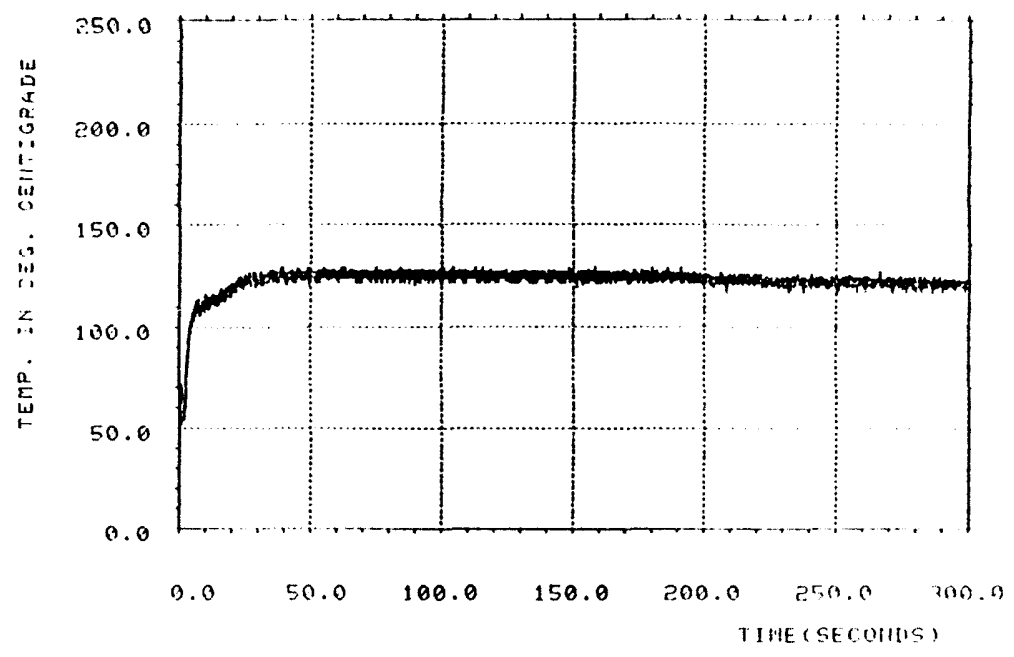

T68-27, CCN-4, DATE 6, . 5? 


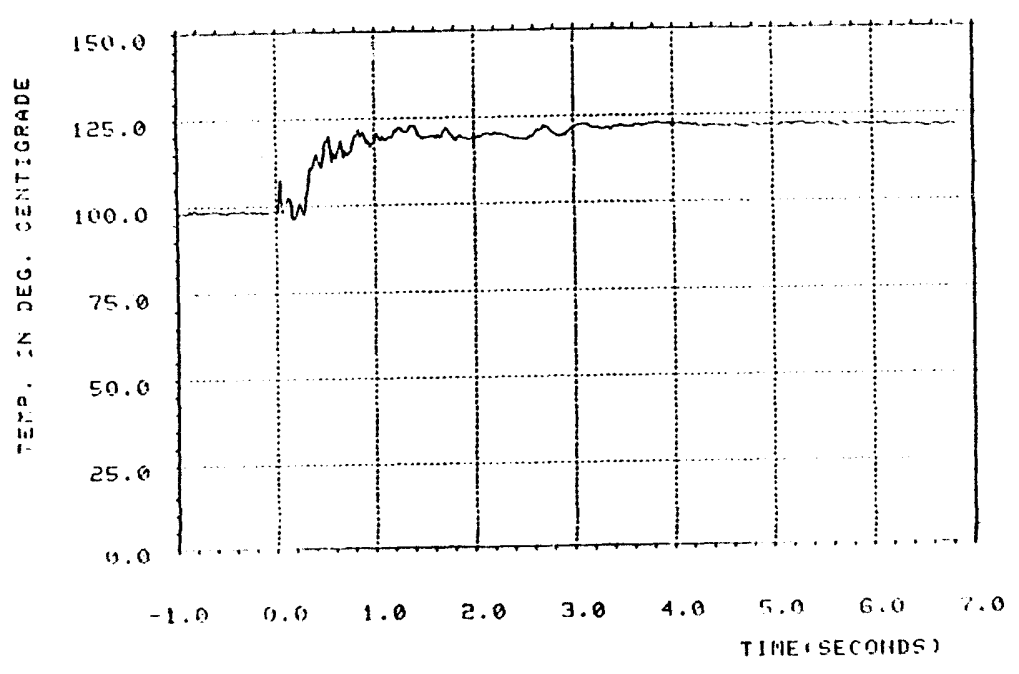

TC8-28, COM-4, DATE 6.4 .37

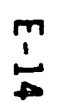

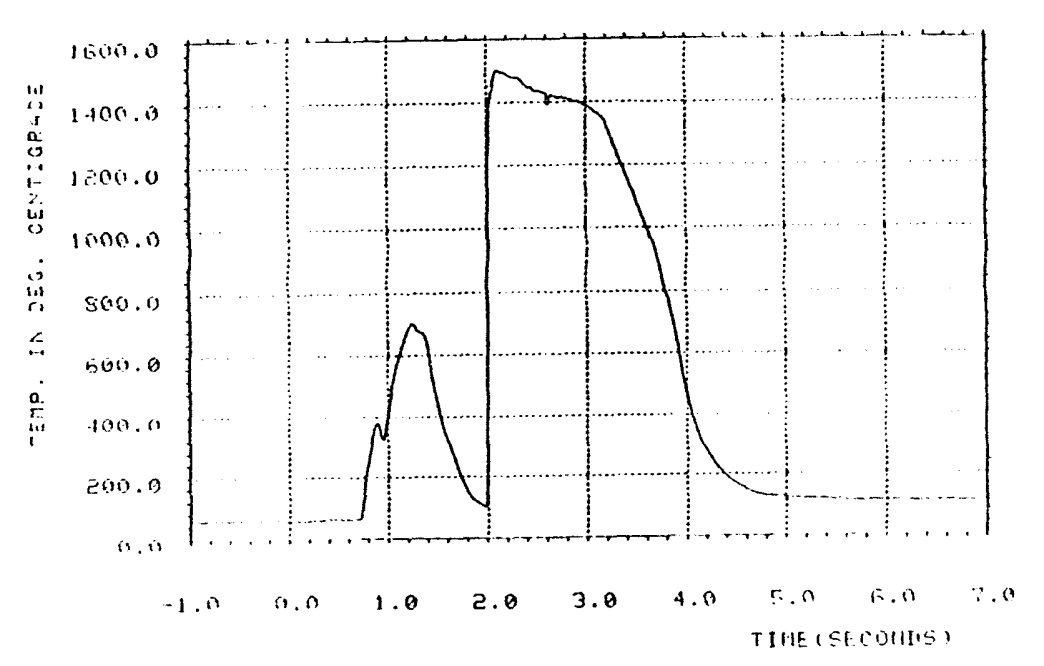

TrE-29, CCN-4, DATE $6: 457$

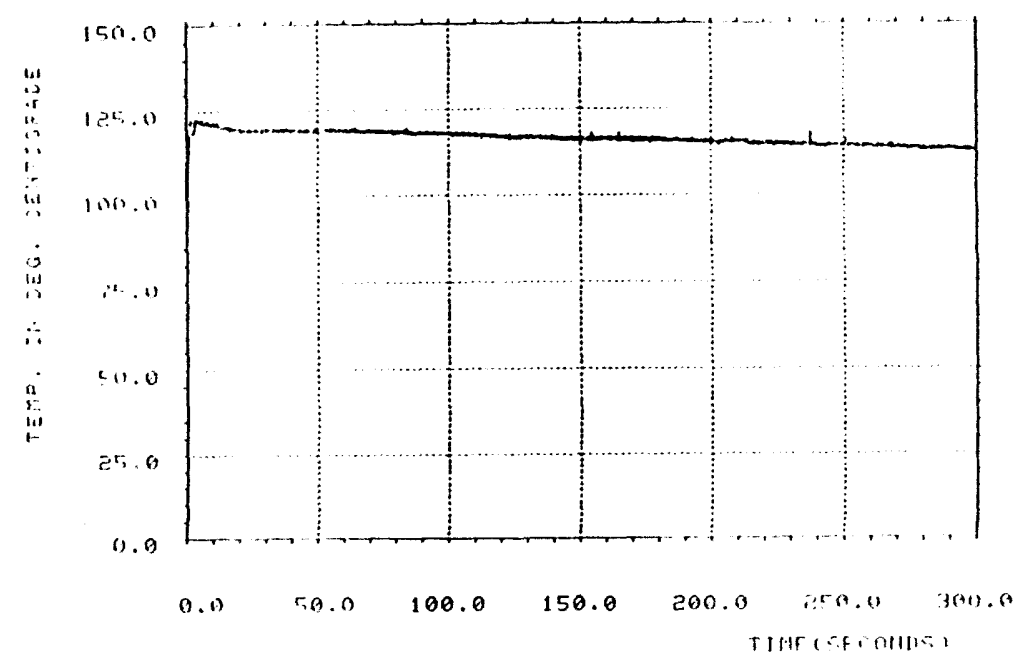

Tr. $3-28$, CCN-4, DATE $5.9 \%$

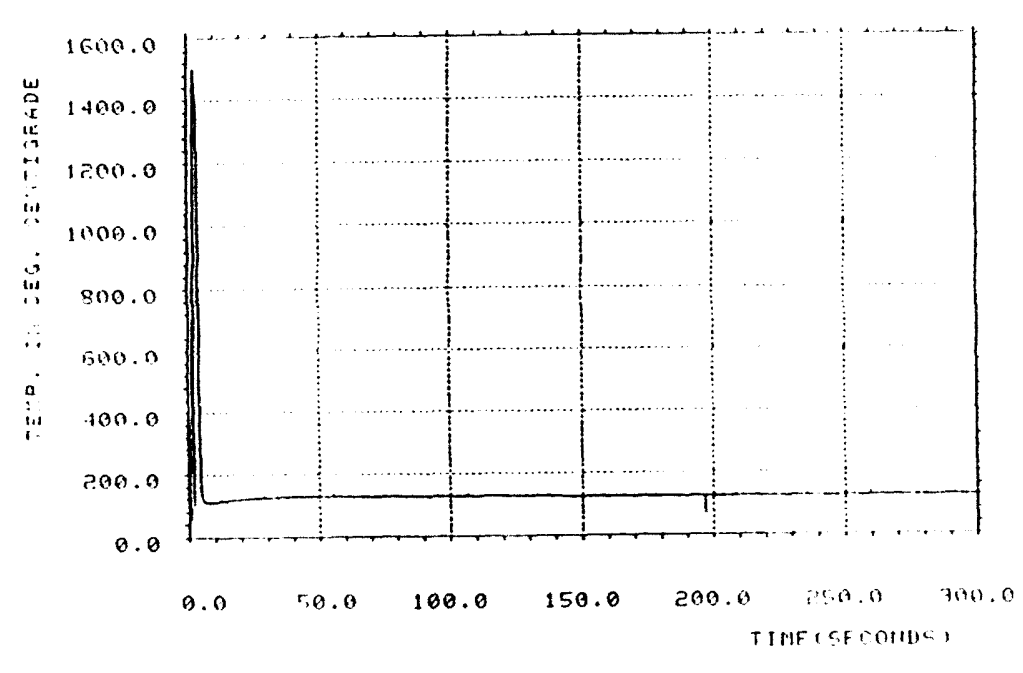

TOS-29, CCH-4, DATE 64 


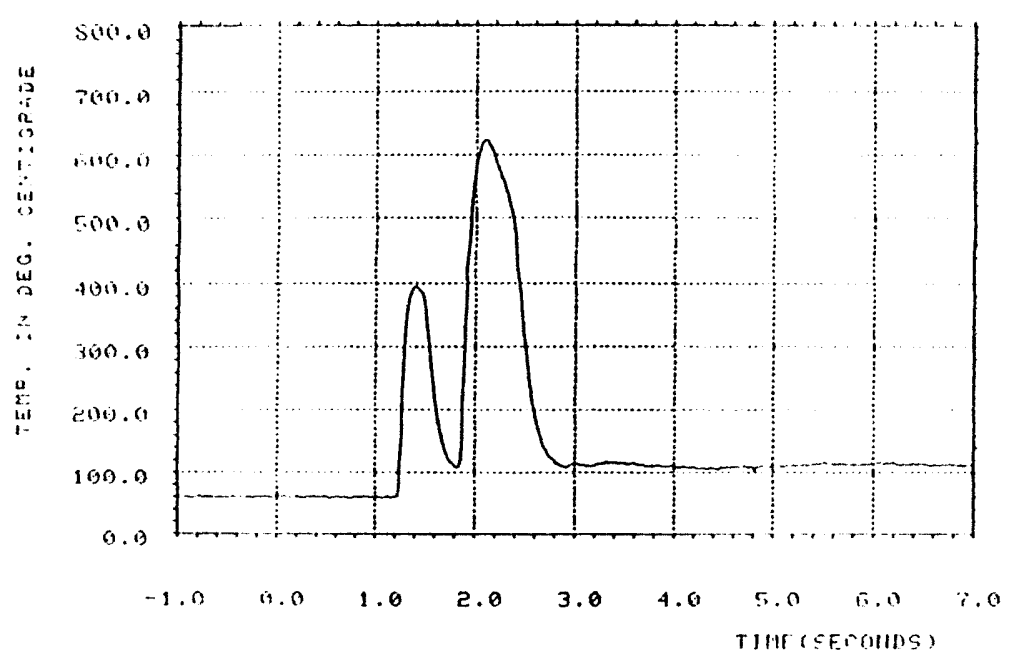

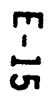

TrS-30, CH-4, DATE B. 13 ;

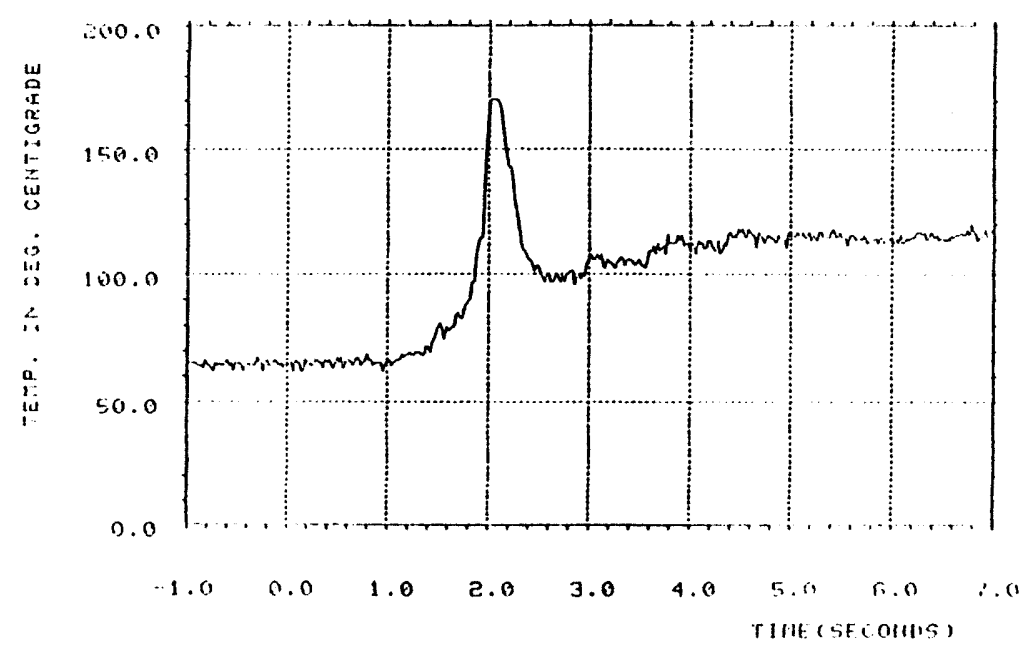

TES-31, CCN-4, DATE $6: 1 \cdot 3 \%$

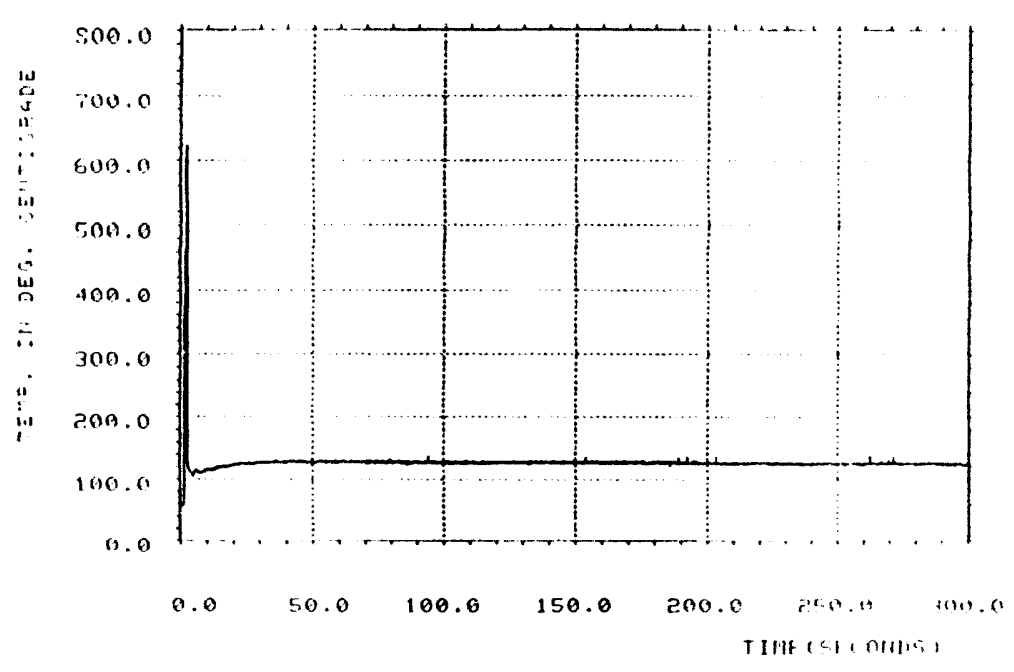

TE-30, COA- DATE $\cdots+\cdots$

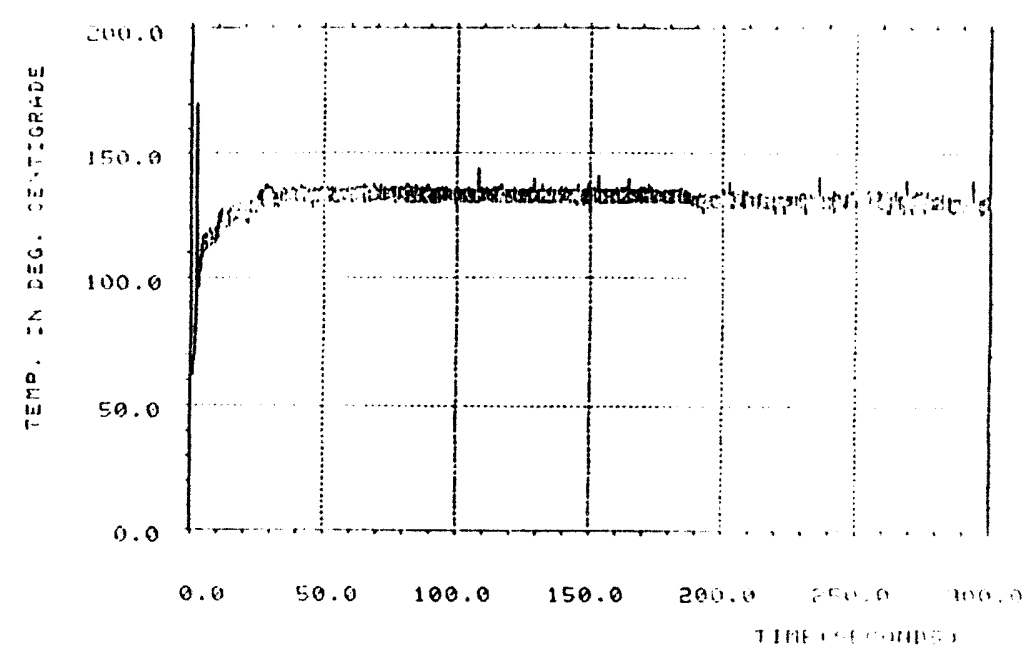

TOQ-31, CCH-1, [HATE 64.9 


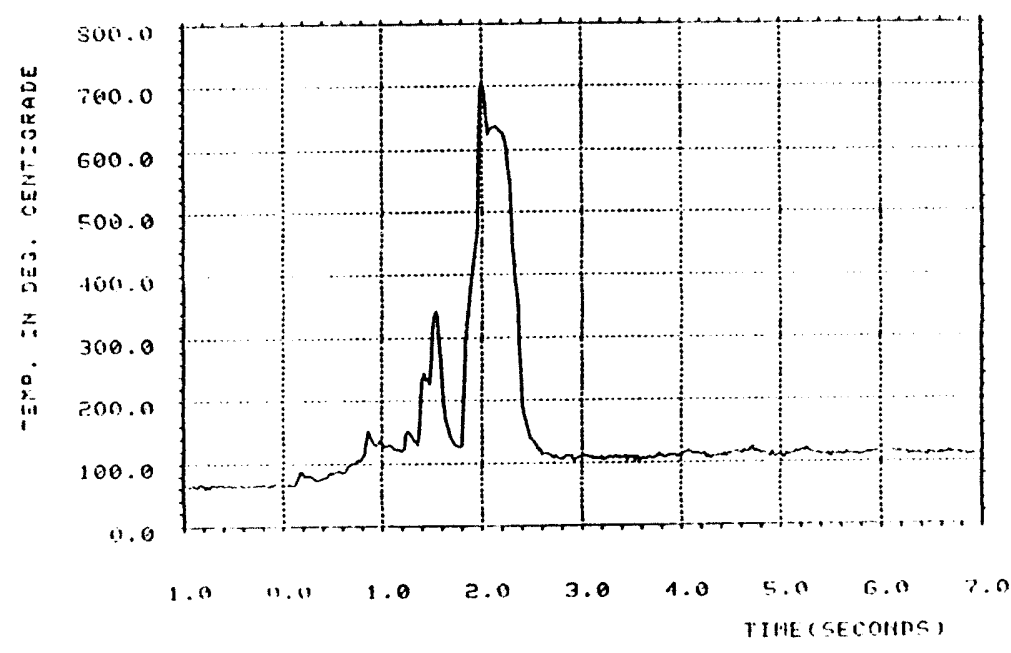

؛ั

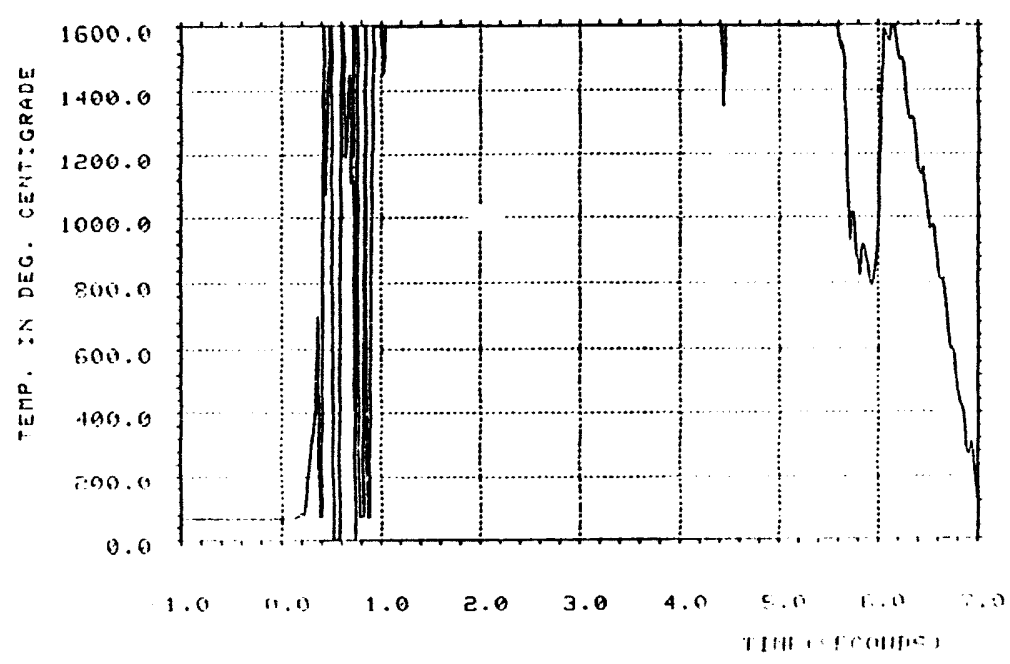

T. $3-33$, CON-4, DATE E. +.3

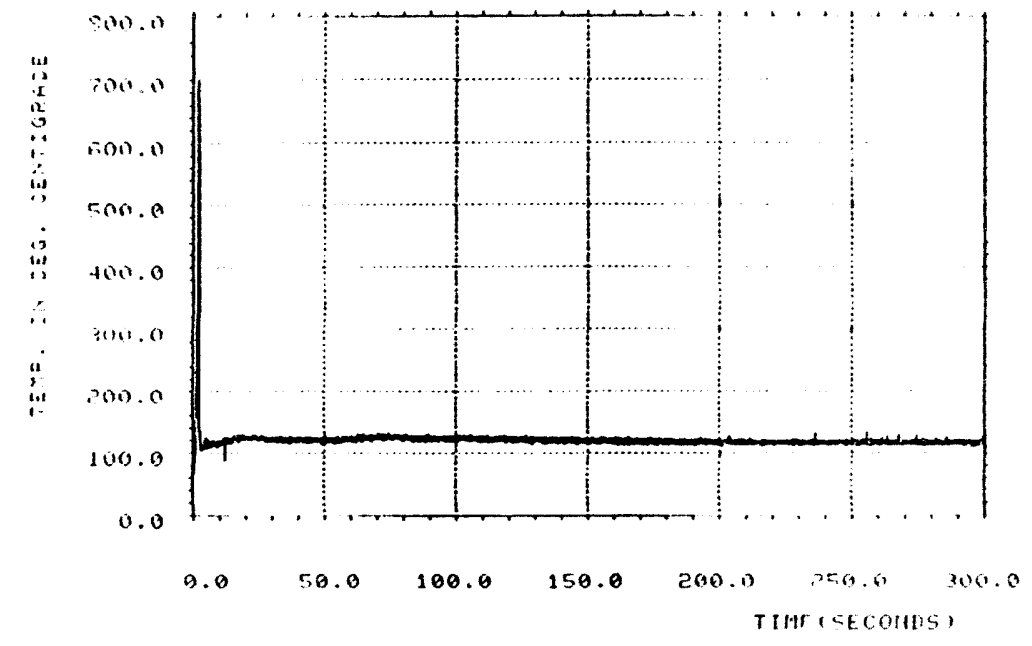

TrS-32, C.CH-4, DATE B.4 87

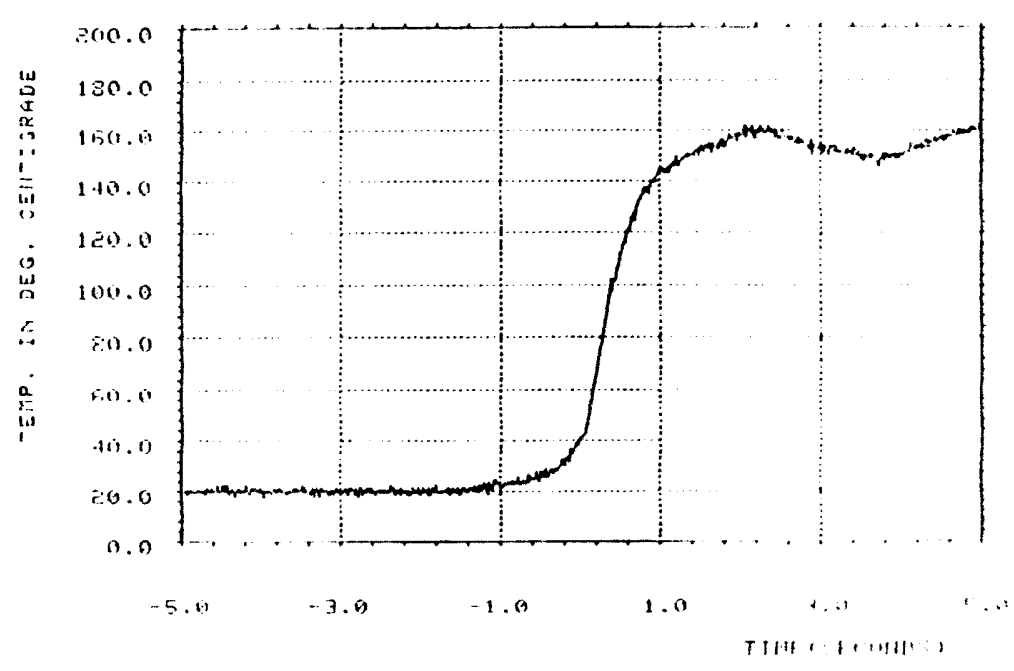

PI9-2, DCN-4. DATE 6.4 


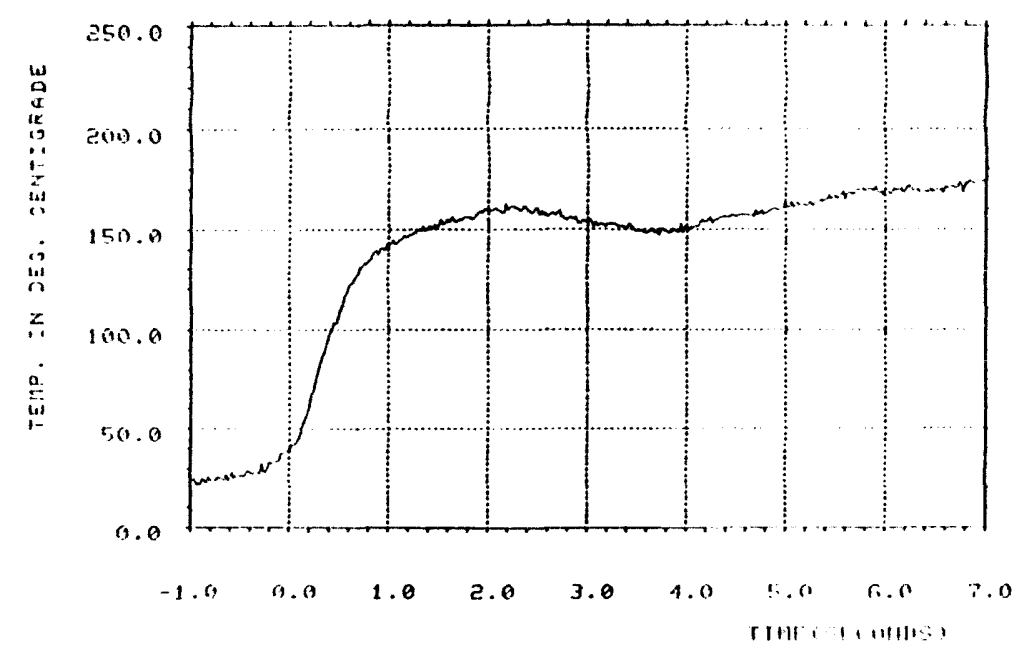

$\stackrel{m}{\check{1}}$

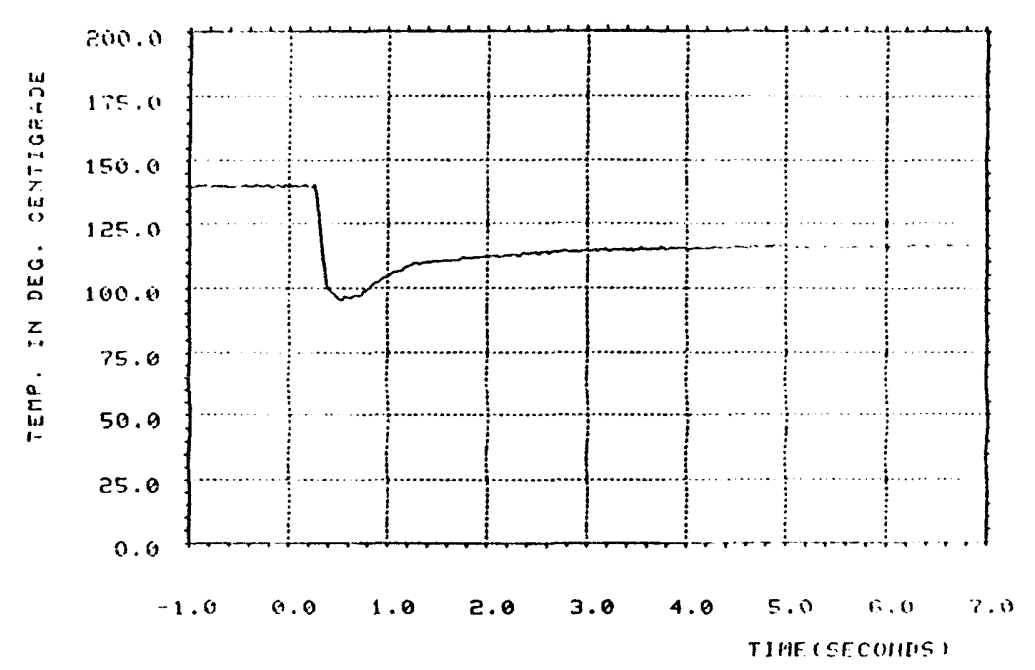

TC12-1, CC.11-4, DATE 6.4,8?

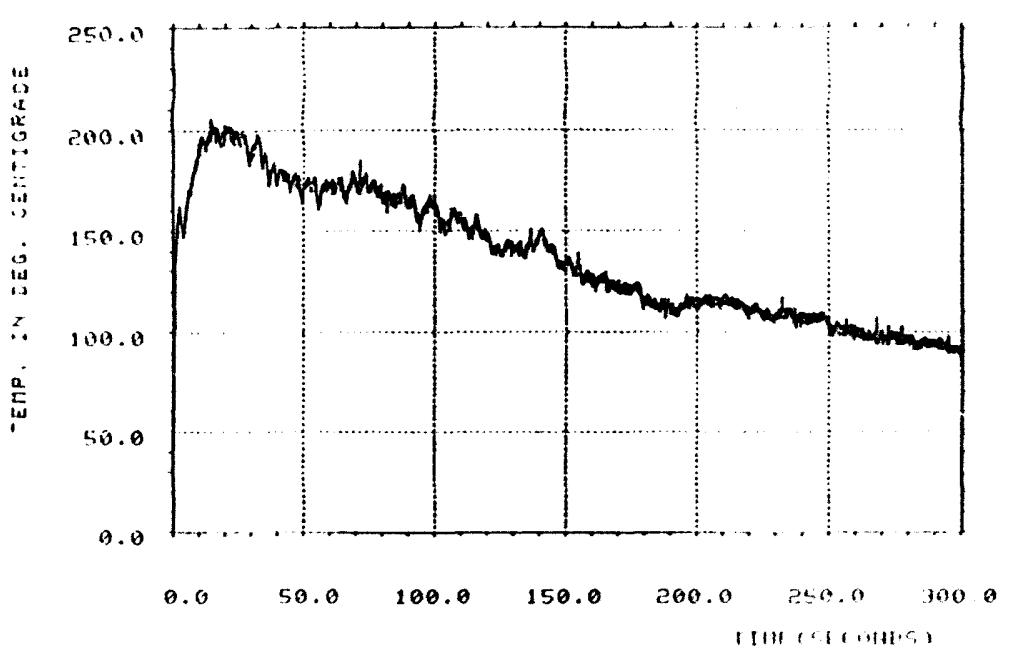

Tro-2, CCM-4, DATE $6,+37$

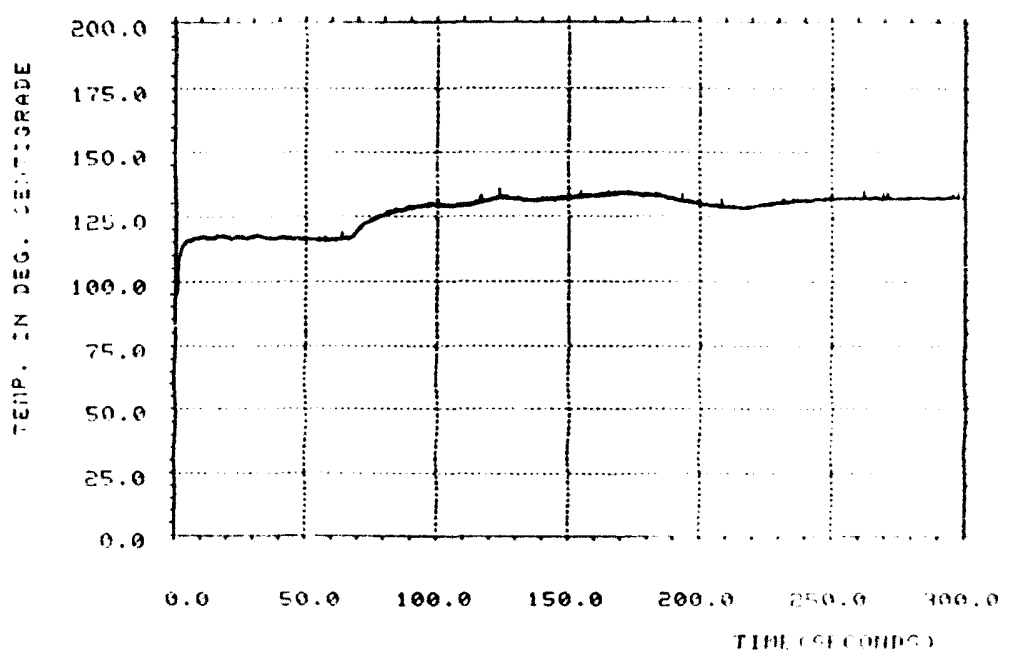

TI: $12-1$, COH-4, DATE F.4 ה? 


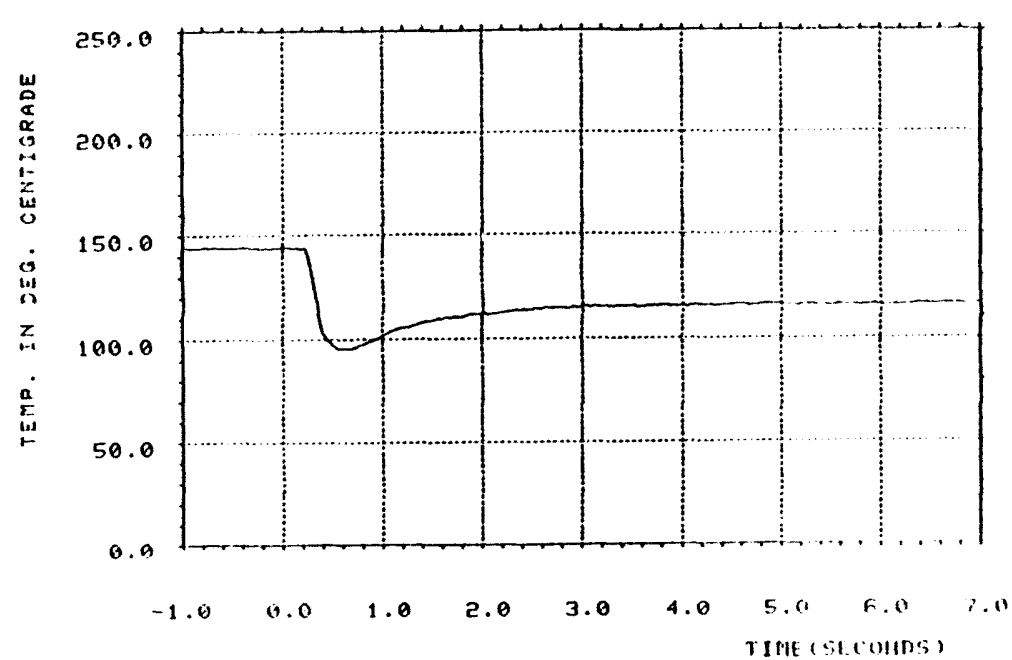

$\stackrel{m}{\stackrel{\infty}{\infty}}$

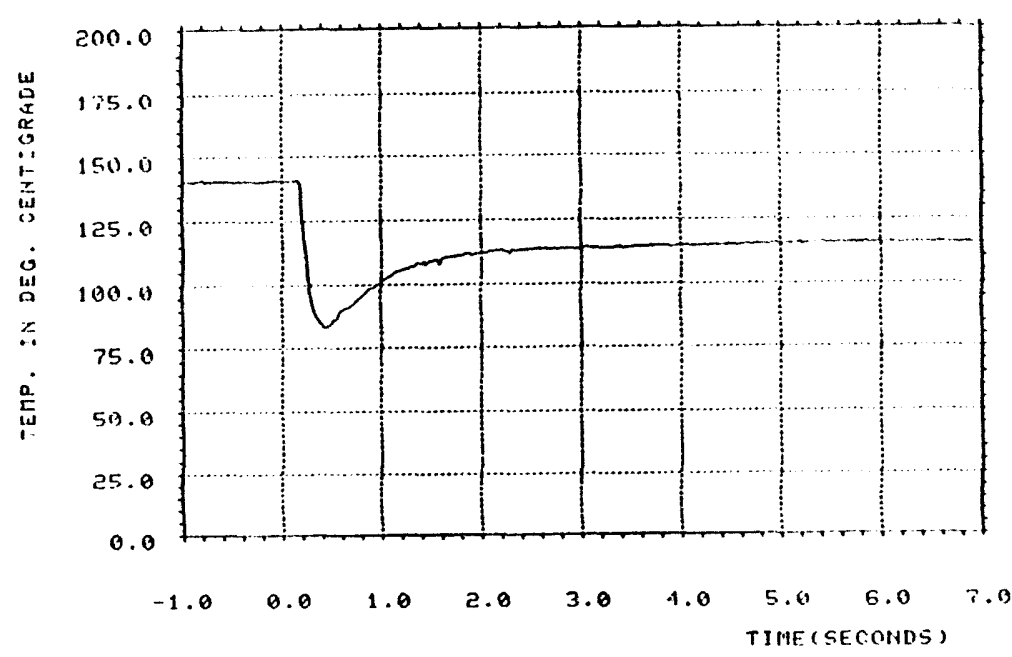

TC12-3, CCHi-4, DATE 6;4:8?

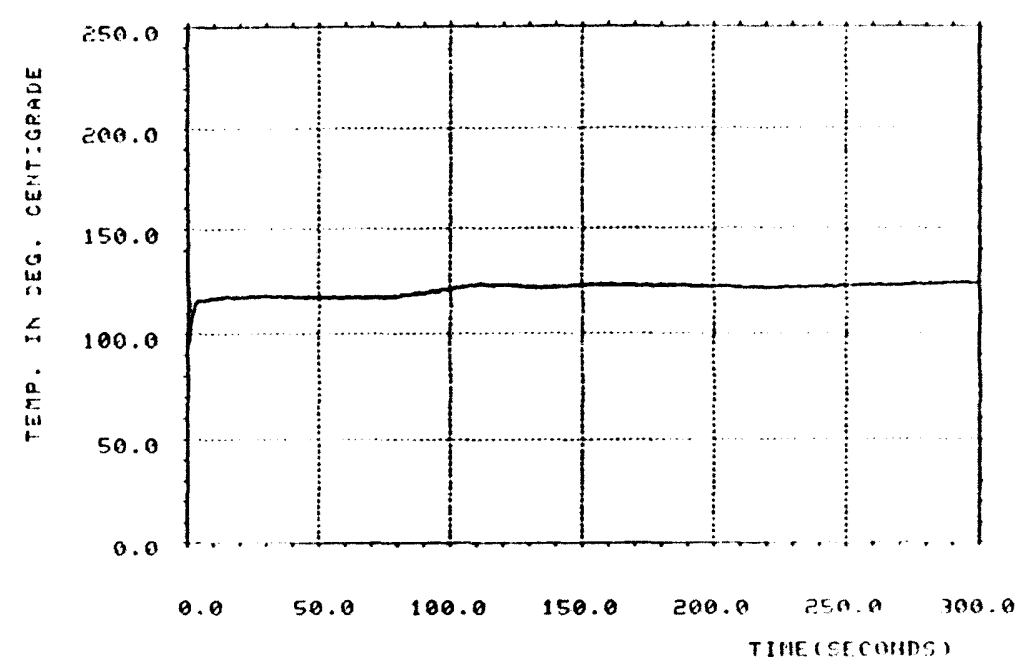

TC12-2, C.M.4, DATE 6/.1.8?

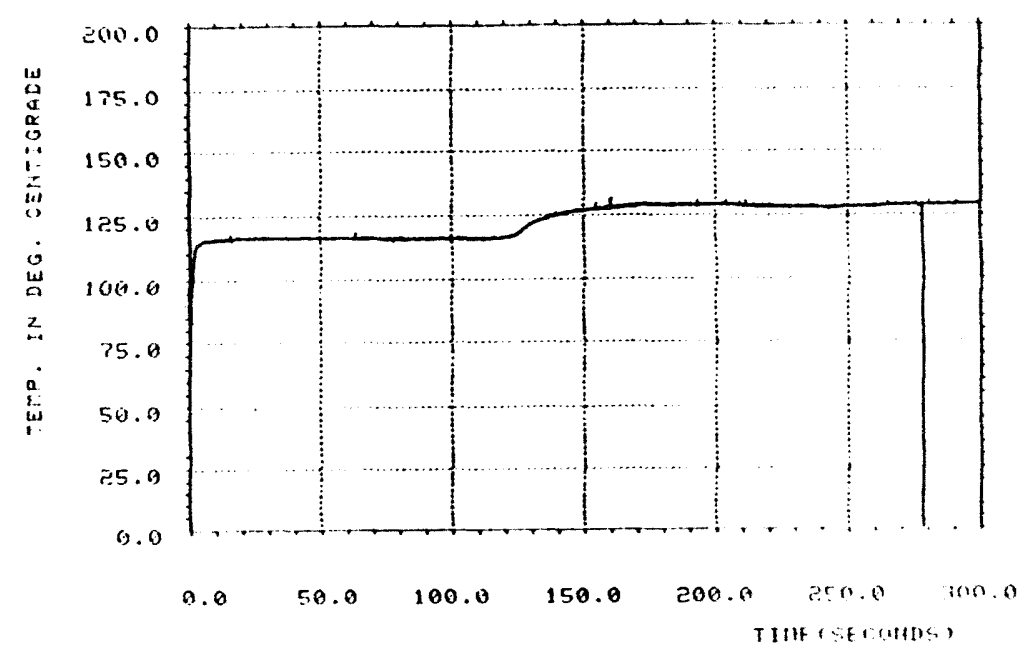

Tr:12-3, CCM-4, DATE 6/4:37 


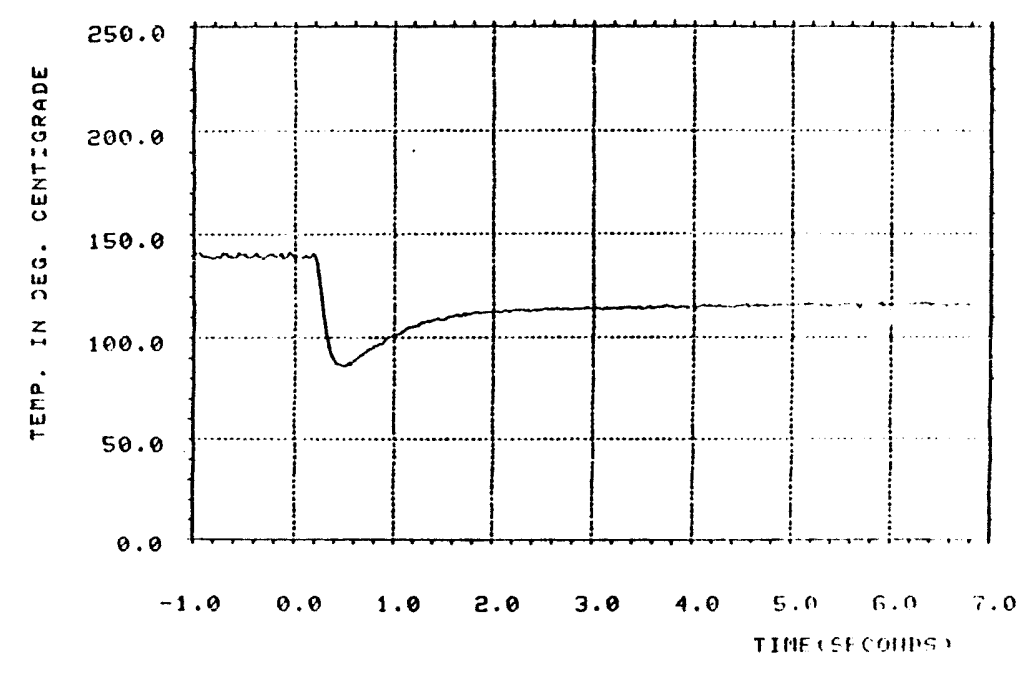

$\stackrel{m}{\grave{\omega}}$

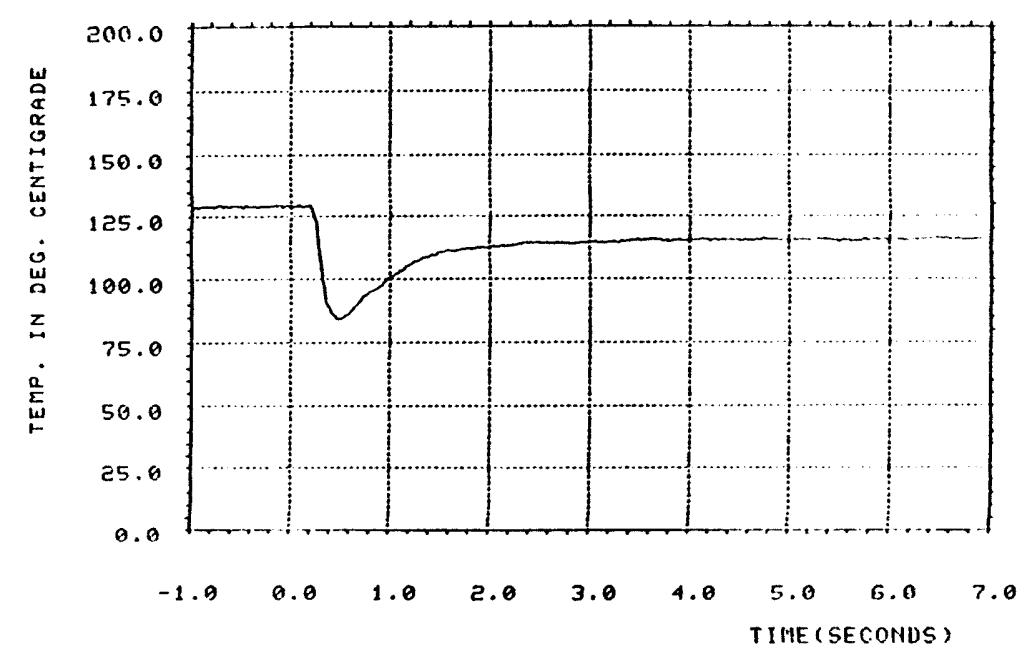

TC12-5, CCI1-4, DATE 6/4.37

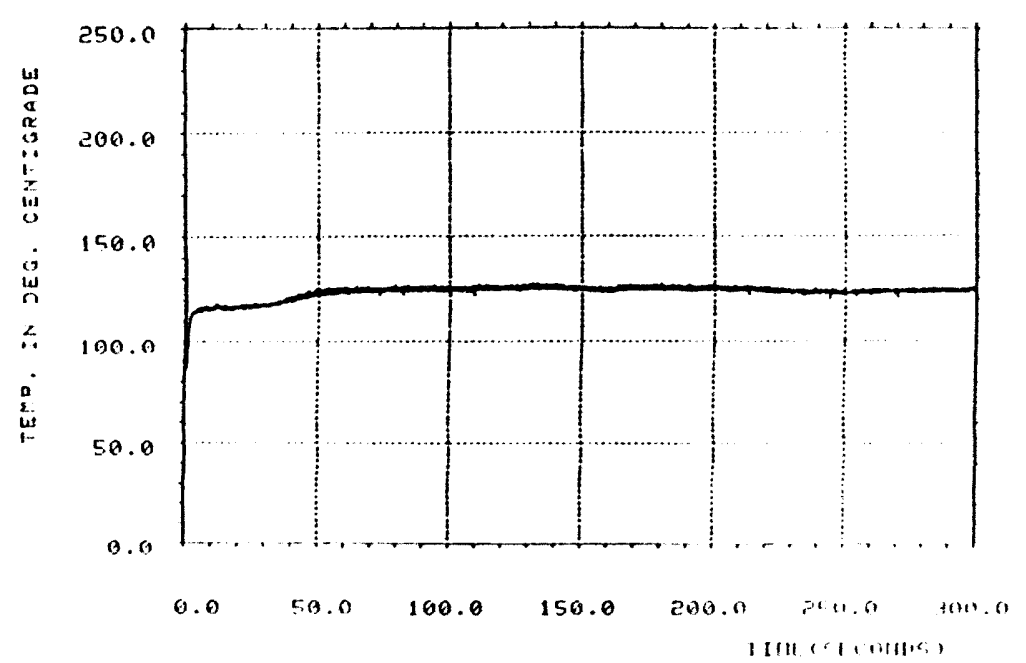

Tr 12̈-4, COA-4, DATE E-4 2 ?

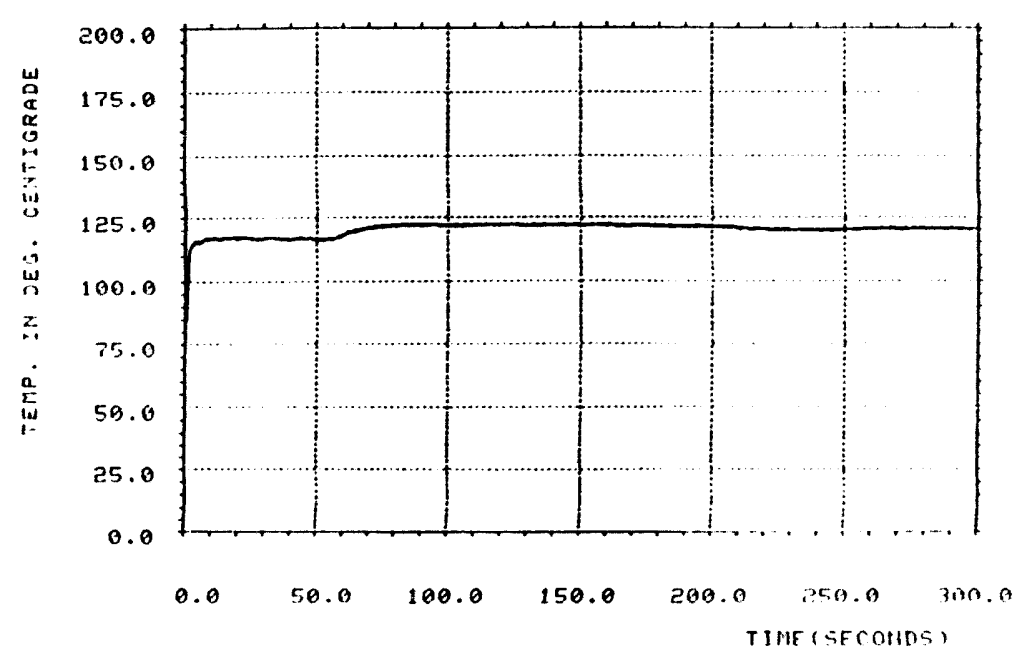

TC12-5, CCN-4, DATE $6,4,37$ 


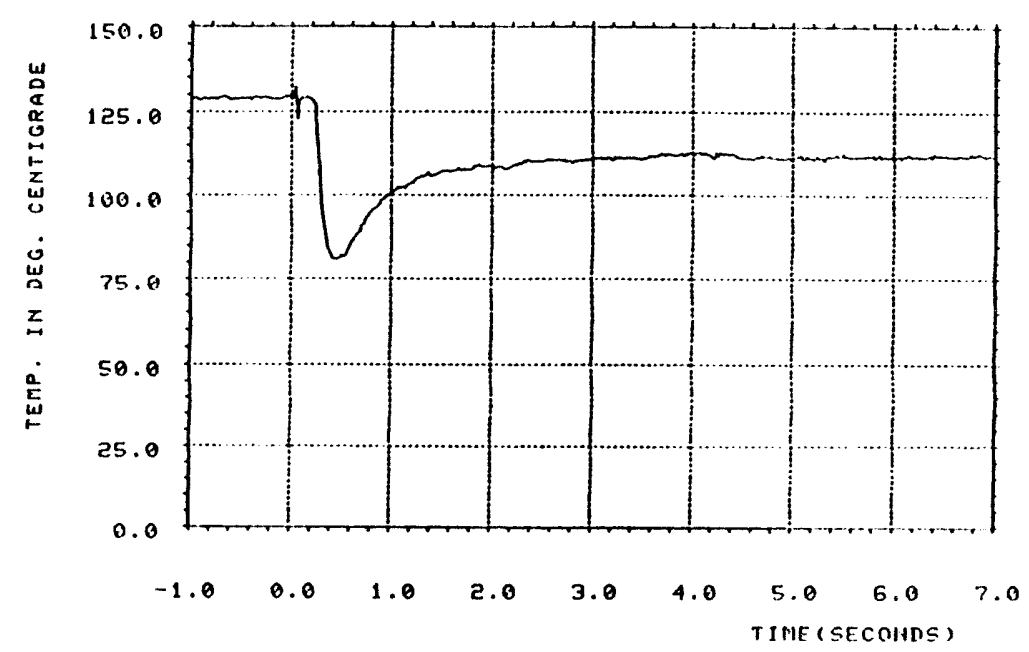

官

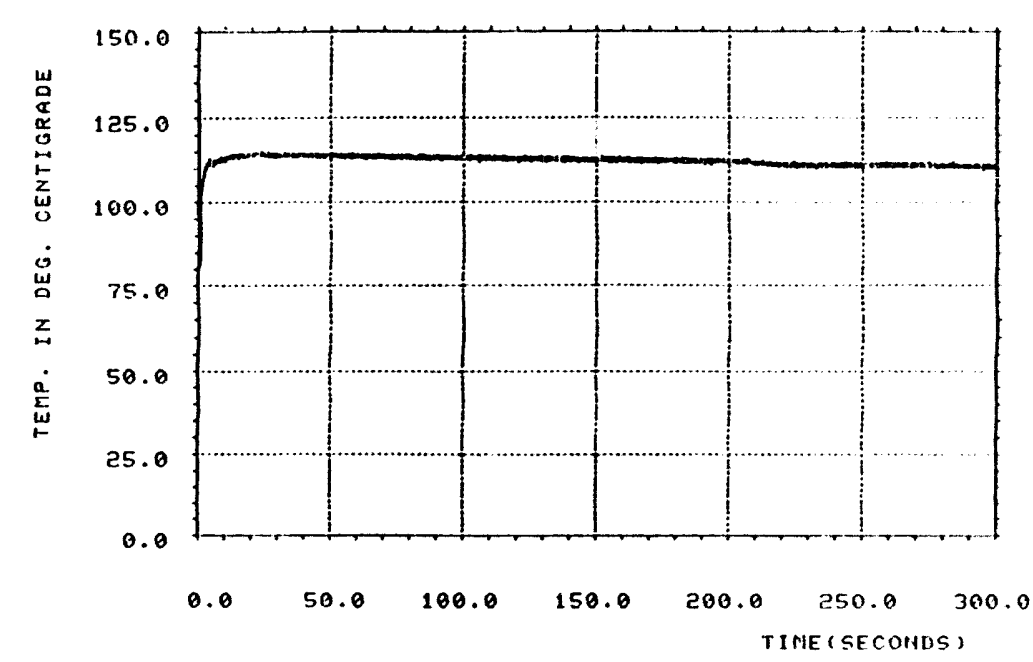

TC12-6, CCH-4, DATE 6/4,87 
APPENDIX F. DATA FOR CCM-5

PTS-1: Thermite-vessel Gas Pressure

PTS-9: Pressure in Top Section of Experiment Vessel

PTS-10: Pressure in Single-view-port Section of Experiment Vessel

PTS-12: Pressure in Single-view-port Section of Experiment Vesse1

TC8-6: Water/Debris Temperature in Catch Pan

TC8 7: Water/Debris Temperature in Catch Pan

TC8-8: Water/Debris Temperature in Catch Pan

TC8 9: Water/Debris Temperature in Catch Pan

TC8-10: Water/Debris Temperature in Catch Pan

TC8-16: Water/Debris Temperature in Catch Pan

TC8-17: Water/Debris Temperature in Catch Pan

TC8-18: Water/Debris Temperature in Catch Pan

TC8-19: Water/Debris Temperature in Catch Pan

TC8-20: Water/Napor Temperature

TC8-21: Water/Napor Temperature

TC8-22: Water/Vapor Temperature

TC8-23: Water/Napor Temperature

TC8-24: Water/Napor Temperature

TC8-26: Water/Napor Temperature

TC8-27: Water/Napor Temperature

TC8-28: Water/Napor Temperature

TC8-29: Water/Vapor Temperature

TC8-30: Water/Vapor Temperature

TC8-31: Water/Vapor Temperature

TC8-32: Water/Napor Temperature

TC8-33: Water/Vapor Temperature

TC8-35: Water/Napor Temperature

TC8-36: Water/Napor Temperature

TC8-37: Water/Napor Temperature

TC8-38: Water/Napor Temperature

TC8-40: Water/Napor Temperature

TC8-41: Water/Vapor Temperature

TC8-42: Water/Napor Temperature

TC8-43: Water/Vapor Temperature 
APPENDIX F. DATA FOR CCM-5 (Cont'd.)

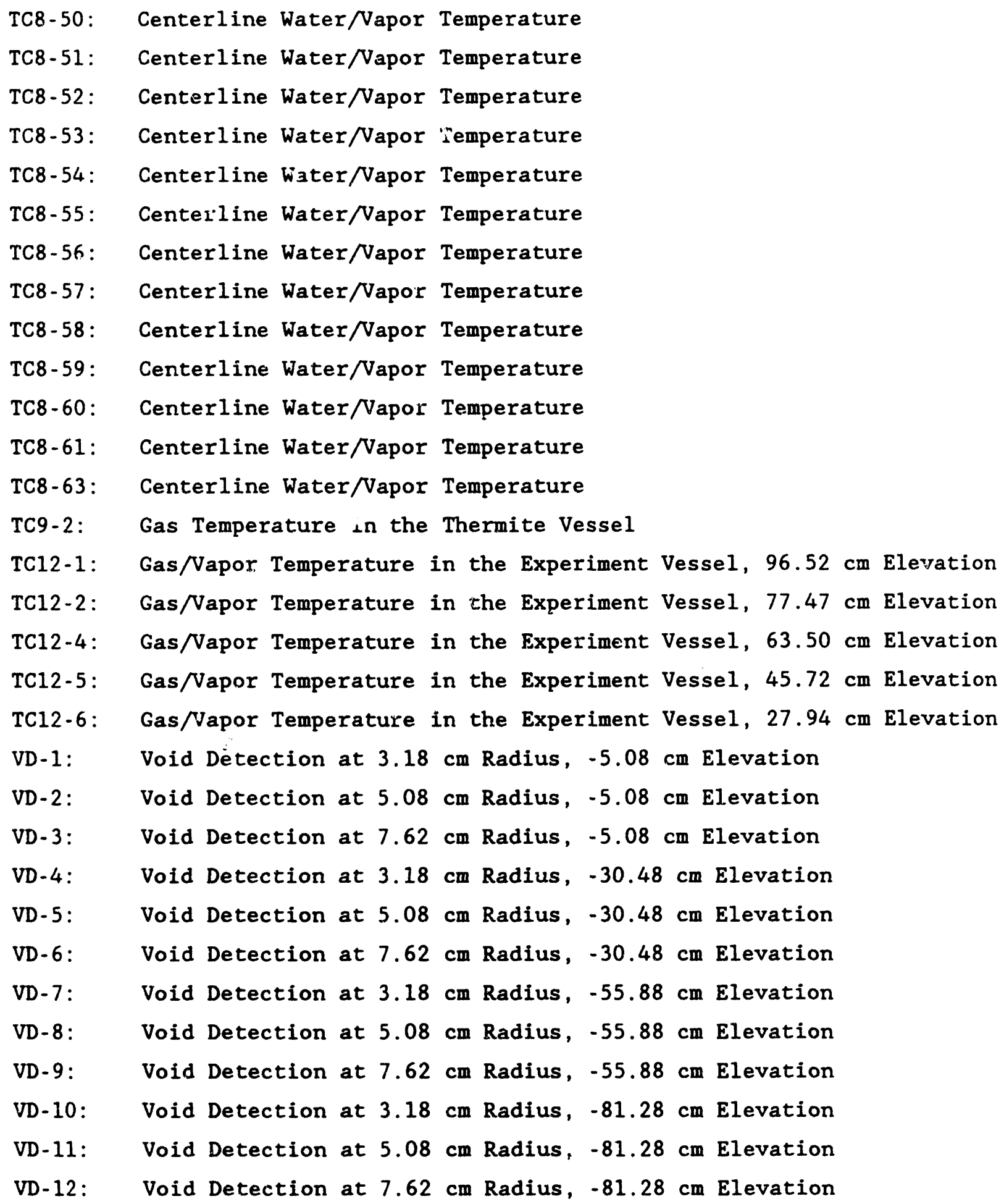




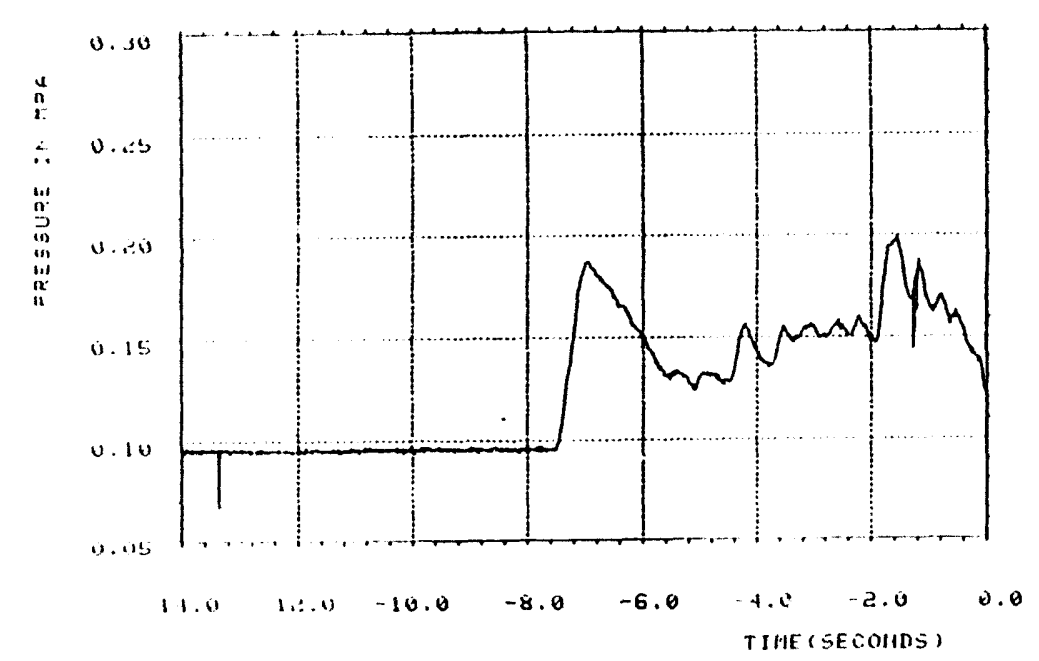

1TS-1, CCN-5, LATE 91?0?

$\tilde{\omega}$

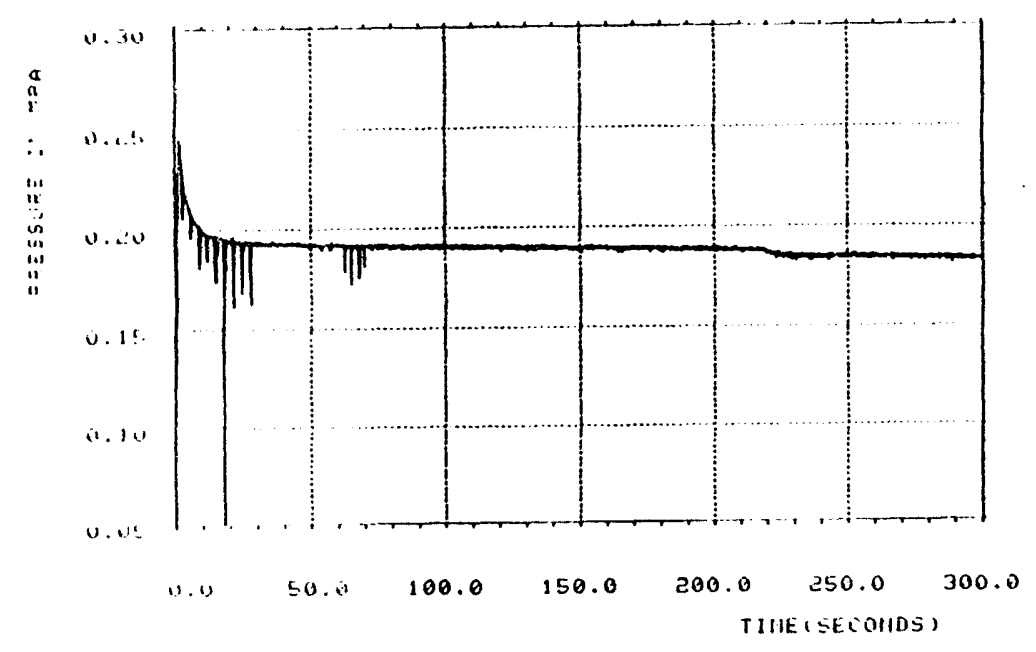

FTS-1, CCM-5, IIATE g 17. S:

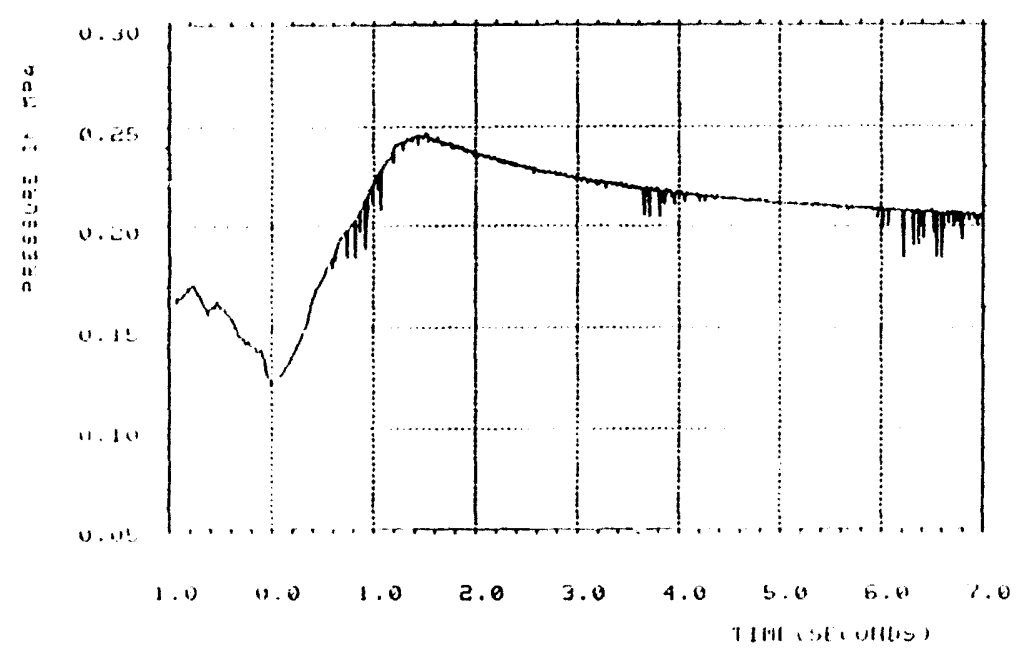

N $\$ 1$, CON-S, WHTE $\subseteq 17 \ldots$

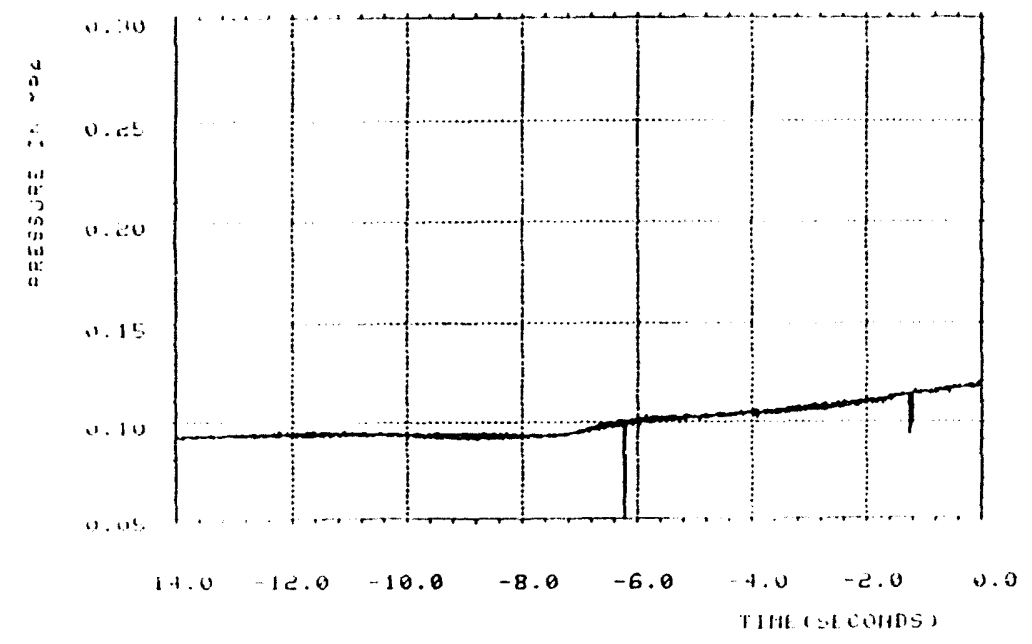

WT-B, CCH-E. DATE \& $1 ; \ldots ;$ 


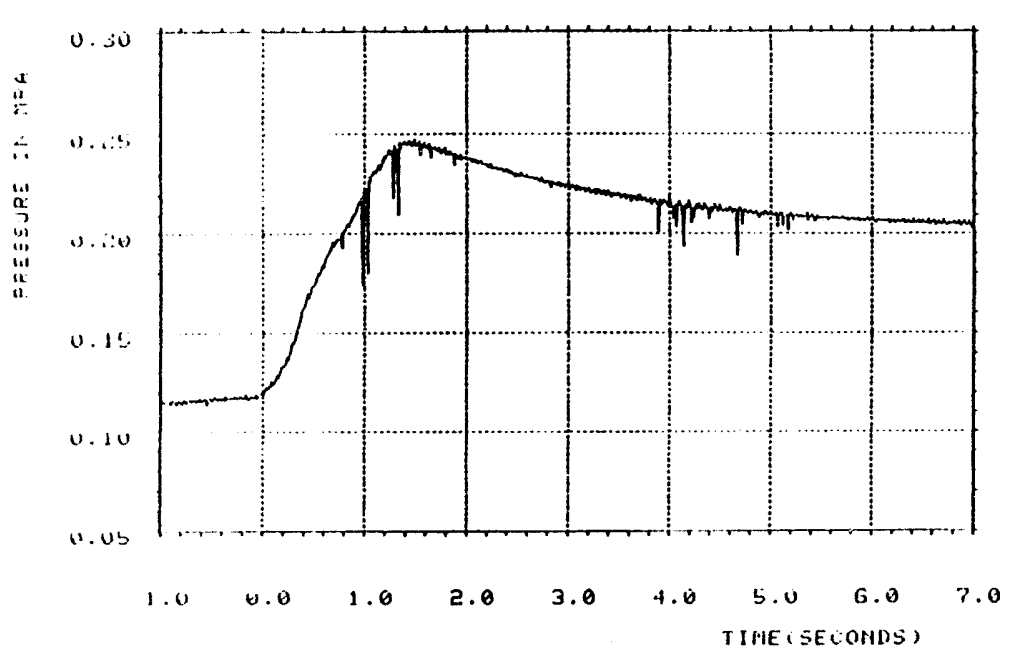

PTS-9, CCM-5, LMATE 9 17-S?

$\overline{5}$

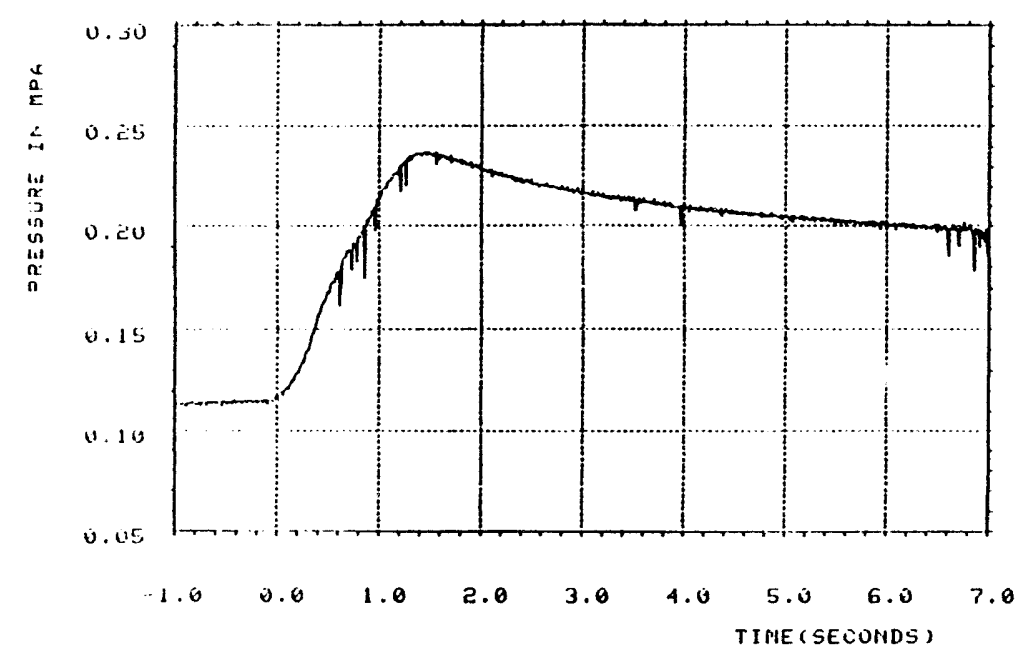

F.T:-10, CCM-5, DATE 9-17.27

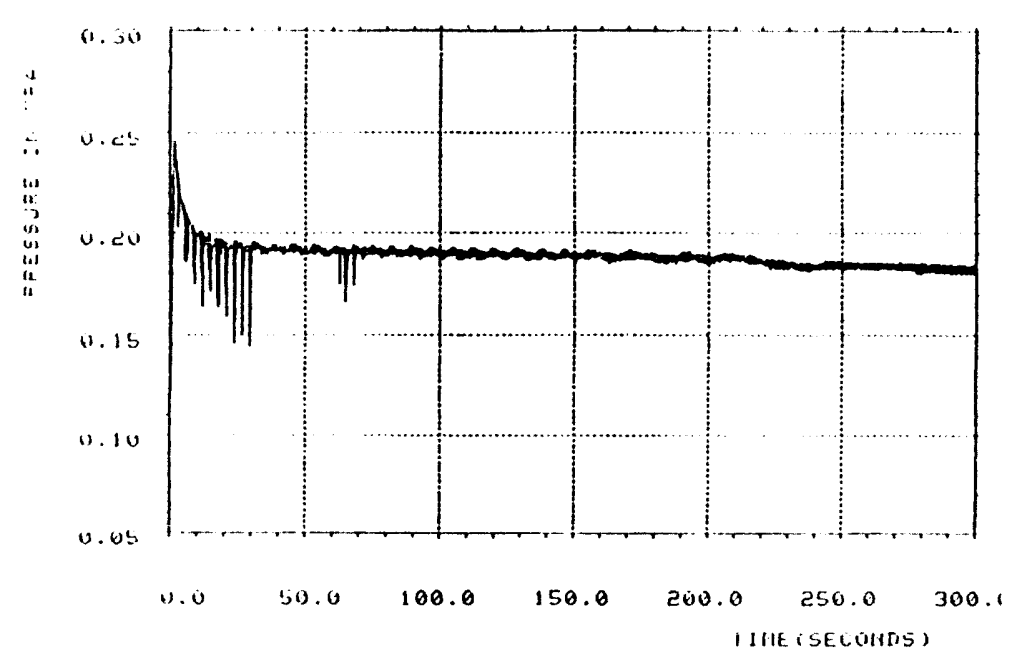

FTS-9. C.CM-5, DATE G.1? :?

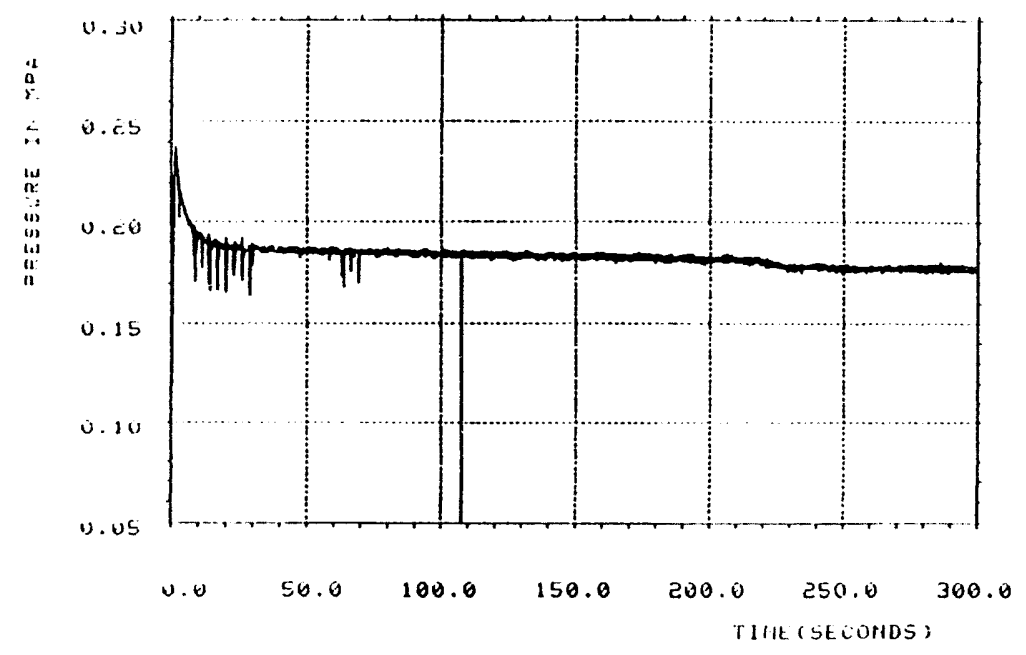

PTS-10, CCH-5, [HATE G 17.8? 


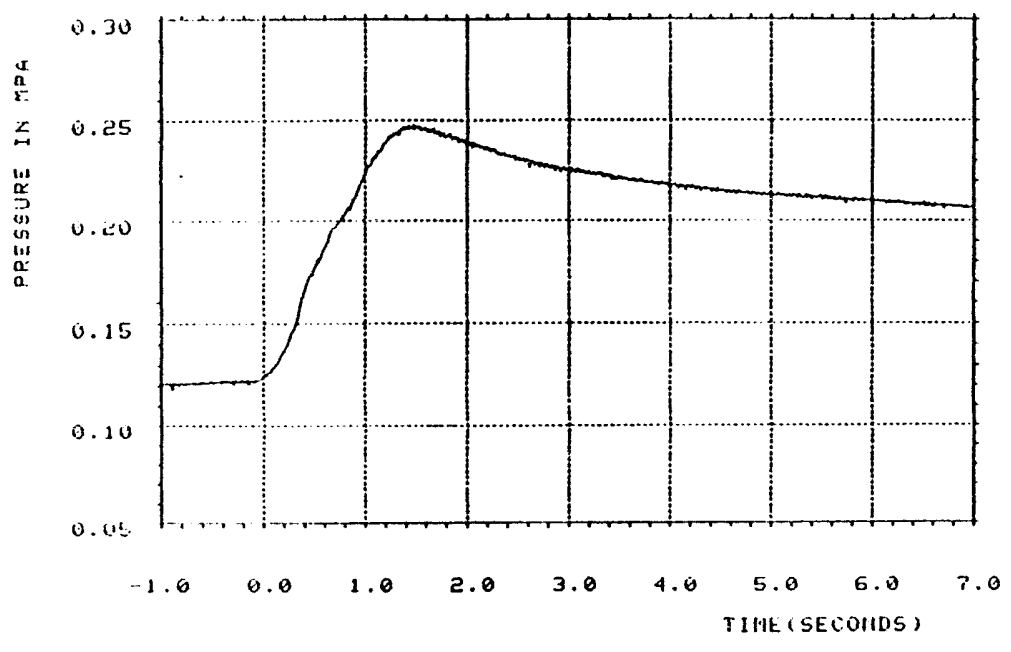

FTs-12, con-5, luate g:17:3;

ín

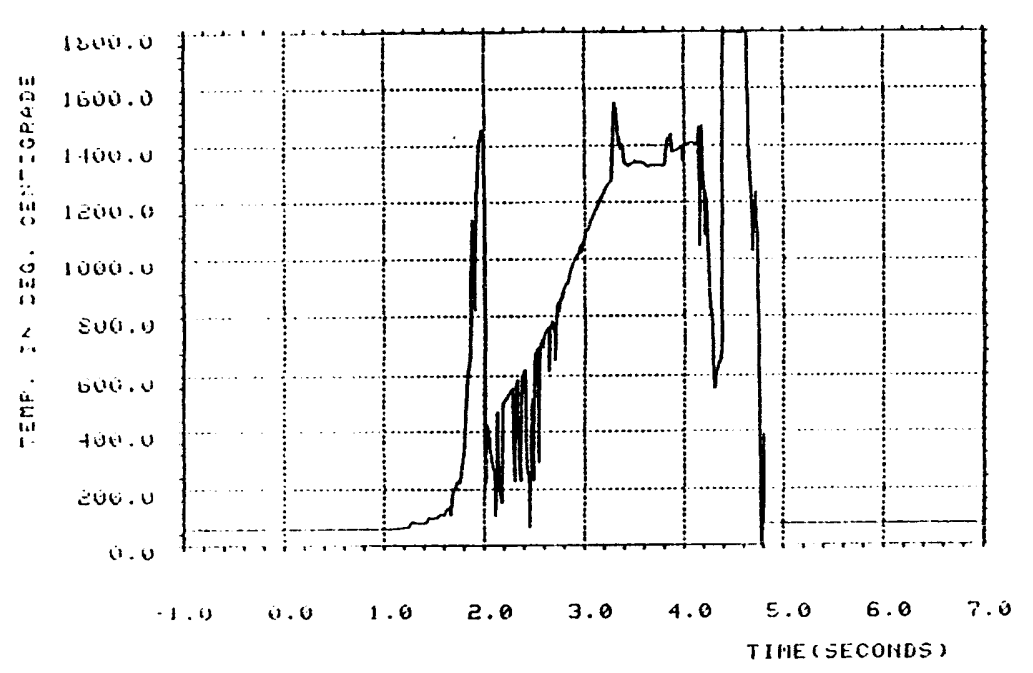

TIS-E, CCN-E, [IATE $9 / 17,87$

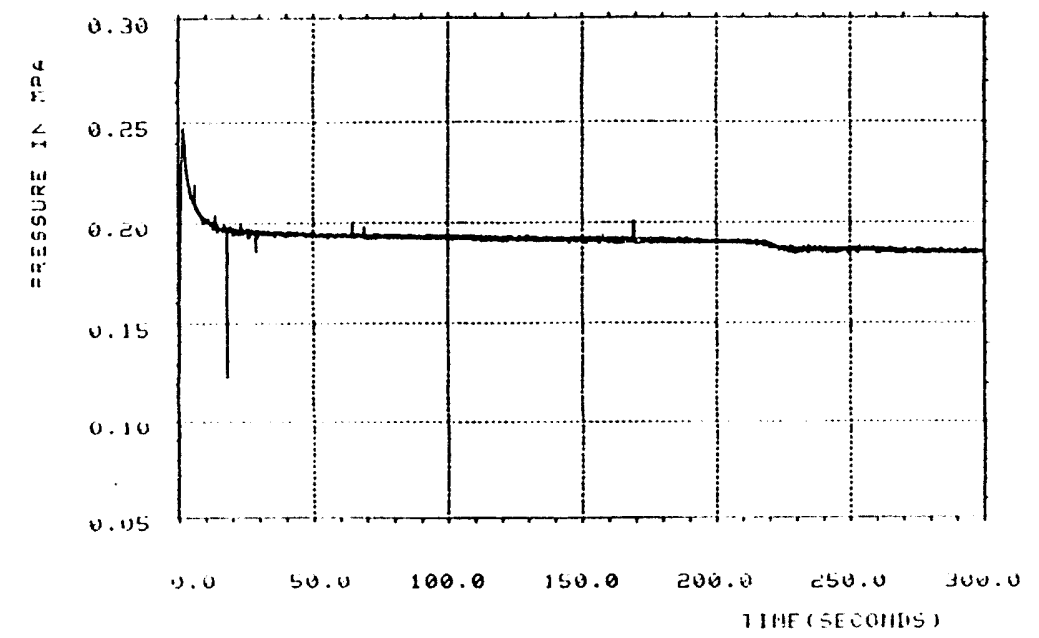

HTS-12, CCH -5, [ATE $y-17,3:$

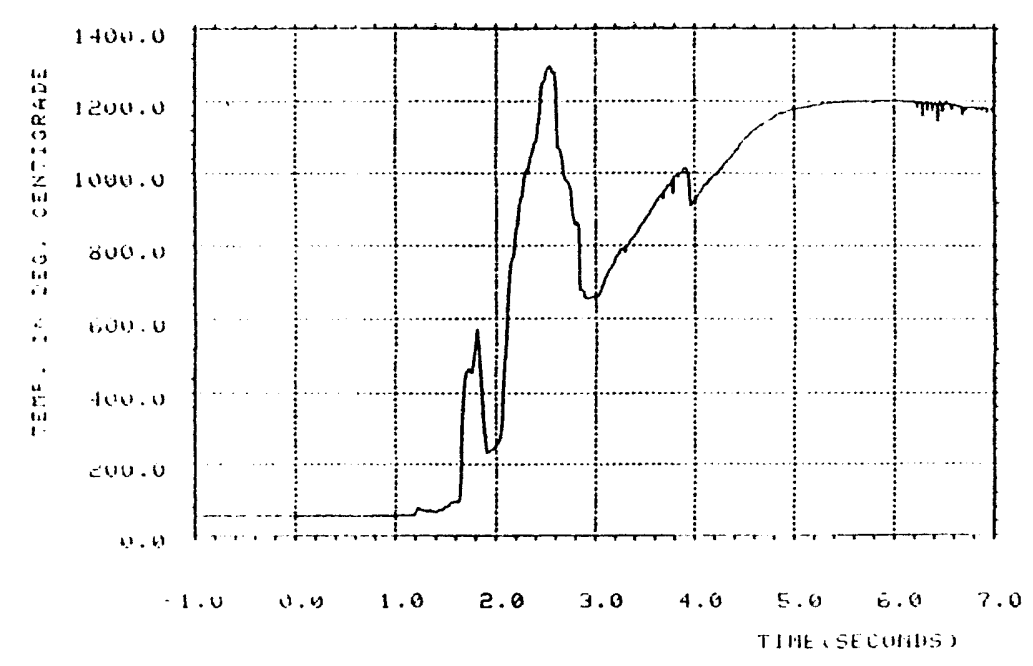

W. $\$-7$, COM-5, [IATE $917 \therefore$ 


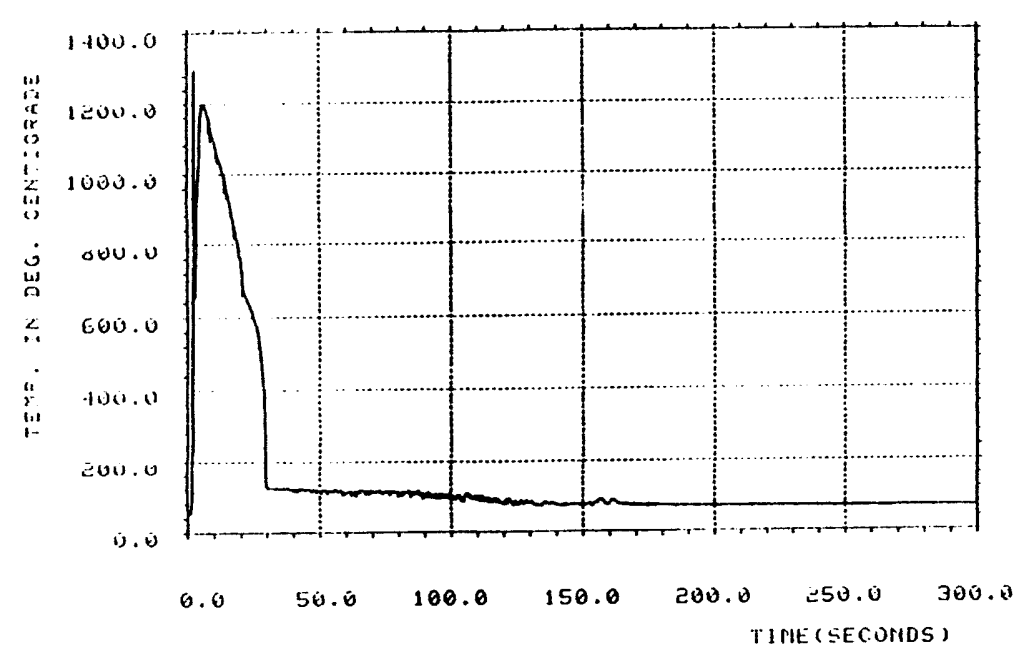

TC8-?, CCH-5, DATE 9:17,8?

$\stackrel{1}{o}$

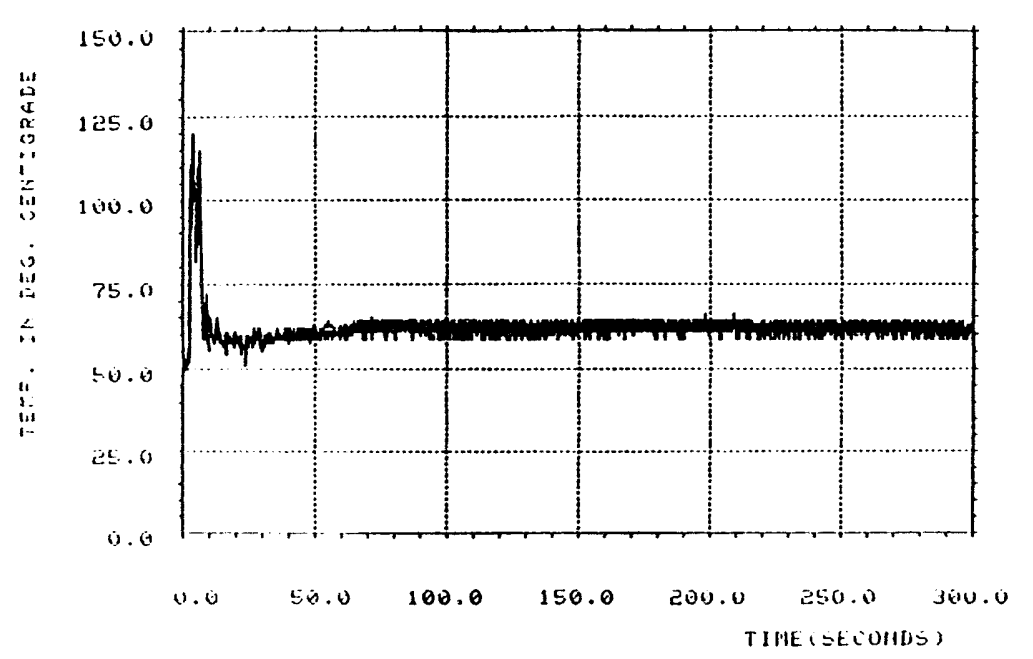

TC3-8, CCI-5, [NATE 9/17-87

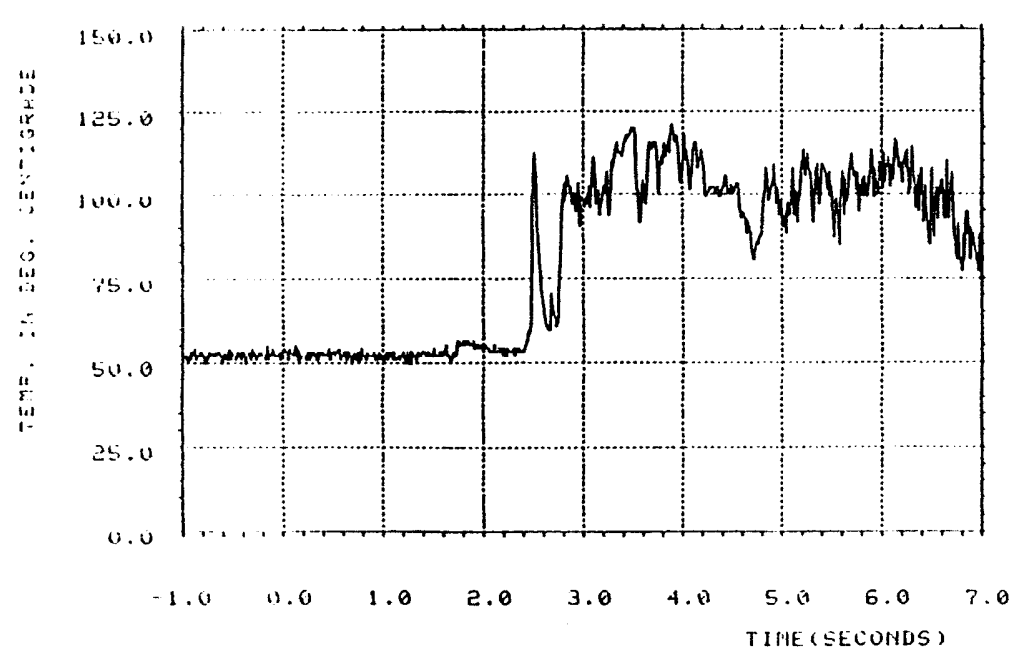

TIS-5, CCII-5, DATE 9,17, 87

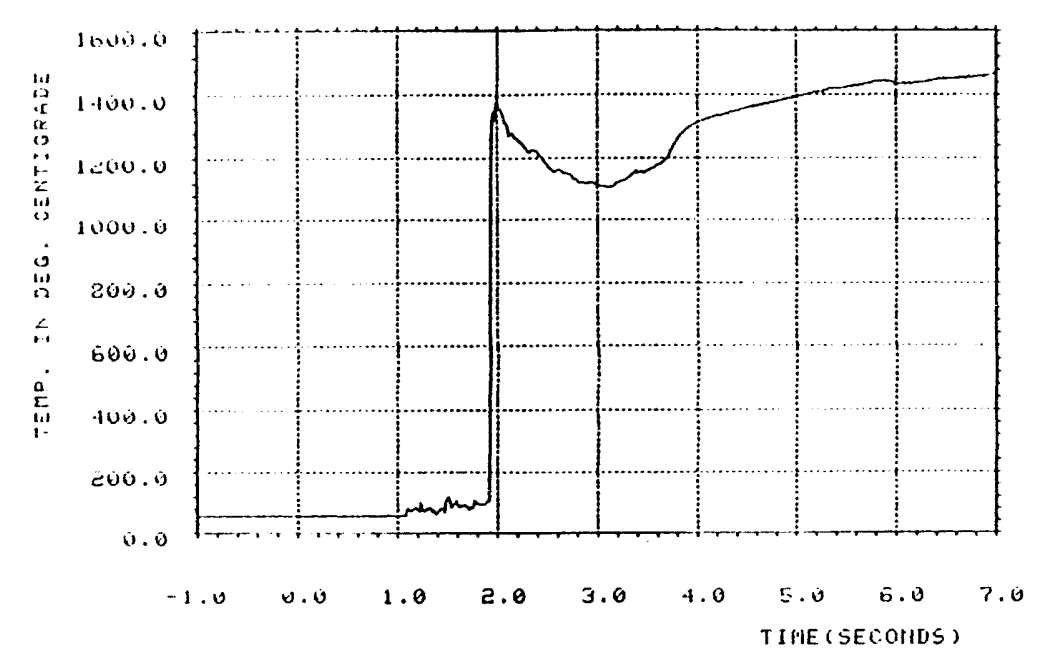

Ti. $8-9$, CCM-5, DATE $9 / 17 / 87$ 


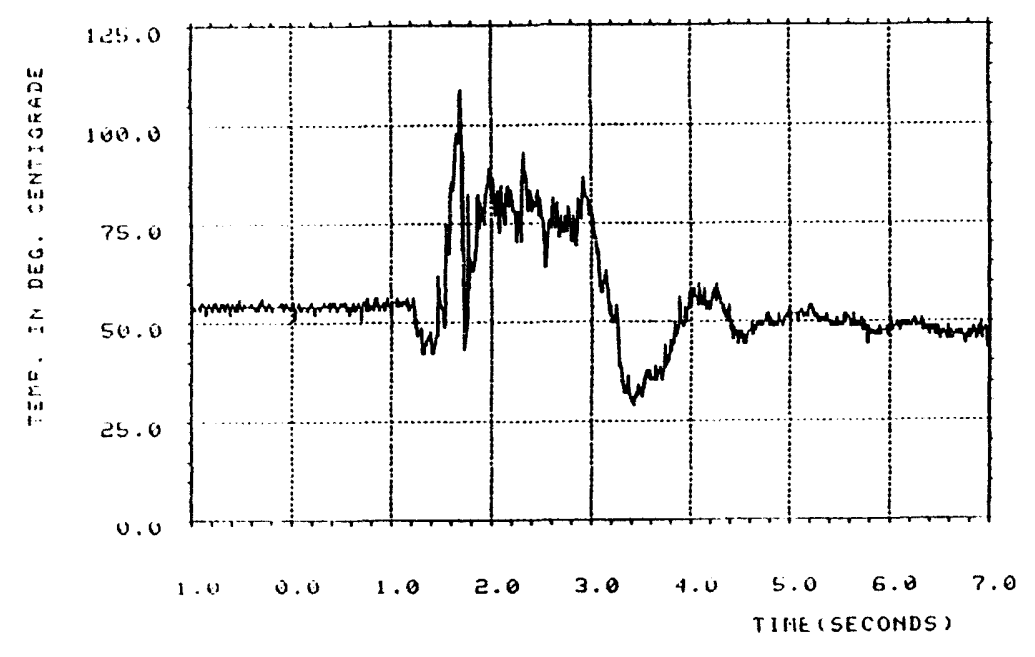

$\stackrel{\pi}{2}$

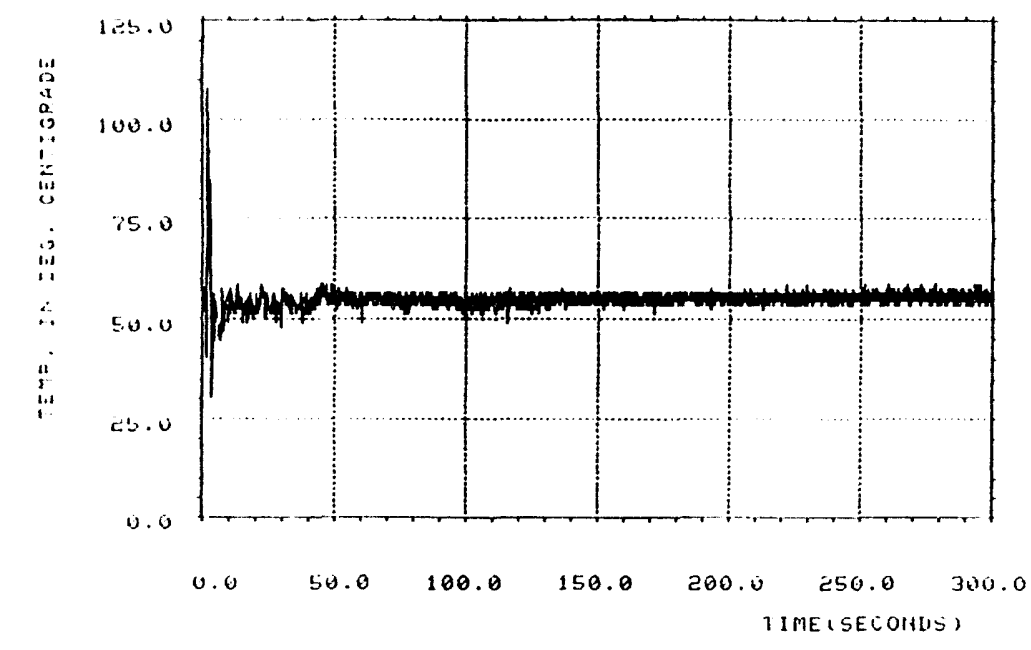

Ti 3-10, CON-5, DATE $9.17 \mathrm{~B}$

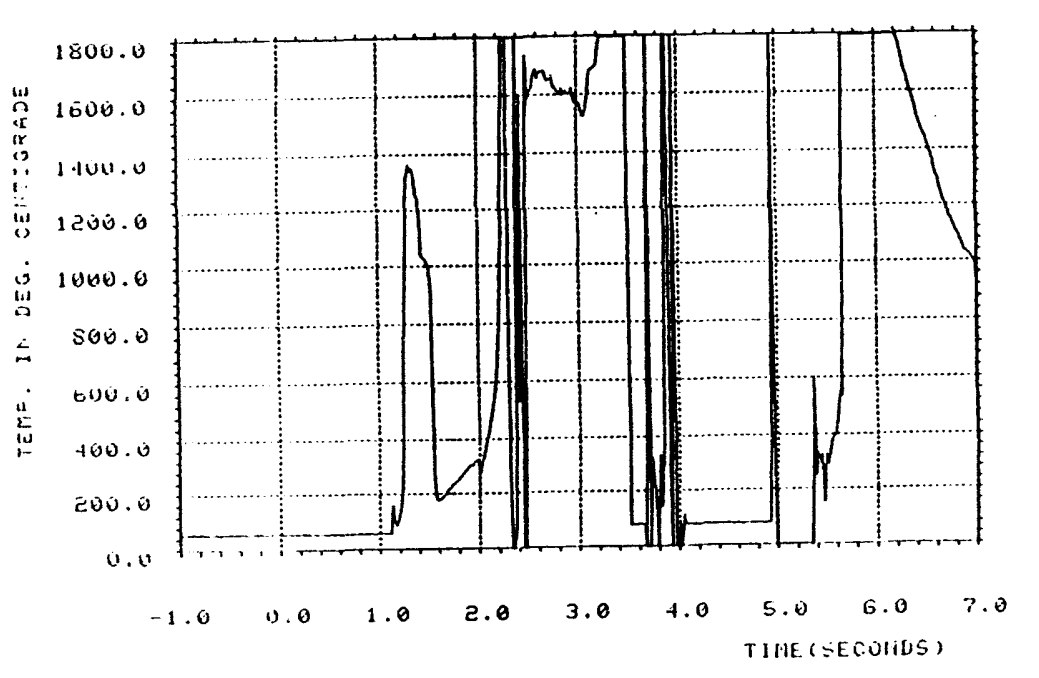

TIE-15, CCM-5, [IATE $9.17 .6 ?$

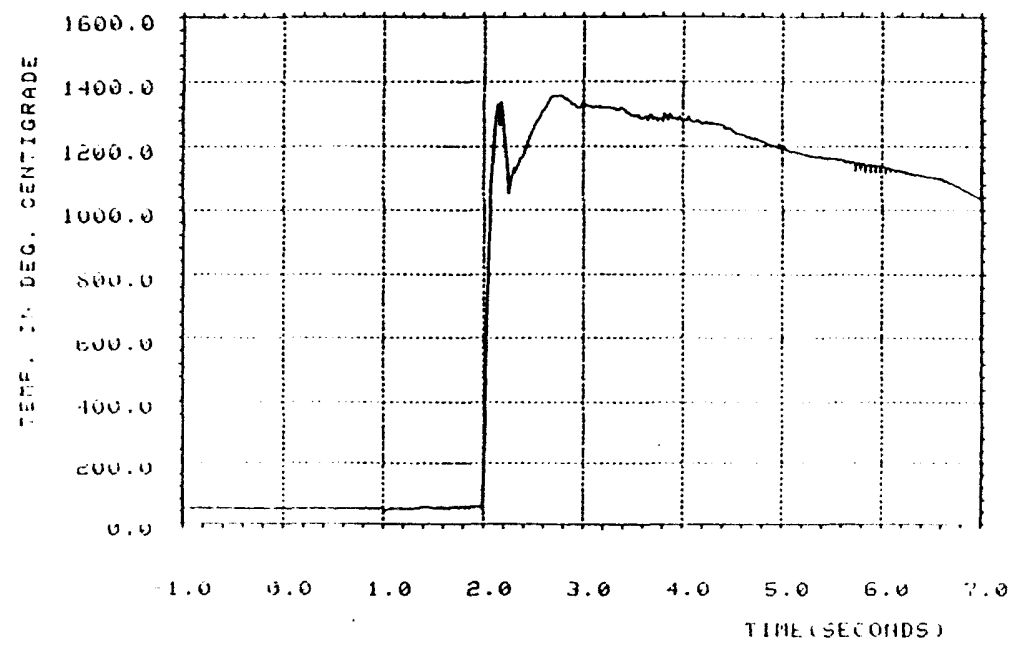

TV8-17, CCH-5, DATE 9.17:? 


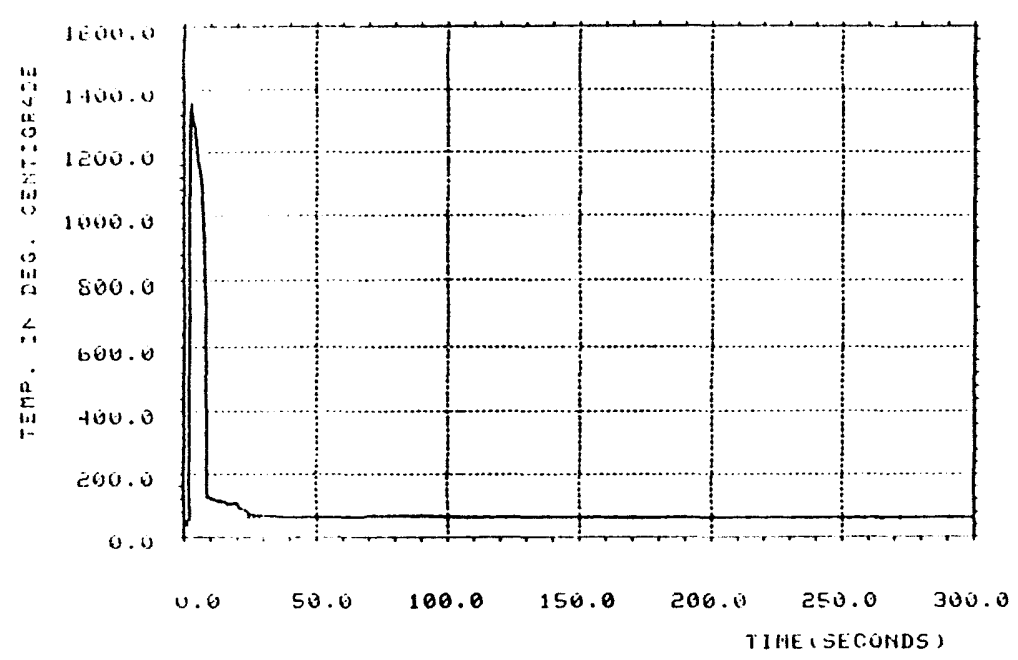

Ti. $3-17$, CCM-5, DATE 9.17 .0

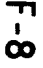

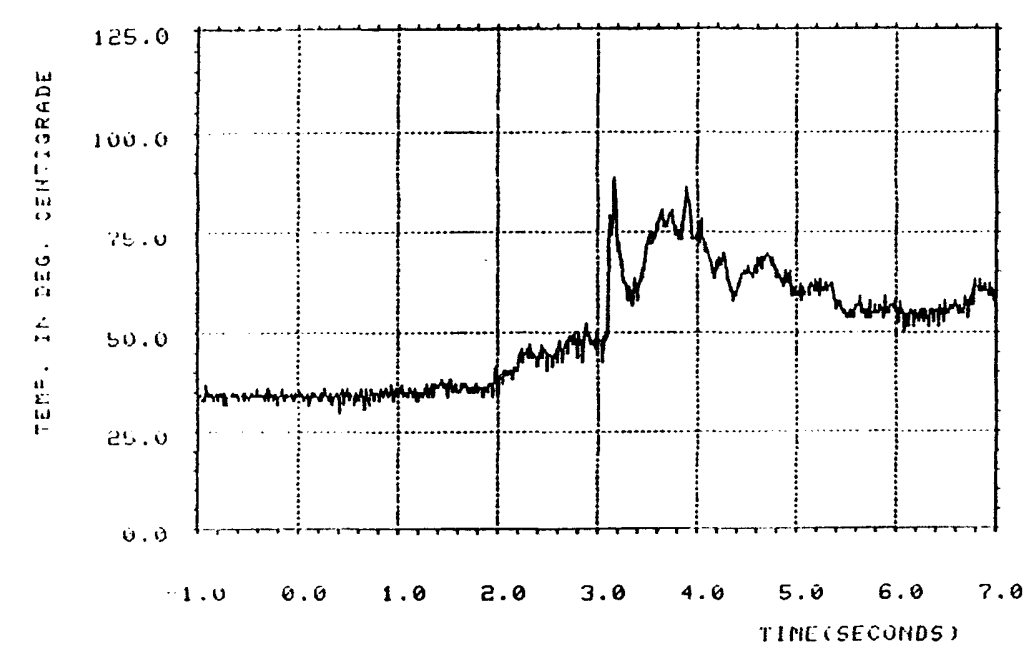

TCE-19, CCM-5, DATE 9.17.87

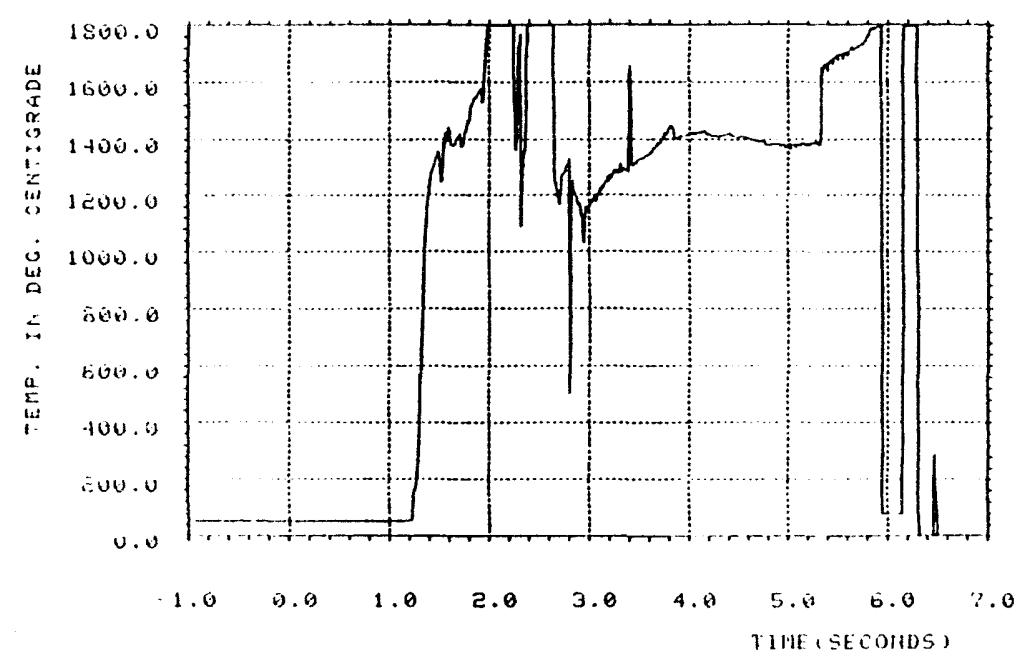

TCE-1E, CCN-5, DATE $91 ?: ?$

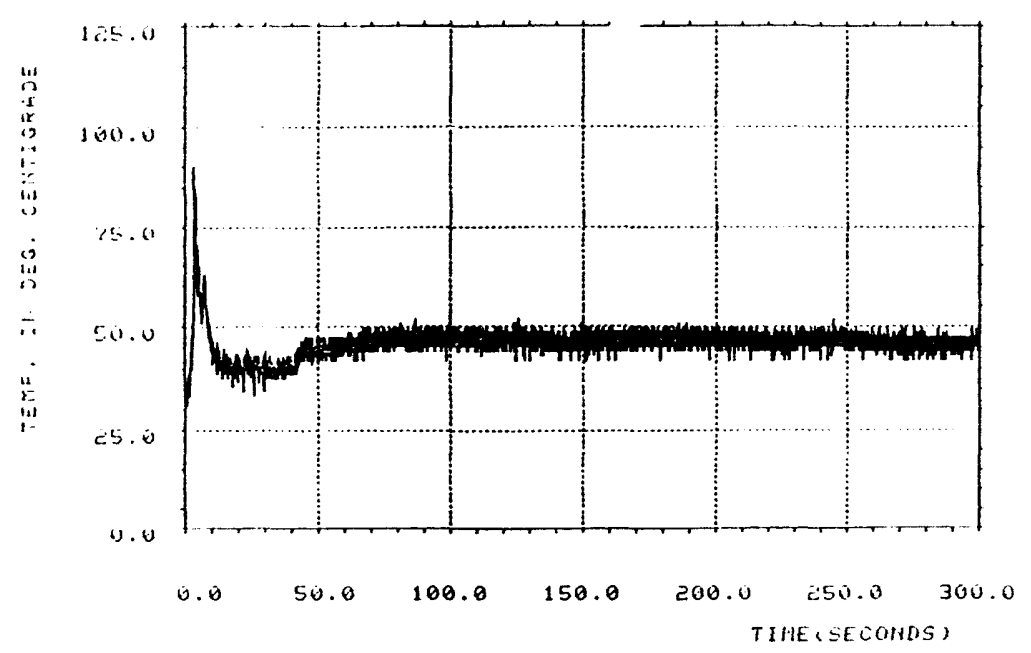

TEE-19, CCM-5, RATE 9.1787 


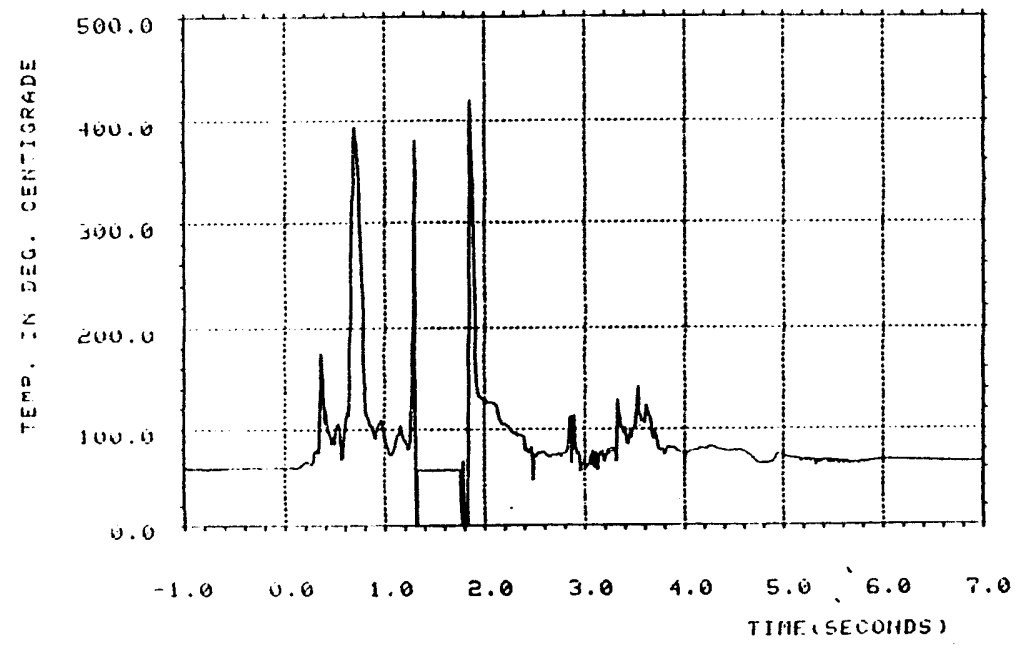

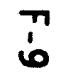

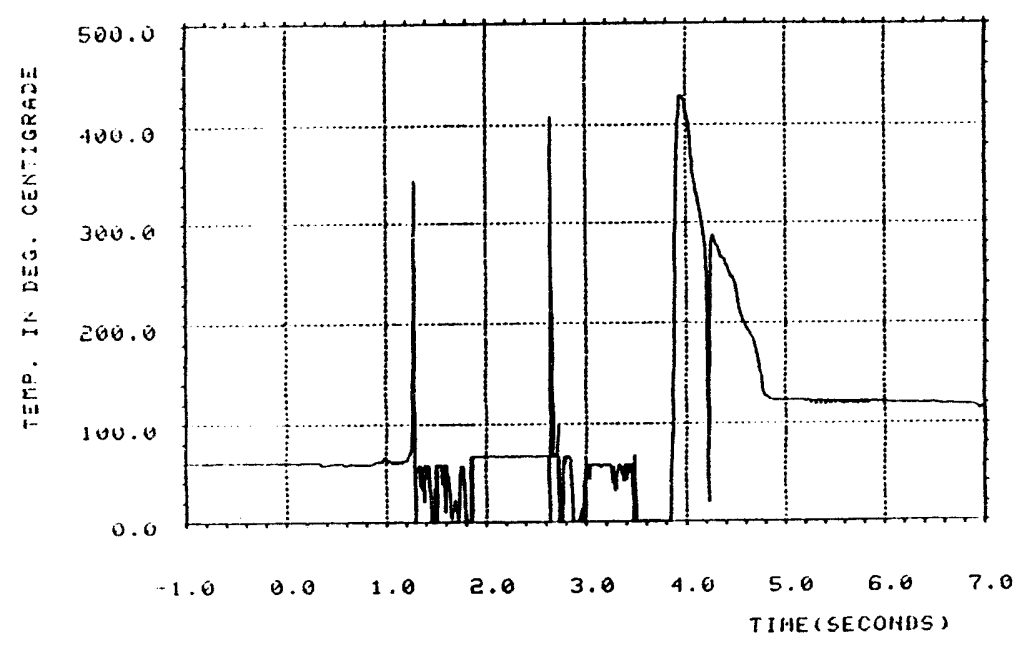

Ti.E-ट1, CCM-5, DATE $9 \cdot 17 \cdot \varepsilon ?$

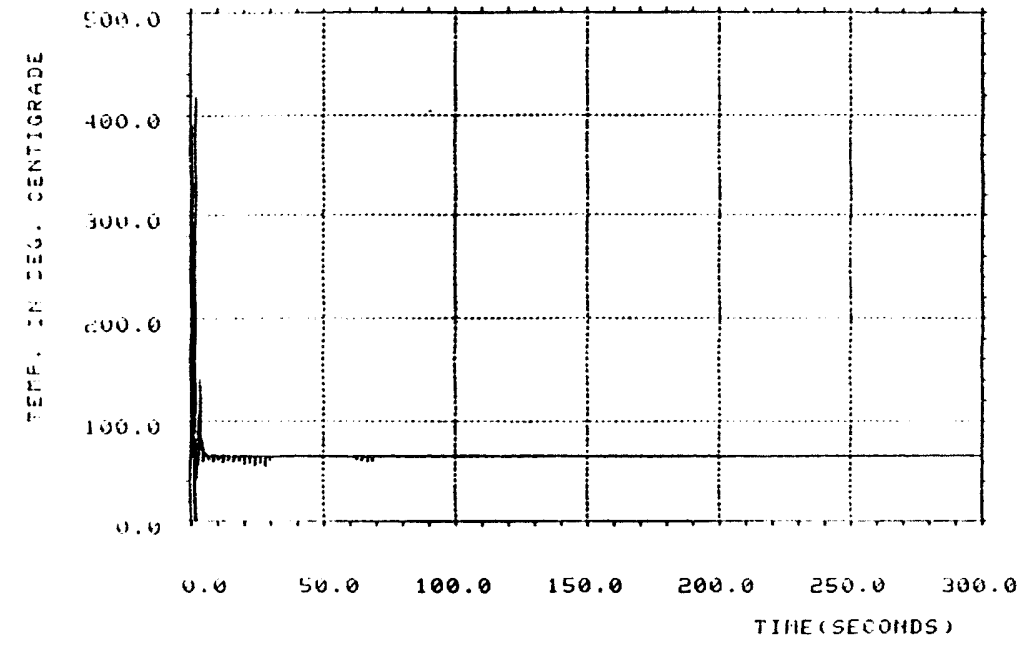

TiE-20, CCN-E, [IATE g.17.8?

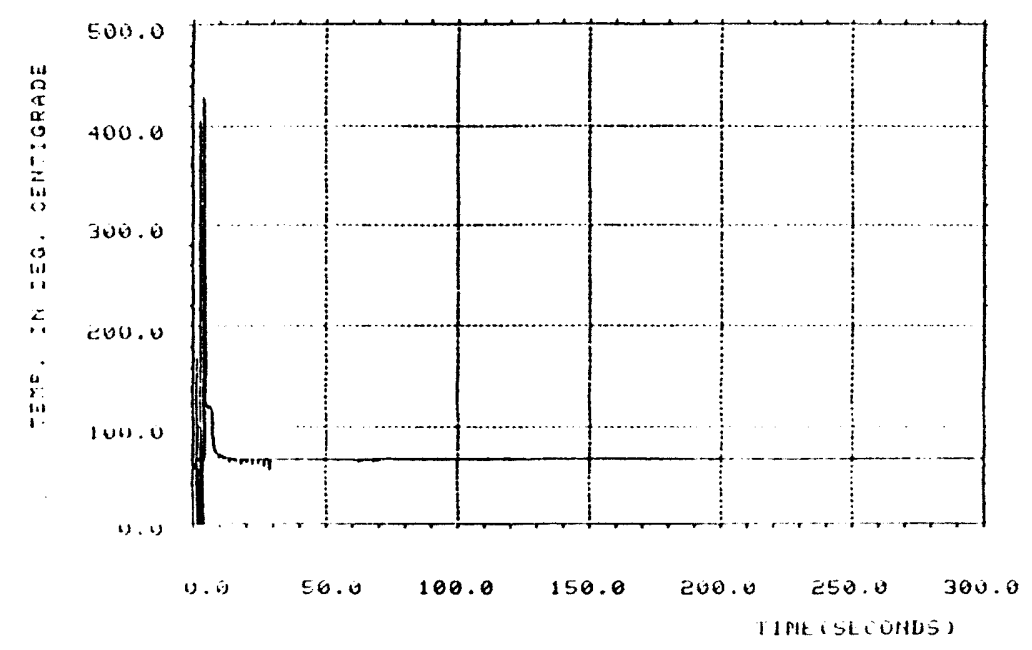

T.8-21, COM-5, DATE 9.17.57 


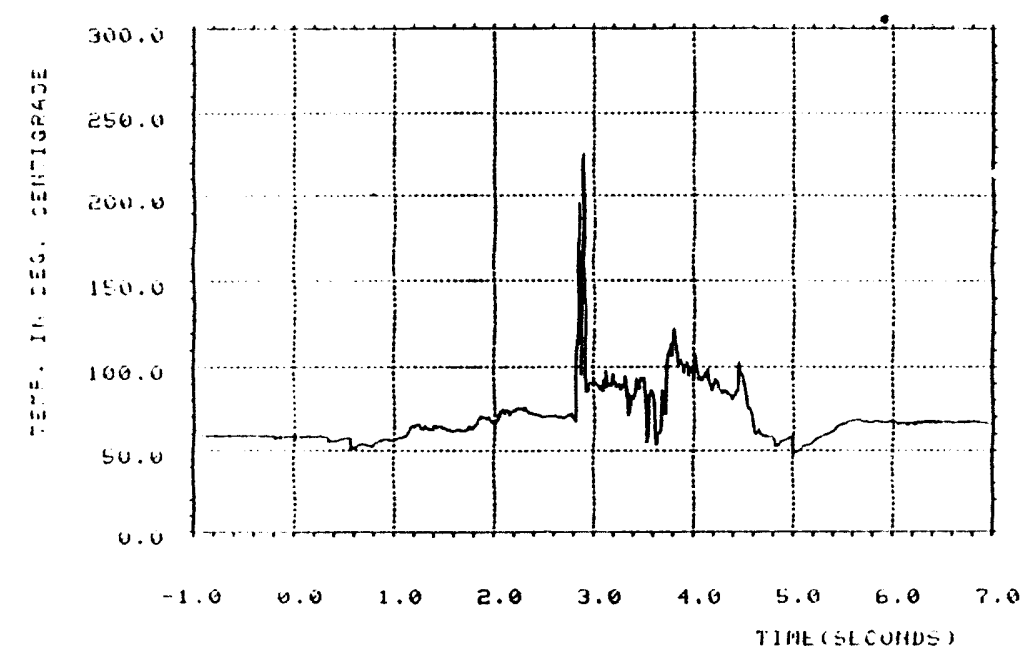

Tiß-22, CCM-5, IHATE 9.17 ॐ

$\stackrel{\pi}{\circ}$

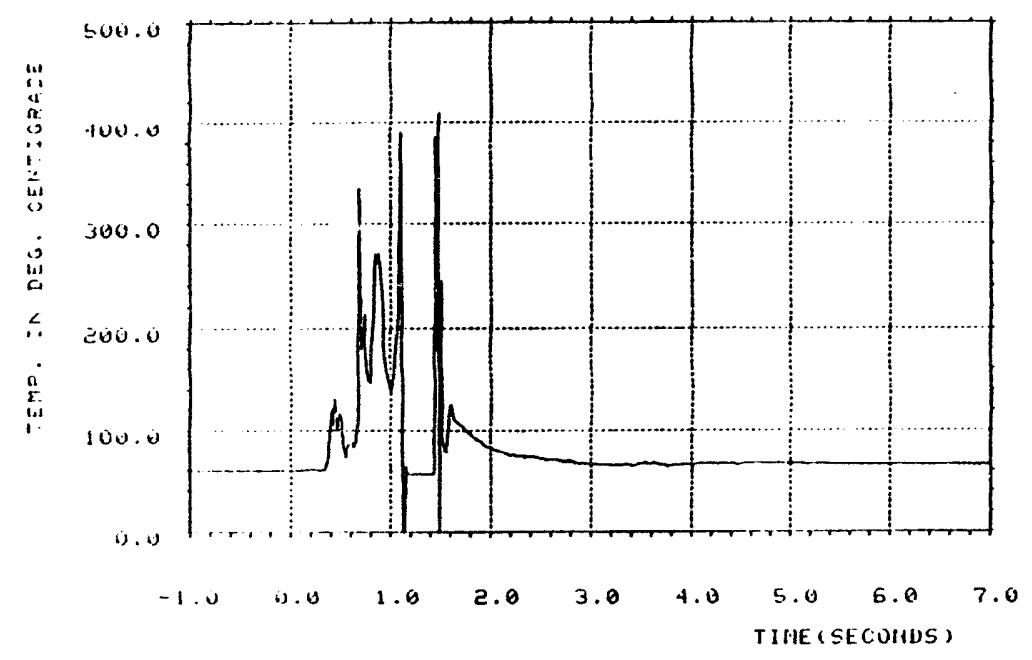

Tis-23, CCIM-5, DATE 9:17:3

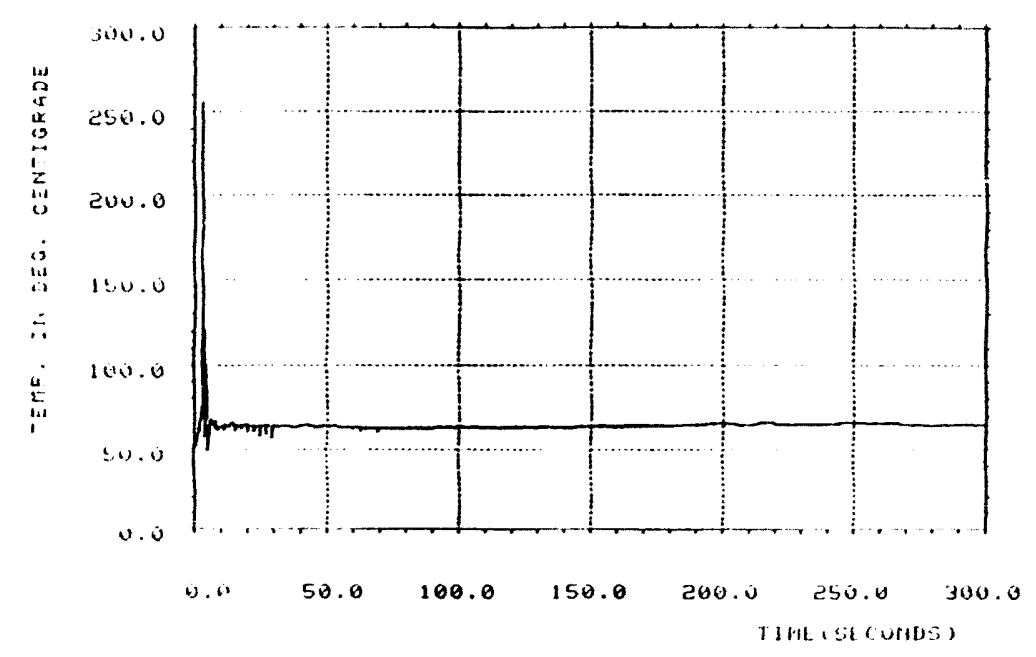

Tiz-22, COH-5, LATE g. $1:-7$

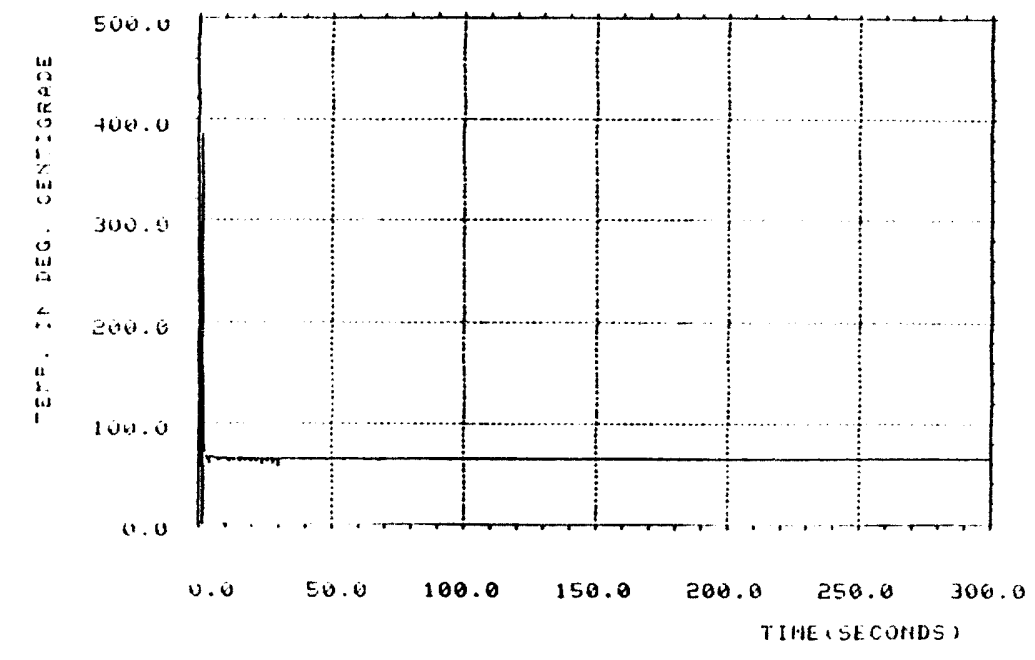

TI. $8-23$, CCM-5, DATE $9.17,67$ 


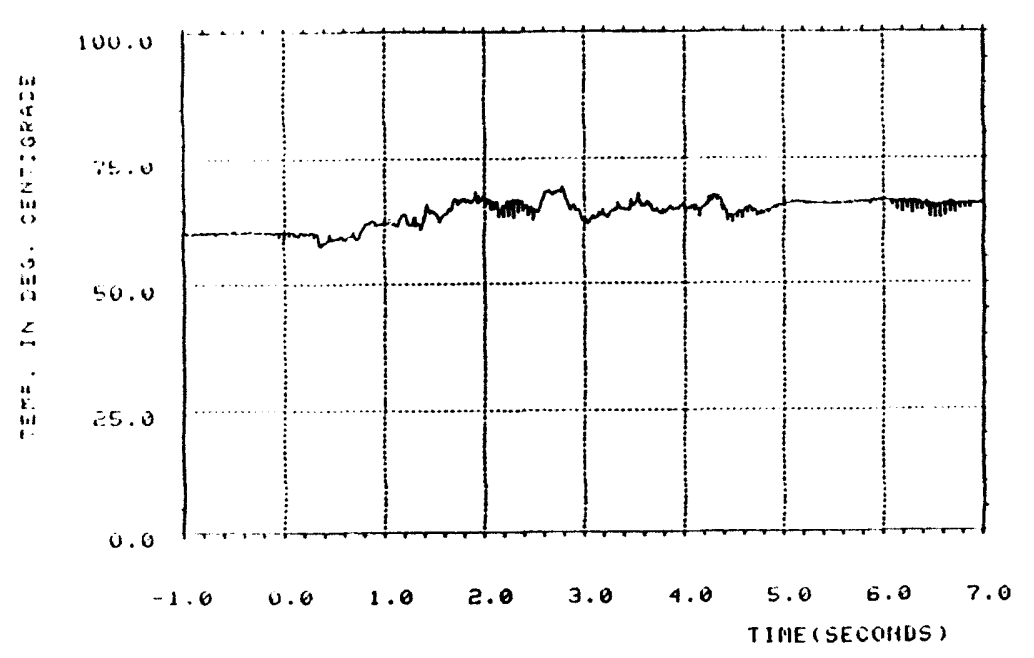

Ti.̄-Z4, CCM-5, DATE 9.17 ह?

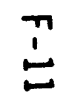

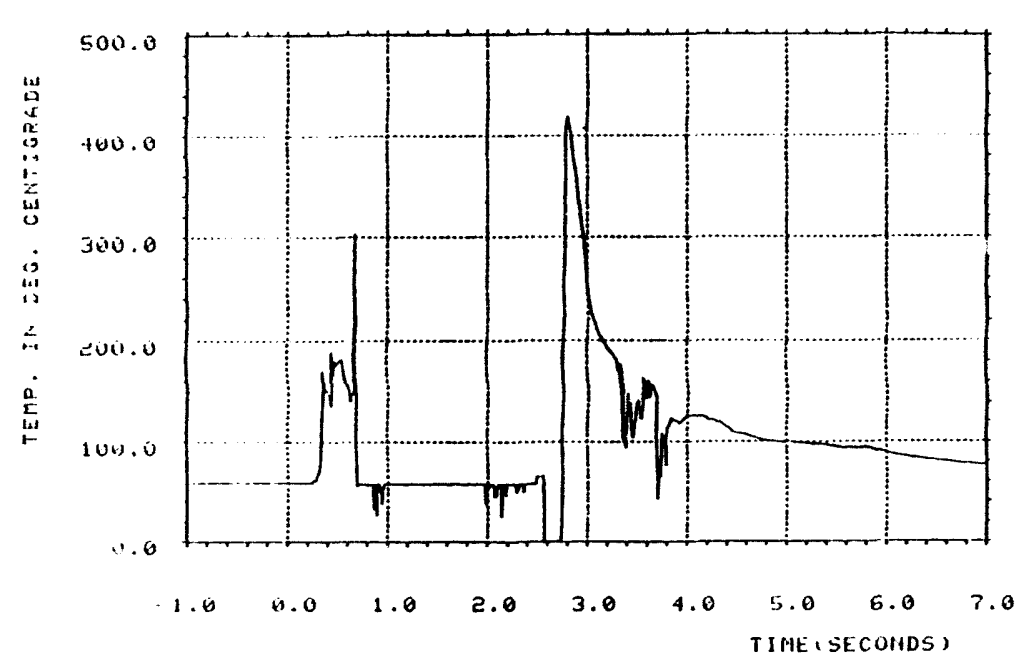

TC8-26, CCM-5, DATE $9 \cdot 17: 57$

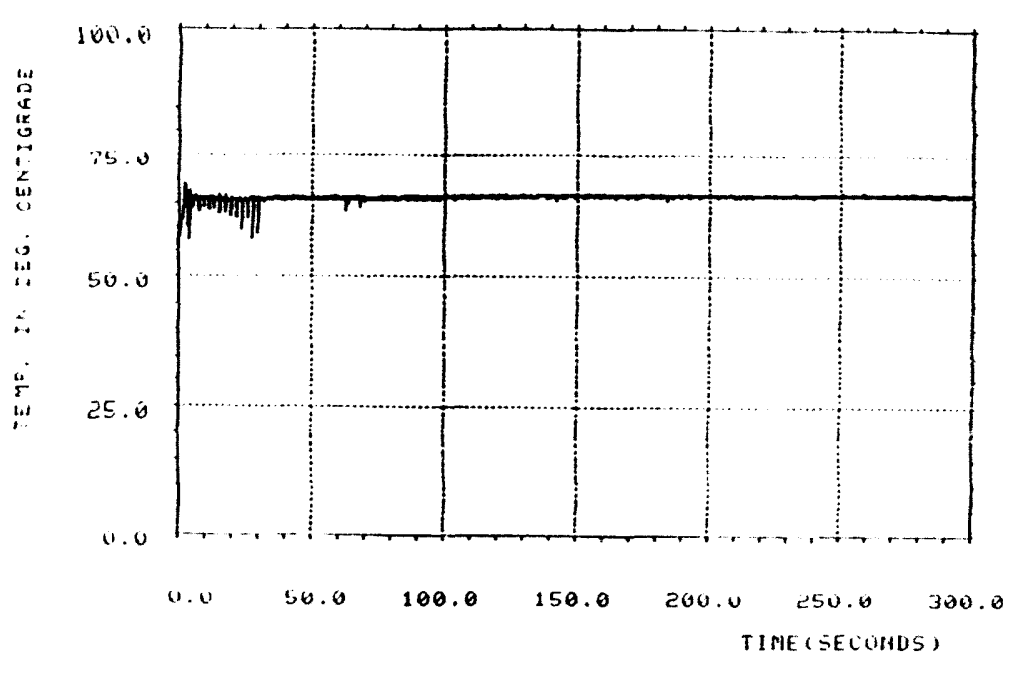

TH.3-24, CCM-5, DATE 9.1\% 2.?

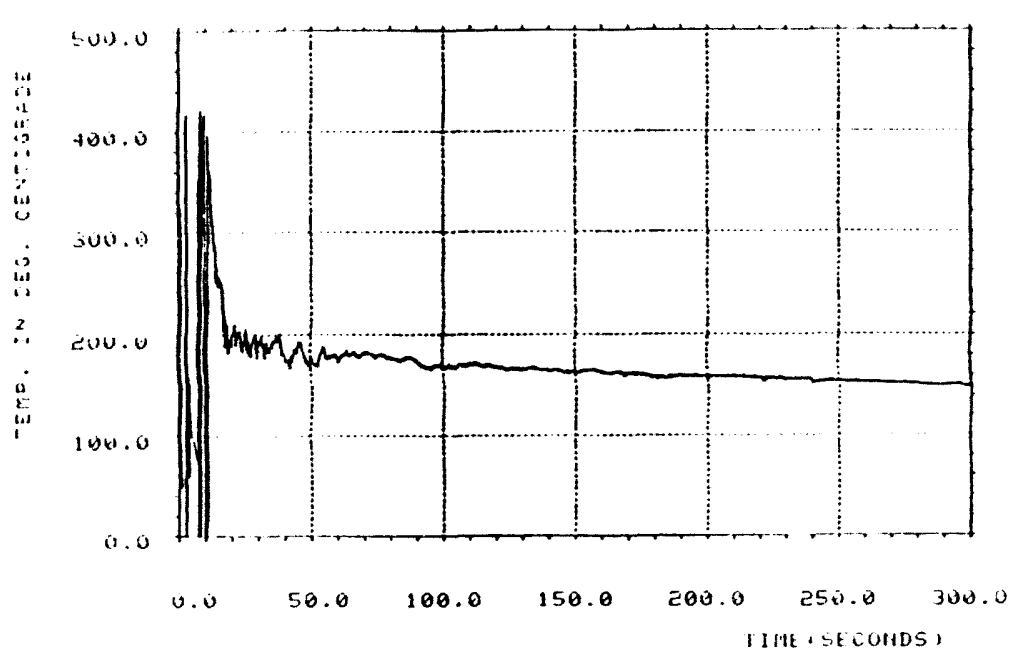

TCS-26, CCM-5, DATE g/17'3: 


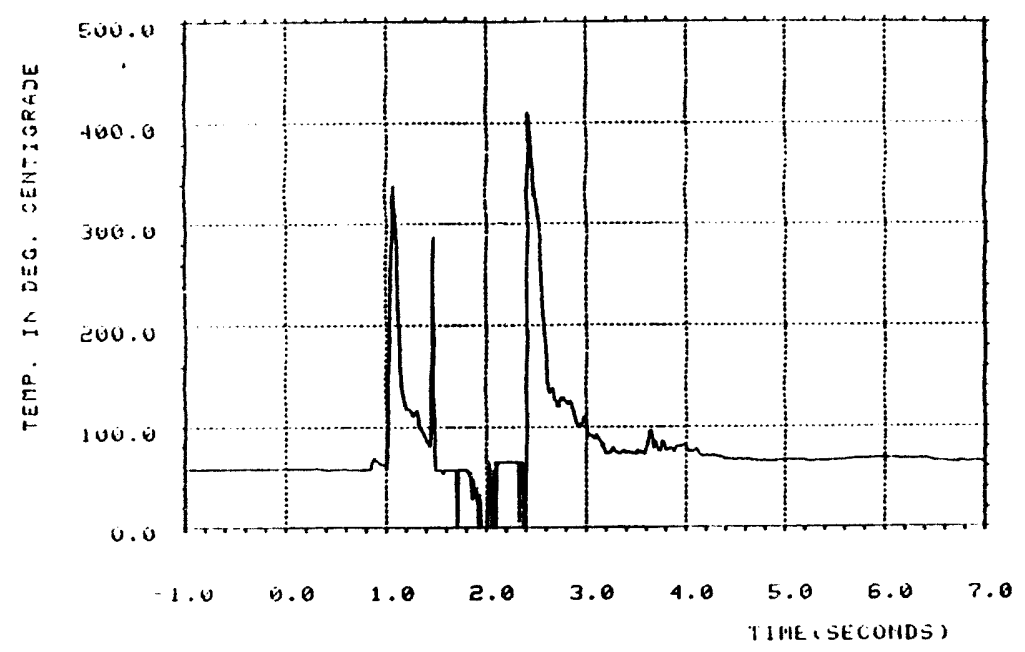

T.8-27, CCM-5, DATE $9,17.87$

$\stackrel{i}{n}$

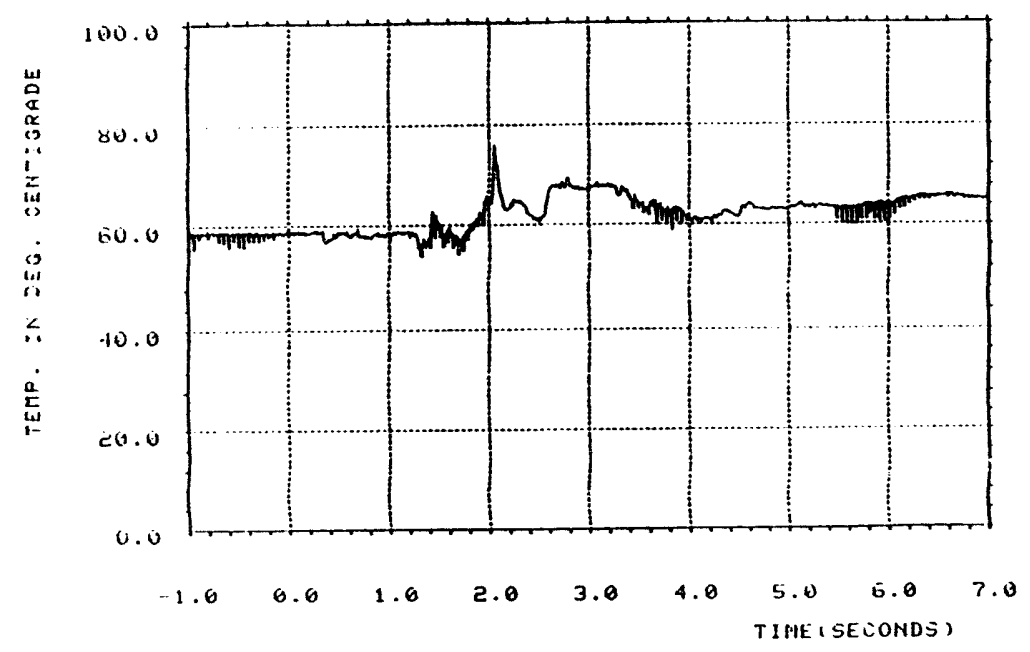

T. $3-28$, CCM-5, LIATE 9.17. 8 ?

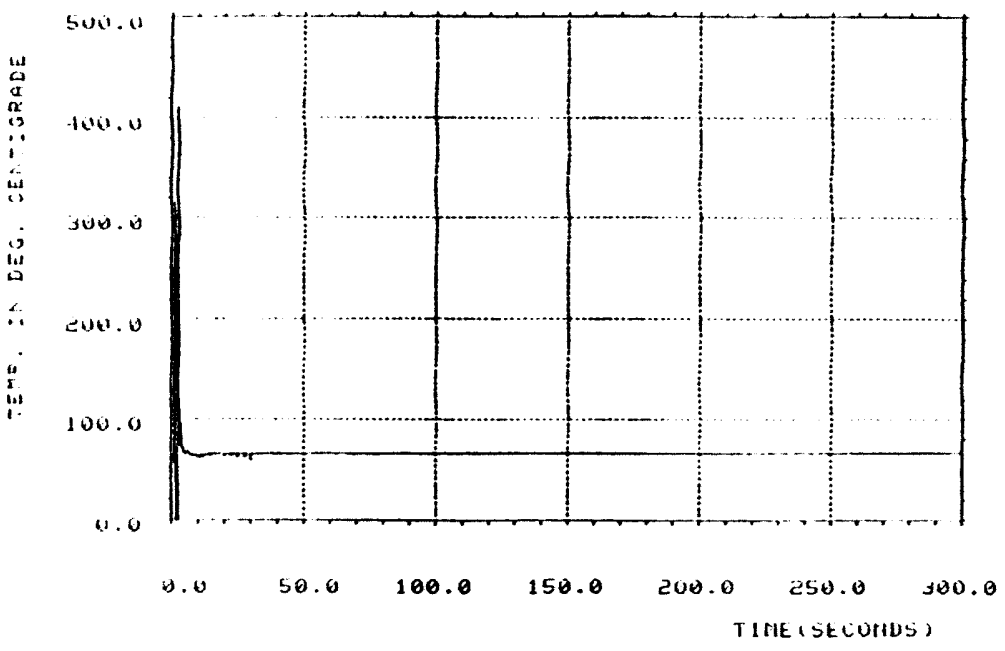

T:8-27, CCH-5, [DATE $9.17: 8$

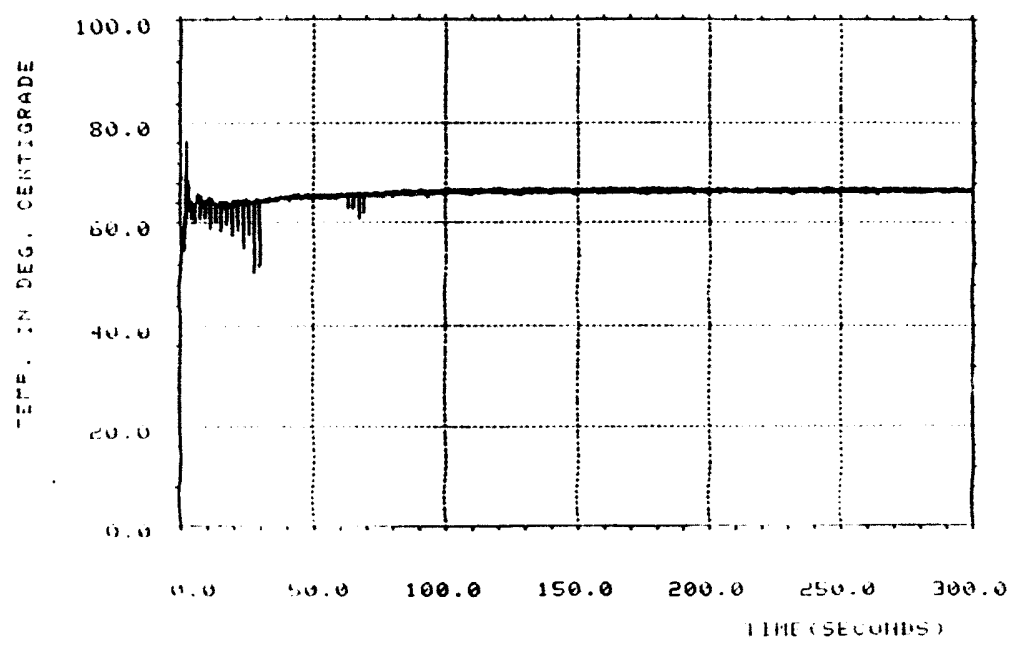

Ti $\overline{-}-\overline{8}$, CCM-5, DHTE $9 / 17: 07$ 


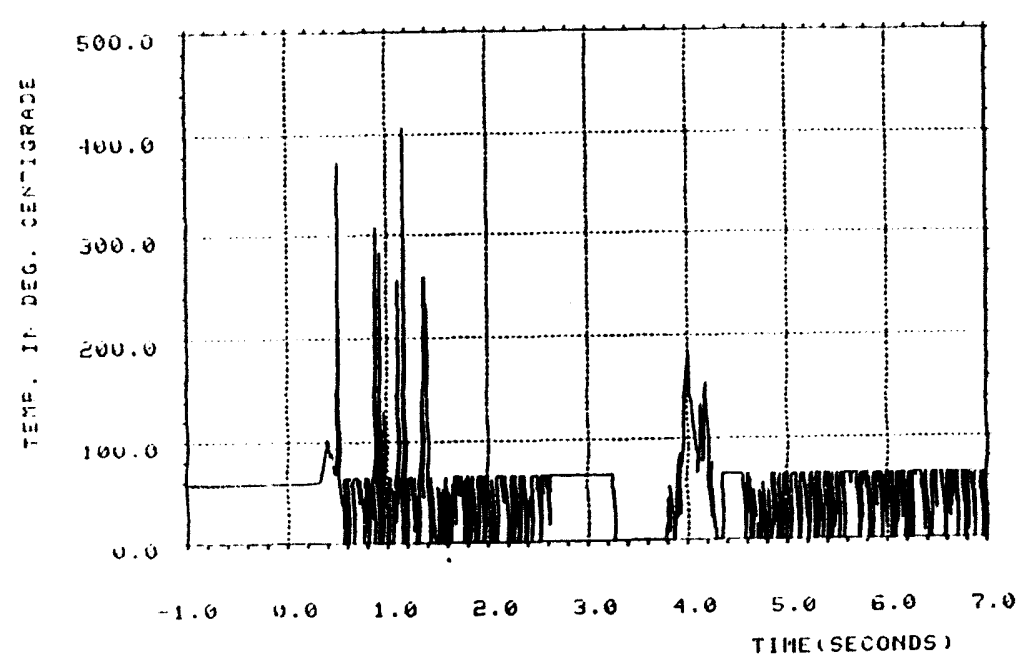

T:8-29, CCM-5, DATE $9 \cdot 17,87$

$\stackrel{T}{\omega}$

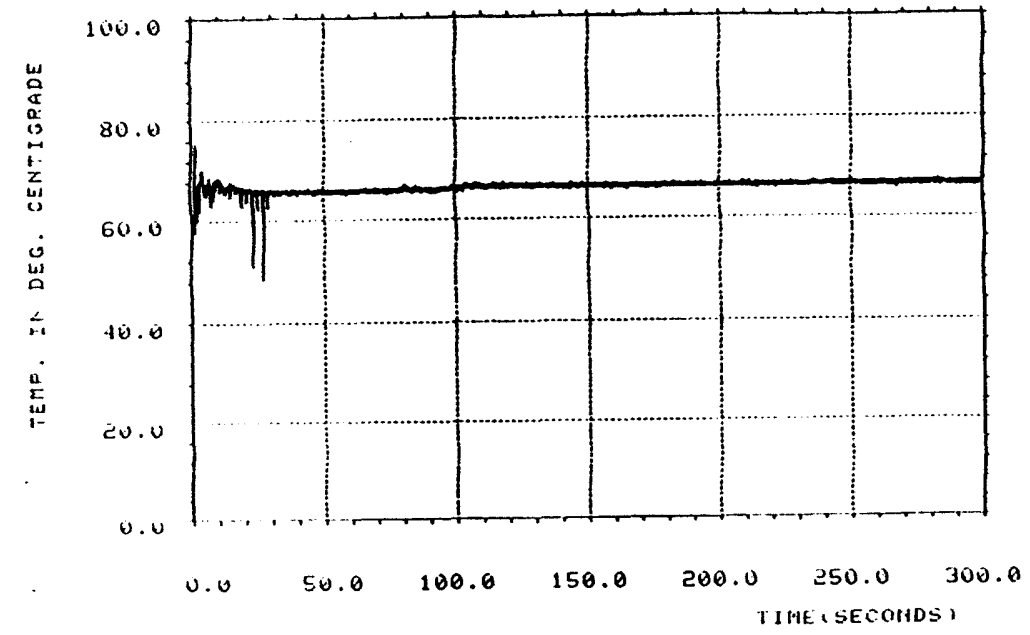

T:8-30, CCM-5, DATE 9.17.87

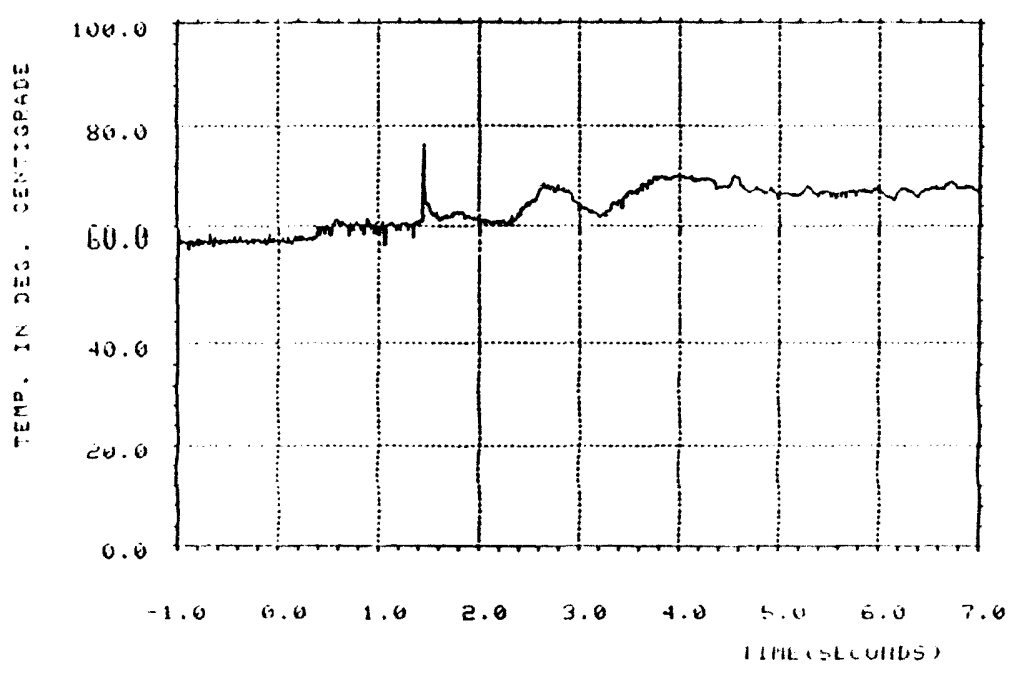

TIE 30, CCIN-5, [HATE $9.17 \%$ ?

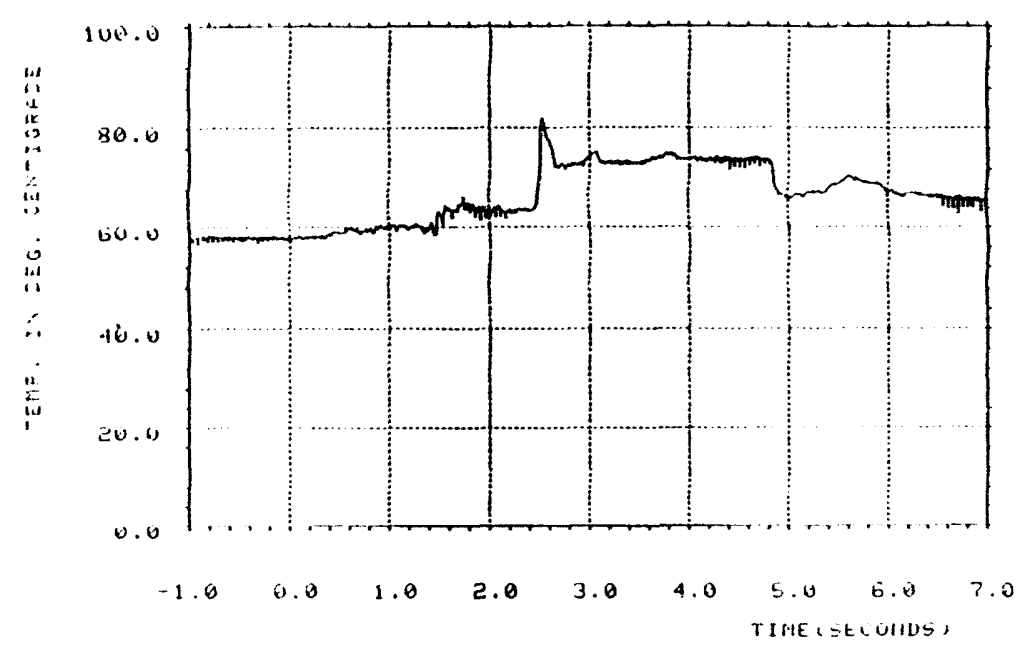

$11 \ldots-31$, CCH-5, VHTE 9.17 . 


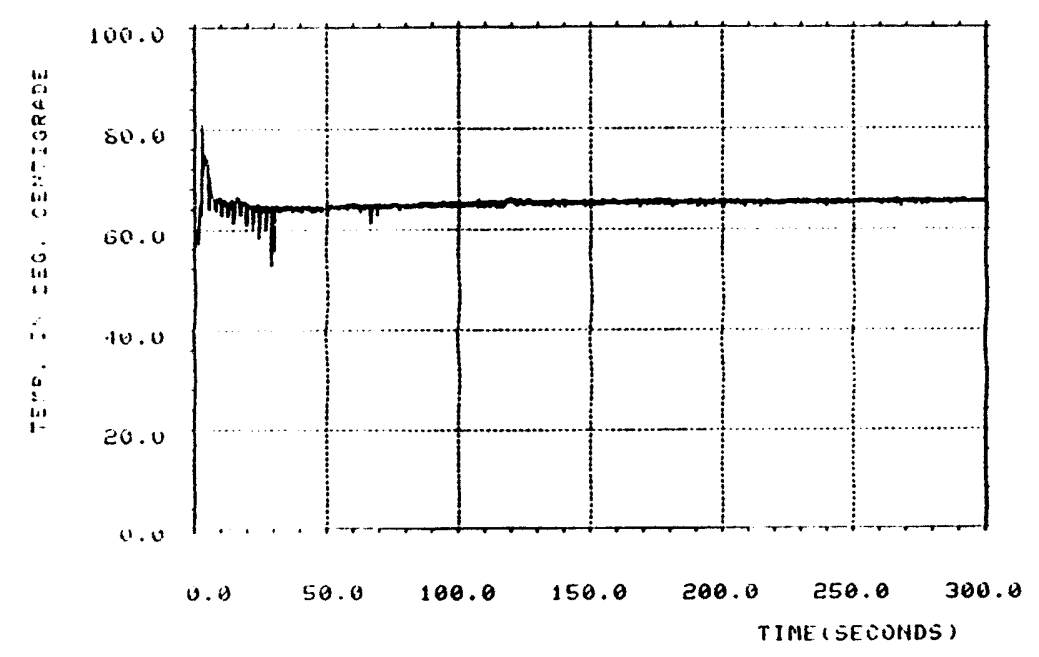

Ti८-31, CCIn-5, DATE $9 / 17.37$

$\stackrel{7}{-1}$

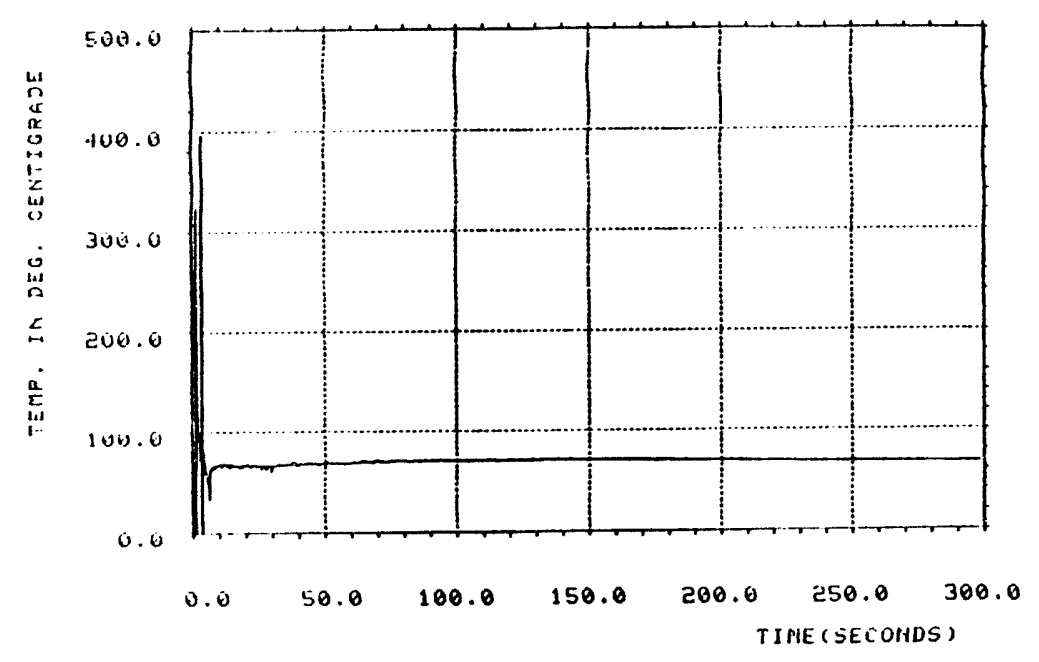

TC8-32, CCM-5, DATE 9,17, 8?

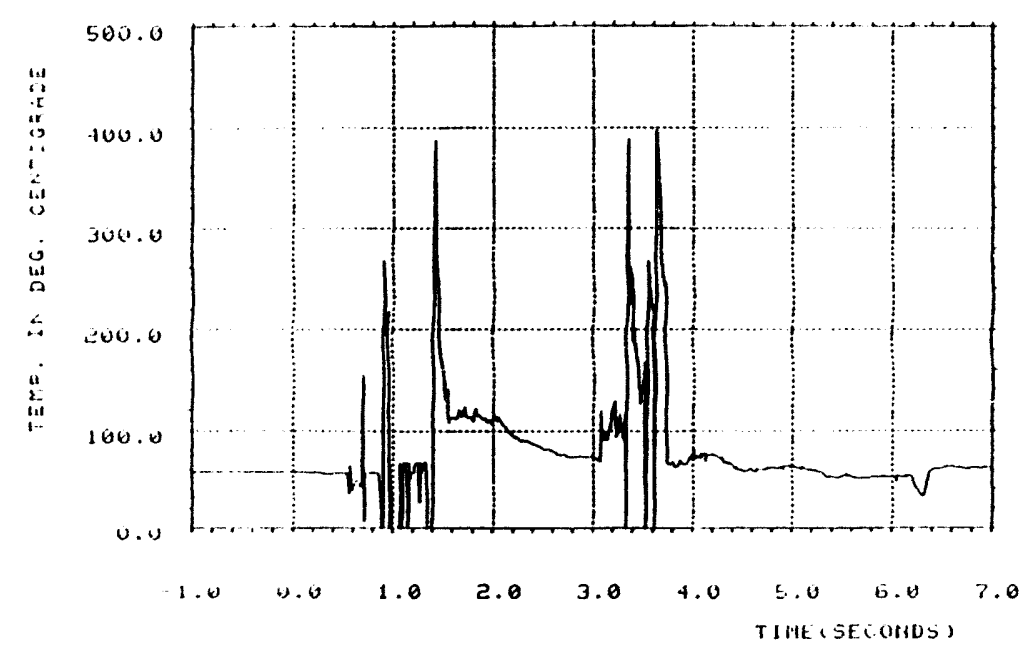

T: $8-32$, CCM-5, DATE 9.17 57

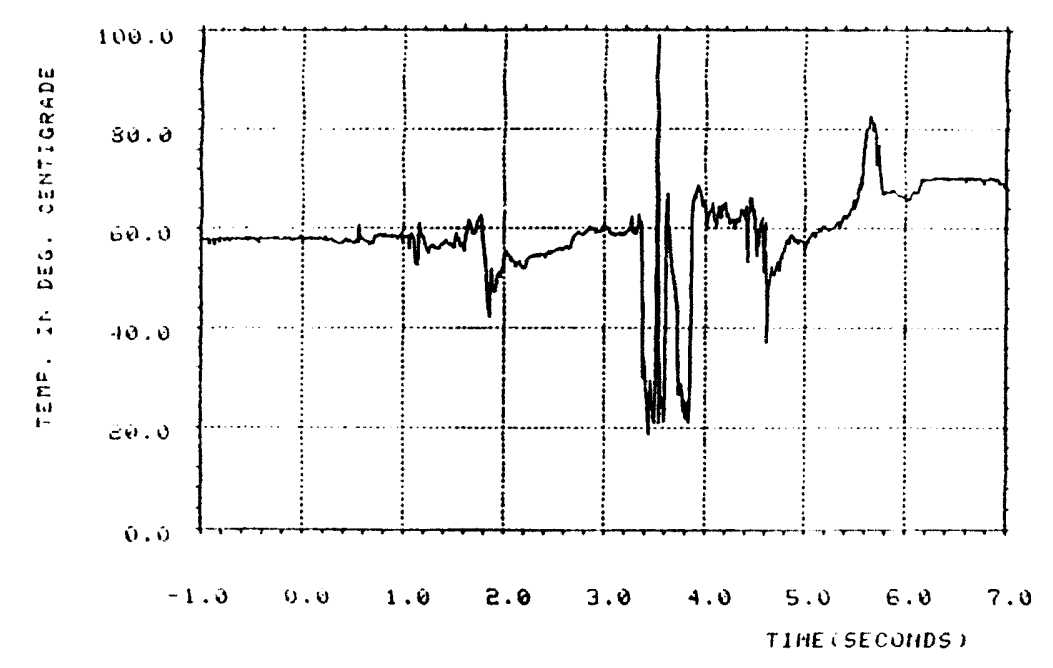

M:3-33, CCN-5, [HATE 9.1\%, 


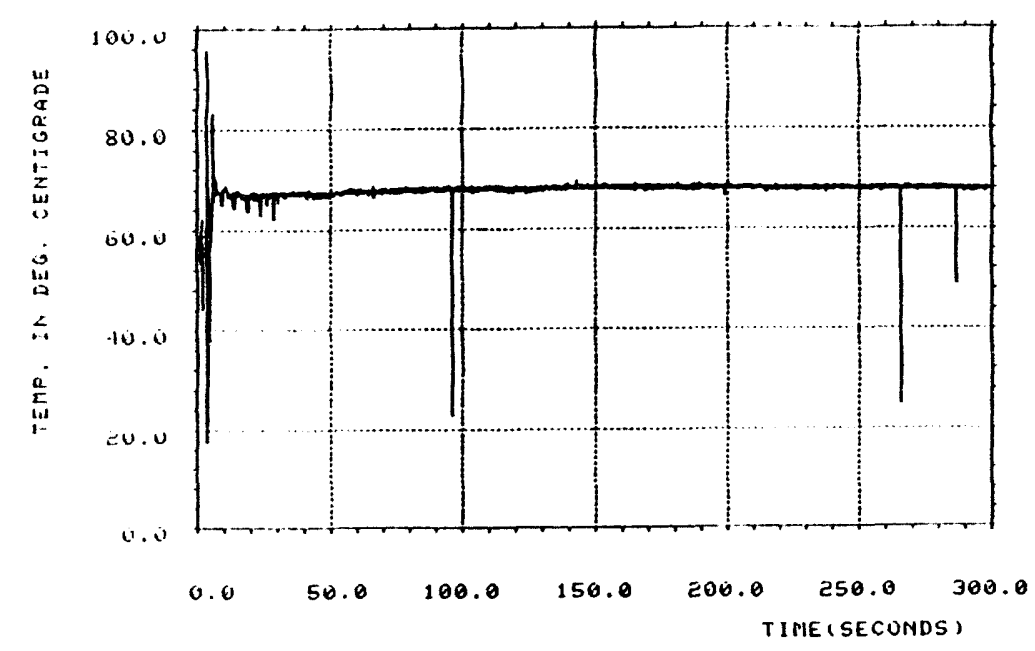

$\stackrel{n}{i}$

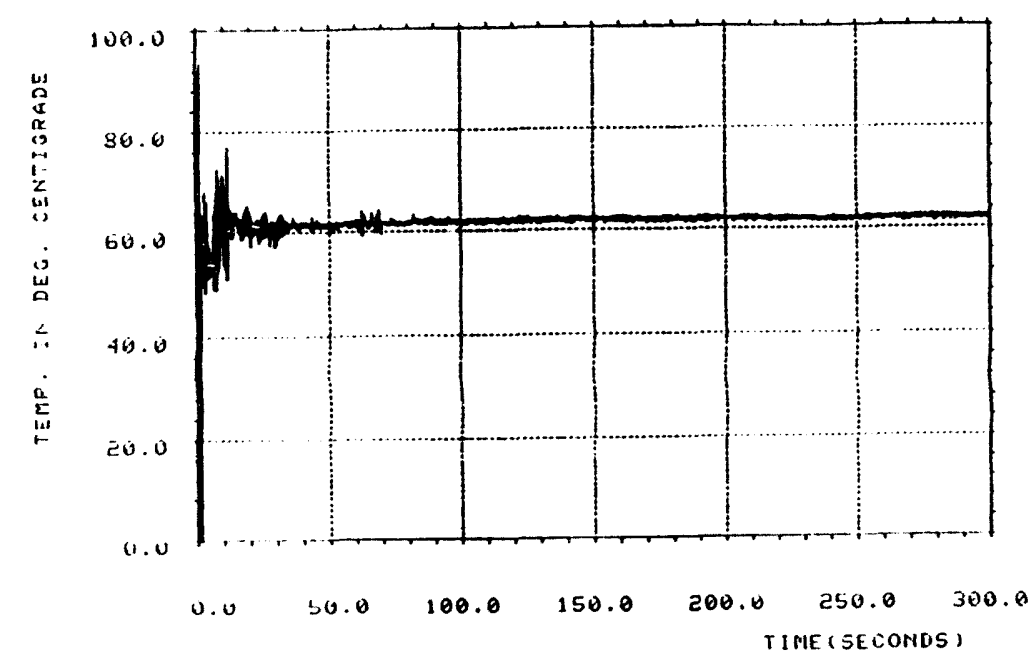

riE-35, CCN-5, IAATE 9/17.27

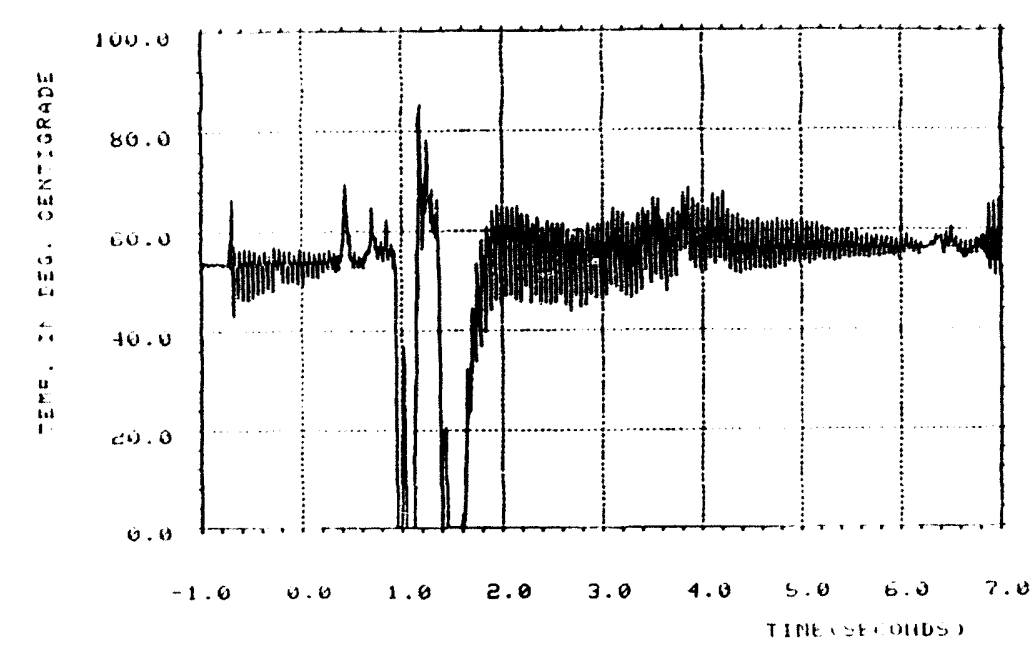

TH 35, CCM-5, HATE 9.17 _.:

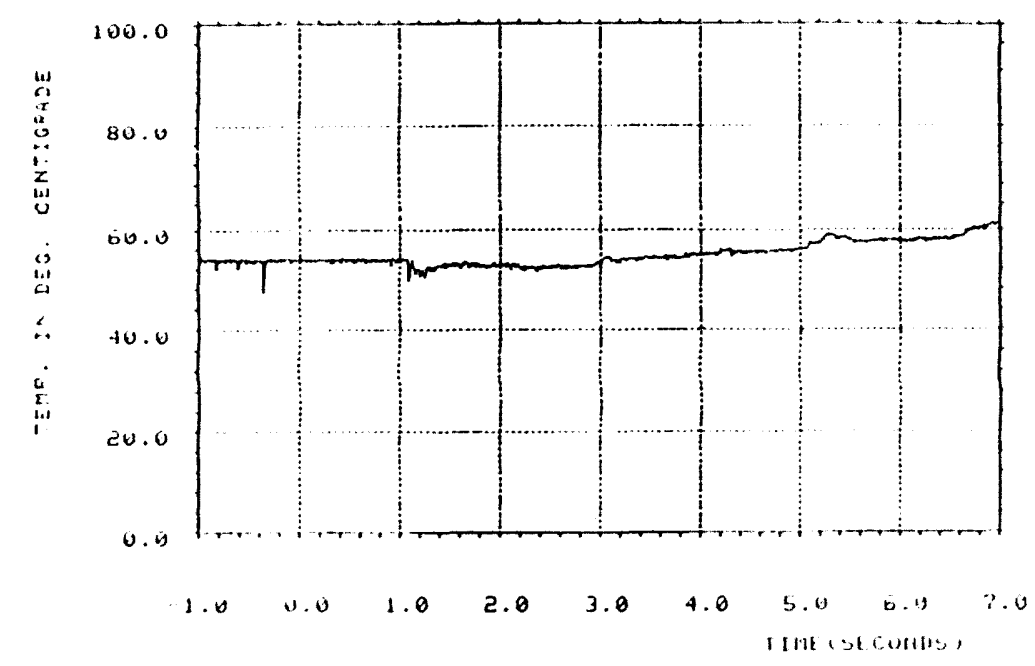

Ti: 3o, CCM-5, WATE gi'i i i 


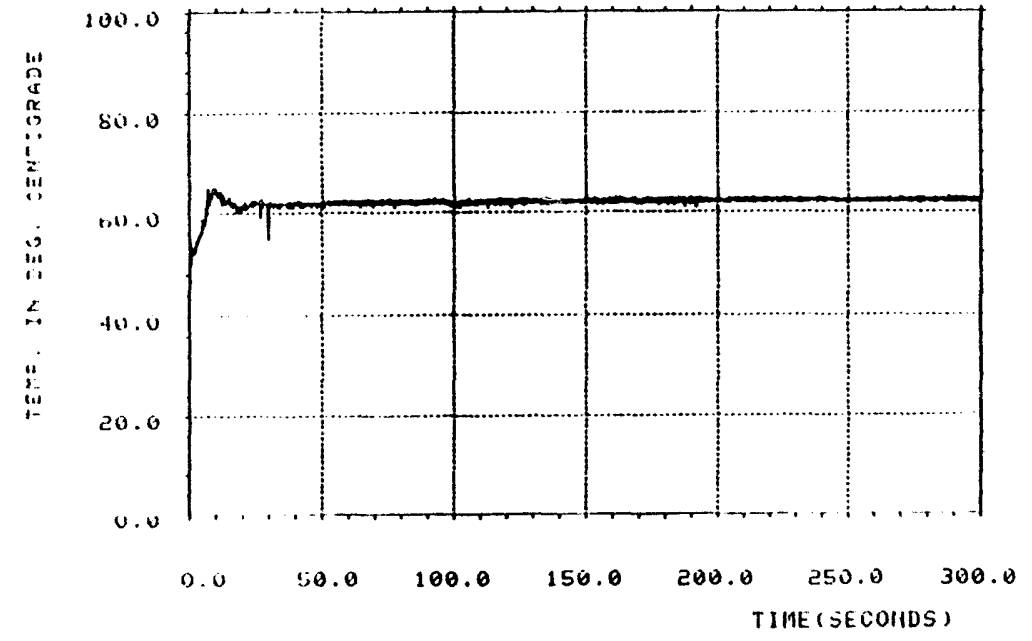

TC, $8-36$, CCM-5, IHATE 9/17:3?

茴

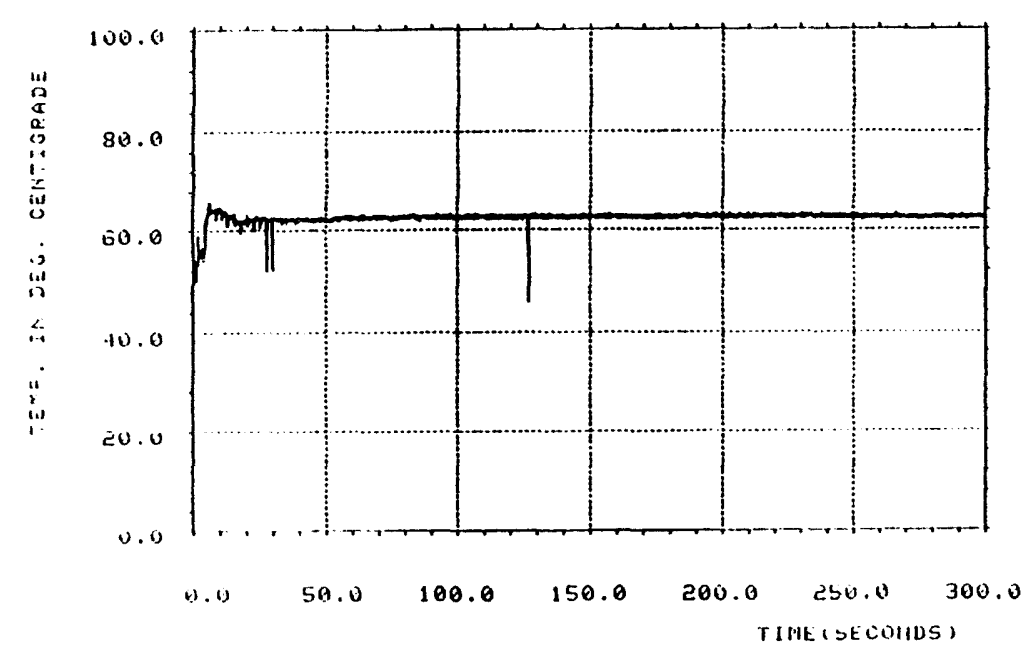

TCE-37, CCIn-5, DATE 9,17:87

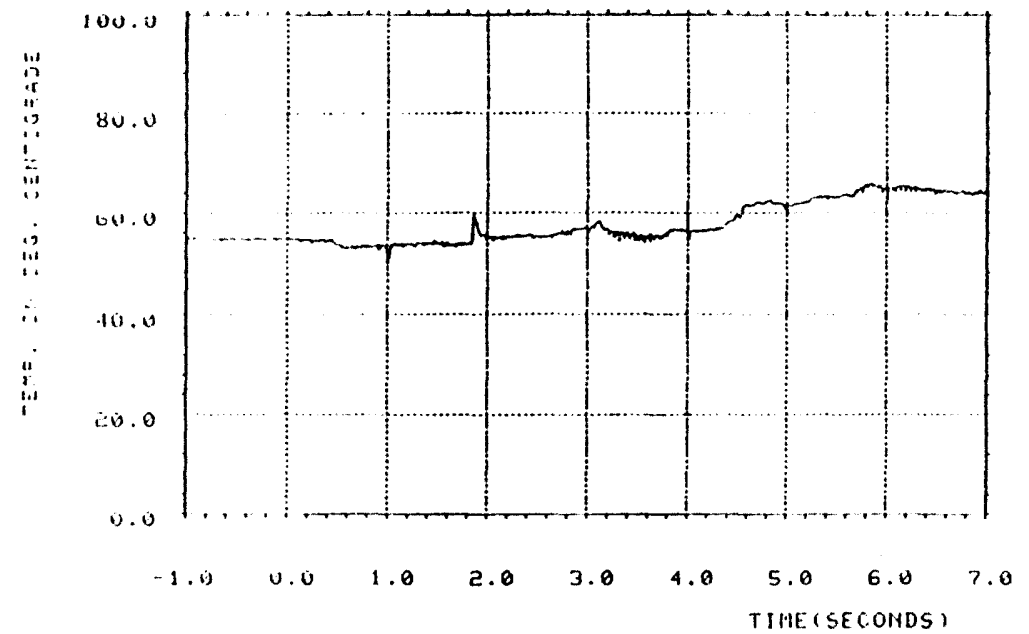

TI $8-37$, CCM-5, [HATE 9/17 67

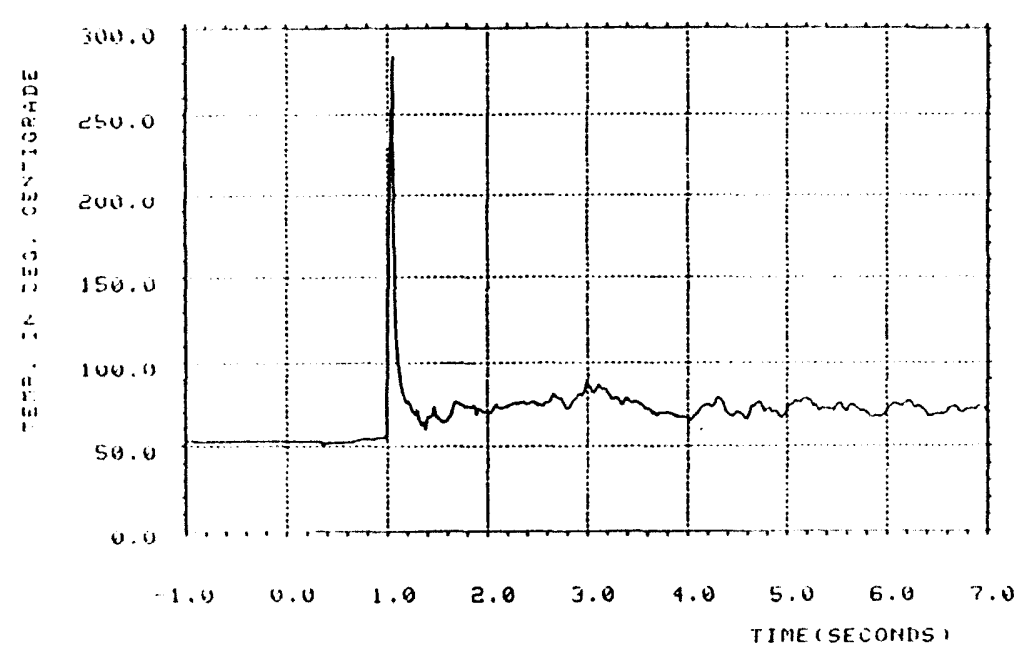

TC8 8 -38, CCM-5, [HATE 9/17/87 


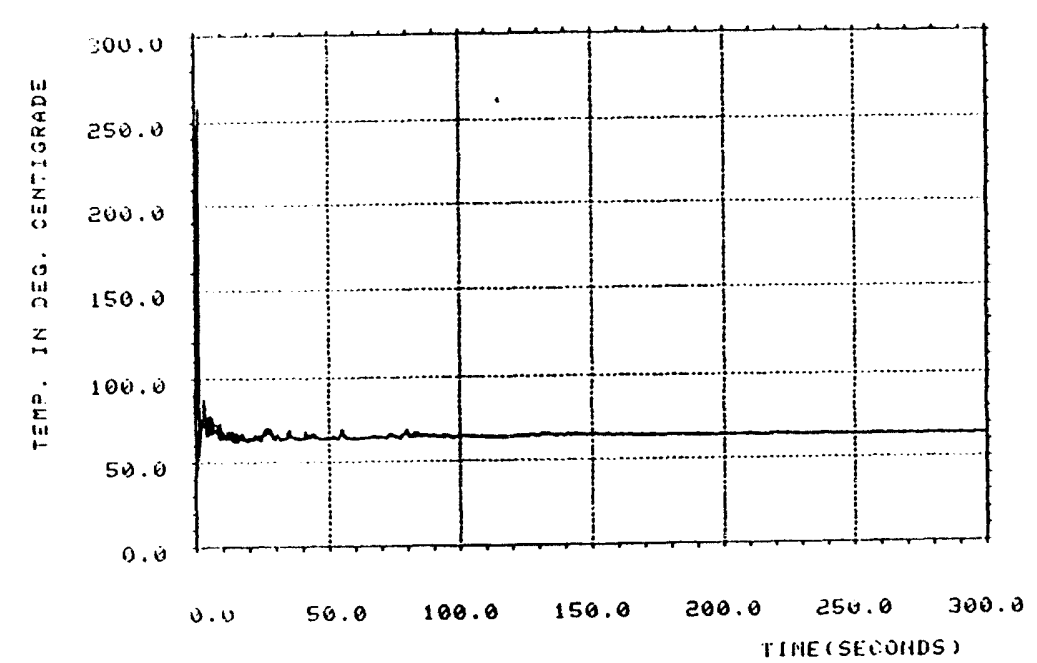

TC8-38, CCM-5, DHTE 9/17:8?

$\stackrel{n}{\Xi}$

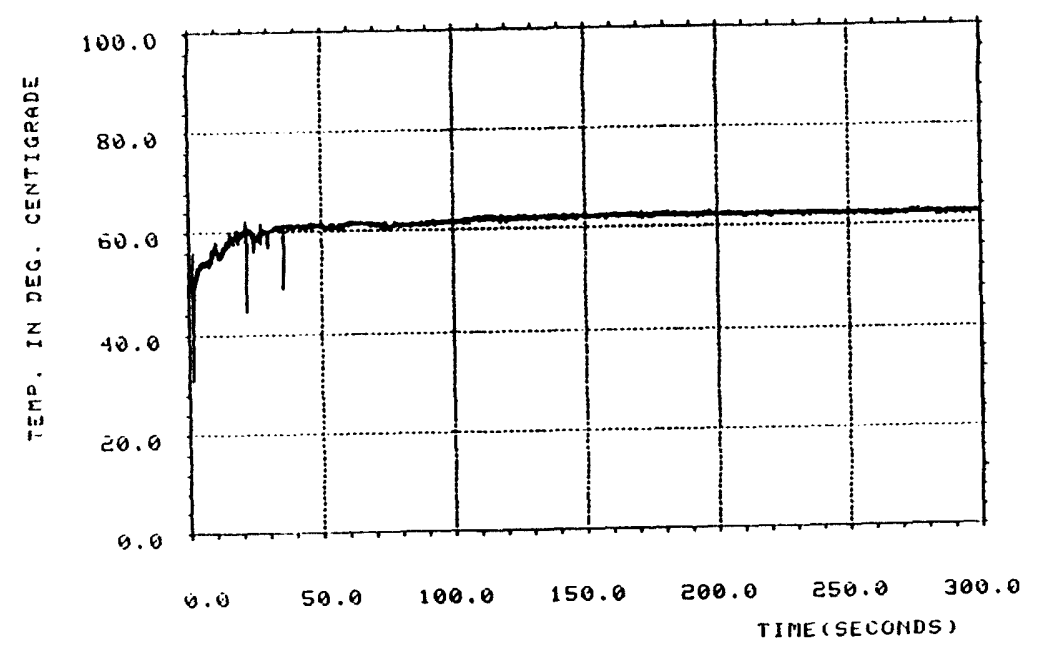

TCS-40, CCM-5, DATE 9,17.87

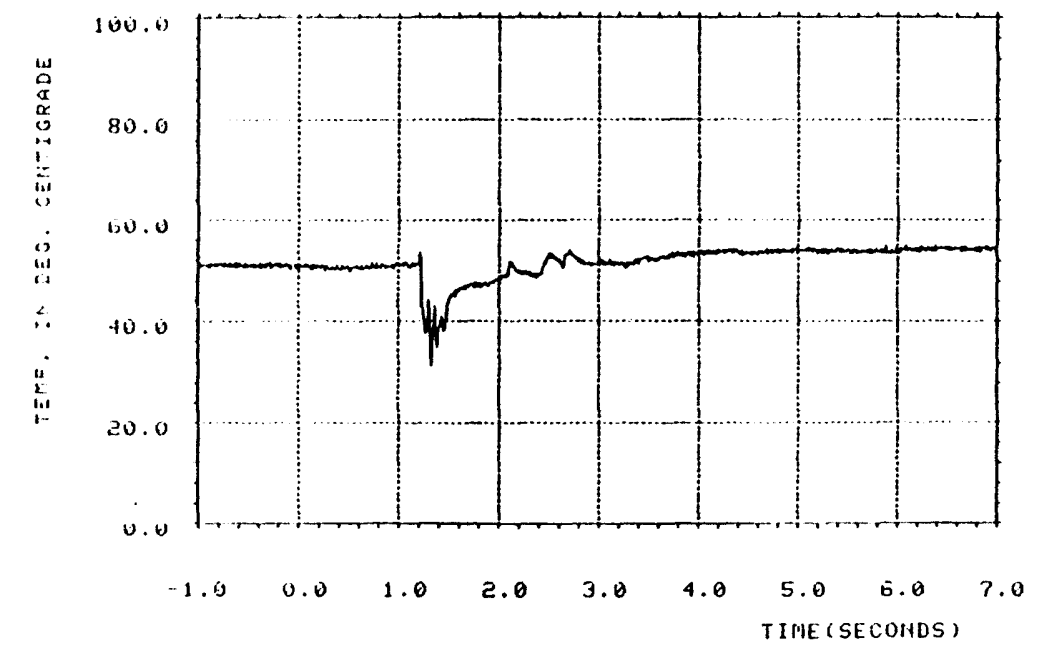

T:S-40, CON-5, INATE 9:17:87

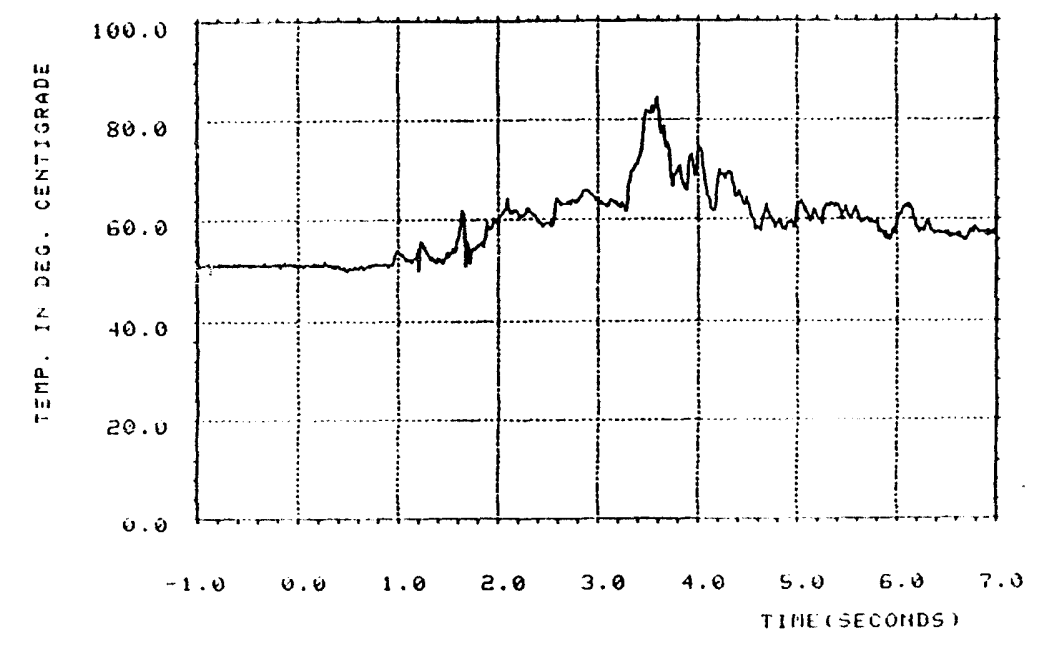

TI.E-11, CCM-5, DATE $9,17: \%$ 


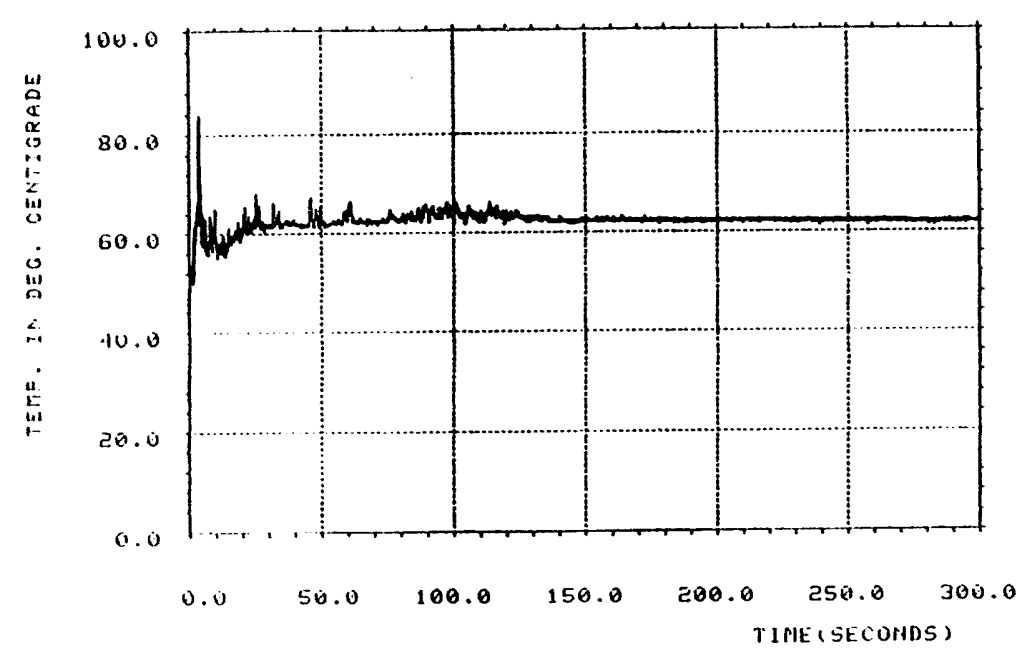

TI8-41, CCM-5, UATE 9.17:S7

$\stackrel{\Gamma}{\dot{\infty}}$

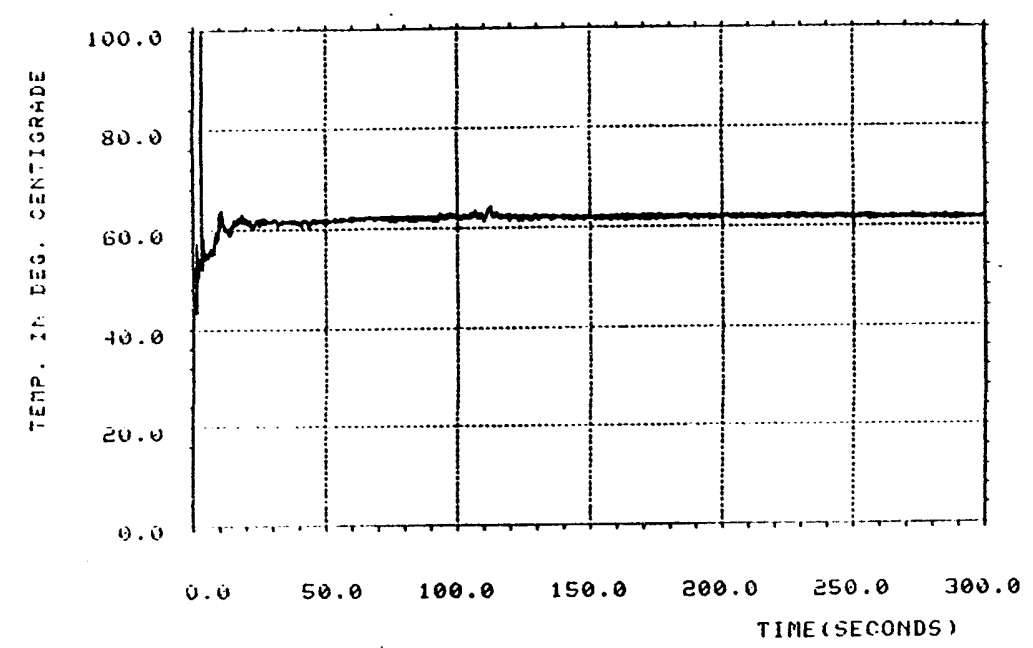

TC $8-42$, CCM-5, IIATE 9/17.'8:

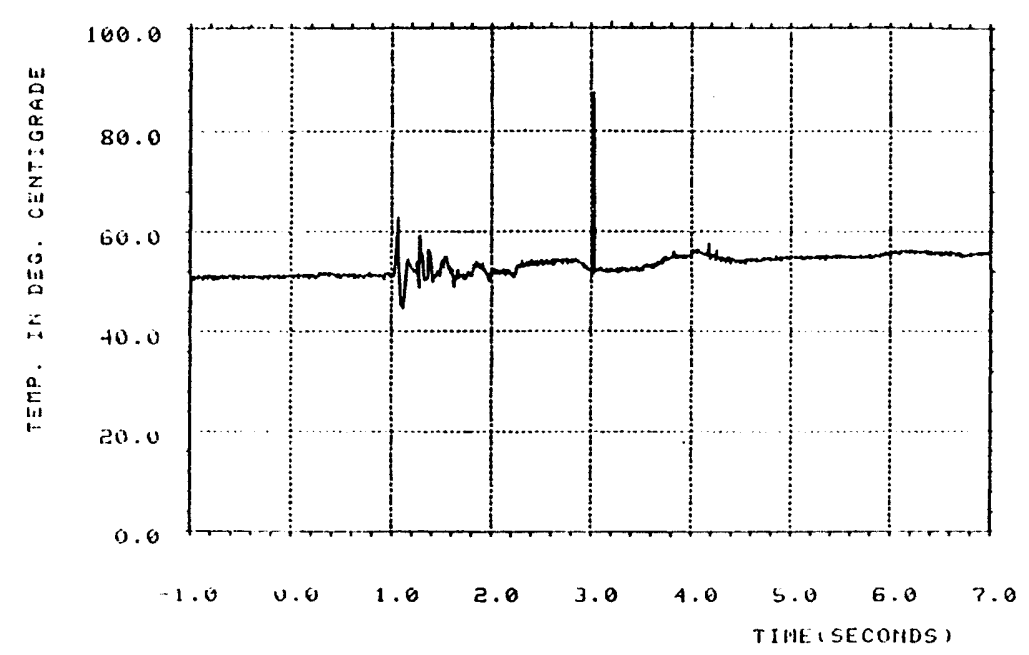

TCS-42, CCM-5, LATE 9:13.8?

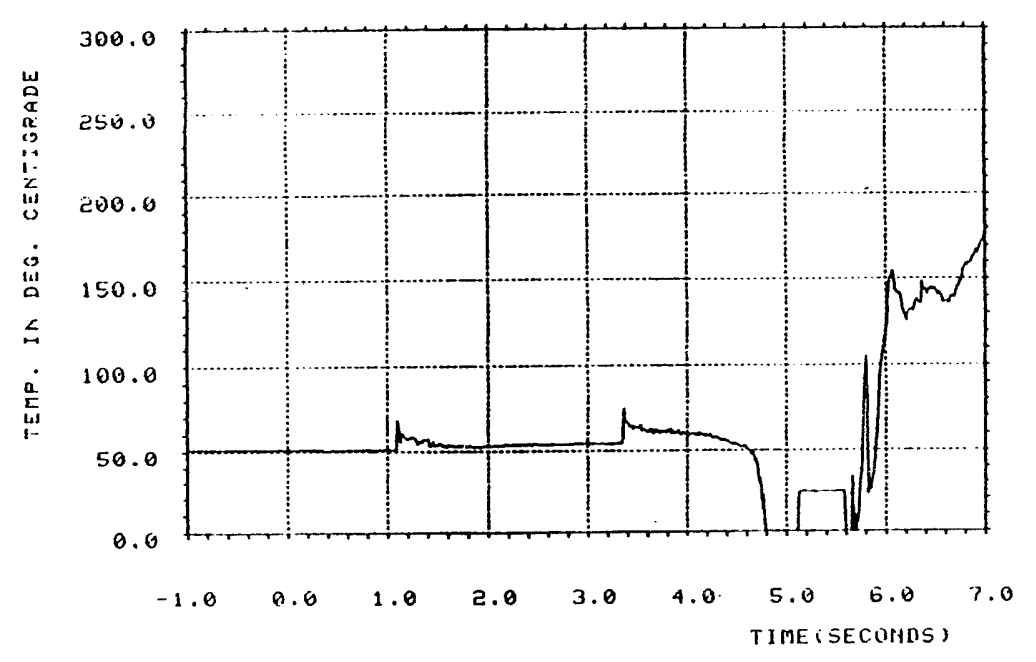

TC8-43, CCN-5, DHTE $9 / 17,87$ 


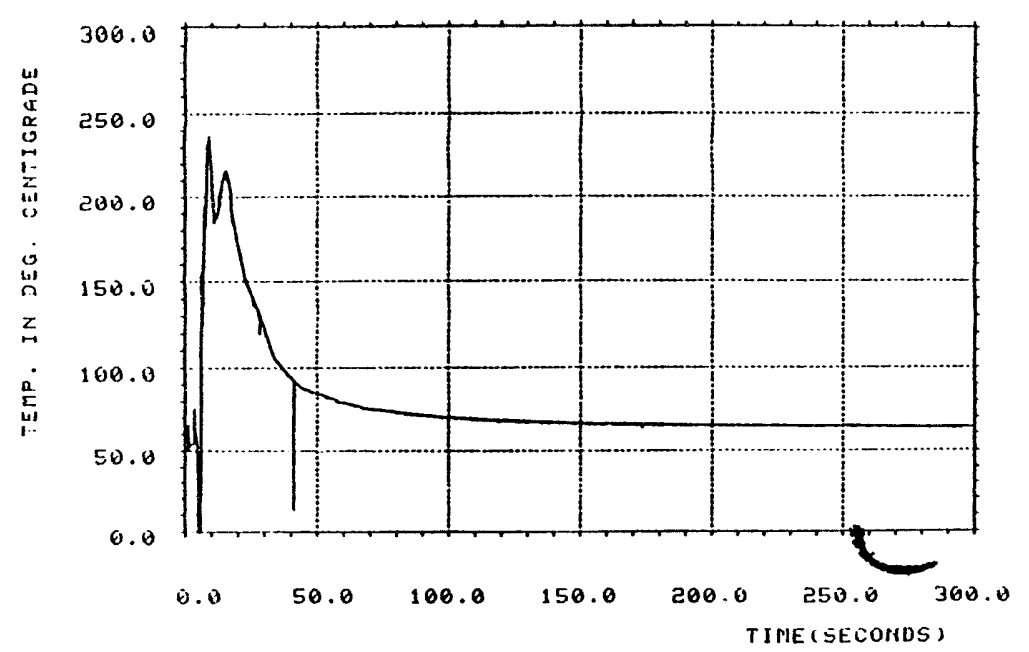

$\frac{7}{6}$

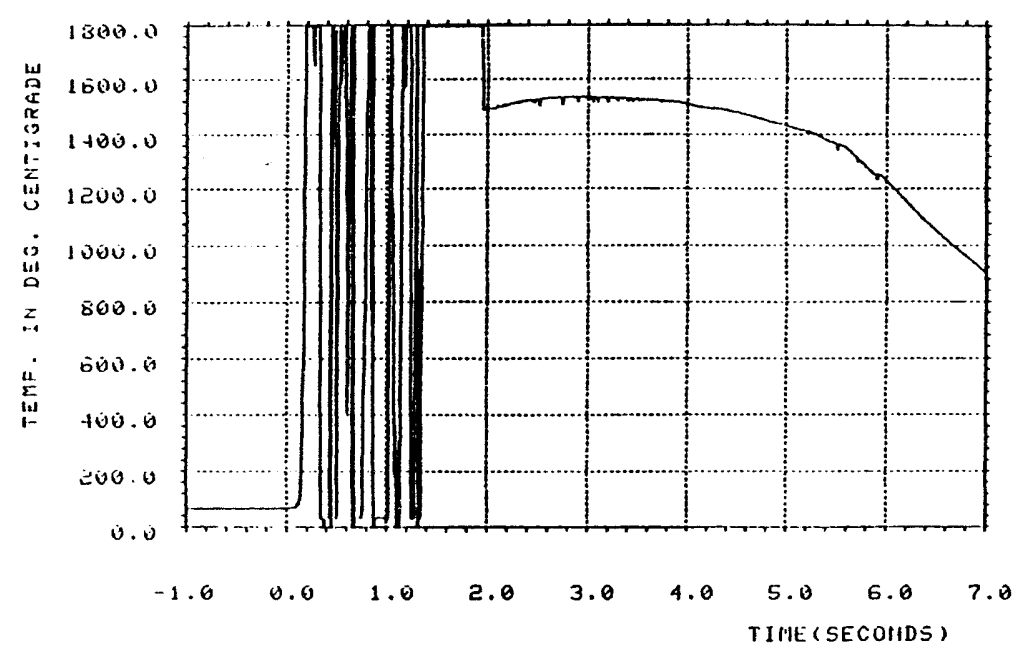

TC8-50, CCII-5, DAATE $9 / 17: 37$

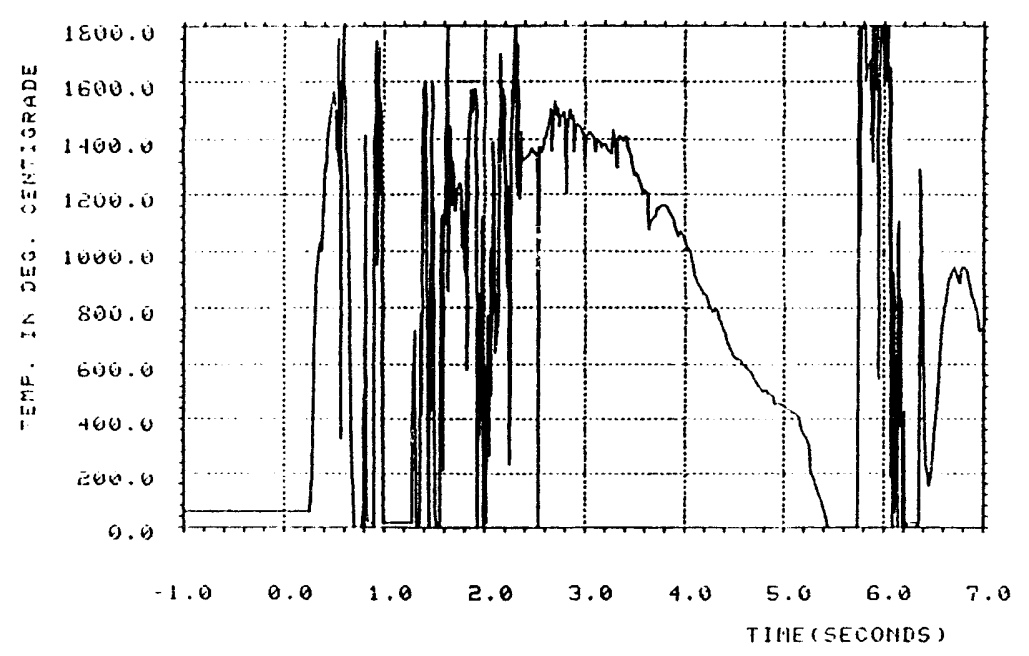

Tr:8-54, CCIn-5, DATE 9/17:-2

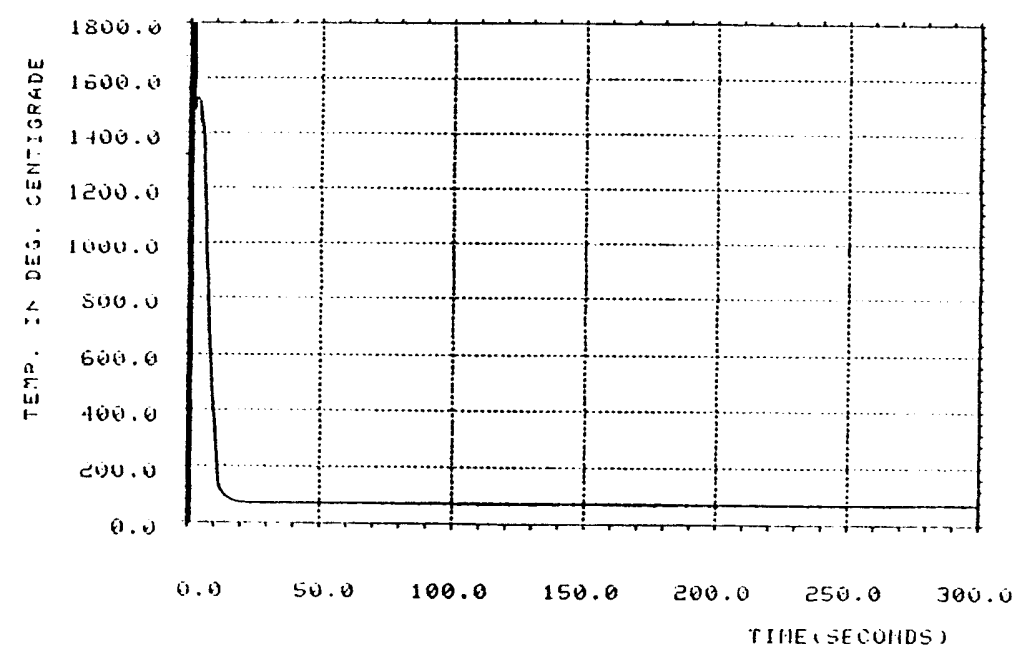

TI,E-50, CON-5, LATE $9,17.57$ 


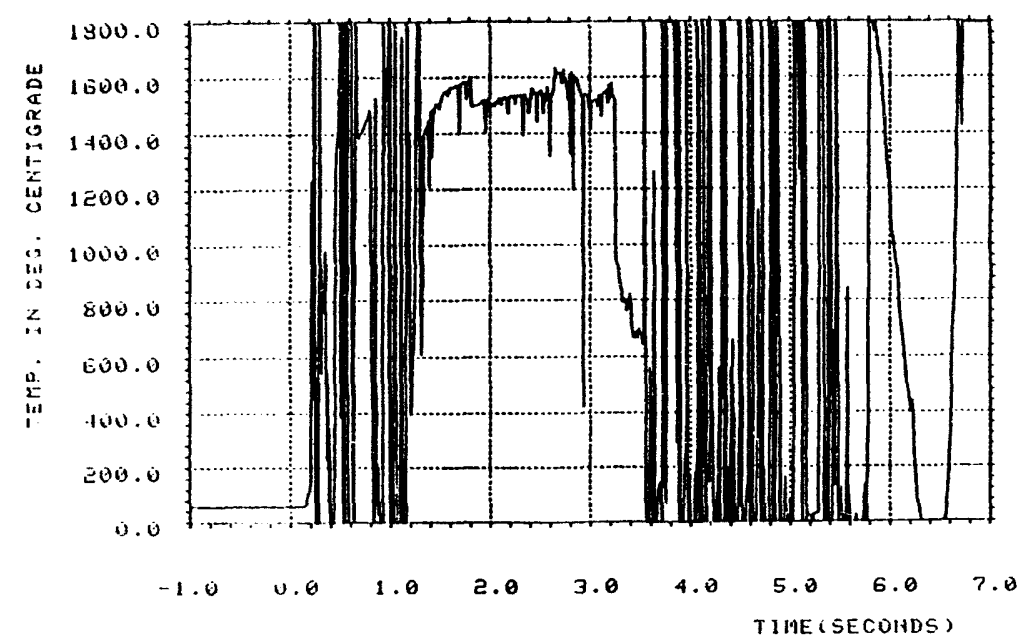

กั

TIS-52, C.N-5, DATE 9,17/3?

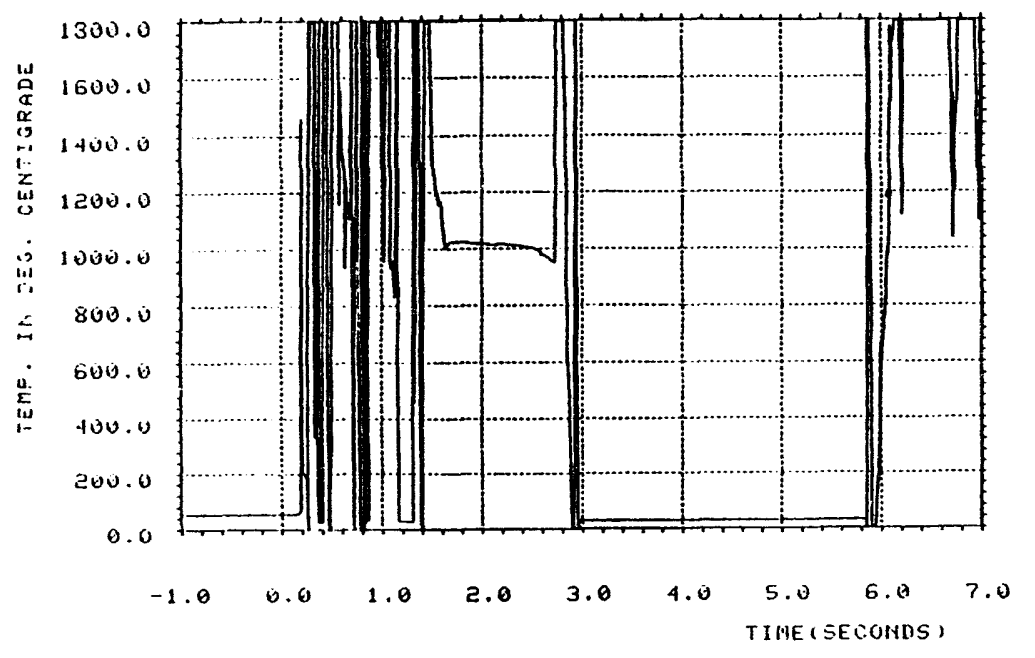

TC8-52, CCM-5, DATE 9,17:57

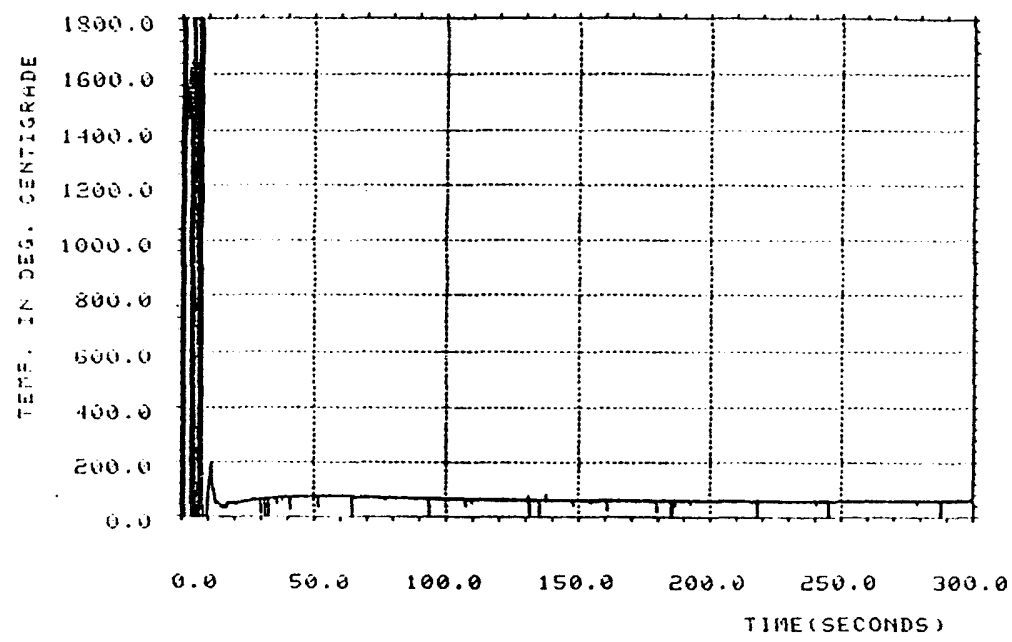

TCB-51, CCM-5, DATE $9 / 17.87$

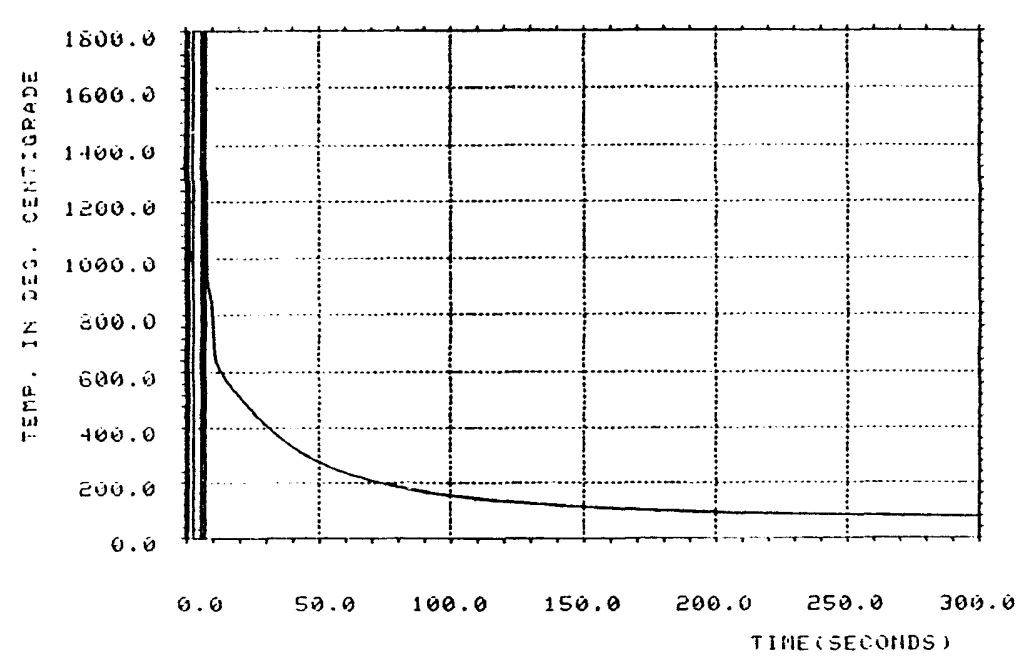




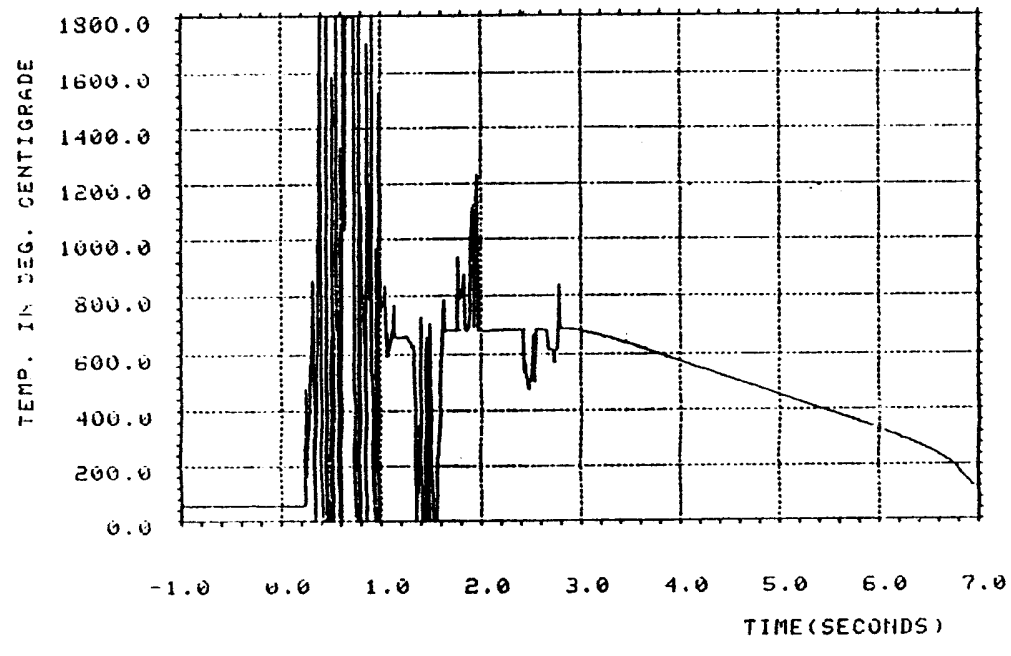

$\stackrel{i}{\tilde{n}}$

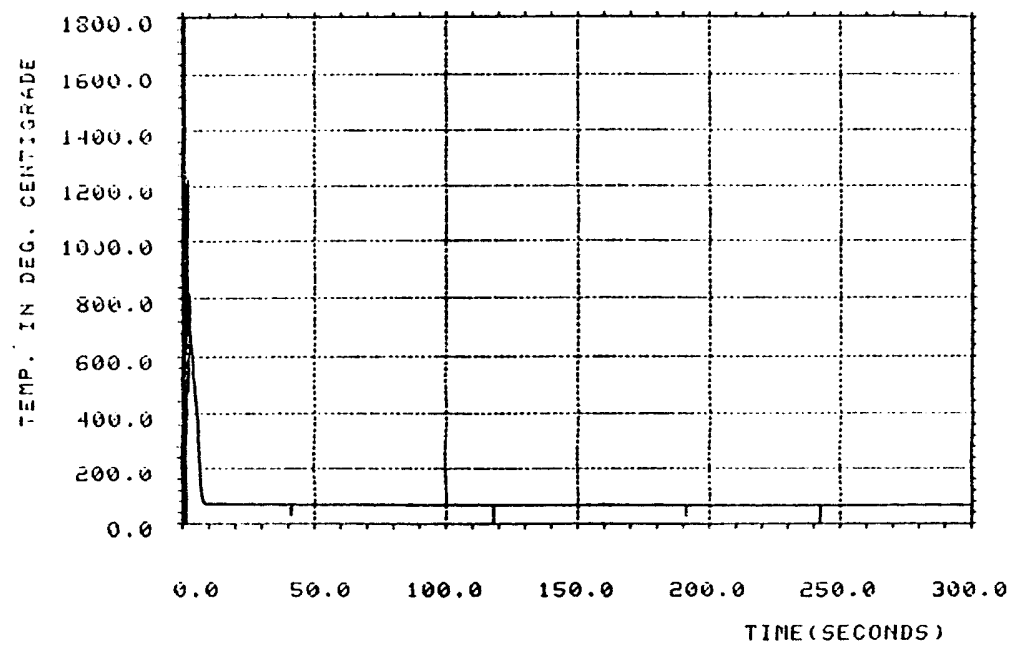

TC3-53, CCM-5, DATE 9/17/8?

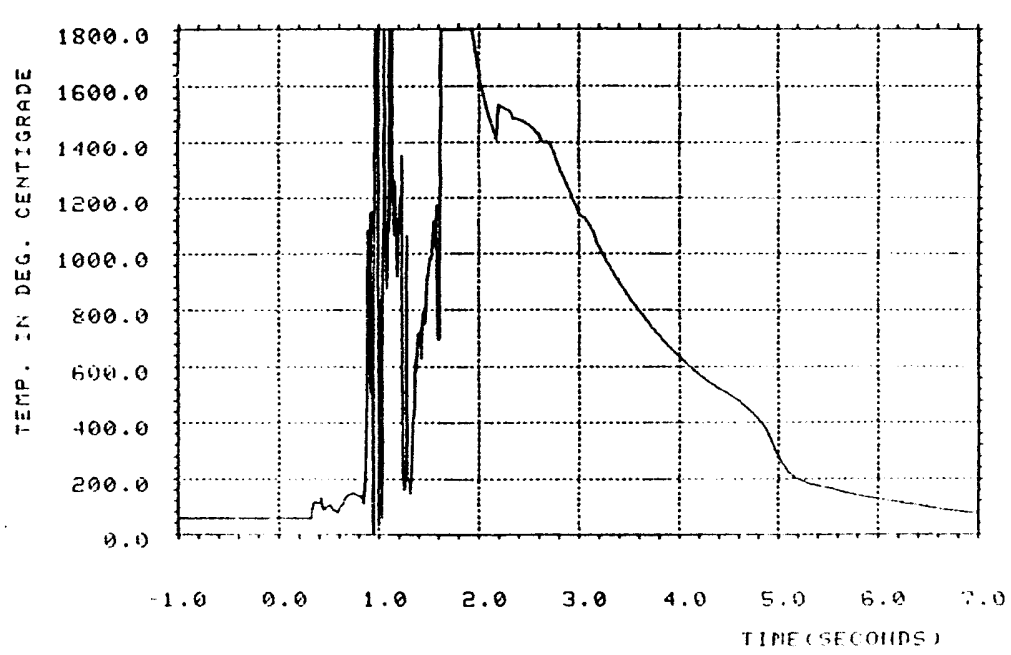

TIS-5.6. CCN-5, LATE 9.17 .87 


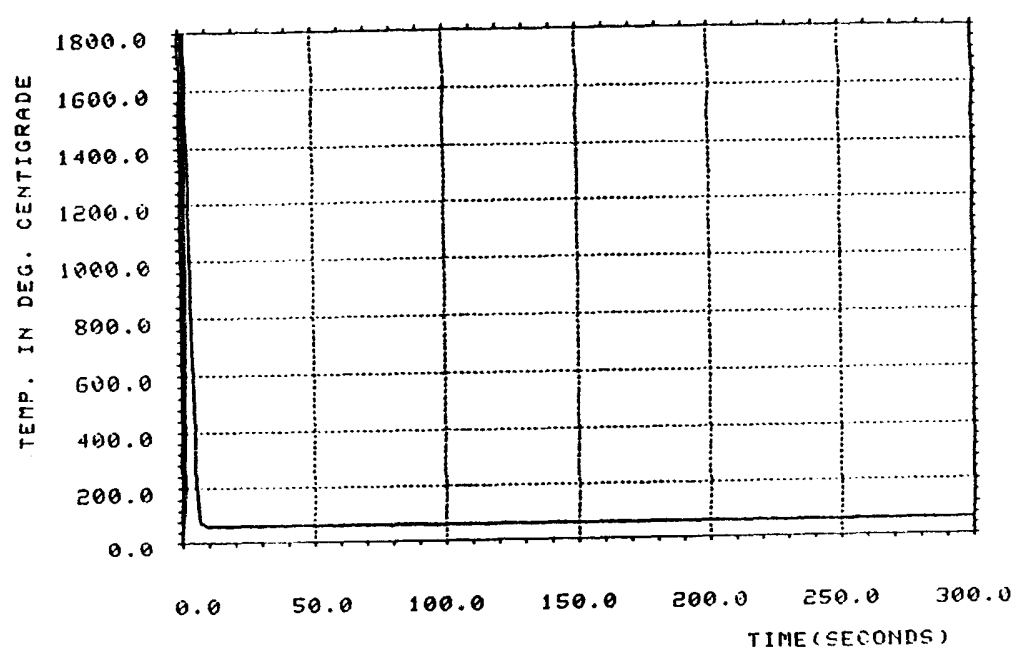

TC8-5., CCM-5, DATE 9,17:8?

$\stackrel{T}{N}$

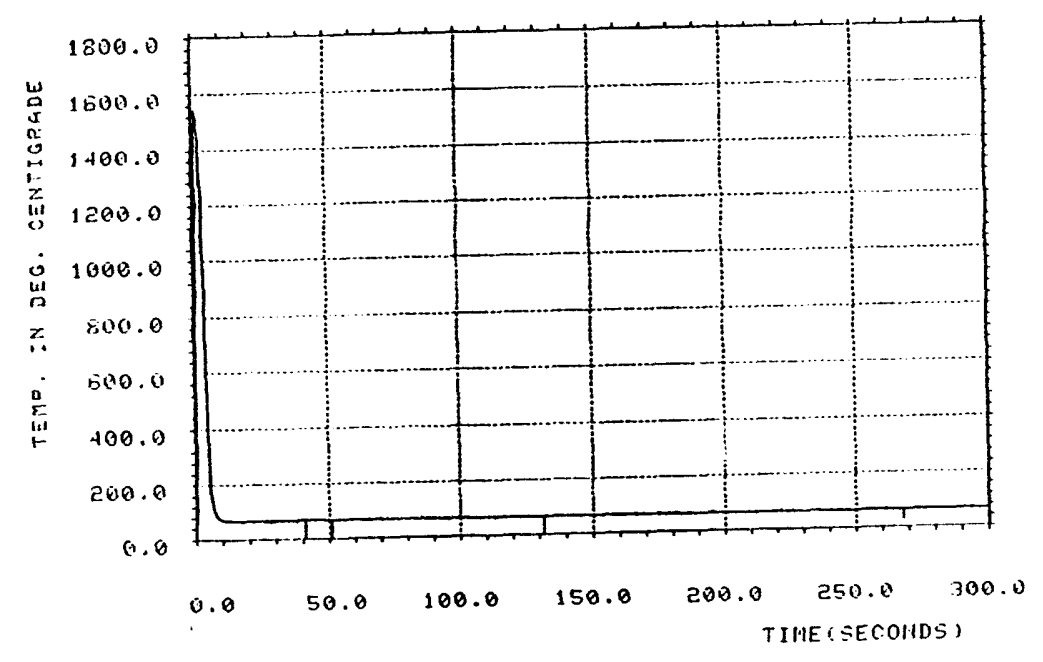

TC8-57, CCN-5, DATE $9 / 17 / 87$

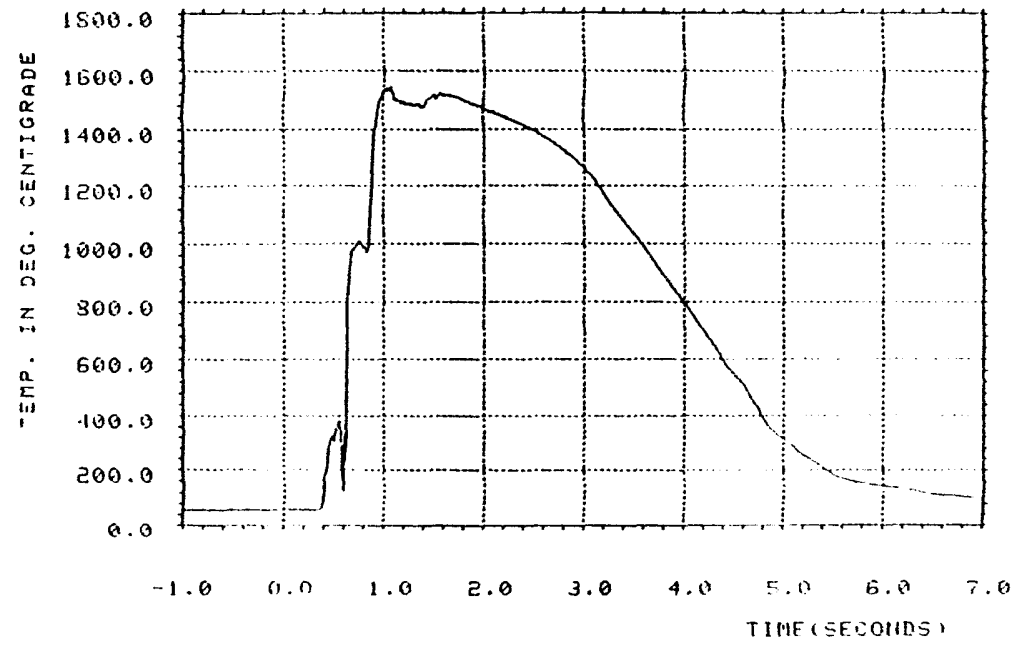

TY3-5. CON-5, [HATE $9: 13,5 \%$

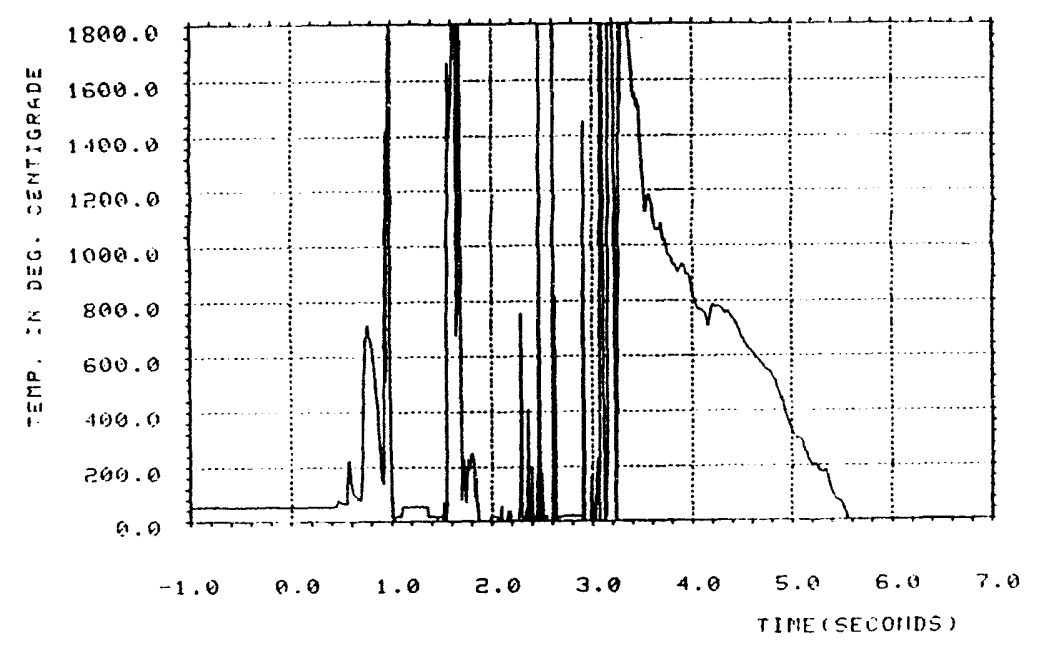

TCE-58, C.CM-5, DATE 9,17.8? 


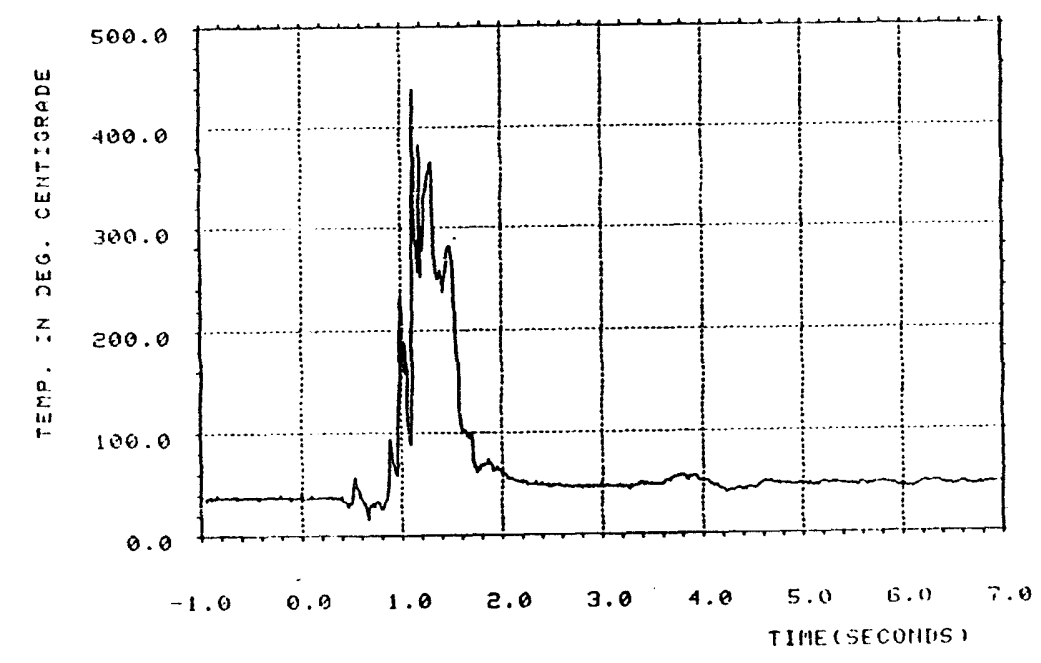

$\ddot{i}$

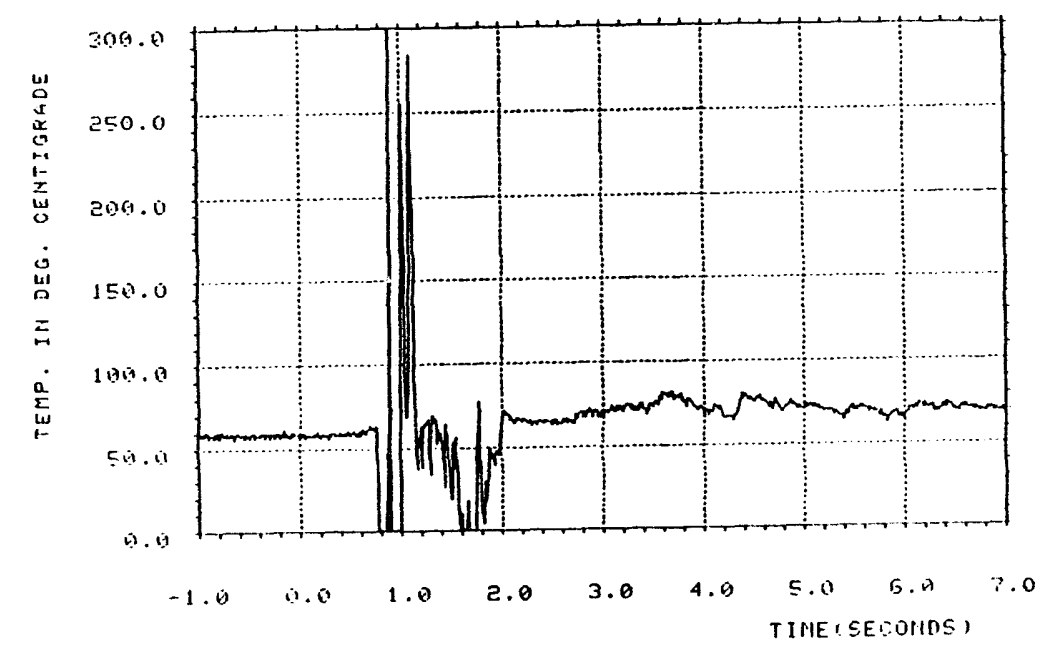

TIS-60, cMIn-5, DATE 9.17 .87

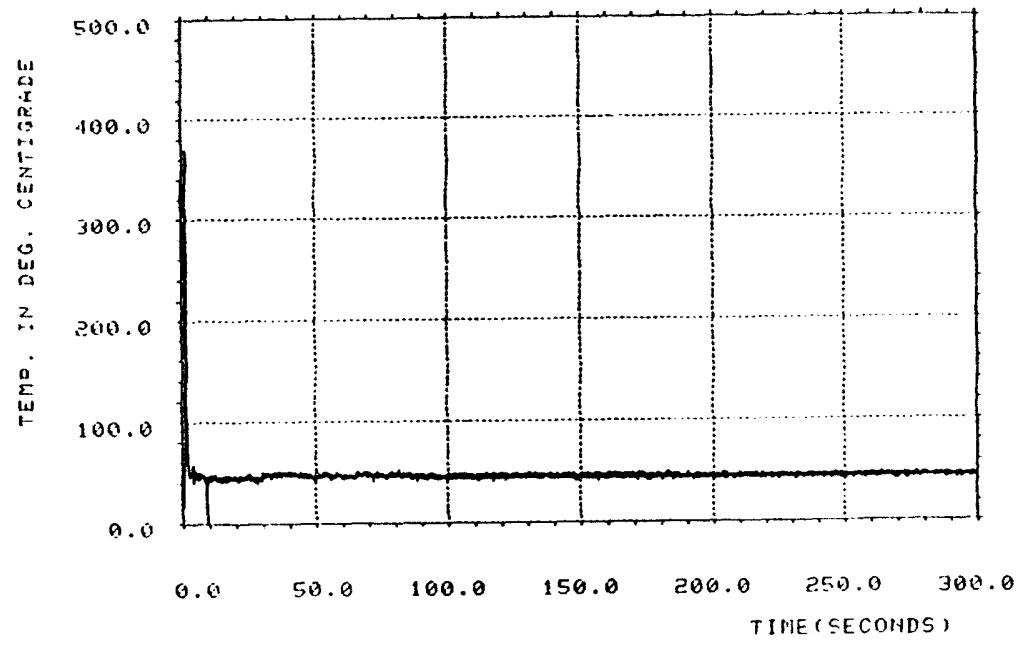

TSE-59, CCN-5, DATE 9:17,:?

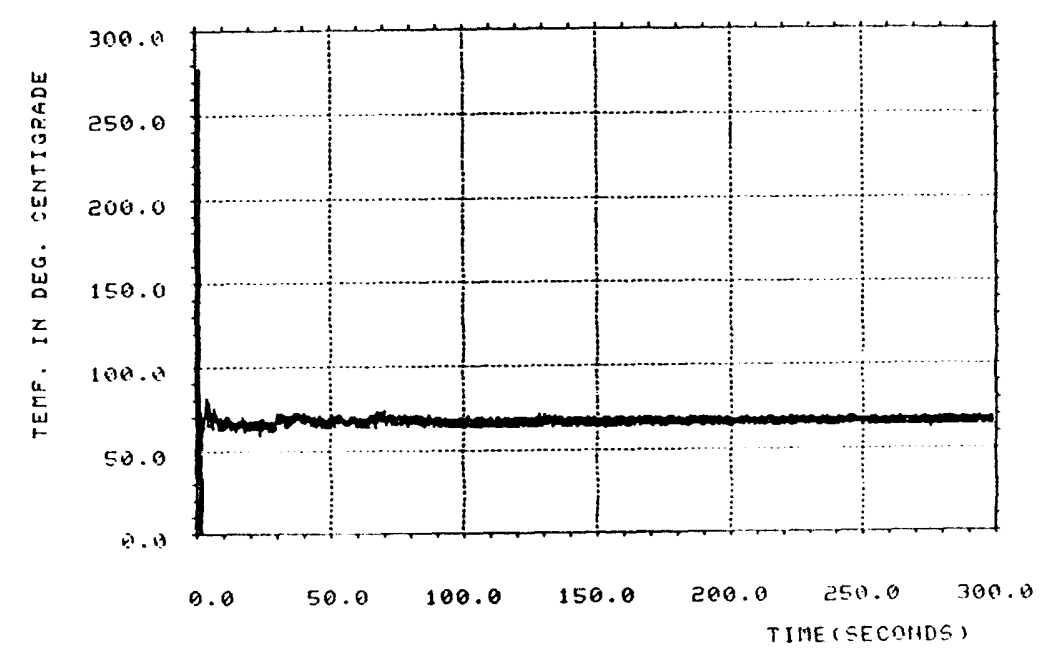

TC8-60, CCN-5, DATE 9.17.87 


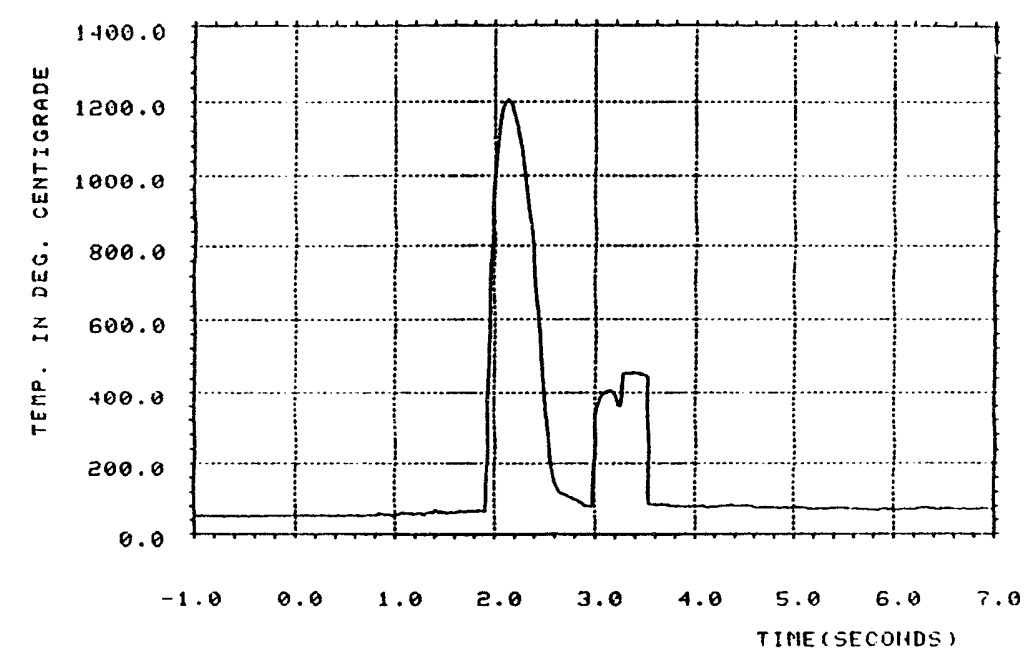

$\stackrel{\grave{n}}{n}$

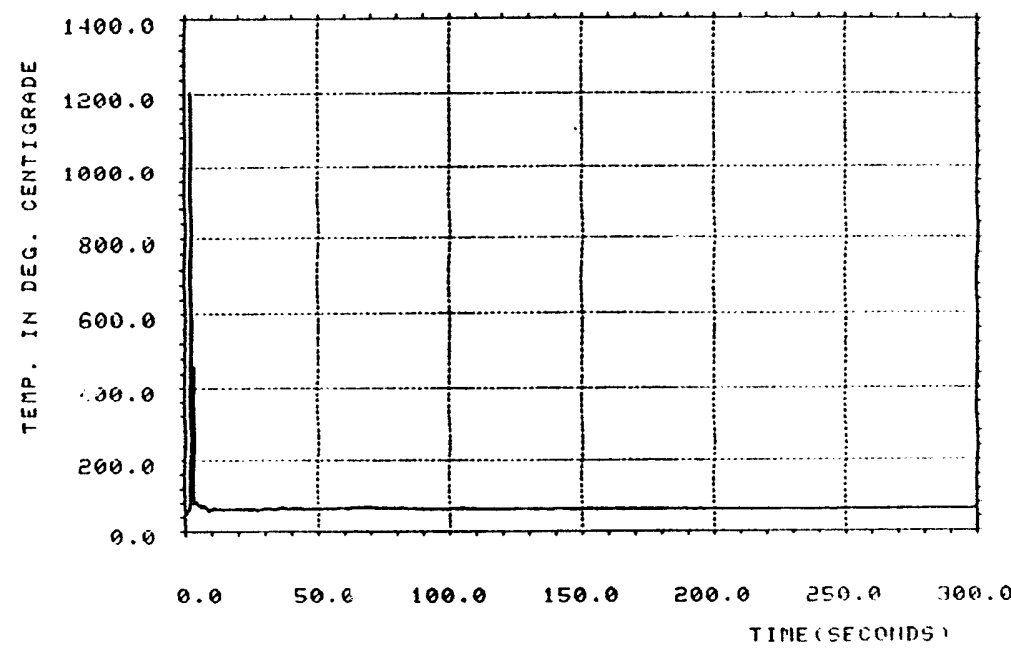

TC:-51, CCM-5, DATE $9: 17.87$

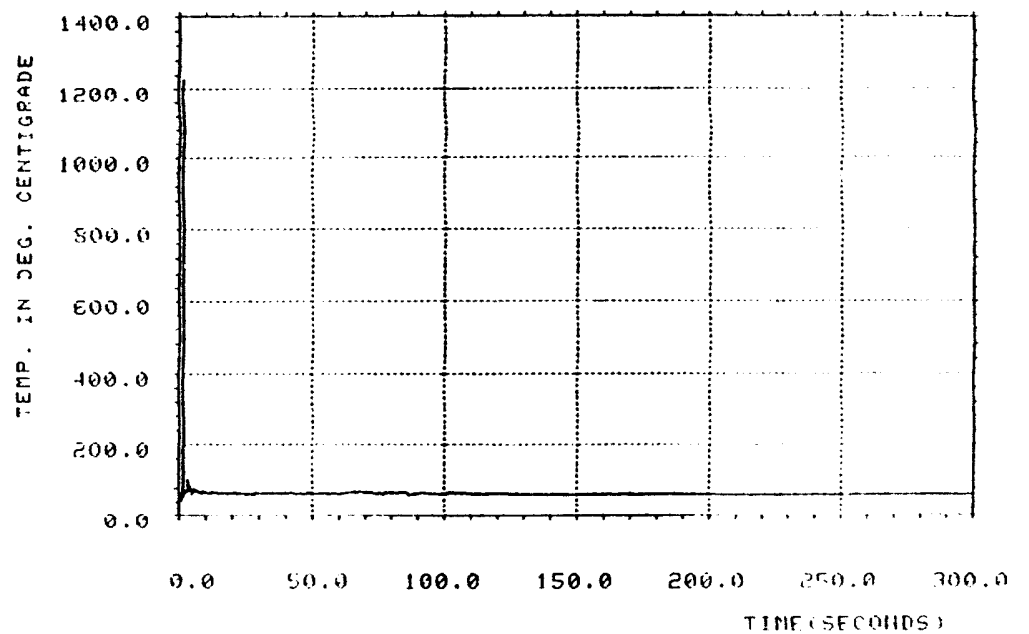

TrS-63, CCII-5, IIATE $9 / 17.87$ 


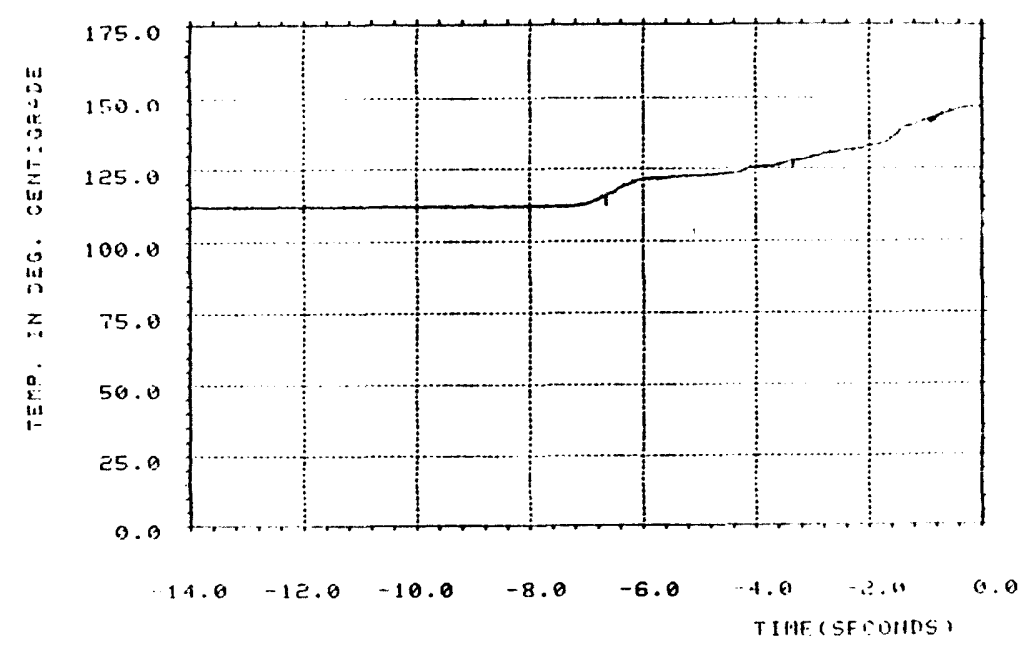

ज̃

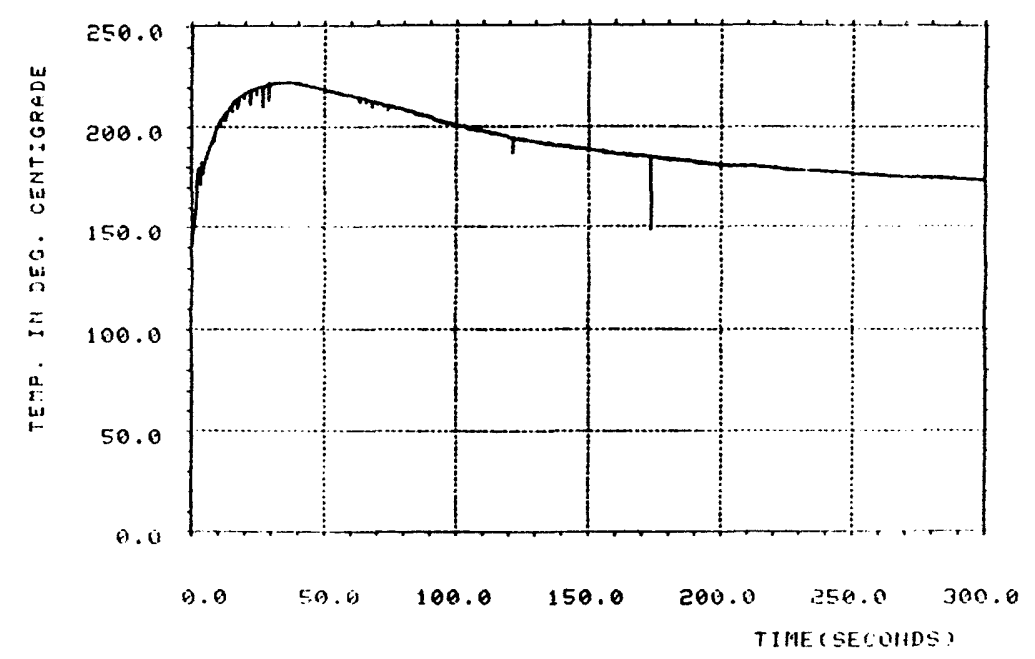

TIO-2, CCM-5, DATE 9/17/8T

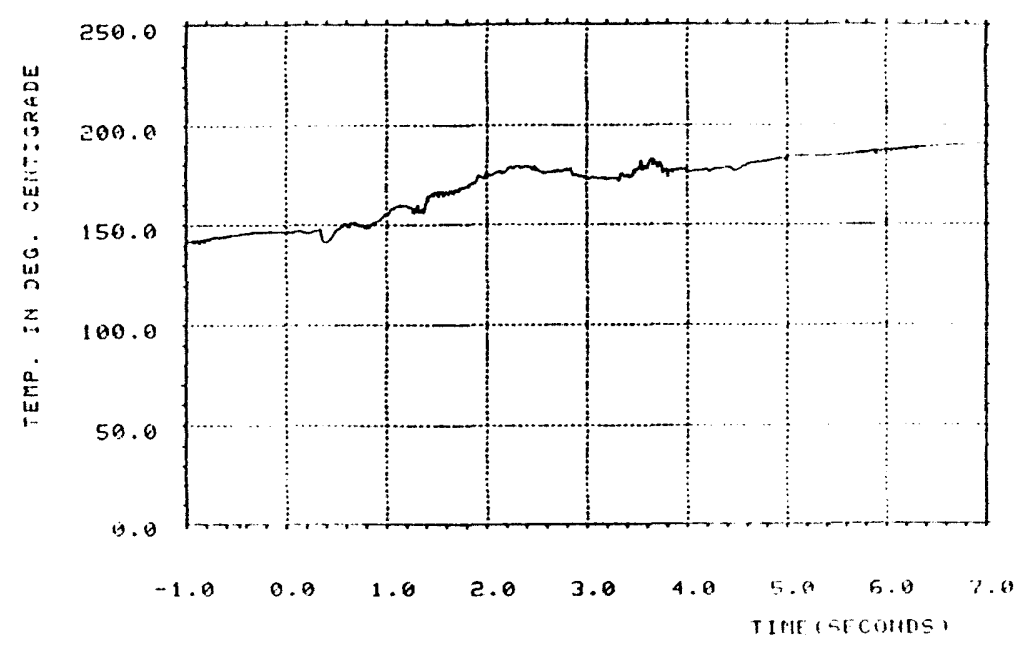

TH:-2, CCN-5, DATE G $1 \%$ :

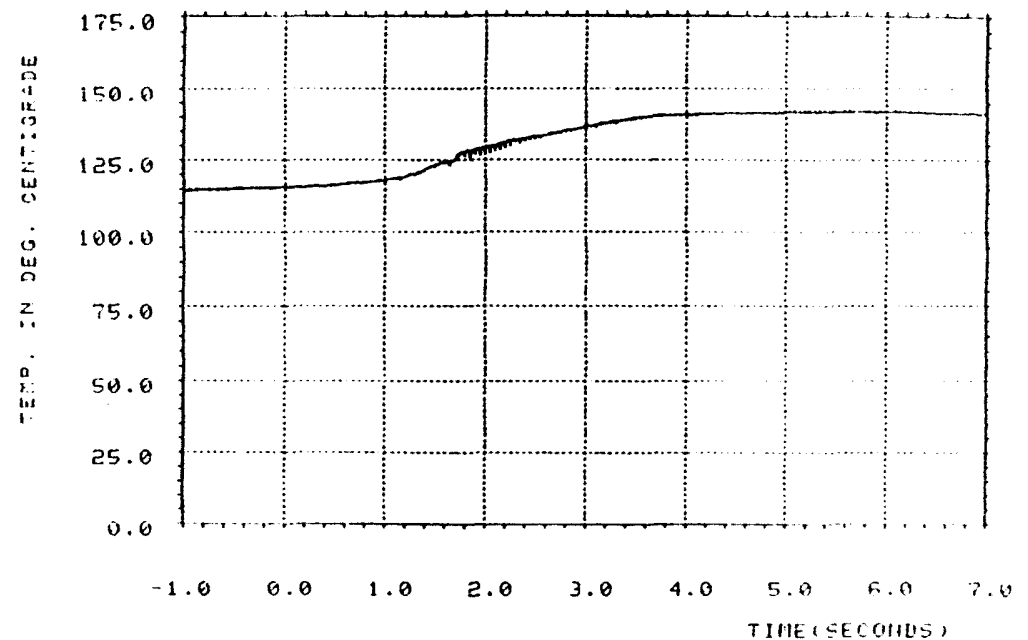

Ti: $12-1$, COM-5, DATE $9.17 \%$ 


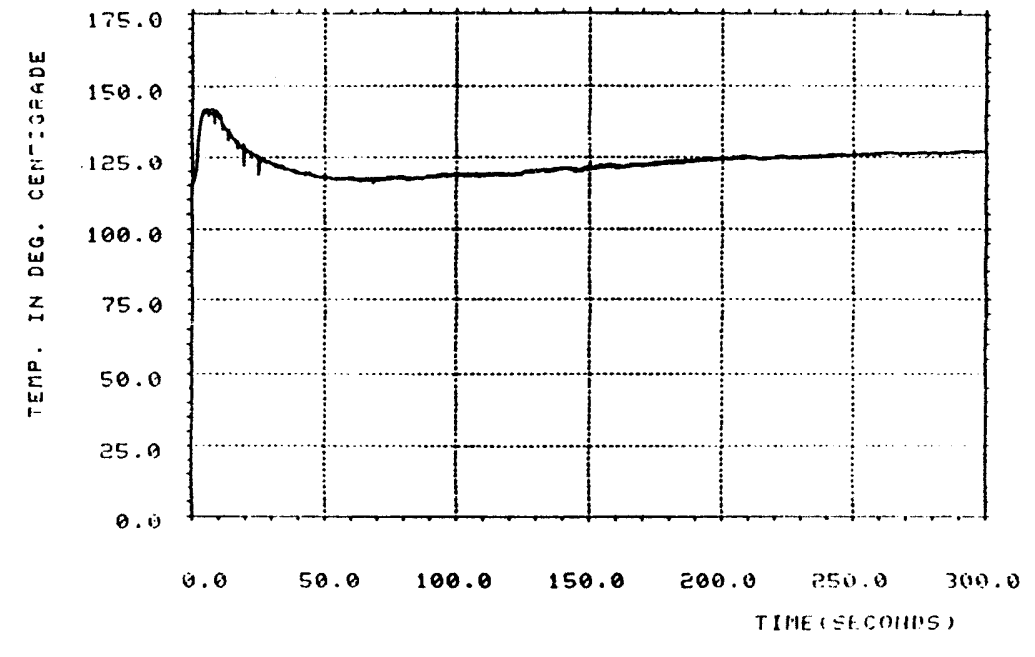

TI.12-1, COM-5, DATE 9.17 .87

ஸ்

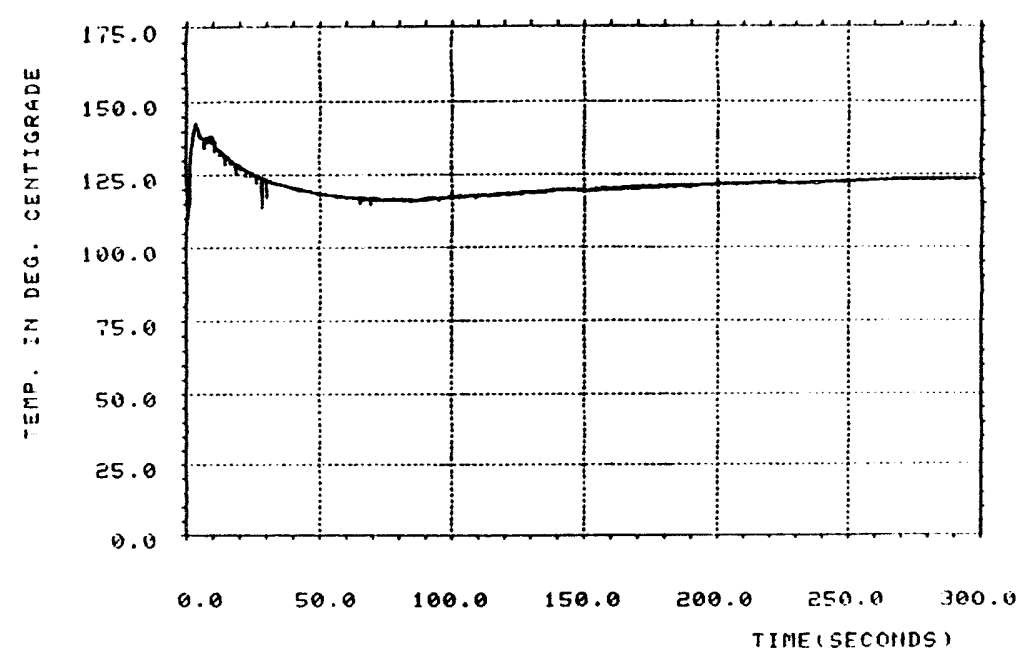

To12-2, CCM-5, [IATE 9,17:87

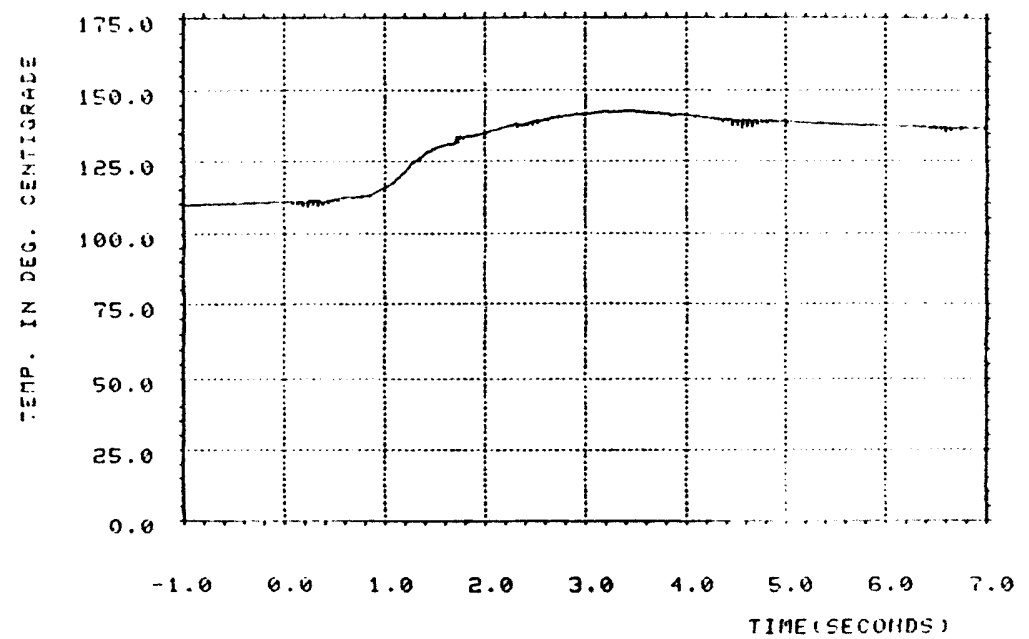

TrI2-2, CCM-5, DATE 9/1787

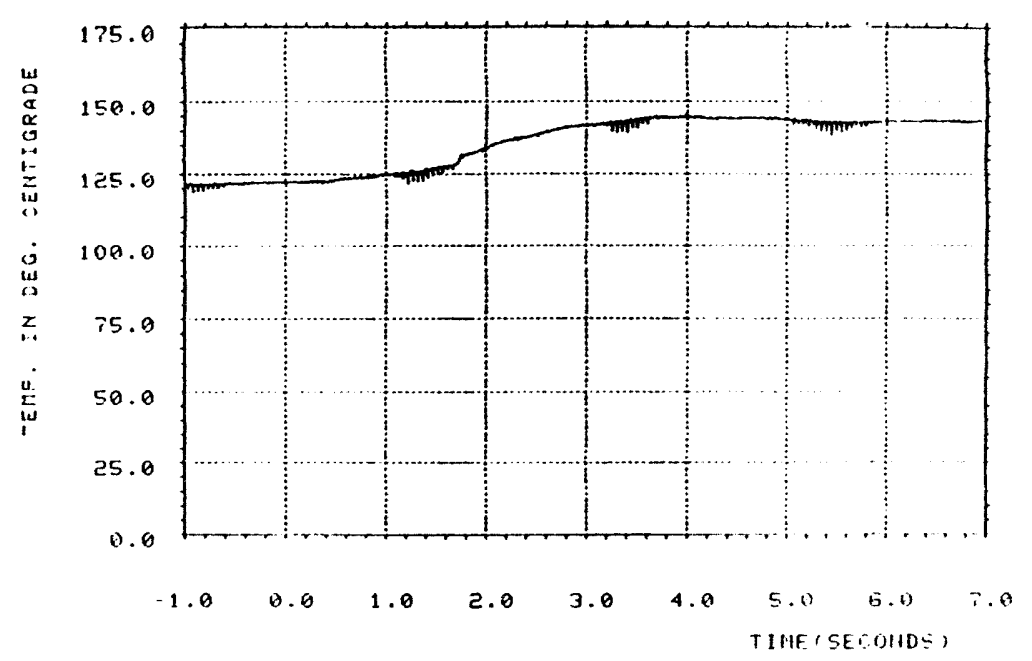

TH12 4, CCN-5, DATE 9.1787 


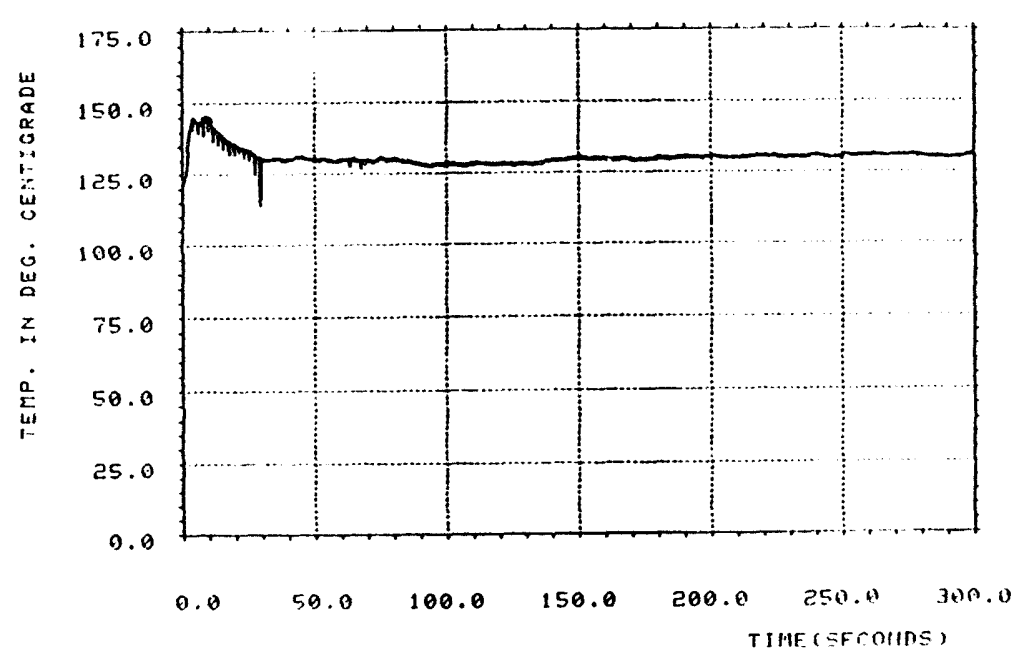

TC12-4, CON-5, IIATE 9,17:S?

$\stackrel{i}{\sim}$

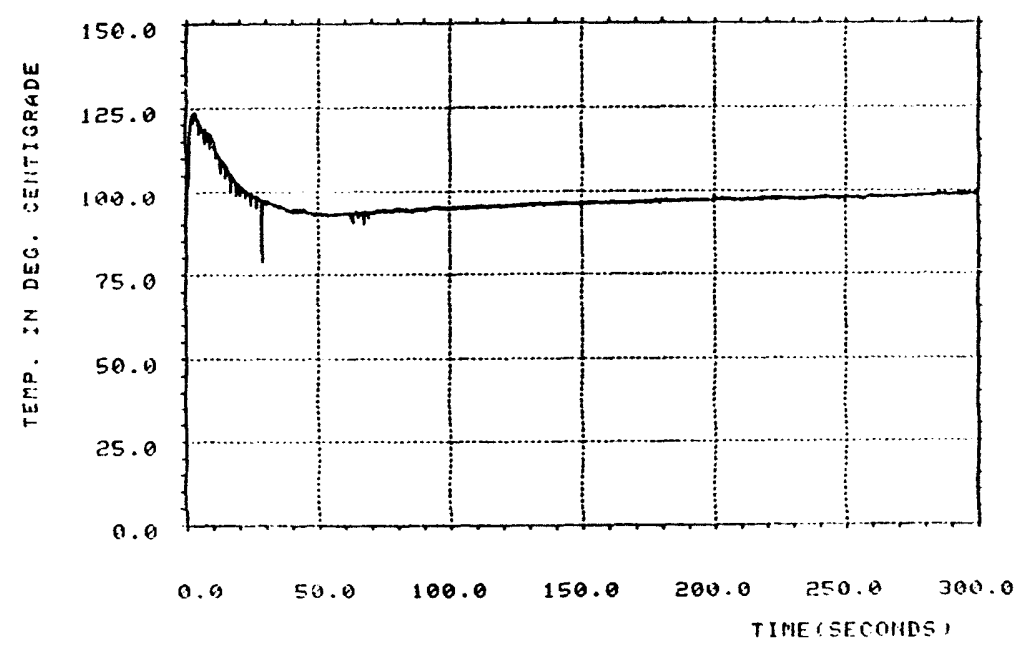

TI2-5, CCN-5, DATE $0.12: 7$

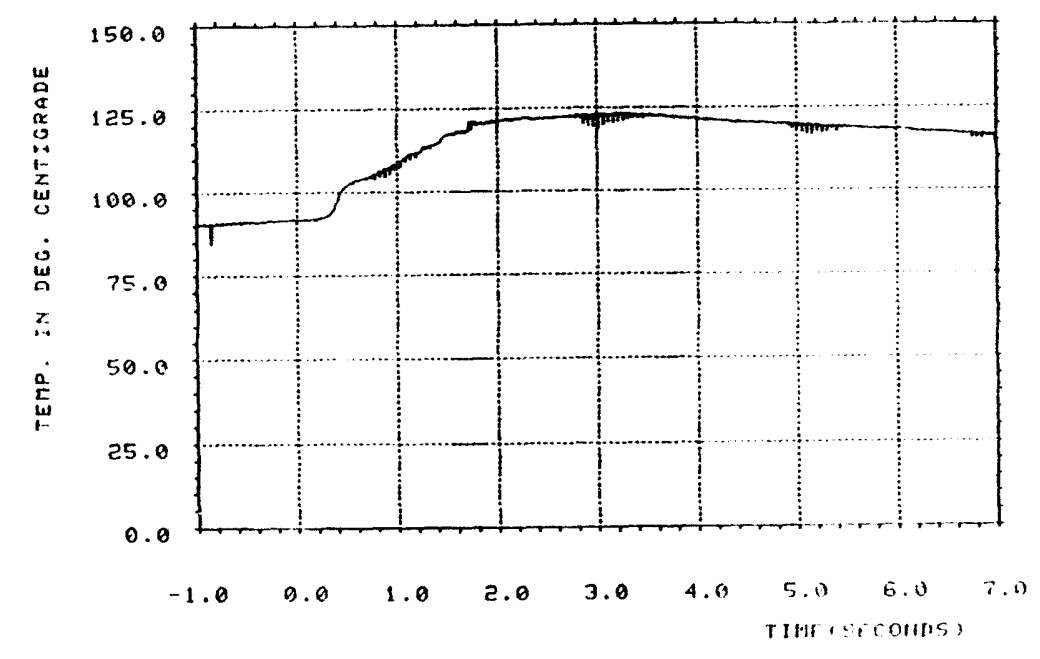

TO1E-5, CON-5, DATE $9.17 \cdot \mathrm{S}$

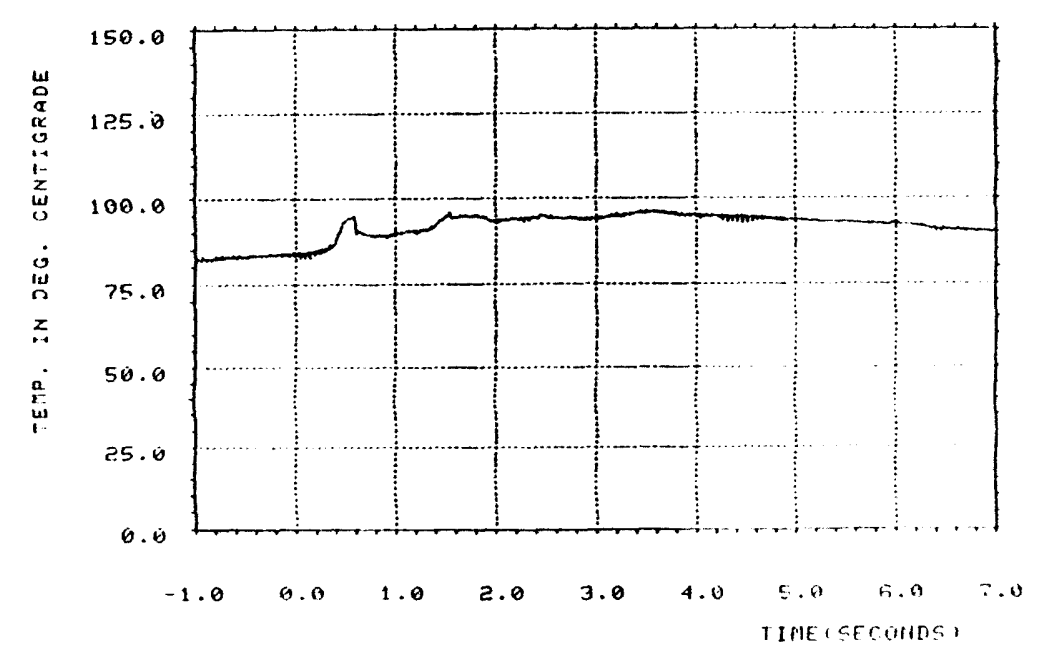

TO12-6, CCM-5, DATE $317 \cdot 87$ 


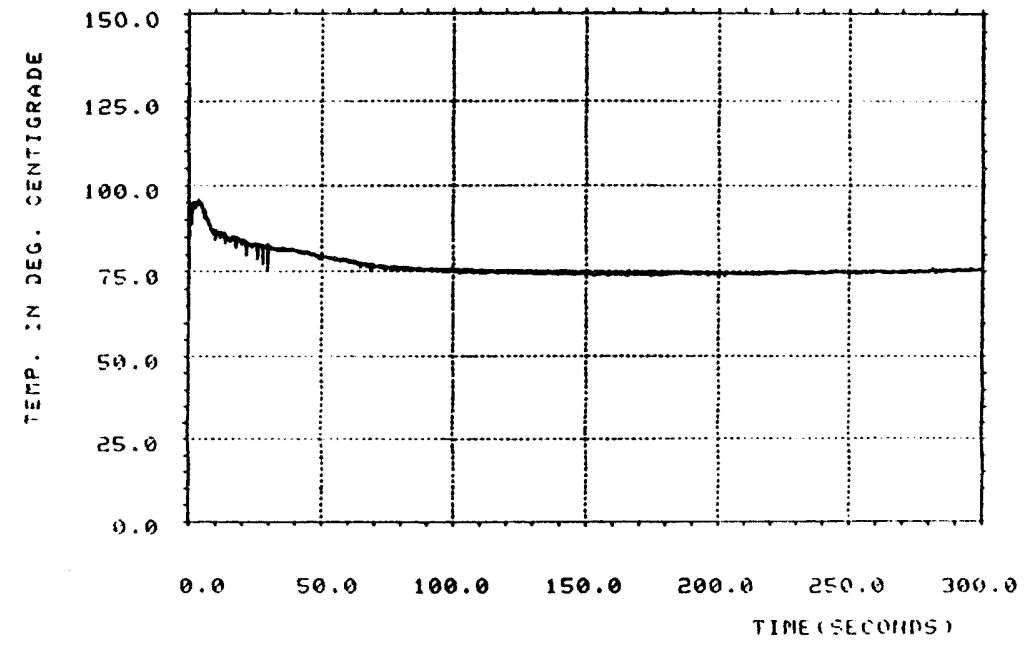

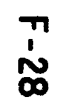

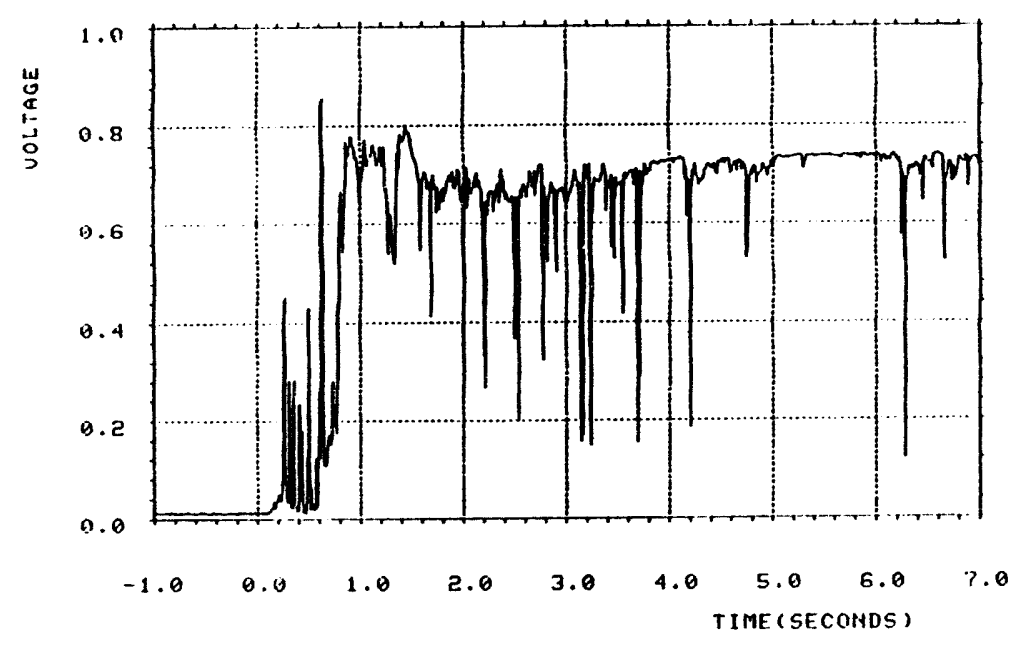

11. D. $* 2$, CCNI-5, DATE $9 / 17 / 87$

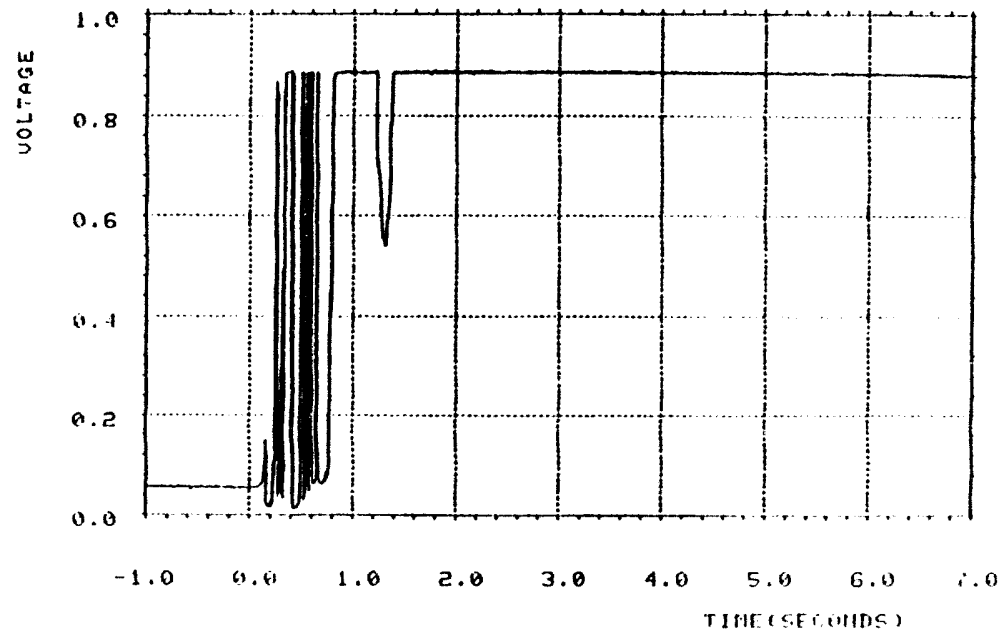

11. D. 11 , Cr:M-5, DATE $9.17: 87$

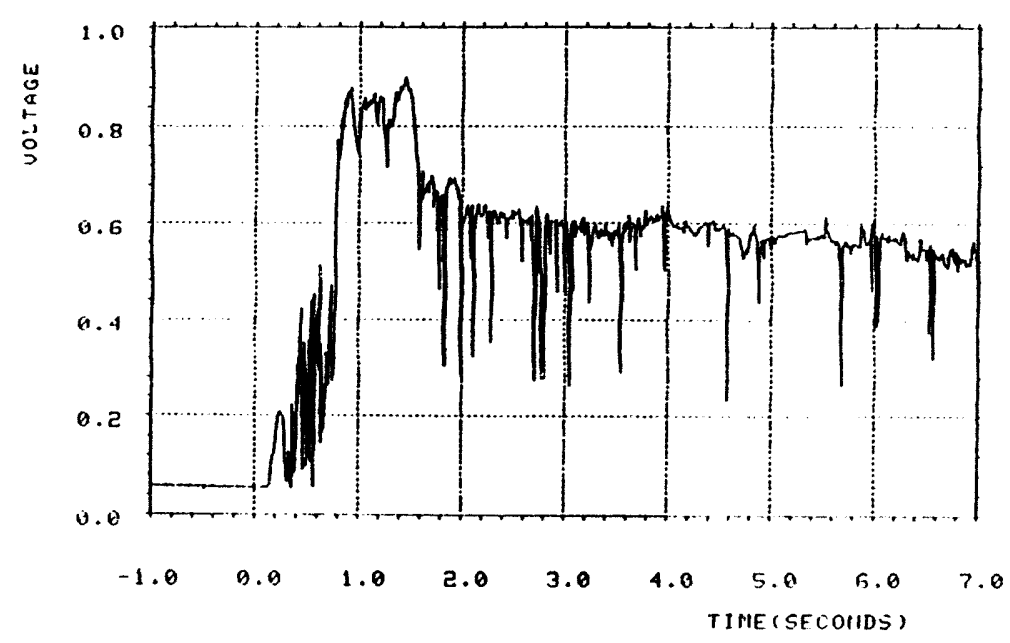

11. 1. 13, cCh-5, DATE 9.17 .87 

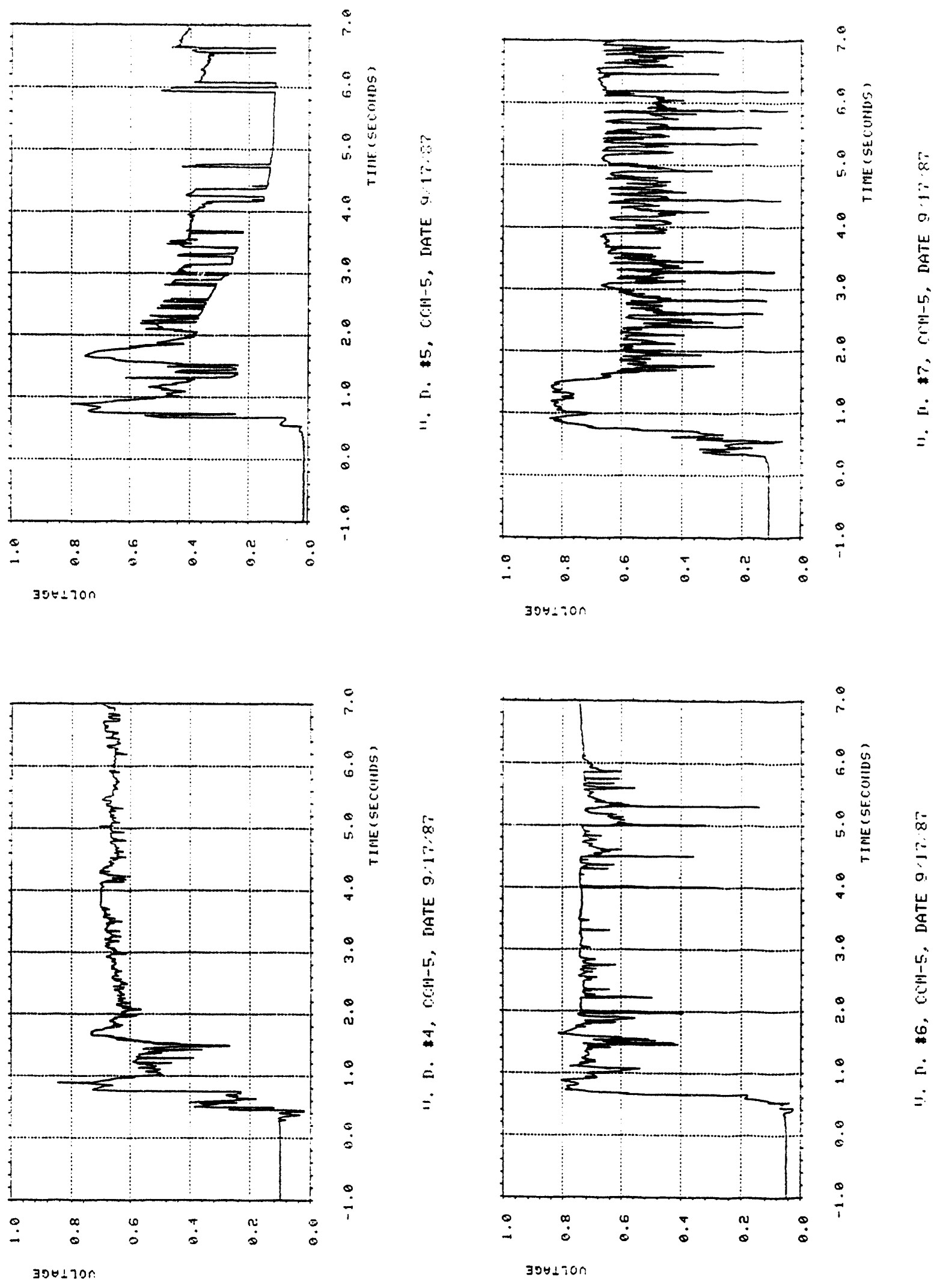


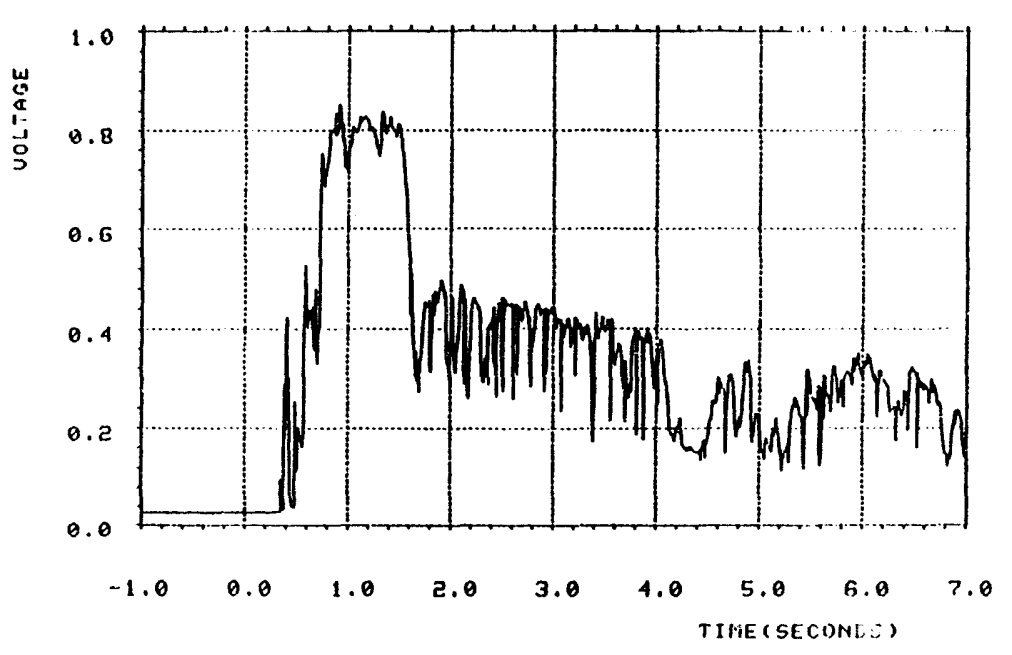

ì.

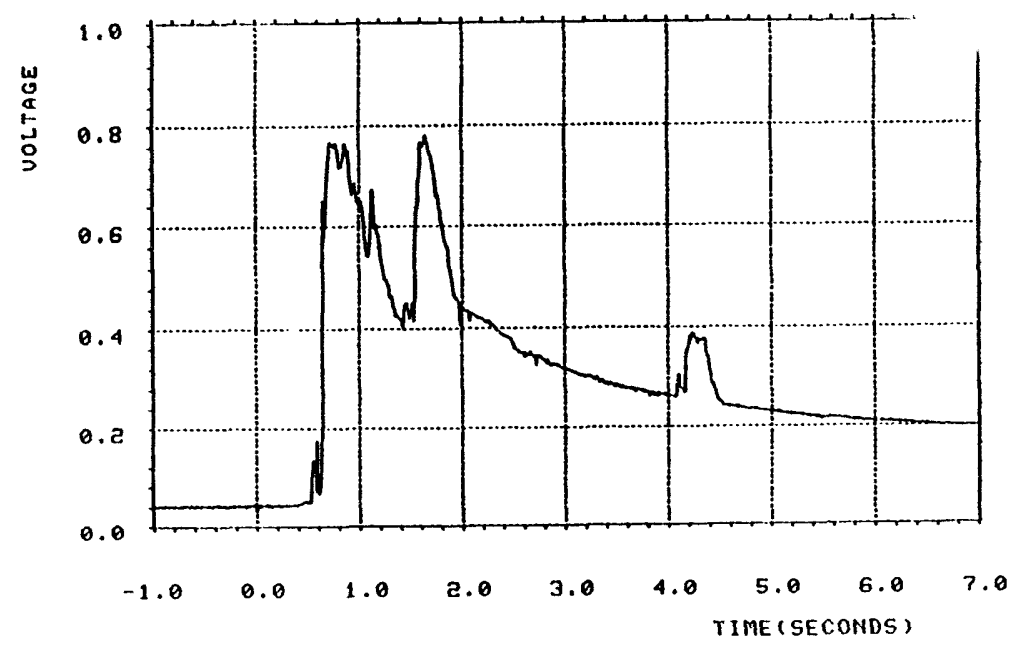

U. D. $\$ 10$, CCN-5, DATE $9: 17 / 87$

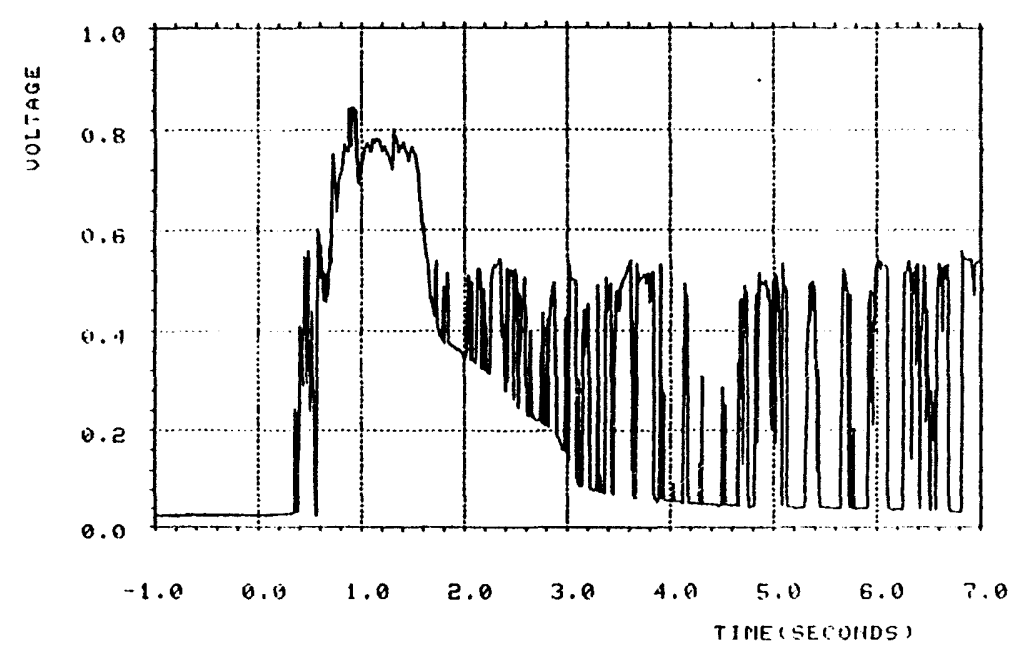

4. 11. 19, CCM-5, DATE 9.1\%:8?

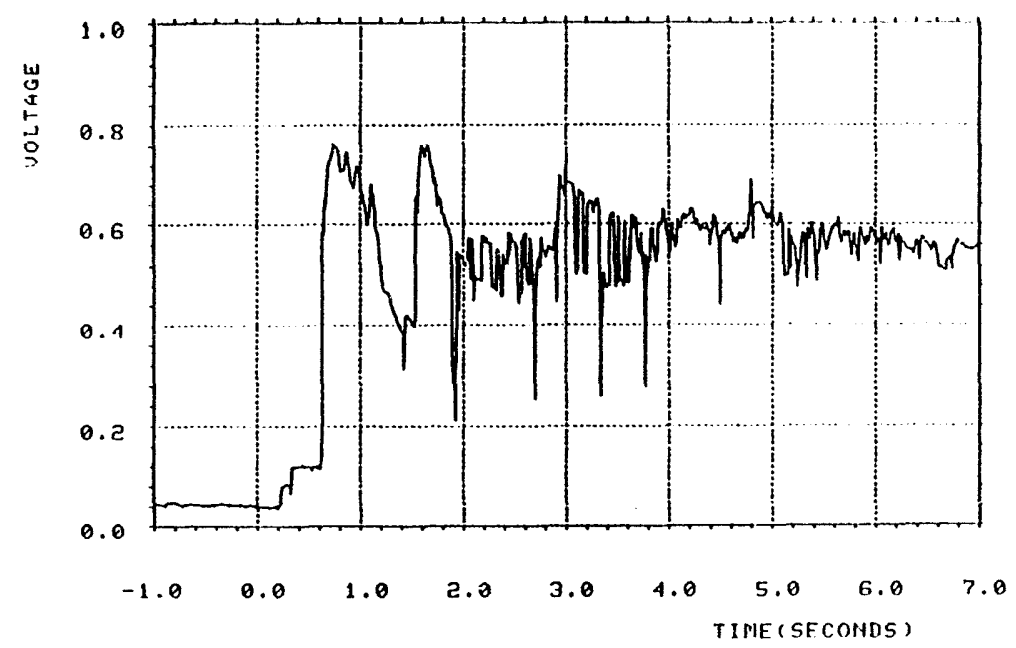

נ. D. $\$ 11$, CON-5, DATE $0: 17,87$ 


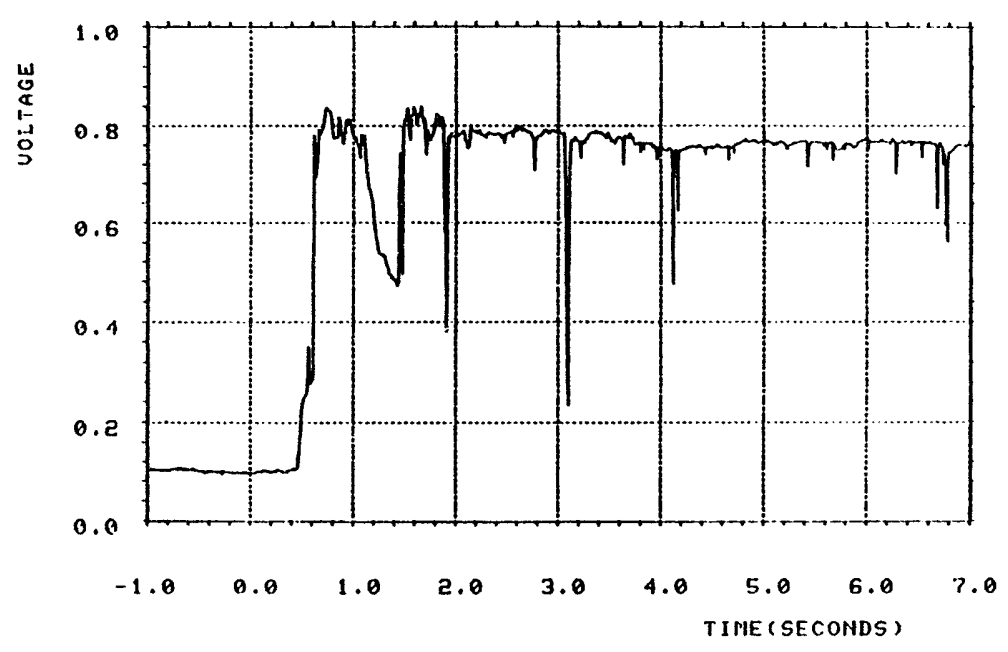

$\bar{\omega}$

4. D. 12 , Cli-5, DATE $917 \% 8$ ? 
APPENDIX G. DATA FOR CCM- 6

PTS-1: Thermite-Vessel Gas Pressure

PTS-12: Pressure in Single-View-Port Section of Experiment Vessel

PTS-13: Pressure in Multi-View-Port Section of Experiment Vessel

PTK-2: Pressure in Single-View-Port Section of Experiment Vessel

TC8-16: Water/Debris Temperature in Catch Pan

TC8-17: Water/Debris Temperature in Catch Pan

TC8-18: Water/Debris Temperature in Catch Pan

TC8-19: Water/Debris Temperature in Catch Pan

TC8-20: Water/Vapor Temperature

TC8-21: Water/Vapor Temperature

TC8-22: Water/Vapor Temperature

TC8-23: Water/Napor Temperature

TC8-24: Water/Napor Temperature

TC8-25: Water/Vapor Temperature

TC8-27: Water/Vapor Temperature

TC8-28: Water/Vapor Temperature

TC8-29 Water/Vapor Temperature

TC8-30: Water/Vapor Temperature

TC8-31: Water/Vapor Temperature

TC8-32: Water/Vapor Temperature

TC8-33: Water/Vapor Temperature

TC8-37: Water/Vapor Temperature

TC8-39: Water/Vapor Temperature

TC8-43: Water/Napor Temperature

TC8-51: Centerline Water/Napor Temperature

TC8-53: Centerline Water/Vapor Temperature

TC8-55: Centerline Water/Vapor Temperature

TC8-56: Centerline Water/Vapor Temperature

TC8-57: Centerline Water/Vapor Temperature

TC8-58: Centerline Water/Vapor Temperature

TC8-59: Centerline Water/Napor Temperature

TC8-60: Centerline Water/Vapor Temperature

TC8-61: Centerline Water/Vapor Temperature

TC8-63: Centerline Water/Vapor Temperature 
APPENDIX G. DATA FOR CCM-6 (Cont'd.)

TC9-2: Tempeature in the Thermite Vesse1

TC12-1: Gas/Napor Temperature in the Experiment Vesse1, $245.7 \mathrm{~cm}$ Elevation TC12-20: Gas/Vapor Temperature in the Experiment Vesse1, $226.7 \mathrm{~cm}$ Elevation TC12-3: Gas/Vapor Temperature in the Experiment Vesse1, $221.6 \mathrm{~cm}$ Elevation TC12-4: Gas/Vapor Temperature in the Expeirment Vesse1, $212.7 \mathrm{~cm}$ Elevation TC12-5: Gas/Napor Temperature in the Expeirment Vesse1, $194.9 \mathrm{~cm}$ Elevation TC12-6: Gas/Vapor Temperature in the Expeirment Vesse1, $177.2 \mathrm{~cm}$ Elevation VD-1: Void Detection at $5.72 \mathrm{~cm}$ Radius, $-5.08 \mathrm{~cm}$ Elevation VD-2: Void Detection at $6.99 \mathrm{~cm}$ Radius, $-5.08 \mathrm{~cm}$ Elevation VD-3: Void Detection at $7.52 \mathrm{~cm}$ Radius, $-5.08 \mathrm{~cm}$ Elevation VD-4: Void Detection at $5.08 \mathrm{~cm}$ Radius, $-30.48 \mathrm{~cm}$ Elevation VD-5: Void Detection at $7.30 \mathrm{~cm}$ Radius, $-30.48 \mathrm{~cm}$ Elevation VD-6: Void Detection at $9.52 \mathrm{~cm}$ Radius, $-30.48 \mathrm{~cm}$ Elevation VD-7: Void Detection at $3.81 \mathrm{~cm}$ Radius, $-55.98 \mathrm{~cm}$ Elevation VD-8: Void Detection at $5.72 \mathrm{~cm}$ Radius, $-55.88 \mathrm{~cm}$ Elevation VD-9: Void Detection at $8.26 \mathrm{~cm}$ Radius, $-55.88 \mathrm{~cm}$ Elevation VD-10: Void Detection at $3.18 \mathrm{~cm}$ Radius, $-81.28 \mathrm{~cm}$ Elevation VD-11: Void Detedtion at $5.72 \mathrm{~cm}$ Radius, $-81.28 \mathrm{~cm}$ Elevation VD-12: Void Detection at $8.26 \mathrm{~cm}$ Radius, $-81.28 \mathrm{~cm}$ Elevation 

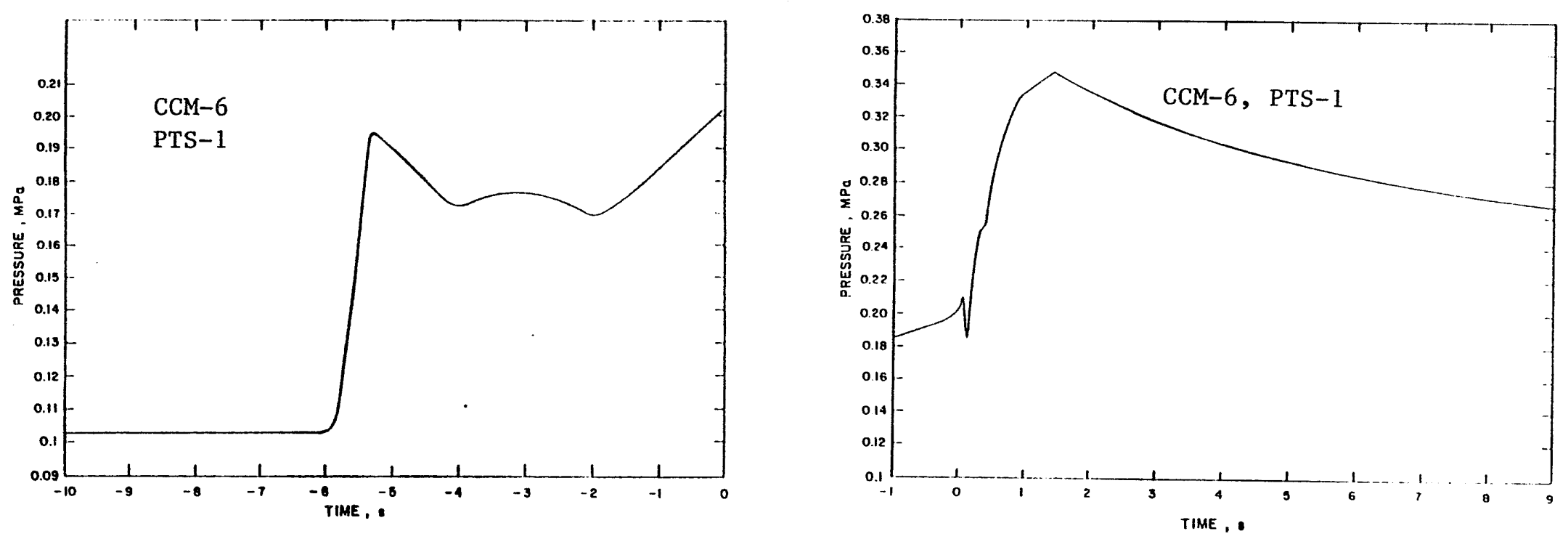

$\stackrel{s}{\dot{\omega}}$
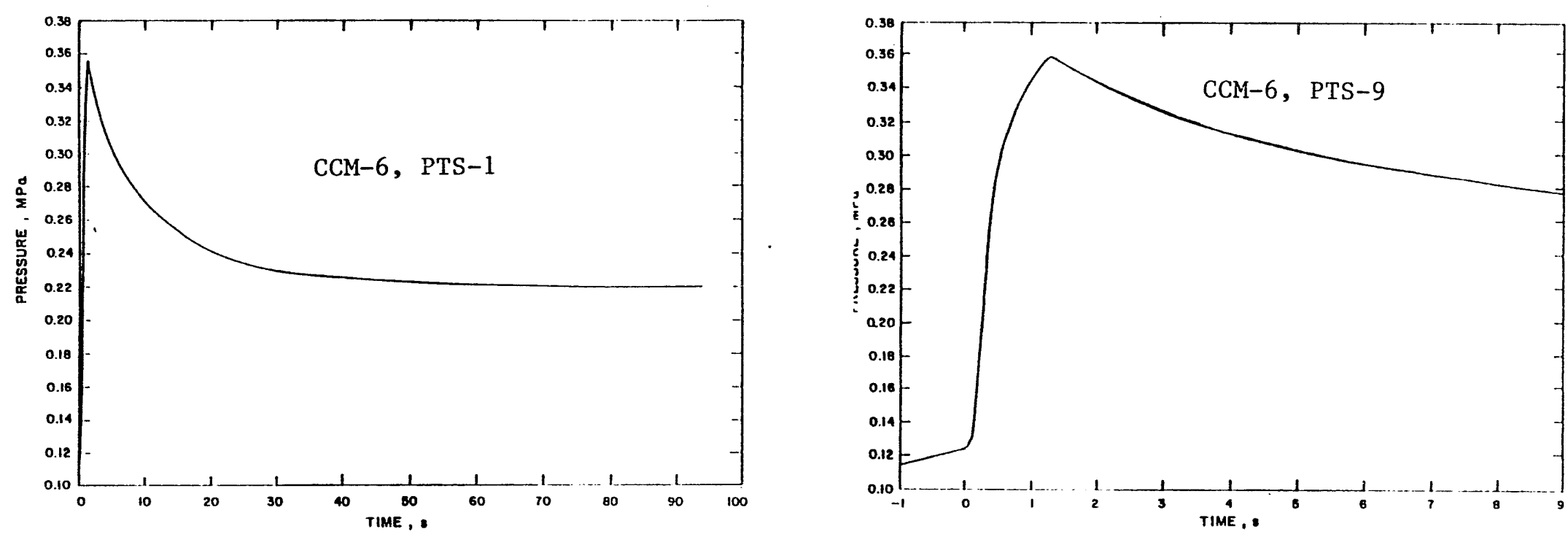

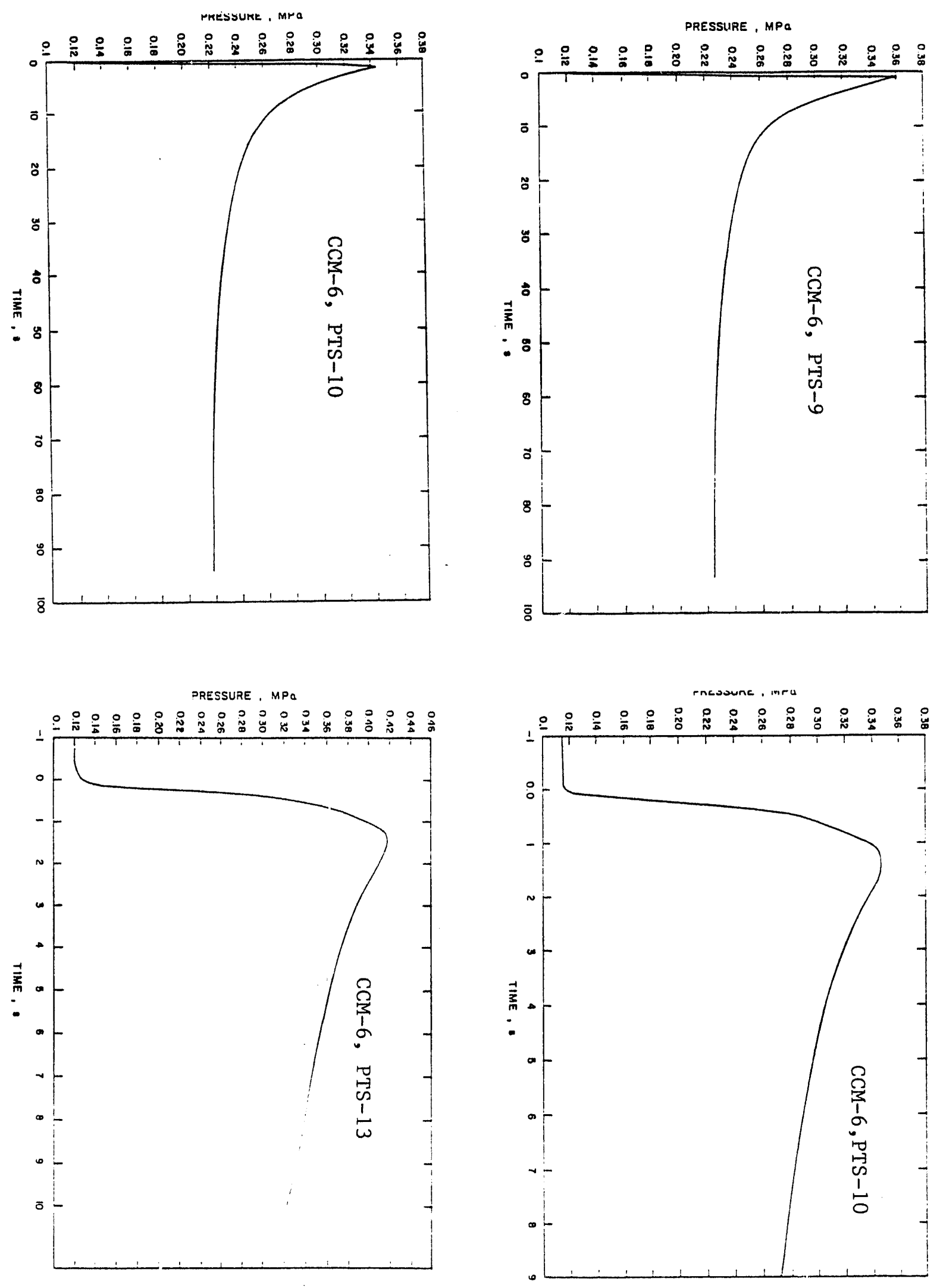

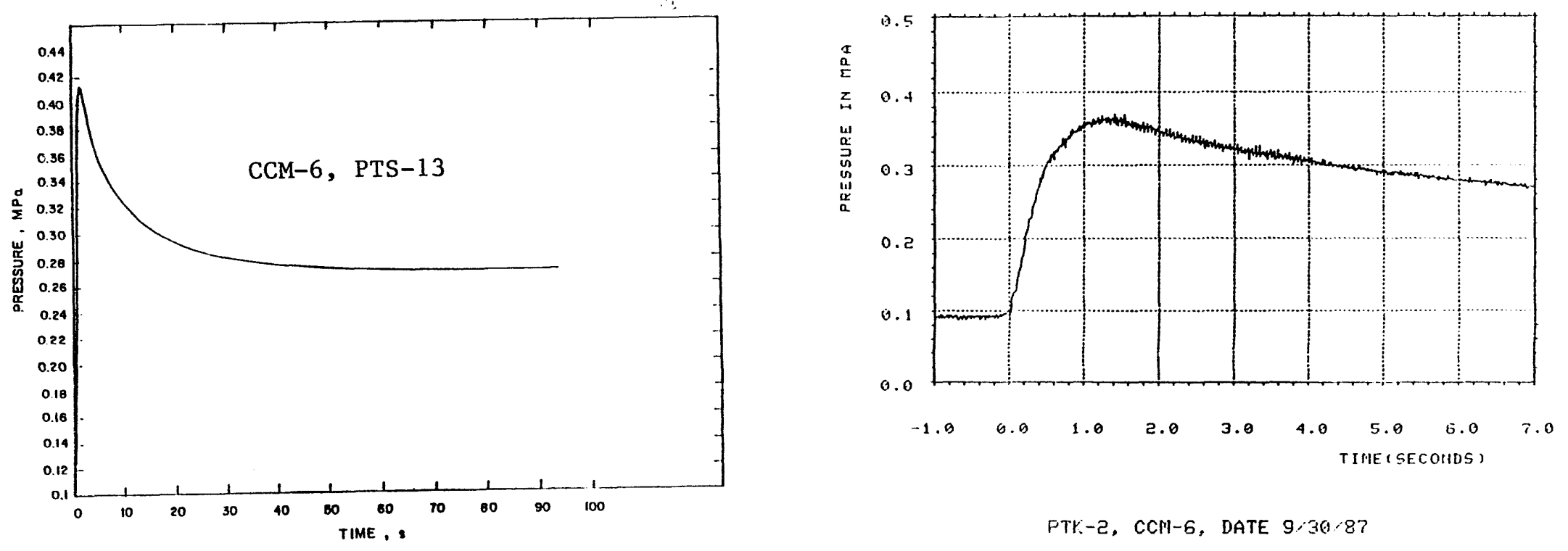

i⿱
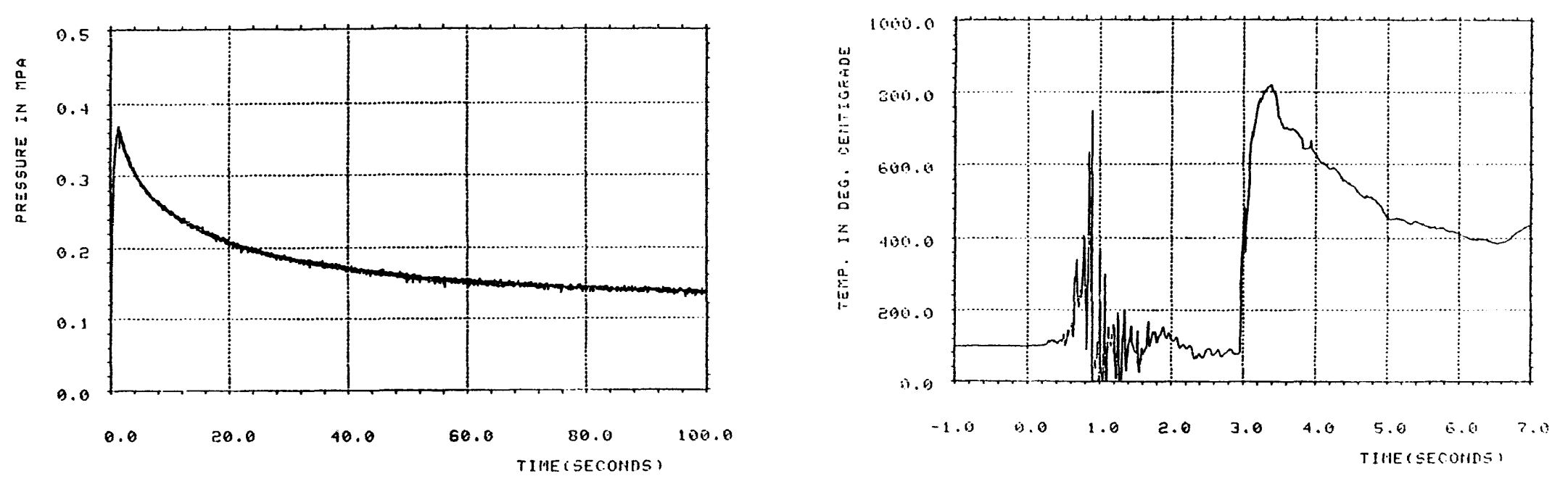

PTK-2, CCM-6, DATE $9 / 30 / 87$

TIE-16, ONHE, DATE 9.30 


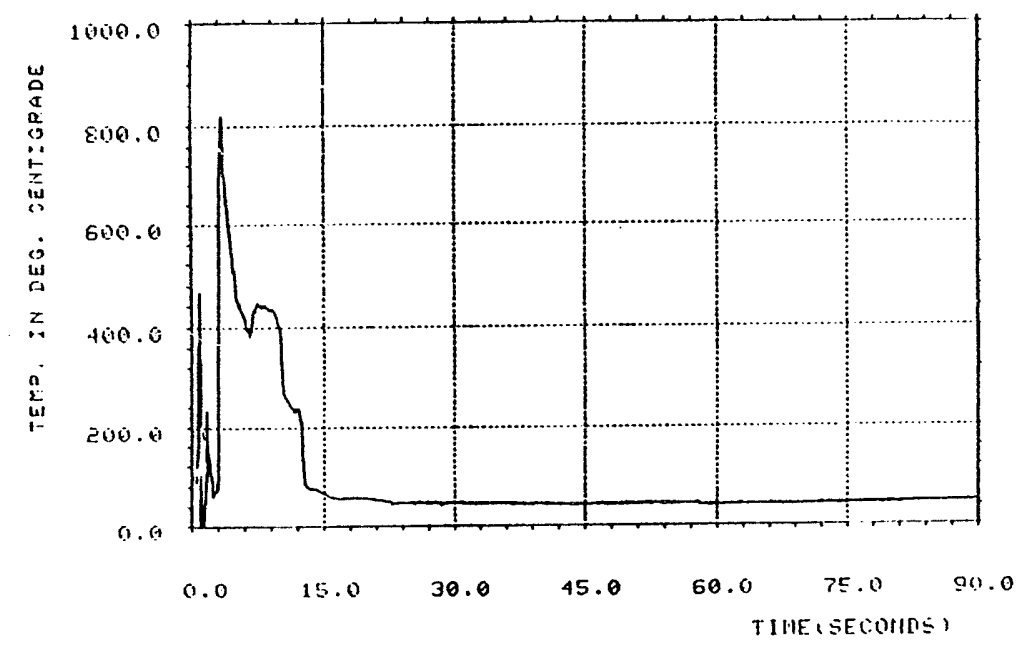

TI3-16. CCN-6, DATE $9,30,57$

$\dot{0}$

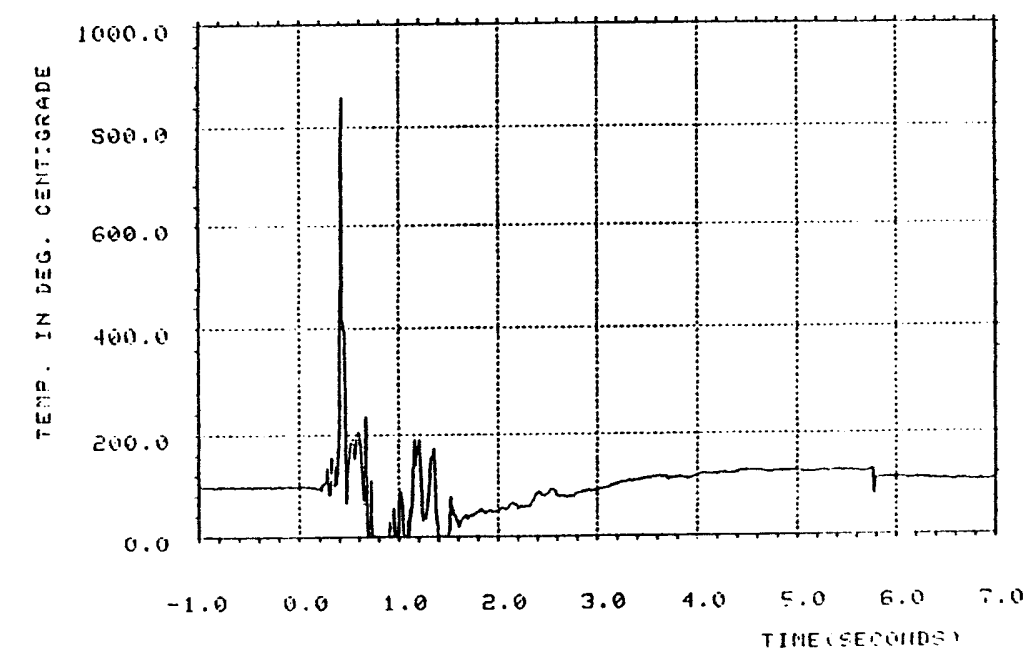

H.-13, CCH-6. [IATE 9.308

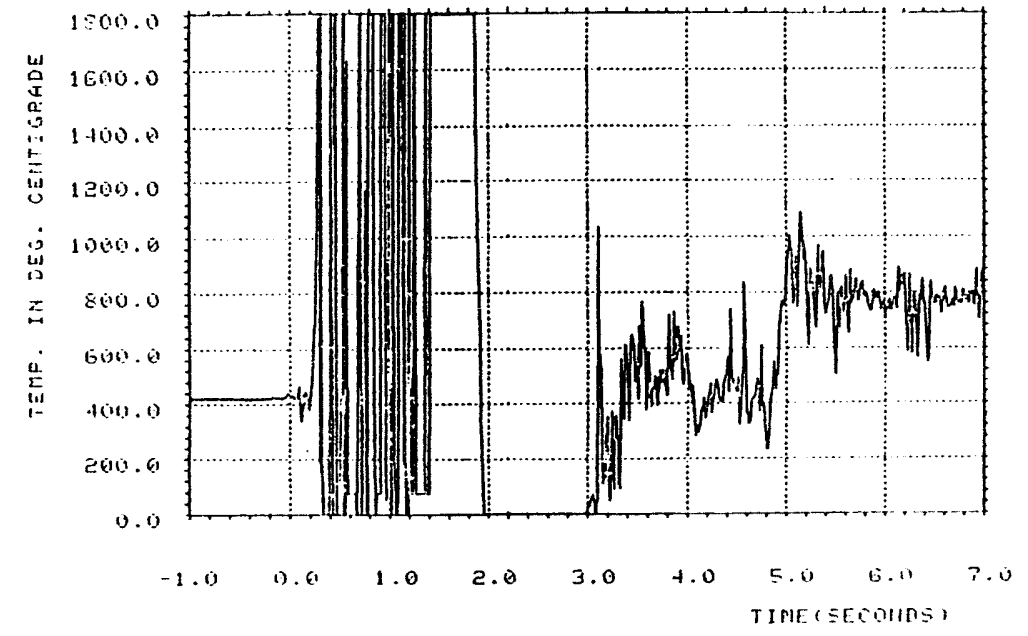

TIZ-17, CON-6, [RATE 9.S11:

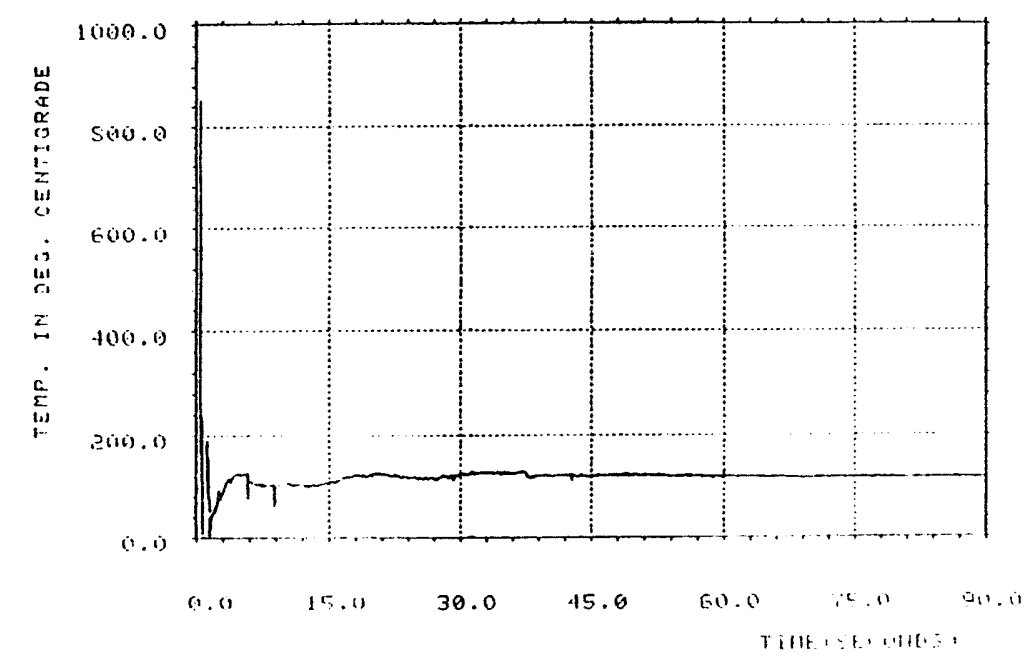

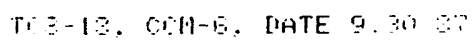




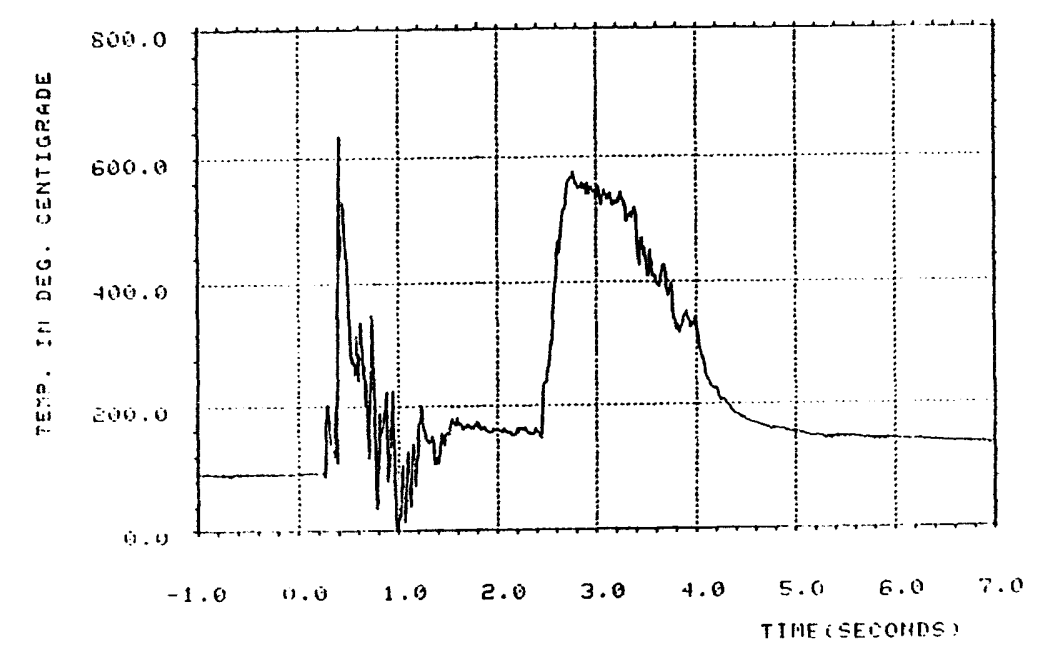

TI8-19, CCH-E, IATE 9,3087

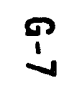

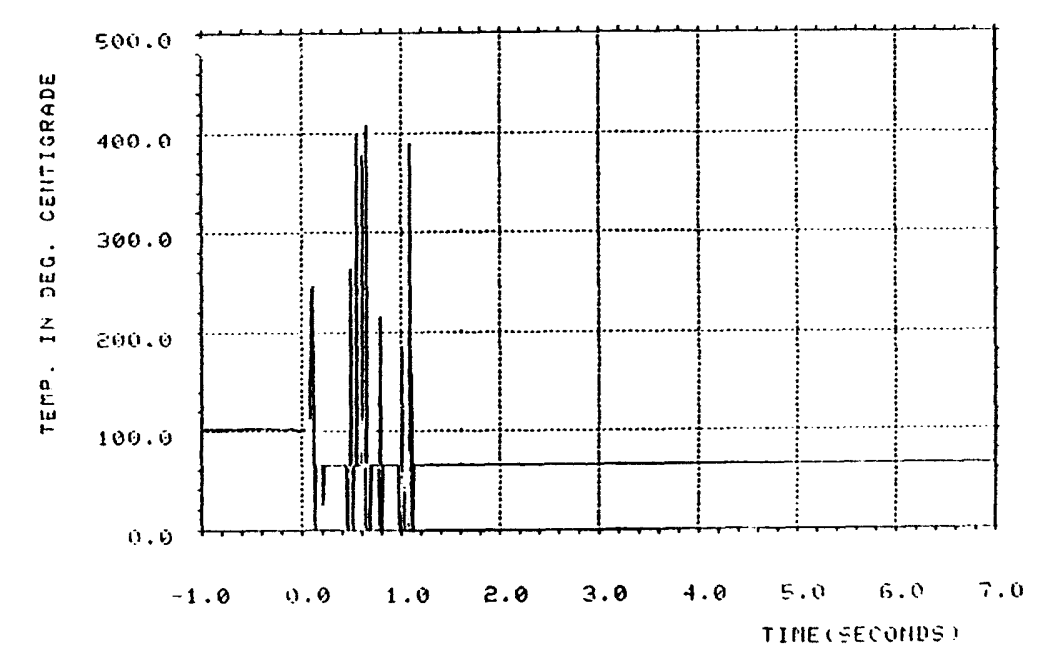

TIS-20, CCH-6, [HATE g. 30 ST

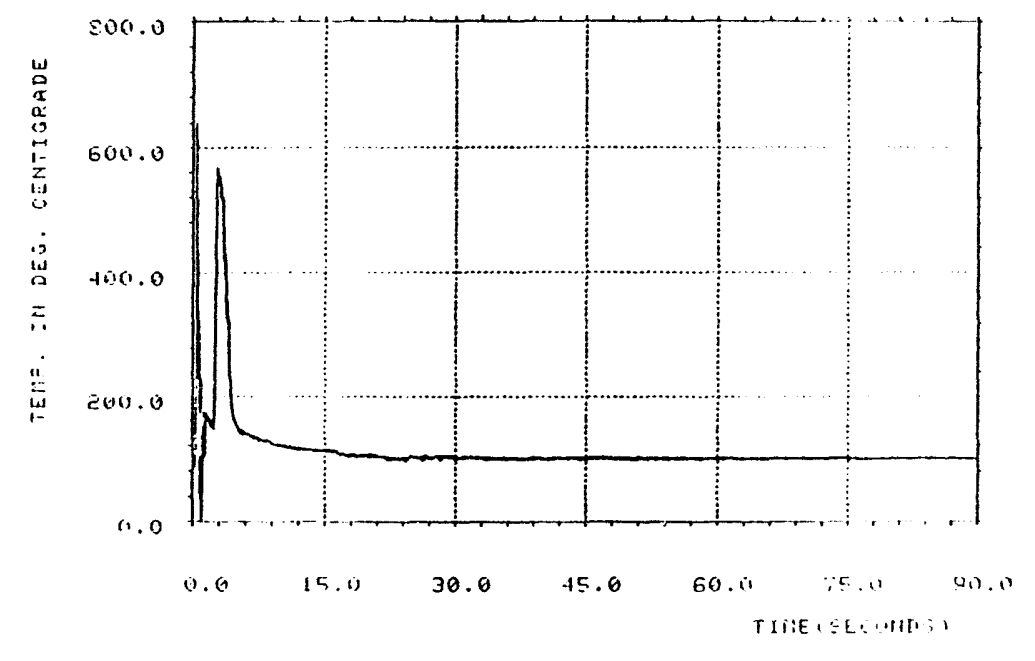

TIB-1?, CCN-6. [IATE 9.300

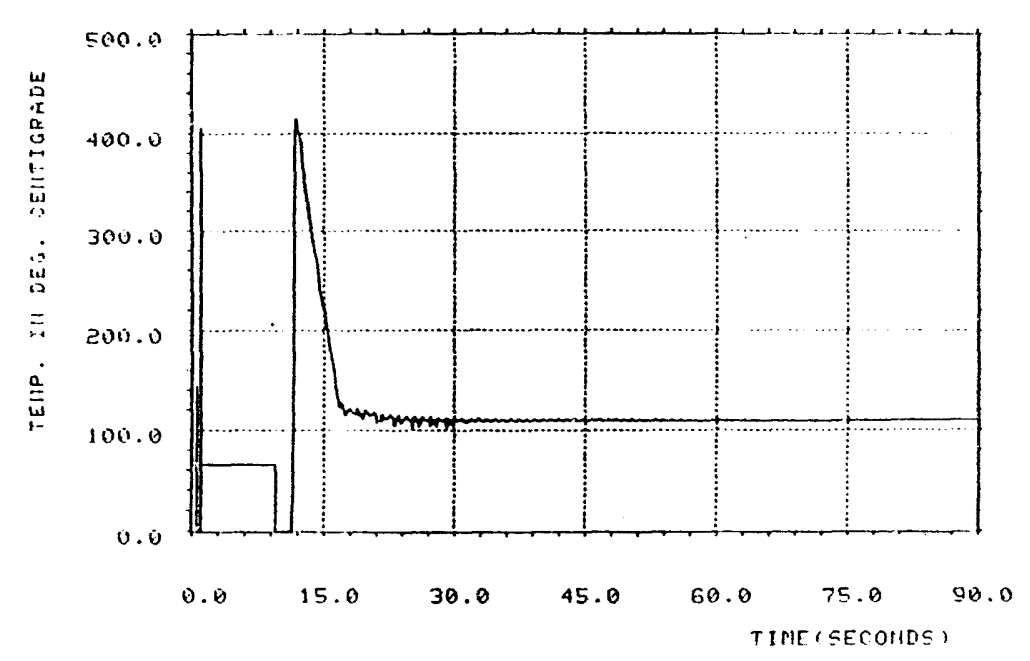

TIS 20, COI-E. LATE 9 30 


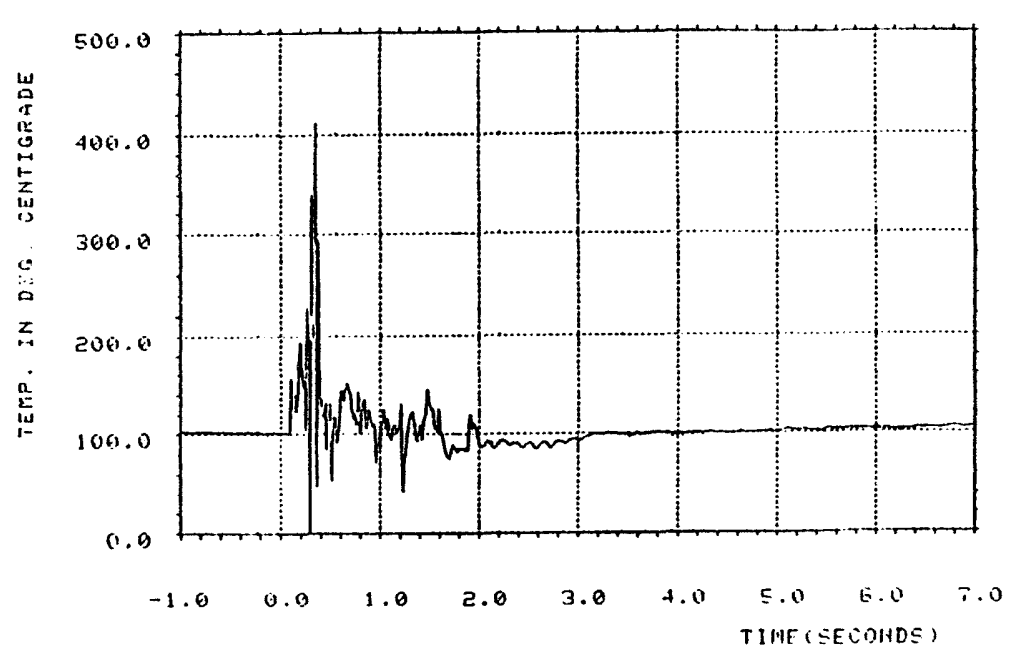

TIZ-21, CCM-5, DATE $O, 30: 5$

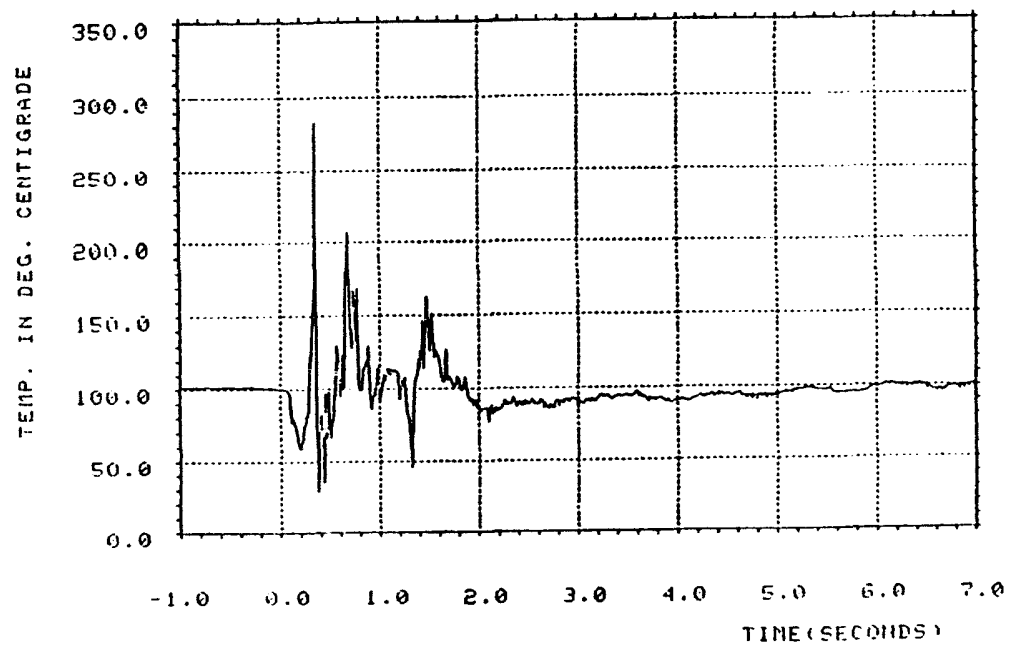

Trs-EZ, CON-6, DATE $9,30 \mathrm{sT}$

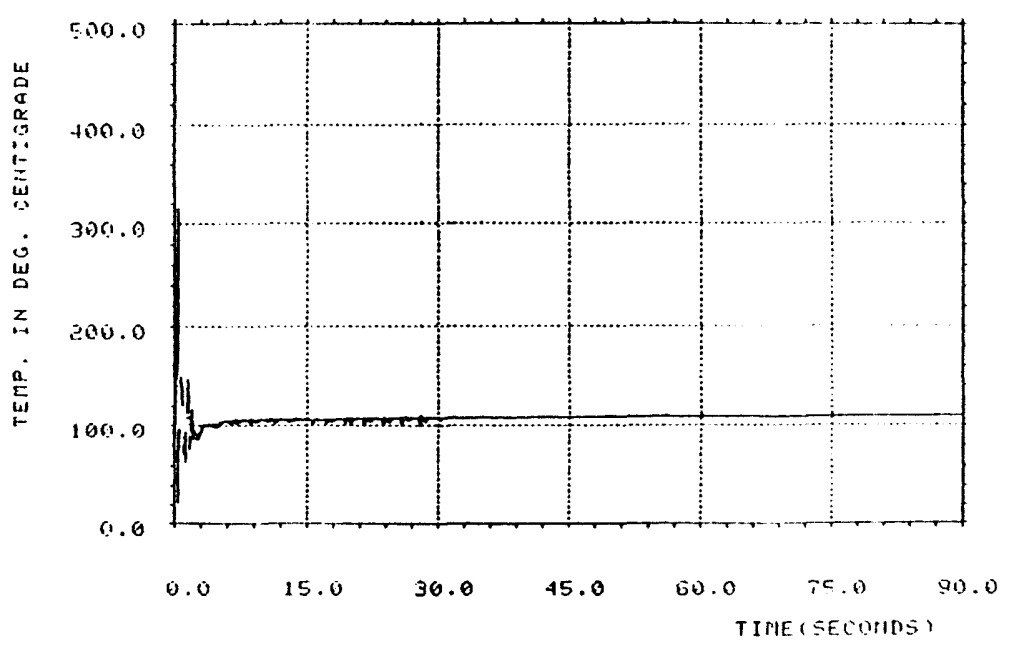

Tr $8-21$, CCM-6, DATE $9 \cdot 3028$

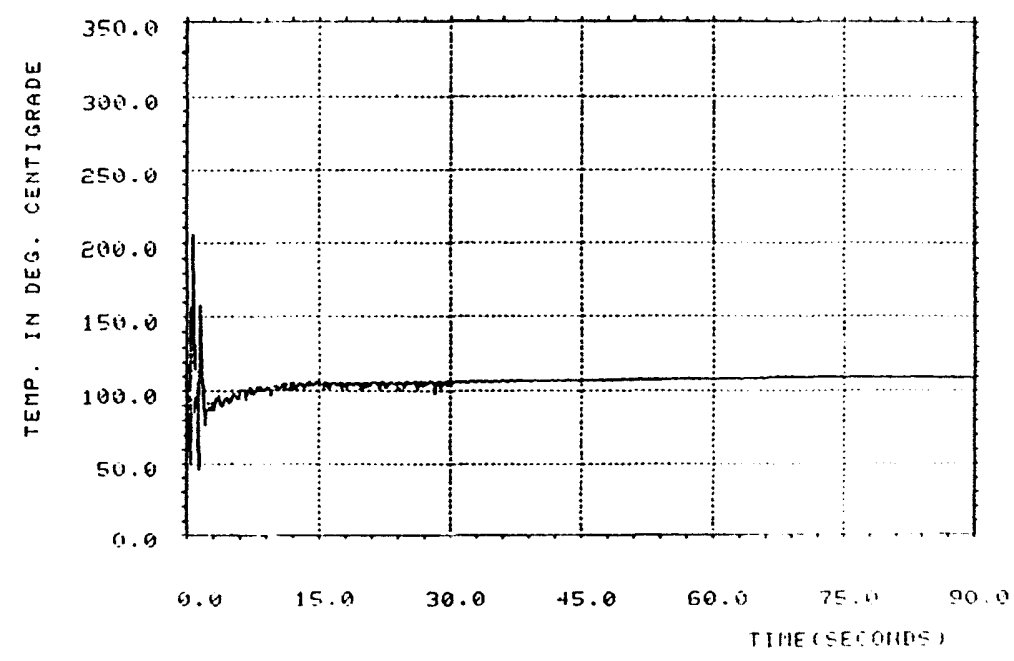

TIZ-Ẽ:2, CN-G. THATE $0.30 \%$ 


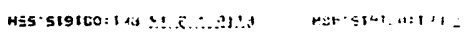

$\therefore \therefore \quad 0 \quad 314[\cdot 9-1.100 \cdot+2-3.11$

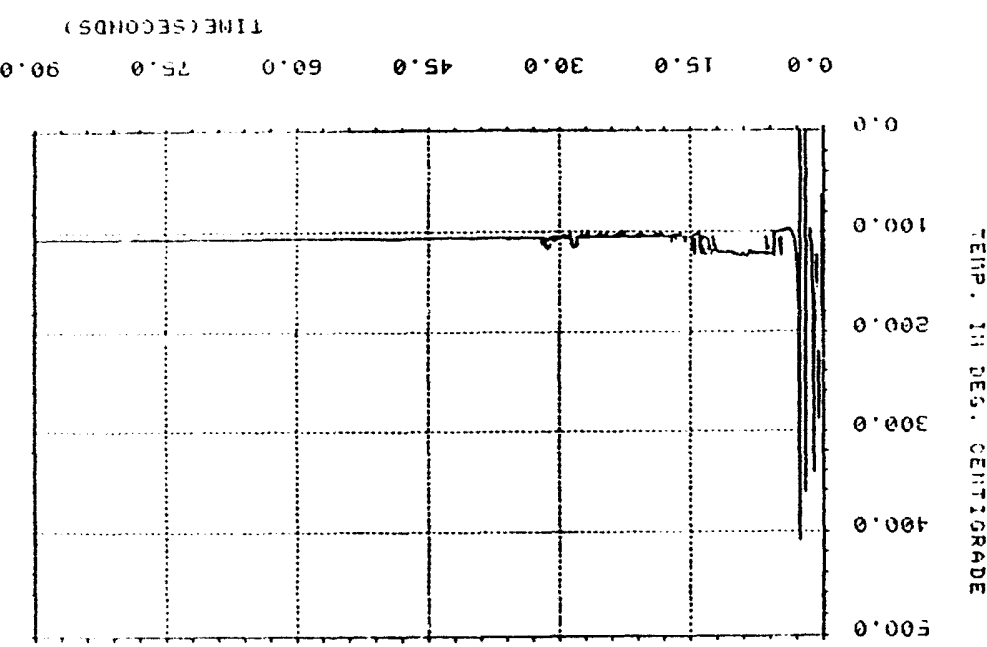

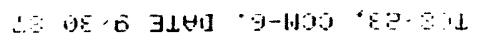

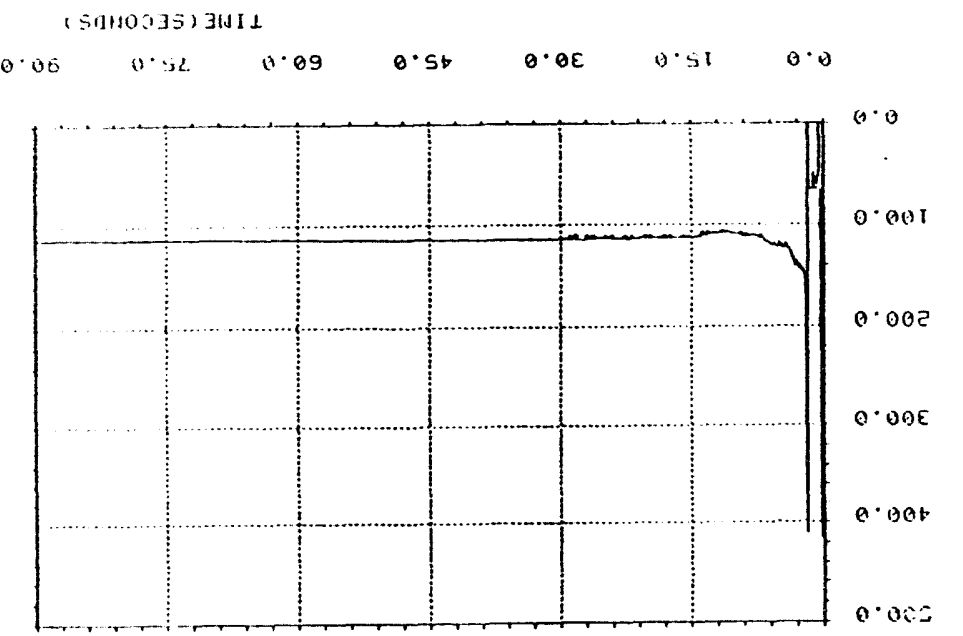

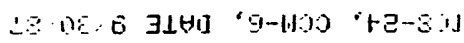

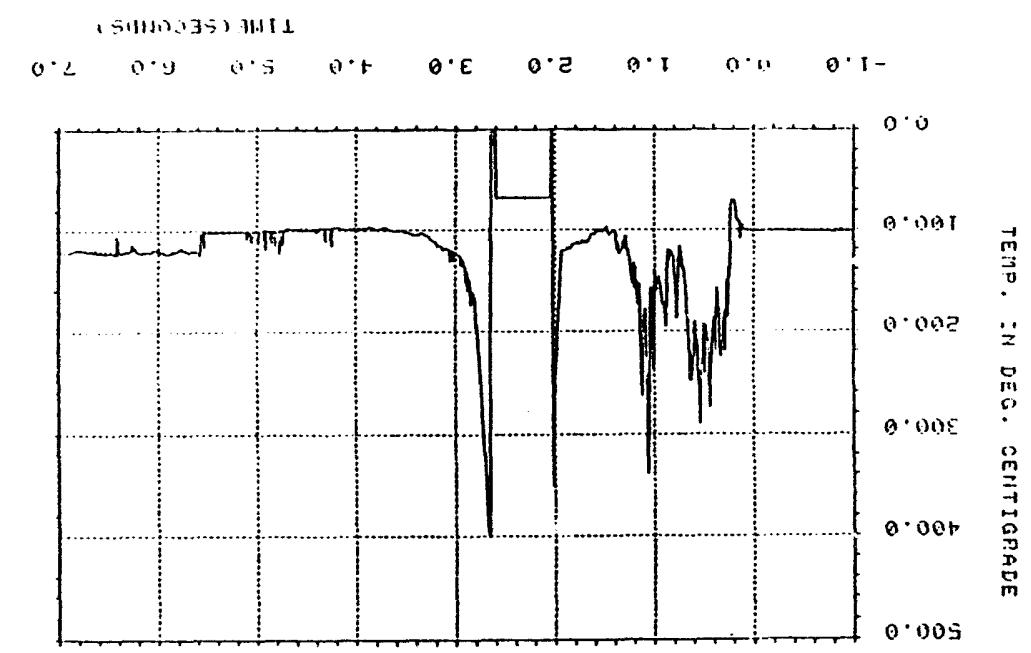

ì
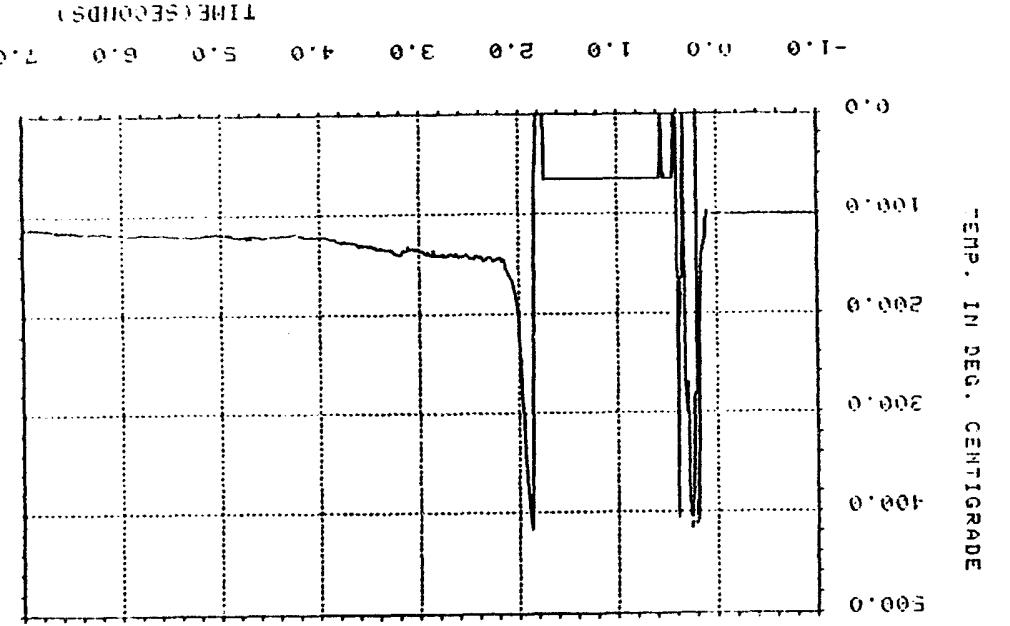


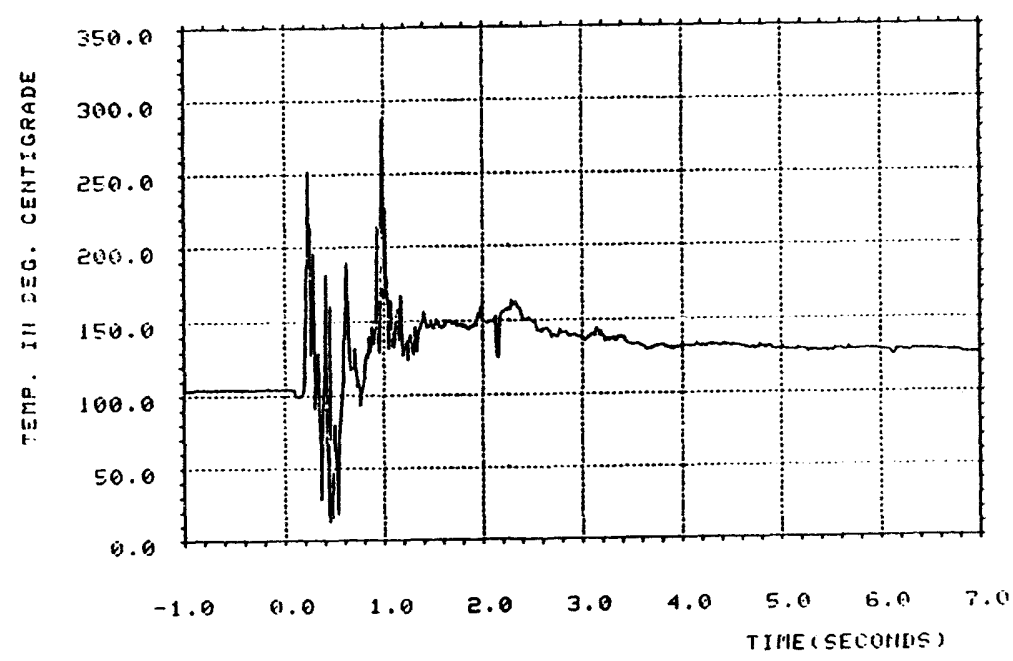

$\stackrel{9}{\circ}$

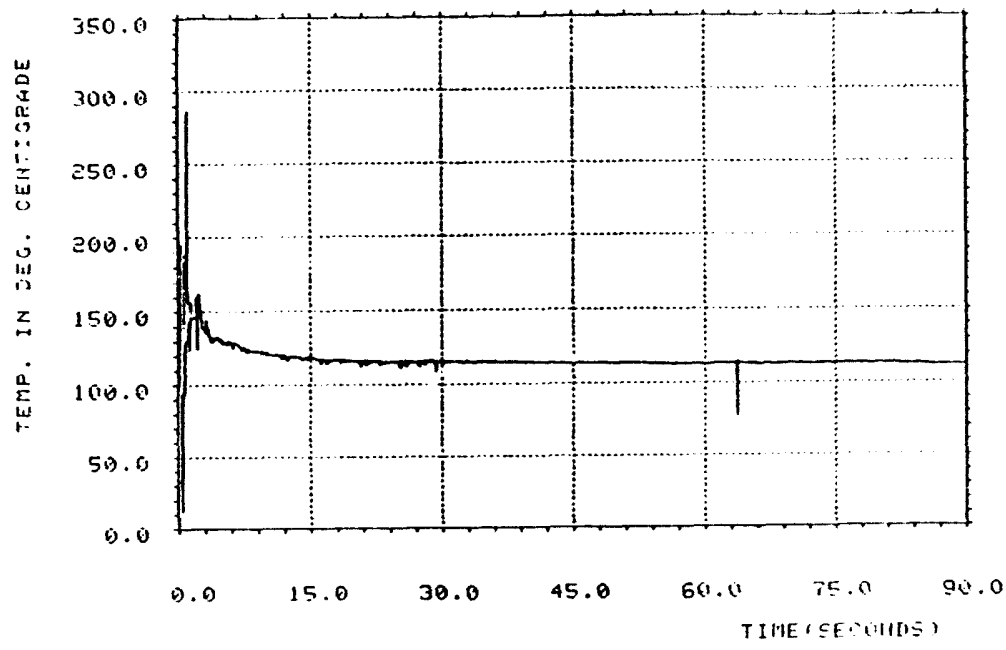

THE-25, COM-5, [IATE $9.30 \cdot 8 \%$

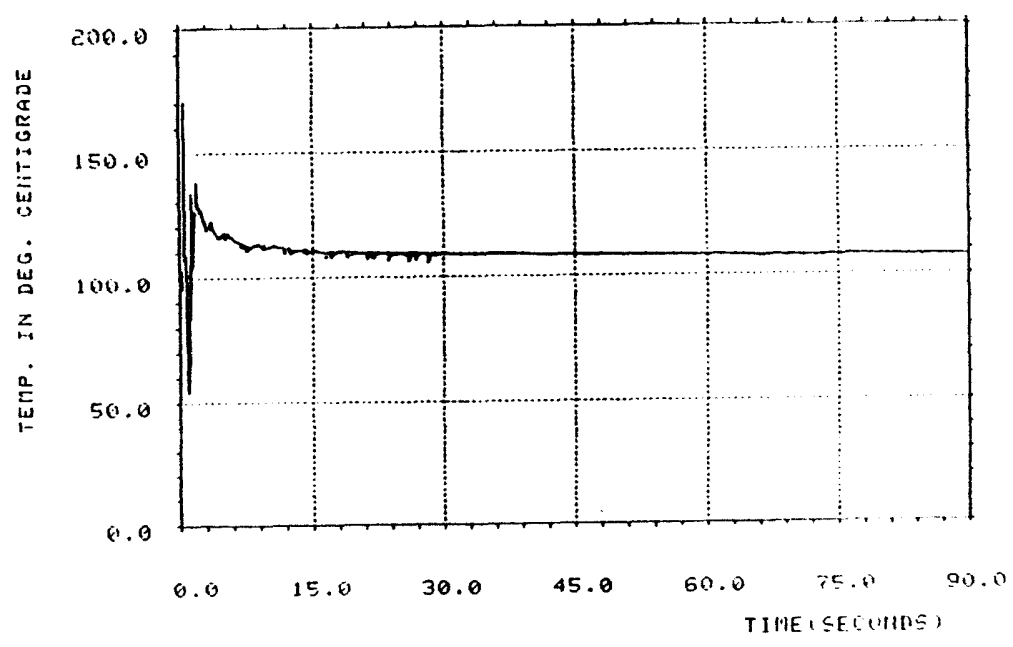

Tis-ż?, CON-6, RATE Q, SO 


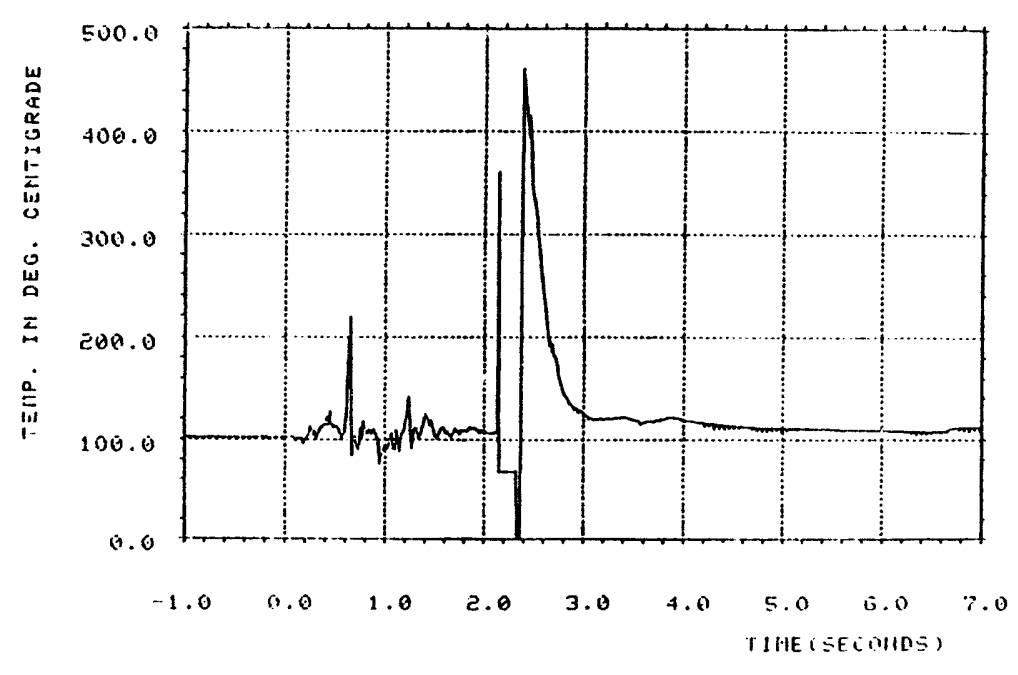

$\stackrel{9}{\vdots}$

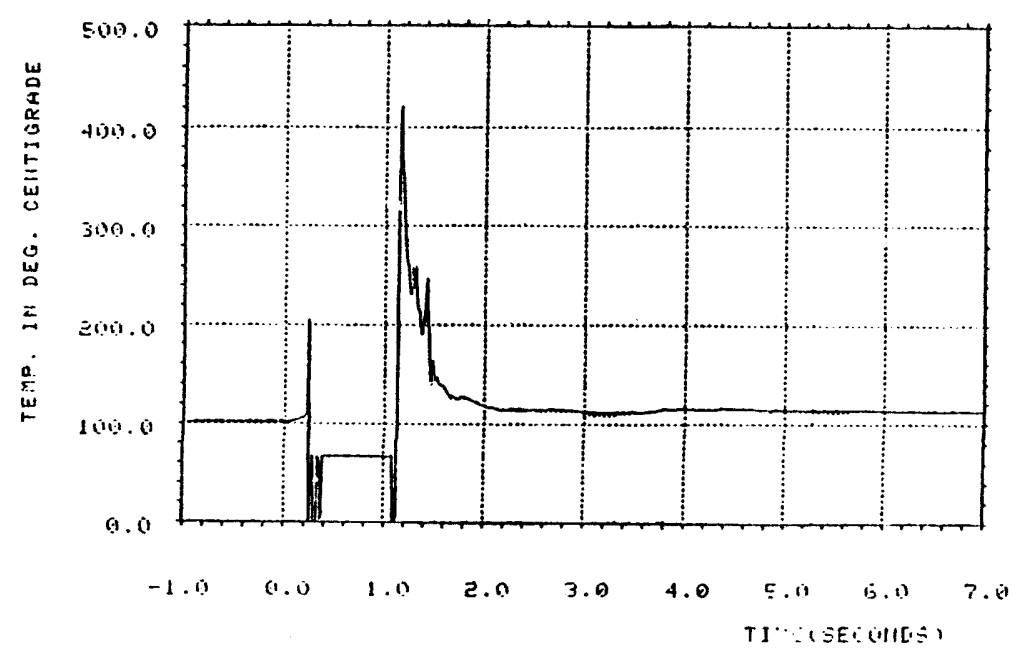

THE-Eด, COH-E, LATE 9 30.07

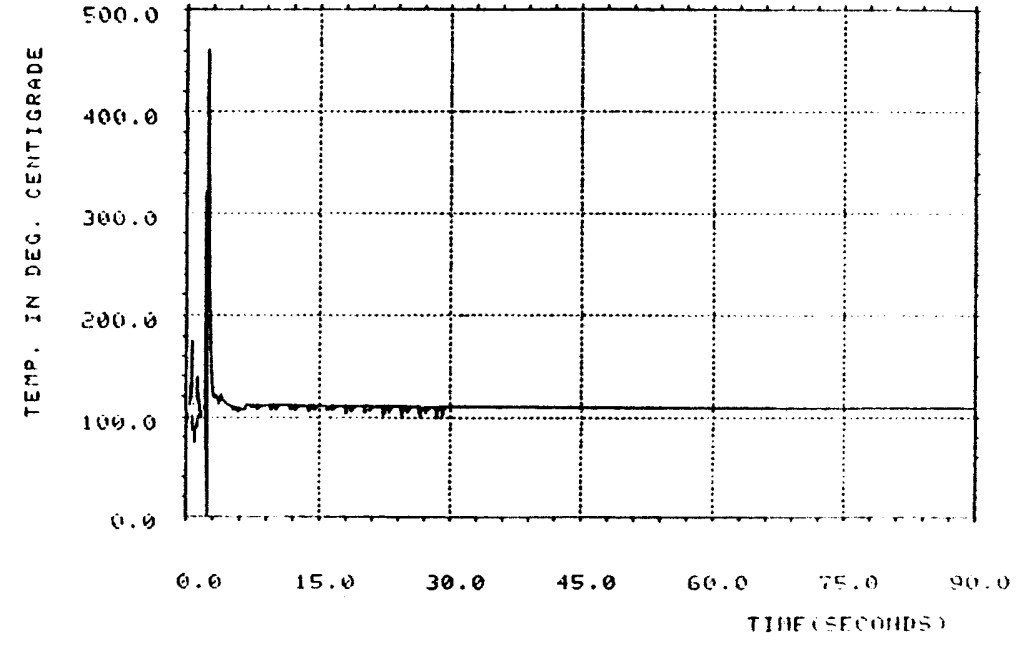

Tre-es, cCh-s, LATE a

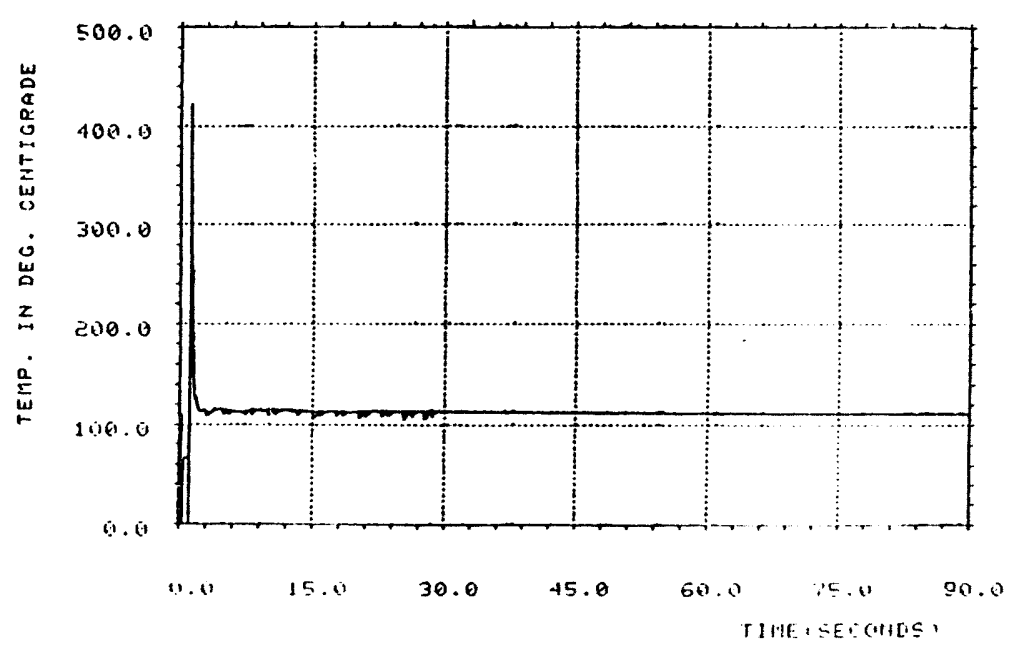

TH-2?, CH-E, DATE 9.30 


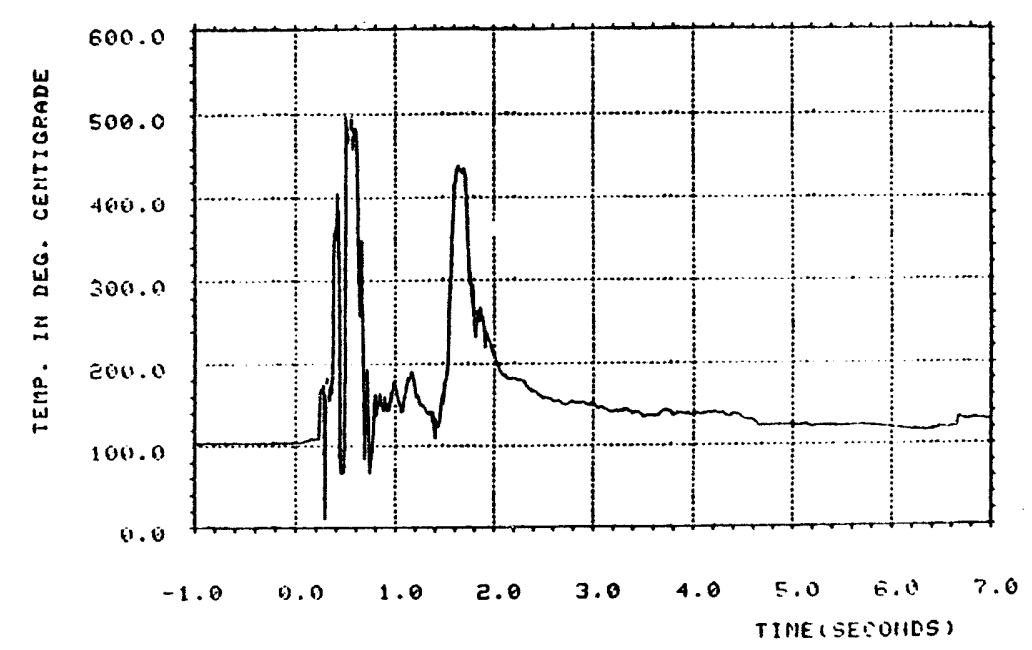

$\stackrel{i}{\sim}$

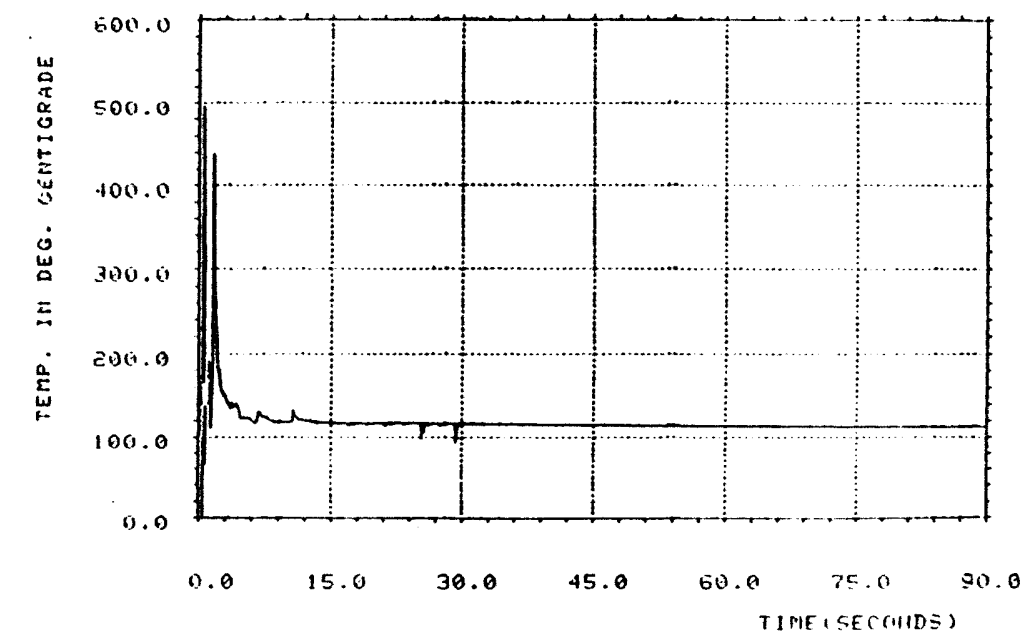

r. $3-30$, cCH-6. DATE Q 30.27

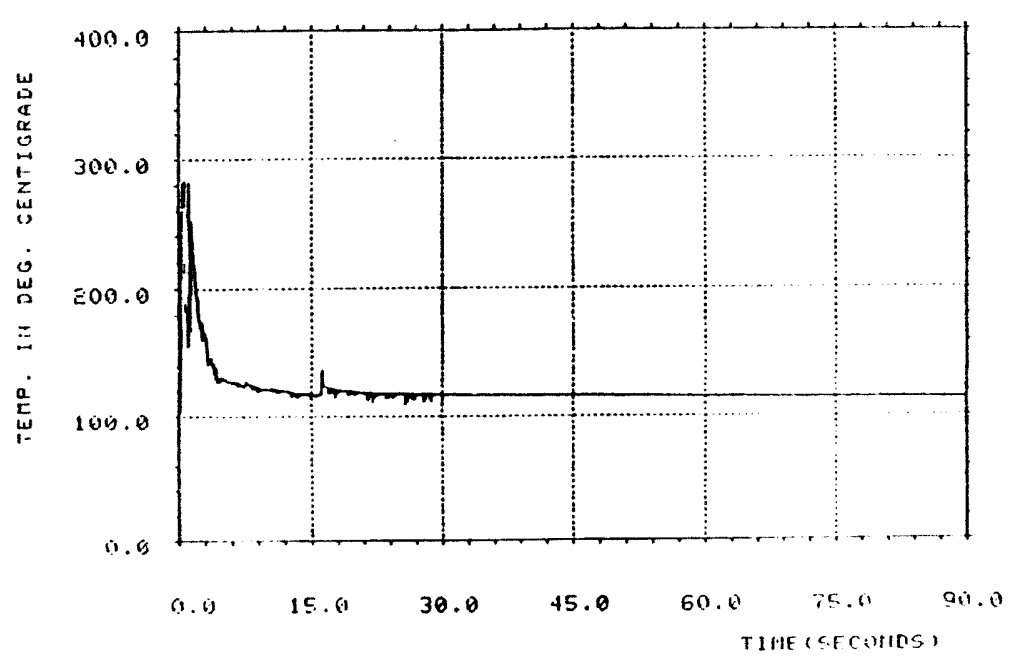

TIE-31. CCPIE. DHTE 9 30 


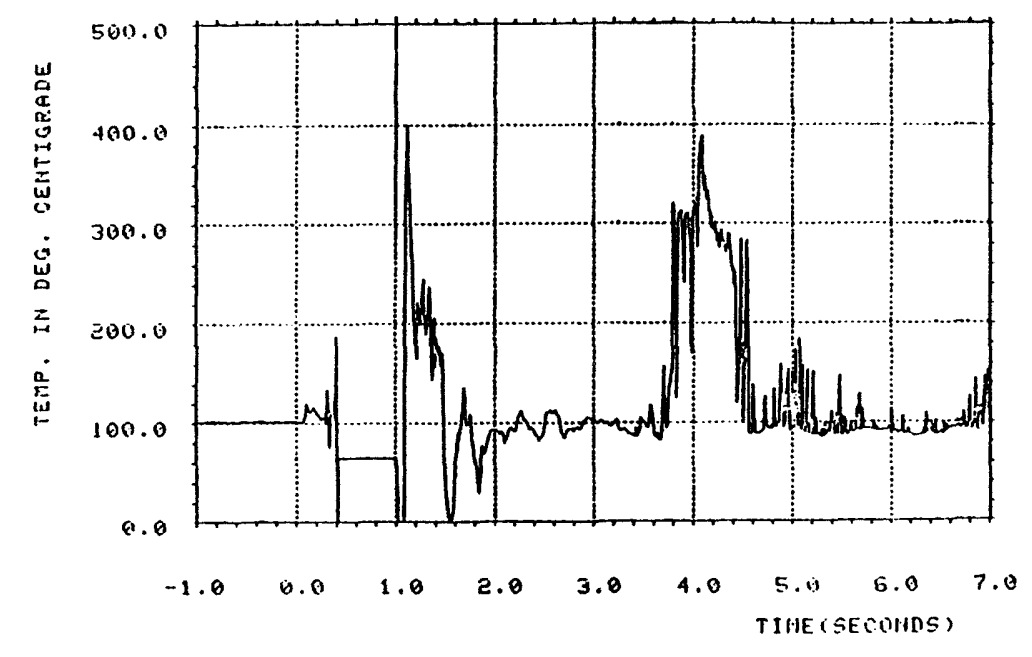

完

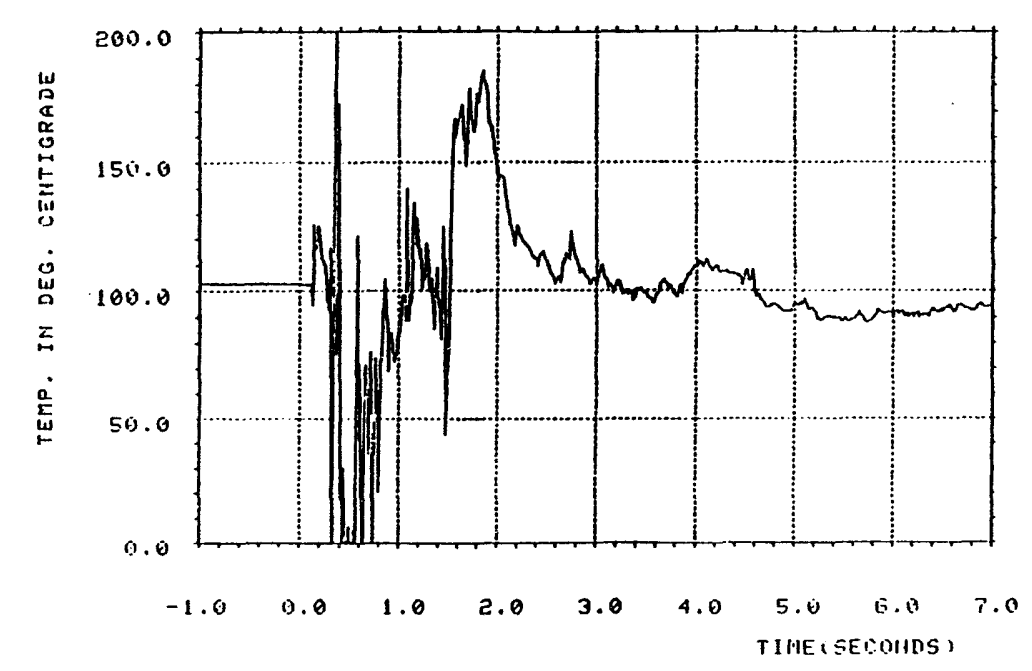

TC8-33, CCH-6, DATE 9.30 .87

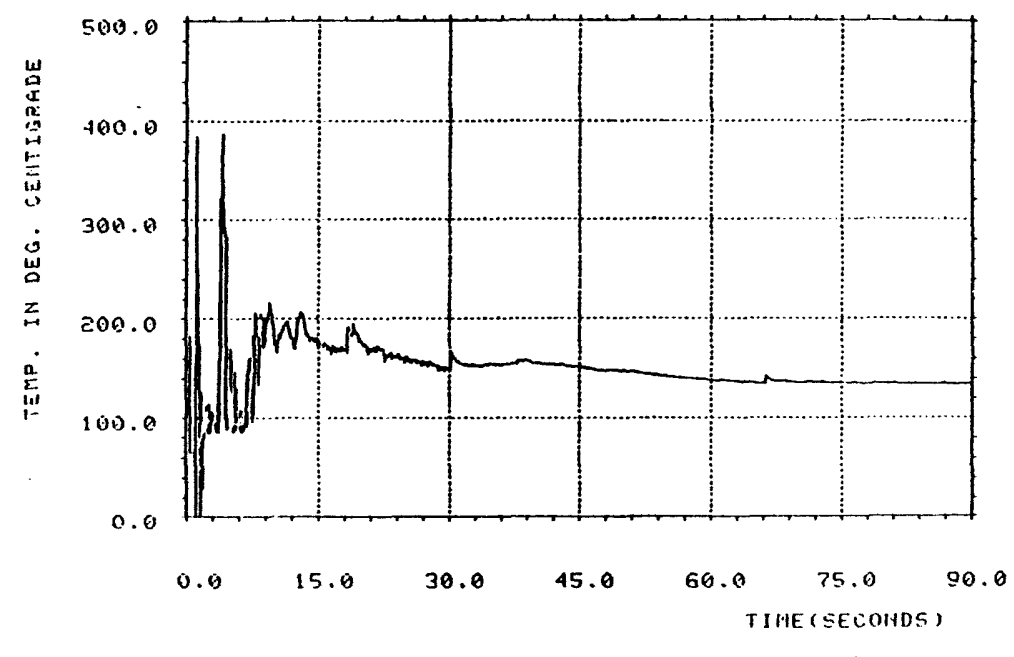

TCE-32, CON-6, IDATE $930 \%$

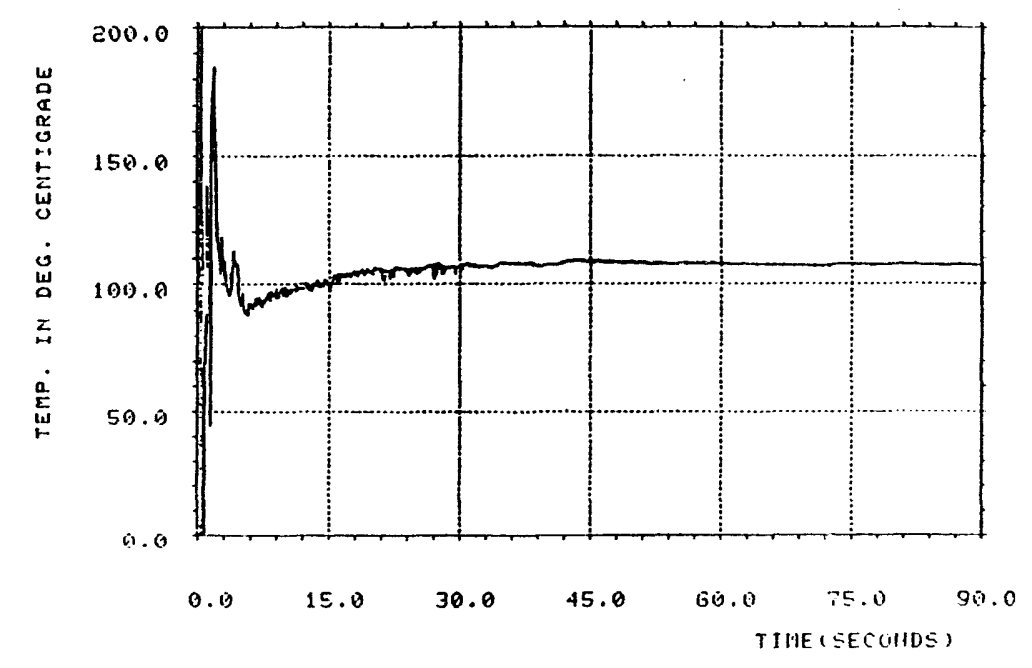

TIS-33, CON-6, LIATE 9 30 


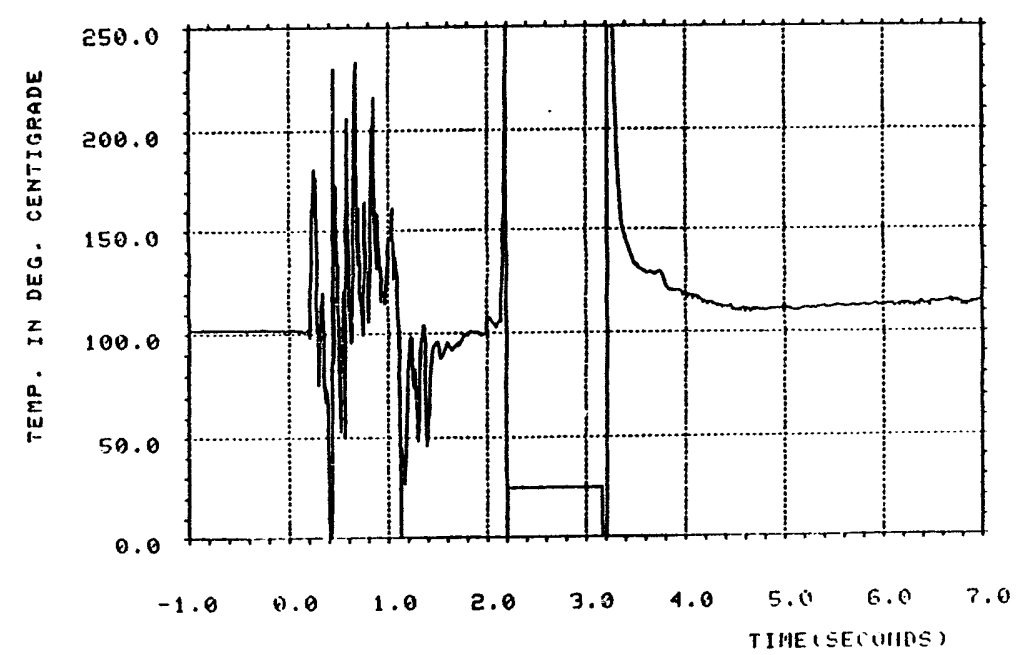

$\stackrel{9}{\ddagger}$

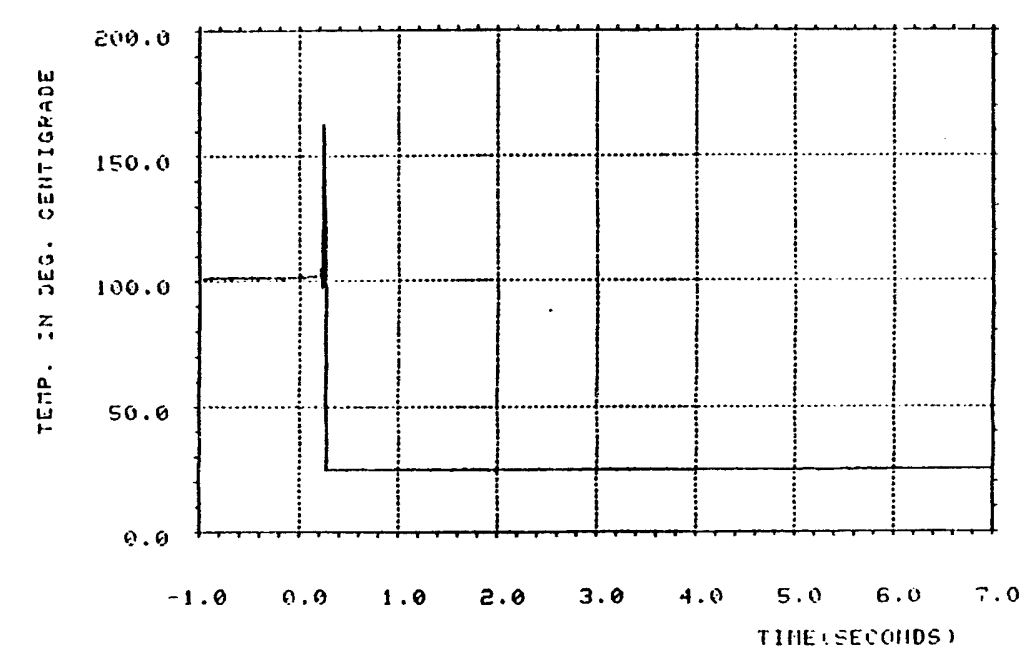

TrS-39, CCH-6, DATE $9: 30 \cdot 87$

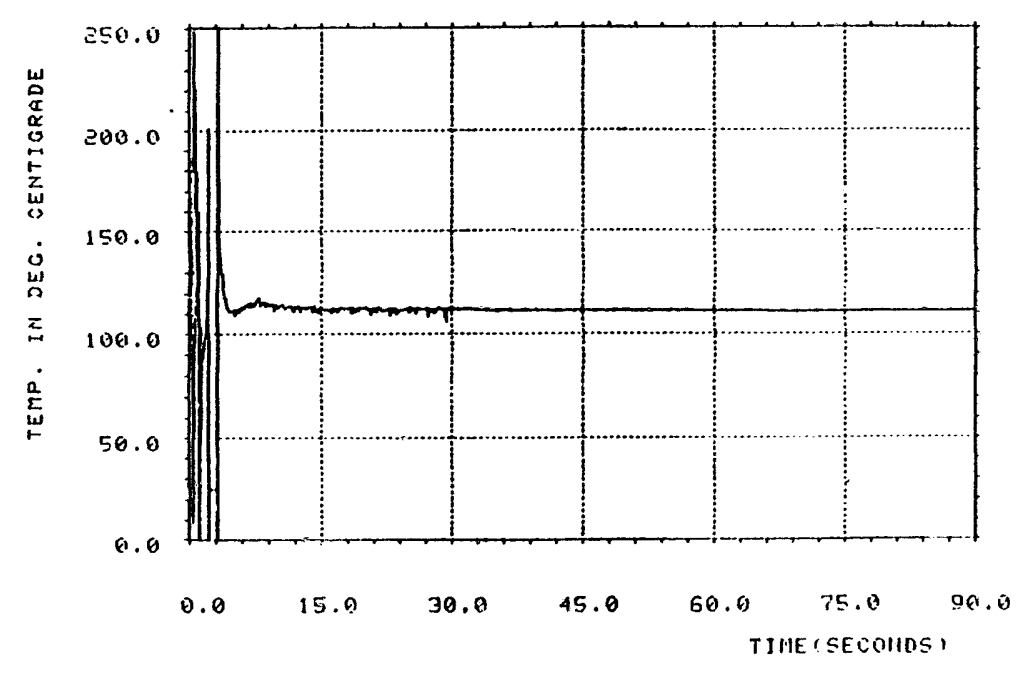

TOB-37, CON-6, DATE 9/30.57

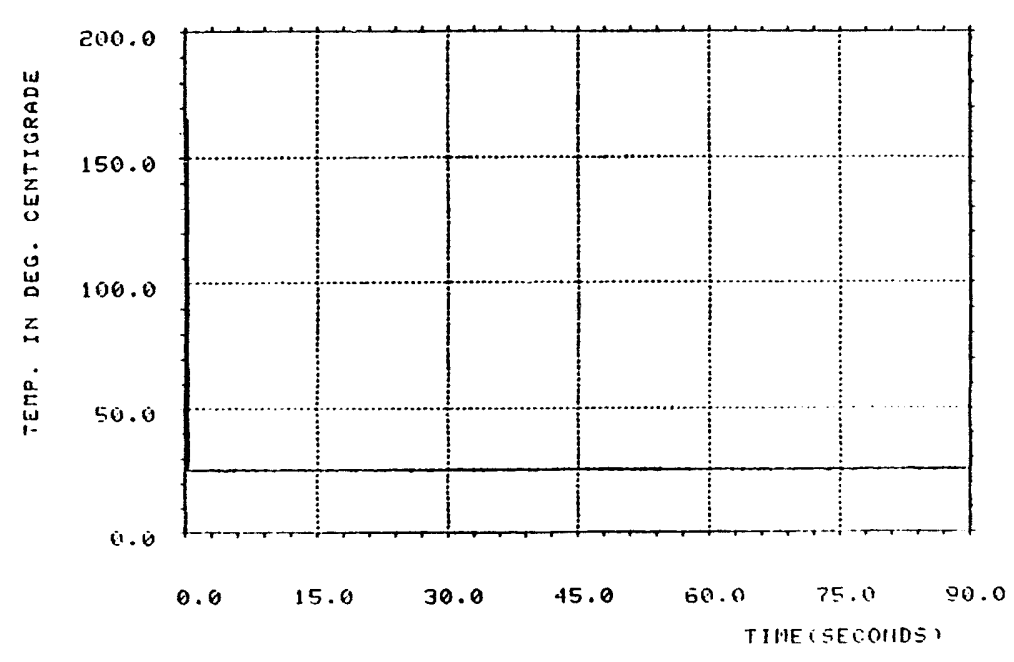

Tr.3-39, CCH-6. DATE $9 / 30.87$ 


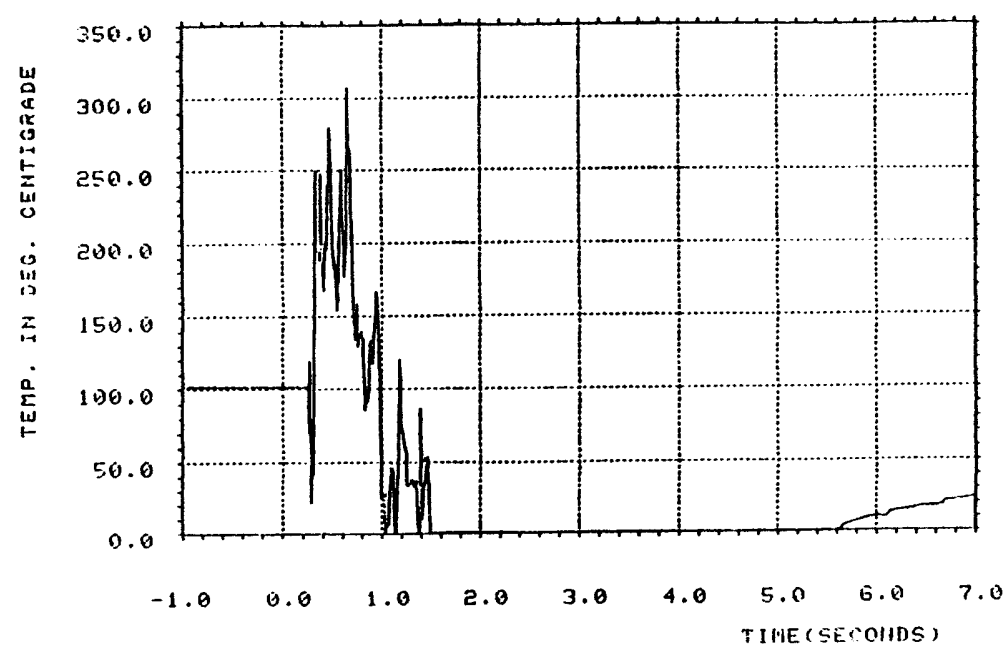

号

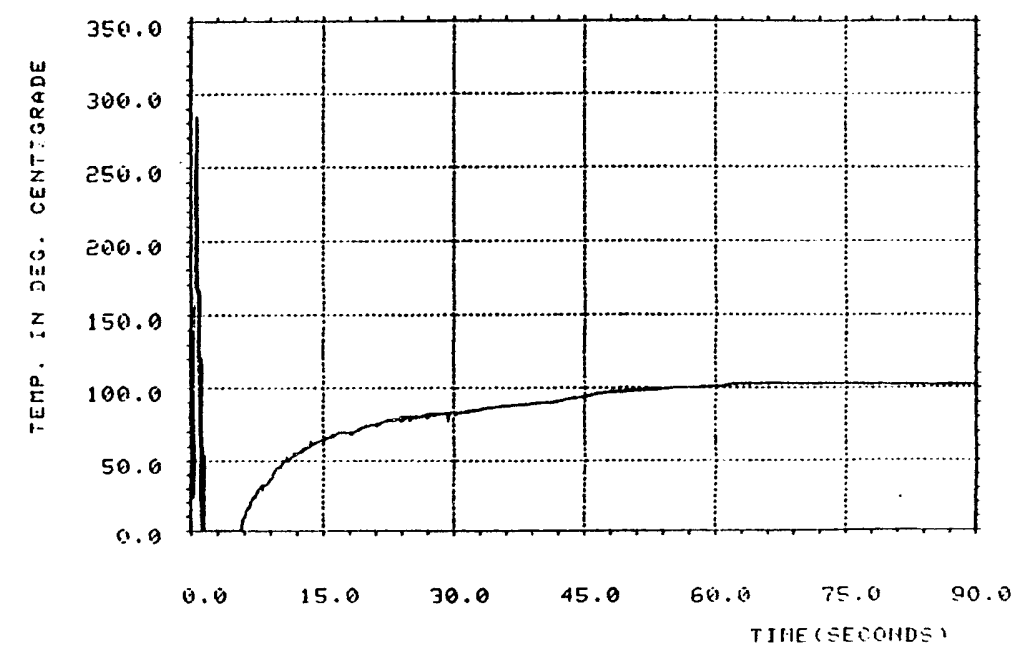

Tr.8-43, CCI1-6, DATE 9,30.8?

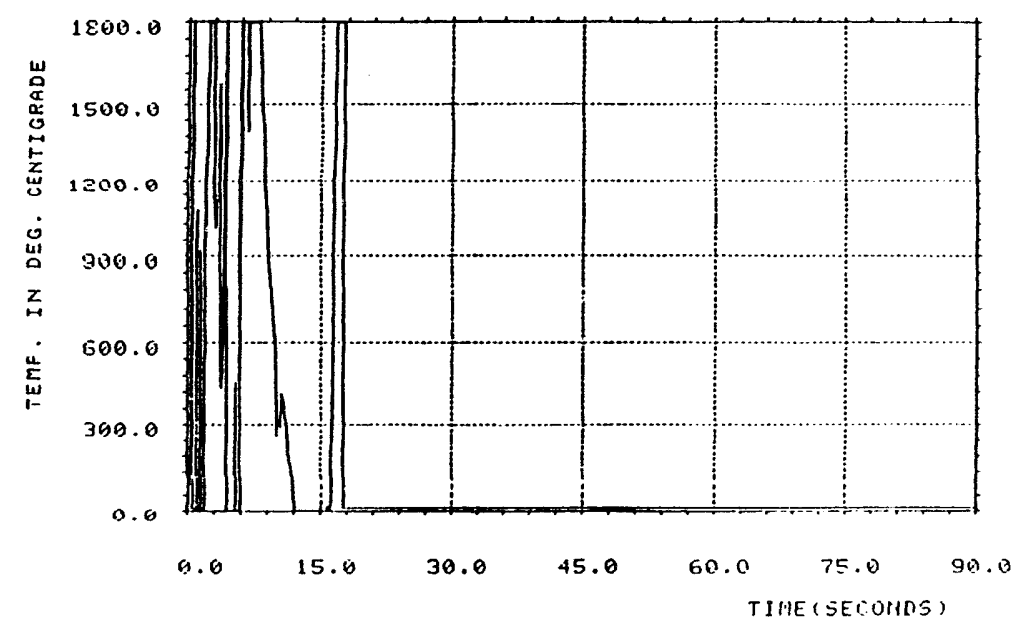

TO9-51, CCIM-6, DATE $9,30,37$

Trg-51, cCM-6, JATE gisa, 37

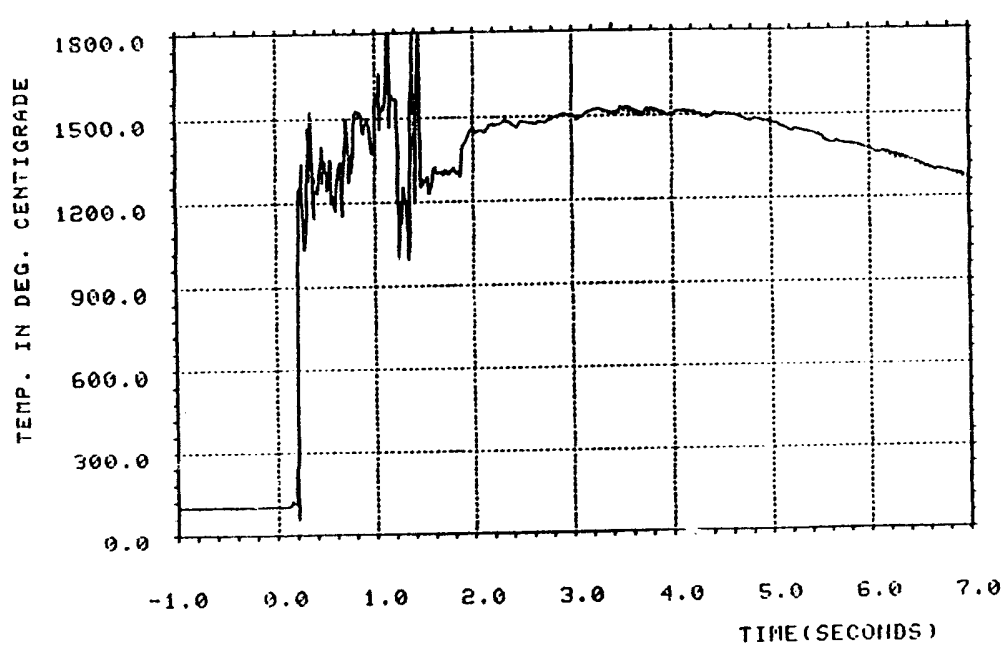

TC3-51, CIN-6, DATE $9 / 30.87$ 


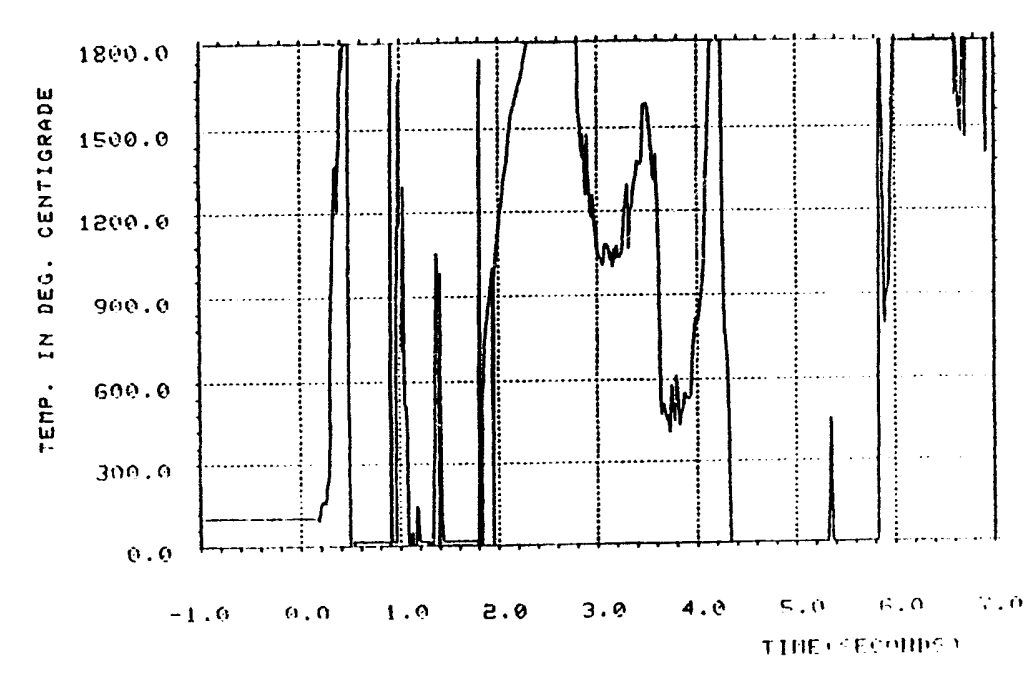

$\stackrel{8}{\dot{\omega}}$

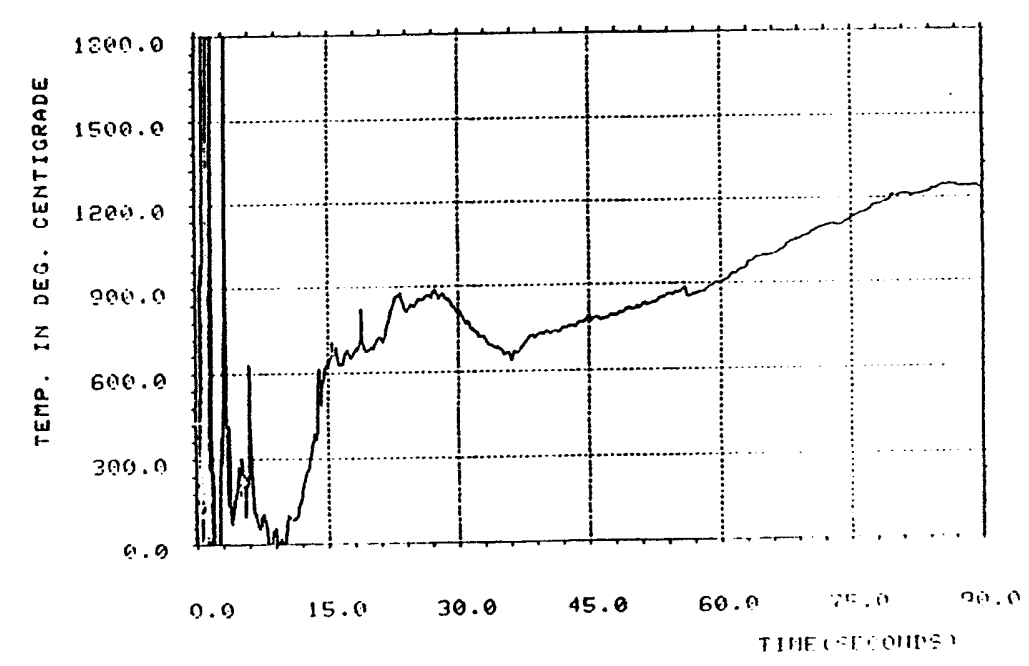

TrE-5.5. COA-6. DATE 0.30

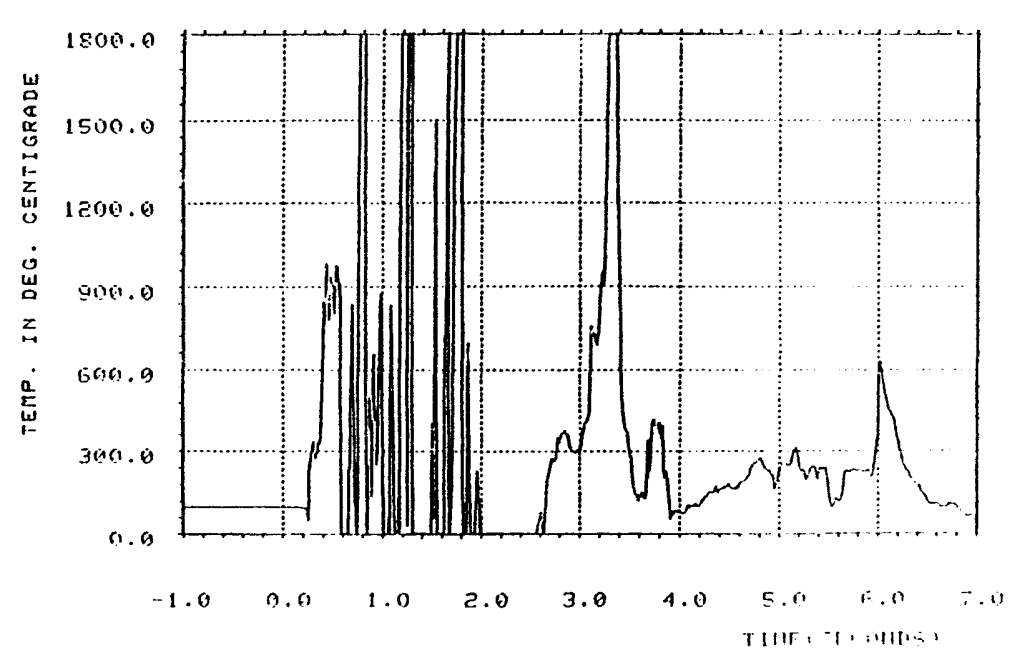

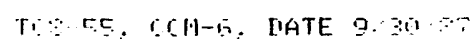

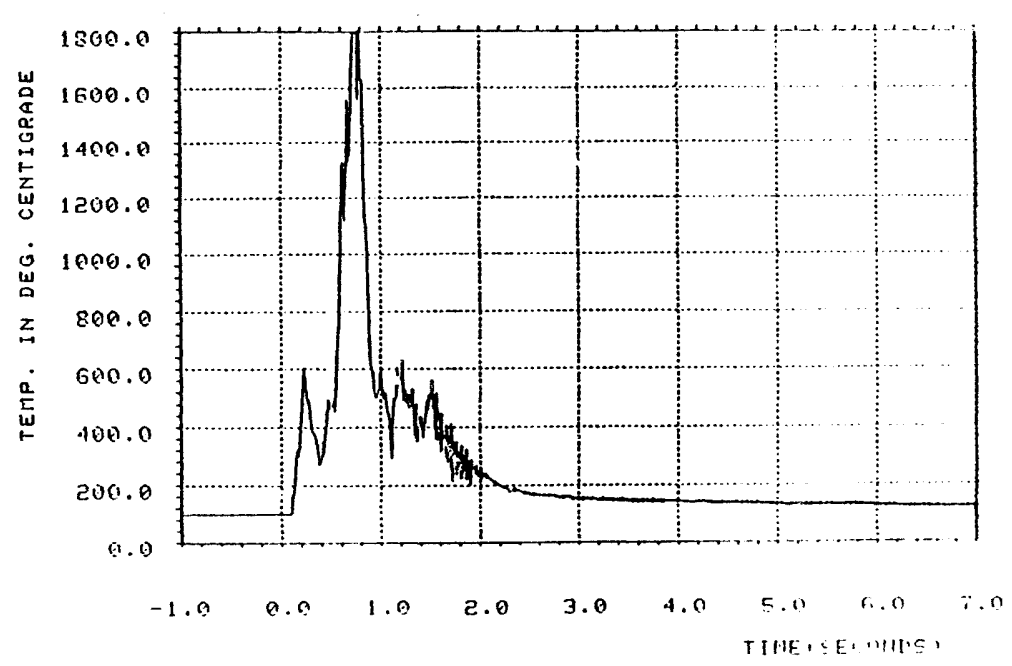

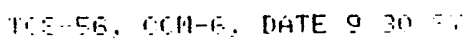



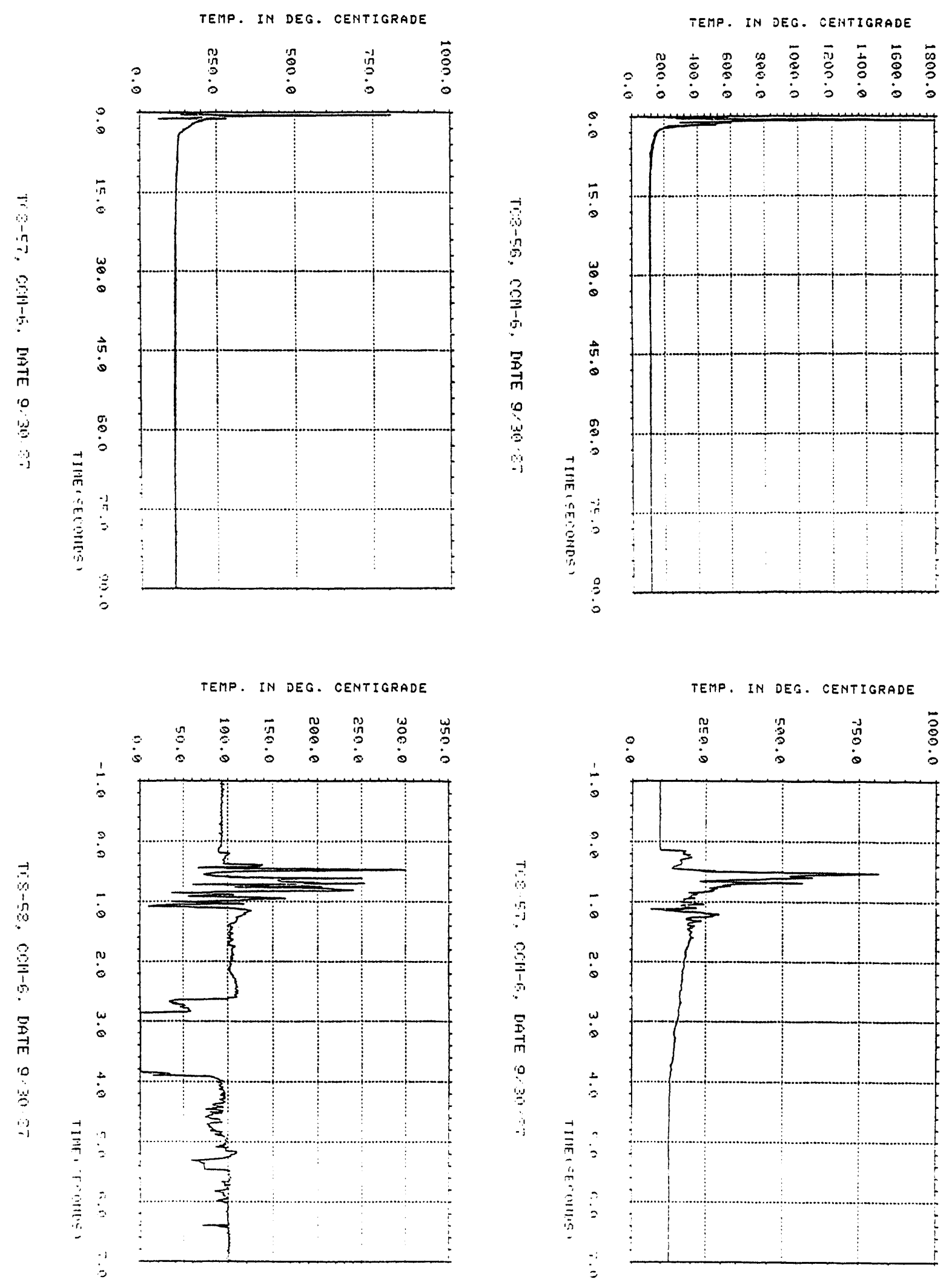


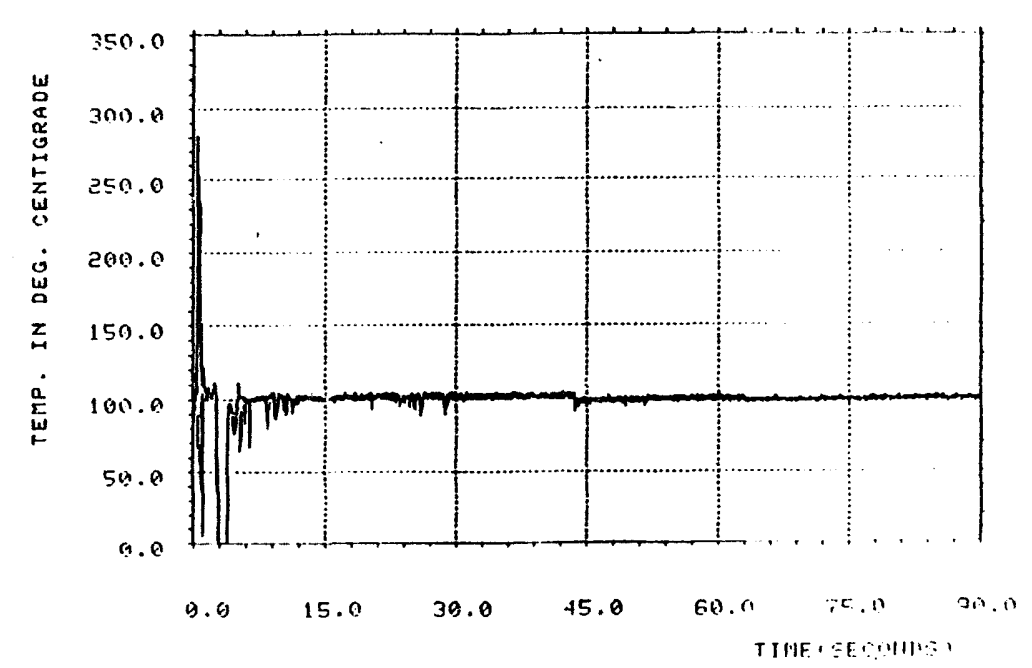

$\frac{P}{\dot{\omega}}$

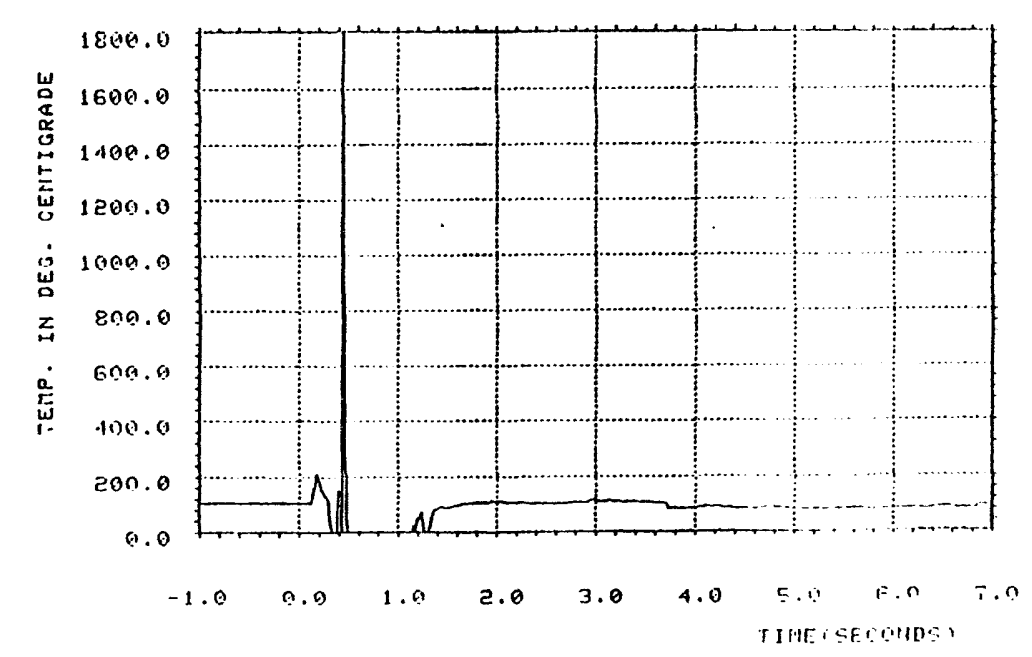

TOS-60. CON-E. DATE $9,30,0$

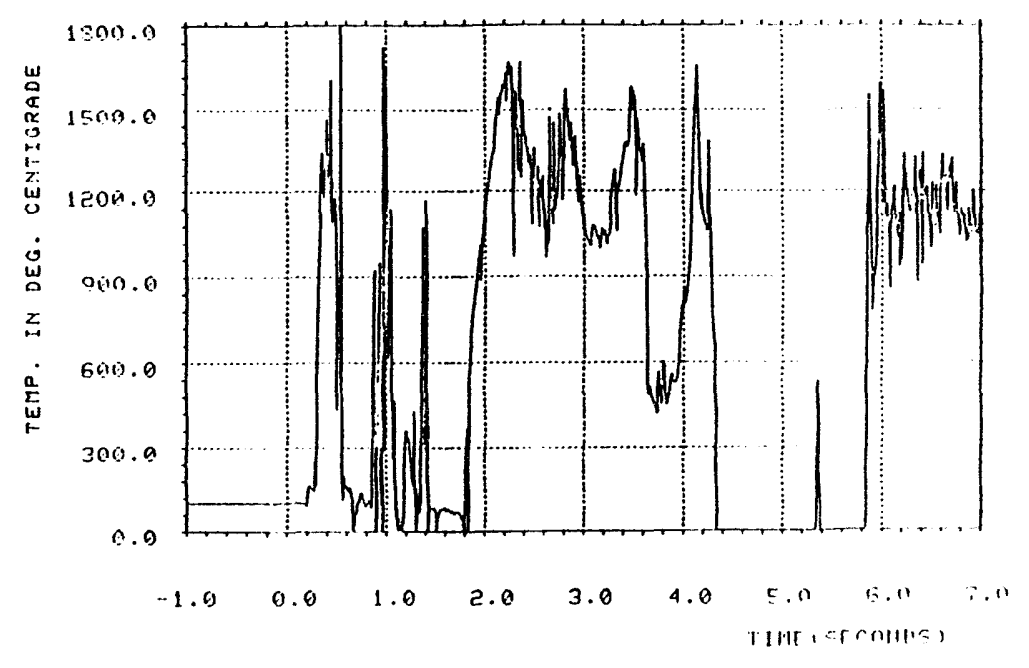

TIO.

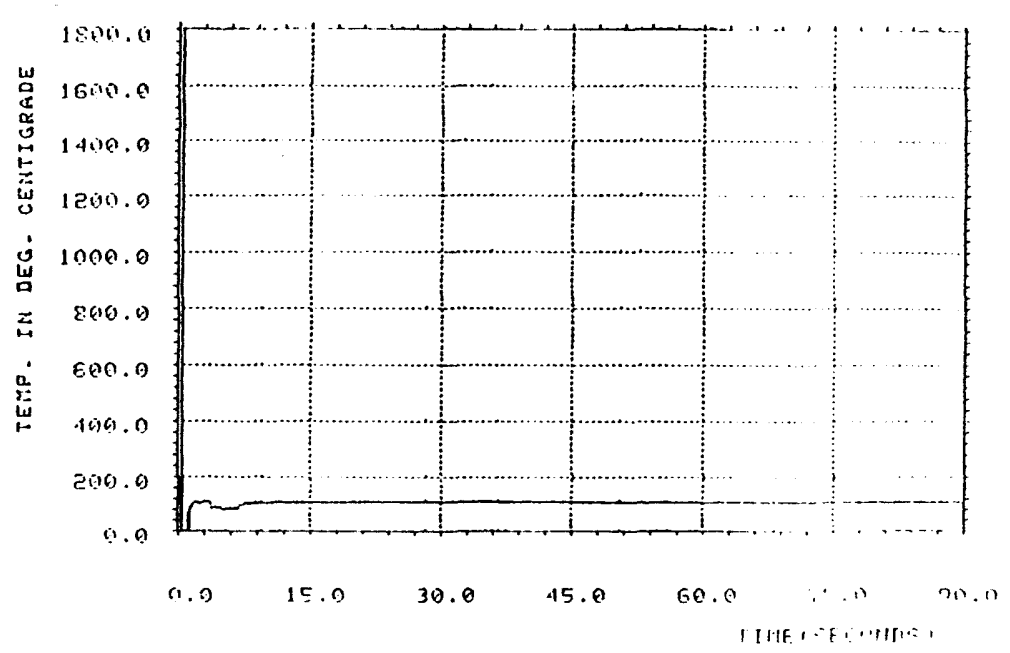

THE-EO, COH-6, [IATE 9. $30, \%$ 

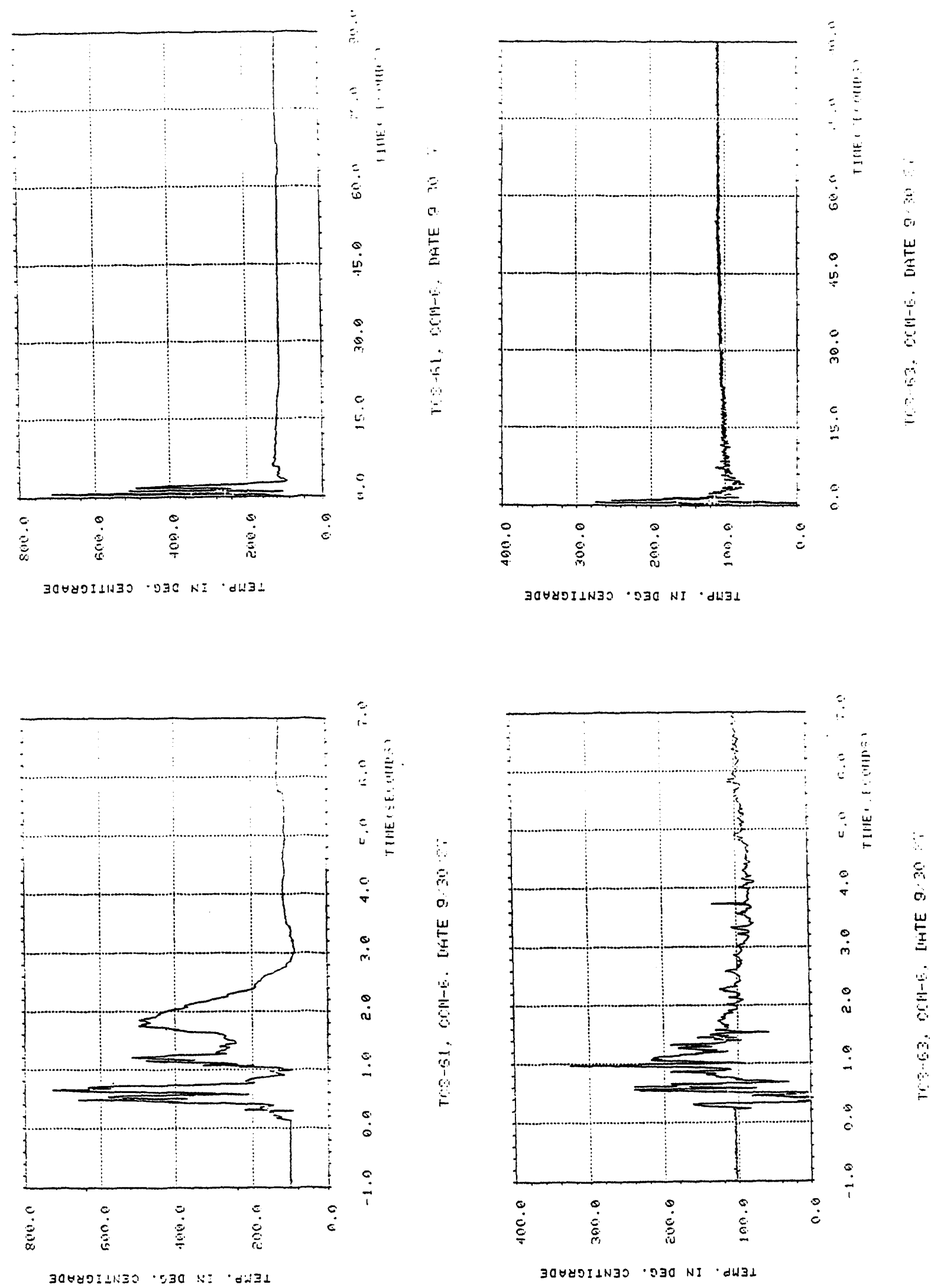

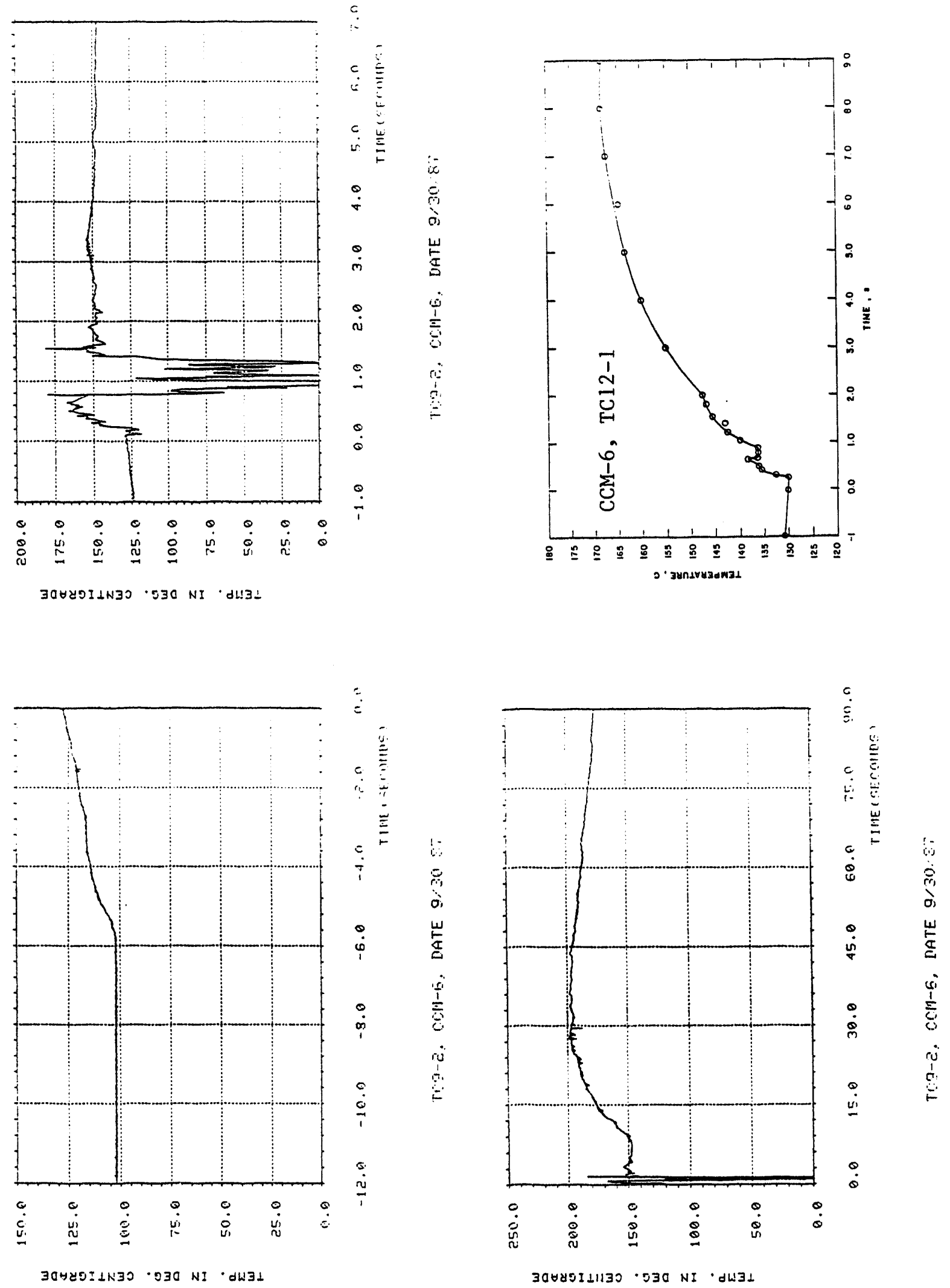


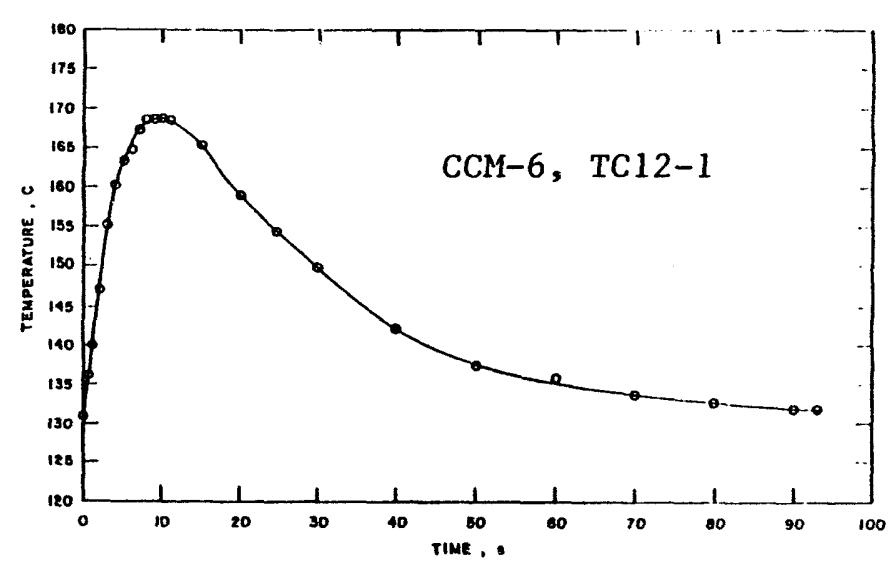

$\stackrel{i}{\sim}$

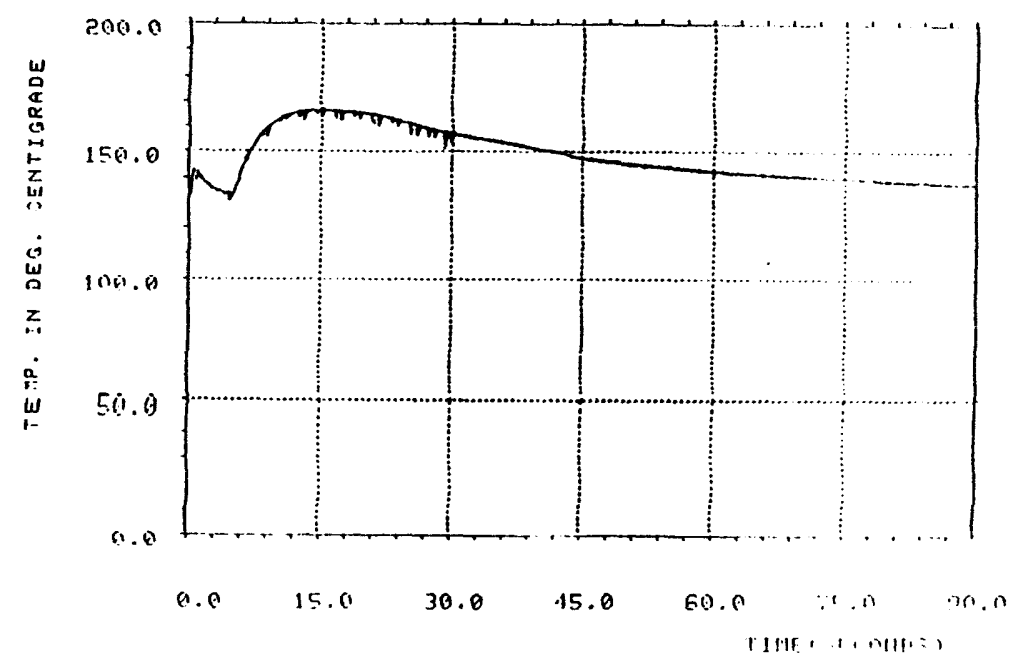

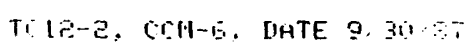

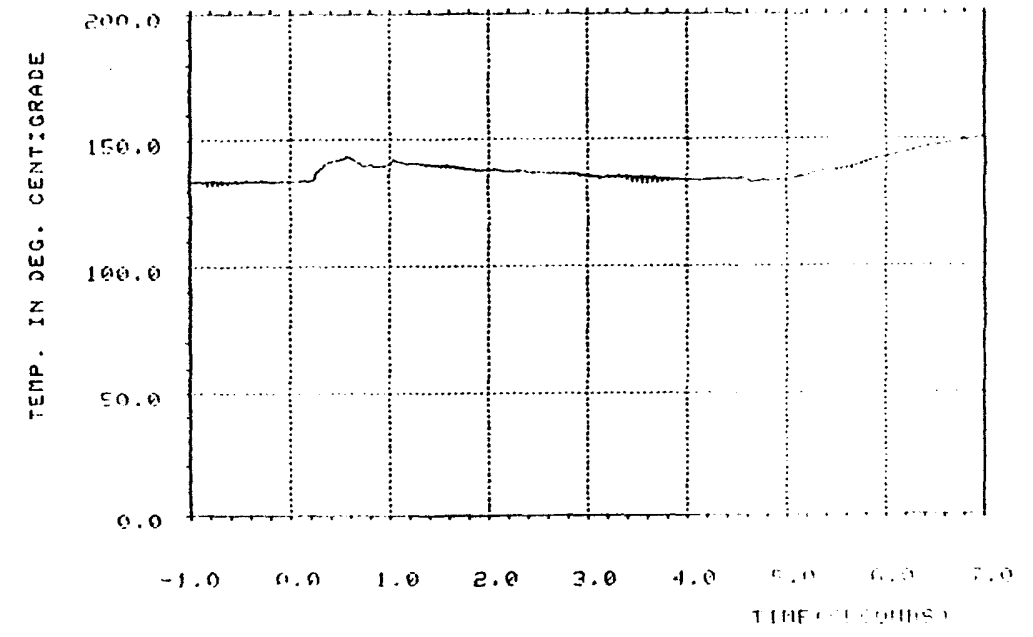

T!!

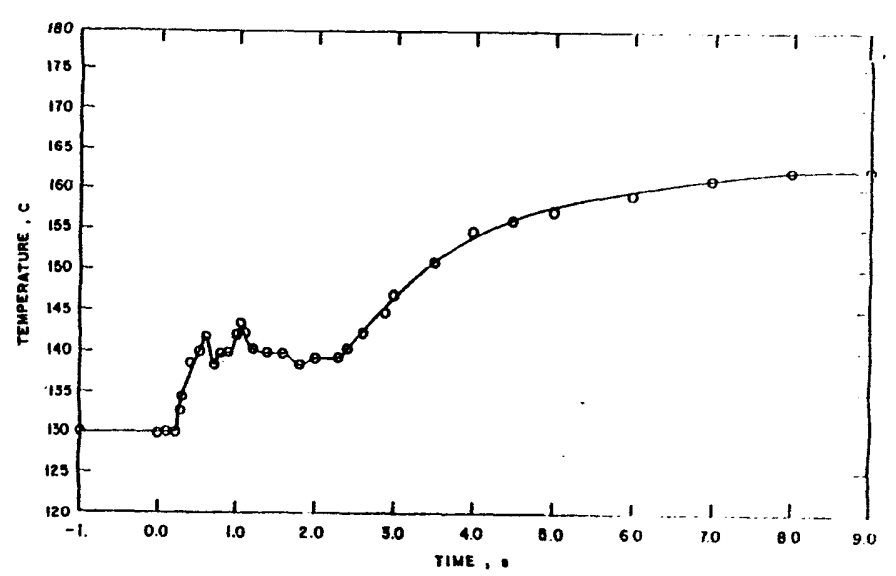




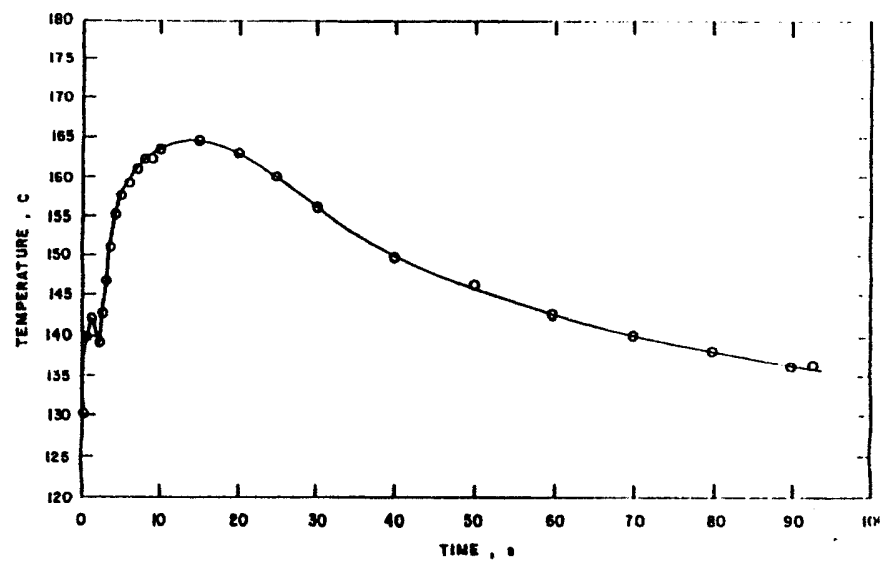

$\stackrel{i}{\text { N }}$

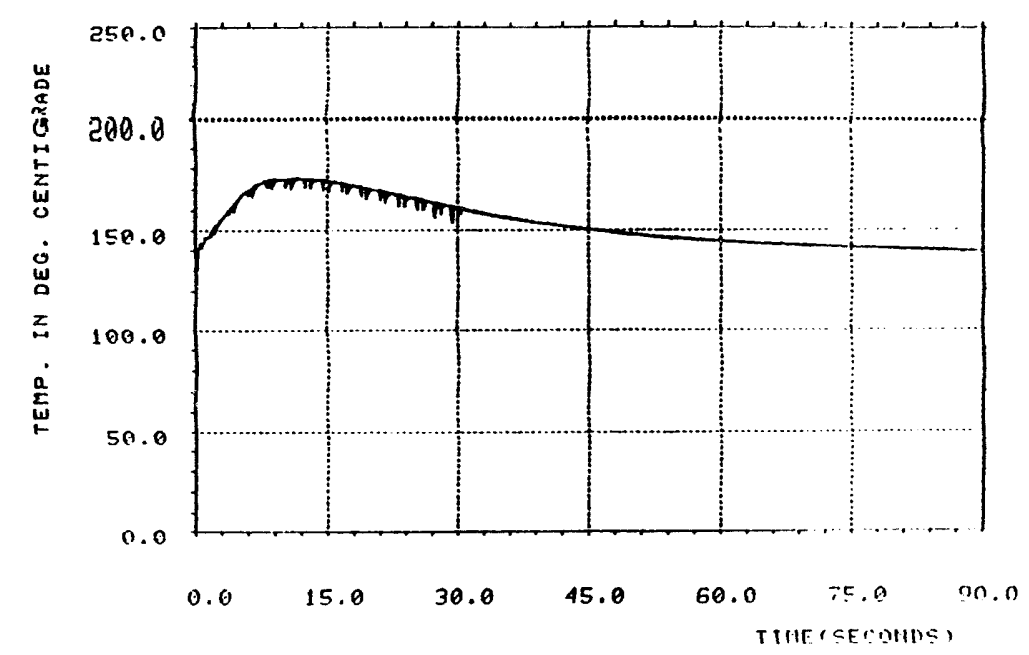

NIEN1. CNA-E. DATE $9.30 \%$

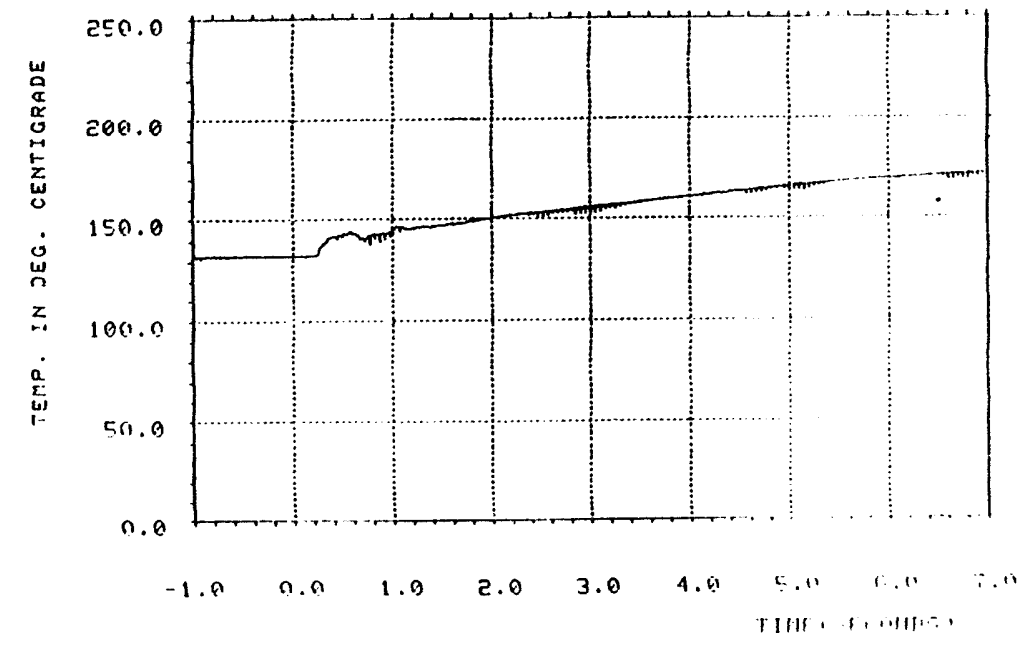

THL-4. OCN-6. [ATE 930

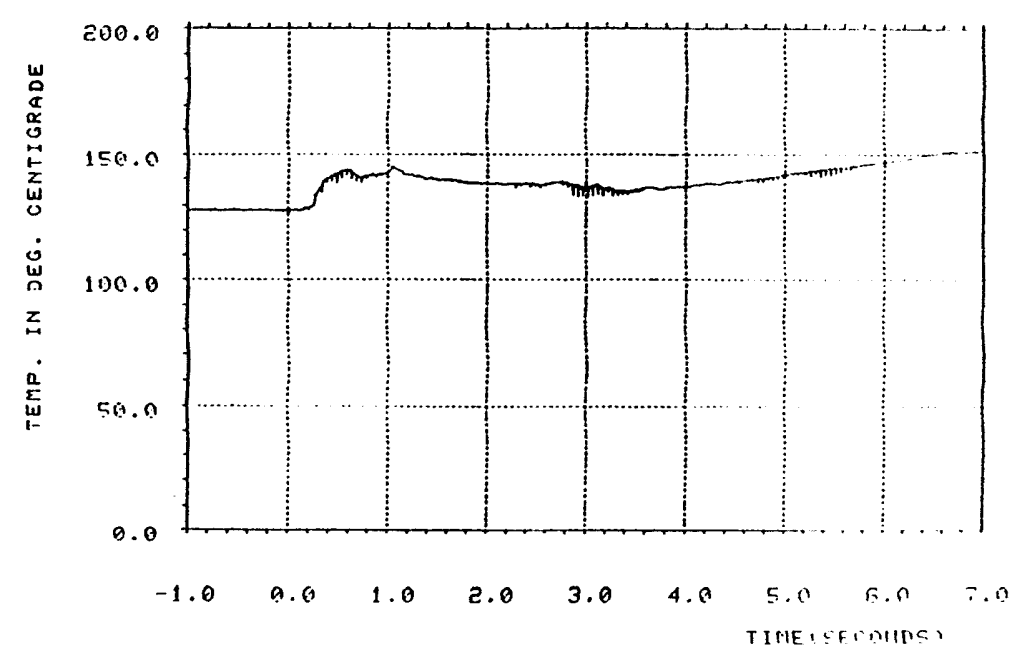

T. le-5, CON-6. LATE $0,30 \mathrm{~B}$ 

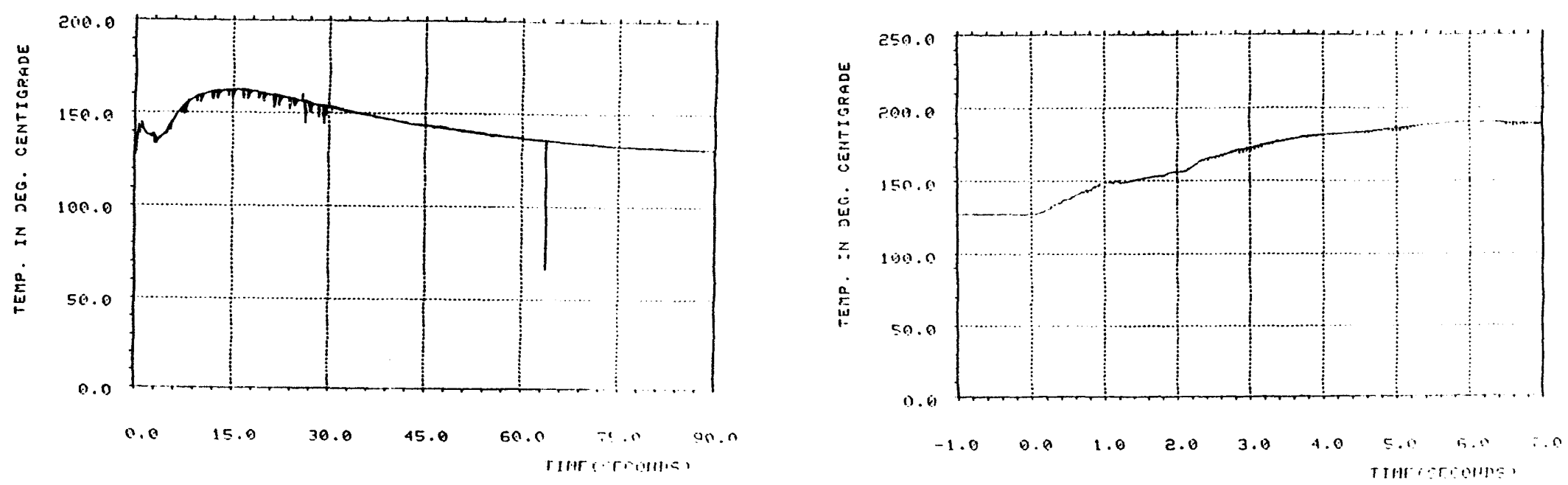

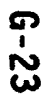

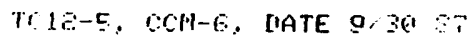

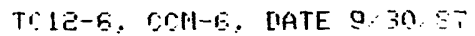

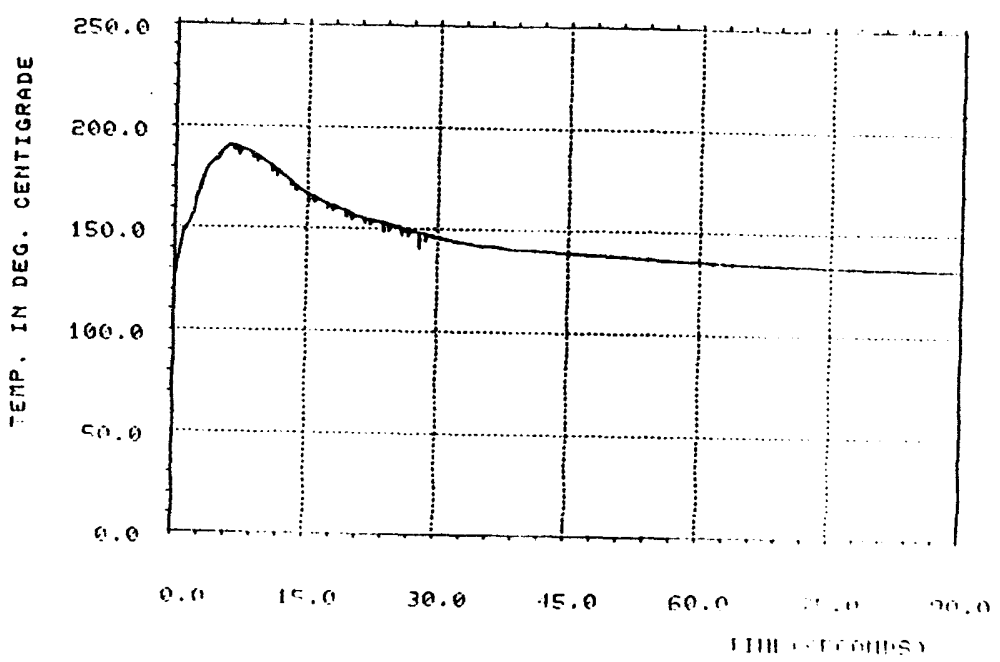

TCLE-5. COM-5. LATE g. 30 
Internal

D. R. Armstrong

TIS Files

L. Baker, Jr.

S. K. Bhattaharyya

C. A. Blomquist (2)

J. E. Cahalan

D. H. Cho

C. C. Chu (2)

L. W. Deitrich

M. T. Farmer

A. E. Klickman

L. M. McUmber

D. R. Pedersen

J. J. Sienicki (2)

B. W. Spencer (5)

J. B. Van Erp

D. C. Wade

\section{External}

ANL Libraries
ANL-E (2)
ANL-W
EDL (10)

B. G. Jones, University of Illinois, Urbana, IL

U.S Nuclear Regulatory Commission, Rockville, MD
R. Barrett
S. Basu
E. S. Beckjord
E. J. Brown
Y-S. Chen
A. Drozd
F. Eltawila
J. H. Flack
R. B. Foulds
C. G. Ginrich
M. D. Houston
T. L. King
R. V. Lee
A. Malliakos
W. Minners
R. L. Palla, Jr.
A. M. Rubin
B. W. Sheron
T. P. Speis
A. C. Thadani
C. E. Tinkler, Jr.
R. W. Walker
T. J. Walker 
U.S. Nuclear Regulatory Commission, Div. of Tehnical Information and Document Control, Bethesda, MD

C. Alexander, Battelle Columbus Laboratory, Columbus, $\mathrm{OH}$

T. Pratt, Brookhaven National Laboratory, Upton, NY

M. Merilo, Electric Power Research Institute, Palo Alto, CA

R. J. Hammersley, Fauske and Associates, Inc., Burr Ridge, IL

Oak Ridge National Laboratory, Oak Ridge, TN
S. Hodge
C. Pugh
L. Oh

F. Panisko, Pacific Northwest Laboratory, Richland, WA

Sandia National Laboratory, Albuquerque, NM

F. David

S. Dingman

R. 0 . Guntt

M. M. Pilch

K. 0. Reil

R. C. Schmidt

1. Catton, University of California, Los Angeles, CA

W. H. Rettig, U.S. Department of Energy, Idaho Falls, ID

Idaho National Engineering Lab, Idaho Falls, ID

C. M. Allison

S. A. Chavez

EG\&G Idaho, Inc., Idaho Falls, ID

E. A. Harvego

C. H. Heath

G. E. Korth

J. L. Rempe

S. P. Snow

L. A. Stickler

G. L. Thinnes

University of Wisconsin-Madison

M. L. Corradini

R. J. Witt

$A B B / C E$, Windson, $C T$

C. Hoffman

R. Schneider

D. Buttermer, PLG, Inc., Encunitas, CA

J. Reed, PLG, Inc., Encunitas, CA

James Metcalf, Stone and Webster, Boston, MA

John Conine, G. E. Knolls Atomic Power Lab, Schenectady, NY

J. W. Wolfe, Westinghouse Bettis Atomic Lab, West Miffl in, PA

S. Inamati, General Atomics, San Diego, C,A

M. Kazimi, Massachusetts Institute of Technology Nuclear Engineering Dept., Cambridge, MA 02139

N. Todreas, Massachusetts Institute of Technology, Cambridge, MA

I. V. Kurchatov Institute of Atomic Energy, Moscow, Russia

V. Asmolov

V. Strishov

M. Li Volant, Institu de Protection et de Surete Nucleair, Cedex, France

G. Cenerino, CEA/ISPN, Cedex, France

Scherrer Institut, Villigan, Switzerland

H. Hirschmann, Paul 
P. Hosemann

W. Kroeger

Thermodynamics and Radiation Physics, Ispra, Italy

P. Fasoli-Stubba

H. Hohmaun

P. Faisnys, VATESI, Lithuania

J. Misak, Nuclear Regulatory Authority, Bratislave, Slovak Republic

H. Bairiot, Belognucleaire, Brussels, Belgium

L. A. Simpson, Whiteshe 11 Laboratories, Pinawa, Manitoba, Canada

Kernforschungszentrum Karlsruhe, Karlsruhe, Germany
S. Hagan
P. Hofmann

J. Sugimoto, Japan Atomic Energy Research Institute, Ibaraki-Ken, Japan

Hee-Dong Kim, Korea Advanced Energy Research Institute, Daeduk-Danji, Korea

Kersji Takumi, Nuclear Power Engineering Center, Japan, Tokyo

S. I. Chang, Institute of Nuclear Energy Research, Lungtan, Taiwan, Republic of China

J. Bagues, Consejo de Seguridad Nuckan, Madrid, Spain

A. Alonso, E. T. S. Ingenieros Industriales, Madrid, Spain

W. Frid, Staten Karnkraftinspektion, Stockholm, Sweden

K. J. Brinkman, Reactor Centrum Nederland, The Netherlands

B. D. Turland, UKAEA, Oxfordshire, England, United Kingdom

Winfrith, Dorset, England, United Kingdom

S. Kinnersly

T. Haste

J. A. Marginez, Consejo Dorado II, Madrid, Spain 


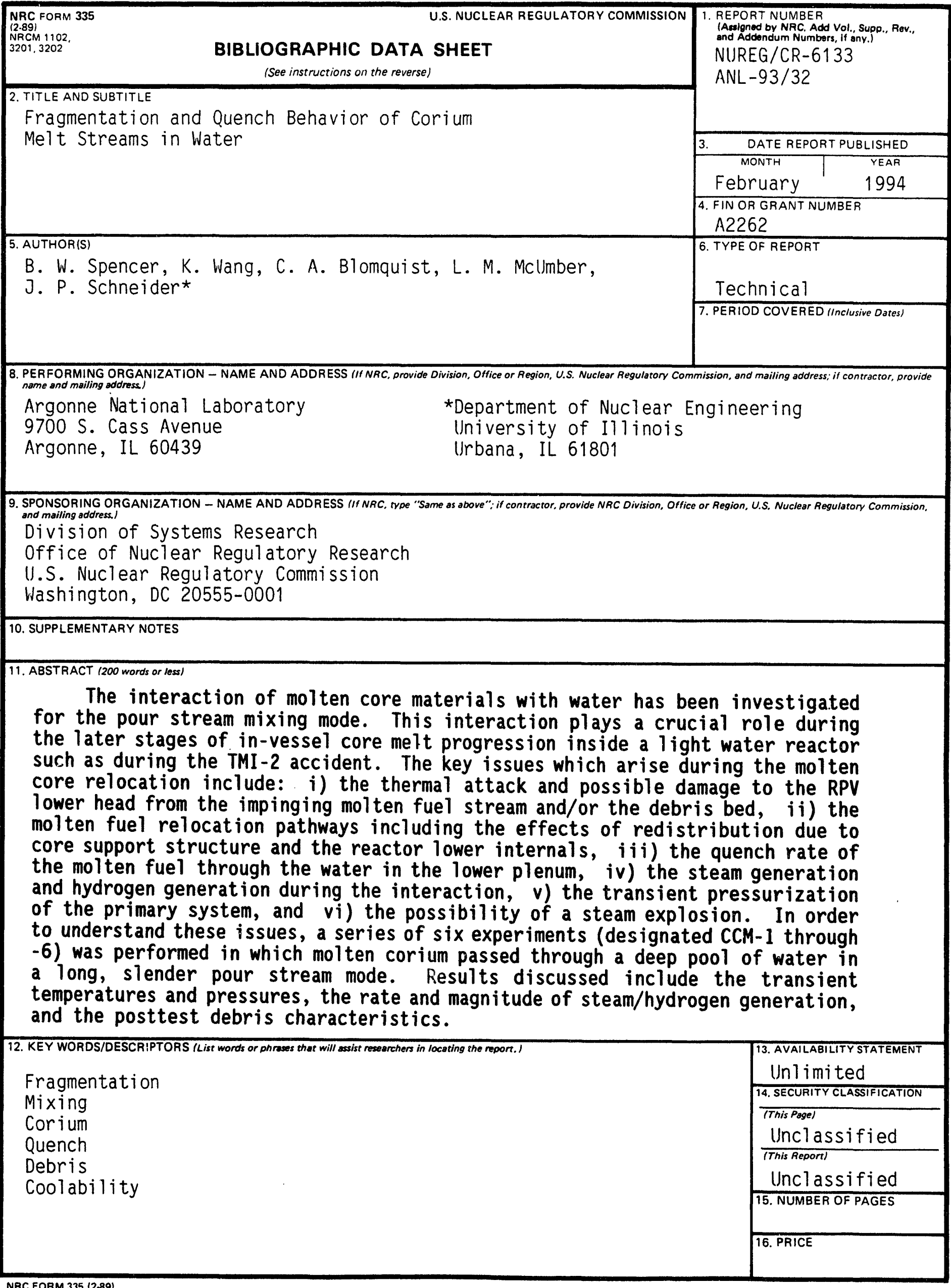



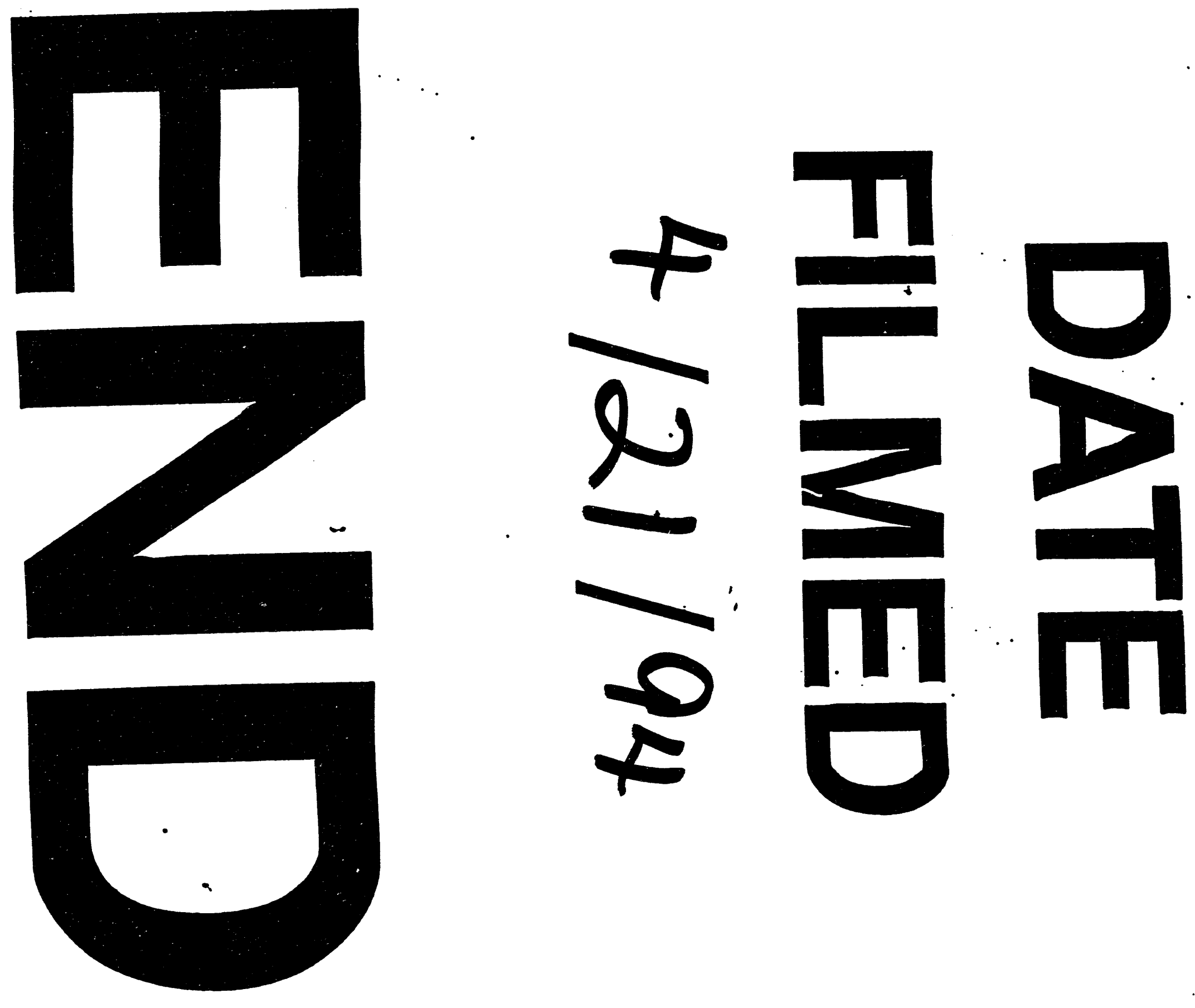
